PhD Dissertation

\title{
Analysis and design of efficient passive components for the millimeter-wave and $\mathrm{THz}$ bands
}

Universidad Politécnica de Valencia

Departamento de Comunicaciones

\author{
Author \\ Antonio José Berenguer Verdú
}

Advisor

Prof. Dr. Mariano Baquero Escudero

Valencia, May 2017 

To my parents, brother and sister. 

"Success is peace of mind which is a direct result of self-satisfaction in knowing you made the effort to become the best of which you are capable."

Jonh Wooden

"Lo que con mucho trabajo se adquiere, más se ama."

Aristóteles 



\section{Agradecimientos}

La realización de esta tesis ha implicado no pocos años de trabajo y esfuerzo. Durante este importante periodo de mi vida han sido muchas las personas han estado a mi lado, brindándome su apoyo, ayuda, cariño y amor. Ya sea de una forma u otra, todas estas personas han contribuido a que este trabajo haya sido posible dando como fruto esta tesis. A todos ellos, me gustaría dedicarles un agradecimiento muy especial de todo corazón.

Sin duda alguna, debo dar las gracias en primer lugar a mi director de tesis Mariano Baquero. Mariano, has sido un padre para mí profesionalmente hablando. Te conocí como alumno en las clases de Microondas, en las cuales me abriste la puerta a un conocimiento que me fascinó. Recuerdo perfectamente cuando fui a verte para preguntarte si podrías dirigirme el Proyecto Final de Carrera, y desde ese año 2009 hemos estado trabajando juntos. Quiero agradecerte no sólo haberme dado la oportunidad de trabajar en cada momento en el área que más me ha gustado, sino el haberte preocupado por mí a nivel personal, velando siempre por mis intereses, ayudándome a desarrollarme personal y profesionalmente. Extiendo en este sentido mi agradecimiento a Miguel Ferrando. Si Mariano es el padre, tu serías sin duda el abuelo. Sin tu ayuda, la beca para la realización de esta tesis y los proyectos que han respaldado la misma no hubieran sido posibles. Tu esfuerzo ha permitido que el Grupo de Radiación Electromagnética (GRE) esté hoy dónde esta y que sus miembros nos beneficiemos de un entorno de trabajo excelente, produciendo trabajo de calidad. Debo agradecerte también tus siempre innovadoras ideas, algunas de las cuales se han convertido en una realidad plasmada en esta tesis.

Una mención especial merece Daniel Sánchez. Dani, aunque tu nombre no figure como co-director bien sabes que podría estarlo. Jamás podré agradecerte lo suficiente la ayuda que me has prestado. Cuántas horas dedicadas a enseñarme en un sin fin de aspectos sin esperar nada a cambio, apoyándome también en los momentos difíciles, siempre presentes en una tesis. Bien sabes que lo mismo has hecho con cada uno tus compañeros, por eso eres el pilar del GRE Dani.

Otra persona a la que estoy muy agradecido es a Vicente Boria. Tu activa colaboración en la dirección de esta tesis ha sido indispensable para la consecución de los resultados más relevantes de la misma. Sugeriste muy acertadamente una estancia en la Queen's University of Belfast, y me abriste la puerta a nuevas líneas de investigación en las cuales seguimos trabajando actualmente. Gracias por preocuparte por mi a nivel profesional y personal.

En el ámbito anterior, extiendo los agradecimientos a todo el GRE. Cada uno de vosotros me ha acompañado y aportado algo en este viaje. De manera especial, Tommy, sabes que eres como un hermano mayor para mí 
a nivel personal. Sólo tú y yo sabemos cuántos momentos buenos y malos hemos pasado juntos, cuántas historias y aventuras, iy las que nos quedan por vivir! Gracias por estar siempre ahí. A Felipe, gracias por hacerme ver las matemáticas de una manera todavía más especial. Gracias a ti me embarqué a estudiarlas aparte, una de las mejores decisiones que he tomado. José Ignacio y Alejandro, gracias por esos consejos, enseñanzas y discusiones tan fructíferas. Toni y Bernat, gracias por estar siempre ahí resolviendo cualquier problema imaginable y fabricando a la perfección hasta el más difícil de los prototipos. Sois unos cracks. Nora, mi vecina, siempre sonriente aunque a veces te invada la mesa :p. Gracias por hacer los días en la oficina tan amenos. Miguel Jr., compañero de viaje, cuántas conversaciones interesantes, risas y anécdotas. Ojalá nuestros doctorados se hubieran solapado más. And, Marko K., I don't forget you my also friend and flatmate. Thank you for your advices and such a good moments we expended.

También quiero dar las gracias a la toda la gente del iTEAM. De forma particular, Alfonso, tu apoyo, especialmente en la fase de escritura de la tesis, es algo que siempre te agradeceré. Has demostrado ser un amigo de verdad. También, Fernando Domene, amigo desde los inicios de la carrera, qué bueno ha sido contar contigo cerca siempre. Por último, Stefano, JJ, Chernan y José Capmany, gracias por vuestros consejos y apoyo. No puedo olvidar a otra gente en la UPV como Joaquín Izquierdo, Julio Benítez y Néstor Thome que me apoyaron en mis tareas docentes.

It is also very important for me to acknowledge Prof. Vince Fusco. Thank you for hosting me in your group in Belfast and guiding me thoughtfully towards the achievement of results that have become fundamental in this thesis. I appreciate very much your advice. I would like to extend my acknowledgements to all ECIT. Specially, Dr. Dmitry Zelenchuk, thank you for your unconditional help and brilliant ideas, which were essential in the success of my research. Also, Dr. Oleksandr Malyuskin and Dr Alexey Shitov, thanks for giving me the opportunity of participating actively in the seminars.

En el terreno personal, sin duda debo agradecer en primer lugar a mi familia, especialmente a mis padres. Habéis sido el apoyo fundamental sin el cual ni esta tesis ni nada de lo anterior hubiera sido posible, siempre velando por mí y ayudándome a levantarme cuando me he caído. Cuánto habéis dado por mi...sois únicos, me faltan palabras para expresaros toda mi gratitud. Gracias también Carolina, mi hermana, siempre dispuesta ayudarme. Y como no, el pequeño Ricardo, compañero de mil aventuras y que siempre me saca una sonrisa. No puedo olvidarme tampoco de mi sobrino Hugo, primos, tíos y abuelos, una familia muy unida cuyo cariño es mi fuerza para mejorar cada día. Me gustaría dedicarle de forma especial esta tesis a aquellos que no están y a quiénes les hubiera hecho mucha ilusión verme completar esta etapa.

Un agradecimiento muy especial va también a mi novia Mar. Te conocí justo cuando menos lo esperaba...a mitad de escribir esta tesis, y te has 
convertido en una persona esencial en mi vida. No puedes imaginar cuán importante ha sido tu apoyo en esta fase de escritura, especialmente dura. Has sabido aguantar todo lo que conlleva, sacrificándote, comprendiéndome como nadie y soportándome en aquellos momentos de estrés en los que no me aguantaba ni yo.

Como no, mis amigos de siempre de Onil, Els Okupes. Gracias por todos esos momentos únicos e irrepetibles que hemos vivido y que para mí han sido la vía de escape a los inevitables momentos de estrés que me han acontecido. Edi, Rafes, Maño, Ximo, Pepet, Cori (gràcies per la portada crack!), Héctor, Davidiu, Andrés, Jairo, Maki, Dani. Sois los mejores, únicos cada uno. Gracias también a ti Picó, prim i amic, por estar siempre ahí.

En Valencia, una persona cuyo apoyo merece mención especial es Jorge Llopis. George, eres como un hermano para mí. Hemos vivido buenos momentos juntos, pero lo que de verdad te agradezco es que hayas estado siempre en los peores. Gracias por ello, y por tu alegría y vivacidad, poniéndole humor al peor de los días. Muchas gracias también Orlando Pereira, has demostrado ser un verdadero amigo durante esta etapa, contando conmigo para todo y haciendo de estos años una época para recordar. Y Vicent Savall, amigo de los de verdad, para ti también va un agradecimiento especial.

Gracias también a mis compañeros matemáticos del IUMPA, a la gente del Gym UPV, a Los Mercenarios, a Iván Tete, Carles Montoliu, Dani G. Algarra, Gonzalo Logroño, Dani Izquiero Co., Llanos, Andrea, Pepe Arsenal y familia, José María Illán, Emilio Pérez, Marco Millo, Javi Ferrero, JuanRa, Laura, Carmen, Rebeca, Juan Serrano, Javi primo, Capitán Rava y amigos, a mis compañeros de piso estos años (Juanmi, Ronald, Alba, Rafa, Orie, y como no, vecino Pablo), a mi gente de Belfast (Jon, Joseba, Jorge Macias, Óscar Murcia, Dani, Consuelo,...), y tantos más que por seguro me dejo.

Y puesto que mi actividad en la moda ha ido paralela a esta tesis, no puedo dejar de agradecer todo lo que han hecho por mí compañeros como Javi Mallol, Gonzalo Cabo, Alim, Dailos; fotógrafos como Rafa Catalá, Pas Ibañez, Raquel Peñaranda; diseñadores como Francis Montesinos, Eduardo Cozera, Viktor Ferrando, Fran Uría; y cómo no a todo el equipo de UNO Models. Todos habéis demostrado una amistad más allá de lo profesional, siendo un apoyo fundamental en esta etapa.

Finalmente, dado que esta tesis ha sido escrita en las ciudades de Estambul, Kuwait, Paris, Valencia, Madrid y La Pobla de Segur (Lleida), quiero agradecer a toda la gente que me ha acompañado en esta fase de la tesis con tanta dedicación.

A todos, de verdad, gracias. 



\section{Abstract}

This thesis tackles issues of particular interest regarding analysis and design of passive components at the mm-wave and Terahertz $(\mathrm{THz})$ bands. Innovative analysis techniques and modeling of complex structures, design procedures, and practical implementation of advanced passive devices are presented.

The first part of the thesis is dedicated to $\mathrm{THz}$ passive components. These days, $\mathrm{THz}$ technology suffers from the lack of suitable waveguiding structures since both, metals and dielectric, are lossy at $\mathrm{THz}$ frequencies. This implies that neither conventional closed metallic structures used at microwave frequencies, nor dielectric waveguides used in the optical regime, are adequate solutions. Among a variety of new proposals, the Single Wire Waveguide (SWW) stands out due to its low attenuation and dispersion. However, this surface waveguide presents difficult excitation and strong radiation on bends. A Dielectric-Coated Single Wire Waveguide (DCSWW) can be used to alleviate these problems, but advantages of the SWW are lost and new problems arise.

Until now, literature has not given proper solution to radiation on bends and, on the other hand, rigorous characterization of these waveguides lacks these days. This thesis provides, for the first time, a complete modal analysis of both waveguides, appropriated for $\mathrm{THz}$ frequencies. This analysis is later applied to solve the problem of radiation on bends. Several structures and design procedures to alleviate radiation losses are presented and experimentally validated.

The second part of the thesis is dedicated to mm-wave passive components. These days, when implementing passive components to operate at such small, millimetric wavelengths, to ensure proper metallic contact and alignment between parts results challenging. In addition, dielectric absorption becomes significant at mmwave frequencies. Consequently, conventional hollow metallic waveguides and planar transmission lines present high attenuation so that new topologies are being considered. Gap Waveguides (GWs), based on a periodic structure introducing an Electromagnetic Bandgap effect, result very suitable since they do not require metallic contacts and avoid dielectric losses.

However, although GWs have great potential, several issues prevent GW technology from becoming consolidated and universally used. On the one hand, the topological complexity of GWs difficulties the design process since full-wave simulations are time-costly and there is a lack of appropriate analysis methods and suitable synthesis procedures. On the other hand, benefits of using GWs instead of conventional structures are required to be more clearly evidenced with high-performance GW components and proper comparatives with conventional structures. This thesis introduces several efficient analysis methods, models, and synthesis techniques that will allow engineers without significant background in GWs to straightforwardly implement GW devices. In addition, several highperformance narrow-band filters operating at Ka-band and V-band, as well as a rigorous comparative with rectangular waveguide topology, are presented. 


\section{Resumen}

Esta tesis aborda problemas actuales en el análisis y diseño de componentes pasivos en las bandas de onda milimétrica y Terahercios $(\mathrm{THz})$. Se presentan nuevas técnicas de análisis y modelado de estructuras complejas, procedimientos de diseño, e implementacion práctica de dispositivos pasivos avanzados.

La primera parte de la tesis se dedica a componentes pasivos de THz. Actualmente no se disponen de guías de onda adecuadas a $\mathrm{THz}$ debido a que ambos, metales y dieléctricos, introducen grandes pérdidas. En consecuencia, no es adecuado escalar las estructuras metálicas cerradas usadas en microondas, ni las guías dieléctricas usadas a frecuencias ópticas. Entre un gran número de recientes propuestas, la Single Wire Waveguide (SWW) destaca por su baja atenuación y casi nula dispersión. No obstante, como guía superficial, la SWW presenta difícil excitación y radiación en curvas. El uso de un recubrimiento dieléctrico, creando la Dielecric-Coated Single Wire Waveguide (DCSWW), alivia estos inconvenientes, pero las ventajas anteriores se pierden y nuevos problemas aparecen.

Hasta la fecha, no se han encontrado soluciones adecuadas para la radiación en curvas de la SWW. Además, se echa en falta una caracterización rigurosa de ambas guías. Esta tesis presenta, por primera vez, un análisis modal completo de SWW y DCSWW, adecuado a la banda de THz. Este análisis es aplicado posteriormente para evitar el problema de la radiación en curvas. Se presentan y validan experimentalmente diversas estructuras y procedimientos de diseño.

La segunda parte de la tesis abarca componentes pasivos de ondas milimétricas. Actualmente, estos componentes sufren una importante degradación de su respuesta debido a que resulta difícil asegurar contacto metálico y alineamiento adecuados para la operación a longitudes de onda tan pequeñas. Además, la absorción dieléctrica incrementa notablemente a estas frecuencias. En consecuencia, tanto guías metálicas huecas como líneas de transmisión planares convencionales presentan gran atenuación, siendo necesario considerar topologías alternativas. Las Gap Waveguides (GWs), basadas en una estructura periódica que introduce un efecto de Electromagnetic Bandgap, resultan muy adecuadas puesto que no requieren contacto entre partes metálicas y evitan las pérdidas en dieléctricos.

No obstante, a pesar del potencial de las GWs, varias barreras impiden la consolidación y uso universal de esta tecnología. Por una parte, la compleja topología de las GWs dificulta el proceso de diseño dado que las simulaciones de onda completa consumen mucho tiempo y no existen actualmente métodos de análisis y diseño apropiados. Por otra parte, es necesario evidenciar el beneficio de usar GWs mediante dispositivos GW de altas prestaciones y comparativas adecuadas con estructuras convencionales. Esta tesis presenta diversos métodos de análisis eficientes, modelos, y técnicas de diseño que permitirán la síntesis de dispositivos GW sin necesidad de un conocimiento profundo de esta tecnología. Asimismo, se presentan varios filtros de banda estrecha operando en las bandas Ka y V con altas prestaciones, así como una comparativa rigurosa con la guía rectangular. 


\section{Resum}

Aquesta tesi aborda problemes actuals en relació a l'anàlisi i disseny de components passius en les bandes d'ona mil-limètrica i Terahercis. Es presenten noves tècniques d'anàlisi i modelatge d'estructures complexes, procediments de disseny, i implementació pràctica de dispositius passius avançats.

La primera part de la tesi es focalitza en components passius de THz. Actualment no es disposen de guies d'ona adequades a $\mathrm{THz}$ causa que tots dos, metalls i dielèctrics, introdueixen grans pèrdues. En conseqüència, no és adequat escalar les estructures metàl-liques tancades usades en microones, ni les guies dielèctriques usades a freqüències òptiques. Entre un gran nombre de propostes recents, la Single Wire Waveguide (SWW) destaca per la seua baixa atenuació i quasi nul-la dispersió. No obstant això, com a guia superficial, la SWW presenta difícil excitació i radiació en corbes. L'ús d'un recobriment dielèctric, creant la Dielecric-Coated Single Wire Waveguide (DCSWW), alleuja aquests inconvenients, però els avantatges anteriors es perden i nous problemes apareixen.

Fins a la data, no s'han trobat solucions adequades per a la radiació en corbes de la SWW. A més, es troba a faltar una caracterització rigorosa d'ambdues guies. Aquesta tesi presenta, per primera vegada, un anàlisi modal complet de SWW i DCSWW, adequat a la banda de THz. Aquest anàlisi és aplicat posteriorment per evitar el problema de la radiació en corbes. Es presenten i validen experimentalment diverses estructures i procediments de disseny.

La segona part de la tesi es centra en components passius d'ones mil-limètriques. Actualment, aquests components pateixen una important degradació de la seua resposta a causa de que resulta difícil assegurar contacte metàl·lic i alineament adequats per a l'operació a longituds d'ona tan menudes. A més, l'absorció dielèctrica incrementa notablement a aquestes freqüències. En conseqüència, tant guies metàl-liques buides com línies de transmissió planars convencionals presenten gran atenuació, sent necessari considerar topologies alternatives. Les Gap Waveguides (GWs), basades en una estructura periòdica que introdueix un efecte de Electromagnetic Bandgap, resulten molt adequades ja que no requereixen contacte entre parts metàl-liques i eviten les pèrdues en dielèctrics.

No obstant, tot i el potencial de les GWs, diverses barreres impedixen la consolidació i ús universal d'aquesta tecnologia. D'una banda, la complexa topologia de les GWs dificulta el procés de disseny atés que les simulacions d'ona completa consumeixen molt de temps i no existeixen actualment mètodes d'anàlisi i disseny apropiats. D'altra banda, és necessari evidenciar el benefici d'utilitzar GWs mitjançant dispositius GW d'altes prestacions i comparatives adequades amb estructures convencionals. Aquesta tesi presenta diversos mètodes d'anàlisi eficients, models, i tècniques de disseny que permetran la síntesi de dispositius GW sense necessitat d'un coneixement profund d'aquesta tecnologia. Així mateix, es presenten diversos filtres de banda estreta operant en les bandes $\mathrm{Ka}$ i $\mathrm{V}$ amb altes prestacions, així com una comparativa rigorosa amb la guia rectangular. 


\section{Acronyms}

4G Fourth Generation (Mobile Communications).

AB Air Bridges.

ABC Asymptotic Boundary Conditions.

ACC Adaptive Cruise Control.

ADS ${ }^{\circledR}$ Advanced Design System ${ }^{\circledR}$.

AE Antenna Emitter.

Ag Silver.

Al Aluminum.

AlGaAs Aluminum Gallium Arsenide.

ARROW Antiresonant Reflecting Optical Waveguide.

ASM Aggressive Space Mapping.

Au Gold.

AWR MO ${ }^{\circledR}$ Applied Wave Research Microwave Office ${ }^{\circledR}$.

BC Backside Conductor.

BCB Benzocylobutene.

BE Beryllium.

BI-RME Boundary Integral-Resonant Mode Expansion.

BiCMOS Bipolar Complementary Metal-Oxide-Semiconductor.

BJT Bipolar Junction Transistor.

BP Bandpass.

BRIC Brazil, Russia, India and China.

BWO Backward Wave Oscillators. 
CAD Computer Aided Design.

CalTech California Institute of Technology.

CKD Clough-Kneizys-Davies.

CMOS Complementary Metal-Oxide-Semiconductor.

CNC Computer Numerically Controlled.

CNT Carbon Nanotubes.

Co Cobalt.

$\mathrm{CO}_{2}$ Carbon Dioxide.

COC Cyclic-Olefin Copolymer.

CPU Central Processing Unit.

CPW Coplanar Waveguide.

CSIP Charge Sensitive Infrared Phototransistor.

CST $^{\circledR}$ Computer Simulation Technology ${ }^{\circledR}$.

CT Computed Tomography.

CTA Cross Traffic Alert.

$\mathrm{Cu}$ Copper.

CUT Chalmers University of Technology.

CW Continuous Wave.

CWG Circular Waveguide.

D2D device-to-device.

dB Decibel.

DC Direct Current.

DCSWW Dielectric-Coated Single Wire Waveguide.

DFB Distributed Feed-Back.

DFG Difference-Frequency Generation.

DGS Defected Ground Structure.

DNA Deoxyribonucleic Acid.

DQD Double Quantum Dot.

DRIE Deep Reactive Ion Etching. 
DSRC Dedicated Short-Range Communication.

DVD Digital Versatile Disc.

e.g. example gratia.

EBG Electromagnetic Bandgap.

ECE Electron Cyclotron Emission.

EDM Electrical Discharge Machining.

EGOS Equal Grade of Service.

EM Electromagnetic.

ENIG Electroless Nickel Immersion.

EO Electro-Optical.

ESIW Empty Substrate Integrated Waveguide.

FCC Federal Communications Comission.

FDTD Finite Differences Time Domain.

Fe Iron.

FEL Free Electron Laser.

FELIX Free Electron Laser for Infrared eXperiments.

FEM Finite Element Method.

FEST3D $^{\circledR}$ Full-wave Electromagnetic Simulation Tool.

FET Field Effect Transistor.

FIB Focused Ion Beam.

FIR Far Infrared.

FIRE First ISCCP Regional Experiment.

FPA Focal Plane Array.

FPU Formación del Profesorado Universitario.

FSO Free Space Optics.

FTIR Fourier Transform Infrared Spectroscopy.

FWHM Full Width at Half Maximum.

GA Genetic Algorithm.

Ga Gallium. 
GaAs Gallium Arsenide.

GaN Gallium Nitride.

GaP Gallium Phosphide.

GaSe Gallium Selenide.

Gbps Gigabit(s) per second.

GDP Gross Domestic Product.

Ge Germanium.

GEO Geostationary Orbit.

GGW Groove Gap Waveguide.

GGW-HP Groove Gap Waveguide Horizontal Polarization.

GGW-VP Groove Gap Waveguide Vertical Polarization.

GL Grating Lobe.

GL Goubau Line.

GRE Electromagnetic Radiation Group / Grupo de Radiación Electromagnética.

GW Gap Waveguide.

HBT Heterojunction Bipolar Transistor.

HD High-Definition.

HDMI High-Definition Multi-media Interface.

HDPE High-Density Polyethylene.

HDTV High-Definition Television.

HE Hybrid Mode type 1.

HEB Hot Electron Bolometer.

HEMT High Electron Mobility Transistor.

HFSS ${ }^{\circledR}$ High Frequency Structural Simulator ${ }^{\circledR}$.

HITRAN High-Resolution Transmission Molecular Absorption Database.

HMSIW Half-Mode Substrate Integrated Waveguide.

HP Horizontal Polarization.

i.e. $i d$ est.

IC Integrated Circuit. 
IEEE Institute of Electrical and Electronics Engineers.

IF Intermediate Frequency.

IL Insertion Loss.

IM Indomethacin.

IMPATT IMPact Ionization Avalanche Transit-Time.

InP Indium Phosphide.

IP Current-Power.

IR Infrared.

ISCCP International Satellite Cloud Climatology Project.

ISM Industrial, Scientific and Medical.

ISW Impenetrable Surface Waveguiding.

ITU International Telecommunication Union.

JCR Journal Citation Reports.

JPL NASA Jet Propulsion Laboratory.

KID Kinetic Inductor Detectors.

LAE Large Area Emitter.

LAN Local Area Network.

LCA Lane Change Assistant.

LCA Large Core Area.

LEO Low-Earth Orbit.

LIDAR LIght Detection and Ranging.

LIGA Lithographie-Galvanoformung-Abformung (Lithography-Electroplating-Molding).

$\mathrm{LiNbO}_{3}$ Lithium Niobate.

$\mathrm{LiTaO}_{3}$ Lithium Tantalate.

LMDS Local Multipoint Distribution Service.

LNA Low Noise Amplifier.

LO Local Oscillator.

LOS Line of Sight.

LP Linearly Polarized. 
LRR Long Range Radar.

LTCC Low Temperature Co-fired Ceramic.

LTG Low Temperature Grown.

LWA Leaky-Wave Antenna.

MASER Microwave Amplification by Stimulated Emission of Radiation.

MATLAB ${ }^{\circledR}$ MATrix LABoratory ${ }^{\circledR}$.

Mbps Migabit(s) per second.

MDCSWW Modified Dielectric-Coated Single Wire Waveguide.

MDMA Methylenedioxymethamphetamine.

MEMS Microelectromechanial Systems.

MGL Modified Goubau Line.

MgO Magnesium oxide.

MIMO Multiple Input Multiple Output.

MMB Millimeter-wave Broadband.

MMIC Monolithic Microwave Integrated Circuits.

Mo Molybdenum.

MoM Method of Moments.

MP3 MPEG-1 and/or MPEG-2 Audio Layer III.

MPEG Moving Picture Experts Group.

MRGW Microstrip Ridge Gap Waveguide.

MRR Medium Range Radar.

MRT Maximum Resolution Temperature.

MSG Metal Strip Grating.

NASA National Aeronautics and Space Administration.

NATO North Atlantic Treaty Organization.

Nb Plumbum (Lead).

NBF Narrow-Band Filters.

NbTiN Niobium Titanium Nitride.

NDR Negative Differential Resistance. 
NEP Noise Equivalent Power.

Ni Nickel.

NLOS Non Line of Sight.

NN Neural Networks.

Np Neper.

NSAID non-steroidal anti-inflammatory drug.

OFDM Orthogonal Frequency Division Multiplexing.

OP Orientation Patterned.

P2P Point-to-Point.

PA Photoconductive Antenna.

PAA Phased Array Antenna.

PBG Photonic Bandgap.

PC Personal Computer.

PC Photoconductive.

PC Polycarbonate.

PCB Printed Circuit Board.

Pd Palladium.

PDA Personal Digital Assistant.

PE Polyethylene.

PEC Perfect Electric Conductor.

PETN Pentaerythritol Tetranitrate.

PF Porous Fiber.

PGF Portable Graphic Format.

PhD Doctor of Philosophy.

PI Power-Current.

PIM Passive Intermodulation.

PMC Perfect Magnetic Conductor.

PMMA Polymethyl Methacrylate.

PMMWC Passive Millimeter Waver. 
PPW Parallel-Plate Waveguide.

PS Polystirene.

PSO Particle Swarm Optimization.

Pt Platinum.

PTFE Polytetrafluoroethylene.

PVC Polyvinyl Chloride.

PVDF Polyvinylidene Fluoride.

QCL Quantum Cascade Laser.

QD Quantum Dot.

QMP Quasi Matched Periods.

QW Quantum Well.

RAM Random-Access Memory.

RBW Relative Bandwidth.

RCS Radar Cross Section.

RDX Cyclotrimethylenetrinitramin.

RF Radio Frequency.

RGW Ridge Gap Waveguide.

RL Return Loss.

RoF Radio over Fiber.

RTD Resonant-tunneling Diode.

RWG Rectangular Waveguide.

SAA Slot Array Antenna.

SBD Schottky-Barrier Diodes.

SCA Small Core Area.

SD Spectral Domain.

SED Spectral Energy Density.

SEM Scanning Electron Microscope.

Si Silicon.

SIC Substrate Integrate Circuits. 
SiGe Silicon-Germanium.

SINRD Substrate Integrated Non-Radiating Dielectric Guide.

SIS Superconductor-Insulator-Superconductor.

SISW Substrate Integrated Slab Waveguide.

SIW Substrate Integrated Waveguide.

SL Side Lobe.

SL Slotline.

SL Sommerfeld Line.

SLED Superlattice Electronic Devices.

SLL Side Lobe Level.

SM Space Mapping.

SMA SubMiniature version A (connector).

SNR Signal to Noise Ratio.

SOG Silion-On-Glass.

SP Symmetry Plane.

SPC Silver Plated Copper.

SRR Short Range Radar.

SRW Slot Rectangular Waveguide.

SSGW Suspended-Strip Gap Waveguide.

SWR Standing Wave Ratio.

SWW Single Wire Waveguide.

SWW-CoB Single Wire Waveguide with Coating on Bend.

TDS Time-Domain Spectroscopy.

TE Transverse Electric.

TED Transferred-Electron Device.

TEM Transverse Electro-Magnetic.

TERASENSE Terahertz technology for electromagnetic sensing applications.

THz Terahertz.

THz-TDS THz Time-Domain Spectroscopy. 
THz-TDSI THz Time-Domain Spectroscopy Imaging.

Ti Titanium.

TikZ kein Zeichenprogramm (not a drawing program).

TiO Titanium Oxide.

TIR Total Internal Reflection.

TM Transverse Magnetic.

TNT Trinitrotoluene.

TPX $^{\circledR}$ Polymethylpentene (PMP).

TRL Thru-Reflect-Line.

TUNNETT Tunnel-injection Transit-Time.

TV Television.

TWW Two Wire Waveguide.

UK United Kingdom.

UPV Polytechnique University of Catalunya / Universitat Politècnica de Catalunya.

UPV Polytechnique University of Valencia / Universidad Politécnica de Valencia.

US United States.

USA United States of America.

USB Universal Serial Bus.

USSR Union of Soviet Socialist Republics.

UT Coaxial cable standard nomenclature.

UV Ultraviolet.

UWB Ultra-Wideband.

V2I Vehicle-to-Infrastructure.

V2V Vehicle-to-Vehicle.

VHF Very High Frequency.

VI Voltage-Current.

VP Vertical Polarization.

VP Voltage-Power.

W Tungsten. 
WiMAX Worldwide Interoperability for Microwave Access.

WIRWG Waffle-Iron Ridge Waveguide.

WR Waveguide Rectangular (nomenclature for standard sizes).

ZnGeP $_{2}$ Zinc-Germanium Diphosphide.

ZnTe Zinc Telluride.

ZOR Zero-Order Resonator. 
Acronyms

xxviii 


\section{Table of contents}

General Introduction $\quad 1$

I THz components

1 Introduction $\quad 7$

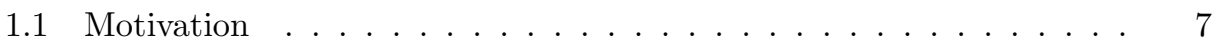

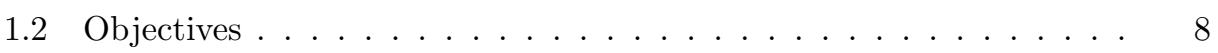

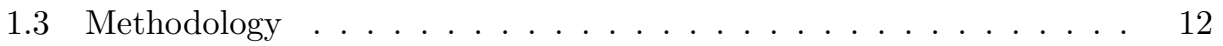

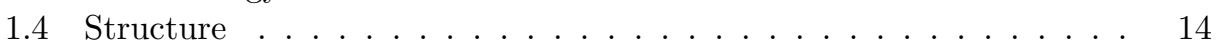

2 State of the art $\quad 17$

2.1 The $\mathrm{THz}$ band . . . . . . . . . . . . . . . . . . . . . 17

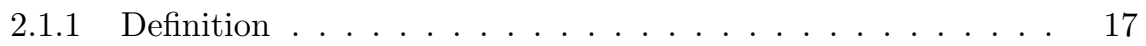

2.1.2 Historical background . . . . . . . . . . . . . . . . 18

2.1.3 $\mathrm{THz}$ band fundamental characteristics . . . . . . . . . . . 19

$2.2 \mathrm{THz}$ Applications . . . . . . . . . . . . . . . . . . 20

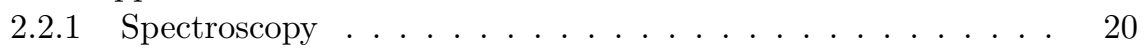

2.2.1.1 Remote Space Study . . . . . . . . . . . . . 21

2.2.1.2 Atmosphere Characterization . . . . . . . . . . 24

2.2.1.3 Molecular Spectroscopy ............. . 24

2.2.2 Plasma Fusion Diagnostic Applications . . . . . . . . . . . . 30

2.2.3 Imaging Applications . . . . . . . . . . . . . . . . . . 31

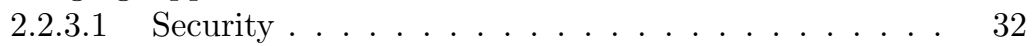

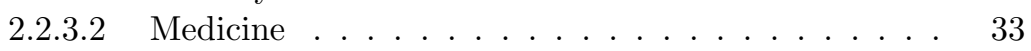

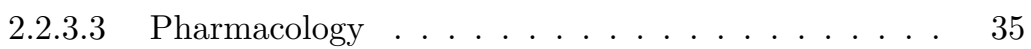

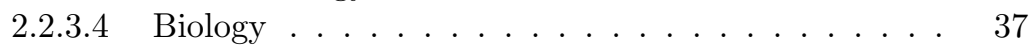

2.2.3.5 Industry . . . . . . . . . . . . . . . . . . . . . . . . . . .

2.2.3.6 Art \& Cultural Heritage Imaging . . . . . . . . . . . 42

2.2.3.7 THz Time Domain Spectroscopy Imaging (THz-TDSI) 48

2.2.4 Radar Applications . . . . . . . . . . . . . . . 51

2.2.5 Communications Applications . . . . . . ...... 55

$2.3 \mathrm{THz}$ Technology . . . . . . . . . . . . . . . . . . . . 57

2.3.1 THz Sensors . . . . . . . . . . . . . . . 58

2.3.1.1 Photon detectors . . . . . . . . . . . 58 
2.3.1.2 Thermal detectors . . . . . . . . . . . . 62

2.3.1.3 Heterodyne (Coherent) vs. Direct (Incoherent De-

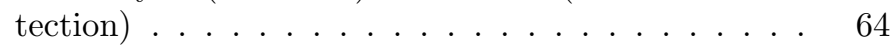

2.3.2 $\mathrm{THz}$ Sources . . . . . . . . . . . . . . . . . . . . 68

2.3.2.1 Electronic Sources . . . . . . . . . . . . . 70

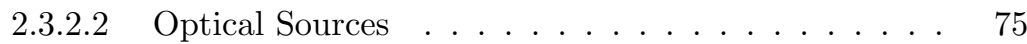

2.3.3 $\mathrm{THz}$ Waveguides ... . . . . . . . . . . . . 87

2.3.3.1 Metallic (Microwave) Waveguides and Transmission Lines . . . . . . . . . . . . . . 88

2.3.3.2 Dielectric (Optic) Waveguides . . . . . . . . . 112

2.3.3.3 Periodic and Electromagnetic Bandgap Structures . 126

2.3.3.4 Surface and Plasmonic Waveguides . . . . . . . . 135

2.3.3.5 Planar Surface and Plasmonic Waveguides . . . . . 149

3 Wire-type Waveguides 157

3.1 Surface Waveguide Theory: . . . . . . . . . . . . . . . . . 157

3.1.1 Imperfect Dielectrics . . . . . . . . . . . . . . . . . . 157

3.1.2 Boundary Conditions ................. 159

3.1.2.1 Boundary at the interface between two mediums . . 159

3.1.2.2 Radiation condition . . . . . . . . . . . . 166

3.1.3 Debye potentials and scalar wave equation . . . . . . . . . . 167

3.1.4 Formal treatment of a surface waveguide problem . . . . . . . 172

3.1.5 The impedance concept . . . . . . . . . . . . . . . . 175

3.1.6 The dispersion diagram . . . . . . . . . . . . . . . . . . . 177

3.1.7 Efficient excitation and curves . . . . . . . . . . . . 179

3.2 Metal properties in the $\mathrm{THz}$ band . . . . . . . . . . . . . . 181

3.2.1 Classical skin-depth model and relaxation-effect model (Drude

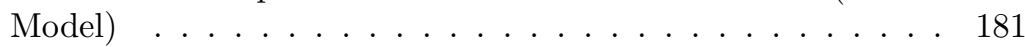

3.2.2 Comparison and examples . . . . . . . . . . . . . . 185

3.3 Dielectric cylinder with real $\varepsilon_{r} \ldots \ldots \ldots$. . . . . . . . . . . 189

3.3.1 Modal analysis . . . . . . . . . . . . . . . 189

3.3.2 Power analysis and attenuation ............... 206

3.4 Dielectric cylinder with complex $\varepsilon_{r} \ldots \ldots$. . . . . . . . . . . 215

3.4.1 Modal analysis equations . . . . . . . . . . . . . 215

3.4.2 Solutions for $\varepsilon_{r}$ real positive in the complex plane $\gamma=\alpha+j \beta \quad 222$ 3.4.2.1 The root-finding problem in the complex plane described by $\gamma \ldots \ldots \ldots \ldots \ldots$. . . . . . . . 226

3.4.3 Solutions for lossy dielectrics: $\varepsilon_{r}$ in the 4 th quadrant of the

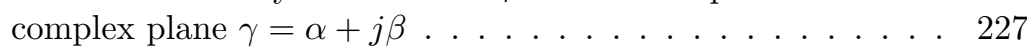

3.4.4 Solutions for $\varepsilon_{r}$ real and negative . . . . . . . . . . . 235

3.4.5 Solutions for metals: $\varepsilon_{r}$ in the 3th quadrant of the complex plane $\gamma=\alpha+j \beta \ldots \ldots . \ldots . \ldots . . \ldots . . \ldots 242$

3.5 Single Wire Waveguide $(\mathrm{SWW})$. . . . . . . . . . . . . . . 250

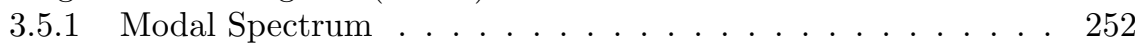

3.5.1.1 Surface modes . . . . . . . . . . . . 254

3.5.1.2 Bulk modes................. . 260

3.5.1.3 Conclusions and important remarks . . . . . . . 263 
3.5.2 Fundamental Mode . . . . . . . . . . . . . . 266

3.5.2.1 Attenuation . . . . . . . . . . . 266

3.5.2.2 Power distribution . . . . . . . . . . . . . . . 268

3.5.2.3 Dispersion .................. . 274

3.5.2.4 Conclusions and important remarks . . . . . . . . 276

3.6 Dielectric-Coated Single Wire Waveguide (DCSWW) . . . . . . . . . 277

3.6.0.5 Justification of the waveguide model . . . . . . . . . 278

3.6.1 Modal analysis equations . . . . . . . . . . . . . . 279

3.6.2 Modal spectrum . . . . . . . . . . . . . . . . . 290

3.6.2.1 Monomode conditions . . . . . . . . . . . . . 297

3.6.3 Power analysis and attenuation . . . . . . . . . . . . . 298

3.6.4 Fundamental mode . . . . . . . . . . . . . . . . . . 300

3.6.4.1 Effect of the coating . . . . . . . . . . . 302

4 Radiation Problems and Proposed Solutions 313

4.1 Problem Description . . . . . . . . . . . . . . . . . . . . 314

4.2 Solution A: Reduction of the wire radius . . . . . . . . . . . . . . 315

4.2.1 Strict monomode condition . . . . . . . . . . . . 315

4.2.2 Numerical simulations and validation of the models . . . . . . 319

4.2.2.1 Conclusions and important remarks . . . . . . . . 325

4.3 Solution B: Control of the curvature phase shift . . . . . . . . . . . . 326

4.4 SWW-DCSWW transitions . . . . . . . . . . . . . . . . . . . . . . . . . . . . . 333

4.4 .1 Tapered coating . . . . . . . . . . . . . . . . . 334

4.4.2 Tapered wire and coating ............. . . 343

5 Experimental results $\quad 349$

5.1 Ka-band prototypes results . . . . . . . . . . . . . . 350

5.1.1 Previous considerations . . . . . . . . . . . . . . 350

5.1 .2 Measurements of straight path losses . . . . . . . . . . . 352

5.1.3 First curved path measurements . . . . . . . . . . . 355

5.1.4 Second curved path measurements . . . . . . . . . . . 357

$5.2 \mathrm{THz}$ prototypes results . . . . . . . . . . . . . . . . . . . . . . . . . . . . . . . 362

5.2.1 Measurement setup . . . . . . . . . . . . . . 363

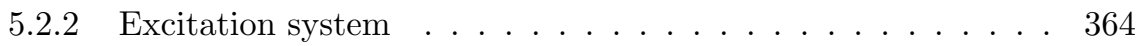

5.2 .3 SWW measurements . . . . . . . . . . . . . . . . . . . . . . . . . . . 368

5.2.4 DCSWW measurements ............... 370

6 Conclusions and future Work $\quad 375$

6.1 Conclusions . . . . . . . . . . . . . . . . . . . 375

6.2 Future Lines . . . . . . . . . . . . . . . . . . . 390

II mm-wave band components 393

7 Introduction $\quad 395$

7.1 Motivation ....................... . . 395

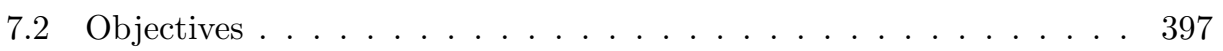


7.3 Methodology . . . . . . . . . . . . . . . . . . . 400

7.4 Structure . . . . . . . . . . . . . . . . . . . 402

8 State of the art 403

8.1 The mm-wave band . . . . . . . . . . . . . . . 403

8.1 .1 Definition . . . . . . . . . . . . . . . . . 403

8.1 .2 Historical background . . . . . . . . . . . . . . . . . 406

8.1 .3 Characteristics . . . . . . . . . . . . . . . 408

8.1.3.1 Antenna beamwidth . . . . . . . . . . . . . 408

8.1.3.2 Propagation characteristics of usual channels . . . . 409

8.1.3.3 Frequency bandwidth and regulation: . . . . . . . . 413

8.1 .4 Applications . . . . . . . . . . . . . . . 416

8.1.4.1 Radar . . . . . . . . . . . . . . 416

8.1.4.2 Communications . . . . . . . . . . . . . . 420

8.1.4.3 Imaging . . . . . . . . . . . . . . . . . . . 428

8.1.4.4 Radiometry . . . . . . . . . . . . . . . . . . 432

8.1.5 Technological problems . . . . . . . . . . . . . . 435

8.1.5.1 Hollow metallic waveguides . . . . . . . . . . . 435

8.1.5.2 Planar transmission lines . . . . . . . . . . . . . 438

8.1.5.3 Narrow-band filters . . . . . . . . . . . . . . . . . 442

8.2 Gap Waveguide Technology . . . . . . . . . . . . . . . . . . . 444

8.2 .1 Gap waveguides . . . . . . . . . . . . . . . . . . . 444

8.2.1.1 Ridge Gap Waveguide (RGW) . . . . . . . . . . 445

8.2.1.2 Suspended-Strip Gap Waveguide (SSGW) . . . . . . 447

8.2.1.3 Groove Gap Waveguide (GGW) . . . . . . . . . 447

8.2.2 Overview of Gap Waveguide Technology . . . . . . . . . . . . 449

8.2.2.1 Study of losses . . . . . . . . . . . . . . 455

8.2 .2 .2 Antennas . . . . . . . . . . . . . . . 458

8.2.2.3 Filters and diplexers . . . . . . . . . . . . . . 468

8.2.2.4 Transition and flanges . . . . . . . . . . . . 473

8.2.2.5 High frequency designs and manufacturing techniques 478

8.2.2.6 Modeling and analysis methods . . . . . . . . . . 482

8.2.2.7 Packaging. . . . . . . . . . . . . . . 491

9 Suspended Strip Gap Waveguide (SSGW) components 499

9.1 Impedance Analysis . . . . . . . . . . . . . . . . . . . 499

9.1.1 Transmission line theory . . . . . . . . . . . . . . . . . . 499

9.1.1.1 The lossless transmission line . . . . . . . . . . . 502

9.1.1.2 The terminated lossless transmission line . . . . . . 503

9.1.1.3 Short circuit terminated line . . . . . . . . . . 507

9.1 .2 Proposed analysis method . . . . . . . . . . . . . 507

9.1.2.1 Basis of the method . . . . . . . . . . . 508

9.1.2.2 Error analysis . . . . . . . . . . . . . . . 511

9.1.2.3 Validation of the method . . . . . . . . . . . . 514

9.2 Constant Impedance SSGWs _ . . . . . . . . . . . . . . . 515

9.3 SSGW coupled lines . . . . . . . . . . . . . . . . . . . 522

9.3.1 Coupled transmission lines theory . . . . . . . . . . . 522 
9.3.2 Analysis ......................... 525

9.3.3 Practical example: SSGW coupled-line filter . . . . . . . . . 530

9.3.3.1 Filter design ....................... 530

9.3.3.2 Results .................. 531

10 Groove Gap Waveguide (GGW) components 535

10.1 Analysis . . . . . . . . . . . . . . . . . 5 536

10.1.1 Groove Gap Waveguide Propagation characteristics . . . . . . 537

10.1.1.1 Operation above cutoff .............. 537

10.1.1.2 Operation below cutoff . . . . . . . . . . 539

10.2 Proposed Analysis Model . . . . . . . . . . . . . . . . . . . . . 544

10.2.1 Analysis Method . . . . . . . . . . . . . . . . 544

10.2.2 Results ........................... 546

10.2.2.1 Cutoff frequency . . . . . . . . . . . . . 546

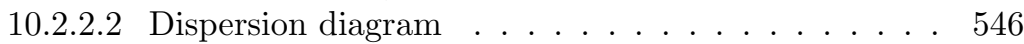

10.2.2.3 Extreme GGW geometries . . . . . . . . . . . . 548

10.2.2.4 Computation efficiency evaluation . . . . . . . . 551

10.2.3 Experimental validation . . . . . . . . . . . . . . . 552

10.3 Groove Gap Waveguide filters . . . . . . . . . . . . . . . . . . . . . . 555

10.3.1 Narrow-band filter design basic theory and procedure . . . . 556

10.3.1.1 Chebyshev filters . . . . . . . . . . . . . . 556

10.3.1.2 Resonance frequency . . . . . . . . . . . . . . . 558

10.3.1.3 Coupling coefficient . . . . . . . . . . . . . . . 558

10.3.1.4 External quality factor . . . . . . . . . . . . . . 560

10.3.1.5 Optimization process . . . . . . . . . . . . 562

10.3.2 GGW all-pole filter topology . . . . . . . . . . . . . 563

10.3.3 Resonating element: GGW cavities . . . . . . . . . . . . 563

10.3.4 Coupling element: GGW inductive windows . . . . . . . . . . 564

10.3.5 Feeding element: Cavity inserted coaxial cable . . . . . . . 565

10.3.6 Design 1: $4^{\text {th }}$-order Ka-band filter at $f=40 \mathrm{GHz}$. . . . . . . 566

10.3.6.1 Filter specifications . . . . . . . . . . . 567

10.3.6.2 Filter design . . . . . . . . . . . . . . . . . 568

10.3.6.3 Manufactured prototype and measurements . . . . . 570

10.3.7 Design 2: $5^{\text {th }}$-order V-band filter at $f=61 \mathrm{GHz}$. . . . . . . 578

10.3.7.1 Filter specifications ................ 579

10.3.7.2 Filter design . . . . . . . . . . . . . . . 580

10.3.7.3 Manufacturing errors sensitivity analysis . . . . . . 583

10.3.7.4 Manufactured prototype and measurements . . . . . 591

10.3.8 Design 3: $5^{\text {th }}$-order V-band filter at $f=60 \mathrm{GHz}$, including post-manufacture tuning metal insertions . . . . . . . . 594

10.3.8.1 Filter specifications . . . . . . . . . . . . 595

10.3.8.2 Tuning insertions topology . . . . . . . . . . 595

10.3.8.3 Aggressive Space Mapping (ASM) . . . . . . . . . 597

10.3.8.4 Simulated response . . . . . . . . . . . . 601

10.3.8.5 Manufacturing and correction process results . . . . 601

10.3.9 Comparative with rectangular waveguide technology at V-band 606

10.3.9.1 Rectangular waveguide filter topology . . . . . . . . 607 
10.3.9.2 Filter design . . . . . . . . . . . . . . 609

10.3.9.3 Manufactured prototype, measurements and comparative..................... 613

10.3.9.4 Re-assembling robustness test: . . . . . . . . . . 615

10.3.10 Results in the context of V-band NBFs state of the art . . . 617

11 Conclusions and future Work $\quad 623$

11.1 Conclusions . . . . . . . . . . . . . . . . . . . . 623

11.2 Future Lines . . . . . . . . . . . . . . . . . . . . 632

$\begin{array}{ll}\text { General Conclusions } & 635\end{array}$

$\begin{array}{ll}\text { A Publications } & \mathbf{6 3 7}\end{array}$

A.1 Indexed JCR journals . . . . . . . . . . . . . . . . . . . . 637

A.2 International Conferences . . . . . . . . . . . . . . . 637

A.3 National Conferences . . . . . . . . . . . . . . . . . . . . . . 640

A.4 Other Publications . . . . . . . . . . . . . . . . . . . . . . . . 642

A.4.1 Indexed JCR journals . . . . . . . . . . . . . . . . . . . . . 642

A.4.2 International Conferences . . . . . . . . . . . . . . . . . 642

A.4.3 National Conferences . . . . . . . . . . . . . . . 643

$\begin{array}{ll}\text { References } & 645\end{array}$ 


\section{General Introduction}

Since Maxwell's equations were established more than 150 years ago, an impressive progress has taken place in the science of manipulating the electromagnetic energy. Step by step, electromagnetic technology has been advancing towards consolidation and general use for radio waves, microwaves, infrared, optics, ultraviolet X-rays and $\gamma$-rays, which progress hits in each one of the aforementioned portions of the electromagnetic spectrum at the different points of the history. Progress always demands on research, with continuously appearing issues to address, possible improvements or new areas to explore. Accordingly to the grade of development of the technology in certain area, research ranges from the very basic research exploring totally unknown concepts and techniques to the very focused research facing an absolutely determined improvement issue in order to reach performance excellence in a specific application of such technology. With some exceptions, the former side has usually belonged to academia whereas the later side has usually belonged to industry, being between both edges a continuous graduation.

This thesis has been developed in the bosom of the Electromagnetic Radiation Group / Grupo de Radiación Electromagnética (GRE) at the Polytechnique University of Valencia / Universidad Politécnica de Valencia (UPV). In our group, it has always been a premise to contribute with innovative research dealing with rather unexplored areas and new challenging problems. Due to the group background at microwave frequencies this innovation has implied a tendency to explore higher bands in the recent years, entering in the millimeter wave (mm-wave) band. Saturation of the spectrum and the possibility of using new features provided by smaller wavelengths has put mm-wave band on the transition from adoption to maturity stage these days. Intense research is being carried out presently in order to complete this transition in benefit of the society welfare. In this revolution, our group has been involved from the beginning together with other reference international research groups in an exciting research line about new waveguiding structures designed $a d$ hoc for mm-wave frequencies: the so-called Gap Waveguides.

On the other hand, the electromagnetic research community has been witness of the emergence of a totally new technology: Terahertz $(\mathrm{THz})$ technology. The previously unexplored $\mathrm{THz}$ region has entered on the transition from novelty to adoption stage. It seems that, from a radiofrequency research point of view, the progress has put mm-wave on the stage that microwaves were some decades ago, and the hunger of knowledge and technology evolution demands from higher frequencies to explore. From a more general electromagnetic scope, we are experiencing this days, the filling of an electromagnetic gap between microwaves and optics that has remained the last 
region to be conquered by applied science. Besides of the available bandwidth at $\mathrm{THz}$ frequencies, unique interaction with matter awaits to be universally used. In this complex scenario, where both, microwave and optic research groups are putting great effort, our group has taken a significant position with a appreciable dedication, collaborating in important projects with a large number of research partners.

In this way, in order to keep participating in first line research, one eye has been put on mm-wave passive components (in the scope of Gap Waveguides) and the other eye has been put on basic, exploring research in the area of $\mathrm{THz}$ passive components. As corroborative evidence of this activity and the importance given by governmental organisms to the considered areas, the following four projects, all of them related with this thesis, have been carried out in the bosom of the GRE:

- Terahertz technology for electromagnetic sensing applications (TERASENSE). Ref: CSD2008-00068.

- Numerical methods applied to mm-wave and THz technology.

Ref: PAID-06-12/SP20120919.

- mm-wave band antennas for high-speed wireless applications.

Ref: TEC-2010-20841-C04-01.

- mm-wave in LTCC for antenna systems.

Ref: TEC-2013-47360-C3-3-P.

The first one, of remarkable entity, is a large collaboration project with participation of an important number of Spanish research groups. It is exclusively dedicated to $\mathrm{THz}$ basic research. The second one focus on analysis techniques applied to passive structures at both bands, mm-wave and $\mathrm{THz}$. The third and fourth one are, to a large extent, focused on different aspects of the use of Gap Waveguide technology to implement passive elements at mm-wave frequencies.

The previously described scenario gave birth 5 years ago to the project of this thesis, with two essential motivation pillars: 1) to provide significant contribution to the research community, facing the very new appearing challenges; 2) to contribute to the achievement of the goals described in the aforementioned projects as a participant member. From what has been described before, it can be easily understood that, in this case, satisfaction of one of them has automatically implied satisfaction of the other one. Therefore, this thesis has been motivated in a clear unique direction by a general challenging objective that has lead to a wide scope research involving both, mm-wave and $\mathrm{THz}$ bands, with the consequent effort that this implies. Although both research lines are closely related, as it will be observed at some points of the thesis, it has been found more conceptually suitable and clean for the reader to split the thesis in two parts. Part I correspond to the analysis and design of $\mathrm{THz}$ components whereas Part II correspond to analysis and design of mm-wave components. This order has been found suitable since it has actually been the followed chronological order and, also in this way, the thesis advances from basic research to more applied one, which is the usual flow. Each one of this parts presents its own particular motivation, objectives, methodology, chapters describing the research work and results, and conclusions. In this way, two thesis appear merged in 
one, that pretends to be, accordingly to the previously commented objectives, the best possible contribution to those necessities found in its origin.

The work that follows this general introduction is not lithe, but it probably has a rarely found extension and detail in $\mathrm{PhD}$ theses. This is motivated by the aim to provide a didactic work, able to transmit with detail complex information to the new incoming researcher, willing to work in the treated topics. From my point of view, this is a suitable procedure in order to ensure efficient continuity in research carried by different people, hence making all the effort put in the research of this thesis to be totally useful. Otherwise, some complex information may be easily lost or misunderstood except for the advanced reader, who always can focus on the details avoiding collateral information, or directly consult related scientific papers. This thesis pretends to be a research work of high quality avoiding common errors. To do so, all the provided information is based on a extensive documentation task; a vast number external works are cited to facilitate the comprehension and research task to the reader; mathematical rigorousness has been a major premise; every result, effect or concept has been deeply studied and justified, treating to avoid unexplained issues or lacking of information; a large quantity of conceptual information and data from different sources is merged and classified for the better comprehension; concepts and results of different sections of the whole thesis have been linked in order to provide a better global vision of the transmitted information. The work in the next chapters is, basically, what I would have liked to find/read when I was starting this thesis.

Finally, it must be pointed out that this work has been developed under the Formación del Profesorado Universitario (FPU) fellowship PhD program AP20104227 of the Education Ministry of the Spanish Government.

\section{Copyright disclaimer}

All the figures extracted from previously published works have the corresponding copyright from the publishing editorial. In all cases, figures and plots are referenced to the work in which they are originally published. 


\section{Part I}

\section{$\mathrm{THz}$ components}





\section{Chapter 1}

\section{Introduction}

\subsection{Motivation}

From the very beginning of the electromagnetic science, human curiosity has always driven pioneer research exploring the electromagnetic spectrum, whereas the increasing technological demand has led to master the use of electromagnetic waves of different regions in the known spectrum in benefit of the human society. This race has steered the electromagnetic technology to an scenario in which it is found technological consistence, on the one hand, at microwave frequencies and below and, on the other hand, at optical frequencies and beyond. Nevertheless, a technological valley is found between both separated sides. In the very bottom of this valley, the Terahertz $(\mathrm{THz})$ band is found, the last realm to explore and technologically consolidate in the electromagnetic spectrum.

The recently considered $\mathrm{THz}$ offers an electromagnetic radiation with unique properties, neither similar to those encountered at microwaves frequencies, nor to those encountered at optical frequencies. Therefore, it is not strange that such unique interaction with the matter and wavelength size has made arise a variety of new possible applications. Possible uses have been clearly postulated in areas such medicine, pharmacology, security, imaging, communications or art and cultural heritage. The potential is, without doubt, vast.

However, the same unique properties of $\mathrm{THz}$ radiation are responsible of the lack of development of $\mathrm{THz}$ technology: neither microwave technology, nor optical technology can be directly scaled with success. This situation has caused till now that development of $\mathrm{THz}$ technology has remained as a very challenging working area, in benefit of other areas demanding from more affordable research, e. g., mmwave technology. Nevertheless, the progressive advances at the surrounding areas of the $\mathrm{THz}$ valley have tighten the vise, this causing increasing attention to the $\mathrm{THz}$ band. As a consequence, from a system perspective, impressive technological advances in receptors, followed by appreciable advances in sources have occurred these last two decades up to the point that some simple $\mathrm{THz}$ systems are being implemented presently. However, there is one essential element that specially lacks of appropriate development: the waveguide. 
At $\mathrm{THz}$ frequencies, metal do not behave as bad as at optical frequencies, however, they are not longer good conductors as it occurs at microwave frequencies. Therefore, ohmic losses are strongly present in any closed structure formed by metals. Similarly, dielectrics are very lossy at $\mathrm{THz}$ frequencies due to the wavelength size, which is very interactive with certain molecular phenomena. Consequently, dielectric waveguides, widely used in optics, present noticeably high attenuation at $\mathrm{THz}$ frequencies. Under this situation, a vast variety of modifications of conventional waveguides and new THz-tailored structures have been proposed. Due to the challenging nature of this problem, none of them has totally imposed as the optimal one, but each solution presents its own advantages and drawbacks that suggest its use for a specific purpose demanding such particular features. Nevertheless, taking into account that a great amount of $\mathrm{THz}$ applications are based in spectroscopy it can be affirmed that, among new waveguiding proposals, plasmonic surface waveguides and, in particular, wire-type waveguides, result specially interesting since they are allow transmission of wide band $\mathrm{THz}$ signals with very low attenuation and dispersion.

Although wire-type waveguides present interesting properties, they also present important drawbacks: they are difficult to excite and they experience strong radiation on perturbations or bends. In addition, these waveguides, which were already considered long time ago, are now recovered from oblivion by means of several works dealing with them with a rather experimental approach. It is found a lack of proper mathematical treatment in order to model these waveguides according to $\mathrm{THz}$ band properties. As a consequence, the maximum potential of this structures is not being exploited. These issues are the main motivation of this part of the thesis, which pretends, in a first step, to establish the basis for the proper analysis and modeling of such waveguides and, in a second step, to solve the radiation issue taking advantage of a well-founded theoretical basis. Additionally, the feeling of certain lack of properly ordered information in the $\mathrm{THz}$ waveguide literature and, in a less extent, in the general $\mathrm{THz}$ technology, motivates this part of the thesis to become a reference document that can be helpful for the incoming researchers in the area of $\mathrm{THz}$ passive components.

\subsection{Objectives}

A good few objectives are involved in this part of the thesis. Classified by sets, they describe the later structure of the thesis. These objectives are:

\section{Related with the general THz technology and THz waveguides scope:}

- To get a general and consistent view of $\mathrm{THz}$ technology and applications. In order to gain knowledge enough to provide arguments, critics and to take decisions with appropriated criteria is necessary to obtain a consistent and general view of the environment where the problem is located. In this way, the intention is to provide valuable research originated from a well neutral position neither conditioned by a more marked radiofrequency, microwave band approach, nor by a more marked infrared band or optics approach. The essence of this objective resides in to be critic and locate with precision the actual existent problems. In this 
way, the approach and procedure of the more specific parts of the thesis, related with concrete structures, will be conditioned by well-founded facts.

- To provide an exhaustive study of the proposed solutions for THz waveguiding. Since $\mathrm{THz}$ technology is still not mature, adequate solutions, procedures and approaches have not been yet well defined. In this way, the contextualization of the environment in which the lately studied structures play their role is not a merely informative process about the state of the art, but becomes an objective itself with its corresponding entity in this thesis. Therefore, the treatment of this question must become as deep as required in order to provide solid arguments that justify the structures chosen to be studied in this thesis.

- To analyze with emphasis the properties of surface and plasmonic waveguides and their recent use in the $\mathrm{THz}$ band. As a direct implication of the previous objective, it becomes necessary to give special attention to the local environment of the structures under study in this part of the thesis. Therefore, the review of the proposals directly related with the wire-type devices, lately analyzed and designed, must be carried out with an additional degree of depth. Furthermore, since the phenomena related to this structures are rather unfamiliar in the environment in which this thesis is developed, they must be identified, understood and disposed in a clear way to be later properly applied.

\section{Related with the analysis of canonical wire-type waveguides:}

- To obtain the adequate physical model to characterize metals as dielectrics in the $\mathbf{T H z}$ band. Since the analysis of the main structure considered in this part of the thesis, the Single Wire Waveguide (SWW) requires considering a metallic conductor as a dielectric in terms of its macroscopic effect on the electromagnetic fields, it becomes necessary to study the available models and to chose the most suitable for the later studies purposes. Since the contribution of this thesis aims to reinforce the basis of wire-type $\mathrm{THz}$ research from the bottom rather than exclusively improve defined structures or techniques, a dedicated attention to this apart becomes necessary. In this way, analysis of several available models must be done, and due to the generality of the question, the conclusions must be focused to the structures to be analyzed, providing in this way the best criteria for the choice.

- To provide a deep and rigorous analysis of the SWW. Originated by the essence of the motivation regarding to this part of the thesis, this objective becomes one of great importance. In order to cover an appreciated lack of information and to solve some controversial questions found in the literature, this analysis must be done carefully and with the largest possible completeness. In this way, the effort must not be directly put on the isolated problem itself, but several steps must be followed with calm, thus obtaining the essential information that describes a conceptual bridge from what is known to what is unknown. Consequently, is 
important to point out that the objective does not focus on the information (or the what), but also on the way to give it (the how). The main intention is not merely contribute with new information, but it is desired this information to be directly available in useful form for the interested researcher, what is the actual valuable feature of research. Therefore, to give enough depth and rigor at each step becomes an objective itself, which if not satisfied jeopardizes the achievement of the general objective described a this point.

- To study in detail the properties of the SWW fundamental mode in practical terms. As a natural consequence of the previous objective, understanding the complete modal spectrum of a waveguiding structure allows to focus on the main transmission mode with the adequate boundary information. In this way, an important aim of this part of the thesis is to understand and provide knowledge about the properties of the fundamental mode supported by the SWW under the specific conditions of the $\mathrm{THz}$ band, not only regarding to the frequency, but dimensions, material properties, etc. Restricted to this particularization, the information given must cover the largest number of cases possible, thus providing a great variety of options available to be chosen at the moment that a design becomes necessary. The idea is not to focus exclusively at the requirements related with the posterior objectives of this part of thesis, but to cover the general case in such way that the given information result useful beyond the scope of this thesis.

- To provide a deep and rigorous analysis of the Dielectric-Coated Single Wire Waveguide (DCSWW). Similarly to the case of the SWW, the aim of this thesis regarding to this point is to provide necessary information that is lacking nowadays. However, the objective regarding to the DCSWW is approached from other perspective, not as deep as the case of the SWW, inasmuch as the DCSWW is contemplated as an auxiliary structure rather than a main one, not only in the scope of this thesis, but in general. This does not mean a loss of rigor, but the idea is to extract the necessary information from the SWW and afford the analysis of a DCSWW from an advanced point, which is the correct and efficient way to proceed. The intention is to provide the necessary information to properly afford the posterior designs in the thesis, facing their corresponding objectives.

- To study in detail the properties of the DCSWW fundamental mode in practical terms. Similarly to the case of the SWW, knowing the complete propagation features of DCSWW allows to focus properly in those of the fundamental mode. In the case of the DCSWW, due to the larger number of parameters defining it, this objective acquires important entity. Of special importance will be to establish the effect of the coating, i.e., the cause of the differences between the SWW and the DCSWW regarding their fundamental mode, and the mechanisms that allow such differences to happen. The main aim involved in the scope of this objective is to provide the necessary information to properly use 
the DCSWW as an auxiliary design to improve the general performance of wire-type waveguides based devices. In this way, the present objective becomes indispensable to allow for the procurement of those later objectives regarding to advanced structures.

\section{Related with the radiation problems of the $S W W$ :}

- To provide a set of analysis and design tools and well-defined procedures for the synthesis of wire-type waveguide based components. Once the analysis tools are generated by the achievement of previous objectives are available, proper design criteria must be provided to properly apply them. Analysis and design processes must be perfectly linked and since both are involved in the scope of this thesis, to give well-founded criteria and procedures to facilitate complex designs is an objective itself. How to decide the best option in front of challenging situations from information provided by the analysis tools, or how to apply commercial solvers to simulate properly wire-type structures, are questions to be answered in the scope of this objective.

- To determine the best design options to avoid the problem of radiation on bends affecting the SWW. Directly mapped from the most relevant motivation of this part of thesis, this objective is an important one to achieve. By using the significant amount of information obtained from the accomplishment of the previous objectives, it is intended to postulate the requirements to minimize the problem of radiation on bends that is present in the SWW and nowadays condition its practical use. As a usual way to proceed in this part of the thesis, generality and exhaustive research of solution must be kept. In this way, the aim is to consider as many scenarios as possible for the problem and to give a variety of possible solutions, well-defined, and at the same time facilitating the foreseeing of their application in problems not directly treated in this thesis.

- To provide advanced structures with entity to be used for $\mathbf{T H z}$ ultra-wide band pulses guided transmission. This objective is, from the conceptual approach the very final objective related with this part of the thesis, or, in other words, that one joining all the previous together. The aim behind this objective is to propose advanced optimized structures that prove how wire-type structures can be used in a thoughtful way, minimizing their disadvantages and maximizing their advantages, and establish them as the most suitable support for guided ultra-wide band $\mathrm{THz}$ pulses, providing a good base of well-argued reasons. The idea is to contribute with innovative designs overcoming the actual performance. Such designs should not be fruit of intuition but supported in the facts thrown by all the analysis work carried out in this part of the thesis. Again, generality must be kept and the objective must be faced allowing every kind of possible scenarios related with the problem motivating it. 


\section{Related with the experimental validation of the treated concepts:}

- To provide experimental evidence of some of the concepts and observed phenomena treated in the analysis and design chapters. Although the major objective of this part of the thesis is to treat in depth analysis and design concepts, it is found very suitable to consider an extra objective regarding the experimental validation to those concepts arising from the carried out research. Pursuing this objective pretends to give further veracity to the presented work. The main goal is to check experimentally the presented analysis tools and design procedures as well as to identify relevant phenomena. As secondary goal, this pretends to be done at $\mathrm{THz}$ frequencies whenever possible, insomuch as it is the actual environment where concepts treated in this part of the thesis are thought to be applied. Additionally, it is pretended to give some useful information about practical aspects regarding $\mathrm{THz}$ experiments with wire-type waveguides.

\subsection{Methodology}

Any self-respecting work is based on strict methodology as a good practice towards success. The large amount of information managed in this part of the thesis and the difference in the nature of it demand from specific methodologies for each type of task. At the same time, similarly grouped information is better afforded by following analogous steps, being that the probability of error is diminished and the conceptual understanding of the problem is facilitated to the reader. This adequate trade-off between diversity and uniformity or systematic features define the methodology followed in this thesis.

Specifically, the elaboration of the state of the art, of great importance in this part of the thesis, would not been possible through the compilation and analysis of numerous scientific works. In this aspect, nowadays technology alleviates such as delicate task. Google Scholar ${ }^{\circledR}$ as source to consult has been the main tool to find the specific papers giving answer or basic information for the posed issues. In the same way, qualified books available in the resources of the universities in which this thesis has been realized have been of important aid. Front such vast quantity of information, a procedure to establish information priorities has been carried out. The read scientific papers have been structured by their importance related to this part of the thesis necessities, and the depth and quantity of notes taken from them has been adapted accordingly. Additionally, when reviewing recent $\mathrm{THz}$ waveguiding structures proposals, some additional analytical calculations have been carried out when possible, in order to contrast the published information and to gain insight in it.

In the analysis of wire-type structures, the aim has been to keep well-founded mathematical and physical bases but not losing the engineering perspective. In this way, the encountered problems are strictly posed, but at the same time that one eye is kept on the solution, the other is kept on the application of it. Regarding to the analysis task, the way to proceed has been to extend as maximum possible the 
analytical resolution. This means that the mathematical problem has been solved on the paper up to the point that numerical tools have been required. In this way two positive results are obtained. On the one hand, invaluable physical insight is gained, allowing to deep understanding of the problem. On the other hand, the numerical problems to be solved, not only are alleviated, but the acquired knowledge prevent from source of error not foreseen at first view. As a tool in the analytical procedures, Mathematica ${ }^{\circledR}$ has been of great aid. In this aspect, it cannot be missed as well the valuable information found in books and specific papers treating the required mathematical and numerical issues.

As a numerical software tool for this stage, mainly mathematical, MATLAB ${ }^{\circledR}$ (MATrix LABoratory) has been extensively used due to its perfect trade-off between speed and user interface. Being that the numerical encountered problems were not critical on the speed at which the algorithms are executed, or how the computer managed the data, and, furthermore, visual information results of extremely importance, $M A T L A B^{\circledR}$ results very suitable. In the programming of the algorithms, the way to proceed has followed a rather prudent tendency. Accuracy and robustness have been always ensured first. Then, efficiency has been optimized, with good results it must be said, however. This seems to be the best option in front to such variety of cases, when the aim is to preserve generality.

Regarding to the more complex wire-type structures, it has been mandatory to draw on general purpose software implementing suitable numerical methods. In this way, HFSS ${ }^{\circledR}$ (High Frequency Structural Simulator), implementing the Finite Element Method (FEM), has been systematically used. The use of this general purpose software has been tested with the developed analytical tools in order to ensure that the obtained numerical results were actually correct. Optimization of the structures has been manually supervised, sometimes implementing optimization algorithms, thus having control on the results in every moment. As well as in the analysis procedures, in the design task it has been proceed step by step in order to every design could take advantage of the previous gained knowledge.

Experimental studies have been carried out with proper equipment, adequate for the employed operation frequency. Since experimental studies are generally more budget demanding, whenever possible, own facilities and material have been used. Nevertheless, for some specific experiments, necessary for the completeness of this part of the thesis, it has been taken advantage external facilities. Both, direct and frequency scaled experiments have been applied in order to recover the maximum possible information with the available resources. During the measurement setup building and data recovering it has been essential to employ the acquired knowledge from theoretical analysis and simulations. On the one hand, experimental systems have been better defined and optimized and, on the other hand, results have been adequately interpreted, avoiding errors introduced by the system, and the desired conceptual link between theory and practice has been provided.

Finally, figures elaborated in this part of the thesis have been created as follow. Base figures have been obtained by the employed software, either $M A T L A B^{\circledR}$ or $H F S S^{\circledR}$. Then, when necessary, additional features have been added or edited by means of Inskape ${ }^{\circledR}$. This software drawing tool has been also used to create very 
specific schematics starting off without any base image. Additionally, $P G F / T i k Z^{1}$ plots have been created from $M A T L A B^{\circledR}$ data in such cases requiring special detail or quality on the figure.

\subsection{Structure}

This part of the thesis is organized as follows:

Chapter 2 reviews the definition of the $\mathrm{THz}$ band and carries out a comprehensive study on the state of the art in this band. The review explores, on the one hand, the $\mathrm{THz}$ band applications and, on the other hand, the $\mathrm{THz}$ technology sustaining those applications. Regarding to the $\mathrm{THz}$ technology, special emphasis is made on $\mathrm{THz}$ waveguides and, particularly, in surface and plasmonic waveguiding structures proposed for transmission of $\mathrm{THz}$ broadband signals, being that the waveguides analyzed in this thesis and the advanced proposed designs belong to this family.

Chapter 3 covers all the analytical study of wire-type structures. First, the theoretical bases of the surface waveguide problem are rigorously established, and observations of the particularities of this kind of waveguide problem are provided. Then, properties of metals at $\mathrm{THz}$ are reviewed through several physical models, and a study is carried out to determine the most suitable choices for the posterior analysis. With all the necessary bases provided, the complete analysis of the SWW is carried out next, passing through several intermedium steps that start at the analysis of a simple cylindrical dielectric waveguide of real permittivity and end with the SWW at $\mathrm{THz}$ frequencies modeled by means of a complex permittivity, providing the intermedium steps valuable conceptual information. Special emphasis is put in the analysis of the SWW fundamental mode features. Finally, the analysis of the DCSWW is faced. Again, established the complete modal spectrum, the fundamental mode receives special attention in order to extract important information for the next chapters. Key remarks provided in this chapter are essential for the rest of this part of the thesis.

Chapter 4 considers the problem of radiation on bends affecting the SWW. First, the problem is described and the critical points are observed. Then, the issue regarding the numerical simulation of wire-type curved structures is treated and suitable procedures to act accurately are established. Then, the extracted concepts from the analytical study carried out in chapter 3 are applied to provide several solutions depending on the design scenario. The problem of radiation on bends is faced by means of two solutions: reducing the wire radius (solution A) and controlling the curvature phase shift (solution B). Once solutions A and B are provided as best options for the waveguiding curved paths, in order to such designs do not condition the rest of the waveguiding sections, SWW-DCSWW transitions are proposed. Several scenarios are contemplated, this leading to the three proposed transitions: 1) tapering the coating; 2 ) tapering the radius; 3 ) tapering the radius and the coating.

\footnotetext{
${ }^{1} \mathrm{PGF} / \mathrm{Ti} k \mathrm{Z}$ is a tandem of languages for producing vector graphics from a geometric/algebraic description. PGF (Portable Graphic Format) is a lower-level language, while TikZ (kein Zeichenprogramm -not a drawing program- in German) is a set of higher-level macros that use PGF.
} 
Chapter 5 presents experimental results that give additional validation to several concepts and observed phenomena described in previous chapters. Two experimental setups are presented: a first one providing measurements at Ka-band frequencies and a second one providing measurements at low $\mathrm{THz}$ frequencies. Implementation, accuracy and type of results are different in the two experimental setups, what provides complementary experimental information that give completeness to this part of the thesis, which has a strong theoretical content. Several wire-type structures, including SWW, DCSWW and SWW with tapered coating, of different electrical radius are tested.

Chapter 6 closes this part of the thesis, summarizing up the most relevant information and listing the conclusions derived from the work procedure and the obtained results in a ordered way. In addition, since there is empty room for further research, several suitable future work lines are described. 
Chapter 1. Introduction 


\section{Chapter 2}

\section{State of the art}

\subsection{The $\mathrm{THz}$ band}

\subsubsection{Definition}

The $\mathrm{THz}$ band, in its widest and most common definition, is the electromagnetic spectrum of frequencies going from $0.1 \mathrm{THz}$ to $10 \mathrm{THz}$ (wavelengths comprised between $30 \mu \mathrm{m}$ and $3 \mathrm{~mm}$ ) [1]-[3], Fig. 2.1. This definition implies, therefore, that the $\mathrm{THz}$ band extends along two decades, including the upper part of the millimeterwave band $(30 \mathrm{GHz}-300 \mathrm{GHz})$, the totality of the submillimeter-wave band $(300 \mathrm{GHz}-$ $3 \mathrm{THz}$ ), and the lower frequencies of the Far Infrared (FIR). In this way, the $\mathrm{THz}$ band becomes a spectrum portion with entity, filling the existent gap between microwaves and optics, between electronics and photonics, and promises to have its own domain, technology and applications. However, since the mm-wave band is nowadays object of several dedicated research, which is successfully developing high performance technology to be commercially used in the next years, the true challenge in the $\mathrm{THz}$ band starts beyond $300 \mathrm{GHz}$, or more precisely, as points out $\mathrm{P}$. Siegel [1], beyond the upper operating frequency of the WR-3 waveguide, i. e., 325 $\mathrm{GHz}$. Some authors [4] elevate even more the inferior limit of the $\mathrm{THz}$ band, locating it at $1 \mathrm{THz}$. Others [5], extend the upper band limit to $30 \mathrm{THz}$, identifying this frequency as the starting point of photonics (since $\mathrm{CO}_{2}$ lasers operate around this frequency). However, beyond $3 \mathrm{THz}$ (or even more if one goes beyond $10 \mathrm{THz}$ ) very few research is found, remaining this part of the band the most unexplored. In this thesis, the first definition will be used as most of the published work assumes it, and the reader should understand that in some referenced works the frontier of this band will be loose due to the strong presence of the well-established microwave and optic bands. Moreover, the diverse profile of the $\mathrm{THz}$ technology researcher, usually having larger background in one of the surrounding bands, either microwave or optics, makes the nomenclature, procedures and information sources very heterogeneous. In this sense, one must keep one foot in each of these two domains. 


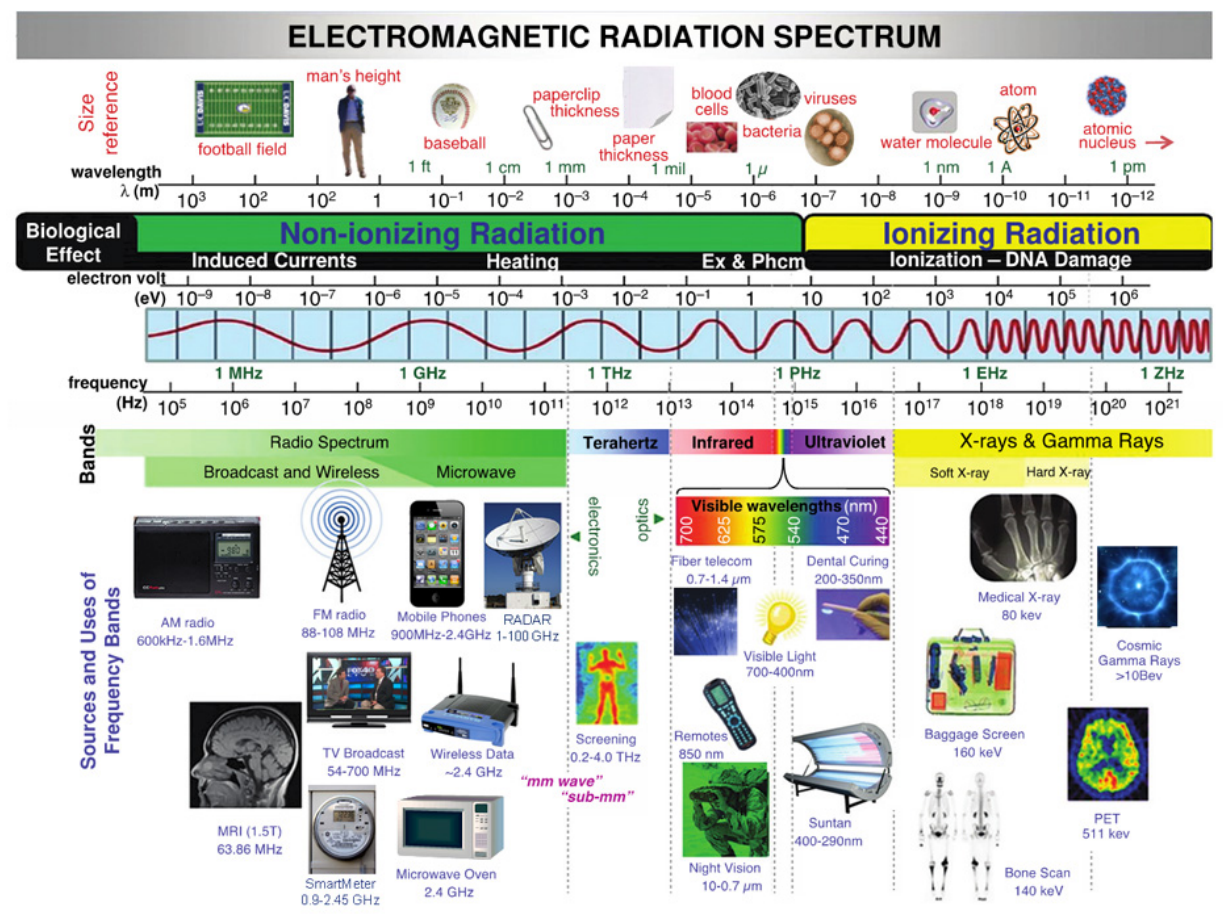

Figure 2.1: Electromagnetic spectrum with the $\mathrm{THz}$ band highlighted.

\subsubsection{Historical background}

Historically (first works can be found as back as at least the 1920s [6]), the $\mathrm{THz}$ frequencies have remained noiseless, being used for very specific or basic research, until some first technological advances (great improvement of sources and sensors [1], [2]) have propitiated the revolution experimented in this field along this $21^{\text {th }}$ century. Spectroscopy down in the Earth, and exploration of the remote universe require the use of a great part of the electromagnetic spectrum frequencies, including the $\mathrm{THz}$ band. Nonetheless, the $\mathrm{THz}$ band plays a key role in these applications, as will be explained later in this chapter. The importance of the $\mathrm{THz}$ role in this applications has been crucial to arrive up to the actual situation, since the necessity of manipulating $\mathrm{THz}$ waves has motivated the effort of using sophisticated and expensive equipment, and exploring new technological possibilities. All these advances finally triggered the $\mathrm{THz}$ Science expansion at the moment that the possibility of considering the $\mathrm{THz}$ band for commercial purposes was technologically and economically reasonable. Some references can approximate the reader to the past state of $\mathrm{THz}$ research, as a very unexplored region [7]. Also, some complete overview papers can be found at the middle of the past century [8], [9], the gross of the research considered therein focused in the lower frequencies of the $\mathrm{THz}$ band, treating the millimeter-wave band, and just grasping the sub-millimeter wave technology.

The first employments of the term terahertz or $\mathrm{THz}$ frequencies/band appeared in the 1970s, to describe the frequency band of operation of point contact diode detectors [10], the resonant frequency of a water laser [11], or the spectral line 
frequency coverage of a Michelson interferometer [12]. The 1990s are much richer on dedicated $\mathrm{THz}$ band research, focused on universe exploration, plasma diagnostics or molecular spectroscopy, all of them more deeply treated later. At the start of this century, some complete overviews appeared with the aim of updating the reader to the current situation [1]-[3], [4], [5], [13]. However, even those good references become, nowadays, rapidly obsolete, and the $\mathrm{THz}$ researcher must be aware to new advances in a wide range of journals and conferences. In the next paragraphs a complete overview of current state of the art in $\mathrm{THz}$ applications is carried out to justify the interest of this thesis. This overview is followed by their counterparts involving $\mathrm{THz}$ sources and sensors, which have technologically made possible those applications and the actual interest for the $\mathrm{THz}$ band. Finally, a deep overview on $\mathrm{THz}$ transmission lines and passive components, the alma mater of this part of the thesis, and one of the big challenges of $\mathrm{THz}$ band, is carried out.

\subsection{3 $\mathrm{THz}$ band fundamental characteristics}

To understand the nature of $\mathrm{THz}$ band applications, some aspects should be presented. Firstly, the $\mathrm{THz}$ band is very opaque for atmospheric transmission, specially below $3 \mathrm{THz}$, Fig. 2.2. Although there is some work still to be done [2], the atmospheric absorption at $\mathrm{THz}$ frequencies is better and better determined by the recent research [14]-[17], showing some transmission windows, but a general highattenuation behavior and a high water vapor continuum absorption spectrum. Thus, $\mathrm{THz}$ frequencies are not suitable for long-distance atmospheric transmission as they can be microwave frequencies. Secondly, due to the involved wavelengths, the main interaction of $\mathrm{THz}$ radiation with matter includes many spectral characteristics related with fundamental physical processes such as molecular rotational transitions, vibrational motions of organic compounds, lattice vibrations in solids, intraband transitions in semiconductors, and energy gaps in superconductors [18]-[20].

Fig. 2.3 shows the main interactions with matter of several spectrum bands, including the $\mathrm{THz}$ band. When the $\mathrm{THz}$ band is considered, one has that the electromagnetic energy is strongly attenuated in the presence of metals (due to the increase of ohmic losses) and dielectrics (due to the higher dielectric absorption because of the aforementioned molecular vibration phenomena) [21]. This issue difficults the guided transmission of $\mathrm{THz}$ waves, and a challenging environment is found when designing a waveguide compared to the surrounding bands, where ohmic losses are low, and good dielectrics are found (microwaves) [22], or transparent dielectrics such as the silicon derivatives exist (optics) [23]. Therefore, much of the considered applications are based in short-range or beyond-the-atmosphere radiated transmission nowadays [1], and the possibility of improving the performance of these applications and facilitate new ones through appropriated $\mathrm{THz}$ transmission lines is a very motivating aspect which drives much of the effort carried out in this thesis.

In order to give to the reader a schematic conceptual map about the strengths and weaknesses of the microwave, terahertz, infrared and X-ray bands, a comparative is carried out in table 2.1. Basically, $\mathrm{THz}$ frequencies provide good resolution whereas enough penetration is achieved, thus filling a functional gap between microwave and infrared regions. Of extreme importance is, furthermore, the special spectral interaction with the matter. Regarding to this point, $\mathrm{THz}$ results more functional 


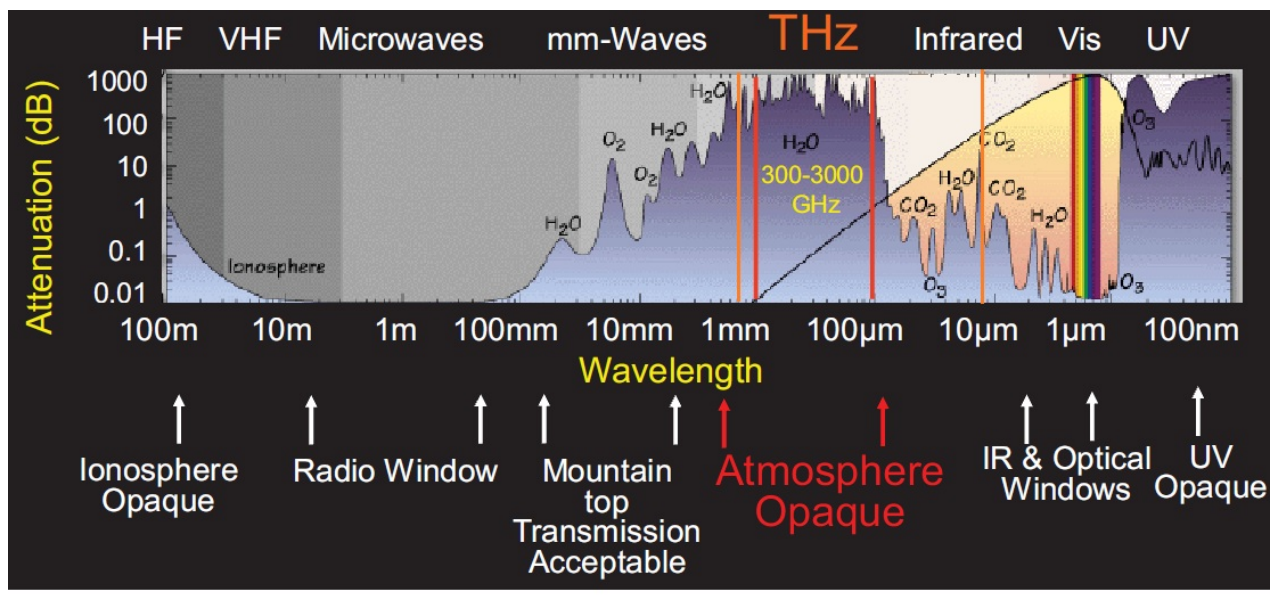

Figure 2.2: Atmospheric transmission along the different electromagnetic spectrum bands.

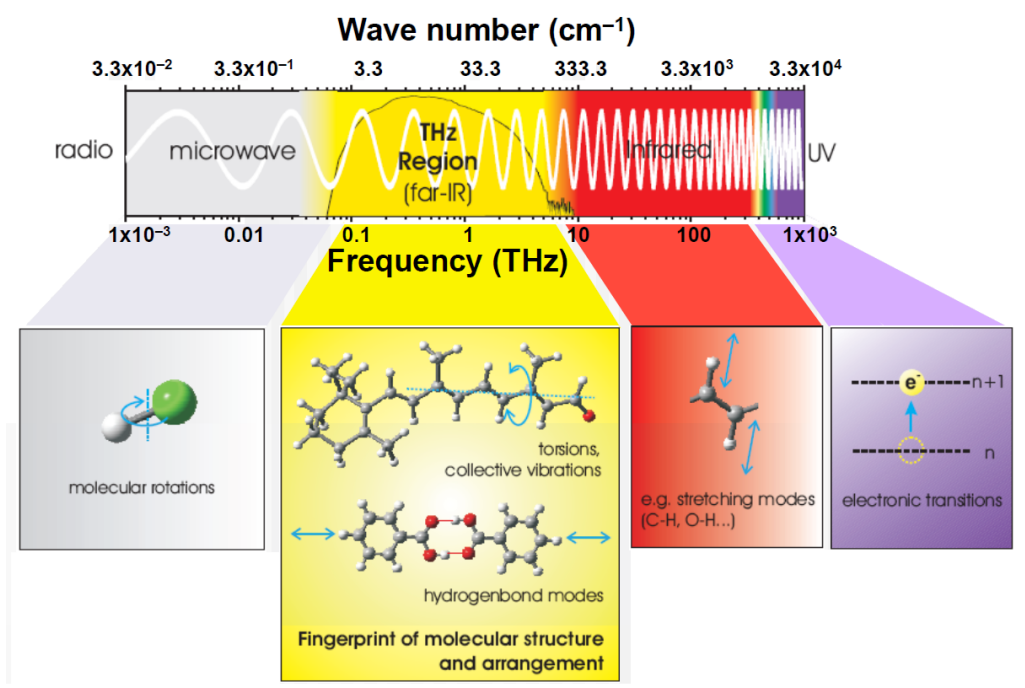

Figure 2.3: Different bands main interaction of radiation with matter.

than X-rays, which, moreover, unlike the other bands, represent a health risk. In some cases $\mathrm{THz}$ will be more suitable than other bands and replace them whereas in other cases the additional information will be added on, providing new systems with enhanced performance [24].

\section{$2.2 \mathrm{THz}$ Applications}

\subsubsection{Spectroscopy}

The applications review starts with the spectroscopy-related applications, which have been the main driving of $\mathrm{THz}$ research. Under the spectroscopy term one must 


\begin{tabular}{|c|c|c|}
\hline & Strengths & Weaknesses \\
\hline$\mu$-waves & $\begin{array}{l}\text { Non-hazardous/Non-contact } \\
\text { High sensitive devices } \\
\text { Good penetration }\end{array}$ & $\begin{array}{l}\text { Low resolution }(\mathrm{cm}) \\
\text { Metals/water block radiation }\end{array}$ \\
\hline Infrared & $\begin{array}{l}\text { Good resolution (mm) } \\
\text { Non-hazardous/Non-contact } \\
\text { High sensitive devices } \\
\text { Optical-based designs }\end{array}$ & $\begin{array}{l}\text { Low penetration }(\mu \mathrm{m}) \\
\text { Thermal noise affected } \\
\text { Needs cryogenic conditions }\end{array}$ \\
\hline X-rays & $\begin{array}{l}\text { Highest penetration }(\mathrm{m}) \\
\text { Excellent resolution (sub-mm) } \\
\text { High sensitive devices } \\
\text { Non-contact }\end{array}$ & $\begin{array}{l}\text { Hazardous } \\
\text { Strict maintenance policies } \\
\text { No spectroscopic information } \\
\text { No functional imaging }\end{array}$ \\
\hline $\mathrm{THz}$ & $\begin{array}{l}\text { Good resolution }(\mathrm{mm}) \\
\text { Moderate penetration }(\mathrm{cm}) \\
\text { Non-hazardous/Non-contact } \\
\text { Frequency information } \\
\text { High sensitive to matter }\end{array}$ & $\begin{array}{l}\text { Low scan rate } \\
\text { Metal/water block radiation }\end{array}$ \\
\hline
\end{tabular}

Table 2.1: Strengths and weakness of different bands radiation.

distinguish separately different applications such as remote space sensing, planetary (planets, asteroids, moons and comets) characterization, Earth atmosphere characterization, and molecular spectroscopy for material characterization.

\subsubsection{Remote Space Study}

The quantity of energy radiated at $\mathrm{THz}$ frequencies at the outer space, and the information that can be obtained from it can surprise the non-familiarized reader. Several examples to illustrate this fact, involving past and new research, are displayed in the Fig. 2.4. In Fig. 2.4(a), from [25] (a recommended science review), it is shown the spectral signature of interstellar dust, light and heavy molecules, including for comparison a $30-\mathrm{K}$ blackbody radiation curve, and the $2.7 \mathrm{~K}$ cosmic background signature. Apart of the continuum spectrum, clearly centered at the $\mathrm{THz}$ band, several thousand of individual lines from molecular compounds are identified. Nonetheless, even much more lines are predicted to be solved once actual signal mask levels are improved. In Fig. 2.4(b) the Spectral Energy Density (SED) of galaxies of different ages are displayed. A great difference between young (E-galaxy is the youngest) and old galaxies (Arp220 is the oldest) is appreciated, and from the energy distribution it is clear that early universe formations have a dominant $\mathrm{THz}$ band content. Indeed, important research is carried out nowadays to clear up the hidden information inside the old universe using $\mathrm{THz}$ frequencies [26], [27].

Fig. 2.4(c), from [27], compares the SED of several old galaxies, including many spiral and dwarf type galaxies. THz-band spectral information results decisive to observe star-forming environments in those galaxies. Another interesting challenge 
for actual THz-band remote space spectroscopy is the direct imaging of a black hole shadow. Promising results have been obtained recently [28]. Closer to the Earth, our Milky Way galaxy (an old galaxy) can be successfully explored at $\mathrm{THz}$ frequencies. Not in vain, one half of the total luminosity and $98 \%$ of the photons emitted since the Big Bang fall into the $\mathrm{THz}$ band [29]. Fig. 2.4(d) shows the spectrum of the Milky Way. In this graph it can be appreciated the importance of $\mathrm{THz}$ frequencies for thermal dust emission, and how abundant is the ionized carbon (see the spectral line at $\lambda=158 \mu \mathrm{m}$, i.e., $f=1.9 \mathrm{THz})$.

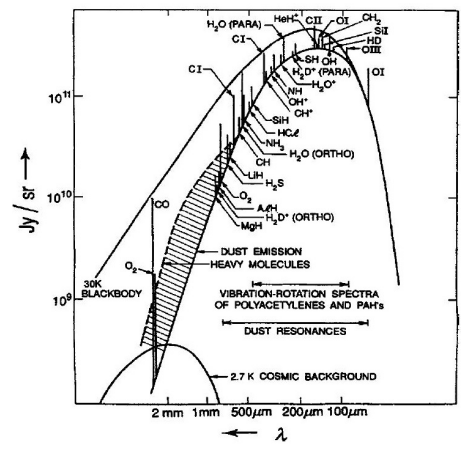

(a)

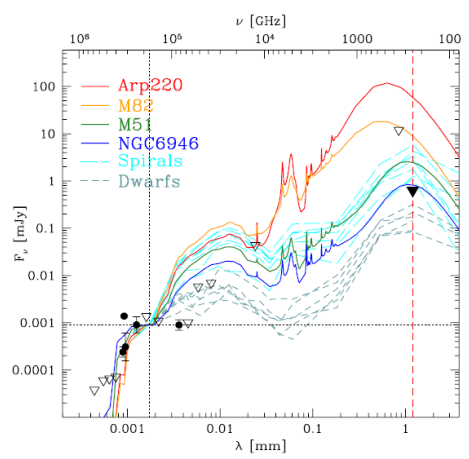

(c)

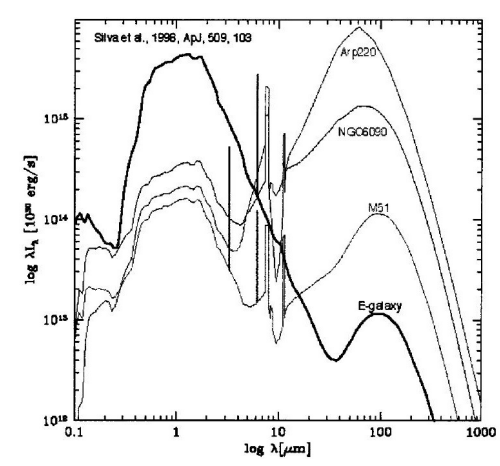

(b)

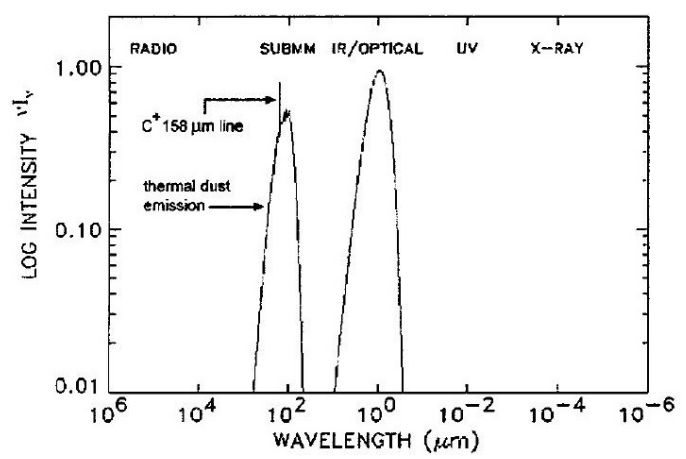

(d)

Figure 2.4: Several examples of employment of spectroscopy for remote space sensing and characterization: a) Spectral content of an interstellar cloud including includes dust continuum and molecular rotation line and atomic fine-structure line emissions assuming $30 \mathrm{~K}$ temperatures [25]; b) Spectral energy density (SED) of galaxies of different ages (E-galaxy the youngest, Arp220 the oldest) [1]; c) Spectral energy density of several known old galaxies and adjacent spiral and dwarf galaxies [27]; d) Spectrum of our galaxy, Milky Way. Half of the total emitted power falls in the $\mathrm{THz}$ region [29].

Continuing with the remote universe exploring, several outer space images are shown ( $\mathrm{THz}$ images and their optical counterpart in some cases) in Fig. 2.5. Although they pertain to $\mathrm{THz}$ imaging rather than spectroscopy, it seems more appropriated treat them now since their finality is reveal the remote universe formations, and the reader is able to better assimilate how important are $\mathrm{THz}$ in this area. The key point in those images is that they show, in a very intuitive way, how the $\mathrm{THz}$ 
can bring information that is hidden for other bands. In Fig. 2.5(a), the Horsehead Nebula in Orion is shown in both versions, the usual optical image (left) and the $\mathrm{THz}$ image (right), specifically observed at $f=345 \mathrm{GHz}$ [13]. Whereas the dust that forms the horse head is dark at optical frequencies and no information of that zone is obtained, at $\mathrm{THz}$ frequencies, the radiation penetrate into the dust, revealing information about the structures therein.

Similarly, the Fig. 2.5(b) shows the optical and THz images (in this case $f=2.1$ $\mathrm{THz}$ ) of Orion constellation [30]. The information given by $\mathrm{THz}$ and optic images is completely different. Particularly, the Terahertz radiation is attributed to thermal radiation from interstellar dust heated up by radiation from young stars in active star-forming regions. Therefore, $\mathrm{THz}$ images are shown to be very suitable to explore the regions with active star-formation. Finally, in 2.5(c) it is shown a image of the S106 Nebula, result of very recent research involving the first supra- $\mathrm{THz}$ heterodyne array of receivers for Astronomy [31]. The good resolution and contrast obtained is clearly discovering valuable information to be added to the usual optical images. Of course, due to the atmospheric absorption, these data must be obtained from satellite stations or high-altitude observatories, aircrafts or balloon platforms), which have a certain life-cycle [1]. Perhaps, in a near future, a new generation of space missions will incorporate the technological advances that are taken place these last years with giant steps, driving a great advance in this vast $\mathrm{THz}$-related research area.

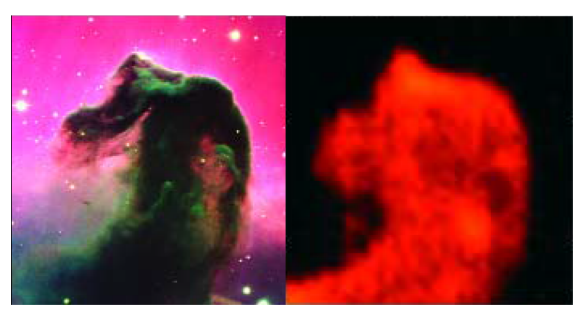

(a)

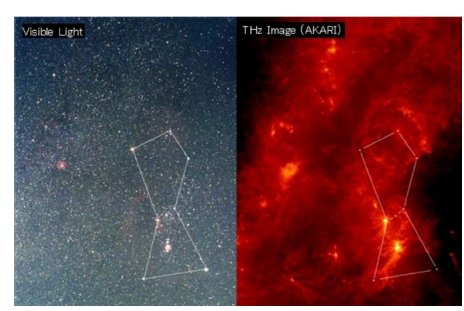

(b)

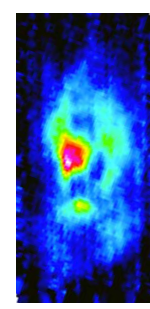

(c)

Figure 2.5: Several $\mathrm{THz}$ images of remote space regions: a) Horsehead Nebula, comparison between optic and $\mathrm{THz}(f=345 \mathrm{GHz})$ images [13]; b) Orion area, comparison between optic and $\mathrm{THz}(f=345 \mathrm{GHz})$ images [30]; c) S106 Nebula image taken by SOFIA airplane at $\lambda=158 \mu \mathrm{m}$, i.e., $f=1.9 \mathrm{THz}$ (ionized atomic carbon -CII- emission) [31].

Other kind of outer space exploration applications are cited now. The small-body (planets, asteroids, moons and comets) $\mathrm{THz}$ characterization helps to improve our information about the evolution of the solar system and refine the current models of the Earths atmosphere. Several missions consisting in surface-based (landers) or orbital remote sensing observations of gaseous species at the atmospheres of our neighbor planets, their moons, and comets, are going on or are planned for the next years [32]-[36]. The small wavelengths of $\mathrm{THz}$ band allow to a compact and light instrumental giving good resolution. Surprising conclusions such those reported in [37], where it was found a new solar burst spectral component which was only emitting in the $\mathrm{THz}$ range, indicate that many information of our solar system is surely coming in the next few years thanks to $\mathrm{THz}$ technology. Even, as suggested in [1], it is not unreasonable to expect that the first notice of extraterrestrial life 
(through the discovery of planets with atmospheric suitable conditions -temperature, pressure, composition, etc.-) take place at $\mathrm{THz}$ frequencies.

\subsubsection{Atmosphere Characterization}

Closer to the Earth, in our atmosphere, there is many information waiting to be discovered at $\mathrm{THz}$ frequencies. Of great important are the thermal emission lines from gases appearing in the Earth stratosphere and upper troposphere, such as water, oxygen, chlorine and nitrogen compounds. These gases directly influence on the levels of ozone destruction, global warming, total radiation balance and atmospheric pollution [1]. Several of these elements have their first resonance in the $\mathrm{THz}$ region, mainly at submillimeter waves [38], as can be seen in the Fig. 2.6(a). In this graph, they are displayed the resonance peaks of the three more abundant elements of the atmosphere like so other elements that participate in the atmospheric composition. Again, due to the atmospheric absorption, these measurements are mandatory taken from outside of our atmosphere through spacial sounders, balloons or aircrafts, and the lower parts of the atmosphere should be better analyzed with lower frequencies in the $\mathrm{THz}$ band.

An intense research is being done these days to proper characterize the $\mathrm{THz}$ propagation in our atmosphere [39]-[42]. On the one hand, calculation of atmospheric transmission requires line positions, intensities, pressure broadening coefficients, and pressure shift coefficients. These parameters need to be carefully determined by precise spectroscopy measurements. On the other hand, continuum absorption, which is defined as the excess of absorption unaccounted by the water vapor resonance spectrum, causes poor agreement with long path observations when it is not taken into account. This absorption remains to be perfectly understood and measured [2], [17]. In Fig. 2.6(b), from [2], it is shown the discrepancy existent between the well-developed models of microwave (NASA JPL ${ }^{1}$ catalogue molecular atmospheric absorption [43] and Liebel model for the continuum [44]), and infrared (HITRAN ${ }^{2}$ line catalogue [45] and $\mathrm{CKD}^{3}$ continuum model [46]). Advances in this area will permit accurate prediction of atmospheric state and phenomena [47], and will establish the proper transmission windows and conditions for $\mathrm{THz}$ communications [48].

\subsubsection{Molecular Spectroscopy}

Spectroscopy applications review ends with the oldest driver of the $\mathrm{THz}$ band usage: the molecular spectroscopy. This field has a long history [49], [50] studying the vibrational molecular phenomena, and it is not surprising that a great amount of interesting commercial applications in a wide range or areas will be based on the information of materials through $\mathrm{THz}$ spectral signatures [1], [2], [5]. The main advantage of $\mathrm{THz}$ spectroscopy to observe molecular phenomena against microwave spectroscopy (simpler to implement) is the larger strength of emission or absorption lines for the rotational and vibrational excitations of the lighter molecules. These

\footnotetext{
${ }^{1}$ NASA Jet Propulsion Laboratory (JPL).

${ }^{2}$ High-Resolution Transmission Molecular Absorption Database (HITRAN).

${ }^{3}$ Clough-Kneizys-Davies (CKD).
} 


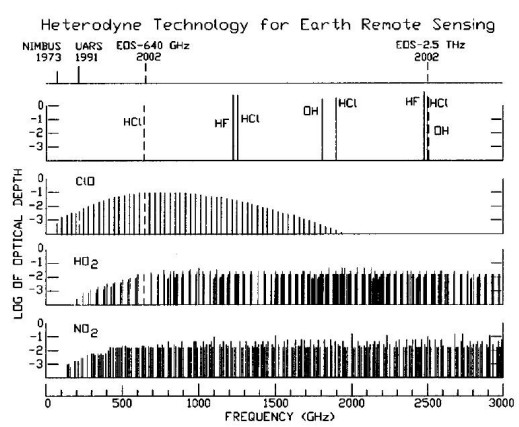

(a)

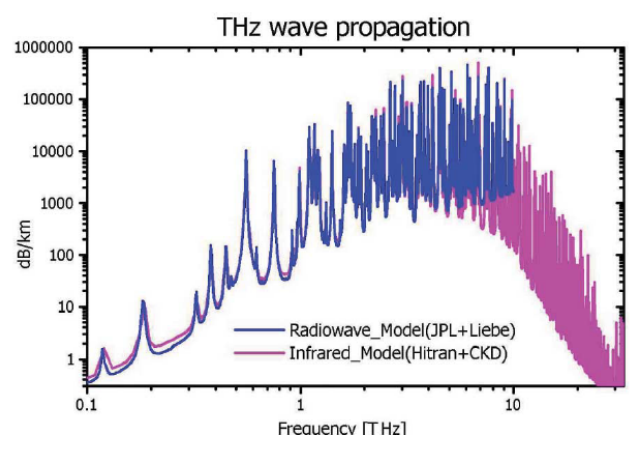

(b)

Figure 2.6: Atmospheric characterization through $\mathrm{THz}$ spectroscopy: a) Spectra of several important molecular gases of the upper part of Earths atmosphera [38]; b) Discrepancy between microwave and infrared wave atmospheric attenuation models at the $\mathrm{THz}$ band [2].

lines strength increase as $f^{2}$ (or even $f^{3}$ ), having their peaks in the submillimeter range.

The natural strong sensitivity and purity advantage of taking spectral information at $\mathrm{THz}$ has motivated and still motivates the development of adequate technology to leverage of this fact. Beyond the basic research of yesteryear based on Fourier Transform Infrared Spectroscopy (FTIR) [51], many more commercial uses are coming from the hand of a new powerful tool: the $\mathrm{THz}$ Time-Domain Spectroscopy (THz-TDS) [52], [53]. Advantages of THz-TDS against FTIR are [2]: 1) The waveform of transmitted and/or reflected terahertz waves are obtained. Therefore, the refractive index and absorption coefficient of the sample are calculated from the phase and amplitude of the waveform. 2) THz-TDS can be applied to investigate multilayer specimens by using information in the time domain waveforms. 3) Cooling the detector is not strictly mandatory. On the other hand, it is well-known that FTIR has a noticeable wider bandwidth than THz-TDS, this last usually ranging from 0.1 to $3 \mathrm{THz}$. However, this may be change with the recent advances in the $\mathrm{THz}$ air photonics. It is worth to mention that promising ultra-broadband THz-TDS have been achieved recently [54].

\section{Pharmacology}

Among the many interesting applications in this field, we start with the big-market [55] of pharmacology industry, probably the responsible of most of the private founding for $\mathrm{THz}$ science in the next years. Several examples of THz-TDS in pharmacology industry are shown in Fig. 2.7. Specifically, in Fig. 2.7(a) it is shown the THz pulsed spectra of binary mixtures of indomethacin ${ }^{4}$ (IM) amorphous and crystalline (with different crystallinity percentages) forms [57]. THz frequencies are especially sensitive to the intermolecular vibration modes of long-range that take place when the compound gets crystalline. Fig. 2.7(b) shows the THz spectra of three commonlyused hydrate forms of lactose, the most widely used excipient in the pharmaceutical

\footnotetext{
${ }^{4}$ Indomethacin is a non-steroidal anti-inflammatory drug (NSAID) commonly used as a prescription medication to reduce fever, pain, stiffness, swelling, and even Alzheimer's disease [56].
} 
industry [58]. Finally, Fig. 2.7(c) and Fig. 2.7(d) show, respectively, the THz absorption spectra of anhydrous D-glucose and D-glucose monohydrate, and the evolution of $\mathrm{THz}$ absorption spectra of D-glucose monohydrate during the dehydration process [59]. Pharmaceutical hydrates have been recognized as playing a significant role in drug formulation. Therefore, studying the kinetics of dehydration or hydration is exceedingly important, and $\mathrm{THz}$ frequencies offer the required sensitivity to accurately supervise these processes.

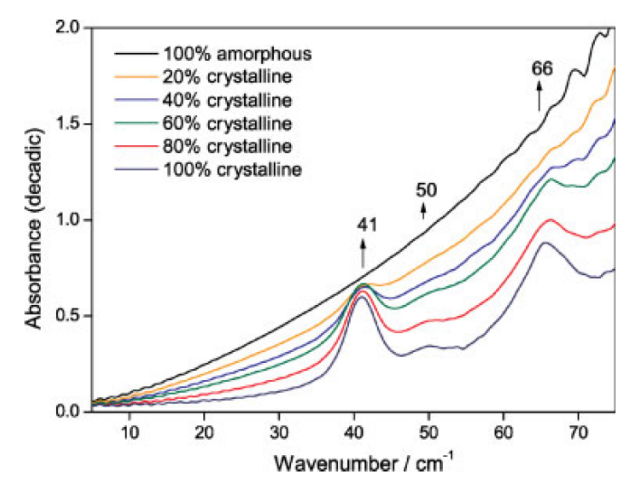

(a)

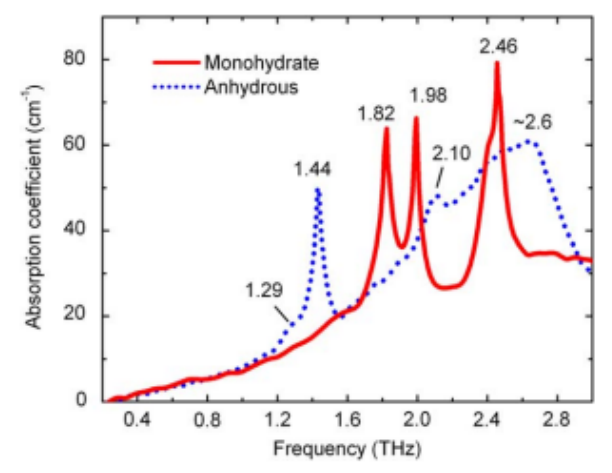

(c)

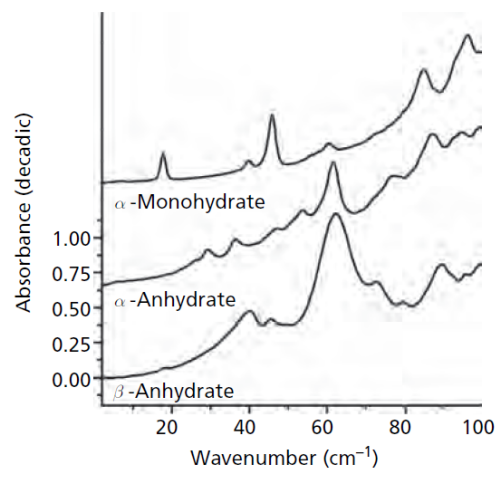

(b)

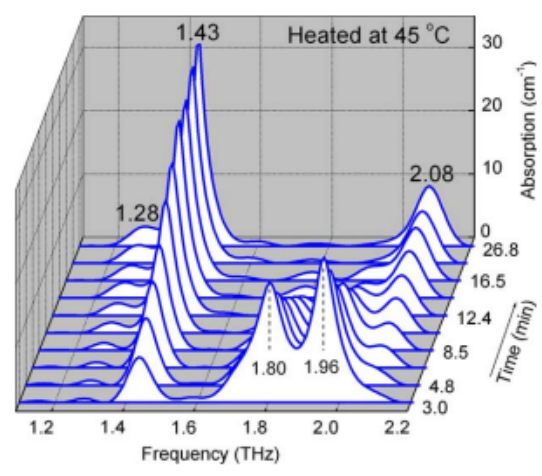

(d)

Figure 2.7: Several examples of employment of THz-TDS in pharmacology: a) Terahertz pulsed spectra of binary mixtures of indomethacin (IM)amorphous and crystalline forms (20\% intervals, 0 to $100 \%$ crystalline form) [57]; b) Terahertz spectra of three commonly-used hydrate forms of lactose [58]. c) $\mathrm{THz}$ absorption spectra of anhydrous D-glucose and D-glucose monohydrate at $25^{\circ}$ [59]; d) Evolution of $\mathrm{THz}$ absorption spectra of D-glucose monohydrate heated at $45^{\circ}$ during 27 min. (dehydration process) [59].

\section{Medical diagnostic}

Other interesting area that will benefit from $\mathrm{THz}$ technology is the medical diagnostic. The high absorption of $\mathrm{THz}$ energy by water implies high sensitivity to some diseases processes, and, although the strong absorption by water molecules causes low body penetration depth, this penetration seems to be enough for several kind of 
diagnostic purposes [60]. The use of THz-TDS has been observed as a powerful tool in studying teeth diseases [61], [62], skin diagnostics [63], [64], breast cancer diagnostic [65], [66], colon tissue cancers [67], or even ophthalmology [68]. As examples, Fig. 2.8(a) shows tooth enamel reflectivity in different demineralization conditions (which indicate the tooth enamel state) in the $\mathrm{THz}$ range, and Fig. 2.8(b) shows spectral signatures of several burnt tissues with a different damage grade.

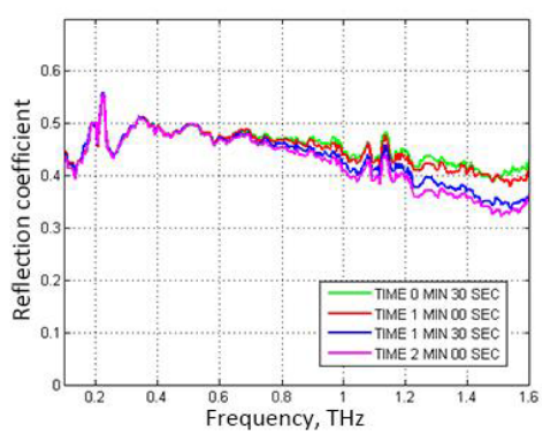

(a)

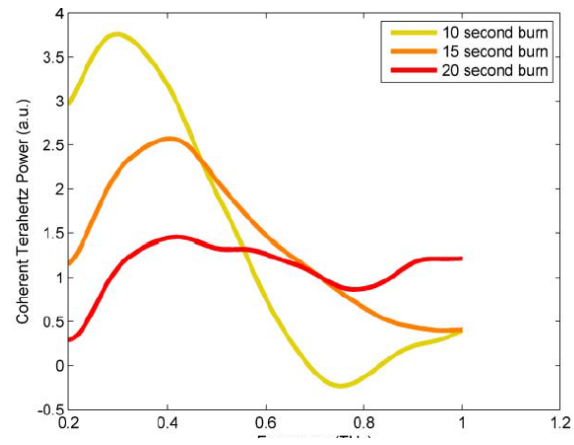

(b)

Figure 2.8: Examples of medical application of THz-TDS in medicine: a) Tooth enamel reflectivity in different demineralization conditions [60]; b) $\mathrm{THz}$ reflection spectral signatures of averaged representative superficial partial (10 s), deep partial (15 s) and fullthickness (20 s) porcine scald burns [64].

\section{Security}

Substance cataloging at $\mathrm{THz}$ is other attractive possibility with promising application in security purposes [69]. The previously mentioned sensitivity of $\mathrm{THz}$ frequencies to molecular phenomena suggest using spectral information as a substance fingerprint. In a similar way that it has been explained for the pharmacology area, different spectral responses permit differentiate some substances to others, leading this to the identification of dangerous substances (explosives, drugs, etc.) from inoffensive ones [69]. Several examples illustrating application of THz-TDS in this area are shown in Fig. 2.9. Particularly, in Fig. 2.9(a) they can be appreciated clear differences between the spectrum of the well-known explosives Trinitrotoluene (TNT) and Pentaerythritol Tetranitrate (PETN) [70]. Distinction of explosive from inoffensive substances is well illustrated in Fig. 2.9(b), from [71]. The Cyclotrimethylenetrinitramin (RDX) absorption spectrum is clear differentiated from flour and polyethylene spectrums. In a similar way, visual distinction of different drugs and agent cuts is not trivial [72], [73]. However, the absorption response at $\mathrm{THz}$ frequencies clearly classifies the studied elements, as shown in Fig. 2.9(c) and $2.9(\mathrm{~d})$.

Based on such interesting results, some more defined applications for security have been recently suggested. In [74], a systematic mail inspection is proposed. Whereas other techniques such X-ray give very accurate image detail about hidden shapes, $\mathrm{THz}$ can obtain chemical information, thus having a non-invasive chemical analysis. Combination of both techniques may result in a powerful tool. Other 

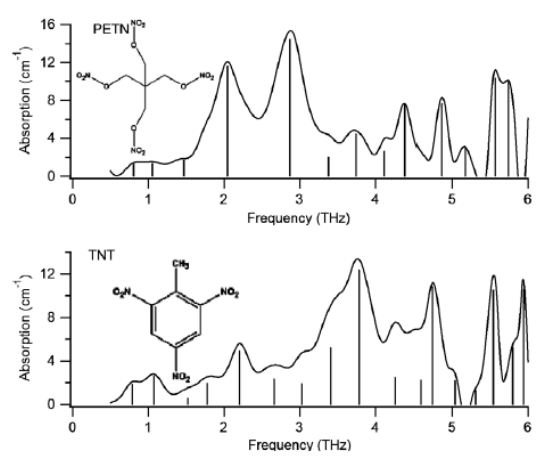

(a)

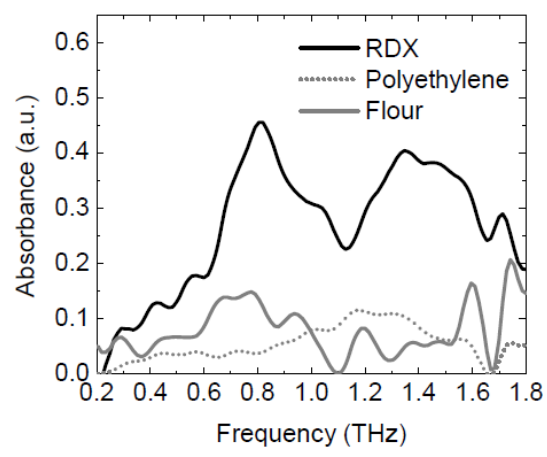

(b)

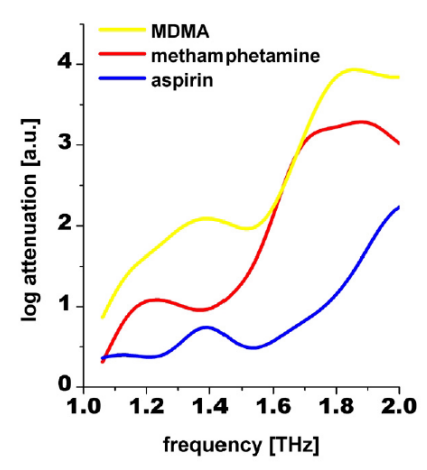

(c)

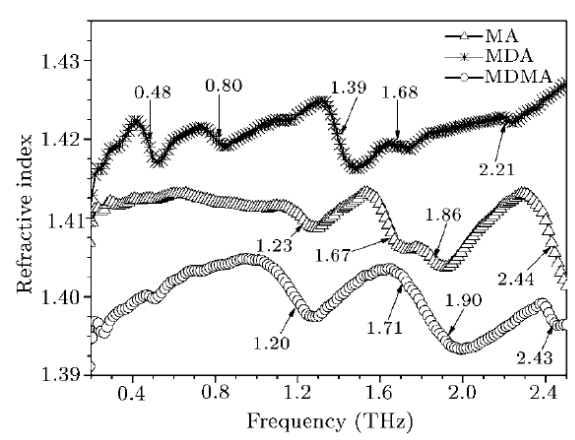

(d)

Figure 2.9: Several examples of employment of $T H z-T D S$ in pharmacology: a) $\mathrm{THz}$ absorption spectra of the explosives PETN and TNT [70]; b) Terahertz absorption spectra of the RDX explosive compared with inoffensive substances [71]. c) THz spectral absorption response of the Methylenedioxymethamphetamine (MDMA) drug, compared with inoffensive compounds [72]; d) Spectral absorption comparison between different amphetamine derived drugs [73].

nice application is the short range target identification that suggested in [75], and illustrated in Fig. 2.10. Although nowadays, the managed power levels are not enough for this purpose, the possibility of implement this system in public areas seems very attractive for the day of tomorrow. Several efforts are being carried out these days to create a reliable chemical database with the spectral responses of the known compounds [2], which is a necessary information to use in the former applications together with appropriated algorithms [76].

\section{Biology}

THz-TDS has been proposed in the biology area to recognize Deoxyribonucleic Acid (DNA) signatures [77]-[79], (an interesting method to find DNA mutation using compact genetic sensors based on compact coupled-line filters can be found in [80]); study protein properties [81], and analyze plant species [82], [83]. Food science may also take advantage from THz-TDS as reported in [84] and [85]. 


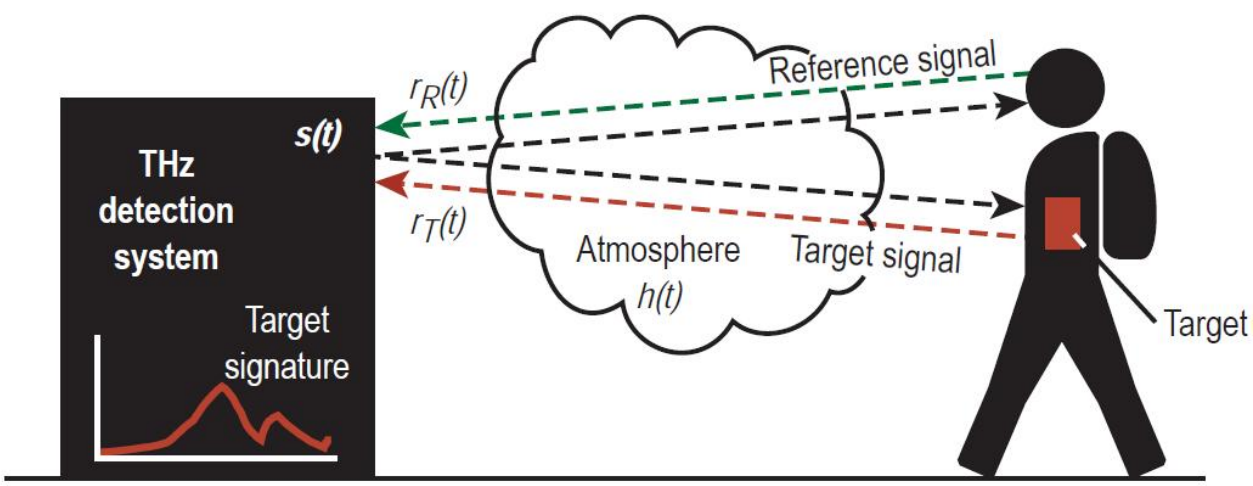

Figure 2.10: Illustration of the THz drug/explosive detection system [75].

\section{Heritage preservation and art sciences}

Also cultural heritage preservation and art sciences will benefit from $\mathrm{THz}$ spectral information in the next years [86]-[88]. Artistic materials of different colors which are similar in the visible range, have $\mathrm{THz}$ spectral information that allows an easy distinction between them. Even character recognition through material identification in medieval manuscripts using THz-TDS has been reported [89].

\section{Temporal information of THz Time Domain Spectroscopy}

Before leaving the THz-TDS, a feature of this technique with essential importance and application should be commented. Apart of the spectral signature in the $\mathrm{THz}$ range, the THz-TDS provides a temporal response full of information. The $\mathrm{THz}$ pulses are reflected and attenuated due to the different layers of the target depending on the thickness and material properties. This information is captured on the temporal response, and a proper analysis reveals extremely important information [53]. Non-destructive analysis of coating thicknesses in solid pharmaceutical tablets [90]-[92], thickness measurement of car paints and supervision of the paint drying [93], [94], or food inspection [95] are only a few examples of application.

Fig. 2.11 illustrates some particular examples of use of the temporal $\mathrm{THz}$ signal. In Fig. 2.11(a) it is shown the waveform of a pulse reflected on a typical multilayer car paint, which usually consist on four distinct layers: electrocoat, primer, basecoat and clearcoat. Conventional methods to measure the thickness of car paint layers such as magnetic gauges, eddy current measurements and ultrasound testing all require direct contact between the measurement sensor and the painted car surface. These measurement techniques can only cover a limited sampling points number on a group of selected cars, hence lack the capability to accurately identify paint defects. In addition, the mentioned techniques merely measure total thickness of the film build and are not capable of resolving the multiple paint layers [93]. Fig. 2.11(b) shows the time domain reflected response of different ibuprofen tablets. The temporal information allows a clear distinction between the two considered cases. This feature is suggested for identification of defective tablets [91] or illegally-sold imita- 
tion drugs [96]. Finally, Fig. 2.11(c) shows another car painting related example, in this case focused on supervising the painting process [94]. The $\mathrm{THz}$ time-domain signal shows different signatures depending on the painting process state, and allows a precise determination of the moment when the process is finish. This might optimize the resources in the expensive car mounting chains.

It is worth to mention that due to the specific material absorption at $\mathrm{THz}$ frequencies, the time-domain signal gives particular information as it occurs with the spectral response. Thus, the reader can imagine that all areas and examples exposed for the spectral signatures can also benefit of the information in the temporal response if layer characterization is involved.

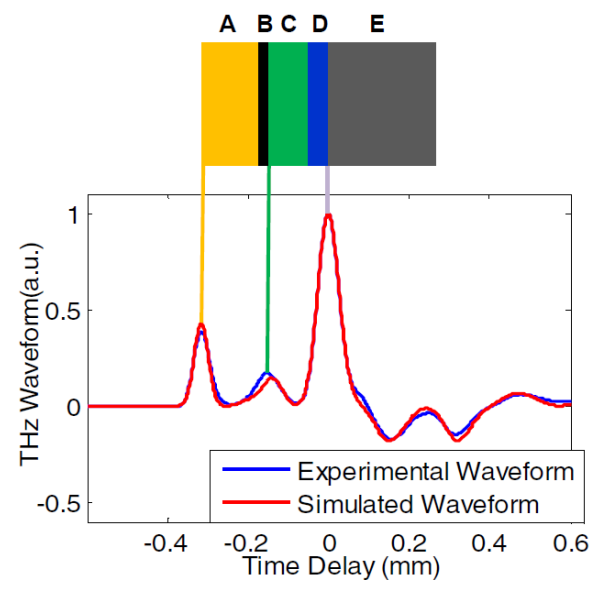

(a)

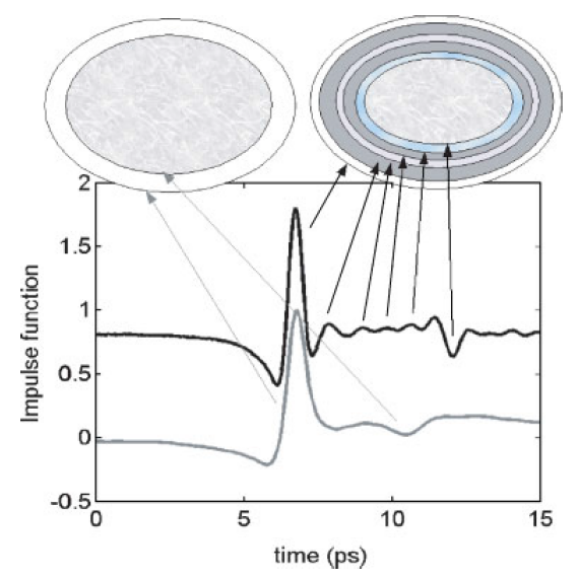

(b)
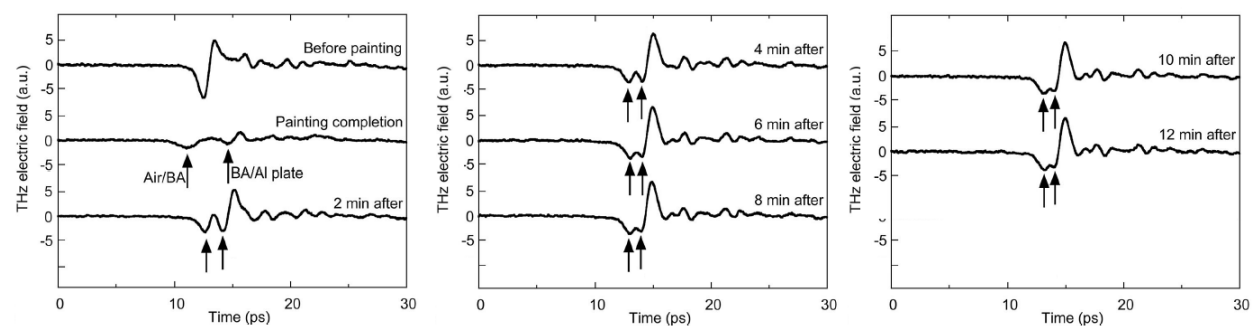

(c)

Figure 2.11: Examples of use of temporal $\mathrm{THz}$ pulse information: a) Reflection waveform of fourlayer paintings on a metallic substrate (car painting application). A: clearcoat, B: basecoat, $C$ : primer, D: electrocoat, E: metallic substrate. [93]; b) Time domain signal reflected by the different layers of ibuprofen tablets [91]; c) Temporal evolution of a $\mathrm{THz}$ pulse-echo signal before and after painting a metallic car sheet [94].

\subsubsection{Plasma Fusion Diagnostic Applications}

After the extensive review on spectroscopy-based applications a stop should be made now in other of the old drivers of $\mathrm{THz}$ technology: the plasma fusion diagnostic [1]. 
A big amount of work carried out the past century in this area is well reviewed in [97], and actual state of the art is well described in [98]. In plasma diagnostics most of the measurements involve determination of the electron density profile as a function of position and time in the plasma core. These measurements are base on the Electron Cyclotron Emission (ECE) effect [99], which, although has the first resonances in the mm-wave, produce $\mathrm{THz}$ harmonics with valuable information [1], [100]. Furthermore, $\mathrm{THz}$ waves allow a wider range of penetration in the plasma core, hence leading a more accurate exploration of it [101]. The plasma fusion diagnostic, although older and less noisy that other more popular areas such medicine or security (since they are more directly affecting the common citizen), is, notwithstanding, plenty of activity these days driven by the technological $\mathrm{THz}$ revolution. Several previously hidden information is being revealed with the recent advances [102], [103].

\subsubsection{Imaging Applications}

Imaging applications are described now. The so-called T-Ray imaging is other of the big markets of $\mathrm{THz}$ technology [1]-[3], in which private companies are strongly betting [104]-[105]. When talking about images at $\mathrm{THz}$ frequencies, the reader just has to remember the characteristics $\mathrm{THz}$ spectral response of many substances and materials of interest. At a proper frequency, the absorption between two materials to be distinguished could be noticeable different, thus producing a image with a clear contrast, that may not be (and it is most of the cases) not as clear as if it was taken using other frequencies, such as the visible ones [106]. T-Rays will have specific performance, being more suitable for determinate applications than other imaging techniques such as ultrasounds (low cost and safe but not giving chemical information), X-Rays (low cost, great resolution and penetration depth, but no chemical information), or Magnetic Resonance Imaging (high sensitivity and deep penetration, but expensive and with low capability of distinguish thin tissues/layers) [107].

Some of the potential of $\mathrm{THz}$ imaging has been already shown when the outer space exploring applications were reviewed. A review of imaging applications in several areas is carried out now, similarly as it was done for spectroscopy. Imaging at $\mathrm{THz}$ frequencies gives very intuitive information that can be complementary to the spectral signatures. Even more, both source of information can converge in what is now as THz Time-Domain Spectroscopy Imaging (THz-TDSI) [108], [109], a promising powerful tool, that will be commented at the end of this imaging dedicated section.

Therefore, it is worth noting now that $\mathrm{THz}$ imaging can be implemented with short pulses of several picoseconds (which allows, among others, THz-TDSI) or using Continuous Wave (CW). Pulsed THz imaging provide more information whereas CW allows for a more compact and simple system. Pulsed imaging provide depth, frequency-domain and time-domain information about the target, which makes it more desirable in order to obtain a complete target analysis, thus being a more powerful tool to extract final conclusions. However, plots of transmitted and reflected energy are enough for several imaging applications. Thus, since $\mathrm{CW}$ is a fast and low-cost option, it should be considered in those cases. In this section, results corresponding to both techniques are considered, being, as it can be understood, more 
noteworthy, thus corresponding to the pulsed technique. An interesting description and comparison of both techniques can be found in [110].

\subsubsection{Security}

Without any doubt, the most popular application in the use of T-ray imaging is the detection of concealed weapons and explosives. Although transmission is strongly limited by the atmospheric absorption, several interesting transmission windows can be found, and short-range imaging below $1 \mathrm{THz}$ is today a reality (although several hoped technological advances should drive it to the desired performance yet) [1], [106]. A big amount of research work has been done [111]-[118], including passive imaging, active imaging, and video; and several patents proposed [119], [120].

To illustrate the $\mathrm{THz}$ properties for security imaging, several examples are shown in Figs. 2.12-2.14. Firstly, in Fig. 2.12(a) it is appreciated the THz imaging potential to detect hidden plastic explosives. Top left image shows a mock pipe bomb made of three 1 inch diameter Polyvinyl Chloride (PVC) pipes. This bomb is attached to the subject torso (top right image), and concealed by cotton jacket (bottom left image). Real time imaging at $625 \mathrm{GHz}$ (see bottom right image) is able to show that some object is intentionally hidden by the subject [116]. Since the THz radiation is nor hazardous as it is the ionizing X-Ray energy, continuous scanning at public spaces could provide an important improvement of the security without affecting the subjects health.

Fig. 2.12(b) shows a THz image of a metal knife and a plastic explosive hidden inside of a shoe sole (left). A visible image taken after removing the sole is shown at the right for comparison. A clear evidence of the hidden dangerous objects is obtained in the $\mathrm{THz}$ image, which exhibits good resolution [112].

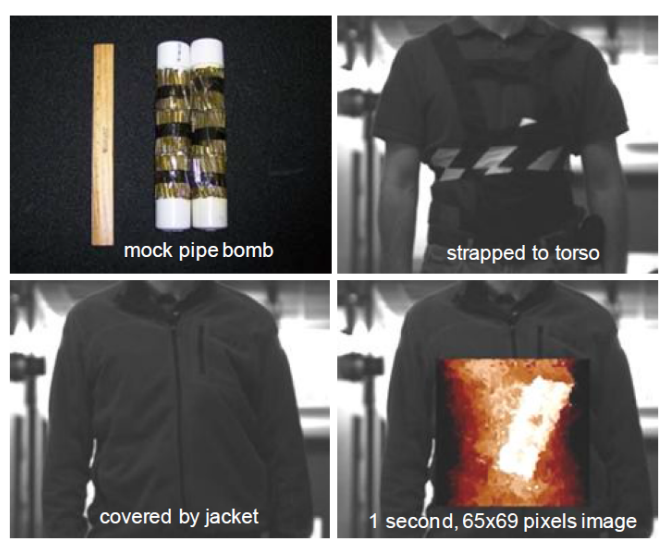

(a)

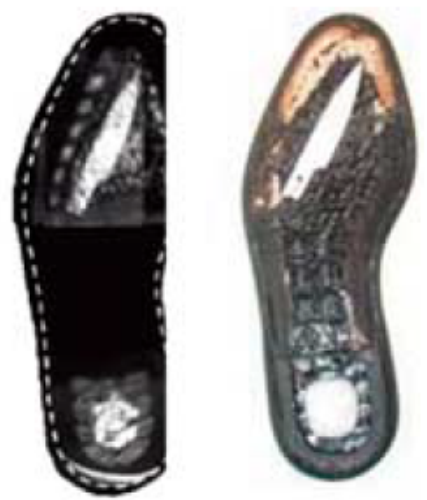

(b)

Figure 2.12: T-ray imaging for concealed weapons and explosives 1 . a) Real time imaging at $625 \mathrm{GHz}$ of a cotton jacket concealing three 1 inch diameter PVC pipes taped to the subject torso [116]; b) THz imaging (left) of a metal knife and a plastic explosive hidden inside of a shoe sole. Visible image (right) is taken after remove the sole shoe [112]. 
In Fig. 2.13(a) they are shown a passive outdoor $94 \mathrm{GHz}$ imaging of a metal knife hidden with a newspaper (left), and an active indoor $640 \mathrm{GHz}$ imaging of a toy gun under a cotton shirt. In the outdoor image, the cold sky leads to a strong target signature whereas indoor conditions require active imaging to identify the hidden gun. Even the gun is appreciated, better contrast and image quality are desirable. Coherent techniques could provide noticeable improvement of the image contrast in the case of active imaging [113].
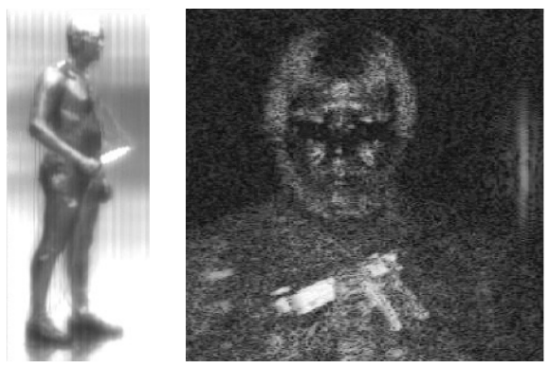

(a)

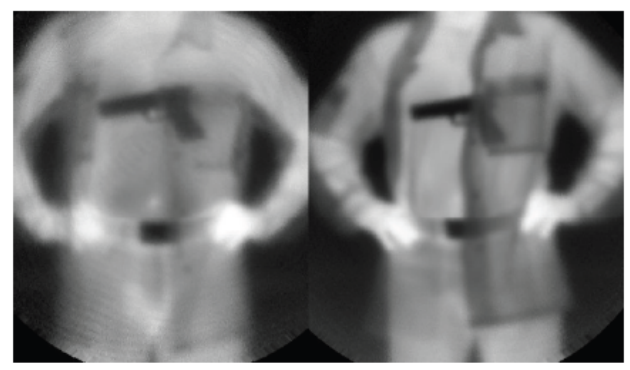

(b)

Figure 2.13: T-ray imaging for concealed weapons and explosives 2. a) Left: Passive outdoor 94 $\mathrm{GHz}$ imaging of a metal knife hidden with a newspaper; Right: Active indoor $640 \mathrm{GHz}$ imaging of a toy gun under a cotton shirt [113]; b) THz active imaging of a hidden gun at $0.35 \mathrm{THz}$ (left) and $0.85 \mathrm{GHz}$ (right) [118].

Fig. 2.13(b) shows THz images of a hidden gun at $0.35 \mathrm{THz}$ (left) and $0.85 \mathrm{GHz}$ (right). The image at $0.35 \mathrm{THz}$ shows lower resolution but less obstruction by the fabrics [118]. The results in that work indicate that current performance suggest better using lower frequencies in order to achieve the required penetration in the weaves. However, future technological advances could provide enough power so that higher frequencies, which lead to a higher resolution, may be successfully used.

Finally, Fig. 2.14 illustrates a computer recreation of the expected $\mathrm{THz}$ imaging quality once the $\mathrm{THz}$ technology reaches its maturity. Properly manipulated, $\mathrm{THz}$ waves could provide very good resolution, together with the desired penetration and health safety.

\subsubsection{Medicine}

Medical imaging is other area where $\mathrm{THz}$ are called to be a great contributor. These days, the evolution of medical imaging is going beyond anatomical and structural imaging, which are well assessed by the mature X-rays and $\gamma$-rays, together with magnetic resonance techniques. The demand for imaging at the molecular and cellular levels is increasing [121], and $\mathrm{THz}$ properties fill well this job [122]. Figs. 2.15-2.16 show several examples of the use of $\mathrm{THz}$ images in the medical area. In Fig. 2.15(a) it is shown the $\mathrm{THz}$ image of a human tooth [107]. The tooth internal structure is clearly revealed at $\mathrm{THz}$ frequencies whereas visible images are not able to penetrate inside, and X-rays can hardly contribute (lack of contrast) to diagnostic internal damages such as caries. 


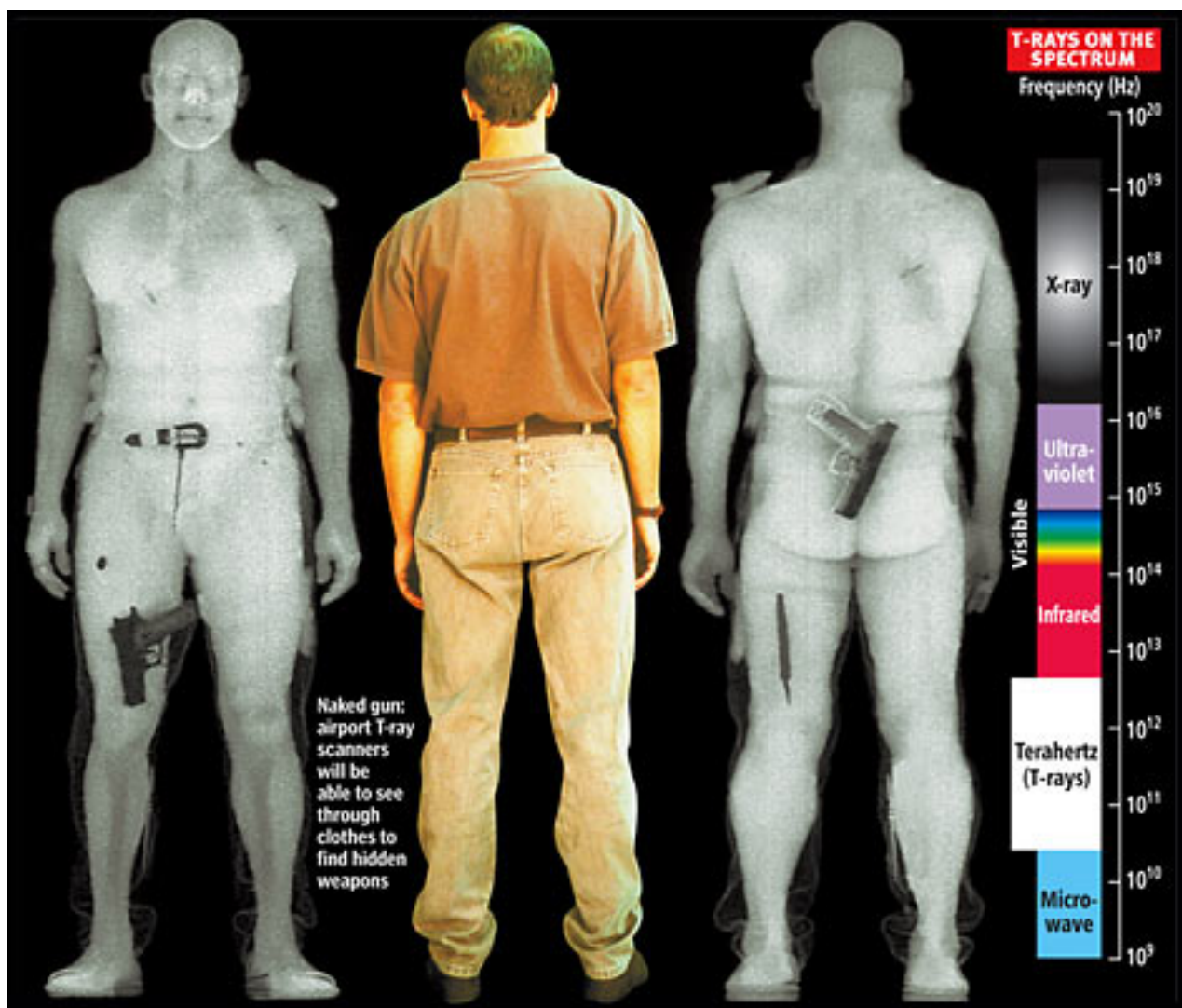

Figure 2.14: Computer recreation of the expected $\mathrm{THz}$ imaging quality once the $\mathrm{THz}$ technology reaches its maturity.

In Fig. 2.15(b) they are shown images of a diseased skin with infiltrating carcinoma taken in the visible (top) and $\mathrm{THz}$ (bottom) ranges. Differences in $\mathrm{THz}$ spectra of healthy and diseased skin lead to appearance of contrast between healthy skin and sick tissues in parametrical images. Contrast is reported even in cases where the cancer lies at a small depth under the skin [60].

In a similar way, Fig. 2.16(a) shows the visible and $\mathrm{THz}$ images of a mastectomy specimen from a patient with an invasive lobular carcinoma, circled in red [123]. It can be appreciated how clear is distinguished the ill region at $\mathrm{THz}$ frequencies whereas the visible image does not give obvious evidence. Comparison of the size and shape of tumor regions in the $\mathrm{THz}$ images with the corresponding histology section gives good correlation. This accuracy is extremely important in order to identify precisely the borders of the cancerous area.

Finally, Fig. 2.16(b) shows images of a subject arm before and in the moments after the application of a freezer burn. THz images, at the top row, clearly identifie the growth of a sub-cutaneous edema whereas the visible images (bottom row) show no evidence of change in the skin surface [124]. These examples show the good contrast and accuracy of $\mathrm{THz}$ imaging for medical diagnostic. It is worth to mention 


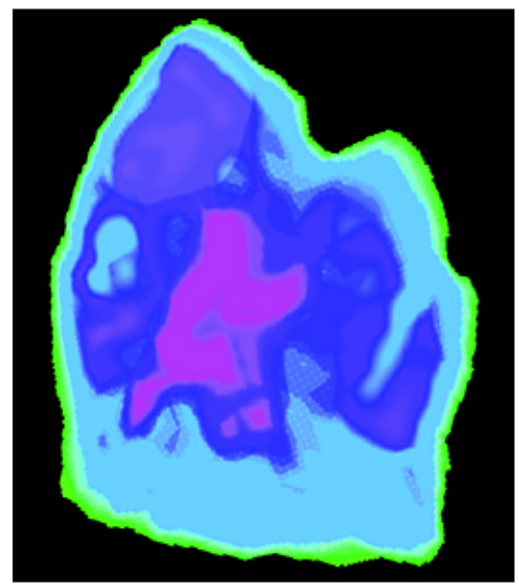

(a)
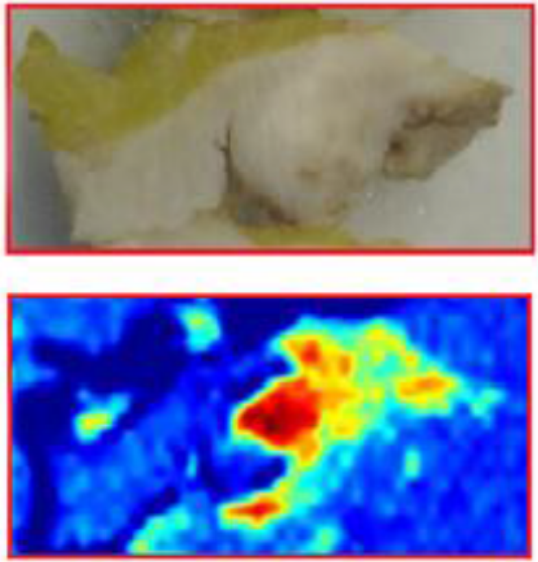

(b)

Figure 2.15: T-ray imaging for medical applications 1. a) THz imaging of a human tooth revealing its interior structure and possible injuries [107]; b) Infiltrating carcinoma. Top image correspond to the photo of in vitro diseased skin and bottom image corresponds to the $\mathrm{THz}$ parametrical image of the cancer [60].

the non-invasive feature of this imaging technique, which furthermore does not imply secondary effects in the patient, as X-rays do.

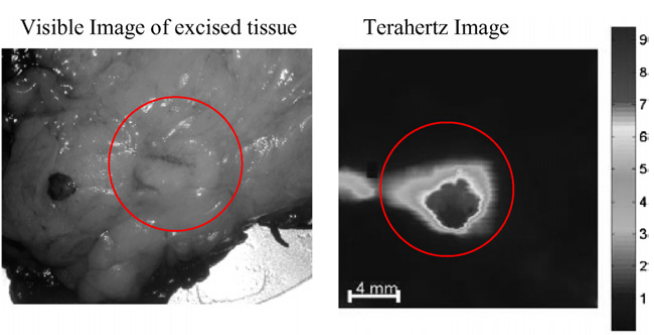

(a)
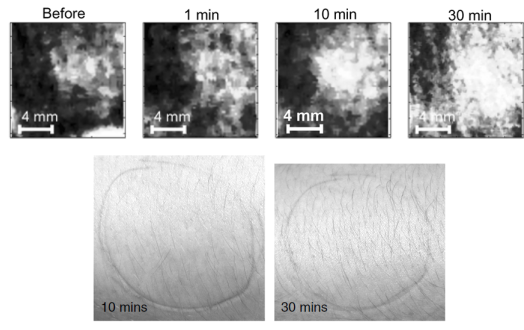

(b)

Figure 2.16: T-ray imaging for medical applications 2. a) Visible and $\mathrm{THz}$ images of a mastectomy specimen from a patient with an invasive lobular carcinoma (circled) [123]; b) A series of THz images (top images) of a subject arm before and in the moments after the application of a freezer burn, clearly identifies the growth of a sub-cutaneous edema. Photographs showing no evident change in the skin surface are shown at bottom [124].

\subsubsection{Pharmacology}

Apart of the multiple possibilities that the $\mathrm{THz}$ band offers to the pharmaceutical industry through spectroscopy, $\mathrm{THz}$ pulsed imaging may become a powerful tool regarding one fundamental aspect in this industry: supervision of tablets manufacturing process. It is well-known that most of the pharmaceutical products are sold in tablet form. Coats for these tablets are used very often due to many reasons such 
as shelf-life improvement, taste and odor purposes, facility of swallow or assimilation process control, e.g., disintegration of the tablet only in the small intestine but not in the stomach. Present coating analyzing methods calculate the amount of applied coating mass from the flow or spray rate of the coating liquid, thus being indirect and giving only average information. Through $\mathrm{THz}$ imaging intrinsic information, several valuable $2-\mathrm{D}$ or $3-\mathrm{D}$ information can be recovered [125]. This could give critical information in order to supervise and improve manufacturing methods.

Figs. 2.17 and 2.18 illustrate some THz imaging results. In Fig. 2.17 it is shown a 6x magnified photography (Fig. 2.17(a)) of a tablet and three $\mathrm{THz}$ images based on different information of the THz pulse (Figs. 2.17(b)-2.17(d)). The coating thickness map, Fig. 2.17(b), is a valuable information of the achieved coating uniformity in the different tablet parts. This information may reveal the origin of systematic errors in the coating process, and avoid defective tablets. Other errors are better appreciated with peak intensity, Fig. 2.17(c), and interface index information, Fig. 2.17(d). For example, the coating cracks, indicated with red arrows in the photograph are clearly observed in the $\mathrm{THz}$ images 3 and 4 [92].

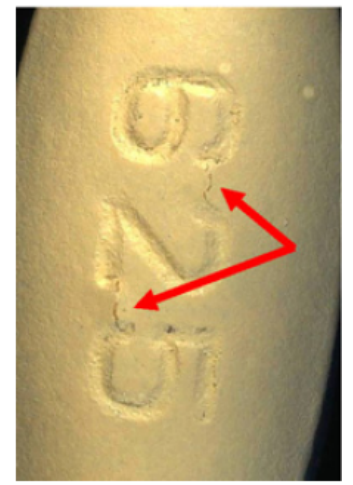

(a)

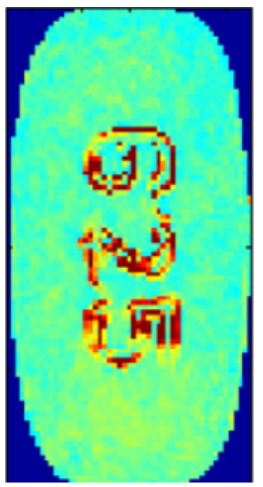

(b)

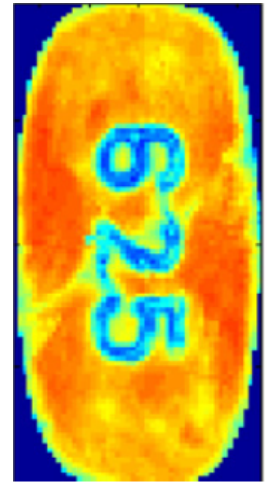

(c)

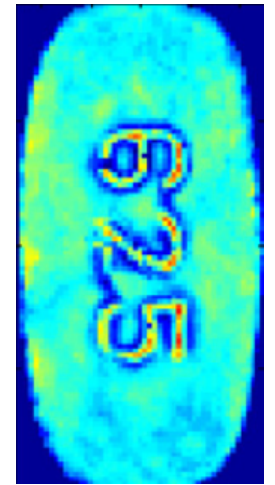

(d)

Figure 2.17: T-ray in pharmaceutical industry 1. Pharmaceutical tablet coating defects study. a) 6x magnified photography (red arrows indicate defects on the coating), b) $\mathrm{THz}$ image showing the coating thickness map, c) $\mathrm{THz}$ image showing the peak intensity map, d) $\mathrm{THz}$ image showing the interface index map [92].

Similarly, Fig. 2.18 illustrates how $\mathrm{THz}$ imaging penetration features of $\mathrm{THz}$ radiation penetration features are used for non-destructive inspection of a packaged tablet. Fig. 2.18(a) shows the visible image of the upper side of the packaged tablet. This tablet has the text "Rorer" engraved at the upper face and the text "Maalox PlusTM" at the bottom face. Figs. 2.18(a) and 2.18(b) correspond to $\mathrm{THz}$ images taken from the upper and bottom sides of the tablet, respectively. $\mathrm{THz}$ radiation penetrates the package and provide the appropriated contrast to recover both engravings. Further technological advances may provide better resolution, which is low at present, and a finer penetration adjust, in order to achieve a more accurate inspection [126]. 


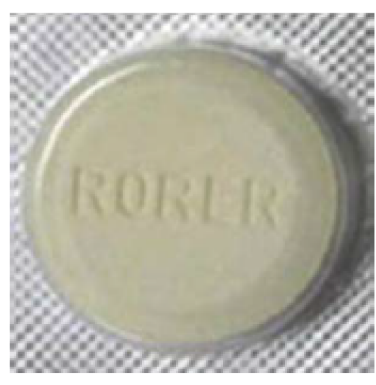

(a)

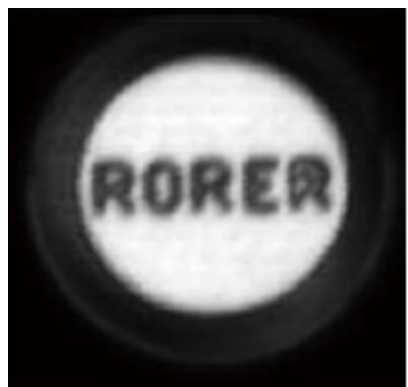

(b)

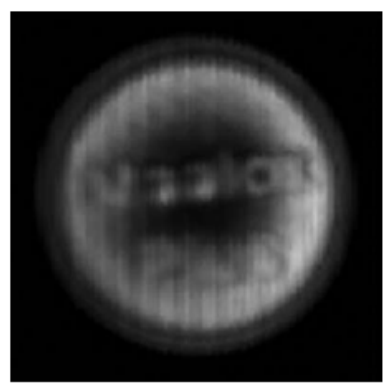

(c)

Figure 2.18: T-ray in pharmaceutical industry 2. Packaged tablet with two engravings: "Rorer" at the upper face, and "Maalox PlusTM" at the bottom face. a) Tablet photography; b) $T H z$ image from the top of the packaged table; c) THz image from the bottom of the packaged table [126].

\subsubsection{Biology}

Due to the sensitivity of the $\mathrm{THz}$ radiation to the target water content, it is previsible that interesting information may be revealed with $\mathrm{THz}$ imaging in the area of biology. Internal structural information of life species can be obtained, specially in those cases where the water does not fill a large volume (since in those cases absorption would be excessive) such as plants leaves. Figs. 2.19-2.21 show some interesting examples of $\mathrm{THz}$ imaging in this area. In Fig. 2.19(a) they can be observed a visible image (1) of a dried seed pod, together with two THz images (2 and 3), taken at a distance larger than $25 \mathrm{~m}$ from the target. Particularly, the configuration used in 3, which optimize the capture system, gives good resolution $(0.75 \mathrm{~mm})$ [127]. Whereas optic image gives only an accurate description of the seed pod surface, $\mathrm{THz}$ reveals its internal structure. One can imagine the possibilities of this kind of imaging, which could lead to supervise in vivo the biological processes that take place in plants. Due to the involved structures size, the required resolution would be better attained with $\mathrm{THz}$ than present microwaves techniques [128].

Fig. 2.19(b) illustrates the imaging of a buried carrot. Two cases are considered: buried in sand (top left visible image), and buried in soil (top right visible image), giving very similar results. Transmission $\mathrm{THz}$ imaging has been applied to this target, buried $5 \mathrm{~cm}$. In the bottom left image, the T-ray imaging result can be appreciated, whereas the bottom right image shows the final T-ray processed image. The shape of the carrot is recovered with good accuracy. A success larger than $96 \%$ was recorded in this experiment [129].

Also from the work in [129], the reflective counterpart experiment is shown in Fig. 2.20. Fig. 2.20(a) is a photo of the used set before being buried. It includes a sweet potato, a turnip, four rocks and a piece of tree branch. Fig. 2.20(b) is the $\mathrm{THz}$ reflection image. Finally, Fig. 2.20(c) is the processed image with which the desired information is recovered. Accuracies going from $87 \%$ to $93 \%$ are recorded in the identification of the different elements. Potential of this technique will noticeable benefit the study of root growth and development, which are critial processes for 


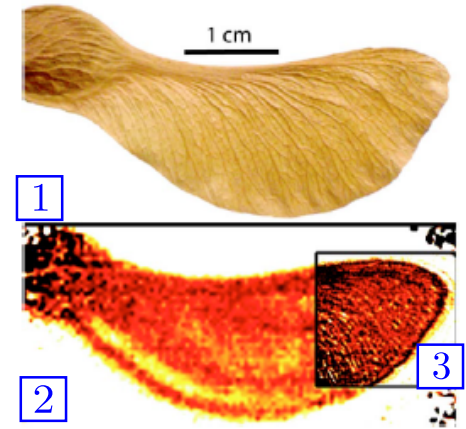

(a)
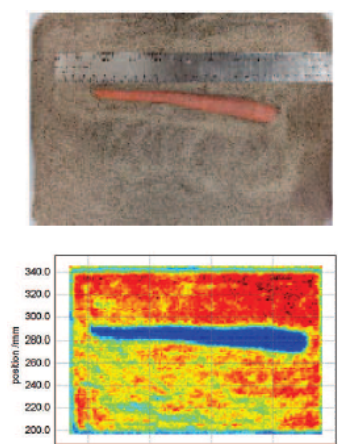
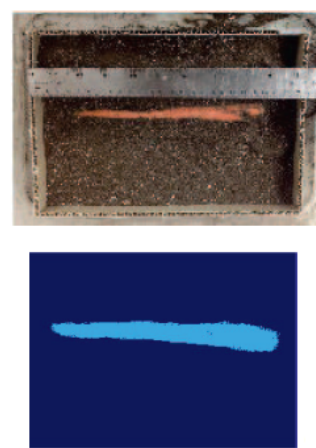

(b)

Figure 2.19: T-ray imaging for biological applications 1. a) Visible (1) and $\mathrm{THz}$ images (2 and 3 ) of a dried seed pod. $\mathrm{THz}$ images are taken from a distance larger than $25 \mathrm{~m}$. Image 3 uses a configuration which allows for a better resolution [127]; b) Visible images of a buried carrot (top left image correspond to a sand buried carrot; top right image correspond to a soil buried carrot) and the corresponding $\mathrm{THz}$ transmission images (bottom left is the $\mathrm{THz}$ image of the sand buried (5cm); bottom right is the $\mathrm{THz}$ final processed image) [129].

plant survival and productivity. Nowadays, there is any non-destructive system able to provide repeatedly high-quality information on roots of field-grown plants.

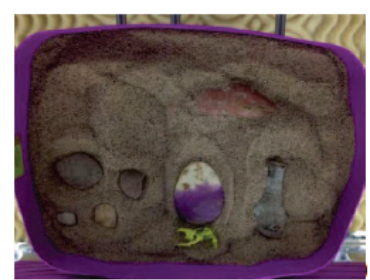

(a)

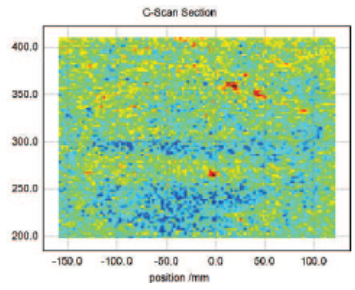

(b)

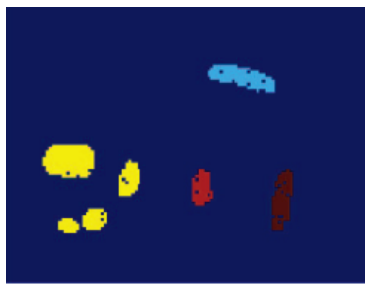

(c)

Figure 2.20: T-ray imaging for biological applications 2. Reflection images for root phenotyping. a) Photo of the samples (a sweet potato, a turnip, four rocks and a piece of tree branch) before being buried; b) $\mathrm{THz}$ reflection image; c) Final processed image [129].

Finally, images revealing the internal structure of a red pepper (left) and a prawn (right) are shown in Fig. 2.21. Optical images are also displayed in each case. Due to the internal composition of these live species $\mathrm{THz}$ results much more selective than $\mathrm{X}$-rays [24]. Furthermore, since $\mathrm{THz}$ is more sensitive to these materials, variation scanning of the imaging frequency could give very different responses, obtaining thus, further information about the specimen.

\subsubsection{Industry}

The quality of several processes in different industrial areas can be also supervised and improved with the aid of $\mathrm{THz}$ imaging. A huge amount of proposals and demonstrations can be found in the literature, with an increasing number tendency during the last years due to the technological advances [130]-[139]. Again, particular $\mathrm{THz}$ 

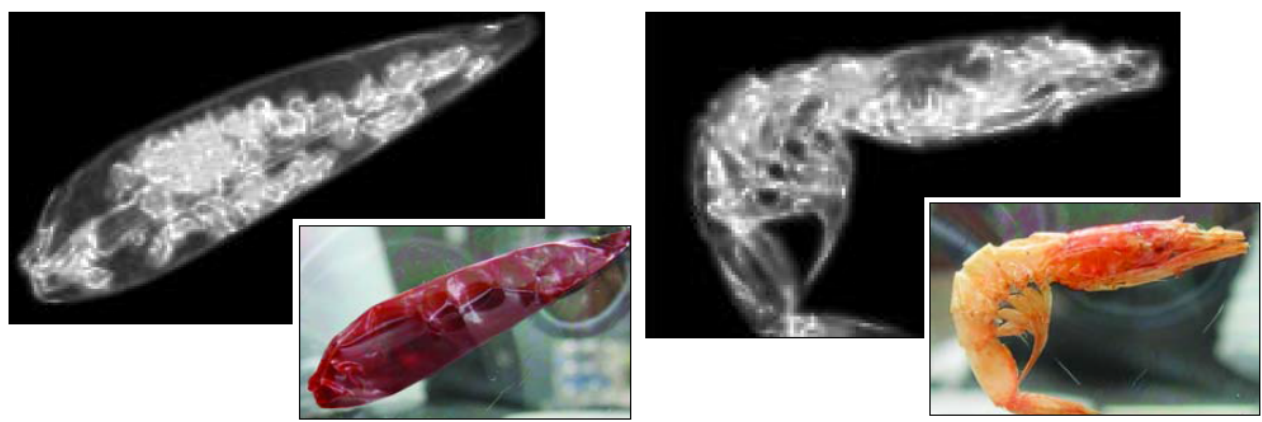

Figure 2.21: $\mathrm{THz}$ and optical images of a red pepper (left) and a prawn (right) [24].

penetration and resolution reveals information not accessible with other imaging techniques.

Some of the many reported examples in the literature are shown in Figs. 2.222.27. An example regarding radar industry is illustrated firstly in Fig. 2.22. In this set of images, it is shown a typical radome, Fig. 2.22(a), and two $\mathrm{THz}$ images which show delamination, Fig. 2.22(b), and water intrusion, Fig. 2.22(c), [134]. Radomes protect the radar against weather conditions. The usual construction consist in a sandwiching fiberglass and structural foam, thus providing strength and transparency to microwaves. When an object strikes the radome it could cause the separation of the fiberglass from the foam, thus implying delamination. The void caused by the delamination allows the water to get in the radome, thus degrading the radar beam. Nowadays, inspection of radomes is manual ("tap test"), and, as can be understood, this is prone to operator and does not provide any way to record the measurement for comparison at a later date. Much thoroughness can be gained with $\mathrm{THz}$ imaging.

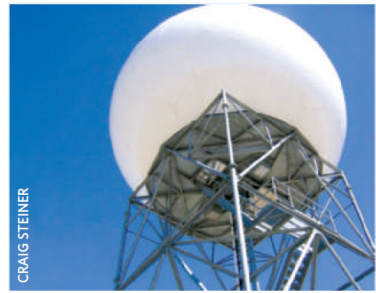

(a)

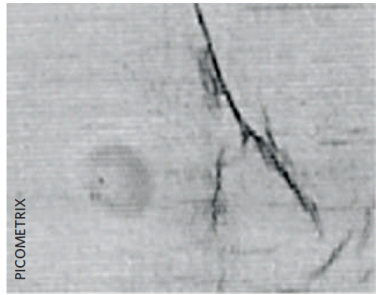

(b)

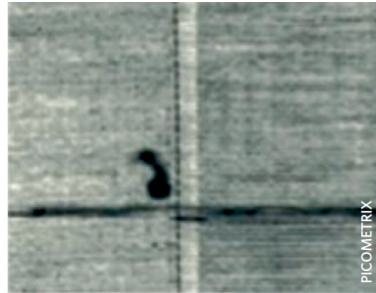

(c)

Figure 2.22: T-ray imaging in industry 1. Example of $\mathrm{THz}$ imaging suitability for non-destructive testing of radomes. a) Photography of a typical radome; b) $\mathrm{THz}$ scan revealing radome delamination; c) is a $\mathrm{THz}$ scan detecting water intrusion in the radome [134].

Fig. 2.23 shows and example of the possibilities of $\mathrm{THz}$ imaging in the examination of automobile dashboards. This element usually consists of a pair of opaque plastic layers which are sandwiched with a $1 \mathrm{~cm}$ thick foam layer. The left image consist on a photograph of a portion of an automobile dashboard. The right image correspond to a $\mathrm{THz}$ capture of the area enclosed by the dashed rectangle of the photograph. An air bubble in the foam, which is invisible in the photograph is 
clearly identified in the $\mathrm{THz}$ image as a region of enhanced transmission. Observed horizontal dark lines in the $\mathrm{THz}$ image are originated by diffraction of the plastic cover edges, located at the back side of the sample. This plastic have screws passing through it which also appear in the $\mathrm{THz}$ image as black circles. When manufacturing dashboards, it is desirable to avoid air voids in the foam since they cause structural instability. It is worth noting that, although foam is a low-density air-polymer composite (very difficult to distinguish it from the air), since the composite voids are comparable in size to the $\mathrm{THz}$ wavelengths, moderate scattering losses take place. This is used as amplitude contrast mechanism, which as has been seen, provides valuable information [124].
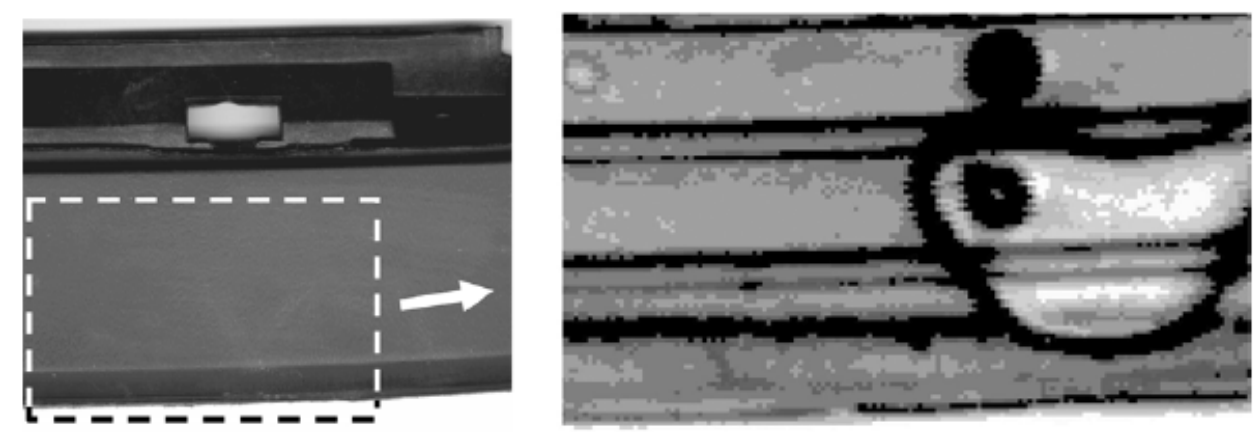

Figure 2.23: Visible image of a section of a car dashboard (left) and a $\mathrm{THz}$ image (right) corresponding to the area enclosed in the dashed rectangle [124].

A case of extreme importance, quality supervision of space shuttle foam structures, is exposed now in Fig. 2.24. It is shown a $\mathrm{THz}$ image which reveals eight built-in defects which include artificial voids and delamination defects [110]. This image was taken using CW configuration. It is worth noting that in this work, better results are obtained for this target by using $\mathrm{CW}$ in comparison with the pulsed imaging. The aforementioned importance of the quality in the foam structure of a space shuttle lies on the sever consequences of small defects in this element. One of the most notorious disasters of the National Aeronautics and Space Administration (NASA), the Columbia Space Shuttle destruction, was originated by defects in the shuttle foam [140], [141].

Other interesting example of industrial application of $\mathrm{THz}$ imaging is the inspection of integrated circuits through emission images. Emission images are not truly a terahertz near-field technique, since the information one obtains is not related to the spectroscopic properties of the sample in the terahertz range. Instead, it shows the ability of the sample to generate terahertz radiation, which involves the local optical absorption coefficient and the ultrafast charge carrier dynamics. A great potential of these images is that if one measures the emitted $\mathrm{THz}$ field as a function of the position of the illumination spot on the emitting substrate, one can form an emission image with spatial resolution determined by the optical spot size, not by the terahertz wavelength [124].

The previous technique is illustrated in Fig. 2.25, where $\mathrm{THz}$ emission images are superimposed to a visible image on a integrated circuit. At the left image the circuit is undamaged whereas at the right image one electrical line has been broken. The 


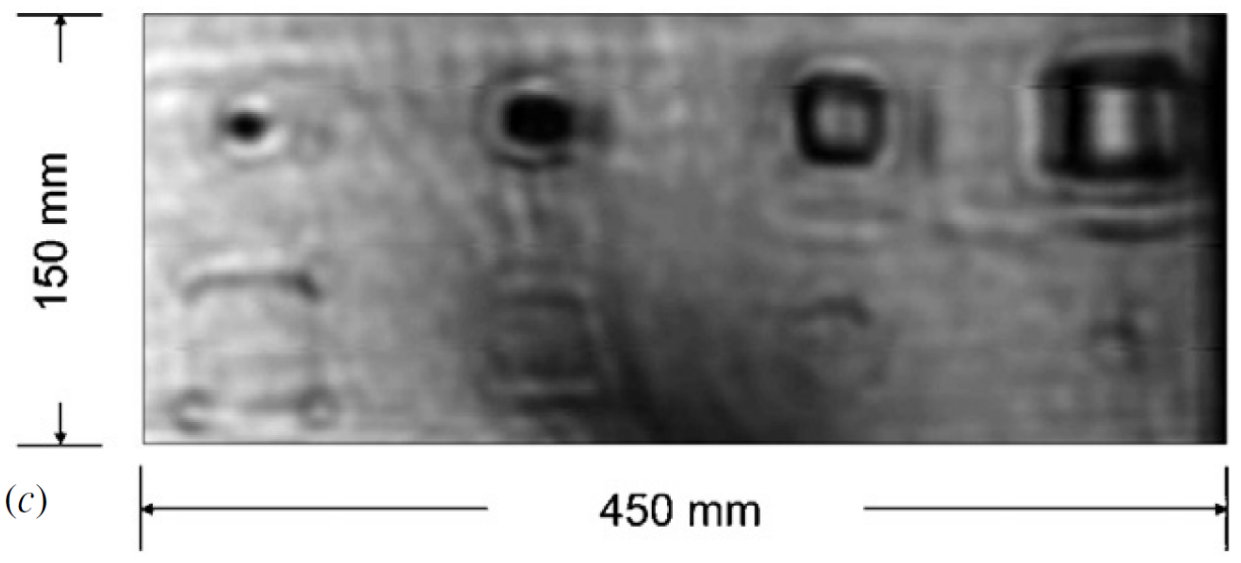

Figure 2.24: T-ray imaging in industry 2. Continuous wave $(C W) T H z$ image of a section of a space shuttle foam sample with eight built-in defects. [110]

$\mathrm{THz}$ emission changes dramatically, indicating a significant change in the electric field. The inset shows a magnification of the region where the circuit has been damaged. A resolution of $3 \mu \mathrm{m}$ has been reported in that work [138]. Application of this technique could easily identify circuit faults with a fast non-invasive inspection.
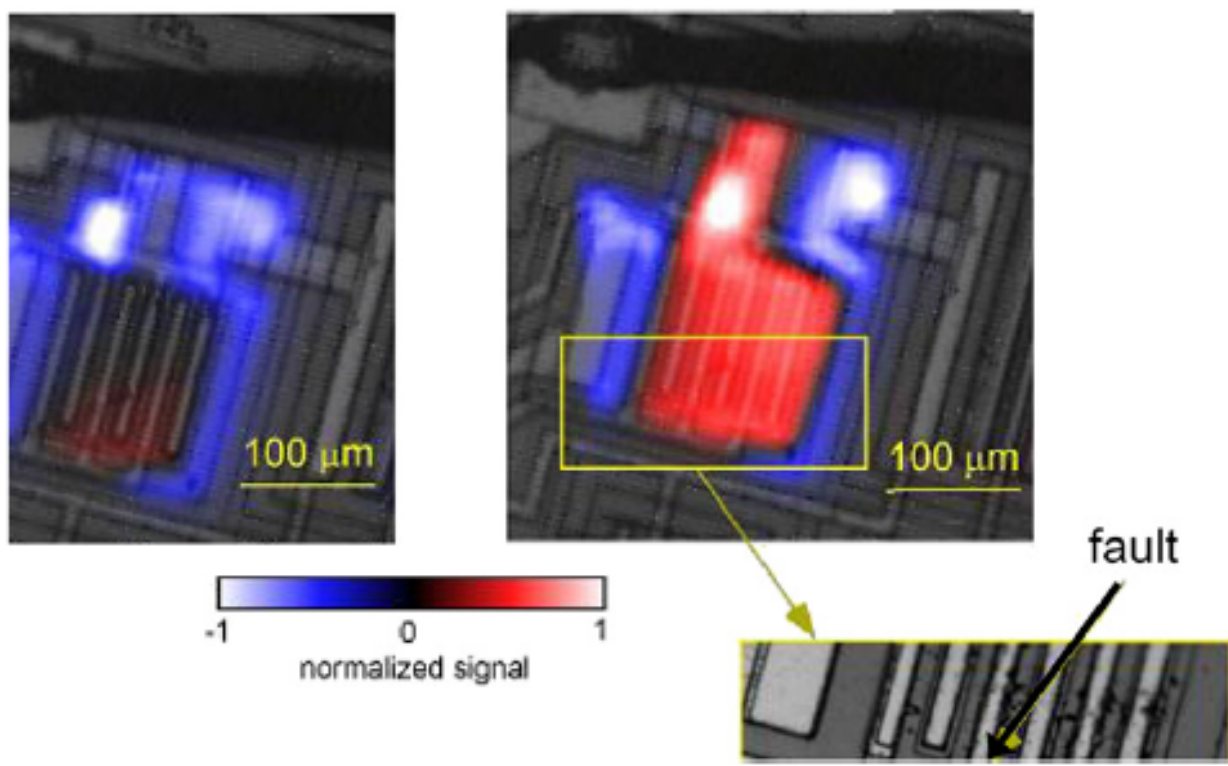

Figure 2.25: T-ray imaging in industry 3. Superimposed $\mathrm{THz}$ emission images over visible images of an integrated circuit. Left image correspond to an undamaged circuit whereas the right image correspond to same circuit after one of its electrical lines has been broken. The inset illustrates the damaged area with more detail [138]. 
Large $\mathrm{THz}$ absorption of dielectrics compared to air and very material dependent produces a great contrast with enormous potential for the plastic manufacturing industry, which has, furthermore, experiment a great revolution with the recently developed 3-D printing techniques. A great market is coming in the next years from the hand of more and more complicated 3-D plastic structures [142], [143].

In Fig. 2.26 it is well appreciated the $\mathrm{THz}$ contrast in transmission images through a plastic piece with drilled air holes. Whereas at the visible image, Fig. 2.26(a), only information of the surface is recovered, the THz images, Figs. 2.26(b) and 2.26(c), reveal with detail the internal structure [144]. A coherent imaging technique was used in that work. The image of Fig. 2.26(b) corresponds to the peak to peak amplitude response of the temporal pulse, and the image of Fig. 2.26(c) is the integrated intensity in the bandwidth comprised between $0.2 \mathrm{THz}$ and $0.3 \mathrm{THz}$. In the next years, pulsed $\mathrm{THz}$ techniques may contribute significantly in this area by providing accurate $3-\mathrm{D}$ structural information.

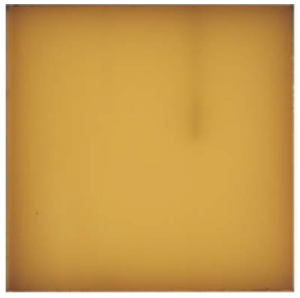

(a)

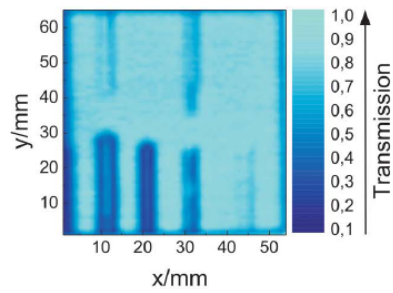

(b)

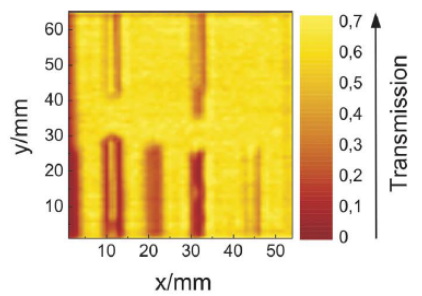

(c)

Figure 2.26: T-ray imaging in industry 4. Plastic piece with drilled air holes [144]. a) Visible image; b) Transmission $\mathrm{THz}$ image correspond to the peak to peak amplitude of the temporal response; c) Integrated intensity image in the range $0.2 \mathrm{THz}-0: 3 \mathrm{THz}$.

Industrial imaging applications end with an example regarding building industry. In Fig. 2.27 they are shown different transmission $\mathrm{THz}$ images (taken at $f=0.2$ $\mathrm{THz}$ ) of a wood sample in which some water has been injected. The large water absorption causes a strong attenuation in the area where it is located (blue areas). A real-time inspection of the evolution of the water distribution is obtained with this system. Also, it can be appreciated how differences of contrast reveal information of the internal structure of the wood. Since timbers obtain the highest strength when they are dry, and free water present inside a timber will accelerate the wood degradation, this technique for water inspection results of very high interest in the wood industry. Similar examples regarding other wood damages (such as wormholes or termite), water and cracks in concrete or defects of ceramic tiles, including transmission and absorption $\mathrm{THz}$ images are all treated in [145].

\subsubsection{Art \& Cultural Heritage Imaging}

Other surprisingly strong area of $\mathrm{THz}$ imaging is the art and cultural heritage conservation science. During the last years a large number of interesting works have been published. A very complete survey can be found in [86]. THz radiation is being recognized as a very interesting tool for dendrochronology (the science of dating woode objects [146]). Also, $\mathrm{THz}$ penetration in optically opaque materials allows 

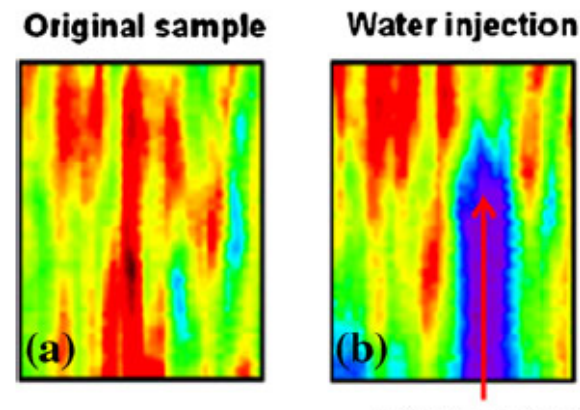

Water injection
Water dry out
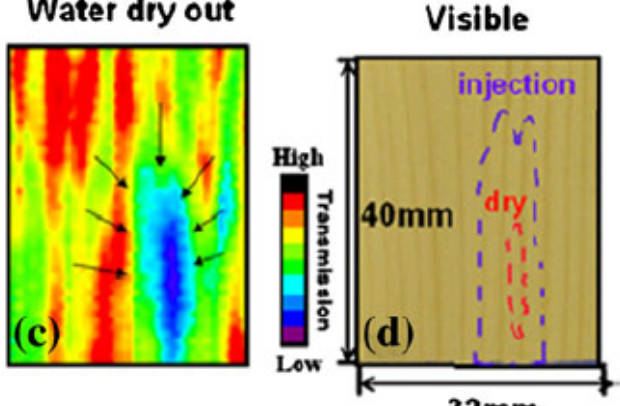

$32 \mathrm{~mm}$

Figure 2.27: T-ray imaging in industry 5. CW THz imaging at $f=0.2$ of a piece of wood with thickness of $14.2 \mathrm{~mm}$. The piece is injected with $1.55 \mathrm{~g}$ of water into an area of $3.5 \mathrm{~cm}^{2}$. a) $\mathrm{THz}$ image of the original sample; b) $\mathrm{THz}$ image of the sample at the moment of the injection; $\mathrm{c}$ ) $\mathrm{THz}$ image of the sample moments later when the water is drying out; d) the visible image. In the $\mathrm{THz}$ images, location an concentration of water is appreciated in blue to the high water absorption [145].

$\mathrm{THz}$ imaging for efficient subsurface imaging of culturally significant objects. The resolution, which goes from micrometers to several millimeters seems to be enough for this kind of purpose.

Other powerful nondestructive forms of radiation for cultural heritage imaging, such as X-rays, $\gamma$-rays, or magnetic resonance imaging present some inconvenient. On the one hand they are only available through large and complex installations intrinsically implying financial and logistical barriers. It is well-known that human health risk complicate the use of these technologies. On the other hand, these techniques may heat up unbound protons inside the sample or reaction with the ferromagnetic parts of the sample under study, thus losing in part their non-invasive feature.

By the above, imaging with the non-ionizing $\mathrm{THz}$ radiation results very suitable to inspect this very delicate, high-value and unique historical targets. In addition, recent technological advances point to hand-size $\mathrm{THz}$ imaging systems [147]. It is reasonable that cultural heritage preservation sciences are provided in the future with simple and compact $\mathrm{THz}$ cameras to carry out their work. $\mathrm{THz}$ imaging has been demonstrated in mummy inspection [148], papyrus and other old manuscripts inquiring [109], sealed vessels and pottery interior non-invasive inspection [149], art painting analysis, including discovery of hidden features [150], proving authenticity [151], or revealing the employed painting techniques through pulsed imaging [88], among others. Figs. 2.28-2.32 illustrate a short overview of this $\mathrm{THz}$ imaging usage.

In Fig. 2.28 two cases of historical art paintings studied with $\mathrm{THz}$ imaging are reported. Case 1, from [88], regards to the Polittico di Badia painting, dating from the XIV century. From left to right they are shown the visible image (with a yellow rectangle marking the studied area), the $\mathrm{THz}$ reflection image obtained by pulsed $\mathrm{THz}$ imaging, and the cross section image of part of the area under study, recovered with the temporal information of the $\mathrm{THz}$ pulse.

At the $\mathrm{THz}$ image they are clearly distinguished hidden gold leaves beneath the paint layers, thanks to the high reflectivity of this metal. In a similar way, it is concluded that the white area of the paint was made with a particular lead white, 
which has, specifically, the strongest reflection of all the common white pigments. Since, in this case, pulsed imaging is used, the temporal waveform provides further information and conclusions about the painting structure. The identified layers indicate that a new wood panel was inserted to compensate deterioration damage, and confirm that the artist followed a usual medieval procedure: he spread a gesso ${ }^{5}$ layer directly on the support to flatter the carved wood base, then the cloth was placed on the gesso, and finally, another gesso layer was used as a preparation layer for the painting.

In the case 2, from [153], it is studied an old wood painting with gold leaf and natural pigment obscured by a $0.5 \mathrm{~mm}$ layer of gesso. The visible image is shown at the top. The analyzed area is marked with a rectangle. A $\mathrm{THz}$ image from this area taken at $f=0.15$ is shown below. The visible opaque gesso zone is very transparent when imaging at $\mathrm{THz}$ frequencies. High reflectivity of gold at $\mathrm{THz}$ frequencies is used to determine the previously obscured shapes. In this work, it is pointed out that the interference between the sample and the end of the micro-probe provides phase information which can be used to enhance the image contrast, monitor the surface topological features or determine the complex refractive index of the target. In addition, pseudo-tomographic measurements can be built by scanning the target at different focal planes. Once more, $\mathrm{THz}$ waves provide a large number of tools to recover information of interest.
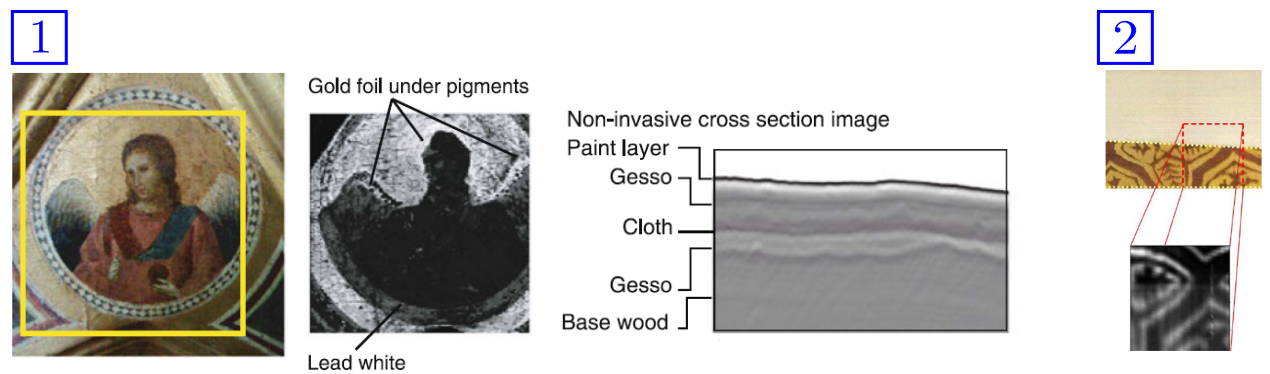

Figure 2.28: $T H z$ imaging in art and cultural heritage 1. Study of historical art painting structures. 1) From left to right: visible image of the Polittico di Badia with a mark indicating the area under study, the $\mathrm{THz}$ reflection image of the area under study, and a non-invasive cross section image recovered by the temporal information of the signal [88]; 2) Old wood painting with gold leaf and natural pigment obscured by a $0.5 \mathrm{~mm}$ layer of gesso. Visible image (top) and $\mathrm{THz}$ image at $f=0.15 \mathrm{THz}[153]$.

In Fig. 2.29, other interesting example regarding art painting is shown now [150]. In this case, previously processed material spectral information is used, and the imaging system is able to reveal buried layer information such as a graphite handmade sketch covered by several layers of painting. In this figure, they are shown a photograph of the prepared graphite sketch, Fig. 2.29(a), a photograph of the previous sketch after being painted with four different colors, Fig. 2.29(b), a $\mathrm{THz}$ transmission image obtained with the maximum value of the $\mathrm{THz}$ waveform,

\footnotetext{
${ }^{5}$ Gesso is a white paint mixture consisting of a binder mixed with chalk, gypsum, pigment, or any combination of these [152]. It is used in artwork as a preparation for any number of substrates such as wood panels, canvas and sculpture as a base for paint and other materials that are applied over it.
} 
Fig. 2.29(c), and a 3D THz map of the sample obtained the main pulse time delay (blue color indicates an increase of the optical pathway), Fig. 2.29(d).

Compared with the previous cases, where gold reflectivity clearly marked the painting shapes, this case results more challenging. In spite of this fact, the original sketch is recovered with good accuracy. It is not possible, however, obtain color information in the $\mathrm{THz}$ transmitted image, since similar absorption spectrum is observed for the different colours.

Again, the pulsed nature of the $\mathrm{THz}$ imaging allows to evaluate the variations of the painting thickness. The temporal information is able to give a little distinction between colours, but not enough. Signal processing techniques applied to the large quantity of information obtained in this technique may facilitate this secondary purpose.

In Fig. 2.30 the study of an ancient Egyptian mummified hand with the use of $\mathrm{THz}$ imaging and comparative with other techniques is illustrated. As aforementioned, this kind of historical rest are very sensitive, and totally non-invasive feature is difficult to achieve even with the well-established X-rays or magnetic resonance techniques. They are shown, the mummified hand as seen in the $\mathrm{THz}$ experimental setup, Fig. 2.30(a), the metacarpal bones (II-IV area) visualized with conventional X-ray Computed Tomography (CT), Fig. 2.30(b), a THz transmission image at $f=0.16 \mathrm{GHz}$, Fig. $2.30(\mathrm{c})$, and a $\mathrm{THz}$ pulse delay image with false colors indicating $\mathrm{THz}$ optical density of the specimen, Fig. 2.30(d). Although the X-rays are providing better spatial resolution and allow to a better tissue identification, the $\mathrm{THz}$ transmission image seems suitable to differentiate bone and cartilaginous structures from surrounding soft tissues. However, in this case, the key advantage of $\mathrm{THz}$ usage is the additional information given by the pulse delay image, which clearly overcomes X-ray CT results.

Other great example of the suitability of $\mathrm{THz}$ images instead of present conventional techniques is shown in Fig. 2.31. The set of images correspond to a work [149] which aim was confirm the ability of $\mathrm{THz}$ radiation to reveal the presence of organic materials embedded into a sealed piece of pottery. To carry out that objective, a red clay pottery of heigth $10.4 \mathrm{~cm}$ and diameter $11.8 \mathrm{~cm}$ coming from Mali was filled with different materials.

Photographs of the different fillings are shown in the first row. From left to right the materials are (1) empty, (2) dried flowers, (3) pine bark, (4) hazel wood slice of $1.1 \mathrm{~cm}$ thick and (5) white sand. Below each photograph they are displayed the X-ray image (b), the $\mathrm{THz}$ transmission image at $f=110 \mathrm{GHz}(\mathrm{c})$, and differential $\mathrm{THz}$ imaging (d), which is built by subtracting the empty jar and filled jar $\mathrm{THz}$ images.

The base case, the empty pot, looks similar in both, $\mathrm{THz}$ and X-ray imaging. It exhibits larger absorption in the lower part, caused by the larger thickness in this region. Regarding to the other images, the X-rays imaging provides a good resolution, however, it is not determined the object shape and/or nature. Organic materials remain practically transparent to this radiation. The $\mathrm{THz}$ images provide more precise information about the shape. Particularly, the differential images provide accurate information. In the case of an inorganic material (5), both X-rays and $\mathrm{THz}$ determine the presence of it inside the pot. Notwithstanding, $\mathrm{THz}$ seems to provide better spatial shaping of the content. It is worth to mention that the operat- 


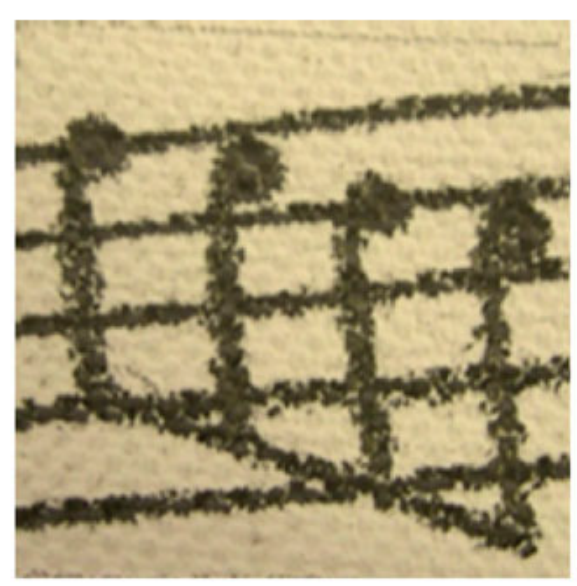

(a)

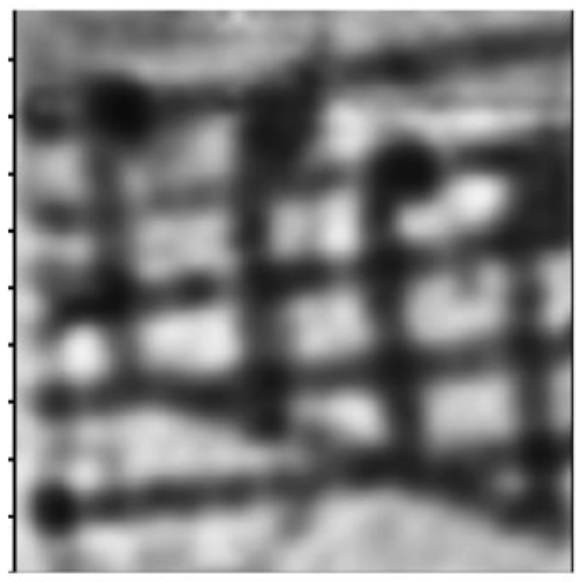

(c)

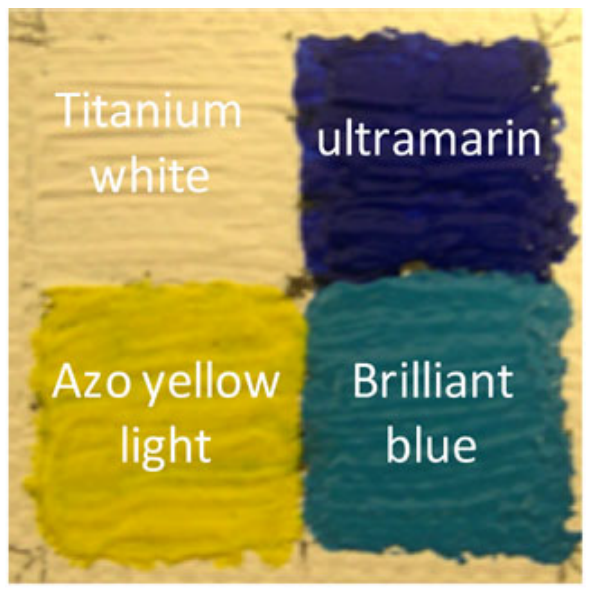

(b)

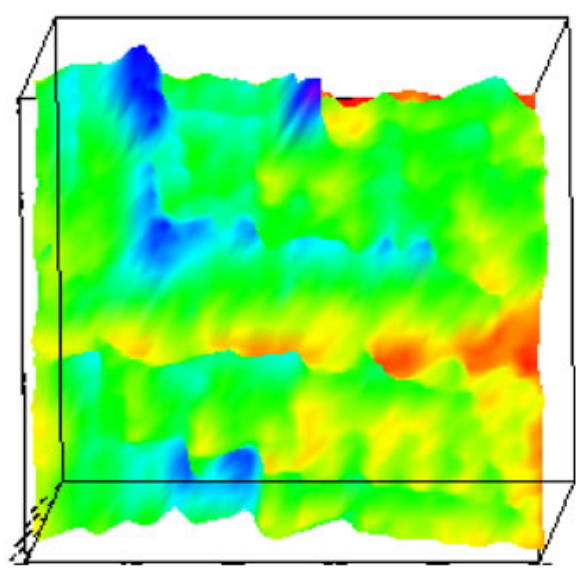

(d)

Figure 2.29: $\mathrm{THz}$ imaging in art and cultural heritage 2. Recovering a buried layer of graphite handmade sketch [150]. a) Photograph of the prepared graphite sketch; b) photograph of the sketch after being painted with four different colors; c) $\mathrm{THz}$ transmission image obtained with the maximum value of the $\mathrm{THz}$ waveform; d) $3 \mathrm{D} \mathrm{THz}$ map of the sample obtained from the main pulse time delay (blue color indicates an increase of the optical pathway).

ing frequency is low ( $f=110 \mathrm{GHz}$, which could be labeled as mm-wave rather than $\mathrm{THz}$ ), thus implying lower resolution than other possible $\mathrm{THz}$ higher frequencies.

Finally, a particularly interesting feature of $\mathrm{THz}$ imaging is presented in Fig. 2.32. The two shown images correspond to work [109], which aim was to prove $\mathrm{THz}$ potential in the full non-invasive inspection of ancient text, including the exploration of different layers/pages through modification the focalization point depth, and usage of temporal waveform information.

The image in Fig. 2.32(a) correspond to a experiment performed with a $8 \mathrm{~mm}$ separated papyrus layers. Both layers were handwritten using Arabic gum and other 


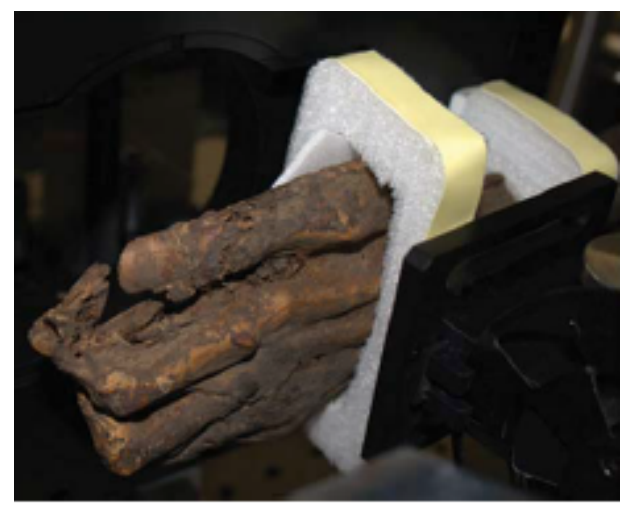

(a)

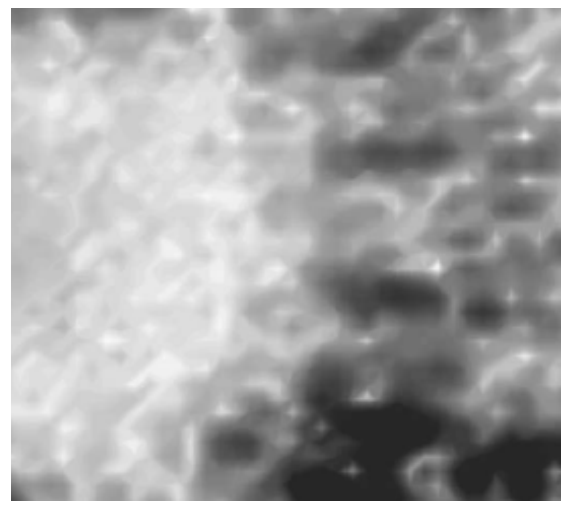

(c)

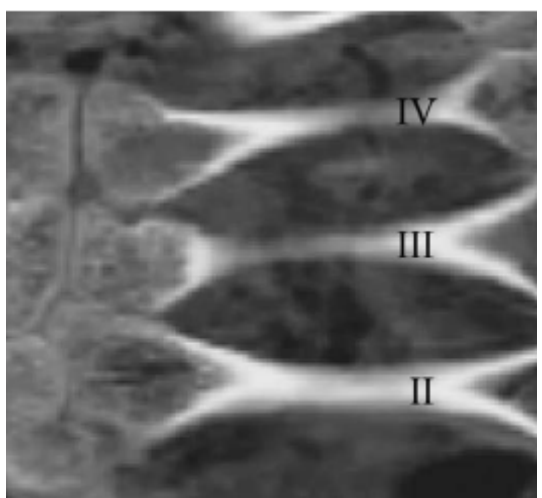

(b)

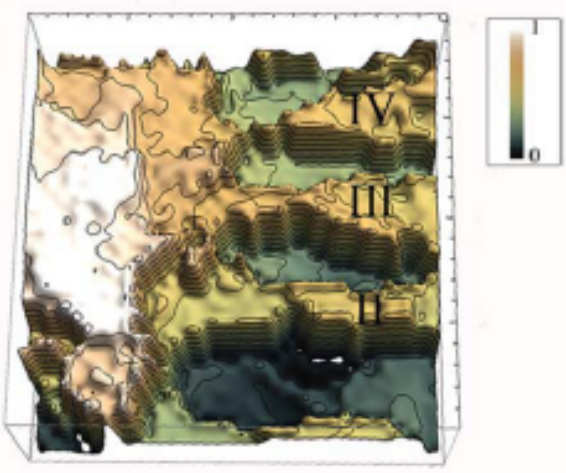

(d)

Figure 2.30: $\mathrm{THz}$ imaging in art and cultural heritage 3. Ancient Egyptian mummified hand inspection [148]. a) THz experimental set up; b) Metacarpal bones (II-IV area) visualized with conventional X-ray computed tomography $(C T)$; c) $T H z$ transmission image at $f=0.16 \mathrm{GHz}$; $)$ $\mathrm{THz}$ pulse delay image with false colors indicating $\mathrm{THz}$ optical density of the specimen.

two common pigments used historically. In the top layer it was written $I L E$ whereas at the bottom layer it was written $C 2 R M F$, the two organizations participating in this project. The used inks present a higher absorption in $\mathrm{THz}$ transmission and higher $\mathrm{THz}$ reflectivity than the base papyrus. The second layer is in focus, therefore, the C2RMF inscription is appreciated through the higher signal reflected. It is appreciated as well the shadow caused by the first layer ink absorption. The ink concentration benefits the quantity of reflected signal of the desired layer, but produces higher absorption when the signal crosses the first layer. The authors report that they are working in an algorithm able to correct this undesirable shadow.

The image in Fig. 2.32(b) correspond to other experiment, where a layer with $I L E$ written on was covered by five layers with different inscription and inks. The target layer was placed at $5 \mathrm{~mm}$ beneath the rest of the stack. Pulse information and proper focalization allow to recognize the desired characters. 
a (1)

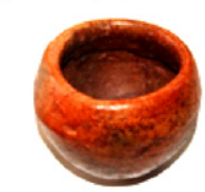

(2)

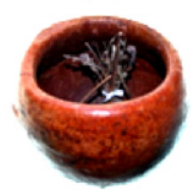

(3)

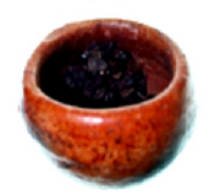

(4)

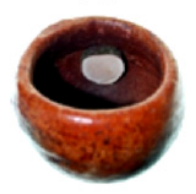

(5)
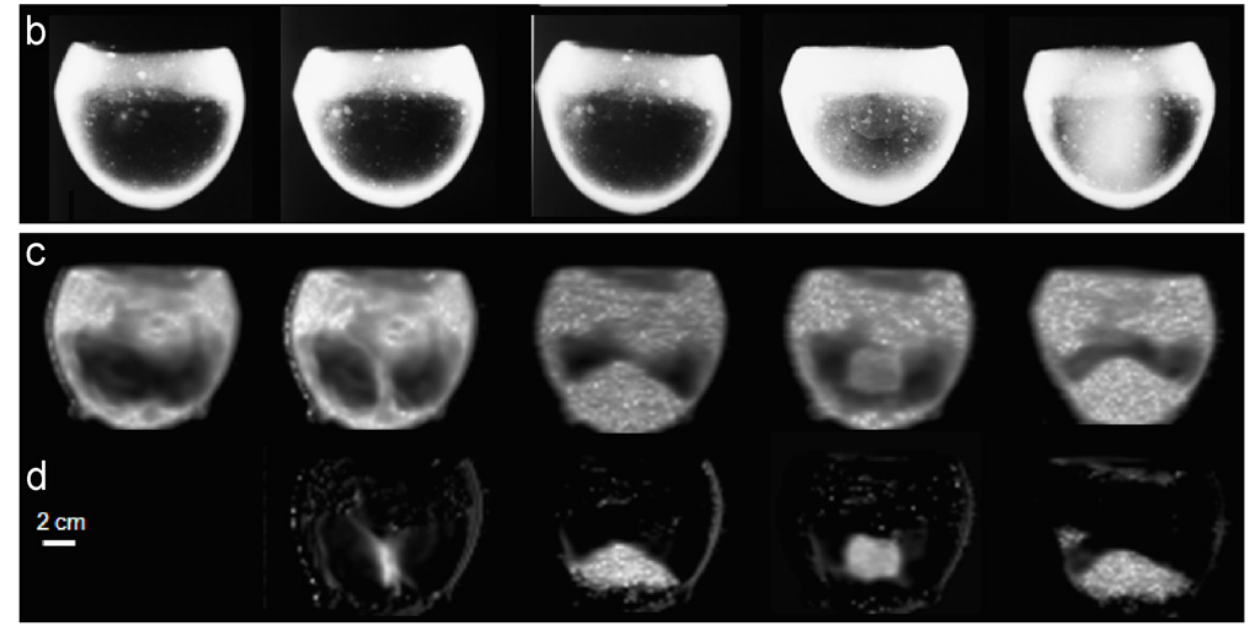

Figure 2.31: $\mathrm{THz}$ imaging in art and cultural heritage 4. Inspection of the interior of ancient pottery [149]. Regarding to the pot content, five cases are studied: (1) empty, (2) dried flowers, (3) pine bark, (4) hazel wood slice of $1.1 \mathrm{~cm}$ thick and (5) white sand. First row (b) show photographs of the considered cases, second row (b) present X-rays images, third row (c) are $f=0.11 \mathrm{THz}$ transmission images and fourth row are differential $\mathrm{THz}$ images created by subtracting the empty jar $\mathrm{THz}$ image to each of the $\mathrm{THz}$ images.

In these experiments the separation between papyrus layers is still large, but further technological improvements may allow this technique operate with distances between papyrus of less than $1 \mathrm{~mm}$. The reader can imagine the suitability of this technique to extract the written information without manipulating the layers or pages of the historic object. Many old manuscripts are nowadays inaccessible to archaeologist and historians due to the difficulty of opening the fragile specimen, which could be easily damaged, or due to the recycling of papyrus sheets, sometimes used in the past to produce book covers.

\subsubsection{THz Time Domain Spectroscopy Imaging (THz-TDSI)}

We conclude the T-ray or $\mathrm{THz}$ imaging review with an explicit illustration of $\mathrm{THz}-$ TDSI, which combine both, THz single frequency properties and spectral sensitivity of $\mathrm{THz}$ band to form set of images. At this point, it has been completely described with several examples that $\mathrm{THz}$ pulsed imaging contains spectral and temporal information, and a large quantity of information is manipulated. The possibility of order the results as a group of images (which may be built in video form) could be really helpful for the interpretation of the results, specially in the cases where the imaging system operator is not completely aware about the system technology. 


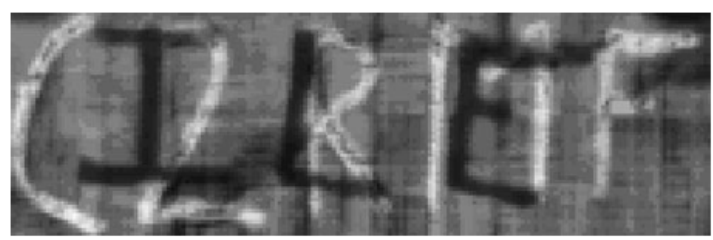

(a)

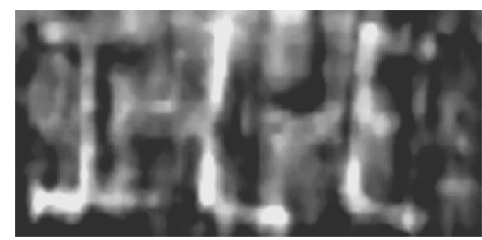

(b)

Figure 2.32: $\mathrm{THz}$ imaging in art and cultural heritage 5 [109]. Fully non-invasive papyrus and old manuscript reading with $\mathrm{THz}$ reflective imaging. a) Two papyrus with $1.3 \mathrm{~cm}$ of separation between them are analyzed. Focus is pointed to the second layer (C2RMF written); b) A papyrus layer (ILE written) beneath under 5 other layers (target layer is separated $5 \mathrm{~mm}$ from the 5-layer stack) is read with a proper focus location.

To better expose this possibility, an example related with security against drugs and explosives, Fig. 2.33, and a example related with pharmacology, Fig. 2.34, are shown.

The first example illustrates an experiment [72] to non-invasively differentiate powder forms of MDMA, methamphetamine and aspirine (the first two, the most employed illegal drugs in Japan, and the third, a pharmacological drug, eventually used as cutting agent). Fig. 2.33(a) shows a photography of the inspected target with the three samples. The observed powder inside the small polyethylene bags contains, from left to right, MDMA, aspirin and methamphetamine. The yellow mark indicates the imaging area, with size 20x38 mm. It can be observed that one bag is slightly longer. This fact is intended, and was used to avoid confusion during the sample manipulation due to the similar appearance between methamphetamine and aspirin.

Fig. 2.33(b) shows the absorption spectrum of the analyzed compounds in the considered band. This graph was previously exposed in this chapter as an example of the spectral sensitivity of $\mathrm{THz}$ to differentiate illegal or pharmaceutical drugs. It is appreciated how, at some frequencies, a pair of substances have similar absorption (see for example methamphetamine and aspirine at the lowest frequency or around $1.4 \mathrm{THz}$ ) and will be hardly distinguishable in an image. However, the same pair of substances present an absorption clearly different around $f=1.7 \mathrm{THz}$.

The previous fact can be appreciated in Fig. 2.33(c), where $\mathrm{THz}$ images of the area under test at seven different frequencies are shown. Regarding to the aforementioned classification of aspirin and methamphetamine, see how the $f=1.39$ $\mathrm{THz}$ results unclear for that purpose, whereas the $f=1.69 \mathrm{THz}$ image shows a determinant contrast between both samples.

A data processing algorithm is applied to the set of images taken at different frequencies and the spectral information. The result is a spacial pattern isolating each one of the substances (MDMA in yellow, aspirin in blue and methamphetamine in red), Fig. 2.33(d). As can be observed the grade of isolation for each case is of great accuracy, showing the capacity of this powerful technique.

The second example is part of the TERASENSE project [154]-[156]. In this particular experiment, the aim was show the advantages of using spectral imaging instead of single-frequency imaging for pharmaceutical compound identification and 


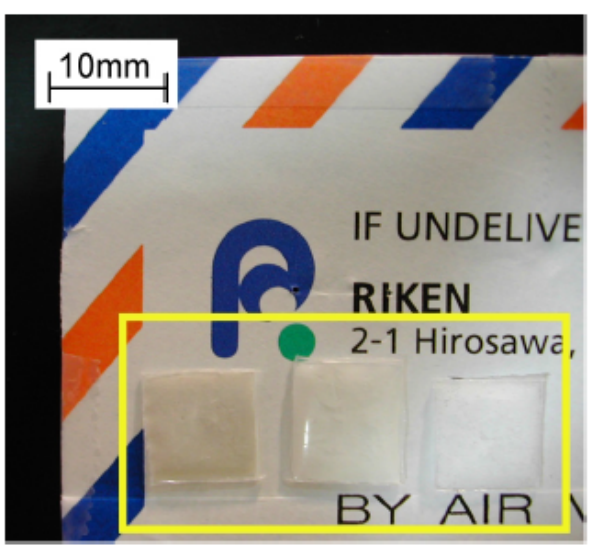

(a)

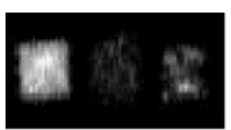

\section{$1.32 \mathrm{THz}$}

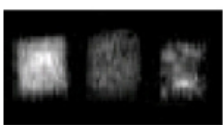

$1.39 \mathrm{THz}$
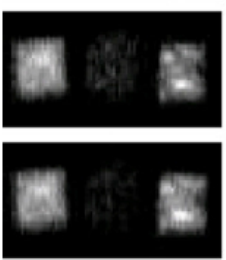

\section{$1.54 \mathrm{THz}$}

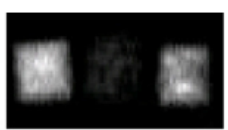

1.69THz

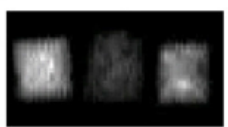

$1.84 \mathrm{THz}$

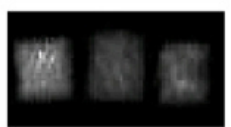

0.0

$1.98 \mathrm{THz}$

3.5

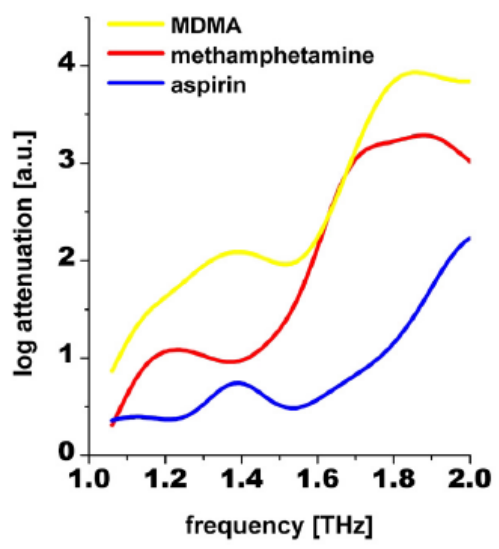

(b)
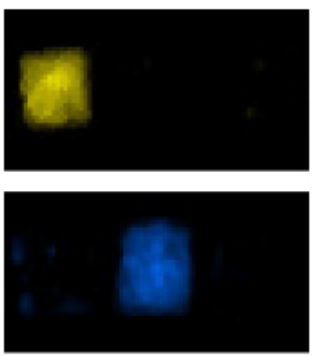

\section{Log attenuation}

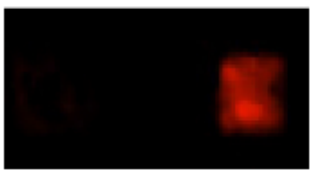

(c)

(d)

Figure 2.33: $T H z-T D S I$ for illegal and pharmaceutical drug identification experiment. a) Photograph of the samples under test. From left to right polyethylene bags filled with MDMA, aspirin and methamphetamine. Yellow mark indicate the imaging target, 20x38 mm in size; b) Absorption spectra of the considered substances in the imaging operating band; c) Seven multiespectral images in the considered frequency range; d) Extracted spatial patters of MDMA (yellow), aspirin (blue) and methamphetamine (red) [72].

spacial determination. In Fig. 2.34 they are shown the images relatives to the analysis of a Rohacell $\left(\varepsilon_{r}=1.05\right)$ cylinder acting as a holder of a cylindrical sample of Polaramine ${ }^{6}$. The Rohacell cylinder is $12 \mathrm{~mm}$ diameter and the inner Polaramide cylinder is $6 \mathrm{~mm}$, and its center is slight displaced in the $x$ coordinate.

Firstly, Fig. 2.34(a) shows the absorption spectrum of the Polaramide. A strong peak is found at $f=525 \mathrm{GHz}$. Fig. 2.34(b) shows the same information (the absorption spectrum of the Polaramide) calculated now by integration of the image

\footnotetext{
${ }^{6}$ Polaramine is the commercial name of Dexchlorpheniramine, an antihistamine with anticholinergic properties used to treat allergic conditions such as hay fever or urticaria. It is the pharmacologically active dextrorotatory isomer of chlorpheniramine [157].
} 
zone where the Polaramide is placed, for different frequencies. In this graph it is also displayed the absorption spectrum of Rohacell calculated by the same procedure. An inset with a photography of the sample under test has been included.

The eight multiespectral images used in the previous calculation are shown in Fig. 2.34(c). The observed central red circle correspond to the Rohacell cylinder. In most of the images, Polaramide cannot be distiguished. However, at $f=525 \mathrm{GHz}$, the peak of absorption of Polaramide modifies noticeably the received level in the image. It that image, it can be appreciated the spacial offset in the $x$ direction of the Polaramide cylinder. The last two images at frequencies $f>600 \mathrm{GHz}$ present a different colour configuration due to the decrease of the Signal to Noise Ratio (SNR). This example is another proof of the importance of capture images at different $\mathrm{THz}$ frequencies, only possible with THz-TDSI systems, in order to properly identify the target composition and its geometry. This example also suggest the possibility of obtaining the spectral signature substances located at certain positions in a similar way than that described in Fig. 2.10.

\subsubsection{Radar Applications}

Radar based applications in the $\mathrm{THz}$ regime are also calling to the attention these days. The use of $\mathrm{THz}$ spectrum supplies noticeable different conditions than those that can be found using microwave frequencies. Available bandwidth, wavelength and material absorption dispose a rather new scenario in terms of resolution, sensitivity, and kind of target.

With the small $\mathrm{THz}$ wavelengths, resolution under $1 \mathrm{~mm}$ is available [158]. This fact suggest the use of $\mathrm{THz}$ to illuminate scale models of large objects, thereby simulating the Radar Cross Section (RCS) of objects that are massive and complex to manipulate such as planes, tanks, and battleships. The savings in anechoic test chamber dimensions justify the use of this scale models and more expensive $\mathrm{THz}$ equipment, rather than use the common lower frequencies at which defense radar systems operate actually. Resolutions up to $\lambda / 100$ (of these small per se wavelengths) have been reported [159].

Two interesting examples from [158] and [160] are shown in Figs. 2.35 and 2.36. In [158] a coherent transceiver is used, with the phase stability of the received signal, sufficient to allow coherent imaging processing. In Fig. 2.35 they are shown the visible image of the scaled target (a 1:72 scale model of a T-80BV tank made of plastic and coated with $1200 \dot{A}$ of aluminum) and the $f=2.4 \mathrm{THz}$ azimuth/elevation imagery of the target, with pixel resolution of $0.4 \mathrm{~mm} \times 0.6 \mathrm{~mm}$. It was found a reflectivity of $99 \%$ for the aluminum in this experiment. The system setup simulates a Ka-band radar at $f=33 \mathrm{GHz}$. As can be seen, the wheels, the treads, the main gun, the machine gun, and the snorkel, and many other small features are easily distinguished.

Fig. 2.36 shows the schematic diagram of the THz RCS setup together with the scaled target (10 cm-long 1:150 metal model of aircraft fighter F-16), and the frequency-averaged-RCS of this target. Clear traces of scattering originated at the airplane nose, wings tips, fuselage, tail and exhaust pipe, are visible. In this experiment [160], a $\mathrm{THz}$ time domain system with freely propagating $\mathrm{THz}$ pulses and sub-picosecond time resolution was used. 


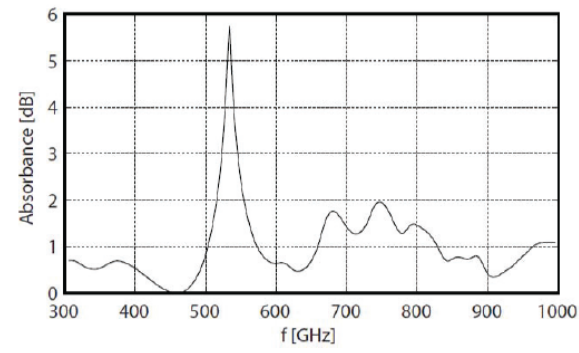

(a)

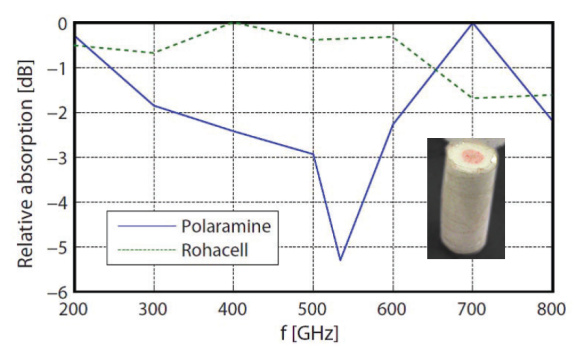

(b)

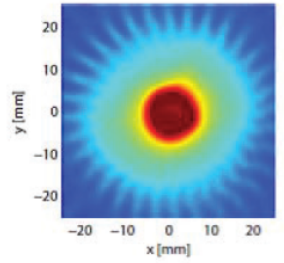

(a) $200 \mathrm{GHz}$

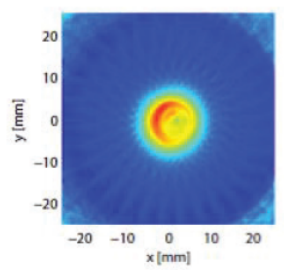

(f) $525 \mathrm{GHz}$

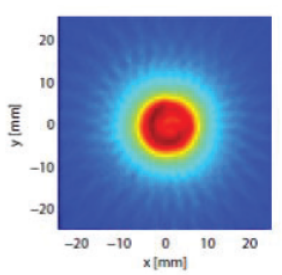

(b) $300 \mathrm{GHz}$

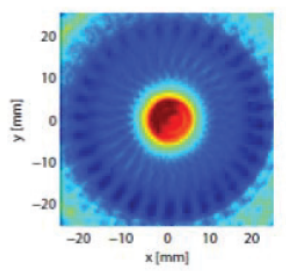

(g) $600 \mathrm{GHz}$

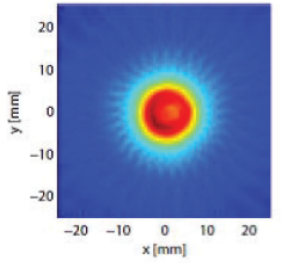

(c) $400 \mathrm{GHz}$

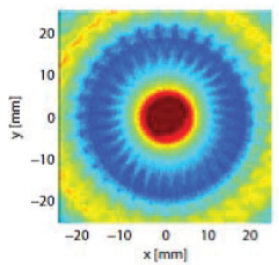

(h) $700 \mathrm{GHz}$

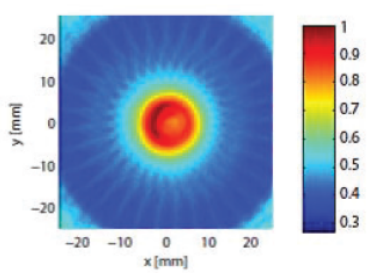

(d) $500 \mathrm{GHz}$

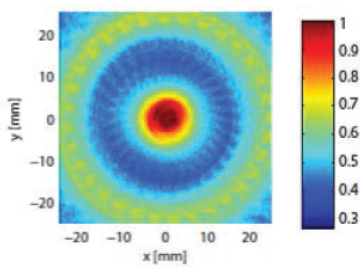

(i) $800 \mathrm{GHz}$

(c)

Figure 2.34: Use of THz-TDSI for pharmaceutical compound spatial and spectral identification. A Rohacell $\left(\varepsilon_{r}=1.05\right)$ cylinder $(12 \mathrm{~mm}$ radius) acting as a holder of a cylindrical sample of Polaramine (6 mm radius and slightly displaced in the $x$ coordinate) is analyzed. a) Absorption spectrum of Polaramide calculated through $\mathrm{THz}-\mathrm{TDS}$; b) Absorption spectrums of Polaramide and Rohacell calculated through integration of the image zone where the compounds are located in several $\mathrm{THz}$ images. Inset shows the cylindrical holder and sample; c) $\mathrm{THz}$ images of the target at eight differnt frequencies the [156].
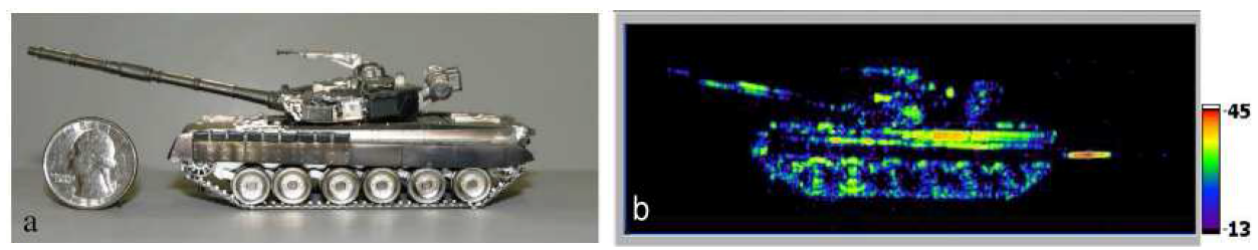

Figure 2.35: Scaled simulation of Ka-band radar. Visible (a) and $2.4 \mathrm{THz}$ azimuth/elevation images (b) of a 1:72 scale model of a T-80BV tank made of plastic and coated with $1200 \dot{A}$ of aluminium. $\mathrm{THz}$ image resolution is $0.4 \mathrm{~mm} \times 0.6 \mathrm{~mm}$. A calibration object (dihedron) is located to the right of the image [158].

These examples show very accurate results using scaled models of the real targets. The small size and weight of the used targets implies easy manipulation and allow 

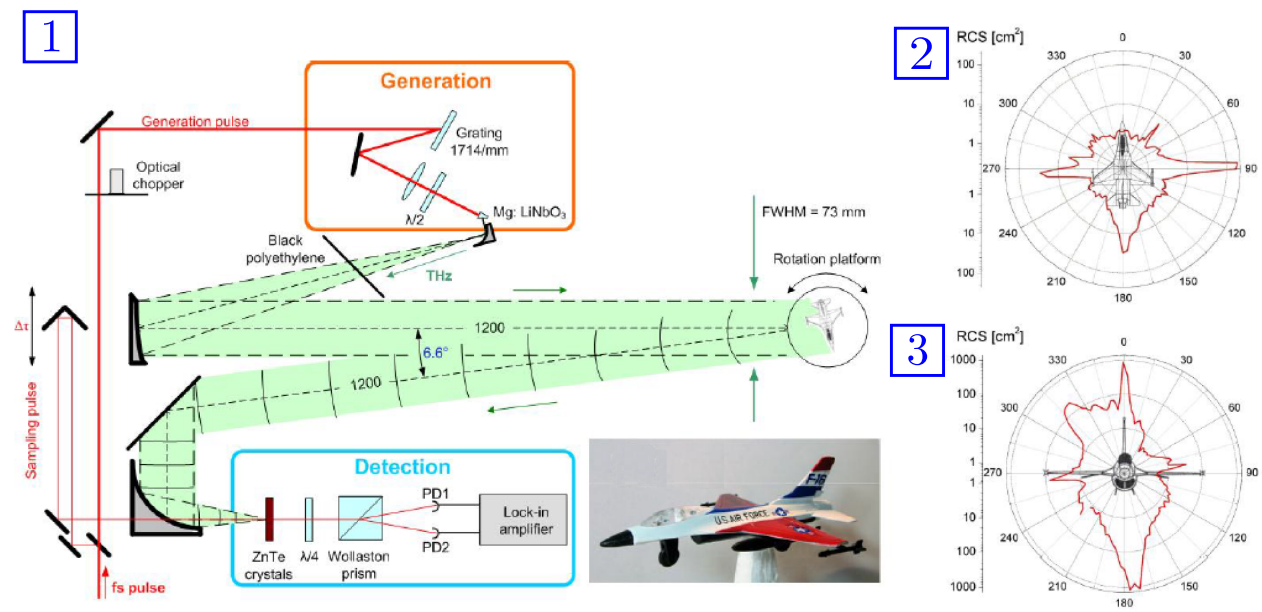

Figure 2.36: $\mathrm{THz}$ time domain radar. 1) Schematic diagram of the THz RCS setup and $10 \mathrm{~cm}$-long 1:150-scale metal model of aircraft fighter F-16; Polar (2) and Azimuthal (3) frequency-averaged RCS [160].

for iterative design and testing procedure, where the test object is manufactured by a rapid prototyping system such as a computer numerically controlled 3D milling machine or a $3 \mathrm{D}$ printer.

By the above, the intense dedicated research is well justified, being carried out presently with the aim of optimizing the employed systems to the $\mathrm{THz}$ conditions. In [161], an extensive experimental study of scattering of dielectrics in time domain is presented, motivated by the actual targets and $\mathrm{THz}$ systems nature. In [162], for example, the influence of Gaussian beam on $\mathrm{THz}$ RCS is deeply studied, since in RCS estimation, usually a plane wave is assumed, whereas in real measurements at $\mathrm{THz}$ frequencies, generally, a Gaussian beam or similar source is adopted. By using a canonical spherical example, errors less than $0.07 \mathrm{~dB}$ are reported with this approach, which seems necessary for $\mathrm{THz}$ radar systems.

Another interesting intrinsic $\mathrm{THz}$ radar feature is the capacity of accurate characterization of micro-Doppler effect [163]. Micro-Doppler effect arises from nonuniform linear motion of some parts of the target, for instance, uniformly variable motion, vibration, rotation, etc. These motions are usual in the rotation of the rotor blades on helicopters, ballistic missile warheads, or even heartbeat, swing of arms and legs and other movements of human beings. These motions induce modulation of the signals returned back from the target, and sideband appears beside the Doppler frequency. Thanks to the smaller wavelengths and higher bandwidth offered by $\mathrm{THz}$ compared to microwaves, this effect can be much better solved at $\mathrm{THz}$ frequencies, thus providing a new degree of target characterization.

Resolution and $\mathrm{THz}$ interaction with matter also suggest other kind of targets, non-metallic, less affordable for microwave bands. The range information can also be used to construct a 3-D map, which is more naturally interpreted by human observers. This is another indicator of how the range dimension of submillimeter imagery might be of great benefit to threat identification with lower probability of false alarms [116]. 
For example, in [164] it is presented an all-THZ fiber-based swept-source imaging radar operated at room temperature for real-time location of concealed moving live objects with high stability. Results of this experiment are shown in Fig. 2.37. They can be observed two sets of images, a) and b), corresponding to two different instants in which a live mouse was at different locations hidden inside an opaque box. Each set of images shows, from left to right, the system setup with the schematic of the mouse location, the radar imaged area corresponded to the fan-shaped region scanned by the rotating mirror, and photographs showing the actual location of the mouse.

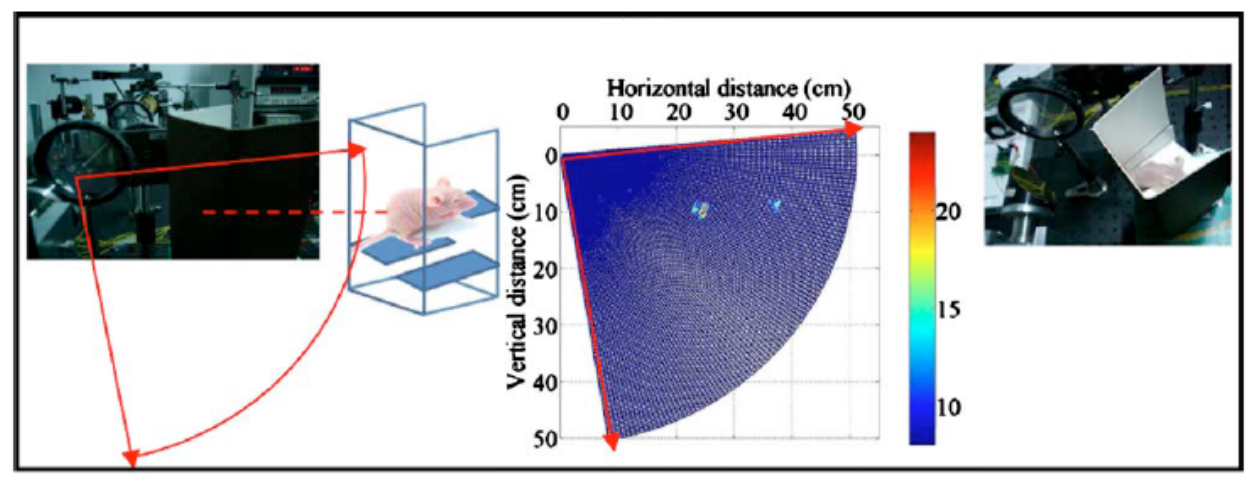

(a)

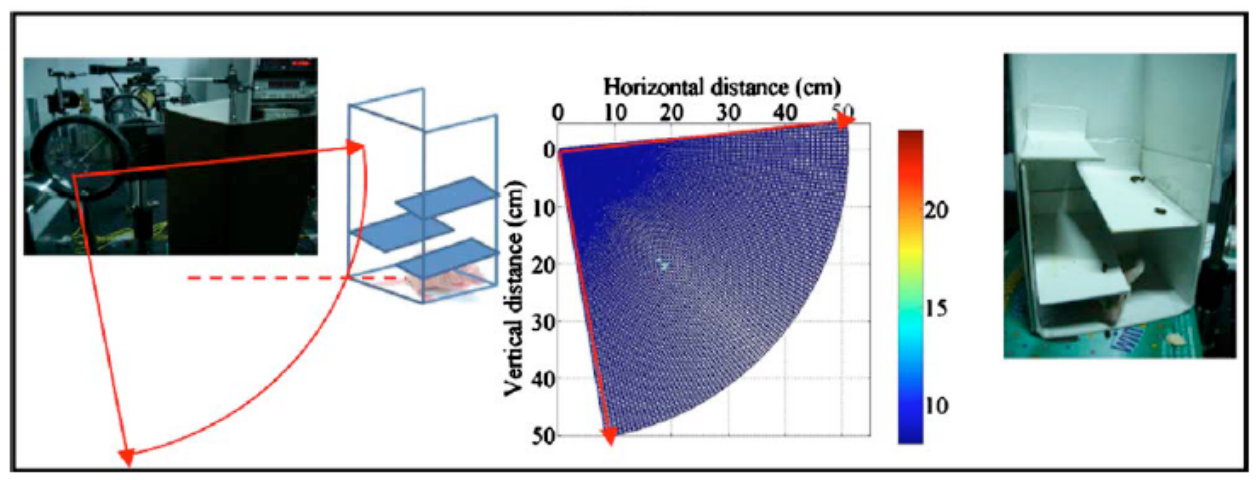

(b)

Figure 2.37: Swept-source $\mathrm{THz}$ radar images. A live mouse is considered as target, occupying two different locations inside an opaque box, as shown in a) and b). For each case they are shown, from left to right, a photography of the system, the radar colormap image, and a photography showing the actual location of the mouse. The radar imaged area corresponds to the fan-shaped region scanned by the rotating mirror. The two radar images were acquired with a time difference of $20 \mathrm{~s}$ [164].

The possibility of detecting and tracking living objects is very motivating. In this experiment, a reflection of $70 \%$ was reported for water in the employed range of frequencies, what causes provides high contrast in the color images. However some issues must be solved first. In the previously described experiment, acquisitions of 
$6.65 \mathrm{~s}$ were obtained, whereas the temporal delay between the two images was 20 s. Velocity is crucial in real-time systems, and lot of effort is carried out to solve this issue. As reported in [165], the lack of heterodyne transceivers arrays due to cost and complexity of constructing many parallel submillimeter-wave sources and receivers, is limiting these days the radar time acquisition velocity. In such work, velocity is doubled by time-delay multiplexing techniques, taking advantage of $\mathrm{THz}$ large available bandwidth. If efficient waveguides are developed next years, much more will be done or the realization of the required arrays.

\subsubsection{Communications Applications}

$\mathrm{THz}$ communications are reviewed now. Normally, the term THz communications may be encountered in the literature to reefer effective data rates exceeding $1 \mathrm{Tbit} / \mathrm{s}$ (usually using optical carriers), or communications with a $\mathrm{THz}$ carrier wave [166]. This last will be the case treated here, endeavoring to consider carriers beyond 300 $\mathrm{GHz}$, i. e., submillimeter wave band, since millimeter wave band will be particularly considered in the other part of this thesis.

The $\mathrm{THz}$ band offer a large range of of spectrum still unused for communications. Compared to microwaves, $\mathrm{THz}$ communications offer greater bandwidth due to the higher frequency of the carriers, and the advantage of smaller component size, which is fundamental for in-movement systems, such as satellites. Furthermore, licenses beyond $250 \mathrm{GHz}$ are inexistent, thus being the $\mathrm{THz}$ band license-free, presently [167].

On the other hand, as mentioned above in this chapter, atmospheric attenuation strongly limits the affordable distance between transmitter and receiver [40], [168]. It is worth to mention also that $\mathrm{THz}$ communications cannot be nowadays intended for large distance high data rate transmission as optics are presently used. Whereas at $\mathrm{THz}$ frequencies there is a lack of effective guided transmission media, at optical frequencies, silicon based fibers are practically lose-less. Furthermore, optical carriers offer even larger associate bandwidth.

Basically, due to the aforementioned features, three kind of applications are very suitable for $\mathrm{THz}$ communications: 1) Inter-satellite communications [169]-[171], 2) Ultra-wide band communications, including short-range indoor communications and "last mile" line-of-sight communications [168]-[173], and 3) Short-range secure tactical communication, mainly for military [174], [175], but also industrial purposes [176]. Some works [177] also suggest the possibility of satellite-Earth communications under dry conditions and using robust modulations, which try to preserve SNR at the expense of using more bandwidth. However, such options seems limited to very specific conditions.

Inter-satellite communications results fundamental nowadays due to the improvement in the whole system performance [178]. For such way of communication, atmospheric attenuation is not a problem for $\mathrm{THz}$ frequencies. Actually, beyond 16 $\mathrm{km}$ from the Earth attenuation at $\mathrm{THz}$ frequencies is practically null [167]. $\mathrm{THz}$ waves have some advantages over laser techniques due to the wider beam of the former, which make it easy the pointing between satellites. Furthermore, the information carried by $\mathrm{THz}$ frequencies cannot be intercepted by the satellite stations on earth since the atmosphere results noticeably opaque. Moreover, the reduction of the system size and weight due to the higher frequency used compared with mi- 
crowaves may have very positive influence in the cost of the satellite launching and maintaining [179].

Short range indoor ultra-wide band communications and "last mile" line-of-sight communications are considerably more claimed than the previous to the increasing date rates demanded by users. The former refer to an scenario where to devices want to share large amount of information in short-time, whereas the second scenario correspond to an alternative to optical wired links to support the huge aggregated bandwidth of the several channels offered by the service provider. Both cases require high date rate, hence bandwidth, and require wireless feature.

In many current wireless systems operating at microwave frequencies, data capacity has been able to be improved by increasing the spectral efficiency through advanced modulation schemes and signal processing technologies, recently developed [168]. However, despite the signal processing advances, the increasing demand of bandwidth will irredeemably require the increasing of carriers frequency.

Historically, individual, i.e., point-to-point, links have been increasing faster than wired links. The comfort and versatility of wireless connections is very attractive to the user, which day after day deal with media containing more information. Actually, according to Edholm's law of bandwidth [180], the demand for bandwidth in wireless short-range communications has doubled every 18 months over the last 25 years. As a example, in 1984 telemetry was offering less than $1 \mathrm{~kb} / \mathrm{s}$ whereas in 2009, Local Area Networks (LANs) were offering more than $100 \mathrm{Mb} / \mathrm{s}$ through the 802.11 standard. Therefore, they are predicted data rates of around $5-10 \mathrm{~Gb} / \mathrm{s}$ in 5 years. It has been suggested that $\mathrm{THz}$ communications system will replace Wireless LAN systems in 2017-2023 [172]. Also, it is expected that wired and wireless communications data rates converge about 2030 [167].

It is worth to mention, notwithstanding, that the term $\mathrm{THz}$ communications has sometimes more nature of mm-wave communications when reviewing the literature [168]-[173], inasmuch the considered frequencies are below the $300 \mathrm{GHz}$ barrier. Since this thesis review both bands, this clarification is necessary. $\mathrm{THz}$ frequencies will become an extension of the mm-wave band when this last begin to be busy for the required communication channels and increasing capacity.

Compared to the microwave band, the $\mathrm{THz}$ band is offering more bandwidth, but, on the other hand, more directivity is required. This last feature is inherent to the less free-space diffraction of waves. Line of sight will be required for $\mathrm{THz}$ systems, which, furthermore, hardly can overcome any obstacle due to the strong material attenuation.

Compared to Infrared Infrared (IR) waves, $\mathrm{THz}$ radiation suffers from less attenuation under certain atmospheric conditions such as fog. Moreover, in the IR band, time varying fluctuations in the real refractive index of the atmospheric path leads to undesired peaks and valleys. This imply that $\mathrm{THz}$ can provide longer links compared to wireless IR.

Regarding to "last mile" line-of-sight communications, THz communications will provide a huge data throughput easy and fast to deploy to cover certain areas or events in the cases that wiring is not suitable solution. As it has been indicated before, is not unreasonable to think that at some point in the future, $\mathrm{THz}$ wireless could be the first option in those cases [173]. 
However, it is worth noting that an important effort should be made to characterize the propagation channel. This imply not only characterizing atmospheric propagation, discussed previously in this section, but also describe properly the reflection and scattering on building materials in order to recreate and simulate accurately the actual scenario. Some works have been carried out these last years to afford this issue [181]-[183].

Also, is important to mention that, to sustain this commercial activity, implied technology not only must be satisfying the sometimes challenging specifications, but also must be cheap enough and compact. Therefore, all these promising expectations depend in the success of present research in Silicon-Germanium (SiGe), BiCMOS ${ }^{7}$ and Indium Phosphide (InP) technologies for planar integrated circuits, amplifiers, or antenna arrays. To date, most of the $\mathrm{THz}$ communications measurements have used alternative hardware, such as $\mathrm{THz}$ time-domain system or microwave mixers. There is still much work to be done (much more compared to mm-wave frequencies) [172].

Finally, secure communications, suggested mainly for military purposes (tactical communications), but also for secure transmission of relevant information in industry/corporation environments, arise from several inherent features of $\mathrm{THz}$ communications [172]-[186]. These features are highly directional beams compared to microwave communications, less scattering than IR (possibility of communicate in dust and smoke enviroments), limited propagation distance due to atmospheric attenuation, encryption of the beam, large channel bandwidth for spread spectrum techniques, which enable antijamming [187], low probability of detection systems, or to hide $\mathrm{THz}$ signals in the background noise.

The $\mathrm{THz}$ applications section ends here, and after the review, the reader can appreciate the huge amount of applications that may be possible at $\mathrm{THz}$ frequencies. Most of them are depending on the evolution of $\mathrm{THz}$ technology at these days. The described scenario can slightly vary next years. Other new applications will arise on the future, as creative ideas coming from the state of the art of $\mathrm{THz}$ technology. As observed through this section, some features that can be an inconvenient for certain purposes, become an advantage to others. Therefore, to consider the THz spectrum will, for sure, contribute noticeably to the society wellness.

\section{$2.3 \mathrm{THz}$ Technology}

The review on the state of the art of $\mathrm{THz}$ technology starts with the indisputable main driver of $\mathrm{THz}$ revolution: $\mathrm{THz}$ sensors. Great advances in this area have without doubt been the major trigger of the actual $\mathrm{THz}$ technology expansion [34], [188]-[190]. It continues with the THz sources, a critical element that is still far from the desired level of performance and that, with the research nowadays, may hopefully give an important impulse to $\mathrm{THz}$ systems. The review ends with the state of the art in $\mathrm{THz}$ waveguides, which are probably the most challenging element to be efficiently implement at $\mathrm{THz}$ frequencies [191], [192] (note that all the aforementioned $\mathrm{THz}$

\footnotetext{
${ }^{7} \mathrm{BiCMOS}$ is an developed semiconductor technology that integrates both, Bipolar Junction Transistor (BJT) and Complementary Metal-Oxide-Semiconductor (CMOS) transistor, semiconductor technologies in a single integrated circuit device [184].
} 
applications are based on radiated transmission, and guided elements are generally avoided to the lack of efficient waveguides). This last part of the section will drive the reader to the understanding of the main aims of this thesis.

\subsubsection{THz Sensors}

Detection systems are usually subdivided into two groups, heterodyne systems and direct systems. Heterodyne systems are coherent, i.e., they allow to detect not only the signal amplitude, but also its phase. Direct systems are non-coherent and they detect only the signal amplitude. This feature is important from the point of view of the information available after detection, which directly influences in important detector parameters such as detector sensitivity and spectral resolution [34], [188][190]. The detectors are used in both detection systems are basically the same, but in some cases, some of them are not suitable for coherent systems due to their relatively long response time. For example, this is the case of most uncooled thermal detectors [188].

Nowadays, there exist a large variety of $\mathrm{THz}$ radiation sensors (detectors). Some of them come from traditional schemes, e.g., all different kind of bolometers ${ }^{8}$, and others are new propositions based on different principles and materials, which have appeared recently due to the $\mathrm{THz}$ technology demand: optical readout-bimaterial based infrared Focal Plane Arrays (FPAs) [194]; heterojunction/homojunction interfacial workfunction internal photoemission detectors [195]; quantum dot detectors [196]-[197]; nanobolometers [198]; cold electron bolometers [199]; plasma wave detection by Field Effect Transistors (FETs) [200]-[201], different kind of antenna detectors [202]; Schottky diodes using semiconducting single-walled nanotubes [203]; carbon nanotube bolometers [204]; room temperature bipolar semiconductor Hot Electron Bolometers (HEBs) [205].

Since the field is vast, only the most common sensors will be described next. Then, main characteristics and use of both, heterodyne and direct, detection will be commented and compared. THz detectors can be classified in two broad categories: photon detectors and thermal detectors. Characteristics of them and few examples of each class are described now.

\subsubsection{Photon detectors}

In photon detectors, the radiation is absorbed within the material by interaction with electron either bound to lattice atoms, to impurity atoms, or with free electrons. The observed electrical output signal results from the changed electronic energy distribution. They exhibit both good signal-to-noise performance and a very fast response. However, to achieve this, the photon detectors require cryogenic cooling. This is necessary to prevent the thermal generation of charge carriers. The thermal transitions compete with the optical ones, making non-cooled devices very noisy. Photoconductive Antennas (PAs) [206]-[208], Schottky-Barrier Diodes (SBD) [203]-[211], Superconductor-Insulator-Superconductor (SIS) [212]-[213], and the most recently

\footnotetext{
${ }^{8} \mathrm{~A}$ bolometer is a device for measuring the power of incident electromagnetic radiation via the heating of a material with a temperature-dependent electrical resistance. It was invented in 1878 by the American astronomer Samuel Pierpont Langley [193].
} 
proposed, Kinetic Inductor Detectorss (KIDs) [214]-[216], and Quantum Dot (QD) detectors [217]-[219], all of them shown in Fig. 2.38, are photon detectors.

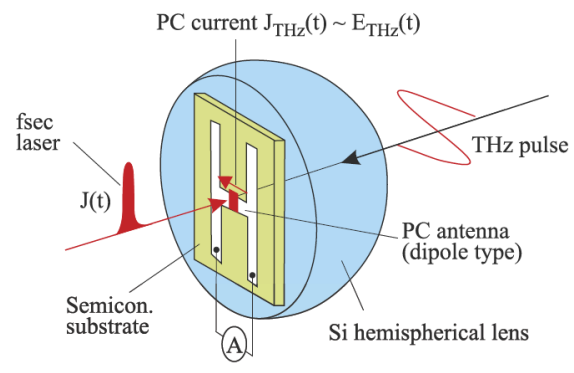

(a)
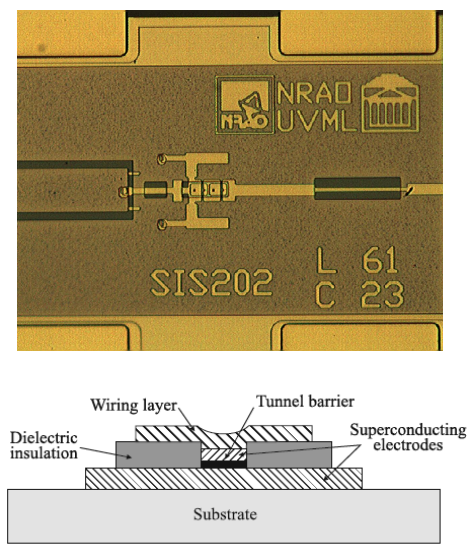

(c)

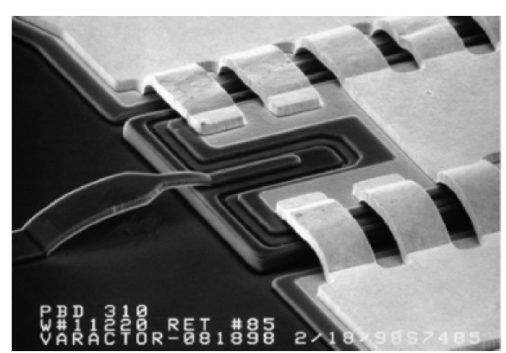

(b)

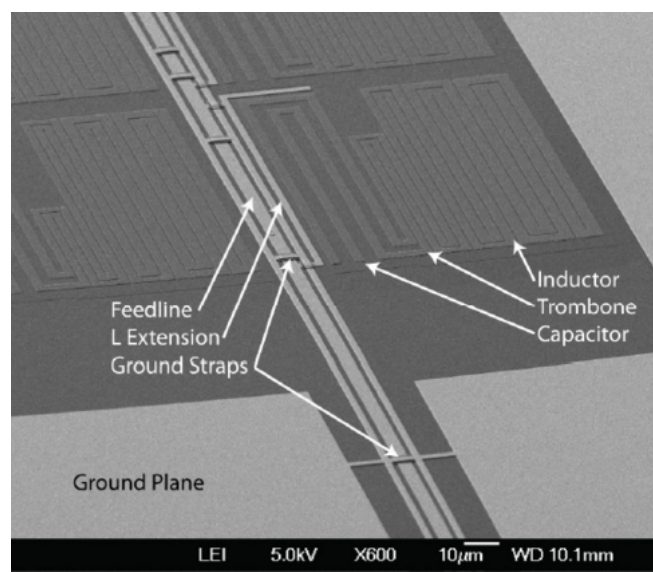

(d)

Figure 2.38: Several THz photon detectors: a) Photoconductive Antenna. Schematic of operation from [189]; b) Schottky Barrier Diode (SBD). Electronic microscope Image of a SBD from [220]; c) Superconductor-Insulator-Superconductor (SIS). Schematic from [189] and experimental device electronic microscope image [221] ; d) Kinetic Inductor Detectors (KID). Electronic microscope image of a single KID pixel, from [222].

- Photoconductive Antennas (PAs): In a photoconductive antenna, each pulse of a femtosecond laser producing an optical-pulse train separates into two path. One reaches the $\mathrm{THz}$ emitter, such as a photoconductive antenna, semiconductor wafer or nonlinear crystal, where the optical pulses are transformed into ultrashort electromagnetic pulses. These pulses propagate in free space, and are focused onto an ultra-fast detector, such as Low Temperature Grown (LTG) Gallium Arsenide (GaAs) photoconductive swith or electro-optic crystal. The second part of the pulse is also delivered onto the detector after passing through a time-delay stage [189].

Fig. 2.38(a) shows a schematic of a photoconductive antenna sensor (detector). It consists of an H-shaped stripline structure deposited on a semiconductor substrate. The electric field of the focused incoming $\mathrm{THz}$ radiation 
from femtosecond laser, induces a transient bias voltage across the $1 \mu \mathrm{m}$ to $5 \mu \mathrm{m}$ gap between the two arms of this receiving antenna. Thus, when the laser pulse coincides spatially and temporally with the $\mathrm{THz}$ electric field of the incoming $\mathrm{THz}$ radiation, a photocurrent is induced that is proportional to the incident electric field. By delaying the laser pulse, relative to the $\mathrm{THz}$ pulse, the time-dependence of the current can be measured. Since the laser pulse is narrow in comparison to the time duration of the $\mathrm{THz}$ pulse from a laser induced semiconductor emitter, the laser acts as a gated sampling signal.

PAs have been one of the main drivers of coherent detection in the past [223], presenting sensitivities orders of magnitude larger than thermal detectors. However, PAs are based on highly resistive semiconductors. Thus, impedance matching is a challenge nowadays [224]. Several topologies including bow-type [206], horn structure [225], fractal [226], etc, have been proposed these last years .This kind of detectors are widely used to detect broadband $\mathrm{THz}$ radiation in applications such as Time-Domain Spectroscopy (TDS) or imaging.

- Schottky-barrier Diodes (SBDs): This kind of diode offers some advantages over more classical p-n junction photodiodes. Specifically, Schottky barrier photodiodes are easier to manufacture due to their simple metal barrier deposition process and their lack of high-temperature diffusion processes. Furthermore, since a SBD is a majority carrier device, minority carrier storage and removal problems do not exist, thus, higher bandwidths can be expected. SBDs have high speed of response and the thermionic emission process in them is much more efficient than the diffusion process. This implies that, for a given built-in voltage, the saturation current in a Schottky diode is several orders of magnitude higher than in the p-n junction. In Fig. 2.38(b) it is shown a microscope image of a bridged SBD [220].

SBDs are widely used as detectors in the $\mathrm{THz}$ band, either in direct detection, or as nonlinear element in heterodyne receiver mixers, operating at temperatures ranging from cold $4 \mathrm{~K}$ to ambient $300 \mathrm{~K}$ [34, 189, 227, 228]. During the 80 s and early 90s cryogenically cooled SBDs were used in mixers preferably. Nowadays they have been widely replaced by SIS or HEB mixers, later reviewed in this section. During the last decades it has been shown that heterodyne SBD receivers are, in general, worse than cooled HEB receivers and SIS mixers. However, SBD receivers operation without cooling is an interesting feature very suitable for many of the applications described in this chapter, mainly those focused to the general public.

- Superconductor-Insulator-Superconductor (SIS): The SIS detector pertain to the class of pair braking photon detectors, based in superconductivity materials. If the temperature is far below the transition temperature, most of electrons in them are banded into Cooper pairs [229]. Photons with energies exceeding the binding Cooper pair energies in superconductor can break these pairs producing quasiparticles (electrons). This process resembles the interband absorption in semiconductors, with a small energy gap. One of the advantages of these detectors is that the fundamental noise due to the random generation and recombination of thermal quasiparticles decreases ex- 
ponentially with temperature. Specifically, the SIS detector is a sandwich of two superconductors separated by a thin $(\approx 20 \dot{A})$ insulating. Fig. 2.38(c) shows an schematic (bottom) and electronic microscope image (top) of a SIS detector [221].

Nowadays, the SIS configuration is mainly used as mixer in heterodyne type $\mathrm{THz}$ receivers because of its strong non-linear I-V characteristics, although it can be used also as direct detection detector. The usual operation temperature of SIS detectors is below $1 \mathrm{~K}$, typically $T<300 \mathrm{mK}$ [189]. Below $f=0.7 \mathrm{THz}$ SIS mixers are among the most sensitive and low intrinsic noise structures, being almost quantum limited. However, it should be noted that beyond this frequency, intrinsic noise increase noticeably due to the losses increment. Gain of sensitivity is possible using multielement or matrix arrays. Notwithstanding, SIS detectors do not present a easy array integration. In spite of this fact, SIS mixers seem to be best solution for the ground-based radio-astronomy in the $\mathrm{THz}$ band for $f<1 \mathrm{THz}$. Compared to the previously described Schottky Barrier Diodes, SIS mixers demand much lower Local Oscillator (LO) power ( $\mu \mathrm{Ws}$ instead of $\mathrm{mWs})[34]$.

- Kinetic Inductor Detectors (KIDs): This kind of detector, also known as Glsmkid was recently developed in the Jet Propulsion Laboratory (JPL) of the California Institute of Technology (CalTech), in 2003 [214]. This device operates at cryogenic temperatures, usually below $1 \mathrm{~K}$, offering hihg-sensitivity, which has been shown very suitable for astronomical signal detection purposes.

In a KID, an incident photon hits the surface of a superconductor and it breaks Cooper pairs (two weakly bound electrons) in the material. The inductance of the material is inversely proportional to the Cooper pair density, so an absorbed photon increases the inductance. Combined with a capacitor, the inductance creates a resonant circuit with a characteristic frequency. This frequency thus changes with the absorption of photons. If the circuit is excited by a microwave probe tuned near the resonant frequency, photons will be seen as changes in the phase and amplitude of the probe signal. These circuits are very high quality resonators (the $\mathrm{Q}$ factor, or center frequency divided by the width of resonator, is about 18000), so photon energy and arrival time can be determined to within several percent and in the order of $\mu \mathrm{s}$, respectively.

Compared to the other photon detectors, KIDs are a more novel concept. It is worth to mention that KIDs can be easily multiplexed for large arrays. Other advantages of this kind of detector are high responsitivity and good power handling. However, although KIDs are much more developed at other bands such as X-rays, THz KID technology still requires further advances to compete with more mature technologies [230]-[231].

- Quantum Dot (QD) Detectors: These detectors are the most popular solution for single-photon counting (other structures such as charge-sensitive IR photransistors -CSIPs- may also afford this task [217]). In the conventional photodetectors, see Fig. 2.39(a), one electron is excited by one photon and is at best carried to the drain of the structure. Under this configuration, the current 
responsivity is bounded by a term which depends on the photon energy, which is inherently low at $\mathrm{THz}$ frequencies compared to higher frequency bands.

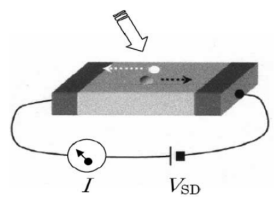

(a)

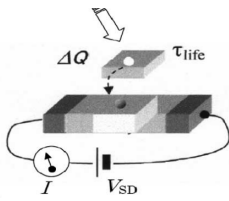

(b)

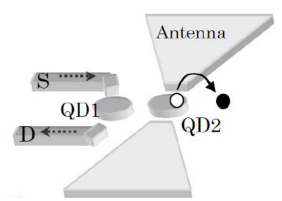

(c)

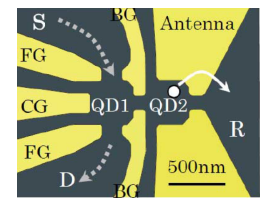

(d)

Figure 2.39: Quantum Dot (QD) Detector [217]. a) Conventional photodetector; b) QD photodetector; c) Double Quantum Dot (DQD) photodetector; d) Practical realization of a Double Quantum Dot (DQD) photodetector.

However, if the scheme of Fig. 2.39(b) is employed instead, a photon is absorbed by an isolated small semiconductor island and an electron tunnels out of the island, so that the island becomes positive charged (a "hole"). The excited electron outside the island is separated from the hole by a potential barrier, yielding a long-recombination lifetime of the excited electron-hole pair. This noticeably increases the number of electrons carried from the source to the drain of the structure per one absorbed photon. Therefore, the responsivity reaches very high values, giving to this structure an outstanding sensitivity The most straightforward realization of a QD detector is through a Double Quantum Dot (DQD), shown in Figs. 2.39(c)-2.39(d) [217].

The single-photon counting, well established in higher frequency bands (X-ray, Ultraviolet (UV), visible and near infrared) was already postulated as a good candidate for high sensitivity sensing at the starts of the past decade [1], [196]. Not so far, these predictions are becoming true through the effort in this area the past decade [217], and new structures, in which detection is carried out on an electrically driven single chip, point towards $\mathrm{THz}$ integrated circuits for on-chip quantum optical experiments [219]. The $\mathrm{THz}$ researcher must keep one eye on new advances of this technology.

\subsubsection{Thermal detectors}

Thermal detectors base their operation in the change of material temperature due to the incident radiation. This change of temperature produces some physical property changes, used to generate an electrical output. Unlike photon detectors, the signal does not depend on the photonic nature of the incident radiation. Thus, thermal effects are generally wavelength independent, i.e., the signal depends on the radiated power (or its rate of change) but not on the spectral content. Since the radiation can be absorbed in a black surface coating, the spectral response can be very broad. Here, three thermal detectors are treated: bolometers, pyroelectrics and Golay cells, all of them shown in the same order in Fig. 2.40. In bolometers, changes are detected in their electrical resistance, in pyroelectric detectors, the internal electrical polarization is the sensitive parameter, and in the case of Golay Cells the sensitive change is an increase of current caused my mechanical movement of mirrors due to gas expansion. When not cooled, these detectors present in general slow response. 
Brief description and main features of these three kinds of thermal detectors is given next.

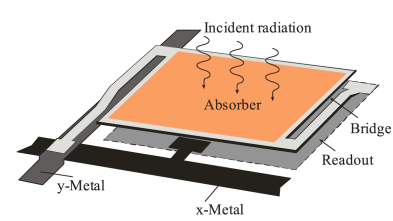

(a)

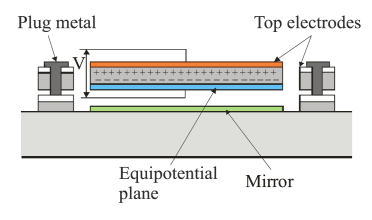

(b)

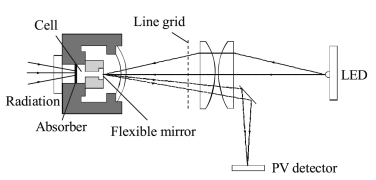

(c)

Figure 2.40: Several THz thermal detectors: a) Bolometer; b) Pyroeletric detector; c) Golay Cell. Images from [189].

- Bolometers: This kind of detector is formed by a resistive element from a material with a very small thermal capacity and large temperature coefficient so that the absorbed radiation produces a large change in resistance. The change in resistance is similar to that of a photoconductor, however, the basic detection mechanism is different. In the case of a bolometer, incoming radiated power produces heat within the material, which in turn produces the resistance change. There is not direct photon-electron interaction. At $\mathrm{THz}$ regime, semiconductor and superconductor bolometers are preferred due to their extremely sensitive low-temperature (specially for these last) [34], [189].

Several conventional semiconductor bolometers based on bismuth or tellurium have been successfully used for direct $\mathrm{THz}$ detection [34], [232]-[233]. Nonwithstanding, $\mathrm{THz}$ frequencies demand that, if the bolometer is to be used as a $\mathrm{THz}$ mixer, it has to be fast enough to follow the Intermediate Frequency (IF), i.e., the overall time constant of the processes involved in the mixing has to be of the order of tens of ps at maximum. Therefore, high heat conductivity and small heat capacity are required. These requirements can be fulfilled by such subsystem as far as electrons in semiconductor or superconductor interact with the lattice (phonons). Electron heat capacity is many orders lower compared to the lattice one. Here arises the hot electron configuration. Whereas in the normal bolometer, the crystal lattice absorbs energy and transfers it to the free carriers via collisions, in hot electron bolometer, the incident radiation power is absorbed directly by free carriers, the crystal lattice temperature remaining essentially constant. HEBs are fast enough to allow $\mathrm{GHz}$ output IF bandwidths.

HEBs based in semiconductor played an important role as a mixers in the early sub-mm wave astronomy of the 80s [234]. However, they were overcome by SIS mixer at the 90s. Nowadays, room-temperature detectors have limited applications outside diagnostic measurements due to sensitivity constrains. It has been the development of the superconducting HEBs [235] which has keep this device as a suitable option for high sensitive detection in $\mathrm{THz}$ band. Superconducting HEBs are significantly more sensitive than SBDs but somewhat less sensitive than SIS mixers. A brief comparison between heterodyne receivers comprising this three kinds is carried later. 
- Pyroeletric Detectors: These detectors are formed by a small capacitor with two conducting electrodes mounted perpendicularly to the direction of spontaneous polarization, see Fig. 2.40(b). During incident radiation, the change in polarization appears as a charge on the capacitor and a current is generated, the magnitude of which depends on the temperature rise and the pyroelectrical coefficient of the material. The signal, however, must be chopped or modulated. The detector sensitivity is limited by either amplifier noise or by loss-tangent noise.

Pyroelectric detectors can be designed in order to be useful for fast laser pulse detection, however with proportional decrease in sensitivity [189]. Therefore, these detectors are used presently for $\mathrm{CW}$ detection in imaging applications at room temperature [236]-[237]. A very recent work [238] concludes that pyroelectric detectors offers lower SNR than microbolometers at high frequencies in the $\mathrm{THz}$ band. Thus, is a candidate to keep an eye on.

- Golay Cells: These detectors consist of a hermetically sealed container filled with gas (usually xenon for its low thermal conductivity) and arranged, so that expansion of the gas under heating by a photon signal distorts a flexible membrane on which a mirror is mounted. The movement of the mirror is used to deflect a beam of light shining on a photocell and, in this way, produces a change in the photocell current as the output. In modern Golay Cells, the photocell is replaced by a solid state photodiode and light emitting diode is used for illumination. The performance of the Golay cell is only limited by the temperature noise associated with the thermal exchange between the absorbing film and the detector gas, consequently the detector can be extremely sensitive. However, the response time is rather slow, being usual a response of the order of ms.

Golay Cells are neither compact or fast. However, they offer high sensitivity for room temperature operation, overcoming pyroeletric detectors [110], and are easy to implement. Several recent works consider this kind of detector in their systems [239]-[240]. Continuous wave imaging at room temperature is a good niche for Golay Cells [241].

\subsubsection{Heterodyne (Coherent) vs. Direct (Incoherent Detection)}

After the short review on $\mathrm{THz}$ sensing structures, most relevant characteristics of these devices are known, and it is easier now afford a brief description and comparative of the two types of detection: heterodyne (coherent) and direct (non-coherent). In heterodyne systems, a heterodyne circuit design is used to give proper amplification to the signal, since so far, efficient amplifiers lack at $\mathrm{THz}$ frequencies. The detected signals are transferred to much lower frequencies, usually ranging from $f_{i}=1 \mathrm{GHz}$ to $f_{i}=30 \mathrm{GHz}$, where low-noise amplifiers do their job, see Fig. 2.41(a). In directs systems, all the detection and amplification process is carried at the operating frequency, i.e. THz frequencies, Fig. 2.41(b). 


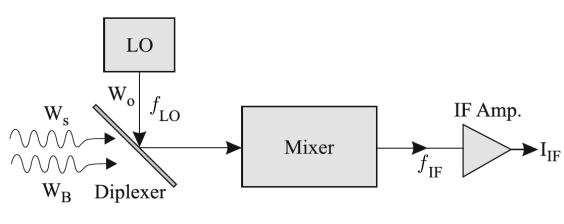

(a)

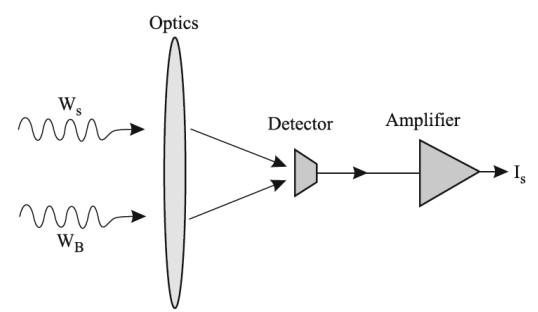

(b)

Figure 2.41: Simplified schematics of heterodyne and direct detection. a) Heterodyne detection. b) Direct detection. $W_{s}, W_{B}$ and $W_{o}$ are the signal power, background radiation power and local oscilator power, respectively. $f_{L O}$ and $f_{I F}$ are the local oscilator and intermediate frequencies, respectively. Schematics based on [189].

Firstly, if one compares $\mathrm{THz}$ band with shorter wavelengths, it is found that photon energies are low, of the order of $\mathrm{meV}$, and the Airy disk ${ }^{9}$ is rather large, diameter is about hundred of $\mu \mathrm{m}$. On the one hand, low photon energies imply that ambient background thermal noise almost always will dominate naturally emitted narrow-band signals, requiring cryogenic cooling of the detector elements or integration time consuming radiometric techniques, if not both. On the other hand, a large Airy disk makes indispensable a mode converter o matched antenna between the signal and the sensor element, i.e., unlike higher frequencies, such as optics, $\mathrm{THz}$ beams are not "naturally" focused in the usual, small, required spot [18], [34], [188], [189].

Additionally, it must be pointed out that the crossover frequency at which an ideal quantum detector (photon detector) overcomes the sensitivity (power spectral density) of an ideal thermal noise limited detector, such as a room-temperature Schottky barrier diode, takes places between $1 \mathrm{THz}$ and $10 \mathrm{THz}$, i.e., $\mathrm{THz}$ frequencies. This fact is also the foundation of the advantage of microwave detection systems over optical detection systems [34].

On the other hand, regarding to lower frequencies, one encounters that $\mathrm{THz}$ band suffers from lack of available electronic components such as lumped resistors, capacitors, inductors, amplifiers and low-loss transmission media. This has remarkably influenced the historical predominance of heterodyne detectors since, as it has been detailed in this chapter, $\mathrm{THz}$ technology has been driven by high resolution spectroscopy. This is changing, however, impulsed by a variety of less sensitivity demanding applications. More and more effort is being carried out these days towards direct detection techniques.

- Heterodyne (coherent): In these systems, the signals with $\mathrm{THz}$ frequencies are down-converted to an IF, located in the microwave regime, and preserving the amplitude and phase. Since amplitude and phase are preserved, it is possible, after the down-conversion, to process (including the most important task, amplification) this information through well-established electronic technology. The coherent feature of this detection allows to obtain higher spectral

\footnotetext{
${ }^{9}$ The Airy disk is the best focused spot of light that a perfect lens with a circular aperture can make, limited by the diffraction of light [242].
} 
resolution, which is a required characteristic of many applications, such as spectroscopic studies [25], remote space sensing [31], and some imaging techniques [243].

The crucial element of a heterodyne system is a mixer, the element that carries out the conversion to the IF. The mixer is responsible of the sensor responsivity and its conversion loss enhancement decreases the contribution into the noise heterodyne receiver temperature, and the consequent IF amplifier. Although signal power losses occur at diplexer and detector, see Fig. 2.41(a), the most noise of a heterodyne receiver comes from the mixer and its distributing circuits [189], [244].

To be used in a THz array, the mixer choice is dictated by the available LO power at these frequencies, the self mixer operating temperature and the required sensitivity. Depending on the availability of suitable LOs, two heterodyne techniques are possible. One uses a tunable LO and a fixed IF amplifier with filters. The other ones uses a fixed frequency LO in combination with IF amplifiers and filters to cover the needed frequency range. The first one is more flexible but tunable continuous wave sources providing enough power are required, something difficult these days as it will be justified later in this chapter.

Any nonlinear electronic device can be used as a mixer. However, efficient conversion and low noise in the $\mathrm{THz}$ band can be only achieved with few types of detectors. Most common choice are devices having strong electric field quadratic nonlinearity. Some examples are the froward biased SBDs, SIS tunnel junctions, and semiconductor and superconducting HEBs, previously described in this section. A comparative of noise temperature as function of the frequency is presented in Fig. 2.42. Before $f=1.3 \mathrm{THz}$, SIS mixers lead the low noise performance close to quantum limit. However, beyond that frequency one must better think in cooled HEBs. As commented before, the historically most used SBDs remain presently one step low in performance compared to the two former ones. This change is, actually, with great probability, the main driver in $\mathrm{THz}$ revolution.

- Direct (non-coherent): These kind of detection is widely used with high performance in spectroscopic applications at the ultraviolet, visible and infrared. At $\mathrm{THz}$, however, direct detection is suitable whenever the application does not require ultrahigh spectral resolution. On the other hand, unlike heterodyne detection systems, direct detection systems do not present the problem of multielement arrays formation conditioned by LO power and fast detector response (usually required to be below 1 ns due to the IF value) [189].

Direct $\mathrm{THz}$ detection systems can use even room temperature detectors with long response time, of the order of ms, whenever only modest sensitivity is required. Thermal detectors play a major role in those cases [189]. However, reasonable performance will usually demand from cooling [34]. Normally, antennas and focusing elements are an indispensable element to couple properly the power to the small thermal absorbing region. 


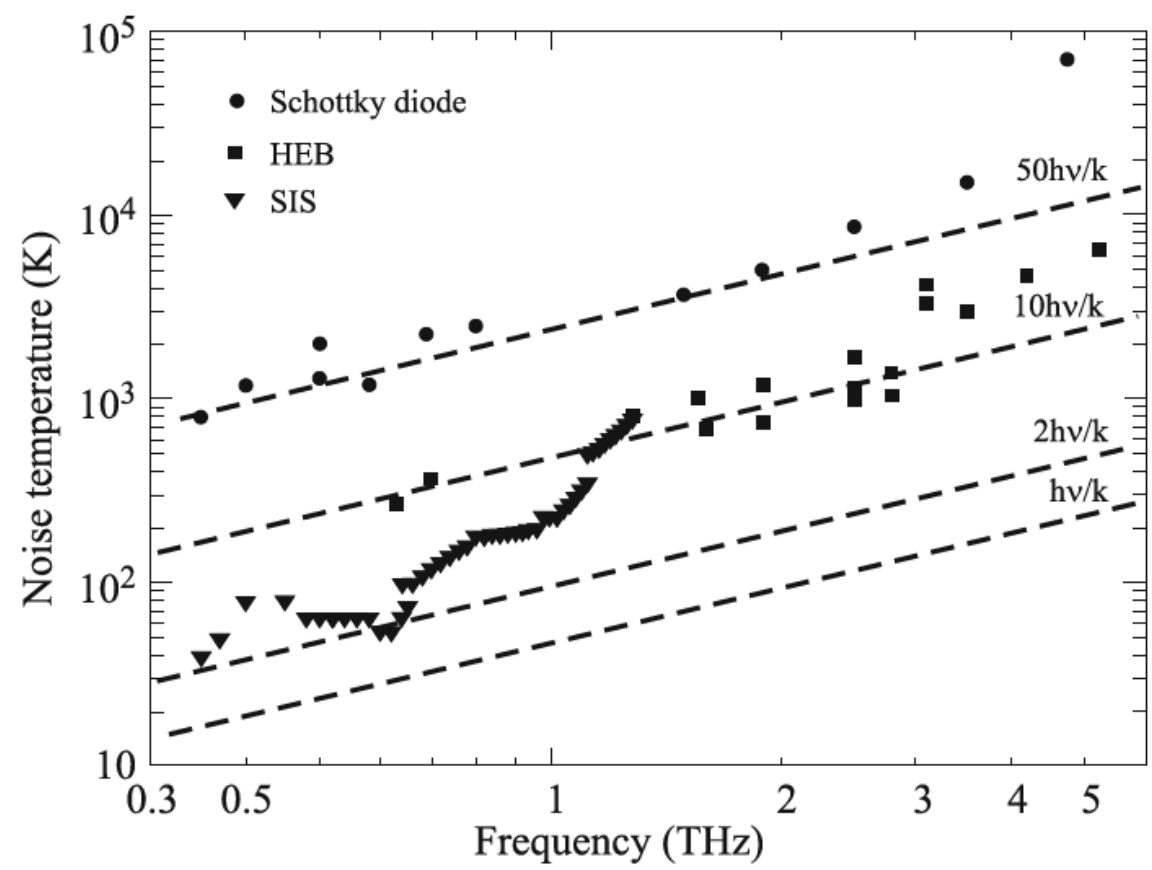

Figure 2.42: Comparative of $S B D, S I S$ and $H E B$ detectors acting as mixers for heterodyne $T H z$ reception [228].

For direct $\mathrm{THz}$ detection, thermal detectors are preferred at rather low frequencies. The use photon detectors is restricted to higher frequencies. For thermal direct detection, when fluctuation noise ${ }^{10}$ (background flux fluctuations) predominates, the minimum detectable signal is limited by the small but never vanishing ground photon noise. Therefore, performance of these detectors is background noise limited compared to the quantum noise limited of heterodyne systems.

In general, the threshold power detected by direct detectors will be higher than heterodyne detectors, mainly due to the stronger noises present by both, the detector itself, and in circuit elements, such the amplifier, operating at $\mathrm{THz}$ frequencies [189].

Summarizing up, heterodyne detectors offer more spectral resolution whereas direct detection detectors can operate in a wider spectral range. Direct detectors are preferable in those applications where spectral resolution does not play a major role. Finally, large format arrays are hardly built for heterodyne detectors whereas it is an easy task for direct detectors [246].

Whereas ultrahigh resolution spectroscopy demand from heterodyne systems, imaging is better afforded with direct systems. Thus, although traditionally heterodyne systems have lead $\mathrm{THz}$ technology, it is reasonable to think in an present and

\footnotetext{
${ }^{10}$ Thermal fluctuation noise, also known as phonon noise arises from the random exchange of energy between a thermal mass and its surrounding environment [245].
} 


\section{Heterodyne (coherent)}

- Can detect frequency modulation and phase modulation.

- More discrimination against background fluctuation noise.

- IF conversion provides gain. Output signal hard to be overrode.

- Conversion gain proportional to $W_{L O} / W_{s}$. Weaker signals detected.

\section{Direct (non-coherent)}

- Easy to produce large format array.

- Do not require beam coincidence on diameter and Pointing vectors.

- Beam waveforms do not require neither same radius of curvature nor same transverse spatial mode structure, nor same polarization.

Table 2.2: Comparative between heterodyne (coherent) and direct (non-coherent) detections.

future scenario where commercial applications impulse the use of direct detection systems. This section finish with the table 2.2 showing the main advantages of each one, heterodyne and direct, kinds of detection.

\subsection{2 $\mathrm{THz}$ Sources}

It is time now for the $\mathrm{THz}$ sources, which together with the sensors conform the basis of any $\mathrm{THz}$ system. It can be said that $\mathrm{THz}$ sources have also experimented a great advance in the last decades, which also contributes to present $\mathrm{THz}$ technology potential. However, although some authors [3] may underline them as the main driver, the advances in $\mathrm{THz}$ sources are not at the same level as those in $\mathrm{THz}$ detectors [1], [13], [189]. Therefore the term of 'THz gap' become a reality when talking about sources. Actually, sources imply an inherent larger difficulty grade than sensors. Several reasons support this fact. One the one hand, electronic solidstate sources based on semiconductors, i.e., oscillators and amplifiers suffer from high frequency degradation due to parasitic reactances and ohmic losses. On the other hand, optical style sources, i.e., solid-state laser, should operate at very low energy levels (of the order of $\mathrm{meV}$ ) for $\mathrm{THz}$ frequencies. These levels are comparable to that of the lattice phonons (relaxation energy of the crystal) and only proper cooling can avoid this problem, at the obvious expense of increasing the cost and complexity of the device. Finally, free electron sources such as tubes suffer from the inherent scaling problem, that implies higher metallic losses originated by the employed extremely high fields (electric and magnetic) [1], [189], [247].

Until not so far, during the last 20 years, most successful techniques for generating terahertz power have come from frequency conversion, either up from microwave (mm-wave bands) or down from the optical and Infrared regions [1]. Like a valley 
in between two mountains, $\mathrm{THz}$ source topic has been attacked from both sides by researches, usually more specialized in only one of these areas, either microwave or optics. Furthermore, the attempts to intrinsically generate $\mathrm{THz}$ power are much less efficient than multiplying (microwaves) or mixing (optics) other frequency signals. Actually, if one display the several source designs according source performance vs. frequency, one obtains a ' $\mathrm{V}$ ' shape, marking a strong valley around $1 \mathrm{THz}$, see Fig. 2.43. For example, in the case of multiplier chains (black circles in Fig. 2.43) any added stage noticeably affects the output power [248], [249]. Also, design a Quantum Cascade Laser (QCL) [250], for such THz "large" wavelengths imply a toll in efficiency [251], see red squares of Fig. 2.43.

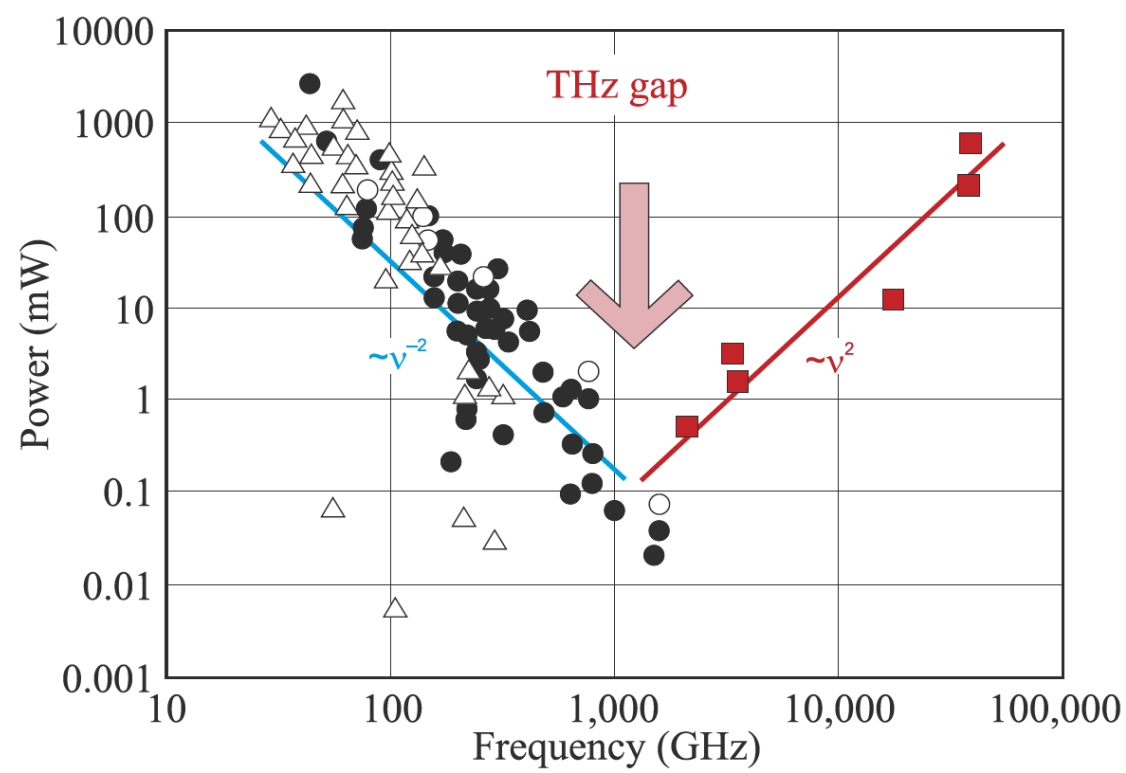

Figure 2.43: Performance of $\mathrm{THz}$ sources. Black circles correspond to frequency multipliers based sources, triangles correspond to other electronic sources, empty circles correspond to electronic sources using cooling systems, and red filled squares correspond to quantum cascade lasers (QCL) progressing downward from high frequencies [189], [227].

It is worth to mention, that Fig. 2.43 is not showing all the reality, and, of course one can find high-power THz sources based on free electron devices [1], [247], [252], such as Backward Wave Oscillators $(\mathrm{BWO})^{11}$ or Gyrotrons ${ }^{12}$ that can generate $\mathrm{kW}$ or even $\mathrm{MW}$ of $\mathrm{THz}$ power. However, it makes any sense to consider these sources beyond very specific applications allowing for huge source size, weight, power consume and budget.

\footnotetext{
${ }^{11} \mathrm{~A}$ backward wave oscillator (BWO), also called carcinotron or backward wave tube, is a vacuum tube that is used to generate microwaves up to the $\mathrm{THz}$ range. It belongs to the traveling-wave tube family, and is an oscillator with a wide electronic tuning range. An electron gun generates an electron beam that is interacting with a slow-wave structure. It sustains the oscillations by propagating a traveling wave backwards against the beam. The generated electromagnetic wave power has its group velocity directed oppositely to the direction of motion of the electrons [253].

${ }^{12} \mathrm{~A}$ Gyrotron is a high-power linear-beam vacuum tube which generates millimeter-wave electromagnetic waves by the cyclotron resonance of electrons in a strong magnetic field [254].
} 
Similarly to the case of sensors, one encounter a large variety of proposals in the literature. With the sources, notwithstanding, the spectrum wides even more due to the different technologies involved in, coming from both, microwave and optic worlds. To facilitate the the overview, again, classification in types and subtypes is carried out know, supported with some pictures and schematics, in order to give a clear summarized idea of this vast field. Other classifications are possible, however, here, sources are spitted in two big groups attending to the origins of the technology used: electronics and optics/photonics. Also, it should be keep in mind that important features that characterize a sources include if it is CW [255] or pulsed [256], narrowband [257] or wide-band [258], coherent [259] or non-coherent [260], tunable [261] or rather fixed [262]. Each kind of application will demand from a specific choice between these features, [110], [166], [172], [247].

\subsubsection{Electronic Sources}

Source designs coming from the microwave regime have lead most of $\mathrm{THz}$ space missions and the early stage $\mathrm{THz}$ revolution in the recent past [1], although optical sources are playing more and more role in the sub-mm range of $\mathrm{THz}$ band these days [263]. Electronic sources include upconverters (multiplication of low frequency oscillators signal), solid-state devices, including transistor based oscillators and amplifiers, as well as two terminal active devices, and free electron based sources, such as Backward Oscillators or Gyrotrons. It must be pointed out now again that this last subtype is reserved to very specific applications, and, between the first two, multiplying chains has played, with difference, the major role so far these decades. However, solid-state $\mathrm{THz}$ designed structures are gaining ground fast, and it has been recently postulated a new era driven by them for the next decade [264].

- Multiplication of low frequency oscillators: This technique has been the most popular technique for producing CW small amounts of power at frequencies between $500 \mathrm{GHz}$ and $1 \mathrm{THz}$ these last to decades [1], [247]. Due to the available electronic technology, the most reasonable approach nowadays is to multiply up from microwave frequencies, ranging from $20 \mathrm{GHz}$ to 40 GHz. These sources have supported important space missions in which to carry bulky and high-power consuming lasers, or short-life, heavy and high-power tube sources was unaffordable. As usual, cooling improves source performance, however, cooling is not an unavoidable requisite for these sources, which are usually demanded to operate at room-temperature. This is a remarkable practical advantage of this multipliers chain sources.

As disadvantages to take into account, it must be said that reach $\mathrm{THz}$ frequencies from microwave frequencies imply expand several octaves, i.e., multiplication factor of several tens. Although, in theory, high-order multipliers can be achieved [248], practical implementations give as a conclusion that doublers, and as much triplers [265] must be used in chain to achieve high multiplication factors [1], [249].

To achieve high multiplication factor by means of low-order multiplication elements chains implies, inherently, some limitations. On the one hand, each 


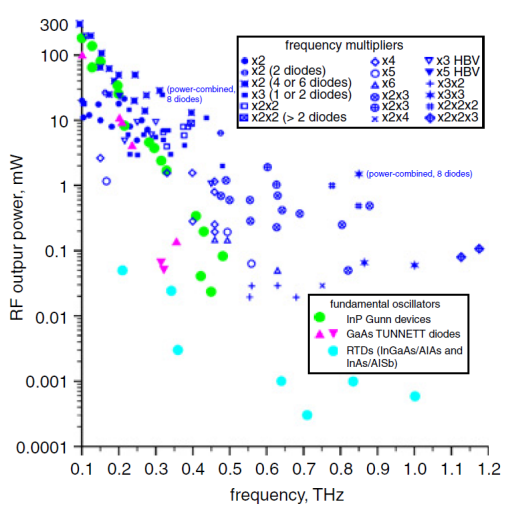

(a)
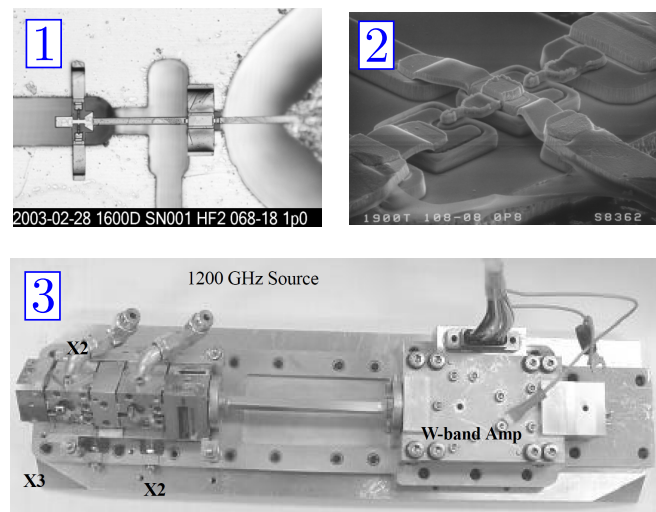

(b)

Figure 2.44: $\mathrm{THz}$ multiplier chain sources. a) State of the art from frequency multipliers and some solid-state two-terminal active devices from $0.1 \mathrm{THz}$ to $1.2 \mathrm{THz}$ at room temperature [264]; b) Practical realizations. 1) Close-up of a $1.9 \mathrm{THz}$ tripler chip. The mesa is less than $15 \mu \mathrm{m}$ long and the anodes area is smaller than $1 \mu \mathrm{m}^{2}$; 2) $1.6 \mathrm{THz}$ doubler placed in a waveguide mount is shown. The chip is only a few $\mu \mathrm{m}$ thick. The machined feature size of the output waveguide is less than $50 \mu \mathrm{m}$; 3) waveguide block where a $1.2 \mathrm{THz}$ multiplier chain chip is packaged. The LO was driving this source giving 1-2 $\mathrm{mW}$ at $W$-band [266].

multiplication will imply a poor conversion efficiency, thus noticeably diminishing the total multiplication element efficiency. On the other hand, output power will be also conditioned by the input power, which is limited to the first stages power handling. Furthermore, multiplier chains critically depend on the highpass characteristics of the sections of waveguides between different stages to eliminate unwanted signals from the strong nonlinearities of the Schottky diodes, and improve stability. The resulting minimum size of waveguide blocks and the need for complex biasing schemes may exclude multiplier chains from portable applications such as wireless communications, where compact sources are needed [166]. Maybe, this kind of applications demand rather from the solid-state sources, next described [264].

In Fig. 2.44(a), it is shown the state of the art from frequency multipliers and, for comparison, some solid-state two-terminal active devices from $0.1 \mathrm{THz}$ to $1.2 \mathrm{THz}$ at room temperature [264]. As it can be appreciated, multipliers offer lower output power when frequency increases. This graph also shows that chains of multipliers overcome direct high-order multiplications. To reach the highest considered frequencies, they are practically the only option considered. The upper bound of $1 \mathrm{~mW}$ at $1 \mathrm{THz}$ at room temperature is to be achieved during the next years.

Traditionally, whisker-contacted GaAs Schottky Diodes have been used for multiplying purposes, although high-performance planar designs have replace them these last years [1], [264]. In Fig. 2.44(b)-1 it is shown a close-up of a $1.9 \mathrm{THz}$ tripper chip from [266]. In this high-frequency $\mathrm{THz}$ design, the mesa is less than $15 \mu \mathrm{m}$ long and the anodes area is smaller than $1 \mu \mathrm{m}^{2}$. In Fig. 2.44(b)-2, a $1.6 \mathrm{THz}$ doubler placed in a waveguide mount is shown. 
The chip is only a few $\mu \mathrm{m}$ thick. The machined feature size of the output waveguide is less than $50 \mu \mathrm{m}$. Finally, Fig. 2.44(b)-3 shows a waveguide block where a $1.2 \mathrm{THz}$ multiplier chain chip is packaged. The LO driving this source was giving 1-2 $\mathrm{mW}$ at the $\mathrm{W}$-band. Output power of more than $100 \mathrm{~mW}$ was obtained for this design. In that work, outstanding powers of $50 \mathrm{~mW}$ were obtained for frequencies as high as $1.8 \mathrm{THz}$. It is also remarked that those thin and delicate chips become difficult to handle and require special precautions, including placement of handling structures on the chip.

- Solid-state devices: At the beginning of this century, solid-state devices operating beyond the commercial microwave frequencies were rare and expensive cases offered by very few companies, with a performance far of what is desirable [1]. Multiplier chains were by far the best solution for the early era of $\mathrm{THz}$ applications, although some advances started to appear, including InP Monolithic Microwave Integrated Circuits (MMIC) power-amplifier chips operating beyond $100 \mathrm{GHz}$ [267], and some attempts to reach the $200 \mathrm{GHz}$ barrier [268]-[269] (a beginning to reach into actual challenging $\mathrm{THz}$ frequencies, i.e., sub-mm waves). The very dedicated research has given its fruits these years and the current state of the art in solid devices justifies a niche for these kind of sources, which are shortening distances with multiplier chains [264].

Solid-state sources include local-oscillators two terminal active devices and amplifiers based on transistors. Two terminal active devices are the first solid state devices ever used in microwave oscillators. These devices generate output power by providing a Negative Differential Resistance (NDR) at the frequency of interest, hence compensating, or even overcompensating the inherent losses in a resonant circuit [270]. Relevant devices of this kind are the Resonanttunneling Diodes (RTDs), Tunnel-injection Transit-Time (TUNNETT) devices, Transferred-Electron Devices (TEDs, also called Gunn devices), Superlattice Electronic Devicess (SLEDs) or IMPact Ionization Avalanche TransitTime (IMPATT) diodes [271].

One of the great advantages of active two-terminal devices is their simpler device fabrication compared to three-terminal devices. They typically have vertical layer structures and no fabrication compromises need to be made, a requirement that could lead to an increase in detrimental contact or series resistances. Actually, in transistor amplifiers the access resistance generally affects the frequency limits [264].

As an example, operation of GaAs TUNNETT diodes up to $755 \mathrm{GHz}$ has been reported [272], with remarkable good results, obtaining $0.33 \mathrm{~mW}$ at $0.41 \mathrm{THz}$ [273]. Furthermore, Gallium Nitride (GaN)-based TUNNETT devices, are promising when applied at lower frequencies (output powers of more than 160 $\mathrm{mW}$ at $200 \mathrm{GHz}$, and more than $20 \mathrm{~mW}$ at $300 \mathrm{GHz}$ from a single device without the use of any power-combining scheme [264]). Since TUNNETT diode is based in tunneling breakdown instead of avalanche breakdown [274], and delay of tunneling breakdown is smaller than that for the avalanche breakdown [275], TUNNETT diodes tend to shown higher operation frequency than IMPATT 
diodes when implementing oscillators. However, output power provided by TUNNETT diodes is smaller.

In spite of the low power levels, the very small size of oscillators with active two-terminal devices makes them ideally suited for portable applications, such as wideband wireless communications. It is worth to mention that design these sources beyond $300 \mathrm{GHz}$ will demand for Computer Aided Design (CAD) tools not found in present available electromagnetic simulators, a new area opened by the $\mathrm{THz}$ regime to numerical methods researchers.

Transistors provide desired output power through non-linear characteristics of semiconductor compounds properly biased. As pointed out in [276], an excellent review of $\mathrm{THz}$ transistors, overcoming the high-frequency limitations of these devices may lead to a strong revolution in $\mathrm{THz}$ systems. An outstanding operation frequency of $0.65 \mathrm{THz}$ has been achieved recently [277]. Actually, MMICs output powers in the low range of $\mathrm{THz}$ band is reaching nowadays 50 $\mathrm{mW}$ at $200 \mathrm{GHz}$ [278], and $10 \mathrm{~mW}$ [279] beyond the $300 \mathrm{GHz}$ barrier. These power levels were so far only possible with two terminal active devices such as IMPATT diodes [271], [264].

In this family of $\mathrm{THz}$ power providers they are generally found High Electron Mobility Transistors (HEMTs), Heterojunction Bipolar Transistors (HBTs), and CMOS transistors, this last emerging nowadays in the $\mathrm{THz}$ band. Although higher power is achieved with GaN, InP leads the gain for high frequencies and seems to be the most reasonable option [276]. From noise perspective, SiGe-based transistor amplifiers have been shown preferable, reporting, furthermore, high integration capability than other amplifiers. However, their practical use still pertains rather to mm-wave than $\mathrm{THz}$. Finally, one must keep an eye in power-combining techniques, known for decades [280]. These techniques may play an important role for solid-state devices [264], [281], [282].

To overview the above information regarding solid-state devices, Fig. 2.45-1 shows the state of the art of solid state sources comparing several single chip transistor amplifiers and a variety of two terminal active devices. Roughly, it can be said that solid state devices output power falls off as $f^{-1}$ up to 100 $\mathrm{GHz}$, then as $f^{-2}$ up to $200 \mathrm{GHz}$, and finally as $f^{-3}$ beyond $200 \mathrm{GHz}[247]$.

InP HEMT based amplifiers (red circles) achieve the best results for reasonable frequencies, however higher frequencies have been reached by two terminal active devices, which inherently to its structure offer a better frequency behavior (for example, the RTDs, have reported operation frequencies beyond $1 \mathrm{THz}$, presenting notwithstanding, very low output power [275]).

- Free Electron Sources: This kind of sources take their name from the fact that they are based on the effect of accelerating electrons which are not bound to the atom but they move freely through a magnetic structure, reaching very high velocities [252]-[253]. This mechanism is used to provide high power sources at frequencies going from microwave to X-rays. Focusing in $\mathrm{THz}$, 

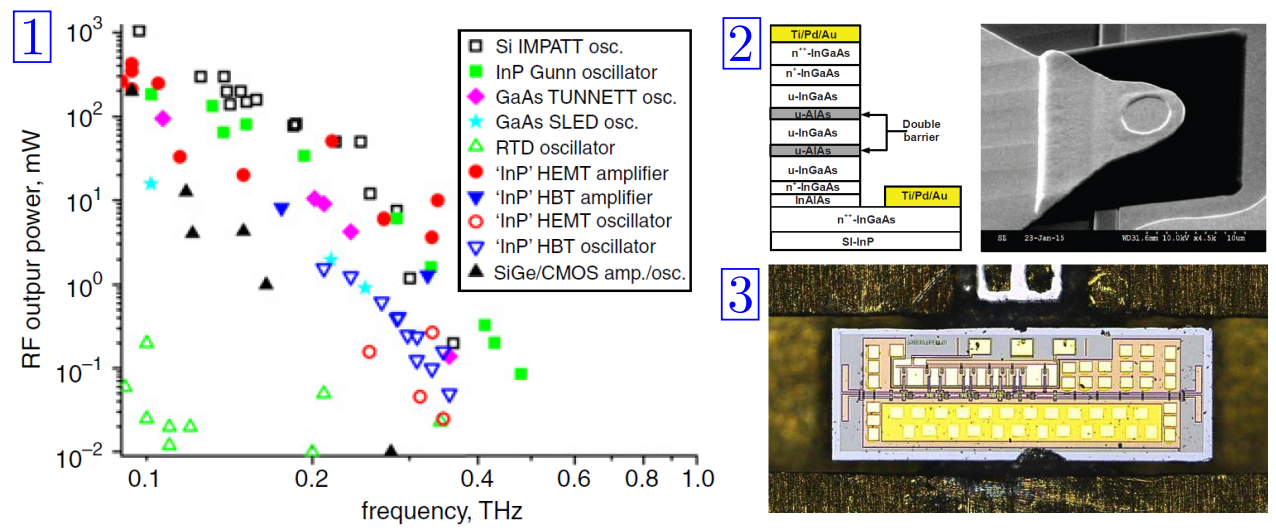

Figure 2.45: Solid-state $\mathrm{THz}$ sources. 1) State of the art from frequency multipliers and some solid-state two-terminal active devices from $0.1 \mathrm{TH}$ to $1.2 \mathrm{THz}$ at room temperature [264]; 2) Microphotograph of a $0.48 \mathrm{THz}$ Low Noise Amplifier (LNA) in split block housing. The signal is electromagnetically coupled to the chip by monolithically integrated dipole probes [281]; 3) Schematic of the layer structure of a Resonant Tunneling Diode (RTD) and micrograph of a $4 \times 4$ $\mu \mathrm{m}^{2}$ device, from [281].

the operation and results of Gyrotrons [254] and BWO [253] (also known as Carcitron ${ }^{13}$ ) will be only briefly introduced.

The gyrotron is a type of free electron MASER ${ }^{14}$ that can produces extremely high power even at the small wavelengths due to its fast-wave nature, which allow the gyrotron dimensions be much larger than the operating wavelength. This behavior differs from other conventional microwave such the aforementioned BWO, which is based on a single-mode resonant cavity and a slow-wave structure, having thus smaller dimensions [252]-[283].

The high-size characteristic of gyrotrons lead to power levels that reach $1 \mathrm{MW}$ at lower $\mathrm{THz}$ frequencies $(170 \mathrm{GHz}$ in [284]). At higher frequencies, they have been reported also outstanding values: $5 \mathrm{~kW}$ at $0.5 \mathrm{THz}$ and $0.5 \mathrm{~kW}$ at 1.3 $\mathrm{THz}$ in [285]. Recently, they having proposed designs to achieve $\mathrm{kW}$ level for broadband $\mathrm{THz}$ emission around $0.335 \mathrm{THz}$, which results more interesting than narrow-band radiation for certain type of applications [286].

In Fig. 2.46-1 they are shown the frequency and time domain simulations of the design proposed in [286] to provide $\mathrm{THz}$ power in the band going from $0.33 \mathrm{THz}$ to $0.34 \mathrm{THz}$, whereas in Fig. $2.46-2$ it is shown a photography of a $0.3 \mathrm{THz}$ gyrotron [285]. As can be noted, a gyrotron is a large device, requiring laboratory bench-size (when not room-size [34]) and manipulating very high magnetic fields. Thus, results obvious that gyrotrons left dedicated to very particular applications, rather related with fundamental research, and far away from mass market. Two examples are plasma diagnostic [287] and dynamic nuclear polarization [288], [289].

\footnotetext{
${ }^{13}$ The widely employed term Carcitron is a trade name for tubes manufactured by CSF, nowadays Thales ${ }^{\circledR}$.

${ }^{14}$ Microwave Amplification by Stimulated Emission of Radiation.
} 
The Backward Wave Oscillator (BWO) is based on a electron gun which generates an electron beam that interacts with a slow-wave structure (usually waveguide involving metal gratings or corrugations). Oscillations appear by propagating a traveling wave backwards against the beam, thus having generated electromagnetic wave power with group velocity directed oppositely to the direction of motion of the electrons [253].

Typically, power of tens to hundreds of $\mathrm{mW}$ have been reported in the $\mathrm{THz}$ region along the last decade [247]. Actually, an interesting feature of BWO is that a number of elements can be implemented in an integrated system to cover altogether a wide frequency range. In Fig. 2.46-3 it is shown the output power and spectral distribution of 13 BWO devices employed in a $\mathrm{CW}$ spectroscopic system.

More recently, output powers up to $20 \mathrm{~W}$ in the $186 \mathrm{GHz}-202 \mathrm{GHz}$ range [290], up to $8.05 \mathrm{~W}$ in the frequency range of $363.4-383.8 \mathrm{GHz}$ [291], and decaying to $150 \mathrm{~mW}$ when the frequency is pushed up to $1.2 \mathrm{THz}$ [292], have been recorded. To achieve such output powers, a BWO require voltages in the order of $\mathrm{kV}$ and a magnetic field reaching $1 \mathrm{~T}$ [34]. Thus, despite BWOs are smaller than Gyrotrons, are still a bulky source, what limits their use for certain applications.

Fig. 2.46-4 shows a BWO structure, being possible to appreciate its corrugated rectangular waveguide acting in slow-wave mode. Actually, this structure support the $\mathrm{TM}_{01}$ mode [290]. Since the power must be coupled to an antenna, appear the problem of low coupling efficiency, being reported that only $1 / 1000$ th fraction of the total power couples to the antenna radiating mode. This fact imposes a significant limitation for BWO [34].

As in the case of Gyrotrons, plasma diagnostic and nuclear fusion are applications where BWO results nowadays interesting as a source [293]. However, the lower complexity of BWO makes possible their use in applications with a more commercial role, such imaging [294]. The high output power level makes BWO attractive to achieve good contrast active images. It must pointed out, notwithstanding, that progress in the compact solid-state sources may provide more handling imaging systems with good performance, relegating the bulky BWOs to a smaller range of applications.

\subsubsection{Optical Sources}

Although electronic sources seem to have led the $\mathrm{THz}$ systems in the past decade, rather operating still far below from $1 \mathrm{THz}$, the tendency of pushing up operating frequencies to exploit unique $\mathrm{THz}$ characteristics close to and beyond $1 \mathrm{THz}$, and the inherent advance of $\mathrm{THz}$ optical technology, have provided that optical sources play today a major role in THz technology [1]-[3], [18], [295].

Actually, commercial-type applications are most commonly driven by optical down-conversion sources either by photomixing or non-linear rectification through large second-order susceptibility crystals. Furthermore, $\mathrm{THz}$ laser sources have experimented an incredible revolution driven mainly by advances in QCLs, which every year report more and more outstanding performance in terms of frequency (towards 

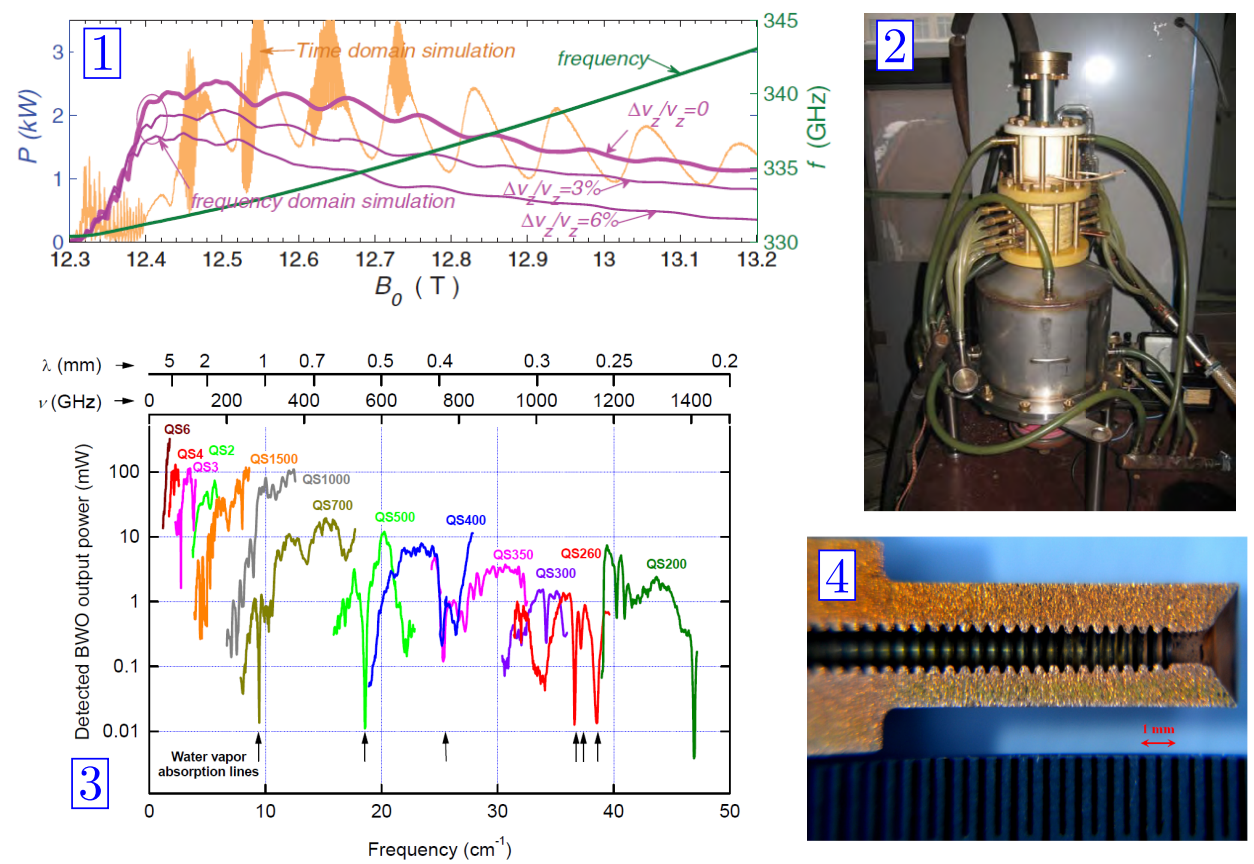

Figure 2.46: Free electron based $\mathrm{THz}$ sources. 1). $0.33 \mathrm{THz}-0.34 \mathrm{THz}$ Gyrotron simulated (frequency and time domains) output power depending on the applied magnetic field [286]; 2) Photograph of a $0.3 \mathrm{THz}$ gyrotron [285]; 3) Output power and spectral distribution of $B W O$ devices employed in a CW spectroscopic system [264]; 4) Photograph of a BWO structure showing the typical waveguide corrugations used for $T M_{01}$ mode propagation [290].

lower frequencies), power, temperature of operation and compactness. Even for very specific high-power-demanding applications one found free electron lasers, which play the same role as gyrotrons or BWO in this approach angle from higher frequencies. When revising the prevalent bibliography, a great variety of optic source schemes can be found, most of them next classified by groups, and briefly described.

- Photomixing: The process of generating $\mathrm{THz}$ radiation through one or two femtosecond (fs) lasers follows the same principle as the photoconductive antennas reviewed in section 2.3.1.1. In the simplest case of using one laser and pulsed excitation [296], [297], generally a Ti-sapphire laser illuminates a gap between closely spaced electrodes on a photoconductor (e.g., silicion-on-sapphire or LTG GaAs), generating carriers, which are then accelerated in an applied electric field, generated by the biased metal structure. The obtained current is coupled then to an Radio Frequency (RF) antenna. The frequency components reflect the pulse duration (several hundreds of $\mathrm{fs}$ ), hence this source provides $\mathrm{THz}$ radiation [1], see Fig. 2.47(a).

However, the described scheme is a simple way to produce pulsed $\mathrm{THz}$ radiation which is becoming rather obsolete these days. Instead, pulsed $\mathrm{THz}$ radiation is much more efficiently produced nowadays in similar schemes using the later detailed nonlinear optical rectification, either with crystals or by 


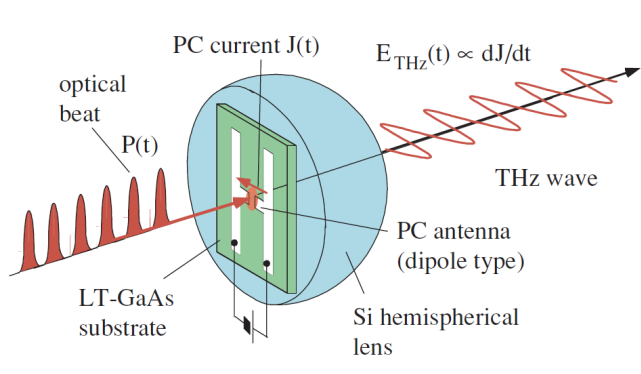

(a)

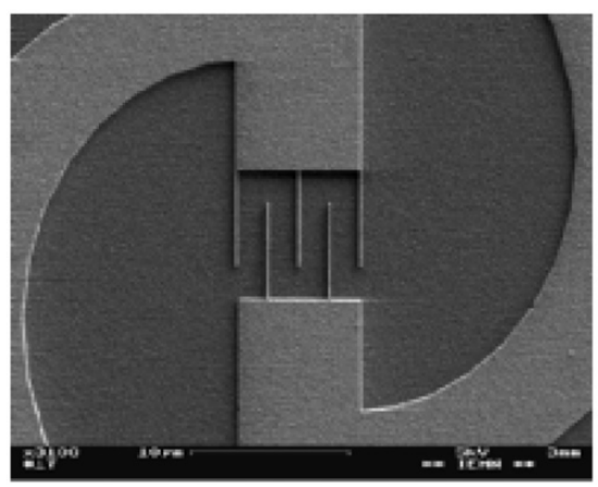

(c)

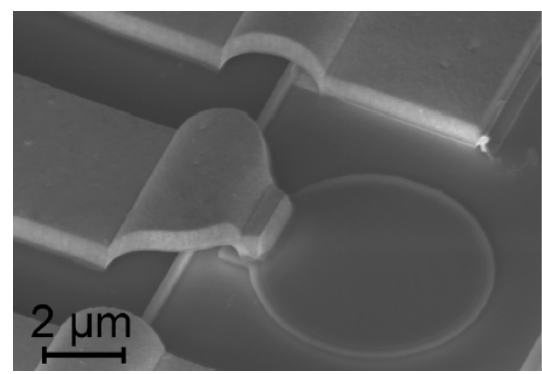

(b)

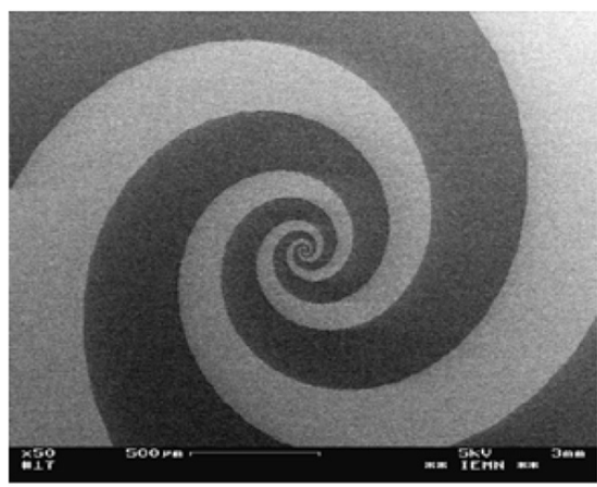

(d)

Figure 2.47: Optical downconversion through photomixing. a) Schematic of the photomixing scheme [298]; b) Electron microscope image of a metallic (gold) Fabry-Perot cavity GaAs photoconductor [299]; d) Interdigitaled nano-electrode array at the center of photomixer devide. Dimensions are $8 \times 8 \mathrm{~mm}^{2}$ [300]; d) Spiral antenna coupled to electrode array, with a maximum radius of 1.5 $\mathrm{mm}$ [300].

plasma generation. In the case of photomixing, continuous-wave terahertz radiation with frequency tunability can be achieved with two single-mode lasers or output modes of a dual-mode laser, provided the optical lasers are tunable [225]. In [301], a frequency range going from $0.4 \mathrm{THz}$ to $1.73 \mathrm{THz}$ is covered with a resolution of $3 \mathrm{MHz}$, able to discover molecular resonances with high precision for athmosphere characterization purposes.

In $\mathrm{THz}$ photomixing sources, all the incident photons are absorbed within a few $\mu \mathrm{m}$ due to the large absorption coefficient of the employed semiconductors [302]. Thus, photomixing is furthermore compact and presents low power consumption, being all its features together ideal for communications [173],[168] or imaging, in which $\mathrm{CW}$ provides higher resolution than pulsed radiation [302]. As a matter of fact, [303] reports a scheme providing $0.8 \mathrm{~mW}$ at $1 \mathrm{THz}$ using optical pumping power of only $150 \mathrm{~mW}$ at $1550 \mathrm{~nm}$.

It is worth to mention that the classical simple gap [52], [297], [298], or the interdigital electrode configuration [300], [304], has developed to more efficient structures these last years. In [299], the metallic mirror Fabry-Pebrot cavity 
shown in Fig. 2.47(b) reported an increment of the output power regarding previous works with GaAs photomixers. Other interesting example are the plasmonic contact electrodes [303] or nano-electrodes [305], this last improving the output power in 2 orders of magnitude when compared with classical interdigital configuration. Photomixer array schemes are also possible to enhance the output power, specially in the case of coherent combination [302].

Regarding the radiation, it must be noted that although the concept of Large Area Emitter (LAE), i.e, the photomixer radiating itself without power coupled to an antenna [302] has been shown possible, practically suffers from low conversion efficiencies compared to those photomixing sources with Antenna Emitter (AE). Fig. 2.47(c) shows a nano interdigital array biasing scheme, whereas Fig. 2.47(d) shows the spiral antenna used to radiate the power more efficiently [300].

In the case of using AE, the bottleneck is the antenna matching bandwidth which limits the range of tunability. Common solutions have been using typically broadband antennas such as spiral or bowtie, although other new proposals are based on high impedance antennas [225]. A result from TERASENSE project [156] affording this issue is the proposal of a meander dipole antenna operating outside of its resonance. Improvement by a factor of 8 regarding to self-complementary antennas was reported.

- Non-linear optical rectification: This way of producing $\mathrm{THz}$ energy is based on the crystals presenting a large second-order susceptibility (fieldinduced polarization) $\chi^{2}$. When two ${ }^{15}$ femtosecond lasers beams cross the crystal the nonlinear effect causes mixing, producing a time-varying polarization with a frequency-response representative of the pulse length, thus obtaining an emitted THz spectra [1], [307], [308].

Since the involved non-linear process is only moderately strong, large laser intensities of several hundred $\mathrm{W} / \mathrm{cm}^{2}$ are demanded [302]. Furthermore, the laser beams interact along the crystal, this interaction usually required to be of the order several hundred of $\mu \mathrm{m}$ or even $\mathrm{mm}$ [257], [309]. Therefore, CW operation is hardly achievable, noting that to ensure phase-matching over this extremely long interaction length, and an acceptable average power results rather challenging [302].

Average powers of these kind of sources were of the order of $\mathrm{nW}$ and rarely reaching few $\mu \mathrm{W}$ at the starting of the $\mathrm{THz}$ revolution [1]. More recent works report $100 \mu \mathrm{W}$ of average power [307], or even $5.3 \mathrm{~mW}$ in the case of pumping power of $15 \mathrm{~W}$ [310], where the employed structure avoided the photorefractive damage of the crystal, an important limitation for efficiency, and thermals damages due to heat deposition.

Regarding to the previous fact, optical rectification is restricted to pulsed lowpower sources, which may achieve enough peak power levels, however, and that can be interesting for recovering 3D spatial information in imaging applications [110], [302]. Although, photomixing scheme usually provides higher average

${ }^{15}$ Single laser schemes can also be found [306]. 
power levels, in [257] and [311] is pointed out that photoconductive antennas are incapable of sustaining the high peak power of strong ps pulses. Furthermore, saturation appears due to their finite capacitance and it is a fact that photoconductive switching drops rapidly beyond $1 \mathrm{THz}$ due to finite carrier relaxation times of the substrate materials $(\approx 0.5 \mathrm{ps})$. Surprising ultra-short intense pulses of $5 \mathrm{MW}$ power are reported in [307].

Several materials (crystals) have been considered as the non-linear element, including GaAs, GaP, ZnGeP 2 , GaSe, ZnTe, $\mathrm{LiNbO}_{3}$ or $\mathrm{LiTaO}_{3}$, being ZnTe slightly more popular due to its transparency to $\mathrm{THz}$ radiation [312]. An excellent review comparing the linear, nonlinear optical properties and figures of merit of such wide range of elements, justified by the pros and cons of each one, can be found in [311]. More recently, new configurations, based on periodically poled crystals (see Figs. 2.48(a) and 2.48(b)) have shown to allow control on the output signal bandwidth [313], producing average power of 1 $\mathrm{mW}$, predicted to be potentially scalable to $100 \mathrm{~mW}$ [311].

Optical rectification has been also recently shown to be achieved also via third order (instead of second order) non linear susceptibility $\chi^{3}$, which is attractive because normal air or other common gases can be used as a rectifying medium [312]. This process was shown to be enhanced by the presence of plasma, that can be easily generated by one of the incident laser pulses, as shown in Fig. $2.48(\mathrm{c})$. The great advantage of this new technique of optical rectification is that the emission takes place in the form of a strongly collimated $\mathrm{THz}$ beam towards forward direction. Very distant targets (kilometers away) may be reached with this technique according to [312].

Finally, before going into the realm of lasers, Fig. 2.49 groups the pulsed $\mathrm{THz}$ optical downconversion techniques using femtosecond lasers, from left to right: photomixing (generally inlcuding a photoconductive antenna), optical rectification, and reflection on semiconductor surface. Except of the last one, which has lost relevance in the last years due to its lower efficiency, caused by the high semiconductor $\mathrm{THz}$ energy absorption [302], the other two, just described, present new advances every year, and is hard to predict if one will impose to the other. At least, in the next few years, the $\mathrm{THz}$ researcher must be aware of advances in both kind of sources.

- Lasers: As solid-state devices are for electronics, $\mathrm{THz}$ laser are the $\mathrm{THz}$ optical sources intrinsically producing $\mathrm{THz}$ energy. It must be pointed out that under the term "laser", different kinds of $\mathrm{THz}$ source are found, and it must distinguished between types.

The first subgroup is the IR-pumped gas lasers, generally based on grating tuned $\mathrm{CO}_{2}$ cavities that lase to produce the $\mathrm{THz}$ output signal. Power levels of this lasers range from 1-20 mW, depending on the chosen line, being the methanol one, at $f=2.522 \mathrm{THz}$, the strongest one (even $30 \mathrm{~mW}$ have been reported using this line). Operation frequencies range from $0.5 \mathrm{THz}$ to $3 \mathrm{THz}$, however, not all lines are covered, and some of the gases required are nowadays under strict environmental safety polices [1]. 


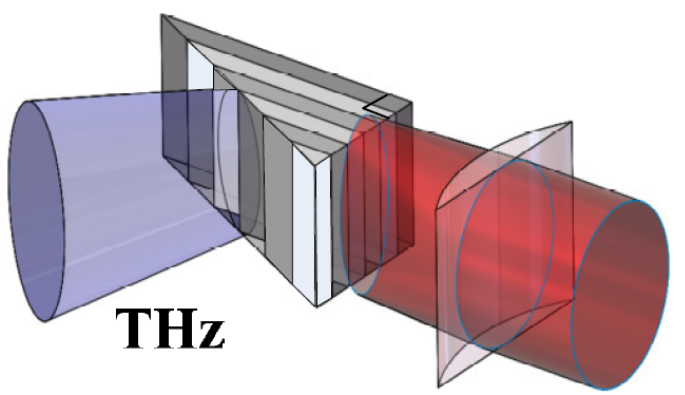

fs laser

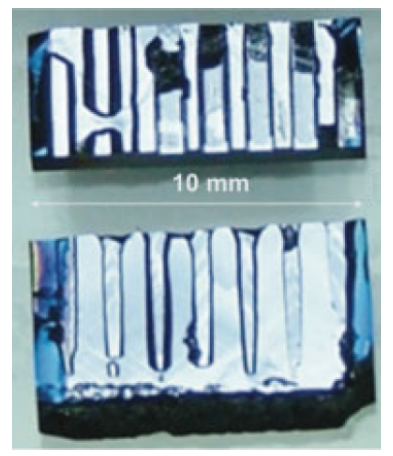

(b)

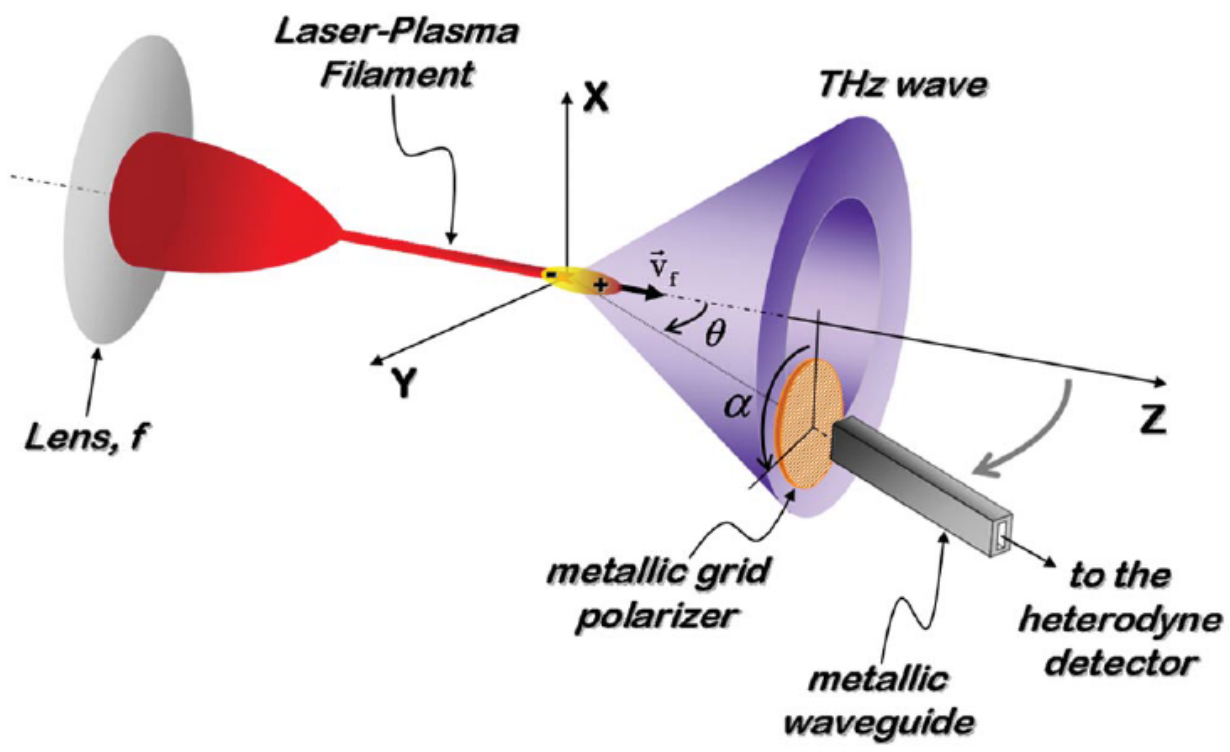

(c)

Figure 2.48: Optical downconversion through optical rectification. a) Schematic of the optical rectification using a periodically poled crystal scheme [313]; b)Top view of Orientation Patterned $(O P)$ GaAs. Sample length is $L=10 \mathrm{~mm}$ having Quasi Matched Periods (QMP) of $1.3 \mathrm{~mm}$; c) Interdigitaled nano-electrode array at the center of photomixer devide. Dimensions are $8 \times 8 \mu \mathrm{m}^{2}$ [300].

This kind of laser have played a major role in the past, sustaining much astronomical and Earth-based spectroscopy research experiments, [314], [315]. However, although at the beginning of the $\mathrm{THz}$ revolution they were still reviewed as essentially the principal kind of $\mathrm{THz}$ laser sources [1], and despite of the fact of their high power levels still attract some specific research [316], they have been displaced these last years by the advances in direct semiconductors lasers and, especially, by the revolution experimented in the area of 


\section{THz Pulse Generation}

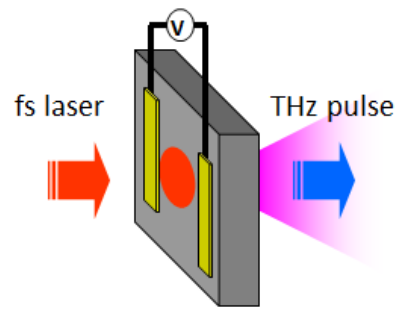

Photoconductive Antenna

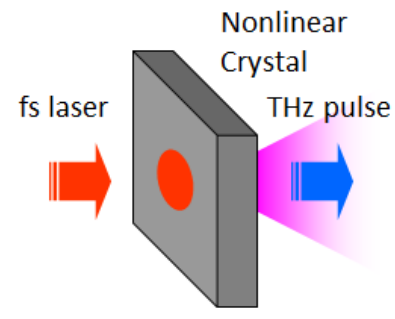

Optical Rectification

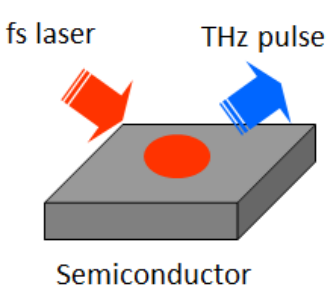

Semiconductor Surface

Figure 2.49: $\mathrm{THz}$ pulse generation techniques.

QCLs. These new THz-designed laser sources, described next, fit better for the emerging $\mathrm{THz}$ commercial applications.

The second subgroup, the aforementioned semiconductor lasers, is based on radiative recombination of electrons and holes due to intersubband transitions. The system needs to be either electrically or optically pumped in order to generate more light by stimulated emission than is lost by absorption. Early $\mathrm{THz}$ semiconductor lasers [317] were based in Germanium (Ge), allowing to very short duty cycle pulsed radiation. The discovery of Beryllium (BE)-doped Ge [318] allowed to increase the duty cycle to $2.5 \%$. Very bright lines, as narrow as $1 \mathrm{MHz}$ were reported at that time with Gallium (Ga)-doped Ge.

Before the end of the past century, CW operation of Ge-based lasers was reported by using the inactive, electric field-free Ge laser material as a heat sink, and exploring new ohmic contact geometries and a new variety of of semideep acceptors [319]. These techniques allowed to reduce power consumption and increase conversion efficiency. At that time, $\mathrm{THz}$ semiconductor laser were mostly optically pumped and gain saturation was an important issue [320].

Nowadays, electrical pumping is preferred, since any external laser source is required [321]. In this case, however, the ideal configuration of an optically pumped central area surrounded by absorbing regions is lost and the use of new structures to avoid lateral spreading is recommendable to overcome this fundamental problem of actual THz semiconductor lasers. A 2D photonic crystal implemented via sole patterning the device top metallization is proposed in [321], see Fig. 2.50(a). In this work, the high boundary absorption is replaced by high reflectivity conditions by removing the top $\mathrm{n}^{+}$contact (red layer), causing this mode mismatch and, therefore, the desired reflection, see 2.50(b). An optical microscope image of the device surface is shown in Fig. 2.50(c).

$\mathrm{THz}$ semiconductor lasers produce the largest output power among conventional (free electron lasers must be treated apart) $\mathrm{THz}$ lasers, generally of several watts (outstanding $10 \mathrm{~W}$ were reported in [322]), although high electric and magnetic fields and some cooling are required for their operation, being this a strong limitation for practical applications. Another advantage is that their unique parameters allow for wide continuous spectral tunability 


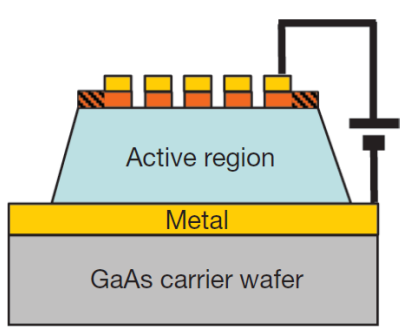

(a)

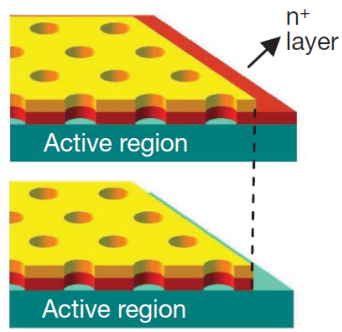

(b)

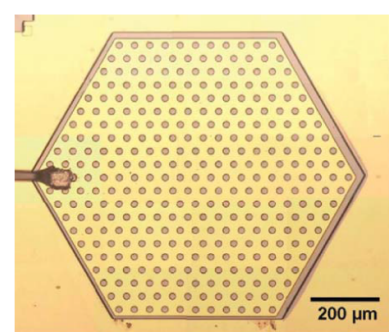

(c)

Figure 2.50: Electrically pumped $\mathrm{THz}$ semiconductor laser scheme enhancing output coupling efficiency via a $2 D$ photonic crystal structure [321]. 1) Schematic cross section of the device. The laser active region (blue) is sandwiched between two metal $\mathrm{Ti} / \mathrm{Au}$ contact layers. The top metal is patterned with the desired photonic-crystal/bandgap structure design; 2) Detailed scheme of the boundary conditions implementation technique: when the top $\mathrm{n}^{+}$contact, represented as a red layer, is left in place between the metal edge and the mesa periphery (top panel), the absorption is high and the boundary behaves like an absorber. When instead it is removed (bottom panel), the mode mismatch at the metal edge introduces a high reflectivity, and therefore mirror boundary conditions; 3) Optical microscope image of the device surface. The wire bonding is applied directly on the top metallization.

(even from $1 \mathrm{THz}$ to $4 \mathrm{THz}$ was reported [323]), which is a feature lacking in previously described gas lasers. Compared to the very popular QCLs, described next, for frequencies enough close to $1 \mathrm{THz}$, semiconductor lasers offer more compactness (although other authors [324] consider that, for reasonable power levels, QCLs total system may result smaller in size due to the required external pumping for Ge-lasers), longer life time and more efficiency [325]. .

The third and most popular device are Quantum Cascade Lasers. QCLs were firstly reported for the mid-infrared (around $70 \mathrm{THz}$ ) in [250], an extensively cited work. THz milestone appeared in 2002 [326], where the first proposal of this configuration for $\mathrm{THz}$, offering $2 \mathrm{~mW}$ at $4.4 \mathrm{THz}$ and $50 \mathrm{~K}$ temperature, triggered an intense race (provided that these structures have been shown to overcome straightforward technological barriers [324]) to develope higher and higher performance QCLs.

The basic QCL principles are displayed in Figs. 2.51 and 2.52. In classical semiconductor lasers, the well-known inter-band transition between conduction and valence bands produces an emitted photon through electron-hole recombination, Fig. 2.51(a). Therefore, the operation is bipolar, having only one photon emitted per each electron-hole pair annihilated [317].

QCLs are based, however, in intra-band (sometimes called inter-subband) transitions ${ }^{16}$. This transition takes places exclusively in the conduction band, being only electrons used. These electrons change their energy states to emit a photon. The photon emission takes place at the desired frequency due to a proper band structure engineering, done by properly choosing the structure layers thickness [326], see Fig. 2.51(b). This emission is produced in an active semi-

\footnotetext{
${ }^{16}$ A good comparative between quantum cascade structures using intra and interband transitions can be found in [328].
} 


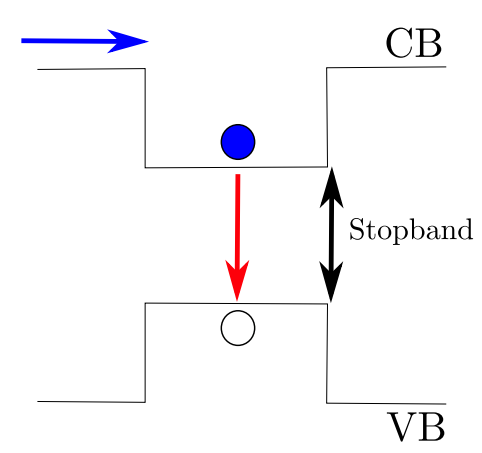

(a)

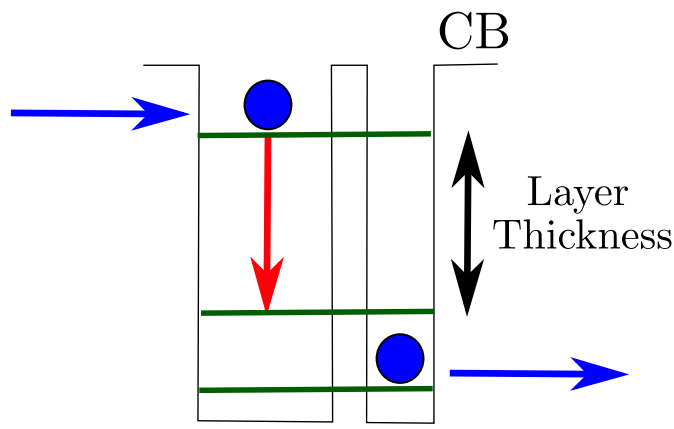

(b)

Figure 2.51: Operational principles of a Quantum Cascade Laser 1. a) Classical inter-band transition between conduction band and valence band. Electrons and holes recombine to emit a photon. The operation is, therefore, bipolar, and each photon emitted annihilates an electron-hole pair; b) QCL typical intra-band (inter-subband) transition, using exclusively conduction band. Only electrons are used, changing their energy states to emit a photon. The operation is unipolar, and each electron can emit in principle $N$ photons for $N$ cascade units; c) Typical band diagram for bound-to-continuum configuration (one of the mostly adopted [324], [327]). The different wells are separated by a think barrier, so electron tunnel between adjacent wells. Proper photon energy chosen by bandstructure engineering allows radiation in the desired $\mathrm{THz}$ frequency at the active regions. Cascading several units allow to each electron produce many photons, so that high powers are possible.

conductor region realized by multiple Quantum Well (QW) heterostructure, being most popular the use of the GaAs/AlGaAs sandwich [251].

A QCL consist of coupled QWs constructed by nm-thick layers of GaAs sandwiched between potential barriers of Aluminum Gallium Arsenide (AlGaAs). The quantum cascade consist of a repeating the structure in which each repeat unit is made up of an injector and an active region, see Fig. 2.52. Since the wells are separated by a thin barrier, wave functions spread across adjacent wells, and electrons can tunnel along them. In the active region, a population inversion exist and electron transitions to a lower energy occurs [329]. N photons are emitted for each electron for a N-units cascade due to the unipolar characteristic of the emission (electrons are recycled), being this the feature which allows for high power levels [250]. Images of practical realizations of a single QCL and a single-chip array of QCLs are shown in Figs. 2.53(a) and $2.53(\mathrm{~b})$, respectively.

Current state of the art in QCL reports output power overcoming the $1 \mathrm{~W}$ threshold at $10 \mathrm{~K}$ operation in pulsed mode [263] (decaying to $0.42 \mathrm{~mW}$ for 77 $\mathrm{K}$ temperature), and certain tunability ( $4 \mathrm{GHz}$ span around $3 \mathrm{THz}$, giving 0.7 $\mathrm{mW}[331])$. This last aspect may be solved by arraying several QCL, which can be done in a single chip [330]. Tunability from $1 \mathrm{THz}$ to $5 \mathrm{THz}$ is reported in that work. Finally, CW and pulsed operation is possible for QCL, demanding the former for lower temperatures of operation and providing lower output powers [332], as it can be expected. 


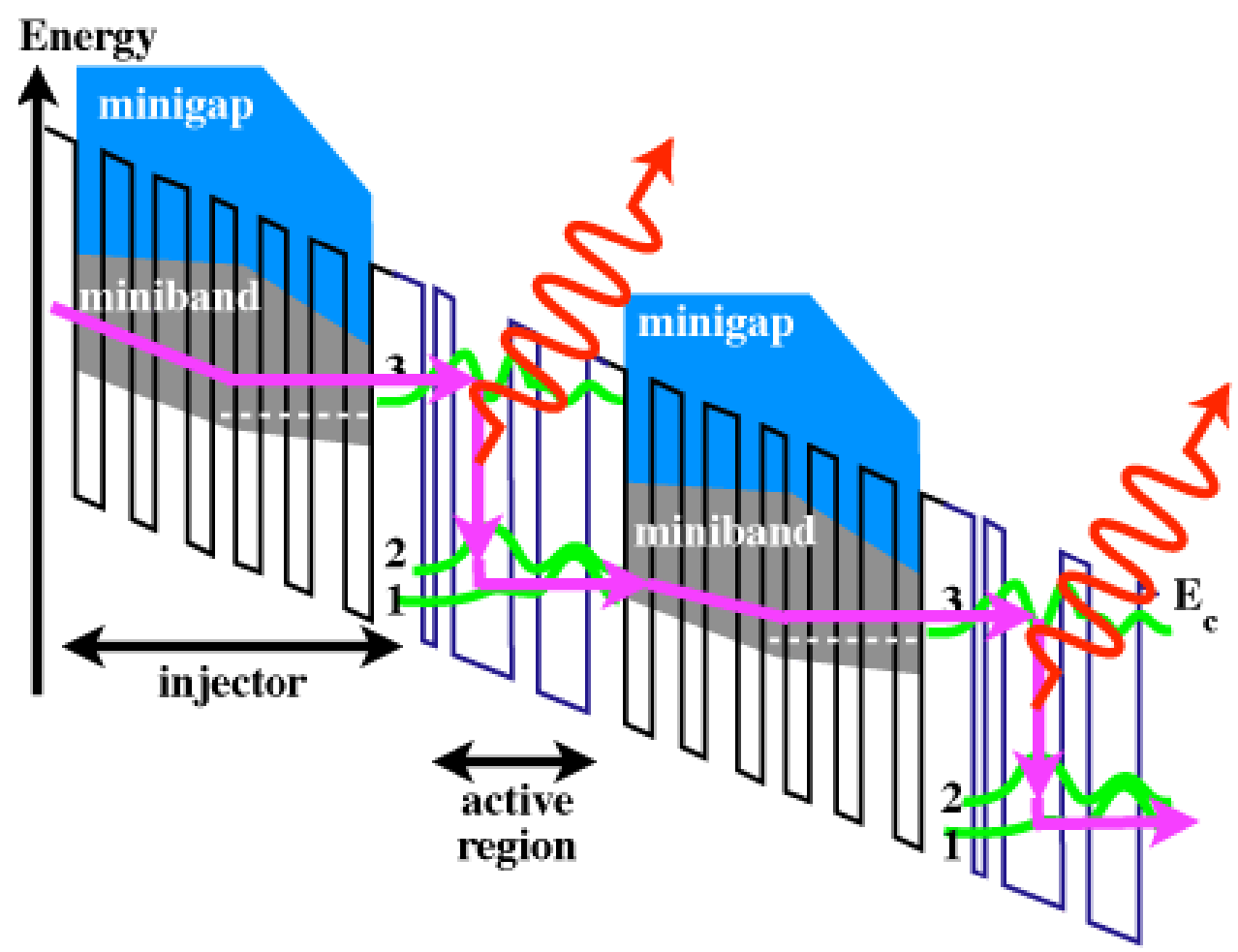

Figure 2.52: Operational principles of a Quantum Cascade Laser 2. Typical band diagram for bound-to-continuum configuration (one of the mostly adopted [324], [327]). The different wells are separated by a think barrier, so electron tunnel between adjacent wells. Proper photon energy chosen by bandstructure engineering allows radiation in the desired $\mathrm{THz}$ frequency at the active regions. Cascading several units allow to each electron produce many photons, so that high powers are possible.

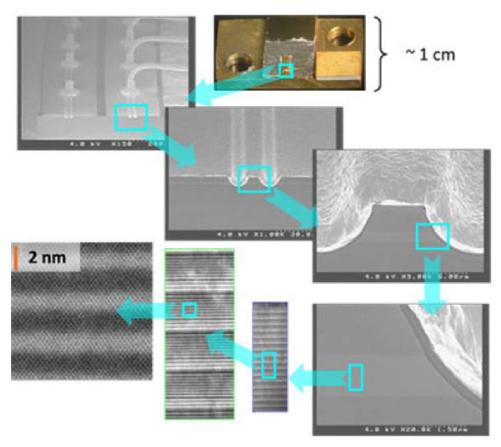

(a)

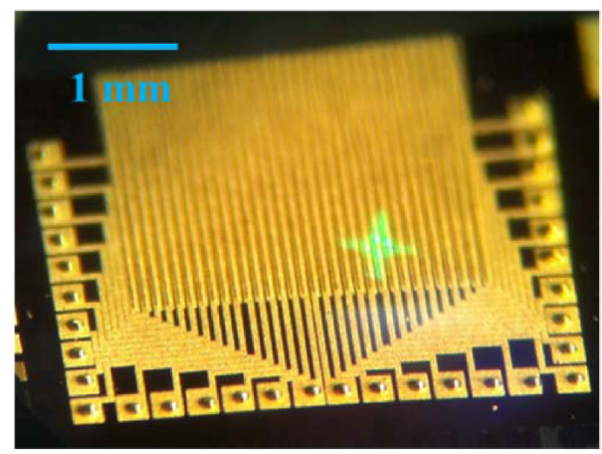

(b)

Figure 2.53: QCL single device and array images [330]. a) Single QCLs images at different magnifications; b) Single-chip Distributed FeedBack (DFB) QCL.

By the above, QCL is nowadays a very promising candidate for $\mathrm{THz}$ power providing in a wide range of applications since competitive output power is deliv- 
ered for relatively small devices with certain cooling. Three main disadvantages must be afforded in order to a larger acceptation of QCLs. The first and most important one is the temperature of operation. Reaching room-temperature operation or at least a temperature high enough for using non-complex cooling systems seems mandatory for mass use of QCLs. A progression in this aspect has been observed following the highest temperatures reported in the bibliography these years, exhibiting, however, some kind of progress saturation for the last period [327]. In that study it is reported how crucial will be the role of the metallic and plasmonic waveguides that extract the power from the QCL in the temperature aspect. Other strong point towards the necessity of study efficient ways of waveguiding $\mathrm{THz}$ energy.

The other two aspects are to achieve frequency/beam control and power efficiency. In QCLs based on metal-metal resonators, the active core thickness is much smaller than the wavelength and a fundamental Transverse ElectroMagnetic (TEM) mode is always present in multiconductor waveguides. This causes poor extraction efficiency as almost all the power is back-reflected at the facet and non-directional, diffractive far-fields, incompatible with applications, appear. Interestingly, [327] points again towards microwave regime solutions, suggesting the use of horn antennas. It seems that microwave techniques may be in the future a necessary tool to drive these optical sources towards high performance levels. If the aforementioned problems are solved, QCL may obtain a dominant position among other $\mathrm{THz}$ sources.

Before leaving QCLs, a special configuration, recently considered is reported. A scheme using two mid-IR QCLs is conceived in order to generate $\mathrm{THz}$ radiation by Difference-Frequency Generation (DFG) [333]. Mid-IR QCLs have also experienced a great development these years, being one step forward than $\mathrm{THz}$ QCL technology due to the higher frequencies employed, easier to be generated. In mid-IR, QCLs can provide high power with high efficiency at room temperature. The key fact is that these devices shown a large nonlinearity, very suitable for THz DFG. With proper wavelength selection mechanism, such Distributed Feed-Back (DFB) for dual mid-IR frequency operation, $\mathrm{THz}$ emission is produced by intracavity generation. Therefore, this type of $\mathrm{THz}$ sources inherits the advantage of mid-IR QCL: room temperature operation, electrical pumping, compact size, as well as the potential for mass production. In [333] $1.4 \mathrm{~mW}$ are achieved at $3.6 \mathrm{THz}$, which is a good value for room temperature.

The section of lasers end with the Free Electron Laser (FEL). In a FEL, a beam of electrons is accelerated to almost the speed of light. Electrons are bunched with magnetic fields to achieve coherent spontaneous emission [247], then compressed and coupled to an undulator (sometimes called wiggler), see Fig. 2.54(a), where the electron bunches are forced to wiggle transversely along a sinusoidal path about the axis of the undulator.

This free-electron source, a kind of photonics equivalent to the Gyrotron is, by far, the largest power provide among $\mathrm{THz}$ lasers, and, more in general, among $\mathrm{THz}$ sources beyond $1 \mathrm{THz}$ [324]. Furthermore, these devices provide also the widest tunability among laser sources [336]. The prize of these suitable 


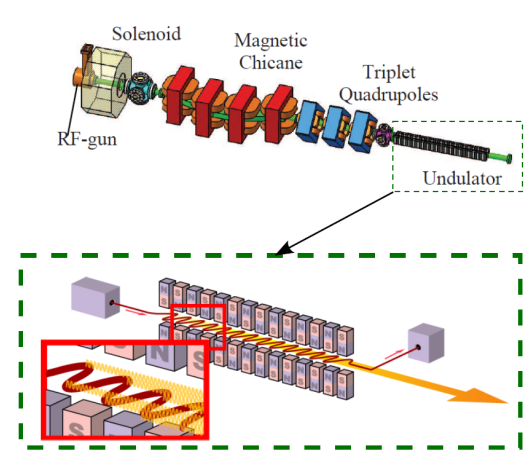

(a)

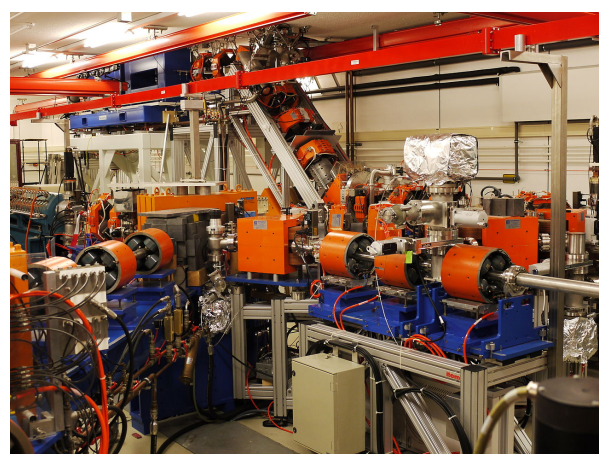

(b)

Figure 2.54: Free Electron Laser (FEL). a) Schematic view of a compact THz-FEL with detail of the undulator structure [334]; b) Image of the Free Electron Laser for Infrared eXperiments (FELIX) [335] at the Institute for Plasma Physics Rijnhuizen, Netherlands.

characteristics, is the enormous complexity and size of such structures. Only few laboratories in the world can afford the expense of built and maintain a FEL, which is dedicated for very specific research experiments. In Fig. 2.54(b) it is shown the FEL of the Institute for Plasma Physics Rijnhuizen, in Netherlands. In the image it can be appreciated the grade of complexity of the devices.

Short pulses ( 2 ps at $1 \mathrm{THz}$ ) of FELs allow for interesting dynamical measurements of $\mathrm{THz}$ systems [324]. Tunable radiation in the region from $120 \mathrm{GHz}$ to $4.8 \mathrm{THz}$, with an outstanding output peak power ranging from $500 \mathrm{~W}$ to $\mathrm{kW}$ for pulses going from $1 \mu \mathrm{s}$ to $20 \mu \mathrm{s}$, has been reported [247]. Nowadays, some efforts are found in the attempt to create compact FELs. In [247], table top devices are mentioned, and the challenge for micro FELs, able to provide short, pulsed power for more conventional spectroscopic experiments, is proposed. The power levels, however, are predicted to stay in the $\mathrm{mW}$ level, where other competitors, already reviewed in this section, may be more suitable. In [334], it is described a $5 \mathrm{~m}$ long, 'compact', FEL design (schematic of Fig. 2.54(a)), under construction at this moment. The authors estimate a power from 0.2 MW to $2 \mathrm{MW}$ in the $1 \mathrm{THz}-3 \mathrm{THz}$ band, quite good for a device relatively accessible for more conventional laboratories.

At this point of the section, scene of $\mathrm{THz}$ sources is much better observed, and it is understood that output power is only one of a wide variety of features when considering a $\mathrm{THz}$ source. Temperature of operation, range of frequencies, tunability, CW or pulsed mode, efficiency, type of pumping, size, complexity, cost...impose, altogether, a fine source research for a specific application. Thus, it is possible to left behind now the simple ' $\mathrm{V}$ ' trend in output power image of the $\mathrm{THz}$ sources and $\mathrm{THz}$ gap, and get a final global comparison through the graph of Fig. 2.55 [324]. State of the art of most of the sources commented in this section is represented in this graph, which helps to better assimilate all read information and get a global picture. 


\section{Wavelength $(\mu \mathrm{m})$}

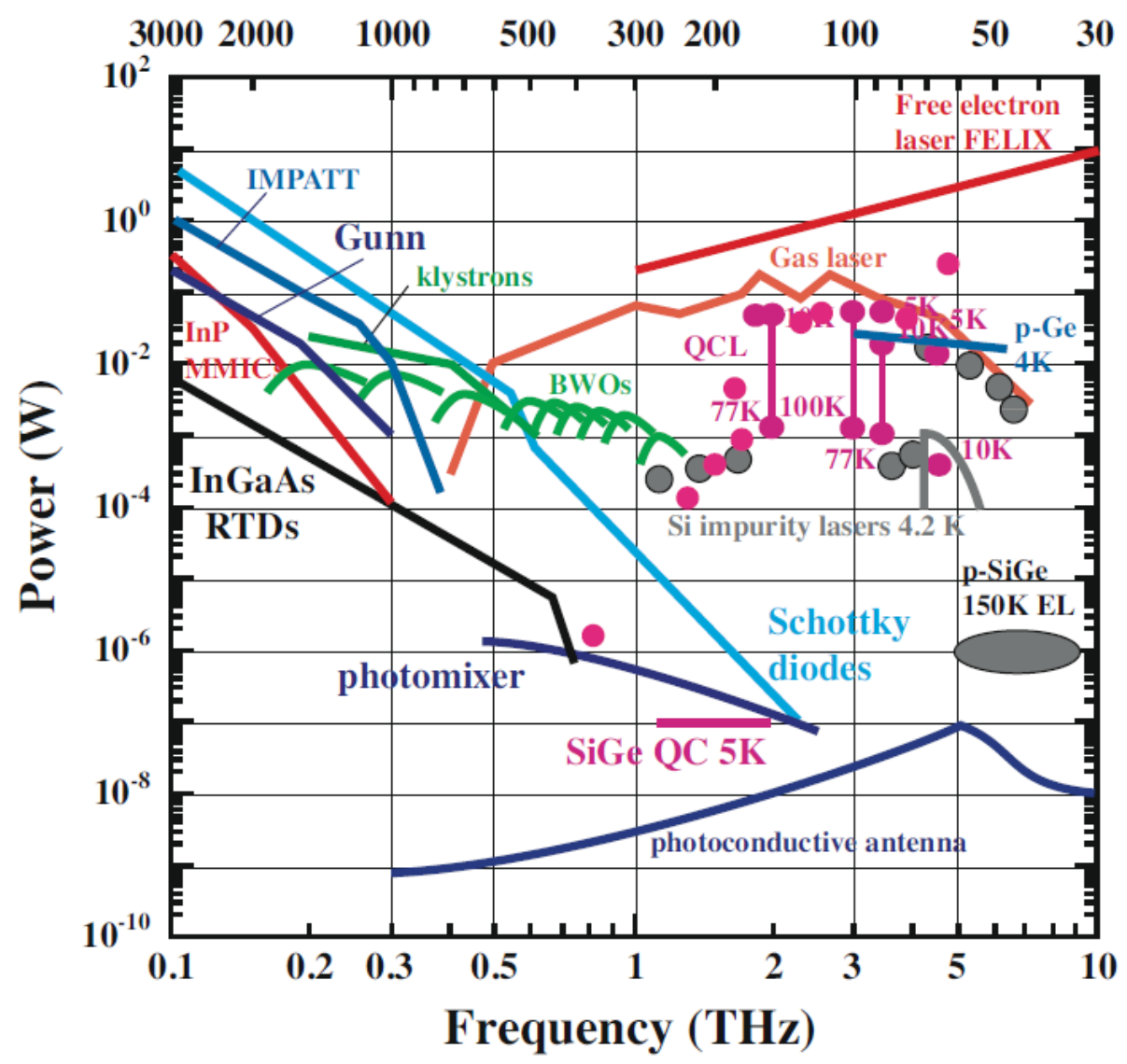

Figure 2.55: Comparative of $\mathrm{THz}$ sources [324].

\subsubsection{THz Waveguides}

Up to this point, $\mathrm{THz}$ applications have motivated the interest of the field. Detectors and sources state of the art has given an idea of the $\mathrm{THz}$ band systems design challenges. Along the review, at several points it has been found an important fact: the importance of the actual lack of $\mathrm{THz}$ waveguides. Furthermore, the immediate benefit of having certain kind of high-performance waveguide has been also glimpsed in some cases. This section will treat the issue of waveguide designing at $\mathrm{THz}$ frequencies through a careful review of the state of the art.

$\mathrm{THz}$ waveguides are the most challenging component of a $\mathrm{THz}$ system [192], and prove of that is the huge amount of different proposals, of very different nature, that have been proposed to the date. Furthermore, for most systems, the question of which design is more appropriate remains still open nowadays.

As usual, many classifications are possible. Here, four groups are distinguished. For each group bulk and planar designs are distinguished separately, since the de- 
manded functionality for each kind is quite different. In a similar way that has been done for the sources, the first two groups contain the designs coming from the microwave and the optic areas. These are the metallic waveguides and the dielectric waveguides, respectively. The third group involves structures where the inclusion of periodic elements plays a major role. The designs in this group have a particular grade of innovation, being very novel designs. Finally, the last group involves surface waveguides, a particular kind of waveguide that weakly guides the energy, railing the waves, it could be said. In this group, designs are not of special novelty, but they recover old theoretical designs and apply them into practice in a very thoughtful way. In this order, the review goes from the more known and classic to the newer, ending with the kind of waveguide which is object of study in this thesis by the reasons that will arise along this section.

\subsubsection{Metallic (Microwave) Waveguides and Transmission Lines}

The waveguide section starts with which is, actually, the first adopted solution for $\mathrm{THz}$ waveguiding for all kind of devices requiring transmission media. Most of the early $\mathrm{THz}$ sytems incorporated scaled microwave designs, the easiest way to afford the firstly used $\mathrm{THz}$ frequencies [1], [79], [80], [167].

The THz interaction with matter (high metal and dielectric losses) and the challenging small dimensions of $\mathrm{THz}$ devices, make the microwave solutions not to be naturally well-suited. Therefore, the increasing demand of system quality together with the low cost feature has impulsed three different lines of research and progress.

First, the manufacturing techniques. Advances in the manufacturing process are the main responsible of reaching the performance that is expected for the design. Also, manufacturing process entails an important part of the technology cost. Second, the structural topology. The way of how materials are geometrically disposed is crucial for waveguide performance. Therefore, one finds new proposals introducing modifications on the conventional designs in order to exploit the geometry to alleviate ohmic losses, dielectric losses and/or dispersion. Third, the materials. Although with less possible range of improvement due to the inherent physical limitations, some progress is achieved by finding better and better materials, the building blocks of the waveguides. For that reason is it also possible see along the bibliography the appearance of new materials (mainly dielectrics) that get of common use in the new waveguide designs.

Progress in all three directions is necessary to achieve high-performance waveguides. Next, $\mathrm{THz}$ advances in the rectangular waveguide, circular waveguide, coaxial cable, and planar microwave transmission lines (parallel-plate waveguide, stripline, microstrip, slotline and coplanar waveguide), reported.

\section{- Rectangular Waveguide (RWG)}

First serious attempts to design THz RWGs came around the year 2000 [192], [337], [338], when several research groups, pioneers in mm-wave, not so technologically mature as these days, started to venture in the sub-mm wave regime, even properly naming these RWG as $\mathrm{THz}$ waveguides. From this starting point, either due to manufacturing limitations [339], [338], or inherent structure losses and performance [192], it has been quite common to introduce some 
modifications on the usual RWG (structure, dimensions and/or modal operation). It is true, notwithstanding, that in more recent works, [340]-[345], conventional use of the RWG starts to be seen, at least for lower frequencies, thanks to the advances in manufacturing processes, being remarkable those involving SU-8 photoresist micromachining technology [346].

The classical RWG structure and its main design parameters are shown in Fig. 2.56(a). Generally, $b$ is taken as $a / 2$ in order to have optimum loss-bandwidth relationship [339]. To avoid dielectric losses (which are specially important at $\mathrm{THz}$ frequencies) the RWG is usually air-filled $\left(\varepsilon=\varepsilon_{0}\right)$. Finally, metal conductivity $\sigma$ results relevant in microwave regime to calculate the losses. At $\mathrm{THz}$, moreover, other parameters related with the conductivity become relevant: the penetration (or skin) depth $\delta=(\pi f \mu \sigma)^{-1 / 2}$, and the surface impedance or metal properties as a dielectric [347], [348]. One reason for the first one is that, although at $\mathrm{THz}$ frequencies the penetration depth is lower, some new manufacturing techniques are based on thin metal layers that may not reach the penetration depth. The other reason is that wall roughness may be comparable the penetration depth [337], [349]-[353]. The second one arises because assumed metal model properties (very high $\sigma$ or perfect conduction) may noticeably change due to manufactured surface roughness [354], [342], or microscopic electromagnetic interaction (Drude model of metals [347]) [355][348].

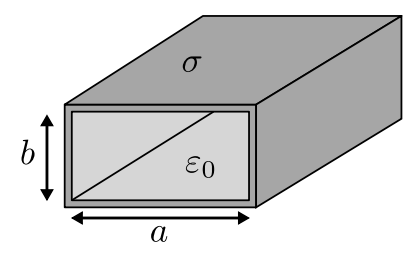

(a)

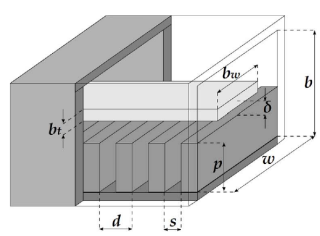

(b)

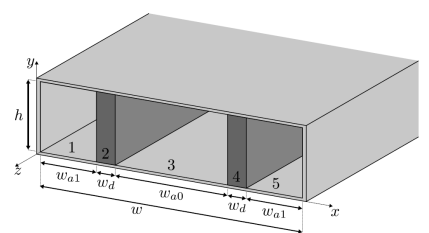

(c)

Figure 2.56: Rectangular Waveguide (RWG) at $T H z$ frequencies 1. a) Schematic of a classical rectangular waveguide, defined by width $a$, height $b$ and conductor material $\sigma$. To reduce losses, inner dielectric is normally air $\left(\varepsilon_{0}\right)$; b) Schematic of corrugated RWG for slow-wave high power propagation in a Backward Wave Oscillator [352], [293]; c) E-plane dielectric layer loaded RWG schematic and prototype detail [357];

By using classical RWG analysis formulas and using standard waveguide dimensions [358], extended to the $\mathrm{THz}$ regime, it is found that, even in the ideal case, having a perfect structure with the conventional cooper conductivity $\left(\sigma=5.8 \cdot 10^{7}\right)$, they are found the following theoretical losses: 0.008 $\mathrm{dB} / \mathrm{mm}(0.1 \mathrm{THz}), 0.024 \mathrm{~dB} / \mathrm{mm}(0.3 \mathrm{THz}), 0.090 \mathrm{~dB} / \mathrm{mm}(1 \mathrm{THz})$ and 0.970 $\mathrm{dB} / \mathrm{mm}(3 \mathrm{THz})$. Note that for $\mathrm{THz}$ frequencies, due to dimensions and attenuation values, units of $\mathrm{dB} / \mathrm{mm}$ are commonly used as attenuation instead of the usual $\mathrm{dB} / \mathrm{cm}$ or even $\mathrm{dB} / \mathrm{m}$. However, along this thesis, $\mathrm{dB} / \mathrm{m}$ will be used sometimes for special cases of very low attenuation.

By the above reflection, it seems that RWG is inherently limited to be used in the lower $\mathrm{THz}$ spectrum despite of its desirable characteristic of be a extensively studied structure. In that spectrum, however, it will be hard that other 
technology displace the RWG in the typical applications in which it is used at microwaves (low-loss filters, horns, BWO, etc.).

Actually, most of the practical systems (mainly in astronomy applications) providing true $\mathrm{THz}$ experimental results until these days, have appeal for the RWG for waveguiding purposes (being waveguiding, actually, avoided as possible) [1], [5], [31], [168]. Filters, antennas, and twist have been prototyped showing relatively good results. Also, in a $\mathrm{THz} \mathrm{BWO}$, a corrugated RWG, Fig. 2.56(b), is very suitable. At $\mathrm{THz}$ frequencies, the high interaction impedance of the corrugations introduces a compensation of losses higher than for lower frequencies [352], which is a positive effect.

Similarly, it is very probable that some modified structures, behaving as a RWG, but tailored to overcome high loss of $\mathrm{THz}$ band, such as the E-plane dielectric layer loaded RWG of [357] (see Fig. 2.56(c)), the H-plane dielectric layer loaded RWG [359] (named as shielded dielectric multiple slot waveguide) or the Groove Gap Waveguide (GGW) [360], [361], gain some ground in the next years.

To better understand the RWG role and practical issues in $\mathrm{THz}$ band, main results, observations and conclusions of the last 15 years of research in this topic are reviewed now. In the pioneer works [337], [338] manufacturing was done through that time lithographic micromachining, with the important drawback of having maximum manufacturable heights, being the ratio $b=a / 2$ not satisfied by far. In [337], a $2.54 \mathrm{~mm}$ x $0.7 \mathrm{~mm}$ gold sputtered RWG exhibited 0.2 $\mathrm{dB} / \mathrm{mm}$ at $0.1 \mathrm{THz}$, much more than the expected value, although overcoming by far previous works. Augmented attenuation was mainly attributed to bad metal contact due to metal roughness in this H-plane manufactured RWG. In [338], the height of the waveguide was even more limited, being the dimensions $2.54 \mathrm{~mm} \times 0.1 \mathrm{~mm}$. Losses ranging from $0.2 \mathrm{~dB} / \mathrm{mm}$ to $5 \mathrm{~dB} / \mathrm{mm}$ were observed at the $\mathrm{W}$-band $(75 \mathrm{GHz}$ to $100 \mathrm{GHz})$, whilst theoretically expected were of only $0.05 \mathrm{~dB} / \mathrm{mm}$ to $0.34 \mathrm{~dB} / \mathrm{mm}$. Imperfections in the surface walls of the waveguide were also pointed in this work as the origin of the additional losses. They were reported as well difficulties in the measurements due to required contact with the measure instrument at these high frequencies.

In [192], it was reported a very different and innovative approach. Whereas in the previous works, RWG very scaled (with limitations) for their use in the mm-wave, this work talks about an overmoded RWG designed to transmit wide-band $\mathrm{THz}$ signals, which is the required case for most of $\mathrm{THz}$ applications. A $0.250 \mathrm{~mm} \times 0.125 \mathrm{~mm}$ (optimized dimensions to achieve optimal trade off between coupling efficiency and losses) RWG was proposed to transport signals in the frequency range extending from $0.65 \mathrm{THz}$ to $3.5 \mathrm{THz}$. This implies a bandwidth an order of magnitude larger than the conventional WR-3 (0.340 $\mathrm{mm} \times 0.170 \mathrm{~mm}$, operating monomode from $0.220 \mathrm{THz}$ to $0.325 \mathrm{THz}$ ). Up to 35 modes were able to propagate in the proposed waveguide, although proper coupling and design allowed to excite mainly the fundamental $\mathrm{TE}_{01}$, and the $\mathrm{TM}_{12}$ modes. Coupling was done quasi-optical using mirrors and silicon lenses to focus a Gaussian beam with a maximum efficiency of $98 \%$, a very high value. 
Brass was used as metal due to its good trade off between conductivity and mechanical robustness. Low losses were affirmed in the paper, however no consistent numerical data are specifically given. A careful analysis reveals that, in time-domain, peak to peak amplitude decays at a rate of roughly $1 \mathrm{~dB} / \mathrm{mm}$. Degradation of the measured response due to manufacturing imperfections (burrs affecting contact between pieces) is mentioned. Low dispersion is also declared, however it is specifically said that the velocity group varies from c to $\mathrm{c} / 4$ (being $\mathrm{c}$ the speed of light in the waveguide medium, in this case air: $c_{0}=$ $3 \cdot 10^{8}$ ) along the transmitted band, what is a strong dispersion characteristic, actually. The time-domain pulse spreads from $0.04 \mathrm{ps}$ to $13 \mathrm{ps}$, being $6 \mathrm{ps}$ of the spreading attributed at the optical feeding system. With similar low-loss premises, in [362], a photomixing generation scheme is implemented via an overmoded RWG. In this case however, the operating band ranges from 0.2 $\mathrm{THz}$ to $0.5 \mathrm{THz}$, being monomode until $0.347 \mathrm{THz}$. The source provided 0.53 $\mathrm{mW}$, which is a remarkable output good power.

Continuing with conventional use of RWG, [349] reports good results in surface finish by employing SU-8 photoresist techniques. Manufacturing is done in E-plane, the total structure being quite bulky to the real waveguide size. Results for the 3 manufactured RWGs are: D-band (0.11 THz to $0.17 \mathrm{THz}$ ) $0.07 \mathrm{~dB} / \mathrm{mm}$ to $0.10 \mathrm{~dB} / \mathrm{mm}$, G-band $(0.11 \mathrm{THz}$ to $0.17 \mathrm{THz}) 0.14 \mathrm{~dB} / \mathrm{mm}$ to $0.22 \mathrm{~dB} / \mathrm{mm}$ and Y-band $(0.22 \mathrm{THz}$ to $0.325 \mathrm{THz}) 0.139 \mathrm{~dB} / \mathrm{mm}$ to 0.20 $\mathrm{dB} / \mathrm{mm}$, which improves previous works. It is indicated, however, that Y-band prototype experiments leakage losses due to the difficulty to ensure perfect metal contact between the two halves. This observation is really important because it confirms that even in E-plane manufacture, requirements of contact are alignment result very challenging at $\mathrm{THz}$ frequencies.

Operating frequency is pushed up to $1.19 \mathrm{THz}-1.87 \mathrm{THz}$ in [351] by using a $150 \mu \mathrm{m} \times 75 \mu \mathrm{m}$ waveguide. Prototypes of microstrip fed RWG with integrated horn are manufactured via SU-8 photoresist and laser-hardened polymer block. A comparison between both techniques is shown in Fig. 2.57. The laserhardened polymer block shows significant distortion of the design dimensions, see Fig. 2.57(a). Except some errors near the backshort, SU-8 exhibits great performance: $\pm 1 \mu \mathrm{m}$ accuracy. It is affirmed that conventional techniques are relegated to as much as $0.6 \mathrm{THz}$ and $\mathrm{SU}-8$ process or similar techniques must be used beyond that threshold.

More recently, the group of Prof. Lancaster has proved feasibility of reasonableperformance RWG in the $\mathrm{THz}$ band, up to $0.75 \mathrm{THz}$, through several works [341]-[343], [345], [353], [364]. In [353], $0.144 \mathrm{~dB} / \mathrm{mm}$ average losses are obtained for an Y-band E-plane manufactured design, used to implement a $\mathrm{H}$ plane bend. Presented losses are still far from the $0.025 \mathrm{~dB} / \mathrm{mm}$ achieved with conventional micromachining, more expensive, time costing, and difficult to scale to higher frequencies. They are reported problems related to the air gaps between manufactured pieces since no convenient way to employ pins and screws to eliminate this gaps is found, causing additional loss. Simulation of chokes and Photonic Bandgap (PBG) structures reveal that results can be improved by including these elements, with best results for the former. Similarly, 


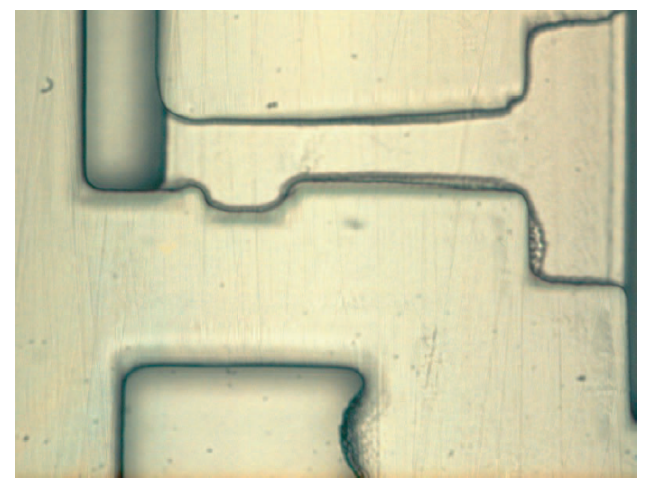

(a)

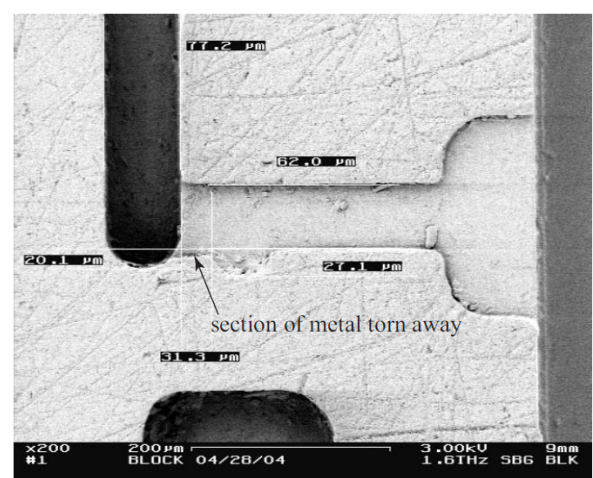

(b)

Figure 2.57: Rectangular Waveguide (RWG) at $\mathrm{THz}$ frequencies 2: manufacturing processes. a) Photomicrograph of the same $1.6 \mathrm{THz}$ waveguide block implemented by laser hardening a polymer base block. Significant distortion of the block dimensions is observed [351]; b) Scanning Electron Microscope (SEM) image of a $1.6 \mathrm{THz}$ Computer Numerically Controlled (CNC) [363] machined waveguide block. Inside corners are rounded for easy of fabrication. It is remarked a section of the metal next to the backshort that resulted damaged during the machining

the mentioned RWG design is used in [342] to implement a 4th order filter with a Relative Bandwidth (RBW) of $8 \%$ centered at $0.3 \mathrm{THz}$. Insertion Losses (IL) of $3.3 \mathrm{~dB}$ which implies an equivalent quality factor of only $Q=82$ (microwave RWG filters have Qs in the order of thousands). Response degradation and frequency shift are justified by manufacturing tolerances in the SU-8 process of $10 \mu \mathrm{m}$ to $15 \mu \mathrm{m}$, quite influential at the small $\mathrm{THz}$ wavelengths.

Much better results are obtained in the subsequent works. In [343], RWG losses go down from previous $0.14 \mathrm{~dB} / \mathrm{mm}$ to $0.03 \mathrm{~dB} / \mathrm{mm}$ (single layer $\mathrm{SU}-8$ process) and $0.05 \mathrm{~dB} / \mathrm{mm}$ (double layer SU-8 process), really close to the $0.021 \mathrm{~dB} / \mathrm{mm}$ of conventional micromachining (experimentally proved with gold plated $\mathrm{CNC}$ machined RWG in the same work), and overcoming any other contemporary designs. Interesting information about manufacturing technologies is found in this paper: to the authors experience SU-8 process overcome other common processes for high frequency RWG manufacturing such as Si Deep Reactive Ion Etching (DRIE) [365] or LIGA ${ }^{17}$. SU-8 is said to provide the best combination of time consume and accuracy among those three techniques.

In [343], several designs are presented with the WR-3, among them, a 5th order filter with the same specifications as the previous (RBW of $8 \%$ and centered at $0.3 \mathrm{THz}$ ). The equivalent $\mathrm{Q}$ is incremented up to near 400 in this design. A photography of the WR-3 prototype is shown in Fig. 2.58(a). They can be appreciated the two E-plane split halves containing the waveguides. Contact is ensured via four screws plus an additional PTFE screw used to push the micromachined circuit towards. Position and schematic of the employed flange

\footnotetext{
${ }^{17}$ LIGA is a German acronym for Lithographie, Galvanoformung, Abformung (Lithography, Electroplating, and Molding) that describes a fabrication technology used to create high-aspect-ratio microstructures [350], [366].
} 


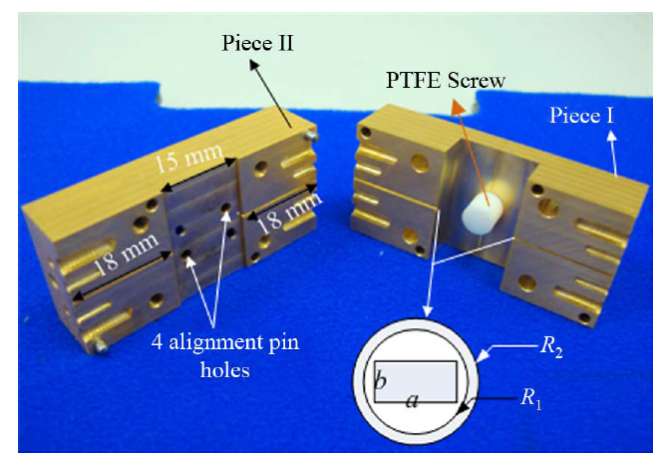

(a)

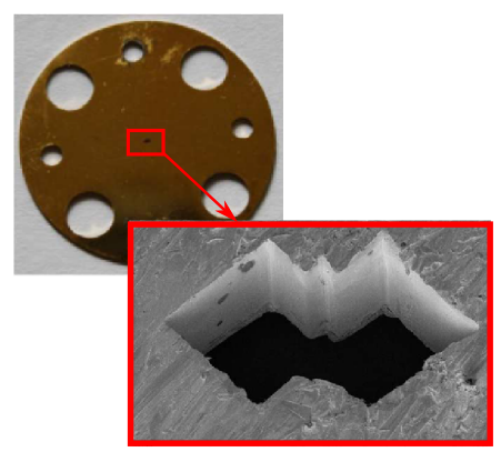

(b)

Figure 2.58: Rectangular Waveguide (RWG) at $\mathrm{THz}$ frequencies 3: manufactured prototypes. a) Photograph of the measurement metal block in [343]. A WR-3 waveguide was split in E-plan, using four screws to achieve contact between pieces. Additionally, a Polytetrafluoroethylene (PTFE) screw is used to push the micromachined circuits towards. Position an schematic of choke rings are indicated; b) Images of the WR-1.5 twist prototype of [340]. Left image shows the entire twist shim and right image shows the SEM capture of the structure.

(standard UG-387) are also indicated. Precision of SU-8 process is reported to be of tens of $\mathrm{nm}$ for the side wall roughness and under $10 \mu \mathrm{m}$ for the filter widths. Therefore, response degradation seems to be again originated by nonperfect assembling of layers, which is more noticeable $(0.05 \mathrm{~dB} / \mathrm{mm}$ against $0.03 \mathrm{~dB} / \mathrm{mm}$ ) for the two-layer designs. In this work, it is again reported how critical is the join contact for the RWG structure performance even for the E-plane manufacturing.

Continuing the RWG up-scaling in frequency, the $0.5 \mathrm{THz}-0.75 \mathrm{THz}$ band is reached, covered by the WR-1.5, which is used in [340] to implement 90 degrees waveguide twist (see Fig. 2.58(b)), and in [345], to implement a 3th order equal bandpass ripple filter with $5 \% \mathrm{RBW}$, centered at $0.65 \mathrm{THz}$. In [340], mean losses of $0.065 \mathrm{~dB} / \mathrm{mm}$ are reported for the presented design, which is a good result for the year of publication. Some mismatch between waveguide and twist, causing ripples in the measurement, was reported. Also, it is appreciated strong influence of metal roughness, which produces differences between the $S_{11}$ and the $S_{22}$. It must be pointed out that, in the presented results high error range is found for the measurements, which indicates that ensuring contact and matching is also critical for the measurement setup. Regarding to this important problem, some new techniques are being proposed [367], however it seems that leave the classical RWG scheme and introduce contactless structures, such as Gap Waveguides (GWs), could be much more effective. In other works, [368] they are developed uncertainty analysis that capture the dominant sources of measurement error: discontinuities caused by changes in the waveguide dimensions, E-plane, H-plane, and angular displacements, corner rounding, burrs, etc.

In [340], outstanding $0.043 \mathrm{~dB} / \mathrm{mm}$ losses are reported for their WR-1.5, and the proposed filter exhibits a Q-factor over 400. However, small manufacturing 
errors causing cavity dimensions variation up to $20 \mu \mathrm{m}$ cause an appreciable response deviation. The achieved metal roughness is under $50 \mathrm{~nm}$, so that equivalent conductivity falls from theoretical $6.3 \cdot 10^{7} \mathrm{~S} / \mathrm{m}$ to $3.9 \cdot 10^{7}$. This is, however, a great improvement in the manufacturing techniques in a short time (in [342] equivalent conductivity was of only $6.5 \cdot 10^{5} \mathrm{~S} / \mathrm{m}, 2$ orders of magnitude less).

Alternatively, in [344], DRIE process is used to explore E-plane and H-plane split-block designs with WR-3. In that work, a gold layer after DRIE is employed, achieving an equivalent conductivity of $3 \cdot 10^{7}$, quite close to the theoretical value for gold $\left(\sigma=4.55 \cdot 10^{7}\right)$. Chebyshev filters of 5 th order with RBW of $6 \%$ and $10 \%$, centered at $0.55 \mathrm{THz}$ and $0.64 \mathrm{THz}$, respectively, are designed and manufactured. Some deviation from original response is found, caused almost totally by the deviation of $2^{\circ}$ of the involved vertical walls, supposed to be of $90^{\circ}$. Apart from this fact, outstanding equivalent $\mathrm{Q}$ of 500 is found for the resonators in the E-plane design. H-plane design suffers more the imperfect contact between metallic parts and the equivalent $\mathrm{Q}$ falls down to 200 .

Finally, thinking in the real $\mathrm{THz}$ challenging range (beyond $1 \mathrm{THz}$ ), packaging techniques have been presented [369]. The authors claim that the proposed scheme may be extended up to $5 \mathrm{THz}$. The presented prototype operating at $0.385 \mathrm{THz}-0.5 \mathrm{THz}$ exhibits $0.05 \mathrm{~dB} / \mathrm{mm}$, which is a not a really outstanding value compared to the reviewed bibliography. More recently, [370] presented a $\mathrm{TE}_{301} / \mathrm{TE}_{102}$ dual mode RWG resonant cavities filter manufactured by means of DRIE process. Involved waveguides showed $0.31 \mathrm{~dB} / \mathrm{mm}-0.83 \mathrm{~dB} / \mathrm{mm}$ in the range $0.75 \mathrm{THz}-1.1 \mathrm{THz}$. In [371] frequencies as high as $3 \mathrm{THz}$ are reached with a multilayer micromachined WR-0.3 $(75 \mu \mathrm{m} \times 37.5 \mu \mathrm{m})$, being the losses at this frequency $1.3 \mathrm{~dB} / \mathrm{mm}$. Although these results are acceptable, the lack of suitability of the classical RWG for these high frequencies is a fact, because, apart of the losses increment caused by the dimensions reduction, assumed bulk conductivy of metals provide an oversimplified model, being real losses much higher than predicted [355]. Therefore, RWG will not be suitable in monomode operation for applications involving practical distances of few $\mathrm{cm}$.

In parallel with this conventional designs, mainly driven by the advances in manufacturing techniques, they have been published some works considering modifications of the RWG structure to alleviate $\mathrm{THz}$ band losses. This is the case of the E-plane loaded RWG [357], [372]-[373], shown in Fig. 2.56(c). By disposing several dielectric layers, a 1-D Electromagnetic Bandgap (EBG) structure is created. As detailed in [357], due to the introduced dielectric layers, the distribution of the $\mathrm{TE}_{03}$ mode posses a field distribution showing high concentration in the central air region. This produces a noticeable reduction of losses respect to the classical RWG case. Position and thickness of the dielectric layers is optimized to minimize the losses. Very low coupling to other modes is reported in the case of a bend. By using high permittivity dielectric layers, the described effect is achieved in large bandwidth.

In [372] it is shown that, although this waveguide concept involves several dielectric layers (multilayer), not much is gained by going beyond the single 
layer structure, which is the actual design shown in in Fig. 2.56(c). The current at side walls is reduced a $20 \%$ respect to the conventional RWG, which theoretically makes the losses at $1 \mathrm{THz}$ to fall from $0.07 \mathrm{~dB} / \mathrm{mm}$ of the classical RWG to $0.057 \mathrm{~dB} / \mathrm{mm}$. With this waveguide, a H-plane horn array operating at $1 \mathrm{THz}$ is designed in [373]. The reduction of losses allows to achieve an antenna efficiency of $91 \%$.

Very recently, they can be also found some works considering the use of graphene [374], [375]. In [374], a RWG is considered in the cases of a) top metal wall consisting in a graphene layer and b) top and bottom layers consisting in a graphene layer. Bulk properties of graphene make the analysis and behaviour understanding of the structure rather complex. By applying electromagnetic fields, the dispersion curves can be controlled. In that work it is affirmed that attenuation and propagation constants similar to the conventional RWG are obtained. However, it is not clear the advance of using graphene. Similar conclusions are obtained in [375]. However, in this work a more concrete advantage is reported: graphene can be used for amplitude and phase modulation purposes.

\section{- Circular Waveguide (CWG):}

The Circular Waveguide (CWG) consist on a metal pipe, usually filled by air, Fig. 2.59(a). This waveguide is known to offer slightly lower losses than the RWG (involving also smaller bandwidth) for its fundamental mode $\left(\mathrm{TE}_{11}\right)$. Furthermore, its $\mathrm{TE}_{01}$ mode experiments an anomalous losses decrement with frequency [376]. This last feature results attractive for some designs. Also, the CWG cylindrical geometry results more suitable than rectangular one for certain applications. This becomes really true in the $\mathrm{THz}$ band since, due to the involved waveguide dimensions, cylindrical geometry offer more robust and flexible topology, able to cover applications that the RWG would not. Contrarily to the RWG, for the CWG is hard to find works considering the monomode design, since the involved bandwidth would be quite limited.

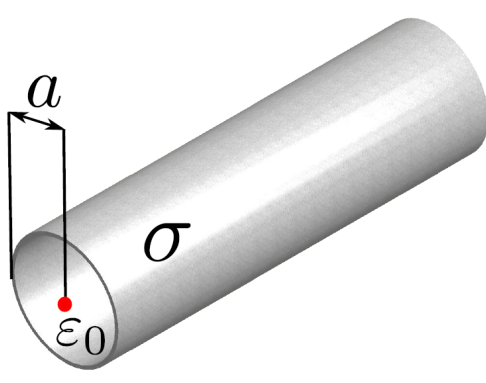

(a)

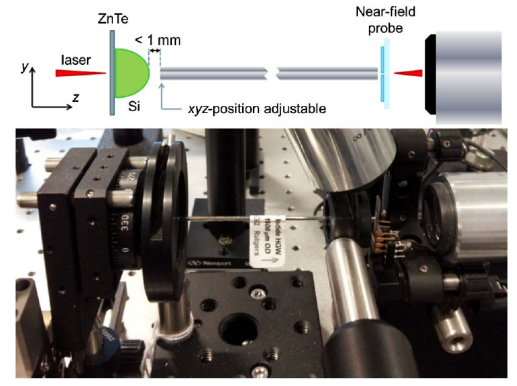

(b)

Figure 2.59: Circular Waveguide (CWG) at $T H z$ frequencies 1. a) Schematic of a classical circular waveguide, defined by its radius $a$, conductor material $\sigma$ and inner dielectric (conventionally air, $\varepsilon_{0}$ ) ; b) Schematic diagram (top) and picture (bottom) of the time-domain experimental setup. The waveguide can be seen in the center of the picture, whereas the subwavelength aperture near-field probe is on the right-hand side [377]. 
A nice first attempt to overcome inherent high frequency losses of CWG was that of [378]. The work traslates to the cylindrical geometry the groove concept [379], which mainly consist on a deformation of the waveguide and the elimination of side walls, to let the mode to spread and to interact less with the metallic walls, thus reducing the conductor losses. Theoretical analysis and measurements are provided. The waveguide results attractive due to its single mode operation, low dispersion and large dimensions and low loss (one order of magnitude less than a RWG). Power handling applications, such gyrotron implementation are pointed as possible uses. Authors remark that the waveguide is easy to manufacture and connect and that the bandwidth is relatively broad. However, $\mathrm{THz}$ usually required bandwidths are much higher. This approach is not found in posterior research, leaded by overmoded CWG, sometimes involving inner coatings.

It was in 1999 when [380] proposed an overmoded CWG to operate in the $0.65 \mathrm{THz}-3.5 \mathrm{THz}$ band, being the employed quasi-optical methods for power coupling a great novelty at that time. An efficiency of $40 \%$ was achieved for this coupling. The $\mathrm{TE}_{11}, \mathrm{TM}_{11}$ and $\mathrm{TE}_{12}$ were the mainly excited modes by linearly polarized incoming $\mathrm{THz}$ pulses. A stainless steal $\left(\sigma=1.32 \cdot 10^{6} \mathrm{~S} / \mathrm{m}\right)$ tube of $r=140 \mu \mathrm{m}$ provided losses of $0.6 \mathrm{~dB} / \mathrm{mm}$ at $1 \mathrm{THz}$. The authors indicate that this is two orders of magnitude less than $\mathrm{THz}$ planar transmission lines used at that time. Furthermore, losses were shown to go down as the frequency increases, contrarily to other transmission media. Velocity group ranges from $\mathrm{c} / 4$ to $\mathrm{c}$, which translates in a very large dispersion behavior. Although this is pointed out by the authors, it is also affirmed that it may be suitable for surface-specific frequency-dependent abortion measurements and low volume sample cells for high pressure, long-path spectroscopic measurements of toxic or reactive gases and liquids.

Similarly, in 2000, [192] considers stainless steel, brass and nickel overmoded waveguides to cover the range from $0.1 \mathrm{THz}$ to $4 \mathrm{THz}$. However, multimode propagation (25 modes able to propagate, 5 of them mainly excited by the incoming pulses) originates serious problems beyond $1.7 \mathrm{THz}$. Brass exhibits half losses than stainless steel. The badly defined shape of the tubes is pointed as source of additional loss. It is indicated that sub-ps transmission involves several octaves, which can be only afforded with these large waveguides. The excess of coupled modes is justified by the linear polarization of the coupler, which is not fully compatible with the boundary conditions of the circular waveguide fundamental mode.

Later, in 2004, [381] started a design tendency that ruled CWG designs in the following years. Based on the authors experience in the mid-IR, where metals are far from being good conductors, a thin dielectric coating added to the internal waveguide walls is considered in order to reduce the interaction of the transmitted fields with the metal walls. Actually, the manufacturing method proposed by the authors consists in coating silica or polymer tubes inner walls with both metal and dielectric, defining in this way the waveguide by means of liquid-phase chemistry methods. Copper was preferred for its good conductivity and manipulability. Firstly, non-coated CWG of radius from $a=2$ 
$\mathrm{mm}$ to $a=6 \mathrm{~mm}$ were considered. The following losses were obtained: 0.006 $\mathrm{dB} / \mathrm{mm}(1.62 \mathrm{THz}), 0.007 \mathrm{~dB} / \mathrm{mm}(1.89 \mathrm{THz})$ and $0.005 \mathrm{~dB} / \mathrm{mm}(2.53 \mathrm{THz})$, one or two orders of magnitude less than those observed in RWG works. Two important effects were observed. First, losses were not scaling as $a^{-3}$ due to multimode effects, but were higher than expected. Second, by adding the coating, the losses increased up to $0.009 \mathrm{~dB} / \mathrm{mm}$. The coating, however, prevents from radiation losses on bends, that arise from mode conversion and from the non-perfect reflectivity of the thin $(700 \mathrm{~nm}) \mathrm{Cu}$ wall at $\mathrm{THz}$ frequencies. In Fig. 2.59(b) it can be seen an schematic diagram (top) and picture (bottom) of a time-domain experimental setup using such kind of CWG [377].

The same authors improved their design in [382]. An $a=2.2 \mathrm{~mm}$ silver CWG coated with Polystirene (PS) exhibit in that work $0.001 \mathrm{~dB} / \mathrm{mm}$ at $2.5 \mathrm{THz}$. Inner coating was optimized (best case was thickness $t=8.2 \mu \mathrm{m}$ ). Authors remarked that as the dielectric coating becomes thicker, TE modes become HE modes, which field configuration is very adequate to reduce metallic losses. Dielectric losses also increase for thicker coatings, however. Multimode and dispersion seem to be still disadvantages of this structure. The same authors treat more carefully the coating design and monomode excitation in [383]. Below $a=1.6 \mathrm{~mm}$ only the fundamental $\mathrm{HE}_{11}$ mode is excited, exhibiting lower losses than the $\mathrm{TE}_{11}$ mode and even the $\mathrm{TE}_{01}$ mode. Fig. 2.60(a) shows $\mathrm{HE}_{11}$ mode E-field pattern and and far-field spatial intensity distribution at $f=2.5 \mathrm{THz}$ for the case of a $a=1.6 \mathrm{~mm}$ silver CWG. Coupling efficiencies of $84 \%$ are reported whereas best losses remain in $0.001 \mathrm{~dB} / \mathrm{mm}$.

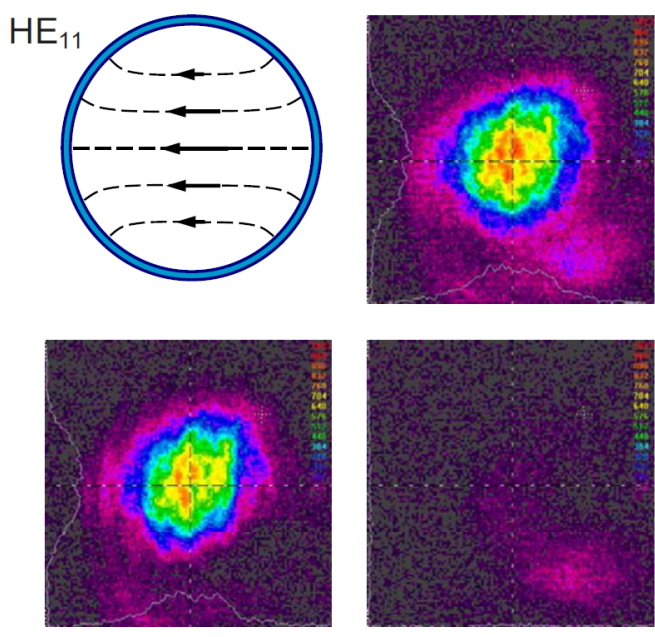

(a)

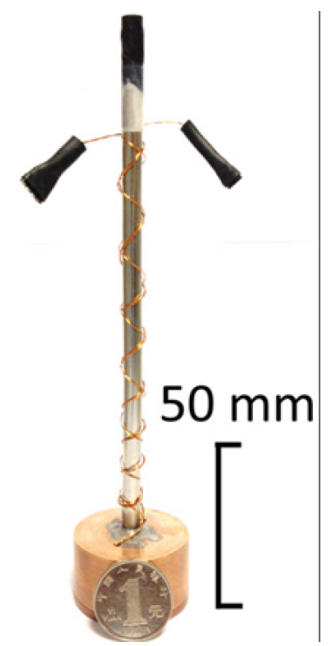

(b)

Figure 2.60: Circular Waveguide (CWG) at $T H z$ frequencies 2. a) $H E_{11}$ mode E-field lines and far-field spatial intensity distribution of a $a=1.6 \mathrm{~mm}$ radius silver $C W G$ at $2.5 \mathrm{THz}$. Field image of top right is the total magnitude, whereas down images correspond to horizontal polarization (left) and vertical polarization (right) [383]; b) Photograph of the probe implemented in [384], which consists of a stainless-steel tube acting as a CWG and a hollow copper container hosting the emitter. 
Other authors [385], [386], used the same concept to implement a very interesting flexible CWGs, with possible uses in remote $\mathrm{THz}$ spectroscopy. Manufacturing process and waveguide structure are schematized in Fig. 2.61. It is highlighted that excitation of the fundamental mode $\mathrm{TE}_{11}$ is preferred instead the $\mathrm{TE}_{01}$ mode due is compatibility with linear polarized coupling and to avoid mode conversion on bends. Bend losses as low as $0.5 \mathrm{~dB}$ are reported even for sharp bends. Best result for these designs is $0.0015 \mathrm{~dB} / \mathrm{mm}$ at $1.67 \mathrm{THz}$ for an $a=1.5 \mathrm{~mm}$. Authors remarked that the metal thickness $(50 \mathrm{~nm}-100 \mathrm{~nm})$ may concern problems related with the skin depth. They evaluate CWG for three cases: 1) no coating; 2) inner coating; 3) outer coating. Best case by far is the inner coating one, and it is indicated that the effect of coating is very noticeable for bend losses reduction, even for the outer case. Also, it is mentioned that one limitation of this structure is that thicker Polyethylenes (PEs) coatings are not possible because its poor solubility in solvent, thus reducing the described design benefits for the lower $\mathrm{THz}$ frequencies.

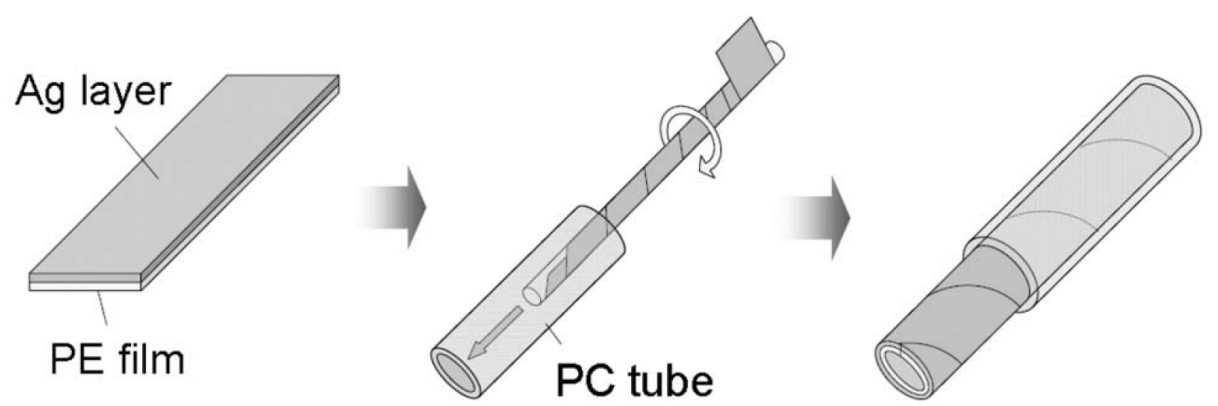

Figure 2.61: Circular Waveguide $(C W G)$ at $T H z$ frequencies 3: fabrication process of polyethylene (PE)-film-insert hollow CWGs. A Polycarbonate (PC) tube is used as base tubing [386];

Some practical uses of the CWG are found in [384], [387]-[389]. In [387], an overmoded CWG was part of a vacuum electronic tube source operating at $f=0.65 \mathrm{THz}$. DRIE process is reported to overcome Electrical Discharge Machining (EDM) [390] in this work. In [388], a corrugated CWG supporting TM modes is visioned as a promising slow-wave structure for high power $\mathrm{THz}$ transmission, specially indicated for plasma characterization. In [389], a very sensitive waveguide technique was applied to the $\mathrm{THz}$ analysis of DNA solutions, requiring the use of a $a=0.59 \mathrm{~mm}, 0.13 \mathrm{~mm}$ thick brass shim. Finally, in [384] a liquid-nitrogen-cooled compact CW source uses an $a=1.75 \mathrm{~mm}$ cooper CWG, Fig. 2.60(b). The work does not pay much attention to the waveguide, but conventional CWG overmoded design was chosen, in which the inner surface was polished in order to achieve acceptable losses $(0.055 \mathrm{~dB} / \mathrm{mm})$.

The circular waveguide section ends with the necessary answer for a fundamental question regarding the use of $\mathrm{CWG}$ at $\mathrm{THz}$ frequencies, giving firstly in the interesting work [348]. On the one hand, metals are very good conductors at microwave frequencies. On the other hand, in IR region metals act rather as a bad dielectric than a metal [21]. Between these disparate points there is a transition that takes place along the $\mathrm{THz}$ band. In the mentioned 
work, it is afforded the question of until which frequency and under which conditions microwave surface impedance approximations are still valid in $\mathrm{THz}$ CWGs. The authors consider the metal as a dielectric through the Drude model [347], having thus a dielectric tube waveguide problem with the dielectric having complex permittivity. Results throw interesting conclusions: for overmoded CWGs, some error is introduced by the classical mode beyond 1 $\mathrm{THz}$, whilst this limit goes up to $10 \mathrm{THz}$ for monomode designs. In fact, for high frequencies the fundamental $\mathrm{HE}_{11}$ mode tends to concentrate near the metal walls, becoming a surface-plasmon mode whereas the $\mathrm{TE}_{01}$ does not exhibit any change.

Similar general conclusions have been also recently reported in [391]. Actually, this CWG problem is the dual problem of the single metal wire (deeply analyzed in the next chapter), if one exchanges metal and air. Although different propagation behavior is found, analysis of both waveguides is related. Results highlight the singularity of $\mathrm{THz}$ frequencies, at which metals behave at half way between good conductors and poor, high permittivity dielectrics. Therefore, one more time, it is found that particular effort must be done in develop adequate analysis models that capture $\mathrm{THz}$ specific effects, in order to be able to predict correctly how the designed devices will act in practice.

\section{- Coaxial Cable:}

The coaxial transmission line or coaxial cable is a two-conductor waveguide consisting in an inner metallic wire of radius $a$, coaxial to a metallic tube of radius $b$, having the role of outer conductor, Fig. 2.62(a). A dielectric must be generally displayed between both conductors to keep the distance between conductors. It is possible to use air as dielectric in very specific designs [392], being the structure quite less unpractical than the conventional coaxial cable in terms of flexibility.

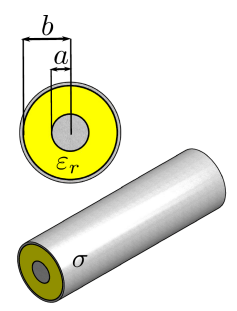

(a)

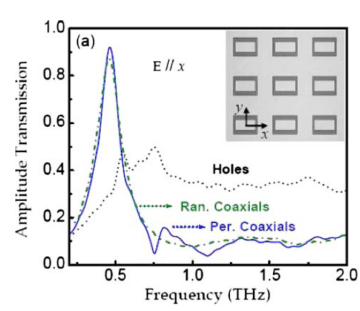

(b)

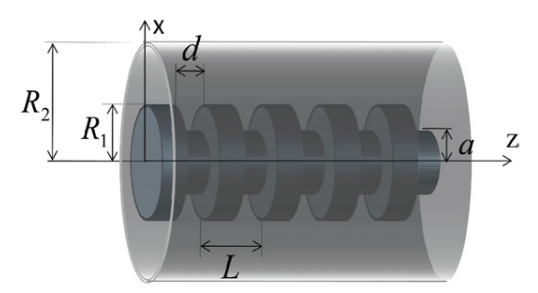

(c)

Figure 2.62: Coaxial transmission line at $T H z$ frequencies 1. a) Schematic of a classical coaxial transmission line, defined by its inner conductor radius $a$, outer conductor radius $b$, conductor material $\sigma$ and inner dielectric characteristics $\varepsilon$. In the case of the coaxial cable dielectric is usually required for mechanical reasons; b) Enhanced transmission at $\mathrm{THz}$ frequencies using rectangular coaxials [393]. The graph correspond to the measured frequency-dependent amplitude transmissions of a layer of periodic (solid curve) and random (dash-dotted curve) coaxials with external dimensions of $100 \times 80 \mu \mathrm{m}^{2}$ and internal dimensions of $80 \times 40 \mu \mathrm{m}^{2}$, together with the hole-only (without inner conductor) case (dotted curve). Employed period is $160 \mu \mathrm{m}$, and applied field is $x$-polarized; c) Schematic of the corrugated waveguide employed in [394]. Corrugation dimensions are small compared to the operating wavelength. 
Thanks to the fact of having two conductors, the coaxial cable supports a fundamental TEM mode with no cutoff and exhibiting theoretically no dispersion under monomode transmission [376]. As drawbacks, losses are higher than in single-conductor due to the higher confinement of the field, which dissipates power by ohmic effect on the metal walls, but also because of the dielectric absorption. Actually, when low-loss features are demanded in microwaves, waveguides are clearly preferred than coaxial cable or other TEM transmission lines.

Despite of its high losses, which even accentuate at $\mathrm{THz}$ due to the lack of good dielectrics and the smaller required dimensions, the coaxial cable has been also considered (in a less extent than the RWG or the CWG) for $\mathrm{THz}$ frequencies. It seems obvious that monomode transmission would require dimensions such any $\mathrm{THz}$ signal would be extremely damped in few millimeters. Thus, overmoded coaxials must be considered at $\mathrm{THz}$ frequencies, therefore losing (in principle) the desired non-dispersive behavior. However, the careful analysis of the structure and some experimental test have shown that the coaxial cable may have its own niche among other $\mathrm{THz}$ waveguides.

First transmission of frequencies well above $0.1 \mathrm{THz}$ using coaxial cables was reported in [395], where a $50 \Omega, 1.85 \mathrm{~mm}$ coaxial, was shown to be able to transmit pulses between two Coplanar Waveguides (CPWs), thus being very suitable for chip interconnection. Very high losses were obtained, however. Also, although is not clearly extracted from the paper, coupling efficiency is supposed to be quite low by the conclusions of posterior works.

The reference paper in the topic of $\mathrm{THz}$ coaxial cables is, without doubts, that presented by Jeon et al. in 2004 [396]. In that work a $50 \Omega$ coaxial is designed by using $a=80 \mu \mathrm{m}$ and $b=330 \mu \mathrm{m}$, and Teflon $\left(\varepsilon_{r}=2.1, \tan \delta=1.5 \cdot 10^{-3}\right.$ at $f=1 \mathrm{THz}$ ) as a dielectric. It was the first time that pulses having frequencies up to $1 \mathrm{THz}$ were launched into a coaxial cable. It was already remarked in this paper that quasi-optical coupling techniques are not effective for the complex field patterns of the TEM mode of a coaxial cable. To overcome this problem, an improved method, using photoconductive material having the proper coaxial shape, was presented. Results showed that monomode transmission takes place for the proposed design until $0.4 \mathrm{THz}$. Beyond this frequency some oscillations and other undesired effects appear in the received signal. Authors conclude that coaxial cable exhibits similar dispersion to CPW (output signals widened only 1 ps are obtained) together with losses comparable to early designs of single-conductor $\mathrm{THz}$ waveguides. At $f=0.5 \mathrm{THz}$ losses of 0.3 $\mathrm{dB} / \mathrm{mm}$ were obtained.

It is difficult to find posterior works of the same magnitude and relevance involving coaxial cables in their conventional use. This may be justified by the first $\mathrm{THz}$ proposals of the Single Wire Waveguide (SWW) and the DielectricCoated Single Wire Waveguide (DCSWW), treated later, which were contemporary to the previous work. These waveguides, basically coaxial cables without external conductor, captured much attention because their lower losses. 
On the other hand, very recent patents [397] indicate the potential use of the coaxial cable in interesting practical applications, thus deserving a particular niche of use for it. In that technical work, Mittleman affirms that coaxial cables are not very much considered at $\mathrm{THz}$ frequencies due to the difficulties in coupling the radiation into the guide, what is justified because of neither linearly nor circulary polarizations can be effectively coupled into a coaxial cable. This has prevent coaxial cable of playing a major role until these days. As a solution, it is presented a radially symmetric antenna that matches the mode profile of the TEM fundamental mode (mode-matching optimization determines the antenna dimensions).

It is worth to mention an interesting fact highlighted in the previous work. Since only the TEM mode is detectable by the presented configuration, even if the launched $\mathrm{THz}$ waves were propagating under multi-mode regime, this would not lead to measurable group velocity dispersion. Instead, high propagation losses would take place for that power coupled to high-order modes. This feature could be very useful for spectroscopy applications.

Finally, some techniques are proposed to achieve an empty (or filled with a gas transparent to $\mathrm{THz}$ frequencies) coaxial, which would be desirable for lowloss condition. However, it must be said that, despite of the fact that very promising use of coaxial is visioned in this patent technical report, it is not clear if losses will be low enough for the supposed applications. The presented transmission media are called to be used to access targets were direct radiation is not possible. Compared to the SWW, also considered in the work, the coaxial cable exhibits lower radiation losses but high propagation losses. Thus, its use maybe limited to short, complex (including sharp bends), waveguiding paths.

It can be also found recently a work studying deeply the $\mathrm{THz}$ propagation on an overmoded coaxial cable [398]. By using the Electro-Optical (EO) experimental set up shown in Fig. 2.63, the authors are able to accurately determine which modes and how the propagate in a $\mathrm{THz}$ coaxial cable. A typical, microwave regime, coaxial cable with $a=0.45 \mathrm{~mm}$ and $b=1.5 \mathrm{~mm}$ is studied. It is found that mainly 4 modes propagate: $\mathrm{TE}_{11}, \mathrm{TE}_{12}, \mathrm{TM}_{11}$ and $\mathrm{TM}_{12}$. Despite of this fact, $\beta$ of this modes seems to be similar enough to have low dispersion behavior. They are not found, however, clear assessments about propagation losses in this work.

As practical uses of $\mathrm{THz}$ coaxial cables, it is interesting that [399] suggests, through a theoretical study, the possibilities of this transmission line for the implementation of $\mathrm{THz}$ gyrotrons. A large size coaxial cavity supporting the $\mathrm{TE}_{04}$ mode is analyzed. Numerical calculations and simulation predict that advantages take place by using a coaxial cable instead of a single-conductor waveguide. The size of coaxial is larger so that the output power can be increased. Moreover, authors indicate that is possible have a large interval between TE modes, thus mode competition is also improved.

It is possible to found less conventional uses of the coaxial transmission line structure at $\mathrm{THz}$ frequencies. For example, in [393] it is proved that square coaxial structure improves by 8 times compared to the RWG the enhanced 


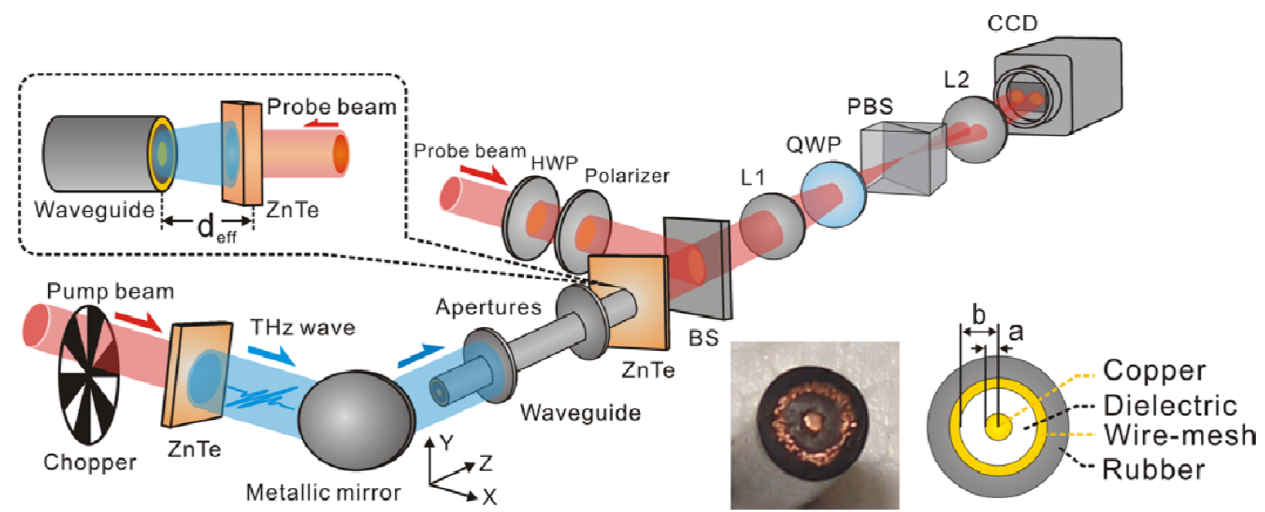

Figure 2.63: Coaxial transmission line at $\mathrm{THz}$ frequencies 2: applications. Schematic of the balanced electro-optical (EO) holographic imaging system employed in [398]. Remarked inset shows the existent distance between the waveguide output end and the measurement plane. A photography an schematic of the employed coaxial cable are shown on the bottom right of the image.

transmission based in plasmonic effects. In Fig. 2.62(b) they are shown measured frequency-dependent amplitude transmissions of a layer of periodic (solid curve) and random (dash-dotted curve) coaxials with external dimensions of $100 \times 80 \mu \mathrm{m}^{2}$ and internal dimensions of $80 \times 40 \mu \mathrm{m}^{2}$, together with the holeonly (without inner conductor) case (dotted curve). The employed period is $160 \mu \mathrm{m}$, and the applied field is $x$-polarized. A sharp peak at the desired frequency $f=0.5 \mathrm{THz}$ is appreciated for the coaxial waveguide case. Furthermore, the array of coaxial transmission lines solves the polarization-dependent transmission discrepancy, found in the RWG arrays. This may be caused by the interaction of the plasmonic waves of inner and outer conductor. It is concluded that its unique resonant characteristics make coaxial structures an excellent candidate in developing some active and passive $\mathrm{THz}$ components.

Other example is the periodically corrugated coaxial waveguide proposed in [394]. An schematic of this waveguide is shown in Fig. 2.62(c). An analysis based on mode-matching is carried out, finding that TM modes highly localized can be propagated in this structure with low velocity group. Since metals have not strong plasmonic behavior at $\mathrm{THz}$ frequencies, the corrugations (small compared to the wavelength) are necessary to this fact. The geometry can be engineered to satisfy the desired grade of the plasmonic effect. As advantages front a corrugated wire, a corrugated coaxial cable is shown to have less crosstalk due to its shielded nature, and lower velocity group can be achieved. On the other hand, there is not clear information about losses, nonetheless, high field concentration near metals of this shielded, plasmonic waveguide points towards very high losses. Therefore, this design get relegated to very specific applications requiring short waveguiding paths and demanding particular effects to be introduced by the waveguide.

\section{- Planar transmission lines:}


Most commonly considered planar waveguides or transmission lines in the microwave regime are the stripline, Fig. 2.64(a), the microstrip, Fig. 2.64(b), the Slotline (SL), Fig. 2.64(c), and the coplanar waveguide (CPW), Fig. 2.64(d), [337], [400]. At $\mathrm{THz}$ frequencies, however, only microstrip or CPW designs are found by several reasons. A deep analysis of these four waveguides for the $\mathrm{THz}$ regime can be found in [401]. Although only simulations are reported, conclusions give a useful general view of advantages and drawbacks of these waveguides.

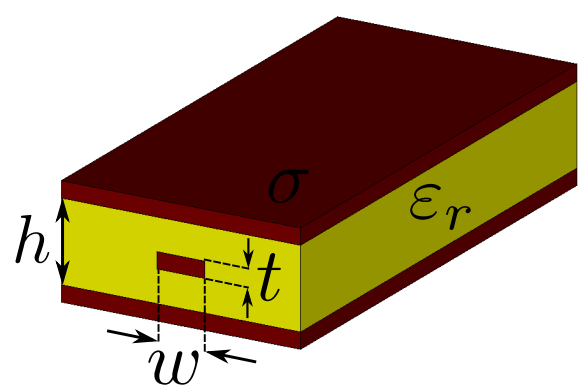

(a)

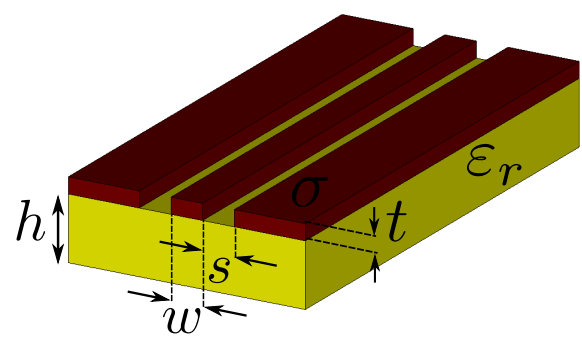

(c)

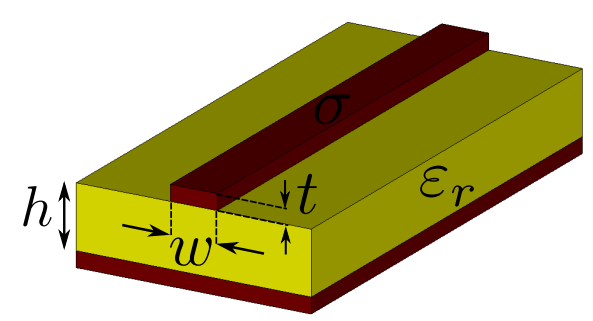

(b)

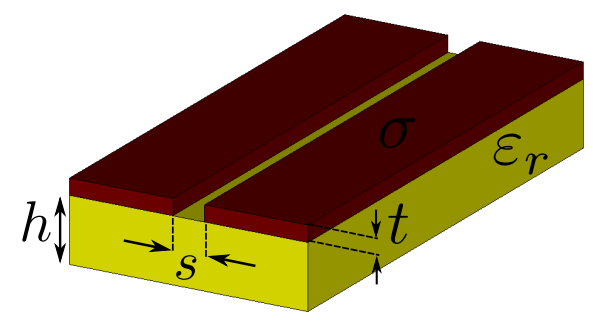

(d)

Figure 2.64: Planar waveguides at $\mathrm{THz}$ frequencies 1. a) Stripline; b) Microstrip; c) Coplanar Waveguide $(C P W) ;$ d) Slotline (SL).

The stripline, which is the earliest transmission line, presents the lowest dispersion, and intermedium losses (theoretically $6 \mathrm{~dB} / \mathrm{mm}$ at $f=1 \mathrm{THz}$ [401]) compared with the other three. However, it requires strong symmetry and thereby presents difficulties in the circuit design, incorporation of elements, and bias circuitry. This becomes a specially undesired feature for the small $\mathrm{THz}$ circuits.

The slot line presents large dispersion radiation losses and high order mode excitation problems. Thus, broadband transmission is not well supported by this waveguide. Since two-conductor transmission lines are considered in $\mathrm{THz}$ mostly for non-dispersive broadband signal transmission, the slotline gets out of the choice.

In the case of microstrip, propagation takes place mainly in the dielectric region between the strip and the ground plane. This implies high confinement of the field, having, therefore, both, large conductor losses and large dielectric 
absorption. Benzocylobutene (BCB), a low permittivity, relative low-loss dielectric at $\mathrm{THz}$ frequencies $\left(\varepsilon_{r}=2.42, \tan \delta=0.007\right.$ [402]), is commonly used [401], [403], [159], also because of its easy grafting. Despite of the high-loss feature of the microstrip at $\mathrm{THz}$ frequencies, its easy integration and compactness has reserved some uses to it in the recent $\mathrm{THz}$ research. Nevertheless, modifications of the original structure to better adapt it to the $\mathrm{THz}$ conditions has been also common, as with other microwave waveguides.

Back in 1991, a sort of microstrip lines were designed using high permittivity dielectric instead of metal, treating to avoid the large conductor losses [404], [405]. In such works, microstrip is called "unpracticable" at sub-mm frequencies justified by its attenuation, as high as $0.1 \mathrm{~dB} / \mathrm{mm}$ already at only 0.1 $\mathrm{THz}$ in a theoretical context. The proposed design reduces classical microstrip losses by a factor between 3 and 6 [404], and diminishes the dispersion [405]. Theoretically, losses under $1 \mathrm{~dB} / \mathrm{mm}$ maybe reached at $f=1 \mathrm{THz}$. It is not considered in those papers that, probably, isolation and substrate modes could be practical problems of that modified structure.

Later in 1997, in [406], thin-film microstrip lines using polymerized cyclotene were experimentally proved to achieve $1 \mathrm{~dB} / \mathrm{mm}$ at $0.1 \mathrm{THz}$ despite of the fact of the substrate high losses (conductor losses still dominating for the design). It is worth to mention that, in this work, it was highlighted that microstrip is preferable to CPW because of the its better isolation against the substrate and its lower radiation losses.

An interesting work showing a successful use of microstrip line and giving strong reasons for its use can be found in [407]. Despite of previous successful works using a CPW to implement a HEB receiver, it is preferred the microstrip line (a common solution for SIS detectors). The microstrip line has a characteristic impedance generally lower, and it can be varied in a wider range, thus being easier to match the low impedance of a Nb HEB. Also, it was already proved up to $1 \mathrm{THz}$ in SIS detectors, and whereas analytical models and closed forms are found for microstrip, CPW often demand from full-wave analysis. Finally, the microstrip line does not disturb the antenna pattern, as it occurs when the CPW is used, since the ground plane is interrupted.

More recently, microstrip has been proved to have strong potential for measuring broadband $\mathrm{THz}$ absorption of polycrystalline materials or sensing DNA in biomedical instruments [403], [408]. The experimental setup used in [408] is shown in Fig. 2.65(a). A lithographically defined microstrip on chip is used to sense a sample displayed above the strip. A Ti-Au coated strip of $30 \mu \mathrm{m}$ over a $6 \mu \mathrm{m}$ BCB substrate is used, being the total microstrip length of $2.8 \mathrm{~mm}$. The evanescent fields propagating above the strip penetrate in the sample and interact with it.

The system is able to cover the $0.1 \mathrm{THz}$ to $0.8 \mathrm{THz}$ band, showing losses of roughly $6 \mathrm{~dB} / \mathrm{mm}$ at $0.8 \mathrm{THz}$. The compactness of the system makes, however, this high attenuation constant be acceptable for the purpose. A good feature of the system is that the samples do not require contact with the strip, thus avoiding a possible deterioration due to repeated experiments. 


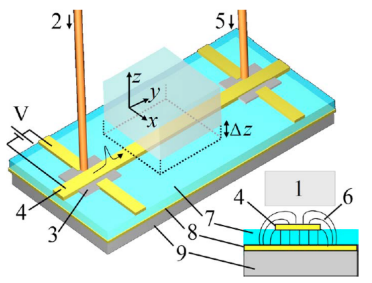

(a)

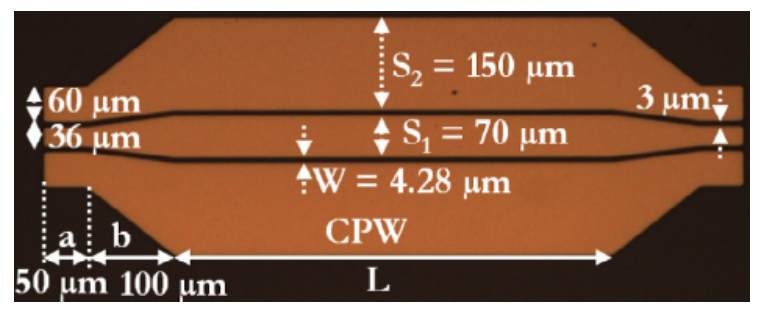

(b)

Figure 2.65: Planar waveguides at $\mathrm{THz}$ frequencies 2. a) Experimental setup implemented for measuring broadband $\mathrm{THz}$ absorption of polycrystalline materials using a microstrip line, from [408]; b) Microscope view of the realized CPW waveguide on BCB substrate, incorporating input and output transitions [409]. Main geometrical parameters and dimensions are sketched on the image.

It is worth to mention that the authors indicate that planar Goubau Line (GL), which is basically a microstrip without ground plane, later reviewed in the surface $\mathrm{THz}$ waveguides section, may be more suitable for more sensitive interaction with the sample. However, since for the microstrip case radiation losses limits the bandwidth, planar Goubau Line may suffer from this effect in a stronger way. Compared to similar experiments using Parallel-Plate Waveguide (PPW), this microstrip based sensor presents 3 times more resolution (2 $\mathrm{GHz}$ ), but half of bandwidth.

In [403], a sharp reject band of about $25 \mathrm{~dB}$ of insertion losses is achieved at $f=1.44 \mathrm{THz}$ through microstrip designs with Defected Ground Structure (DGS), i.e., microstrip lines where some modifications are practiced on the ground conductor [410]. Again, BCB is preferred as dielectric. Thanks to the DGS, neither stubs nor stepped impedance structures are required. Instead, a very compact $50 \Omega \lambda / 4$ microstrip line with hexagonal shape DGS is achieving the desired reject-band functionality. The sharpness of the rejection band could allow very sensitive sensing and detection in biomedical instruments. Although the idea is interesting and it is contrasted with several simulation software $\left(\operatorname{HFSS}^{\circledR}[411], \mathrm{CST}^{\circledR}[412]\right.$ and $\left.\operatorname{ADS}^{\circledR}[413]\right)$, it would be desirable some experimental confirmation.

Finally, some attempts are found recently to avoid dielectric losses by using suspended microstrip lines. For example, in [414] it is described the case of a microstrip in which only a very thin dielectric layer is paced under the metal strip, thus having mostly air between strip and ground plane. Simulated losses are of $0.077 \mathrm{~dB} / \mathrm{mm}$ at $0.3 \mathrm{THz}$. The authors propose, furthermore, the use of a thin coating for the ground plane in order to minimize conductors losses. However, losses reduce only to $0.073 \mathrm{~dB} / \mathrm{mm}$.

The coplanar waveguide presents structurally two main differences regarding to the microstrip line. First, CPW is a single layer structure, i.e., strip and ground plane are in the same plane. Second, conductor is split in two parts, this implying a total of three conductors. The first structural feature drives to a more equal power distribution between dielectric and air, and a lower 
confinement between strip and ground. Thus, dielectric losses are reduced and radiation losses are increased. Analysis and experiments of the last decades bibliography (see for instance [401], [415]-[409]) indicate that the second effect is much more influential at $\mathrm{THz}$ frequencies, despite of the high dielectric absorption. Furthermore, to achieve some confinement CPW requires the distance $w$ (see Fig. 2.64(d)) be quite small. This affects ohmic losses.

The second feature implies the main drawback according published works: the possibility of excite the slotline mode (SL mode, even mode or asymmetric mode). This can be solved by maintaining circuit symmetry (complex restriction) or by introducing Air Bridges (AB) [417], [418], what leads to lose the single-layer easy of manufacture.

By the above, in general, CPW presents higher losses at $\mathrm{THz}$ frequencies than the microstrip line. However, in the still open contest [409] of choosing a concrete waveguide (even when the desired features are quite concrete) one founds some works strongly defending and properly justifying the preference of CPWs. Some relevant or interesting works are reviewed now from an objective point of view.

CPW research of this century must thanks two great works, [416]-[417], in which the CPW analysis and experimental test was done in a very rigorous and detailed form. Obtained conclusions gave a good starting point for more recent designs. The fact of this works were published indicates the relevant role of CPW from the beginning of the $\mathrm{THz}$ revolution.

The work done in [416] was motivated by the necessity of both, designing a high-performance $\mathrm{THz}$ waveguide and having proper analysis models for it, in order to afford material characterization in the $\mathrm{THz}$ band.

Analytical formulas were obtained fitting full-wave simulations (costly at that time). In addition, experimental time-domain measurements using sub-ps pulses provided verification to the derived formulation, and provide a practical test for $\mathrm{CPW}$ use in the $\mathrm{THz}$ regime. The great agreement between the new proposed model and experiments indicates a good improvement from the significant inaccuracy of conventional quasi-static approximations at $\mathrm{THz}$ frequencies. Specifically, the predicted growing of attenuation as $f^{3}$ is, in reality, much lower.

The attenuation is leaded by radiative loss, which clearly predominate to power loss coupled to substrate modes. Regarding to this, the CPW presents one unusual characteristic. Contrarily to most of dielectric waveguides, in the CPW, radiation losses increase (with a large rate) with the frequency.

The experimental test was done by means of a $500 \mu \mathrm{m}$ thick GaAs realized by standard photolithography, with a $50 \mathrm{~nm} / 350 \mathrm{~nm} \mathrm{Ti} / \mathrm{Au}$ conductor. A $0.6 \mathrm{ps}$ pulse $(0.5 \mathrm{THz}$ bandwidth) was through to propagate up to $5.2 \mathrm{~mm}$. Losses of $0.6 \mathrm{~dB} / \mathrm{mm}$ were obtained at $0.5 \mathrm{THz}$ and $2 \mathrm{~dB} / \mathrm{mm}$ at $1 \mathrm{THz}(10 \mathrm{~dB} / \mathrm{mm}$ were predicted for the inaccurate quasi-static model). As major drawbacks, one founds that losses are very frequency dependent, and some pulse broadening is appreciated in the received signal. 
The work carried out in [417] was also motivated by the procurement of an adequate semiempyrical model and, additionally, tries to give good insight in the even and odd CPW modes. In this work, a special emphasis was done on the loss problem and frequency distortion, originated by the high contrast between dielectric and air. This contrast makes the guided waves radiate some of their energy into the substrate as a shock wave. A microwave adopted alternative is to use an additional dielectric over the conductors. At $\mathrm{THz}$ frequencies, however, on the one hand, it would difficult to not create small gaps in the assembling and, on the other hand, dielectric absorption will increase.

Therefore, the authors proposed an interesting structure. Instead a bulky substrate, under the conductors zone, only a thin $(1.4 \mu \mathrm{m})$ membrane of composite dielectric is placed. Effectively, this is like to have a very low permittivity substrate, since most of the power is propagated with very low dispersion in the air regions. It was found that this configuration not only reduces radiation losses and dielectric losses, but also conductor losses. Main observed drawback was the difficulty of achieving enough low impedance. Another disadvantage that may be intuited is the possible mechanical instability.

Experimental tests were done considering a CPW with conductor width of $s_{1}=40 \mu \mathrm{m}$ and slot width $w=25 \mu \mathrm{m}$, considering both, the conventional CPW and the proposed membrane-based CPW. Whereas for the conventional CPW mean attenuation was of $2 \mathrm{~dB} / \mathrm{mm}$ and the original pulse $(0.8 \mathrm{ps} / 0.6$ $\mathrm{THz}$ bandwidth) was broadened $2.7 \mathrm{ps}$, the proposed structure exhibits 0.24 $\mathrm{dB} / \mathrm{mm}$ and negligible dispersion. This is a great improvement. Furthermore, the membrane-structure was shown to experience less influence from differences between even and odd modes. Whereas in the conventional CPW group velocity difference between even and odd modes was about $20 \%$, in the proposed design, this difference was reduced to $5 \%$. Therefore, air bridges to prevent the even mode may be even unnecessary. However, the authors pointed out that achieving a $50 \Omega$ line is very geometrically restricting, hence the design lose its good properties for the required dimensions. This implies that the proposed structure be only slightly better than the conventional case for this commonly employed impedance.

Later, in the 2000 waveguide overview of Grischkowsky [415], the CPW case was considered with the objective of achieving high-speed circuits transmitting sub-ps pulses at least $10 \mathrm{~mm}$. Again, radiation losses appeared as the major threat, and the authors discard many common dielectrics such as sapphire, fused silica, semi-insulating GaAs or $\mathrm{MgO}$ due to absorption reasons. As alternatives, crystalline quartz, high-density polyethylene or $\mathrm{TPX}^{\circledR}$ are considered.

In order to reduce the radiation losses, approximation of the conductors was considered. Since this increases ohmic losses, superconductors were considered. At $T=2.5 \mathrm{~K}$, the used aluminum presented 12 times less resistivity. As a result, attenuation at $1 \mathrm{THz}$ was $0.7 \mathrm{~dB} / \mathrm{mm}$, instead of the $0.9 \mathrm{~dB} / \mathrm{mm}$ of room temperature, what is, however, not a great improvement. At $f=0.8 \mathrm{THz}$, even mode exhibits $1 \mathrm{~dB} / \mathrm{mm}$ front the $0.4 \mathrm{~dB} / \mathrm{mm}$ of the odd-mode. Again it is seen how important is avoid the former mode. Since the velocity group 
dispersion of the CPW is of opposite sign to that of conventional waveguides and dielectric fibers, the authors suggested possible interconnecting of them to achieve non-distorted pulses. However, possible losses due to mode mismatch and mode coupling fall this into question, since they would imply a too high price for non-dispersive transmission, maybe attainable by other ways.

More recently, some works have gone again into deep analysis and fruitful experiments using BCB dielectric [401], [418], [409]. It is highlighted that planar waveguides characterization that fit the observed experimental results is hard at $\mathrm{THz}$ frequencies. The sources of loss (ohmic, dielectric, radiative and even mode) are deeply and separately studied. Whereas the two last are the major contribution, dielectric losses have low contribution. Theoretical calculations give $3 \mathrm{~dB} / \mathrm{mm}$ at $0.5 \mathrm{THz}$ and $6 \mathrm{~dB} / \mathrm{mm}$ at $1 \mathrm{THz}$ when the CPW chacteristic impedance is forced to be $50 \Omega$. The presented prototype, Fig. 2.65(b), achieves a best value of $3.5 \mathrm{~dB} / \mathrm{mm}$ at $0.5 \mathrm{THz}$ what proves good agreement between theory and experiments, and shows CPW competitiveness front the microstrip line.

The authors highlight that the main advantages of the CPW front the microstrip line are the easy connection with Field Effect Transistors (FETs) and Photoconductive (PC) switches, and that CPW does not require via holes. Moreover, reduction of radiation losses is proposed by adding a Backside Conductor (BC). This, however, causes some undesired resonances. The resonances may be eliminated through via holes (complicating the manufacturing). Other good idea to solve this problem could be add some periodic pattern to the ground plane to achieve some EBG effect, as occurs with some designs reviewed in the Part II of this thesis.

Finally, a very recent publication [419], reports low-loss filters implemented with superconducting NbTiN CPW to provide an on-chip filterbank spectrometers. A total of 9 channels are implemented, being the maximum resonator Q over 800 at $0.35 \mathrm{THz}$, which is an outstanding value. The mean resonators quality factor is $Q=550$, however, being the minimum value $Q=94$, which indicates that some control about the prototypes quality is still necessary.

Authors incise in that, although the microstrip line has much lower radiation losses, it has its own challenge with material losses. CPW is considered as a good alternative to microstrip in large scale $\mathrm{THz}$ electronic circuits due the following advantages: 1) it can be based on metal film deposited on crystalline dielectric, thus avoiding amorphous lossy dielectric; 2) it is trivial to make a short to the ground, making it easier to realize quarter-wavelength resonators (this being less true when ABs are present, however).

Planar waveguides review ends with the Paralel-Plate Waveguide (PPW). Whereas microstrip and coplanar waveguides represent a direct scaled microwave solution, looking for low dispersion and integration features at the cost of very high loss, the PPW is rather a rediscovered waveguide with promising properties for the $\mathrm{THz}$ band. The classical PPW consist simply on two metal plates separated a distance $h$ [376], Fig. 2.66(a). Practical implementation of this concept may require some mechanical system to keep the distance between 
plates, electrically isolating them. One possible solution, as proposed in [420], consist on placing two electrical layers or slabs at the sides, in order to left a propagation channel of width $w$ (see Fig. 2.66(a)).

The PPW structure allows for TEM mode transmission, with the great advantage of avoiding dielectric losses. Furthermore, mode distribution has specially low interaction with the metal plates compared to other planar waveguides. Thus, the PPW is intrinsically prepared for low-loss and non-dispersive transmission, therefore capturing the attention of $\mathrm{THz}$ technology researchers.

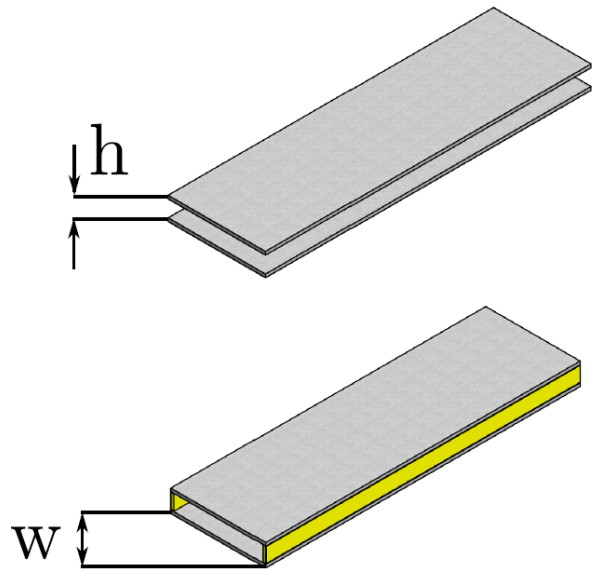

(a)

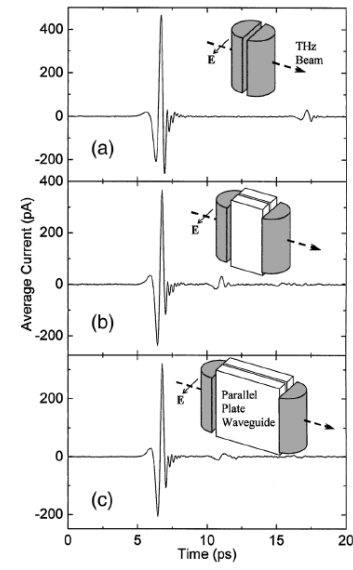

(b)

Figure 2.66: Parallel-Plate Waveguide $(P P W)$ at $T H z$ frequencies 1. a) Schematic of a classical $P P W$, defined by the distance between plates $h$, and a practical implementation version, were dielectric dielectric layers are disposed at the sides for electrical isolation of plates. This second structure is defined, additionally, by the propagation channel width $w$. Normally air is preferred as dielectric between plates for low-loss purposes; b) From up to down: reference pulse and schematic of the confocal lens system, pulse through a short $(12.6 \mathrm{~mm})$ path $P P W$ and schematic of the experimental setup, and pulse through a short $(24.4 \mathrm{~mm})$ path $P P W$ and schematic of the experimental setup [421].

At the starting of this century, Mendis and Grischkowsky [420], [421] proved theoretical and experimentally the great characteristics of $\mathrm{PPW}$ for $\mathrm{THz}$ frequencies, considered at the moment for the demand of wideband structures solving classical waveguides limitations. More specifically, in [420], tow thin coppers strips together with dielectric slabs at sides were used to create a $w=15 \mathrm{~mm}, h=90 \mu \mathrm{m}$ PPW. The employed reference transmission signal was a 0.22 ps pulse with spectral content going from $\mathrm{DC}$ to $4 \mathrm{THz}$ (mostly concentrated between $0.5 \mathrm{THz}$ and $2.5 \mathrm{THz}$ ).

The waveguide was shown to operate mostly monomode, with negligible velocity group dispersion. In [421] it was pointed out that, although from $1.5 \mathrm{THz}$ other modes can propagate, power is hardly coupled to the second mode, the $\mathrm{TM}_{1}$, and in case of coupling to the next, the $\mathrm{TM}_{2}$, high losses of this mode will make this coupled power vanish. Good correspondence between theory and experiment is obtained in these works. The reported velocity group dispersion is 
under $0.1 \%$. This fact is reflected on the obtained pulses, Fig. 2.66(b). Thus, the small widening of the received pulse, of $0.39 \mathrm{ps}$ wide, is justified by the experimented frequency-dependent propagation losses. $\mathrm{Tb} / \mathrm{s}$ transmission with PPW are visioned by the authors. Mean observed losses are of $0.04 \mathrm{~dB} / \mathrm{mm}$, and are caused not only by the ohmic effect, but also because of the pulse spreading in the unguided dimensions, which is, clearly, the main limitation of this waveguide.

Inspired in this good results, the work in [422] proposes a transmitter based on the PPW. This transmitter requires, however, to fill the propagation channel with a semiconductor dielectric ( $\mathrm{GaAs}$ ), thus losing the feature of dielectric air. Employed dimensions are $h=120 \mu \mathrm{m}$ and $w=10 \mathrm{~mm}$. The authors obtain good results in transmitting a $0.31 \mathrm{ps}$ pulse (spectra extending up to $3 \mathrm{THz}$, approximately). However, the use of dielectric implies, on one hand, that higher order modes are more easily excited for the same PPW dimensions. On the other hand, the PPW ends its low-loss property compared with other planar waveguides.

Other promising paper appeared in 2007 [423]. In this work, a dielectric slab was sawed and, then, the sawed dielectric faces were gold-metalized. As a result a $(h=270 \mu \mathrm{m}, w=300 \mu \mathrm{m})$ PPW with a propagation length of $40 \mathrm{~mm}$, was obtained. In this case, the propagation channel has an aspect ratio much less planar, appearing rather as a square. Propagated energy confinement is lower, however, the authors showed it has reasonable extension. Actually, the effective transmission area is about 40 times smaller than for other lowloss, low dispersion waveguides, such as the Single Wire Waveguide. Clear single-mode transmission was achieved over the range $0.1 \mathrm{THz}-1 \mathrm{THz}$. Results showed good agreement with calculations, obtaining outstanding losses, going from $0.01 \mathrm{~dB} / \mathrm{mm}$ to $0.03 \mathrm{~dB} / \mathrm{mm}$ in the aforementioned bandwidth.

Later, in 2009, Mendis and Mittleman proposed a totally different approach for the use of the PPW [424]. In this work, the use of the $\mathrm{TE}_{1}$ was proposed instead of the fundamental TEM mode (also called $\mathrm{TM}_{0}$ mode). Whereas the fundamental mode experiences the usual increasing attenuation with frequency, the $\mathrm{TE}_{1}$ mode, with E-field parallel to the plates, shows the contrary behavior. Authors indicated that this phenomena is not observed in any other waveguide. However, it is true that the $\mathrm{TE}_{01}$ mode of CWG has similar behavior, and, at some frequency range, metallic waveguide modes experience also decreasing attenuation with frequency.

In that work, the case of $h=0.5 \mathrm{~mm}$ was firstly considered, providing 0.005 $\mathrm{dB} / \mathrm{mm}$ at $f=0.5 \mathrm{THz}$ and $0.002 \mathrm{~dB} / \mathrm{mm}$ at $f=1 \mathrm{THz}$, which are very good figures. However, a noticeable chirp is observed in the received pulse for this case, mainly originated by the dispersive behavior of the $\mathrm{TE}_{1}$ mode. The authors solve this problem by increasing the plate separation up to $h=5$ $\mathrm{mm}$. Although this overmoded PPW is able to propagate up to 17 modes, the power was nearly $100 \%$ coupled to the desired $\mathrm{TE}_{1}$ mode, thanks mainly to the good matching between the input Gaussian beam and the sinusoidal field pattern of the mode. 
Dispersion was reduced to negligible levels for this case. Furthermore, theoretical calculations predict an incredible low-loss feature: $2.6 \mathrm{~dB} / \mathrm{Km}$. The authors were not able to measure such low levels since a large path would be required. Regarding to this, the problem of pulse broadening in the unguided dimension arose as an important drawback. It is also proposed in this paper a possible solution to mitigate the problem: folding the plates at the sides, generating concave shapes. This is, curiously, very similar to the groove CWG of [378]. To test this, $2 \mathrm{~cm}$-wide Gaussian beams are launched into a $h=10$ $\mathrm{mm}, 1 \mathrm{~m}$ long PPW. Whereas the conventional PPW spatially widens the pulse up to $20 \mathrm{~cm}$ in the transversal direction, the proposed design keeps it under $3 \mathrm{~cm}$. Despite of the promising results obtained, further work seems necessary to check the expected results, and observe the practical limitations of the structure. For example, the solution of curving the plates or other similar perturbations may lead to the excitation of high-order modes.

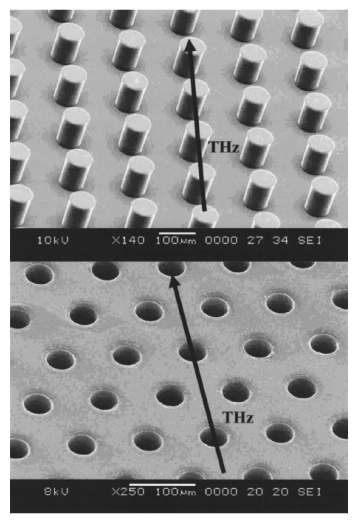

(a)

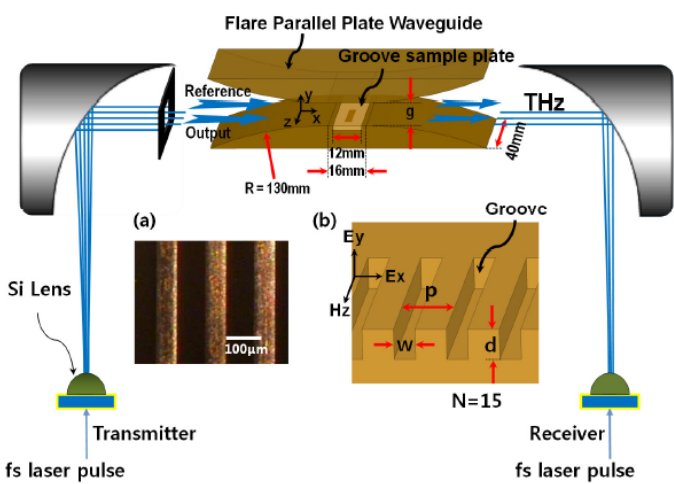

(b)

Figure 2.67: Parallel-Plate Waveguide $(\mathrm{PPW})$ at $\mathrm{THz}$ frequencies 2: filtering applications a) SEM images of the employed Photonic Bandgap structures in [425], consisting in metal pillars and its dual: holes. They were used to implement filtering functions in PPW; b) Experimental setup of [426] consisting in a quasi-optical system using a fs laser, and geometry $(p=142 \mu \mathrm{m}, w=58 \mu \mathrm{m}$, $d=84 \mu \mathrm{m})$ and photography of the studied groove structure. The number of grooves is 15.

The PPW has been also proved for filtering purposes by introducing some PBG structures[425], [427]. In [425] the disposal of two types of PBG structures, pilars and holes (see. Fig. 2.67(a)), in one of the plates is studied. The authors highlight the suitability of the PPW for the implementation, due to the lack of spatial dependence of TEM fundamental mode, so that the 3D PBG structures can be replicated 2D. The manufacturing is done by means of photolithography and metal deposition, avoiding traditional etching methods, maybe inaccurate for this design.

The PPW is $h=100 \mu \mathrm{m}$, and the cylinders and holes have a height of $70 \mu \mathrm{m}$. These structures offer dual responses, approximately, being the cylinders case narrow-band pass and the holes narrow-band reject. Cylinders imply higher losses in the bandpass, and the authors remark that very high losses are found for larger cylinder heights. Also this type of PBG is more dispersive than the 
holes one. Experimental response shows contrasts going from $65 \mathrm{~dB}$ to 100 $\mathrm{dB}$ from pass and rejected bands, whereas total losses are $15 \mathrm{~dB}$ for $25 \mathrm{~mm}$, which means $0.6 \mathrm{~dB} / \mathrm{mm}$. Although sharp skirts and good reject is obtained, it seems that a more compact filtering structure would provide better results since, in this case, low-loss characteristics of the PPW are lost and one has large size filter with excess of insertion losses.

In [427], the quasi-optical experimental setup shown in Fig. 2.67(b) is used to shown the filtering properties of PPWs when some grooves are created in one of its faces (a schematic and a photography showing the grooves details can be appreciated also in Fig. 2.67(b)). A total of 15 grooves are displayed, with deep $d=84 \mu \mathrm{m}$, wide $w=58 \mu \mathrm{m}$, and periodically spaced in the propagation direction $z$ with period $p=142 \mu \mathrm{m}$. This structure provides some narrow rejection bands (as it consists in grooves rather than obstacles) with $30 \mathrm{~dB}$ contrast. The results show $3 \mathrm{~dB}$ of insertion losses, and the authors give a good value of equivalent $\mathrm{Q}$ for the passbands: 400. It is surprisingly affirmed that assuming metal as perfect conductor is a good model for THz. Despite of this approximation, good agreement between simulation and results is found.

One can also find some graphene uses in the PPW. In [427], PPWs with one or both plates implemented with a graphene structure are studied. In the analytical study, hybrid modes are found, and the compromise between losses and confinement become important due the new boundary conditions. The interest of the structure relies on the high control of the propagation constant via applied bias fields. Maybe in the future, these structures will be chip-integrated to provide complex, very specific responses. Communications applications would strongly benefit from this feature.

The research carried out provides strong reasons to consider the PPW as the guided media for many applications. The research continue on, and, in these days, PPW sustain several pioneer experiments using THz-TDS spectroscopy to characterize materials [428]-[429].

\subsubsection{Dielectric (Optic) Waveguides}

In the optical regime, metals interact with the matter in a very different way than at lower frequencies. Actually, in the optical regime, a metal is rather a bad dielectric with moderate permittivity, than a conductor [430]. This idea will be better understood in the next chapter of this thesis. Therefore, at the very high optical frequencies, manipulate the light for low-loss guidance must involve dielectric waveguides, where no metals are present.

It was first, in 1961, when Snitzer [431] considered the concept of a cylindrical dielectric waveguide with two layers (core and cladding) having similar permittivity. Propagation of first modes was numerically analyzed. The fact of very low contrast between core and cladding permittivity allowed to have the modes more spaced, i.e., easier monomode transmission, even for a waveguide being very large in terms of the wavelength. This was eminently practical at those extremely small wavelengths.

Inspired in the theoretical predictions, in 1966, Kao and Haockham [432] recognized that, if impurities were removed of the dielectric materials, a very low-loss 
media would be achieved. Progressively, silica-derived materials were being built more and more transparent to optical waves the following years until reaching the high-performance $0.17 \mathrm{~dB} / \mathrm{Km}$ of nowadays [21].

At $\mathrm{THz}$ frequencies, seems evident that, at least from current dielectrics known, a similar solution will not be possible. It is true, however, that the presence of metals is an important source of losses in the usual microwave waveguides, when applied to $\mathrm{THz}$ frequencies. Therefore, research coming from the optical side has been trying to create low-loss all-dielectric transmission lines these last two decades with the same or more intensity than microwave research groups.

The limitation of having a lack of transparent $\mathrm{THz}$ dielectrics has stimulated the wit to create more and more complex and sophisticated structures to manipulate the $\mathrm{THz}$ light in an optimum way. A huge number of designs have been proposed, in such way that some classifications are necessary [433], [434]. A pass closer can be done to look more in detail the waveguiding mechanisms involved, so that, in this thesis, some dielectric waveguides are classified attending to their involved photonic bandgap effects, or impenetrable surface waveguiding, later described.

Therefore, in this section, they are found dielectric waveguide designs trying to force the guided energy to travel through air regions using different phenomena such as Total Internal Reflection (TIR) or resonant effects, as it is the case of the so called Antiresonant Reflecting Optical Waveguides (ARROWs) [435]. The distinction between bulk and planar become specially important here since, for dielectric waveguides, the volume of non-planar designs is specially large. Furthermore, Porous Fibers (PFs) are named as an apart class due to its relevance. Dielectric waveguides, more difficult to analyze than conventional microwave waveguides per se, will be shown in this section to adopt very challenging geometrical forms. One more time, it will be appreciated how desirable is to have proper analysis methods, which is a major objective in this thesis, to proper design, optimize and choose.

\section{- Solid-core Fibers and Tubes:}

It was in 2000 when the group of Grischkowsky considered, as a part or their research in $\mathrm{THz}$ waveguides (including, as it has been reviewed RWGs, CWGs and PPW), the possibility of using a cylindrical sapphire $\operatorname{rod}\left(\varepsilon_{r}=9.6, \tan \delta=\right.$ $1.7 \cdot 10^{-3}$ at $\left.f=0.9 \mathrm{THz}[21]\right)$ in monomode operation [436]. Fig. 2.68(a) shows the schematic of a classical dielectric cylindrical waveguide. The authors justify the interest in dielectric waveguides because, in their words, with good materials, these waveguides may overcome the performance of metallic ones. As an example, high-resistive silicon $\left(\varepsilon_{r}=11.66, \tan \delta<4 \cdot 10^{-4}\right.$ from $f=1$ $\mathrm{THz}$ to $f=4 \mathrm{THz}$ [437]) is mentioned. However, propagation of $\mathrm{THz}$ waves in bulk high resistive silicon would imply losses as high as $0.04 \mathrm{~dB} / \mathrm{mm}$.

Pulses of $0.6 \mathrm{ps}$ (considered frequency range is $0.8 \mathrm{THz}-3.5 \mathrm{THz}$ ) were linearly coupled with high efficiency to the fundamental $\mathrm{HE}_{11}$ mode of the fiber. This results much more efficient than coupling $\mathrm{THz}$ waves to the $\mathrm{TE}_{11}$ mode of a CWG. Although more modes could propagate, $\mathrm{HE}_{11}$ was clearly dominant in the excitation. It is questionable, however, the possible coupling in bends or other perturbations to other modes, very easy to happen in this kind of waveguides [439]. 


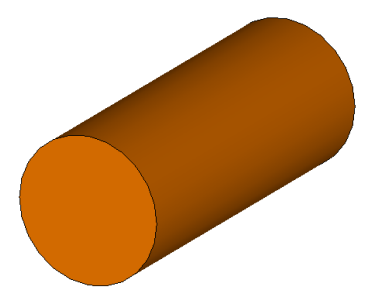

(a)

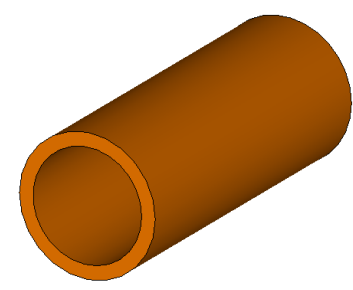

(b)

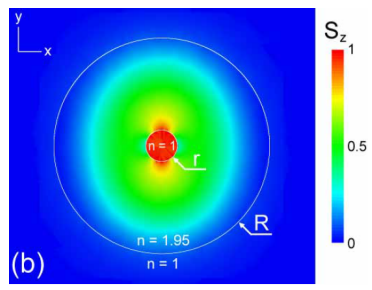

(c)

Figure 2.68: Dielectric fibers and tubes at $\mathrm{THz}$ frequencies 1. a) Basic dielectric cylindrical waveguide consisting in a rod of radius $a$; b) Dielectric tube defined by the its inner radius a and the dielectric thickness $t$; c) Dielectric tube having small air inner region, proposed in [438], and E-field magnitude inside the structure.

Thanks to the high contrast between core (high-resistivity silicon) and cladding (air), sizes are quite electrically smaller than those used in the optical regime. Three radius were considered: $a=325 \mu \mathrm{m}, a=250 \mu \mathrm{m}$ and $a=150 \mu \mathrm{m}$. However, it is known that, the higher the contrast, the larger the frequency dispersion: lower frequencies experience external region propagation whereas higher frequencies propagate confined inside the core, which is denser [21], [440], [441]. This justifies the high velocity group $\left(v_{g}\right)$ dispersion observed: $v_{g}$ ranges from $\mathrm{c} / 3$ to $\mathrm{c}$. Regarding to the losses, $0.3 \mathrm{~dB} / \mathrm{mm}$ were obtained at $f=1 \mathrm{THz}$. Therefore, although efficient coupling was achieved, this first attempt to use dielectrics for waveguiding purposes seems to be far from $\mathrm{THz}$ requirements.

First step forward was done in 2006 by Chen [442]. In that work, it was considered using subwavelength fibers in order to reduce confinement so that most of the power propagate in the outer air region, thus reducing the losses caused by the dielectric core. Polyethilene $\left(\varepsilon_{r}=2.3, \tan \delta=1.4 \cdot 10^{-3}\right.$ at $\left.f=1 \mathrm{THz}[21]\right)$ was used to implement the fiber. The low permittivity of polyethilene allowed also for lower losses and lower dispersion than in [390]. However, the attenuation dependence with the frequency (attenuation dispersion), characteristic of dielectric waveguides, was still present: $8.6 \cdot 10^{-4} \mathrm{~dB} / \mathrm{mm}$ were obtained at $0.31 \mathrm{THz}$, whereas, at $0.36 \mathrm{THz}$, the attenuation grow up to $0.026 \mathrm{~dB} / \mathrm{mm}$.

The coupling efficiency is also highlighted in this work. An efficiency of $20 \%$ is reported by using off-axis parabolic mirror. Other indicated advantage is that the employed fibers are commercially available (easy manufacturing). Finally, since the wave extends well in the air region, sensing properties of this waveguide are remarked. However, the virtual noticeable low-loss is caused by the very weak guidance of the waveguide. Problems at bends and some frequency dispersion are predicted for this still improvable design.

More interesting resulted the contemporary proposal of Nagel [438]. The work was motivated by the recent advances in integrated optics. Some waveguides concentrating power in low-permittivity media were achieved by considering small regions of low-permittivity enclosed or surrounded by high-permittivity regions [443], [444]. In this way, although guidance by TIR implies a slow 
exponential decay in the low permittivity media, since the boundaries where TIR occur are very close, an enhanced field is obtained. A more mathematical way to understand this effect is that, for those modes which E-field polarization is rather orthogonal to the boundary, since the displacement current $\vec{D}$ must be continuum, the E-field experiments an enhancement in the low-permittivity media, close to the boundary. If a low-permittivity media region is trapped between boundaries, a E-field concentration spot is achieved.

In this work, a dielectric tube, Fig. 2.68(b), was proposed to guide $\mathrm{THz}$ waves mostly in air, but confined. This work considered tubes with $a$ comparable or smaller than $t$, then obtaining high confinement in the inner air region due to the effect explained before. In Fig. 2.68(c) it is shown an schematic of the tube used in [438] and the E-field magnitude distribution in it. By this, a great trade-off between losses and confinement is achieved. The authors gave several reasons to defend that the presented design is preferable than other options like Bragg or photonic bandgap fibers. These last imply complex manufacturing due to their geometrical features, and some undesired effects: losses to variations in the fibers or surface roughness, scattering from multiple surfaces and deformation of the geometry at bends.

Fused silica $\left(\varepsilon_{r}=3.8, \tan \delta=0.001\right.$ at $f=0.5 \mathrm{THz}$ [445]) was considered as a dielectric. Although the work did not go very deep into the tube structure analysis, a good optimization procedure is carried out. It must be highlighted that proper $a$ and $t$ give quite improvement from rather random values. Thus, optimization is a must for this structure. The key point is to maxime the field in the inner air region. Through this, not only dielectric losses are reduced, but also radiation losses. This is contrary to the previously commented bulk dielectric rods, for which the main air region is external.

On the other hand, optimization was done for a single frequency (intrinsically, it is the usual way, rather than averaging for broadband), so that the design experimented dispersion features. For example, simulation results indicated that the dielectric tube shows $0.04 \mathrm{~dB} / \mathrm{mm}$ losses at $0.5 \mathrm{THz}$, instead of 0.09 $\mathrm{dB} / \mathrm{mm}$ of a dielectric rod of the same material. However, at $0.3 \mathrm{THz}$ and 0.9 $\mathrm{THz}$, losses of both waveguides are pretty much the same. The improved performance in bends is much clear. The dielectric tube exhibits only additional $0.005 \mathrm{~dB} / \mathrm{mm}$ losses, two orders of magnitude less than the Single Wire Waveguide (but also propagation losses two orders of magnitude larger), information given as a justifying example.

Experimentally, the structure exhibited $0.18 \mathrm{~dB} / \mathrm{mm}$ at $0.5 \mathrm{THz}$, and it was appreciated that the dielectric absorption was double than predicted. Therefore, although the idea was good, results are not improving those obtained with CWGs, a structure that offers equal or greater confinement for bends.

Other interesting work, with more successful results, is described in [446]. With the idea of avoiding metal losses reported in those works considering CWGs with inner coatings, a similar structure is proposed without metal, and using low permittivity material. Thus, in the proposed design $t<a$ (see Fig. 2.68(b)). The authors use commercially available Teflon tubes $\left(\varepsilon_{r}=2.1\right.$, 
$\tan \delta=1.5 \cdot 10^{-3}$ at $\left.f=0.9 \mathrm{THz}[21]\right)$ of 3 meters long, with $a=4.5 \mathrm{~mm}$ and $t=0.5 \mathrm{~mm}$.

The design is based on the resonance phenomena caused due to the 2-D dielectric cavity formed by the waveguide, the same phenomena in which ARROW are based [435], [447]. This phenomena provides confinement (narrow-band, of course), so that the authors indicate that neither multilayer nor metal coatings are necessary.

A good feature of the design is that $84 \%$ coupling efficiency is obtained. Experimental losses are $0.015 \mathrm{~dB} / \mathrm{mm}$ at $0.55 \mathrm{THz}$ and as low as $6.84 \cdot 10^{-4} \mathrm{~dB} / \mathrm{mm}$ at the antiresonance of $f=0.4 \mathrm{THz}$. There is strong dispersion, however. Multimode operation is caused by the large waveguide size, which is critical since losses grow as $a^{-4}$. It is found also that the bandwidth is proportional to $1 / t$, however, decreasing $t$ would imply manufacturing complexity and poor mechanical robustness.

As in the previous work, many studies with dielectric waveguides were lacking of studies of the radiation issue on bends. In [448], the sources of losses in dielectric subwavelength rods are breakdown concluding that losses originated by surface roughness are negligible and highlighting the relevance of bend losses. In [449], the bend problem for the same dielectric tube of [446] is afforded considering different frequencies, polarizations, core diameters and cladding thicknesses. It is highlighted the importance of properly afford the bend losses issue. Surprisingly, low bending losses are reported in such work, despite of the fact that the structure is a dielectric tube with a large air core and a low permittivity thin cladding. It is found that lower $t$ provides lower propagation losses and lower radiation on bends but in this case, also weaker resonant peaks, so the drawback of narrow band for thin cladding is not found in this work.

The structure is tested in the range $0.32 \mathrm{THz}-0.42 \mathrm{THz}$ with a gun oscillator, obtaining $0.005 \mathrm{~dB} / \mathrm{mm}$ additional losses for a $R=60 \mathrm{~cm}$ bend, which are comparable with that of the CWGs with thin metal cladding reviewed before. This losses are independent of whether the polarization is parallel or perpendicular to the plane where the bend takes place. In general, performance of the waveguide is quite good except of the dispersive attenuation and, probably, the large size of the waveguide.

More recently, an interesting work considering the deliberate use of a thick, lossy cladding has been presented [450]. Apparently, this configuration, attenuates the field in the cladding, reducing interferences and damping the resonant effects present in ARROW-based waveguides. Since most of the power still propagates in the air region, this dielectric tube preserves the low-loss characteristic. Furthermore, since the cladding is thick, the structure is mechanically robust and easy to handle.

The proposed fibers are made from Polymethyl Methacrylate (PMMA), which has low permittivity and large absorption at $\mathrm{THz}$ frequencies [451]. Radius is fixed at $a=4 \mathrm{~mm}$ and claddings of $t=1.29 \mathrm{~mm}, t=1.97 \mathrm{~mm}$ and $t=2.95$ $\mathrm{mm}$ are considered. When the thickness $t$ is small, the resonant attenuation 
peaks every $0.1 \mathrm{THz}$ are still present. However, they damp considerably for the largest thickness considered at the expense of only a slight increment of losses in average (of course, at the anti-resonances the standard dielectric tube has lower losses).

Experimental results show $0.16 \mathrm{~dB} / \mathrm{mm}$ at $f=0.5 \mathrm{THz}$ that go down to $0.04 \mathrm{~dB} / \mathrm{mm}$ at $f=1 \mathrm{THz}$. It is observed that losses decrease with the frequency as $1 / f$. This fact is interesting when this waveguide is compared with hollow metallic waveguides, which experience strong increasing of losses with the frequency. Curiously, on bends, losses are quite equalized which is a good feature. For a curvature radius of $r=30 \mathrm{~cm}$ bend, the additional losses are of $0.017 \mathrm{~dB} / \mathrm{mm}$, and they grow up to $0.132 \mathrm{~dB} / \mathrm{mm}$ if the curvature radius is reduced to $r=10 \mathrm{~cm}$.

Dispersion characteristics of this waveguide are as well interesting. The proposed structure present a velocity group between $0.98 c$ and $c$ for the considered bandwidth, just presenting some oscillations caused by the the resonant effects when the cladding thickness is not enough thick. Surprisingly, by adding water to the cladding, extremely flat velocity group is found arrond $v_{g}=0.99 \mathrm{c}$.

It is worth to mention that some interesting comparisons with the best considered $\mathrm{THz}$ waveguides are carried out in that work. For example, the authors indicate that excitation efficiency of their structure is superior to that of the Single Wire Waveguide, and that mode pattern is much more stable than the Parallel Plate Waveguide one, which suffers from broadening in the unguided direction. Also, it is indicated that hollow-core dielectric fibers are preferred to solid or porous core ones since these last have more absorption and are sensitive to external perturbations and bends. Furthermore, it is pointed out that PBG hollow-core fibers require from high air filling fraction to increase the bandwidth. This complicates the manufacturing process. The same can be said regarding Bragg fibers and the required high permittivity contrast.

Regarding to practical uses, dielectric tubes have been shown effective to transform the multimode and highly diverging beam of a THz QCL into the tube $\mathrm{HE}_{11}$, which couple easily to free-space in linear polarization [452]. A Pyrex $\left(\varepsilon_{r}=2.15,7.8 \mathrm{~dB} / \mathrm{mm}\right.$ absorption coefficient at $f=0.8 \mathrm{THz}$ [445]) tube $a=1.8 \mathrm{~mm}$ is considered. Similarly to [450], high dielectric absorption is not a problem, since most of the power is guided in the air core. At $f=3 \mathrm{THz}$, losses of only $0.026 \mathrm{~dB} / \mathrm{mm}$ are obtained. However, no information about performance in bends is given.

The authors of that work report 35\% coupling efficiency and indicate that it can be increased by proper optimization. The good beam transformation may be employed in applications where a spatial coherent beam is required. It is highlighted that, in contrast to metallic waveguides, dielectric waveguides provide excellent transverse mode control (due to the weaker boundaries). Specifically, the dielectric tube is incorporated in many optically pumped submm wave molecular gas lasers.

Parallel to the structure engineering, it is still possible to find some works that try to base the low-loss feature on the advances in materials. Recently, high 


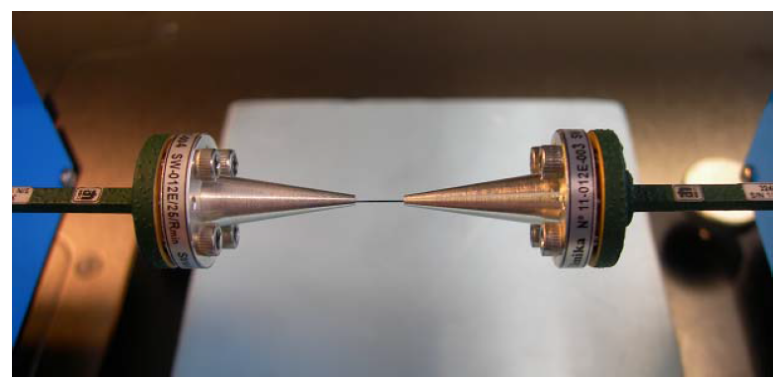

(a)

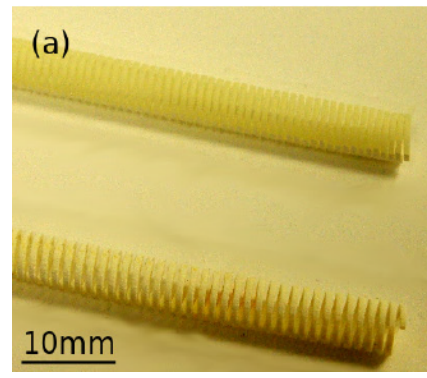

(b)

Figure 2.69: Dielectric fibers and tubes at $\mathrm{THz}$ frequencies 2. a) Basic dielectric cylindrical waveguide consisting in a rod of radius $a$; b) Dielectric tube defined by the its inner radius $a$ and the dielectric thickness $t$; c) Dielectric tube having small air inner region, proposed in [438], and E-field magnitude inside the structure.

resistivity silicon and sapphire rectangular $(0.61 \mathrm{~mm} \times 0.31 \mathrm{~mm})$ dielectric rods have been considered for the lowest frequencies of the $\mathrm{THz}$ band [453]. In this work, it is affirmed that with good dielectrics, lower losses than metallic waveguides, with the additional facility of integrating active devices on the waveguide surface. Experiments show, however, that this may be only partially true. Using WR-3 to WR-6 waveguides for the feeding, Fig. 2.69(a), the dielectric rods are tested in the $0.11 \mathrm{THz}-0.33 \mathrm{THz}$ range. Sapphire rods achieve the best results with $0.25 \mathrm{~dB} / \mathrm{mm}$ at $0.3 \mathrm{THz}$, which is still far from the best performance of metallic waveguides. It seems that, a big step forward in $\mathrm{THz}$ materials is necessary before using this configuration.

Finally, it is commented the very recently proposed helical dielectric waveguide [454], Fig. 2.69(b). This waveguide is easily manufactured with conventional 3-D printing techniques, so it is low cost. Furthermore, structure is flexible and may result practical for some applications. The helix is made by means of a $0.6 \mathrm{~mm}$ side square rod. The helix radius is $a=4 \mathrm{~mm}$, whereas pitches (helical period) of $90 \mu \mathrm{m}$ and $120 \mu \mathrm{m}$ are considered.

The employed polymer presents an absorption coefficient of $2.17 \mathrm{~dB} / \mathrm{mm}$ at $0.2 \mathrm{THz}$ and $19.5 \mathrm{~dB} / \mathrm{mm}$ at $1 \mathrm{THz}$, which is quite high. The interesting feature that the helix configuration introduce is the mitigation of the ARROW resonant effect, in a similar way to lossy cladding tubes. As a counterpart, an increment of average losses (more increment than in the lossy cladding tubes) is experienced. Attenuation of $0.8 \mathrm{~dB} / \mathrm{mm}$ is found at $f=0.2 \mathrm{THz}$ and 0.06 $\mathrm{dB} / \mathrm{mm}$ for $f=1 \mathrm{THz}$. Nevertheless, using a better dielectric may reduce this values, keeping the non-resonant effect, which is provided by the structure according to the authors. In the paper, a deeper structure analysis is missed, since it could provide better exploration of this helix dielectric waveguide possibilities.

\section{- Porous Fibers:}

Porous Fibers (PFs), proposed less than one decade ago, are one of the most popular classes of $\mathrm{THz}$ waveguides. These structures try to provide confined 
waveguiding, concentrating the power in inner air regions manufactured in a bulky rod, thus giving to it the porosity feature. Researchers in this topic defend that this type of waveguides provide the best overall performance for practical applications. Advantages and drawbacks of PFs are reviewed next, giving comparison with other structures.

Practically in parallel, the first to proposals of porous fibers are found in [455], [456] and [457]. In all of them it is found that the main motivation is to diminish as maximum main problem in dielectric subwavelenght rods and partially also found in dielectric tubes: bends radiation.

In [455], the arranging of sub-wavelength holes shown in Fig. 2.70(a) is used to provide true waveguiding, alternative to the barely wave "railing" of small dielectric rods and other surface waveguides. The triangular lattice is chosen since it gives higher porosity than other patterns. Porosity and fiber diameter and studied to enhance the percentage of power transmitted in the air regions, see Figs. 2.70(b)-2.70(c) . The authors indicate that the $26 \%$ of power transmitted in the inner air region, achieved by the dielectric tube in [438], is not enough for low-loss feature. As a reference, it is presented a conceptual design with hole radius of $20 \mu \mathrm{m}$ and total core radius of $200 \mu \mathrm{m}$, achieving $37 \%$ porosity. In Fig. 2.70(c) it is shown how the E-fields tends to concentrate in the sub-wavelength air holes.

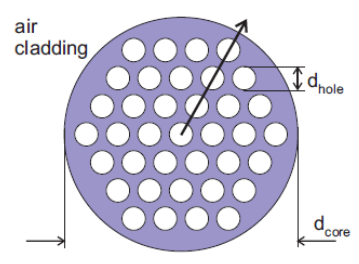

(a)

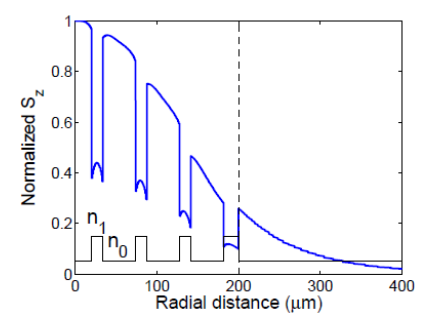

(b)

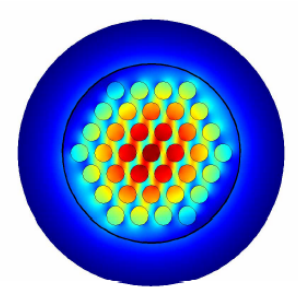

(c)

Figure 2.70: Porous Fibers (PFs) at $\mathrm{THz}$ frequencies 1: Design proposed in [455] a) Schematic; b) Transversal E-field magnitude vs. radial distance; c) 2-D cross section E-field magnitude.

In that work it is found that to achieve confinement in the porous core, a minimum radius is necessary. Fibers with larger porosity have a larger minimum radius. Simulations indicate that PFs are less sensitive to radius variations due to manufacturing process. Under good confinement conditions, the proposed PMMA-based PF, achieves $0.8 \mathrm{~dB} / \mathrm{mm}$ at $f=0.5 \mathrm{THz}$ front of the $6 \mathrm{~dB} / \mathrm{mm}$ of a solid fiber. Furthermore, a PF exhibits less attenuation dispersion with frequency. As drawbacks, manufacturing challenging, which is expected to be proportional to the porosity, and coupling efficiency, are mentioned.

A similar structure is proposed in [457] together with a Bragg-type hollowcore fiber (a periodic structure based waveguide kind, reviewed later), giving comparison between both. Teflon (bulky absorption of $0.13 \mathrm{~dB} / \mathrm{mm}$ according to the authors) is used as dielectric in the conceptual designs. In the used hexagonal array, holes are about $0.1 \lambda$ for the design central frequency $(f=1$ 
$\mathrm{THz}$ ) and 9 periods are used to conform the core. A Finite Element Method (FEM) eigenmode analysis is carried out to study the proposed PF.

It is found that it exist a optimum ratio between hole diameter and period, $d / p$, which gives the maximum power concentration in the air holes. On the one hand, more separation of holes (less porosity) causes higher concentration in the dielectric medium. On the other hand, less separation of holes (more porosity) causes filed concentrate in the outer air medium, thus increasing radiation losses. It is observed that for fibers with conventional porosity $(d / p<$ 0.9), absorption is the dominant loss mechanism. For the same absorption losses, much less radiation losses are observed compared to sub-wavelength rods.

Best results are losses under $0.015 \mathrm{~dB} / \mathrm{mm}$ in the $0.75 \mathrm{THz}-1.5 \mathrm{THz}$ band, even with the presence of bends sharp as $R=3 \mathrm{~cm}$. In comparison, the studied Bragg fiber achieves better losses $(0.005 \mathrm{~dB} / \mathrm{mm})$, however in a small bandwidth $(0.1 \mathrm{THz})$ and allowing for less sharp bends $(R=12 \mathrm{~cm})$. Therefore, according to this work, overall behavior points towards PFs as better solution.

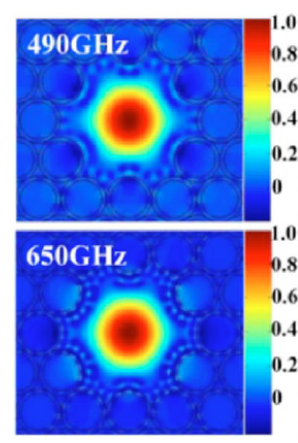

(a)

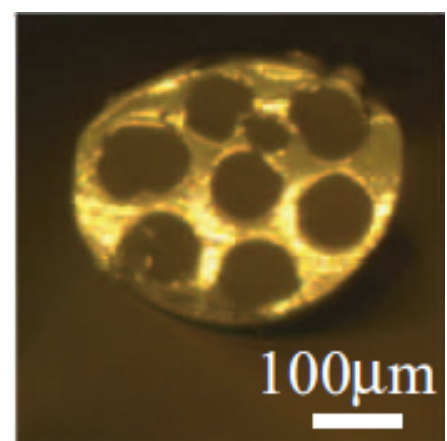

(b)

Figure 2.71: Porous Fibers (PFs) at THz frequencies 2. a) PF proposed in [456] and detail of the E-field enhancement in the air core region; b) PF manufactured in [458] from an initial PE and PMMA (sacrificial) structure.

In [456], a different design, shown in Fig. 2.71(a) is proposed. This works provides additionally experimental results with good agreement with simulations. Teflon tubes of inner and outer radius $a=1.68 \mathrm{~mm}$ and $b=2.08 \mathrm{~mm}$, respectively, are stacked together to form a structure with a central air core of $5.5 \mathrm{~mm}$ of diameter. Although the structure aspect suggest PBG effects, simulations using 1 and 3 layers indicate that propagation is that observed in ARROW structures, having no influence the number of layers. Experiments confirm this fact. This structure shows $\beta<k_{0}$ characteristics, something common to big, empty core structures (similar behaviour is found in [450] and [454], but not in [438]).

The proposed PF achieves best losses of only $1.6 \cdot 10^{-3} \mathrm{~dB} / \mathrm{mm}$ at $0.77 \mathrm{THz}$ and coupling efficiency of $50 \%$, which are good figures. However, low-loss behavior is narrow-band. They are found high-loss peaks caused by resonances of the 
tubes employed. The key point is that, even if a narrow-band application is required, it will be hard to precisely manufacturing the structure to locate the noticeably narrow transmission peak. Furthermore, total diameter of these fibers is about $25 \mathrm{~mm}$, being too bulky for many applications. Therefore, a deep study of the results indicate that this way to produce PFs may be not the best.

Further technological advances are reported in [458], work in which PE large porosity fibers of total radius $r=190 \mu \mathrm{m}$, are achieved. An initial preform having PE with sacrificial PMMA regions is treated to create the fibers shown in Fig. 2.71(b). Whereas reported bulky PE loss is about $0.8 \mathrm{~dB} / \mathrm{mm}$, the proposed fibers achieve $0.008 \mathrm{~dB} / \mathrm{mm}$ at $f=0.3 \mathrm{THz}$, which is 100 times less than a solid fiber of same radius. The problem in this work is the considered radius is small, which cause that an important fraction of the power be weakly guided in the outer air medium. The authors also propose a coupling structure, coupling power (in the transversal direction of propgation) between the designed PF and a solid-core fiber. This coupler has, however, high attenuation and propagation dispersion, acting as a low-pass filter.

The same authors of [455] come back with further advances in [459] very quickly. By exploiting optical fiber fabrication techniques, Atakaramians et al. state good manufacturing bases for the complex PF geometries. In this work, it is highlighted that basic stacking methods achieve porosity of $8 \%-18 \%$, and subtraction methods such that of [458] reach, in the best case, $45 \%$. Since this method involves submerging the fiber in solvent to etch away material in holes and a posterior drying, days and even weeks are necessary.

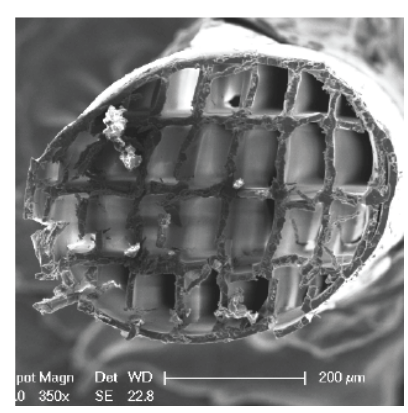

(a)

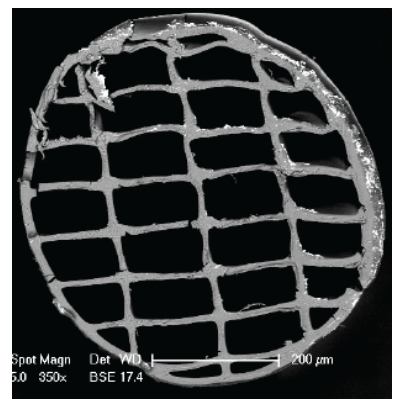

(b)

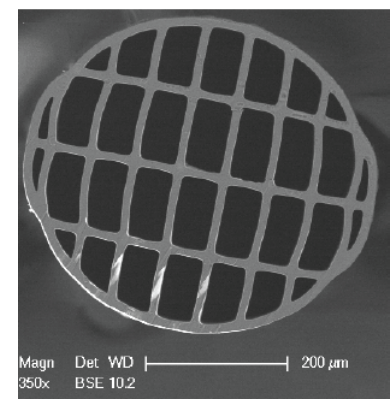

(c)

Figure 2.72: Porous Fibers (PFs) at $\mathrm{THz}$ frequencies 3: Cross sections of several rectangular grid PFs, cleaved with different techniques in [459]. a) Conventional cleaving; b) Cleaving with heated blades; c) Focused Ion Beam (FIB) cleaving.

The proposed method in the paper is based on an extrusion technique, which is valid not only for soft glasses but also for polymers. The advances in the cleaving part are very relevant. In Fig. 2.72, they are show the transverse cuts of a rectangular grid $\mathrm{PF}$, cleaved with different techniques. By using conventional cleaving blades (Fig. 2.72(a)) important damage is done to the fiber. Heating the blades, alleviate the damage (Fig. 2.72(b)), however, important 
errors are still present. Finally, noticeably better results are obtained by using the Focused Ion Beam (FIB) [460] techniques (Fig. 2.72(c)). A possible drawback of this last technique is the process time, close to one day long.

With the proposed method $57 \%$ porosity is achieved for a spider-web pattern (67\% in the conceptual design) and up to $65 \%$ porosity is achieved for the aforementioned rectangular pattern ( $71 \%$ in the conceptual design). The lower porosity of manufactured prototypes regarding to the initial designs is caused by the still slightly present round corners and thickened struts. Fibers of more than $30 \mathrm{~mm}$ are tested experimentally, exhibiting losses under $0.21 \mathrm{~dB} / \mathrm{mm}$ up to $0.8 \mathrm{THz}$, with single-mode operation. Also, a slight birefringence is found at some frequencies. The authors indicate that advances of manufacturing techniques will result crucial to achieve the theoretically expected good properties of PFs.

Finally, a very recent design in [461] treats to overcome major drawbacks of the PFs waveguide family. Whereas previous works report not enough low loss levels and barely flat dispersion inside a narrow-band, this work affords low-dispersive and low-loss propagation from $1 \mathrm{THz}$ to $5 \mathrm{THz}$. To do that, a structure with two different porous zones is proposed, having the core less porosity, Fig. 2.73. Used dimensions are: $r_{1}=117.9 \mu \mathrm{m}, r_{1}=171.4 \mu \mathrm{m}$, $\Lambda_{1}=350 \mu \mathrm{m}, \Lambda_{2}=380 \mu \mathrm{m}$ and $D_{\text {core }}=2.54 \mathrm{~mm}$. Core porosity is $33 \%$.

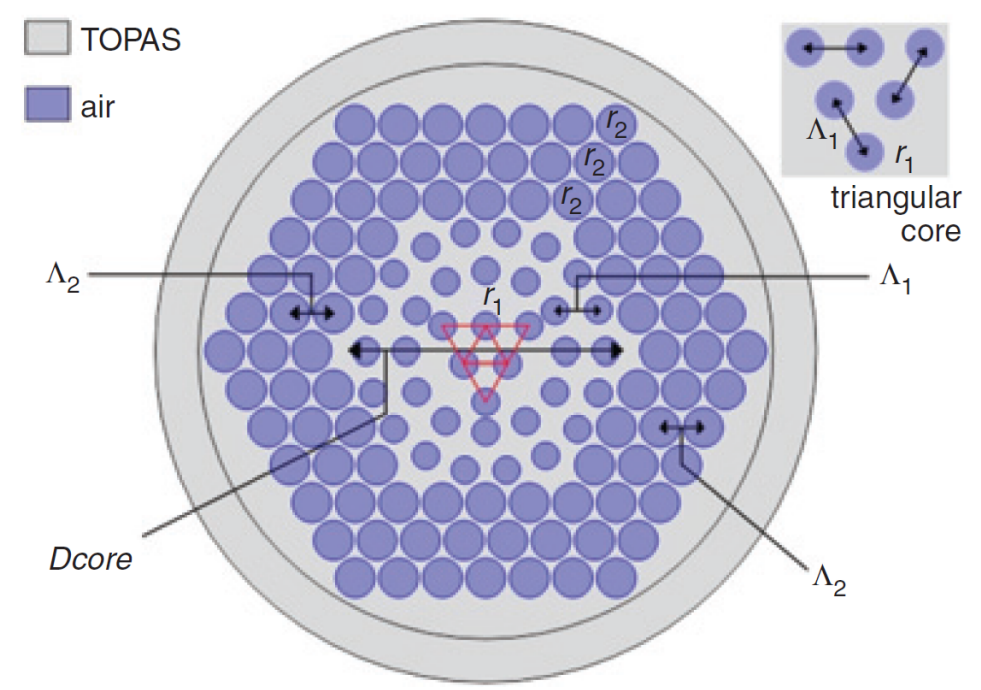

Figure 2.73: Porous Fibers (PFs) at $\mathrm{THz}$ frequencies 4: Design in [461], consisting in a PF of two regions with different porosity to provide broadband near zero dispersion characteristics.

The considered material is TOPAS ${ }^{\circledR}$, a Cyclic-Olefin Copolymer (COC) [462] with relatively low $\mathrm{THz}$ absorption: $0.16 \mathrm{~dB} / \mathrm{mm}$. Over all the considered band $(1 \mathrm{THz}-5 \mathrm{THz})$ losses are under $0.03 \mathrm{~dB} / \mathrm{mm}$, and dispersion is about $0.4 \mathrm{ps} / \mathrm{THz} / \mathrm{cm}$, an acceptable pulse broadening rate for propagation lengths of several cms. The design exhibits very interesting results, however it lacks experimental evidence through prototypes. Although current techniques are 
reliable, the structure complexity may imply a challenging manufacturing process and, probably, lower performance than theoretically predicted.

\section{- Planar Dielectric Waveguides:}

Planar dielectric waveguides have received much less attention than dielectric fibers. Whereas bulky dielectric fibers are motivated by low-loss transmission of $\mathrm{THz}$ waves along guided paths of certain practical lengths, that can be of several $\mathrm{cm}$, planar waveguides are intended to realize compact, integrated inter-chip or even intra-chip interconnections. Therefore, higher values of loss are tolerated for this transmissions lines. This have implied that common planar lines, such CPW or microstrip line, have been the usual choice to implement some acceptable performance applications, despite their obvious lack of suitability for $\mathrm{THz}$ frequencies (attenuation of several $\mathrm{dB} / \mathrm{mm}$ ). Displace microwave conventional planar lines, has been hard, to the moment, for their dielectric counterparts. However, one eye must be keep in the advances coming from integrated optics. Revolution will come next years for the planar interconnections, always more challenging. Here, it is primarily mentioned the first look into these waveguides for $\mathrm{THz}$ transmission purposes, then they are reviewed some interesting attempts one decade ago, and finally, the very recent proposals, that may open the door to changes for planar technology, are commented.

It is not surprising that the first proposal came from the group of Grischkowsky in the year 2000 [463]. In that work, plastic ribbons of High-Density Polyethylene (HDPE) were proposed for confined $\mathrm{THz}$ waveguiding. Two ribbons of 2 $\mathrm{mm}$ wide $10 \mathrm{~mm}(20 \mathrm{~mm})$ long, and $150 \mu \mathrm{m}(120 \mu \mathrm{m})$ were manufactured and tested. A thickness variation (roughness) of less than $\lambda / 10^{5}$ is reported, so that it can be affirmed that roughness is not contributing to radiation losses [464].

Excellent quasi-optical coupling was achieved, and simulation and experiments showed good agreement. Quite flat losses are obtained between $0.5 \mathrm{THz}$ and $1 \mathrm{THz}$, with $0.2 \mathrm{~dB} / \mathrm{mm}$ and at the band edges and $0.3 \mathrm{~dB} / \mathrm{mm}$ at $0.75 \mathrm{THz}$, something unusual since linear or even stronger grow could be expected [21]. In the considered band $(0.1 \mathrm{THz}$ to $3.5 \mathrm{THz})$ the waveguide is dispersive, as is common for solid, large enough dielectric waveguides. However, the fundamental mode is guided with strong confinement between $1 \mathrm{THz}$ and $3.5 \mathrm{THz}$, so the velocity group at these frequencies is quite stable around $0.65 \mathrm{c}$.

Although this attenuation is good for a planar waveguide the authors suggest further reduction by decreasing the ribbon thickness, in such way that the energy propagate in the outer air region. This is the foundation of the surface waveguiding with high-permittivity substrates, reviewed later.

A quite interesting proposal is found in [438]. Together with the small air-core tube, previously commented, the authors propose a dielectric Slot Rectangular Waveguide (SRW), as the planar version of this kind of waveguides, that enhance the field in a enclosed or partially enclosed small air region. The schematic and the field distribution in this waveguide can be appreciated in Fig. 2.74(a). It is observed a high field confinement in the air region. In fact, 
up to $56 \%$ of the transmitted power is transmitted in this central region, much more than the $26 \%$ achieved with the tube proposed in the same work.

The high energy concentration is possible thanks to the E-field polarization, totally orthogonal to the dielectric walls, which is not the case for the fundamental $\mathrm{HE}_{11}$ mode in the tube. Furthermore, in the case of the of the SRW, higher permittivity is considered, being, actually, the commented effect proportional to $\sqrt{\varepsilon_{r}}$.

Optimization of the structure is quite important and peaks of performance are observed when proper size and geometrical parameter ratios are chosen. For example, it is convenient to have small gaps $(g<50 \mu \mathrm{m})$. Simulations give losses below $0.01 \mathrm{~dB} / \mathrm{mm}$ from $0.5 \mathrm{THz}$ to $1 \mathrm{THz}$. However, some dispersion is found, caused by the high contrast between the dielectric and the air. Another powerful feature of this structure is the low radiation on bends $\left(4 \cdot 10^{-4} \mathrm{~dB} /{ }^{\circ}\right)$, four times less than the tube. Furthermore, the high power confinement provides enough isolation to implement arrays of adjacent SRW with negligible crosstalk.

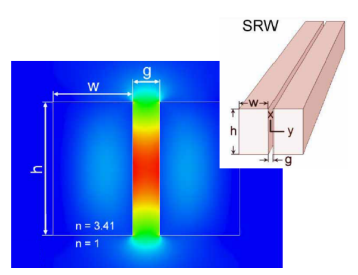

(a)
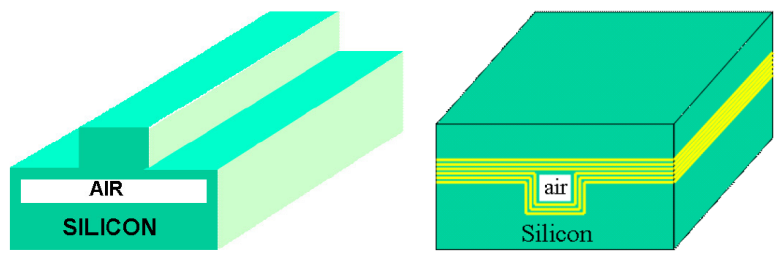

(b)

Figure 2.74: Planar dielectric waveguides at $\mathrm{THz}$ frequencies 1 a) Schematic and simulated E-field magnitude in the high resistivity silicon $\left(\varepsilon_{r}=11.7\right)$ Slot Rectangular Waveguide (SRW) proposed in [438]; b) Some planar structures considered in [465] for waveguiding high $\mathrm{THz}$ frequencies. Left waveguide is a suspended rib-channel consisting of a silicon membrane clad below and above by air. Right waveguide is an ARROW hollow-core rectangular channel with the clad consisting in alternative layers of $\mathrm{SiGe}$ and $\mathrm{Si}$.

Other work to take into account is that of [465]. Although this review of new planar waveguides proposals, based on silicon and germanium derivatives, is rather focused to the FIR region, frequencies in the range $1 \mathrm{THz}-10 \mathrm{THz}$ are considered as a part of the study in many cases. The structures shown in Fig. 2.74(b) are proposed in the paper. The left waveguide in Fig. 2.74(b) is based on a suspended rib-channel consisting of a silicon membrane clad below and above by air. The employed crystal silicon exhibits $0.3 \mathrm{~dB} / \mathrm{mm}$ for $f>1 \mathrm{THz}$ whilst the reported waveguide loss is below $0.1 \mathrm{~dB} / \mathrm{mm}$.

The right waveguide in Fig. 2.74(b) is an ARROW hollow-core rectangular channel with the clad consisting in alternative layers of SiGe and Si. This waveguide achieves losses under $0.165 \mathrm{~dB} / \mathrm{mm}$ beyond $3 \mathrm{THz}$, something unthinkable for the CPW or the microstrip line. However, some spikes are found due to material absorption resonances. The interest in the work of [465] relies in the good information about optical techniques and material advances, that may be really useful when the frequency is pushed up to high $\mathrm{THz}$ frequen- 
cies and the bottleneck of some conventional microwave structures becomes so tight.

Finally, the structure recently proposed in [466], Fig. 2.75 is commented. The authors employ recent advances in Silion-On-Glass (SOG) technology to provide a serious alternative to common planar transmission lines, and even suggesting the proposed structure as an alternative to the RWG, of problematic manufacturing according to them.

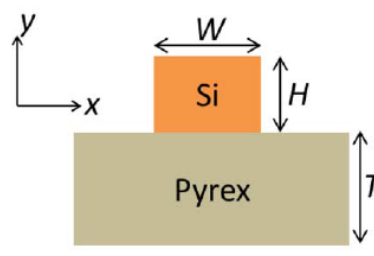

(a)

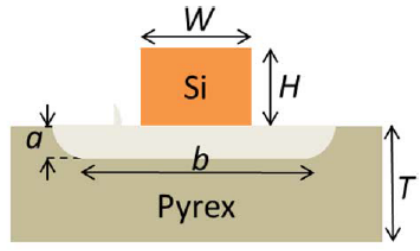

)

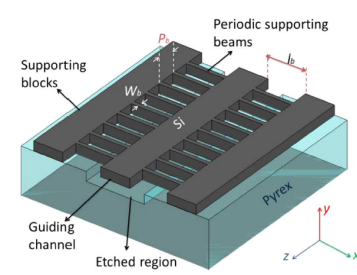

(b)

Figure 2.75: Planar dielectric waveguides at $\mathrm{THz}$ frequencies 2: Silicon-on-Glass (SOG) dielectric waveguide proposed in [466]. a) Original design cross section and proposed modification with an air region under the main waveguide channel; b) $3 D$-view schematic of the complete structure.

A high resistivity silicon ribbon is placed over a Pyrex substrate of $\varepsilon_{r}=4.4$, as con be observed in the left image of Fig. 2.75(a). Since the absorption of Pyrex raises quite high beyond $0.2 \mathrm{THz}$, and much more beyond $0.5 \mathrm{THz}$, an air channel is etched below the silicon region, thus allowing an air region providing almost null absorption (see right image of Fig. 2.75(a)). To enable mechanical stability, the complete structure is based on additional supporting blocks and beams placed at the sides, lying on the Pyrex substrate, Fig. 2.75(b).

The simulations in that work show that most of the power is guided inside the silicon region, however, some concentration is observed in the air channel. Losses go from $0.1 \mathrm{~dB} / \mathrm{mm}(0.3 \mathrm{THz})$ to $0.007 \mathrm{~dB} / \mathrm{mm}(0.5 \mathrm{THz})$. A well-finished prototype is manufactured through current photolithography and DRIE techniques, having $W=310 \mu \mathrm{m}, H=100 \mu \mathrm{m}$ and $b=3 \mathrm{~mm}$. From 0.44 $\mathrm{THz}$ to $0.5 \mathrm{THz}$, obtained losses are between $0.02 \mathrm{~dB} / \mathrm{mm}$ and $0.1 \mathrm{~dB} / \mathrm{mm}$, and some disagreement is observed between simulations and experiment.

A good feature of the design is that it relies in current manufacturing lowcost techniques. However, despite the acceptable attenuation, the dispersive behavior and the narrow-band considered put into question the application of this compact structure in spectroscopic sensing chips.

In general, planar dielectric waveguides must be taken into account for future compact interconnections. The proposal in [438], if practically implementable may provide very interesting low-loss, high confinement and component integration characteristics. Large work in improving dispersion characteristics of dielectric waveguide proposes must be still done, however, to overcome the quasi-TEM properties of conventional planar lines. 


\subsubsection{Periodic and Electromagnetic Bandgap Structures}

The first serious study of electromagnetic wave propagation in periodic structures was done in 1953 by Brillouin [467]. In particular, the analogy found between uni or multi-dimensionally periodic structures and real crystals resulted in a very fruitful and thoughtful geometry engineering to have control over the light, providing the well-known Photonic Bandgap (PBG) effect, also called Electromagnetic Bandgap (EBG) [468], [469]. The field in periodic structures is vast, with a huge work driven first by the photonic crystals used in optics, opening later the door to a huge new class of microwave and optics EBG components, and revolutionized more recently by the metamaterial world [21].

At $\mathrm{THz}$ frequencies, the interest of periodic structures arises from the fact that, properly engineered, these structures can provide a desired behavior or performance, not achievable but simpler geometries of plane, bulky materials. More specifically, the $\mathrm{THz}$ electromagnetic energy can be forced to propagate confined in low-loss air regions by a boundary that, furthermore, does not cause high losses, either by reducing currents in metals or the concentration of the field inside the dielectrics present. The proposed $\mathrm{THz}$ waveguides mainly motivated by this have been left to this section.

The first kind of PBG waveguides to be reviewed are the PBG dielectric fibers, which are strongly related with porous fibers (up to the point that sometimes the separation line becomes blurry), but their propagation mechanism are different. Then some more innovative metallo-dielectric structures, very unique of $\mathrm{THz}$ frequencies are commented. Planar designs are also briefly overviewed.

\section{- Photonic Bandgap Fibers:}

The driven idea of PBG fibers is the same as porous fibers: maximize the propagation in confined air-core region. However, whereas porous fibers use subwavelength holes to provide field enhancement via TIR effect, in the PBG fibers, a periodic dielectric structure forms the cladding, creating an EBG condition in such way that, for certain bandwidth, waves can not escape from the core, since propagation is not allowed in the cladding for such frequencies.

For example, in [470] a hollow-core plastic Bragg ${ }^{18}$ fiber with cobweb shape, Fig. 2.76(a), is proposed. The authors choose this particular shape due to its better performance compared to classical Bragg fibers or more modern ring-structured Bragg fibers. $\operatorname{HDPE}\left(\varepsilon_{r}=2.3\right)$ is chosen as dielectric, and radius from $r=8 \mathrm{~mm}$ to $r=15 \mathrm{~mm}$ are considered, thus having multimode operation.

Simulated losses are below $8.5 \cdot 10^{-4} \mathrm{~dB} / \mathrm{mm}$ in the wide band going from 0.1 $\mathrm{THz}$ to $4.3 \mathrm{THz}$. However, this promising results gets under question when some practical implementation issues are considered in the paper. The authors recognize that avoiding water in the dielectric material and prevent moisture at the manipulation and storage may result crucial to keep this loss level. Furthermore, material purity, non-uniformity of the fiber and absorption of

\footnotetext{
${ }^{18}$ Bragg fibers are 1-D periodic structure based fibers, consisting in alternative superposition of concentric cylindrical layers of high and low permittivity dielectrics [471].
} 


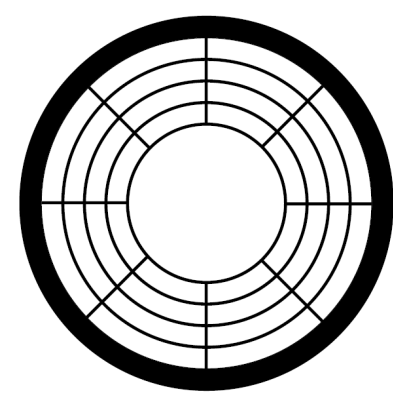

(a)

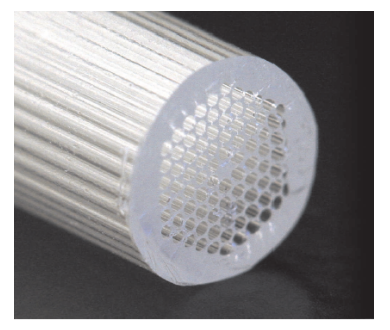

$-0$.

(b)

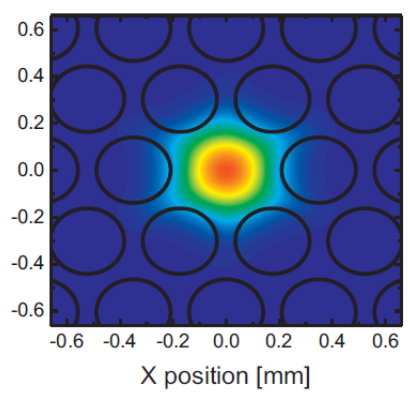

Figure 2.76: Photonic Bandgap (PBG) Fibers at THz frequencies 1: a) Schematic of the hollowcore cobweb Bragg Fiber used in [470]. Three designs with inner radius $r_{A}=8 \mathrm{~mm}, r_{B}=10 \mathrm{~mm}$ and $r_{C}=15 \mathrm{~mm}$ are used; b) Manufactured Topas ${ }^{\circledR} P B G$ fiber and simulated field in the air core [472].

supporting strips are factors that will contribute to loss and are not taken into account in the simulation. Behavior at bends is not detailed, although multimode excitation is one foreseen problem. Thus, it is already observed through this first work that the theoretically well-suited PBG fibers have important drawbacks in practice.

In [472], a proposal with experimental results can be found. In 2.76(b), it can be seen the manufactured prototype and the simulated E-field magnitude in the air core. In this case, the periodic structure follows an hexagonal 2D pattern, and the core size is noticeable reduced. Two designs are proposed: Large Core Area (LCA) with $r_{\text {core }}=280 \mu \mathrm{m}$ and Small Core Area (SCA) with $r_{\text {core }}=140 \mu \mathrm{m}$. However, the SCA design appears to be excessively lossy and dispersive. Single-mode is achieved, but it is very restricted to size, frequency and porosity (period to hole diameter ratio $p / d_{\text {hole }}$ ).

TOPAS ${ }^{\circledR}$ is found, one more time, as used polymer. In the paper, bulk losses of $0.05 \mathrm{~dB} / \mathrm{mm}$ and $0.5 \mathrm{~dB} / \mathrm{mm}$ at $1.5 \mathrm{THz}$ are indicated. The proposed design achieves $0.04 \mathrm{~dB} / \mathrm{mm}$ in average in the $0.2 \mathrm{THz}-0.8 \mathrm{THz}$ band, but the attenuation is quite variable in the mentioned range and the measurement error uncertainly is considerable. They are highlighted the high confinement and the possibility of mass-production. However, in this case, with experimental results, much less outstanding losses are appreciated for the considered PBG fiber.

Another Bragg fiber work, in this case with experimental implementation, is found in [473]. Two Bragg fiber types are considered, see Fig. 2.77. The first one alternates PTFE $127 \mu \mathrm{m}$ films with air layers. In practice, this is done by placing powder over the polymer film (see Fig. 2.77). The second one alternates PE films with Titanium Oxide (TiO) doped PE films (the bulk losses of the $\mathrm{TiO}$ doped $\mathrm{PE}$ are $1 \mathrm{~dB} / \mathrm{mm}$ at $1 \mathrm{THz}$ and $4 \mathrm{~dB} / \mathrm{mm}$ at $2 \mathrm{THz}$ ). Measured inner diameter for both designs is of approximately $6 \mathrm{~mm}$, thus being multimode fibers. 

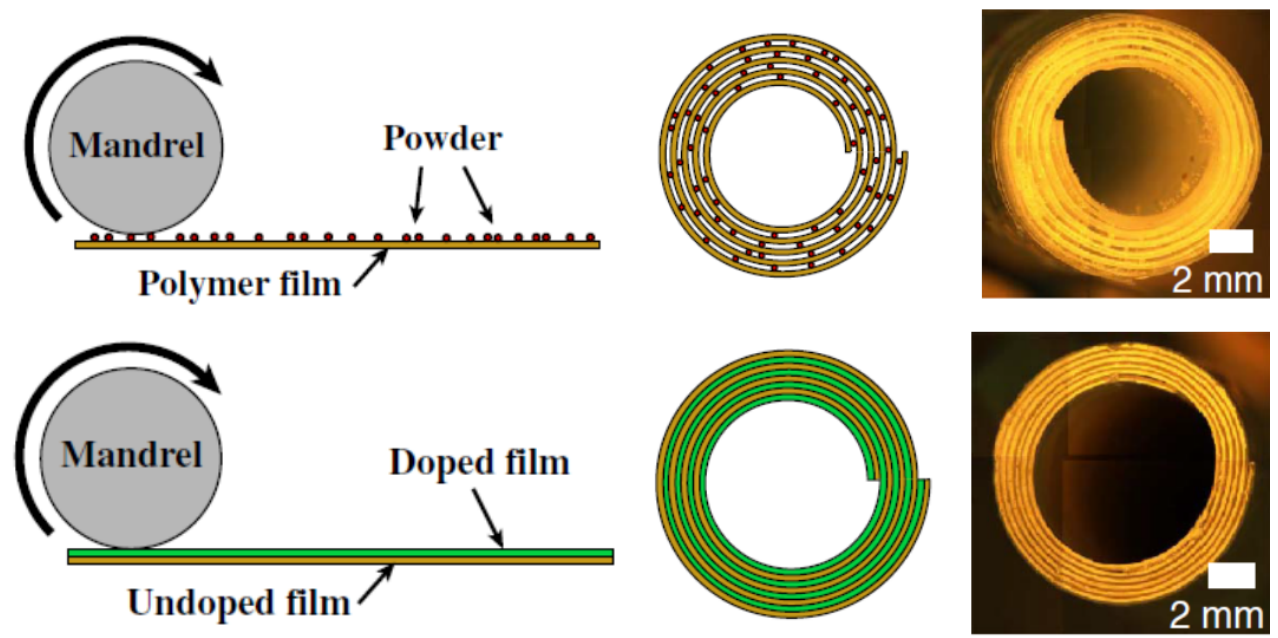

Figure 2.77: Photonic Bandgap (PBG) Fibers at THz frequencies 2: Bragg fibers of [458]. From left to right: manufacturing process, cross section schematic and manufactured prototype of a hollow-core Bragg fibers. The top row correspond to an air-polymer (PTFE) Bragg fiber and the bottom row to a PE-TiO doped PE Bragg Fiber.

Due to the effects of powder, and the final spiral-shape obtained, the first design behaves worse. The obtained experimental attenuation for the second one is under $0.16 \mathrm{~dB} / \mathrm{mm}$ from $0.1 \mathrm{THz}$ to $2 \mathrm{THz}$, being under $0.08 \mathrm{~dB} / \mathrm{mm}$ in most of the band. It is worth to appreciate the detailed theoretical analysis found in this work, which could provide very optimized designs if manufacturing techniques achieve the challenging required grade of precision. By the moment, the metallic CWG presents much better results with similar geometry and flexibility.

In order to reduce the dispersion, an octogonal pattern TOPAS ${ }^{\circledR}$ PBG fiber has been also recently proposed [474]. Numerical results using FEM provide losses of $0.04 \mathrm{~dB} / \mathrm{mm}$ at $f=1 \mathrm{THz}$, exhibiting linear grow with frequency, and dispersion less than $0.18 \mathrm{ps} / \mathrm{THz} / \mathrm{cm}$ in the range $1 \mathrm{THz}-1.8 \mathrm{THz}$. The core diameter is shown to have poor influence on the performance, however, obtained porosity results very influential. Thus, fabrication errors may imply losses higher than predicted.

Other interesting new geometry with similar intentions is the rotated porous core with hexagonal patter PBG fiber proposed in [475], Fig. 2.78. Singlemode propagation is one of the main objectives of the design in the paper (core diameter is keep below $380 \mu \mathrm{m}$ for this objective), being highlight the poor broadband properties of other alternatives, such as Bragg fibers. The authors indicate that, thanks to the proposed core rotation, which provides concealing more area with air holes, a reduction of losses and dispersion is achieved, thus overcoming results of similar PBG fibers.

The best design achieves (in simulation) $0.05 \mathrm{~dB} / \mathrm{mm}$ at $1 \mathrm{THz}$. It must be pointed, notwithstanding, that losses increase to $0.14 \mathrm{~dB} / \mathrm{mm}$ when the frequency moves to $1.3 \mathrm{THz}$. Again, porosity ratio has strong influence on 

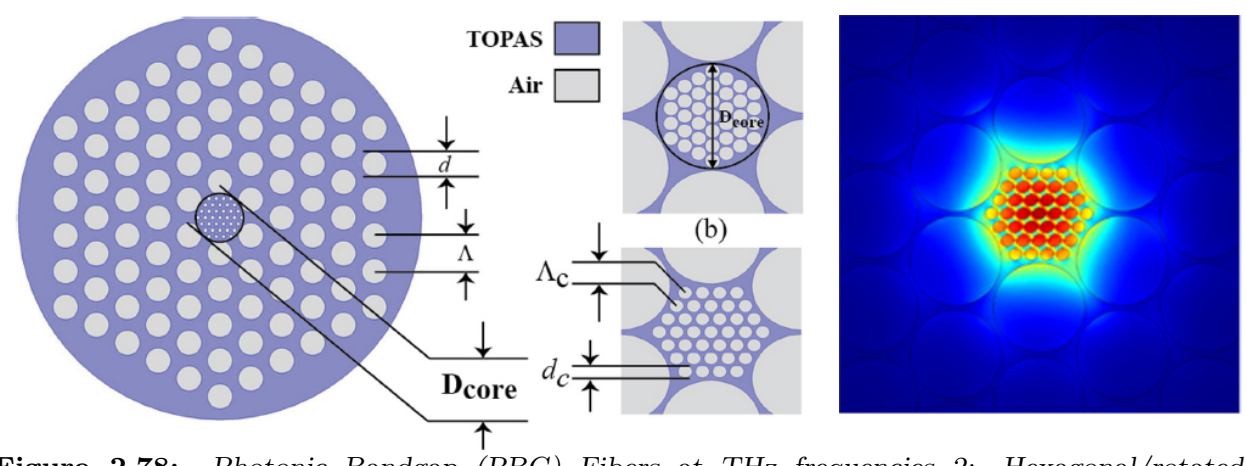

Figure 2.78: Photonic Bandgap (PBG) Fibers at $\mathrm{THz}$ frequencies 2: Hexagonal/rotatedhexagonal porous core PBG fiber of [475]. Schematic and E-field magnitude.

losses. Regarding to dispersion, stable $1 \mathrm{ps} / \mathrm{THz} / \mathrm{cm}$ is found between 0.5 $\mathrm{THz}$ and $1.1 \mathrm{THz}$, which is acceptable, but not excellent. Bend losses below $0.006 \mathrm{~dB} / \mathrm{mm}$ are reported for bends of $R=3 \mathrm{~cm}$. These losses increase to 0.08 $\mathrm{dB} / \mathrm{mm}$ when $R=1 \mathrm{~cm}$, and are also very sensitive to the porosity obtained, thus the performance relying againg in a precise manufacturing process.

The presented work is complete in general, however, experimental results that may convince the $\mathrm{THz}$ waveguide community lack in this and other similar papers. In general, PBG fibers offer a solution that can be, in theory, interesting under certain conditions. The geometrical complexity of the structures and the many dielectrics available, allow to design several kind of waveguides at the prize, it must be said, of a very complex analysis [476]. However, the intrinsic manufacturing complexity and the vast amount of additional losses sources in this waveguides, make unprovable a major role of them in $\mathrm{THz}$ waveguiding the next years.

\section{- EBG metallo-dielectric structures:}

Until now, most of the commented designs are conceptual inspired in microwave or optic waveguides, having slight modifications in order to be better adapted to challenging $\mathrm{THz}$ waveguiding environment. In this section, two structures are reviewed which, although they are inspired in some conventional geometry, they arise from a careful synthesis process involving metals and dielectrics proper disposition to provide a specifically tailored $\mathrm{THz}$ waveguide. These are two examples of how innovating the geometry can affect the performance when is almost nothing to do to improve employed materials.

The first metallo-dielectric EBG structure is that proposed by Llombart in [191], Fig. 2.79(a). This novel design can be viewed as a result of two approaches. On the one hand, one can think in a Bragg fiber [471]. Since Bragg fibers require many layers and have an electrically large core, high contrast between layers is inflicted by using fused quartz $\left(\varepsilon_{r}=4.6, \tan \delta=8 \cdot 10^{-4}\right.$ at $f=1 \mathrm{THz}[21])$ and air $\left(\varepsilon_{r} \approx 1\right)$. Furthermore, an outer metallic coated is placed, thus preventing any radiation. By this approach, the structure requires only 2-4 dielectric layers (simulations on the paper work with only 2), 
and the central air-core is about $4 \lambda_{0}$ diameter, which means $d_{\text {core }}=1.63 \mathrm{~mm}$, an acceptable size for flexibility and handling in practical applications.

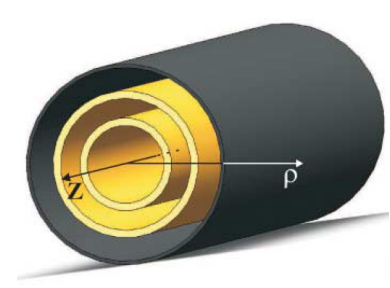

(a)

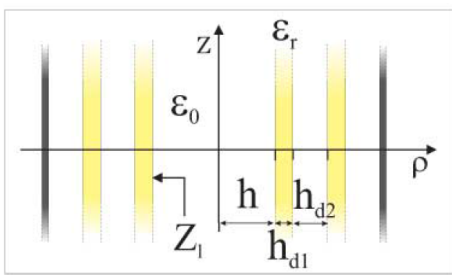

(b)

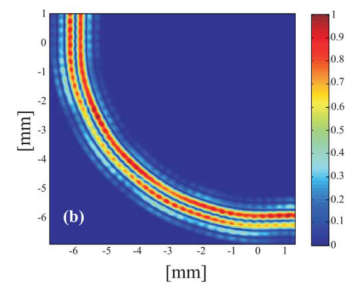

(c)

Figure 2.79: Metallo-dielectric EBG structures at $\mathrm{THz}$ frequencies 1: Cylindrical EBG waveguide proposed in [191], consisting in concentric two layers of fused-quartz separated by air and outer coated by metal. a) $3 D$ schematic; b) Conceptual view as a radial transmission line for design purposes; c) E-field magnitude on a $R=4 \mathrm{~mm}$ bend.

On the other hand, thinking first in the outer metallic coat, a metallic CWG comes to mind. It may be say that, whereas in [381]-[386] a simple inner dielectric coating is placed, in the EBG CWG, a much more sophisticated design is carried out taking into account the waveguide properties as a radial transmission line, see the central image of Fig. 2.79(b). Each air spacing is a quarter-wavelength step impedance transformer that provides a null of the E-field at the start of each period. Actually, the outer metal coating is placed in a radial point of very low impedance, thus barely affecting the guided mode. With these two approaches one can understand previous words about the novelty of the design, which seems to merge them.

One controversial aspect of the design is that it uses the low-loss $\mathrm{TE}_{01}$ mode which is degenerated with the $\mathrm{EH}_{11}$ in this complex, hybrid-mode structure. Therefore, after a first stage of design using transmission line theory, nonlinear optimization is applied to full-wave simulations in order to minimize both, ohmic and dielectric losses, and maximize the difference between the propagation constant of both degenerated modes in order to reduce coupling between them.

The proposed design achieves losses of $0.006 \mathrm{~dB} / \mathrm{mm}$ at $f=0.6 \mathrm{THz}$, that decrease with the frequency, getting close to $0.001 \mathrm{~dB} / \mathrm{mm}$ at $f=0.9 \mathrm{THz}$. This behavior is opposite to the classical CWG, which losses are displayed in the work for comparison, exhibiting larger values and a linear increment with the frequency. The structure presents also great performance at bends, with less than $1 \mathrm{~dB}$ losses in the band from $0.63 \mathrm{THz}$ to $0.87 \mathrm{THz}$ when the bend radius is of only $R=4 \mathrm{~mm}$.

The E-field magnitude distribution in such bend is shown for $f=0.75 \mathrm{THz}$ in the right image of Fig. 2.79(c). In this image it can be appreciated the large confinement of this structure that prevent radiation losses. However, the E-field patter irregularities also shows some mode coupling, even for this frequency, at which the design has been optimized. This coupling reaches -9 $\mathrm{dB}$ at some frequencies. 
Although the idea es innovative and the waveguide is carefully designed, there are some practical aspects to consider. Intrinsically, this waveguide is narrowband due to the implied resonant conditions of the EBG, aspect that can be also viewed from the transmission line approach. Also, although the authors suggest feeding coupling using serpentine quasi-optical mode converters, from the rest of the literature it can be deduced that excite with high efficiency an azimuthal polarized E-field with such a complex magnitude distribution will be complex.

Other important issue regards to the multimode feature of the waveguide. The dispersion diagram in the paper suggest that there are more modes apart of the $\mathrm{EH}_{11}$ that are above cutoff, although they are not shown. A cylindrical cable is susceptible of many perturbations including bending and torsion, that can deform the structure and cause strong mode coupling although the design try to minimize it. Finally, practical manufacturing of the structure may require dielectric bridges to keep the distance between the concentric dielectric layers or similar solutions previously seen in the PBG fibers. This will introduce additional losses, may cause resonant effects, and since the design is very sensitive to this distance between dielectric layers, from conclusions in the previous PBGs review, deterioration is expected.

To overcome the previous problems and exploit EBG possibilities in the THz, a similar solution, considering rectangular geometry is presented by Sánchez in [477]-[481]. The structure consists on the E-loaded RWG of [357], [372]-[373], where top and bottom metallic walls have been substituted by a woodpile [482] structure, Fig. 2.80(a). This EBG RWG is not introducing a small modification to slightly reduce losses of the RWG as in [477]-[481], but tries to control the mode propagation in an omnidirectional way, as it is done in [191].

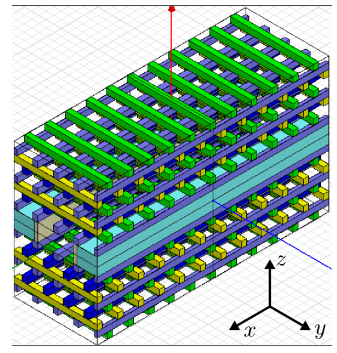

(a)

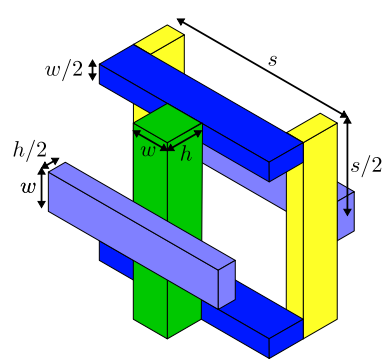

(b)

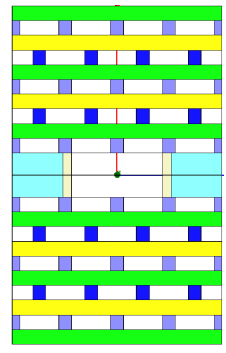

(c)

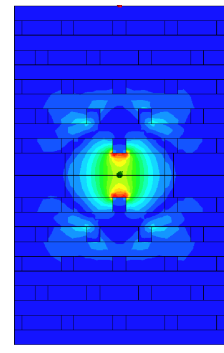

(d)

Figure 2.80: Metallo-dielectric EBG structures at $\mathrm{THz}$ frequencies 2: Rectangular EBG waveguide proposed in [477], consisting in a RWG with side walls loaded by E-plane dielectric layers (1D EBG) [357], [372]-[373], and top and bottom walls substituted by a woodpile structure (3D $E B G)$ [482]. a) $3 D$ schematic; b) Unit cell of the woodpile structure; c) Cross section; d) E-field magnitude.

The rectangular geometry, however, implies some additional difficulties [477]. For the top and bottom walls, the E-field is not parallel as for the side walls, and one has grazing incidence. Therefore, the 1D EGB used for the sides is not valid for them. In this case, a 3D EBG that forbids propagation in any 
direction, is required. The woodpile structure [482] (the unit cell is shown in Fig. 2.80(b)) is chosen due to its success in implementing EBG waveguides, and its reported unique properties in the mm-wave band regime [483]. Compared to other 3D periodic EBG structures, the woodpile provides better performance, robustness and manufacturing simplicity.

On the other hand, the rectangular geometry is much more suitable for component design than the circular one. Furthermore, rectangular geometries suffer less from perturbations caused by bends and torsions, at the lack of having a less mechanical flexibility. The possibilities of the EBG RWG to implement $\mathrm{THz}$ components are well shown in [481], where efficient bends, H-plane dividers and $\mathrm{H}$-plane horns and horn arrays are demonstrated.

To have more concrete figures, in [478], a woodpile structure having its bandgap centered at $f_{0}=0.95 \mathrm{THz}$, with bandwidth $0.17 \mathrm{THz}(17,9 \%)$, is designed by choosing woodpile parameters $w=35 \mu \mathrm{m}, h=40 \mu \mathrm{m}$ and $s=140 \mu \mathrm{m}$. The waveguide is designed to strictly operate single-mode, thus being the air channel dimensions of $245 \mu \mathrm{m} \times 120 \mu \mathrm{m}$, see Figs. 2.80(c)-2.80(d). The simulated losses of the structure are $0.04 \mathrm{~dB} / \mathrm{mm}$ at $0.95 \mathrm{THz}$, whereas the classical RWG presents losses of $0.09 \mathrm{~dB} / \mathrm{mm}$, and the RWG including only 1D EBG at the sides presents losses of $0.07 \mathrm{~dB} / \mathrm{mm}$. A 90 degrees bend is presented in this work, however, since the woodpile pattern is not modified for the bend, some leakage is produced.

Better results are obtained in [479] and [480], with a small, further optimization of the structure dimensions. Losses of $0.03 \mathrm{~dB} / \mathrm{mm}$ are obtained at $0.95 \mathrm{THz}$ ( $66 \%$ less than the classical RWG) and $0.2 \mathrm{~dB} / \mathrm{mm}$ when the design is scaled to operate at $f=4 \mathrm{THz}$. This means $80 \%$ less losses than a RWG. Those works show, furthermore, that the same design using only woodpile and not 1D EBG at the lateral sides exhibits $0.043 \mathrm{~dB} / \mathrm{mm}$ at $0.95 \mathrm{THz}$, and that using all woodpile boundary (no metal, as proposed in [483]) produces a waveguide with $0.068 \mathrm{~dB} / \mathrm{mm}$, which is, surprisingly, more than 2 times the losses of the proposed design. Moreover, it is highlighted that having a simple 1D EBG structure at sides facilitates $\mathrm{H}$-plane bending and component design in this dimension, since the woodpile requires a careful design when the path is not longer straight.

To end this section, the possibilities of the EBG RWG for component design are commented. In [481] they are designed a power divider, a horn antenna and the final horn antenna array, implemented all of them in the H-plane. In this paper, a 90 degree bend with excellent confinement and low-loss is presented. Unlike in [478], the woodpile structure is modified, being adapted to the bend so that undesired EBG modes coupling is under $-20 \mathrm{~dB}$ level, see Figs. 2.81(a)2.81(c). The designed horns have a directivity of $D=12$ and efficiency close to $99 \%$, whereas the total array, Fig. 2.82(a), presents an efficiency of $95 \%$, which is very high for $\mathrm{THz}$ frequencies. A prototype scaled for the $\mathrm{X}$-band is shown in Fig. 2.82(c). Lateral metallic walls are added later by covering all the design with copper film. 


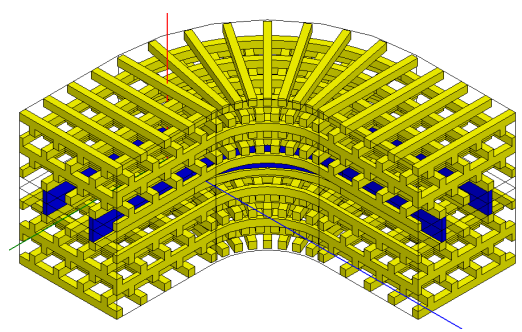

(a)

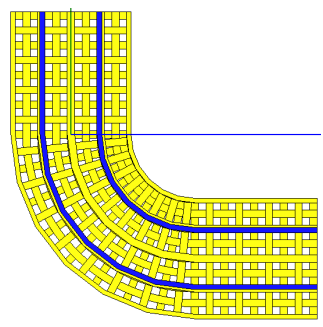

(b)

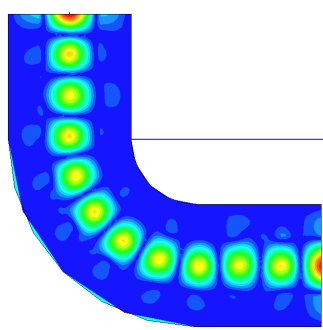

(c)

Figure 2.81: Metallo-dielectric EBG structures at $\mathrm{THz}$ frequencies 3: 90 degrees bend implemented with the rectangular EBG waveguide [481]. a) $3 D$ view; b) top view; c) E-field magnitude.

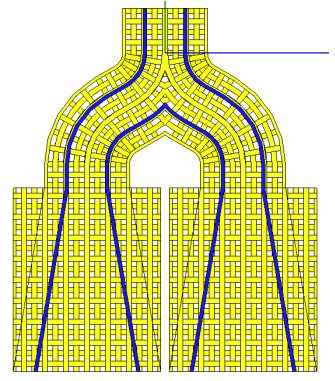

(a)

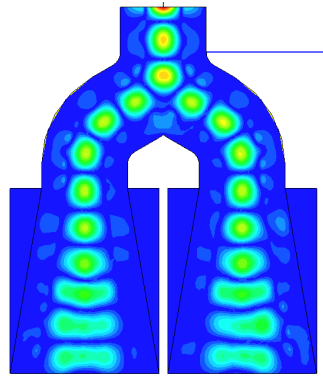

(b)

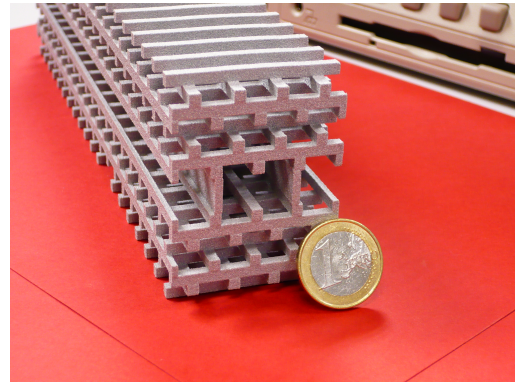

(c)

Figure 2.82: Metallo-dielectric EBG structures at $\mathrm{THz}$ frequencies 4. a) Top view schematic of $H$-plane horn array implemented with the rectangular EBG waveguide [481]; b) E-field magnitude; c) Scaled prototype for the X-band.

- EBG planar waveguides: The use of EBG structures has been also conceived to provide high performance planar designs, more suitable for applications requiring compactness, a feature that the previous bulky designs do not provide.

In [484], it is considered the possibility of arraying metal cylinders in a square lattice to create a $2 \mathrm{D}$ EBG structure. Then, by removing one or two rows, a propagation channel is created, see Fig. 2.83(a). Conceptually, the presented structure involves a 2D scenario, i.e., the structure is assumed infinite in the cylinders axial direction. However, any way to provide confinement in this direction is enough to obtain an implementable waveguide. The authors suggest using metal plates, thus having a PPW with boundary conditions at the lateral sides provided by the EBG. Waveguide heights from $25 \mu \mathrm{m}$ to $100 \mu \mathrm{m}$ are suggested depending the desired grade of compactness, and good agreement with $2 \mathrm{D}$ simulations is found.

By using cylinders of radius $r=10 \mu \mathrm{m}$, separated $p=50 \mu \mathrm{m}$, a bandgap is created for frequencies beyond $3.7 \mathrm{THz}$. Single mode operation is achieved between $1.2 \mathrm{THz}$ and $3.7 \mathrm{THz}$ when only one row is removed (waveguide width of $a=80 \mu \mathrm{m}$ ) and from $1 \mathrm{THz}$ to $2 \mathrm{THz}$ when two rows are removed (waveguide width of $a=130 \mu \mathrm{m}$ ). The typical trade off between bandwidth and losses is 


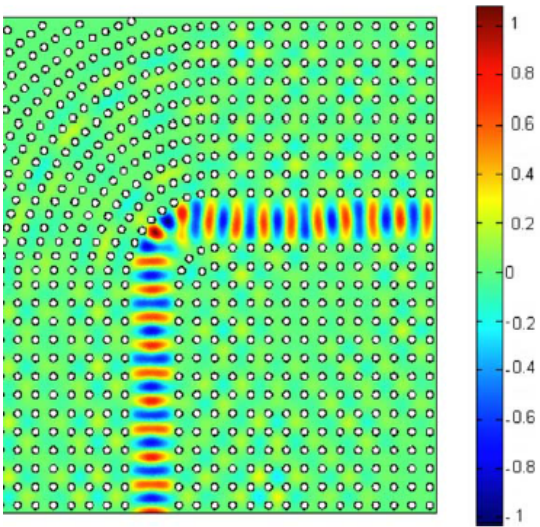

(a)

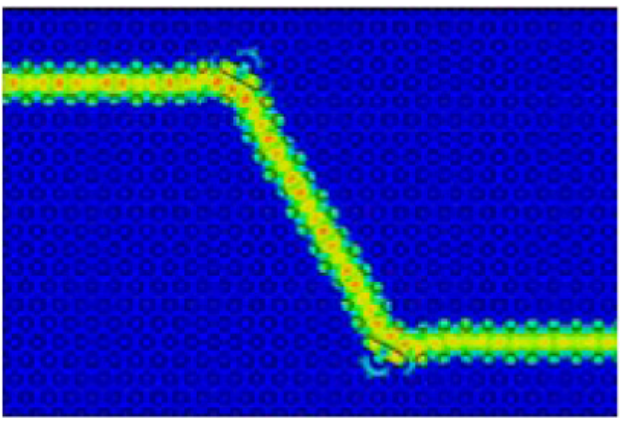

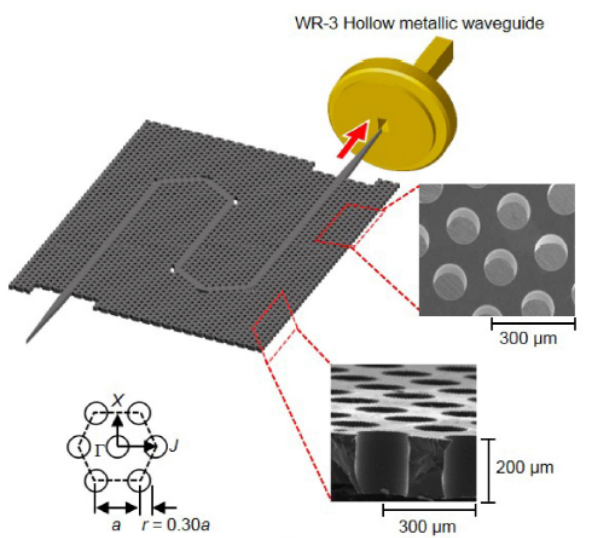

(b)

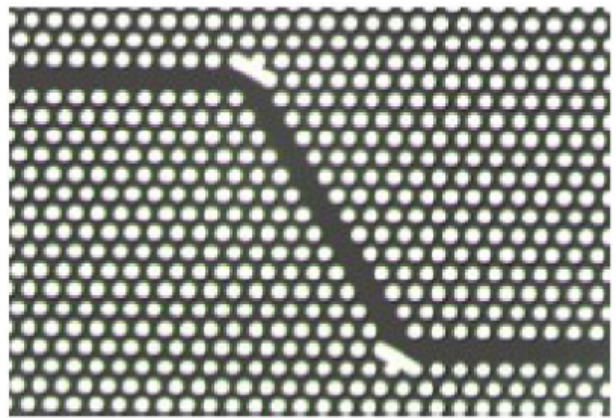

(c)

Figure 2.83: Planar EBG structures at $\mathrm{THz}$ frequencies. a) Optimized bend implemented with a EBG waveguide based on a lateral 2D EBG made of metallic cylinders, proposed in [484]. Cylinder radius is $r=10 \mathrm{mum}$ and standard period is $p=50 \mathrm{mum}$. The E-field magnitude at $f=4.1 \mathrm{THz}$ is also shown; b) EBG waveguide proposed in [485], consisting in a silicon slab where dielectric holes are practiced in triangular lattice to form a $2 D$ EBG structures. The hole radius is $r=90 \mathrm{mum}$, the period is $a=300 \mu$ and the slab thickness is $h=200 \mu \mathrm{m}$. In the image they are shown details of the feeding with WR-3, schematic of the triangular periodic lattice and involved parameters and photographs of the top and side of the structure; c) 60 degree bend implemented with the EBG silicon waveguide of [485] and E-field magnitude in it.

found also in this waveguide. However the planar nature of the design suggest the narrow waveguide as more appropriate since the slight reduction in losses of the wide channel waveguide not justifies the noticeable reduction of the operation band.

Simulations use the Drude model for the metal cylinders, which ensures high accuracy for the calculated losses. However, top and metal plates are considered ideal. Under this conditions, losses of approximately $1.1 \mathrm{~dB} / \mathrm{mm}$ are obtained at $f=3 \mathrm{THz}$ for any of the considered heights. Given the high frequency, it is a good performance. Furthermore, the structure provides high confinement and low bend losses, as it can be appreciated in Fig. 2.83(a). Several 90 degrees sharp bends are studied including the null radius case and 
some mitered examples. Best results are obtained for a sharp bend with small but not null radius, having $98 \%$ transmission (less than $0.01 \mathrm{~dB}$ losses) without any dip for the whole $1.2 \mathrm{THz}-3.7 \mathrm{THz}$ band.

Still not experimentally proved, the design looks interesting. They lack some assessments about possible manufacturing process options, and it seems that the conditions in the vertical direction may still be improved. When not ideal, ohmic losses will appear at the top and bottom walls, and some structure to diminish this losses may be necessary in order to keep the losses at the level that make this waveguide an interesting planar candidate.

The other reviewed proposal [485], although in lower frequencies, gives convincing evidence of its possibilities through practical results. A high resistivity silicon slab of $h=200 \mu \mathrm{m}$ is perforated to obtain a triangular lattice of holes (radius $r=90 \mu \mathrm{m}$, period $a=300 \mu \mathrm{m}$ ). In such way, a 2D EBG is created except in the drawn paths with no holes, where the waves are guided, see Fig. 2.83(b). In the vertical dimensions, guidance is provided by the TIR effect in the silicon-air top and bottom interfaces.

One possible drawback of the proposal is its narrow-band character and low frequency considered. The considered band ranges from $0.32 \mathrm{THz}$ to $0.33 \mathrm{THz}$. However, some good features are appreciated. The experimental prototype losses in this band are under $0.01 \mathrm{~dB} / \mathrm{mm}$, being the minimum losses of 0.004 $\mathrm{dB} / \mathrm{mm}$. This is 2 or 3 orders of magnitude less than common planar waveguide designs, and the best record for similar planar EBG structures. Furthermore, easy coupling from a WR-3 (see Fig. 2.83(b)) is demonstrated through a tapered connection with adiabatic change, providing less than $0.2 \mathrm{~dB}$ insertion losses.

Furthermore, the structure provides high confinement, as it is shown in Fig. $2.83(\mathrm{c})$, where a 60 degrees bend and the E-field magnitude distribution in it can be appreciated. It must be highlighted that in this work, transmission along $50 \mathrm{~cm}$ including 28 bends like the previous one is carried out with only $12 \mathrm{~dB}$ losses. An 1.5 Gbps $\mathrm{THz}$ link is created by using such path, providing uncompressed high quality video error-free transmission. Therefore, although the structure is inherently narrow-band, its suitability for communications applications is well demonstrated. Accurate optic band manufacturing technology is employed, therefore the authors point to material unexpected losses to find the origin of some disagreement between simulations and experiment. From what has been reviewed in this section, considering a somehow air-filled channel could enhance the good transmission properties of this line.

\subsubsection{Surface and Plasmonic Waveguides}

The waveguide section ends with surface and plasmonic waveguides. Both kinds of waveguides are basically to specifications of the same phenomena: impenetrable surface waveguiding [21]. More generally, surface waveguides include also dielectric waveguides, or, actually, any open waveguide. However, when talking about impenetrable surface waveguides, one refers to those structures which rather rail the transmitted power, i.e., the wave is weakly guided, just attached to the waveguide 
surface, with most of the power located in the outer air region surrounding the surface.

The term plasmonic comes from the optical regime, defining the strong field concentration near metal surfaces for the very high frequencies [486]. Actually, impenetrable surface waveguiding requires from high contrast between media, usually provided by high permittivity dielectrics, and, in the optical regime, a metal acts as a high permittivity, very lossy dielectric [21]. At $\mathrm{THz}$ frequencies, metals are not as bad conductor as in optics, however, they can be modeled as extremely high permittivity, lossy dielectrics [21], [348], [356].

This results very interesting if one notes that surface waveguiding is not possible on perfect conductors (or at least results unpractical in very good conductors, as metals are in the microwave regime) [487]-[490]. Therefore, at $\mathrm{THz}$ frequencies, metal surface waveguiding implies low penetration in the metal, but still enough to achieve a practical (moderately large) field extension, thus providing low attenuated, low dispersive waveguiding with a adequate propagation area dimensions.

This kind of waveguiding, is, actually, the oldest considered [487]. However, it resulted unpractical in the microwave regime due to the extremely large field extension [490]. On the other hand, it is very lossy at optic frequencies [430](plasmonics are used recently, mostly to provide high field enhancement for sensing or causing non-linear effects [486], but not for low-loss transmission). At $\mathrm{THz}$ frequencies, it provides unique characteristics that have call the attention of many researchers.

Here, proposed cylindrical structures based on simple wires and their planar counterparts are reviewed. The scientific activity has been intense, justified by the promising performance of such structures. As the whole review of the $\mathrm{THz}$ science justifies the interest of investigate $\mathrm{THz}$ waveguides, this section will justify, why in this part of the thesis, strong effort has been put, first, on carrying out a rigorous analysis of wire-type surface waveguides, and second, on proving proper designs exploiting their best characteristics. These are the two major found lacks when the published bibliography is reviewed.

\section{- Wire-type waveguides:}

The wire-type waveguides proposals are schematized in Fig. 2.84. In Fig. 2.84(a) it is shown the Single Wire Waveguide (SWW) or Sommerfeld Line (SL) [487], consisting in a bare metal wire of radius $a$. If a dielectric coating of thickness $t$ is added for field confinement purposes, one obtains the DielectricCoated Single Wire Waveguide (DCSWW) or Goubau Line (GL) [490], Fig. 2.84(b). Additionally, an air gap can be left between the wire and the coating to enhance confinement and reduce losses, Fig. 2.84(b), thus obtaining the Modified Dielectric-Coated Single Wire Waveguide (MDCSWW) or Modified Goubau Line (MGL) [491]. Finally, by arranging two wires of radius $a$ and separation between them $d$, it is formed the Two Wire Waveguide (TWW), Fig. 2.84(d).

The big trigger of wire-type waveguides came in 2004 when Wang and Mittleman reported experimentally outstanding low-loss and low-dispersion in [492] by using a simple metal wire (SWW). Pulses with spectral content from 0.1 $\mathrm{THz}$ to $0.5 \mathrm{THz}$ were shown to propagate up to $24 \mathrm{~cm}$ with negligible dispersion and average attenuation of only $0.002 \mathrm{~dB} / \mathrm{mm}$ along an $a=0.45 \mathrm{~mm}$ 


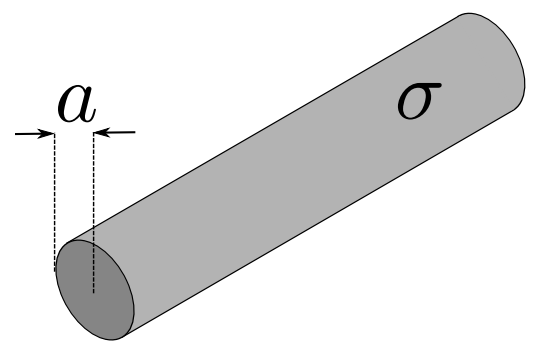

(a)

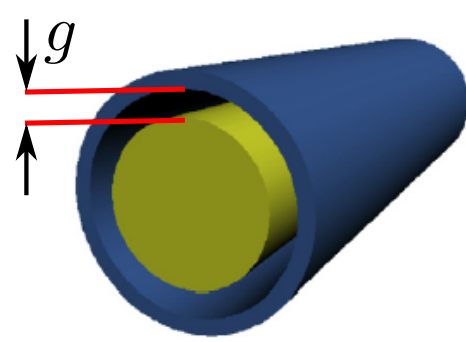

(c)

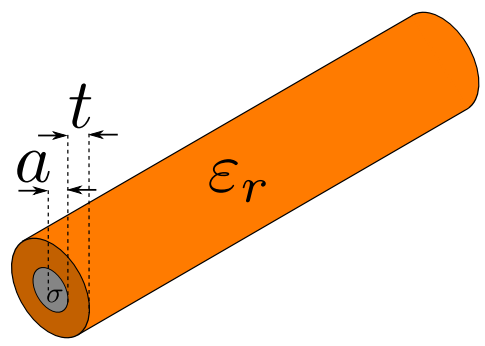

(b)

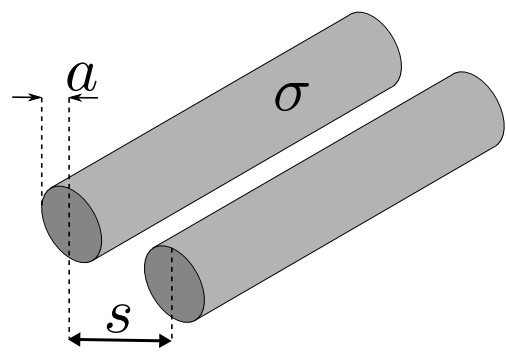

(d)

Figure 2.84: Wire-type surface waveguides at $\mathrm{THz}$ frequencies 1. a) Single Wire Waveguide $(S W W)$ or Sommerfeld Line $(S L)$ described by its radius $a$; b) Dielectric-Coated Single Wire Waveguide $(D C S W W)$ or Goubau Line (GL) consisting in a $S W W$ coated with a dielectric with thickness $t$ and permittivity $\varepsilon_{r}$; c) Modified Dielectric-Coated Single Wire Waveguide (MDCSWW) or Modified Goubau Line (MGL), where an air gap of thickness $g$ is left between the wire surface and the coating; d) Two Wire Waveguide $(T W W)$, consisting in two wires of radius a separated a distance $d$.

SWW. The employed excitation scheme is shown in Fig. 2.85(a). An auxiliary wire was used to couple the incident beam. The received pulses and spectra are shown in Fig. 2.85(b). The low attenuation and dispersion is clearly appreciated. A practical example of $\mathrm{THz}$ endoscope using coupled SWW was also demonstrated in this work.

The impact of the work was very high, being it, actually, the most cited publication on $\mathrm{THz}$ waveguides by far. Nevertheless, some important issues to address remained. The excitation efficiency was very low. Actually, the SWW has a difficult excitation, inherent to its fundamental mode radial polarization (note that linear polarized beams are commonly used for coupling $\mathrm{THz}$ energy), the low confinement of the field and its exponential amplitude decay from the wire surface. Furthermore, the work was eminently practical, with little theoretical base. One controversial point was the decreasing attenuation with frequency which, after posterior assessments seemed to be caused by the excitation scheme, thus discarding it could be a feature of the SWW.

Therefore, a huge number of researchers came with improved experimental setups, some investigations on the propagation characteristics of this waveguide, and studies on the effect of adding a dielectric coating on the wire (DCSWW). 
The work, resumed next, after a brief historical review of the wire-type waveguides, is still very experimental, and although much research has been done, some aspects are still to be addressed.

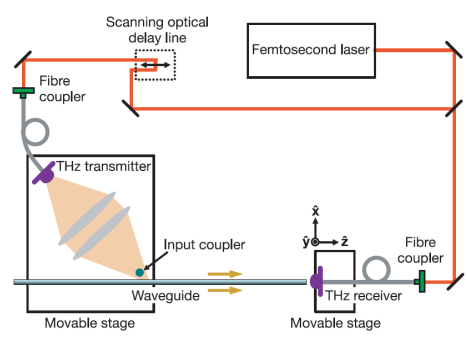

(a)

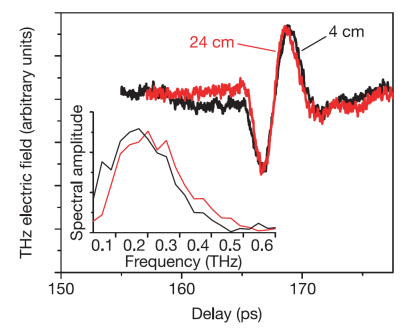

(b)

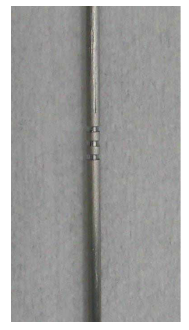

(c)

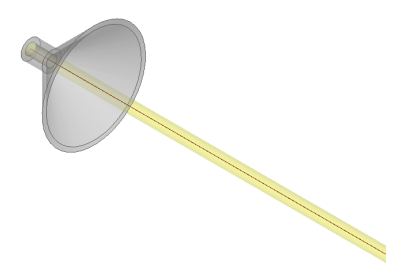

(d)
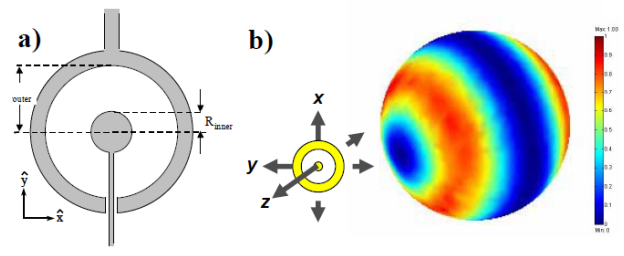

(e)

Figure 2.85: Wire-type surface waveguides at $\mathrm{THz}$ frequencies 2: excitation schemes and results. a) Excitation quasi-optical scheme used in [492] to excite the $S W W$ by using a perpendicular wire to couple the energy; b) Received pulses and spectra after $4 \mathrm{~cm}$ and $24 \mathrm{~cm}$ propagation [492]; c) Milled grooves practiced on a $S W W$ surface to enhance the excitation efficiency [493]; d) Horn launcher used to excite a DCSWW by opening with a taper the outer conductor of a coaxial [490], [494]; Photoconductive antenna with coaxial shape and 3D radiation pattern [495]. The shape of the antenna provides efficient excitation of the $S W W$ fundamental mode by creating a similar mode pattern.

The SWW was not a novel structure in 2004, but the oldest waveguide proposed. In the early of the $20^{t h}$ century, Sommerfeld studied the propagation of surface cylindrical waves on metal wires [487], [488], whereas Zenneck did the same for plane waves on metal plates [496]. Later, Stratton [489] put it in a formal way and gave further appreciations. Stratton described how a principal wave (mode $\left.\mathrm{TM}_{01}\right)$ with E-field mostly radial to the wire posses very low attenuation and dispersion whereas high-order modes are extremely damped since they travel confined inside the bulk metal of the wire. The lack of the required mathematical tools made impossible a proper analysis, and some concepts and affirmations are unclear, unfortunately.

Later, at the middle of $20^{\text {th }}$ Goubau [490], [497], [498], [499] rescued the Sommerfeld idea for frequencies of several $\mathrm{GHz}$, at that time named as kMcycles/s. Goubau found that, still at these high frequencies, the extension of the field guided by the SWW was of non-practical size, even close to $0.1 \mathrm{THz}$, for the considered wire sizes. Furthermore, the problem was of complex analysis for the available numerical tools, since waveguiding can only occur if finite conductivity is considered, being a singular problem otherwise. His proposal was to add a coating to form the waveguide that inherited his name, mentioning that 
the same result could be obtained by modifying the metal surface (roughness, groves, etc.). The fact of adding a coating (or modifying the surface) allows the problem to be solved without the necessity of having a finite conductivity, thus being easier to most deeply the propagation characteristics through a more affordable analysis, which was still made with several approximations at that time.

He found that a SWW of $a=1 \mathrm{~mm}$ may present an attenuation of only $0.05 \mathrm{~dB} / \mathrm{m}$ at $f=10 \mathrm{GHz}$, being, however, the field extension very large (a transversal cylindrical area of $10 \mathrm{~cm}$ radius only encompassed $75 \%$ of total guided power along the surface of the wire) [490]. Adding the coating allowed more practical values and possible excitation of the fundamental mode. The most popular scheme for excitation was a horn launcher based on a coaxial cable which outer conductor was opened with a taper to adapt the mode of a DCSWW (which can actually be roughly regarded as a coaxial cable with the outer conductor at the infinity) [490], [497],[499]. An schematic of this kind of excitation is shown in Fig. 2.85(d). The excitation using a dipole as that used by Wang and Mittleman [492] was also considered at that time [498]. In that work, the possibility of using two wires as a low loss, high frequency transmission line, is also found for the first time.

A decade later, King came [500] with a new analysis with less approximations. In this work, it is highlighted that the coating increases the attenuation in one order of magnitude, however, the field extension is reduced in more than one order of magnitude. A good conclusion of the mathematical calculations on this work was that the propagation depends on the term $f^{3} / \sigma$ which means that a bad conductor SWW behave as a good conductor SWW at higher frequencies, thus being possible reduce the extension of the field by using lower conductivity metals. The SWW was also shown to support high power levels.

However, the enough low loss feature of hollow metallic waveguides, the good and more practical characteristics of coaxial cable, and later, planar transmission lines, sentenced wire-type waveguides to be just a curiosity without tangible practical uses. They can be found just some works in the 70s. Chiba [501] studied radiation losses on bends of DCSWW, which is indeed, a major problem of these open structures. An interesting point studied in this work are the antenna properties of a bent DCSWW.

Despite of the fact the DCSWW propagation characteristics were not totally clear, the more complex modified DCSWW was deeply analyzed in [502]. In such rigorous work, it was theoretically proved that the MDCSWW provides more confinement, lower losses (up to 7 times less), and low dispersion than the DCSWW. However, keeping the gap between the wire and the coating was very unpractical for real experiments at that time, and no further use was reported up to these days. With less detail, in [503], the cutoff wave numbers of a DCSWW, which are frequency and geometrically dependent, were reported. Curiously, it is found that the $\mathrm{HE}_{11}$ is a fundamental mode of the DCSWW, however, it is not appreciated the true low-loss $\mathrm{TM}_{01}$ fundamental mode (both are, actually, degenerated). 
From that point until nowadays, uses of the DCSWW are hardly found (and less for the SWW). A patent was presented in 1995 [504], describing how some perturbations can be added over a DCSWW to create a leaky wave antenna for security purposes (detection of intruders). At the starting of this century, Alonso [505] published an interesting work on the excitation of a DCSWW by means of an improved tapered coaxial which outer conductor becomes a horn. In the proposed design, unlike in [490], [497], [499], the DCSWW is smartly tapered to a small radius $(a=10 \mu \mathrm{m})$. This causes field confinement and avoids mode matching losses when the horn ends. It was also observed that, for the high $\mathrm{THz}$ frequencies, the taper has an optimum length, since long, soft tapering would imply high propagation losses in an small radius DCSWW.

In our days, after [492], several works afford the excitation scheme [493], [506][508]. In [506], unlike direct generation an launching, pulses are optoelectronic generated with a specialized photoconductive antenna transmitter chip similar to that shown in Fig. 2.85(e). The SWW is tapered close to the transmitter to enhance the efficiency. At the reception, a gap PC antenna is placed over the wire. Experimentally, it is observed that a SWW with $a=260 \mu \mathrm{m}$ presents similar E-field distribution (and lower lossses) than a coaxial cable with same inner conductor radius and an outer conductor of $9 \mathrm{~mm}$, however, such a coaxial would be multimode beyond $0.02 \mathrm{THz}$ which is not the case of a SWW. It is also studied the effect of curvatures. By approximating the SWW ends (thus curving the wire), the signal attenuation is analyzed, founding a rapid drop of several dBs for such small considered curves.

In [493] milled groves are practiced over the wire SWW surface (see Fig. 2.85(c)), allowing field enhancement in such area, and thus, better coupling through direct launching schemes. The incidence is normal to the grooved wire. Nevertheless, it is observed that each groove produces one oscillation on the transmitted pulse, causing pulse distortion. Thus, a single groove may provide broadband signals, but multiple grooves reduces noticeably the bandwidth. On the other hand, adjusting the groove distribution and shape allows to manipulate the central frequency and the bandwidth, what can be very useful.

In [507], a PC antenna similar to that of [506] is employed. However, the reception is done by means of a probe placed few $\mu \mathrm{m}$ from the wire surface. This avoids alignment errors and provides more flexibility and accuracy for loss calculations, since power at several positions can be systematically obtained. Actually, this is the main objective of this work, in order to clear up the strange attenuation dispersion obtained in [492]. Smaller wires, and the use of coatings are considered. By this, relative errors in measurements are alleviated, since losses are higher for small or coated wires. Good agreement between theoretical models, HFSS ${ }^{\circledR}$ simulations and experiments are found for a small $a=25 \mu \mathrm{m}$ SWW with and without a $t=10 \mu \mathrm{m}$ Polyurethane $\left(\varepsilon_{r}=2.1, \tan \delta=0.001\right)$ coating. Experimentally, a SWW with $a=25 \mu \mathrm{m}$ exhibits less than 0.02 $\mathrm{dB} / \mathrm{mm}$ in the range $0.02 \mathrm{THz}-0.4 \mathrm{THz}$, one order of magnitude more than a $a=450 \mu \mathrm{m}$. Dispersion is also higher for the considered radius, but still in very low levels. 
A further improvement (mainly experienced by the lower frequencies of a broadband pulse) is found in [508], by forcing the SWW tip to touch the inner conductor of the PC antenna. In this work, an interesting practical use of the SWW is found: it is carried out a spectroscopy analysis of $1 \mathrm{mg}$ lactose powder dispersed on the top the SWW. A clear absorption peak at $f=0.53$ $\mathrm{THz}$ is observed. It is highlighted that this scheme is much more efficient than free-wave, radiating spectroscopy, which large beam require from excessively large samples, not always available. Furthermore, other transmission lines are to lossy or dispersive so that the propagation path is limited, therefore the interaction region. This inhibits investigation of weakly absorbing materials or the use of long thin films. A clear unique niche of application of wire structures is foreshadowed.

The group of Mittleman continues their work in [509] and [495]. In [509], they are obtained, basically, the same conclusions as [492], but curves are studied. It is found that $90^{\circ}$ degree bends of curvature radius ranging from $R=20 \mathrm{~cm}$ to $R=90 \mathrm{~cm}$ cause additional losses ranging from $0.14 \mathrm{~dB} / \mathrm{mm}$ to $0.08 \mathrm{~dB} / \mathrm{mm}$, having an increase of attenuation increasing exponentially with $R^{-1}$. The authors point to the difference of paths between inner and outer way around the curve, which distorts the axialsymmetric fundamental mode. Excitation efficiency with the same scheme of [492] is quantified in $1 \%$.

In [495], a deep study on the excitation of the SWW is carried out with simulations and some experiments. Conclusions establish that a proper excitation of the SWW is a challenging problem. On the one hand direct launching and coupling through an auxiliary wire produces as maximum $0.42 \%$ according to simulations. On the other hand, a PC coaxial antenna allows for a $56 \%$ efficiency. However, this is only the coupling efficiency from the antenna to the SWW. Actually, the PC has itself low efficiency, therefore the improvement is not as high as expected when regarding coupling efficiency figures. A main controversial point of the work is that simulations are carried out using PEC conditions for the SWW surface. Therefore, the software is considering very high conductivities, probably calculating less confined field distribution and propagation losses than those expected in reality.

An interesting work on controlling the field distribution on the SWW by using subwavelength grooves and varying the radius is found in [510]. Simulations predict that adiabatic changes allow for large and precise control over the field extent. The authors indicate that the structure is low-loss, however, the high field confinement suggest the contrary, at least, for reasonable distances of propagation. Nevertheless, such modifications on the SWW could be very interesting for excitation, sensing, and preventing radiation on bends. A simulation showing the E-field along the proposed tapered, grooved SWW, can be observed in Fig. 2.86(a).

Step by step, some attempts to optimize the use of the SWW have come by understanding better the propagation features of this waveguide. Wang and Mittleman observe experimentally in [512] the stronger waveguiding (appreciated on the larger difference between the propagation constant from that of a plane wave) of a SWW of $a=18 \mu \mathrm{m}$. In [513] a conical SWW is used to 


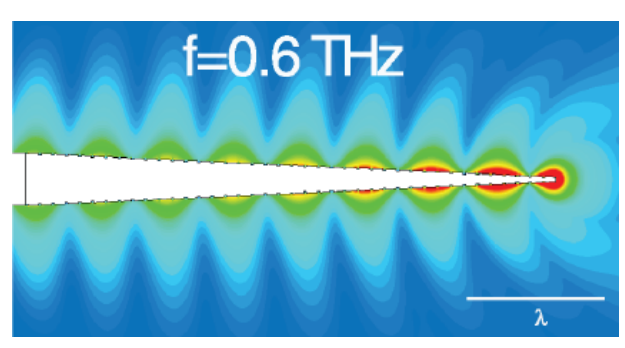

(a)
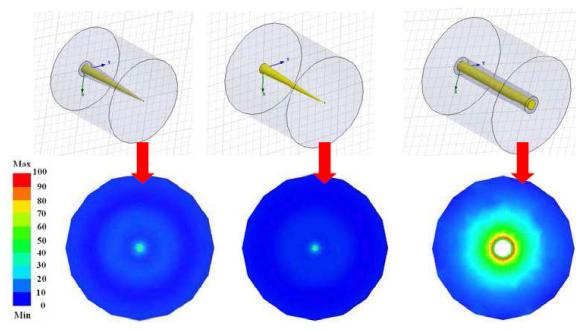

(b)

Figure 2.86: Wire-type surface waveguides at $\mathrm{THz}$ frequencies 3: grooved and conical wires a) Conical $S W W$ with subwavelength grooves proposed in [510] to provide larger confinement and field enhancement for sensing purposes; b) From left to right, E-field distribution in a conical DCSWW, a conical $S W W$ and a conventional DCSWW. Best confinement is observed for a conical $S W W$ [511].

enhance the excitation. A SWW of diameter $d=2 a=500 \mu \mathrm{m}$ is tapered to a tip of $30 \mu \mathrm{m}$ of diameter. The received power is 4.5 times than that with a conventional SWW.

Similarly, in [514] a SWW of diameter $d=2 a=500 \mu \mathrm{m}$ is tapered to a tip of $20 \mu \mathrm{m}$ of diameter, obtaining that the E-field is confined in a radius one order of magnitude less than for the conventional thick SWW and two orders of magnitude less than free space. Specifically, near the tip, the Full Width at Half Maximum (FWHM) of the E-field is located at $r=30 \mu \mathrm{m}$.

In [515], a careful characterization of the conical SWW problem is done. Again, it is observed that reducing the radius has an strong effect, not previously reported in optics. This may be justified by the larger impenetrability of the metal surface. Therefore, unlike dielectric wires, metal wires do not lose waveguiding properties when they become subwavelength. The authors found that propagation in a conical SWW is very sensitive to the metal conductivity, suggesting a possible application of this waveguide for temperature sensing purposes.

Deep and interesting results also the work in [511], affording with detail the problem of a transition from a coaxial to a DCSWW or a SWW. Conical version of these two waveguides and the propagation on them are simulated. The most interesting point is that, contrarily that could be though, a conical SWW provides more field confinement than a DCSWW, see Fig. 2.86(b). It seems that, for the considered coating permittivity and thickness, there is an effect of surface waveguiding in the dielectric-air interface. Therefore, a concentration of E-field takes place in the outer air region surrounding the dielectric. In the conical SWW the field is concentrated around the metal tip, in a more compact area.

The case of extremely small metallic nanowires ( $a$ ranging from $100 \mathrm{~nm}$ to 1 $\mu \mathrm{m})$ has been also considered [516]. According to the authors this small radius, cause interaction of the fundamental mode with the bulk modes traveling confined inside the metal since, for example, the penetration depth of copper 
is of $\delta_{C u}=51 \mathrm{~nm}$ at $f=1 \mathrm{THz}$. Involved equations are solved with approximations regarding the small radius employed, and for very small wires it is found a singular behavior of the attenuation, that decreases with frequency, contrary to the usual case. However, the calculated attenuation values are very small (less than $0.0008 \mathrm{~dB} / \mathrm{mm}$ ), thus falling the results into question, which, furthermore, have not been endorsed by posterior works.

Additional conclusions on coupling and bends are reported by the group of Wang and Mittleman in [517]. It is interesting that a gap between two axial aligned wires has almost no return losses when the radius is large enough $(a=875 \mathrm{~nm})$, due to the similarity between the propagation on a SWW and the free space. A mirror is shown to be a good solution instead bends for two unaligned SWWs. However, this solution may be quite unpractical. It is also studied the total attenuation on bends depending on the curvature radius. A maximum occurs since very large $R$ implying soft bends reduces radiation losses but also implies high propagation losses. Unfortunately, the best result is a loss of $7.5 \mathrm{~dB}$, which is quite high for the frequency range considered (up to $0.4 \mathrm{THz}$ ).

A new excitation scheme has been recently presented in [518]. A double dielectric slab waveguide is used to provide accurate mode-matching in the coupling area. The thickness and separation of the slabs are optimized to provide the best field exponential decay likening the SWW field profile. Polarization is linear, however. The coupling is simulated to be $38 \%$ at $1 \mathrm{THz}$, what is a good figure taking into account that any PC antenna is used, and the power is quasi-optically coupled to the double slab with good efficiency. A possible problem of this proposal is that the mode matching (and therefore, the efficiency) is very sensitive to the electrical thickness of the slabs. This implies that the design is narrow-band and somehow sensitive to the slab manufacturing tolerances, which may misspend the good broadband characteristic of the SWW, one of its strongest features.

Works mainly focused in the DCSWW (in a main role sense, and not as complementary case of a set of experiments with the SWW) are much less prominent, since this structure is neither low-loss (as the desired levels) nor low dispersive, and still is an open waveguide. In [519], which is contemporary to the first work of Wang and Mittleman, theoretical analysis and simulations are carried out for the fundamental mode of the DCSWW estimulated by the results of [505].

At this work some interesting assessments are done. It is found how high permittivity coatings increase the ohmic losses due to the larger confinement, and that there is a compromise with $t$ regarding radiation losses one the one hand, and propagation losses and bandwidth on the other. Also, it is appreciated for the first time that smaller wires provide less radiation losses on bends. With a small radius $(a=50 \mu \mathrm{m})$ and a thin coating $(a=10 \mu \mathrm{m})$ of $\varepsilon_{r}=2$, good compromise between propagation losses $\left(\alpha_{p}=0.08 \mathrm{~dB} / \mathrm{mm}\right.$ at $\left.1 \mathrm{THz}\right)$ and radiation losses $\left(\alpha_{r}=0.008 \mathrm{~dB} / \mathrm{mm}\right.$ at $\left.1 \mathrm{THz}\right)$ is found. The propagation losses are, however, in the level of a RWG, therefore losing sense the chose of this DCSWW for practical medium distance guided paths. The coaxial transition 
to the DCSWW is studied, founding that return losses, of $3 \mathrm{~dB}$ when direct connection between waveguides is applied, can be improved to $15 \mathrm{~dB}$ with a $L=6 \mathrm{~mm}$ taper.

Although some properties of DCSWW are clarified, the study is not deep enough to clearly quantify the influences of the design parameters of the DCSWW. Furthermore, the important question of high-order modes, which are more evident in the DCSWW due to the presence of the coating, continues to remain untreated.

Continuing with the DCSWW work, in [520], it is found an equivalent between a DCSWW with a SWW with subwavelength grooves. The theoretical analysis of the grooves obtains an equivalent coating with given thickness and permittivity, that produces the same propagation, see Fig. 2.87. The authors, probably comparing with the optical regime, consider that the structure is low loss due to the metals good behavior at THz. However, due to the high plasmonic field concentration at the grooves, again noticeable larger losses than the dielectric case are expected. Moreover, the equivalent narrow-band due to the resonant nature of the grooves effect. Despite of this facts, this kind of structure and analysis may have its application, allowing filtering features over wire structures. In addition, a fine control on the energy coupling process may be achieved if the grooves are manufactured with enough precision.

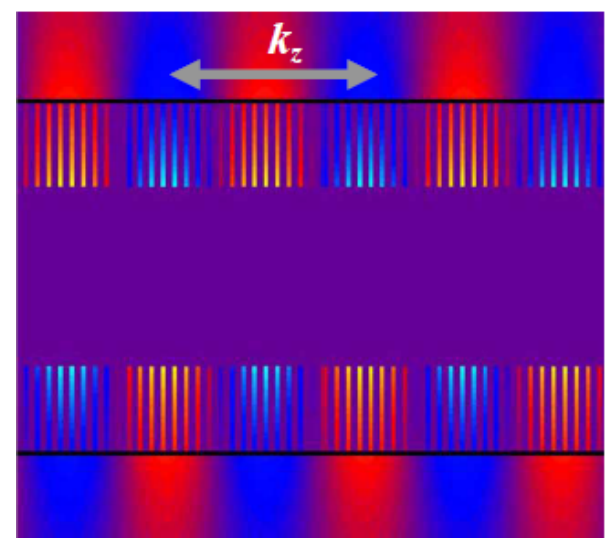

(a)

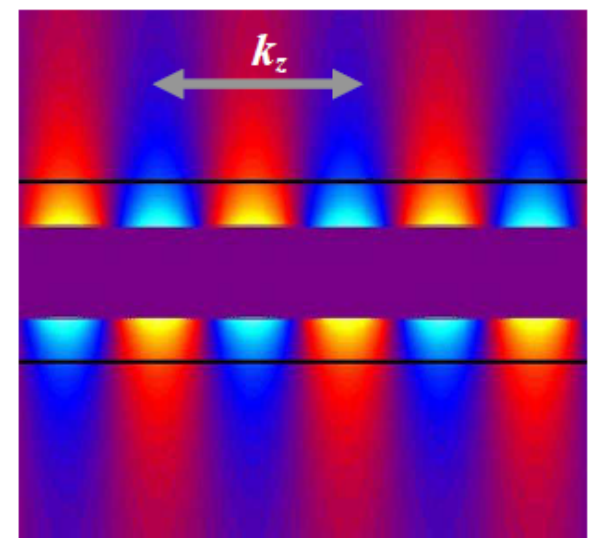

(b)

Figure 2.87: Wire-type surface waveguides at $\mathrm{THz}$ frequencies 4: grooves and coating equivalent in [520] a) Lateral view of the proposed $S W W$ with subwavelength grooves and field distribution; b) Equivalent DCSWW with same field extension and propagation constant. The equivalent is inherently narrow-band, and expected propagation losses are higher in the grooved $S W W$ due to the high ohmic losses caused by the field concentration in the vicinity of the grooves.

A curious work is found in [521], where a DCSWW with a metamaterial dielectric is analyzed and the propagation behavior is compared with the classical DCSWW. The phenomena involved are quite singular, thus being, at this moment, hard to visualize future applications. It is worth to mention that, despite of the complex theoretical work carried out, high-order modes lack in the anal- 
ysis, founding one more time a study concentrated uniquely in the fundamental $\mathrm{TM}_{01}$ mode.

In [522], a quite rigorous analysis is applied to the modified DCSWW operating at THz. In this work, the Drude model for metals is applied, thus providing a more accurate characterization for the high, $\mathrm{THz}$ frequencies. In the paper it is highlighted that common simulations with a commercial solver such HFSS ${ }^{\circledR}$ may provide some errors since the common skin depth model is applied. However, looking into detail, it is found that the error is quite small.

By considering copper wires and dielectric Teflon, they are shown the theoretical benefits of having the air gap provided by the modified DCSWW. With a radius of $a=100 \mu \mathrm{m}$ and a coating of thickness $t=80 \mu \mathrm{m}$, the DCSWW has an attenuation of $0.1 \mathrm{~dB} / \mathrm{mm}$ at $f=0.7 \mathrm{THz}$, which is reduced to 0.06 $\mathrm{dB} / \mathrm{mm}$ if a gap of $g=20 \mu \mathrm{m}$ is left between conductor and coating. This gap enhances, furthermore, the confinement in the inner air region, thus preventing radiation, in a similar way that a dielectric tube does. Actually, TIR effect occurs in the inner dielectric face and in the outer dielectric face. Larger gaps provide even lower losses: with $g=60 \mu \mathrm{m}, 0.01 \mathrm{~dB} / \mathrm{mm}$ are obtained at $f=0.7 \mathrm{THz}$. However, although the waveguide concept is interesting, its practical implementation is of great complexity: additional supporting elements would be necessary as was the case of [191]. Furthermore, high-order modes, not considered, may considerable affect this kind of structure, imposing limitation on the gap and coating thicknesses in a structure dispersive per se.

As a practical application of single wire structures, the very recent work in [523] is given as an example. The experimental setup scheme is shown in Fig. 2.88(a). A copper wire of radius $a=100 \mu \mathrm{m}$ is coated with a $t=442 \mu \mathrm{m}$ layer of PMMA, and a $t=10 \mu \mathrm{m}$ layer of Polyvinylidene Fluoride (PVDF), a natural thermoelectric semi-crystalline dielectric having a frequency-dependent permittivity at $\mathrm{THz}$ frequencies. This cable is shown to be very sensitive to characterize liquids in the $\mathrm{THz}$ band. In Fig. 2.88(b) they can be appreciated the strong attenuation peaks (imaginary part of the effective refractive index). On the one hand, the attenuation at the peaking frequencies, caused by the analyte liquid, is much larger than the air case (green curve). On the other hand, the peak frequency is very dependent on the refractive index of the liquid (in this example a difference less than $1 \%$ is clearly recognized by an enough large frequency displacement).

Along the commented proposals with the SWW and DCSWW, several experiments, different coupling schemes, and solutions to reduce radiation loss problems in bends and perturbations have been proposed, rather as patches, in a try and error improving system. Some interesting practical uses have been also demonstrated. However, although in some works, such as [510], [516] or [511], some theoretical self work supports the posterior simulations and/ or experiments, most of the literature relies and the work did more than 50 years ago by Goubau and King, which at the same time reference back Stratton and Sommerfeld. 


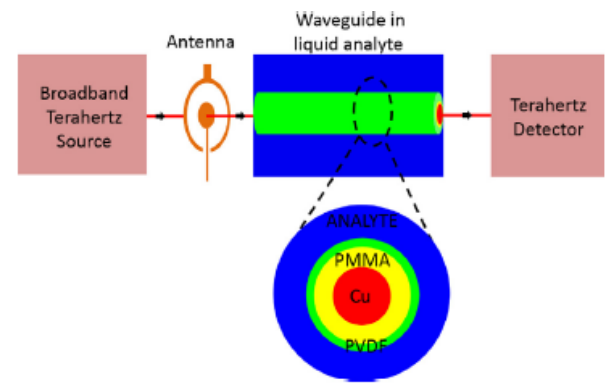

(a)

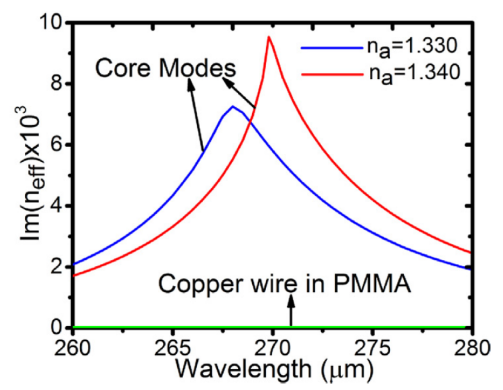

(b)

Figure 2.88: Wire-type surface waveguides at $T H z$ frequencies 5: DCSWW for sensing liquids [523] a) Schematic of the proposed experimental setup and cross section of the sensor; b) Imaginary part of the dispersion curves related with losses for liquids with refractive index of $n=1.33$ and $n=1.34$. The green curve corresponds to the case with no liquid. The observed strong peaks and the resolution in frequency for the small variation of the refractive index (less than 1\%) proves the are indicators of the high sensitivity of the device.

The fundamental surface mode of SWW and DCSWW is, up to some level, well-known, and some characteristics are exploited. There is found even some work dealing with the propagation characteristics of this mode. In [524], it is developed an explicit formula for the SWW complex propagation constant, in order to avoid a routine including a zero-finding task. However, the use of the formula reaches the complexity of the original problem, which is analytic, and errors up to $5 \%$ are found.

Despite the possible utility of the proposed formulas, issues common to all the other publications are found. First, the high-order modes are not considered neither in the SWW nor in the DCSWW. It is not clear the nature of these modes and the possible negative influence to the fundamental low loss $\mathrm{TM}_{01}$ mode. Secondly, the depth of the theoretical studies on the fundamental mode propagation in single wire structures has not a depth proportional to the relevance that the structure has acquired in the $\mathrm{THz}$ waveguing scenario. Third, the possibility of jointly exploiting the benefits of each one of the commented structures through its proper interconnection and optimization has not been considered at all. Only the proposals regarding conical wires exploit some features. However, since the problem is not deeply studied, it could be expected that some improvement is possible through proper optimization. This are the issues that call to be solved in this thesis, becoming thus, part of its objectives.

Although at this point, the text has driven the reader to the motivational origin of this part of the thesis, it would not be fair leave apart some recent surface/plasmonic waveguide proposals. This section ends with the Two Wire Waveguide, which arose as a possible solution for SWW radiation problems. Nevertheless, the popularity of the TWW is not comparable to that obtained by the SWW.

The first proposal came in 2009, again by the group of Mittleman [525]. In this work, the TWW obtains similar results than the SWW in simulation, however, 
the measurements show higher losses: two wires of $a=150 \mu \mathrm{m}$ separated $d=0.5 \mathrm{~cm}$ exhibited about $0.015 \mathrm{~dB} / \mathrm{mm}$ in the $0.1 \mathrm{THz}-0.5 \mathrm{THz}$ range. The authors conclude that the TWW may be used to transport signals up to $0.2 \mathrm{THz}$ with low bending losses (approximately $4 \mathrm{~dB}$ for a $90^{\circ}$ bend with curvature radius $R=8.6 \mathrm{~cm}$ ). Therefore, the conclusions do not state any relevant improvement from the SWW general behavior.

Much more deep is the work provided by Pahlevaninezhad [526], [527] one year later. First, in [526], a rigorous analysis of the structure is done for the fundamental mode: since the structure has more than one conductor, the thoughtful Conformal Mapping can be applied [528], i.e., a complicated geometry is mapped to a simpler one through a complex analytic function. Numerically, the complex function imposes no problem, and afterward, the solution is transformed to the original geometry.

The only attributable problem to this approach is that consider the wires as perfect conductors, thus introducing an error hard to quantify. On the other hand, a theoretical analysis assuming the metal as a complex dielectric would be excessively complex. Considering wires of $a=200 \mu \mathrm{m}$, separated $d=1$ $\mathrm{mm}$, ohmic losses of $0.01 \mathrm{~dB} / \mathrm{mm}$ at $f=1 \mathrm{THz}$ are estimated by perturbative theory [376]. Reducing the separation $d$ increases the losses exponentially, and some loss reduction can be achieved with larger wires, however not improvement is found beyond $a=300 \mu \mathrm{m}$, which is, actually, the wavelength size at the frequency of operation. However, a possible problem that the value of this distance between wires $d$ conditions the higher TE and TM modes, not considered in the analysis.

In [527], excitation of the TWW is afforded. It is shown that focusing the excitation beam in a bounded area, mainly focused between the wires can provide high efficiency coupling, up to $70 \%$. However, this efficiency is very sensitive to the wire separation $d$. Therefore, it is expected that it noticeably aggravates in practice.

Apart of its inherent disadvantages, the TWW has a difficult practical implementation: it requires some mechanical structure to hold the two wires and let them be equally separated along the waveguiding path. This problem is well addressed in [529]. Two different dielectric porous supports are considered, and comparison with the original TWW is provided. The analyzed structures and the field distribution in them are shown in Fig. 2.89.

As can be observed, the structures are designed to allow the field travel mostly in the inner air region, confined and partly isolated from exterior, and giving good mechanical consistence to the wires. As it can be foreseen, losses are higher in the proposed structures, which add dielectric losses. However, the deep analysis carried out in the paper indicates that the proposed design has a better performance than porous fibers, highlighting the lower propagation and attenuation dispersion.

The authors affirm that the TWW has 5 times lower losses at bends than a common SWW, and their study on the coupling efficiency concludes that is possible to achieve good efficiency in a relatively wide range of frequencies 


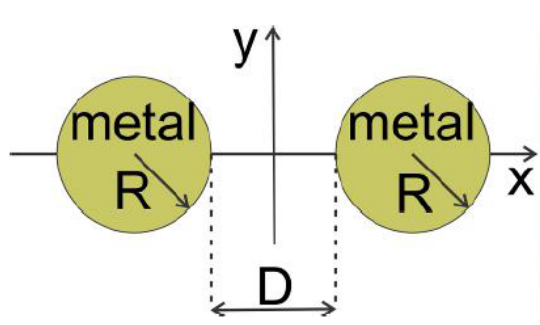

(a)

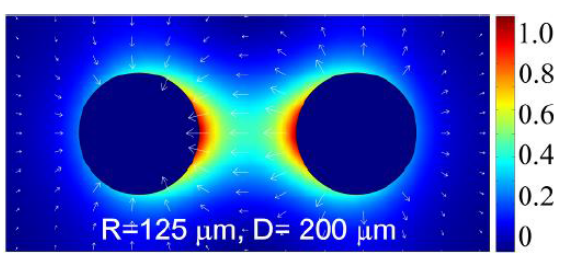

(d)

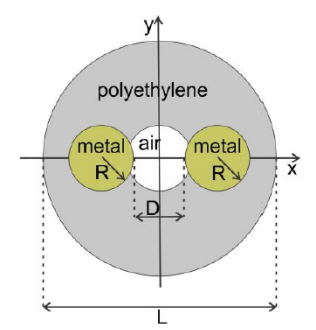

(b)

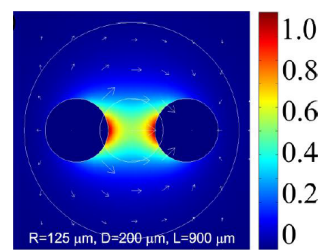

(e)

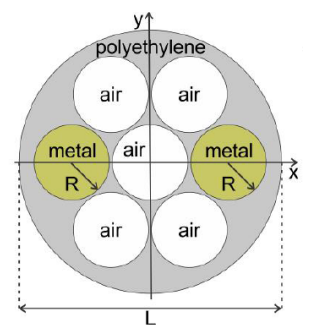

(c)

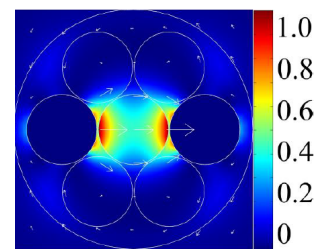

(f)

Figure 2.89: Wire-type surface waveguides at $\mathrm{THz}$ frequencies 6: Two-wire waveguide $(T W W)$ a) Basic $T W W$ immersed in a outer air region b) Practical implementation 1 proposed in [529], consisting in a tube enclosing the wires with a central air region for low loss propagation; $c$ ) Practical implementation 2 proposed in [529] consisting in a porous fiber supporting the wires with several air holes, to avoid dielectric losses as possible; d)-f) E-field magnitude distribution of the fundamental mode for the structures above.

with a proper design. However, except some valleys at very low frequencies, losses are not competitive for the proposed design, which furthermore shows to propagate several high-order modes. Furthermore, the presence of the dielectric causes field pattern variations when the frequencies increase, with the mode tending to be concentrated inside the dielectric. Although the structure with more holes alleviates this effect, in general, it could be said that the proposed structure is closer to a porous fiber in propagation characteristics than a TWW. It should be pointed out, however, that further improvements may be possible using more complex structures involving dielectric or metallo-dielectric EBGs. This could be a nice line to research in.

With more experimental character, [530] has recently tested the TWW structure to transmit broadband pulses. The experimental setup consist on two wires of radius $a=125 \mu \mathrm{m}$, separated a distance $d=300 \mu \mathrm{m}$. The $20 \mathrm{~cm}$ long TWW is hold at both ends with a dielectric slab. They are obtained losses of $0.075 \mathrm{~dB} / \mathrm{mm}$ at $f=1 \mathrm{THz}$, which is a good figure for the proven broadband transmission $(0.1 \mathrm{THz}$ to $4 \mathrm{THz})$.

More recently, as occurs with many waveguides, the TWW has been also proposed to interact with graphene samples. In this case the considered separation is only $d=24 \mu \mathrm{m}$, thus being the desired effects, different from the aforementioned works. With such small separation, losses would result prohibitive. 


\subsubsection{Planar Surface and Plasmonic Waveguides}

As with the other kinds of waveguides, a planar version of the proposals is always found. Planar impenetrable surface waveguides came firstly with a plastic ribbon made of high-permitivity alumina $\left(\varepsilon_{r}=9.9, \tan \delta=2.6 \cdot 10^{-3}\right.$ [21]) ribbon proposed in 2005 by Yeh [531], see Fig. 2.90(a). In such a nice and complete work, surface waveguiding advantages, solution to possibles drawbacks, and comparison with other structures is well a addressed.

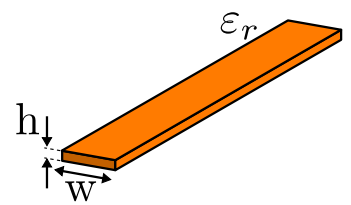

(a)

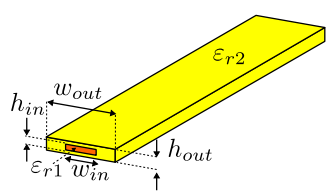

(d)

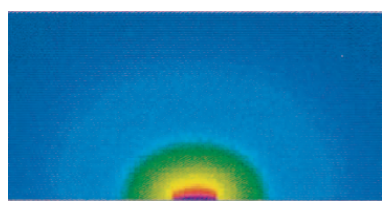

(b)

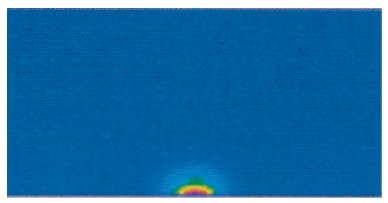

(e)

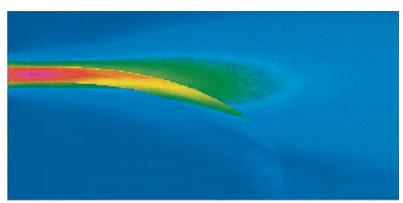

(c)

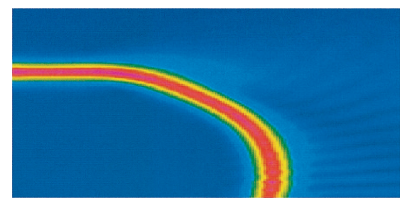

(f)

Figure 2.90: Planar surface waveguides at $\mathrm{THz}$ frequencies 1: high permittivity dielectric ribbon. a) $3 D$ schematic b) Cross section E-field magnitude distribution; c) E-field magnitude on a 90 degrees bend d)-f) Same as a)-c) for a low-permittivity coated dielectric ribbon. The confinement noticeably increases when the low permittivity coating is added to the ribbon.

By using a high permittivity dielectric, the surface waveguiding is more impenetrable, thus allowing strong field concentration in the outer air region, close to the dielectric interface. The effect is not as strong as with metal-air interfaces, but allowed to provide simulated losses a hundred times lower than other metallic and dielectric structures. Actually, simulations in the paper showed, at $f=1 \mathrm{THz}$, losses of several $\mathrm{dBs} / \mathrm{mm}$ for the microstrip line, of $0.1 \mathrm{~dB} / \mathrm{mm}$ for the RWG, $0.02 \mathrm{~dB} / \mathrm{mm}$ for the dielectric rod (propagation predominantly inside the dielectric region), but only $5 \cdot 10^{-4} \mathrm{~dB} / \mathrm{mm}$ for the proposed dielectric ribbon.

Furthermore, high power handling capability and easy active component integration should be affordable for this waveguide, since no metallic parts are involved. The authors suggested that the structure may be the base of ultra high-speed electronic $\mathrm{THz}$ circuits, and solve the problem of the bulky, fixed path, hardly scalable, and sometimes lossy, quasi-optical systems avoiding waveguides at that time.

The extreme low losses are achieved by using a ribbon with width $w=0.625 \lambda$ and height $h=0.0625 \lambda$, clearly subwavelength. The drawback of this configuration is that the field extent in the outer air region is huge and both, the attenuation dispersion and propagation dispersion, are noticeably high. As a consequence, excitation, radiation losses on bends, and signal distortion are 
important issues. As an example, Fig. 2.90(c) shows the E-field magnitude on a bend. As the ribbon starts curve its path, practically all the power is radiated, as it is only weakly attached to the surface. Furthermore, the concept of impedance gets loose for this kind of waveguides, thus being not possible apply the well-developed transmission line design theory and techniques.

Nevertheless, it must appreciated the thoughtful solutions proposed in this work. Firstly, the field extension and radiation on bends is afforded by using a low permittivity, relatively low-loss dielectric coating, see Fig. 2.90(d). A Teflon coating of thickness $t=\lambda / 4$ is applied over the ribbon, causing that the power distribution roughly became (ribbon: $5 \%$, Teflon: $85 \%$, air: 10\%). In such configuration, the energy is confined transversely in a much more compact area, see Fig. 2.90(e). This solves completely the radiation loss problem in bends. As can be appreciated in Fig. 2.90(f), when the coating is present, practically all the power reaches the end of the curve. Simulations indicate that only $0.2 \mathrm{~dB}$ would be lost in such bend.

The prize of the larger confinement is an increment of the losses in two orders of magnitude, originated by the absorption in the Teflon coating. This losses may be even larger in practice due to the optimistic model employed for the Teflon, and possible manufacturing issues. In this way, the waveguide is not longer lowloss. However, a very interesting solution is proposed: use the coating when it is strictly necessary. To achieve that, transitions between the bare ribbon and the coated ribbon are proposed. Three cases are studied: no transition, direct and inverted. The first, is rather a discontinuity, however, only $0.5 \mathrm{~dB}$ losses are reported for such a rough solution. Probably, this occurs for a single frequency, and is narrow-band. The authors suggest this configuration for filtering purposes, actually. The direct transition is a linear taper consisting in a coating that adiabatically gets thicker from the top surface of the ribbon. The loss is $0.3 \mathrm{~dB}$ in this case. Finally the inverted linear tapper consist on the vertical flip of the previous: a linear tapper growing downwards from the final top surface of the coating. This is the best case ( $0.2 \mathrm{~dB}$ losses $)$, however, it looks unpractical regarding manufacturing and robustness.

The coated ribbon results also useful in the excitation process. When this coating is present, a good matching can be achieved between a microstrip line and the coated ribbon by matching the microstip dielectric with the coating. Both, polarization and field distribution are very similar so that only 0.24 $\mathrm{dB}$ are lost by missmatch. This would be no longer possible if an uncoated ribbon was used, since any way to achieve good matching is possible: field concentration in the ribbon is very low, and field extension in the air is very large. Furthermore, the microstip substrate is of different permittivity, lower than the ribbon, larger than the air. In addition, the possibility of exciting the coated and the bare ribbon with a RWG is suggested, by means of proper tapers. It should be pointed out that the scheme is very different for each case, coated and uncoated.

The work lacks from experimental results and a more specific description of a practical use of the proposed designs. However, the main ideas are of great importance, which justifies the space dedicated to it. Through the proposed 
solutions it has been show how, with two designs, one showing great features for straight, long path propagation, and the other for excitation and propagation in curves, it possible to create high performance waveguide structures inheriting only the good characteristics. Unfortunately, continuation of the described work is not found, probably caused by the issues regarding the practical implementation of this waveguide, which, one must not forget, is very frequent-dependent due to its dielectric nature. Nevertheless, the ideas in this work has been seed on some designs proposed in the next chapter. The possibility of combining the properties of the SWW and the DCSWW through proper interconnection is a promising solution that has not been well exploited to date.

The more recent works commented now in this section are much more practical, but the results are not as flamboyant. Features of planar surface and plasmonic waveguides are not clearly overcoming the previous planar designs. In any case, some unique features are still found do to its particular nature.

In [532] a planar version version of the Goubau Line is proposed, i.e., a metal plate covered by a dielectric coating. The main work is focused in the design of transition between a CPW and the proposed transmission line by means of Vivaldi horn taper. The designed transition imposes $1.85 \mathrm{~dB}$ of insertion losses. A possible problem of the transition may be the lack of good mode matching between the waveguides, due tho polarization reasons. The planar GL itself exhibits losses as high as $0.4 \mathrm{~dB} / \mathrm{mm}$ at only $0.2 \mathrm{THz}$ in the experimental results, what questions the interest in this line in detriment of the conventional planar lines. Sharp 90 degrees bends are afforded with estimated losses of $5 \mathrm{~dB}$, however, dielectric losses are not taken into account. In general, it seems that the design, confining the power inside the dielectric, does not exploit the good features of surface waveguides. The same authors propose in [533] a similar design using two cascade linear tapers in the transition, see Fig. 2.91(a). In this work the transition losses are reduced to $1.25 \mathrm{~dB}$, and the propagation losses are shown to be $2 \mathrm{~dB} / \mathrm{mm}$ at $f=0.22 \mathrm{THz}$, better but still very high.

In [534], the Zenneck surface wave [496], i.e., a surface wave propagating on a metal sheet (the planar version of a SWW) is studied. In the past, this waveguide has generated much discussion, since proving its existent is very challenging. Whereas in the cylindrical geometry, the curvature causes certain confinement that can be even controlled and made larger though small wires, in the planar case the extension is extremely unpractical, and its almost impossible to distinguish it from a plane wave. The authors afford the issue using aluminum (actually a not good conductor in the $\mathrm{THz}$ band), to design a metallic ribbon of $10 \mathrm{~cm}$ wide and $51 \mu \mathrm{m}$ thick. The faces are smooth but not polished.

The excitation is made by means of a PPW, with the sheet attached to its bottom plate, a good way to provide good polarization matching. At $f=0.4$ $\mathrm{THz}$, the attenuation is $0.018 \mathrm{~dB} / \mathrm{mm}, 900$ times larger than predicted in theory, even using the Drude model. Probably some lateral spreading may be contributing to the losses. The authors point out that the Zenneck wave is 


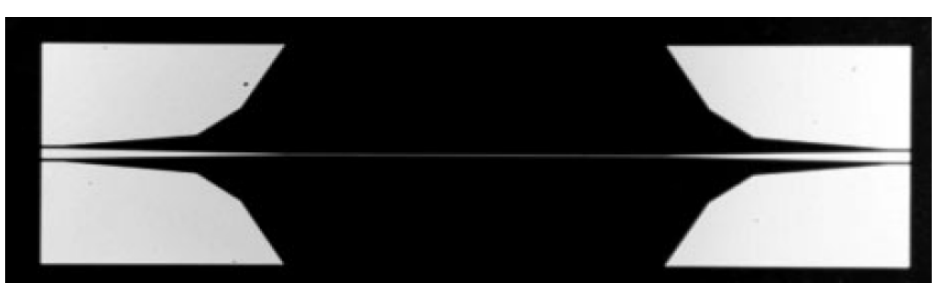

(a)

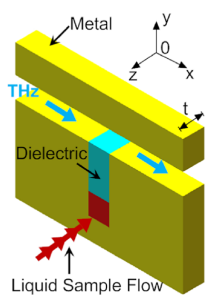

(b)

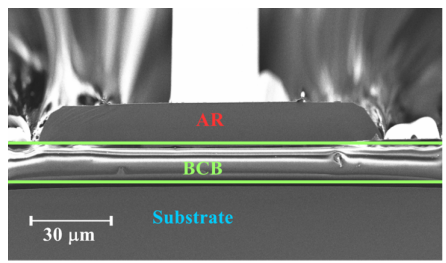

(c)

(x $\left.10^{-6} \mathrm{~m}\right)$

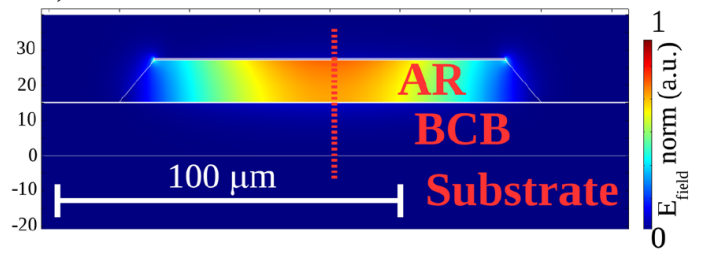

(d)

Figure 2.91: Planar surface waveguides at $\mathrm{THz}$ frequencies 2: practical implementations a) Photography of the top view of the device designed in [533] for the excitation of a planar GL and test the properties of this line at $\mathrm{THz}$ frequencies. It consists on a transition from a $\mathrm{CPW}$ to a planar GL, made by a cascade of two linear tapers; b)

difficult to establish since it is still of unpractical extent at $\mathrm{THz}$ frequencies. This will be better understood in the next chapter.

With similar intentions, in [535], it is proposed to add a thin coating to a metallic sheet in order to provide enough confinement for the Zenneck wave, in such way that simulations and experimental results agree, thus proving the existence of this mode and exhibiting design control over it. With a coating as thin as $\lambda / 50$, the field collapses up to 200 times. In the experimental test, a polyethilene coating of $t=12.5 \mu \mathrm{m}$ is displayed over an aluminum sheet of $10 \mathrm{~cm}$ wide per $100 \mu \mathrm{m}$ thick. The excitation is done by means of a PPW, adiabatically open until $1.2 \mathrm{~mm}$, however, it is pointed out that, if a very good match was required, the waveguide end should be opened until $15 \mathrm{~cm}$, which is an exaggerated distance for a practical implementation.

The experimental results are quite good since, at $f=1 \mathrm{THz}$, propagation losses are of $0.017 \mathrm{~dB} / \mathrm{mm}$, which split in $0.013 \mathrm{~dB} / \mathrm{mm}$ losses caused by the metal and $0.004 \mathrm{~dB} / \mathrm{mm}$ caused by the dielectric. The figure is quite competitive for a planar design. Regarding confinement, $65 \%$ of the field is concentrated under $1.5 \mathrm{~mm}$ from the sheet surface from $0.5 \mathrm{THz}$ to $1.5 \mathrm{THz}$. The figure is good if one compares it with a SWW, however, when talking about planar waveguides, it may imply losing the planar feature. The transmission line is also low dispersive since the velocity group varies between $0.93 \mathrm{c}$ and $0.99 \mathrm{c}$. However, the authors indicate that there are some other leaky modes which appear sometimes in both, numerical simulations and experiments. This issue is not treated deep but it seems could be a hidden threat for the performance 
of this waveguide. Finally, it is highlighted that grooves may be practiced to the sheet instead of using dielectric, however this solution is narrow-band whereas the dielectric coating offer broadband features. Sensing applications are foreseen for the proposed design, which is, at least, one to take into account.

The planar surface review end with two examples of application. In [536], a microfluidic sensor is designed by means of a plasmonic transmission line containing a stub through the liquid flows, see Fig. 2.91(b). Although the designed transmission line adopts a form similar to a PPW, the plasmonic effects of high field enhancement near the metal faces predominate due to the proximity of them. Actually, the main channel height is only $20 \mu \mathrm{m}$, and the stub is only $20 \mu \mathrm{m}$ wide, and $30 \mu \mathrm{m}$ deep, from which $20 \mu \mathrm{m}$ correspond to the dielectric and $10 \mu \mathrm{m}$ to the fluid under test. The Drude model is used together with transmission line theory, showing, at least in simulation, that the proposed structure is very suitable for identifying the signatures of samples in liquid, as for example cancer cells.

The second example regards to the work in [537], where a new design of a metalmetal plasmonic waveguide, including a special configuration of dielectric layers (see Figs. 2.91(c) and 2.91(d)) is incorporated in a QCL to improve the far-field emission. This planar design is only $125 \mu \mathrm{m}$ wide, and the vertical separation between the metal layers is about $15 \mu \mathrm{m}$. Again, BCB appears as a suitable dielectric exhibiting acceptable absorption. A $15 \mu \mathrm{m}$ layer of this substrate is displayed under the bottom plate, which is only $300 \mathrm{~nm}$ thick, thus being some field leakage through it. The frequency of operation is $f=2.9 \mathrm{THz}$. The authors highlight the powerful possibilities of combine metals and dielectrics in what they call hybrid plasmonic waveguides, which make a wide range of structural designs to be possible.

To close this deep review of $\mathrm{THz}$ waveguides, and given that many information and details have been provided, a schematic comparative among all the waveguides reviewed in this chapter is presented in table 2.3. In the table, waveguides are classified by groups, which are separated by a triple line, in the same way as it has been done in this chapter: Metallic (microwave); Dielectric (optic); Periodic/EBG; Surface/Plasmonic. For each group, bulk and planar subgroup designs are distinguished, separated by a double line. For each waveguide, the best reported performance according to the state of the art, if enough representative of the class, has been considered. The parameters taken into account have been: propagation losses $(\alpha)$; dispersion of propagation losses $(\alpha(f))$; dispersion $(\beta(f))$; single mode operation (SM); confinement (Cnf.); Manufacturing difficulty (Mfr.); and difficulty of use (Use). The scale used is: $5:=$ very good; 4:=good; $3:=$ fair; $2:=$ poor; $1:=$ very poor. To keep homogeneity, higher values mean better behavior referring to any parameter, i.e., for each parameter 5 is the closest to the ideal desired behavior. Although the results are displayed in a qualitative way for the easy of comprehension, most of them are based in careful quantitative comparison.

The table results quite helpful to synthesize information. In a compact form, this list summarizes the most relevant information regarding $\mathrm{THz}$ waveguides: 
- Metallic waveguides hardly offer good propagation losses (specially bad for planar cases) unless they operate under multimode regime, which complicate their use in practical environments. Manufacturing is challenging, but alleviated if the waveguide is overmoded. On the other hand, their attenuation is quite stable with the frequency, dispersion is acceptable, confinement is excellent and their use straightforward. The PPW waveguide is an exception providing a very good overall behavior except of the confinement problems due to the lateral spread of the guided waves.

- Dielectric waveguides are generally lossy unless high percentage of the power is transmitted through an (several) air region(s). The soft boundary conditions in these waveguides (compared to a metallic one) cause that dispersion in both, attenuation and propagation constant, to be specially high, and rather poor confinement. However, some topological configuration can improve some one of these features. Manufacturing process follows quite standard criteria and is straightforward except for complicated geometries. Their use presents medium difficulty.

- Periodic and EBG structures present low propagation losses and good confinement in general, but dispersion in both, attenuation and propagation constant, is appreciable due to multimode behavior except in the topology is forced to single mode operation. The structures are quite versatile and the information in the table might be showing only part of their possibilities. In must be highlighted that manufacturing difficulty may range from simple to very complex depending on the particular topology, which also conditions the difficulty of use, rather complex in general.

- Surface waveguides are able to achieve at the same time low loss, nondispersive behavior (including this single mode operation) and easy manufacturing. However, this performance is at the cost of very poor confinement and consequent difficulty of use, the largest among all waveguides. Dielectric or other mechanisms can be used to alleviate this problems at an appreciable increment of either losses or dispersion, or both.

From the above it can be said that metallic waveguides represent what is standard, but limited and does not contribute with specially good features for $\mathrm{THz}$; dielectric waveguides can be topologically engineered to perform well, but narrow-band; periodic/EBG structures offer a high versatility and may cover special niches of use; and surface waveguides are a very potential solution which exhibits at the same time, outstanding features and prohibitive disadvantages.

The state of the art of this part of the thesis, focused on the $\mathrm{THz}$ ends here. The detailed review has allow to understand the minor and key details that make this region different from both, microwave and optics. Step by step, the shape of the problem to be afforded has become more defined. At this point, it has been seen that $\mathrm{THz}$ applications rely many times on the use of broadband $\mathrm{THz}$ pulses. Therefore, for these cases, a low-loss, non-dispersive waveguide is necessary. From what has been reviewed and recently empathized in the $\mathrm{THz}$ waveguides comparative, the SWW results very suitable for such demanded features, being, furthermore, a simple structure. However, it has been found 
that excitation and radiation on bends are problems to be solve mandatorily for its practical use.

The DCSWW can be seen as the dual waveguide regarding these features, since it may enhance the excitation efficiency and reduce the bend losses. However, this waveguide in neither low-loss nor low-dispersive. In order to develop designs with each one of these two waveguides, or combine them when necessary to take advantage of the good features of each one, propagation characteristics of both structures must be known, and this implies a rigorous analysis. A lack of such a proper analysis is found along in the reviewed work, so this thesis aims to contribute to $\mathrm{THz}$ technology in this aspect. In the next chapter, a rigorous analysis of both structures will be presented, whilst a further chapter will introduce some proposals of combined use of them. Regarding to the previous table, this part of the thesis contributes, on the one hand, to give specific information to each performance parameter depending on the waveguide parameters and frequency of operation, studying deep the structures, and, on the other hand, to improve those features clearly limiting the enormous potential of these waveguides. 
Waveguide Image $\quad \begin{array}{lllllll}\alpha & \alpha(f) & \beta(f) & \text { SM } & \text { Cnf. } & \text { Mfr. Use }\end{array}$

\begin{tabular}{|c|c|c|c|c|c|c|c|c|}
\hline RWG & Ex & 3 & 4 & 3 & 5 & 5 & 1 & 5 \\
\hline CWG (Overmoded) & & 5 & 4 & 3 & 1 & 5 & 3 & 4 \\
\hline Coaxial & & 2 & 4 & 5 & 5 & 5 & 1 & 5 \\
\hline Microstrip & $\varepsilon_{r}$ & 1 & 3 & 4 & 4 & 4 & 2 & 4 \\
\hline CPW & & 2 & 3 & 4 & 4 & 3 & 2 & 4 \\
\hline PPW (TEM) & $\overline{\overline{\mid 1 \uparrow \uparrow \uparrow \uparrow \uparrow \uparrow 1 \uparrow}}$ & 4 & 5 & 5 & 5 & 3 & 5 & 4 \\
\hline $\mathrm{PPW}\left(\mathrm{TE}_{1}\right)$ & 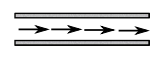 & 5 & 5 & 3 & 1 & 3 & 5 & 3 \\
\hline Solid fiber & & 2 & 2 & 1 & 4 & 2 & 5 & 3 \\
\hline Dielectric tube & & 3 & 1 & 1 & 1 & 3 & 4 & 3 \\
\hline
\end{tabular}

Porous fiber

$\begin{array}{lllllll}4 & 1 & 4 & 5 & 3 & 3 & 3\end{array}$

Dielectric slot

$4 \quad 2$

\begin{tabular}{ccccccccc}
\hline \hline PBG fiber & 4 & 1 & 2 & 4 & 3 & 2 & 3 \\
\hline CWG metal-diel & 5 & 2 & 3 & 1 & 5 & 1 & 2 \\
\hline RWG EBG-woodpile & 4 & 2 & 5 & 4 & 5 & 4 & 4 \\
\hline \hline Planar EBG & 4 & 2 & 5 & 4 & 5 & 4 & 4 \\
\hline \hline SWW & 5 & 5 & 5 & 5 & 1 & 5 & 2 \\
\hline DCSWW & 3 & 2 & 2 & 2 & 3 & 4 & 3 \\
\hline TWW & 4 & 5 & 5 & 2 & 3 & 4 & 4 \\
\hline \hline Dielectric ribbon & 5 & 1 & 1 & 5 & 1 & 5 & 2 \\
\hline Planar GL & 4 & 5 & 4 & 2 & 2 & 5 & 2 \\
\hline \hline
\end{tabular}

Table 2.3: Comparative of $\mathrm{THz}$ waveguides. The parameters taken into account for the comparative are: propagation losses $(\alpha)$; dispersion of propagation losses $(\alpha(f))$; dispersion $(\beta(f)$; single mode operation (SM); confinement (Cnf.); Manufacturing dificulty (Mfr.); and difficulty of use (Use). Scale: 5:=very good; 4:=good; 3:=fair; 2:=poor; 1:=very poor; To keep homogeneity, higher values mean better behavior referring to any parameter. 


\section{Chapter 3}

\section{Wire-type Waveguides}

\subsection{Surface Waveguide Theory:}

Surface waveguides are open waveguides that exhibit a waveguiding phenomena that could be described by the particular characteristic of that waves are railed over the surface rather than guided (in the sense of closed waveguides, in which all the energy is strongly guided along the desired path, travelling very confined in the interior of the waveguide). The variety of possible surfaces that provide this kind of mechanism (low permittivity dielectrics, high permittivity dielectrics, metals, metamaterials, etc.) cause a large variety in the grade that surface waveguiding is provided, ranging from the soft, sometimes called penetrable surface waveguiding, offered by low permittivity dielectrics, to the strong, sometimes called impenetrable surface waveguiding, offered by metal surfaces. This last case is also usually named as plasmonic waveguiding. In the penetrable surface case, depending on the electrical size of the waveguide, the waves are guided mainly in the external medium (electrically smaller waveguides) or confined in the internal medium (electrically larger waveguides), and the transition between both states is soft. In the case of impenetrable surfaces, the waves are guided more clearly attached to the surface, in the external medium, with very low penetration into the internal medium. In any case, these waveguides present a particular kind of electromagnetic problem, with its specific boundary conditions, solutions, propagation characteristics, etc. In this section, the main features of the surface waveguide theory are reviewed to provide the necessary theoretical foundation for the later treated analysis problems.

\subsubsection{Imperfect Dielectrics}

Dielectrics are mandatorily present in surface waveguide problems, since some boundary must be an open boundary, i.e, a change between two dielectric mediums, either because both of them are dielectrics or one ${ }^{1}$ of them is a metal modeled as a dielectric. Since the mathematical modelling of metals will have in the section $3.2 \mathrm{a}$ deeper review, now, only the dielectric materials formalisms are afforded.

\footnotetext{
${ }^{1}$ It is possible consider the interface between two metals modeled as dielectrics, however it lacks of interest in the scope of this thesis.
} 
Dielectrics are characterized by its dielectric permittivity $\varepsilon$, being usual the employment of the relative permittivity $\varepsilon_{r}=\frac{\varepsilon}{\varepsilon_{0}}$, which allows to compare them with the vacuum media $\left(\varepsilon=\varepsilon_{0}, \varepsilon_{r}=1\right)$. Losses at dielectrics occur by several reasons: material conductivity, relaxation phenomena, molecular resonances and molecular structure of the material [21]. Mathematically, these losses are introduced in the Maxwell equations via adding an imaginary term in the permittivity, so it becomes complex number with this form:

$$
\varepsilon=\varepsilon^{\prime}-j \varepsilon^{\prime \prime}
$$

The imaginary part the permittivity is always negative, otherwise gain would be obtained instead loss, what violates the energy conservation principle. In this way, the previous expression is chosen, having $\varepsilon^{\prime \prime}$ real and positive. The ratio between imaginary and real parts is known as loss tangent, with the following expression:

$$
\tan \delta=\frac{\varepsilon^{\prime \prime}}{\varepsilon^{\prime}}
$$

and therefore,

$$
\varepsilon=\varepsilon^{\prime}(1-j \tan \delta)
$$

At the microwave and millimeter-wave bands, it is considered that the main loss contribution is that coming from the finite conductivity of the dielectric material, $\sigma$, which is small but not null (as it is in the ideal case). Therefore, the imaginary part of the dielectric permittivity is assumed to be $\varepsilon^{\prime \prime}=\frac{\sigma}{\omega}$, and, according to (3.1) and (3.3), one has that

$$
\tan \delta=\frac{\sigma}{\omega \varepsilon^{\prime}}
$$

and

$$
\varepsilon=\varepsilon^{\prime}\left(1-j \frac{\sigma}{\omega \varepsilon^{\prime}}\right)
$$

From the previous expressions, it may be understood that the dielectric losses decrease with the frequency, however, the power dissipated in a lossy dielectric region is

$$
P_{L d}=\frac{\omega \varepsilon^{\prime \prime}}{2} \iiint_{v}|\vec{E}|^{2} \mathrm{~d} V
$$

where $V$ is the volume of the lossy dielectric. In the previous expression it can be appreciated that the term $\omega$ compensates that appearing in the denominator of (3.5). In fact, it is generaally found that both parameters $\varepsilon^{\prime}$ and $\varepsilon^{\prime \prime}$ are relatively stable with the frequency inside a certain band. This means that common dielectric materials experience an increase of its absorption, that compensates the predicted decrement of the losses term $\varepsilon^{\prime \prime}$ with the frequency.

Indeed, as the decades are expanded from microwaves to the $\mathrm{THz}$ band, the model of dielectrics, obtained experimentally, generally implies a higher $\tan \delta$. Since losses 
inside a dielectric medium grow with the frequency according to (3.6), in the cases studied in this thesis, an increment of frequency will always produces a noticeable increment of dielectric losses, given by both terms in (3.6), $\omega$ and $\varepsilon^{\prime \prime}$. This is the mathematical point of view of the high losses experienced at $\mathrm{THz}$ frequencies.

\subsubsection{Boundary Conditions}

In this section the possible boundary conditions that can appear in a surface waveguide problem are formally treated. These are boundary conditions regarding the interface between two mediums, and the radiation condition, necessary by the open nature of the structure, [21], [538].

\subsubsection{Boundary at the interface between two mediums}

In the problems treated in this thesis the following two cases appear:

1. Both mediums are dielectrics.

2. The medium 1 is a perfect conductor and the medium 2 is a dielectric.

A third condition may be considered, regarding to the case of having one dielectric medium and an imperfect metal medium, applying the particular surface impedance of the medium under question [21]. However, this a simplification of the case 1, when one of the dielectrics is really an imperfect conductor modeled with its equivalent complex permittivity. Therefore, only the two enumerated cases will be described. With the first one, they can be afforded all the boundary conditions involved in the analysis of the SWW and in all the simpler cases considered before (dielectric cylinders with $\varepsilon_{r}$ of a variety of kinds), as well as the boundary existent between the dielectric and the air external medium that is found in the DCSWW. The second case, appears exclusively in the treatment of the metallic cylinder present in the DCSWW, and its use will facilitate in a great extent the analysis of this structure, more complex geometrically.

\section{Both mediums are dielectrics.}

To understand the boundary conditions to which the quantities $\vec{E}, \vec{D}, \vec{H}$ y $\vec{B}$ are subjected for the first case, three scenarios are analyzed.

\section{- Scenario A}

The scenario A is represented in Fig. 3.1. Two rectangular parallel surfaces have been located, being each one a specular image from the other one. The upper rectangle is in the medium 1, whereas the lower rectangle is in the medium 2. The sides of each rectangle are $\Delta s_{1}$ and $\Delta s_{2}$. The vectors $\hat{x}, \hat{y}$ and $\hat{z}$ are the three unitary vectors in the $x, y$ and $z$ directions, respectively. $P$ is the plane that separates both mediums.

Maxwell equations are: 


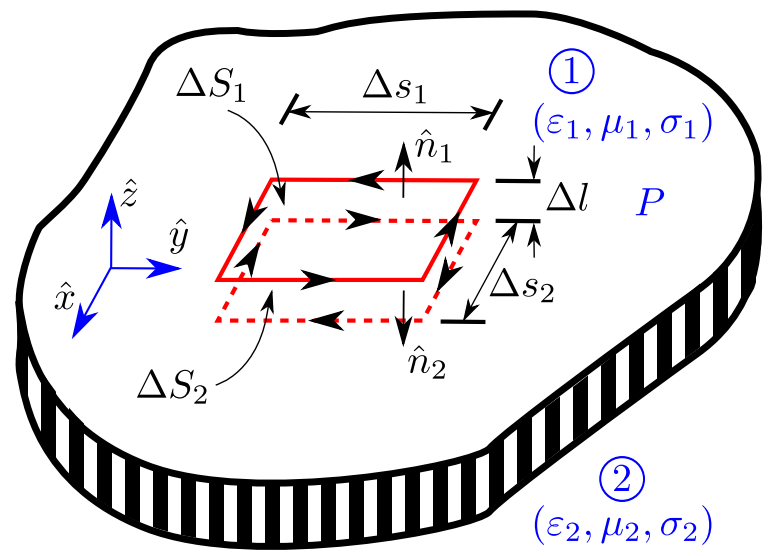

Figure 3.1: Geometry of scenario A. This scenario shows the relationship between the tangential components of the electric field with the normal component of the magnetic flux, as well as the relationship between the tangential components of the magnetic field with the normal component of the electric displacement vector when a transition between two dielectric mediums occur. The rectangular area $\Delta S_{1}$ is parallel to the rectangular area $\Delta S_{2}$. The sides of each rectangle are $\Delta s_{1}$ and $\Delta s_{2}$, and the separation between them is $\Delta l . P$ is the interface between medium 1 and medium 2 .

$$
\begin{aligned}
\nabla \times \vec{E}(r, t) & =-\frac{\partial \vec{B}(r, t)}{\partial t} \\
\nabla \times \vec{H}(r, t) & =\vec{J}(r, t)+\frac{\partial \vec{D}(r, t)}{\partial t} \\
\nabla \cdot \vec{B}(r, t) & =0 \\
\nabla \cdot \vec{D}(r, t) & =\rho(r, t)
\end{aligned}
$$

Integrating (3.7) on the rectangular area $\Delta S_{1}$ of region 1 :

$$
\int_{\Delta S_{1}}\left(\nabla \times \vec{E}_{1}\right) \cdot \hat{n}_{1} \mathrm{~d} S=-\frac{\partial}{\partial t}\left(\int_{\Delta S_{1}} \vec{B}_{1} \cdot \hat{n}_{1} \mathrm{~d} S\right)
$$

whereas that the same equation is integarted on the rectangular area $\Delta S_{2}$ of region 2:

$$
\int_{\Delta S_{2}}\left(\nabla \times \vec{E}_{2}\right) \cdot \hat{n}_{2} \mathrm{~d} S=-\frac{\partial}{\partial t}\left(\int_{\Delta S_{2}} \vec{B}_{2} \cdot \hat{n}_{2} \mathrm{~d} S\right)
$$

Applying now the Stokes Theorem to (3.11) and (3.12), and adding the result, it is obtained:

$$
\int_{c_{1}} \vec{E}_{1} \cdot \hat{s}_{1} \mathrm{~d} s_{1}+\int_{c_{2}} \vec{E}_{2} \cdot \hat{s}_{2} \mathrm{~d} s_{2}=-\left[\frac{\partial}{\partial t}\left(\int_{\Delta S_{1}} \vec{B}_{1} \cdot \hat{n}_{1} \mathrm{~d} S\right)+\frac{\partial}{\partial t}\left(\int_{\Delta S_{2}} \vec{B}_{2} \cdot \hat{n}_{2} \mathrm{~d} S\right)\right]
$$


where $c_{1}$ and $c_{2}$ are, respectively, the curves that enclose the areas $\Delta S_{1}$ and $\Delta S_{2}$. In rectangular coordinates, if $\Delta s_{1} \rightarrow 0$ and $\Delta s_{2} \rightarrow 0, \vec{E}$ has a constant value at each side. Now, if the separation between both rectangular surfaces tends to 0 , it is obtained

$$
\left(E_{1 x}-E_{2 x}\right) 2 \Delta x+\left(E_{1 y}-E_{2 y}\right) 2 \Delta y=-\frac{\partial}{\partial t}\left(B_{1 z}-B_{2 z}\right) \Delta x \Delta y
$$

where the following assignments have taken place:

$$
\begin{array}{r}
\Delta s_{1}=\Delta x \\
\Delta s_{2}=\Delta y \\
\hat{s}_{1}=\hat{x} \\
\hat{s}_{2}=\hat{y} \\
\hat{n}_{1}=-\hat{n}_{2}=\hat{n}=\hat{z}
\end{array}
$$

with the subindex of vectors $\vec{E}$ and $\vec{B}$ indicates the corresponding field component and the medium.

In a similar way, following the relation (3.8) for the tangential components of $\vec{H}$ and the normal component of $\vec{D}$ on the surface $P$ :

$$
\begin{aligned}
& \left(H_{1 x}-H_{2 x}\right) 2 \Delta x+\left(H_{1 y}-H_{2 y}\right) 2 \Delta y \\
& \quad=\frac{\partial}{\partial t}\left(D_{1 z}-D_{2 z}\right) \Delta x \Delta y+\left(J_{1 z}-J_{2 z}\right) \Delta x \Delta y
\end{aligned}
$$

The expressions (3.14) and (3.20) collect the conclusions from this first scenario and their implications will be treated after the description of the other two scenarios.

\section{- Scenario B}

Consider now a rectangular path $c$ like it is described in Fig. 3.2, crossing the interface between two dielectric media, $P$. The two sides $\Delta s$ of this rectangle are parallel to $P$ and the separation between them (or the short side of this rectangle) is $\Delta l$, being the rectangle area $\Delta S$. The unity vector $\hat{n}_{0}$ is normal to the described rectangle, and the vectors $\vec{s}_{1}$ and $\vec{s}_{2}$ are parallel to the surface $P$, and orthogonal to $\hat{n}_{0}$ and $\hat{n}$, with $\hat{n}$ the unity vector orthogonal to the surface $P$. In addition, $\vec{s}_{1}=-\vec{s}_{2}, \hat{s}_{1}=\hat{n}_{0} \times \hat{n}$, and $\hat{s}_{2}=-\hat{n}_{0} \times \hat{n}$. Integrating (3.7) over the rectangular area,

$$
\int_{\Delta S}(\nabla \times \vec{E}) \cdot \hat{n}_{0} \mathrm{~d} S=-\frac{\partial}{\partial t}\left(\int_{\Delta S} \vec{B} \cdot \hat{n}_{0} \mathrm{~d} S\right)
$$




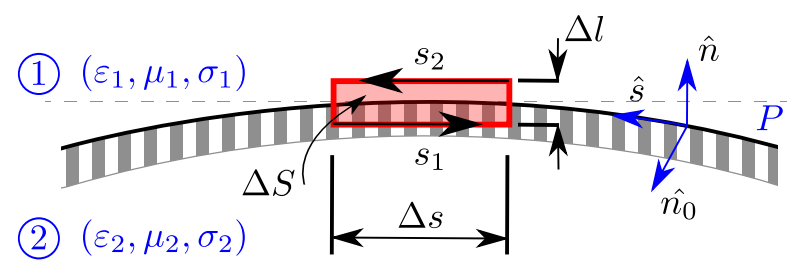

Figure 3.2: Geometry of the scenario B, showing the continuity of the tangential electric and magnetic fields across a surface $P$, between two dielectric mediums.

and by the Stokes Theorem:

$$
\int_{c_{1}} \vec{E} \cdot \hat{s} \mathrm{~d} s=-\frac{\partial}{\partial t}\left(\int_{\Delta S} \vec{B} \cdot \hat{n}_{0} \mathrm{~d} S\right)
$$

If in this last expressions it is considered a very small rectangle, such that $\Delta s \rightarrow 0$ y $\Delta l \rightarrow 0$, then $\vec{E}$ has a constant value at each side of the surface, therefore

$$
\begin{aligned}
\left(\hat{s}_{1} \cdot \vec{E}_{1}+\hat{s}_{2} \cdot \vec{E}_{2}\right) \Delta s+A & =-\frac{\partial \vec{B}}{\partial t} \cdot \hat{n}_{0} \Delta s \\
& =-\frac{\partial \vec{B}}{\partial t} \cdot \hat{n}_{0} \Delta s \Delta l
\end{aligned}
$$

where $A$ is the contribution of the rectangle small sides. Taking the limit $\Delta s \rightarrow 0 \mathrm{y} \Delta l \rightarrow 0$, it is found that

$$
\left(\hat{n}_{0} \times \hat{n} \cdot \vec{E}_{1}-\hat{n}_{0} \times \hat{n} \cdot \vec{E}_{2}\right)=-\lim _{\Delta l \rightarrow 0}\left(\frac{\partial \vec{B}}{\partial t} \cdot \hat{n}_{0} \Delta l\right)
$$

that can be expressed as

$$
\hat{n}_{0} \cdot\left[\hat{n} \times\left(\vec{E}_{1}-\vec{E}_{2}\right)\right]=\lim _{\Delta l \rightarrow 0}\left(\hat{n}_{0} \cdot \frac{\partial \vec{B}}{\partial t}\right) \Delta l
$$

Since in (3.23) $A \rightarrow 0$ when $\Delta l \rightarrow 0$, it is finally obtained that.

$$
\hat{n} \times\left(\vec{E}_{1}-\vec{E}_{2}\right)=0
$$

Applying an analogous procedure for the equation (3.8) it is concluded that

$$
\hat{n} \times\left(\vec{H}_{1}-\vec{H}_{2}\right)=\vec{J}_{s}
$$

where $\vec{J}_{s}$ is the surface current density at the boundary surface. It has been shown that the tangential components of the electric and magnetic fields must 
be continuous across the dielectric boundary. That these boundary conditions on the tangential components of the electric and magnetic fields at the boundary of two dissimilar dielectric media are necessary and sufficient boundary condition will be shown later.

\section{- Scenario $\mathrm{C}$}

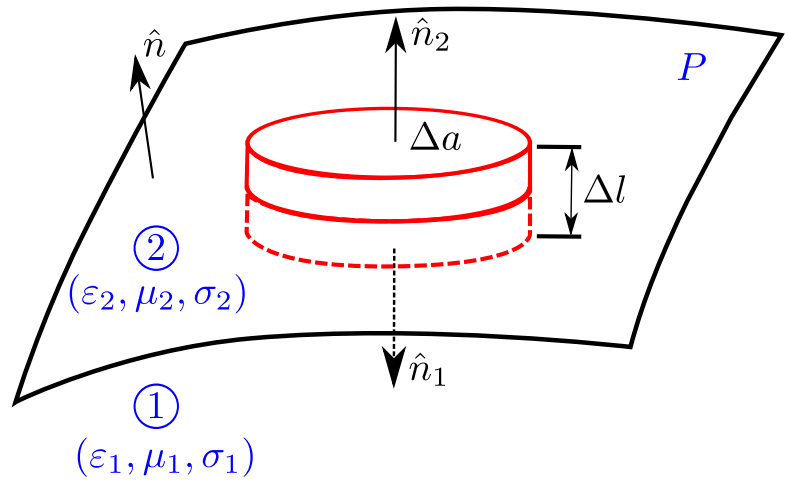

Figure 3.3: Geometry of the scenario C. By means of this scenario they are shown the continuity of the normal components of the magnetic flux and the displacement vector across a surface $P$ separating two dielectric mediums.

This scenario consist in a small cylinder, with height $\Delta l$, and having its top and bottom faces an area $\Delta a$. The cylinder is crossing a interface $S$ between the medium 1 and the medium 2, as it is shown in Fig. 3.3. The vectors $\hat{n}_{1}$ y $\hat{n}_{2}$ are normal to the cylinder top and bottom faces, hence normal to the surface $S$. The volume of the cylinder is $\Delta V$. If the equation (3.9) is integrated over that close volume, it is found that

$$
\int_{\Delta V} \nabla \cdot \vec{B} \mathrm{~d} V=0
$$

and by applying the Divergence Theorem

$$
\int_{\Delta A_{c}} \vec{B} \cdot \hat{n} \mathrm{~d} S=0
$$

where the integration area $\Delta A_{c}$ represents the total area of the considered cylinder. If such cylinder is made very small, by doing $\Delta a \rightarrow 0$ y $\Delta l \rightarrow 0$, then $\vec{B}$ will have a constant value for each of the implied surfaces. Therefore, proceeding with (3.29) it is found that

$$
\left(\hat{n}_{1} \cdot \vec{B}_{1}+\hat{n}_{2} \cdot \vec{B}_{2}\right) \Delta a+A=0
$$

where $A$ is the contribution of the lateral face of the cylinder to the integral. Since $\hat{n}_{2}=-\hat{n}_{1}=-\hat{n}$, in the limit, (3.30) becomes 


$$
\left(\vec{B}_{1}-\vec{B}_{2}\right) \cdot \hat{n}=0
$$

The same treatment of (3.10) gives

$$
\left(\vec{D}_{1}-\vec{D}_{2}\right) \cdot \hat{n}=\rho_{s}
$$

where $\rho_{s}$ is the surface charge density at the interface between the two mediums. These equations indicate that, across the interface between two different mediums, the normal component of $\vec{B}$ must be continuous, and the normal component of $\vec{D}$ must be discontinuous due to the surface charge density. That these conditions are necessary, but not sufficient is shown next.

Along the discussion carried out with the scenarios A, B and C, the following six boundary conditions have been obtained for an interface between two dielectric media:

$$
\begin{gathered}
\left(E_{1 x}-E_{2 x}\right) 2 \Delta x+\left(E_{1 y}-E_{2 y}\right) 2 \Delta y=-\frac{\partial}{\partial t}\left(B_{1 z}-B_{2 z}\right) \Delta x \Delta y \\
\left(H_{1 x}-H_{2 x}\right) 2 \Delta x+\left(H_{1 y}-H_{2 y}\right) 2 \Delta y \\
=\frac{\partial}{\partial t}\left(D_{1 z}-D_{2 z}\right) \Delta x \Delta y+\left(J_{1 z}-J_{2 z}\right) \Delta x \Delta y \\
\hat{n} \times\left(\vec{E}_{1}-\vec{E}_{2}\right)=0 \\
\hat{n} \times\left(\vec{H}_{1}-\vec{H}_{2}\right)=\vec{J}_{s} \\
\left(\vec{B}_{1}-\vec{B}_{2}\right) \cdot \hat{n}=0 \\
\left(\vec{D}_{1}-\vec{D}_{2}\right) \cdot \hat{n}=\rho_{s}
\end{gathered}
$$

Let consider the expressions (3.33) and (3.34), for a problem with harmonic variation, described by a pulsation $\omega=2 \pi f$. In this case, the temporal derivatives $\partial / \partial t$ may be replaced by $j \omega$, adopting the problem in this way complex phasorial expressions independent of the time for the fields. Under such situation, (3.33) and (3.34) become

$$
\left(E_{1 x}-E_{2 x}\right) 2 \Delta x+\left(E_{1 y}-E_{2 y}\right) 2 \Delta y=-j \omega\left(B_{1 z}-B_{2 z}\right) \Delta x \Delta y
$$

and 


$$
\begin{aligned}
& \left(H_{1 x}-H_{2 x}\right) 2 \Delta x+\left(H_{1 y}-H_{2 y}\right) 2 \Delta y \\
& \quad=j \omega\left(D_{1 z}-D_{2 z}\right) \Delta x \Delta y+\left(J_{1 z}-J_{2 z}\right) \Delta x \Delta y
\end{aligned}
$$

These equations, must be satisfied at the interface between both dielectric mediums. The equation (3.39) shows that if the boundary condition (3.35), of continuity of the tangential electric field is satisfied, i.e., $E_{1 x}=E_{2 x} \mathrm{y}$ $E_{1 y}=E_{2 y}$, then the left term vanishes. Since $\omega \neq 0$ and $\Delta x \Delta y \neq 0$, it is mandatory that $B_{1 z}=B_{2 z}$, hence being satisfied the boundary condition on the normal component of $\vec{B}$, given by (3.37). However, the fact that the boundary condition on the normal component of $\vec{B}\left(B_{1 z}=B_{2 z}\right)$, given by (3.31), is satisfied, only means that:

$$
\left(E_{1 x}-E_{2 x}\right) 2 \Delta x+\left(E_{1 y}-E_{2 y}\right) 2 \Delta y=0
$$

so it is not possible to conclude that (3.35) is satisfied, since (3.41) implies that the sum of the terms $\left(E_{1 x}-E_{2 x}\right) 2 \Delta x$ and $\left(E_{1 y}-E_{2 y}\right) 2 \Delta y$ must be null, but each term needs not to be null.

With an analogous procedure for equation (3.40) it is concluded that, if the boundary condition of the magnetic field tangential components, given by (3.36), is satisfied, then the boundary condition on the normal component of $\vec{D}$, given by (3.38), is automatically satisfied. However, the reciprocal is not true.

Therefore, in order to satisfy the boundary conditions on a interface between two dielectric mediums it is necessary that the boundary conditions on the tangential components of the electromagnetic fields are satisfied. This is, the tangential electric field must be continuous, and the magnetic field must be continuous except in the case that surface currents appear in such interface, an special case that not appears in the problems under study in this thesis. In this way, the necessary and sufficient boundary conditions for the case 1 are proven.

It must be pointed out that any constitutive relationship between $\vec{E}, \vec{D}, \vec{H}$ and $\vec{B}$, through $\mu$ and $\varepsilon$ has been used. Therefore, the enunciated principles are valid for mediums of isotropic or anisotropic nature, dispersive or not dispersive, in movement or stationary, linear or non-linear, and for any complex value of $\mu$ and $\varepsilon$. Therefore, the conclusions are lawfully applicable to an interface between a metal modeled as a dielectric, and a dielectric, which is one of the case of interest in this thesis.

\section{The medium 1 is a perfect conductor and the medium 2 is a dielec- tric.}

Maxwell equations state that electromagnetic fields cannot exist inside a perfect conductor, named here as medium or region 1 . The potential on a perfect electric 
conductor is a constant, therefore, no tangential electric field can exist on the surface of that perfect conductor, whilst, by the Ampere's Law, given by (3.8), the tangential magnetic field must be equal to the surface current density and must be in a direction normal to that surface current. All the electric field lines must terminate normally on the perfectly conducting surface. Hence, the boundary conditions are:

$$
\begin{aligned}
\hat{n} \times \overrightarrow{E_{1}} & =0 \\
\hat{n} \times \overrightarrow{H_{1}} & =\overrightarrow{J_{s}} \\
\hat{n} \cdot \overrightarrow{B_{1}} & =0 \\
\hat{n} \cdot \overrightarrow{D_{1}} & =\rho_{s}
\end{aligned}
$$

where $J_{s}$ is the total electric surface current density, which consists of the induced surface current density due to an incident electromagnetic field in medium 1 and any other existing impressed currents due to presence of sources, and $\rho_{s}$ is the surface charge density.

\subsubsection{Radiation condition}

The field associated to a finite distribution of sources, or the field scattered from obstacles must satisfy some conditions at infinity, which are related to the finiteness of the energy radiated by the sources or scattered by obstacles, as well as the assurance that the field at infinity represents an outgoing wave. This is, the radiation condition requires that:

1. For a finite three-dimensional (3D) the field intensities must vanish at infinity such that $\left(r^{2}|\vec{E}|\right)$ and $\left(r^{2}|\vec{H}|\right)$ are bounded when $r \rightarrow \infty$, where $r$ is the 3D radial distance from the origin.

2. For a finite two-dimensional (2D), the field intensities must vanish at infinity such that $(r|\vec{E}|)$ and $(r|\vec{H}|)$ are bounded when $r \rightarrow \infty$, where $r$ is the distance $2 \mathrm{D}$ radial distance from the origin.

3. The electromagnetic wave represented by $\vec{E}$ y $\vec{H}$ must behave as an outgoing divergent traveling wave at great distances from the source.

The finiteness of the energy radiated by the source is assured by the condition 1 for a $3 \mathrm{D}$ source, and by the condition 2 for a $2 \mathrm{D}$ source. For a time-periodic field in a homogeneous medium the $3 \mathrm{D}$ radiation condition at infinity takes the form [21]:

$$
\begin{aligned}
\lim _{r \rightarrow \infty} r\left[\vec{H}-\left(\frac{\varepsilon_{0}}{\mu_{0}}\right)^{1 / 2}(\hat{r} \times E)\right] & =0 \\
\lim _{r \rightarrow \infty} r \vec{E} & =C
\end{aligned}
$$

where $r$ is the radial distance from an arbitrary origin in the neighborhood of the sources, $\hat{r}$ is a unit vector directed from the origin in the radial direction, and 
$C$ is a constant. In the cases studied in this thesis, which involve cylindrical surface waveguides, i. e., a $2 \mathrm{D}$ electromagnetic problem in an infinite region, the $2 \mathrm{D}$ radiation conditions will result determinant in order to choose the appropriate solution in the external infinite medium.

\subsubsection{Debye potentials and scalar wave equation}

If Maxwell equations are manipulated in the context of a problem of harmonic variation, free of sources, by using the constitutive parameters $\mu$ y $\varepsilon$, they are easily found the well-known vectorial wave equations for the electric and magnetic fields:

$$
\begin{aligned}
\nabla \times \nabla \times \vec{E}(r)-\omega^{2} \mu \varepsilon \vec{E}(r) & =0 \\
\nabla \times \nabla \times \vec{H}(r)-\omega^{2} \mu \varepsilon \vec{H}(r) & =0
\end{aligned}
$$

These equations imply a quite high complexity in their resolution due to their vectorial nature. In the case of closed metallic waveguides, involving only an inner finite medium, TE and TM modes are totally determined by their unique not null axial component of the field, magnetic for the TE modes, electric for the TM modes. In the case of two $^{2}$ conductor waveguides, the TEM mode appears, and the procedure is even simpler, since both the axial components, electric and magnetic, are null, and it is possible to proceed by using Laplace equation from a potential $V$, that satisfies $\nabla_{t} V=\vec{E}$. Even very complex geometries can be easily solved in this case by the use of conformal mapping [528].

In this section, it is pretended to give a formal approach to this procedure in the general case of having the all 6 components from the electromagnetic field not null. This will be done for the most general case of arbitrary curvilinear coordinates, considering all type of possible modes. From this point, particularizations and simplifications will be introduced later in this thesis when to the problems to be afforded require treatment. In order to do this, the Debye potentials [539], $\Psi(r)$ y $\Phi(r)$, are introduced now:

$$
\begin{aligned}
& E(r)=\nabla \times(\hat{a} \Psi(r))-(j / \omega \varepsilon) \nabla \times \nabla \times(\hat{a} \Phi(r)) \\
& H(r)=\nabla \times(\hat{a} \Phi(r))-(j / \omega \mu) \nabla \times \nabla \times(\hat{a} \Psi(r))
\end{aligned}
$$

where $\hat{a}$ is the unity vector in the direction of the position vector $\vec{r}$. For example, in spherical coordinates $\hat{a}=\hat{r}$, in cylindrical coordinates $\hat{a}=\hat{z}$, the axial vector, and in rectangular coordinates $\hat{a}=\hat{x}, \hat{a}=\hat{y}$ or $\hat{a}=\hat{z}$, depending on which choice is more suitable for the problem to be solved. Since the divergence of a curl is null, $\nabla \cdot(\nabla \times \vec{A})=0$, if the divergence operator is applied to (3.50) and (3.51), it is found that $\nabla \cdot \vec{E}=0$ and $\nabla \cdot \vec{H}=0$. This means that the representation of $\vec{E}$ and $\vec{H}$ by the Debye potentials satisfy the divergence equations for the electric and magnetic field. The two scalar functions $\Psi(r)$ and $\Phi(r)$ satisfy a pair differential equations of second order, obtained by substituing (3.50) and (3.51) in the vectorial

\footnotetext{
${ }^{2}$ In general they will appear $N-1$ TEM modes if the waveguide structure present $N$ independent conductors [376].
} 
wave equations (3.48) and (3.49), respectively. Therefore, for a homogenous and isotropic medium, $\Psi(r)$ y $\Phi(r)$ satisfy the Helmholz scalar equation:

$$
\begin{aligned}
& \left(\nabla^{2}+k^{2}\right) \Psi(r)=0 \\
& \left(\nabla^{2}+k^{2}\right) \Phi(r)=0
\end{aligned}
$$

with $k^{2}=\omega^{2} \mu \varepsilon$. This means that the use of the Debye potentials reduce the two vectorial wave equations, given by (3.48) and (3.49), to two escalar equations given by (3.52) and (3.53). Since the solution of the Helmholtz scalar equation is known for a large variety of cases, it is possible to found for all of them the solution to the vectorial wave equation through (3.50) and (3.51). Furthermore, since the vectorial wave equations for homogeneous and isotropic mediums are linear, by means of the superposition theorem, it is possible to obtain the complete solution of the electromagnetic fields from (3.50) and (3.51). This means that these equations represent the complete electromagnetic solution in a simple medium by means of the Debye potentials $\Psi(r)$ and $\Phi(r)$.

From a historical point of view, it is possible to identify the vector $\hat{a} \Phi(r)$ ) as the electrical Hertz vector, and the $\hat{a} \Psi(r)$ ) vector as the magnetic Hertz vector, where $\hat{a}$ is the constant unitary vector in both cases [21]. When $\hat{a}$ is taken as the unitary vector in the direction $\hat{z}$, then, the Hertz vectors are $\hat{z} \Phi(r)$ and $\hat{z} \Psi(r)$ for the electric and magnetic type, respectively. According to (3.50) and (3.51), all the electromagnetic field components can be known from the axial Hertz vectors, $\hat{z} \Phi(r)$ y $\hat{z} \Psi(r)$. Therefore, it is only necessary to obtain $\Phi(r)$ and $\Psi(r)$ from the scalar wave equations, (3.52) and (3.53), in order to obtain all the field components.

As commented before, the linearity of the electromagnetic fields in a simple medium allows the use of the superposition theorem in order to obtain the complete electromagnetic fields from knowing only some of their components. Hence, depending on the nature of the known components of the field, it is possible to distinguish between several basic kinds of waves:

- Transversal Electromagnetic (TEM) Waves. This kind of wave has not longitudinal components, neither electric nor magnetic. $E_{z}$ and $H_{z}$ are null if $\hat{z}$ is the propagation direction. This meas that $\Phi(r)=0$ and $\Psi(r)=0$.

- Transversal Magnetic (TM) waves. This kind of waves has longitudinal electric field component, but has not longitudinal magnetic field component. $H_{z}$ is null if $\hat{z}$ is the propagation direction. Therefore, $\Psi(r)=0$.

- Transversal Electric (TE) waves. This kind of waves has longitudinal magnetic field component, but has not longitudinal electric field component. $E_{z}$ is null if $\hat{z}$ is the propagation direction. Therefore, $\Phi(r)=0$.

- Hybrid waves (HE or EH). This kind of wave has all the components of electric and magnetic fields. These waves are a linear superposition of TE and TM waves, therefore $\Phi(r) \neq 0$ and $\Psi(r) \neq 0$. Generally, it is adopted the HE notation when $H_{z}$ predominates over $E_{z}$ (TE wave predominates), and $\mathrm{EH}$ in the contrary case, [376], [540]. However, this notation is ambiguous in some 
problems or contexts [541]. In the cases treated in this thesis, it will be shown that some remarks are required regarding to this issue.

The possibility of separating the totally of the fields in partial fields and particular types of waves that satisfy the wave equation, allows to obtain a direct way to use the scalar wave equation for the components of the field in the problem of waveguided propagation with harmonic conditions, by means of the Maxwell equations, and without the necessity of using direct the Debye potentials or the Hertz vectors. When the guided modes propagate along a perfect path, totally straight in the axial direction, it can be assumed that each field of the electromagnetic wave can be represented by the form $f(u, v) e^{-j \beta z+j \omega t}$, where $\hat{z}$ has been chosen as the propagation direction, $\beta$ is the propagation constant, and $u, v$ are generalized orthogonal coordinates in the plane transversal to the propagation direction. In the following steps, the factor $e^{j(\omega t-\beta z)}$ will be omitted since it appears in all the field components. Maxwell equations for a harmonic field variation in a isotropic and homogenous medium $(\mu, \varepsilon)$ free of sources are, by components [21]:

$$
\begin{aligned}
\frac{1}{h_{2}}\left[\frac{\partial E_{z}}{\partial v}+j \beta h_{2} E_{v}\right]+j \omega \mu H_{u} & =0 \\
\frac{1}{h_{1}}\left[-j \beta h_{1} E_{u}-\frac{\partial E_{z}}{\partial u}\right]+j \omega \mu H_{v} & =0 \\
\frac{1}{h_{1} h_{2}}\left[\frac{\partial h_{2} E_{v}}{\partial u}-\frac{\partial h_{1} E_{u}}{\partial v}\right]+j \omega \mu H_{z} & =0 \\
\frac{1}{h_{2}}\left[\frac{\partial H_{z}}{\partial v}+j \beta h_{2} H_{v}\right]-j \omega \varepsilon E_{u} & =0 \\
\frac{1}{h_{1}}\left[-j \beta h_{1} H_{u}-\frac{\partial H_{z}}{\partial u}\right]+j \omega \varepsilon E_{v} & =0 \\
\frac{1}{h_{1} h_{2}}\left[\frac{\partial h_{2} H_{v}}{\partial u}-\frac{\partial h_{1} H_{u}}{\partial v}\right]-j \omega \varepsilon E_{z} & =0
\end{aligned}
$$

where $h_{1}$ and $h_{2}$ are the curvilinear coefficients of the coordinate system employed. If (3.55) and (3.57) are combined, and solved for $E_{u}$ and $H_{v}$ in terms of $\partial E_{z} / \partial u$ and $\partial H_{z} / \partial v$, they are obtained the expressions (3.60) and (3.63). On the other hand, by combining (3.54) and (3.58) and solving $E_{v}$ and $H_{u}$ in terms of $\partial E_{z} / \partial v$ and $\partial H_{z} / \partial u$ the expressions (3.61) and (3.62) are found. These results are shown below:

$$
\begin{aligned}
E_{u} & =\frac{1}{p^{2}}\left(\frac{j \beta}{h_{1}} \frac{\partial E_{z}}{\partial u}-\frac{j \omega \mu}{h_{2}} \frac{\partial H_{z}}{\partial v}\right) \\
E_{v} & =\frac{1}{p^{2}}\left(-\frac{j \beta}{h_{2}} \frac{\partial E_{z}}{\partial v}+\frac{j \omega \mu}{h_{1}} \frac{\partial H_{z}}{\partial u}\right) \\
H_{u} & =\frac{1}{p^{2}}\left(\frac{j \omega \varepsilon}{h_{2}} \frac{\partial E_{z}}{\partial v}-\frac{j \beta}{h_{1}} \frac{\partial H_{z}}{\partial u}\right) \\
H_{v} & =\frac{1}{p^{2}}\left(-\frac{j \omega \varepsilon}{h_{1}} \frac{\partial E_{z}}{\partial u}-\frac{j \beta}{h_{2}} \frac{\partial H_{z}}{\partial v}\right)
\end{aligned}
$$


where $p^{2}=\omega^{2} \mu \varepsilon-\beta^{2}$. If now (3.60) and (3.61) are substituted in (3.56), it is found that

$$
\left[\frac{1}{h_{1} h_{2}}\left(\frac{\partial}{\partial u} \frac{h_{2}}{h_{1}} \frac{\partial}{\partial u}+\frac{\partial}{\partial v} \frac{h_{1}}{h_{2}} \frac{\partial}{\partial v}\right)+p^{2}\right] E_{z}=0
$$

and substituing (3.62) and (3.63) in (3.59)

$$
\left[\frac{1}{h_{1} h_{2}}\left(\frac{\partial}{\partial u} \frac{h_{2}}{h_{1}} \frac{\partial}{\partial u}+\frac{\partial}{\partial v} \frac{h_{1}}{h_{2}} \frac{\partial}{\partial v}\right)+p^{2}\right] H_{z}=0
$$

which are the scalar wave equations for $E_{z}$ and $H_{z}$. Using vectorial notation, it is possible to write (3.64) and (3.65) in the following way:

$$
\left[\nabla_{t}^{2}+p^{2}\right]\left[\begin{array}{c}
E_{z} \\
H_{z}
\end{array}\right]=0
$$

where the Laplacian operator $\nabla_{t}^{2}$ in curvilinear coordinates is given by

$$
\nabla_{t}^{2}=\frac{1}{h_{1} h_{2}}\left(\frac{\partial}{\partial u} \frac{h_{2}}{h_{1}} \frac{\partial}{\partial u}+\frac{\partial}{\partial v} \frac{h_{1}}{h_{2}} \frac{\partial}{\partial v}\right)
$$

According to (3.60)-(3.63), all the transversal components of the field, $E_{u}, E_{v}, H_{u}$ and $H_{v}$, of a wave propagating in a simple medium $(\mu, \varepsilon)$ following a perfect straight path in the propagation direction $\hat{z}$, can be obtained from the axial/longitudinal fields $\left(E_{z}, H_{z}\right)$. These fields are governed by (3.64) y (3.65), according to the previous steps.

Debye potentials, $\Phi(r)$ and $\Psi(r)$, are recovered now. The relationship between Hertz vector and Debye potentials has been already treated in this section, but it still lacks to ascertain if there is a direct correspondence between the axial components of the field $\left(E_{z}, H_{z}\right)$ and the Debye potentials $(\Phi(r), \Psi(r))$. From the field expressions derived from the Debye potentials (3.48) and (3.49), it is possible to obtain, for $\hat{a}=\hat{z}$, the following expressions:

$$
\begin{gathered}
E_{z}(u, v)=-\frac{j p^{2}}{\omega \varepsilon} \Phi(u, v) \\
H_{z}(u, v)=\frac{j p^{2}}{\omega \mu} \Psi(u, v)
\end{gathered}
$$

where they have been used the scalar wave equations for the Debye potentials (3.52) and (3.53). It is proven, therefore, the correspondence one to one between the Debye potentials and the longitudinal fields of the wave. Since the notation $E_{u, v, z}$ and $H_{u, v, z}$ is much more evident, it is the notation found usually in the bibliography, and it will be also the case of this thesis. 
To keep the formalism and completeness, the following issue is addressed. From the previous statements, TM or E (Transversal Magnetic) modes, concern $E_{z}=0$, TE or H (Transversal Electric) modes, concern $H_{z}=0$, HE and EH (hybrid modes) imply both, $E_{z} \neq 0$ and $H_{z} \neq 0$, and finally, TEM (Transversal Electromagnetic) modes concern $E_{z}=H_{z}=0$. For this last case, a different procedure is required. From (3.60)-(3.65), when $E_{z}$ and $H_{z}$ are both null, the only non trivial solution for the transversal field components occurs when $p^{2}=0$, this implying that $\beta=\omega \sqrt{\mu \varepsilon}$. This is the origin of the well-know fact that, for TEM modes, the propagation constant is equal to the wavenumber in the media that they propagate: $\beta=k_{d}=$ $\omega \sqrt{\mu \varepsilon}$. By replacing $E_{z}=0$ and $H_{z}=0$ in (3.54)-(3.59) it is obtained that

$$
\begin{array}{r}
H_{u}=-\sqrt{\frac{\varepsilon}{\mu}} E_{v} \\
H_{v}=-\sqrt{\frac{\varepsilon}{\mu}} E_{u} \\
\frac{\partial h_{2} E_{v}}{\partial u}-\frac{\partial h_{1} E_{u}}{\partial v}=0 \\
\frac{\partial h_{2} H_{v}}{\partial u}-\frac{\partial h_{1} H_{u}}{\partial v}=0
\end{array}
$$

Consider now the following relation:

$$
\vec{E}=\nabla_{t} V=\frac{1}{h_{1}} \frac{\partial V}{\partial u} \hat{u}+\frac{1}{h_{2}} \frac{\partial V}{\partial v} \hat{v}
$$

where $V$ is a scalar function, $\nabla_{t}$ is the transversal gradient operator, and $\hat{u} \mathrm{y}$ $\hat{v}$ are the unitary vectors in the transversal coordinates. If now (3.70), (3.72) and (3.74) are applied to (3.72),

$$
\vec{E}=\frac{\partial}{\partial u}\left(\frac{h_{2}}{h_{1}} \frac{\partial V}{\partial u}\right)+\frac{\partial}{\partial v}\left(\frac{h_{1}}{h_{2}} \frac{\partial V}{\partial v}\right)=0
$$

or, in a more compact form:

$$
\nabla_{t}^{2} V=0
$$

which is the Laplace wave equation.

For the rest of the cases, which are the cases to appear in this part of the thesis, it is possible to reduce the partial derivatives equation, either (3.64) or (3.65), to an ordinary differential equation through the well-known technique of separation of variables [376]:

$$
\left[\begin{array}{l}
E_{z} \\
H_{z}
\end{array}\right]=U(u) V(v)
$$

Where $U(u)$ and $V(v)$ are functions of only one variable, satisfying the ordinary differential equations of second order. The use of this technique is possible for 
four coordinate systems with longitudinal coordinate $z$ : Rectangular (or Cartesian) coordinates, Circular Cylindrical coordinates, Elliptical Cylindrical coordinates, and Parabolic Cylindrical coordinates. The solution for such differential equations in the mentioned coordinate systems are well-known, and can be easily found in the bibliography [21], [542]. In this part of the thesis, due to the cylindrical nature of the wire-type structures, the Circular Cylindrical coordinates will be employed. Particularities of the solution for these will be extensively detailed along this chapter when the corresponding waveguides are analyzed.

The longitudinal components expansion to obtain the rest of the electromagnetic field components has been addressed and formalized along this section and it has been shown to be a very helpful simplification in the resolution of electromagnetic waveguiding problems. Several types of modes have been reviewed, with the most general case corresponding the hybrid modes, each one being a superposition of a TE mode and a TM mode. The choice of the differential equation solutions in each region, or the type of the modes considered will depend on the boundary conditions, including the radiation condition, of the problem under analysis. In some simple cases it will be possible to split the solution in TE and TM modes separately, however, in other cases this will be not possible, and the more general case of hybrid modes will be necessary. Nevertheless, even in such general case, physical insight will be obtained by looking in detail to their transversal and axial components. At that moment, the breakdown carried out in this section will recover all its sense.

\subsubsection{Formal treatment of a surface waveguide problem}

Along the previous sections, the basic principles regarding the resolution of the field equations, and how particular boundary conditions must be applied over the obtained solution, have been established. Moreover, this task has been carried out trying to give the most appropriated approach for a surface waveguiding problem. In this section, it will be schematized, in a simple and brief way, the considerations and the steps to follow in the resolution procedure of a surface waveguiding problem. These are the steps that will be followed in the subsequent sections, where a more particular emphasis on the physical meaning of the wave equation solutions will be given, highlighting the main differences respect to the case of closed metallic waveguides, probably more handy to the reader. The four considerations that must be taken into account in a surface waveguiding problem are:

1. For the guided modes that are propagated along a perfect straight path in the direction $\hat{z}$, all the field components are able to be represented in the following form:

$$
f(u, v) e^{-j \beta z} e^{j \omega t}
$$

where $u$ and $v$ are the transversal coordinates, $\beta$ the propagation constant, and $\omega$ the angular frequency of the wave. 
2. The solution of the longitudinal fields $\left(E_{z}, H_{z}\right)$ must satisfy the scalar wave equation

$$
\left[\nabla_{t}^{2}+\left(\omega^{2} \mu \varepsilon-\beta^{2}\right)\right]\left[\begin{array}{l}
E_{z} \\
H_{z}
\end{array}\right]=0
$$

where $\nabla_{t}$ is the transversal gradient operator, defined in (3.74), and $(\mu, \varepsilon)$ are the constitutive parameters of the medium. It is noted that the previous equation must be modified when the mediums are inhomogeneous, anisotropic or nonlinear, which is not the case of the problems studied in this part of the thesis.

3. The obtained electromagnetic fields as solution of a certain structure must satisfy the appropriated boundary conditions, which are the following:

- The tangential componentes of $\vec{E}$ and $\vec{H}$ must be continuous across the interface between two dielectric medium.

- The tangential components of $\vec{E}$ must vanish on the surface of a perfect conductor.

- The radiation condition must be satisfied at the infinity.

When the boundary conditions are imposed to the possible solutions for the electromagnetic fields of a certain problem, it is arrived to an equation, generally transcendental in $f$ and $\beta$ for the guided modes. For a given frequency $f$, such equation will have a finite number a roots for $\beta$. Directly associated to the obtained eigenvalues (the values of $\beta$ that satisfy the equation) are the eigenfunctions, which are the functions describing the fields. Under the usual procedure, assuming the ideal case of perfect conductors and lossless dielectric mediums, these roots result to be real ${ }^{3}$. Once solutions are found, the perturbation method is applied as a second step in order to find the losses caused by the real materials forming the waveguide. In the perturbation method, the losses features of either imperfect metals or dielectric materials are applied to the problem considering the fields solution of the ideal problem [376].

Contrarily, if the real problem is afforded directly, either by mathematical requirements or by desired accuracy, a complex propagation constant $\gamma=$ $\alpha+j \beta$ must be considered, i.e., the obtained dispersion equation, relating in this case $f$ and $\gamma$, will have, in general, complex solutions for $\gamma$ given a frequency of operation. When $\gamma$ differs noticeably from $\beta$ it can be understood that the corresponding eigenfunctions, the fields, will also differ. Thus, if the real case with losses is very different from the ideal lossless case, the second option to solve a problem must be considered.

It must be pointed out that the completeness of the solution of the problem is not totally given by the guided modes found as the finite set of solutions of the dispersion equation. The radiation condition must be included, and in a more general solution of the equation, i. e., without restricting the values of $\beta$ and $\gamma$ to the particular mode behavior (guided in the sense of dielectric

\footnotetext{
${ }^{3}$ Some very exceptional cases may appear, but are beyond the scope of this thesis [543]
} 
waveguides), modes that are radiated but not guided are found for this kind of waveguides. This case is very different from the observed solutions in an ideal closed metallic waveguide, where the modes propagate $(\beta$ real) or are evanescent ( $\beta$ pure imaginary). The case of complex $\gamma$ involves even more conceptual difficulties regarding the nature of the solutions [430]. As a part of the objectives of this thesis, this issue will be particularly afforded over more concrete geometries, since without exemplification it results difficult to give the required insight.

4. In general, the transversal electromagnetic fields inside an open structure satisfy certain orthogonality condition, that can be expressed as follows [21], [339]:

$$
\vec{E}_{t}(u, v, z)=C E_{t}^{\text {radiated }}(u, v, z)+\sum_{p} A_{p} \vec{E}_{t p}^{\text {guided }}(u, v) e^{-\beta_{p} z}
$$

where the subscript $t$ means transversal, $C$ is the amplitude coefficient of the radiated wave, the index $p$ represents the index of the finite summation of the surface modes that propagate at a given frequency, $A_{p}$ is the amplitude of the guided mode $p$, and $\beta_{p}$ is the propagation constant of the mode $p$. It can be proved that, at any plane $z$, the following relation holds:

$$
\begin{array}{rl}
\int_{-\infty}^{\infty}\left(\vec{E}_{t p} \cdot \vec{E}_{t q}^{*}\right) h_{1} h_{2} \mathrm{~d} u \mathrm{~d} v=0 & p \neq q \\
\int_{-\infty}^{\infty}\left(\vec{E}_{t p} \times \vec{H}_{t q}^{*}\right) \cdot \hat{z} h_{1} h_{2} \mathrm{~d} u \mathrm{~d} v=0 & p \neq q
\end{array}
$$

where $p$ and $q$ can both be either a guided or a radiated mode, thus having all possible combinations. This means that (3.81) are (3.82) valid for any two modes, regardless of the nature of each one, either guided or radiative. This equations are the so called orthogonality relations for surface waves [21]. By means of these expressions in a surface waveguiding problem, any field distribution in a given plane $z$ can be expressed as a linear combination of the orthogonal wave functions of the modes (eigenfunctions). The coefficients $C$ or $A_{p}$ can be obtained by means of the orthogonality relations (3.81) y (3.82). The presence of such orthogonality relations also implies that the power of each guided mode and the power of each radiative mode are additive and independent. This kind of relationships are well-known for closed metallic waveguides, used in the well-established modal techniques, applied in such structures with very successful results [544]. With the described approach, it is pretended to give a link or a parallelism between both kinds of problems. Although modal techniques have been recently considered for open problems [545]-[547], there is still much work to do in order to clear up the particular features of these modal techniques when the involved regions are not finite. In this way, the careful modal study of surface waveguide may give the necessary foundation required to apply the rigorous and powerful modal techniques. This is another research niche foreseen in this thesis. 


\subsubsection{The impedance concept}

The particular case of TEM solutions in a waveguiding structure allows for the conventional analysis through circuit theory, by means of voltages and currents calculated by integration of the electromagnetic fields in the structure, through the three typical formulations: Voltage-Current (VI), Voltage-Power (VP) or Current-Power (IP) [376]. The noticeable simplicity that circuit theory offers in the design process, and the powerful methods developed under the foundations of this theory, makes very suitable to find a transmission line equivalent for a given waveguiding structure. This has been the case of the usual two conductor lines either bulky, e.g., the coaxial line, or planar, e.g., the microstrip line. This procedure gives as a result that a transmission line model for a certain waveguiding structure is defined by its characteristic impedance $Z_{c}$ and its propagation constant $\beta$, both depending on waveguide geometrical parameters and involved dielectrics. Characterization of transmission lines themselves, or any more general $N$-port network by means of the usual impedance matrix $Z$ (or admittance matrix $Y=Z^{-1}$ ), the scattering matrix $S$ or the transmission matrix $A$, rely on $Z_{c}$ and $\beta$ of the involved transmission lines.

When the waveguide does not support TEM modes, but at least one axial field component is required (TE or TM modes), the previous concept of impedance becomes ambiguous and rather useless, since the integrals required to obtain either, voltages or currents, become dependent on the integration path. Therefore, the characteristic impedance $Z_{c}$ is not uniquely defined anymore. It is possible, however, to extend the concept of impedance to a more loose one by defining the wave impedance $Z_{w}$ of a mode as the ratio between the transversal electric and magnetic fields. In closed single conductor metallic waveguides the expressions are the following:

$$
Z_{w}^{(T E)}=\left|\frac{E_{t}^{(T E)}}{H_{t}^{(T E)}}\right|=\frac{\omega \mu_{0}}{\beta^{(T E)}}
$$

and

$$
Z_{w}^{(T M)}=\left|\frac{E_{t}^{(T M)}}{H_{t}^{(T E)}}\right|=\frac{\beta^{(T M)}}{\omega \varepsilon_{0}}
$$

where $\beta^{(T E)}$ and $\beta^{(T M)}$ are the propagation constants of TE and TM modes, respectively. Note that $Z_{w}$ is dispersive with the frequency. It can not be in other way to capture the dispersive nature of the TE and TM modes. It must be highlighted that the wave impedance, introduced to extend the concept of impedance to TE and TM modes, does not provide relevant information for TEM modes. This occurs because for them, the expression results too simple, independent of the geometrical parameters of the waveguide, and relying only on the electric and magnetic properties of the homogeneous ${ }^{4}$ medium between the conductors:

\footnotetext{
${ }^{4}$ Note that TEM modes can exist only in homogeneous mediums. Cases such as the microstrip or the CPW imply quasi-TEM modes, for which the conclusions extracted for TEM modes are almost, but not totally true.
} 


$$
Z_{c}^{(T E M)}=\sqrt{\frac{\mu}{\varepsilon}}
$$

Note that the above expression would be the same for a plane wave traveling in an infinite medium characterized by the couple $(\mu, \varepsilon)$.

Now, the concept of impedance is posed for hybrid modes. The fact that the two axial components of the field are not null has a critical implication on the transversal components of the field through (3.60)-(3.63). Note that in this case, the transversal components of the fields have two terms, each one coming from an axial component, therefore the quotient defining the wave impedance is not longer independent of the transversal coordinates:

$$
\begin{aligned}
& \left|\frac{E_{u}}{H_{v}}\right|=\left|\frac{\left(-\frac{j \beta}{h_{1}} \frac{\partial E_{z}}{\partial u}-\frac{j \omega \mu}{h_{2}} \frac{\partial H_{z}}{\partial v}\right)}{\left(\frac{j \omega \varepsilon}{h_{1}} \frac{\partial E_{z}}{\partial u}-\frac{j \beta}{h_{2}} \frac{\partial H_{z}}{\partial v}\right)}\right| \\
& \left|\frac{E_{v}}{H_{u}}\right|=\left|\frac{\left(-\frac{j \beta}{h_{2}} \frac{\partial E_{z}}{\partial v}-\frac{j \omega \mu}{h_{1}} \frac{\partial H_{z}}{\partial u}\right)}{\left(\frac{j \omega \varepsilon}{h_{2}} \frac{\partial E_{z}}{\partial v}-\frac{j \beta}{h_{1}} \frac{\partial H_{z}}{\partial u}\right)}\right|
\end{aligned}
$$

The dependence of the above expressions on the transversal coordinates $(u, v)$ is obvious. It is clear that, for the hybrid modes, the transversal fields adopt its more complex form and, accordingly, the wave impedance concept is a rather complex to handle with. Nevertheless, the wave impedance concept becomes also difficult to handle even for TE or TM in surface waveguides. Unlike closed metallic waveguides, having one unique region and constant field patterns, e. g., sinusoidal patterns in the RWG, dielectric waveguides involve more than one region, and a different boundary condition (continuity of tangential EM fields instead of nullity of tangential E-field). The result is a field distribution between mediums that depends on the frequency, and an impedance that is not constant in each of the regions. Thus, it is not possible to obtain an unidimensional transmission line equivalent. Furthermore, the presence of discontinuities, or not homogeneous zones or curvatures, produces radiated waves that take the power away from the waveguide, and that cannot be taken into account with a simple transmission line model. In the best case, what can be done is to obtain an approximate value of the wave impedance by using an averaged mean that take into account the distribution of power in the waveguide, i. e., averaging each region according the percentage of power in it. This rough approach may be useful in a first step of the design, and it is widely used. As an example, a very similar approach is systematically used to estimate the attenuation in dielectric waveguides, as it has been obseved in the review of the state of the art [436], [438], [442], [448], [450], [454]. 
In the case of impenetrable surface waveguides, such as the SWW, it is true that for the fundamental quasi-TEM mode, it could be assumed that

$$
Z_{w} \approx Z_{w}^{\left(T E M_{0}\right)}=\sqrt{\frac{\mu_{0}}{\varepsilon_{0}}}=120 \pi
$$

however, this impedance will remain quite constant even for important changes on the field pattern, and this will be also the case of any attempt of using the concept of characteristic impedance. It seems that the best option for surface waveguides, which involve more than one region, one of them infinite (or at least very large so it can be assumed infinite in practice), and that support hybrid modes, is to take a step of generalization forward, and keep the fields patterns in mind along any circuit design. Probably, complex models based on transmission lines may be developed, however, these models would be very structure dependent, and, at the end, they would rely on the complexity of the field patterns, which would be carried in a direct or indirect way along all the design process.

\subsubsection{The dispersion diagram}

The dispersion diagram of a waveguide is a fundamental tool on its analysis process. In it, the frequency dependence of the propagation constant for the possible solutions (modes) is represented. Directly, from the dispersion diagram, they can be obtained $\partial \beta / \partial \omega$ (group velocity $v_{g}$ ) and $\partial^{2} \beta / \partial \omega^{2}$, which are indicators of how a pulse containing certain frequency spectra may be broadened and distorted when is propagated along the waveguide. Also, the phase velocity $\omega / \beta$ is directly obtained.

Let now to take a reflection on the nature of the propagation constant $\beta$ in a simple dielectric waveguide, consisting in two regions of different permittivity. For a guided mode, it is clear that $\beta$ is the same in both regions. Therefore, intuitively it must have a value in between the propagation constant of a plane wave in the medium $1, k_{1}$, and the propagation constant of the medium $2, k_{2}$. Assume now that the medium 1 is denser, i. e., $\varepsilon_{r_{1}}>\varepsilon_{r_{2}}$, therefore $k_{1}>k_{2}$. In the proposed scenario, it is known that a dielectric region can confine the waves to act as a waveguide in as much it is denser than the surrounding dielectric. The larger the contrast between both media, and the electrical size of the dielectric region, the larger the confinement, hence the waveguiding properties. Therefore, for dielectric waveguides $k_{2}<\beta<k_{1}$, and it could be roughly said that $\beta$ depends on the power distribution between the two mediums. When the mode start to propagate (the waveguide has low waveguiding properties) almost all power travels in the low permittivity region, therefore $\beta \approx k_{2}$. As the dielectric region 1 gains waveguiding properties, since it becomes electrically larger when the frequency increases, the mode becomes trapped inside it, so it could be said that it practically "sees" only this region, thus occurring $\beta \approx k_{1}$. In the particular case of impenetrable surface waveguides, the former state $\left(\beta \approx k_{2}\right)$ remains longer, in the best cases, almost independent of the frequency, e. g., the SWW. The grade of impenetrability will depend on the boundary conditions offered by the interface between the two media, and the mode pattern, i. e. how 
the polarization and magnitude of electric and magnetic fields interact with the boundary.

The previous description of the dispersion properties of a dielectric waveguide is very approximated, and the reader can understand that the variety of possible cases involved with this kind of waveguides require specific comments for each one. However, the main features have been mentioned, and the differences between the conventional, more deeply studied closed metallic waveguides can be understood. To give a first impression, the dispersion diagram of a conventional dielectric waveguide, and some its typical features are displayed in Fig. 3.4. In the diagram they are shown the fundamental mode and the first three high-order modes. The modes start to propagate with $\beta=k_{2}$, since for values $\beta<k_{2}$ the mode becomes leaky or radiative. All the modes tend asymptotically to $\beta=k_{1}$ as the frequency increases. It is quite usual to found that the fundamental mode has not theoretical cutoff, but experiment a practical cutoff, from which effective waveguiding occurs. Note that although solution exist, it can be in a form similar to a plane wave in the external medium. The third higher order mode experiences Impenetrable Surface Waveguiding (ISW) in a certain bandwith. This is appreciated in the fact that it propagates but $\beta$ remains close to $k_{2}$. The shape of the curve indicates this characteristic effect, not present on the others (the fundamental mode may also experience this effect, but it is obscured but the feature commented before, so that it is difficult to figure out in the graph which effect predominates.).

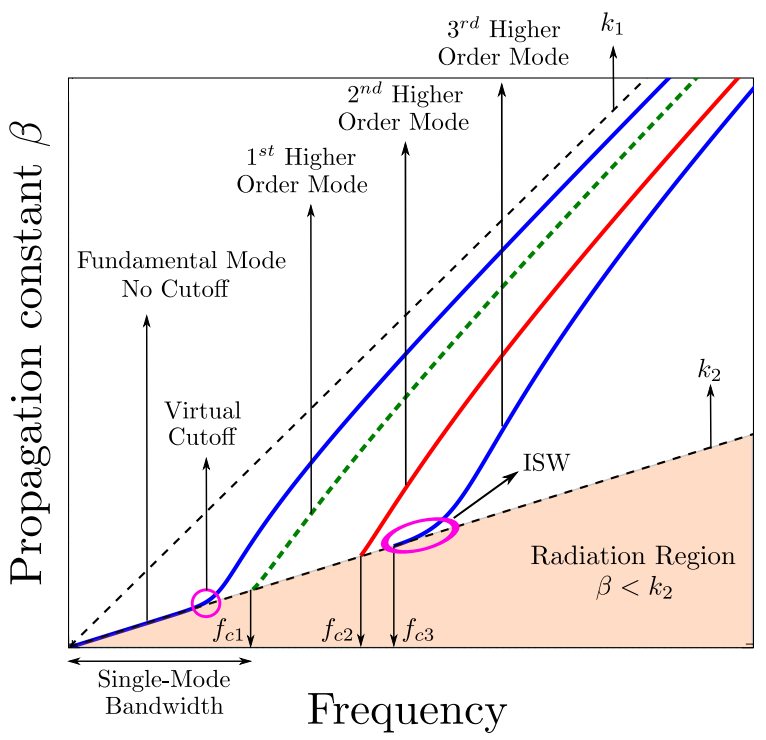

Figure 3.4: Typical dispersion diagram of a dielectric waveguide. In the diagram they are shown the fundamental mode and the first three high order modes. The modes start to propagate with $\beta=k_{2}$. For values $\beta<k_{2}$ the mode becomes leaky or radiative. All the modes tend asymptotically to $\beta=k_{1}$ as the frequency increases. The fundamental mode has not theoretical cutoff, but experiment a practical, from which effective waveguiding occurs. The third higher order mode experiences Impenetrable Surface Waveguiding (ISW) in a certain bandwidth. This is appreciated in the fact that it propagates but $\beta$ remains close to $k_{2}$, indicating that most of the power travels in the external medium. 
Let the external medium to have $\varepsilon_{r_{2}}=1$ for clarity purposes. Note that $\beta>k_{0}$. This is contrary to single-conductor closed metallic waveguides, in which the modes start to propagate with $\beta=0$ and asymptotically tend to $\beta=k_{0}$. In fact, the region of the dispersion diagram were these kind of modes would exhibit their curves is the orange region of the dispersion diagram shown in 3.4, in which the dielectric waveguide modes are radiative. Note also that $v_{p}=\omega / \beta$, and observing the diagram it is found that $\frac{c}{\sqrt{\varepsilon_{r_{1}}}}<v_{p}<c$, with $c$ the speed of light in the vacuum. Therefore, the phase velocity will be always lower than the speed of light. Since this is the contrary behavior to the conventional waveguides such as RWG or CWG, the modes propagating in dielectric waveguides are usually called slow-wave modes. Of course, the group velocity, given by

$$
v_{g}=\frac{\partial \omega}{\partial \beta}
$$

is also lower than $c$ and it has the same bounds: $\frac{c}{\sqrt{\varepsilon_{r_{1}}}}<v_{g}<c$. The group velocity decreases with the frequency, since the region 1 is denser. Finally, it must be mentioned that, since the curve of $\beta$ takes place between two asymptotic limits ( $\beta=k_{2}$ and $\beta=k_{1}$, the two straight lines that delimit the propagation area), it is quite usual to represent $\beta / k_{0}$ instead of $\beta$, to have horizontal limits, and better appreciate the variations of $\beta$. This will be one of the main forms to represent dispersion diagrams in this thesis.

Particularities of the dispersion diagram under certain situations and for certain modes will be better understood by treating particular examples, what will be do later in this chapter. For the moment, the reader has now a preliminary image of the main features of this kind of waveguides, and it can be now understood that the usual techniques used with closed waveguides must be reviewed for this different kind of waveguides. Also, it is easily foreseen that interconnection of both kinds of waveguides requires a careful design process since propagation in each one is of very different nature.

\subsubsection{Efficient excitation and curves}

So far, the main theoretical aspects of dielectric, and, in particular, surface waveguides have been reviewed: the boundary conditions, the types of solutions, the impedance concept issue, and the general dispersion diagram. Taking advantage of the obtained conclusions, some practical aspects are briefly commented now. These aspects are the efficient excitation of surface waveguides and the effect of curves on these waveguides. In general, the following three conditions must be satisfied to provide efficient power coupling into o from a waveguide:

1. Impedance matching. The impedance of the incident wave (or mode) must be as close as possible to the impedance of the guided mode that is desired to be excited or transmitted in order to provide good matching. Losses caused by Fresnel reflections due to longitudinal separations or transversal misalignment follow in this category [21]. Gradual transitions between different impedances is a well-known technique to alleviate the mismatching. 
2. Field pattern correspondence. The field pattern of the incident wave (or mode) must be as close as possible to the guided mode that is desired to be excited or transmitted in order to provide matching. In the conventional sense, polarization and maximums and minimums of the magnitude pattern conform the total information to take into account to prevent mismatch losses, caused by differences between field patterns. In the case of surface waveguides, at the region where fields extent to infinity, by means of the radiation condition, an exponential decay of the fields from the surface takes place. Since very different ratios of exponential decrement of magnitude may occur (the field distribution ranges from confined states to expanded states), this feature must be taken into account. This means, it appears an additional feature to look at, which is how fields ruled by the same eigenfunction (giving exponential decay) distribute. When the incident wave pattern matches with any high order mode, coupling of power to it or them occurs. This is an undesired effect to prevent.

3. Propagation constant (phase velocity) correspondence. The phase velocity of the incident wave (or mode) must be as close as possible to the guided mode that is desired to be excited or transmitted. This kind of correspondence becomes special important in those cases considering excitation of open waveguides by means of transversal coupling instead of longitudinal coupling. As it can be understood, the possible coupling between two open waveguiding structures, close to each other, has a large dependence on the similitude of the phase evolution. This procedure has been widely used with planar structures such as the microstrip line [376], [339], [22].

When dealing with surface waveguides, the complexity of the impedance concept causes that the condition on the impedance rely directly on the field condition. In fact, this condition will be the most important of the three in this thesis, since, although in some cases differences on the propagation constant may be found, e. g., coating or uncoating the waveguide, most of the time, achieve a good matching in $\beta$ will not be the main issue. On the other hand, matching field patterns is complicated in these waveguides because, as mentioned before, not only polarization and proper magnitude distribution is required in the usual way (matching maximums and minimums of the field over a particular fixed region), but matching the magnitude distribution between the regions forming the waveguide, and matching the exponential decay in the outer media must be also achieved. Since these parameters are, generally, very frequency dependent (specially the exponential decay), one can figure out how challenging may become the efficient excitation of surface waveguides.

In relation with the previous issue are the curvatures or perturbations. It is known that curvatures or bends can cause mode coupling in closed structures. However, in the case of surface waveguides the effect is larger and less controllable, since the fields are not confined, but with large extensions, hence the patterns are more deformed by the curve. Moreover, the use of elements to prevent the radiations is less obvious and more difficult, again due to the extension of fields.

These two brief reflections give a clear conclusion: to properly excite a surface waveguide, and to prevent losses in curves caused by mode conversion (that can be 
radiative), a configuration that provide a confinement comparable to closed waveguides is required. This can be afforded by modification of the waveguide structure, sometimes requiring an auxiliary dielectric waveguide. Furthermore, both, original and modified structures, must be similar, and proper transitions between them must be provided. A good example, already reviewed in the state of the art, is the ribbon proposed in [531]. In that case, for excitation and curves the coating is applied, thus having an open waveguide with confinement features. The coating is removed when is not necessary to have a low-loss, low dispersive surface waveguide for straight paths.

From a contrary approach, one can take the positive part the characteristic features of surface waveguides. As these waveguides act rather as antennas when they are curved, by the reciprocity theorem, it would be in principle possible to couple power to them with the same efficiency as it is lost. It is true that, to carry out this procedure, not only the radiation characteristics of a curve must be carefully studied, but also a system to replicate the radiation pattern in the inverse direction must be designed. Nevertheless, it is an option rather unexplored, and that may play its role in the race to improve the nowadays poor excitation techniques.

It is a fact that despite the great effort and the quality work published, the surface waveguide theory and the propagation characteristics of these kind of waveguides are less known than for other more usual waveguides. Since the structures are complex, there are as well more unexplored possibilities, and although many issues are found, the possibility of discovering an efficient solution to the main intrinsic problems is a very motivating one, since, at $\mathrm{THz}$, it is clear that surface waveguides have much to say.

\subsection{Metal properties in the $\mathrm{THz}$ band}

\subsubsection{Classical skin-depth model and relaxation-effect model (Drude Model)}

In the previous section, the main characteristics of the mathematical modeling of a dielectric, and the corresponding implied boundary conditions have been reviewed, since this kind of material appears in one way or another in the wire-type waveguides studied in this thesis. Also, a metal acting as a perfect conductor has been reviewed from the boundary conditions point of view. Now, metals as not perfect conductors are modeled considering that they act macroscopically as a dielectric with very particular characteristics. This issue requires special detail because, on the one hand, it will be an essential mathematical tool for the analysis of the SWW, and, on the other hand, it shows a reality in the $\mathrm{THz}$ band: to consider metals as perfect conductors is not a banal decision when solving a electromagnetic problem.

At microwaves, to solve the fields in most of the electromagnetic problems, metals are assumed to be perfect electric conductors, i.e., their conductivity $\sigma$ is assumed to be infinite. In this way, the problem is simplified due to the simple boundary condition that a perfect conductor imposes: the inner volume is not longer region of the problem (fields are null inside), and the tangential electric field at the external faces vanishes. Once fields are solved, calculation of losses requires to consider the 
real material that is present in the problem, i.e., assume the finite value of $\sigma$ that the employed metal has. The perturbative theory allows to consider the fields of the ideal case (obtained in an easier way) and calculate the interaction with this finite value of $\sigma$. This first step towards considering non-ideal metals is known as skin-effect model, which describes how, at enough high frequencies, the current density in a bulk conductor tends to to concentrate near the surface (and not equally distributed as occurs for DC, i.e, $f=0 \mathrm{~Hz}$ ). This model involves the well-known parameter of skin depth:

$$
\delta=\frac{1}{\sqrt{\pi f \mu \sigma}}
$$

where $f$ is the frequency of operation and $\mu$ the magnetic permeability of the conductor media. Good conductors and high frequencies imply the skin depth to be small, however the effect of this parameters in the losses is contrary. This can be better observed considering the the surface resistance, the parameter which directly determine ohmic losses caused by finite conductivity:

$$
R_{s}=\sqrt{\frac{\pi f \mu}{2 \sigma}}=\frac{\delta}{\sigma}
$$

The larger $R_{s}$, the larger the ohmic losses. More specifically, the following expression gives the ohmic losses per unit length by perturbative theory on a conductor surface:

$$
P_{L c}=\frac{R_{s}}{2} \oint_{C}\left|\vec{J}_{s}\right|^{2} \mathrm{~d} l
$$

where $\vec{J}_{s}$ is the current of the conductor surface, and $C$ is a curve along the path described by a transversal cut of metallic region. Since ohmic losses are proportional to $R_{s}$, high frequencies and low conductivity increase the losses caused by an imperfect metal. In this model, by applying Maxwell equations, the metal can be regarded as a dielectric whenever the following permittivity is considered:

$$
\varepsilon_{m}=\varepsilon_{0}\left(1+\frac{\sigma}{j \omega \varepsilon_{0}}\right)
$$

This consideration allows to see a metallic material as a dielectric. Note that $\omega \varepsilon_{0}$ is equal to the unity at $f \approx 18 \mathrm{GHz}$, and for good conductors $\sigma$ is beyond $10^{6} \mathrm{~S} / \mathrm{m}$. This means, that, at the microwave regime, this model implies a relative permittivity with a very large negative imaginary part, and a positive real part that is negligible in magnitude compared to the imaginary part. Therefore, the following simplification is always assumed

$$
\varepsilon_{m} \approx-j \frac{\sigma}{\omega}
$$

In this way, the electromagnetic problem under study can be solved in a more exact way, considering the real fields (in the approximated skin-effect model) inside the metal, now modeled as a dielectric through (3.94). In fact, it is the same that assuming a finite $\sigma$ and solving Maxwells equations, however, it is seen in a easier way 
when one thinks in a dielectric media. This treatment is rarely found in microwave electromagnetic problems, since the perturbative method gives enough accuracy.

On the other hand, in the infrared regime, interaction of metals with the electromagnetic waves becomes more complex, and the previously described model is not longer valid. In a further step, the metal is treated as a free electron gas and the conductivity is not longer a constant, but it is ruled by the following definition ${ }^{5}$ [21], [347]:

$$
\sigma=\frac{\omega_{p}^{2} \varepsilon_{0}}{j \omega+\omega_{\tau}}=2 \pi \frac{f_{p}^{2} \varepsilon_{0}}{j f+f_{\tau}}
$$

where $f_{p}$ is the plasma frequency of the metal (following its description as a free electron gas), and $f_{\tau}$ is the collision frequency of the electrons, sometimes called as the inverse of the electrons lifetime. Therefore, by substitution of this new $\sigma$ in (3.93) it is obtained ${ }^{6}$

$$
\varepsilon_{m}=\varepsilon_{0}\left(1-\frac{f_{p}^{2}}{f^{2}-j f f_{\tau}}\right)
$$

what implies, for the real and imaginary parts of the permittivity, that:

$$
\begin{aligned}
& \varepsilon_{m}^{\prime}=\varepsilon_{0}\left(1-\frac{f_{p}^{2}}{f^{2}+f_{\tau}^{2}}\right) \\
& \varepsilon_{m}^{\prime \prime}=-\varepsilon_{0}\left(\frac{f_{\tau} f_{p}^{2}}{f\left(f^{2}+f_{\tau}^{2}\right)}\right)
\end{aligned}
$$

This is the relaxation-effect model or Drude Model, which is commonly used in the infrared region, but it has been also shown to be valid for frequencies in the $\mathrm{THz}$ range [347]. The Drude model can be refined to take into account other effects, e. g., the fact that the electrons are bounded, however, this is only useful for the high frequencies of the IR region and the optical region [348]. Therefore, the expression of (3.96) will be assumed as the most exact choice to characterize a metal in this thesis. It should be mentioned that, in the Drude model, the relative permittivity of a metal $\left(\varepsilon_{r_{m}}\right)$ has also a large negative imaginary part. However, in it appears a negative real part, smaller in magnitude, but comparable with the imaginary part. Also, the imaginary part is smaller in magnitude than predicted by the skin-effect model. These are the main differences between both models.

Since at microwaves the skin-effect model is used, whereas at IR the Drude model is used, the question about the characterization of metals at $\mathrm{THz}$ arises. The problem is that the Drude model complicates the analysis of electromagnetic problems (sometimes in a very appreciable way [348]), however, it is clear that at

\footnotetext{
${ }^{5}$ In the research area of modeling metals at IR and optic frequencies is very usual to work with $\omega$ in $\mathrm{cm}^{-1}$ units, however, to keep a nomenclature more consistent with that of radiofrequency engineering, all the expressions have been left as a function of frequency in $\mathrm{THz}$.

${ }^{6}$ In the literature the expression for a metal permittivity by means of the Drude model is usually found in terms of $\omega$ instead of $f$. However, in this thesis the use of $f$ is preferred since it gives more direct information for engineering purposes.
} 
some point in the $\mathrm{THz}$ band, for certain electromagnetic problems, the skin-depth effect model will be too rough. The question has not unique and simple answer, as can be observed reviewing the bibliography.

Generally, most of designs involving metallic materials are simulated with the simple skin-depth effect model, widely used by commercial solvers. This has a simple reason: there are not simple and direct ways to do it in a more accurate way, and the simulation techniques employed in the microwave regime drag the researcher with an obvious inertia. However, a small group of researches is providing evidences that the Drude model is both, necessary and sufficient, [348], [355], [356], [391], [548]. Nevertheless, those works have not achieved yet the necessary impact to impulse an appreciable change in the analysis techniques.

Planar metallic surfaces are affected, [549], but when dealing with them, it seems that the issue is not critical [534], [535]. However, in curved surfaces, the change in the result is noticeable. For instance, in [348], the mode patterns of a CWG change dramatically from their typical shape to a surface plasmonic pattern, with the fields totally concentrated near the metallic surface, forming a thin ring shape, thus leaving empty the rest of the region. In that work, it is observed that, for monomode CWG, this occurs beyond $f=10 \mathrm{THz}$, however, for the widely used $\mathrm{THz}$ overmoded CWGs, the frontier goes down to $f=1 \mathrm{THz}$.

Even if the review is done more specifically fore wire-type waveguides, the treatment of metals offer a variety of employed solutions. In some works, the wire surface is assumed as a perfect conductor ${ }^{7}$ [495], [513]. This is acceptable when the dielectric is present, but it will be shown to have important consequences for bare wires, since in this last case, the conductivity (or the metal equivalent permittivity) totally determines the solution of the problem. Other works like [507] use the classical formulation of the analytical SWW problem employed by Goubau an King [490], [497], [500], obtaining good correspondence between analytical calculations, commercial solver simulations and measurements. However, it is true that the considered frequencies are still low $(f<0.4 \mathrm{THz})$ and that, at $\mathrm{THz}$, manufacturing and measurement errors are still obscuring the validation of analysis/simulation tools. Finally, one found some works were the Drude model is used to solve the SWW fundamental mode dispersion properties and fields [512], [524].

It must be noted that for the case of a SWW, the characterization of a metal as a dielectric is not an option of accuracy, but is mandatory in order to have a non-singular electromagnetic problem, able to be solved. Although Stratton [489] talks about the case of infinite conductivity SWW, but barely giving details, King and Goubau [490], [497], [500] showed clearly that the problem becomes singular as $\sigma \rightarrow \infty$ (the guided mode becomes a plane wave in an infinite air medium, and in the limit, radiation condition is violated). The fact of using the simpler skin-effect model or the more exact Drude model has some implications in the fundamental mode, since more exact models are required if either bad metals or small wires are used [512]. It is true that, under certain conditions, it may be unnecessary to use the Drude model. Nevertheless, from an analytical point of view, the employment of Drude model causes a great difference, because the nature of the complex value of the permittivity will determine the nature of the general solutions of the problem, i.

\footnotetext{
${ }^{7}$ The commercial solver takes a finite, very large value.
} 


\begin{tabular}{lccccc}
\multicolumn{1}{c}{ Metal } & Symbol & $10^{-6} \sigma_{D C}(\mathrm{~S} / \mathrm{m})$ & $f_{p}(\mathrm{THz})$ & $f_{\tau}(\mathrm{THz})$ & $10^{-4} \varepsilon_{r}(f=1 \mathrm{THz})$ \\
\hline Aluminum & $\mathrm{Al}$ & 38 & 3570 & 19.8 & $-3.24-64.2 j$ \\
Cobalt & $\mathrm{Co}$ & 17 & 960 & 8.9 & $-1.16-10.3 j$ \\
Copper & $\mathrm{Cu}$ & 58 & 1788 & 2.2 & $-54.9-121 j$ \\
Gold & $\mathrm{Au}$ & 41 & 2184 & 6.45 & $-1.12-72.2 j$ \\
Iron & $\mathrm{Fe}$ & 10 & 990 & 4.41 & $-4.79-21.1 j$ \\
Lead & $\mathrm{Nb}$ & 47 & 1782 & 48.9 & $-0.13-6.49 j$ \\
Molybdenum & $\mathrm{Mo}$ & 18.7 & 1806 & 12.3 & $-2.12-13.1 j$ \\
Nickel & $\mathrm{Ni}$ & 14.3 & 1182 & 10.6 & $-1.24-13 j$ \\
Palladium & $\mathrm{Pd}$ & 9.5 & 1320 & 3.7 & $-1.17-43 j$ \\
Platinum & $\mathrm{Pt}$ & 9.3 & 1245 & 16.7 & $-0.55-9.23 j$ \\
Silver & $\mathrm{Ag}$ & 63 & 2181 & 4.4 & $-24-104 j$ \\
Titanium & $\mathrm{Ti}$ & 2.4 & 609 & 11.5 & $-0.28-3.21 j$ \\
Tungsten & $\mathrm{W}$ & 18 & 1551 & 14.6 & $-1.12-16.4 j$ \\
\hline
\end{tabular}

Table 3.1: $D C$ conductivity and Drude Model at $\mathrm{THz}$ for several common metals.

e., the high order modes, which are extremely necessary to properly characterize a structure. Since the main aim of this part of the thesis is give a rigorous analysis of wire-type structures and clarify some unresolved issues present in the current state of the art regarding these structures, not only the most exact model must be used for the SWW, but also conclusions about the differences between the solutions with one model and the other must be given.

\subsubsection{Comparison and examples}

Propagation characteristics on a SWW will be strongly determined by the nature of the equivalent permittivity of the metal. In fact, to solve analytically a dielectric cylinder of complex permittivity requires a careful study on the effect of the real and imaginary parts of the permittivity. Therefore, now the typical values of relative permittivity that common metals can adopt in the $\mathrm{THz}$ regime, $\varepsilon_{r_{m}}$, will be reviewed.

First, Table 3.1 shows the DC conductivity $\sigma_{D C}$, the plasma frequency $f_{p}$, the electron collision frequency $f_{\tau}$, and the permittivity at $f=1 \mathrm{THz}$ for 12 well-known metals. Roughly, the plasma frequency represents the frequency at which the metal behaves macroscopically like a near-zero permittivity material, an interesting feature that is pursued with metamaterials [550]-[552]. As can be observed in the table, for bulk metals this occurs in the optical regime. The electron collision frequency is in the order of $\mathrm{THz}$, but larger than usual operating frequencies of the band. All this causes that, at $\mathrm{THz}$ frequencies, the metal have both, real and imaginary parts, large in magnitude and negative. For example, at $f=1 \mathrm{THz}$, it is observed that the imaginary part magnitude for the reviewed metals is on the order of $10^{5}$, and the real part is approximately one order of magnitude smaller.

Second, to better understand the differences between the conventional skin-depth model and the Drude model, and the physical implications of Drude model, several 
graphs are displayed in Fig. 3.5. The range of frequencies considered is $0.1 \mathrm{THz}-100$ $\mathrm{THz}$, extending the upper limit into the mid IR, in order to give a better figure of the effects. The metals considered are Silver and Aluminum, a very good conductor and a moderate-good conductor below $\mathrm{THz}$, respectively.

In Figs. 3.5(a) and 3.5(b), it is shown the magnitude (notice that all the considered parameters are negative) of real and imaginary parts of the equivalent permittivity of these metals for both, skin-depth effect and Drude models. Note that the conventionally assumed approximation of the skin-depth model has only imaginary part, according to (3.94). As can be observed in these graphs, the skin-depth effect model gives good accuracy for the imaginary part up to some frequency in the $\mathrm{THz}$ band, frequency from which the Drude model curve does not follow a lineal law anymore and starts to diverge to smaller values in magnitude. One has that, at the starting of the $\mathrm{THz}$ band (high frequencies of the microwave band), both curves are equal, then the difference must be taken into account for accuracy in the $\mathrm{THz}$ regime, and finally, in the IR, the calculated imaginary part with the conventional skin-depth effect model is several orders of magnitude larger (in the magnitude sense) than the accurate one provided by the Drude model. Let comment now the real part of the permittivity. According to the Drude model, at the microwave regime, the real part of the permittivity remains small compared to the imaginary part, so neglecting it may be assumed, however, when the frequency goes high in the $\mathrm{THz}$ band, both curves converges, and from a certain frequency, the real part becomes even larger in magnitude than the imaginary part. This effect of the increasing contribution of the real part, totally avoided in the skin-depth effect model, has an important influence in the sense that, the propagation characteristics propitiated by a certain metallic structure, given in the last term by Maxwell equations, will be of different nature than those predicted by the skin-depth effect model.

To better view the ratios of interest, in Fig. 3.5(c) it is represented the quotient between the imaginary parts of the skin-effect model and the Drude model, $\operatorname{Im}\left\{\varepsilon_{r_{\text {skin }}}\right\} / \operatorname{Im}\left\{\varepsilon_{r_{\text {Drude }}}\right\}$, and in Fig. 3.5(d) it is represented the quotient between the imaginary and real parts of the permittivity provided by the Drude Model, $\left(\operatorname{Im}\left\{\varepsilon_{r_{\text {Drude }}}\right\} / \operatorname{Re}\left\{\varepsilon_{r_{\text {Drude }}}\right\}\right)$. In Fig. 3.5(c), it is appreciated how fast takes places the difference between both models as the frequency increase. In the case of Silver, the top frequency for the skin-depth model regarding this part of the permittivity may be establish at $f=1 \mathrm{THz}$. From that point, the difference between both models increase exponentially. In Fig. 3.5(d) the contribution of the real part is perfectly determined. In this case, the progression is softer with the frequency, even so, notice that both axis are logarithmic. From this graph it is obvious that at $\mathrm{THz}$, the real part cannot be neglected.

Something that results surprising at the first view in Figs. 3.5(a) and 3.5(b) is the better behavior of the simple skin-effect model in the Aluminum, which is, in principle, a worse conductor than Silver. It is appreciated that the for the Aluminum case, Fig. 3.5(b), the divergence between both models appears later, and the real part in the Drude model remains relative small compared to the imaginary part until the frequency reach larger values. However, if the values of the table 3.1 are reviewed for both metals, one founds that for Aluminum $f_{p}=3570 \mathrm{THz}$ and $f_{\tau}=19.8 \mathrm{THz}$, whereas for Silver $f_{p}=2181 \mathrm{THz}$ and $f_{\tau}=4.4 \mathrm{THz}$. By inspection of (3.96)-(3.98), 


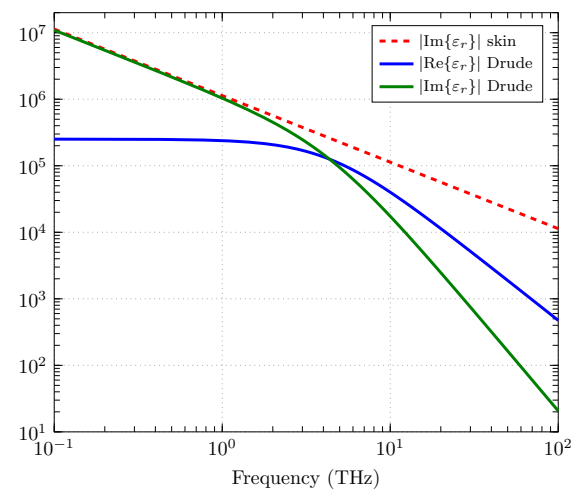

(a)

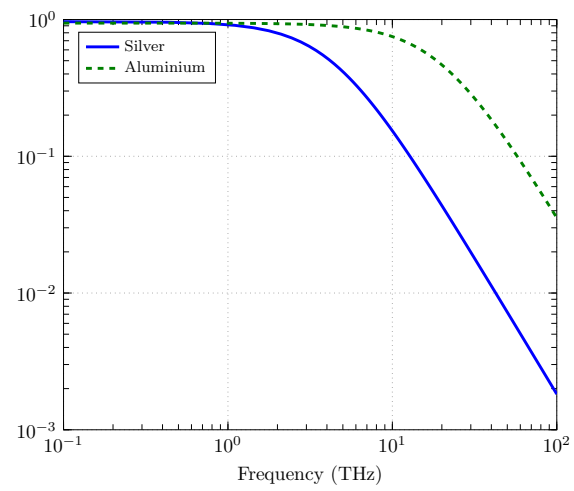

(c)

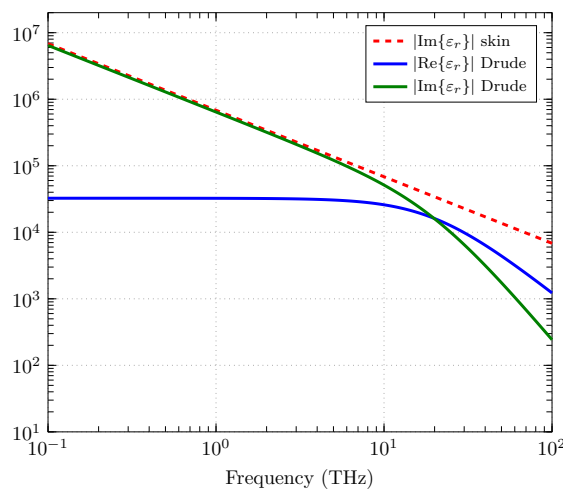

(b)

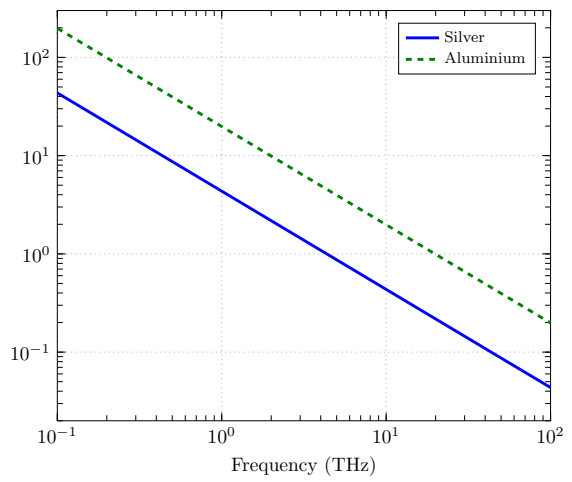

(d)

Figure 3.5: Study of dielectric properties of metals at $\mathrm{THz}$ frequencies (range extended to 100 $\mathrm{THz}$ to observe better the effects). a) Comparative between the relative permittivities provided by the skin-effect model (only imaginary part) and the Drude model for Silver; b) Same as a) for Aluminum; c) Quotient between the imaginary parts of the skin-effect model and the Drude model, $\left(\operatorname{Im}\left\{\varepsilon_{r_{\text {skin }}}\right\} / \operatorname{Im}\left\{\varepsilon_{r_{\text {Drude }}}\right\}\right)$, for Silver and Aluminum; d) Quotient between the imaginary and real parts of the permittivity provided by the Drude Model, $\left(\operatorname{Im}\left\{\varepsilon_{r_{\text {Drude }}}\right\} / \operatorname{Re}\left\{\varepsilon_{r_{\text {Drude }}}\right\}\right.$ ), for Silver and Aluminum.

the value of $f_{p}$ indicates ${ }^{8}$ the frequency at which the real part of the permittivity, blue curve in Figs. 3.5(a) and 3.5(b), reaches the zero value, while the imaginary part, green curve in the previous plots, reaches $\varepsilon_{r_{m}}^{\prime \prime}=f_{\tau} / f_{p}$, which is a small value. This means that $f_{p}$ acts as an anchor in the model, imposing where plasma conditions take place. On the other hand, whenever $f_{p}>>f_{\tau}$, which is always the case, the value of $f_{\tau}$ is a good indicator of the frequency at which real and imaginary parts of the Drude model permittivity become equal, see the expressions (3.96)-(3.98) and Fig. $3.5(\mathrm{~d})$. Since $\left|\varepsilon_{r_{m}}^{\prime \prime}\right|$ is upper bounded by $1-\left(f_{p}^{2}-f_{\tau}^{2}\right)^{2}$ when $f \rightarrow 0$ and $\varepsilon_{r_{m}}^{\prime}$ does not since $\left|\varepsilon_{r_{m}}^{\prime}\right|$ continues growing as $f$ decreases, converging to the skin-effect

${ }^{8}$ According to $(3.97)$, the exact frequency at which $\varepsilon_{r_{m}}^{\prime}=0$ is $f_{\varepsilon_{r_{m}}^{\prime}}=0=\sqrt{f_{p}^{2}-f_{t}^{2}}$, which satisfies $f_{\varepsilon_{r}^{\prime}=0} \approx f_{p}$. 
model, $f_{\tau}$ is a clear indicator of the frequency from which $\left|\varepsilon_{r_{m}}^{\prime \prime}\right|$ folds down, and appreciable differences between both models occur. Therefore, a larger value of $f_{\tau}$ implies that the skin-effect model holds until higher frequencies and the real part can be assumed negligible, whereas the position of $f_{p}$ helps to understand how fast both models diverge. A lower $f_{p}$ implies higher rate of divergence

From these observations it is obvious that, at THz, according to the empirical data [347], the microscopic effects are playing a major role in Silver than Aluminum, exhibiting this last better properties. According to (3.93), a perfect conductor implies $\varepsilon_{r}=-j \infty$. Therefore, the larger the magnitude of the imaginary part, and the smaller the magnitude of the real part, the better is the conductor under study. The comparison between both metals made here does not indicate that Aluminum is better conductor than Silver at $\mathrm{THz}$, since this last presents a larger imaginary part. However, is not as easy as microwaves to predict which conductor will offer better results, since now the real part issue appears.

The complexity of the model, affecting both, material properties and solution of the problem, does not allow to known, a priori, which is the better conductor. Nevertheless, what is true is that, with high probability, usual commercial solvers will predict solutions or behaviors far from reality as the skin-effect model becomes poor with the increasing frequency. This fact, together with other sources of error, e.g. manufacturing, measurements, implies a loss of control on the design process. This explains the usual disagreement between simulations and experiments found on most of the $\mathrm{THz}$ bibliography. It is easy to understand now that, even simple problems, already solved at the microwave regime must be reviewed at $\mathrm{THz}$ frequencies due to the new intervening materials properties, not treated before (see for instance the case of metallic waveguides [356], [348]). It must be noted also that Aluminum is the most suitable metal to be characterized by the skin-depth model without excessive error due to its high values of $f_{p}$ and $f_{\tau}$. However, most of the metals in the table 3.1 exhibit values of $f_{p}$ and $f_{\tau}$ that really question the applicability of the skin-effect model at $\mathrm{THz}$ frequencies in those cases in which the metal modeling is critical (RWG, CWG, SWW, etc.). See for instance the copper features: at $f=1 \mathrm{THz}$, the real part, neglected by the skin-depth effect model is almost one half of the imaginary part. An effort becomes necessary in both, developing adequate analysis tools and getting physical insight into the obtained numerical solutions.

By the previous study it is found that the SWW at $\mathrm{THz}$ will be the problem of a dielectric cylinder waveguide with complex permittivity with large, negative, real and imaginary parts. The dielectric cylinder is a well-known structure for the case of being embedded in an infinite external medium with similar permittivity of the inner region or core, both small and real, since this is the case of the very popular optical fiber [553]. However, as an open waveguide, and even the cylindrical geometry provides the simplest analytical case (rectangular open 2D waveguides are not analytical [21]), the complexity of the solutions of the dielectric cylinder is larger than for usual metallic waveguides, and even the simple case of having high permittivity has not been extensively reviewed, at least compared with the low permittivity case. In the problem under study in this thesis, regarding the analysis of the SWW, not only the permittivity will be extremely high in absolute value, but complex, and having a negative real part, which differs with the case of lossy cylinders. Therefore, to really understand the problem from its roots, getting 
insight in the effects caused by the material (permittivity) and the electrical size of the structure it seems necessary to follow several steps. It is proposed then, to review the following problems, each one with its particular grade of detail, required to obtain the desired information and allowing to link each step to next one:

1. Dielectric cylinder waveguide with high, real $\varepsilon_{r}$.

2. Dielectric cylinder waveguide with complex $\varepsilon_{r}$ (positive real part).

3. Dielectric cylinder waveguide with negative $\varepsilon_{r}$.

4. Dielectric cylinder waveguide with complex $\varepsilon_{r}$ (same complex phase as metals in $\mathrm{THz}$, but with simple, manageable values).

5. Dielectric cylinder waveguide with complex $\varepsilon_{r}$ of metals at $\mathrm{THz}(\mathrm{SWW})$.

The first item is the ideal case of a lossless dielectric cylinder, just making a larger incise in the magnitude of the permittivity. The second case involves the direct analysis of a real cylinder, including the dielectric losses. The third case implies a real $\varepsilon_{r}$, but negative, and it has its practical applications in modeling metals in the optical regime, where the real part is negative and larger than the imaginary part [554], see Fig. 3.5(d). In this case, the implications of the negative sign in the real part of $\varepsilon_{r}$ will be obtained. The fourth step is considered to avoid the less manageable permittivity values obtained for metals at $\mathrm{THz}$, that may obscure the nature of the effects of having a permittivity belonging to the third quadrant of the complex plane. Small and simple numbers will be considered. Finally, the SWW will be afforded with a big amount of information in form of conclusions of previous cases. Note also that, for the analysis of the DCSWW, although perfect conductor will be assumed for this case, the results of the first and second cases will be of utility regarding to the effect of the dielectric. Fortunately, for this waveguide, the presence of the dielectric alleviates the model used for the conductor [490], [497], [500], [522], which will be easily treated as a perfect conductor. The tremendous complexity of a complete modal analysis of a Drude Model based DCSWW, and the small benefit of it (the complete modal information, including modes confined in the metal, are obtained with the analysis of a Drude model based SWW, and the behavior of the DCSWW is estimated to be very similar) make sense to opt for this simplified choice.

\subsection{Dielectric cylinder with real $\varepsilon_{r}$}

\subsubsection{Modal analysis}

The electromagnetic problem of solving the propagation of waveguided modes in a uniform solid core dielectric circular cylinder, Fig. 3.6(a), can be described as follows. Two regions are distinguished when the cross section of the waveguide is observed, Fig. 3.6(b). Circular cylindrical coordinates $(r, \theta, \phi)$, named simply as cylindrical coordinates in the following, are used. Formally: 
- Region 1: $r \leq a$

- Region 2: $r \geq a$

The region 1 is formed by a finite volume dielectric with permittivity $\varepsilon_{r_{1}}$, and, since any magnetic property will be considered, the wavenumber in this media is described by:

$$
k_{1}=\omega \sqrt{\mu 0 \varepsilon_{0} \varepsilon_{r_{1}}}=k_{0} \sqrt{\varepsilon_{r_{1}}}
$$

where $k_{0}$ is the wavenumber in the free space. The region 2 is formed by an infinite volume of dielectric with permittivity $\varepsilon_{r_{2}}$, surrounding the dielectric region 1. It must be pointed out that, generally, $\varepsilon_{r_{2}}=1$, since, except some specific examples given in this section for didactic purposes, the medium of region 2 will be air. However, to keep for the moment a general formulation, the only restriction for the medium 2 will be $\varepsilon_{r_{2}}<\varepsilon_{r_{1}}$, although it will be assumed $\varepsilon_{r_{2}}=1$ unless it is specified. In its more general case, the wavenumber in region 2 is:

$$
k_{2}=\omega \sqrt{\mu 0 \varepsilon_{0} \varepsilon_{r_{2}}}=k_{0} \sqrt{\varepsilon_{r_{2}}}
$$

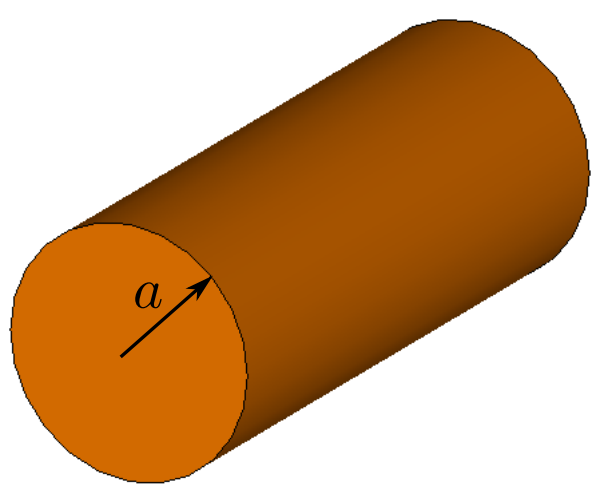

(a)

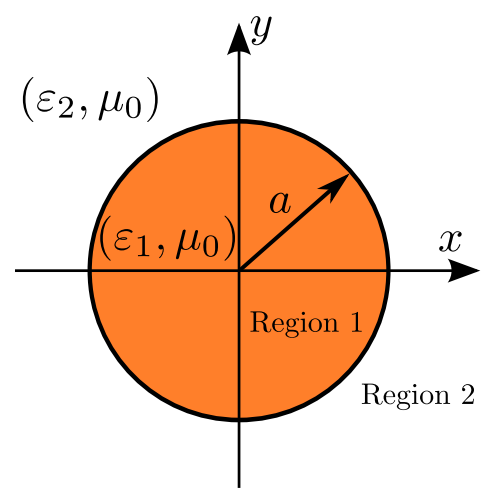

(b)

Figure 3.6: Dielectric Cylinder Waveguide. a) 3D view; b) Problem description.

Let consider now the parameters describing the behavior of the fields in each region by being the argument of the cylindrical functions solution of the problem. In the hollow metallic waveguides, which are described by a unique inner region, this parameter is usually called cutoff wavenumber, since it indicates the wavenumber (and by extension the frequency) from which a particular mode starts to propagate. For single-conductor closed waveguides, the cutoff wavenumber is a constant (therefore the cutoff frequencies and the modal field patterns) as it is provided by the strong Perfect Electric Conductor (PEC) condition of the boundary. This is not the case for a dielectric rod since the boundary condition of continuity of the tangential fields provides that the wavenumber will depend on the frequency. Thus, wavenumbers on the two considered regions will be better called transversal propagation constants. In addition, since $k_{2} \leq \beta \leq k_{1}$ (provided that $\varepsilon_{r_{2}}<\varepsilon_{r_{1}}$ ) it makes sense (to keep working with real numbers for the moment) to define them as follows: 


$$
\begin{aligned}
& \beta_{r_{1}}^{2}=k_{1}^{2}-\beta^{2} \\
& \alpha_{r_{2}}^{2}=\beta^{2}-k_{2}^{2}
\end{aligned}
$$

Note that, in the external medium, the definition of $\alpha_{r_{2}}$ implies a sign change. Now, applying the Maxwell equations, and expressing the Helmholtz equations for electric and magnetic fields, particularized for cylindrical coordinates one has:

$$
\begin{aligned}
& {\left[\frac{1}{r}\left(\frac{\partial}{\partial r} r \frac{\partial}{\partial r}+\frac{\partial}{\partial \phi} \frac{1}{r} \frac{\partial}{\partial \phi}\right)+p^{2}\right] E_{z}=0} \\
& {\left[\frac{1}{r}\left(\frac{\partial}{\partial r} r \frac{\partial}{\partial r}+\frac{\partial}{\partial \phi} \frac{1}{r} \frac{\partial}{\partial \phi}\right)+p^{2}\right] H_{z}=0}
\end{aligned}
$$

where $p=\beta_{r_{1}}$ in the region 1 , and $p=\alpha_{r_{2}}$ in the region 2 . The term $e^{j(\omega t-\beta z)}$ has been suppressed since is common to all the components of the field. Therefore, solution for the axial electric and magnetic field in the region 1 will be given by the standard Bessel functions, whereas for the region 2, the modified Bessel functions are the solution instead [542], due to the change of sign in (3.102). The rest of the six electromagnetic field components are described by:

$$
\begin{aligned}
E_{r} & =\frac{1}{p^{2}}\left(j \beta \frac{\partial E_{z}}{\partial r}-\frac{j \omega \mu}{r} \frac{\partial H_{z}}{\partial \phi}\right) \\
E_{\phi} & =\frac{1}{p^{2}}\left(-\frac{j \beta}{r} \frac{\partial E_{z}}{\partial \phi}+j \omega \mu \frac{\partial H_{z}}{\partial r}\right) \\
H_{r} & =\frac{1}{p^{2}}\left(\frac{j \omega \varepsilon}{r} \frac{\partial E_{z}}{\partial \phi}-j \beta \frac{\partial H_{z}}{\partial r}\right) \\
H_{\phi} & =\frac{1}{p^{2}}\left(-j \omega \varepsilon \frac{\partial E_{z}}{\partial r}-\frac{j \beta}{r} \frac{\partial H_{z}}{\partial \phi}\right)
\end{aligned}
$$

with the corresponding substitution of $p$ for each region. In general, the six components of the field are necessary to fulfill the boundary condition of the problems. These solutions (modes), are therefore hybrid, having both axial components $E_{z}$ and $H_{z}$. The predominance of one or other will indicate the dominant character of the mode (Transversal Electric -TE/H- or Transveral Magnetic -TM/E-) [553]. These hybrid modes will be named as $\mathrm{HE}_{n m}$ and $\mathrm{EH}_{n m}$, where the index $n$ is associated with the azimuthal coordinate $\phi$, and the index $m$ is associated with the radial coordinate. In the case of the index $n$, the complete set comprise both, positive and negative numbers. However, it only influences the term $e^{j n \phi}$, solution of the azimuthal variable. Therefore, it will be assumed $n \geq 0$, and one must take into account that each $n>0$, implies two degenerated modes with a different origen of phase, something usual in cylindrical waveguides, [21], [376]. The eigenfunctions that describe the field of the modes are, therefore:

\section{$\mathbf{H E}_{n m}$ and $\mathbf{E H}_{n m}$ modes:}


$\underline{\text { Region } 1, r \leq a \text { : }}$

$$
\begin{aligned}
E_{z_{1}} & =A \mathrm{~J}_{n}\left(\beta_{r_{1}} r\right) e^{j n \phi} \\
H_{z_{1}} & =B \mathrm{~J}_{n}\left(\beta_{r_{1}} r\right) e^{j n \phi} \\
E_{r_{1}} & =\frac{-j \beta}{\beta_{r_{1}}^{2}}\left[\beta_{r_{1}} A \mathrm{~J}_{n}^{\prime}\left(\beta_{r_{1}} r\right)+\frac{j \omega \mu_{0} n}{\beta r} B \mathrm{~J}_{n}\left(\beta_{r_{1}} r\right)\right] e^{j n \phi} \\
E_{\phi_{1}} & =\frac{-j \beta}{\beta_{r_{1}}^{2}}\left[\frac{j n}{r} A \mathrm{~J}_{n}\left(\beta_{r_{1}} r\right)-\frac{\omega \mu_{0}}{\beta} B \mathrm{~J}_{n}^{\prime}\left(\beta_{r_{1}} r\right)\right] e^{j n \phi} \\
H_{r_{1}} & =\frac{-j \beta}{\beta_{r_{1}}^{2}}\left[\beta_{r_{1}} B \mathrm{~J}_{n}^{\prime}\left(\beta_{r_{1}} r\right)-\frac{j \omega \varepsilon_{1} n}{\beta r} A \mathrm{~J}_{n}\left(\beta_{r_{1}} r\right)\right] e^{j n \phi} \\
H_{\phi_{1}} & =\frac{-j \beta}{\beta_{r_{1}}^{2}}\left[\frac{j n}{r} B \mathrm{~J}_{n}\left(\beta_{r_{1}} r\right)+\frac{\omega \varepsilon_{1}}{\beta} A \mathrm{~J}_{n}^{\prime}\left(\beta_{r_{1}} r\right)\right] e^{j n \phi}
\end{aligned}
$$

$\underline{\text { Region } 2, r \geq a \text { : }}$

$$
\begin{aligned}
E_{z_{2}} & =C \mathrm{~K}_{n}\left(\alpha_{r_{2}} r\right) e^{j n \phi} \\
H z_{2} & =D \mathrm{~K}_{n}\left(\alpha_{r_{2}} r\right) e^{j n \phi} \\
E_{r_{2}} & =\frac{j \beta}{\alpha_{r_{2}}^{2}}\left[\alpha_{r_{2}} C \mathrm{~K}_{n}^{\prime}\left(\alpha_{r_{2}} r\right)+\frac{j \omega \mu_{0} n}{\beta r} D \mathrm{~K}_{n}\left(\alpha_{r_{2}} r\right)\right] e^{j n \phi} \\
E_{\phi_{2}} & =\frac{j \beta}{\alpha_{r_{2}}^{2}}\left[\frac{j n}{r} C \mathrm{~K}_{n}\left(\alpha_{r_{2}} r\right)-\frac{\omega \mu_{0}}{\beta} D \mathrm{~K}_{n}^{\prime}\left(\alpha_{r_{2}} r\right)\right] e^{j n \phi} \\
H_{r_{2}} & =\frac{j \beta}{\alpha_{r_{2}}^{2}}\left[\alpha_{r_{2}} D \mathrm{~K}_{n}^{\prime}\left(\alpha_{r_{2}} r\right)-\frac{j \omega \varepsilon_{2} n}{\beta r} C \mathrm{~K}_{n}\left(\alpha_{r_{2}} r\right)\right] e^{j n \phi} \\
H_{\phi_{2}} & =\frac{j \beta}{\alpha_{r_{2}}^{2}}\left[\frac{j n}{r} D \mathrm{~K}_{n}\left(\alpha_{r_{2}} r\right)+\frac{\omega \varepsilon_{2}}{\beta} C \mathrm{~K}_{n}^{\prime}\left(\alpha_{r_{2}} r\right)\right] e^{j n \phi}
\end{aligned}
$$

where $\mathrm{J}_{n}(x)$ is the Bessel function of first kind, $\mathrm{K}_{n}(x)$ is the modified Bessel function of second kind, respectively, and the primes over $\mathrm{J}_{n}^{\prime}$ and $\mathrm{K}_{n}^{\prime}$ indicate derivation with respect to their arguments, $\beta_{r_{1}} r$ and $\alpha_{r_{2}} r$, respectively. Note that the Bessel function of second kind $\mathrm{Y}_{n}(x)$ cannot be considered for the region 1 since it is singular at the origin, and the modified Bessel function of second kind $\mathrm{I}_{n}(x)$ cannot be considered for the outer region since it describes and exponential grow, thus not satisfying the radiation condition of region $2 .(A, B, C, D)$ are arbitrary constants. Now, applying the following boundary conditions, which describe the continuity of the tangential fields:

$$
\begin{aligned}
E_{z_{1}}(a, \phi) & =E_{z_{2}}(a, \phi) \\
H_{z_{1}}(a, \phi) & =H z_{2}(a, \phi) \\
E_{\phi_{1}}(a, \phi) & =E_{\phi_{2}}(a, \phi) \\
H_{\phi_{1}}(a, \phi) & =H_{\phi_{2}}(a, \phi),
\end{aligned}
$$

it is found that 


$$
A \mathrm{~J}_{n}\left(\beta_{r_{1}} a\right)-C \mathrm{~K}_{n}\left(\alpha_{r_{2}} a\right)=0
$$

$$
B \mathrm{~J}_{n}\left(\beta_{r_{1}} a\right)-D \mathrm{~K}_{n}\left(\alpha_{r_{2}} a\right)=0
$$

$$
\begin{aligned}
A\left(\frac{j n}{\beta_{r_{1}}^{2} a} \mathrm{~J}_{n}\left(\beta_{r_{1}} a\right)\right) & +B\left(-\frac{\omega \mu_{0}}{\beta_{r_{1}} \beta} \mathrm{J}_{n}^{\prime}\left(\beta_{r_{1}} a\right)\right)+ \\
& +C\left(\frac{j n}{\alpha_{r_{2}}^{2} a} \mathrm{~K}_{n}\left(\alpha_{r_{2}} a\right)\right)+D\left(-\frac{\omega \mu_{0}}{\alpha_{r_{2}} \beta} \mathrm{K}_{n}^{\prime}\left(\alpha_{r_{2}} a\right)\right)=0
\end{aligned}
$$

$$
\begin{aligned}
A\left(\frac{\omega \varepsilon_{1}}{\beta_{r_{1}} \beta} \mathrm{J}_{n}^{\prime}\left(\beta_{r_{1}} a\right)\right)+ & B\left(\frac{j n}{\beta_{r_{1}}^{2} a} \mathrm{~J}_{n}\left(\beta_{r_{1}} a\right)\right)+ \\
& +C\left(\frac{\omega \varepsilon_{2}}{\alpha_{r_{2}} \beta} \mathrm{K}_{n}^{\prime}\left(\alpha_{r_{2}} a\right)\right)+D\left(\frac{j n}{\alpha_{r_{2}}^{2} a} \mathrm{~K}_{n}\left(\alpha_{r_{2}} a\right)\right)=0
\end{aligned}
$$

Note at this point the necessity of the six components of the field unless $n=0$. If the mode was TM, $B=D=0$, and this would make (3.127) to be incompatible with (3.125). In a dual way, if the mode was TE, $A=C=0$, and this would make (3.128) to be incompatible with (3.126). Also, notice that this has its origin on the more complex nature of the continuity boundary condition respect to the simpler one imposed by perfect conductors in metallic, single-conductor, closed waveguides. These four equations (3.125)-(3.128) describe a homogeneous linear system on the unknowns $(A, B, C, D)$ that can be expressed in its matrix form as:

$$
\left[\begin{array}{cccc}
m_{11} & 0 & m_{13} & 0 \\
0 & m_{22} & 0 & m_{24} \\
m_{31} & m_{32} & m_{33} & m_{34} \\
m_{41} & m_{42} & m_{43} & m_{44}
\end{array}\right]\left[\begin{array}{l}
A \\
B \\
C \\
D
\end{array}\right]=\left[\begin{array}{l}
0 \\
0 \\
0 \\
0
\end{array}\right]
$$

where 


$$
\begin{aligned}
m_{11} & =\mathrm{J}_{n}\left(\beta_{r_{1}} a\right) \\
m_{13} & =-\mathrm{K}_{n}\left(\alpha_{r_{2}} a\right) \\
m_{22} & =\mathrm{J}_{n}\left(\beta_{r_{1}} a\right) \\
m_{24} & =-\mathrm{K}_{n}\left(\alpha_{r_{2}} b\right) \\
m_{31} & =\frac{j n}{\beta_{r_{1}}^{2} a} \mathrm{~J}_{n}\left(\beta_{r_{1}} a\right) \\
m_{32} & =-\frac{\omega \mu_{0}}{\beta_{r_{1}} \beta} \mathrm{J}_{n}^{\prime}\left(\beta_{r_{1}} a\right) \\
m_{33} & =\frac{j n}{\alpha_{r_{2}}^{2} a} \mathrm{~K}_{n}\left(\alpha_{r_{2}} a\right) \\
m_{34} & =-\frac{\omega \mu_{0}}{\alpha_{r_{2}} \beta} \mathrm{K}_{n}^{\prime}\left(\alpha_{r_{2}} a\right) \\
m_{41} & =\frac{\omega \varepsilon_{1}}{\beta_{r_{1}} \beta} \mathrm{J}_{n}^{\prime}\left(\beta_{r_{1}} a\right) \\
m_{42} & =\frac{j n}{\beta_{r_{1}}^{2} a} \mathrm{~J}_{n}\left(\beta_{r_{1}} a\right) \\
m_{43} & =\frac{\omega \varepsilon_{2}}{\alpha_{r_{2}} \beta} \mathrm{K}_{n}^{\prime}\left(\alpha_{r_{2}} a\right) \\
m_{44} & =\frac{j n}{\alpha_{r_{2}}^{2} a} \mathrm{~K}_{n}\left(\alpha_{r_{2}} a\right)
\end{aligned}
$$

Due to its homogenous nature, the system in (3.129) provide non trivial solutions only in the case its determinant vanish. Therefore, given the waveguide parameters and the operation frequency, only certain discrete values of the propagation constant $\beta$ allow for non-zero $(A, B, C, D)$. The expression for the determinant of the matrix in $(3.129)$ is

$$
\begin{aligned}
& \frac{\varepsilon_{r_{1}}}{\left(\beta_{r_{1}} a\right)^{2}} \cdot \frac{\mathrm{J}_{n}^{\prime 2}\left(\beta_{r_{1}} a\right)}{\mathrm{J}_{n}^{2}\left(\beta_{r_{1}} a\right)}+\frac{\varepsilon_{r_{2}}}{\left(\alpha_{r_{2}} a\right)^{2}} \cdot \frac{\mathrm{K}_{n}^{\prime 2}\left(\alpha_{r_{2}} a\right)}{\mathrm{K}_{n}^{2}\left(\alpha_{r_{2}} a\right)}+\frac{\varepsilon_{r_{1}}}{\beta_{r_{1}} \alpha_{r_{2}} a^{2}} \cdot \frac{\mathrm{J}_{n}^{\prime}\left(\beta_{r_{1}} a\right) \mathrm{K}_{n}^{\prime}\left(\alpha_{r_{2}} a\right)}{\mathrm{J}_{n}\left(\beta_{r_{1}} a\right) \mathrm{K}_{n}\left(\alpha_{r_{2}} a\right)} \\
& +\frac{\varepsilon_{r_{2}}}{\beta_{r_{1}} \alpha_{r_{2}} a^{2}} \cdot \frac{\mathrm{J}_{n}^{\prime}\left(\beta_{r_{1}} a\right) \mathrm{K}_{n}^{\prime}\left(\alpha_{r_{2}} a\right)}{\mathrm{J}_{n}\left(\beta_{r_{1}} a\right) \mathrm{K}_{n}\left(\alpha_{r_{2}} a\right)}-n^{2}\left(\frac{\beta}{k_{0}}\right)^{2} \cdot\left(\frac{1}{\left(\beta_{r_{1}} a\right)^{2}}+\frac{1}{\left(\alpha_{r_{2}} a\right)^{2}}\right)^{2}=0
\end{aligned}
$$

which is a transcendental equation, i.e., any closed form must be obtained for $\beta$ as a function of the waveguide parameters and the frequency, but must be solved numerically for each case. It must be mentioned that in some of the involved terms, they have been multiplied both numerator and denominator by the factor $a$ in order to leave the expressions as a function of the parameters $\beta_{r_{1}} a$ and $\alpha_{r_{2}} a$, which gives more physical insight and results helpful in the numerical resolution of the problem.

Once (3.142) is solved and a certain eigenvalue $\beta$ of a particular mode is obtained, since the system is not full-rank, $A$ is left as a parameter ${ }^{9}$ and the ratios $B / A, C / A$

\footnotetext{
${ }^{9}$ The value that $A$ takes for a certain mode will depend on the excitation. The real interest resides in the ratios $B / A, C / A$ and $D / A$ since they describe the relation between components of a determinate mode.
} 
and $D / A$ can be solved. This ratios are of physical importance since they govern the mode field pattern. Particularly, $B / A$ is of great importance, since it relates the electric and magnetic axial field components. The expressions for these constants are:

$$
\begin{aligned}
\frac{B}{A} & =\frac{j \beta n}{\omega \mu_{0}}\left[\frac{1}{\left(\beta_{r_{1}} a\right)^{2}}+\frac{1}{\left(\alpha_{r_{2}} a\right)^{2}}\right]\left[\frac{\mathrm{J}_{n}^{\prime}\left(\beta_{r_{1}} a\right)}{\left(\beta_{r_{1}} a\right) \mathrm{J}_{n}\left(\beta_{r_{1}} a\right)}+\frac{\mathrm{K}_{n}^{\prime}\left(\alpha_{r_{2}} a\right)}{\left(\alpha_{r_{2}} a\right) \mathrm{K}_{n}\left(\alpha_{r_{2}} a\right)}\right]^{-1} \\
\frac{C}{A} & =\frac{\mathrm{J}_{n}\left(\beta_{r_{1}} a\right)}{\mathrm{K}_{n}\left(\alpha_{r_{2}} a\right)} \\
\frac{D}{A} & =\frac{\mathrm{J}_{n}\left(\beta_{r_{1}} a\right)}{\mathrm{K}_{n}\left(\alpha_{r_{2}} a\right)} \frac{B}{A}
\end{aligned}
$$

Therefore, by (3.142), the dispersion diagram of the dielectric cylinder waveguide can be obtained. In addition, by (3.143)-(3.145), the field pattern of each mode can be solved. Thus, at this point, the first stage of a waveguide analysis could be already carried out. However, several reasons justify few more algebraic manipulations and some particularization to distinguish the nature of the involved modes, classified in four groups: HE, EH, TM and TE.

Firstly, the waveguide under analysis is simple in such way that analyzing the involved equations more carefully gives very interesting physical insight, that otherwise would be obscured by the equations complexity, caused by having more complex waveguide parameters, either geometrical or constitutive. Since the later studied structures in this thesis are more complex, some conclusions extracted thanks to this simpler effort now will be applied then, as when a room gets darker a candle that one brings gives light. Secondly, it results much more convenient for numerical calculations in every of the considered cases in this thesis. This occurs not only because of the reduced complexity of some particular cases, but also because in order cases helps to avoid numerical errors and issues on the programming of the involved algorithms, something that becomes challenging when complex variables enter in scene. Finally, the fundamental mode of the SWW and the main fundamental mode of the DCSWW are of TM nature, thus being interesting to look carefully in the particular characteristics of this kind of mode.

Firstly, separation of $\mathrm{HE}$ and $\mathrm{EH}$ modes is carried out. The equation (3.142) is a quadratic equation (some algebraic manipulations are necessary to directly observe this fact) on the term ${ }^{10} \frac{\mathrm{J}_{n}^{\prime}\left(\beta_{r_{1}} a\right)}{\left(\beta_{r_{1}} a\right) \mathrm{J}_{n}^{\prime}\left(\beta_{r_{1}} a\right)}$, thus, solved for this term it gives

$$
\frac{\mathrm{J}_{n}^{\prime}\left(\beta_{r_{1}} a\right)}{\beta_{r_{1}} a \mathrm{~J}_{n}^{\prime}\left(\beta_{r_{1}} a\right)}=-\left(\frac{\varepsilon_{r_{1}}+\varepsilon_{r_{2}}}{2 \varepsilon_{r_{1}}}\right) \frac{\mathrm{K}_{n}^{\prime}\left(\alpha_{r_{2}} a\right)}{\alpha_{r_{2}} a \mathrm{~K}_{n}^{\prime}\left(\alpha_{r_{2}} a\right)} \pm R
$$

with $R$ given by

\footnotetext{
${ }^{10} \mathrm{It}$ may be postulated as quadratic on the term $\frac{\mathrm{K}_{n}^{\prime}\left(\alpha_{r_{2}} a\right)}{\left(\alpha_{r_{2}} a\right) \mathrm{K}_{n}^{\prime}\left(\alpha_{r_{2}} a\right)}$, this has been observed, however, less convenient in practice)
} 


$$
\begin{aligned}
R=\left[\left(\frac{\varepsilon_{r_{1}}-\varepsilon_{r_{2}}}{2 \varepsilon_{r_{1}}}\right)^{2}\left(\frac{\mathrm{K}_{n}^{\prime}\left(\alpha_{r_{2}} a\right)}{\alpha_{r_{2}} a \mathrm{~K}_{n}^{\prime}\left(\alpha_{r_{2}} a\right)}\right)^{2}\right. & \\
& \left.\quad+\frac{n^{2}}{\varepsilon_{r_{1}}}\left(\frac{\beta}{k_{0}}\right)^{2}\left(\frac{1}{\left(\beta_{r_{1}} a\right)^{2}}+\frac{1}{\left(\alpha_{r_{2}} a\right)^{2}}\right)^{2}\right]^{1 / 2}
\end{aligned}
$$

Now, by using the derivative relations of Bessel functions

$$
\begin{aligned}
\mathrm{J}_{n}^{\prime}(x) & =-J_{n+1}(x)+\frac{n}{x} \mathrm{~J}_{n}(x) \\
\mathrm{J}_{n}^{\prime}(x) & =J_{n-1}(x)-\frac{n}{x} \mathrm{~J}_{n}(x)
\end{aligned}
$$

one has, for the positive sign, the equation for EH modes:

$$
\frac{\mathrm{J}_{n+1}\left(\beta_{r_{1}} a\right)}{\left(\beta_{r_{1}} a\right) \mathrm{J}_{n}^{\prime}\left(\beta_{r_{1}} a\right)}=\left(\frac{\varepsilon_{r_{1}}+\varepsilon_{r_{2}}}{2 \varepsilon_{r_{1}}}\right) \frac{\mathrm{K}_{n}^{\prime}\left(\alpha_{r_{2}} a\right)}{\left(\alpha_{r_{2}} a\right) \mathrm{K}_{n}^{\prime}\left(\alpha_{r_{2}} a\right)}+\left(\frac{n}{\left(\alpha_{r_{2}}\right)^{2}}-R\right)
$$

and for the negative sign, the equation for HE modes:

$$
\frac{\mathrm{J}_{n-1}\left(\beta_{r_{1}} a\right)}{\left(\beta_{r_{1}} a\right) \mathrm{J}_{n}^{\prime}\left(\beta_{r_{1}} a\right)}=-\left(\frac{\varepsilon_{r_{1}}+\varepsilon_{r_{2}}}{2 \varepsilon_{r_{1}}}\right) \frac{\mathrm{K}_{n}^{\prime}\left(\alpha_{r_{2}} a\right)}{\left(\alpha_{r_{2}} a\right) \mathrm{K}_{n}^{\prime}\left(\alpha_{r_{2}} a\right)}+\left(\frac{n}{\left(\alpha_{r_{2}}\right)^{2}}-R\right)
$$

In this way the set of solutions of (3.142) has been separated in two independent sets, given now by (3.152) and (3.153).

Let now consider the particular case of $n=0$, the axialsymmetric $\left(\frac{\partial}{\partial \phi}=0\right)$ modes. Whereas in the general case of $n \neq 0$ the boundary conditions described by (3.125)-(3.128) require from the six components of the electromagnetic field to not vanish, when $n=0$ some terms in the expressions become null, thus being possible a TM or TE solution satisfying these boundary conditions. Therefore, for the axialsymmetric modes, the 4 x 4 matrix of (3.130) decouples in two $2 \times 2$ independent matrix. The process is better understood following the same previous steps:

\section{$\mathrm{TM}_{0 m}$ modes:}

$\underline{\text { Region } 1, r \leq a \text { : }}$ 


$$
\begin{aligned}
E_{z_{1}} & =A \mathrm{~J}_{0}\left(\beta_{r_{1}} r\right) \\
H_{z_{1}} & =0 \\
E_{r_{1}} & =\frac{j \beta}{\beta_{r_{1}}} A \mathrm{~J}_{1}\left(\beta_{r_{1}} r\right) \\
E_{\phi_{1}} & =0 \\
H_{r_{1}} & =0 \\
H_{\phi_{1}} & =\frac{j \omega \varepsilon_{1}}{\beta_{r_{1}}} A \mathrm{~J}_{1}\left(\beta_{r_{1}} r\right)
\end{aligned}
$$

$\underline{\text { Region } 2, r \geq a \text { : }}$

$$
\begin{aligned}
E_{z_{2}} & =C \mathrm{~K}_{0}\left(\alpha_{r_{2}} r\right) \\
H z_{2} & =0 \\
E_{r_{2}} & =-\frac{j \beta}{\alpha_{r_{2}}} C \mathrm{~K}_{1}\left(\alpha_{r_{2}} r\right) \\
E_{\phi_{1}} 2 & =0 \\
H_{r_{1}} 2 & =0 \\
H_{\phi_{2}} & =-\frac{j \omega \varepsilon_{2}}{\alpha_{r_{2}}} C \mathrm{~K}_{1}\left(\alpha_{r_{2}} r\right)
\end{aligned}
$$

note in the signs that, for this particular case, since $n$ is defined, derivatives are already applied and incorporated, i.e., $\mathrm{J}_{0}^{\prime}(x)=-\mathrm{J}_{1}(x)$ and $\mathrm{K}_{0}^{\prime}(x)=-\mathrm{K}_{1}(x)$. In this case, the boundary conditions are specified over $E_{z}$ and $H_{\phi}$ :

$$
\begin{aligned}
A \mathrm{~J}_{0}\left(\beta_{r_{1}} a\right) & =C \mathrm{~K}_{0}\left(\alpha_{r_{2}} a\right) \\
\frac{\varepsilon_{1}}{\beta_{r_{1}}} A \mathrm{~J}_{1}\left(\beta_{r_{1}} a\right) & =-\frac{\varepsilon_{2}}{\alpha_{r_{2}}} C \mathrm{~K}_{1}\left(\alpha_{r_{2}} a\right),
\end{aligned}
$$

expressed in matrix form:

$$
\left[\begin{array}{cc}
\mathrm{J}_{0}\left(\beta_{r_{1}} a\right) & -\mathrm{K}_{1}\left(\alpha_{r_{2}} a\right) \\
\frac{\varepsilon_{1}}{\beta_{r_{1}}} \mathrm{~J}_{1}\left(\beta_{r_{1}} a\right) & \frac{\varepsilon_{2}}{\alpha_{r_{2}}} \mathrm{~K}_{1}\left(\alpha_{r_{2}} a\right)
\end{array}\right]\left[\begin{array}{l}
A \\
C
\end{array}\right]=\left[\begin{array}{l}
0 \\
0
\end{array}\right]
$$

which determinant, after some algebraic manipulations, gives the following transcendental dispersion equation for the $\mathrm{TM}_{0 m}$ modes:

$$
\frac{\mathrm{K}_{1}\left(\alpha_{r_{2}} a\right)}{\left(\alpha_{r_{2}} a\right) \mathrm{K}_{0}\left(\alpha_{r_{2}} a\right)}+\frac{\varepsilon_{r_{1}}}{\varepsilon_{r_{2}}} \frac{\mathrm{J}_{1}\left(\beta_{r_{1}} a\right)}{\left(\beta_{r_{1}} a\right) \mathrm{J}_{0}\left(\beta_{r_{1}} a\right)}=0
$$


which results much simpler than (3.142). Once solved, the fields are determined from the value of $\frac{C}{A}$, obtained from $(3.166)$ as

$$
\frac{C}{A}=\frac{\mathrm{J}_{0}\left(\beta_{r_{1}} a\right)}{\mathrm{K}_{0}\left(\alpha_{r_{2}} a\right)}
$$

In a similar way,

\section{$\underline{\mathrm{TE}_{0 m} \text { modes: }}$}

$\underline{\text { Region } 1, r \leq a \text { : }}$

$$
\begin{aligned}
E_{z_{1}} & =0 \\
H_{z_{1}} & =B \mathrm{~J}_{0}\left(\beta_{r_{1}} r\right) \\
E_{r_{1}} & =0 \\
E_{\phi_{1}} & =-\frac{j \omega \mu_{0}}{\beta_{r_{1}}} B \mathrm{~J}_{1}\left(\beta_{r_{1}} r\right) \\
H_{r_{1}} & =\frac{j \beta}{\beta_{r_{1}}} B \mathrm{~J}_{1}\left(\beta_{r_{1}} r\right) \\
H_{\phi_{1}} & =0
\end{aligned}
$$

$\underline{\text { Region } 2, r \geq a \text { : }}$

$$
\begin{aligned}
E_{z_{2}} & =0 \\
H z_{2} & =D \mathrm{~K}_{0}\left(\alpha_{r_{2}} r\right) \\
E_{r_{2}} & =0 \\
E_{\phi_{2}} & =\frac{j \omega \mu_{0}}{\alpha_{r_{2}}} D \mathrm{~K}_{1}\left(\alpha_{r_{2}} r\right) \\
H_{r_{2}} & =-\frac{j \beta}{\alpha_{r_{2}}} D \mathrm{~K}_{1}\left(\alpha_{r_{2}} r\right) \\
H_{\phi_{1}} 2 & =0
\end{aligned}
$$

with boundary conditions equations

$$
\begin{gathered}
B \mathrm{~J}_{0}\left(\beta_{r_{1}} a\right)=D \mathrm{~K}_{0}\left(\alpha_{r_{2}} a\right) \\
\frac{1}{\beta_{r_{1}}} B \mathrm{~J}_{1}\left(\beta_{r_{1}} a\right)=-\frac{1}{\alpha_{r_{2}}} D \mathrm{~K}_{1}\left(\alpha_{r_{2}} a\right), \\
{\left[\begin{array}{cc}
\mathrm{J}_{0}\left(\beta_{r_{1}} a\right) & -\mathrm{K}_{1}\left(\alpha_{r_{2}} a\right) \\
\frac{1}{\beta_{r_{1}}} \mathrm{~J}_{1}\left(\beta_{r_{1}} a\right) & \frac{1}{\alpha_{r_{2}}} \mathrm{~K}_{1}\left(\alpha_{r_{2}} a\right)
\end{array}\right]\left[\begin{array}{l}
B \\
D
\end{array}\right]=\left[\begin{array}{l}
0 \\
0
\end{array}\right]}
\end{gathered}
$$


which determinant, after some algebraic manipulations, gives the following transcendental dispersion equation for the $\mathrm{TE}_{0 m}$ modes:

$$
\frac{\mathrm{K}_{1}\left(\alpha_{r_{2}} a\right)}{\left(\alpha_{r_{2}} a\right) \mathrm{K}_{0}\left(\alpha_{r_{2}} a\right)}+\frac{\mathrm{J}_{1}\left(\beta_{r_{1}} a\right)}{\left(\beta_{r_{1}} a\right) \mathrm{J}_{0}\left(\beta_{r_{1}} a\right) 0}=0
$$

Once solved, the relationship of the constants $B$ and $D$ is the same as it was $\frac{C}{A}$ for $\mathrm{TM}_{0 m}$ modes:

$$
\frac{D}{B}=\frac{\mathrm{J}_{0}\left(\beta_{r_{1}} a\right)}{\mathrm{K}_{0}\left(\alpha_{r_{2}} a\right)}
$$

The dispersion equation of $\mathrm{TE}_{0 m}$ modes is very similar to that of $\mathrm{TM}_{0 m}$ modes, however, the small difference between them results to be a key point. Note that, whereas in the dispersion equation for $\mathrm{TM}_{0 m}$ modes the factor $\frac{\varepsilon_{r_{1}}}{\varepsilon_{r_{2}}}$ (dielectric contrast between media) multiplies the second term, this factor does not appear in the dispersion equation for $\mathrm{TE}_{0 m}$ modes. Thus, it can be foreseen that, for the cases were $\varepsilon_{r_{1}} \approx \varepsilon_{r_{2}}, \mathrm{TM}_{0 m}$ and $\mathrm{TE}_{0 m}$ will propagate very similar, e.g, optical fibers [553]. However, for the cases were $\varepsilon_{r_{1}}>>\varepsilon_{r_{2}}$, which is the case of interest for the posterior analysis, the difference will be accentuated. Note also that this difference does not regards exclusively to the propagation constant, but will be directly translated to the field components, something that can be easily understood taking into account the expressions for the transversal propagation constant of each region, (3.101) and (3.102), and looking to the fields expression for the considered modes.

Regarding to this question, let recover now the dispersion equations for $\mathrm{HE}_{n m}$ and $\mathrm{EH}_{n m}$ modes, (3.153) and (3.152). If we let $n$ to be 0 in those equations, firstly, $R$ becomes

$$
R=\left(\frac{\varepsilon_{r_{1}}-\varepsilon_{r_{2}}}{2 \varepsilon_{r_{1}}}\right) \frac{\mathrm{K}_{n}^{\prime}\left(\alpha_{r_{2}} a\right)}{\alpha_{r_{2}} a \mathrm{~K}_{n}^{\prime}\left(\alpha_{r_{2}} a\right)}
$$

and now is easy to realize that $\mathrm{HE}_{n m}$ modes dispersion equation becomes that of $\mathrm{TM}_{0 m}$ modes, and that $\mathrm{EH}_{n m}$ modes dispersion equation becomes that of $\mathrm{TE}_{0 m}$ modes. Thus, at this point, it is observed that $\left(\mathrm{TM}_{0 m} / \mathrm{HE}_{n m}\right)$ modes are related, and share some characteristics. The same occur for $\left(\mathrm{TE}_{0 m} / \mathrm{EH}_{n m}\right)$ modes. In fact, with some inspection in (3.153) and (3.152) it is found that $\varepsilon_{r_{1}} / \varepsilon_{r_{2}}$ has much more influence in (3.153), the equation for $\mathrm{HE}_{n m}$ modes.

From the previous statements, one could say that the nomenclature may result contradictory, since it seems that, for example, HE makes more sense to design $\mathrm{H}$ predominant modes, i.e., TE modes. This question is quite controversial, and although hybrid modes have been treated since quite far, some recent works affording the nomenclature issue can still be found [541]. What occurs is that HE modes 
evolve to TE (or H modes) when the cylindrical dielectric rod problem evolves to the cylindrical waveguide problem. However, the previous analysis has given evidence that, at least, while dealing with the cylindrical dielectric rod, HE modes have more TM modes features than TE modes features. Despite of this fact, the presented nomenclature will be maintained to keep consistence with the classical studies of the problem [553], something that is also found in works dealing with the SWW [430].

At this point, given the radius of the waveguide $a$, the frequency of operation $f$, and the permittivity $\varepsilon_{r}$, a complete dispersion analysis could be already afforded. However, for this simple, canonical case, all this three parameters can be synthesized into a unique one which posses all the information regarding the waveguiding process. It is defined as normalized frequency $V$ :

$$
V=\frac{2 \pi a}{\lambda_{0}} \sqrt{\varepsilon_{r_{1}}-\varepsilon_{r_{2}}}=\left(\beta_{r_{1}} a\right)^{2}+\left(\alpha_{r_{2}} a\right)^{2}
$$

the last equality easily obtained from (3.101) and (3.102). This parameter results very useful to get physical insight since it indicates the electrical size of the waveguide respect to the external medium, which is the capacity of the waveguide to confine the energy and to have waveguiding features.

When some mode starts to propagate, the propagation constant of this mode is $\beta=k_{2}$. Progressively, as the frequency (or the radius) increases, the propagation constant grows towards the asymptotic limit of $\beta=k_{1}$, the propagation constant in the dielectric medium, as has been pointed out in the section 3.1. Therefore, when $\beta=k_{2}$, by (3.102) $\alpha_{r_{2}}=0$, i. e., the transversal field attenuation is null and the mode is a plane wave in the external medium. According to (3.189), in this situation, $V=\beta_{r_{1}} a$. In any other case, it is also obvious that $V<\beta_{r_{1}} a$. Therefore, the condition $0 \leq \beta_{r_{1}} a \leq V$ will be always satisfied, and $V$ establishes in this way the propagation properties of the mode. As a matter of fact, this parameter allows to have a constant cutoff frequency, since, at it will be seen, for most of the modes, there is a constant value of $V$ from which they start to propagate in the surface waveguide sense. This is the reason why this parameter is commonly used in the analysis of optical fibers [553]. It can be now easily understood how optical fibers can operate in single mode in spite to be electrically large: inner and outer region have very similar permittivity, therefore $\varepsilon_{r_{1}}-\varepsilon_{r_{2}}$ is very small so the value of $V$ remains small for large $a$ according to (3.189).

In Fig. 3.7 the dispersion diagrams for several cases of the permittivity are displayed, including the first 12 modes that propagate. Note that, as is usual in dielectric waveguides, the normalized propagation constant $\beta / k_{0}$ is displayed instead of $\beta$. In this way, the dispersion diagram encompasses the range $\sqrt{\varepsilon_{r_{2}}}<\beta / k_{0}<$ $\sqrt{\varepsilon_{r_{1}}}$, being here $\varepsilon_{r_{2}}=1$ for most of the cases, as indicated before. Horizontal dashed lines are displayed at $\beta / k_{0}=\sqrt{\varepsilon_{r_{2}}}$ and $\beta / k_{0}=\sqrt{\varepsilon_{r_{1}}}$, the lower and upper limits of $\beta / k_{0}$, respectively.

The first case, Fig. 3.7(a), correspond to the typical case of an optical fiber. Exceptionally, in this case, the outer medium is a dielectric different from air, adopting usual values used in practice [553]: $\varepsilon_{r_{1}} \approx \varepsilon_{r_{2}}=2$ (numerically: $\varepsilon_{r_{1}}=2.001$ and $\left.\varepsilon_{r_{1}}=2\right)$. In the graph, it can be appreciated that there is a clear modal degeneration by groups. This is the origin of the well-know Linearly Polarized (LP) [553] modes $\mathrm{LP}_{n m}: \mathrm{LP}_{01}\left(2 \mathrm{xHE}_{11}\right), \mathrm{LP}_{11}\left(2 \mathrm{xHE}_{21}, \mathrm{TM}_{01}, \mathrm{TE}_{01}\right), \mathrm{LP}_{21}\left(2 \mathrm{xEH}_{11}, 2 \mathrm{xHE}_{31}\right)$, 
$\mathrm{LP}_{02}\left(2 \mathrm{xHE}_{12}\right), \mathrm{LP}_{31}\left(2 \mathrm{xEH}_{21}, 2 \mathrm{xHE}_{41}\right), \mathrm{LP}_{12}\left(2 \mathrm{xHE}_{22}, \mathrm{TM}_{02}, \mathrm{TE}_{02}\right)$, where the factor $2 \mathrm{x}$ arises due to the aforementioned degeneration in the index $n$ when this is not 0 .

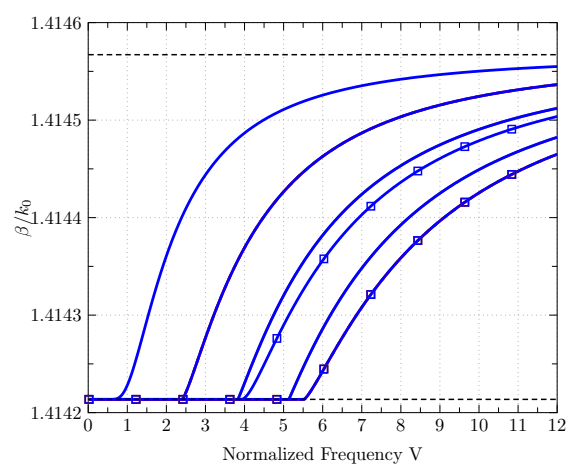

(a)

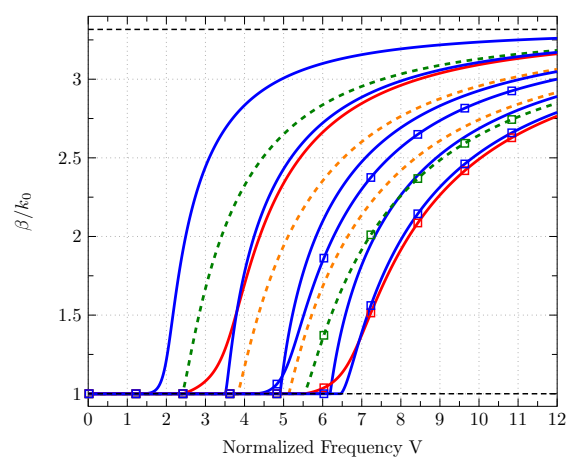

(c)

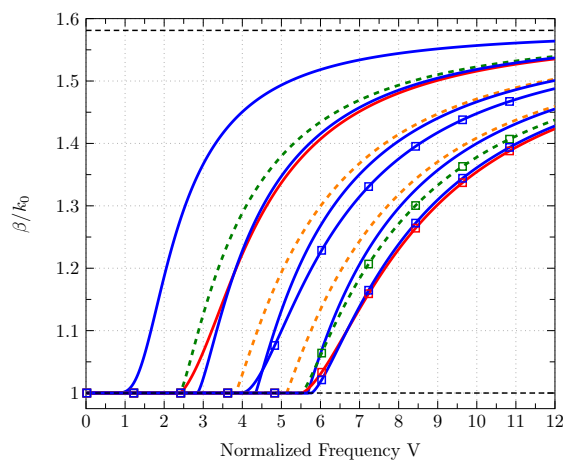

(b)

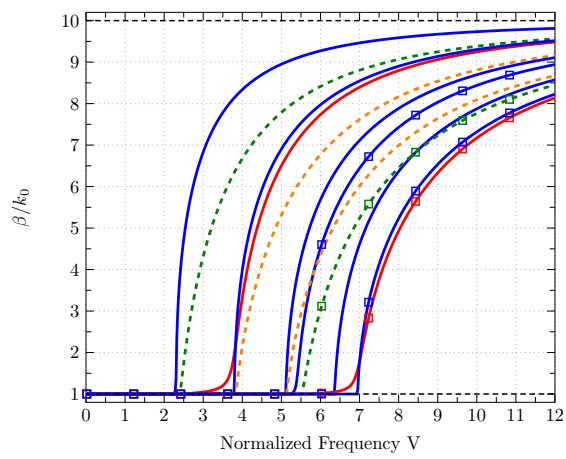

(d)

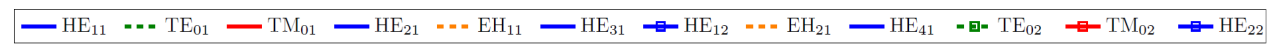

(e)

Figure 3.7: Dispersion diagram of a cylindrical dielectric waveguide. a) $\varepsilon_{r} \approx \varepsilon_{r 2}$. This is the typical case of an optical fiber, with inner and outer region having similar permittivity (low permittivity dielectrics); b) $\varepsilon_{r}=2.5$; c) $\varepsilon_{r}=11$; d) $\varepsilon_{r}=100$; e) Mode legend. For conceptual clarity, the same curve color is used for all modes of the same type $\left(\mathrm{TM}_{0 m}, \mathrm{TE}_{0 m}, \mathrm{HE}_{n m}, \mathrm{EH}_{n m}\right)$ no matter the index $n$. Markers are added to distinguish the index $m$.

As the contrast between the inner and outer regions increases (for the following cases $\varepsilon_{r_{2}}=1$ ), the curves start to forsake the degeneracy, being this effect proportional to the contrast. See the cases of low permittivity, $\varepsilon_{r_{1}}=2.5$ (for example, polymers), in Fig. 3.7(b), and high permittivity, $\varepsilon_{r}=11$ (for example, crystallines), in Fig. 3.7(c). Additionally, the extreme case of $\varepsilon_{r_{1}}=100$ has been added to give better understanding of the observable effects, Fig. 3.7(d). The curves of every mode variate as the permittivity increases, but this is much more evident for certain modes. These modes are the $\mathrm{TM}_{0 m}$ modes and the $\mathrm{HE}_{n m}$ modes. In fact, for the general $\mathrm{HE}_{n m}$ modes there is even a change of the value of $V$ from they start to 
propagate. In the case of $\mathrm{TM}_{0 m}$ this change does not take place, but it is observed how the mode, when starts to propagate, still remains having $\beta$ close to $k_{2}\left(k_{0}\right.$ in the curves) for a certain range, the typical surface waveguiding behavior. There is also a similar singular case. Mathematically, the $\mathrm{HE}_{11}$ mode always offer a solution for the dispersion equation, i. e., the mode has no cutoff frequency, $V_{c}=0$. However, $\beta \approx k_{0}$ until some frequency $V$ where $\beta$ starts to raise and the mode is guided in a practical sense. For this singular case, a larger contrast causes an increment in the virtual cutoff, from which a real change in the propagation takes place.

Since for the different considered contrasts the range of variation of $\beta$ is not the same, to compare the considered cases for a particular mode still implies some effort. Therefore, it is proposed now use the following parameter

$$
b_{n}=\frac{\frac{\beta}{k_{0}}-\sqrt{\varepsilon_{r_{2}}}}{\sqrt{\varepsilon_{r_{1}}}-\sqrt{\varepsilon_{r_{2}}}},
$$

that can be understood as a further normalization of the propagation constant in such way that, independently of the permittivity of the cylinder, it will be satisfied $b_{n} \in[0,1]$. Specifically, when the mode starts to propagate it occurs that $\beta=k_{2}$ and $b_{n}=0$, and, when the frequency (or the radius) grows, in the limit, the mode satisfies $\beta=k_{1}$ and $b_{n}=1$. Let examine now the propagation properties of the first mode of each kind with this new parameter. In the Fig. 3.8, the renormalized propagation constant $b_{n}$ is displayed for the $\mathrm{TM}_{01}, \mathrm{HE}_{11}, \mathrm{TE}_{01}$ and $\mathrm{EH}_{11}$ modes. The case of $\varepsilon_{r_{1}} / \varepsilon_{r_{2}} \rightarrow \infty$ is added to better appreciate the effect.

It is observed how the $\mathrm{TM}_{01}$ and $\mathrm{HE}_{11}$ mode experience a sharpening of the curve slope, which physically means an abrupt change between propagating mostly in the air region to propagate inside the dielectric medium. More specifically, the $\mathrm{HE}_{11}$ experience an increase of its virtual cutoff normalized frequency. In the case of the $\mathrm{TM}_{01}$ mode, there is not change in the cutoff frequency, which remains in $V_{c}=2.405$, however, it is now clearly observed that the mode remains near cutoff conditions for a certain range of $V$. This is very evident for the case $\varepsilon_{r_{1}} / \varepsilon_{r_{2}} \rightarrow \infty$. As the graphic shows, the $\mathrm{TM}_{01}$ mode starts to propagate but experiencing $\beta \approx k_{0}$ up to near $V=3.8$. In fact, this last value of $V$ may be said to be its practical cutoff, in a similar way that is said for the $\mathrm{HE}_{11}$ case, but observed with detail, a clear surface waveguiding effect takes place. Mathematically, this situation translates in that, a solution is found for (3.169) for $V \geq 2.405$, but the large value of $\varepsilon_{r_{1}}$ influences in the smoothness of the function, making it stepper, so that changes in $V$ do not affect up to some point, but after it, the solution rapidly changes with $V$. Numerically, the algorithms should be robust against this especially singular situation. On the other hand, for the $\mathrm{TE}_{01}$ and $\mathrm{EH}_{11}$ modes, not only there is not change in the cutoff normalized frequency, but the evolution of the propagation constant to the confinement state is done much softer.

The reason for this relies in the E-field polarization. For those modes with radial dominant polarization, the waveguiding becomes of the type impenetrable surface. This is caused by the boundary condition on the continuity of the current displacement vector $\vec{D}(3.38)$ : 


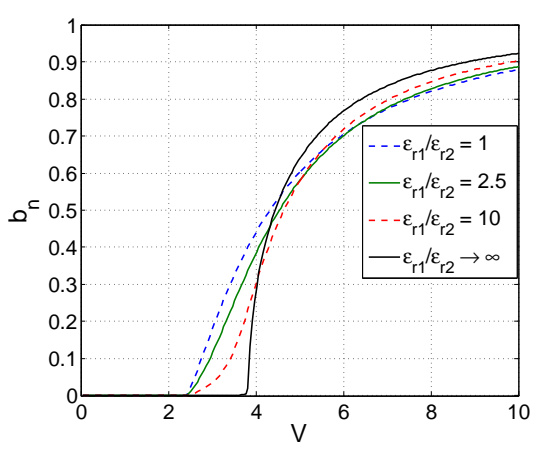

(a)

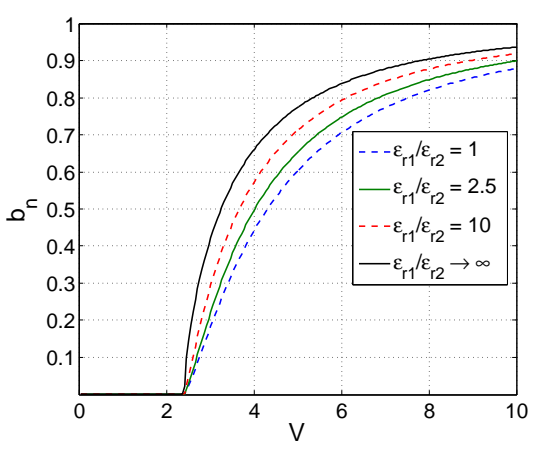

(c)

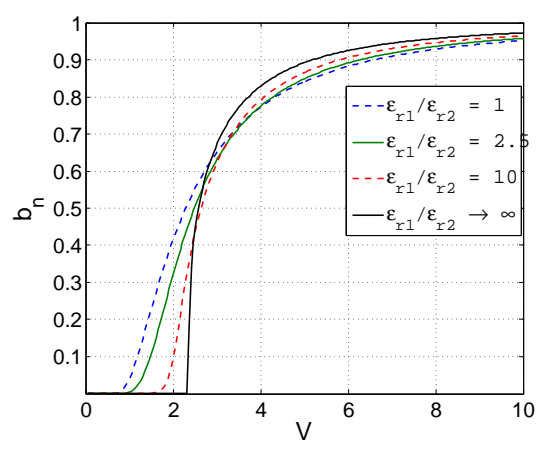

(b)

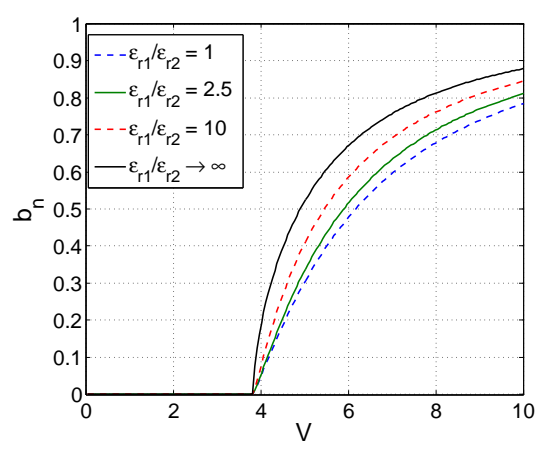

(d)

Figure 3.8: Proposed renormalized propagation constant $b_{n}$ for the first mode of each type and several cases of contrast. a) $T M_{01}$; b) $H E_{11}$; c) $T E_{01}$; d) $E_{11}$.

$$
\begin{aligned}
& \hat{n}_{1} \cdot \vec{D}_{1}(a, \phi)=-\hat{n}_{0} \cdot \vec{D}_{2}(a, \phi) \quad \rightarrow \\
& \rightarrow \quad \varepsilon_{1} E_{r 1}(a, \phi)=\varepsilon_{0} E_{r 2}(a, \phi) \quad \rightarrow \quad \frac{E_{r 2}(a, \phi)}{E_{r 1}(a, \phi)}=\varepsilon_{r}
\end{aligned}
$$

By the above equation, the discontinuity that experience the radial component of the E-field is directly proportional to the dielectric contrast between the inner and outer region. This means that it appears a field enhancement in the outer region, just close to the boundary, since from that surface the field decay exponentially.

The previous fact is directly observable in the axialsymmetric modes, $\mathrm{TM}_{01}$ and $\mathrm{TE}_{01}$ in as much as the former has a clearly dominant radial polarization $\left(E_{z}\right.$ is always several orders of mangnitude smaller than $E_{r}$ ), and the second has only azimuthal E-field component. To better visualize what occurs when the dielectric contrast increases, in Fig. 3.9 it is represented the E-field magnitude for these two modes at a normalized frequency of $V=3.5$, a frequency of transition at which $\beta$ adopts an intermedium value in its range. The first column of images correspond for to the $\mathrm{TM}_{01}$, whereas the second column correspond to the $\mathrm{TE}_{01}$ mode. From up to 
down, a) and b) are the case of $\varepsilon_{r_{1}} \approx \varepsilon_{r_{2}}=2$, typical of optical fibers, in c) and d) the inner region permittivity is $\varepsilon_{r_{1}}=2.5$, and, finally, in e) and $\mathrm{f}$ ) the permittivity is $\varepsilon_{r_{1}}=11$.

The difference is clear. When there is almost no dielectric contrast, the mode patterns are very similar. It is true that a slight concentration of the field surrounding the dielectric rod takes place for the $\mathrm{TM}_{01}$ mode, but the effect is almost negligible. When there is a moderate jump of permittivity, Fig. 3.9(c), it is appreciated that the peak of the field takes place in the outer medium close to the boundary, and they are found both, surface waveguiding and confined waveguiding. At this point, the mode is in the transition between both states. For this low permittivity, of $\varepsilon_{r_{1}}=2.5$, the transition is smooth with $V$. See also how no change is appreciated for the $\mathrm{TE}_{01}$ mode. If the contrast is increased, Fig. 3.9(e), then the surface waveguiding is clearly appreciated for the $\mathrm{TM}_{01}$ mode. For cases such this, when the mode starts to propagate, it is propagated attached to the surface, with most of the power traveling in the external air medium. The inner region is almost empty of electromagnetic energy. When the frequency increases, the mode finally becomes confined, in an abrupt transition, and the mode pattern seems to that of Fig. 3.9(a). The key point is that, the larger the permittivity, the more impenetrable remains the dielectric surface, so the $\mathrm{TM}_{01}$ mode remains as a surface mode despite of increasing the frequency of operation. At some point, this effect collapses and the mode is abruptly trapped inside the dielectric region, becoming a confined one. See for instance the slope of the $\frac{\varepsilon_{r_{1}}}{\varepsilon_{r_{2}}} \rightarrow \infty$ curve of Fig. 3.8(a). It is worth to mention that the value of propagation constant $\beta$ or any of its renormalization is just an estimation of the state of the field patterns and, for example, $b_{n}=0.5$ does not mean that the field patterns are at the middle of their limit states at $b_{n}=0$ and $b_{n}=1$.

In the case of the hybrid modes, it is not as direct to observe the dominant polarization regarding to the field expressions since all components of the field are present in the both cases, $\mathrm{HE}_{11}$ and $\mathrm{EH}_{11}$. Although the propagation constant curves give some intuition about the radial polarization predominance in $\mathrm{HE}_{11}$, and the azimuthal polarization predominance in $\mathrm{EH}_{11}$, representation of the vectorial transversal E-field gives a more intuitive view for each case.

In Figs. 3.10 and 3.11 they are represented the E-field transversal polarization and magnitude for the $\mathrm{HE}_{11}$ and $\mathrm{EH}_{11}$ modes. For the $\mathrm{HE}_{11}$ mode, $V=2.4$ is chosen, whilst for the $\mathrm{EH}_{11}$ mode, $V=5$ is chosen, i. e., an intermedium frequency regarding each mode dispersion curve, see Figs. 3.8(b) and 3.8(d). The considered contrasts are the same as in the axialsymmetric modes case. As can be observed, now, the effect is more complex. In these cases, apart of the modification of the magnitude pattern, that occur for both, $\mathrm{HE}_{11}$ and $\mathrm{EH}_{11}$, there is a modification in the polarization.

Starting with the $\mathrm{HE}_{11}$ mode, it is observed that, for the very small contrast of typical optical fibers, the mode is linearly polarized, and the field is concentrated in the very central core of the rod, far from the boundary, and not as a ring like in the $\mathrm{TM}_{01}$ and $\mathrm{TE}_{01}$ cases. As it can be appreciated, the mode pattern is axialsymmetric due to the equilibrium between radial and azimuthal polarizations that provides the 


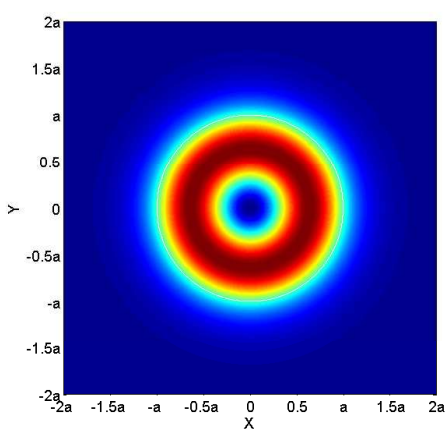

(a)

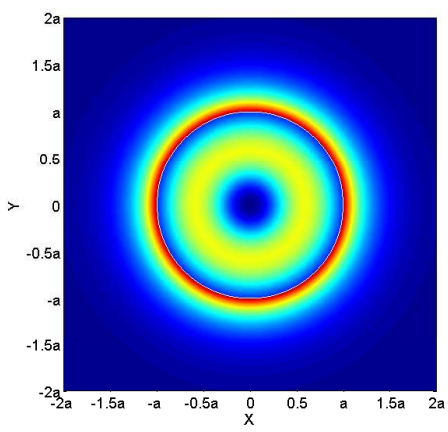

(c)

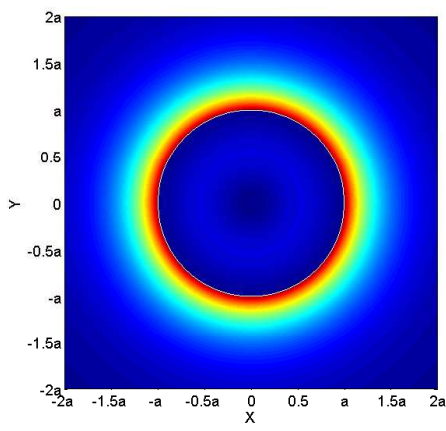

(e)

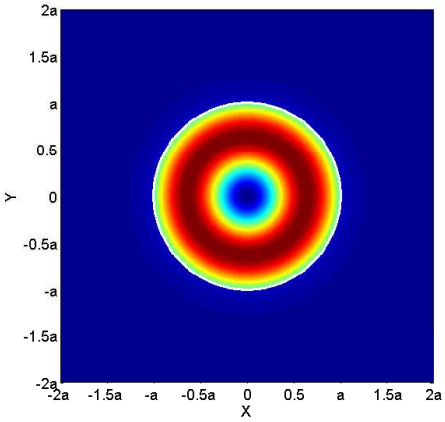

(b)

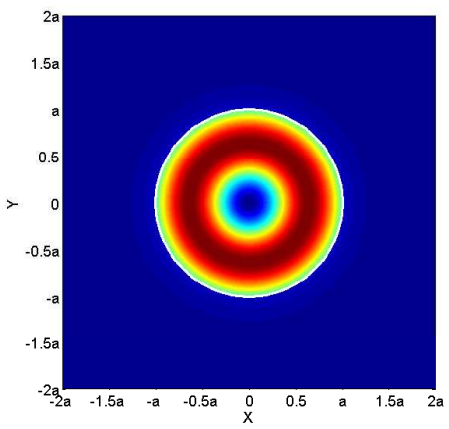

(d)

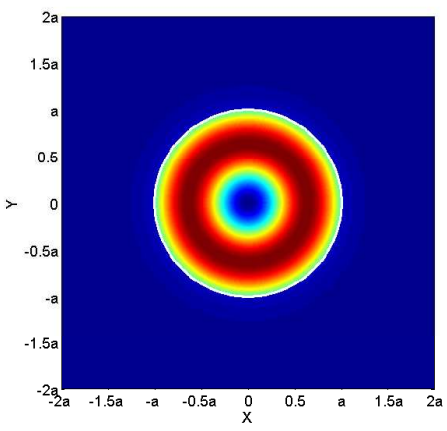

(f)

Figure 3.9: E-field magntide of the $T M_{01}$ and $T E_{01}$ modes at $V=3.5$ for different dielectric contrasts between inner and outer mediums. The first column correspond to the TM $M_{01}$ mode and second row to the $T E_{01}$ mode. a) and b) $\varepsilon_{r_{1}} \approx \varepsilon_{r_{2}}=2$; c) and d) $\varepsilon_{r_{1}}=2.5$; e) and f) $\varepsilon_{r_{1}}=11$.

linear polarization. This equilibrium is caused by the very similar contribution of the coupled $\mathrm{TE}_{11}$ and $\mathrm{TM}_{11}$ modes that form the $\mathrm{HE}_{11}$ mode. 
When the contrast increases, inside the region 1 , the polarization remains quite invariant, and the axialsymmetry is maintained. However, near the inner surface, and even more surrounding the outer surface, the change is appreciated. In Figs. $3.10(\mathrm{e})$ and $3.10(\mathrm{f})$, that correspond to $\varepsilon_{r_{1}}=11$, this is very evident. What occurs is that only the radial component experiments enhancement at the exterior part of the boundary. In this way, the equilibrium between radial and azimuthal polarization is broken, and the polarization is not linear any more. The azimuthal variation, of order $n=1$, is very evident in the E-field magnitude pattern. The mode is predominantly surface waveguided, but still a part of the energy remains in the core. It is noted one appreciation. The E-field lines in the outer medium seem to be the continuation of a $\mathrm{TE}_{11}$ lines of a CWG. This can be explained as follows. For the fields outside, the cylinder surface is view as a low impedance (as for the fields inside as an high impedance [540]). Therefore, inside the mode pattern looks like that of a CWG with perfect magnetic walls, and outside, the mode pattern looks as a direct continuation of a metallic CWG.

In the case of the $\mathrm{EH}_{11}$ mode, for very low contrast, Figs. 3.11(a) and 3.11(b), the mode is concentrated in the core, but following an axialsymmetric ring pattern, similar of that of the $\mathrm{TE}_{01}$ and $\mathrm{TM}_{01}$ modes, but leaving even more empty area in the central core. At the top and bottom, radial polarization predominates, whereas, azimuthal polarization dominates at the sides. There is an equilibrium, but in this case contributes in an opposite way as the $\mathrm{HE}_{11}$ mode, thus having a curly polarization, close to, but not exactly circular. When the contrast increase, and the equilibrium is broken (see for instance Figs. 3.11(e) and 3.11(f), $\varepsilon_{r_{1}}=11$ ), the $\mathrm{EH}_{11}$ mode presents enhancement of the azimuthal contribution (in fact, getting the detrimental part of the $\mathrm{HE}_{11}$ mode), and a detriment in the radial contribution (which goes to the $\mathrm{HE}_{11}$ mode). Therefore, the axialsymmetry provided by similar contributions of the coupled $\mathrm{TE}_{11}$ and $\mathrm{TM}_{11}$ modes is broken, and the azimuthal variation of order $n=1$ is appreciated. In this case, the change is better appreciated in the inner region. As $E_{\phi}$ dominates in the $\mathrm{EH}_{11}$ mode for a reasonable value of $\varepsilon_{r_{1}}$, the mode is propagated confined, and does not adopt surface waveguiding features.

\subsubsection{Power analysis and attenuation}

The power transmission and the attenuation features of the cylindrical dielectric rod are addressed now. This involves the calculation of the total transmitted power of each region by integration of the Poynting vector, which gives the power distribution of a certain mode in the waveguide. Then, if the losses of the dielectric, ideal until the moment, are considered, the power absorbed by the dielectric can be calculated by the pertubative method [376], i. e., assuming that the field patterns are those of the ideal case. By this, the attenuation ${ }^{11}$ of the waveguide is finally obtained.

The total mean power transmitted by the waveguide is given by

$$
P_{T}=\frac{1}{2} \operatorname{Re}\left\{\iint_{S}\left(\vec{E} \times \vec{H}^{*}\right) \cdot \hat{z} \mathrm{~d} S\right\},
$$

\footnotetext{
${ }^{11}$ Note that in this case, the dielectric losses in the region 1 are the unique possible contribution of losses.
} 


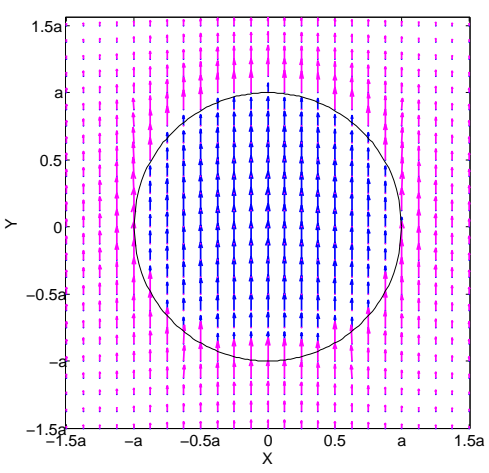

(a)

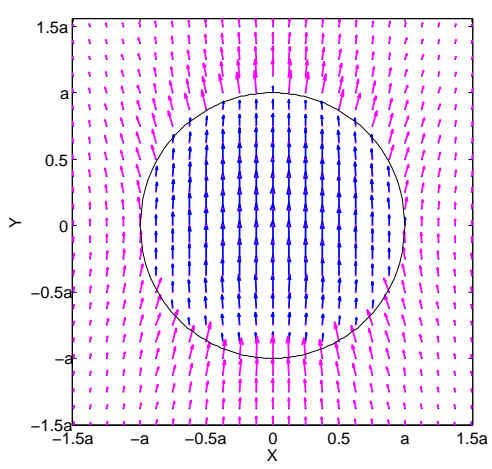

(c)

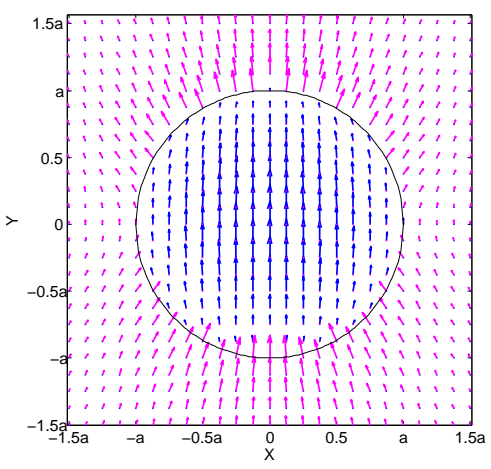

(e)

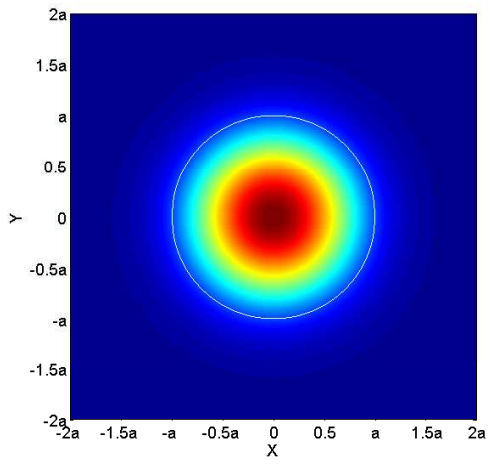

(b)

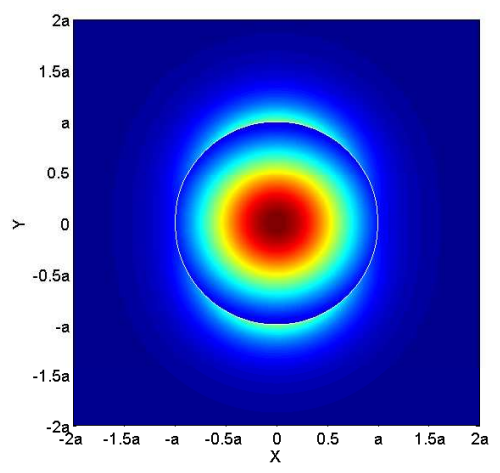

(d)

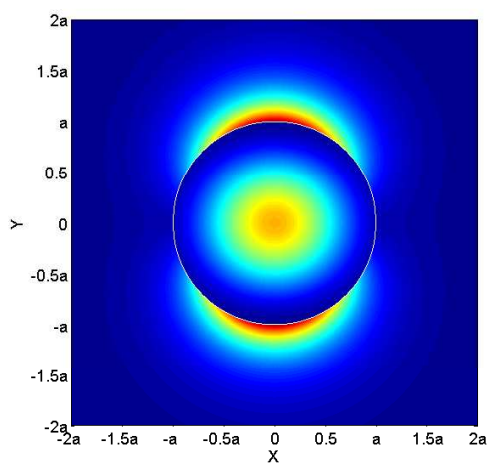

(f)

Figure 3.10: E-field lines and magnitude of the $H E_{11}$ mode at $V=2.4$ for several cases of dielectric contrast between inner and outer medium. a) and b) $\varepsilon_{r_{1}} \approx \varepsilon_{r_{2}}=2$; c) and d) $\varepsilon_{r_{1}}=2.5$; e) and f) $\varepsilon_{r_{1}}=11$. 


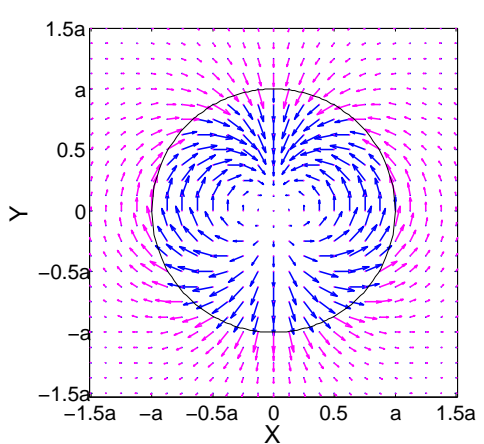

(a)

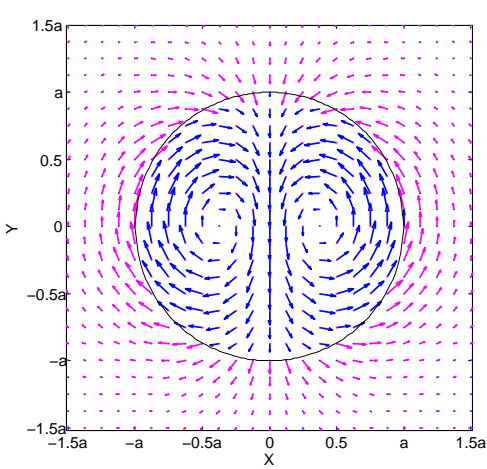

(c)

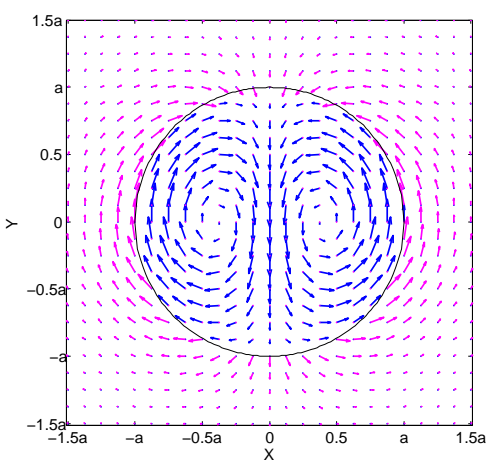

(e)

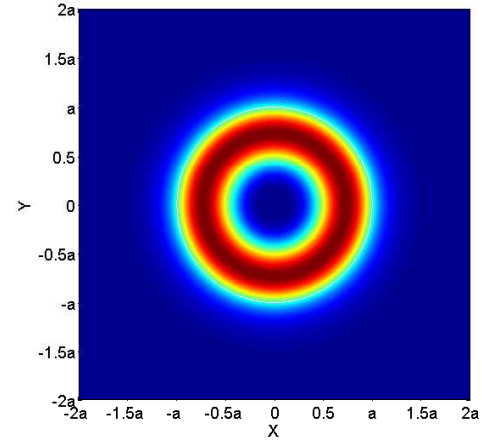

(b)

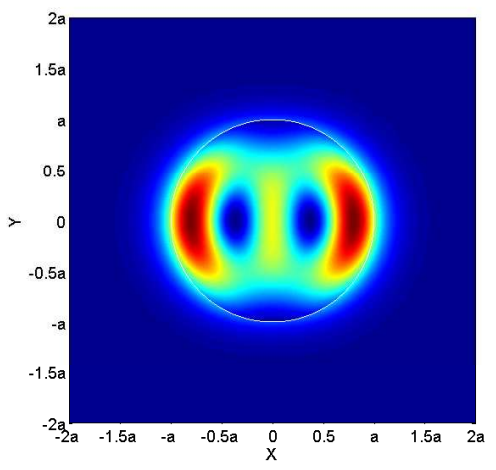

(d)

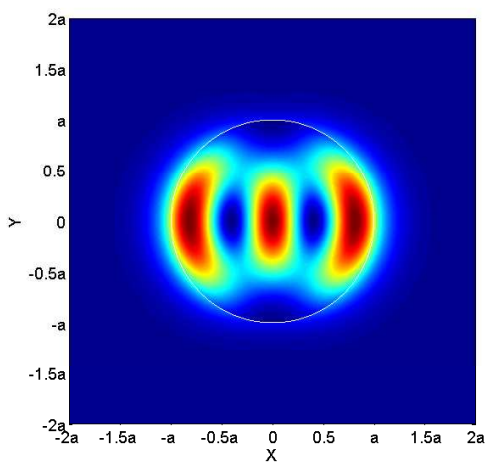

(f)

Figure 3.11: E-field lines and magnitude of the $E H_{11}$ mode at $V=5$ for several cases of dielectric contrast between inner and outer medium. a) and b) $\varepsilon_{r_{1}} \approx \varepsilon_{r_{2}}=2$; c) and d) $\varepsilon_{r_{1}}=2.5$; e) and f) $\varepsilon_{r_{1}}=11$. 
where $S$ is the transversal surface of the waveguide, in this case, extending to the infinity, i. e., the whole $\mathbb{R}^{2}$. It is possible to particularize the integration for each region, thus obtaining the fraction of power traveling in each one of the regions. In the geometry under study:

$$
\begin{aligned}
& P_{1}=\frac{1}{2} \operatorname{Re}\left\{\int_{0}^{2 \pi} \int_{0}^{a}\left(E_{r_{1}} H_{\phi_{1}}^{*}-H_{r_{1}}^{*} E_{\phi_{1}}\right) r \mathrm{~d} r \mathrm{~d} \phi\right\} \\
& P_{2}=\frac{1}{2} \operatorname{Re}\left\{\int_{0}^{2 \pi} \int_{a}^{\infty}\left(E_{r_{2}} H_{\phi_{2}}^{*}-H_{r_{2}}^{*} E_{\phi_{2}}\right) r \mathrm{~d} r \mathrm{~d} \phi\right\}
\end{aligned}
$$

and

$$
P_{T}=P_{1}+P_{2}
$$

where $P_{1}$ and $P_{2}$ are the power transmitted in regions 1 and 2 , respectively. If the propagation constant has been found, by applying the field expressions of the particular mode under study, just the integration in (3.193) and (3.194) remains. Except some further analytic simplifications for the axialsymmetric modes $(n=0)$, the integrals must be carried out numerically. However, since the involved functions are sufficiently smooth, by appropriate quadratures the computational time is negligible. More specifically, the typical Gauss-Legendre quadrature is used for the region 1 , and the Gauss-Laguerre quadrature, suitable for functions exhibiting exponential decays in an infinite integration domain, is chosen for region 2 [528]. Less than 20 nodes are used for each integral. Since the integration in the azimuthal component is trivial, the true problem remains only in the radial component. Therefore, the integrals are simple, and the computational cost is very small.

The information in $P_{1}$ and $P_{2}$ results relevant since it exactly describes how power is distributed for a guided mode. It is possible to define the following parameter:

$$
\eta=\frac{P_{1}}{P_{T}}=\frac{P_{1}}{P_{1}+P_{2}} \quad \in[0,1]
$$

which gives the fraction of total power being transmitted in the inner dielectric region. This parameter gives a more precise information than the propagation constant $\beta$ about attenuation and confinement. In order to see how $\beta$, or more clearly, the proposed normalization $b_{n}$, can give only a first approximated idea of the power distribution, better given by the core fraction power $\eta$, in Fig. 3.12 these last two parameters are compared for a high permittivity dielectric case, $\varepsilon_{r_{1}}=11$.

It can be observed that the fraction of power in the core is larger than expected when $\beta$ is considered. See for instance that, at $V=2.2$, the $\mathrm{HE}_{11}$ mode presents $b_{n}=0.3$, however $\eta=0.8$. This accentuated difference occurs in the transition from weakly to confined waveguiding, which is more clear for radial dominant Efield polarization modes $\left(\mathrm{TM}_{0 m}, \mathrm{HE}_{n m}\right)$ and is caused by the TIR effect. This issue is later briefly described.

Another fact that throws Fig. 3.12(b) is that azimuthal-dominant E-field polarization modes $\left(\mathrm{TE}_{0 m}, \mathrm{EH}_{n m}\right)$ have already some fraction of power traveling in the dielectric when they start to propagate. Physically this means that these modes 


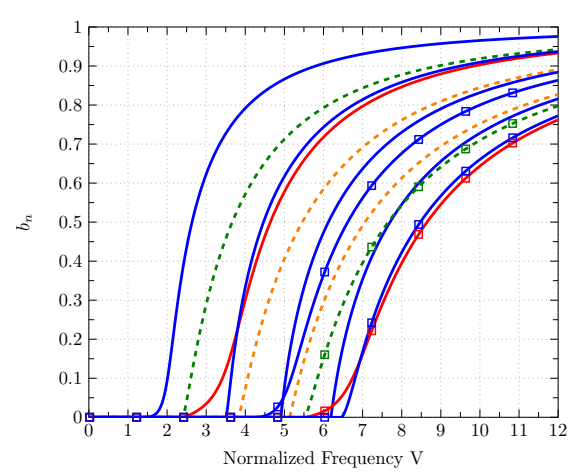

(a)

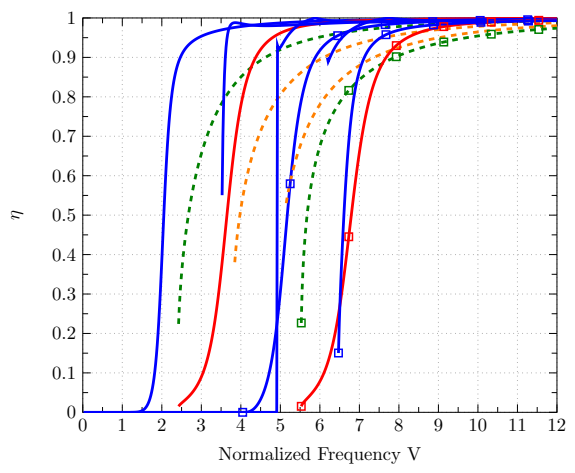

(b)

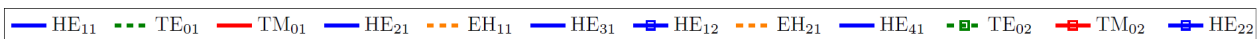

(c)

Figure 3.12: Normalized propagation constant $b_{n}$ and fraction power guided in the region $1 \eta$, for the first 12 modes of a high permittivity cylinder, $\varepsilon_{r_{1}}=11$. a) $b_{n} v s . V$; b) $\eta v s V$; c) Mode legend.

does not experience surface waveguiding. Also the slope is softer for this modes, and they can be easily tracked. However, the radial dominant E-field polarization modes $\left(\mathrm{TM}_{0 m}, \mathrm{HE}_{n m}\right)$ experience an abrupt change between surface and confined guidance. This is very evident for the $\mathrm{HE}_{n m}$ modes with large $n$, the curve is almost vertical in the transition.

There are some strange features in some curves related with the higher $\mathrm{HE}_{n m}$ modes. They seem to start already confined and do not increase the confinement monotonically, as expected, in what could be understood as a numerical error. However, a deep analysis of the problem reveals a complex phenomena that occurs. It is mathematically proven that high permittivity dielectric can break the orthogonality between the set of modes [543]. It comes out that, at very specific frequencies, there is power coupling between $\mathrm{HE}_{n m}$ and $\mathrm{EH}_{n m}$ modes even any perturbation is present in the waveguide. They become complex modes. In the cases of interest in this thesis that motivate the study of the dielectric cylindrical waveguide, this phenomena, characteristic of high permittivity loseless dielectrics, has not been appreciated. The interested reader can check some works related with the complex phenomena generated by the presence of dielectrics in waveguides [555]-[556], which are the analytical prize to pay for a more complex boundary when it is compared with a perfect electric or magnetic conductor.

To better appreciate the effect of the contrast in the power distribution, in Fig. 3.13(a), the parameter $\eta$ is compared for the fundamental mode $\mathrm{HE}_{11}$ considering four cases of contrast, $\varepsilon_{r_{1}} \approx \varepsilon_{r_{2}}=2$ (optical fibers), $\varepsilon_{r_{1}}=2.5$ (low permittivity), $\varepsilon_{r_{1}}=11$ (high permittivity) and $\varepsilon_{r_{1}}=40$ (extreme permittivity case), which give a complete vision of this effect. The graphs reflect well the general surface dielectric waveguide reality. When high permittivities are used, surfaces modes (as it is the $\mathrm{HE}_{11}$ mode) remain surface guided, but then, when they collapse into the waveguide, the confinement is larger than for lower permittivities. Therefore, the strong change 
of medium at the boundary provides both, impenetrability of the surface regarding the modes traveling outside, and more confinement via TIR for the modes traveling inside. Therefore, a high dielectric constant creates larger differences between the mode patterns, regarding their state of propagation. This isolation effect may have its own utility when perturbations occur, including the excitation process. Again, the effect of complex modes is observed for the extreme case of $\varepsilon_{r_{1}}=40$.

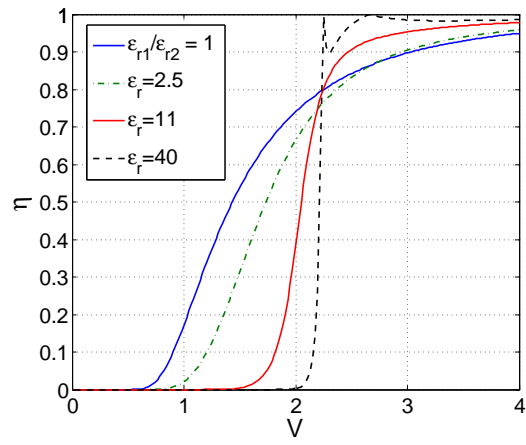

(a) $\eta(V)$.

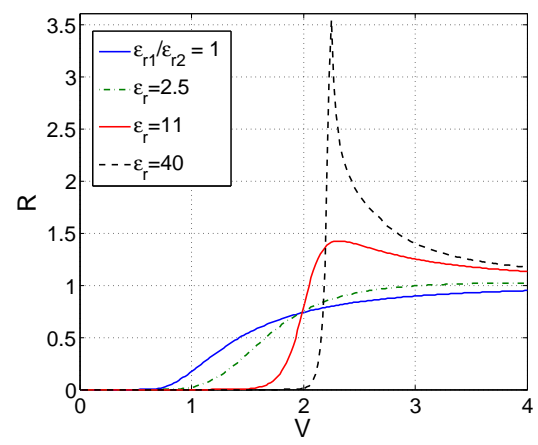

(b) $R(V)$.

Figure 3.13: Comparison between fraction power in region $1 \eta$ and normalized attenuation constant $R$ for several cases of permittivity: $\varepsilon_{r_{1}} \approx \varepsilon_{r_{2}}=2, \varepsilon_{r_{1}}=2.5, \varepsilon_{r_{1}}=11$ and $\varepsilon_{r_{1}}=40$. a) $\eta(V)$; b) $R(V)$.

It is the moment now to afford the issue of the attenuation in the waveguide, in this case provided exclusively by the dielectric medium of region 1 . Considering now that this dielectric is not longer ideal and the permittivity has an imaginary term of negative sign of magnitude $\varepsilon_{1}^{\prime \prime}$, one has that the total power absorbed by the dielectric in a volume $V$ is given by

$$
P_{L d}=\frac{\omega \varepsilon_{1}^{\prime \prime}}{2} \iiint_{v}|\vec{E}|^{2} \mathrm{~d} V
$$

or, more specifically, considering the power absorbed per unit length in the region of interest, and the usual parameter indicating the losses of dielectrics, $\tan \delta=\frac{\varepsilon^{\prime \prime}}{\varepsilon^{\prime}}$ :

$$
P_{L d}=\frac{\omega \varepsilon_{1} \tan \delta}{2} \int_{0}^{a} \int_{0}^{2 \pi}\left(\left|E_{r_{1}}\right|^{2}+\left|E_{\phi_{1}}\right|^{2}+\left|E_{z_{1}}\right|^{2}\right) r \mathrm{~d} \phi \mathrm{d} r
$$

The inclusion of losses in the ideal problem implies that the axial variation of fields not longer is described by $e^{-j \beta}$, but $e^{-j \gamma}$, with $\gamma=\alpha+j \beta$, including now the term $\alpha$ indicated the losses in Nepers per unit length. As a consequence, the power falls as $e^{-2 \alpha}$, this ruled by the dielectric absorption, hence:

$$
\frac{\mathrm{d} P_{T}}{\mathrm{~d} z}=-P_{L d}=-2 \alpha P_{T} \quad \Longrightarrow \quad \alpha=\frac{P_{L d}}{2 P_{T}}
$$

which allows to calculate $\alpha$. From the expression in (3.198), it can be deduced that $\alpha$ has lineal dependence with the frequency for a fixed field distribution, and it is directly proportional to $\varepsilon_{1}$ and $\tan \delta$. At $\mathrm{THz}$ frequencies, this implies high losses 
since $\tan \delta$ is not compensating the inherent increase of losses with frequency, but this parameter is even larger at $\mathrm{THz}$ frequencies. Also, it is directly observed that one consequence of using high permittivity dielectrics is an increase of losses with respect to a lower permittivity case with the same $\tan \delta$.

As it has been done renormalizing the propagation constant, up to use $b_{n}$, it is usual when dealing with dielectric waveguides, the employment of a parameter that describes waveguide attenuation independently from the previous variables. The normalized attenuation $R$ is described $\operatorname{similar}^{12}$ as in [21]:

$$
R=\frac{\alpha(\mathrm{Np} / \mathrm{m}) \lambda_{0}}{\pi \tan \delta \sqrt{\varepsilon_{r_{1}}}}
$$

In this way, it is obtained valuable information related with how the losses change as the E-field experiences a change in the distribution, therefore being able to capture some effects that previous parameters, $b_{n}$ and $\eta$ do not give. At first view, one may think that at least $\eta$ gives a quite good estimation of the dielectric losses, just by multiplying this factor to the bulk losses of the considered dielectric. In fact, this is a very common applied calculation [436], [438], [442], [448], [450], [454]. Note that $R=1$ means that the considered mode experiences the same attenuation as a plane wave in an infinite dielectric medium of the region 1 material and this value may be firstly understood as an upper limit for $R$. However, in the transition between weak and confined guidance this is not longer true for enough large contrast between mediums, and some curious effect takes place.

In Fig. 3.13(b), the normalized attenuation constant $\mathrm{R}$ for the fundamental mode $\mathrm{HE}_{11}$ is shown, considering the same cases of permittivity of Fig. 3.13(a) so both parameters can be compared. For low permittivities, both parameters, $\eta$ and $R$, increase monotonically with $V$, as expected. They have practically the same values, indeed. However, when the dielectric constant increases, it is found an overattenuation region for which $R>1$, i. e., the losses are higher than the losses of a plane wave propagating inside an infinite medium constituted by the dielectric material. More specifically, looking into 3.13(b), a peak is observed, then $R$ decreases monotonically to the unity value. The larger the permittivity, the higher this peak, and the more abrupt the change between the negligible losses of a mode guided mainly in the outer medium, and the overattenuated behaviour.

This behavior is observed for every mode, not only for those with surface waveguiding features. However, these last experience the described effect with larger intensity due to their more abrupt changes between surface and confined guidance. Something that is common for every curve of Fig. 3.13(b) is that all of them converge to $R=1$. This is what is expected since when the cylinder becomes infinitely large (in terms of the wavelength), the situation is the same of a plane wave traveling in an infinite medium filled with the region 1 material, and $\beta=k_{1}$.

Looking with detail this effect, the following explanation is found. Assume now a dielectric cylinder surrounded by air. By taking into account (3.101) and that $k_{0}<\beta<k_{1}$, it is deduced that, when the propagation starts $\left(\beta=k_{0}\right)$ one has $\beta_{r_{1}}=\sqrt{k_{1}^{2}-k_{0}^{2}}=k_{0} \sqrt{\varepsilon_{r_{1}}-1}$, and as $V$ increases, one has that, asymptotically,

\footnotetext{
${ }^{12}$ In [21] $\varepsilon_{r_{1}}$ appears instead of $\sqrt{\varepsilon_{r_{1}}}$, however, the former option causes $R \in\left[0, \varepsilon_{r_{1}}^{-1 / 2}\right]$, which seems less desirable than $R \in[0,1]$, this last providing a uniform domain, independent of $\varepsilon_{r}$.
} 
$\beta \rightarrow k_{1}$, hence $\beta_{r_{1}} \rightarrow 0$ (more exactly, $\beta_{r_{1}}$ tends asymtotically to a constant value and $\beta$ continues growing with the frequency so that $\beta_{r_{1}}$ becomes relatively very small to $\beta$ ). This implies that, when the mode starts to propagate, $\beta_{r_{1}}>>\beta$, what makes the propagation to be very transversal.

To better illustrate what this physically implies, in Fig. 3.14(a) it is displayed the visual representation of the trigonometric relationship between $\beta$ (solid blue) and $\beta_{r_{1}}$ (dashed red), according to (3.101). Note that the larger the permittivity of the region 1 , the larger $k_{1}$, and by extension the larger the circumference, hence providing larger differences between $\beta$ and $\beta_{r_{1}}$.

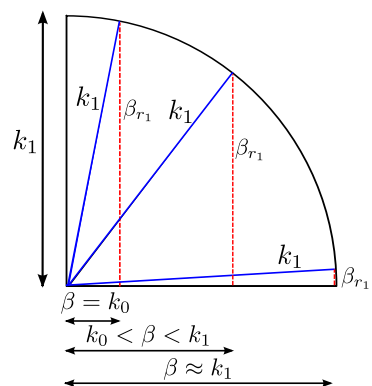

(a)

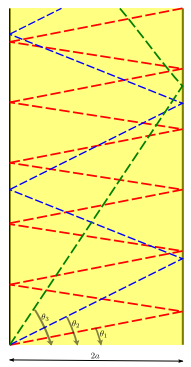

(b)

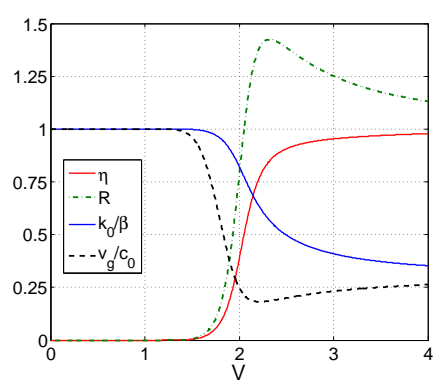

(c)

Figure 3.14: Analysis of the overattenuation effect $R>1$ in high permittivity dielectrics. Calculations are made for the fundamental mode $H E_{11}$ considering $\varepsilon_{r_{1}}=11$. a) Visual representation of the trigonometric relationship by (3.101) between $\beta$ (solid blue) and $\beta_{r_{1}}$ (dashed red), governed by $k_{1}$. b) Visual representation of the propagation considering trace of rays for several angles of incidence. Lower angles occur when the mode starts to propagate, implying an effective longer traveled path by the mode; c) Influence of this effect in the main waveguide propagation parameters in the case of $\varepsilon_{r_{1}}=11$.

In a first approximation, by ray theory, the angle of incidence against the waveguide dielectric walls of the waves conforming the mode is $\theta=\arcsin \left(k_{1} / \beta\right)$, therefore, the real traveled distance per unit length will be approximately $k_{1} / \beta$ (in fact, regarding the case of the $\mathrm{HE}_{11}$ mode, there is some contribution of the $\mathrm{TE}_{11}$ mode implying the rays to have certain helical features [553]). To appreciate the influence of the value of $\beta$ respect $k_{1}$ in this effect, an schematic of the described ray propagation is displayed in Fig. 3.14(b). Several cases of propagation are displayed in the image. Low angle of incidence (red dashed lines) occur when $\left(\beta \approx k_{0}<<k_{1}\right)$, i. e., when the mode starts to propagate in a high permittivity dielectric. Note that for this case, the effective path traveled by the waves is indeed much longer than the real path of waveguiding, indicated by the advanced length in the axial direction.

When the frequency increases, $\beta$ increases towards $k_{1}$, and the angle of incidence increases up to the point that the rays are axial. At this point the effective length is the same as the real length, and all the wave is confined. This gives $R=1$. However, when the angle of incidence is very low, it is possible that, although the mode has a distribution power such that the mode is not completely confined, the factor $k_{1} / \beta$ provide an effective length enough large, in such way that the rays become even more attenuated that in the total confinement case, thus giving $R>1$. The peak is very clear for a surface mode propagating in a high permittivity dielectric by 
two reasons. First, the factor $k_{1} / \beta$ can reach very high values. Second, the mode becomes suddenly confined in terms of power distribution. At this point, there is a disagreement between $b_{n}$ and $\eta$, having thus the maximum loss combination: large effective path given by $k_{1} / \beta$, and high attenuation per unit length traveled by the rays, given by the larger confinement in the dielectric medium (parameter $\eta$ ).

Associated to this effect, the axial component of the fields also experiences an increment of its relative contribution to the mode pattern, i.e., the modes become further away from the TEM features, experienced up to some extent for enough large contrast. This can be easily observed by thinking in the the ray trace description. The effects described result important in the sense that they must be taken into account in the excitation process, to avoid or enhance possible perturbations and mode coupling, properly designing the waveguide for certain functionality, etc.

A graph that resumes the consequences of this effect of enhanced transversal propagation on the main characteristics of a guided mode is shown in Fig. 3.14(c). The chosen permittivity is $\varepsilon_{r_{1}}=11$. In this graph they are displayed the quantities $k_{0} / \beta$ (note that it is the inverse of the usual $\beta / k_{0}$ ), $\eta, R$, and the normalized group velocity $v_{g} / c$. Note that all this quantities, a priori, should be comprised between 0 and 1. Neither the propagation constant nor the power distribution reveal the effect for the considered contrast. However, the normalized group velocity and, even in a more accentuated way, the normalized attenuation, shown an anomalous behavior. The effect in both, the velocity at which the energy is transmitted, and its attenuation, may be regarded as an undesired effect or contrarily as a powerful tool to implement certain responses.

Since the cylindrical dielectric waveguide electromagnetic problem has been almost only considered to analyze and design the optical fiber problem, the case of larger contrast remains much less understood (some efforts have been done for the well-known high Q dielectric resonators [540]). As observed, the effects caused at the interface between both regions are not obvious, and understanding them requires some reflections. For instance, to the author knowledge, the overattenuation effect has not been clearly studied in the literature. The conclusions given here about how the modes behave attending to their field components, and the effect of the contrast, help to understand the propagation characteristics of complex $\varepsilon_{r}$ dielectrics, and by extension, the complex case of a SWW. Otherwise, observed phenomena could be attributed to the wrong cause. In addition, these conclusions allow to better understand the dispersion diagram of the DCSWW and to develop proper designs with it. Furthermore, the overattenuation effects observed here, are directly implied in any interface involving a change on a dielectric medium, with more or less strength. To do it with the cylindrical dielectric waveguide is crucial, since it is the simplest two-dimensional dielectric waveguide (bring back that an infinite dielectric Ribbon is only a 1D waveguide concept, very simple, whereas the rectangular dielectric waveguide has not analytical solution [21], [557], [558]).

Along this section is has been observed that the problem of a dielectric cylinder gives results that strongly depend on dielectric permittivity (considering external medium air). Notice that the situation at the boundary covers cases ranging from almost no discontinuity of dielectric properties to abrupt changes of media. Hence, the casuistic is large, much more than that of a simple PEC boundary condition offered by usual hollow metallic waveguides. Since the basics for the analysis of this 
kind of waveguide have been already established, the step to contemplate not $\beta$, i. e., solving the real problem, but $\gamma=\alpha+j \beta$, i. e., the complex problem involving the search of eigenvalues in the complex plane, is ready to be afforded.

\subsection{Dielectric cylinder with complex $\varepsilon_{r}$}

Once the simplest case of having a dielectric cylinder waveguide with real, positive permittivity (ideal dielectrics) has been analyzed and the main characteristics of this waveguide have been understood, is the moment to analyze the cases $2-4$ mentioned in the section 3.2: lossy dielectrics ( $\varepsilon_{r}$ in the first quadrant of the complex plane), $\varepsilon_{r}$ real and negative, and $\varepsilon_{r}$ in the third quadrant of the complex plane. The case of the SWW analysis is left for the next section. For this task, the considered problem will be now that described in Fig. 3.15. The region 2 will be air for all the considered cases and, since now the only permittivity of relevance is that of the region 1 , the subscript 1 referring to it will be obviated. This will follow along all the next formulation, clarifying the expressions.

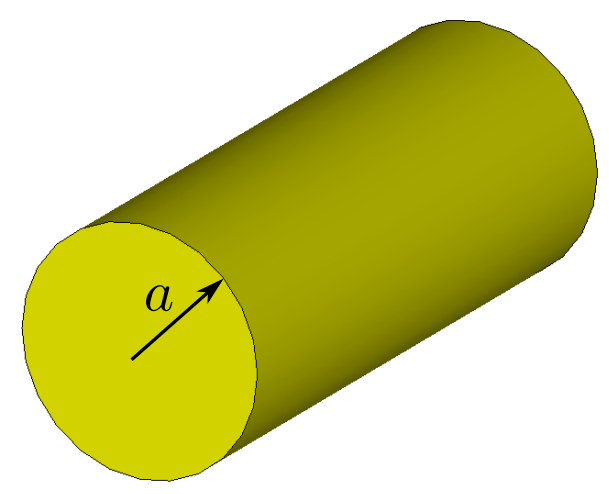

(a)

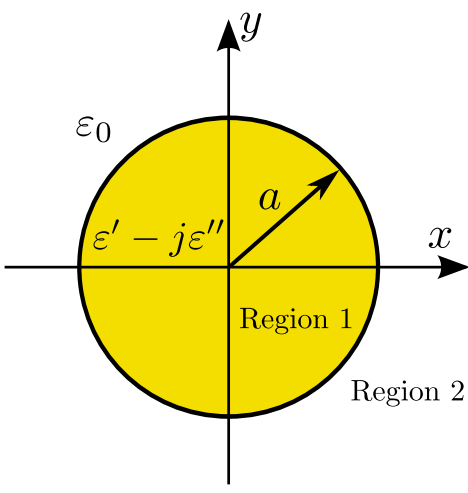

(b)

Figure 3.15: Dielectric Cylinder Waveguide with complex permittivity. a) $3 D$ view; b) Problem description.

\subsubsection{Modal analysis equations}

In the analysis carried out in the previous section two clearly separated stages were distinguished. First, the dielectric was ideal, $\varepsilon_{r}$ was considered real and the propagation constant $\beta$, unknown of the problem, was found. It was a real variable problem. Second, the losses were added considering $\varepsilon_{r}=\varepsilon_{r}^{\prime}-j \varepsilon_{r}^{\prime \prime}$, so that the propagation constant became $\gamma=\alpha+j \beta$ (and the longitudinal field variation is ruled then by $\left.e^{-\gamma z}\right), \alpha$ accounting the losses per unit length, and $\beta$ having the same role and the value(s) calculated in the first stage. The value(s) of $\alpha$ were calculated using the field expressions obtained in the stage 1 and the provided value of $\varepsilon_{r}^{\prime \prime}$.

In this section, the procedure will be done all in one. Indeed, this is the exact way to solve a lossy dielectric problem. Now, from the first moment, it will be assumed 
that $\gamma=\alpha+j \beta$, being this the unique unknown of the problem, since the rest of required elements for the waveguide analysis (dispersion diagram, fields, power distribution, and attenuation) can be extracted from $\gamma$. Therefore, the problem becomes now of complex variable. This is the prize of the obtained accuracy. Note that, in this case, the calculated fields and the calculated attenuation will be the exact one, since Maxwell equations are solved directly assuming $\gamma=\alpha+j \beta$, and any perturbation method is applied. For lossy dielectrics, this means that, even for extremely large losses $\left(\varepsilon_{r}^{\prime \prime} \uparrow \uparrow\right)$, the calculations will be exact, something that cannot be affirmed for the perturbation method.

Next the problem will be formulated. The expressions are very similar to those of the real permittivity dielectric waveguide, however, it seems appropriated to have them explicitly written in order consult them when it is necessary, and be able to take some insight as the results are commented. Previously $\gamma=j \beta$, so $\beta=\gamma / j=$ $-j \gamma$. Basically this is the main change in the formulation. Now, in the region 1 , $k=k_{1}=k_{0} \sqrt{\varepsilon_{r}}$, and in the region $2, k=k_{0}$. Therefore, the transversal propagation constant become:

$$
\begin{gathered}
\beta_{r_{1}}^{2}=k_{1}^{2}+\gamma^{2} \\
\alpha_{r_{2}}^{2}=-\gamma^{2}-k_{0}^{2}
\end{gathered}
$$

Note that $\beta_{r_{1}}$ and $\alpha_{r_{2}}$ are now complex. This mean that the argument of the implied functions, the ordinary Bessel function of first kind $\mathrm{J}_{n}(x)$ in the region 1 , and the modified Bessel function of second kind $\mathrm{K}_{n}(x)$ in the region 2, will be complex, hence the behavior of the fields will be neither purely oscillatory in the region 1 nor purely exponential (decaying) in the region 2. In fact, the following relationships of Bessel function may clarify this issue:

$$
\begin{aligned}
\mathrm{J}_{n}(j x) & =j^{n} \mathrm{I}_{n}(x) \\
\mathrm{K}_{n}(j x) & =\frac{j^{n \pi}}{2} \mathrm{H}_{n}^{(2)}(x)
\end{aligned}
$$

Therefore, in the region 1 , the contribution of the imaginary part of $\beta_{r_{1}}$ will be an exponential grow from the origin (whatever be the sign, since $\mathrm{I}_{n}(x)$ is an even function). This can be seen as an exponential decay from the boundary to the origin: a surface wave or plasmonic behavior. In the region 2, the imaginary part of $\alpha_{r_{2}}$ will cause phase variation on the field pattern, caused by the outgoing wave behavior of $\mathrm{H}_{n}^{(2)}(x)$ : leaky wave mode behavior. Therefore, some oscillatory behavior can occur in the outer region (apart of the necessary exponential decay in order to achieve the radiation condition). It is pointed out that now, the change of sign in (3.202), responsible of having the modified (instead of the ordinary) Bessel differential equation in the region 2 , is not longer motivated by keep working with real numbers, since now the complex nature of $\gamma$ forces the problem to be intrinsically complex. However, keeping the notation of (3.201) and (3.202) is very useful to gain physical insight since in this way, the imaginary part of $\beta_{r_{1}}$ and $\alpha_{r_{2}}$ will indicate the anomalous behavior respect to the real $\varepsilon_{r}$ case. Note that, independently of the 
employed functions the solution for the fields will be, obviously, the same, but the transversal propagation constant would be different if different functions are used, in order to be consistent with this fact. Assessments about the magnitude and phase of the appearing complex quantities will be very important along the results of this section in order to extract conclusions about the propagation characteristics of the waveguides under study.

If the differential equations (3.103) and (3.104) are solved for this new problem, and the transversal field components are accordingly calculated from the longitudinal ones, the following results are obtained.

\section{$\mathbf{H E}_{n m}$ and $\mathbf{E H}_{n m}$ modes:}

$\underline{\text { Region } 1, r \leq a \text { : }}$

$$
\begin{aligned}
E_{z_{1}} & =A \mathrm{~J}_{n}\left(\beta_{r_{1}} r\right) e^{j n \phi} \\
H_{z_{1}} & =B \mathrm{~J}_{n}\left(\beta_{r_{1}} r\right) e^{j n \phi} \\
E_{r_{1}} & =\frac{-\gamma}{\beta_{r_{1}}^{2}}\left[\beta_{r_{1}} A \mathrm{~J}_{n}^{\prime}\left(\beta_{r_{1}} r\right)-\frac{\omega \mu_{0} n}{\gamma r} B \mathrm{~J}_{n}\left(\beta_{r_{1}} r\right)\right] e^{j n \phi} \\
E_{\phi_{1}} & =\frac{-\gamma}{\beta_{r_{1}}^{2}}\left[\frac{j n}{r} A \mathrm{~J}_{n}\left(\beta_{r_{1}} r\right)-\frac{j \omega \mu_{0}}{\gamma} B \mathrm{~J}_{n}^{\prime}\left(\beta_{r_{1}} r\right)\right] e^{j n \phi} \\
H_{r_{1}} & =\frac{-\gamma}{\beta_{r_{1}}^{2}}\left[\beta_{r_{1}} B \mathrm{~J}_{n}^{\prime}\left(\beta_{r_{1}} r\right)+\frac{\omega \varepsilon n}{\gamma r} A \mathrm{~J}_{n}\left(\beta_{r_{1}} r\right)\right] e^{j n \phi} \\
H_{\phi_{1}} & =\frac{-\gamma}{\beta_{r_{1}}^{2}}\left[\frac{j n}{r} B \mathrm{~J}_{n}\left(\beta_{r_{1}} r\right)+\frac{j \omega \varepsilon}{\gamma} A \mathrm{~J}_{n}^{\prime}\left(\beta_{r_{1}} r\right)\right] e^{j n \phi}
\end{aligned}
$$

$\underline{\text { Region } 2, r \geq a \text { : }}$

$$
\begin{aligned}
E_{z_{2}} & =C \mathrm{~K}_{n}\left(\alpha_{r_{2}} r\right) e^{j n \phi} \\
H z_{2} & =D \mathrm{~K}_{n}\left(\alpha_{r_{2}} r\right) e^{j n \phi} \\
E_{r_{2}} & =\frac{\gamma}{\alpha_{r_{2}}^{2}}\left[\alpha_{r_{2}} C \mathrm{~K}_{n}^{\prime}\left(\alpha_{r_{2}} r\right)-\frac{\omega \mu_{0} n}{\gamma r} D \mathrm{~K}_{n}\left(\alpha_{r_{2}} r\right)\right] e^{j n \phi} \\
E_{\phi_{2}} & =\frac{\gamma}{\alpha_{r_{2}}^{2}}\left[\frac{j n}{r} C \mathrm{~K}_{n}\left(\alpha_{r_{2}} r\right)-\frac{j \omega \mu_{0}}{\gamma} D \mathrm{~K}_{n}^{\prime}\left(\alpha_{r_{2}} r\right)\right] e^{j n \phi} \\
H_{r_{2}} & =\frac{\gamma}{\alpha_{r_{2}}^{2}}\left[\alpha_{r_{2}} D \mathrm{~K}_{n}^{\prime}\left(\alpha_{r_{2}} r\right)+\frac{\omega \varepsilon_{0} n}{\gamma r} C \mathrm{~K}_{n}\left(\alpha_{r_{2}} r\right)\right] e^{j n \phi} \\
H_{\phi_{2}} & =\frac{\gamma}{\alpha_{r_{2}}^{2}}\left[\frac{j n}{r} D \mathrm{~K}_{n}\left(\alpha_{r_{2}} r\right)+\frac{j \omega \varepsilon_{0}}{\gamma} C \mathrm{~K}_{n}^{\prime}\left(\alpha_{r_{2}} r\right)\right] e^{j n \phi}
\end{aligned}
$$

With the same procedure as with the dielectric cylinder of real $\varepsilon_{r}$, the boundary condition of continuity of the tangential fields is applied, and the following system is obtained 


$$
\left[\begin{array}{cccc}
m_{11} & 0 & m_{13} & 0 \\
0 & m_{22} & 0 & m_{24} \\
m_{31} & m_{32} & m_{33} & m_{34} \\
m_{41} & m_{42} & m_{43} & m_{44}
\end{array}\right]\left[\begin{array}{l}
A \\
B \\
C \\
D
\end{array}\right]=\left[\begin{array}{l}
0 \\
0 \\
0 \\
0
\end{array}\right]
$$

where

$$
\begin{aligned}
m_{11} & =\mathrm{J}_{n}\left(\beta_{r_{1}} a\right) \\
m_{13} & =-\mathrm{K}_{n}\left(\alpha_{r_{2}} a\right) \\
m_{22} & =\mathrm{J}_{n}\left(\beta_{r_{1}} a\right) \\
m_{24} & =-\mathrm{K}_{n}\left(\alpha_{r_{2}} b\right) \\
m_{31} & =\frac{j n}{\beta_{r_{1}}^{2} a} \mathrm{~J}_{n}\left(\beta_{r_{1}} a\right) \\
m_{32} & =-\frac{j \omega \mu_{0}}{\beta_{r_{1}} \gamma} \mathrm{J}_{n}^{\prime}\left(\beta_{r_{1}} a\right) \\
m_{33} & =\frac{j n}{\alpha_{r_{2}}^{2} a} \mathrm{~K}_{n}\left(\alpha_{r_{2}} a\right) \\
m_{34} & =-\frac{j \omega \mu_{0}}{\alpha_{r_{2}} \gamma} \mathrm{K}_{n}^{\prime}\left(\alpha_{r_{2}} a\right) \\
m_{41} & =\frac{j \omega \varepsilon}{\beta_{r_{1}} \gamma} \mathrm{J}_{n}^{\prime}\left(\beta_{r_{1}} a\right) \\
m_{42} & =\frac{j n}{\beta_{r_{1}}^{2} a} \mathrm{~J}_{n}\left(\beta_{r_{1}} a\right) \\
m_{43} & =\frac{j \omega \varepsilon_{0}}{\alpha_{r_{2}} \gamma} \mathrm{K}_{n}^{\prime}\left(\alpha_{r_{2}} a\right) \\
m_{44} & =\frac{j n}{\alpha_{r_{2}}^{2} a} \mathrm{~K}_{n}\left(\alpha_{r_{2}} a\right)
\end{aligned}
$$

The determinant of the previous homogenous system gives finally the following complex transcendental dispersion equation:

$$
\begin{aligned}
& \frac{\varepsilon_{r}}{\left(\beta_{r_{1}} a\right)^{2}} \cdot \frac{\mathrm{J}_{n}^{\prime 2}\left(\beta_{r_{1}} a\right)}{\mathrm{J}_{n}^{2}\left(\beta_{r_{1}} a\right)}+\frac{1}{\left(\alpha_{r_{2}} a\right)^{2}} \cdot \frac{\mathrm{K}_{n}^{\prime 2}\left(\alpha_{r_{2}} a\right)}{\mathrm{K}_{n}^{2}\left(\alpha_{r_{2}} a\right)}+\frac{\varepsilon_{r}}{\beta_{r_{1}} \alpha_{r_{2}} a^{2}} \cdot \frac{\mathrm{J}_{n}^{\prime}\left(\beta_{r_{1}} a\right) \mathrm{K}_{n}^{\prime}\left(\alpha_{r_{2}} a\right)}{\mathrm{J}_{n}\left(\beta_{r_{1}} a\right) \mathrm{K}_{n}\left(\alpha_{r_{2}} a\right)} \\
& +\frac{1}{\beta_{r_{1}} \alpha_{r_{2}} a^{2}} \cdot \frac{\mathrm{J}_{n}^{\prime}\left(\beta_{r_{1}} a\right) \mathrm{K}_{n}^{\prime}\left(\alpha_{r_{2}} a\right)}{\mathrm{J}_{n}\left(\beta_{r_{1}} a\right) \mathrm{K}_{n}\left(\alpha_{r_{2}} a\right)}+n^{2}\left(\frac{\gamma}{k_{0}}\right)^{2} \cdot\left(\frac{1}{\left(\beta_{r_{1}} a\right)^{2}}+\frac{1}{\left(\alpha_{r_{2}} a\right)^{2}}\right)^{2}=0
\end{aligned}
$$

The ratios relating $(A, B, C, D)$ are now 


$$
\begin{aligned}
\frac{B}{A} & =\frac{\gamma n}{\omega \mu_{0}}\left[\frac{1}{\left(\beta_{r_{1}} a\right)^{2}}+\frac{1}{\left(\alpha_{r_{2}} a\right)^{2}}\right]\left[\frac{\mathrm{J}_{n}^{\prime}\left(\beta_{r_{1}} a\right)}{\left(\beta_{r_{1}} a\right) \mathrm{J}_{n}\left(\beta_{r_{1}} a\right)}+\frac{\mathrm{K}_{n}^{\prime}\left(\alpha_{r_{2}} a\right)}{\left(\alpha_{r_{2}} a\right) \mathrm{K}_{n}\left(\alpha_{r_{2}} a\right)}\right]^{-1} \\
\frac{C}{A} & =\frac{\mathrm{J}_{n}\left(\beta_{r_{1}} a\right)}{\mathrm{K}_{n}\left(\alpha_{r_{2}} a\right)} \\
\frac{D}{A} & =\frac{\mathrm{J}_{n}\left(\beta_{r_{1}} a\right)}{\mathrm{K}_{n}\left(\alpha_{r_{2}} a\right)} \frac{B}{A}
\end{aligned}
$$

and, finally, the split of (3.230) in the separated sets of $\mathrm{HE}$ and $\mathrm{EH}$ modes gives that the transcendental dispersion equation for $\mathrm{EH}$ modes is:

$$
\frac{\mathrm{J}_{n+1}\left(\beta_{r_{1}} a\right)}{\left(\beta_{r_{1}} a\right) \mathrm{J}_{n}^{\prime}\left(\beta_{r_{1}} a\right)}=\left(\frac{\varepsilon_{r}+1}{2 \varepsilon_{r}}\right) \frac{\mathrm{K}_{n}^{\prime}\left(\alpha_{r_{2}} a\right)}{\left(\alpha_{r_{2}} a\right) \mathrm{K}_{n}^{\prime}\left(\alpha_{r_{2}} a\right)}+\left(\frac{n}{\left(\alpha_{r_{2}}\right)^{2}}-R\right)
$$

and for for HE modes:

$$
\frac{\mathrm{J}_{n-1}\left(\beta_{r_{1}} a\right)}{\left(\beta_{r_{1}} a\right) \mathrm{J}_{n}^{\prime}\left(\beta_{r_{1}} a\right)}=-\left(\frac{\varepsilon_{r}+1}{2 \varepsilon_{r_{1}}}\right) \frac{\mathrm{K}_{n}^{\prime}\left(\alpha_{r_{2}} a\right)}{\left(\alpha_{r_{2}} a\right) \mathrm{K}_{n}^{\prime}\left(\alpha_{r_{2}} a\right)}+\left(\frac{n}{\left(\alpha_{r_{2}}\right)^{2}}-R\right),
$$

with

$$
\begin{aligned}
R= & {\left[\left(\frac{\varepsilon_{r}-1}{2 \varepsilon_{r}}\right)^{2}\left(\frac{\mathrm{K}_{n}^{\prime}\left(\alpha_{r_{2}} a\right)}{\left(\alpha_{r_{2}} a\right) \mathrm{K}_{n}^{\prime}\left(\alpha_{r_{2}} a\right)}\right)^{2}\right.} \\
& \left.\quad-\frac{n^{2}}{\varepsilon_{r}}\left(\frac{\gamma}{k_{0}}\right)^{2}\left(\frac{1}{\left(\beta_{r_{1}} a\right)^{2}}+\frac{1}{\left(\alpha_{r_{2}} a\right)^{2}}\right)^{2}\right]^{1 / 2}
\end{aligned}
$$

Continuing now with the axialsymmetric modes $(n=0)$, the following expressions are found.

\section{$\mathrm{TM}_{0 m}$ modes:}

$\underline{\text { Region } 1, r \leq a \text { : }}$

$$
\begin{aligned}
E_{z_{1}} & =A \mathrm{~J}_{0}\left(\beta_{r_{1}} r\right) \\
H_{z_{1}} & =0 \\
E_{r_{1}} & =\frac{\gamma}{\beta_{r_{1}}} A \mathrm{~J}_{1}\left(\beta_{r_{1}} r\right) \\
E_{\phi_{1}} & =0 \\
H_{r_{1}} & =0 \\
H_{\phi_{1}} & =\frac{j \omega \varepsilon}{\beta_{r_{1}}} A \mathrm{~J}_{1}\left(\beta_{r_{1}} r\right)
\end{aligned}
$$


$\underline{\text { Region } 2, r \geq a \text { : }}$

$$
\begin{aligned}
E_{z_{2}} & =C \mathrm{~K}_{0}\left(\alpha_{r_{2}} r\right) \\
H z_{2} & =0 \\
E_{r_{2}} & =-\frac{\gamma}{\alpha_{r_{2}}} C \mathrm{~K}_{1}\left(\alpha_{r_{2}} r\right) \\
E_{\phi_{2}} & =0 \\
H_{r_{2}} & =0 \\
H_{\phi_{2}} & =-\frac{j \omega \varepsilon_{0}}{\alpha_{r_{2}}} C \mathrm{~K}_{1}\left(\alpha_{r_{2}} r\right)
\end{aligned}
$$

The boundary conditions of continuity on the tangential components of the field give the system

$$
\left[\begin{array}{cc}
\mathrm{J}_{0}\left(\beta_{r_{1}} a\right) & -\mathrm{K}_{1}\left(\alpha_{r_{2}} a\right) \\
\frac{\varepsilon}{\beta_{r_{1}}} \mathrm{~J}_{1}\left(\beta_{r_{1}} a\right) & \frac{\varepsilon_{0}}{\alpha_{r_{2}}} \mathrm{~K}_{1}\left(\alpha_{r_{2}} a\right)
\end{array}\right]\left[\begin{array}{l}
A \\
C
\end{array}\right]=\left[\begin{array}{l}
0 \\
0
\end{array}\right]
$$

which determinant gives the following transcendental dispersion equation for the $\mathrm{TM}_{0 m}$ modes:

$$
\frac{\mathrm{K}_{1}\left(\alpha_{r_{2}} a\right)}{\left(\alpha_{r_{2}} a\right) \mathrm{K}_{0}\left(\alpha_{r_{2}} a\right)}+\varepsilon_{r} \frac{\mathrm{J}_{1}\left(\beta_{r_{1}} a\right)}{\left(\beta_{r_{1}} a\right) \mathrm{J}_{0}\left(\beta_{r_{1}} a\right)}=0
$$

The constants $A$ and $C$ are related by

$$
\frac{C}{A}=\frac{\mathrm{J}_{0}\left(\beta_{r_{1}} a\right)}{\mathrm{K}_{0}\left(\alpha_{r_{2}} a\right)}
$$

In a similar way,

\section{$\underline{\mathrm{TE}_{0 m} \text { modes: }}$}

$\underline{\text { Region } 1, r \leq a \text { : }}$ 


$$
\begin{aligned}
E_{z_{1}} & =0 \\
H_{z_{1}} & =B \mathrm{~J}_{0}\left(\beta_{r_{1}} r\right) \\
E_{r_{1}} & =0 \\
E_{\phi_{1}} & =-\frac{j \omega \mu_{0}}{\beta_{r_{1}}} B \mathrm{~J}_{1}\left(\beta_{r_{1}} r\right) \\
H_{r_{1}} & =\frac{\gamma}{\beta_{r_{1}}} B \mathrm{~J}_{1}\left(\beta_{r_{1}} r\right) \\
H_{\phi_{1}} & =0
\end{aligned}
$$

$\underline{\text { Region } 2, r \geq a \text { : }}$

$$
\begin{aligned}
E_{z_{2}} & =0 \\
H z_{2} & =D \mathrm{~K}_{0}\left(\alpha_{r_{2}} r\right) \\
E_{r_{1}} & =0 \\
E_{\phi_{2}} & =\frac{j \omega \mu_{0}}{\alpha_{r_{2}}} D \mathrm{~K}_{1}\left(\alpha_{r_{2}} r\right) \\
H_{r_{2}} & =-\frac{\gamma}{\alpha_{r_{2}}} D \mathrm{~K}_{1}\left(\alpha_{r_{2}} r\right) \\
H_{\phi_{2}} & =0
\end{aligned}
$$

with boundary conditions equations

$$
\begin{aligned}
B \mathrm{~J}_{0}\left(\beta_{r_{1}} a\right) & =D \mathrm{~K}_{0}\left(\alpha_{r_{2}} a\right) \\
\frac{1}{\beta_{r_{1}}} B \mathrm{~J}_{1}\left(\beta_{r_{1}} a\right) & =-\frac{1}{\alpha_{r_{2}}} D \mathrm{~K}_{1}\left(\alpha_{r_{2}} a\right),
\end{aligned}
$$

and associated sytem

$$
\left[\begin{array}{cc}
\mathrm{J}_{0}\left(\beta_{r_{1}} a\right) & -\mathrm{K}_{1}\left(\alpha_{r_{2}} a\right) \\
\frac{1}{\beta_{r_{1}}} \mathrm{~J}_{1}\left(\beta_{r_{1}} a\right) & \frac{1}{\alpha_{r_{2}}} \mathrm{~K}_{1}\left(\alpha_{r_{2}} a\right)
\end{array}\right]\left[\begin{array}{l}
B \\
D
\end{array}\right]=\left[\begin{array}{l}
0 \\
0
\end{array}\right]
$$

which determinant, after some algebraic manipulations, gives the following transcendental dispersion equation for the $\mathrm{TE}_{0 m}$ modes:

$$
\frac{\mathrm{K}_{1}\left(\alpha_{r_{2}} a\right)}{\left(\alpha_{r_{2}} a\right) \mathrm{K}_{0}\left(\alpha_{r_{2}} a\right)}+\frac{\mathrm{J}_{1}\left(\beta_{r_{1}} a\right)}{\left(\beta_{r_{1}} a\right) \mathrm{J}_{0}\left(\beta_{r_{1}} a\right) 0}=0
$$

Finally, the constants $D$ and $B$ are related by 


$$
\frac{D}{B}=\frac{\mathrm{J}_{0}\left(\beta_{r_{1}} a\right)}{\mathrm{K}_{0}\left(\alpha_{r_{2}} a\right)}
$$

The set of equations detailed above give all the necessary tools to proceed with the modal analysis and the attenuation analysis. The power distribution features of the waveguide must be calculated using (3.193), (3.194) and (3.196). However, since the following studied cases are only suitable intermedium steps with didactic intention, to better understand the complex modal spectrum of the SWW, tracking the solutions from the rather familiar concepts of the classical dielectric cylindrical waveguide as it evolves to the SWW, in the following sections, only the issues dealing with the solutions of the transcendental equations (modes) and they behavior in terms of propagation and attenuation will be afforded.

\subsubsection{Solutions for $\varepsilon_{r}$ real positive in the complex plane $\gamma=$ $\alpha+j \beta$}

As a prelude, let first briefly consider that $\varepsilon_{r}$ is real, but now as a particular and simple case of a complex $\varepsilon_{r}$. Previously, only a portion of the axis $\gamma=j \beta$ of the complex $\gamma=\alpha+j \beta$ was observed. Specifically, to find propagating modes, the usual range $k_{0} \leq \beta \leq k_{1}$ was considered. Now, it makes sense to observe the whole first quadrant of the $\gamma$ plane to get a more general perspective (note that a negative $\alpha$ would imply amplified modes violating the energy principle, and a negative $\beta$ is just the symmetric solution considering propagation in the $-\hat{z}$ direction, except some anomalous cases involving complex and backward modes [555]-[556], not considered for the moment). To deal with simple values and clarify the text, the normalized quantities (by dividing by the factor $k_{0}$ ) will be considered. They will noted as $\gamma_{N}=\alpha_{N}+j \beta_{N}, \beta_{r_{1} N}$ and $\alpha_{r_{2} N}$.

The case $\varepsilon_{r}=4$ is considered as the base case, which give also simple values. For instance, it is known from the previous section that as the electrical size of the cylinder grows (either by increasing $a$ or $f$ ) the normalized propagation constant $\gamma_{N}=j \beta_{N} \rightarrow \sqrt{\varepsilon_{r}}$, giving in this simple case $\gamma_{N} \rightarrow 2$. In Figs. 3.16 and 3.17, it is represented $G=1 /\left|F\left(\gamma_{N}\right)\right|$, for the cases of $F$ described by (3.230) (HEM modes), (3.234) (EH modes), (3.235) (HE modes), (3.250) (TM modes), and (3.267) (TE modes). The radius of the cylinder is set to $a=3 \lambda$ in order to allow propagation to many modes. Notice that not only the domain is complex, but the determinant, $F\left(\gamma_{N}\right)$, has also its image in the complex space. Therefore, both, real and imaginary parts of $F\left(\gamma_{N}\right)$ must vanish, a couple of conditions that can be merged in one: nullity of the absolute $\left|F\left(\gamma_{N}\right)\right|$. The choice of representing $1 / \mid F\left(\gamma_{N} \mid\right.$ instead of $\left|F\left(\gamma_{N}\right)\right|$ is due to the larger contrast of a pole compared with a zero. Other options such logarithmic representation are as well suitable.

In the plots, the solutions of the transcendental equation for each case are appreciated as bright points, being dark red in their cuspid, sometimes too small and sharp to be appreciated. The $\mathrm{TM}_{0 m}$ and $\mathrm{TE}_{0 m}$ modes, Figs. 3.16(a) and 3.16(b), respectively, exhibit a quite handle transcendental function in the complex plane of $\gamma_{N}$. They are observed the guided modes in the range $1<\beta_{N}<\sqrt{\varepsilon_{r}}=2$, with the tendency of getting accumulated in the limit, i. e., $\beta_{N}=\sqrt{\varepsilon_{r}}=2$. Note that these 


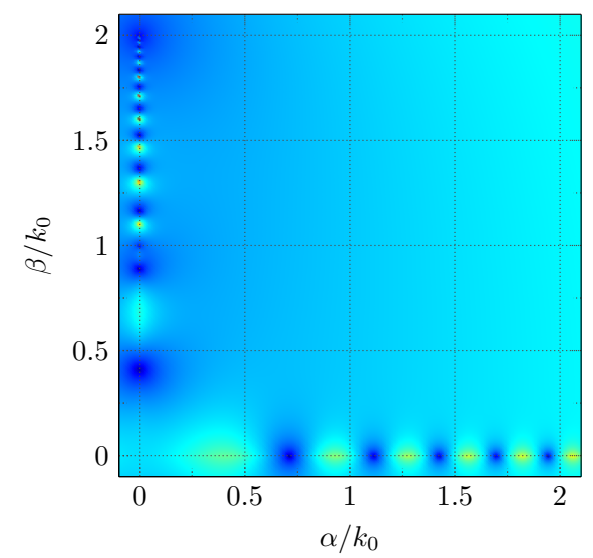

(a)

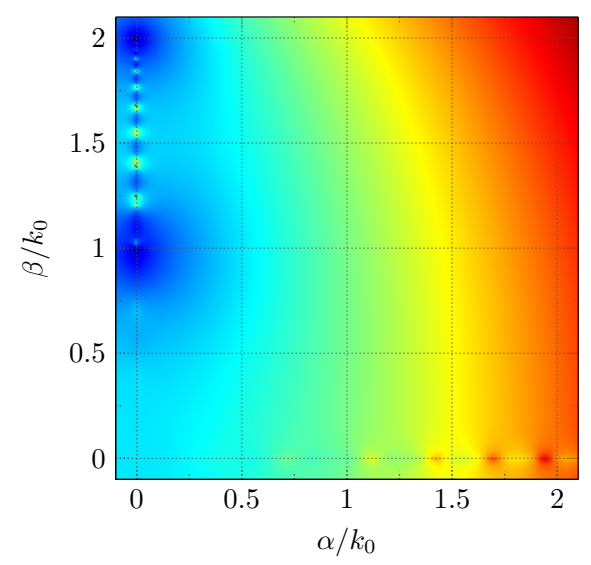

(c)

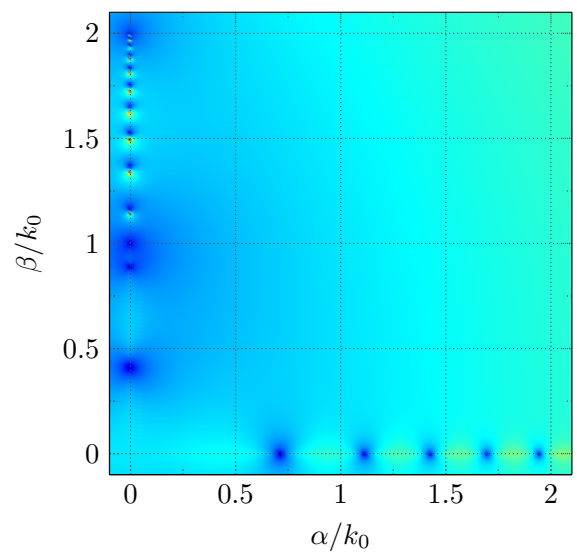

(b)

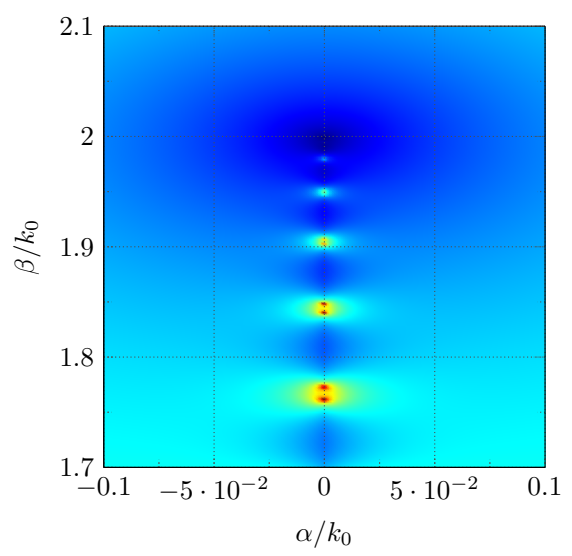

(d)

Figure 3.16: Colormap representation of $1 /\left|F\left(\gamma / k_{0}\right)\right|$ with $F$ described by (3.230) (HEM modes), (3.250) (TM modes), and (3.267) (TE modes), for the case of $\varepsilon_{r}=4$ and $a=3 \lambda_{0}$. a) $\mathrm{TM}_{0 \mathrm{~m}}$ modes; b) $\mathrm{TE}_{0 m}$ modes; c) HEM modes ( $\mathrm{HE}_{1 \mathrm{~m}}$ and $\mathrm{EH}_{1 \mathrm{~m}}$ modes together); d) HEM detail in $[-0.1,0.1] \times[1.7,2.1]$.

2D plot are, actually, the complex version of a vertical cut in the real plots of Fig. 3.7 .

However, now, they are also observed some solutions that do not satisfy the waveguiding conditions in the sense of surface waveguides, as established in section 3.1. There are two types of non-propagating modes. On the one hand, they are observed modes that satisfy $\alpha_{N}=0$ and $\beta_{N}<1$. These are leaky wave modes, with their angle of radiation given by $\arcsin \left(\beta_{N}\right)$. On the other hand, there is a set of evanescent modes having certain value of $\alpha_{N}$, but $\beta_{N}=0$. These modes are exponentially damped out with any kind of propagation, neither guided nor radiative. All the modes start as evanescent, and as the electrical size of the cylinder increases they become less and less damped until the moment they become leaky, 
starting from a radiation very transversal $\left(\beta_{N} \approx 0\right)$ and progressing to a more razing one $\left(\beta_{N} \approx 1\right)$. Then, they are propagated by the waveguide, and, at the end, they tend asymptotically to $\beta_{N}=\sqrt{\varepsilon_{r}}=2$.

By the above, the cutoff issue must be pointed now again. In the real variable problem, it is clear that $\alpha_{r_{2}}=0$ provides the condition, and modes below cutoff exhibit purely imaginary $\alpha_{r_{2}}$ : transversal outgoing waves. Notice that when the waves are not exponentially attenuated from the surface of the waveguide, they are not attached, guided any more. When $\alpha_{r_{2}}$ becomes complex, this is translated in the following condition [430]: $\operatorname{Re}\left(\alpha_{r_{2}}\right)=0$, since it is the real part of $\alpha_{r_{2}}$ which provides the transversal attenuation. However, in this case, the complex nature of $\alpha_{r_{2}}$ may give also some additional transversal propagation features through $\operatorname{Im}\left(\alpha_{r_{2}}\right)$, similar to those provided by $\operatorname{Re}\left(\beta_{r_{1}}\right)$ in the region 1 . Now, accordingly to (3.202) they are found to cases when $\operatorname{Re}\left(\alpha_{r_{2}}\right)=0$ is satisfied:

1. $\boldsymbol{\alpha}=\mathbf{0}$ and $\boldsymbol{\beta} \leq \boldsymbol{k}_{\mathbf{0}}$. If $\alpha=0$ (3.202) turns into (3.102), which gives the cutoff condition $\beta=\bar{k}_{0}$. Therefore, the modes lying in the semiaxis given by $\gamma=j \beta$, with $\beta \leq k_{0}$ will be at cutoff. These are the radiative modes.

2. $\boldsymbol{\beta}=\mathbf{0}$. If $\beta=0$, then $\gamma^{2}=\alpha^{2}$ and (3.202) gives pure positive imaginary $\alpha_{r_{2}}$. This is a deeper cutoff, the same that occurs for closed metallic waveguides.

By the above, they are more deeply understood the propagation characteristics of a dielectric waveguide, and, in particular, those of a surface waveguide. Note that there is a true cutoff given by $\beta=0$, which corresponds to that found for closed metallic waveguides. However, when the mode starts to propagate in the sense of the longitudinal direction, it is still not attached to the waveguide, and propagates in a radiative purport. This changes when $\beta$ reaches $k_{0}$, and, since for $\beta>k_{0}$ the structure acts as a waveguide, this is usually assumed as the cutoff of interest. Nonetheless, it can foreseen how important can be to take into account these reactive and radiative energy of the modes below this cutoff in order to estimate local effects. To apply modal methods to this kind of waveguides, this information must be known. It is very interesting to observe how the complex consideration of the propagation constant provides good physical insight even still the ideal case is assumed. Nevertheless, this makes sense thanks to the fact that, at this point of the thesis, the previous analysis considering a real variable problem is well understood.

In Fig. 3.16(c) the case for $n=1$, involving the hybrid modes $\left(\mathrm{HE}_{1 m}\right.$ and $\mathrm{EH}_{1 m}$ altogether) is represented. In this case the $G\left(\gamma_{N}\right)$ function has a more cumbersome aspect, exhibiting an exponential grow as $\alpha_{N}$ and $\beta_{N}$ assume large values, this making difficult the visualization the peaks corresponding to some solutions. Since (3.230) is used to express $F(\gamma)$, both, $\mathrm{HE}_{1 m}$ and $\mathrm{EH}_{1 m}$ solutions appear in the plot. This is better appreciated in the detailed plot of Fig. 3.16(d), in which a zoom of the zone $[-0.1,0.1] \times[1.7,2.1]$ is provided. The HE and EH modes appear as nearby couples. However, the following particularity occurs. The mode $\mathrm{HE}_{11}$ appears isolated, as it is the fundamental mode of the waveguide, with no cutoff. This mode is followed by the couples $\left(\mathrm{HE}_{n m+1}, \mathrm{EH}_{n m}\right)$. This phenomena is well appreciated in the simpler real plot of Fig. 3.7(b), for example, if a vertical cut is taken at some frequency $V$. 
These two sets of $\mathrm{HE}_{1 m}$ and $\mathrm{EH}_{1 m}$ modes can be splitted by using (3.235) and (3.234), respectively. The result is shown in Fig. 3.17(a) for the $\mathrm{HE}_{1 m}$ modes, and in Fig. 3.17(b) for the $\mathrm{EH}_{1 m}$ modes. With this procedure is easier to isolate the roots of the transcendental equation. Furthermore, $G\left(\gamma_{N}\right)$ takes a more suitable aspect, giving more resolution to the peaks. On the other hand, due to the presence of the root given by $R$ in (3.235) and (3.234), some branch cuts appear (see the discontinuities with a shape similar to the function $f(x)=x^{-1}$ ), due to the wellknown discontinuity of $f(z)=\sqrt{z}$ in the negative real semiaxis of the complex plane [542]. This is not affecting the propagating solutions, but hinders the finding of radiative and evanescent solutions. This is specially true for the $\mathrm{HE}_{1 \mathrm{~m}}$ modes, see Fig. 3.17(a). Since (3.235) takes the negative sign of $R$, the phase jump leave the solutions of $G(\gamma)$ in the improper Riemann sheet. Nevertheless, it is always possible to define other phase origin for $f(z)=\sqrt{z}$ in order to move the branch cut and observe the function in the correct Riemann sheet, as a mantle is removed to observe the content of a paint.

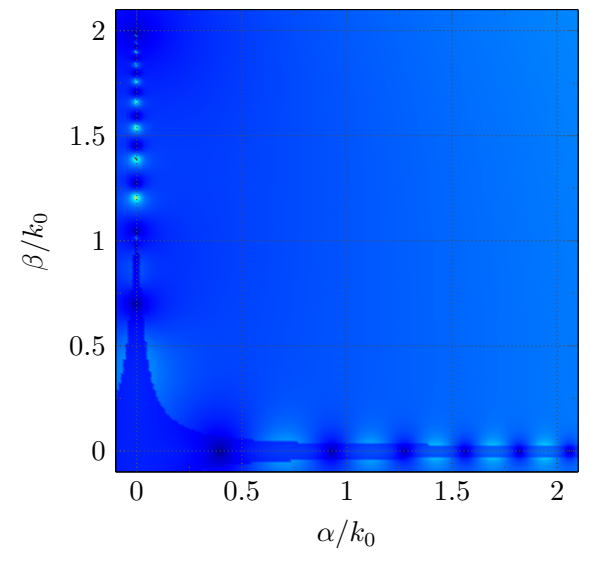

(a)

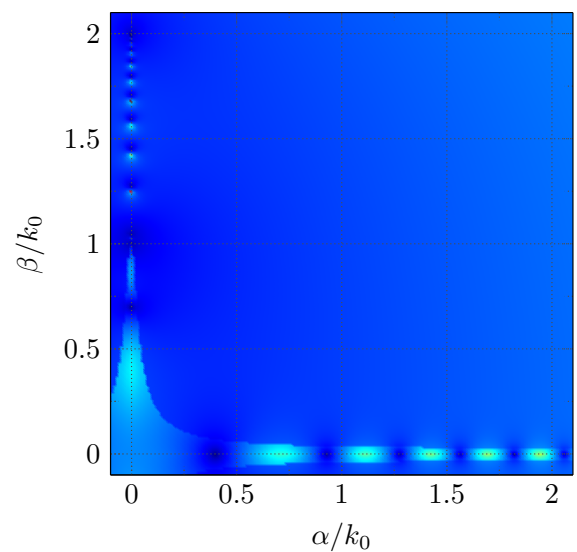

(b)

Figure 3.17: Split of $1 / F\left(\gamma / k_{0}\right)$ for the hybrid modes with $n=1$ given by (3.234) (EH modes) and (3.235) (HE modes), for the case of $\varepsilon_{r}=4$ and $a=3 \lambda_{0}$. a) $H E_{1 m}$ modes; b) $E_{1 m}$ modes.

Despite of the branch cut issues, the previous plots are a good example of the utility of manipulating the initial dispersion equation given (3.230) to obtain particularized equations for each kind of modes. When working with complex variable this become of great importance. Note that the root-finding problem turns 2-dimensional, thus having a wider case of possible features adopted by the function under study, and a larger number of possible points, which makes more difficult and slow the process. In this environment, the robustness of the algorithm gets threaten by roots that come very proximal to each other, something that is true in practice even advanced methods are used, unless some physical insight is gained previously in the problem. Imagine how difficult may become to distinguish between solutions when they get accumulated close to $\gamma_{N}=\sqrt{\varepsilon_{r}}$. The problem of complex root finding is an old and well documented one [528], [559]-[561], still in progress [562]-[563], and quite usual in some electromagnetic problems [564]-[566]. 


\subsubsection{The root-finding problem in the complex plane described by $\gamma$}

For the root-finding problem, one thoughtful possibility is to use such family of procedures described in [560]-[561], where poles of a function are located by doing a curvilinear integral on the $\gamma_{N}$ complex plane such that it encloses the poles. Advantages of these procedures are the systematically inspection of the complex domain of $G(\gamma)$ and the suitability of some quadrature methods (see for instance the extraordinary efficiency of trapezoids quadrature in the complex plane [564]). However, when many roots are involved in an exploring area (and this will be the case when they accumulate), since a prudent, enough large area must be considered inasmuch as the distribution the poles is not known a priori, the method present some issues. On the one hand, the integration path may pass close or go through a pole, this involving numerical errors. On the other hand, the large set of found solutions must be check one by one with additional information (analyze its $\alpha_{N}, \beta_{N}$, fields, etc.) in order to distinguish them. The tracking of a particular mode when any of the waveguide parameters is varied may be, hence, cumbersome.

By these aforementioned issues, in this work is has been found more suitable other procedure, relying one more time in the physical insight or a priori information available from the problem. On the one hand, the problematic case of having proximal couples of $\left(\mathrm{HE}_{n m+1}, \mathrm{EH}_{n m}\right)$ is avoided by splitting the equation with the use of (3.235) and (3.234). The appearing branch cut issue is a delicate one, but totally straightforward or even avoidable if particular information of the problem is used. On the other hand, the isolation of roots in accumulation zones is done by the following argumentation. Note that, for a given $n$, the different modes with index $m$ appear due to the oscillations provided by the oscillatory ${ }^{13}$ character of $\mathrm{J}_{n}\left(\beta_{r_{1}} r\right)$ in the region 1. As the waveguide enlarges in terms of the wavelength, more oscillations can take place, appearing modes of higher index $m$. Since the roots of $\mathrm{J}_{n}\left(\beta_{r_{1}} r\right)$ are known, it is possible to consider windows that isolate only one solution. Mathematically, there is a mapping from $\gamma$ to $\beta_{r_{1}}$ through 3.202 that makes the small distance (in relative terms for $\gamma$ ) between roots, to become well distinguished in $\beta_{r_{1}}$, thanks to the subtraction of $k_{1}$. Since bounds on $\beta_{r_{1}}$ can be set to isolate a single root by knowing $\mathrm{J}_{n}\left(\beta_{r_{1}} r\right)$ roots, the complexity of the problem is noticeably reduced. In summary, since $\mathrm{J}_{n}\left(\beta_{r_{1}} r\right)$ is quasi-periodic, and each quasi-period provides one ${ }^{14}$ solution, a simple procedure is restrict the research area to that quasi-period containing the desired solution. This becomes specially important when large quantities are involved, which will be the case of a SWW operating in the $\mathrm{THz}$ regime.

Finally, isolated the root, a good starting point is achieved, and Muller's method [528] is very suitable in terms of efficiency. In practice, the best option has been found by using combination of Muller method together with tracking the solution along the parameter which is varied with small enough steps from a known point. For instance, the asymptotic tendency $\gamma_{N} \rightarrow j \sqrt{\varepsilon_{r}}$, together with the root isolation procedure described before is a good performance option. For electrically large

\footnotetext{
${ }^{13}$ When $\beta_{r_{1}}$ and $\alpha_{r_{2}}$ are complex, both $\mathrm{J}_{n}\left(\beta_{r_{1}} r\right)$ and $\mathrm{K}_{n}\left(\alpha_{r_{2}}\right)$ provide a mixed nature, oscillatory and exponential, character. However, for small imaginary parts of the propagation constant (and this is the case for $\beta_{r_{1}}$ of the considered modes), the main oscillatory features are associated to $\mathrm{J}_{n}\left(\beta_{r_{1}} r\right)$.

${ }^{14}$ If the expression (3.230) for HEM modes is used, they will appear a couple of solutions, $\left(\mathrm{HE}_{n m+1}, \mathrm{EH}_{n m}\right)$.
} 
enough cylinders, the root is found to be close to $\gamma_{N}=j \sqrt{\varepsilon_{r}}$, therefore, it can be track for decreasing electrical length, even in those cases where the followed path is quite complex, as it will be shown next. In front of cases where the robustness of Muller method can be in risk, the algorithm is endorsed by inspection of the function through sampling of the predicted success zone by the tracking procedure. It is worth to mention that errors in the tracking process result critical inasmuch as when the solution jumps to a incorrect one the rest of points of the parametric analysis will give wrong solutions.

The final synthesized algorithm is efficient and robust. Between 50 and 100 frequency points per second are analyzed with no error for a large variety of cases. The larger the order $n$ the larger the time employed, mainly by the involved numerical evaluation of Bessel functions, which is more costly for larger orders of the function. The set of procedures and algorithms previously described is a good example of why it is worth to examine the analytical problem carefully. The a priori information not only provides the proper conclusions, but it is essential for a correct numerical resolution.

\subsubsection{Solutions for lossy dielectrics: $\varepsilon_{r}$ in the 4th quadrant of the complex plane $\gamma=\alpha+j \beta$}

Once the practical issues in the modes root finding have been commented, it is considered now the true problem where the previous formulation and procedures make sense. Now, $\varepsilon_{r}=\varepsilon_{r}^{\prime}-j \varepsilon_{r}^{\prime \prime}$, with both, $\varepsilon_{r}^{\prime}>0$ a $\varepsilon_{r}^{\prime \prime}>0$, thus $\varepsilon_{r}$ laying in the fourth quadrant of the complex plane described by $\gamma=\alpha+j \beta$. Accordingly to what was established in section 3.1.1, this is the case of a lossy. The initial point is given by $\varepsilon_{r}=4$ and then losses are added. Therefore, the following cases are afforded:

$$
\begin{aligned}
& \text { 1. } \varepsilon_{r}=4 \\
& \text { 2. } \varepsilon_{r}=4-0.2 j \\
& \text { 3. } \varepsilon_{r}=4-j \\
& \text { 4. } \varepsilon_{r}=4-2 j
\end{aligned}
$$

In the lossy cases, the imaginary part $\varepsilon_{r}^{\prime \prime}$ has been considered of the order of the real part $\varepsilon_{r}^{\prime}$ (usual ${ }^{15}$ dielectrics have lower $\varepsilon_{r}^{\prime \prime}$ ) to better appreciate the effect of the losses. In Fig. 3.18, it is shown the colormap representation of $G(\gamma)$ for the considered cases of permittivity in the case of $\mathrm{TM}_{0 m}$ modes (the following study has been made for every kind of mode, but explanation will be giving using results for $\mathrm{TM}_{0 m}$ modes; at the end of the study clarifications for $\mathrm{HE}_{n m}, \mathrm{EH}_{n m}$ and $\mathrm{TE}_{0 m}$ will be given). First of all, in these plots, for $\varepsilon_{r}$ non real, they are appreciated some discontinuities at the axis. This effect, rather related with mathematical aspect of the problem than the physical one, is briefly commented now as an important issue of the practical implementation.

\footnotetext{
${ }^{15}$ Those employed as low-loss dielectrics. However common materials may reach loss levels such that $\varepsilon_{r}^{\prime}$ and $\varepsilon_{r}^{\prime \prime}$ are of the same order.
} 


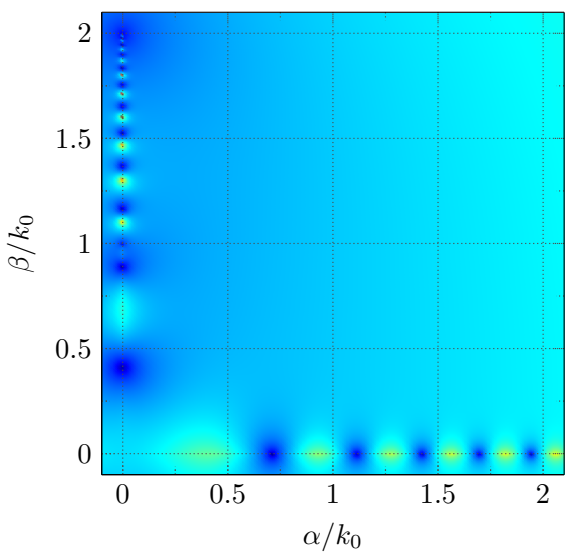

(a)

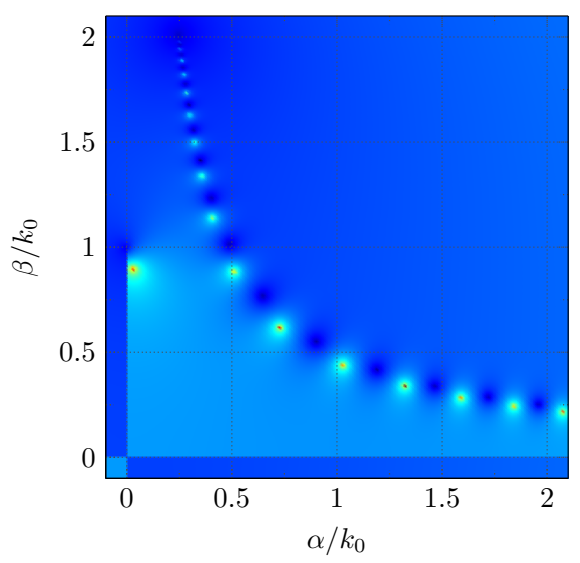

(c)

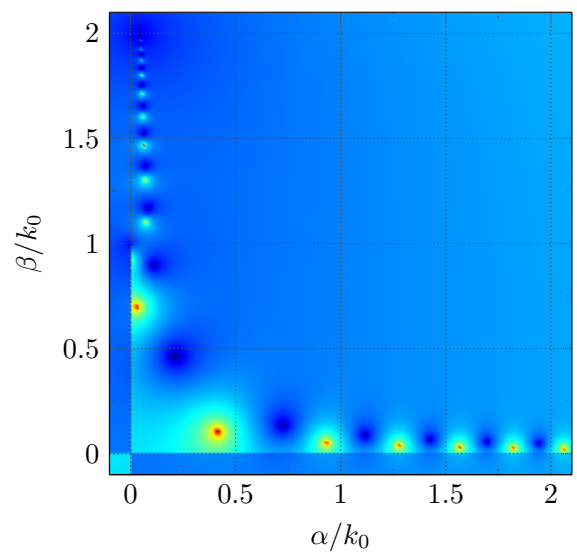

(b)

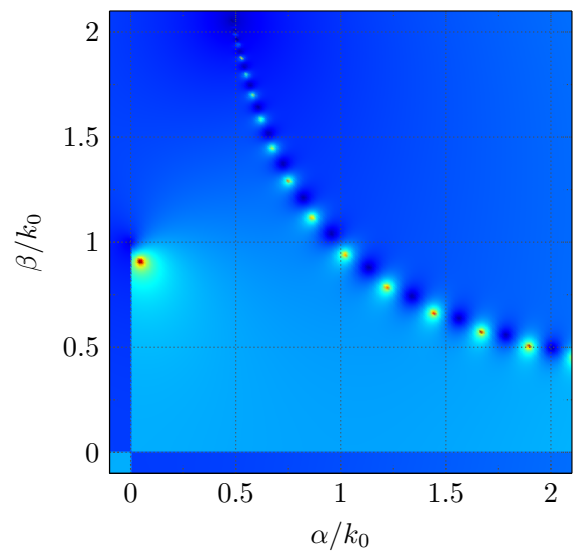

(d)

Figure 3.18: Colormap representation of $G\left(\gamma / k_{0}\right)$ for $\mathrm{TM}_{0 m}$ modes of a lossy dielectric cylinder of $a=3 \lambda_{0}$. a) $\varepsilon_{r}=4$; b) $\varepsilon_{r}=4-j 0.2$; c) $\varepsilon_{r}=4-j$; d) $\varepsilon_{r}=4-2 j$.

Specifically, these discontinuities are branch cuts that are present in the whole real axis $\alpha_{N}$, and in the imaginary axis $\beta_{N}$ in the range $[-1,1]$. These branch cuts have their origin in the square root involved in $\alpha_{r_{2}}$, defined in (3.202). The argument of the root defining $\alpha_{r_{2}}$ becomes real and negative when $\beta=0$ or when $\alpha=0$ and $\beta<k_{0}$. This conditions are the same as the cutoff conditions, which makes sense, since an argument real and negative in the root defining $\alpha_{r_{2}}$ implies $\alpha_{r_{2}}$ to be purely imaginary. Note that any branch cut associated to the root of $\beta_{r_{1}}$ is found on the graphs, however it is obvious that (3.201) causes a branch cut when the square root is applied to find $\beta_{r_{1}}$. Forcing the argument of the square root defining $\beta_{r_{1}}$ to be real and negative implies $\varepsilon_{r}^{\prime}+\alpha_{N}^{2}-\beta_{N}^{2}<0$ and $\beta_{N}=\varepsilon_{r}^{\prime \prime} /\left(2 \alpha_{N}\right)$ to be simultaneously satisfied, what gives a branch cut curve in the first ${ }^{16}$ quadrant of the complex plane.

${ }^{16}$ and in the third quadrant by symmetry, however this is not a region of interest for the problem. 
However, $\mathrm{J}_{n}(z)$ is even when $n$ is even, and odd when $n$ is odd. Since $f(z)=z$ is trivially odd, the phase jump of $\pi$ over the branch cut given by the square root of $\beta_{r_{1}}$ is always compensated in the second term of (3.250).

Conversely, $\mathrm{K}_{n}(z)$ has much less symmetries, and it can be only said that, over the curves defined by the branch cuts of $\alpha_{r_{2}}$, its real part is even and its imaginary part is odd, therefore acting as a conjugation over its argument. Properties of division in the complex plane makes that the total effect of $K_{1}\left(\alpha_{r_{2}} a\right) / K_{0}\left(\alpha_{r_{2}} a\right)$ is the same. Furthermore, since the term $\left(\alpha_{r_{2}} a\right)$ is purely imaginary over the branch cuts, and it only has a change of sign when crossing them, the total effect of the first term in (3.250) is exactly the same described: acts as a conjugation when the branch cuts of $\alpha_{r_{2}}$ are crossed. Note now that the absolute value of $z$ and $\bar{z}$ is the same, and that $|z+p|=|\bar{z}+p|$ its true if and only if $p$ is real. Since the quotient of the second term in (3.250) is real over the branch cuts of $\alpha_{r_{2}}$, when $\varepsilon_{r}$ is real, the second term in (3.250) is real. Therefore, when the absolute value is taken over $F\left(\gamma_{N}\right)$, any discontinuity is appreciated although it is present in $F\left(\gamma_{N}\right)$. This is the reason why, for the real $\varepsilon_{r}$ case, any discontinuity is found for $G\left(\gamma_{N}\right)$. The same behavior is observed for all kind of modes, being the argumentation exactly the same as the just described one.

Continuing with the physical effects of losses, as can be observed in the images, the increment of losses complicates the propagation phenomena in the dielectric cylindrical waveguide. When the case is non ideal, it is not longer possible to classify the modes as purely evanescent, radiative, or propagative. The perfect $L$ shape path followed by the modes as the waveguide electrical size increases (in decreasing direction in the $\gamma_{N}=\alpha_{N}$ axis towards the origin, and then, continuing along the $\gamma_{N}=j \beta_{N}$ axis) is deformed by the losses, turning into a more curved one. The larger the losses, the softer turns the path.

For relative small imaginary part, $\varepsilon_{r}=4-0.2 j$, it is still possible to loosely classify the modes as more propagative, more radiative or more evanescent. However, when $\varepsilon_{r}^{\prime \prime}$ increases, the nature of each mode is a mixed one, which certain characteristics, only valuables when the particular $\left(\gamma, \beta_{r_{1}}, \alpha_{r_{2}}\right)$ are known. The possible behaviors are much more in those cases. When the electrical size $a / \lambda_{0}$ grows, the modes still tend to accumulate at $\gamma_{N}=j \sqrt{\varepsilon_{r}}$. However, note that this value is not longer purely imaginary, but has a real term accounting for the losses. It is actually the complex propagation constant of a plane wave in a complex $\varepsilon_{r}$ infinity medium.

The limiting values of $\gamma_{N}$ for the considered cases are listed in the first row of table 3.2. Note the large losses due to the high values of $\tan \delta$ considered, since the values of the table show the normalized propagation constant $\gamma_{N}$. This is, the attenuation in $\mathrm{Np}$ per wavelength is obtained as $2 \pi \alpha_{N}$, or what is the same, in $\mathrm{dB}$ per wavelength by multiplying the values of $\alpha_{N}$ in the table by a factor close to 55 .

Apart of the first commented phenomena of in the graphs of Fig. 3.2, there is a second one that even attracts more the attention. No matter the increment of $\varepsilon_{r}^{\prime \prime}$ there is found a solution close to the semiaxis $\gamma_{N}=j \beta_{N}$, more specifically, close to $\beta_{N}=1$. This means, that there is a mode that, even for very lossy cylinders, is propagated with a propagation constant very similar to a plane wave, and with low attenuation. In fact, the losses for this mode are, at least, one order of magnitude less than for the rest. A careful numerical study of the problem reveals that this anomalous solution is always close to the propagation constant of a planar surface 


\begin{tabular}{lcccc} 
Parameter/Expression & $\varepsilon_{r}=4$ & $\varepsilon_{r}=4-0.2 j$ & $\varepsilon_{r}=4-j$ & $\varepsilon_{r}=4-2 j$ \\
\hline$\gamma_{N b}=j \sqrt{\varepsilon_{r}}$ & $2 \mathrm{j}$ & $0.05+2 \mathrm{j}$ & $0.248+2.015 \mathrm{j}$ & $0.496+2.059 \mathrm{j}$ \\
$\gamma_{N s}=j \sqrt{\frac{\varepsilon_{r}}{\varepsilon_{r}+1}}$ & $0.89 \mathrm{j}$ & $0.005+0.895 \mathrm{j}$ & $0.021+0.9 \mathrm{j}$ & $0.038+0.91 \mathrm{j}$ \\
$\beta_{r_{1} N}\left(\beta_{N}=1.5\right)$ & 1.33 & 1.336 & 1.363 & $1.489-0.001 \mathrm{j}$ \\
$\alpha_{r_{2} N}\left(\beta_{N}=1.5\right)$ & 1.11 & $1.121-0.094 \mathrm{j}$ & $1.151-0.43 \mathrm{j}$ & $1.213-0.823 \mathrm{j}$ \\
$\beta_{r_{1} N}\left(\gamma_{N s}\right)$ & & & & \\
$\alpha_{r_{2} N}\left(\gamma_{N s}\right)$ & 1.781 & $1.79-0.054 \mathrm{j}$ & $1.806-0.27 \mathrm{j}$ & $1.856-0.52 \mathrm{j}$
\end{tabular}

Table 3.2: Complex values of the normalized propagation constants, longitudinal $\left(\gamma_{N}\right)$, transversal in region $1\left(\beta_{r_{1} N}\right)$, and transversal in region $2\left(\alpha_{r_{2} N}\right)$, for several cases of interest, and different permittivities. $\gamma_{N b}$ is the normalized propagation constant of a plane wave in an infinite bulky dielectric material of $\varepsilon_{r}$ and it is the limit for bulk modes as a/ $\lambda_{0}$ increases, and $\gamma_{N s}$ is the normalized propagation constant of a planar surface (or plasmonic) mode traveling attached to an interface between a dielectric region with $\varepsilon_{r}$ and an air region.

mode (usually called surface plasmon [21], [348], [430], [512], [554], [567]-[571]) in the interface between two dielectric mediums, one of them having complex $\varepsilon_{r}$. The expression for this parameter is, for the normalized case:

$$
\gamma_{N s}=\frac{\gamma_{s}}{k_{0}}=j \sqrt{\frac{\varepsilon_{r}}{\varepsilon_{r}+1}}
$$

In the limit $a / \lambda \rightarrow \infty, \gamma_{N}$ tends to reach this asymptotic value inasmuch as when the radius of the cylindrical rod becomes large in terms of the wavelength, the curvature of it becomes locally null, as the propagation took place along a planar interface. However, the features associated to this mode are more complicated than they can be thought in a fist view. This solution is not independent of the rest of the set for a given $n$, as in principle it could be understood, at least in Figs. 3.18(c) and $3.18(\mathrm{~d})$, where this mode is very isolated, exhibiting a pair $\left(\alpha_{N}, \beta_{N}\right)$ very different from the others.

The actual propagation behavior of modes is as follows. For the first modes (explanation given in terms of the normalized propagation constants used in the plots), $\gamma_{N}$ starts with very large $\alpha_{N}$ and small $\beta_{N}$, so that, in this situation, they are rapidly damped out. With such small values of $\beta_{N}$, the energy is rather reactive. Progressively, as $a / \lambda_{0}$ increases, there is an increase on $\beta_{N}$ and a decrement on $\alpha_{N}$, hence the mode propagates with less and less losses. However, the curve is not exactly the same obtained if one links the points observed in the plots of, for example, Figs. 3.18(c) and 3.18(d). In fact, the path followed by the modes tends 
towards the cutoff condition given by $\alpha_{N}=0$. The modes reach the line and are at cutoff for a range of $a / \lambda_{0}$. After this situation of cutoff, from a larger $a / \lambda_{0}$, they start propagating again from the same cutoff condition $\left(\alpha_{N}=0\right)$, but with larger $\beta_{N}$. They stay in the condition of plasmonic mode, with a propagation constant close to that given by (3.269), and, finally, with the continued increasing of $a / \lambda_{0}$, they left this condition to become confined modes.

As the order $m$ of the mode increases, the previously described path shows a less exaggerated zig-zag shape. For example, the mode reaches the $\left(\alpha_{N}=0, \beta_{N}<1\right)$ cutoff segment in a larger $\beta_{N}$ and remains at cutoff a smaller range of $a / \lambda_{0}$. For some value of $m$, what occurs is that the mode approaching the plasmonic zone of the $\gamma_{N}$ plane just get close to the last mode in that zone, that remains there. The incoming mode, just pass close and continues its path to $\gamma_{N b}=j \sqrt{\varepsilon_{r}}$. This means, from some value $m$ the plasmonic solution remains there, and does not continue to become a confined one. The incoming modes pass further away as $m$ continue increasing, up to some point, for large enough $a / \lambda_{0}$, they follow a soft curved path without any zigzag, similar to that one that would be obtained if the points (apart of the plasmonic solution) of Figs. 3.18(c) and 3.18(d) were linked.

The process is rather a complicated to understand one. In order to clarify it, some auxiliary plots are displayed in Figs. 3.19 and 3.20. In Figs. 3.19(a) and 3.19(b) it is represented $G\left(\gamma_{N}\right)\left(\mathrm{TM}_{0 m}\right.$ modes) for $\varepsilon_{r}=4-0.5 j$ and $\varepsilon_{r}=4-j$, respectively, when $a=1.5 \lambda_{0}$. The previous particular permittivities are chosen since they offer good visualization of the effect under study. In both plots, it can be observed that the $\mathrm{TM}_{06}$ mode is in the plasmonic zone (considering $m=6$, counting the solutions in decreasing $\left.\beta_{N}\right)$. $\mathrm{TM}_{0 m}$ modes with lower $m$ are on their way to the limit of bulk modes $\gamma_{N b}=j \sqrt{\varepsilon_{r}}$. $\mathrm{TM}_{0 m}$ modes with larger $m$ are on their way to get less and less damped and reach the plasmonic zone. A tracking of the actual $\mathrm{TM}_{06}$ mode in both cases, shown in Fig. 3.20(a) for $\varepsilon_{r}=4-0.5 j$, and in Fig. 3.20(a) for $\varepsilon_{r}=4-0.5 j$, reveals the aforementioned behavior followed by the modes. In those plots, the limit $\gamma_{N b}=j \sqrt{\varepsilon_{r}}$, and the limit $\gamma_{N s}$ given by (3.269), are marked with a blue and a red square, respectively.

Note how for the $\varepsilon_{r}=4-0.5 j$ case, as $a / \lambda$ increases, the $\mathrm{TM}_{06}$ progress towards the plasmonic zone, reaching the cutoff $\alpha_{N}=0$. Then, it starts to propagate again, starting on the plasmonic zone and progressing towards $\gamma_{N b}=j \sqrt{\varepsilon_{r}}$. For the considered case, the cutoff state is a very short one, i.e, the range $a / \lambda_{0}$ in which the mode does not exist is quite narrow. In the analyzed curve, the overattenuation effect described in the section 3.3.2 is also appreciated: note that $\alpha_{N}$ is larger at some points than in the limit $\gamma_{N b}=j \sqrt{\varepsilon_{r}}$. The explanation is exactly the same as that given for the lossless case. The curve corresponding to the case $\varepsilon_{r}=4-1 j$, in Fig. 3.20(a), is totally different. In this case, the mode just progress monotonically towards lower $\alpha_{N}$ and larger $\beta_{N}$, until reaching $\gamma_{N b}=j \sqrt{\varepsilon_{r}}$. This curve correspond to the mode located at the point $\left(\alpha_{N}=0.75, \beta_{N}=0.5\right)$ in Fig. 3.19(a), which is actually the sixth mode following the path to $\gamma_{N b}=j \sqrt{\varepsilon_{r}}$.

Therefore, the question of what is the order $m$ of the surface plasmonic mode arises. What exactly happens is the following. When, from some order $m$, the plasmonic solution remains there and is not substituted by the following mode, there is still some interaction. The first mode that does not clearly stay as a plasmonic mode, passes, however, quite close to the plasmonic mode. When this occurs, the 


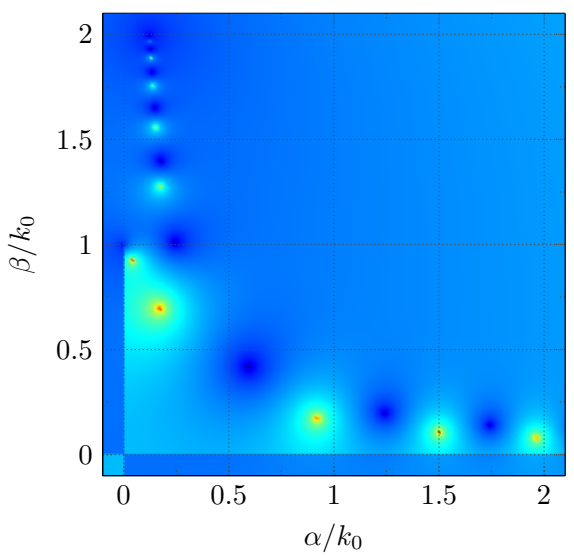

(a)

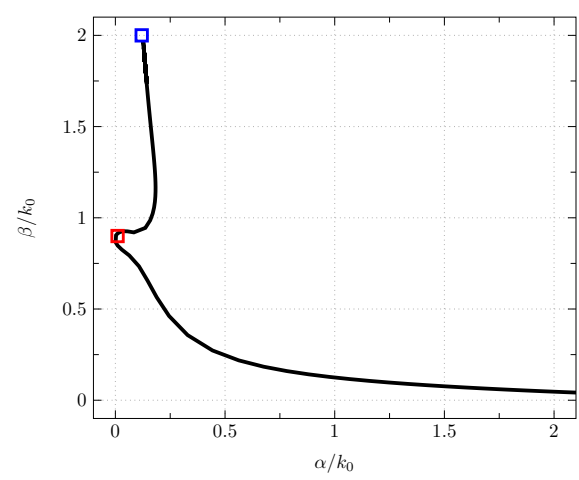

(c)

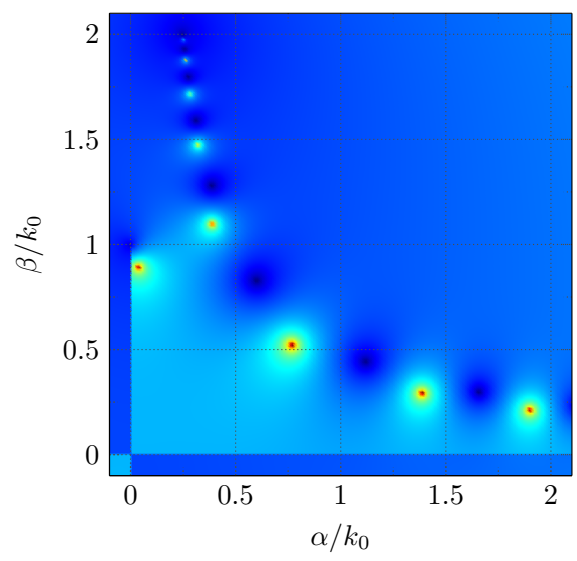

(b)

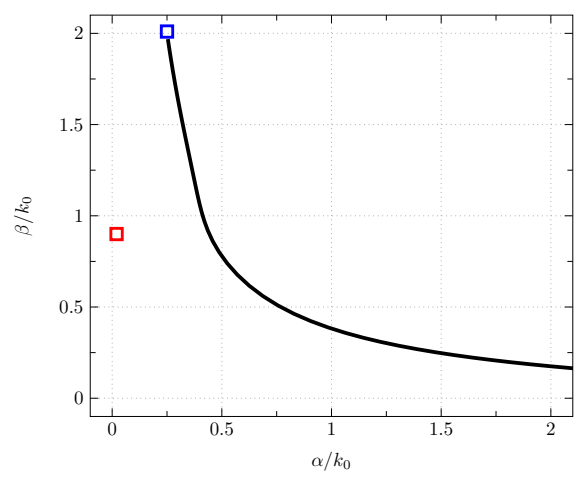

(d)

Figure 3.19: Mode path evolution in the complex plane of $\gamma_{N}$ as $a / \lambda_{0}$ increases. a) and b) show $G\left(\gamma_{N}\right)$ for $a=1.5 \lambda_{0}$. a) $\varepsilon_{r}=4-0.5 j$; b) $\varepsilon_{r}=4-j$. The $T M_{06}$ mode is in the plasmonic zone in those plots. c) and $d$ ) are the paths that follow the $T M_{06}$ in the $\gamma_{N}$ plane as $a / \lambda_{0}$ increases. c) $\varepsilon_{r}=4-0.5 j$; d) $\varepsilon_{r}=4-j$.

plasmonic solution slightly moves from the proximity of the red square point approximating the incoming mode (slightly losing the surface plasmonic features). Both modes interact. The field patter of the plasmonic mode becomes similar of that of the incoming mode that passes close to it. As $m$ increases, the modes pass further away, so that the interaction becomes weaker, and this effect is less appreciated. The plasmonic mode field pattern adopts in the region 1 the number of oscillations $m$ of the nearest mode passing close to it. However, as the interaction is lower, the character of the field becomes exponential rather than oscillatory, so that these oscillations are less appreciated.

See in Figs. 3.20(a) and 3.20(b) for instance the E-field magnitude mode patterns of the plasmonic modes shown in Figs. 3.19(a) and 3.19(b), respectively. In both cases, $m=6$ oscillations occur in the radial coordinate. However, in Fig. 3.19(b), corresponding to $\varepsilon_{r}=4-j$, they are much less evident, since and exponential decay 


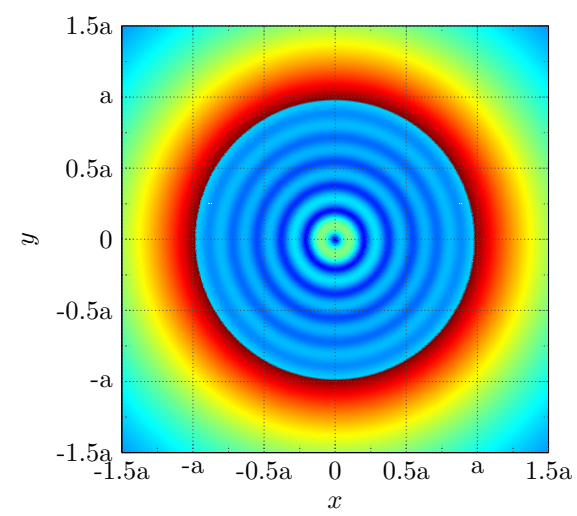

(a)

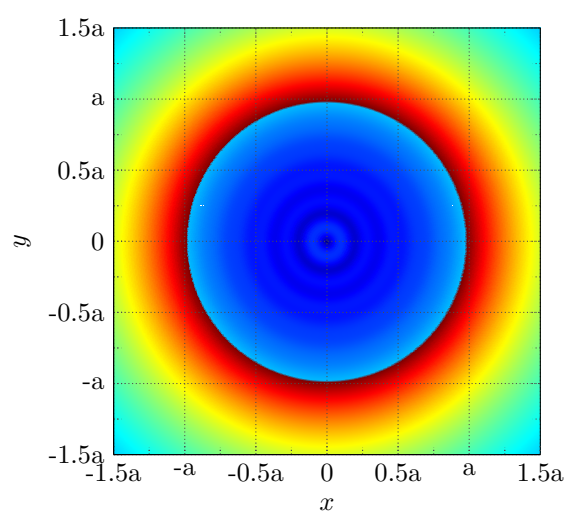

(b)

Figure 3.20: E-field magnitude of the plasmonic mode obtained from $G\left(\gamma_{N}\right)$ for a radius of $a=1.5 \lambda_{0}$. a) $\varepsilon_{r}=4-0.5 j$; b) $\varepsilon_{r}=4-j$.

from $r=a$ to the center of the cylinder predominates. The larger the losses, the more purely plasmonic become the mode in such zone. This is well appreciated in the column corresponding to $\beta_{r_{1} N}\left(\gamma_{N s}\right)$ in the table 3.2. It is bethought that the imaginary part of $\beta_{r_{1} N}$ causes the exponential decay in the region 1, typical of surface plasmons, and this contribution is larger as the losses increase.

It is obvious that the larger the losses, the set of modes apart from the surface plasmonic one, from now named as the set of bulk ${ }^{17}$ modes, follows a path passing further away from the plasmonic zone. Actually, as losses increase, the index $m$ from which there is no substitution of the plasmonic mode by the incoming one, decreases. With, for example, $\varepsilon_{r}=4-8 j$, the first mode is the only one that reach the plasmonic zone, and then it stays there. The rest of modes follow a monotone ( $\alpha_{N}$ decreasing, $\beta_{N}$ increasing) path towards $\gamma_{N b}=j \sqrt{\varepsilon_{r}}$ that passes quite far from the plasmonic zone.

The complexity of the observed phenomena, regarding the propagation features of an infinite set of bulk modes plus a plasmonic surface mode, both interrelated, has required a detailed explanation, that concludes in the following. Introducing the losses to the problem causes that non pure features are found any more. Therefore:

1. The modes are not exactly classified as: a) Evanescent $\left(\beta_{N}=0\right)$; b) Radiative $\left(\alpha_{N}=0, \beta_{N} \leq 1\right)$; c) Propagative $\left(\alpha_{N}=0, \beta_{N} \geq 1\right)$. They have more or less features of each kind along its path on the $\gamma_{N}$ complex plane as $a / \lambda$ increases. The larger the losses the blurrier becomes the frontier between the three diffent kinds.

2. There is a plasmonic zone $\left(\gamma_{N}\right.$ close to $\left.j\right)$, however, there is neither a purely plasmonic state that every mode experience, nor a total isolated plasmonic surface mode independent of the bulk modes, but occurs a continuous transition between these two behaviors. This transition can take any intermediate

\footnotetext{
${ }^{17}$ Bulk is a term commonly used to name those modes that are not experiment surface plasmonic behaviour, and travel more confined in the bulky dielectric inner medium.
} 
features (a great variety of cases), as the losses are varied. In a lossless dielectric cylinder waveguide every mode experience plasmonic features for a range of $a / \lambda_{0}$. If, contrarily, the dielectric is quite lossy, only the first mode $\left(\mathrm{TM}_{01}\right)$ reaches the plasmonic zone and stays there as an almost independent mode from the rest of modes, of bulky character. For intermedium losses, some modes experience a transient plasmonic feature, other with intermedium $m$ pass close and get influenced with some plasmonic features, and, finally, others with large $m$ pass far away from the plasmonic zone with almost no influence.

It is crucial to point out now that, the observed plasmonic behavior is only experienced by $\mathrm{TM}_{0 m}$ and $\mathrm{HE}_{n m}$ modes. For the $\mathrm{TE}_{0 m}$ and $\mathrm{EH}_{n m}$ modes the path followed is just that corresponding to a soft monotonically increasing in $\beta_{N}$ and decreasing in $\alpha_{N}$. For such cases, all of them are always bulk modes. This is the same as observed in the lossless dielectric cylindrical waveguide, but along the previous study the general view of the effect has been obtained, with a deep physical insight. Now the surface mode behaviour is totally understood. Note that the surface plasmononic limit $\gamma_{N s}$, for lossy real dielectrics $\left(\varepsilon_{r}\right.$ in the fourth quadrant of the complex plane), implies $\beta_{N}<1$. This means that, over the plasmonic zone, the surface mode is rather a leaky mode, radiative. This is, it has both, a field pattern with density of power predominantly in region 2 , and radiative character described by its propagation constant, which results to be less than that of a plane wave, therefore radiating with a real angle $\theta=\arcsin \left(\beta_{N}\right)$. Therefore, what was observed in the lossless dielectric case, was the tail of the surface state of the mode, which was already in the beginning of its way towards the total confinement at $\gamma_{N b}=j \sqrt{\varepsilon_{r}}$. The larger the dielectric contrast, the more difficult for the modes to escape from the plasmonic or surface features zone, this caused by the lower smoothness of $F\left(\gamma_{N}\right)$ for larger values of $\varepsilon_{r}$. Thus, larger contrasts allowed then to observe clearly the surface features in the propagating zone in as much the mode remained for a range of $a / \lambda_{0}$ in a segment of $\beta_{N}$ that was propagative $\left(\beta_{N} \geq 1\right)$ but enough close to $\beta_{N s}$ to still preserve surface waveguiding features.

Before leaving this section, the rest of observable features from the data in table 3.2 are briefly commented. In the second row, the values of $\gamma_{N}$ in the surface plasmon limit $\left(\gamma_{N s}\right)$ are studied. It is appreciated an increment of the propagation losses as the dielectric losses increase. However, this increment in propagation losses is lower than that predicted by the increment in the dielectric losses. For example, comparing $\varepsilon_{r}=4-1 j$ and $\varepsilon_{r}=4-2 j$, in the first row $\left(\gamma_{N b}\right)$, the losses are multiplied by a factor 2, which is, indeed, the factor that describes the increment of losses between the two dielectric cases. However, in the second row $\left(\gamma_{N s}\right)$, the factor is lower than 2. Now, looking the $5^{\text {th }}$ and $6^{\text {th }}$ rows, showing the transversal normalized propagation constants $\beta_{r_{1} N}$ and $\alpha_{r_{2} N}$ when $\gamma_{N}=\gamma_{N s}$, the following qualitative description is obtained. The main change in $\beta_{r_{1} N}$ has already been commented: as losses increase, the imaginary part becomes large, indicating an exponential decay from the interface $r=a$ to the inner region. The mode becomes confined near the surface in region 1. Regarding to $\alpha_{r_{2} N}$, the main feature is the increase of its real part, which describes how fast the fields decay exponentially from the interface $r=a$ to the infinity. Therefore, losses upgrade the surface waveguiding features of the mode 
that lies in the surface plasmonic zone, becoming the power concentrated near the interface between the two mediums, at both sides. Note also that the imaginary part of $\alpha_{r_{2} N}$ is larger than the real part, a fact that comes from the radiative character of the mode. To obtain the quantitative results, the integrals in (3.193) and (3.194) should be calculated. However, for qualitative conclusions is enough just to observe aforementioned issues. The final conclusion is that losses enhance noticeably the confinement near the interface, and the surface mode experience an increment of losses slightly lower than that predicted by the increment of the losses in the dielectric.

Regarding to the bulk modes, the rows $3^{\text {th }}$ and $4^{\text {th }}$ show how are the normalized transversal propagation constants, $\beta_{r_{1} N}$ and $\alpha_{r_{2} N}$, when the mode is, approximately, on its half way to the limit $\gamma_{N b}$. They have been calculated for the situation when the mode exhibits $\gamma_{N}=\alpha_{N}+1.5 j$, i. e., $\beta_{N}=1.5$. The most relevant feature to comment resides in $\beta_{r_{1} N}$, which is practically real. This means that bulk modes behave in the region 1 very similar to the lossless case. The character in this region is purely oscillatory. At the same time, there is an increment of the confinement of the power in the outer region, given by the increment of the real part of $\alpha_{r_{2} N}$. The imaginary part of $\alpha_{r_{2} N}$ experience a noticeable increment in relative terms, denoting oscillatory character in region 2, which can be interpreted as a radiative feature. However, in this case, the real part of $\alpha_{r_{2} N}$ imposes a strong damping factor en the radial direction, so the oscillatory effect is practically canceled. Therefore, for this kind of propagation, losses contribute also for a larger confinement of the mode. However, for bulk modes, the increment of confinement is less obvious and takes place only in the region 2 .

To the author knowledge, this rigorous study of the propagation characteristics of a lossy dielectric cylinder is not found in the literature. The conclusions extracted here about how the wide range of cases possibles for $\tan \delta$ cause a large variety of modal characteristics has not been reported. Although the analysis is left here because it forms part of a didactic process and numerical tool for the analysis of the SWW, the results obtained here might open the other to an interesting research line. Although dielectris used in microwave waveguides and transmission lines have lower $\varepsilon_{r}^{\prime \prime}$ than considered here (two or three orders of magnitude less), common materials have losses comparable to the dielectric materials treated here. The area of dielectric characterization [572]-[574] usually deal with high losses, and the cases involving cylindrical structures may benefit in accuracy from the particularities of the lossy dielectric cylindrical waveguide observed here. In the same way, extensions to include dielectric elements of well-known modal analysis techniques such as described in [575] and [576], or improvement of actual numerical analysis techniques in dielectric waveguides [577]-[578], must rely on the conclusions obtained in the simplest structures, such is a bulk cylinder.

\subsubsection{Solutions for $\varepsilon_{r}$ real and negative}

The third case to be analyzed is the first that comprises a permittivity that cannot be found in the nature for dielectric materials. A real and negative $\varepsilon_{r}$ will be considered. This artificial situation of having a virtual negative $\varepsilon_{r}$ is well-known our days due to popular topic of metamaterials, which has opened the possibilities 
of design by providing chart designed constitutive parameters $(\mu, \varepsilon)$ [579]. Nevertheless, the possibility of having a negative $\varepsilon_{r}$ in the microwave range comes from creating periodic microstructures (with a period smaller than the operating wavelength) that allowed to push down in frequency an effect that is typical for metals in the optical regime [580]-[581]. As commented in section 3.2, the Drude model shows clearly through (3.96) that metals in the optical regime are modelled with a complex permittivity, located in the third quadrant of the complex plane, and with a predominant negative real part, something that may be better appreciated by observing the values in the table 3.1, or observing the curves tendency in Fig. 3.5 .

In fact, the proposal of analyzing a dielectric cylindrical waveguide with negative real $\varepsilon_{r}$ came before of the metamaterials revolution. In 1994, Prade [554], carried out a rigorous analysis of this case as an approximation of a metal equivalent permittivity, neglecting the imaginary part. In this way, the analysis is much simpler since, with some considerations, it can be done by solving a real variable problem, similar to that of a lossless dielectric. It could be say that it is the case in which losses of the metal are neglected. Strong points of the analysis in [554] are the thoughtful disposal of some expressions, the use of auxiliary parameters that give physical insight, and a proper mathematical treatment. Here, some of the conclusions of that work will be taken, and the analysis will be expanded by taking advantage of using the complex $\gamma_{N}$ formulation, which indeed has been already shown to provide additional physical insight. The addition information revealed here, will naturally follow an easy incorporation of the imaginary part of $\varepsilon_{r}$ in the following sections.

Respecting the simplicity carried out in previous sections, the manageable value of $\varepsilon_{r}=-4$ is considered. Lets see the aspect of $G\left(\gamma_{N}\right)$ for the different types of solutions. First, in Figs. 3.21(a) and 3.21(b) they are shown the colormaps of $G\left(\gamma_{N}\right)$ for the TM and TE modes, respectively, for a cylinder of radius $a=\lambda_{0}$, chosen to provide a good example for modal properties explanation. The aspect of the function for each case is totally different. In the case of TM modes it is observed an isolated solution on the imaginary axis, hence lossless. This solution is a surface mode which lies in what before has been named as plasmonic zone. However, note that now $\beta_{N}>1$ so this mode is propagative instead of radiative, what was the case for positive real part of $\varepsilon_{r}$. This is easy to understand if one follows the propagation constant expression of a surface plasmonic mode traveling on a planar interface (3.269). Since now $\varepsilon_{r}$ is negative, in the denominator of the square root, the ${ }^{\prime}+1^{\prime}$ coming for the permittivity of the air is reducing the magnitude of the denominator, hence the quotient becoming larger than the unity. This fact is the most relevant of the analysis, since it says that, for a negative $\varepsilon_{r}$, the surface plasmonic solution is propagative, so it becomes a solution with more relevance in terms of waveguiding. The lossless character of this solution results obvious from the lack of losses term in $\varepsilon_{r}$.

Additionally, it is found a set of bulky $\mathrm{TM}_{0 m}$ modes close to, but not lying in the real axis. There is found a duplicity with symmetry in the real axis for each solution. This is nothing more that the duplicity of the mathematical problem, which is another apart of the symmetry of the problem in the $z$ direction. Since any branch cut appear on the real axis due to the real nature of $\varepsilon_{r}$ by the arguments given in the previous section, solution of both Riemann sheets are appreciated. This 


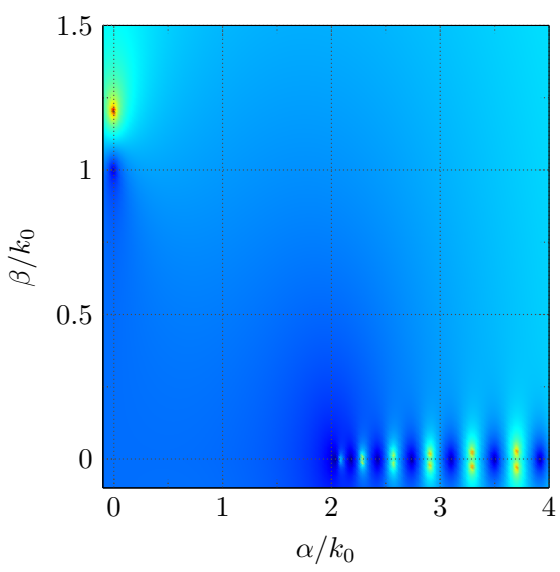

(a)

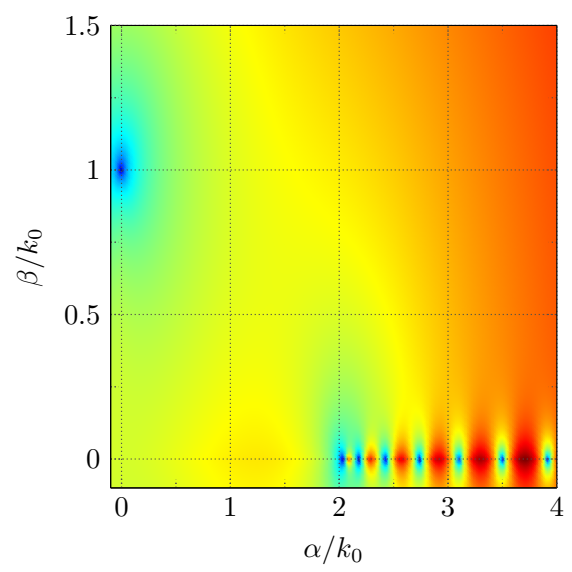

(b)

Figure 3.21: Colormap representation of $G\left(\gamma / k_{0}\right)$ of a virtual dielectric cylinder of permittivity $\varepsilon_{r}=-4$ and radius $a=\lambda_{0}$. a) TM modes; b) TE modes.

strange mathematical situation come from the anomalous solutions obtained, which exhibit complex propagation constant despite of the fact that the problem is losses by $\varepsilon_{r}$. It makes sense to take positive $\beta$ in order to follow attenuation direction. The posterior section confirm that the negative $\beta$ solutions disappear as losses are introduced.

The limit of bulk modes is given now by $\gamma_{N b}=j \sqrt{\varepsilon_{r}}=2$, where the negative sign of the square root has been taken since the lossless case is the limiting case $\varepsilon_{r}^{\prime \prime} \rightarrow 0$, the limit approaching from negative values, hence from that corresponding side of the branch cut discontinuity. Therefore, as $a / \lambda_{0}$ increases, bulk modes tend to a purely evanescent situation, which is, actually, how a plane wave behaves in a negative $\varepsilon_{r}$ infinite medium ${ }^{18}$. If $a / \lambda_{0}$ decreases, every bulk mode experience an increase of $\alpha_{N}$, and a slight increase of $\beta_{N}$, this last meaning that the imaginary part of $\gamma_{N}$ is necessary to the mode exist. The increase of $\beta_{N}$ is evident from the limit to the right of the plot, however, it asymptotically tends to $\beta_{N}=0.04$, therefore bounded. In must be highlighted that, for negative $\varepsilon_{r}$, the two kind of modes are independent, since any bulk mode reach the plasmonic zone, where the surface mode always is found. Therefore, it makes sense to call from now the surface mode as $\mathrm{TM}_{0}$ mode, and the rest of bulk modes as $\mathrm{TM}_{0 m}$. Particularities of this situation and the corresponding argumentation will be later given.

Regarding the TE modes case, the most important fact is that any surface mode solution is found. Note that solutions are given by red points (indicating a pole of $G\left(\gamma_{N}\right)$ ), and the point observed in Fig. 3.21(b) is a zero of $G\left(\gamma_{N}\right)$ (singularity in the determinant $F\left(\gamma_{N}\right)$, present in every case. Therefore, as in the previous cases, no surface modes or features are observed for TE modes. In this problem, the previous fact can be mathematically argued in an obvious way. Following [554], it results

\footnotetext{
${ }^{18}$ Consider a plane wave at optical frequencies propagating inside an infinite metallic medium. It is very intuitive to think that the propagation will be evanescent.
} 
interesting, in order to convert the problem in a real one (if this is desired), to observe that $k_{1}^{2}=k_{0}^{2} \varepsilon_{r}$, is now negative. Therefore, to make $\beta_{r_{1}}$ real, one must define it as:

$$
\left(\beta_{r_{1}}^{-}\right)^{2}=\beta^{2}-k_{1}^{2}
$$

or what is the same, inverting the sign in (3.101) in the same way as it is done in (3.102). With this change, both transversal propagation constants remain real, and the modified Bessel functions must be used also in region 1. Since the region 1 contains the origin, where $\mathrm{K}_{n}(x)$ is singular, and does not extent to infinity, where $\mathrm{I}_{n}(x)$ is singular, it is determined that $\mathrm{I}_{n}(x)$ functions must be used in region 1 if negative $\varepsilon_{r}$ is considered. From the formulation followed along the complex $\varepsilon_{r}$ case, this is the same as letting $\beta_{r_{1}}$ to be defined as in (3.201), and due to the negative nature of $k_{1}$, obtain purely imaginary values for $\beta_{r_{1}}$ if $\gamma$ is purely imaginary, as it is the case of the surface mode. In such described case the imaginary character of $\beta_{r_{1}}$ infers the $\mathrm{I}_{n}(x)$ behavior to the $\mathrm{J}_{n}(x)$ functions, thus having the usual surface mode situation.

It is noted now that, under this situation, the two terms in the dispersion equation of TE modes (3.266) are positive and present similar exponential behavior. Since both terms are of the same sign and monotones (any oscillation is present), there is no possible solution. However, for TM modes, the presence of a negative $\varepsilon_{r}$ in (3.249) allows to have a solution, since one of the terms changes its sign an the function crosses zero once. In [554], this is proved mathematically. The uniqueness of the TM mode solution is not proved, however. Nevertheless, it seems obvious that any additional solution can be considered in the $\gamma=j \beta$ axis since both terms in (3.249) are monotones due to the fact that any oscillatory character is present.

Since in [554] only purely imaginary propagation constants are considered, bulk modes are totally missed. Note that if $\gamma_{N}$ is considered in the real axis or its vicinity $\left(\gamma_{N} \approx \alpha_{N}\right), \beta_{r_{1}}$ become predominantly real (or $\beta_{r_{1}}^{-}$predominantly imaginary) in as much as $\alpha_{N}$ is larger than $\sqrt{\varepsilon_{r}}$. Therefore, oscillatory behavior of in the second term of (3.266) and (3.249) is found and an infinity number of $\mathrm{TM}_{0 m}$ and $\mathrm{TE}_{0 m}$ solutions are recovered. In the case of $\mathrm{TE}_{0 m}$ modes, the bulk solutions are also different than those for $\mathrm{TM}_{0 m}$ modes. They are found on the real axis (purely evanescent), and less defined, being that $G\left(\gamma_{N}\right)$ does not show a clear peak. Mathematically speaking, there is not solution. However, the problem is in the limit of this solution appear, just requiring a small, but not null imaginary part in $\varepsilon_{r}$. Physically speaking, and even more in engineering terms, they must be considered as solution since they will actually be whenever a small loss is considered. A similar situation was observed, with less clarity, in the case of a $\varepsilon_{r}=4$ dielectric cylinder, see Fig. 3.18(a). Some of the radiative and evanescent solution are not well defined peaks. However, it is observed, that a small loss, moving them from the axis, allow to observe clear modes, see Fig. 3.18(b).

The modes with higher number $n$ of azimuthal variation (the hybrid HEM modes) exhibit similar behavior, with a slightly more complex $G\left(\gamma_{N}\right)$ function. In Fig. $3.22(\mathrm{a}) G\left(\gamma_{N}\right)$ is represented for $n=1$ modes, without any separation in HE or EH modes. A surface mode and a set bulk modes are appreciated, however, representing them in the same plot makes difficult to clearly observe them due to the bad contrast 
provided by the properties of $G\left(\gamma_{N}\right)$ in the considered domain. A detail of the surface mode is given in Fig. 3.22(b). It is clearly appreciated how this solution lies in the imaginary axis of $\gamma_{N}$, with $\beta_{N}$ close to 1.2 , clearly propagative. Separation of the dispersion equation for hybrid modes in $\mathrm{HE}$ and $\mathrm{EH}$ modes, or a deep analysis of this solution, indicates it is the $\mathrm{HE}_{1}$, where the radial index $m$ is removed by the same reasons as before: only one solution is found and there are not radial variations.

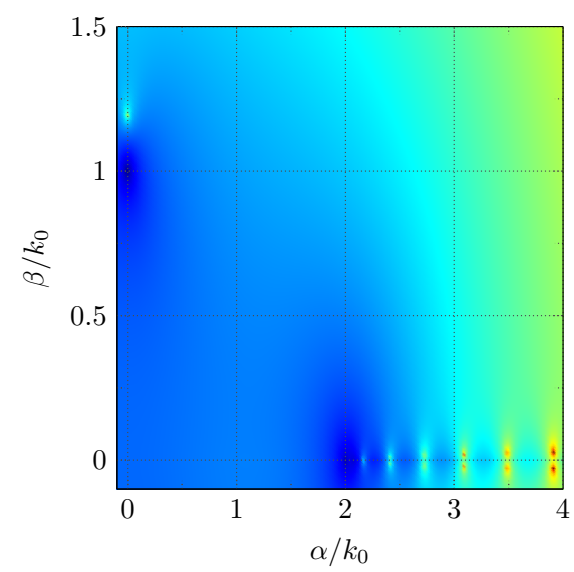

(a)

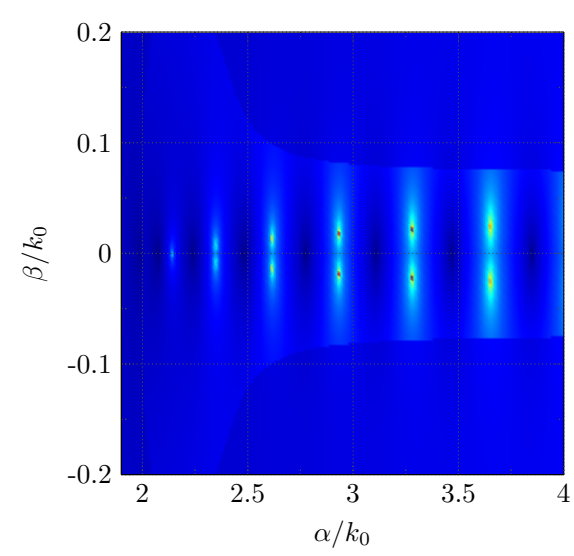

(c)

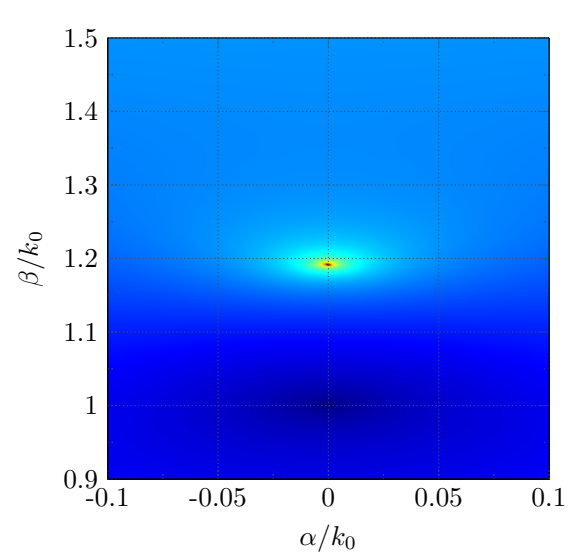

(b)

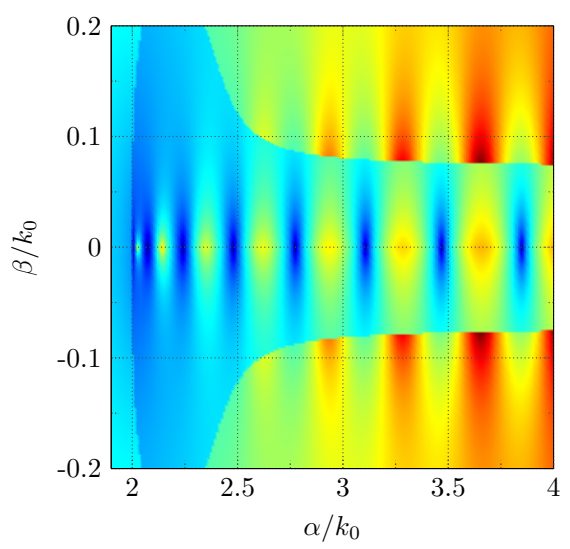

(d)

Figure 3.22: Colormap representation of $G\left(\gamma / k_{0}\right)$ of a virtual dielectric cylinder of permittivity $\varepsilon_{r}=-4$ and radius $a=\lambda_{0}$. a) All HEM modes for $n=1$; b) Detail of the HE $E_{1}$ surface mode; c) Detail of the $\mathrm{HE}_{1 \mathrm{~m}}$ bulk modes; d) Detail of the $\mathrm{EH}_{1 \mathrm{~m}}$ bulk modes.

Regarding to the bulk modes, details of $G\left(\gamma_{N}\right)$ in this zone are given in Fig. 3.22(c) for $\mathrm{HE}_{1 m}$ modes, and in Fig. 3.22(d) for $\mathrm{EH}_{1 m}$ modes. The branch cuts of the term $R$ described in (3.236) are clearly observed. In the case of $\mathrm{HE}_{1 m}$, the same behavior as for $\mathrm{TM}_{0 m}$ modes is observed: they have non-zero $\beta_{N}$ and only the positive $\beta_{N}$ must be considered. In a similar way, a parallelism is observed between $\mathrm{EH}_{1 m}$ modes and $\mathrm{TE}_{0 m}$ modes: the solutions lie in the real axis, and are not well 
defined. Note how, for bulk modes, $\mathrm{HE}_{1 m}$ solutions totally mask $\mathrm{EH}_{1 m}$ solutions in Fig. 3.22(a) due to the lack of definition of these last. This effect can be appreciated also in Fig. 3.22(d), where the strength of the $\mathrm{HE}_{1 m}$ solutions is observed beyond the branch cut. When no separation is made, the skirts of the $\mathrm{HE}_{1 m}$ peaks mask the $\mathrm{EH}_{1 m}$ weaker peaks.

In the waveguide under study it seems obvious that the modes of interest are the surface modes, which provides unattenuated waveguiding. From the previous argumentation it is clear that for each index $n$ a surface mode appears. In the case of the $\mathrm{TM}_{0}$ mode it is obvious that the dominant polarization is the radial one, since the field is purely radial. However, the hybrid modes $\mathrm{HE}_{n}$ also exhibit a clear dominance of the radial polarization, being $E_{r_{1}}$ more than 2 orders of magnitude larger than $E_{\phi_{1}}$. It can be perfectly said that those hybrids modes are quasi-TM modes. This is the usual surface plasmon mode features, which has polarization orthogonal to the interface between the two regions, and exponential decay at both sides of such interface. In Fig. 3.23 the E-field magnitude mode pattern for the first 4 surface modes $\left(\mathrm{TM}_{0}, \mathrm{HE}_{1}, \mathrm{HE}_{2}\right.$ and $\left.\mathrm{HE}_{3}\right)$ is shown. To appreciate the strong field decay from the interface, the considered scale is logarithmic, ranging from $-100 \mathrm{~dB}$ to $0 \mathrm{~dB}$. For the considered radius, $a=\lambda_{0}$, the same radial behavior is observed in every mode, i.e., how fields depend on $r$ through $\beta_{r_{1}}$ and $\alpha_{r_{2}}$. Thus, only the azimuthal variations determine the mode. This, however, will be shown to change when the radius is diminished. The curvature of the structure allows to a different interaction when $n$ is not null, a phenomena that has been object of discussion in several works dealing with optical plasmons [430], [554], [567]-[569]. A fact to be also observed in the previous plots is the large damping of the fields towards the inner region, much more evident than the damping towards the outer region.

To better understand the propagation nature of these surface modes, $a / \lambda$ is parametrized between 0 and 2 , and $\beta_{N}$ is obtained for the first 5 surface modes $\left(\mathrm{TM}_{0}, \mathrm{HE}_{1}, \mathrm{HE}_{2}, \mathrm{HE}_{3}\right.$ and $\left.\mathrm{HE}_{4}\right)$. In Fig. 3.24, the dispersion diagrams for the cases of $\varepsilon_{r}=-4$, Fig. 3.24(a), and $\varepsilon_{r}=-2$, Fig. 3.24(b), are shown for these modes. In both plots the main observed fact is that the $\mathrm{TM}_{0}$ mode presents a singular behavior when $a / \lambda_{0}$ is decreased. Instead of experiencing a decrement of $\beta_{N}$ until the mode become radiative, $\beta_{N}$ grows exponentially towards infinity. Actually, the problem is singular as $a / \lambda_{0}$ tends to 0 . Since for $n=0$ there is no azimuthal phase variation, the fields in the inner region become enhanced when the radius is decreased, causing a larger confinement of the surface mode, something that cannot occur for $n \neq 0$.

When $a / \lambda_{0}$ becomes very large, every mode tends towards the limit of a propagation constant equal to that of a surface plasmon on a planar interface given in (3.269), being that the local curvature experienced by the mode is very large. This limit is shown in the plots with a horizontal dashed black line. As can be observed this limit is more evident (in the propagative sense of differing from a plane wave in the outer medium $\beta_{N}=1$ ) when the absolute value of the permittivity is lower (case $\left.\varepsilon_{r}=-2\right)$.

The propagation phenomena are also more exaggerated for $\varepsilon_{r}=-2$, as can be observed comparing both plots of Fig. 3.24. Apart of the singularity of the $\mathrm{TM}_{0}$ mode, which due to this fact has virtually no cutoff, a particular behavior is observed for the $\mathrm{HE}_{1}$ mode, and with less intensity as $n$ increase: when $a / \lambda_{0}$ decreases, before going to the cutoff condition given by $b_{n}=1$ (the modes become radiative), there is 


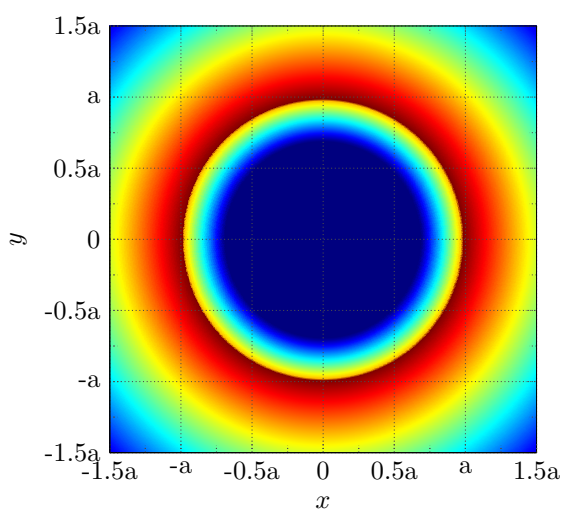

(a)

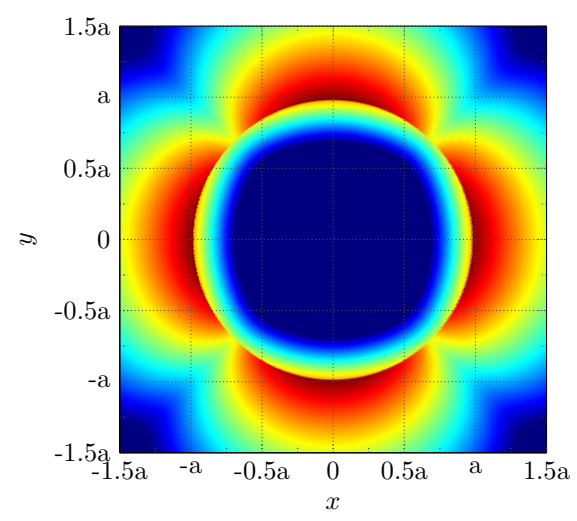

(c)

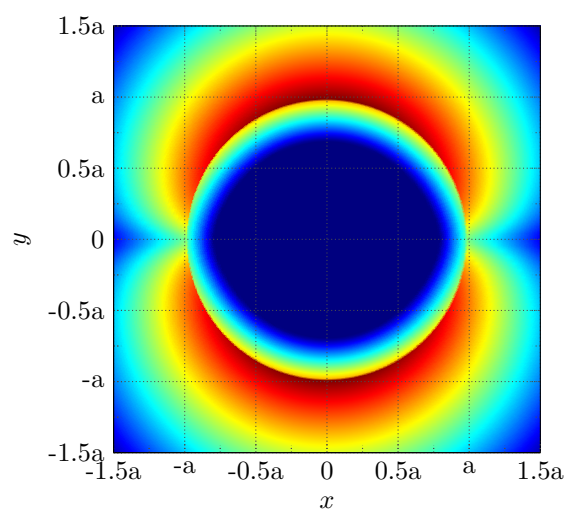

(b)

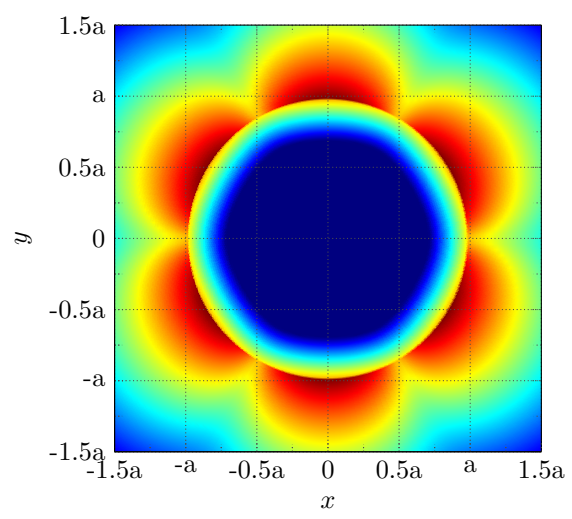

(d)

Figure 3.23: E-field magnitude mode pattern in logarithmic scale with 100 dB range for the first surface modes of a dielectric cylinder of permittivity $\varepsilon_{r}=-4$ and $a=\lambda_{0}$. a) $T M_{0}$; a) $H E_{1}$; a) $\mathrm{HE}_{2}$; a) $\mathrm{HE}_{3}$.

an unusual grow of $b_{n}$ that reaches its maximum value and then decreases towards the cutoff. Every mode experience this behavior, however, the peak in $\beta_{N}$ becomes less evident as the order $n$ increases. This can be affirmed since every curve overcomes the limit given by $\beta_{N s}$, thus having a local maximum for some $a / \lambda_{0}$.

The $\mathrm{HE}_{1}$ continues having not cutoff, mathematically, however it is again found a virtual cutoff at which the mode becomes essentially a plane wave in the outer medium. The rest of $\mathrm{HE}_{n}$ have a quite evident cutoff, and it is clearly appreciated that the cutoff between modes is equally spaced. Note also that the case of $\varepsilon_{r}=-2$ results electrically larger than the case of $\varepsilon_{r}=-4$, since smaller radius are required for propagation. This is contrary to what would occur for positive values of the permittivity. Actually, what is obtained is that the closer $\varepsilon_{r}$ to -1 , the larger the effective electrical size of the waveguide.

The singular behavior of the $\mathrm{TM}_{0}$ can be argued as follows. When $a / \lambda_{0}$ becomes small, the second term in (3.250) becomes very large, however, a large enough $\beta$ 


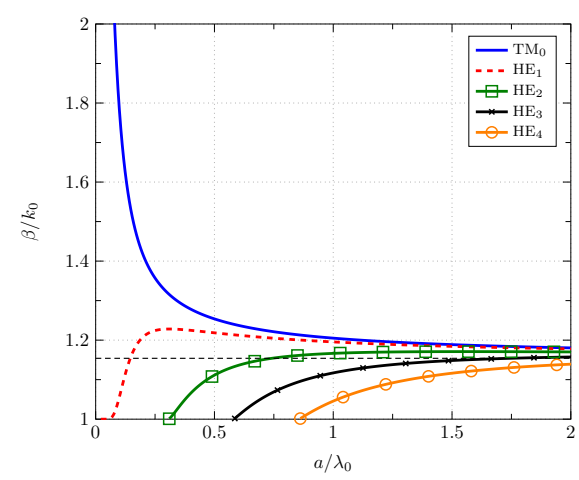

(a)

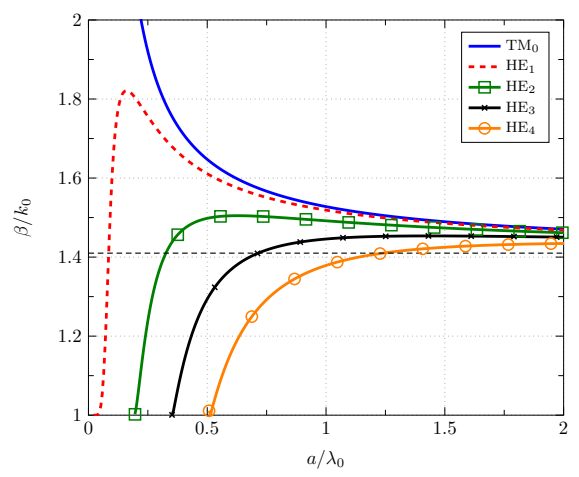

(b)

Figure 3.24: Dispersion diagram of the first 5 surface modes $\left(\mathrm{TM}_{0}, H \mathrm{E}_{1}, \mathrm{HE}_{2}, \mathrm{HE}_{3}\right.$ and $\left.H E_{4}\right)$ of dielectric cylinder of negative permittivity. The horizontal dashed line marks the propagation constant of a surface plasmonic mode in a planar interface $\beta_{N s}$. a) $\varepsilon_{r}=-4$; b) $\varepsilon_{r}=-2$.

in the term $\gamma$ allows to continue satisfying the equation. This also happens when $n \neq 0$, nonetheless, the additional terms in (3.235) predominate with contrary effect. Thus, the higher order surface modes tend to follow the singular behavior of the $\mathrm{TM}_{0}$ mode, but the effect of the additional terms becomes strong and send them to the cutoff condition when the electrical size of the waveguide is decreased.

Note that surface modes exist in any case of $\varepsilon_{r}$, but the particular value of $\varepsilon_{r}$, real and negative in this case, disposes special features for them. Specifically, it is observed that surface modes are propagative, hence of interest for waveguiding purposes, and, furthermore, independent from the bulk modes. Additionally, the negative value of $\varepsilon_{r}$ disposes that now the fundamental mode of the waveguide is the $\mathrm{TM}_{0}$ mode, with a singular behavior. The $\mathrm{HE}_{1}$ still is, mathematically, a fundamental mode, but its role belongs to the high-order modes set. The behavior observed in this case is very close to that of SWW in the optical regime. It has been already observed an interesting independence between bulk and surface modes, and that there is possible to operate under monomode conditions if $a / \lambda_{0}$ is reduced. Small wires allow to clearly distinguish the $\mathrm{TM}_{0}$ mode propagation from the rest of the modes, the surface modes, which become radiative, and the bulk modes, which become even more damped out.

\subsubsection{Solutions for metals: $\varepsilon_{r}$ in the 3th quadrant of the com- plex plane $\gamma=\alpha+j \beta$}

The last case of permittivity before dealing with the complex values of $\varepsilon_{r}$ characterizing the SWW at THz frequencies is afforded now. So far, it has been observed that the values of $\varepsilon_{r}^{\prime}$ and $\varepsilon_{r}^{\prime \prime}$ create a modal spectrum with particular characteristics. Propagation characteristics of the obtained solutions are not obvious and step by step they have been established some common features that allow to map more complex cases to easier ones. For instance, it is clear that surface and bulk modes exist, and its location and path followed in the complex plane described by $\gamma_{N}$ is 


\begin{tabular}{ccc} 
Permittivity $\varepsilon_{r}$ & Surface modes limit $\gamma_{N s}$ & Bulk modes limit $\gamma_{N b}$ \\
\hline$-3-4 \mathrm{j}$ & $0.095+1.0531 \mathrm{j}$ & $2+\mathrm{j}$ \\
$-30-40 \mathrm{j}$ & $0.0081+1.0060 \mathrm{i}$ & $6.3246+3.1623 \mathrm{j}$ \\
$-300-400 \mathrm{j}$ & $0.0008+1.0006 \mathrm{j}$ & $20+10 \mathrm{j}$
\end{tabular}

Table 3.3: Limit values of $\gamma_{N}$ when $a / \lambda_{0} \rightarrow \infty$ for surface modes $\left(\gamma_{N s}\right)$ and bulk modes $\left(\gamma_{N b}\right)$ for the considered permittivity of $\varepsilon_{r}=-3-4 j$. The limits for $10 \varepsilon_{r}$ and $100 \varepsilon_{r}$ are also displayed in the table in order to show the effect of increasing $\left|\varepsilon_{r}\right|$.

very conditioned by the limiting values of a surface plasmon propagating on a planar interface $\left(\gamma_{N s}\right)$ and a plane wave propagating in a bulky, infinite dielectric medium $\left(\gamma_{N b}\right)$, respectively. For instance, when $\varepsilon_{r}^{\prime}>0$ both zones interact and a particular mode may have bulk or surface features depending on the value of $a / \lambda_{0}$, whereas if $\varepsilon_{r}^{\prime}<0$ it has been shown that both zones and the solutions in them (modes) are independent.

In this section, the chosen permittivity is $\varepsilon_{r}=-3-4 j$. This complex number is located in the third quadrant of the complex plane, which is the quadrant in where equivalent dielectric permittivities of metals through the Drude model are located (see (3.96)). In fact, this permittivity could be perfectly associated to a metal in the optical regime. For example, $\varepsilon_{r}=-34.5-8.5 j$ is the equivalent permittivity for aluminum at $f=614.75 \mathrm{THz}$ [430]. In the case treated here, $\varepsilon_{r}$ has a phase similar to that of metals at $\mathrm{THz}$, being as a scaled permittivity. The limiting values of $\gamma_{N}, \beta_{r_{1} N}$, and $\alpha_{r_{2} N}$ result very manageable for this permittivity, reasonably small in magnitude. In the first row of table 3.3 they are shown $\left(\gamma_{N s}\right)$ and $\left(\gamma_{N b}\right)$ for the considered case. Note that $\varepsilon_{r}$ has been chosen in such way $\gamma_{N b}$ is very simple, and $\gamma_{N s}$ differs enough from a plane wave propagating in the free space.

In order to identify the numerical issue as the permittivity becomes large in magnitude, the cases $10 \varepsilon_{r}$ and $100 \varepsilon_{r}$ has been also incorporated to the table. For larger permittivities it is clear that $\gamma_{N s}$ tends to be a plane wave, and $\gamma_{N b}$ increases proportionally to $\sqrt{\varepsilon_{r}}$. These effects are clearly understood through the expressions of $\gamma_{N s}$ and $\gamma_{N b}$. It seems obvious that at $\mathrm{THz}$ frequencies, where metals have even larger $\varepsilon_{r}^{\prime}$ and $\varepsilon_{r}^{\prime \prime}$ the figures will become less pleasant to deal with. Therefore, it is preferable to describe the propagation in a SWW for simpler values, and then extrapolate the conclusions to THz. In fact, in this section the actual propagation characteristics of a SWW will be perfect understood and only the numerical values will change in the next section, where the SWW is analyzed at THz frequencies.

Firstly, in Fig. 3.25 it is shown the colormap representation of $G\left(\gamma_{N}\right)$ for the TM and TE modes of the considered dielectric cylinder, when $a=\lambda_{0}$. In both cases, as occurred with real dielectrics, the inclusion of losses induce the modes to have a mixed nature, going away from the axis. However, for the negative $\varepsilon_{r}^{\prime}$ case, the influence is not as noticeable. For example, in the case of TM modes, Fig. 3.25(a), the surface mode $\mathrm{TM}_{0}$ still remains independent of the set of bulk modes $\mathrm{TM}_{0 m}$. These bulk modes continue experiencing a large damping compared to the lossless case, since $\alpha_{N}>2$, but now they have larger $\beta_{N}$. It is worth to insist in that these modes converge to $\gamma_{N}=j \sqrt{\varepsilon_{r}}$, now $\gamma_{N b}=2+j$, and they approach this point from larger $\alpha_{N}$ and lower $\beta_{N}$ (particular anomalous effects will be commented later). 
Therefore, $\varepsilon_{r}$ has total influence on this point and the propagation characteristics. For the considered case of permittivity, $\beta_{N}$ remains below 1 (leaky modes), but since the image of $\gamma_{N b}$ as a function with domain $\varepsilon_{r}$, it is practically no restricted (as it occurs for $\gamma_{N s}$ ). Thus, it is pointed out that almost any mode feature can be achieved if it is possible to engineer $\varepsilon_{r}$. It must be comment, however, that unless $\varepsilon_{r}$ has a clearly predominant positive $\varepsilon_{r}^{\prime}$ (case of real dielectrics with low losses), $\alpha_{N}$ will be large in $\gamma_{N b}$. Therefore, although some propagation features can be understood considering the values of $\beta_{N}$, bulk modes are predominantly evanescent due to their large $\alpha_{N}$, and the are damped out very fast.

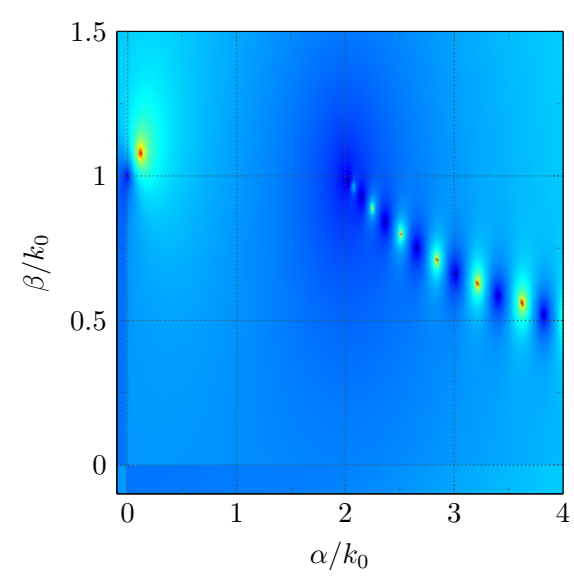

(a)

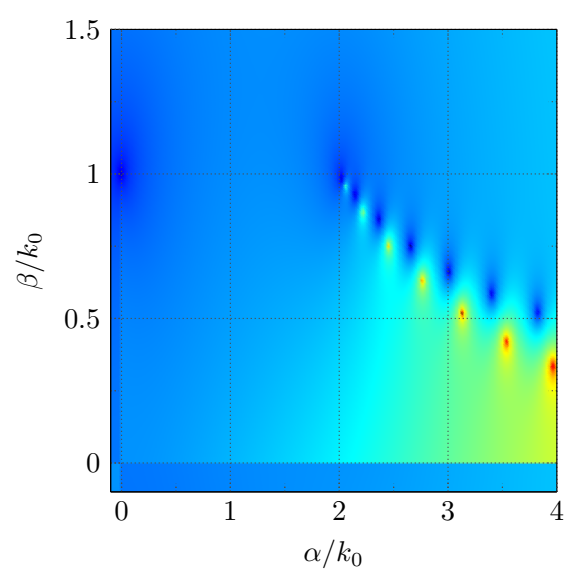

(b)

Figure 3.25: Colormap representation of $G\left(\gamma_{N}\right)$ for a virtual dielectric cylinder of permittivity $\varepsilon_{r}=-3-4 j$ and radius $a=\lambda_{0}$. a) TM modes; b) TE modes.

Regarding the surface $\mathrm{TM}_{0}$ mode, in this case, it does not lie any more on the imaginary $j \beta_{N}$ axis, but has some losses due to the imaginary part of $\varepsilon_{r}$. Nevertheless, the losses of this mode are much lower than those of bulk modes, and the propagation is quite close to that of an unattenuated plane wave in the external air medium. For waveguiding purposes this is, indeed, the unique solution that makes sense to consider.

In the case of TE modes, it is not found any surface solution. The bulk $\mathrm{TE}_{0 m}$ modes are, for this lossy case, more clearly defined. However, it can be appreciated that, for the same values of $\alpha_{N}$, they have lower $\beta_{N}$ than the bulk $\mathrm{TM}_{0 m}$ modes. Note that the blue points correspond to the zeros of $G\left(\gamma_{N}\right)$ caused by the oscillatory nature of $\mathrm{J}_{n}(x)$ over the path described by them. This curve corresponds to the conditions $\varepsilon_{r}^{\prime}+\alpha_{N}^{2}-\beta_{N}^{2}>0$ and $\beta_{N}=\varepsilon_{r}^{\prime \prime} /\left(2 \alpha_{N}\right)$, which makes $\beta_{r_{1}}$ real and positive. Therefore, for $\mathrm{TM}_{0 m}$ modes, $\beta_{r_{1}}$ is real, and the character in the inner region is purely oscillatory, but $\mathrm{TE}_{0 m}$ modes have a $\beta_{r_{1}}$ with imaginary part, having some exponential decay. This effect has it origin in the polarization of the modes. Since $\mathrm{TM}_{0 m}$ modes have radial polarization, they are strongly influenced by the boundary at $r=a$, and, in as much bulk modes travel rather confined in the region $1, \mathrm{TM}_{0 m}$ modes experience more strongly transversal resonances that cause this pure oscillatory behavior in the radial direction. 
Figs. 3.26(a) and 3.26(b) show $G\left(\gamma_{N}\right)$ for HE and EH modes, respectively, in the case of $n=1$. The similarities of $G\left(\gamma_{N}\right)$ between HE and TM modes, and between EH and TE modes are evident, and the same assessments can be established for the hybrid mode case. Inspection of the fields confirm what is apparent in the previous figures: $\mathrm{HE}_{n m}$ modes have a clear radial dominant polarization whereas $\mathrm{EH}_{n m}$ modes have a clear azimuthal dominant polarization.

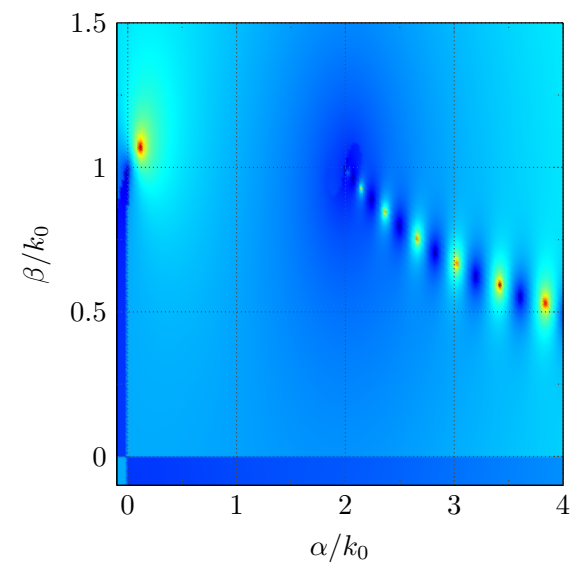

(a)

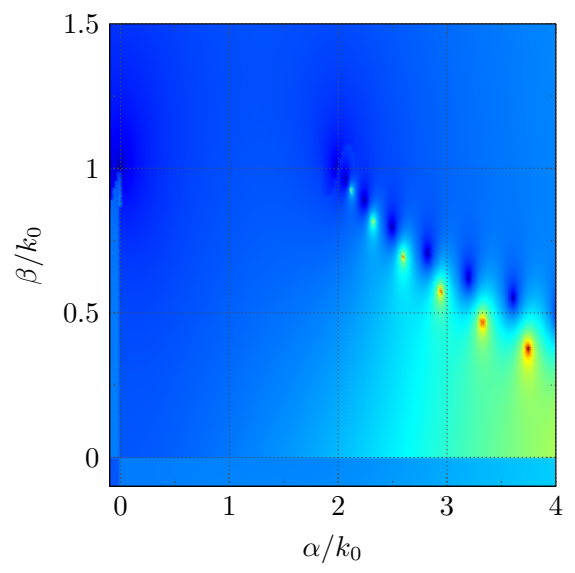

(b)

Figure 3.26: Colormap representation of $G\left(\gamma_{N}\right)$ for a virtual dielectric cylinder of permittivity $\varepsilon_{r}=-3-4 j$ and radius $a=\lambda_{0}$. a) HE modes; b) $E H$ modes.

Additionally, Fig. 3.27 shows $G\left(\gamma_{N}\right)$ for all HEM modes with $n=1$, but extending the range of $\alpha_{N}$, in order to appreciate an interesting effect. As the modal numer associated to the radial index $m$ increases, the $\mathrm{EH}_{n m}$ modes tend to the cutoff condition given by $\beta_{N}=0$, i. e., they become purely evanescent. Therefore, there is a finite number of $\mathrm{EH}_{n m}$ bulky modes. Conversely, $\mathrm{HE}_{n m}$ modes never reach the cutoff condition. From the plot, it seems that they approach the cutoff condition asymptotically, but detailed examination of complex dispersion diagram reveals anomalous features in $\beta_{N}$ that will be particularly examined later. However, even $\beta_{N}$ does not decrease monotonically (this anomalous effect not observable in the plot of Fig. 3.27), $\alpha_{N}$ does, giving the main feature to the propagation, which is a rather evanescent one. Basically, all bulk modes consist in reactive energy, and their influence on the electromagnetic problem under question will depend on how local are the calculations.

Now, the complex propagation constant of the two sets of modes, surface and bulk, is analyzed as a function of the electrical size of the waveguide $a / \lambda_{0}$. First of all, the attenuation and propagation constants of the first 5 surface modes $\left(\mathrm{TM}_{0}\right.$, $\mathrm{HE}_{1}, \mathrm{HE}_{2}, \mathrm{HE}_{3}$ and $\mathrm{HE}_{4}$ ) are represented as a function of $a / \lambda_{0}$ in Figs. 3.28(a) and 3.28 (c), vertically aligned for better analysis of the data. As can be observed, in both graphs, the surface modes converge, for large $a / \lambda_{0}$ to the values specified by $\gamma_{N s}$. When decreasing $a / \lambda_{0}$, in the same way as in the losses case, the $\mathrm{TM}_{0}$ mode behaves in a singular way, exhibiting larger values of both, $\alpha_{N}$ and $\beta_{N}$, and having no cutoff. 


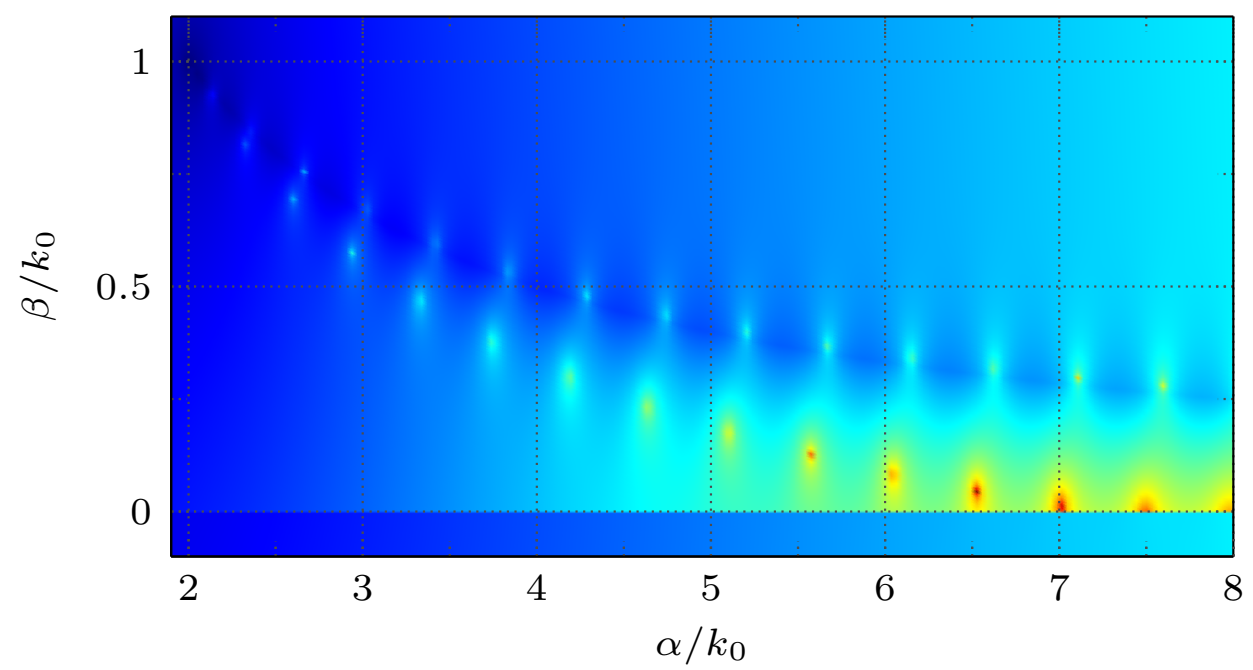

Figure 3.27: Detail of of $G\left(\gamma_{N}\right)$ for all HEM $(n=1)$ modes considering a wider range of $\alpha_{N}$. Dielectric permittivity is $\varepsilon_{r}=-3-4 j$ and the radius $a=\lambda_{0}$. Upper set of modes correspond to the bulk $\mathrm{HE}_{n m}$, whereas lower set of modes correspond to the bulk $\mathrm{EH}_{n m}$. Tendency to cutoff is observed for $\mathrm{EH}_{n m}$ modes as the radial index $m$ increases.

Regarding to the higher order modes, they exhibit, for this complex $\varepsilon_{r}$ case, a more sophisticated behavior than in the losses case. They do not converge to the singular point given by $\gamma_{N}=j$ following the imaginary axis, but they describe a path in the complex plane with non-zero $\alpha_{N}$. In fact, it is appreciated that, for $n \neq 0$ surface modes, the cutoff condition is given by $\alpha_{N}=0$. As it has been for the previous permittivities, the case $\mathrm{HE}_{1}$ is singular, having no theoretical cutoff, but still a practical one, also in the condition $\alpha_{N}=0$. For small $a / \lambda_{0}$, this mode becomes a plane wave in the outer medium, but having $n=1$ azimuthal variation. This is a crucial information since this mode is the most susceptible to couple the fundamental mode of interest, the $\mathrm{TM}_{0}$. An additional feature observed in the $\mathrm{HE}_{1}$ mode is that, decreasing in $a / \lambda_{0}$, before reaching the plane wave status, it becomes slightly radiative, something that was not observed in [430], were the case $\varepsilon_{r}=-34.5-8.5 j$ was considered.

The rest of high order modes behave all of them similarly. As they approach the cutoff in decreasing $a / \lambda_{0}$, they cross the $\beta_{N}=1$ line towards a radiative behavior. The larger the order $n$, the lower the value of $\beta_{N}$ at cutoff. It is worth to mention that an enough small radius not only makes each curve diverge from each other, but can cause all ${ }^{19}$ higher modes disappear. Therefore, these results suggest the necessary condition to provide monomode transmission and, in any case, to minimize the undesired effects of high order modes: reduce the radius $a$.

The complex dispersion diagram, composed by the attenuation diagram and the dispersion diagram is merged in Fig. 3.28(b) to show the path that the aforementioned modes follow in the complex plane described by $\gamma_{N}$, thus having the information of Figs. 3.28(a) and 3.28(c) all in one plot. In this plot it is clearly observed

${ }^{19}$ Considering that the $\mathrm{HE}_{1}$ mode has a practical cutoff 


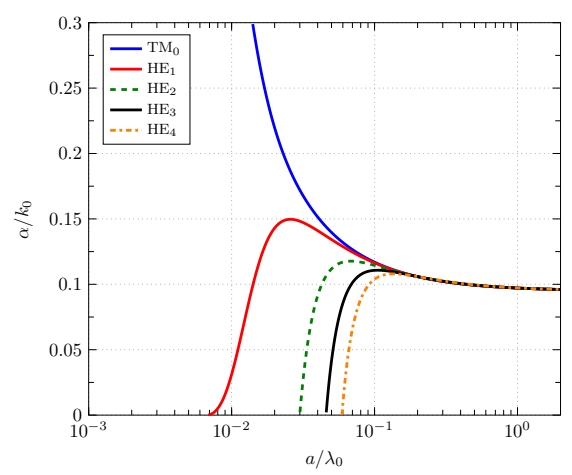

(a)

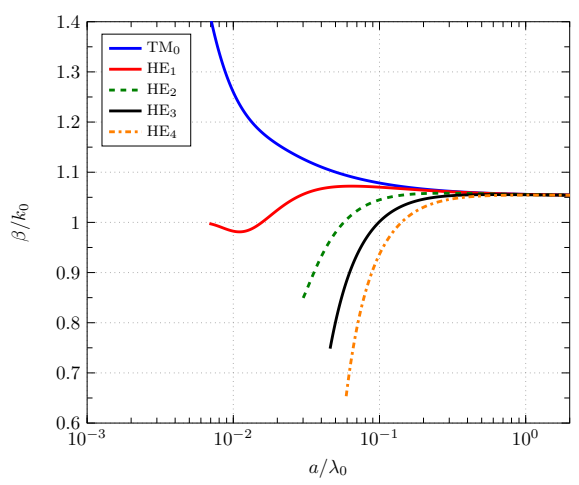

(c)

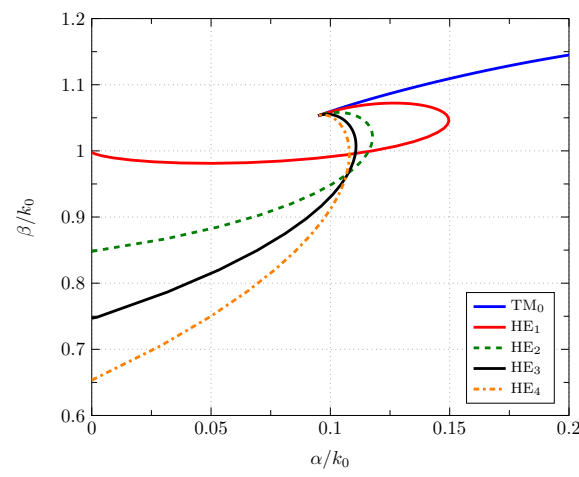

(b)

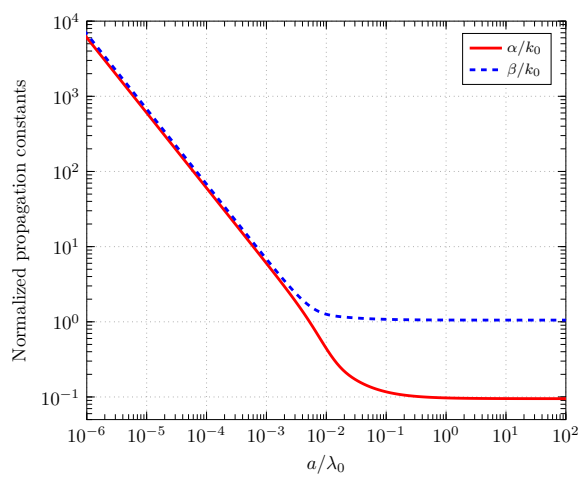

(d)

Figure 3.28: Complex dispersion diagram of the first 5 surface modes $\left(\mathrm{TM}_{0}, \mathrm{HE}_{1}, \mathrm{HE}_{2}, \mathrm{HE}_{3}\right.$ and $\left.H_{4}\right)$ propagating in a dielectric cylindrical waveguide of permittivity $\varepsilon_{r}=-3-4 j$. a) Attenuation diagram: $\alpha_{N}$ vs. $a / \lambda_{0}$; b) Dispersion diagram: $\beta_{N}$ vs. $a / \lambda_{0}$.

the divergence of the modes from the limiting point $\gamma_{N}=\gamma_{N s}$, and the anomalous behavior of the $\mathrm{TM}_{0}$ mode. In this plot it is easier to observe that, indeed, all modes experience the singular behavior of the $\mathrm{TM}_{0}$ mode when $a / \lambda_{0}$ decreases. However, the azimuthal phase changes in $n \neq 0$ modes cancel the effect of proximity of the interfaces, that it is understood to be negligible for large $n$.

The fundamental mode $\mathrm{TM}_{0}$ follows a path covering much more range of $\alpha_{N}$ and $\beta_{N}$ than the higher order modes. The propagation features of this mode have been calculated and represented in Fig. 3.28(d), where $\alpha_{N}$ and $\beta_{N}$ of the $\mathrm{TM}_{0}$ are shown for a wide range of $a / \lambda_{0}$. The inflexion point of both curves take place between $a / \lambda_{0}=0.01$ and $a / \lambda_{0}=0.01$. Looking the graph towards lower values of $a / \lambda_{0}$, in the aforementioned range, it is observed how $\alpha_{N}$ and $\beta_{N}$ start to grow exponentially, a behavior that continues with decreasing $a / \lambda_{0}$. It is noted, however, that $\alpha_{N}$ experience a larger grow, almost reaching $\beta_{N}$. A careful study of both curves reveals that, except in the transition moment, the quotient $\alpha_{N} / \beta_{N}$ remains constant, being 0.9 in the exponential grow zone, and 0.1 in the constant zone, approximately. This effect seems to be originated by the not direct influence of 
the propagation constant on the power distribution, something that was already observed in a lossless, real dielectric cylinder of section 3.3. This means, $\beta$ and $\eta$ do not match exactly, hence $\beta_{N}$ and $\alpha_{N}$ (which depend on $\eta$ ) do not. It is worth noting that, although very high values of both, $\alpha_{N}$ and $\beta_{N}$, are reached, the $\mathrm{TM}_{0}$ still keeps its surface plasmonic mode features, i. e., exponential decay of fields towards inner and outer region. However, a small radius causes the local effects of the boundary to be very close to each other, enhancing the field in the inner region, and causing a larger confinement at both sides of the interface. This means that the exponential decay towards inner and outer region become more similar.

From the propagation characteristics of the fundamental mode, and regarding to those of the higher order modes, it can be concluded that is convenient to chose a electrically small radius to cause difference in $\gamma_{N}$ between the fundamental and the higher order modes (and in this way reduce coupling, according of what was reviewed in section 3.1.7). However, a very small radius implies losses that may be too large. Nevertheless, most of the main interesting effects of the propagation occur in the range from $a / \lambda_{0}=0.01$ to $a / \lambda_{0}=0.01$. In this range, high order modes vanish, and the $\mathrm{TM}_{0}$ mode still preserve low-loss and low dispersion characteristics. Therefore, it is concluded that, there is range of radius (frequencies) in which the SWW can perform in an optimal way in terms of monomode operation.

The modal analysis is completed, finally, with the propagation characteristics of bulk modes. Although, in principle, the effect of bulk modes if of secondary order, less relevant than the higher order surface modes, it result necessary to know and have control over its propagation characteristics in order to keep away undesired effects. Furthermore, they become relevant during excitation or discontinuities, and any modal method should consider them. Its contribution has been shown to be relevant in the simpler planar case [570]. The case of $\mathrm{TM}_{0 m}$ bulk modes is of particular importance, since these modes have exactly the same polarization and azimuthal pattern than the fundamental surface mode $\mathrm{TM}_{0}$.

In Fig. 3.29, they are given the complex propagation characteristics of the first bulk modes that propagate confined inside an dielectric cylinder with $\varepsilon_{r}=-3-4 j$. First, Figs. 3.29(a) and 3.29(c) show the attenuation and propagation constant, respectively, as a function of $a / \lambda_{0}$. The attenuation constant shows an exponential growth when $a / \lambda_{0}$ decreases. The curves of azimuthal dominant E-field modes, $\mathrm{TE}_{0 m}$ and $\mathrm{EH}_{n m}$, stop when these modes reach the real axis $\left(\beta_{N}=0\right.$, pure evanescent modes) for some value of $\alpha_{N}$, something that was already predicted by analyzing $G\left(\gamma_{N}\right)$ in Fig. 3.27. On the other hand, the curves of $\beta_{N}$ show an anomalous behavior not easy to be foreseen by a simple inspection of $G\left(\gamma_{N}\right)$. It is observed that $\mathrm{TE}_{0 m}$ and $\mathrm{EH}_{n m}$ modes rapidly approach the cutoff condition given by $\beta_{N}=0$ as $a / \lambda_{0}$ is decreased. Conversely, for $\mathrm{TM}_{0 m}$ and $\mathrm{HE}_{n m}$ modes, although $\beta_{N}$ experience a decrement tendency for decreasing $a / \lambda_{0}$ (looking the curves from the limit $\beta_{N b}$ ), there is an inflexion point, from which $\beta_{N}$ starts grow and diverges for small $a / \lambda_{0}$. The larger the general order $(n, m)$ of the mode, the deeper the valley in $\beta_{N}$, which reaches lower values in its minimum.

When bulk modes were treated in [430] for $\varepsilon_{r}=-34.5-8.5 j$, this phenomena was only observed for the $\mathrm{TM}_{01}$, justifying it by some parallelism between this singularity and that observed for the $\mathrm{TM}_{0}$ surface mode. However, a careful inspection of that case reveals that what actually occurs is that the rest of the curves reach 


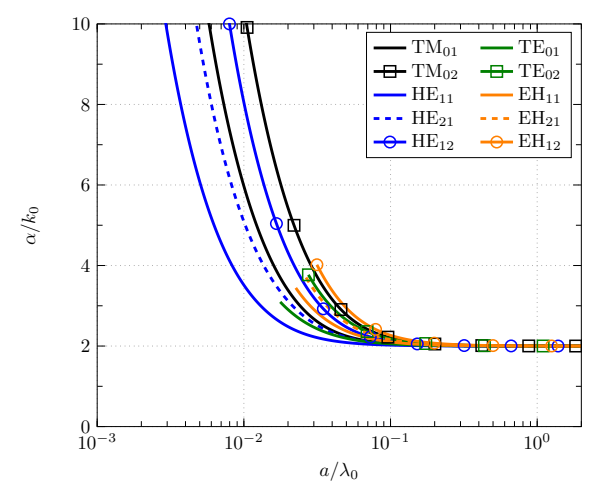

(a)

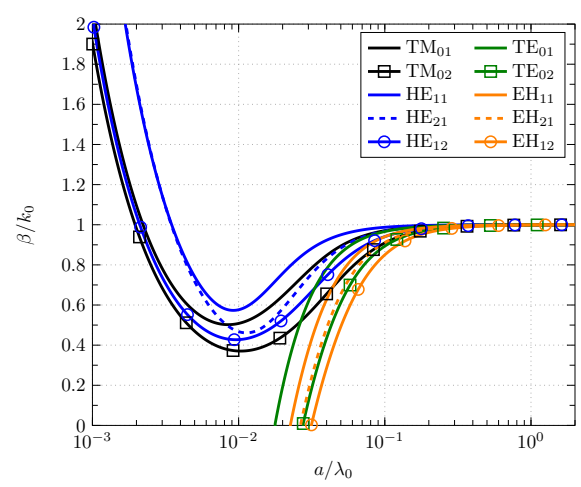

(c)

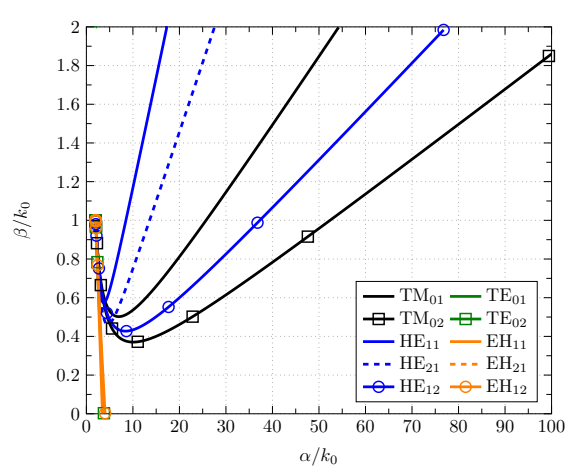

(b)

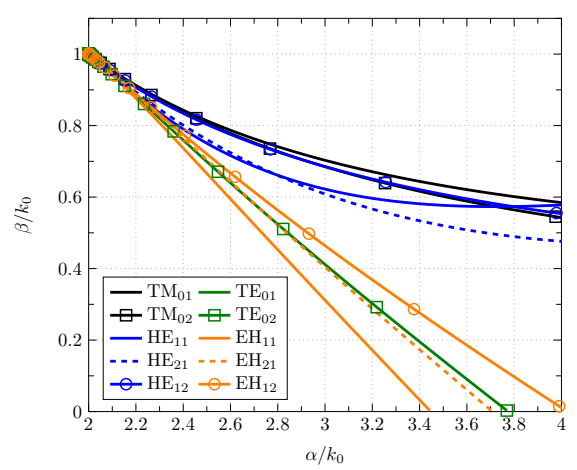

(d)

Figure 3.29: Complex dispersion diagrams of the first bulk modes propagating on dielectric cylindrical waveguide of permittivity $\varepsilon_{r}=-3-4 j$. Attenuation diagram: $\alpha_{N}$ vs. $a / \lambda_{0} ; b$ ) Dispersion diagram: $\beta_{N}$ vs. a/ $\lambda_{0}$. c) Complex dispersion diagram: path followed by the considered bulk modes in the complex plane described by $\gamma_{N}$. d) Detail of the path bifurcation between $\left(\mathrm{TM}_{0 m}, \mathrm{HE}_{n m}\right)$ modes and $\left(\mathrm{TE}_{0 m}, \mathrm{EH}_{n m}\right)$ modes in the complex plane described by $\gamma_{N}$.

$\beta_{N}=0$ before the inflexion occur, thus being understood a simple cutoff to happen. However, if the solution is tracked across the branch cut of the real axis it is obtained that it comes again to values of $\gamma_{N}$ in the first quadrant, i.e, the inflexion point takes place for negative $\beta_{N}$. Therefore, it seems possible that the structure under study is able to support backward modes for certain order $(n, m)$ and permittivity $\varepsilon_{r}$.

As before, representing a parametric plot of $\left(\alpha_{N}, \beta_{N}\right)$, see Fig. 3.29(b) helps to figure out the propagation features of the studied modes by giving an alternative, integral view of $\gamma_{N}$. The $\mathrm{TE}_{0 m}$ and $\mathrm{EH}_{n m}$ modes follow a direct linear path to cutoff and the covered range of this path is quite bounded in the $\gamma_{N}$ plane. A zoom of the area where those paths take place is shown in Fig. 3.29(d). From those plots it is also observed that the $\mathrm{TM}_{0 m}$ and $\mathrm{HE}_{n m}$ not only diverge suddenly after certain range of $a / \lambda_{0}$ showing a tendency of reaching the cutoff, but the followed path by them is quite different to that of $\mathrm{TE}_{0 m}$ and $\mathrm{EH}_{n m}$ modes even for very large $a / \lambda_{0}$, when the curves are close to their limit $\gamma_{N}=\gamma_{N b}$. For higher order modes, the slope of the 
curves is lower, indicating that the growth in $\alpha_{N}$ becomes more important than the growth of $\beta_{N}$ compared to lower order modes. However, from the numeration in the plots axis, it can be understood that in any case the growth is clearly predominant in $\alpha_{N}$.

Recovering now the zone comprised between $a / \lambda_{0}=0.01$ and $a / \lambda_{0}=0.01$, it is again observed that the main changes in the propagation characteristics occur also in this zone for bulk modes. This waveguide electrical size range seems to be the critical for the considered permittivity. In the case of bulk $\mathrm{TM}_{0 m}$ and $\mathrm{HE}_{n m}$ modes, when $a / \lambda_{0}$ reaches those small values, due to their dominant radial E-field and the fact that they travel mostly in the inner region, they are comprised by the boundary. Since the mode tends to preserve its oscillatory behavior in the inner region, when the boundary comprises this region, the real part of $\beta_{r_{1} N}$ is forced to grow, causing $\gamma_{N}$ to grow in the same way, mainly in its $\alpha_{N}$ component. Actually, $\beta_{r_{1} N}$ and $\gamma_{N}$ become very similar, what through ray theory translates in $45^{\circ}$ degrees transversal incidence of the rays, hence having the modes similar $E_{r_{1}}$ and $E_{z_{1}}$.

The main characteristics of a $\varepsilon_{r}=-3-4 j$ have been reviewed in a study that perfectly cover the behavior of the SWW in the optical regime, giving some additional information and correcting the answer to some unclear issues regarding to previous works [430]. The main obtained result resides on the observed range of $a / \lambda_{0}$ where propagation features of all modes experience a change in a more or less extent. Engineering the electrical size of the waveguide provides through this fact the possibility of avoiding undesired degeneration or similarities in $\gamma_{N}$, in order to avoid coupling. It is possible to choose $a / \lambda_{0}$ such any surface mode apart of the fundamental exist, and still obtaining good loss and dispersion features. Furthermore, it has been proven that this makes $\gamma_{N}$ of bulk modes to get even further away of the $\gamma_{N}$ zone of the fundamental mode, which is specially important for the bulk $\mathrm{TM}_{0 m}$ modes, which exhibit the same azimuthal pattern and radial polarization as the fundamental mode.

\subsection{Single Wire Waveguide (SWW)}

One of the most important objectives of this thesis is afforded now. The Single Wire Waveguide (SWW) is analyzed in this section. Its modal spectrum consisting in bulk and surface modes is determined, and particularities of the singular fundamental mode, the surface mode $\mathrm{TM}_{0}$, are determined in the $\mathrm{THz}$ range. The information treated next is the essential analytical base support that it requires a structure which has been treated with such intensity in the last decade for its use in the $\mathrm{THz}$ band, as reviewed in section 2.3.3.4. Furthermore, the controversial question of its modal spectrum, practically untreated, will be clearly solved, closing a discussion that comes for more than one century, originated by Sommerfeld [488] and popularly established by Stratton [489], works which have been the reference for the analytical base of this structure to date.

The description of the problem follows Fig. 3.30. The waveguide under study is a metallic cylinder surrounded by an infinite air region. The interior of the cylinder forms the region 1 whereas the outer air region is named region 2 . The mathematical analysis of the problem uses the Drude Model of section 3.2, specifically through 
equation (3.96), to convert the problem in that reviewed in last sections, with the only particularity of having a complex permittivity that, now, is frequency dependent. The formulation is essentially the same presented in section 3.4, so it will not be repeated here, but the aforementioned dispersion in $\varepsilon_{r}$ must be kept in mind.

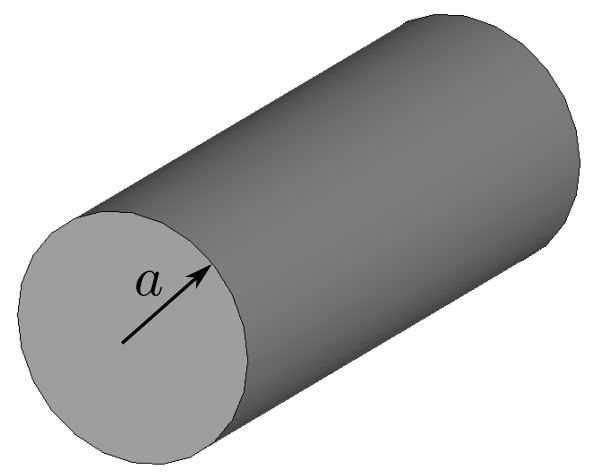

(a)

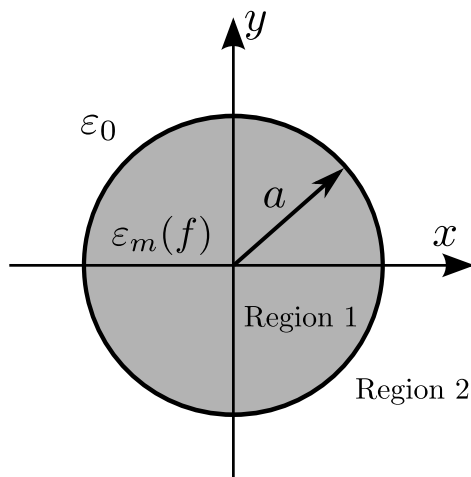

(b)

Figure 3.30: Single Wire Waveguide $(S W W)$ as a metallic cylinder modeled with a complex permittivity $\varepsilon_{m}(f)$, given by the Drude Model. a) $3 D$ view; b) Problem description.

In fact, the complexity of the propagation characteristics of a dielectric cylindrical waveguide modes, specially for those case of $\varepsilon_{r}$ belonging to the third quadrant of the complex plane, i.e., $\varepsilon_{r}$ of metals, has been partially alleviated in the last sections. In this way, this section has been avoided to be excessively dense conceptually and the ideas desired to be transmitted to be masked by the numbers complexity, what is caused by the extreme situation at $\mathrm{THz}$ frequencies, to close to the limit in many cases and, therefore, blurring the important ideas. Note also that, the additional complexity added in this section regarding to the last one, where a simple permittivity of the third quadrant was considered $\left(\varepsilon_{r}=-3-4 j\right)$, is, on the one hand, numerical, by the large values of $\varepsilon_{r}^{\prime}$ and $\varepsilon_{r}^{\prime \prime}$ for metals at $\mathrm{THz}$ frequencies. On the other hand, these values are frequency dependent. This last implies that the parameter describing the electrical size of the waveguide, $a / \lambda_{0}$, splits its meaning in radius $a$ and $\lambda_{0}$ or, in other words, radius $a$ and frequency $f$. If the radius is varied, the same situation as in previous section will take place, with the particularity of $\varepsilon_{r}^{\prime}$ and $\varepsilon_{r}^{\prime \prime}$ being large values. However, if the frequency $f$ is varied the permittivity will change with this parameter, thus having a new situation. Therefore, the SWW dependence on its geometrical parameter $a / \lambda_{0}$ must be mandatorily analyzed separately for radius and frequency. Since it is more common to have a fixed radius (or a set of them to consider) and regard a frequency spectrum of operation, an eye must be put on the new phenomena appearing in this section through the frequency parametrization.

In order to quantify the problem of the numerical complexity and establish the analytical environment to deal with, the table 3.4 collects the equivalent permittivity values for gold $\left(f_{p}=2184 \mathrm{THz}, f_{\tau}=6.45 \mathrm{THz}\right)$ and aluminum $\left(f_{p}=3570 \mathrm{THz}\right.$, $f_{\tau}=19.8 \mathrm{THz}$ ), split in real and imaginary parts, $\varepsilon_{r}^{\prime}$ and $\varepsilon_{r}^{\prime}$, for the lowest frequency $(f=0.1 \mathrm{THz})$, the central frequency in logarithmic terms $(f=1 \mathrm{THz})$, and the 
highest frequency $(f=10 \mathrm{THz})$ of the $\mathrm{THz}$ range. Additionally, the limiting values of the propagation constant for surface modes $\left(\gamma_{N s}=\alpha_{N s}+j \beta_{N s}\right)$ and bulk modes $\left(\gamma_{N b}=\alpha_{N b}+j \beta_{N b}\right)$ are also displayed, given information of how the modal solutions will be distributed in the complex plane defined by the normalized propagation constant $\gamma_{N}$. The conclusions to be extracted from this table are pretty much the same for both metals. The large value of $\left|\varepsilon_{r}\right|$ causes that $\gamma_{N s} \approx j$ (since the quotient inside the square root describing $\gamma_{N s}$ is very close to the unity), the normalized propagation constant of a plane wave in the region 2. Note that $\beta_{N s}$ is described as $\beta_{N s}=1+x \cdot 10^{-p}, p \in \mathbb{N}^{+}$, since its value is to close to the unity that the usual decimal representation results unpractical. Therefore, it will make sense along this section to consider most of the time the following normalization:

$$
\beta_{N N}=\left(\frac{\beta}{k_{0}}-1\right)
$$

and, since $\alpha_{N}$ is very small, requiring also from exponential notation, in this section it will be important to pay attention to the magnitude of the exponents involved in quantifying how small is the distance to the point $(0,1)$ in the complex plane described by $\gamma_{N}$ of the surface mode solutions. From the table, it is appreciated that $\alpha_{N s}$ scales with $f$, whereas $\left(\beta_{N s}-1\right)$ does with $f^{2}$. However, it seems evident that a large $a / \lambda_{0}$ will imply, for any frequency, practically the situation of a plane wave. Since $\alpha_{r_{2} N}^{2}=-\gamma_{N}^{2}-1$, and $\gamma_{N} \approx j$, it can be understood that, for such situation, $\alpha_{r_{2}} \approx 0$, hence the extension of the fields will be huge.

Regarding to the bulk modes, the large values of both $\varepsilon_{r}^{\prime}$ and $\varepsilon_{r}^{\prime \prime}$, send $\gamma_{N b}$ very far from both, real and imaginary axis. Particularly, the very large values of $\alpha_{N b}$ suggest a propagation that can be considered practically evanescent, inasmuch as this value multiplied by a factor close to 55 gives the attenuation in $\mathrm{dB}$ per wavelength in free space. Note that the attenuation of a bulk mode will be, at least, that of the limit, accordingly to what was observed in last section. This fortunately extreme location in the $\gamma_{N}$ complex plane of bulk modes, endorses the independence with surface modes, and the difficulty of coupling between both sets.

A feature that can be also commented regarding the values of $\gamma_{N b}$ is that this parameter present similar real and imaginary parts, which is specially true for lower frequencies. Note that, for low frequencies, the Drude model infer a small value in magnitude to $\varepsilon_{r}^{\prime}$, therefore the phase of $\varepsilon_{r}$ being close to $\pi / 2$, hence its positive sign root having a phase close to $\pi / 4$ : equal real and imaginary part. In fact, if the simpler skin model is considered, as some works do in the literature, $\varepsilon_{r}$ would be purely imaginary and this situation would be obtained. Note how for high frequencies the situation provided by the Drude model differs in an evident way for this simpler skin-depth effect model case, hence different results must be expected.

\subsubsection{Modal Spectrum}

The modal spectrum study starts with the study of the aspect of $G\left(\gamma_{N}\right)$. Now, the distance between the location of the bulk mode zone and the surface mode zone in the complex plane $\gamma_{N}$ implies the inspection of $G\left(\gamma_{N}\right)$ to be necessarily separated. 
Gold

\begin{tabular}{lccc}
\hline \hline Frequency & $0.1 \mathrm{THz}$ & $1 \mathrm{THz}$ & $10 \mathrm{THz}$ \\
\hline \hline$\varepsilon_{r}^{\prime}$ & $-1.15 \cdot 10^{5}$ & $-1.12 \cdot 10^{5}$ & $-3.37 \cdot 10^{4}$ \\
$\varepsilon_{r}^{\prime \prime}$ & $-7.39 \cdot 10^{6}$ & $-7.22 \cdot 10^{5}$ & $-2.17 \cdot 10^{4}$ \\
\hline$\alpha_{N s}$ & $6.76 \cdot 10^{-8}$ & $6.76 \cdot 10^{-7}$ & $6.76 \cdot 10^{-6}$ \\
$\beta_{N s}$ & $1+1.05 \cdot 10^{-9}$ & $1+1.04 \cdot 10^{-7}$ & $1+1.04 \cdot 10^{-5}$ \\
\hline$\alpha_{N b}$ & 1937 & 649.3 & 192.1 \\
$\beta_{N b}$ & 1908 & 556.3 & 56.57 \\
\hline
\end{tabular}

Aluminum

\begin{tabular}{lccc}
\hline Frequency & $0.1 \mathrm{THz}$ & $1 \mathrm{THz}$ & $10 \mathrm{THz}$ \\
\hline \hline$\varepsilon_{r}^{\prime}$ & $-3.25 \cdot 10^{4}$ & $-3.24 \cdot 10^{4}$ & $-2.59 \cdot 10^{4}$ \\
$\varepsilon_{r}^{\prime \prime}$ & $-6.44 \cdot 10^{6}$ & $-6.42 \cdot 10^{5}$ & $-5.13 \cdot 10^{4}$ \\
\hline$\alpha_{N s}$ & $7.77 \cdot 10^{-8}$ & $7.77 \cdot 10^{-7}$ & $7.77 \cdot 10^{-6}$ \\
$\beta_{N s}$ & $1+3.92 \cdot 10^{-10}$ & $1+3.92 \cdot 10^{-8}$ & $1+3.92 \cdot 10^{-6}$ \\
\hline$\alpha_{N b}$ & 1798 & 649.3 & 204.5 \\
$\beta_{N b}$ & 1789 & 581.1 & 125.61 \\
\hline
\end{tabular}

Table 3.4: Complex permittivity $\varepsilon_{r}$ modeled with Drude Model and associated limiting normalized propagation constant values for surface modes $\left(\gamma_{N s}=\alpha_{N s}+j \beta_{N s}\right)$ and bulk modes $\left(\gamma_{N b}=\alpha_{N b}+j \beta_{N b}\right)$ for gold $\left(f_{p}=2184 \mathrm{THz}, f_{\tau}=6.45 \mathrm{THz}\right)$ and aluminum $\left(f_{p}=3570 \mathrm{THz}\right.$, $\left.f_{\tau}=19.8 \mathrm{THz}\right)$. 


\subsubsection{Surface modes}

The analysis of the surface mode zone of $G\left(\gamma_{N}\right)$, which is of more interest, is done first. In Fig. 3.31(a), it is plotted the colormap representation of $G\left(\gamma_{N}\right)$ of TM modes restricted to the surface mode zone $\left(\mathrm{TM}_{0}\right.$ mode), for a gold SWW of radius $a=\lambda_{0} / 40$ at $f=1 \mathrm{THz}(a=7.5 \mu \mathrm{m})$. Such a small radius allows, as in the case of bulk modes, the singular surface mode to be enough differentiated of a plane wave in propagation terms, and to be, relatively easy, to be observed. Note that, nevertheless, since the $\gamma_{N}=j$ is a singular point in $G\left(\gamma_{N}\right)$ (as it causes a pole in $F\left(\gamma_{N}\right)$ ), now, the surface solution and this singular point are very close, what is a numerically issue to take into account. A thoughtful decision, which is very practical in terms of visual representation of the solution area, is to do a logarithmic mapping of the neighborhood of the singular point $\gamma_{N}=j$, in order to be able to inspection the function as close as desired to this singularity. To do that, $\alpha_{N}$ and the recently defined $\beta_{N N}$, both small around this point, are represented ${ }^{20}$. The result of this mapping, is shown in Fig. 3.31(b) in the area $[-5,-2] \times[-5,-2]$. The solution is, in this case, much more visually evident.

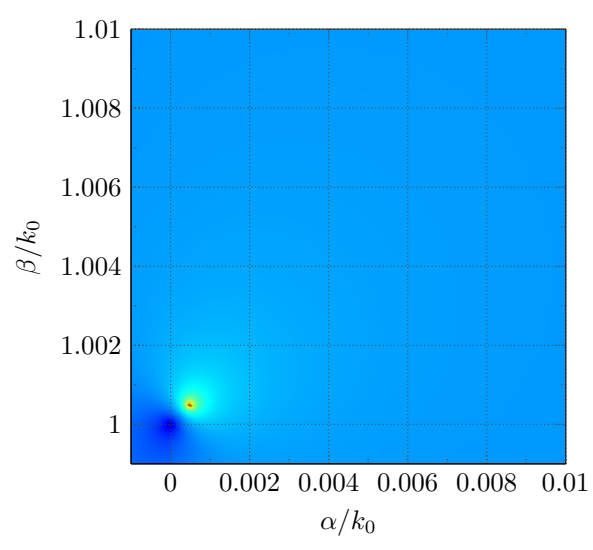

(a)

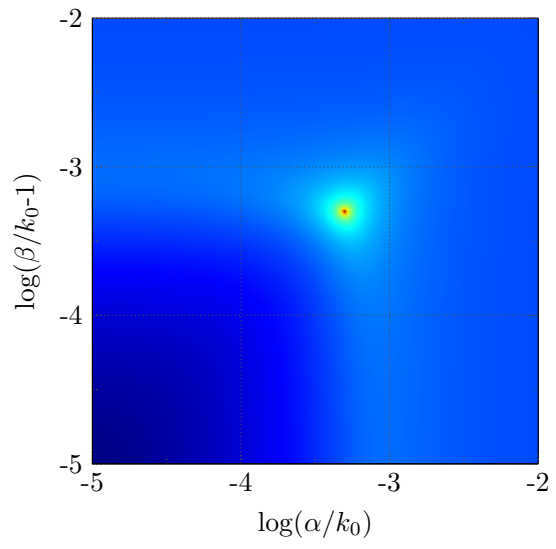

(b)

Figure 3.31: Colormap representation of $G\left(\gamma_{N}\right)$ of TM modes restricted to the surface mode zone ( $T M_{0}$ mode) for a gold $S W W$ of radius. a) Linear domain; b) Logarithmic domain representation of the neighborhood of $\gamma_{N}=j$, the point $(0,1)$.

The use of this tool takes all its sense when the solution is close to its limiting value $\gamma_{N s}$, which will be always particularly true for $\mathrm{HE}_{n}$ modes. See for instance Fig. 3.32(a), where $G\left(\gamma_{N}\right)$ is represented for a large gold SWW of radius $a=10^{3} \lambda_{0}$ at $f=1 \mathrm{THz}(a=30 \mathrm{~cm})$, in the area of the $\mathrm{TM}_{0}$ mode solution. By consulting the table 3.4 it is clearly observed how $\gamma_{N} \approx \gamma_{N s}$, adopting numbers not very suitable for linear domain representation.

\footnotetext{
${ }^{20}$ Note that logarithmic functions only admit positive arguments, therefore, for leaky solutions $\beta_{N}<1$, hence $\beta_{N N}<0$, it is not possible to provide a mapping unless absolute value is taken over $\beta_{N N}$. However, this last option breaks the injectivity of the mapping, causing positive and negative $\beta_{N N}$ areas to be mapped over the same zone, a fact to take into account.
} 
The case of the higher order surface modes is even more delicate. See for instance Fig. 3.32(b), which offer the same representation as just commented, but for the case of $n=1$ (mode $\mathrm{HE}_{1}$ ). In this case, the solution is a peak located between a hill and a valley which are, indeed, very close to each other, causing the root finding and tracking to be numerically difficult. This different and singular shape of $G\left(\gamma_{N}\right)$ is, actually, what makes higher order modes to disappear when $a / \lambda_{0}$ diminishes.

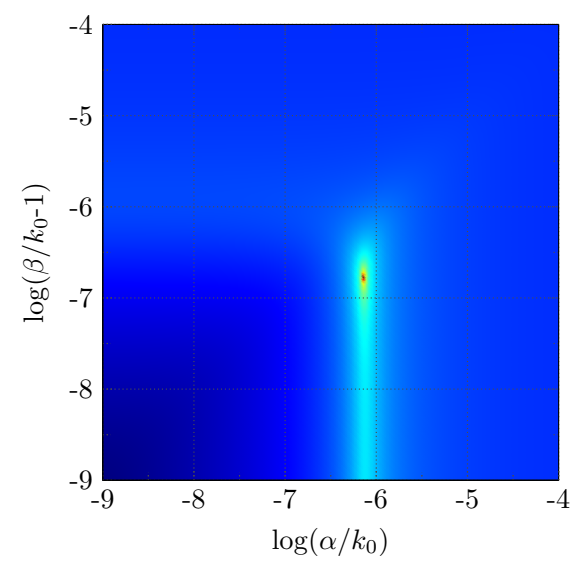

(a)

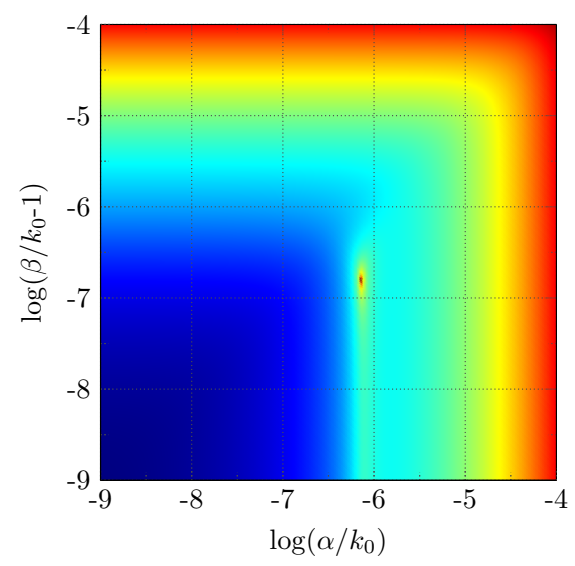

(b)

Figure 3.32: Colormap logarithmic representation of $G\left(\gamma_{N}\right)$ in the neighborhood of $\gamma_{N}=j$ for a large gold $S W W$ of radius $a=10^{3} \lambda_{0}$ at $f=1 \mathrm{THz}(a=30 \mathrm{~cm})$, causing the solution to be $\gamma_{N} \approx \gamma_{N s}$. a) $T M_{0}$ mode; b) $H E_{1}$ mode.

The propagation characteristics of the first 5 surface modes $\left(\mathrm{TM}_{0}, \mathrm{HE}_{1}, \mathrm{HE}_{2}\right.$, $\mathrm{HE}_{3}$ and $\mathrm{HE}_{4}$ ) is studied now as a function of $a / \lambda_{0}$. In this first approach, the frequency is fixed at $f=1 \mathrm{THz}$, and the radius $a$ is varied. The graphs are displayed using the usual form (with $a / \lambda_{0}$ as abscissa variable), but now they are directly related to real values is as much as $\lambda_{0}=300 \mu \mathrm{m}$. Results are shown in Fig. 3.33, organized as usual. The attenuation and dispersion diagrams are displayed vertically aligned in Figs. 3.33(a) and 3.33(c), respectively, and this information is merged, in Fig. 3.33(b) for the same domain range of $\alpha_{N}$ and $\beta_{N N}$. Two things must be noted: now, $\beta_{N N}$ is used instead of $\beta_{N}$, and the axis involving either $\alpha_{N}$ or $\beta_{N N}$ are multiplied by a factor $10^{6}$. Despite of this fact, the shape of the curves is very similar to that observed in the last section.

It must be noted, however, that the cutoff values of $a / \lambda_{0}$ for the higher order modes are different now. In the SWW operating at $\mathrm{THz}$ frequencies, the electrical size of the waveguide has to be larger in order to this modes appear. Specifically, the first higher order mode, $\mathrm{HE}_{1}$ does not propagate for $a<30 \lambda_{0}$, which for the analyzed case means $a<9 \mathrm{~mm}$, a rather large radius. In addition, despite that higher order modes describe some path in the complex plane defined by $\gamma_{N}$, these modes are, whatever the radius is, practically a plane wave when they are above the cutoff condition.

Although the fundamental $\mathrm{TM}_{0}$ mode propagation can become also very close to that of a plane wave for large $a$, its singular behavior allows to have also an enough 


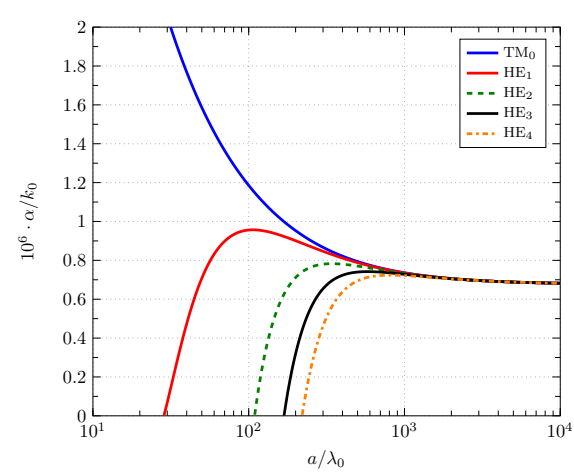

(a)

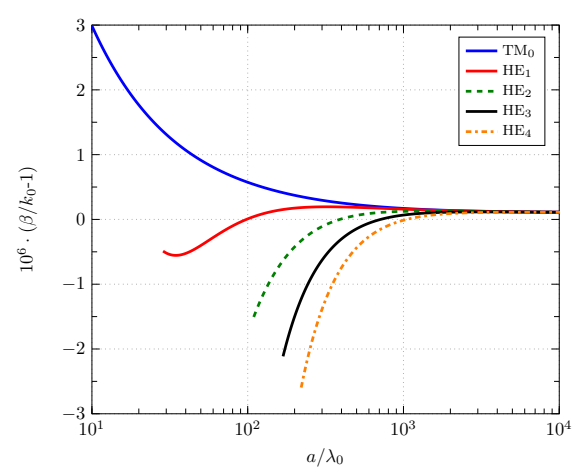

(c)

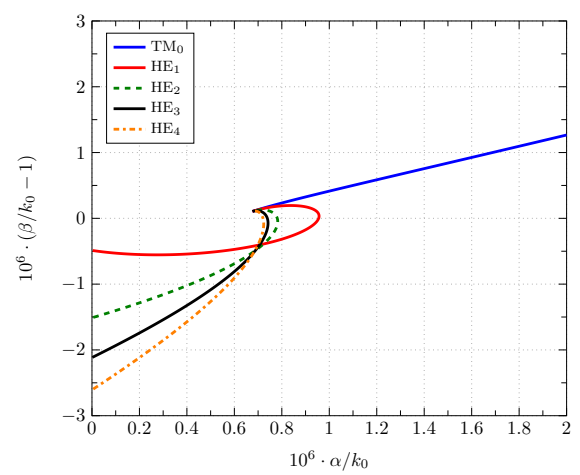

(b)

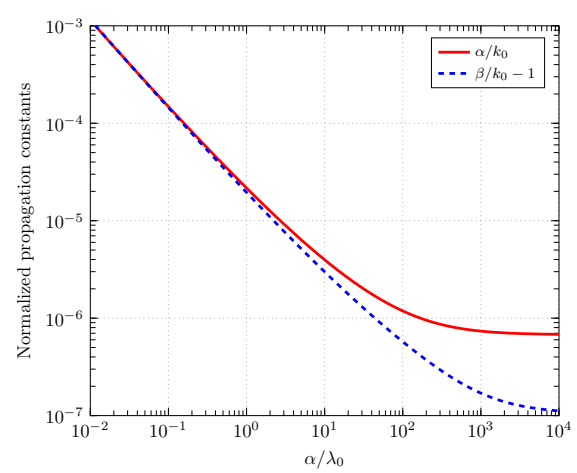

(d)

Figure 3.33: Complex dispersion diagram of the first 5 surface modes $\left(\mathrm{TM}_{0}, H E_{1}, H E_{2}, H E_{3}\right.$ and $\mathrm{HE}_{4}$ ) propagating on a gold $\mathrm{SWW}$, with operating frequency $f=1 \mathrm{THz}$, as a function of the electrical size of the wire $a / \lambda_{0}$. a) Attenuation diagram: $\alpha_{N}$ vs. $\left.a / \lambda_{0} ; b\right)$ Complex dispersion diagram: $\gamma_{N}$ vs. $a / \lambda_{0}$ c) Dispersion diagram: $\beta_{N}$ vs. $\left.a / \lambda_{0} ; d\right) \alpha_{N}$ and $\beta_{N}$ for the fundamental $T M_{0}$ mode.

different situation if a small radius is chosen. See for instance Fig. 3.33(d), where $\alpha_{N}$ and $\beta_{N N}$ are displayed for this mode, considering a wide range of $a / \lambda_{0}$. Note that the axis for these parameters is not multiplied now, and the employed scale is logarithmic due to their considerable range of variation. Due to the large values of $\varepsilon_{r}^{\prime}$ and $\varepsilon_{r}^{\prime \prime}$, the surface is quite impenetrable and it can be said that the propagation still keep some characteristics of a plane wave even for small radius, however, specially the losses term become considerable, as the $\mathrm{TM}_{0}$ mode can experience a not negligible attenuation. For example, a SWW with $a=0.1 \lambda_{0}=30 \mu \mathrm{m}$ would cause $\alpha_{N}=$ $1.5 \cdot 10^{-4}$, which means $0.027 \mathrm{~dB} / \mathrm{mm}$, a low loss, but to be considered. In the graph it is observed that reduction of the radius affect more $\beta_{N N}$, which in the limit $\gamma_{N s}$ is lower $\alpha_{N}$, converging both curves when $a / \lambda_{0}$ decreases. Nevertheless, this has not relevance on the propagation constant, since $\beta_{N} \approx 1$ in any case. Therefore, in practical terms, the variation of the radius has only influence on the losses in 
as much the SWW will remain dispersionless due to the small variation of $\beta_{N}$ in absolute terms, even in the case the losses become important.

The previous results prove that the undesired surface high order modes may be kept away if practical radius are used (it is not conceived the use of a SWW of several mm radius in $\mathrm{THz}$ applications). Nevertheless, it cannot be easily affirmed they will not take part as an issue in SWW designs. Note that the previous analysis is done for perfectly smooth metallic surfaces properties, whilst any roughness on the wire surface will decrease its conductivity, which means lower $\varepsilon_{r}^{\prime}$ and $\varepsilon_{r}^{\prime \prime}$. For qualitative assessments, it is licit to consider the affirmation given in [500], which establish that, if the term $f^{3} / \sigma$ remains constant, the behavior of a SWW does, something that is roughly true. This means that the real conductivity of the metal forming the SWW is a very influential parameter on the propagation characteristics of this waveguide, as a change of one order of magnitude in this parameter is equivalent to move three decades in frequency.

In order to assess the possible effects of reduction of $\varepsilon_{r}^{\prime}$ and $\varepsilon_{r}^{\prime \prime}$, it is considered now the same problem, but the frequency is increased to the top frequency in the THz band: $f=10 \mathrm{THz}$. The $\varepsilon_{r}$ is reduced in this way one order of magnitude. The results for this analysis are shown in Fig. 3.34 in the same disposition as it was done in the previously commented Fig. 3.33. Note that the axis associated to $\alpha_{N}$ and $\beta_{N N}$ are now multiplied by a factor $10^{5}$, one order of magnitude less than for the case of $f=1 \mathrm{THz}$. Notice also that the abscissas axis in the left column plots, Fig. 3.34(a) and Fig. 3.34(c) is moved as well one decade down. Therefore, when comparing 3.34(a) with 3.33(a), it is found that now, the cutoff of the $\mathrm{HE}_{1}$ is lower, since this mode requires for $f=10 \mathrm{THz}, a / \lambda>3$ to propagate, exactly 10 times less than for the previous case. Since the wavelength is also one order of magnitude lower, this means that a radius of $a=90 \mu \mathrm{m}$ is required, which is not, indeed, a large radius. Therefore, the decrement of $\varepsilon_{r}^{\prime}$ and $\varepsilon_{r}^{\prime \prime}$ has caused the surface become more penetrable, so that the cutoff of high order modes, and specially that of the $\mathrm{HE}_{1}$, has become smaller. Since it is possible that imperfections on the SWW have similar effect of decreasing the impenetrability of the surface (decreasing $\varepsilon_{r}^{\prime}$ and $\varepsilon_{r}^{\prime \prime}$ ) as that presented here by increasing $f$, attention must be paid in the strategies to minimize possible undesired coupling of this high order modes.

The influence of reducing $\varepsilon_{r}^{\prime}$ and $\varepsilon_{r}^{\prime \prime}$ is observed to be more evident for $\beta_{N N}$ than for $\alpha_{N}$ (see the more pronounced slopes in Fig. 3.34(b), or the predominance of $\beta_{N}$ in Fig. 3.34(d)), something in agreement with the observed change of $\gamma_{N s}$ with the frequency in the table 3.4. However, the small difference that this cause on the magnitude of $\beta_{N}$, indicates that attention must be paid only on $\alpha_{N}$. Compared to the case of $f=1 \mathrm{THz}$, the attenuation parameter $\alpha_{N}$ is exactly 10 times larger for $f=10 \mathrm{THz}$ when the radius is very large, and a slightly smaller factor if the radius is small, indicating that smaller radius are slightly less affected by the inherent increment of losses with the frequency. This fact is a positive feature, since it is a compensatory factor: losses increase due to the use of smaller radius (sometimes necessary to avoid high order modes and to confine the $\mathrm{TM}_{0}$ power in a practical transmission area), but the attenuation dispersion (the dependence of the attenuation with the frequency) becomes smaller.

The analysis considering the surface modal spectrum ends with the most complicated case: frequency variation. The analysis is done over a gold SWW with fixed 


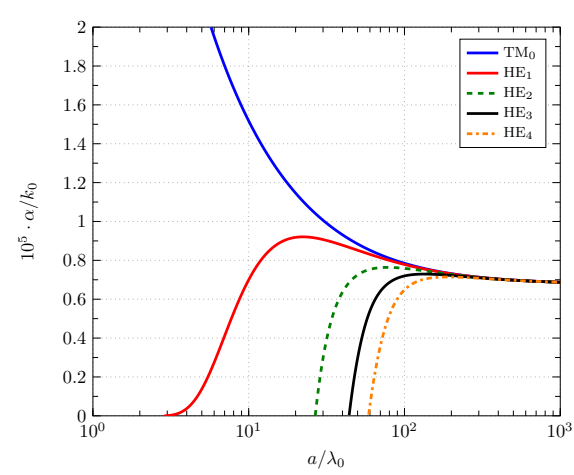

(a)

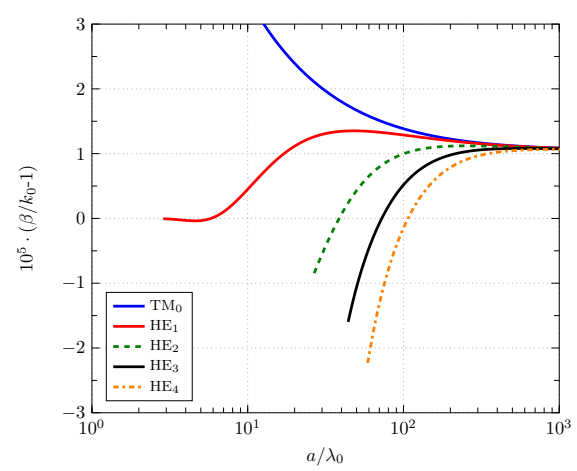

(c)

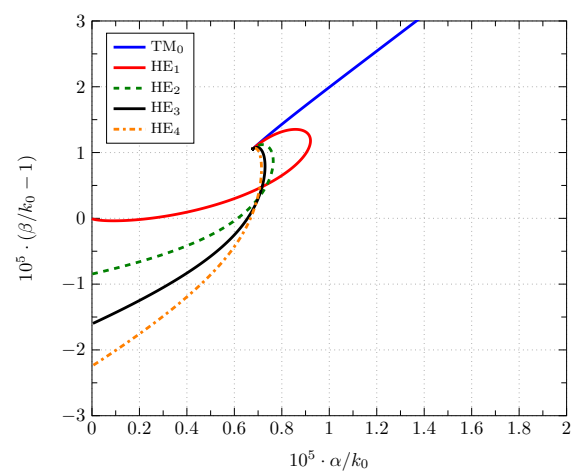

(b)

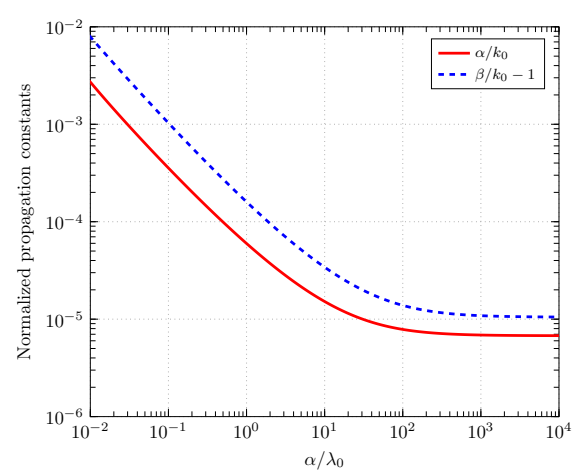

(d)

Figure 3.34: Complex dispersion diagram of the first 5 surface modes $\left(\mathrm{TM}_{0}, H E_{1}, H E_{2}, H E_{3}\right.$ and $\mathrm{HE}_{4}$ ) propagating on a gold $S W W$, with operating frequency $f=10 \mathrm{THz}$, as a function of the electrical size of the wire $a / \lambda_{0}$. a) Attenuation diagram: $\alpha_{N}$ vs. $a / \lambda_{0}$; b) Complex dispersion diagram: $\gamma_{N}$ vs. $a / \lambda_{0}$ c) Dispersion diagram: $\beta_{N}$ vs. $\left.a / \lambda_{0} ; d\right) \alpha_{N}$ and $\beta_{N}$ for the fundamental $T M_{0}$ mode.

radius $a=100 \mu \mathrm{m}$, which implies $a=\lambda_{0} / 3$ at $f=1 \mathrm{THz}$, to give a reference. Now, as the frequency varies $a / \lambda_{0}$ changes proportionally, however same conclusions as with the previous analysis are not longer valid in as much as the permittivity also varies, so both, the structure electrical size and the structure material properties, change at the same time. To better appreciate the frequency dispersion effects the frequency range has been expanded one decade more from each limit of the $\mathrm{THz}$ band, being the analyzed spectrum that comprised between $0.01 \mathrm{THz}$ and $100 \mathrm{THz}$.

Results of this analysis are shown in Fig. 3.35, where, as habitual, $\alpha_{N}$ and $\beta_{N N}$ are vertically aligned in the left column, Figs. 3.35(a) and 3.35(c). The axis are multiplied now by $10^{4}$. The change of the curves regarding to the previous studies is quite evident, becoming a bit more difficult to be interpreted, specially for the fundamental mode. The higher order modes exhibit propagation beyond $f=10$ $\mathrm{THz}$, which means the $\mathrm{THz}$ range is free of high order modes for the considered perfectly smooth gold wire. The propagation characteristics of these modes are very 
close to those of a plane wave in the considered range, but the attenuation is to be considered in the highest frequency limit. The first high order mode, the $\mathrm{HE}_{1}$, goes slower to cutoff as frequency decreases due to its singular characteristic, whereas the rest of the modes do faster. From the observed behavior it is concluded that the decrement on $f$ has more influence on the impenetrability of the surface, which grows by growing $\varepsilon_{r}^{\prime}$ and $\varepsilon_{r}^{\prime \prime}$, than on the electrical size $a / \lambda_{0}$ (which actually increases when $f$ decreases), causing the first effect dominate, and sending the modes to cutoff. This is in concordance with aforementioned affirmation of [500], regarding the influence of conductivity, three times superior to that of the electrical size of the wire.

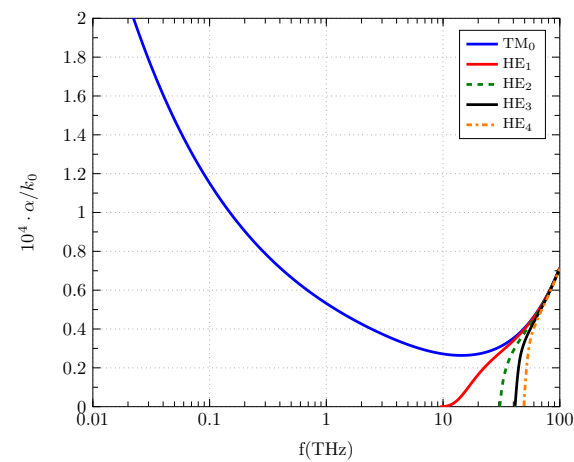

(a)

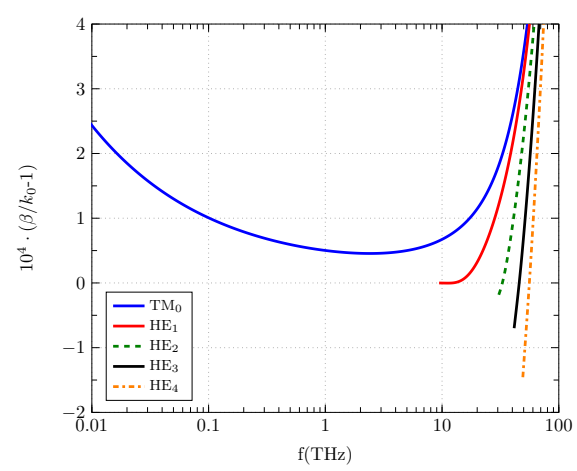

(c)

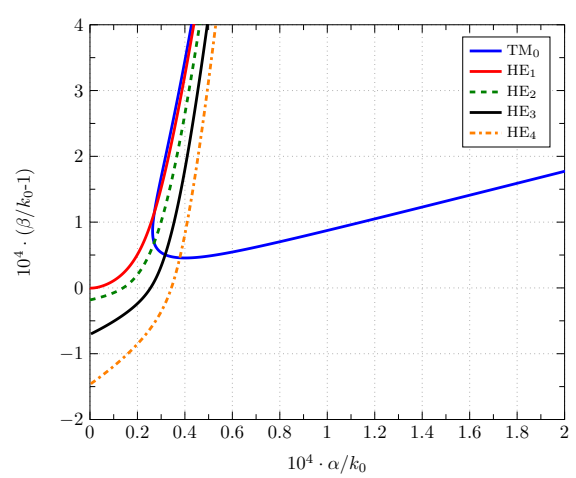

(b)

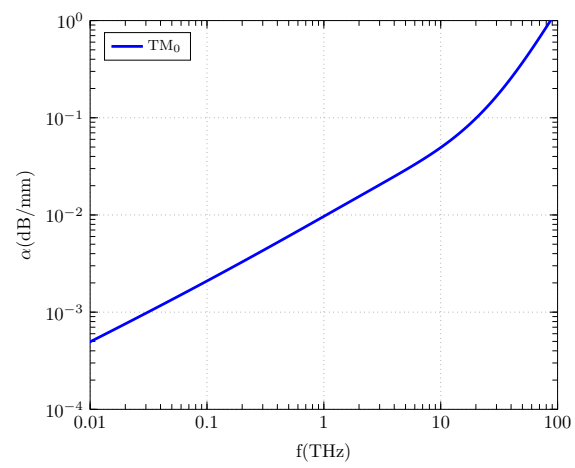

(d)

Figure 3.35: Complex dispersion diagram of the first 5 surface modes $\left(T M_{0}, H E_{1}, H E_{2}, H E_{3}\right.$ and $H E_{4}$ ) propagating on a gold $S W W$ of $a=100 \mu \mathrm{m}$ as a function of the frequency in the range going from $0.01 \mathrm{THz}$ to $100 \mathrm{THz}$. a) Attenuation diagram: $\alpha_{N}$ vs. a/ $\lambda_{0}$; b) Complex dispersion diagram: $\gamma_{N}$ vs. $a / \lambda_{0}$ c) Dispersion diagram: $\beta_{N}$ vs. $\left.a / \lambda_{0} ; d\right)$ Attenuation constant of the fundamental mode $T M_{0}$ in $\mathrm{dB} / \mathrm{mm}$.

The fundamental mode characteristics are treated now. It calls into attention that neither $\alpha_{N}$ nor $\beta_{N N}$ are monotone, exhibiting a local minimum in some frequency of the band, which is not the same for each parameter. The explanation is as follows. If the frequency is low, the wire is electrically small as the operation wavelength is large. From the previous analysis on the radius, it is concluded that 
small radius cause $\gamma_{N}$ to be far from its limiting value for the fundamental mode, i. e., $\alpha_{N}$ and $\beta_{N N}$ are larger. As the frequency is pushed up, $\gamma_{N}$ follows its way towards $\gamma_{N s}$ since the wire becomes electrically larger, and $\alpha_{N}$ and $\beta_{N N}$ decrease. However, as the frequency increases, $\alpha_{N s}$ and $\beta_{N s}$ in $\gamma_{N s}$ also do (see table 3.4). Therefore, although $\gamma_{N}$ goes towards $\gamma_{N s}$ as the frequency increase, this point moves so that, from some frequency, $\alpha_{N s}$ and $\beta_{N s}$ start to grow, inverting the the usual tendency with $a / \lambda_{0}$. Since $\beta_{N N}$ suffers more the reduction in $\varepsilon_{r}^{\prime}$ and $\varepsilon_{r}^{\prime}$ with the increasing frequency, the inflexion point occurs before.

The previous plots are merged in Fig. 3.35(b), where $\alpha_{N}$ and $\beta_{N N}$ are represented in the same range as a parametric plot, with the frequency as parameter. It is observed that the minimum geometrical distance to $\gamma_{N}=j$ occurs in an intermedium point of the curve, hence at some frequency inside the band.

The shape of $\alpha_{N}(f)$ may wrongly suggest that losses decay with the frequency, which would be desirable for some applications. However, the final losses, in $\mathrm{dB} / \mathrm{mm}$ or any similar unit are obtained by denormalizing $\alpha_{N}$, which imply a multiplication by $k_{0}$ at some point, hence multiplying by $f$. Nevertheless, the particular behavior caused by the dispersion of $\varepsilon_{r}$ of a metallic SWW, compensates the linear factor of this multiplication, so that the growth of the real losses in a SWW is lower than linear, hence being a waveguide, not only exhibiting almost zero dispersion, but with small attenuation dispersion, one recurrent problem when dealing with $\mathrm{THz}$ waveguides, accordingly to the state of the art. It worth to mention that, for instance, dielectric waveguides will exhibit always an attenuation dispersion larger than linear in as much the permittivity is constant with the frequency, the dielectric losses scale linearly with frequency, and the mode distribution tends to increase the fraction of power exposed to dielectric losses as the frequency increases. The analysis of the dielectric cylinder shows clearly this fact, which is unavoidable in any dielectric structure, as observed repeatedly in the state of the art review.

By the above it has been rigorously proved that the SWW fundamental mode has, inherently to its structure, good propagation and attenuation dispersion characteristics. Apart of that, low values for the attenuation constant are obtained. In Fig. 3.35(d), the attenuation in $\mathrm{dB} / \mathrm{mm}$ of the fundamental mode is shown. As can be observed, the losses are less than $0.05 \mathrm{~dB} / \mathrm{mm}$ for the whole $\mathrm{THz}$ band, and less than $0.01 \mathrm{~dB} / \mathrm{mm}$ for the first decade, [0.1THz-1THz]. Designs exhibiting even lower losses by increasing the radius will be shown to be possible in next section.

\subsubsection{Bulk modes}

The bulk mode zone is studied, completing in this way the SWW propagation characteristics study. In Fig. 3.37, it is shown the colormap representation of $G\left(\gamma_{N}\right)$ of all HEM modes with $n=1$ in the bulk mode zone of the complex plane $\gamma_{N}$ for a gold SWW of radius $a=\lambda_{0} / 40$ at $f=1 \mathrm{THz}(a=7.5 \mu \mathrm{m})$. It can be observed how the hybrid bulk modes (both, $\mathrm{HE}_{n m}$ and $\mathrm{EH}_{n m}$ ) converge to $\gamma_{N b}$ forming a quite dense line. In fact, a very small radius has been chosen in order to appreciated those modes, since, otherwise, the proximity of the points would describe an almost continuous spectrum of modes. Thinking in the large value of $\left|\varepsilon_{r}\right|$, it could be roughly said that the cylinder is becomes electrically enlarged due to the small value of the wavelength in the metallic medium. 


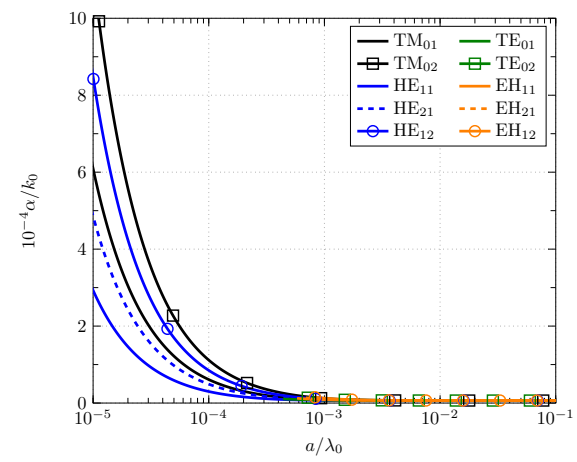

(a)

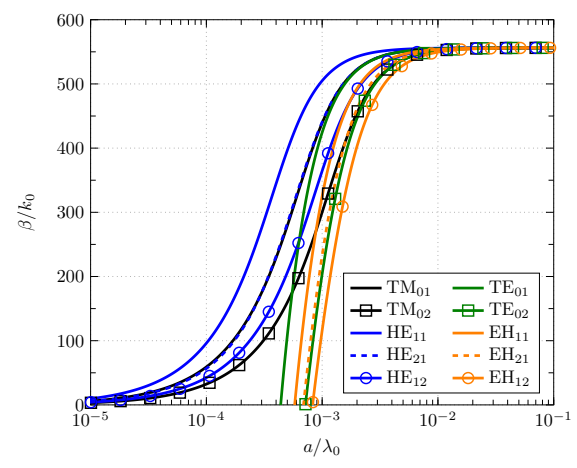

(c)

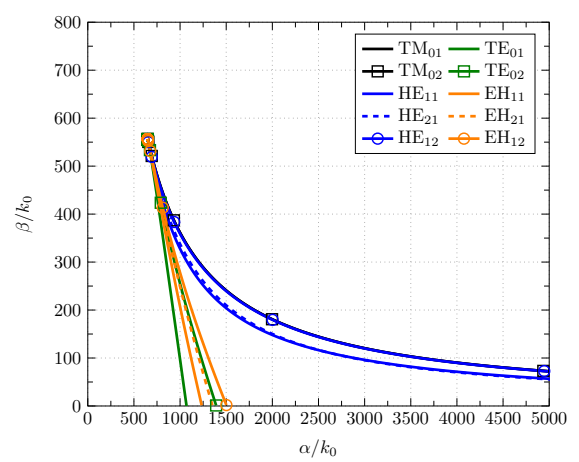

(b)

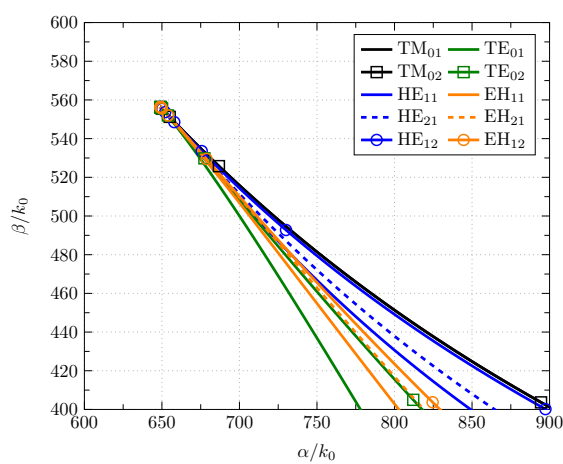

(d)

Figure 3.36: Complex dispersion diagrams of the first bulk modes propagating on propagating in a gold $S W W$, with operating frequency $f=1 \mathrm{THz}$, as a function of the electrical size of the wire $a / \lambda_{0}$. a) Attenuation diagram: $\alpha_{N}$ vs. $\left.a / \lambda_{0} ; b\right)$ Complex dispersion diagram: $\gamma_{N}$ vs. $a / \lambda_{0}$ c) Dispersion diagram: $\beta_{N}$ vs. $a / \lambda_{0}$.

As the radial index $m$ of the solution increase, the points goes towards large $\alpha_{N}$ and $\beta_{N}$, thus becoming (even more) evanescent modes. Note that the large distance to the real axis of $\gamma_{N s}$ causes now that $\mathrm{HE}_{n m}$ and $\mathrm{EH}_{n m}$ modes follow a similar path, being almost degenerated by couples $\left(\mathrm{HE}_{1 m+1}, \mathrm{EH}_{1 m}\right)$. Again, the first bulk mode, the $\mathrm{HE}_{11}$, as usual for this geometry, is singular and has no cutoff. Both sets of modes can be distinguished if a zoom is done. In Fig. 3.37(b) a detail of the bottom right corner of Fig. 3.37 is plotted. Note how, as observed in the last section, $\mathrm{HE}_{n m}$ modes lie in the curve implying $\beta_{r_{1} N}$ to be positive and real, whereas $\mathrm{EH}_{n m}$ modes below it, now only slightly. Despite of the relative proximity of the solutions, caused by the large value of $k_{1}$, inferred by $\varepsilon_{r}$, in absolute terms, the difference is enough to provide different characteristics to each set of modes. Close to the limit point $\gamma_{N b}$, both modes have similar composition of $E_{r_{1}}$ and $E_{\phi_{1}}$, however $\mathrm{HE}_{n m}$ modes have a rather linear polarization, and $\mathrm{EH}_{n m}$ exhibit a curly one, exactly the same behavior of a simple real $\varepsilon_{r}$ dielectric cylinder. When they are closer to the real axis $\left(a / \lambda_{0}\right.$ enough small for the particular mode under question), for $\mathrm{HE}_{n m}$ modes, the polarization turns practically radial, whereas for $\mathrm{EH}_{n m}$ modes it turns 
practically azimuthal. As has been observed along this thesis for this structure in all its versions, $\mathrm{TM}_{0 m}$ bulk modes follow similar path to that of $\mathrm{HE}_{n m}$, and the same can be said regarding $\mathrm{TE}_{0 m}$ and $\mathrm{EH}_{n m}$ modes.

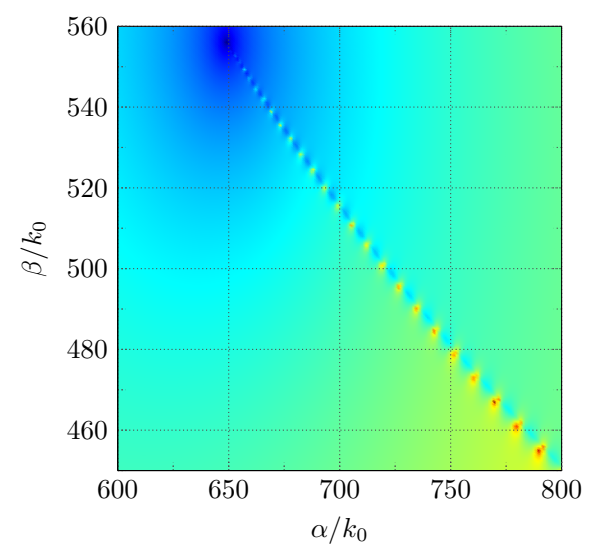

(a)

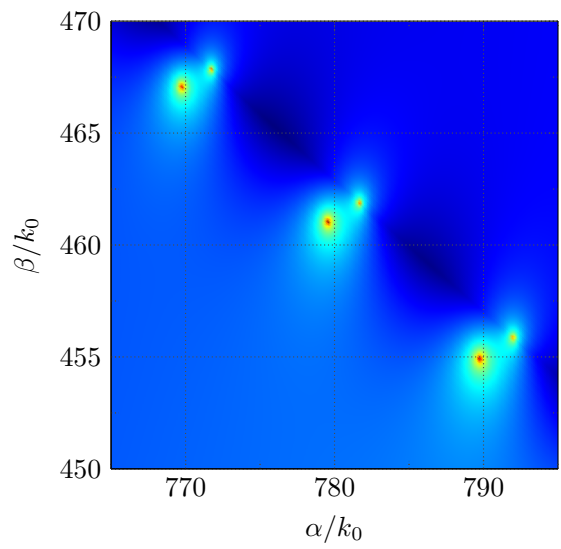

(b)

Figure 3.37: Colormap representation of $G\left(\gamma_{N}\right)$ for HEM modes with $n=1$ in the bulk mode zone of the complex plane $\gamma_{N}$ for a gold $S W W$ of radius $a=\lambda_{0} / 40$ at $f=1 \mathrm{THz}(a=7.5 \mu \mathrm{m})$. a) Set of propagating $\mathrm{HE}_{1 \mathrm{~m}}$ and $\mathrm{EH}_{1 \mathrm{~m}}$ forming a dense spectrum; b) Zoom of the bottom right corner, showing pairs of $\left(\mathrm{HE}_{1 m+1}, \mathrm{EH}_{1 m}\right)$ modes, almost degenerated. $H E_{1 m+1}$ are those lying in the curve described by $\beta_{r_{1} N} \in \mathbb{R}$, and $\mathrm{EH}_{1 m}$ those lying slightly below this curve.

The dispersion diagram of bulk modes is afforded, analyzing the propagation properties of the first modes of each kind $\left(\mathrm{TM}_{0 m}, \mathrm{TE}_{0 m}, \mathrm{HE}_{n m}\right.$ and $\left.\mathrm{EH}_{n m}\right)$ for a gold SWW operating at $f=1 \mathrm{THz}$, as a function of $a / \lambda_{0}$. The normalized attenuation constant $\alpha_{N}$ is shown in Fig. 3.36(a), and the usual normalized propagation constant, $\beta_{N}$, is displayed in Fig. $3.36(\mathrm{c})$, being both parameters, parametrically plotted in Fig. 3.36(b) using the same ranges.

Note first that, for bulk modes, the $\alpha_{N}$ axis is multiplied by a factor less than the unity in this case, $10^{-4}$. For $\alpha_{N}$, the observed behavior is the same than that obtained with simple $\varepsilon_{r}$ case treated in the previous section, $\varepsilon_{r}=-3-4 j$. As $a / \lambda_{0}$ decreases, $\alpha_{N}$ grows exponentially. As indicated at the start of this section, $\alpha_{N}$ has a large lower bound for gold at $f=1 \mathrm{THz}: \alpha_{N b} \leq 649.3$. Therefore the values of the attenuation are as large that bulk modes are immediately damped out inside the inner metallic region of a SWW, which is easily understood in as much the metal is supposed to act rather as a good conductor.

In the case of $\beta_{N}, \mathrm{TE}_{0 m}$ and $\mathrm{EH}_{n m}$ modes behave in the same wave as for $\varepsilon_{r}=-3-4 j$, exhibiting a clear cutoff tendency towards $\beta_{N}=0$ when $a / \lambda_{0}$ is decreased. However, $\mathrm{TM}_{0 m}$ and $\mathrm{HE}_{n m}$ modes do not show for a $\mathrm{SWW}$ at $\mathrm{THz}$ frequencies the anomalous inflexion tendency in $\beta_{N}$ causing a local minimum and a growing behavior as $a / \lambda_{0}$ becomes small, at least in the considered range ${ }^{21} \cdot \mathrm{TM}_{0 m}$ and $\mathrm{HE}_{n m}$ approach the cutoff condition in a much more soft way, exhibiting a shape

\footnotetext{
${ }^{21}$ This behavior still exist, however very small radius should be considered to it become manifested. This is, as $a / \lambda_{0}$ increases, $\beta_{N}$ becomes negative and the curve continues under $\beta_{N}=0$ until the inflexion point is reached, thus $\beta_{N}$ reaching positive values again for very small $a / \lambda_{0}$
} 
in $\beta_{N}$ similar to that of a conventional lossless dielectric cylinder. In fact, Fig. 3.36(c) resembles to some extent the dispersion diagram of a dielectric cylinder, when it is very lossy, and $\alpha_{N}$ decreases monotonically with the increase of $a / \lambda_{0}$ (see again Fig. 3.18(d) or Fig. 3.19(d) for instance).

The difference between the path followed in the complex plane defined by $\gamma_{N}$ between $\mathrm{TE}_{0 m} / \mathrm{EH}_{n m}$ modes and $\mathrm{TM}_{0 m} / \mathrm{HE}_{n m}$ modes is well illustrated in Fig. 3.36(b). However, it must be said that, compared with the $\varepsilon_{r}=-3-4 j$ case, both sets of modes are quite more degenerated when they approach the limit $\gamma_{N b}$, see Fig. $3.36(\mathrm{~d})$. The general idea that the previous plots give is that there is a parallelism on the described paths with the case of a real lossy dielectric with high permittivity and very large losses (see Fig. 3.18(d) for instance). Bulk modes experience the metallic material in this way.

\subsubsection{Conclusions and important remarks}

The previous results indicate that large independence will take place between the fundamental surface mode $\mathrm{TM}_{0}$ and the bulk modes, and undesired coupling will not take place easily due to the very different propagation constant $\gamma$, differing in both, $\alpha$ and $\beta$, for each kind of mode. Observe also that, whereas for $\varepsilon_{r}=-3-4 j$ relevant changes in $\gamma_{N}$ occurred between $a / \lambda_{0}=0.01$ and $a / \lambda_{0}=0.1$ for both, bulk and surface modes, in the analyzed case of a gold SWW at $f=1 \mathrm{THz}$ there is a strong difference: for bulk modes the relevant changes in $\gamma_{N}$ take place between $a / \lambda_{0}=10^{-4}$ and $a / \lambda_{0}=10^{-3}$, whereas for surface modes this happens between $a / \lambda_{0}=50$ and $a / \lambda_{0}=500$. Due to the larger electrical density of the inner region at $\mathrm{THz}$, bulk modes require to be comprised inside a much smaller area of propagation to experience change in their propagation features. Although this effect could be thought to be directly translated to surface modes, due to the very singular limit $\gamma_{N s}$, they are much more sensitive to the rapprochement of the boundaries. Therefore, both kind of modes do not experience any more the same effects with the change of waveguide geometry, a proof of their larger independence.

Furthermore, a fact that helps to avoid coupling is that power distribution is complementary between the fundamental mode and the undesired bulk modes: the $\mathrm{TM}_{0}$ is concentrated in the outer medium attached to the wire surface, and bulk modes are confined in the inner region, sometimes having even larger concentration in the very center of the cylinder.

Nevertheless, for some bulk modes, such as the $\mathrm{TM}_{0 m}$ modes, the polarization will be very similar than that of $\mathrm{TM}_{0}$. This fact translates in practice in that illuminating the face of a cut SWW during excitation must be avoided, i.e., incident field must be forced to be shaped with a ring pattern, having almost no field density in the circle described by the metallic wire.

This section involving the study of the complete modal spectrum of a SWW ends giving answer to a question that has been very controversial, and which must be solved these days due to the relevance of the SWW. Physically, the SWW becomes an interesting waveguiding structure in as much its fundamental surface mode $\left(\mathrm{TM}_{0}\right)$ is in the propagative region, exhibiting, furthermore, an anomalous behavior that allow it to be far enough from the conditions of a plane wave, becoming a practical support for $\mathrm{THz}$ energy. Mathematically, this has its origin in the fact the the 


\begin{tabular}{|c|c|c|c|}
\hline \multirow[b]{2}{*}{ Parameter } & \multicolumn{3}{|c|}{ Frequency } \\
\hline & $f=0.1 \mathrm{THz}$ & $f=1 \mathrm{THz}$ & $f=10 \mathrm{THz}$ \\
\hline$\alpha_{N s}$ (Drude) & $6.76 \cdot 10^{-8}$ & $6.76 \cdot 10^{-7}$ & $6.76 \cdot 10^{-6}$ \\
\hline$\alpha_{N s}($ Skin-depth) & $6.78 \cdot 10^{-8}$ & $6.78 \cdot 10^{-7}$ & $6.78 \cdot 10^{-6}$ \\
\hline$\beta_{N N s}$ (Drude) & $1.05 \cdot 10^{-9}$ & $1.07 \cdot 10^{-7}$ & $1.04 \cdot 10^{-5}$ \\
\hline$\beta_{N N s}$ (Skin-depth) & $-6.88 \cdot 10^{-15}$ & $-6.9 \cdot 10^{-13}$ & $-6.9 \cdot 10^{-11}$ \\
\hline
\end{tabular}

Table 3.5: Comparison between Drude model and skin-depth model limiting normalized propagation constant values for surface modes $\left(\gamma_{N s}=\alpha_{N s}+j \beta_{N s}\right)$ for gold $\left(f_{p}=2184 \mathrm{THz}, f_{\tau}=6.45\right.$ $\left.\mathrm{THz} / \sigma_{D C}=4.1 \cdot 10^{7}\right)$.

real part of $\varepsilon_{r}$ is negative, as was essentially shown in section 3.4.4. Note now the following. If $\varepsilon_{r}$ would be described by the classical skin depth effect model, it would be $\varepsilon_{r}=-j \sigma /\left(\omega \varepsilon_{0}\right)$, thus having zero real part. In such case, in the expression (3.269) describing $\gamma_{N s}$, it is clear that $\left|\varepsilon_{r}+1\right|>\left|\varepsilon_{r}\right|$, and by this $\left|\gamma_{N s}\right|<1$, this last inequality implying that both $\alpha_{N}<1$ and $\beta_{N}<1$. Therefore, since $\beta_{N}<1$, the classical skin depth effect model implies that the SWW structure has its surface plasmonic modes limit in the radiative region.

The limiting values for $\gamma_{N s}=\alpha_{N s}+j \beta_{N s}$ (in the following $\beta_{N N s}=\beta_{N s}-1$ is used for clarity purposes) have been calculated for both models in the case of the metallic material being gold, and they are displayed in table 3.5 to compare them. The first two rows compare $\alpha_{N s}$, which does not present any significant change. The second two rows, comparing $\beta_{N N s}$ show the critical difference. Whereas the Drude model implies $\beta_{N N s}$ being small, but positive (propagating mode), the skindepth effect model has an almost negligible value of $\beta_{N N s}$, but negative (radiating mode). It can be said that in both, skin-depth effect model and Drude model $\gamma_{N s}$ is extremely close to $\gamma_{N}=j$ ( $\gamma_{N}$ of a plane wave in the external air medium), however, being rigorous in mathematical terms, in the skin-depth effect model this limit is approached from the radiative zone, whereas in the Drude model this limit is approached from the propagative zone.

It was seen in Fig. 3.33(a) that higher order modes describe some path, achieving a pair $\left(\alpha_{N}, \beta_{N N}\right)$ with maximum modulus, which is the point $\gamma_{N}$ with more entity waveguiding features (differing from a plane wave). Since, for the skin-depth model, the limiting point $\gamma_{N s}$ has radiative features, although part of the path described by $\left(\alpha_{N}, \beta_{N N}\right)$ enters in the propagating zone due to the typical behavior of the parametric $\left(\alpha_{N}, \beta_{N N}\right)$ curve, most of the path is contained in the radiative zone. Since even when $\beta_{N N}>1$ during the $\mathrm{HE}_{n}$ mode path in the complex $\gamma_{N}$ plane, $\left(\alpha_{N}, \beta_{N N}\right) \approx(0,1)$, which is a singular point, it is numerically challenging to capture these high order $\mathrm{HE}_{n}$ modes (see the aspect of $G\left(\gamma_{N}\right)$ in the previously commented Fig. 3.32(b) as a good prove of this fact). This becomes specially true if one thinks in the computational resources at the time of Sommerfeld or Stratton, when [488] or [489] were published.

In the most relevant works [488], [489], [490], [500], that conform the base that has been referenced by any other paper dealing with the SWW, what is affirmed is that there is one fundamental low-loss mode with $\beta \approx k_{0}$, named as $\mathrm{TM}_{01}$, and that for $n \neq 0$ all modes are damped out rapidly, therefore do not play a significant role. 
It is pointed out that discontinuities can cause loss via coupling to this lossy modes or by radiation [500]. These affirmations are true, but do not make evidence of the actual modal spectrum. In fact, firstly, it could be understood that the fundamental mode is of the same nature of bulk modes, which are considered possible only in the case $n \neq 0$ or if they are $\mathrm{TE}_{0 m}$. Note that, actually, the most relevant (if it could be under very specific situations) bulk mode is the $\mathrm{TM}_{01}$. In this sense, it becomes necessary to establish a proper nomenclature and distinction of the $\mathrm{TM}_{0}$ solution, which is the only one possible in the surface zone (there is not any additional surface solution requiring the use of the index $m$ ). Secondly, higher order modes surface modes, which are the true threat of undesired coupling, are totally missed. Note that, fortunately, large radius are required to these high order modes to have appreciable entity when considering a Drude modeled SWW, but ideal in terms of surface smoothness. However, it has been show than reduction of $\varepsilon_{r}$ by increasing the frequency disposes these modes more suitable to disturb. Since the metal conductivity has an influence 3 times larger than the frequency, it is expected that unavoidable imperfections on the wire surface, make $\mathrm{HE}_{n}$ modes to become relevant, so strategies to prevent them are really necessary.

Thus, an important question has been solved providing rigorous analysis, and by means of the facts established in this section, results in recent experiments can be explained clearly. For example, it can be understood why ring shaped radial incident E-field excitation over small radius SWW like that presented in [506] works better than the initial proposed by Wang and Mittleman in [492]. In the former work, the excitation is tailored for the fundamental mode and, at the same time, prevents the coupling to high-order modes. Also, it can be explained why in [492] anomalous attenuation decaying with the frequency was obtained: at higher frequencies the surface is less impenetrable, hence the fundamental $\mathrm{TM}_{0}$ interacts more with it, being more confined, thus reaching more transmitted power the reception area. Similar affirmations can be done for any structure based on the SWW, regarding excitation, curves, coupling between two SWW (vertical or horizontal), etc.

The key point is that the complete study presented here is the necessary tool to provide accurate optimization of the structure under design according to the operating band, geometrical constrains of the problem, etc. In this way, the SWW design tools, based on the knowledge about the structure, approach more the desired level, that of the well-known structures such as the RWG, CWG or microstrip line, to mention a few of them. Therefore, design strategies can be applied under quantitative bases, and not longer under roughly qualitative evidences, something that has been happening up to date. This is mandatory in order to properly design more advanced structures such as couplers, filters, antennas, etc. In addition, the application of the powerful modal methods is suggested in order to provide very accurate analysis tools for such more complicated structures.

Other important research gateway opened by the rigorous analysis of the SWW is the characterization of similar structures involving the metals modeled as dielectrics through appropriate models, such the Drude model one. Note that the SWW is the simplest open structure of this kind. Understood the principles of the SWW, more complex structures can be afforded. Examples of direct evidence are different cross area structures, e.g., a rectangular metallic wire, or multilayer cylindrical structures (as these examples were the next step when the dielectric cylinder was solved [21]). In 
a similar way, commercial tools should incorporate in their algorithms, strategies to proper characterize the complex effects caused by complex values of $\varepsilon_{r}$ in dielectrics, that can be either metals at high frequencies, as afforded here, or metamaterials, also of great interest. In this way, errors that have been evidenced in [355], [354], [348], or [391], would be avoided. This last suggested work line is mandatory in order that $\mathrm{THz}$ devices can be properly designed with the same efficiency level as in the microwave or optical bands.

\subsubsection{Fundamental Mode}

The rather general perspective adopted to give a rigorous and complete analysis is left now to quantify better the fundamental $\mathrm{TM}_{0}$ mode properties, inasmuch as this mode will be the transmission support in the SWW structure. In this section attention will be focused in the attenuation, power distribution, and dispersion of the $\mathrm{TM}_{0}$ mode, from a more practical perspective than that provided by the study of the normalized propagation constant $\gamma_{N}$. Parametrization to cover a wide spectrum of possible situations is done as follows. Regarding the operation frequency, the whole $\mathrm{THz}$ band $0.1 \mathrm{THz} \leq f \leq 10 \mathrm{THz}$ is considered. Seven radius $a$ ranging from $12 \mu \mathrm{m}$ to $1000 \mu \mathrm{m}$ (specifically: $12 \mu \mathrm{m}, 25 \mu \mathrm{m}, 50 \mu \mathrm{m}, 125 \mu \mathrm{m}, 250 \mu \mathrm{m}, 500 \mu \mathrm{m}$ and $1000 \mu \mathrm{m})$ are contemplated, covering in such a way a great variety of possibilities. Finally two possible metals are considered: gold $\left(f_{p}=2184 \mathrm{THz}, f_{\tau}=6.45 \mathrm{THz}\right.$ $\left.\sigma_{D C}=4.1 \cdot 10^{7} \mathrm{~S} / \mathrm{m}\right)$, and nickel $\left(f_{p}=1182 \mathrm{THz}, f_{\tau}=10.6 \mathrm{THz} / \sigma_{D C}=1.45 \cdot 10^{7}\right.$ $\mathrm{S} / \mathrm{m})$.

\subsubsection{Attenuation}

The losses of the waveguide are extracted from $\alpha_{N}$ through denormalization. Usually it would be interesting to consider them in $\mathrm{dB} / \mathrm{mm}$, since most of the published work consider this unit, and $\mathrm{THz}$ devices are expected to size few millimeters. Since the units of $\alpha=\alpha_{N} k_{0}$ are $\mathrm{Np} / \mathrm{m}$,

$$
\alpha(\mathrm{dB} / \mathrm{mm})=10^{-3} \alpha_{N} k_{0} 10 \log \left(e^{2}\right)=8.686 \cdot 10^{-3} k_{0} \alpha_{N}
$$

where log is the base 10 logarithm.

First, $\alpha(\mathrm{dB} / \mathrm{mm})$ as a function of frequency is shown in Fig. 3.38 for the several radius aforementioned. It is appreciated that attenuation dispersion is quite low. In fact, losses expand as much two decades in the whole $\mathrm{THz}$ band (see the case of largest radius, $a=1000 \mu \mathrm{m}$ ), being possible them to range bounded in an interval expanding only a bit more than one decade (see the case of smallest radius, $a=$ $12 \mu \mathrm{m})$. Larger radius present lower losses and the product $a \alpha$ is quite constant. See for instance that $a=12 \mu \mathrm{m}$ and $a=1000 \mu \mathrm{m}$ differ two orders of magnitude, and the associated losses also do.

The larger attenuation dispersion of larger radius is caused by the lower impenetrability of the metallic surface at high frequencies, which become more evident when the losses are low. However, the difference in attenuation dispersion observed for different $a$ is much less significant than the difference in losses. In this point it must mentioned that, as proved in last section, attenuation dispersion is less than linear until the impenetrability collapses and there is a larger growth. However, 
since in the range in which $\alpha$ grows less than linear with $f$, the growth is almost linear, it is not clearly appreciated in the graphs.

Regarding the material, it can be said that results for both metals, gold and nickel, give similar conclusions, however, the best quality of gold as a conductor implies lower losses, and a slightly lower attenuation dispersion caused by the more stable impenetrability.

Considering gold as metal, it could be said that radius larger than $a=125 \mu \mathrm{m}$ offer competitive attenuation at $\mathrm{THz}$ frequencies since they imply losses below $0.01 \mathrm{~dB} / \mathrm{mm}$ up to $f=1 \mathrm{THz}$. In order to obtain a similar attenuation level at $f=10 \mathrm{THz}$, the radius should be multiplied by a factor 10 , the same as the frequency. The SWW acts in this way contrarily to dielectric structures, that should be scaled in the usual (electrical size of the waveguide) since losses increase with the size in dielectric waveguides, as the mode becomes confined in the lossy dielectric core (or regions ${ }^{22}$ ).

On the other hand, compared with metallic hollow waveguides such as the RWG or the CWG, one found that in such cases an increase of size also reduce the losses. However, those structures are very sensitive to this size increment in multimode propagation terms, whereas in the SWW the propagation of high order modes is not as easy, and the possibility of coupling to them is less probable being that the mode patterns in the SWW do not match with the same grade than for closed structures. To have an infinite region of propagation is helpful in this way. Similar affirmations can be done to any other closed or semi-closed structure involving metals as main element of confinement (PPW, stripline, microstrip line, CPW, etc.). Regarding to periodic structures, these are very frequency dependent in geometrical terms, so there is almost not margin to engineer in their size.

By the above, the SWW presents a particular behavior different from the generic behaviors of other kind of waveguide types. In attenuation terms, the choice of the metal seems to be not very relevant, as it is the choice of the radius. This will be extended directly to the distribution of power, in as much this parameter is very related with the attenuation. For ideal, smooth surface wires, high order modes are not a real threat, engineering the radius can be afforded with a wide variety of possibilities. In practical realizations, prevention to multimode operation may be afforded with simple solutions that will be given along the next chapters.

Another way to see the previous results is to display the losses as a function of the radius, for several frequencies. This is done in Fig. 3.39. In this graph, the curves behavior similar to $f(a)=a^{-1}$ show clearly that this is, roughly, the dependence of the losses with the radius, as commented before. Comparing the curves, it is found that higher frequencies exhibit lower attenuation dispersion, what is normal in as much as the range of radius considered is not implying a wide variety of situations. For example, $f=10 \mathrm{THz}$ experience as small most of the considered radius. In general, there is an important increment of losses if $a<100 \mu \mathrm{m}$.

\footnotetext{
${ }^{22}$ Complex structures as tubes, multilayer fibers, or ARROW structures may present a non monotone increase of losses with frequency due to resonances, however, the general tendency as the frequency increase is that the dielectric regions gain confinement capacity due to their larger electrical size, hence increasing the dielectric absorption losses of the structure.
} 


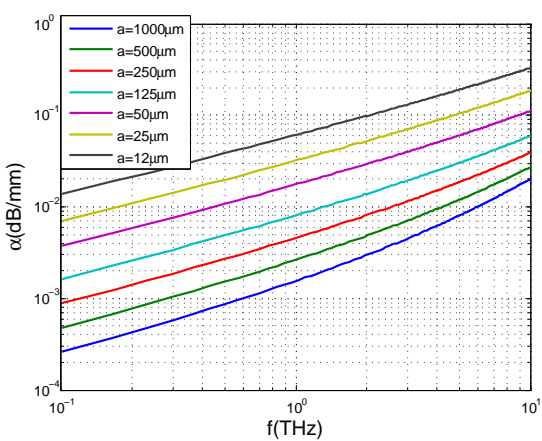

(a)

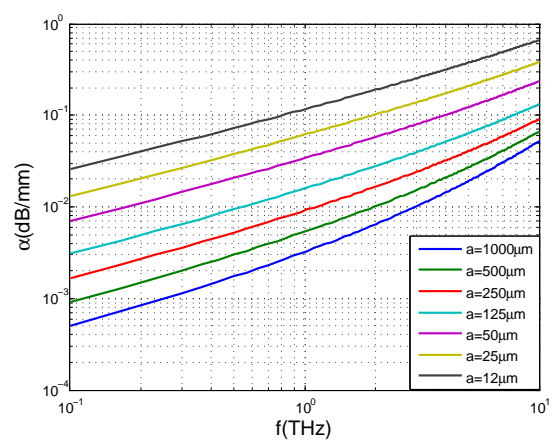

(b)

Figure 3.38: Attenuation in $\mathrm{dB} / \mathrm{mm}$ of the fundamental mode $T M_{0}$ vs. frequency in the $\mathrm{THz}$ range for several values of the radius a. a) Gold; b) Nickel.

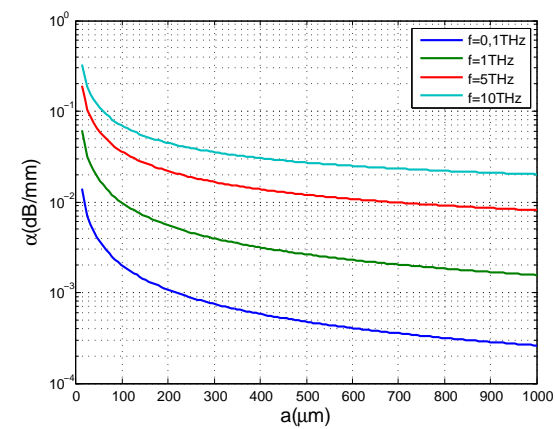

(a)

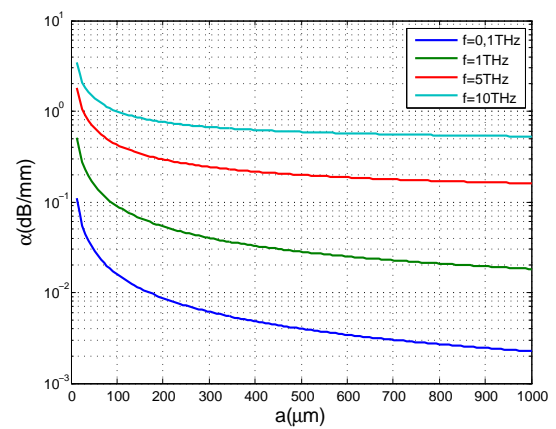

(b)

Figure 3.39: Attenuation in $\mathrm{dB} / \mathrm{mm}$ of the fundamental mode $T M_{0}$ vs. the radius a for several frequencies in the $\mathrm{THz}$ range. a) Gold; b) Nickel.

\subsubsection{Power distribution}

In the SWW, always related to the attenuation, it must be considered the power distribution. Since the external air region, where the propagation takes places, is infinite, very different power distributions can be found. In fact, the direct relationship between $\alpha$ and $\alpha_{r_{2}}$, this last directly determining the extension of the fields, implies that the smaller the losses, the larger the area required to transmit certain fraction of power. Therefore, although it is possible to choose a radius enough large to force the losses to be under certain limit, the extension of the transmitted wave may be rather unpractical, making no sense to consider such design.

A usual way to quantify the power distribution or power extension is that proposed by Goubau [490] and King [500], which consist in determine the required area (cylindrical area determined by its radius $r$ ) to transmit certain fraction or percentage of the total power. Note that, mathematically, whatever the radius a of the SWW is, all the external infinite medium is required to transmit strictly all 
the power. Therefore, depending on the application or the situation, a significant amount of power is establish, e.g., $80 \%$, and the finite area related to the transmission of this power fraction is considered as the practical area of transmission.

Since the total transmitted power is

$$
P_{T}=\frac{1}{2} \operatorname{Re}\left\{\iint_{S}\left(\vec{E} \times \vec{H}^{*}\right) \cdot \hat{z} \mathrm{~d} S\right\}
$$

where $S$ encompass both regions, i. e., the total $\mathbb{R}^{2}$. Cylindrical coordinates allow for an easy particularization of the previous equation in order to calculate the total power transmitted through a transversal area from certain radius $r$ to infinity (a circular ring with inner radius $r$ and infinite outer radius):

$$
P_{T}(r)=\operatorname{Re}\left\{\pi \int_{r}^{\infty} r E_{r} H_{\phi}^{*} \mathrm{~d} r\right\}
$$

Taking into account that it makes sense to consider $r$ only in the case $r>a$, and substituting in the previous expression the fields of the fundamental $\mathrm{TM}_{0}$ mode (see the expressions (3.243)-(3.248)):

$$
P_{T}(r)=\operatorname{Re}\left\{\pi C C^{*} \frac{\gamma k_{0}^{2}}{\omega \mu_{0} \alpha_{r_{2}} \alpha_{r_{2}}^{*}} \int_{r}^{\infty} r \mathrm{~K}_{1}\left(\alpha_{r_{2}} r\right) \mathrm{K}_{1}^{*}\left(\alpha_{r_{2}} r\right) \mathrm{d} r\right\},
$$

integral which results to be analytical (see [542]), obtaining that

$$
\begin{aligned}
P_{T}(r)=-\operatorname{Re}\{\pi & C C^{*} \frac{\gamma k_{0}^{2}}{\omega \mu_{0} \alpha_{r_{2}} \alpha_{r_{2}}^{*}\left[\alpha_{r_{2}}^{2}-\left(\alpha_{r_{2}}^{*}\right)^{2}\right]} \cdot \\
& {\left.\left[\alpha_{r_{2}}^{*} r \mathrm{~K}_{1}\left(\alpha_{r_{2}} r\right) \mathrm{K}_{0}^{*}\left(\alpha_{r_{2}} r\right)-\alpha_{r_{2}} r \mathrm{~K}_{1}^{*}\left(\alpha_{r_{2}} r\right) \mathrm{K}_{0}\left(\alpha_{r_{2}} r\right)\right]\right\} }
\end{aligned}
$$

The last closed form expression gives the total power transmitted on a transversal area from certain radius $r$ to infinity. Particularly, $P_{T}(a)$ describes the total power transmitted in the region 2 , i. e., $P_{2}=P_{T}(a)$. This last implies that the total power transmitted in a circular ring with inner radius $a$ and outer radius $r$ is given by $P_{T}(a)-P_{T}(r)$. Due to the large impenetrability of metals at $\mathrm{THz}$ frequencies, the total power that the fundamental mode transmit inside the metallic region is very small. Therefore, it makes sense to neglect this power, and consider that all the power is transmitted in the external region, thus keeping valuable simplicity with almost not loss of accuracy. Under the described assumption, it is possible to say that the percentage of the total transmitted power by the fundamental mode in a circular ring with inner radius $a$ and outer radius $r$ is:

$$
p(\%)=100 \cdot \frac{P_{T}(a)-P_{T}(r)}{P_{T}(a)}=100 \cdot\left(1-\frac{P_{T}(r)}{P_{T}(a)}\right)
$$

where the quotient $P_{T}(r) / P_{T}(a)$, accordingly to $(3.276)$, is: 


$$
\frac{P_{T}(r)}{P_{T}(a)}=\operatorname{Re}\left\{\frac{\left[\alpha_{r_{2}}^{*} r \mathrm{~K}_{1}\left(\alpha_{r_{2}} r\right) \mathrm{K}_{0}^{*}\left(\alpha_{r_{2}} r\right)-\alpha_{r_{2}} r \mathrm{~K}_{1}^{*}\left(\alpha_{r_{2}} r\right) \mathrm{K}_{0}\left(\alpha_{r_{2}} r\right)\right]}{\left[\alpha_{r_{2}}^{*} a \mathrm{~K}_{1}\left(\alpha_{r_{2}} a\right) \mathrm{K}_{0}^{*}\left(\alpha_{r_{2}} a\right)-\alpha_{r_{2}} a \mathrm{~K}_{1}^{*}\left(\alpha_{r_{2}} a\right) \mathrm{K}_{0}\left(\alpha_{r_{2}} a\right)\right]}\right\}
$$

expression that after some algebraic manipulations becomes more compact:

$$
\frac{P_{T}(r)}{P_{T}(a)}=\operatorname{Im}\left\{\frac{\alpha_{r_{2}} r \mathrm{~K}_{1}\left(\alpha_{r_{2}} r\right) \mathrm{K}_{0}^{*}\left(\alpha_{r_{2}} r\right)}{\alpha_{r_{2}} a \mathrm{~K}_{1}^{*}\left(\alpha_{r_{2}} a\right) \mathrm{K}_{0}\left(\alpha_{r_{2}} a\right)}\right\}
$$

Therefore, (3.277) is a closed form function describing $p(\%)$ as a function of the radius $r$. Although the inverse cannot be obtained, i. e., isolating $r$ to get a function describing $r$ as a function of $p(\%),(3.279)$ is very simple so that numerical routines such as described in [528] result very fast to obtain the required radius given a percentage $p(\%)$ of total transmitted power, which is usually the required data for desing purposes. In this way $(100-p) \%$ is assumed to be lost, and an effective area to do that is the restriction of the design.

The practical study presented here considers a significant percentage of $p(\%)=$ $80 \%$, and the area required to transmit it is defined by finding the associated $r_{80 \%}$. The analysis considers exactly the same cases as the previously afforded attenuation analysis. Firstly, in Fig. 3.40, the radial extension of $80 \%$ of the total transmitted power of the fundamental mode $\mathrm{TM}_{0}$ is presented as a function of frequency for the several radius considered. As it is expected taking into account the curves of $\alpha(f)$, it is found that the highest the frequency the larger the confinement (lower $r_{80 \%}$ ), caused by the larger interaction with the metallic surface (hence larger losses). However, the variation of $r_{80 \%}$ with the frequency is more bounded than that of $\alpha$. In fact, the radial extension only varies one order of magnitude in the $\mathrm{THz}$ band.

Regarding to the effect of the radius it is found again proportionality, but with less influence compared with the attenuation. For instance, $r_{80 \%}$ decreases only one order of magnitude when the radius is diminished by two orders of magnitude. It must be noted that $r$ describes the distance to the origin, not from the SWW surface. This means that, if the extension from the surface would be considered, in each curve of Fig. 3.40 the corresponding radius should be substracted. This would give even less variation, inasmuch as the curves exhibiting larger $r_{80 \%}$ are those having also larger radius. However, it makes sense to consider $r$ from the origin, since in practical realizations the constrain will be usually found in the total area including the wire (think for example in a SWW probe introduced in a human body through a limited area orifice for scanning purposes).

Again $a=125 \mu \mathrm{m}$ could be mentioned as a good reference point between what is acceptable and what is excessive in general terms (this assessment will obviously depend always on the final application). This radius offer an extension of approximately $r_{80 \%}=2 \mathrm{~mm}$ at $f=1 \mathrm{THz}$. However, when talking about the extension on fields, are the larger radius those under question since they may present excessive area of transmission. Therefore, it easy to foreseen the major drawback constrain in the SWW structure: the existent trade off between attenuation and radial extension. This constrain may compared with the well-known trade off between attenuation and bandwidth in closed waveguides.

Although the attenuation dispersion is larger than the extension dispersion, qualitatively the former is more accepted (probably because its recurrent appearance in 


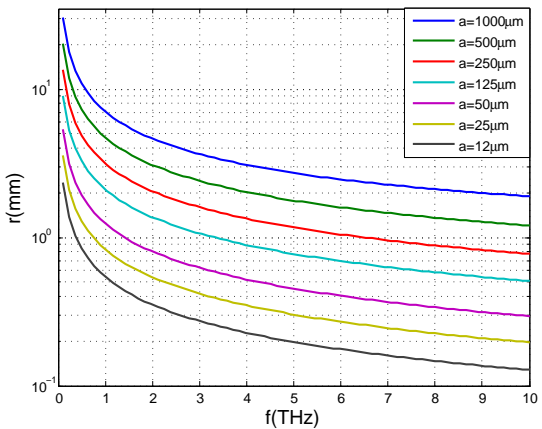

(a)

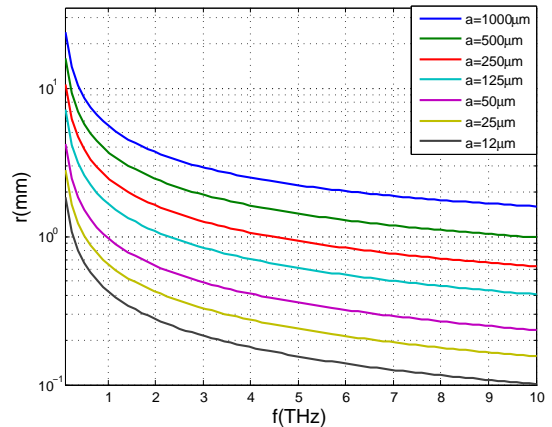

(b)

Figure 3.40: Radial extension of $80 \%$ of the total transmitted power of the fundamental mode $T M_{0}$ vs. frequency in the $\mathrm{THz}$ range for several values of the radius a. a) Gold; b) Nickel.

dielectric structures). When regarding to the use of a SWW operating in a wide spectrum, the differences in power distribution between the maximum and minimum frequencies suggest a challenging problem to be addressed. Think for instance in the excitation or reception. However, a positive fact is that those frequencies more confined, are as well the more attenuated. This means, that whereas higher frequencies will be more damped during the waveguiding path, they are easily to be recovered or excited. Therefore, in a natural manner, the features of the SWW compensate both dispersions, given a more stable behavior inherently.

In addition to previous fact, it is possible to make all frequencies to collapse into a small area if the radius is chosen small enough. This is well appreciated in the plots of Fig. 3.41. The value of $r_{80 \%}$ dramatically drops when $a$ is diminished to small values. Of course, this imply noticeably high losses, but such small radius may be used only for certain purposes like excitation or reception, and by means of properly designed transitions, to allow the use a more convenient low-loss SWW during the rest of the waveguiding path.

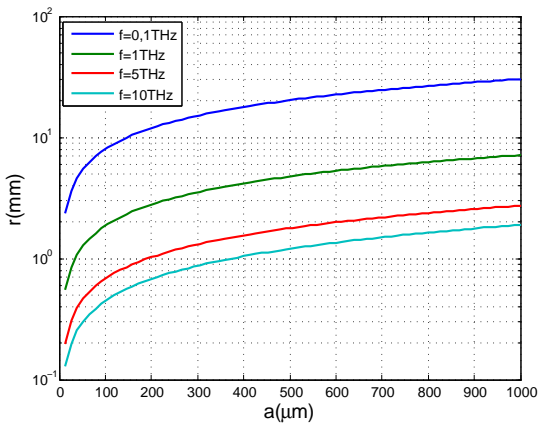

(a)

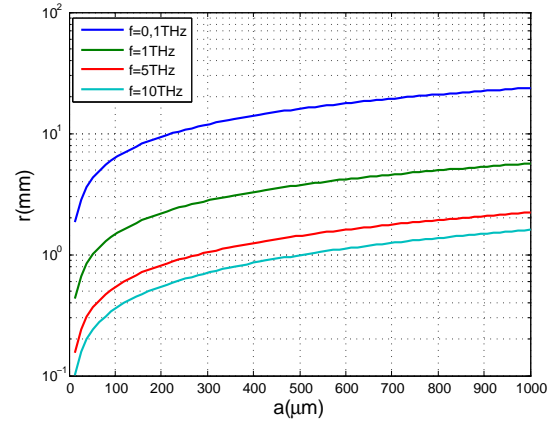

(b)

Figure 3.41: Radial extension of $80 \%$ of the total transmitted power of the fundamental mode $T M_{0}$ vs. the radius a for several frequencies in the THz range. a) Gold; b) Nickel. 
The power distribution and the aspect of field pattern can be better understood through $1 \mathrm{D}$ and $2 \mathrm{D}$ plots of the E-field magnitude of the fundamental mode $\mathrm{TM}_{0}$ in the vecinity of the wire surface. It must be mentioned that this mode behave as a quasi-TEM mode, exhibiting an E-field that is almost in its totally radial, i.e., $\vec{E} \approx E_{r}$. Fig. 3.42 displays the E-field magnitude in logarithmic representation with $15 \mathrm{~dB}$ range for a SWW of $a=450 \mu \mathrm{m}$, assuming gold as metal (left column) and nickel (right column). First row, Figs. 3.42(a) and 3.42(b) correspond to $f=0.1$ $\mathrm{THz}$, whereas second row, Figs. 3.42(c) and 3.42(d) correspond to $f=10 \mathrm{THz}$.

It is appreciated how at low frequencies the fields are more expanded and there is negligible influence of the material (both metals offer very impenetrable surfaces at low frequencies). However, at high frequencies fields collapse in a very small area, an effect that become very evident for worse metals (compare gold and nickel through Figs. 3.42(c) and 3.42(d)). Specifically, in the case of nickel at $f=10 \mathrm{THz}$, Fig. $3.42(\mathrm{~d})$, the field patter is a very thin ring. Employing this area for sensing purposes may be very effective. In this way, making the surface penetrable, and this can be achieved not only by employing a worse metallic material but also reducing the surface smoothness, grooving it or adding a dielectric coating [490], [500], [21], very sensitive SWW probes may be built. The results of tapered periodically corrugated wires in [510] is a good prove of this fact.

Figs. 3.43(a) and 3.43(b) show the same information in $1 \mathrm{D}$ by disposing the E-field magnitude as a function of the radius $r$. In the left image, Fig. 3.43(a), the case $f=0.1 \mathrm{THz}$ is represented. Curves of both metals overlap in this case; the field pattern is the same. Contrarily, in the case of $f=10 \mathrm{THz}$, Fig. 3.43(b), there is a clear difference between both materials. The worse conducting properties of Nickel imply the field to collapse on the vicinity of the wire surface. Note how this situation may be advantageous in such cases in which the mode is required to be excited by small area annular shaped source. Regarding Fig. 3.43(b), think for example in a annular photoconductive antenna extending from the wire surface to $1 \mathrm{~mm}$ beyond it. The E-field tail not interacting with the device would be noticeably higher in the gold SWW case. However, it must be also noted that good conductors present a more stable pattern with frequency, thus enabling a better broadband response.

As it has been already observed in this section by means of Figs. 3.40 and 3.41 , reduction of the extension is much easily afforded by reducing the radius than choosing a worse conductor (unless a very bad conductor is chosen or a quite rough surface is provided). Accordingly, the same field analysis is presented now for an $a=20 \mu \mathrm{m}$ radius SWW in Figs. 3.44 and 3.45. The observation area in the plots is the same as in the case of $a=450 \mu \mathrm{m}$. However, in the $2 \mathrm{D}$ plot the dynamic range has been extended to $45 \mathrm{~dB}$ in order to have better visualization for this case, which exhibits larger power confinement.

As before, any difference between gold and nickel is found for $f=0.1 \mathrm{THz}$, see Figs. 3.44(a) and 3.44(b), whereas at $f=10 \mathrm{THz}$ the lower impenetrability of nickel makes evidence when looking the 2D plots of Figs. 3.44(c) and 3.44(d). However, thinking in absolute area terms, i. e., regarding that both designs, $a=450 \mu \mathrm{m}$ and $a=20 \mu \mathrm{m}$, are considered for the same application or functionality, specified over the same area, the difference is quite evident. Note that if the radius $a$ is big, there is an appreciable empty area with no fields corresponding to the SWW body. In this way, the power distribution patter presents a clear ring shape. Contrarily, small 


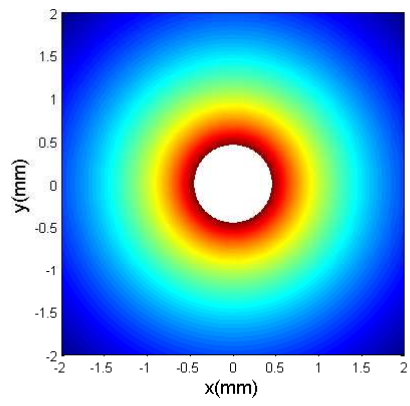

(a)

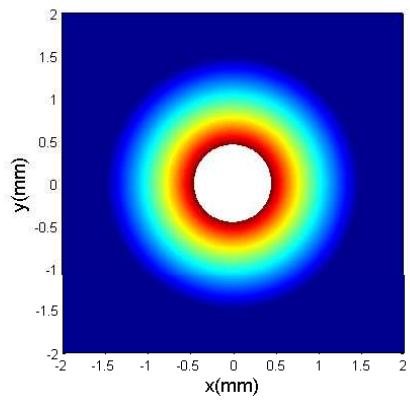

(c)

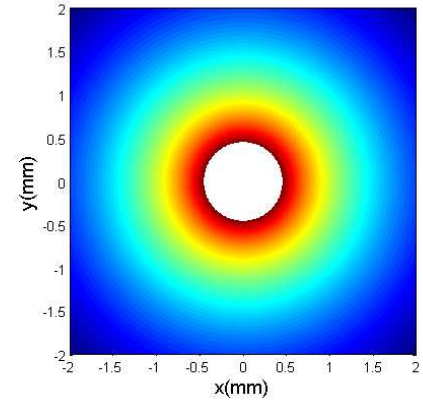

(b)

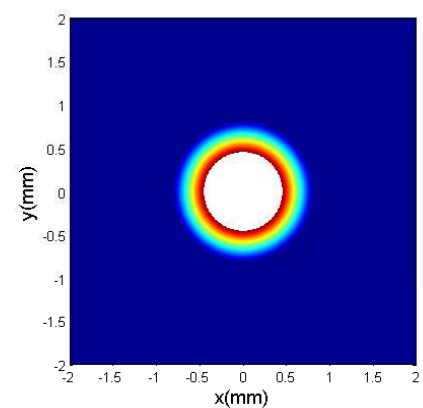

(d)

Figure 3.42: E-field magnitude pattern of the fundamental mode $T M_{0}$ in logarithmic scale with $15 \mathrm{~dB}$ range in an $a=450 \mu \mathrm{m} S W W$ a) Gold, $f=0.1 \mathrm{THz} ; b)$ Nickel, $f=0.1 \mathrm{THz}$; c) Gold, $f=10 \mathrm{THz}$; d) Nickel, $f=10 \mathrm{THz}$

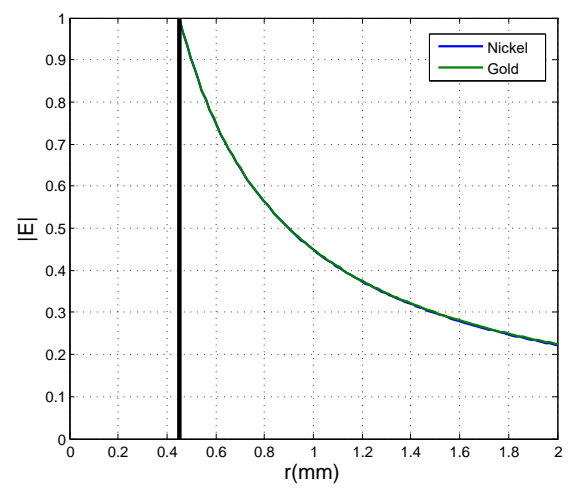

(a)

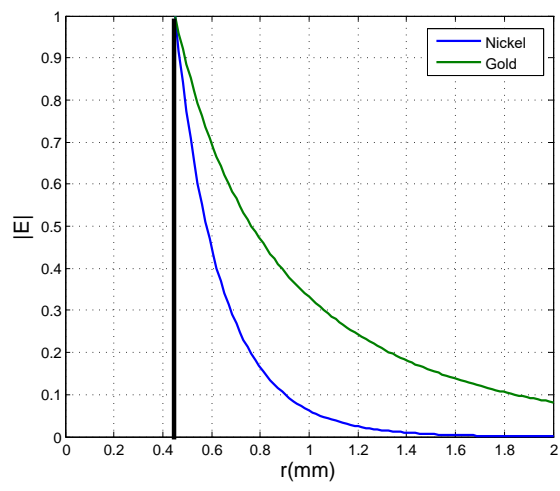

(b)

Figure 3.43: E-field magnitude pattern of the fundamental mode $T M_{0}$ in the radial coordinate $r$ in an $a=450 \mu \mathrm{m} S W W$ a) $f=0.1 \mathrm{THz}$; b) $f=10 \mathrm{THz}$. 


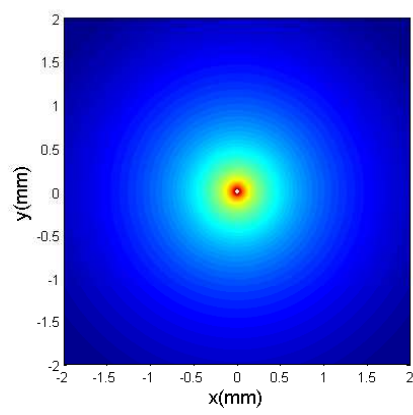

(a)

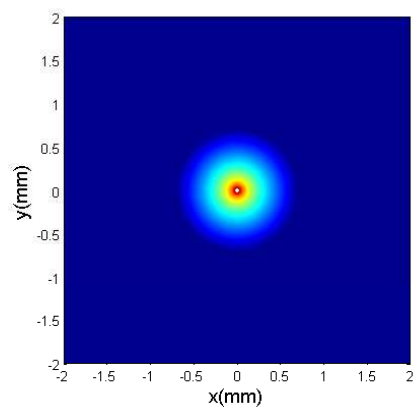

(c)

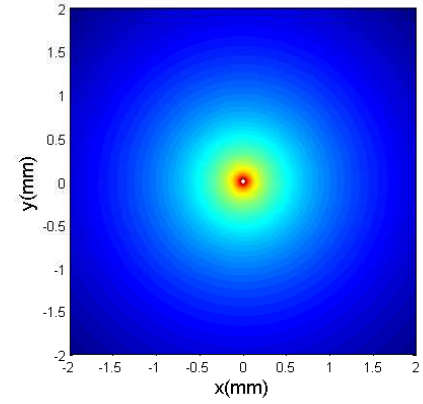

(b)

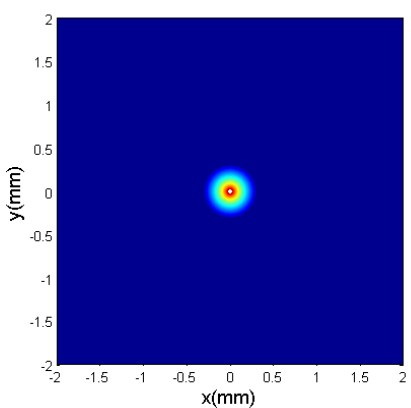

(d)

Figure 3.44: E-field magnitude pattern of the fundamental mode $T M_{0}$ in logarithmic scale with $45 \mathrm{~dB}$ range in an $a=450 \mu \mathrm{m} S W W$ a) Gold, $f=0.1 \mathrm{THz}$; b) Nickel, $f=0.1 \mathrm{THz}$; c) Gold, $f=10 \mathrm{THz}$; d) Nickel, $f=10 \mathrm{THz}$

radius imply a power distribution with a negligible inner area of null transmission. Again, if one or other is a more suitable choice ultimately depends on the required functionality demanded by the application.

Additionally, note how, for the small radius case $(a=20 \mu \mathrm{m})$, when looking at the considered operation area, the difference between gold and nickel wires is not as pronounced than in the $f=10 \mathrm{THz}$ case. Even more, note how the difference between $f=0.1 \mathrm{THz}$ and $f=10 \mathrm{THz}$ cases is quite smaller than for larger radius. This results quite evident to the eyesight by observing the E-field patter displayed as function of the radial distance in Figs. 3.45(a) and 3.45(b). For instance, in these graphs it may be intuitively postulated that an area extending only $r=0.5$ mm maybe enough to consider a good fraction of power for any frequency, with any metal in the whole $\mathrm{THz}$ band.

\subsubsection{Dispersion}

The dispersive properties of the SWW are finally quantified now. Although it seems clear from the analysis in section 3.5 that the small values of $\beta_{N N}$ imply almost zero dispersion (observe that a waveguide with zero dispersion shows $\beta_{N N}=0$ constant 


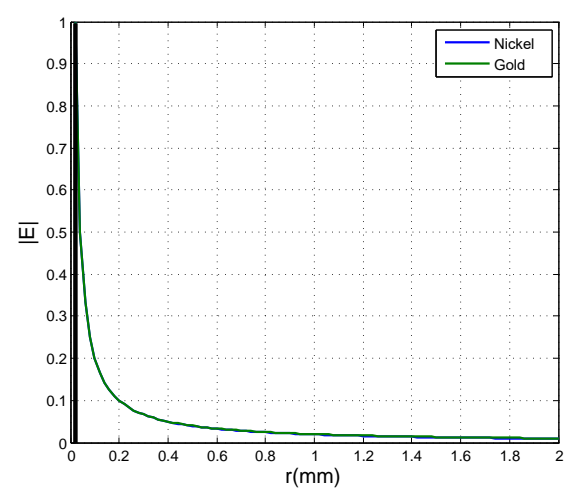

(a)

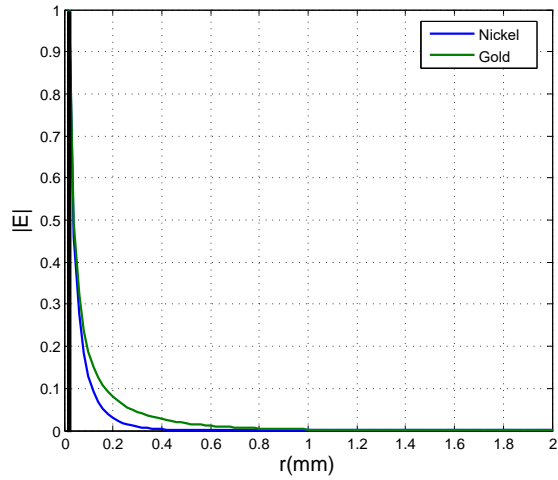

(b)

Figure 3.45: E-field magnitude pattern of the fundamental mode $T M_{0}$ in the radial coordinate $r$ in an $a=450 \mu \mathrm{m} S W W$ a) $f=0.1 \mathrm{THz}$; b) $f=10 \mathrm{THz}$.

with the frequency), it becomes necessary to make evidence of this fact through practical values of SWW realizations. Therefore, in this dispersion analysis, the normalized group velocity $\left(v_{g} / c_{0}\right)$ in a gold and a nickel SWW are characterized in the $\mathrm{THz}$ for the several radius $a$ considered.

Results of this calculation are shown in Fig. 3.46. By observing the graphs, it results obvious that all the frequencies in the wide considered band travel practically with the same velocity, the light velocity in the external medium. In this way, as mentioned before, the fundamental mode resembles in both, field components and propagation constant, to a TEM mode. Although a detailed analysis gives that worse conductors are more dispersive (nickel presents a variation less than $0.07 \%$ front the variation of gold, less than $0.04 \%$ ), in practical terms the dispersion is negligible in both cases. In fact, dispersion is not a problem to consider in the SWW structure, being this a very strong point of it inasmuch as many $\mathrm{THz}$ applications demand from ultra-wide band transmission. Consider for instance spectroscopy in any of the areas described in the state of the art chapter.

Once the attenuation, power distribution and dispersion of the fundamental $\mathrm{TM}_{0}$ mode of the SWW have been well characterized in the $\mathrm{THz}$ band, the possible role of this waveguide is more clearly established. Notice that the whole $\mathrm{THz}$ band has been considered as the operation band and, despite of this fact, reasonable figures have been obtained for the considered parameters/characteristics. The structure shows almost zero dispersion regarding the propagation constant or group velocity. Moreover, frequency dependence of the attenuation and the power distribution are quite acceptable, with the additional advantage of that the possible negative effects of each one of these last compensate: higher frequencies suffer more propagation losses but are better confined, thus being better excited, or prevented from possible radiation (think in bends for example). 


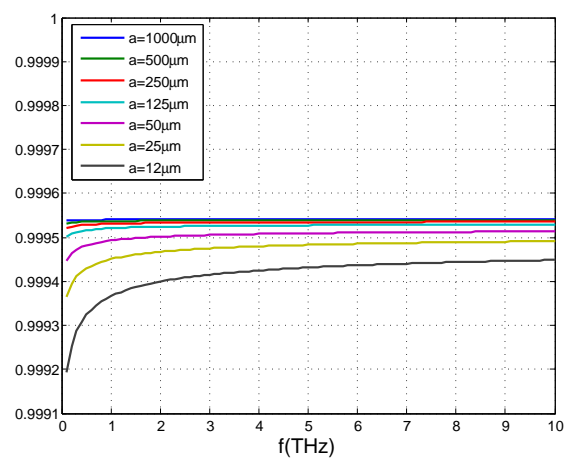

(a)

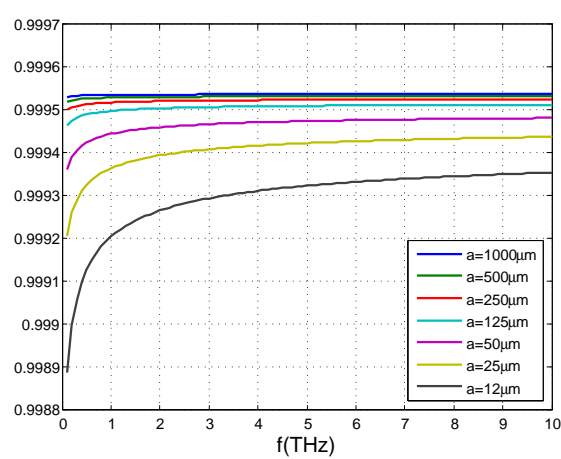

(b)

Figure 3.46: Normalized group velocity $v_{g} / c_{0}$ of the fundamental mode $T M_{0}$ vs. frequency in the $\mathrm{THz}$ range for several values of the radius a. a) Gold; b) Nickel.

\subsubsection{Conclusions and important remarks}

It has been shown that engineering the radius over common values ranging from $a=12 \mu \mathrm{m}$ to $a=1000 \mu \mathrm{m}$ gives lot of control on the attenuation and power distribution. Low losses are achievable, and very reasonable extensions as well for this open structure. All this argumentation provides firmly that the SWW is a very suitable structure for transmission of $\mathrm{THz}$ wide band pulses, which are the base for many spectroscopic based applications. Although structures with very low losses are found in the literature, they are very dispersive and narrow-band. It can be affirmed that the SWW is the most complete structure in terms of attenuation and dispersion.

However, the previous facts do not imply that the SWW is the panacea of $\mathrm{THz}$ waveguiding. Nevertheless, the open character of the structure, and the lack of bounds in the transmission region imply important practical drawbacks that can condition its use unless they are carefully treated. Excitation of the waveguide and reception of the incoming power is an easy one to be foreseen. This possible problem has been qualitatively considered in this section, and seems quite affordable by proper radius engineering and adequate transitions. For example, a small radius SWW implies a field pattern quite affordable to be matched with nowadays technology.

The case of bends may be less obvious. How a curvature affects transmitted waves that travel just attached to the surface, and quantifying the effect of the surface impenetrability on the radiation losses seems less direct. Furthermore, it seems intuitive to think that a sharp bend will cause noticeable losses even in the cases that the field collapse around the SWW since any bound or element is present to avoid it to escape from the confinement zone.

Although the SWW is not such a structure to design complex waveguiding path like those of microstrip circuits, for example, it is clear that guiding the energy along some curved paths is a feature that is mandatorily required for a waveguiding structure in most of applications. Therefore, this is a problem to be afforded. To 
use worse metals, to increase the surface roughness, or to perturb it via grooves or similar elements, makes the field to be more confined, collapsing near the SWW surface. However, still any element is present to prevent radiation of the escaping surface waves when the waveguide is perturbed by bending it. In this way, the use of a dielectric coating seems a more reasonable option being that the TIR effect will prevent radiation of such power traveling inside the dielectric medium. Furthermore, a dielectric offers functionality in a wider band than elements such as grooves, very electrical-size dependent.

The aforementioned solution is the so-called Dielectric-Coated Single Wire Waveguide (DCSWW) or Goubau line (GL), already reviewed in the state of the art chapter. It must be mentioned that, although the consideration of this structure may share some motivation with the original proposals of Goubau [490] and King [500], there are clear differences. In those works, the main problem was the field extension, totally unpractical for the very low, microwave frequencies considered. Since the possibility of manufacturing a very small radius was not considered, probably because it made no sense technologically, a dielectric coating was proposed as a good solution to provide guided power transmission bounded in a reasonable area.

Here, the main reason to consider the dielectric is the TIR effect introduced by it to prevent radiation. In addition, dielectric losses are much more relevant at $\mathrm{THz}$ frequencies than they are at microwave frequencies. Therefore, the confinement inside the dielectric will be paid with high dielectric absorption, something specially true for the very high frequencies of the $\mathrm{THz}$ band. Thus, proper optimized designs intended to maximize the advantages and minimize the possible negative effects of the dielectric coating become necessary. This justifies a detailed analysis of the DCSWW structure, the last base block for the designs lately proposed in this thesis.

\subsection{Dielectric-Coated Single Wire Waveguide (DC- SWW)}

The structure treated in this section, the Dielectric-Coated Single Wire Waveguide (DCSWW), is shown in 3D view in Fig. 3.47(a). The problem description is presented in Fig. 3.47(b). Although, physically, three regions can be appreciated in such schematic (metal, dielectric and air $^{23}$ ), the mathematical problem is reduced to two regions (dielectric and air) in as much the metal is assumed to be a PEC, i.e., all fields vanish inside. The conductor radius is $a$, whereas the dielectric thickness is $t$. This means that the dielectric-air interface is located at $r=a+t$, or alternatively $r=b$, see Fig. 3.47(b). Any of these two expressions will be used indistinctly. Formally:

- Region 1: $a \leq r \leq b$

- Region 2: $r \geq b$

\footnotetext{
${ }^{23}$ The most general case consider for region 2 any dielectric, different from that of region 1 , thus not necessarily air $\left(\varepsilon_{r_{2}}=1\right)$, and the general formulation allowing this case will be presented in this section. However, in all the posterior designs, and usually in the works presented in the literature, region 2 is conformed by air.
} 
The region 1 is formed by a finite volume of dielectric with relative permittivity $\varepsilon_{r_{1}}$, which is considered lossless in a first stage (lossess will be considered later through the perturbative method). In this medium, the wavenumber is $k_{1}=k_{0} \sqrt{\varepsilon_{r_{1}}}$. The region 2 is formed by an infinite volume of dielectric with relative permittivity $\varepsilon_{r_{2}}$ in the general case $\left(\varepsilon_{r_{2}}=1\right.$ along this thesis). Therefore, $k_{2}=k_{0} \sqrt{\varepsilon_{r_{2}}}$, although it must be kept in mind that, unless it is specified, $k_{2}=k_{0}$. Note by this definition that the problem presents the same aspect than the dielectric cylinder problem of section 3.3, with the only difference of having an empty region which is not longer part of the problem. Therefore, procedures, conclusions and phenomena of the dielectric cylinder with real $\varepsilon_{r}$ analysis must be kept in mind in this section, as they will make the new appearing concepts and results to be more easily understood.

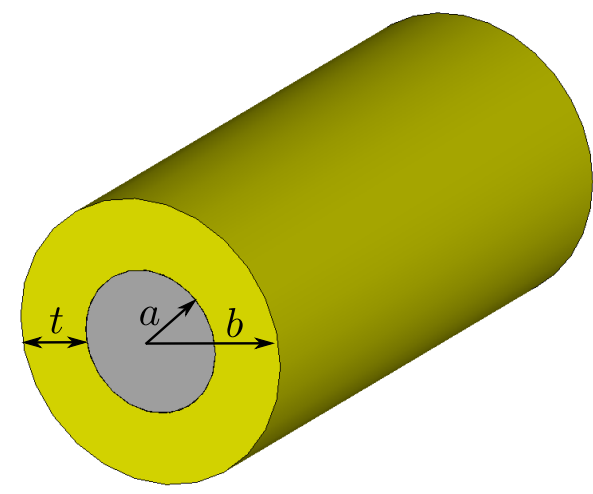

(a)

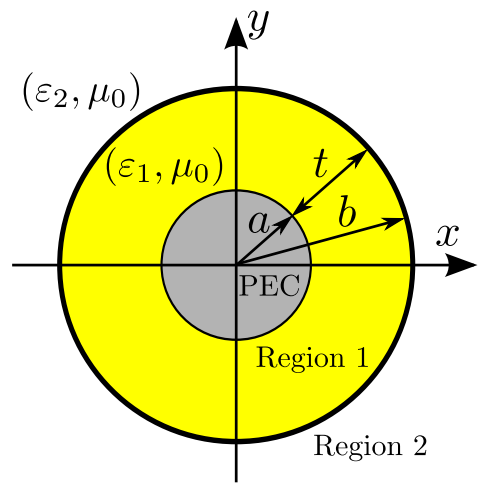

(b)

Figure 3.47: Dielectric-Coated Single Wire Waveguide (DCSWW). a) $3 D$ view; b) Problem description.

\subsubsection{Justification of the waveguide model}

At this point one question must be treated. In section 3.2 it was shown that metals at $\mathrm{THz}$ show a behavior that is better predicted by the Drude model than the simple skin-effect model. Therefore, it can be understood that going further in the simplification of the problem and assuming a metal to be a perfect conductor may be rather presumptuous. In fact, in the case of the SWW it has been observed a strong influence of metal properties on the modal spectrum and propagation characteristics of the fundamental mode. Actually, if the metal is assumed to be perfect conductor, the problem cannot be solved as it becomes mathematically singular [500] (from a physical point of view, note that the wire surface would be totally impenetrable, and any wave could be guided attached to the surface).

There are, however, many reasons that make licit the decision of assuming the metal as a perfect conductor in the case of the DCSWW. The first and most important reason is that accuracy is guaranteed. Note that in the case of a SWW the guidance phenomena is totally described by the contrast between the metallic medium and the external air medium, together with the SWW size. As it has been check, changes in the metallic material cause changes in the propagation features. 
However, when the dielectric is added, the dielectric region has itself waveguiding capacity, and the characteristics of the metal become an influence of second order, lawfully to be neglected with enough accuracy for the final proposal of the analysis.

Of course, ohmic losses will depend on the metal conductivity, however, the field pattern and the propagation constant not as much. Note that in the DCSWW the field will drastically collapse due to the presence of the dielectric, which is a perturbation of the surface stronger than the finite conductivity of the metallic medium. Additionally, note that DCSWW is rather classifiable as a conventional dielectric waveguide, thus presenting $k_{2} \leq \beta \leq k_{1}$, the particular value of $\beta$ in this range determined by the electrical size of the dielectric medium. A support for these facts is found in [522], where losses of the fundamental mode of the DCSWW are calculated through the perturbative method after the fields being calculated assuming the metallic wire as a PEC. Furthermore, differences between the Drude Model and the skin-depth effect model are found to be small for the DCSWW in that work.

Justified that the accuracy is good enough, the second reason is the avoided complexity. The problem, as displayed now, is a conventional real $\varepsilon_{r}$ dielectric problem with some additional geometrical complexity (observe that compared to the dielectric cylinder, in the DCSWW a wider variety of geometries can be obtained in as much two parameters, $a$ and $b$, define the structure) and some physical complexity (the presence of the metal influence the field in such way that special features not common in pure dielectric waveguides are found in the DCSWW). If the metal was regarded as a complex dielectric medium, not only the aforementioned additional complexity will be added, but even more: the problem would become one of complex variable over a three region waveguide. Note that this implies 2 interfaces where the boundary conditions must be satisfy, what produces 8 equations. The determinant of such system is not, for sure, an easy function to deal with. Since the previous sections have shown how complex can become a modal spectrum when complex $\varepsilon_{r}$ is involved, it is easy to figure out the required detail in the analysis of a DCSWW under these conditions, specially if one takes into account the larger casuistic to be considered, provided by the larger number of involved parameters: $\left(\varepsilon_{r m}, \varepsilon_{r 1}, a, b\right)$ front $\left(\varepsilon_{r m}, a\right)$. The possible additional insight of this approach does not justify such effort in the scope of this thesis, although it is left as an interesting future line.

Related to the last affirmations, comes the third reason. In this thesis, the analysis of the DCSWW is not an objective itself as it is the SWW analysis due to its major relevance as a $\mathrm{THz}$ waveguiding structure, but the DCSWW is considered as an alternative possibility under some situations, being the objective in these cases to find solution to some inherent problems of the SWW. As the results in the next chapter confirm, the analysis of the simple PEC wire DCSWW that will be provided next, is enough detailed and rigorous for the involved objectives. Nevertheless, to the author knowledge, a similar modal analysis with the same level of detail is not found in the literature, even for this simplified DCSWW case.

\subsubsection{Modal analysis equations}

Afforded the question of the lawfully of considering the metal as a PEC, the analysis of the problem described in Fig. 3.47(b) is treated now. To keep the section formu- 
lation self-contained, the real variable transversal propagation constants describing the fields variation are recovered again:

$$
\begin{aligned}
& \beta_{r_{1}}^{2}=k_{1}^{2}-\beta^{2} \\
& \alpha_{r_{2}}^{2}=\beta^{2}-k_{2}^{2}
\end{aligned}
$$

As usual in cylindrical problems involving more than one region and the boundary condition of continuity, in the general case, the all six components of the field will be required to satisfy those boundary conditions, being the modes of hybrid nature. By applying the expressions (3.103)-(3.104), eigenfunctions solving the involved differential equations are found, describing the axial components $E_{z}$ and $H_{z}$, and by applying (3.105)-(3.108), the rest of the field components are obtained. This process leads to:

\section{$\mathbf{H E}_{n m}$ and $\mathbf{E H}_{n m}$ modes:}

Region $1, a \leq r \leq b$ :

$$
\begin{aligned}
& E_{z_{1}}(r, \phi)=\left[A_{1} \mathrm{~J}_{n}\left(\beta_{r_{1}} r\right)+A_{2} \mathrm{Y}_{n}\left(\beta_{r_{1}} r\right)\right] e^{j n \phi} \\
& H_{z_{1}}(r, \phi)=\left[B_{1} \mathrm{~J}_{n}\left(\beta_{r_{1}} r\right)+B_{2} \mathrm{Y}_{n}\left(\beta_{r_{1}} r\right)\right] e^{j n \phi} \\
& E_{r_{1}}(r, \phi)=\frac{-j \beta}{\beta_{r_{1}}^{2}}\left[\beta_{r_{1}}\left(A_{1} \mathrm{~J}_{n}^{\prime}\left(\beta_{r_{1}} r\right)+A_{2} \mathrm{Y}_{n}^{\prime}\left(\beta_{r_{1}} r\right)\right)\right. \\
& \left.+\frac{j \omega \mu_{0} n}{\beta r}\left(B_{1} \mathrm{~J}_{n}\left(\beta_{r_{1}} r\right)+B_{2} \mathrm{Y}_{n}\left(\beta_{r_{1}} r\right)\right)\right] e^{j n \phi} \\
& E_{\phi_{1}}(r, \phi)=\frac{-j \beta}{\beta_{r_{1}}^{2}}\left[\frac{j n}{r}\left(A_{1} \mathrm{~J}_{n}\left(\beta_{r_{1}} r\right)+A_{2} \mathrm{Y}_{n}\left(\beta_{r_{1}} r\right)\right)\right. \\
& \left.-\frac{\omega \mu_{0}}{\beta}\left(B_{1} \mathrm{~J}_{n}^{\prime}\left(\beta_{r_{1}} r\right)+B_{2} \mathrm{Y}_{n}^{\prime}\left(\beta_{r_{1}} r\right)\right)\right] e^{j n \phi} \\
& H_{r_{1}}(r, \phi)=\frac{-j \beta}{\beta_{r_{1}}^{2}}\left[\beta_{r_{1}}\left(B_{1} \mathrm{~J}_{n}^{\prime}\left(\beta_{r_{1}} r\right)+B_{2} \mathrm{Y}_{n}^{\prime}\left(\beta_{r_{1}} r\right)\right)\right. \\
& \left.-\frac{j \omega \varepsilon_{1} n}{\beta r}\left(A_{1} \mathrm{~J}_{n}\left(\beta_{r_{1}} r\right)+A_{2} \mathrm{Y}_{n}\left(\beta_{r_{1}} r\right)\right)\right] e^{j n \phi} \\
& H_{\phi_{1}}(r, \phi)=\frac{-j \beta}{\beta_{r_{1}}^{2}}\left[\frac{j n}{r}\left(B_{1} \mathrm{~J}_{n}\left(\beta_{r_{1}} r\right)+B_{2} \mathrm{Y}_{n}\left(\beta_{r_{1}} r\right)\right)\right. \\
& \left.+\frac{\omega \varepsilon_{1} n}{\beta}\left(A_{1} \mathrm{~J}_{n}^{\prime}\left(\beta_{r_{1}} r\right)+A_{2} \mathrm{Y}_{n}^{\prime}\left(\beta_{r_{1}} r\right)\right)\right] e^{j n \phi}
\end{aligned}
$$


Region $2, r \geq b$ :

$$
\begin{aligned}
E_{z_{2}} & =C \mathrm{~K}_{n}\left(\alpha_{r_{2}} r\right) e^{j n \phi} \\
H z_{2} & =D \mathrm{~K}_{n}\left(\alpha_{r_{2}} r\right) e^{j n \phi} \\
E_{r_{2}} & =\frac{j \beta}{\alpha_{r_{2}}^{2}}\left[\alpha_{r_{2}} C \mathrm{~K}_{n}^{\prime}\left(\alpha_{r_{2}} r\right)+\frac{j \omega \mu_{0} n}{\beta r} D \mathrm{~K}_{n}\left(\alpha_{r_{2}} r\right)\right] e^{j n \phi} \\
E_{\phi_{2}} & =\frac{j \beta}{\alpha_{r_{2}}^{2}}\left[\frac{j n}{r} C \mathrm{~K}_{n}\left(\alpha_{r_{2}} r\right)-\frac{\omega \mu_{0}}{\beta} D \mathrm{~K}_{n}^{\prime}\left(\alpha_{r_{2}} r\right)\right] e^{j n \phi} \\
H_{r_{2}} & =\frac{j \beta}{\alpha_{r_{2}}^{2}}\left[\alpha_{r_{2}} D \mathrm{~K}_{n}^{\prime}\left(\alpha_{r_{2}} r\right)-\frac{j \omega \varepsilon_{2} n}{\beta r} C \mathrm{~K}_{n}\left(\alpha_{r_{2}} r\right)\right] e^{j n \phi} \\
H_{\phi_{2}} & =\frac{j \beta}{\alpha_{r_{2}}^{2}}\left[\frac{j n}{r} D \mathrm{~K}_{n}\left(\alpha_{r_{2}} r\right)+\frac{\omega \varepsilon_{2}}{\beta} C \mathrm{~K}_{n}^{\prime}\left(\alpha_{r_{2}} r\right)\right] e^{j n \phi}
\end{aligned}
$$

where the notation is the same followed along this chapter, and the only novelty in the previous expressions is the appearance of the Bessel function of second kind (sometimes called Neumann or Weber function) $\mathrm{Y}_{n}(x)$, and its derivative $\mathrm{Y}_{n}^{\prime}(x)$. Note that the origin (where $\mathrm{Y}_{n}(x)$ is singular) is not part of the problem under analysis and this function must be considered for the region 1 . The similarity with the dielectric cylinder problem expressions is obvious. For the region 2 there is not any change, whereas in the region 1 , where it appeared $A \mathrm{~J}_{n}\left(\beta_{r_{1}} r\right)$, now appears $\left[A_{1} \mathrm{~J}_{n}\left(\beta_{r_{1}} r\right)+A_{2} \mathrm{Y}_{n}\left(\beta_{r_{1}} r\right)\right]$ (and the same can be affirmed for the terms involving $B$ and/or the derivative of Bessel function of first kind $\left.\mathrm{J}_{n}^{\prime}\left(\beta_{r_{1}} r\right)\right)$. Now, the boundary condition equations are six, two caused by the PEC boundary condition at $r=a$ (nullity of tangential electric fields), and four caused by the continuity boundary condition at the interface between dielectric media located at $r=b$ (continuity of all tangential fields):

1. Tangential electric fields must vanish on the PEC surface:

$$
\begin{aligned}
& E_{z_{1}}(a, \phi)=0 \\
& E_{\phi_{1}}(a, \phi)=0
\end{aligned}
$$

2. Tangential electric and magnetic fields must be continuous along an interface between the two dielectric media:

$$
\begin{aligned}
E_{z_{1}}(b, \phi) & =E_{z_{2}}(b, \phi) \\
E_{\phi_{1}}(b, \phi) & =E_{\phi_{2}}(b, \phi) \\
H_{z_{1}}(b, \phi) & =H z_{2}(b, \phi) \\
H_{\phi_{1}}(b, \phi) & =H_{\phi_{2}}(b, \phi)
\end{aligned}
$$


In principle, these equations dispose the necessary system to determine the eigenvalues $\beta$ and the six unknowns of the problem $\left(A_{1}, A_{2}, B_{1}, B_{2}, C, D\right)$ by the usual procedure. However, since the equations associated to the PEC condition at $r=a$ are simple, it is possible to obtain some unknowns as a function of the others. First, from (3.282) and (3.295) it is found that

$$
\left[A_{1} \mathrm{~J}_{n}\left(\beta_{r_{1}} r\right)+A_{2} \mathrm{Y}_{n}\left(\beta_{r_{1}} r\right)\right] e^{j n \phi}=0
$$

what implies

$$
A_{2}=-A_{1} \frac{\mathrm{J}_{n}\left(\beta_{r_{1}} a\right.}{\mathrm{Y}_{n}\left(\beta_{r_{1}} a\right)}
$$

Now, taking into account (3.301), from (3.285) and (3.296), it is trivially obtained that

$$
B_{2}=-B_{1} \frac{\mathrm{J}_{n}^{\prime}\left(\beta_{r_{1}} a\right.}{\mathrm{Y}_{n}^{\prime}\left(\beta_{r_{1}} a\right)}
$$

With these algebraic manipulations, the unknowns of the problem are now four $\left(A_{1}, B_{1}, C, D\right)$. Under these conditions, the second set of boundary conditions (3.297)-(3.300) are applied over the tangential fields giving

$$
\begin{aligned}
& A_{1}\left[\mathrm{~J}_{n}\left(\beta_{r_{1}} b\right)-\frac{\mathrm{J}_{n}\left(\beta_{r_{1}} a\right)}{\mathrm{Y}_{n}\left(\beta_{r_{1}} a\right)} \mathrm{Y}_{n}\left(\beta_{r_{1}} b\right)\right]-C \mathrm{~K}_{n}\left(\alpha_{r_{2}} b\right)=0 \\
& B_{1}\left[\mathrm{~J}_{n}\left(\beta_{r_{1}} b\right)-\frac{\mathrm{J}_{n}^{\prime}\left(\beta_{r_{1}} a\right)}{\mathrm{Y}_{n}^{\prime}\left(\beta_{r_{1}} a\right)} \mathrm{Y}_{n}\left(\beta_{r_{1}} b\right)\right]-D \mathrm{~K}_{n}\left(\alpha_{r_{2}} b\right)=0 \\
& A_{1} \frac{\beta n}{\beta_{r_{1}}^{2} b}\left[\mathrm{~J}_{n}\left(\beta_{r_{1}} b\right)-\frac{\mathrm{J}_{n}\left(\beta_{r_{1}} a\right)}{\mathrm{Y}_{n}\left(\beta_{r_{1}} a\right)} \mathrm{Y}_{n}\left(\beta_{r_{1}} b\right)\right]+ \\
& +B_{1} \frac{j \omega \mu_{0}}{\beta_{r_{1}}}\left[\mathrm{~J}_{n}^{\prime}\left(\beta_{r_{1}} b\right)-\frac{\mathrm{J}_{n}^{\prime}\left(\beta_{r_{1}} a\right)}{\mathrm{Y}_{n}^{\prime}\left(\beta_{r_{1}} a\right)} \mathrm{Y}_{n}^{\prime}\left(\beta_{r_{1}} b\right)\right]+ \\
& +C \frac{\beta n}{\alpha_{r_{2}}^{2} b} \mathrm{~K}_{n}\left(\alpha_{r_{2}} b\right)+D \frac{j \omega \mu_{0}}{\alpha_{r_{2}}} \mathrm{~K}_{n}^{\prime}\left(\alpha_{r_{2}} b\right)=0 \\
& A_{1} \frac{\left(-j \omega \varepsilon_{1}\right)}{\beta_{r_{1}}}\left[\mathrm{~J}_{n}^{\prime}\left(\beta_{r_{1}} b\right)-\frac{\mathrm{J}_{n}\left(\beta_{r_{1}} a\right)}{\mathrm{Y}_{n}\left(\beta_{r_{1}} a\right)} \mathrm{Y}_{n}^{\prime}\left(\beta_{r_{1}} b\right)\right]+ \\
& +B_{1} \frac{\beta n}{\beta_{r_{1}}^{2} b}\left[\mathrm{~J}_{n}\left(\beta_{r_{1}} b\right)-\frac{\mathrm{J}_{n}^{\prime}\left(\beta_{r_{1}} a\right)}{\mathrm{Y}_{n}^{\prime}\left(\beta_{r_{1}} a\right)} \mathrm{Y}_{n}\left(\beta_{r_{1}} b\right)\right]+ \\
& +C \frac{j \omega \varepsilon_{2}}{\alpha_{r_{2}} b} \mathrm{~K}_{n}^{\prime}\left(\alpha_{r_{2}} b\right)+D \frac{\beta n}{\alpha_{r_{2}}^{2} b} \mathrm{~K}_{n}\left(\alpha_{r_{2}} b\right)=0
\end{aligned}
$$

which describe the final linear system of the problem under study. As usual, the system is expressed in matrix form giving 


$$
\left[\begin{array}{cccc}
m_{11} & 0 & m_{13} & 0 \\
0 & m_{22} & 0 & m_{24} \\
m_{31} & m_{32} & m_{33} & m_{34} \\
m_{41} & m_{42} & m_{43} & m_{44}
\end{array}\right]\left[\begin{array}{l}
A_{1} \\
B_{1} \\
C \\
D
\end{array}\right]=\left[\begin{array}{l}
0 \\
0 \\
0 \\
0
\end{array}\right]
$$

where

$$
\begin{aligned}
& m_{11}=\mathrm{J}_{n}\left(\beta_{r_{1}} b\right)-\frac{\mathrm{J}_{n}\left(\beta_{r_{1}} a\right)}{\mathrm{Y}_{n}\left(\beta_{r_{1}} a\right)} \mathrm{Y}_{n}\left(\beta_{r_{1}} b\right) \\
& m_{13}=-\mathrm{K}_{n}\left(\alpha_{r_{2}} b\right) \\
& m_{22}=\mathrm{J}_{n}\left(\beta_{r_{1}} b\right)-\frac{\mathrm{J}_{n}^{\prime}\left(\beta_{r_{1}} a\right)}{\mathrm{Y}_{n}^{\prime}\left(\beta_{r_{1}} a\right)} \mathrm{Y}_{n}\left(\beta_{r_{1}} b\right) \\
& m_{24}=-\mathrm{K}_{n}\left(\alpha_{r_{2}} b\right) \\
& m_{31}=\frac{\beta n}{\beta_{r_{1}}^{2} b}\left[\mathrm{~J}_{n}\left(\beta_{r_{1}} b\right)-\frac{\mathrm{J}_{n}\left(\beta_{r_{1}} a\right)}{\mathrm{Y}_{n}\left(\beta_{r_{1}} a\right)} \mathrm{Y}_{n}\left(\beta_{r_{1}} b\right)\right] \\
& m_{32}=\frac{j \omega \mu_{0}}{\beta_{r_{1}}}\left[\mathrm{~J}_{n}^{\prime}\left(\beta_{r_{1}} b\right)-\frac{\mathrm{J}_{n}^{\prime}\left(\beta_{r_{1}} a\right)}{\mathrm{Y}_{n}^{\prime}\left(\beta_{r_{1}} a\right)} \mathrm{Y}_{n}^{\prime}\left(\beta_{r_{1}} b\right)\right] \\
& m_{33}=\frac{\beta n}{\alpha_{r_{2}}^{2} b} \mathrm{~K}_{n}\left(\alpha_{r_{2}} b\right) \\
& m_{34}=\frac{j \omega \mu_{0}}{\alpha_{r_{2}}} \mathrm{~K}_{n}^{\prime}\left(\alpha_{r_{2}} b\right) \\
& m_{41}=\frac{-j \omega \varepsilon_{1}}{\beta_{r_{1}}}\left[\mathrm{~J}_{n}^{\prime}\left(\beta_{r_{1}} b\right)-\frac{\mathrm{J}_{n}\left(\beta_{r_{1}} a\right)}{\mathrm{Y}_{n}\left(\beta_{r_{1}} a\right)} \mathrm{Y}_{n}^{\prime}\left(\beta_{r_{1}} b\right)\right] \\
& m_{42}=\frac{\beta n}{\beta_{r_{1}}^{2} b}\left[\mathrm{~J}_{n}\left(\beta_{r_{1}} b\right)-\frac{\mathrm{J}_{n}^{\prime}\left(\beta_{r_{1}} a\right)}{\mathrm{Y}_{n}^{\prime}\left(\beta_{r_{1}} a\right)} \mathrm{Y}_{n}\left(\beta_{r_{1}} b\right)\right] \\
& m_{43}=-\frac{j \omega \varepsilon_{2}}{\alpha_{r_{2}} b} \mathrm{~K}_{n}^{\prime}\left(\alpha_{r_{2}} b\right) \\
& m_{44}=\frac{\beta n}{\alpha_{r_{2}}^{2} b} \mathrm{~K}_{n}\left(\alpha_{r_{2}} b\right)
\end{aligned}
$$

The determinant of (3.308) provides the transcendental equation necessary to determine the propagation constant $\beta$ of the modes of the DCSWW. Since the expressions may be cumbersome to deal with, the following definitions are proposed: 


$$
\begin{aligned}
F & =\mathrm{J}_{n}\left(\beta_{r_{1}} b\right)-\frac{\mathrm{J}_{n}\left(\beta_{r_{1}} a\right)}{\mathrm{Y}_{n}\left(\beta_{r_{1}} a\right)} \mathrm{Y}_{n}\left(\beta_{r_{1}} b\right) \\
G & =\mathrm{J}_{n}\left(\beta_{r_{1}} b\right)-\frac{\mathrm{J}_{n}^{\prime}\left(\beta_{r_{1}} a\right)}{\mathrm{Y}_{n}^{\prime}\left(\beta_{r_{1}} a\right)} \mathrm{Y}_{n}\left(\beta_{r_{1}} b\right) \\
M & =\mathrm{J}_{n}^{\prime}\left(\beta_{r_{1}} b\right)-\frac{\mathrm{J}_{n}\left(\beta_{r_{1}} a\right)}{\mathrm{Y}_{n}\left(\beta_{r_{1}} a\right)} \mathrm{Y}_{n}^{\prime}\left(\beta_{r_{1}} b\right) \\
N & =\mathrm{J}_{n}^{\prime}\left(\beta_{r_{1}} b\right)-\frac{\mathrm{J}_{n}^{\prime}\left(\beta_{r_{1}} a\right)}{\mathrm{Y}_{n}^{\prime}\left(\beta_{r_{1}} a\right)} \mathrm{Y}_{n}^{\prime}\left(\beta_{r_{1}} b\right)
\end{aligned}
$$

By using the above expressions, the matrix $\overline{\bar{M}}$ of the described system adopts a more compact expression:

$$
\overline{\bar{M}}=\left[\begin{array}{cccc}
F & 0 & -\mathrm{K}_{n}\left(\alpha_{r_{2}} b\right) & 0 \\
0 & G & 0 & -\mathrm{K}_{n}\left(\alpha_{r_{2}} b\right) \\
\frac{\beta n}{\beta_{r_{1}}^{2} b} F & \frac{j \omega \mu_{0}}{\beta_{r_{1}}} N & \frac{\beta n}{\alpha_{r_{2}}^{2} b} \mathrm{~K}_{n}\left(\alpha_{r_{2}} b\right) & \frac{j \omega \mu_{0}}{\alpha_{r_{2}}} \mathrm{~K}_{n}^{\prime}\left(\alpha_{r_{2}} b\right) \\
\frac{-j \omega \varepsilon_{1}}{\beta_{r_{1}}} M & \frac{\beta n}{\beta_{r_{1}}^{2} b} G & -\frac{j \omega \varepsilon_{2}}{\alpha_{r_{2}} b} \mathrm{~K}_{n}^{\prime}\left(\alpha_{r_{2}} b\right) & \frac{\beta n}{\alpha_{r_{2}}^{2} b} \mathrm{~K}_{n}\left(\alpha_{r_{2}} b\right)
\end{array}\right]
$$

The determinant of this matrix, expanded by columns is:

$$
\begin{aligned}
&|\overline{\bar{M}}|= F\left\{G\left[\left(\frac{\beta n}{\alpha_{r_{2}}^{2} b}\right)^{2}\left(\mathrm{~K}_{n}\left(\alpha_{r_{2}} b\right)\right)^{2}-\frac{w^{2} \mu_{0} \varepsilon_{2}}{\alpha_{r_{2}}^{2}}\left(\mathrm{~K}_{n}^{\prime}\left(\alpha_{r_{2}} b\right)\right)^{2}\right]\right. \\
&\left.-\mathrm{K}_{n}\left(\alpha_{r_{2}} b\right)\left[\frac{\omega^{2} \mu_{0} \varepsilon_{2}}{\beta_{r_{1}} \alpha_{r_{2}}} N \mathrm{~K}_{n}^{\prime}\left(\alpha_{r_{2}} b\right)-\left(\frac{\beta n}{\beta_{r_{1}} \alpha_{r_{2}} b}\right)^{2} G \mathrm{~K}_{n}\left(\alpha_{r_{2}} b\right)\right]\right\} \\
&- \mathrm{K}_{n}\left(\alpha_{r_{2}} b\right)\left\{-G\left[\left(\frac{\beta n}{\beta_{r_{1}} \alpha_{r_{2}} b}\right)^{2} F \mathrm{~K}_{n}\left(\alpha_{r_{2}} b\right)-\frac{w^{2} \mu_{0} \varepsilon_{1}}{\beta_{r_{1}} \alpha_{r_{2}}} M \mathrm{~K}_{n}^{\prime}\left(\alpha_{r_{2}} b\right)\right]\right. \\
&\left.-\mathrm{K}_{n}\left(\alpha_{r_{2}} b\right)\left[\left(\frac{\beta n}{\beta_{r_{1}}^{2} b}\right)^{2} F G-\frac{w^{2} \mu_{0} \varepsilon_{1}}{\beta_{r_{1}}^{2}} M N\right]\right\}
\end{aligned}
$$

Equating this expression to zero and doing some algebraic manipulations, following the same criteria to order the terms as with the dielectric cylindrical waveguide:

$$
\begin{aligned}
\frac{\varepsilon_{r_{1}}}{\left(\beta_{r_{1}} b\right)^{2}} \cdot \frac{M N}{F G}+\frac{\varepsilon_{r_{2}}}{\left(\alpha_{r_{2}} b\right)^{2}} \cdot \frac{\mathrm{K}_{n}^{\prime 2}\left(\alpha_{r_{2}} b\right)}{\mathrm{K}_{n}^{2}\left(\alpha_{r_{2}} b\right)}+\frac{\varepsilon_{r_{1}}}{\beta_{r_{1}} \alpha_{r_{2}} b^{2}} \cdot \frac{M \mathrm{~K}_{n}^{\prime}\left(\alpha_{r_{2}} b\right)}{F \mathrm{~K}_{n}\left(\alpha_{r_{2}} b\right)} \\
+\frac{\varepsilon_{r_{2}}}{\beta_{r_{1}} \alpha_{r_{2}} b^{2}} \cdot \frac{N \mathrm{~K}_{n}^{\prime}\left(\alpha_{r_{2}} b\right)}{G \mathrm{~K}_{n}\left(\alpha_{r_{2}} b\right)}-n^{2}\left(\frac{\beta}{k_{0}}\right)^{2} \cdot\left(\frac{1}{\left(\beta_{r_{1}} b\right)^{2}}+\frac{1}{\left(\alpha_{r_{2}} b\right)^{2}}\right)^{2}=0
\end{aligned}
$$


The previous procedure results interesting in as much it gives good parallelism between the DCSWW and the basic dielectric cylindrical waveguide, a tool that can be used to gain physical insight through the mathematical expressions. Observe that, if the second term in each equation of (3.321)-(3.324) is not considered, (3.325) and (3.327) become the matrix of the system provided by the boundary conditions and the transcendental equation of the dielectric cylinder problem.

Once (3.327) is solved and the corresponding eigenvalue $\beta$ is determined, as usual, $A_{1}$ is left as a parameter of the previous non full-rank system, and the ratios $B_{1} / A_{1}, C / A_{1}$ and $D / A$ can be solved to determine the fields, or eigenfunctions, of the problem. The expressions for these ratios are:

$$
\begin{aligned}
\frac{B_{1}}{A_{1}} & =\frac{j \beta n}{\omega \mu_{0}} \frac{F}{G}\left[\frac{1}{\left(\beta_{r_{1}} b\right)^{2}}+\frac{1}{\left(\alpha_{r_{2}} b\right)^{2}}\right]\left[\frac{N}{\left(\beta_{r_{1}} b\right) G}+\frac{\mathrm{K}_{n}^{\prime}\left(\alpha_{r_{2}} b\right)}{\left(\alpha_{r_{2}} b\right) \mathrm{K}_{n}\left(\alpha_{r_{2}} b\right)}\right]^{-1} \\
\frac{C}{A} & =\frac{F}{\mathrm{~K}_{n}\left(\alpha_{r_{2}} b\right)} \\
\frac{D}{A} & =\frac{G}{\mathrm{~K}_{n}\left(\alpha_{r_{2}} b\right)} \frac{B_{1}}{A_{1}}
\end{aligned}
$$

Note that together with these expressions, the other to unknowns, $A_{2}$ and $B_{2}$ must be recovered with (3.302) and (3.303). At this point, it would be desirable to split (3.327) in two equations describing $\mathrm{HE}_{n m}$ and $\mathrm{EH}_{n m}$ modes separately, which will be shown to have very different properties. Unfortunately, the presence of the PEC boundary condition breaks the symmetry present in the equations of the region 1, and the only that can be said no about (3.327) is that is quadratic in $\frac{\mathrm{K}_{n}^{\prime}\left(\alpha_{r_{2}} b\right)}{\left(\alpha_{r_{2}} b\right) \mathrm{K}_{n}^{\prime}\left(\alpha_{r_{2}} b\right)}$. Unfortunately, developing (3.327) in this way does not split the solutions according to their dominant axial component, as it occurs for simpler problem of a dielectric cylinder.

In this case, several alternative options are available for such suitable classification. Note that $n$ is fixed for solving (3.327), and several solutions with different $m$ appear (let still keep $m$ as usual, indicating the number of radial oscillations), the number of them depending on the dielectric thickness $t$ in as much this parameter determines how many transversal oscillations are possible ${ }^{24}$. Since it is obvious that modes with larger number $m$ will require larger thickness $t$ to appear, the modes will appear sorted as pairs with same $m$, and the only issue to classify them will be discover which is the $\mathrm{HE}_{n m}$ mode (or the $\mathrm{EH}_{n m}$ mode) of the couple. The $\mathrm{HE}_{n m}$ will be shown to be the first to appear always by their nature and the physic of the structure. Therefore, the classification is trivial. Additionally, inspection of their axial components through (3.328) when the modes are far from cutoff is also a very determinant tool (although less fast than previous, which is direct), since $\mathrm{HE}_{n m}$

\footnotetext{
${ }^{24}$ Notice that these modes are not related with the bulk modes propagating evanescent inside the metal, but are provided by the presence of the dielectric coating. In fact, the simplification of assuming PEC imply that bulk modes inside the conductor are totally missed. However, it is reasonable to think they are similar to those of the SWW.
} 
modes will be shown to be quasi-TM, whereas $\mathrm{EH}_{n m}$ modes will be shown to be quasi-TE.

Contrarily, it is still possible in the case $n=0$, as usual with cylindrical structures, to analyze separately $\mathrm{TM}_{0 m}$ and $\mathrm{TE}_{0 m}$ modes. Following the same steps as for hybrid modes:

\section{$\underline{\mathrm{TM}_{0 m} \text { modes: }}$}

Region $1, a \leq r \leq b$ :

$$
\begin{aligned}
E_{z_{1}} & =A_{1} \mathrm{~J}_{0}\left(\beta_{r_{1}} r\right)+A_{2} \mathrm{Y}_{0}\left(\beta_{r_{1}} r\right) \\
H_{z_{1}} & =0 \\
E_{r_{1}} & =\frac{j \beta}{\beta_{r_{1}}}\left[A_{1} \mathrm{~J}_{1}\left(\beta_{r_{1}} r\right)+A_{2} \mathrm{Y}_{1}\left(\beta_{r_{1}} r\right)\right] \\
E_{\phi_{1}} & =0 \\
H_{r_{1}} & =0 \\
H_{\phi_{1}} & =\frac{j \omega \varepsilon_{1}}{\beta_{r_{1}}}\left[A_{1} \mathrm{~J}_{1}\left(\beta_{r_{1}} r\right)+A_{2} \mathrm{Y}_{1}\left(\beta_{r_{1}} r\right)\right]
\end{aligned}
$$

$\underline{\text { Region } 2, r \geq b \text { : }}$

$$
\begin{aligned}
E_{z_{2}} & =C \mathrm{~K}_{0}\left(\alpha_{r_{2}} r\right) \\
H z_{2} & =0 \\
E_{r_{2}} & =-\frac{j \beta}{\alpha_{r_{2}}} C \mathrm{~K}_{1}\left(\alpha_{r_{2}} r\right) \\
E_{\phi_{1}} 2 & =0 \\
H_{r_{1}} 2 & =0 \\
H_{\phi_{2}} & =-\frac{j \omega \varepsilon_{2}}{\alpha_{r_{2}}} C \mathrm{~K}_{1}\left(\alpha_{r_{2}} r\right)
\end{aligned}
$$

For the above fields configuration, the boundary conditions become:

1. Tangential electric fields must vanish on the PEC surface:

$$
E_{z_{1}}(a, \phi)=0
$$

2. Tangential electric and magnetic fields must be continuous along an interface between the two dielectric media:

$$
\begin{aligned}
E_{z_{1}}(b, \phi) & =E_{z_{2}}(b, \phi) \\
H_{\phi_{1}}(b, \phi) & =H_{\phi_{2}}(b, \phi)
\end{aligned}
$$


Solving first (3.344) it is obtained

$$
A_{2}=-A_{1} \frac{\mathrm{J}_{0}\left(\beta_{r_{1}} a\right)}{\mathrm{Y}_{0}\left(\beta_{r_{1}} a\right)}
$$

Now, using this last expression together with (3.345) and (3.346) it is found that

$$
A_{1} \frac{1}{\mathrm{Y}_{0}\left(\beta_{r_{1}} a\right)}\left[\mathrm{J}_{0}\left(\beta_{r_{1}} b\right) \mathrm{Y}_{0}\left(\beta_{r_{1}} a\right)-\mathrm{J}_{0}\left(\beta_{r_{1}} a\right) \mathrm{Y}_{0}\left(\beta_{r_{1}} b\right)\right]-C \mathrm{~K}_{0}\left(\alpha_{r_{2}} b\right)=0
$$

and

$$
\begin{aligned}
A_{1} \frac{\varepsilon_{1}}{\beta_{r_{1}}} \frac{1}{\mathrm{Y}_{0}\left(\beta_{r_{1}} a\right)}\left[\mathrm{J}_{1}\left(\beta_{r_{1}} b\right) \mathrm{Y}_{0}\left(\beta_{r_{1}} a\right)-\mathrm{J}_{0}\left(\beta_{r_{1}} a\right) \mathrm{Y}_{1}\left(\beta_{r_{1}} b\right)\right] & \\
& +C \frac{\varepsilon_{2}}{\alpha_{r_{2}}} \mathrm{~K}_{1}\left(\alpha_{r_{2}} b\right)=0
\end{aligned}
$$

which are the two equations that describes the matrix system of the problem, which can be written in the usual way as

$$
\left[\begin{array}{cc}
\frac{1}{\mathrm{Y}_{0}\left(\beta_{r_{1}} a\right)}\left(\mathrm{J}_{0}\left(\beta_{r_{1}} b\right) \mathrm{Y}_{0}\left(\beta_{r_{1}} a\right)-\mathrm{J}_{0}\left(\beta_{r_{1}} a\right) \mathrm{Y}_{0}\left(\beta_{r_{1}} b\right)\right) & -\mathrm{K}_{0}\left(\alpha_{r_{2}} b\right) \\
\frac{\varepsilon_{1}}{\beta_{r_{1}}} \frac{1}{\mathrm{Y}_{0}\left(\beta_{r_{1}} a\right)}\left(\mathrm{J}_{1}\left(\beta_{r_{1}} b\right) \mathrm{Y}_{0}\left(\beta_{r_{1}} a\right)-\mathrm{J}_{0}\left(\beta_{r_{1}} a\right) \mathrm{Y}_{1}\left(\beta_{r_{1}} b\right)\right) & \frac{\varepsilon_{2}}{\alpha_{r_{2}}} \mathrm{~K}_{1}\left(\alpha_{r_{2}} b\right)
\end{array}\right]\left[\begin{array}{l}
A_{1} \\
C
\end{array}\right]=\left[\begin{array}{l}
0 \\
0
\end{array}\right]
$$

The transcendental equation is now obtained by equating the determinant of the previous system to zero:

$$
\begin{aligned}
\frac{\varepsilon_{2}}{\alpha_{r_{2}}} \frac{\mathrm{K}_{1}\left(\alpha_{r_{2}} b\right)}{\mathrm{Y}_{0}\left(\beta_{r_{1}} a\right)}\left(\mathrm{J}_{0}\left(\beta_{r_{1}} b\right) \mathrm{Y}_{0}\left(\beta_{r_{1}} a\right)-\mathrm{J}_{0}\left(\beta_{r_{1}} a\right) \mathrm{Y}_{0}\left(\beta_{r_{1}} b\right)\right) \\
\quad+\frac{\varepsilon_{1}}{\beta_{r_{1}}} \frac{\mathrm{K}_{0}\left(\alpha_{r_{2}} b\right)}{\mathrm{Y}_{0}\left(\beta_{r_{1}} a\right)}\left(\mathrm{J}_{1}\left(\beta_{r_{1}} b\right) \mathrm{Y}_{0}\left(\beta_{r_{1}} a\right)-\mathrm{J}_{0}\left(\beta_{r_{1}} a\right) \mathrm{Y}_{1}\left(\beta_{r_{1}} b\right)\right)=0
\end{aligned}
$$

which properly manipulated gives the more compact expression

$$
\frac{\varepsilon_{r_{1}}}{\varepsilon_{r_{2}}} \frac{\mathrm{J}_{1}\left(\beta_{r_{1}} b\right) \mathrm{Y}_{0}\left(\beta_{r_{1}} a\right)-\mathrm{J}_{0}\left(\beta_{r_{1}} a\right) \mathrm{Y}_{1}\left(\beta_{r_{1}} b\right)}{\mathrm{J}_{0} b\left(\beta_{r_{1}} a\right)-\mathrm{J}_{0}\left(\beta_{r_{1}} a\right) \mathrm{Y}_{0}\left(\beta_{r_{1}} b\right)}+\frac{\beta_{r_{1}}}{\alpha_{r_{2}}} \frac{\mathrm{K}_{1}\left(\alpha_{r_{2}} b\right)}{\mathrm{K}_{0}\left(\alpha_{r_{2}} b\right)}=0
$$

which is quite simpler than the general transcendental equation (3.327). The two unknown constants are related in this case as

$$
\frac{C}{A_{1}}=\frac{1}{\mathrm{Y}_{0}\left(\beta_{r_{1}} a\right) \mathrm{K}_{0}\left(\alpha_{r_{2}} b\right)}\left[\mathrm{J}_{0}\left(\beta_{r_{1}} b\right) \mathrm{Y}_{0}\left(\beta_{r_{1}} a\right)-\mathrm{J}_{0}\left(\beta_{r_{1}} a\right) \mathrm{Y}_{0}\left(\beta_{r_{1}} b\right)\right]
$$


expression that together with (3.347) determines totally the fields associated to the solution $\beta$.

Similarly:

$\underline{\mathrm{TE}_{0 m} \text { modes: }}$

$\underline{\text { Region } 1, a \leq r \leq b}$

$$
\begin{aligned}
E_{z_{1}} & =0 \\
H_{z_{1}} & =B_{1} \mathrm{~J}_{0}\left(\beta_{r_{1}} r\right)+B_{2} \mathrm{Y}_{0}\left(\beta_{r_{1}} r\right) \\
E_{r_{1}} & =0 \\
E_{\phi_{1}} & =-\frac{j \omega \mu_{0}}{\beta_{r_{1}}}\left[B_{1} \mathrm{~J}_{1}\left(\beta_{r_{1}} r\right)+B_{2} \mathrm{Y}_{1}\left(\beta_{r_{1}} r\right)\right] \\
H_{r_{1}} & =\frac{j \beta}{\beta_{r_{1}}}\left[B_{1} \mathrm{~J}_{0}\left(\beta_{r_{1}} r\right)+B_{2} \mathrm{Y}_{0}\left(\beta_{r_{1}} r\right)\right] \\
H_{\phi_{1}} & =0
\end{aligned}
$$

$\underline{\text { Region } 2, r \geq b \text { : }}$

$$
\begin{aligned}
E_{z_{2}} & =0 \\
H z_{2} & =D \mathrm{~K}_{0}\left(\alpha_{r_{2}} r\right) \\
E_{r_{2}} & =0 \\
E_{\phi_{2}} & =\frac{j \omega \mu_{0}}{\alpha_{r_{2}}} D \mathrm{~K}_{1}\left(\alpha_{r_{2}} r\right) \\
H_{r_{2}} & =-\frac{j \beta}{\alpha_{r_{2}}} D \mathrm{~K}_{1}\left(\alpha_{r_{2}} r\right) \\
H_{\phi_{1}} 2 & =0
\end{aligned}
$$

For the above fields configuration, the boundary conditions become:

1. Tangential electric fields must vanish on the PEC surface:

$$
E_{\phi_{1}}(a, \phi)=0
$$

2. Tangential electric and magnetic fields must be continuous along an interface between the two dielectric media:

$$
\begin{aligned}
& H_{z_{1}}(b, \phi)=H z_{2}(b, \phi) \\
& E_{\phi_{1}}(b, \phi)=E_{\phi_{2}}(b, \phi)
\end{aligned}
$$


Solving first (3.366) it is obtained

$$
B_{2}=-B_{1} \frac{\mathrm{J}_{1}\left(\beta_{r_{1}} a\right)}{\mathrm{Y}_{1}\left(\beta_{r_{1}} a\right)}
$$

If this last expression is used together with (3.367) and (3.368) it is obtained that

$$
B_{1} \frac{1}{\mathrm{Y}_{1}\left(\beta_{r_{1}} a\right)}\left[\mathrm{J}_{0}\left(\beta_{r_{1}} b\right) \mathrm{Y}_{1}\left(\beta_{r_{1}} a\right)-\mathrm{J}_{1}\left(\beta_{r_{1}} a\right) \mathrm{Y}_{0}\left(\beta_{r_{1}} b\right)\right]-D \mathrm{~K}_{0}\left(\alpha_{r_{2}} b\right)=0
$$

and

$$
\begin{aligned}
B_{1} \frac{1}{\beta_{r_{1}}} \frac{1}{\mathrm{Y}_{1}\left(\beta_{r_{1}} a\right)}\left[\mathrm{J}_{1}\left(\beta_{r_{1}} b\right) \mathrm{Y}_{1}\left(\beta_{r_{1}} a\right)-\mathrm{J}_{1}\left(\beta_{r_{1}} a\right) \mathrm{Y}_{1}\left(\beta_{r_{1}} b\right)\right] & \\
& +D \frac{1}{\alpha_{r_{2}}} \mathrm{~K}_{1}\left(\alpha_{r_{2}} b\right)=0
\end{aligned}
$$

these last two equation describing the matrix system

$$
\left[\begin{array}{cc}
\frac{1}{\mathrm{Y}_{1}\left(\beta_{r_{1}} a\right)}\left(\mathrm{J}_{0}\left(\beta_{r_{1}} b\right) \mathrm{Y}_{1}\left(\beta_{r_{1}} a\right)-\mathrm{J}_{1}\left(\beta_{r_{1}} a\right) \mathrm{Y}_{0}\left(\beta_{r_{1}} b\right)\right) & -\mathrm{K}_{0}\left(\alpha_{r_{2}} b\right) \\
\frac{1}{\beta_{r_{1}}} \frac{1}{\mathrm{Y}_{1}\left(\beta_{r_{1}} a\right)}\left(\mathrm{J}_{1}\left(\beta_{r_{1}} b\right) \mathrm{Y}_{1}\left(\beta_{r_{1}} a\right)-\mathrm{J}_{1}\left(\beta_{r_{1}} a\right) \mathrm{Y}_{1}\left(\beta_{r_{1}} b\right)\right) & \frac{1}{\alpha_{r_{2}}} \mathrm{~K}_{1}\left(\alpha_{r_{2}} b\right)
\end{array}\right]\left[\begin{array}{l}
B_{1} \\
D
\end{array}\right]=\left[\begin{array}{l}
0 \\
0
\end{array}\right]
$$

Now, by equating the determinant of such system to zero, the transcendental equation is obtained as

$$
\frac{\mathrm{J}_{1}\left(\beta_{r_{1}} b\right) \mathrm{Y}_{1}\left(\beta_{r_{1}} a\right)-\mathrm{J}_{1}\left(\beta_{r_{1}} a\right) \mathrm{Y}_{1}\left(\beta_{r_{1}} b\right)}{\mathrm{J}_{0}\left(\beta_{r_{1}} b\right) \mathrm{Y}_{1}\left(\beta_{r_{1}} a\right)-\mathrm{J}_{1}\left(\beta_{r_{1}} a\right) \mathrm{Y}_{0}\left(\beta_{r_{1}} b\right)}+\frac{\beta_{r_{1}}}{\alpha_{r_{2}}} \frac{\mathrm{K}_{1}\left(\alpha_{r_{2}} b\right)}{\mathrm{K}_{0}\left(\alpha_{r_{2}} b\right)}=0
$$

Finally, once $\beta$ is solved in the previous equation, the relationship between $B_{1}$ and $D_{1}$ is calculated now by the following expression:

$$
\frac{D}{B_{1}}=\frac{1}{\mathrm{Y}_{1}\left(\beta_{r_{1}} a\right) \mathrm{K}_{0}\left(\alpha_{r_{2}} b\right)}\left[\mathrm{J}_{0}\left(\beta_{r_{1}} b\right) \mathrm{Y}_{1}\left(\beta_{r_{1}} a\right)-\mathrm{J}_{1}\left(\beta_{r_{1}} a\right) \mathrm{Y}_{0}\left(\beta_{r_{1}} b\right)\right]
$$

By looking the transcendental equations of $\mathrm{TM}_{0 m}$ and $\mathrm{TE}_{0 m}$ modes, (3.352) and (3.373), it is found that for the DCSWW they not only differ on the dielectric contrast factor $\varepsilon_{r_{1}} / \varepsilon_{r_{2}}$, but the quotient associated to the region 1 (first term in the equations) presents different form. This fact will cause larger difference between the propagation features of $\mathrm{TM}_{0 m}$ and $\mathrm{TE}_{0 m}$ modes than in the dielectric cylinder, since now, even for small dielectric contrasts, propagation of these modes will be clearly differentiated. This difference is caused by the presence of the PEC wire surface. 


\subsubsection{Modal spectrum}

Considering the presented formulation, the modal spectrum can be calculated straightforward. Since now the transcendental equation is a function of real variable $\beta$, attention will be directly displayed on the dispersion diagram. In addition, being that the effects of the permittivity in dielectric waveguiding structures have been already observed through the analysis of the simple dielectric cylinder, in this first section considering not only the fundamental mode, but the whole modal spectrum, the coating permittivity will be fixed to $\varepsilon_{r_{1}}=2.5$, a typical value in the range of polymers. Since from this point, the external infinite medium will be assumed to be air, $\varepsilon_{r_{2}}=1$, when referring to the coating, it will directly used $\varepsilon_{r}$, with any subscript. By fixing the permittivity, attention will be focused in the rather novel aspect of this structure regarding to what has been treated in this thesis so far: its geometrical configuration. In this sense, during this generalized modal analysis, the parameter proposed in [503] will be helpful. The aspect ratio of the DCSWW is defined as

$$
R=\frac{b}{a}
$$

Observe that the domain of $\mathrm{R}$ is $[0,1]$ and, according to its definition, the SWW structure corresponds to the case $R=1$, whereas the dielectric rod corresponds to the case $R=0$. Therefore, the DCSWW lies somehow in between of these two previously analyzed waveguides. Since the problem is able to be classified as a dielectric waveguide problem, to keep the generality, the normalized frequency $V$ is recovered

$$
V=\frac{2 \pi b}{\lambda_{0}} \sqrt{\varepsilon_{r}-1}=\left(\beta_{r_{1}} b\right)^{2}+\left(\alpha_{r_{2}} b\right)^{2}
$$

Note however that, in this usual definition, the normalized frequency $V$ may be not as general as desired since for the same $b$ a great variety of cases are obtained by varying $R$ along its domain. Although for comparison of the dispersion diagrams of different cases of $R$ the use of $V$ is interesting since they must be equally compared, in order to gain insight in the mathematical problem and alleviate numerical calculations it has been found that the following normalization results more suitable:

$$
V^{\prime}=\frac{V}{1-R}
$$

being that $(1-R)$ is, actually, the fraction of dielectric over a radial segment of length $b$. For calculations, it has proceeded with $V^{\prime}$, but the final results are represented as a function of $V$ as it gives the idea of the total electrical size of the waveguide, ruled by $b$. The dispersion diagram of the first modes of the DCSWW are represented for several cases of $R$ in Fig. 3.48. As usual, the normalized propagation $\operatorname{constant} \beta_{N}=\beta / k_{0}$ is represented. Schematics of the DCSWW for such cases of $R$ are included in Fig. 3.48(f) to better figure out the real aspect of the waveguide. The modes that are represented are the following:

- For $n=0\left(\mathrm{TM}_{0 m}\right.$ and $\left.\mathrm{TE}_{0 m}\right)$, the first two radial variations for each kind of mode: $\mathrm{TM}_{01}$ and $\mathrm{TM}_{02}$ (black curves), $\mathrm{TE}_{01}$ and $\mathrm{TE}_{02}$ (green curves). 
- For $n>0\left(\mathrm{HE}_{n m}\right.$ and $\left.\mathrm{EH}_{n m}\right)$, only the first radial variation is represented for each kind of mode up to $n=4$ : $\mathrm{HE}_{11}, \mathrm{HE}_{21}, \mathrm{HE}_{31}$ and $\mathrm{HE}_{41}$ (blue curves), $\mathrm{EH}_{11}, \mathrm{EH}_{21}, \mathrm{EH}_{31}, \mathrm{EH}_{41}$ (red curves).

Due to the strong change in the propagation features when $R$ varies and the singular modal spectrum observed for some cases, there are many high order modes that should appear on some of the graphs, which are not displayed for clarity purposes. The location of the curves will be easily intuited along the following explanation.

The DCSWW present, theoretically, two fundamental modes, the $\mathrm{TM}_{01}$ and the $\mathrm{HE}_{11}$. However, for this last, it is observed a practical cutoff. This is very evident when $R$ is small, see for instance the case $R=0.1$ in Fig. 3.48(a). This situation keeps similarity with the SWW problem, where the $\mathrm{HE}_{1}$ mode is always found as a solution if the singular point $\gamma_{N}=j$ is considered in the domain, however the $\mathrm{TM}_{0}$ is the real fundamental mode with practical waveguiding features. Therefore, for the DCSWW, the $\mathrm{TM}_{01}$ will be considered as the main fundamental mode, and the practical cutoff of $\mathrm{HE}_{11}$ will be fully considered, i. e., the $\mathrm{HE}_{11}$ will be assumed to have a cutoff. Notice how the $\mathrm{TM}_{0}$ mode of the $\mathrm{SWW}$ and $\mathrm{TM}_{01}$ mode of the DCSWW are the same mode (if $t \rightarrow 0$ ), however, in the case of the DCSWW will be necessary to use the radial index $m$ in as much the dielectric coating allows the apparition of modes with different number of radial oscillations and not only a unique mode, with simple exponential decay, as occurs with the SWW.

The aspect of Figs. 3.48(a)-3.48(d) is noticeable different. If $R \rightarrow 1$ (very thin coating compared with the conductor, i.e., close to SWW situation), the modes tend to degenerate by groups, see Fig. 3.48(d) corresponding to $R=0.95$. These groups are formed regarding their radial index $m$ as follows. First, they appear, for $m=1$, the $\mathrm{TM}_{01}$ mode and all ${ }^{25}$ the $\mathrm{HE}_{n 1}$ modes (see the conglomerate of blue curves). The second group still correspond $m=1$, but in this case is formed by the $\mathrm{TE}_{01}$ mode and all the $\mathrm{EH}_{n 1}$ modes (see the conglomerate of red curves). Again the relationships TM-HE and TE-EH are found. The third group is formed by the $\mathrm{TM}_{02}$ (see black dashed line) and all $\mathrm{HE}_{n 2}$ modes (not represented), and the fourth group is formed by the $\mathrm{TE}_{02}$ (see green dashed line) and all $\mathrm{EH}_{n 2}$ modes (not represented). As $m$ increase groups are appearing with the same configuration.

If the $V$ abscissas axis is observed in the aforementioned Fig. 3.48(d) it is found that, except for the first group, the cutoff of the groups becomes quite high. This means that the superior groups are forced to disappear when the coating becomes very thin. Furthermore, for the first group, although the modes are degenerated for $V$ large enough (when they become confined inside the dielectric), close to cutoff, different values of cutoff normalized frequency $V_{c}$ are found for the different modes. The larger $n$ the larger $V_{c}$. The reason to these values $V_{c}$ to be very close in the graph is just the expansion of the $V$ axis to represent all the groups, however a zoom of the zone under question will reveal significant different in relative terms of $V$. This mean that, except of the fundamental $\mathrm{TM}_{01}$ mode, all the $\mathrm{HE}_{n 1}$ modes are forced to disappear, although with much less intensity than those of the superior groups. Therefore, a parallelism is found with the SWW, inasmuch as, in a first step, only

\footnotetext{
${ }^{25}$ The term all would make sense only in the limiting case $R \rightarrow 1$. In the case of $R$ close to but not equal to 1 , it is understood that a large number of $\mathrm{HE}_{n m}$ propagate before the first mode of the next group.
} 


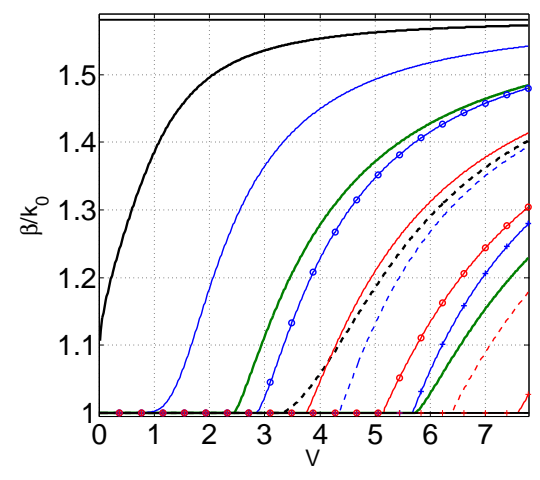

(a)

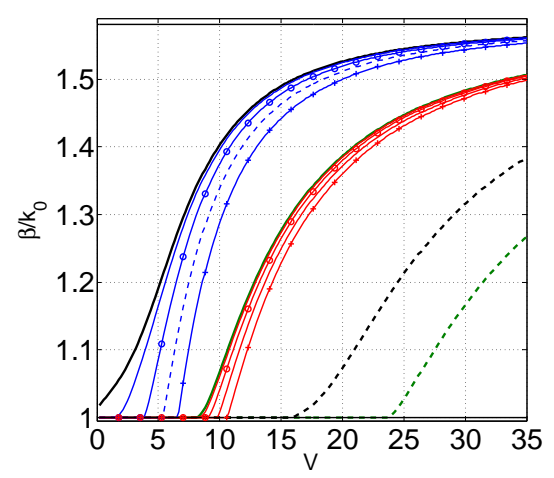

(c)

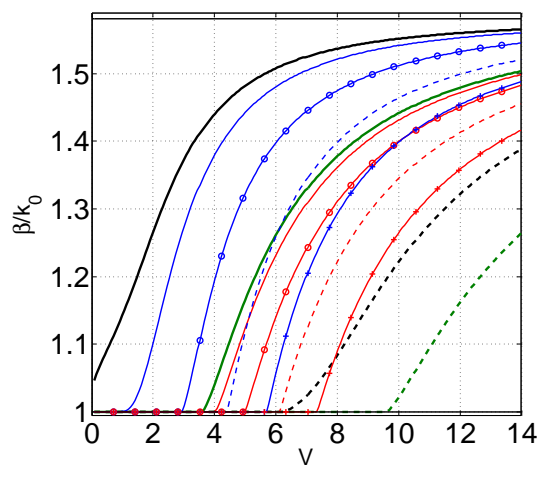

(b)

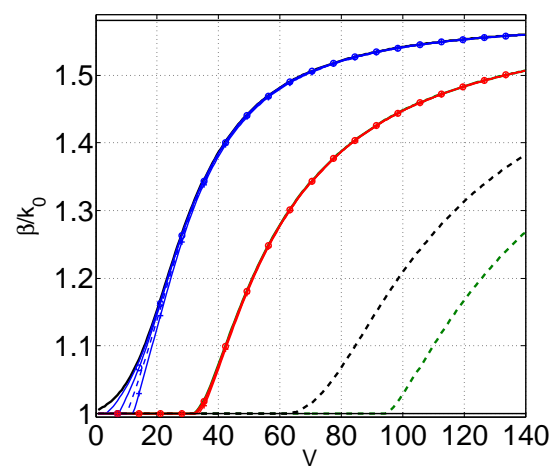

(d)

$-\mathrm{TE}_{01}$---- $\mathrm{TE}_{02}-\mathrm{TM}_{01}$---- $\mathrm{TM}_{02}-\mathrm{HE}_{11}-\mathrm{EH}_{11} \rightarrow \mathrm{HE}_{21} \rightarrow \mathrm{EH}_{21}$---- $\mathrm{HE}_{31}$---- $\mathrm{EH}_{31} \rightarrow \mathrm{HE}_{41}$ 十 $\mathrm{EH}_{41}$

(e)

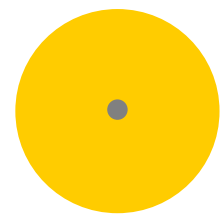

$$
R=0.1
$$

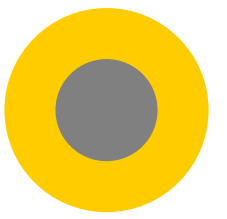

$$
R=0.5
$$

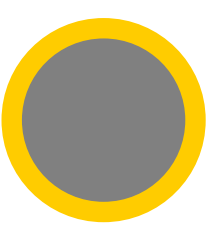

$R=0.8$

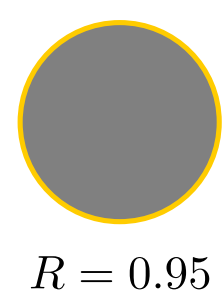

(f)

Figure 3.48: Dispersion diagram of a DCSWW with coating permittivity $\varepsilon_{r}=2.5$. a) $R=0.1$; b) $R=0.5$; c) $R=0.8$; d) $R=0.95$; e) Mode legend; f) DCSWW cross section for the different considered values of $R$.

the first radial variation $m=1$ of TM and HE modes remain propagating when the coating is made thin. In fact, the modes of the first group are the DCSWW version of the surface modes in the SWW, and by this, special attention over these modes must be paid. However, any exact direct matching between the two structures in 
the limit can be rigorously done (in mathematical terms) in as much the DCSWW problem becomes singular if $t \rightarrow 0$. To do that, the DCSWW must be analyzed assuming the conductor as a dielectric. Observation of the phenomena occurring when $t \rightarrow 0$ through that more complicated model is left as an interesting future work line.

The conclusion obtained by the study of results in Fig. 3.48(d) is that for $R$ close to the unity, when the coating is thin compared with the wire, all mode cases of azimuthal variation will appear before any mode with larger number of radial oscillations appear, and for the same $m$, all $\mathrm{TM}_{0 m}$ and $\mathrm{HE}_{n m}$ modes will appear before any $\mathrm{TE}_{0 m}$ or $\mathrm{EH}_{n m}$ mode. In this sense, a thin coating allow the propagation of those modes of high-order groups, whenever enough large $V$ is considered. However, those of the first group, which are the really dominant ones are exactly the modes that survive when the coating is removed, at least, if enough radius $a$ allow for that, inasmuch as they are the surface modes of a SWW.

Following the contrary tendency, i. e., if $R \rightarrow 0$ (thick coating compared with the conductor) the curves of modes of different group become to intertwine, and the previously observed degeneration is broken, see Fig. 3.48(a), corresponding to $R=0.1$. Whereas in the previous case the index $m$ of radial variations was the element of mode sorting, when $R \rightarrow 0$, the index $n$ of azimuthal variations becomes much more relevant for sorting purposes. Note that with $m=1$, from the first group, apart of the fundamental mode, only the singular (no theoretical cutoff) $\mathrm{HE}_{11}$ mode appear before that the $\mathrm{TE}_{01}$ modes, which was the first mode of the second group in the case $R=0.95$. The rest of $\mathrm{HE}_{n 1}$ modes require in this case larger $V$. The particular interest of a DCSWW with small $R$ is observed to be the clear difference in propagation terms between the $\mathrm{TM}_{01}$ mode and the $\mathrm{HE}_{11}$ mode, otherwise more degenerated.

Note that the $\mathrm{HE}_{11}$ mode is a clear candidate to couple to the $\mathrm{TM}_{01}$ mode if the waveguide is perturbed (which is the case of bends), not only because it is the first high order mode, but also due to its similar polarization, which will be shown in this section to be practically radial in its totality, i. e., the $\mathrm{HE}_{11}$ is a quasi-TM mode. Therefore, the strategy to follow in order to minimize the described coupling, if there is found risk to it occur, is to reduce $R$. Since given a design, $V$ will be first fixed, hence $b$, this will be done by reducing the radius $a$.

A particular feature that call into attention in Fig. 3.48(a) is that the $\mathrm{TM}_{01}$ mode starts to propagate with $\beta$ strictly larger than $k_{0}$. Inspection of the fields in such case indicate that this mode always exist, and even $V$ is small, there is a certain concentration of electric field around the wire. Note also that when $R \rightarrow 0$ is not possible to found a good parallelism between the DCSWW and the dielectric cylinder. For instance the $\mathrm{TM}_{01}$ is the fundamental mode and its propagation constant differs noticeably from the $\mathrm{TE}_{01}$, whereas in a dielectric cylinder these two modes are quite degenerated. This is caused by the presence of the PEC condition, which always takes place, even for infinitesimal values of $a$, having strong influence in the fields polarization, hence in the propagation. In mathematical terms, it could be said that the DCSWW problem has not its limit in the dielectric cylinder problem as $R \rightarrow 0$, contrarily with the case of $R \rightarrow 1$, that seems to be more evident. In other words, when $R=0$ the DCSWW is a dielectric cylinder, however, the limit 
$R \rightarrow 0$ does not converge to that situation inasmuch as an infinitesimal value of $a$ is enough to provide the PEC condition and appreciably influence the solution.

The E-field patterns of the most relevant modes in the previous analysis are reviewed now. As it can be understood there is a clear correspondence between their polarization and field distribution and the observed propagation phenomena. For the representation, the case of $R=0.5$ is chosen as it provides good visualization areas for each region and, at the same time, gives an intermedium situation between the aforementioned extreme cases, see Fig. 3.48(b).

First, in Fig. 3.49, the polarization (first row) and magnitude (second row) of the fundamental $\mathrm{TM}_{01}$ mode, and the $\mathrm{TE}_{01}$ mode are shown. In this case, due to the simpler expressions of the field, is trivially obtained that the $\mathrm{TM}_{01}$ exhibit pure radial polarization whereas the the $\mathrm{TE}_{01}$ mode exhibit pure azimuthal polarization. The presence of the conductor has a critical effect on the fields distribution ${ }^{26}$, see Figs. $3.49(\mathrm{c})$ and $3.49(\mathrm{~d})$. Whereas the $\mathrm{TM}_{01}$ has its maximum E-field on the conductor surface and decay as $r$ increases, the $\mathrm{TE}_{01}$ E-field vanishes on the conductor and present its maximum concentration for $r$ approximately in the middle between $a$ and $b$, i. e., confined in the dielectric medium as it was the only waveguiding element of the structure. This clearly explains the larger effect experienced by the $\mathrm{TE}_{01}$ mode when the coating becomes thin compared to the conductor, compared with the $\mathrm{TM}_{01}$. Actually, when this occur, the waveguiding support for the $\mathrm{TE}_{01}$ disappears.

For the high-order modes, inspection of the polarization results necessary. In Fig. 3.50 E-field polarization for the most relevant high order modes of each type in the previous dispersion study is presented. It can be appreciated how the presence of the PEC wire breaks the hybrid nature of the modes, having large purity of radial polarization in the case of $\mathrm{HE}_{n m}$ modes (see Figs. 3.50(a) and 3.50(c), corresponding to the $\mathrm{HE}_{11}$ and $\mathrm{HE}_{21}$ modes, respectively), and having large purity of azimuthal polarization in the case of $\mathrm{EH}_{n m}$ modes (see Figs. 3.50(b) and 3.50(d), corresponding to the $\mathrm{EH}_{11}$ and $\mathrm{EH}_{21}$ modes, respectively). From these plots it seems obvious that $\mathrm{HE}_{n m}$ modes present quasi-TM features and $\mathrm{EH}_{n m}$ modes present quasi-TE features.

Accordingly to the observed polarization, E-field magnitude mode patterns of previous modes present the distribution shown in Fig. 3.50: $\mathrm{HE}_{11}$ and $\mathrm{HE}_{21}$ modes have E-field concentrated near the wire surface whereas $\mathrm{EH}_{11}$ and $\mathrm{EH}_{21}$ modes have E-field vanishing on the wire surface and the field concentrated in the very center of the dielectric region. The same conclusions as for $\mathrm{TM}_{01}$ and $\mathrm{TE}_{01}$ apply here. It is clear that $\mathrm{EH}_{n 1}$ modes are much more forced to cutoff if the coating is made thinner than $\mathrm{HE}_{n 1}$ modes. Furthermore, it is pointed out that the larger $m$ imply a larger number of radial oscillations, being that the effect of the coating is even larger than the described for $m=1$ modes.

The aforementioned fact that $\mathrm{HE}_{n m}$ modes and $\mathrm{EH}_{n m}$ modes are quasi-TM and quasi-TE, respectively, is susceptible to be validated and quantified by analysis of the ratio between the axial components. As suggested in [553], the following parameter gives the proper tool of analysis for this purpose

\footnotetext{
${ }^{26}$ In the E-field vectorial plots, E-field of each region is normalized separately for visualization purposes, therefore fields in the external region may appear amplified. Magnitude plots represent the actual E-field magnitude distribution.
} 


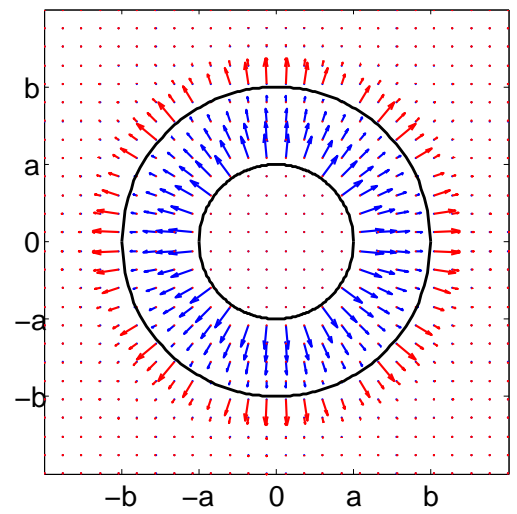

(a)

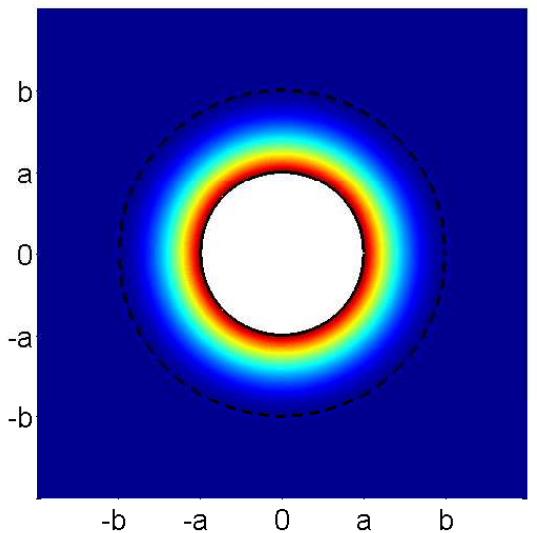

(c)

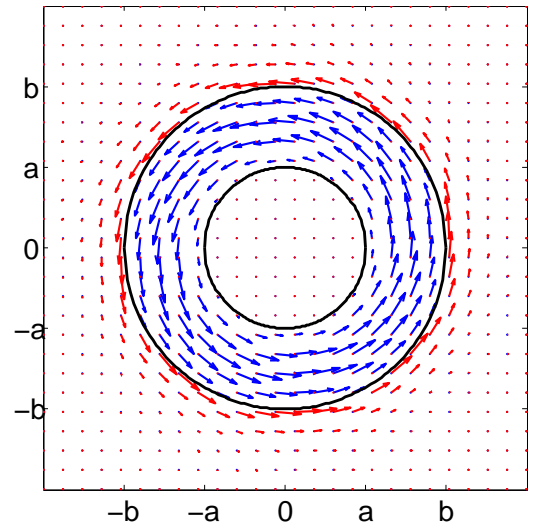

(b)

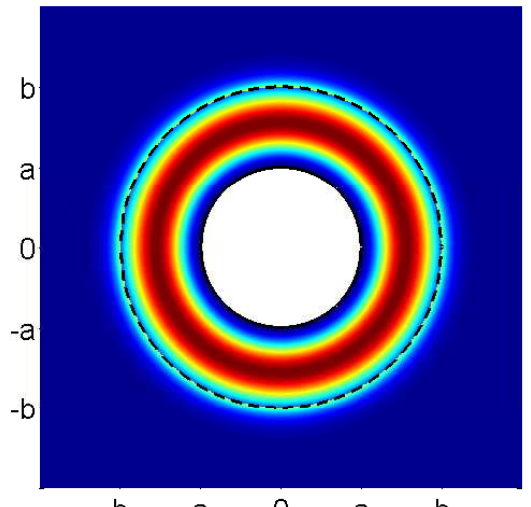

(d)

Figure 3.49: Transversal E-field polarization and magnitude of the fundamental TM $M_{01}$ mode, a) and $c)$, and the $T E_{01}$ mode, b) and $\left.d\right)$. The coating permittivity is $\varepsilon_{r}=2.5$ and the aspect ratio is $R=0.5$.

$$
\xi_{i}=\frac{\mu_{i}\left|H_{z i}\right|^{2}}{\varepsilon_{i}\left|E_{z i}\right|^{2}}
$$

With that expression, for each medium $(i=1$ or $i=2), \xi_{i}$ will adopt values above 1 if the mode is predominantly TE and below 1 if the mode is predominantly TM. Pure TE modes would provide $\xi_{i}=\infty$ whereas pure TM modes would provide $\xi_{i}=0$. Therefore, the use of a logarithmic scale seems the best option of representation for this parameter. Analysis of several hybrid modes is shown in Fig. 3.52 for the cases of $R=0.5$ and $R=0.95$. When the modes are far from cutoff it is appreciated how $\mathrm{HE}$ modes have clear TM predominance whilst EH modes have clear TE predominance, being that the numerator and the denominator in (3.378) differ in several orders of 


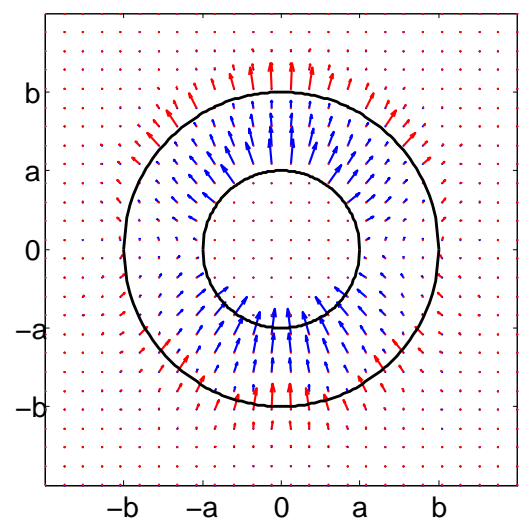

(a)

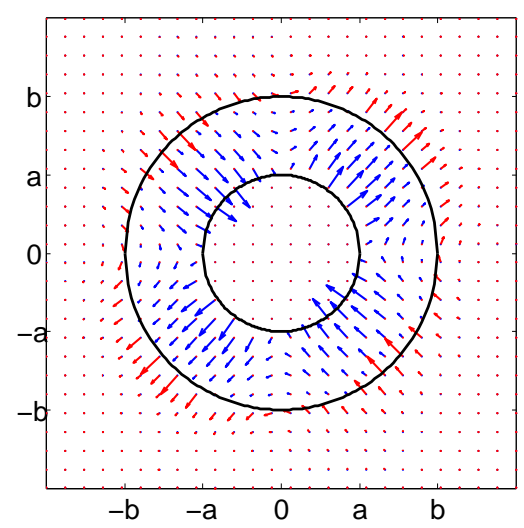

(c)

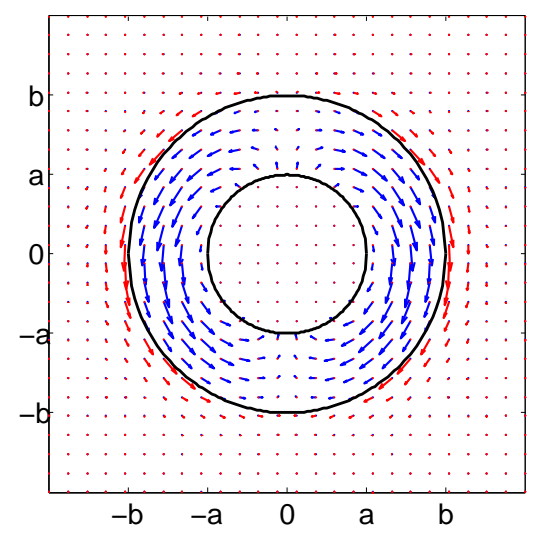

(b)

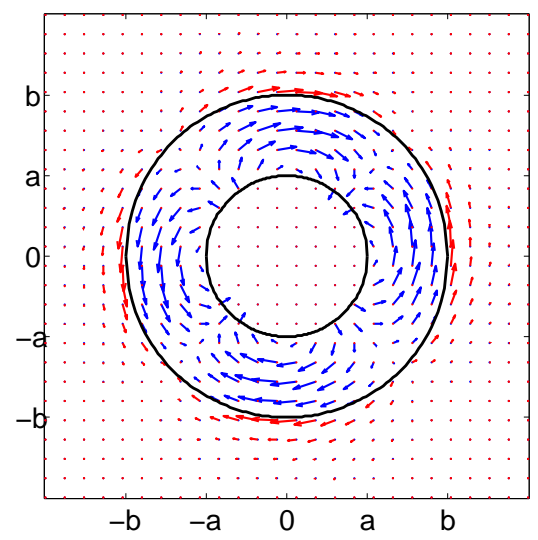

(d)

Figure 3.50: Transversal E-field polarization of the first hybrids modes of each type. a) $H E_{11}$; b) $E H_{11}$; c) $\mathrm{HE}_{21}$; d) $\mathrm{EH}_{21}$.

magnitude. Notice that for conventional optical fibers, $\xi_{i}$ is very close to unity for any considered case [553]. It is also observed that this effect is enhanced when $R$ is close to its upper bound $(R=1)$, as the PEC condition gains relevance. This dependence with $R$ is more obvious for HE modes. It is interesting to find that EH modes are predominantly TM when they start to propagate, then becoming quasi-TE as the coating becomes electrically larger. This situation is provided by a $t$ electrically small that forces the fields of these modes to be very close to the wire, hence being influenced by it. 


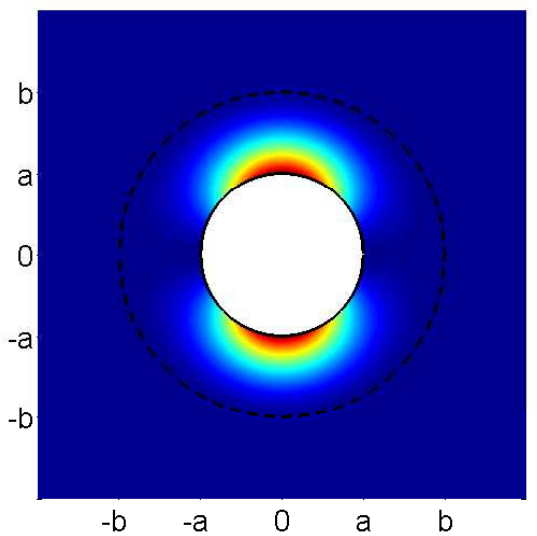

(a)

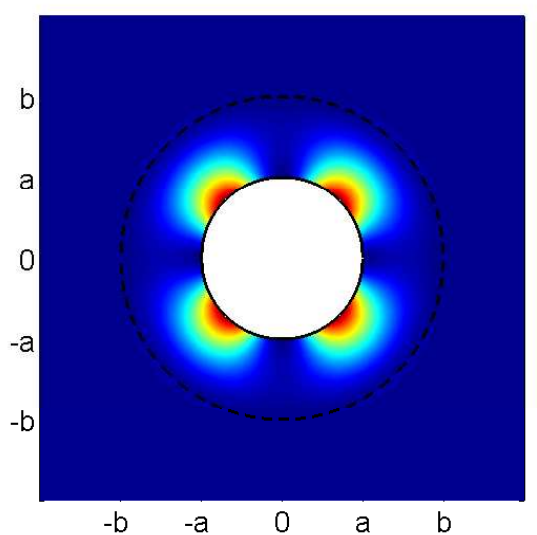

(c)

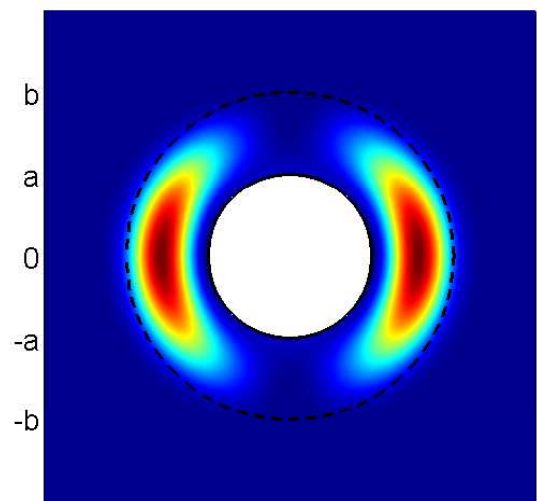

$\begin{array}{lllll}-b & -a & 0 & a & b\end{array}$

(b)

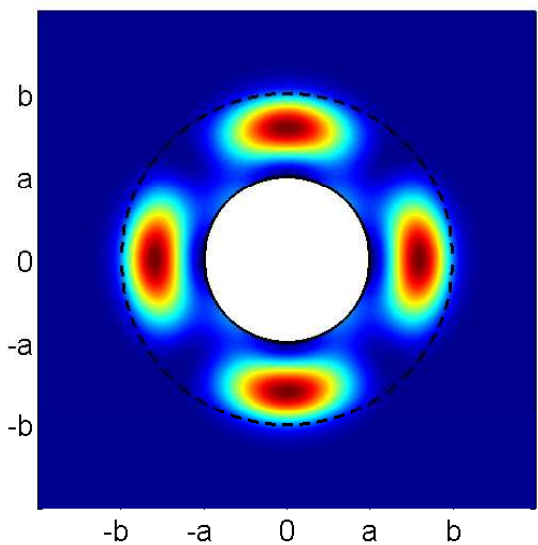

(d)

Figure 3.51: Transversal E-field magnitude of the first hybrids modes of each type. a) $H E_{11}$; b) $E H_{11}$; c) $\mathrm{HE}_{21}$; d) $\mathrm{EH}_{21}$.

\subsubsection{Monomode conditions}

By the above modal spectrum analysis, seems obvious that neither $\mathrm{TE}_{0 m}$ modes nor $\mathrm{EH}_{n m}$ are suitable to couple to the fundamental mode in any case, and they lack of interest in these terms. Regarding to the E-field radial polarized modes, there are to cases two consider. Firstly, it is clear that the group formed by all $\mathrm{HE}_{n 1}$ is suitable to couple the $\mathrm{TM}_{01}$ under certain conditions. The case of $\mathrm{HE}_{11}$ is very obvious. It could be proposed certain design, with $R$ close enough to 1 , allowing multimode transmission. The rather degeneration of the modes of the first group in such case may suggest transmission with almost no dispersion. Nevertheless, it must be pointed that $n$ range in the whole $\mathbb{N}$ set, hence it would be always possible to find modes with $\beta$ similar enough to be coupled, and different enough to cause 


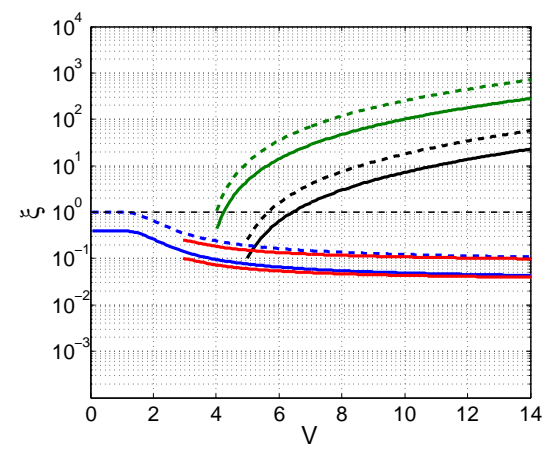

(a)

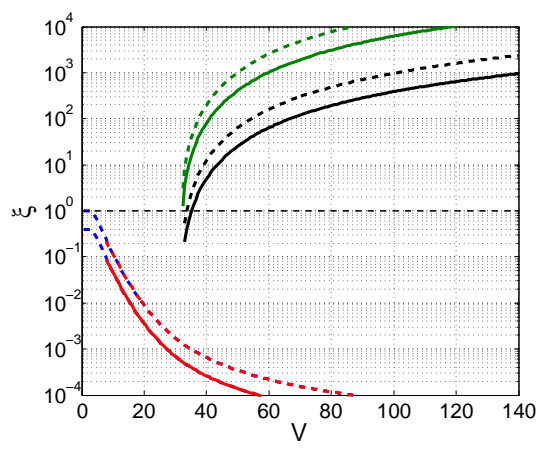

(b)

$$
-\xi_{1}\left(\mathrm{HE}_{11}\right)-\xi_{1}\left(\mathrm{EH}_{11}\right)-\xi_{1}\left(\mathrm{HE}_{21}\right)-\xi_{1}\left(\mathrm{EH}_{21}\right)---\xi_{2}\left(\mathrm{HE}_{11}\right)---\xi_{2}\left(\mathrm{EH}_{11}\right)---\xi_{2}\left(\mathrm{HE}_{21}\right)---\xi_{2}\left(\mathrm{EH}_{21}\right)
$$

Figure 3.52: Ratio $\xi_{i}$ in regions $i=1$ (solid lines) and $i=2$ (dashed lines) for the first hybrid modes of each type: $H E_{11}$ (blue), $E H_{11}$ (green), $H E_{21}$ (red) and $E H_{21}$ (black). a) $R=0.5 ; b$ ) $R=0.95$ c) Mode legend.

dispersion. Moreover, the DCSWW is though as a remedy for the inherent problems of the SWW, hence the coating is though to be present when necessary and removed when not, something that will be better understood when the losses and dispersion of this waveguide are quantified in the next section. If $\mathrm{HE}_{n 1}$ modes are excited, they will become radiative (or propagative with impractical extension for their reception) surface modes when the coating is retired, according to section 3.5. Thus, monomode operation is a feature to keep.

Alternatively, it might be possible to keep the axial symmetry during the perturbation with some element or configuration during the use of the DCSWW, thus preventing the coupling to $\mathrm{HE}_{n 1}$ modes. In such case, the mode to look at would be the $\mathrm{TM}_{02}$ mode, sharing polarization and azimuthal distribution with the fundamental mode. This is a more relaxed condition in as much as avoiding the $\mathrm{HE}_{11}$ requires to force the design to more critical parameters than those required to avoid the $\mathrm{TM}_{02}$ mode, which propagation ultimately depends on whether the coating is electrically large enough or not, leaving more freedom for the radius $a$. These two criteria will be kept in mind when bends are designed with the DCSWW structure in the next chapter.

\subsubsection{Power analysis and attenuation}

At this point, the knowledge about the modal spectrum of the DCSWW is deep enough for the purposes in this thesis, and it results interesting to carry out a deeper study of the fundamental $\mathrm{TM}_{01}$ mode. Since the analysis has been done over a lossless structure in the classical way, both, power distribution and attenuation analysis require from a further analysis of the obtained fields in the previous section, and, in the case of the attenuation, applying the perturbative method.

First, the total power transmitted in the waveguide is: 


$$
P_{T}=\frac{1}{2} \operatorname{Re}\left\{\iint_{S}\left(\vec{E} \times \vec{H}^{*}\right) \cdot \hat{z} \mathrm{~d} S\right\}
$$

where $S$ is the transversal surface through which the guided waves are transmitted. In the case of the DCSSW this surface is defined as the hole $\mathbb{R}^{2}$ except of the circular area defined by the PEC wire, i.e., a circle centered in the origen, with radius $a$. The total transmitted power can be split as usual in the two regions of this dielectric waveguide:

$$
\begin{gathered}
P_{T}=P_{1}+P_{0} \\
P_{1}=\frac{1}{2} \operatorname{Re}\left\{\int_{0}^{2 \pi} \int_{a}^{b} E_{r_{1}} H_{\phi_{1}}^{*} r \mathrm{~d} r \mathrm{~d} \phi\right\} \\
P_{2}=\frac{1}{2} \operatorname{Re}\left\{\int_{0}^{2 \pi} \int_{b}^{\infty} E_{r_{0}} H_{\phi_{0}}^{*} r \mathrm{~d} r \mathrm{~d} \phi\right\}
\end{gathered}
$$

Some analytic work can be done with the above integrals, specially for the simpler case of the fundamental $\mathrm{TM}_{01}$ mode. However, some terms require from numerical integration methods. Being that the only integral to be solve is that for the variable $r$ (on the variable $\phi$ it is trivial), the usual Gauss quadratures work extremely fast inasmuch as the fields are smooth functions. Gauss-Legendre is suitable for the finite region 1, whereas Gauss-Laguerre fits well for infinite region 2, where fields decay exponentially (see [528] for details of these methods).

It is recovered now the parameter summing up the distribution of power in a numerical quantity:

$$
\eta=\frac{P_{1}}{P_{T}}=\frac{P_{1}}{P_{1}+P_{2}}
$$

which is the fraction of power, sometimes expressed as percentage, that is propagated in the region 1 . In the case of the DCSWW this quantity is specially important in as much it gives a very good estimation of the power that will not be lost in a bend or similar in as much as it is confined in the interior of the dielectric, being prevented from radiation by the TIR effect.

Finally, it is also possible to consider for the DCSSW, the radius $r_{p(\%)}$ that define the transversal circumference through certain amount of power $p$ is transmitted. Defining the following function

$$
P_{T}(r)=\frac{1}{2} \operatorname{Re}\left\{\int_{0}^{2 \pi} \int_{r}^{\infty} E_{r_{0}} H_{\phi_{0}}^{*} r \mathrm{~d} r \mathrm{~d} \phi\right\}
$$

it is possible to obtain the percentage of power transmitted through a circumference centered at the origin and with radius $r$ as

$$
p(\%)=100 \cdot \frac{P_{T}(a)-P_{T}(r)}{P(a)}=100 \cdot\left(1-\frac{P_{T}(r)}{P_{T}(a)}\right)
$$


As explained for the SWW, fixed a desired reference $p(\%)$, the corresponding $r=r_{p(\%)}$ can be obtained by fast numerical routines (remember that $r$ cannot be isolated from (3.385)), specially for the fundamental mode since (3.384) is analytical for this mode.

In the case of the DCSWW $r=r_{p(\%)}$ is less relevant, inasmuch as it is usual that an important amount of power is confined in $r<b$, given by the more relevant parameter $\eta$, and the significant area of transmission is noticeably reduced compared to the SWW. In this way, some times $r$ will be specified as the additional radius required to receive a percentage of power, usually demanded to be larger than in the SWW case, e. g., $95 \%$ of the total transmitted power.

Known the total transmitted power $P_{T}$ it is straightforward to obtain the losses by the perturbative theory. Note however that, for the DCSWW, the procedure is applied to both, conductor and dielectric, which when considering real materials will introduce ohmic and dielectric absorption lossess, respectively. These losses are defined as:

$$
\alpha_{c}=\frac{P_{L c}}{2 P_{T}} \quad \alpha_{d}=\frac{P_{L d}}{2 P_{T}}
$$

where, for the DCSWW geometry,

$$
P_{L c}=\frac{R_{s}}{2} \int_{0}^{2 \pi}\left|\vec{J}_{s}\right|^{2} a \mathrm{~d} \phi
$$

and

$$
P_{L d}=\frac{\omega \epsilon_{1} \tan \delta}{2} \int_{a}^{b} \int_{0}^{2 \pi}|\vec{E}|^{2} r \mathrm{~d} \phi \mathrm{d} r
$$

beign the total losses in this way $\alpha=\alpha_{c}+\alpha_{d}$

\subsubsection{Fundamental mode}

Properties of the fundamental mode are studied now. Attenuation and dispersion will be important features to check since the SWW shows good performance in these terms, hence it must be quantified how these features are worsened when the coating is present. The percentage of power in the dielectric medium $\eta(\%)$ results relevant being that it is an important indicator of the percentage of power that will not be radiated on a bend. Finally, the radial extension power $r=r_{p(\%)}$ provides important information for excitation and reception purposes.

First of all, since many parameters define the DCSWW, some assessments will be done regarding the conductor radius $a$ and the dielectric thickness $t$. Then, special attention will be put in $t$, which is the parameter that origins more casuistic in the propagation features, and some assessment about the influence of the dielectric characteristics, permittivity and loss tangent, will be given. Later, in the next chapter, the dependence of DCSWW properties with $a$ will be recovered when necessary, over a more particular scenario.

In Fig. 3.53 the total attenuation $\alpha$ of a DCSWW is presented for different coatings: $t=1 \mu \mathrm{m}$, Fig. 3.53(a), $t=5 \mu \mathrm{m}$, Fig. 3.53(b), $t=10 \mu \mathrm{m}$, Fig. 3.53(c), 
and $t=20 \mu \mathrm{m}$, Fig. 3.53(d). Dielectric characteristics are chosen to be $\left(\varepsilon_{r}=2.5\right.$, $\tan \delta=0.003)$, which are usual values for polymers at $\mathrm{THz}$ frequencies [21], [437], [582]-[583], and copper is chosen as material for the wire $\left(\sigma=5,81 \cdot 10^{7} \mathrm{~S} / \mathrm{m}\right)$. In each graph, the same range of radius $a$ studied in the section 3.5.2 is again considered in order to assess the influence of $a$ in the losses of a DCSWW.

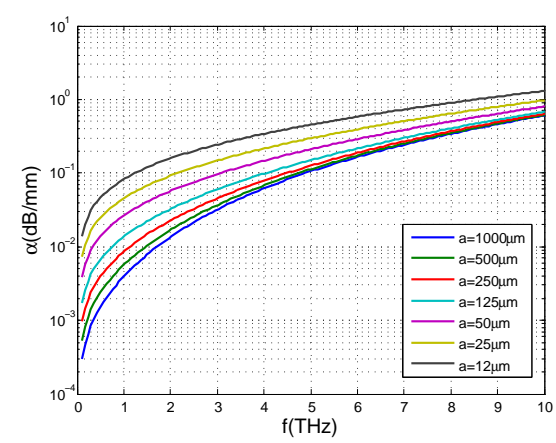

(a)

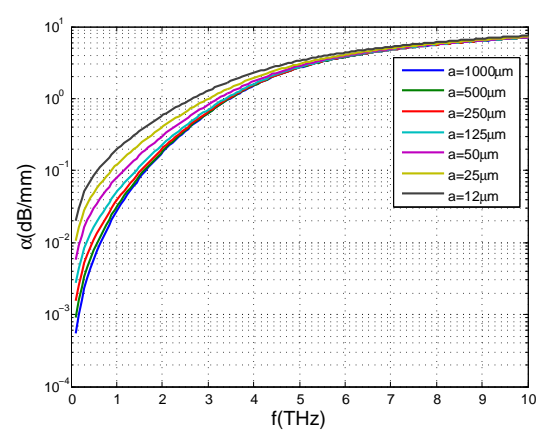

(c)

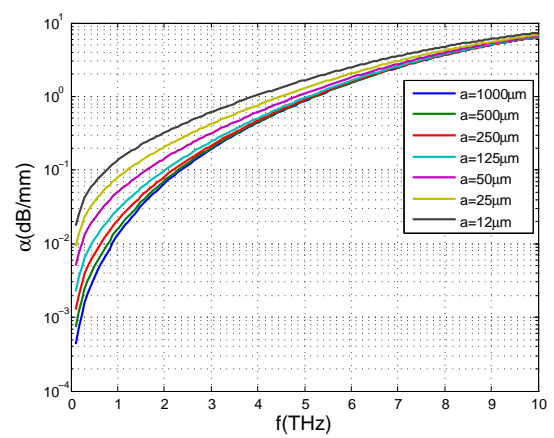

(b)

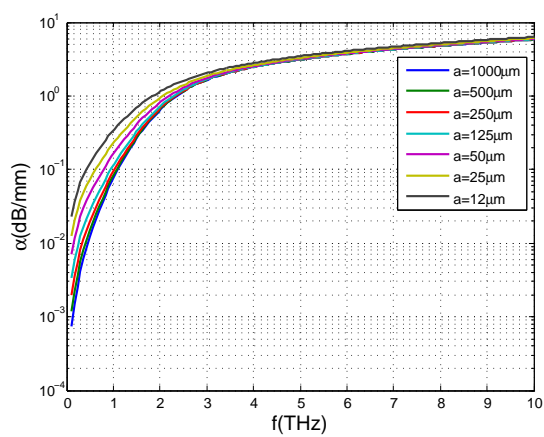

(d)

Figure 3.53: Total attenuation $\alpha$ in $\mathrm{dB} / \mathrm{mm}$, considering a dielectric coating with characteristics $\left(\varepsilon_{r}=2.5, \tan \delta=0.003\right)$, and several radius for the conductor $(a=12 \mu \mathrm{m}, a=25 \mu \mathrm{m}, a=50 \mu \mathrm{m}$, $a=125 \mu \mathrm{m}, a=250 \mu \mathrm{m}, a=500 \mu \mathrm{m}, a=1000 \mu \mathrm{m}$,$) . Copper \left(\sigma=5,81 \cdot 10^{7} \mathrm{~S} / \mathrm{m}\right)$ is chosen as the metal for the conductor. a) $t=1 \mu \mathrm{m}$; b) $t=5 \mu \mathrm{m}$; c) $t=10 \mu \mathrm{m}$; d) $t=20 \mu \mathrm{m}$;

As the aforementioned plots show, the electrical size of $t$ is very determinant in the lossess. Note how for those frequencies that imply $t$ to be large enough to the coating provide confinement for the fundamental mode, the losses are noticeably large (beyond the $1 \mathrm{~dB} / \mathrm{mm}$ barrier). In this configuration, the DCSWW acts as a dielectric waveguide providing full confinement, with the additional handicap of introducing ohmic losses due to the presence of the metallic wire. Under this configuration, the radius of the conductor $a$ has not any influence in the losses in as much the dielectric itself has a strong waveguiding capacity.

Looking now to the opposite case, when the coating is too thin to confine the energy (see for instance the case $t=1 \mu \mathrm{m}$ in Fig. 3.53(a)), the radius $a$ results determinant, as it was the case in the SWW. Attenuation values spread as wide as 
one decade and a half in such situation as the radius varies. Note that under such configuration, the waveguiding properties rely mostly on the wire properties, acting the DCSWW in a similar way to the SWW (as it should be expected). Nevertheless, note that, in the present analysis, the conductor material affects directly to $\alpha_{c}$, but not to the field pattern, which relies directly on $a$ and and the electrical size of $t$. Therefore, $a$ plays an important role exclusively when $t$ is small. A role that, however, could be used for interesting engineering purposes in such case.

\subsubsection{Effect of the coating}

For the moment $a$ will remain fixed, recovering its influence when necessary and a deep study in $t$ will be carried now. This study motivated the work published in [441] in the scope of this thesis. The analysis is focused in the band ranging from $0.5 \mathrm{THz}$ to $1 \mathrm{THz}$, the first interval of real challenging frequencies in the $\mathrm{THz}$ band, which supposes a rather broad relative bandwidth: $R B W=66.67 \%$. The highest frequency, $f=1 \mathrm{THz}$ is taken as main reference. A copper $\left(\sigma=5,81 \cdot 10^{7} \mathrm{~S} / \mathrm{m}\right)$ wire of $a=200 \mu \mathrm{m}$ is chosen, lying in between of the usual considered radius $(20 \mu \mathrm{m}$ to $450 \mu \mathrm{m}$ [492], [506], [507], [511]). Two permittivities are considered: $\varepsilon_{r}=2$ (low, e.g., polymer dielectrics) and $\varepsilon_{r}=10$ (high, e.g., crystalline dielectrics). In each case $\tan \delta=0.001$ is assumed as the loss indicator, which implies rather high quality dielectrics [21], [437], [582]-[583]. As interesting available information for posterior comparison purposes, Fig. 3.54 shows the SWW properties in the considered band for the study, analyzing several radius. As can be observed, the chosen radius, $a=200 \mu \mathrm{m}$, offers an interesting trade off between losses (under $0.005 \mathrm{~dB} / \mathrm{mm}$ in the whole band), and radial extension ( $95 \%$ of power confined in a $1 \mathrm{~cm}$ circumference).

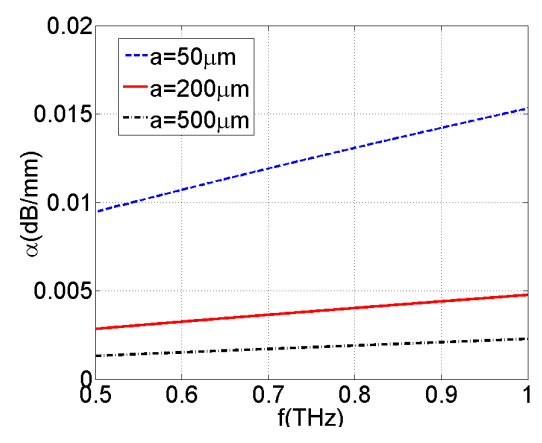

(a)

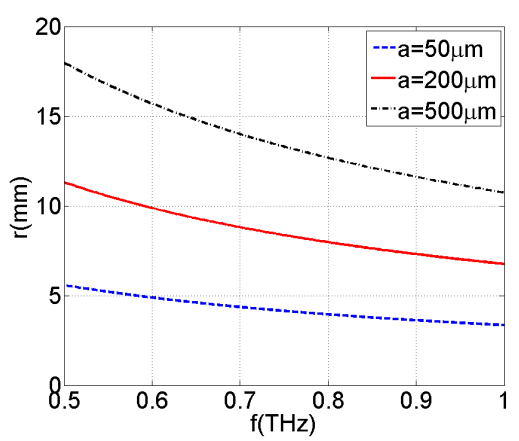

(b)

Figure 3.54: Attenuation and confinement properties of the $S W W$ in the operation band of the study [0.5 THz-1 THz], considering several radius ( $a=50 \mu \mathrm{m}, a=200 \mu \mathrm{m}, a=500 \mu \mathrm{m}$, ). a) Attenuation in $\mathrm{dB} / \mathrm{mm}$; b) Required radius to transmit $95 \%$ of the total power, $r=r_{95 \%}$.

First of all, the range of $t$ to be considered is put under question, in order to be coherent with the monomode operation requirements. In this first approach, the less restrictive condition will be considered, i. e., to avoid the propagation of the first axialsymmetric high order mode with radial propagation: the $\mathrm{TM}_{02}$. The transversal E-field polarization of this mode is shown in Fig. 3.55(a). Note that, 
under similar power distribution conditions, the only difference regarding to the fundamental mode is the null of E-field at some radius $a \leq b$, forming a ring area inside the dielectric with null E-field. To calculate the aforementioned restriction over the DCSWW parameters, it is possible to set $\beta=k_{0}$ in (3.352) to obtain the cutoff condition of $\mathrm{TM}_{0 m}$ modes:

$$
\mathrm{J}_{0}\left(\beta_{r_{1}} b\right) \mathrm{Y}_{0}\left(\beta_{r_{1}} a\right)-\mathrm{J}_{0}\left(\beta_{r_{1}} a\right) \mathrm{Y}_{0}\left(\beta_{r_{1}} b\right)
$$

The results of analyzing the previous expression as a function of $a$ and $t$ are shown in Fig. $3.55(\mathrm{~b})$. In this plot, it is provided the maximum value of $t$, called $t_{\max }$, for monomode operation in the whole operating band (regarding to the propagation of the $\mathrm{TM}_{02}$ mode) as a function of $a$ for the two permittivities of the present study, $\varepsilon_{r}=2$ and $\varepsilon_{r}=10$. Observe how, actually, $a$ has not any influence on the propagation of this high order mode except when it is very small, case in which has a small, but not relevant influence. Therefore, it is found that $t$ rules totally the propagation of the $\mathrm{TM}_{02}$ mode, and analyzing the limiting values for the considered permittivities, $t_{\varepsilon_{r}=2}=150 \mu \mathrm{m}$ and $t_{\varepsilon_{r}=10}=50 \mu \mathrm{m}$, it is found that $t_{\text {max }}$ can be very well estimated as:

$$
t_{\max }=\frac{\lambda_{0}}{2 \sqrt{\varepsilon_{r}-1}}
$$

which coincides with that limit of the infinite planar version of the DCSWW, i. e., except for very small radius, the $\mathrm{TM}_{0 m}$ modes do not experience the curvature introduced by the cylindrical geometry. Note also how $t_{\max }$ depends on the electrical size of the coating regarding the dielectric contrast between region 1 and region 2 , a classical characteristic of dielectric waveguides.

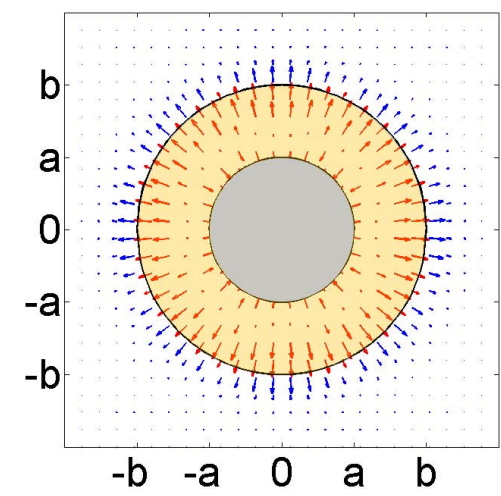

(a)

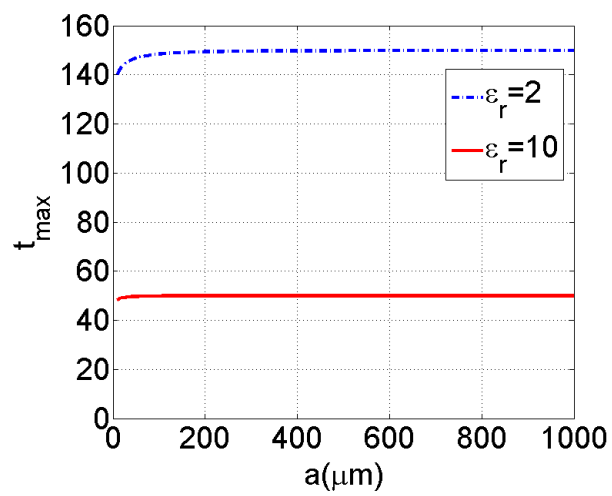

(b)

Figure 3.55: $T M_{02}$ mode. a) E-field transversal polarization; b) Maximum applicable coating $t$ as a function of $a$ in order to avoid the propagation of the $T M_{02}$ mode for the two cases of permittivity considered in the study, $\varepsilon_{r}=2$ and $\varepsilon_{r}=10$.

Once the limiting values for $t$ have been set, the study on the effect of the coating starts with the power confinement. The parameter $\eta(\%)$ indicating the percentage of power in the dielectric region 1 is shown for several equally spaced frequencies in 
the considered band in Figs. 3.56(a) and 3.56(b) for the cases of $\varepsilon_{r}=2$ and $\varepsilon_{r}=10$, respectively. It is well observed how for the frequency that establishes the limit of $t$, i.e., $f=1 \mathrm{THz}$, ranging $t$ in its domain allows to see exactly the full confinement cycle evolution, from $0 \%$ to almost $100 \%$.

As observed in section 3.3, higher permittivities imply a more abrupt transition being that the electrical size of the coating grows faster with its real physical size growth. Since the electrical size of the coating is obviously different for the different frequencies of the band, when $t$ is in its intermedium values, there is found some dispersion in as much as highest frequencies are rather confined and lowest frequencies have still most part of the total power being transmitted in the external air medium. Since the curves of the $\varepsilon_{r}=10$ case are more abrupt, this effect becomes very evident on them. Regarding to this case, see in Fig. 3.56(b) how between $t=25 \mu \mathrm{m}$ and $t=45 \mu \mathrm{m}$ the difference in confinement between the limiting frequencies of the band is very large. Therefore, although higher permittivities provide larger confinement with thinner coatings, they also imply a strong confinement dispersion if intermedium values of $t$ are used. This, translated to a curvature would cause strong attenuation dispersion in as much lower frequencies would radiate much more than higher frequencies. Moreover, even in the case that $t$ is used very close to its limiting value, a lower permittivity exhibits less confinement dispersion. By this argumentation, seems that low permittivities are more suitable in general regarding the confinement features. It is true, however, high permittivity coatings are thinner (interesting feature only whether there is size limitation), and the TIR effect is stronger.

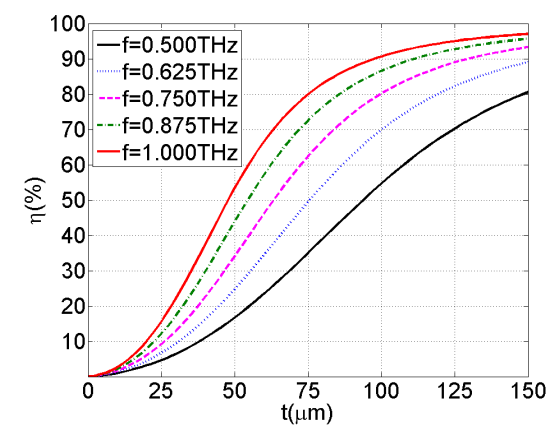

(a)

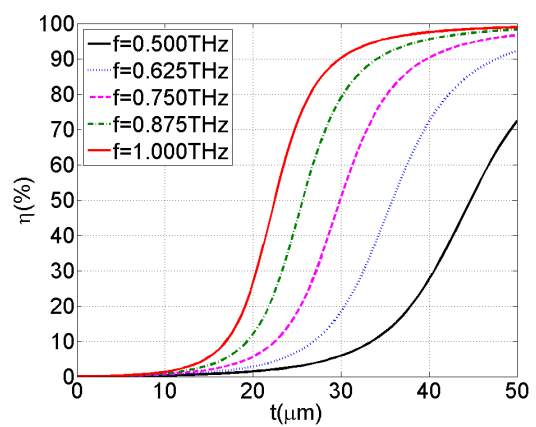

(b)

Figure 3.56: Percentage of transmitted power inside the dielectric coating, $\eta(\%)$ as a function of the coating thickness $t$. a) $\varepsilon_{r}=2$; b) $\varepsilon_{r}=10$.

Continuing with the power distribution features, the radial extension is studied now. In Fig. 3.57 it is displayed the required distance from the dielectric coating external interface, located at $r=b$, to include $95 \%$ of the total transmitted power as a function of the coating thickness $t$ for the same equally spaced frequencies of the operating band as before. In Fig. 3.57(a), which correspond to the case $\varepsilon_{r}=2$, an inset has been included for clarify the definition of the aforementioned distance $r$. By this definition, $r$ indicates how much additional radial distance beyond the DCSWW transversal cut is necessary to afford certain amount of power, which is 
an appreciable information for excitation or reception purposes. Note that a very significant amount, $95 \%$ has been considered in this study.

Figs. 3.57(a) and 3.57(b) are very similar, indicating that, regarding the radial extension, the dielectric permittivity is not a very influential parameter. Moreover very assumable values are achieved even for not very high $t$. Specifically, the additional extension is less than $r=0.5 \mathrm{~mm}$ for the whole band for intermedium values of $t$ in both permittivity cases. This means that, whenever a reasonable coating is present, the transmitted power collapses in a very compact area, what can be affirmed as a good advantage of the DCSWW.

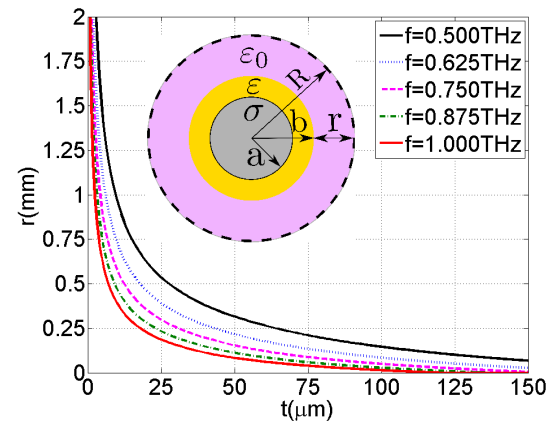

(a)

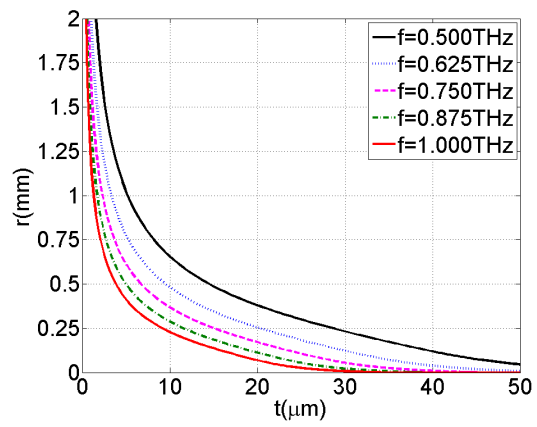

(b)

Figure 3.57: Radial extension from the dielectric coating interface $r=b$, necessary to include $95 \%$ of the total transmitted power as a function of the coating thickness $t$. a) $\varepsilon_{r}=2$; b) $\varepsilon_{r}=10$.

Attenuation features are afforded next. In this case, the frequency is fixed to $f=1 \mathrm{THz}$, the maximum of the band, and the different contribution of loss, ohmic and dielectric are analyzed separately in order to understand the origin of the observed total attenuation. Assessments regarding how a broadband signal would be attenuated will be easily understood from what has been reviewed up to now and the information of the graphs. In Fig. 3.58, the total attenuation $\alpha$ in $\mathrm{dB} / \mathrm{mm}$ is decomposed in the contributions from the imperfect conductor, $\alpha_{c}$, and the imperfect dielectric, $\alpha_{d}$, and it shown as a function of $t$ for the two considered cases of permittivity.

In this case, the differences between both graphs are very evident. First, regarding the total attenuation level (black curves), it could be roughly said that the case of $\varepsilon_{r}=10$, Fig. 3.58(b), present losses one order of magnitude larger than the case of $\varepsilon_{r}=2$, Fig. 3.58(a). Interestingly, this is mainly caused by the ohmic losses contribution, which results to be the major contribution to total losses in the case of $\varepsilon_{r}=10$. Note that, since the thickness $t$ is lower in this case, the electromagnetic fields are comprised in a smaller volume, hence the currents being more intense on the conductor surface. Furthermore, there is a pronounced peak for intermedium values of $t$ in the considered monomode range for this permittivity. The reason for this peak is based in the same phenomena. Note that, for such intermedium values, $t$ is enough large to cause the fundamental mode to be rather confined inside the dielectric (see Fig. 3.56). Since a considerable amount of power is confined in even a smaller volume, the surface currents become even more intense on the conductor. 


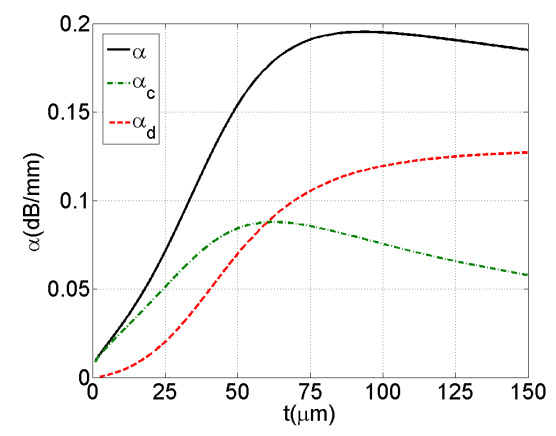

(a)

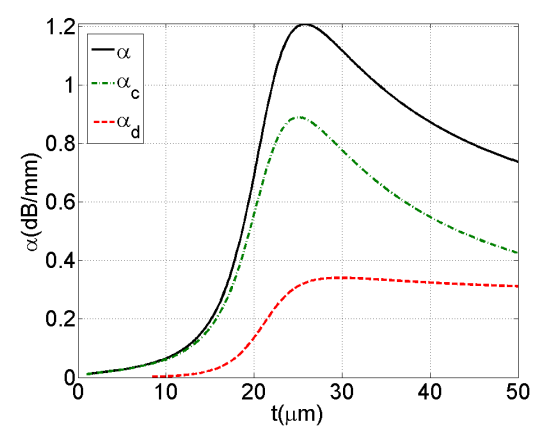

(b)

Figure 3.58: Attenuation vs. coating thickness $t$ at $f=1 \mathrm{THz}$. The total attenuation ((black solid line) is broken down in its ohmic $\left(\alpha_{c}\right.$, green dashed line) and dielectric absorption ( $\alpha_{d}$, red dashed line) contributions. a) $\varepsilon_{r}=2$; b) $\varepsilon_{r}=10$.

Observe that this peak in $\alpha_{c}$ is also appreciated in the $\varepsilon_{r}=2$ case, Fig. 3.58(a). However, since the coating is thicker and $\eta$ is less sensitive to $t$, the peak is much less pronounced.

Second, dielectric losses are analyzed. As usual, since $\tan \delta$ is the same for both cases, dielectric losses are proportional to the permittivity in the same sense at it was observed when the dielectric cylinder was analyzed. Particularly, from the definition (3.200) of the normalized attenuation constant in $R$, it could be roughly said that the losses scale as $\sqrt{\varepsilon_{r}}$. Therefore, contrarily of what could be intuitively though at a fist view, the use of a high permittivity is not as disadvantageous because of the scaling of dielectric losses but it is due the necessary use of lower thickness $t$ causing the ohmic losses to soar. Notice that even the undesired overattenuation peak in the dielectric losses, described in section 3.3, is present, it is practically negligible to the very undesired peak in ohmic losses. It can be also said that, comparing the DCSWW with a conventional dielectric waveguide with no metallic elements, losses are higher in the DCSWW case due to the ohmic loss contribution. This difference is perfectly described by the difference between the black $(\alpha)$ and red curves $\left(\alpha_{d}\right)$, which is quite large for reasonable, intermedium to large, values of $t$.

By the above, it can be affirmed that its large attenuation is an important drawback of DCSWW, and seems clear that the coating must be used only when necessary (bends, excitation, etc.), this suggesting the use of transitions between the SWW and DCSWW. Even in such cases, note that the losses caused by high permittivity dielectric DCSWW may be prohibitive depending of how long the required DCSWW path is. The low permittivity case, keeps on much more acceptable levels (under 0.2 $\mathrm{dB} / \mathrm{mm}$, see Fig. 3.58(a)). It must be also mentioned that $\tan \delta=0.001$ is a rather optimistic presumption that depend on the manufacturing process of the dielectric coating, and that is not extreme to think in that this factor may be easily doubled or even tripled in some real implementations, hence $\alpha_{d}$, since it is directly proportional to $\tan \delta$. In such case, dielectric losses would be predominant and the total losses may reach more challenging levels. 
Finally, dispersion of the DCSWW is studied. Unlike the SWW, where the metallic wire imposes an very impenetrable surface, hence obtaining $\eta \approx 0$ and $\beta \approx k_{0}$, in the DCSWW, the conventional dielectric used for the coating results penetrable as long as the thickness $t$ is electrically large enough, hence exhibiting the usual condition for the propagation constant of dielectric waveguides confined modes: $k_{0} \leq \beta \leq k_{1}$. In Fig. 3.59, it is shown the normalized group velocity $\left(v_{g} / c_{0}\right)$ as a function of the frequency in the operation band, for several values of $t$, equally spaced in the range $\left[0, t_{\max }\right]$ for the two considered permittivities.

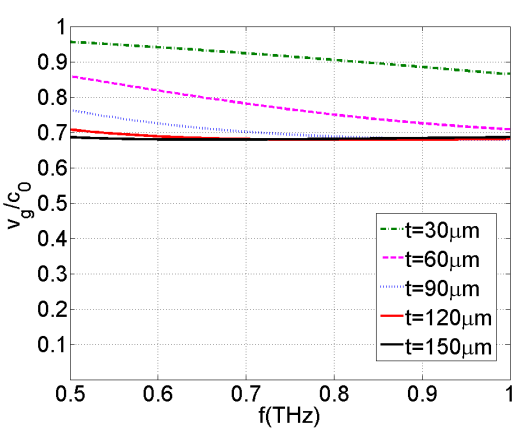

(a)

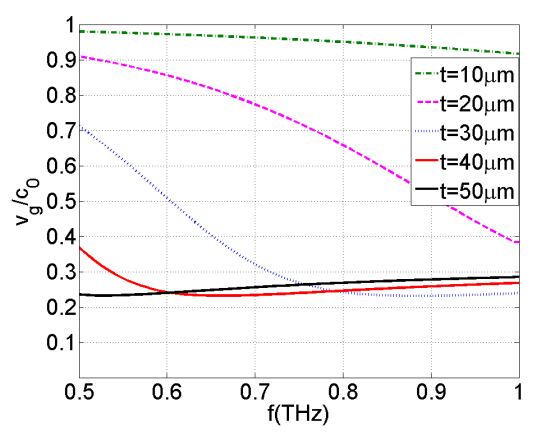

(b)

Figure 3.59: Normalized group velocity $v_{g n}=v_{g} / c_{0}$ vs. frequency in the considered band $[0.5$ $\mathrm{THz}-1 \mathrm{THz}]$ for several equally spaced values of $t$ in the range $\left[0, t_{\text {max }}\right]$. a) $\varepsilon_{r}=2$; $\left.b\right) \varepsilon_{r}=10$.

Since the normalized group velocity of a plane wave in a dielectric medium is $v_{g n}=1 / \sqrt{\varepsilon_{r}}$ (whereas $v_{g n}=1$ for a plane wave in the external air medium), some variation in $v_{g n}$ is experienced by the change in power distribution $\left(v_{g n}\right.$ is, roughly, inversely proportional to $\eta$ ). Therefore, the following situation occurs. If $t$ is small, almost not confinement is produced for all frequencies and $v_{g n} \approx 1$. Contrarily, if $t \rightarrow t_{\max }$, all frequencies become quite confined and $v_{g n} \approx 1 / \sqrt{\varepsilon_{r}}$. However, in between these cases, for intermedium values of $t$, highest frequencies are confined but lowest not, as was observed in Fig. 3.56. In such case, $v_{g}$ ranges between is extreme values, $1 / \sqrt{\varepsilon_{r}}$ and 1 , causing dispersion. By this reason, when comparing Figs. $3.59(\mathrm{a})$ and $3.59(\mathrm{~b})$, for this last $\left(\varepsilon_{r}=10\right)$ it is found that the range of values that $v_{g n}$ takes is very wide, thus indicating that high permittivity coatings with intermediate values cause a strong dispersion in the DCSWW.

If low attenuation is a good SWW feature to preserve, the almost zero dispersion is even more, inasmuch as it is the more genuine characteristic of the SWW, and what mainly justifies the large interest in this structure. Therefore, it is considerably important on practical implementation to check how much dispersion can support an ultra broadband $\mathrm{THz}$ pulse and try minimize it when the coating is present. According to Figs. 3.59 (a) and 3.59 (b), by using $t \approx t_{\max }$, the dispersion problem virtually disappear, no matter the dielectric permittivity. Since $t \approx t_{\text {max }}$ provides also better results in attenuation and the best confinement situation (maximum confinement and lowest confinement dispersion), the previous study indicate that, if the monomode condition can be guaranteed by avoiding the high order mode $\mathrm{TM}_{02}$, $t$ must be maximized up to the limiting value that provides propagation of this mode, 
i.e., the described $t_{\max }$ in Fig. 3.55. Furthermore, using $t=t_{\max }$ causes the radial extension to be minimum, collapsing almost the power in the area described by the waveguide transversal cut, which is a positive feature.

Regarding to the permittivity, the attenuation features clearly demand, in general, for the use of low permittivity dielectrics, since the more compact and with strong TIR effect high permittivity coatings impose losses that will be prohibitive for most of the designs (as usual, the final solution will depend on the design requirements, e. g., if a very compact design requiring a very short transmission path with the coating present is demanded, high $\varepsilon_{r}$ may provide a good solution.). With second order, power confinement is less dispersive for lower permittivity coatings. Finally, if $t$ is taken as maximum, $\varepsilon_{r}$ is influence-less, but if it is not the case, low $\varepsilon_{r}$ also provides better solution for the DCSWW general performance.

To end this section, 2D plots of the E-field magnitude in the cross sectional area of the main transmission zone of the DCSWW, together with 1D plots of the field components of the fundamental $\mathrm{TM}_{01}$ mode as a function as function of the radial coordinate $r$, are provided for several $t$ in Figs. 3.60-3.66 with the purpose of giving a very intuitive vision of the physics related with the aforementioned effects, thus helping to consolidate the recently given concepts. Following the previous conclusions, $\varepsilon_{r}=2$ is chosen for this study, and $t$ is varied from $t=0 \mu \mathrm{m}$ (SWW) to $t=150 \mu \mathrm{m}$ (DCSWW with $t=t_{\max }$ ) with steps of $t=25 \mu \mathrm{m}$, allowing to observe the evolution of the fields in the structure as $t$ increases. The maximum operation of the band, $f=1 \mathrm{THz}$, is the chosen frequency for the computation of the fields, and, as before, $a=200 \mu \mathrm{m}$. The 2D plots of the E-field magnitude are in logarithmic scale with $60 \mathrm{~dB}$ range. In the $1 \mathrm{D}$ plots of the EM field components, the fields are normalized to the maximum component. However, due to the usual large difference between electric and magnetic fields, $\left(E_{z}, E_{r}\right)$ and $H_{\phi}$ are normalized separately. This means that the magnitudes of $E_{z}$ and $E_{r}$ can be compared in the plots, however $H_{\phi}$ is, actually, several orders of magnitude smaller than these components. In these plots, the conductor zone is displayed with gray color whereas the dielectric coating zone is highlighted with yellow color.

The plots corresponding to $t=0 \mu \mathrm{m}$, Fig. 3.60, are calculated with the analysis tools corresponding to the SWW case being that this case is singular for the DCSWW problem. By comparing Figs. 3.60 and 3.61 it is clearly appreciated that the merely fact of having a coating, even it is rather small, is critical in the collapsing of the fields. From that point, each step of increasing $t$ gives a smaller confinement contribution.

An special attention must be focused to the case $t=50 \mu \mathrm{m}$, Fig. 3.62. This intermedium thickness causes, at $f=1 \mathrm{THz}$, the fundamental mode to be equally distributed between region 1 and region $2(\eta(\%)=50 \%$, see Fig. 3.56(a)), and origins the ohmic peak losses in Fig. 3.58(a). Inspection of the fields indicate that there is actually equally distribution between both regions. Specifying on the components, it is found that $E_{r}$ suffers a jump on the interface at $r=b$, indicating that the coating is acting as well as a surface waveguide. In this way, both interfaces $r=a$ and $r=b$ present similar behavior. Regarding to $E_{z}$, it is found that it is maximum for this thickness. In fact, if $t$ is small or large, the mode is quasi-TEM, however, for this intermedium case, $E_{z}$ peaks, indicating the more transversal incidence of the rays, an effect explained in Fig. 3.14 of section 3.3. If the permittivity was higher, this effect 
would be more noticeably, being the origin of the overattenuation peak of $\alpha_{d}$, which is observed in the case $\varepsilon_{r}=10$. Finally, $H_{\phi}$ experience its maximum confinement for this case of $t$. Note that a very significant part of this component is contained in only $t=50 \mu \mathrm{m}$. This is the origin of the attenuation peak in $\alpha_{c}$, inasmuch as surface currents are directly described by $H_{\phi}(a)$, being this value, relatively maximum for this case due to the normalized distribution in $r$. Contrarily, the dielectric losses increase monotonically as the fraction of total E-field inside the dielectric increase with each step of $t$.

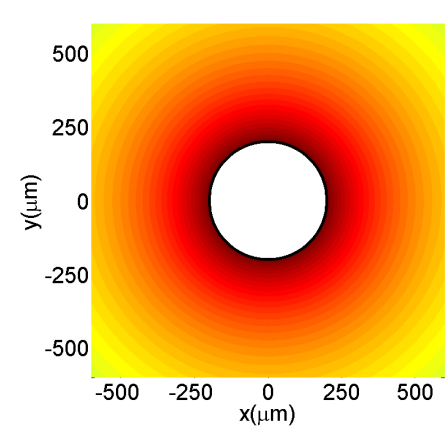

(a)

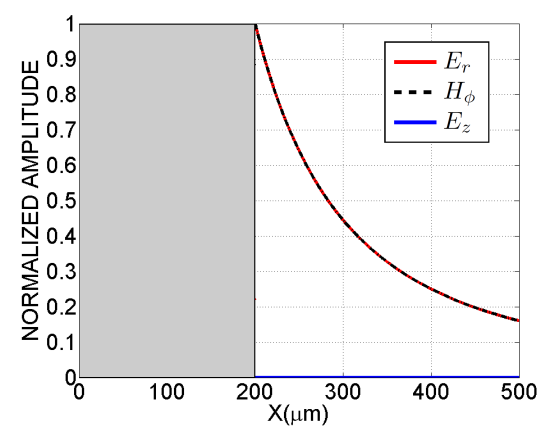

(b)

Figure 3.60: Case $t=0 \mu \mathrm{m}$. a) E-field magnitude; b) EM Field components vs. $r$.

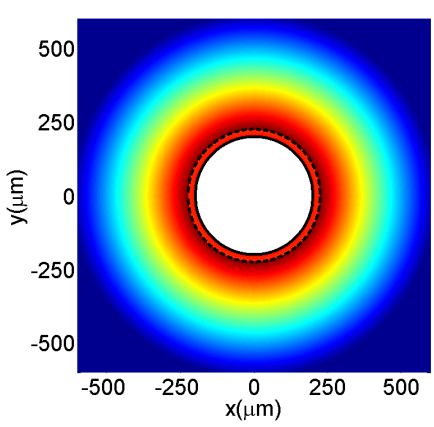

(a)

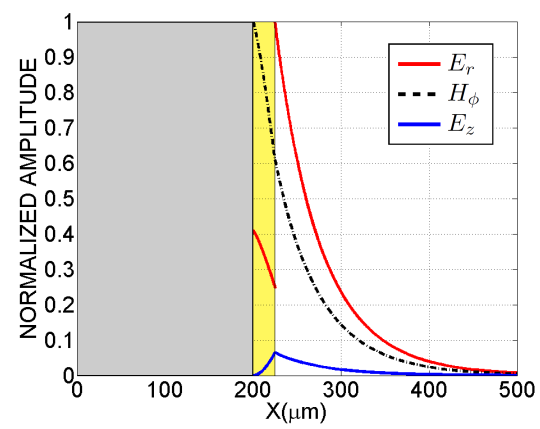

(b)

Figure 3.61: Case $t=25 \mu \mathrm{m}$. a) E-field magnitude; b) EM Field components vs. $r$.

With the previous results and plots the effect of the coating when it is placed over a SWW to form a DCSWW is well understood. At this point, it is desirable to go closer to the objective of solving the bend issue in the SWW structure by checking more exactly the performance of both wire-type structure when they are curved, and how the coating influences in that case. Therefore, the features of the analyzed SWW and DCSWW when they are bent are the object of study of the next chapter. 


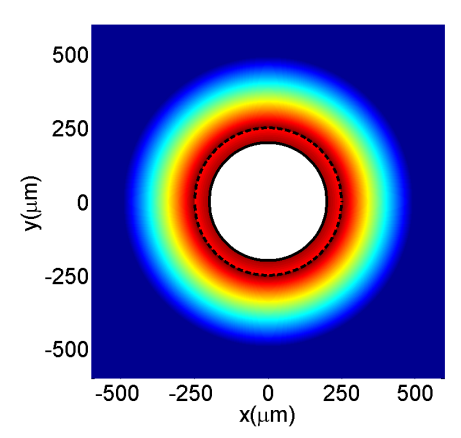

(a)

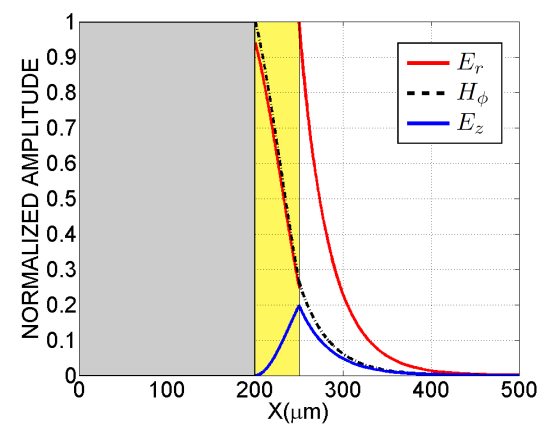

(b)

Figure 3.62: Case $t=50 \mu \mathrm{m}$. a) E-field magnitude; b) EM Field components vs. $r$.

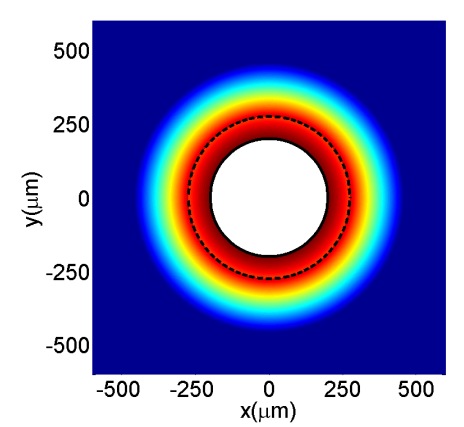

(a)

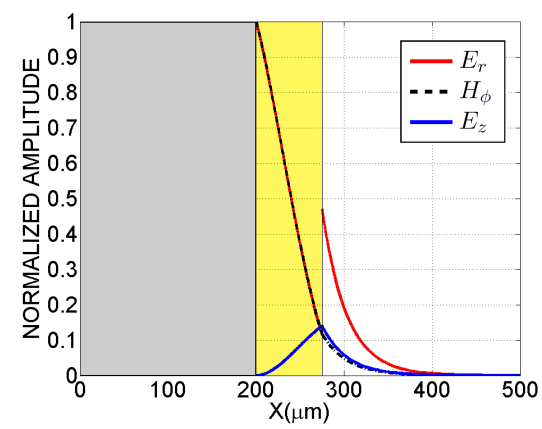

(b)

Figure 3.63: Case $t=75 \mu \mathrm{m}$. a) E-field magnitude; b) EM Field components vs. $r$.

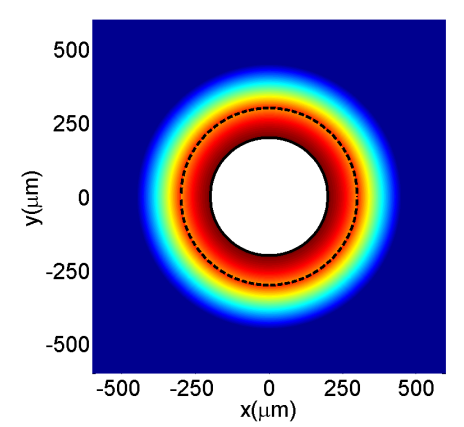

(a)

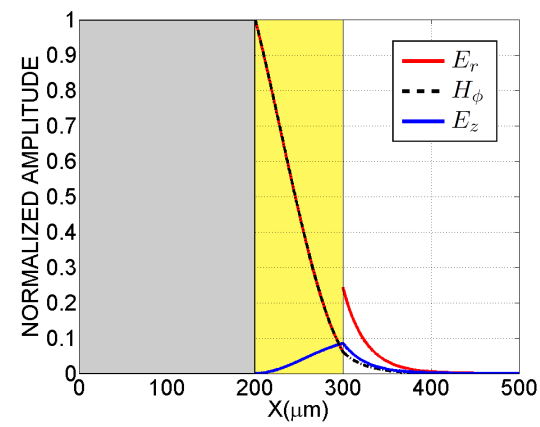

(b)

Figure 3.64: Case $t=100 \mu \mathrm{m}$. a) E-field magnitude; b) EM Field components vs. $r$. 


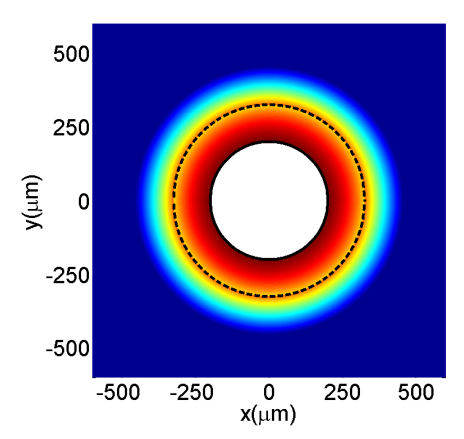

(a)

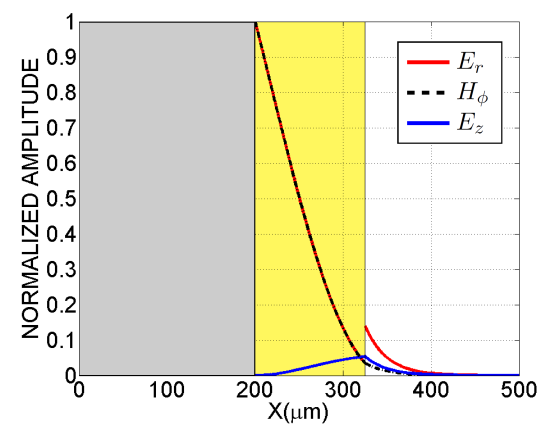

(b)

Figure 3.65: Case $t=125 \mu \mathrm{m}$. a) E-field magnitude; b) EM Field components vs. $r$.

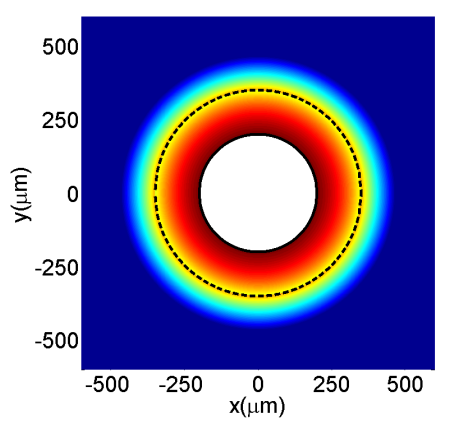

(a)

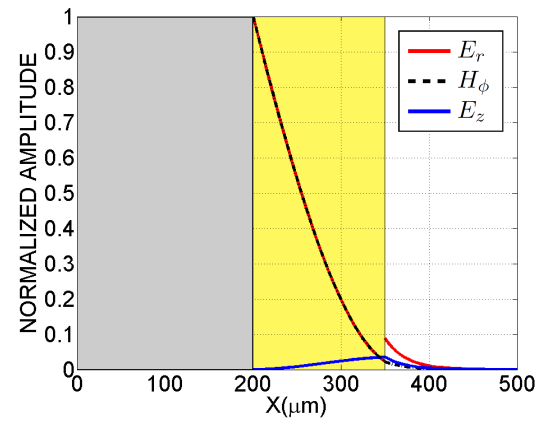

(b)

Figure 3.66: Case $t=150 \mu \mathrm{m}$. a) E-field magnitude; b) EM Field components vs. $r$. 


\section{Chapter 4}

\section{Radiation Problems and Proposed Solutions}

Open waveguides present the inherent problem of exhibiting certain radiation of the electromagnetic waves which are desired to be guided when the waveguide is bent by requirements of the path. This effect occurs even in open waveguides providing a rather strong guidance with metallic and dielectric elements. For instance, this is the case of the coplanar waveguide (CPW) and the slotline (SL) [416]-[418], or, with less relevance, even the microstrip line [404], [409]. In a similar way, dielectric waveguides must consider this source of loss, even in the case they act confining the energy and guiding it by means of the TIR effect [21], [438], [448]-[449]. However, in both cases, radiation losses are not the main problem to look at when a design must be done.

When talking about surface waveguides, the radiation loss problem reach a superior level. This is specially true when the surface waveguiding is forced to be over an impenetrable surface: high permittivity dielectrics surfaces [531], or with more evidence, metallic surfaces, as is the case of the SWW. The propagating waves in a SWW are totally unbounded, hence any perturbation easily causes the guided waves to be radiated and not to follow the path described by the waveguide. To illustrate this fact, Fig. 4.1 shows the power distribution of the fundamental $\mathrm{TM}_{0}$ mode in a SWW, and the E-field magnitude on a 90 degrees bend implemented with a typical SWW. Since for this waveguide there are not any element that force the waves to keep guided when the path changes abruptly, practically all the energy escapes from the waveguide in form of radiated waves. This means that, even a simple, unique bend, may cause extremely large losses in a SWW. In this way, it can be understood how severe is this problem inasmuch as a waveguiding structure is normally supposed to cover paths with some change of direction.

Therefore, the same impenetrable surface waveguiding nature of the SWW, which provides outstanding low losses and dispersion, is the responsible of the important drawback of strong radiation on bends. In this chapter, this problem will be afforded, trying to minimize it, in as much it is one of the key points conditioning the universal using of wire-type structures for the guiding of $\mathrm{THz}$ broadband signals. The analysis 


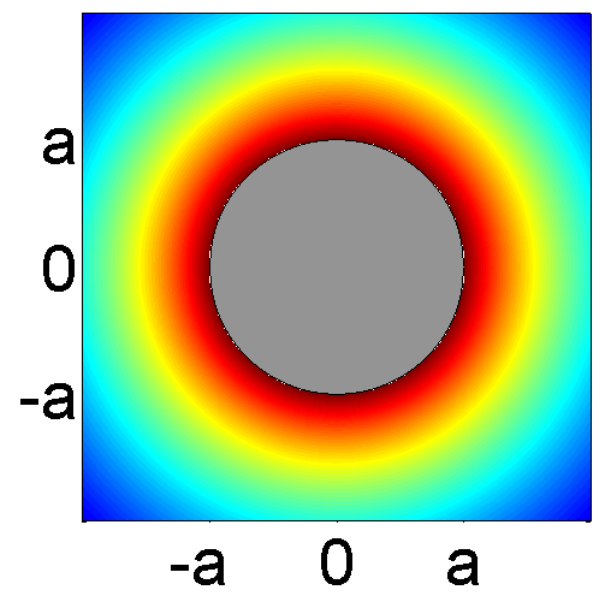

(a)

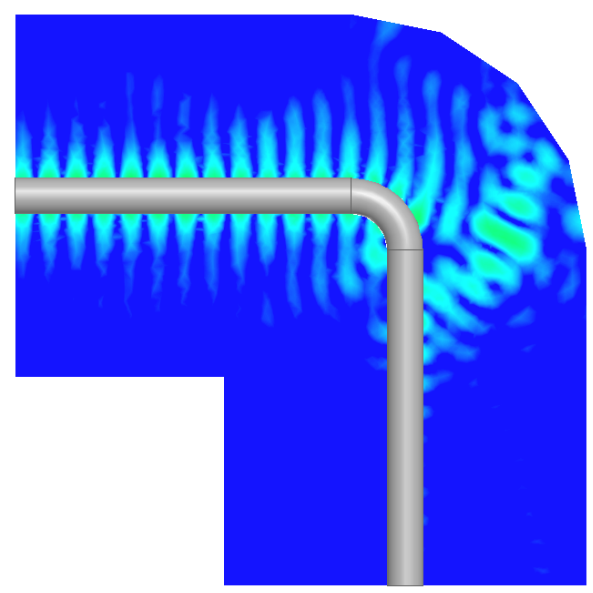

(b)

Figure 4.1: Problem of radiation on bends with the $S W W$. a) Fundamental $T M_{0}$ mode power distribution. b) Usual E-field magnitude on a 90 degree bend implemented with a $S W W$.

carried out in the previous section will give the answer about the physical origin of the radiation in these structures, and will suggest the proposed solutions by proper design of SWW and DCSWW structures, and optimized combination of them. Next, the general problem of a SWW and DCSWW curved paths is briefly described through its main parameters. Then, the two proposed solutions for bends in the scope of this thesis are postulated: a) Reduction of the wire radius [439], [584]; b) control of the curvature phase shift [585], [584]. Finally, being that the bend design should not condition the rest of the waveguiding sections, appropriate transitions are proposed for such cases in which the bend design noticeably differs from the straight path sections design [586]-[588].

\subsection{Problem Description}

The electromagnetic problem of a bend or curved path in SWW and DCSWW is illustrated in Figs. 4.2(a) and 4.2(b), respectively. Apart of the usual parameters describing the waveguide, the bend is mainly described in terms of its angle $\theta$, which indicates the change of direction, and its radius of curvature $R_{c}$, which indicates how sharp or abrupt is made this change of direction.

Along the following sections, the behavior of the SWW and the DCSWW on bends will be studied and optimized. The problem will be mainly focus from the waveguide parameters perspective, but this will not be always the case, and in some solutions the approach will come from the curve parameters $\theta$ and $R_{c}$. Generally, $\theta=90$ degrees bends will be considered insomuch as they suppose both, a common demand, and a quite challenging case. Regarding to the curvature radius $R_{c}$, it will be commonly chosen to have reasonable compact bends. Notice that the larger $R_{c}$, the softer the curve, but also the larger the curved path, which is the part of the structure in between of the dashed lines that describe $\theta$ in Figs. 4.2(a) and 


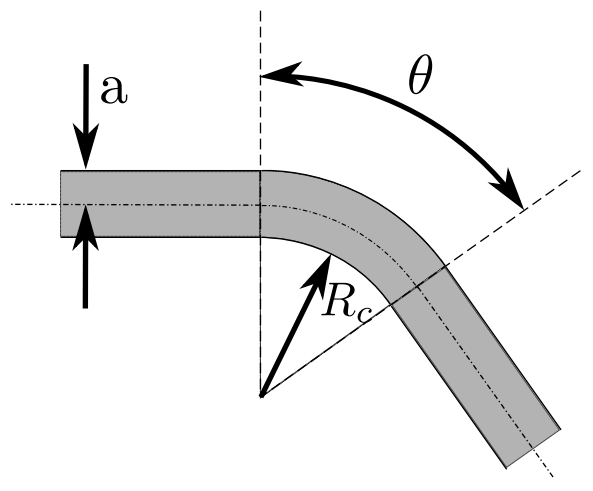

(a)

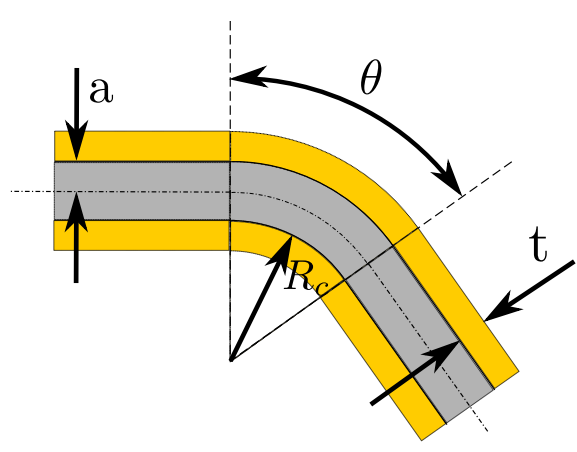

(b)

Figure 4.2: Bend problem description with general angle $\theta$ and radius of curvature $R_{c}$ for wiretype waveguides. a) $S W W$; b) $D C S W W$.

4.2(b). Very large $R_{c}$ may relax the perturbation imposed by the bend, causing less radiation, but also dispose a larger path to be covered by the waves. Since, at the bend, the waves must be more attached/confined to the waveguide, this may cause the increment in propagation losses to be as high that the solution may be counterproductive [517].

The general idea of this chapter is to exploit the fact that SWW and DCSWW have advantages and disadvantages that are complementary, i. e., whereas the SWW is low-loss and low dispersive but presents high radiation on curves, the DCSWW exhibit questionable loss and dispersion, prohibitive in some cases, but is more prepared for bends. Although, the specific solutions proposed in this thesis will appear step by step in the following, to give a first approach, the general underlying concept is illustrated in Fig. 4.3. The idea is to provide a specific solution on the bend, such as shown in Fig. 4.3(b), trying in this way that the design demanded by the bend do not excessively condition the design on the straight path. Keeping this image in mind will result helpful to understand every step of the overall process as is presented next.

\subsection{Solution A: Reduction of the wire radius}

\subsubsection{Strict monomode condition}

In section 3.6.4.1, monomode condition was ensured in its weaker form, i.e, regarding only for first axialsymmetric mode with radial polarization, the $\mathrm{TM}_{02}$. This allowed to consider coatings as thick as $t_{\max } \approx \lambda_{0} / 2 \sqrt{\varepsilon_{r}-1}$, and complete a quite wide study in such parameter. In that study, it was found that low permittivity coatings with the maximum thickness, $t=t_{\max }$, were the best option. However, a bend is quite prone to break the axialsymmetry of the fundamental mode due to the different length of the interior and exterior paths of the curvature (see the curved region in Figs. 4.2(a) and 4.2(b)). In this way, the propagation of any mode with 


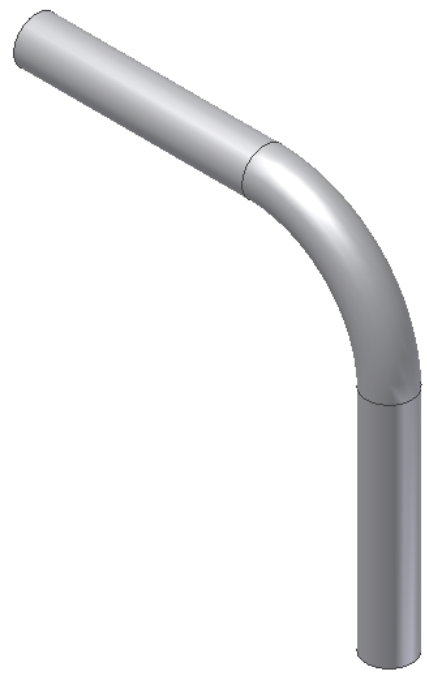

(a)

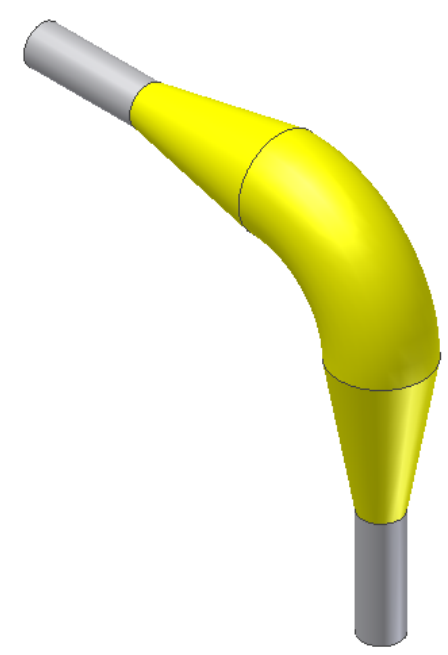

(b)

Figure 4.3: Schematic of the conceptual idea of optimal combination of $S W W$ and DCSWW structures by using the coating (DCSWW) only when necessary, and using proper transitions to interconnect both wire-type waveguides. a) Conventional bend with $S W W$; b) Optimized bend using the coating only during the curved path to reduce the radiation.

similar polarization to the $\mathrm{TM}_{01}$ should be considered, and strategies to prevent or minimize the excitation of such undesired modes must be studied.

In the study carried out next, it will be considered again the band ranging from $0.5 \mathrm{THz}$ to $1 \mathrm{THz}$, focusing the main interest when the frequency is particularized to the maximum frequency, $f=1 \mathrm{THz}$. Attending to the low permittivity criteria, $\operatorname{TPX}^{\circledR}\left(\varepsilon_{r}=2.1, \tan \delta=0.001\right.$ at $\left.f=1 \mathrm{THz}[21]\right)$ is chosen as dielectric. Copper $\left(\sigma=5.81 \cdot 10^{7} \mathrm{~S} / \mathrm{m}\right)$ is chosen as conductor.

First, in Fig. 4.4(a) it is shown the percentage of power $\eta(\%)$ inside the dielectric medium as a function of the coating thickness $t$ for the first propagating modes of a DCSWW with $a=100 \mu \mathrm{m}$. The parameter $\eta(\%)$ gives a good estimation of the matching between the mode patterns in terms of power distribution, hence estimating the probability of coupling by this factor. It is observed how, for this moderate radius, in between the curves of the $\mathrm{TM}_{01}$ and $\mathrm{TM}_{02}$ modes, this last starting at $t_{\max }=142 \mu \mathrm{m}$, there are many other modes appearing. Green and red curves correspond to TE and HE nature modes, which polarization is orthogonal to that of the fundamental mode, thus providing very low polarization matching for coupling. However, they are observed the first three $\mathrm{HE}_{n 1}$ modes, which due to their radial polarization are very suitable to couple when they have $\eta(\%)$ values close to that of the fundamental mode. Particularly, the $\mathrm{HE}_{11}$ mode practically degenerates with the fundamental mode, and the $\mathrm{HE}_{21}$ mode is very suitable to couple for intermedium values of $t$ in the considered range. In the case in which coupling to these modes occurred during the bend, some frequency dispersion would be experienced by the different $\beta$ of the $\mathrm{HE}_{21}$ mode. Furthermore, when the coating was removed, all the power being transmitted by both, the $\mathrm{HE}_{11}$ mode and the 
$\mathrm{HE}_{21}$ mode, would be lost in as much they convert to the $\mathrm{HE}_{1}$ mode and the $\mathrm{HE}_{2}$ mode of the SWW. The same can be extended to the $\mathrm{HE}_{31}$ in the case that suitable conditions for its coupling are provided, i. e., choosing large enough $t$.

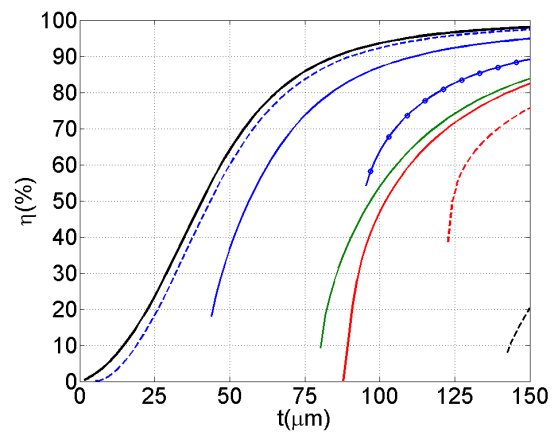

(a)

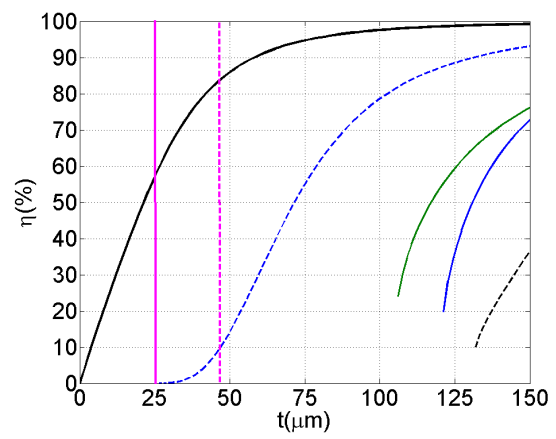

(c)

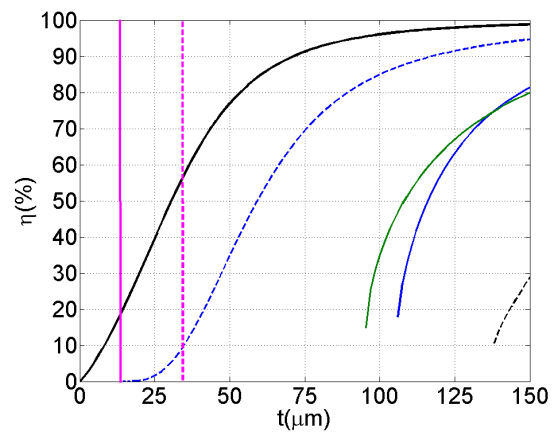

(b)

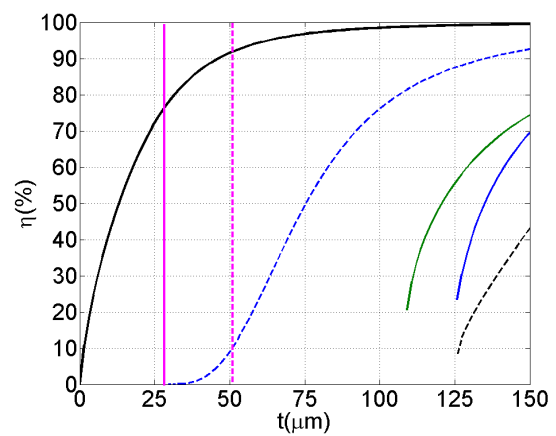

(d)

\section{$-\mathrm{TM}_{01}-\mathrm{HE}_{11}-\mathrm{HE}_{21}-\mathrm{HE}_{31}-\mathrm{TE}_{01}-\mathrm{EH}_{11}-\mathrm{EH}_{21}--\mathrm{TM}_{02}$}

(e)

Figure 4.4: Percentage of power inside the dielectric coating $\eta(\%)$ vs. coating thickness $t$ for the first modes in a DCSWW operating at $f=1 \mathrm{THz}$. Conductor is copper $\left(\sigma=5.81 \cdot 10^{7} \mathrm{~S} / \mathrm{m}\right)$ and dielectric is $\operatorname{TPX}^{\circledR}\left(\varepsilon_{r}=2.1, \tan \delta=0.001\right)$. a) $a=100 \mu \mathrm{m}$; b) $a=20 \mu \mathrm{m}$; c) $a=5 \mu \mathrm{m}$; d) $a=1 \mu \mathrm{m} ;$ e) Mode legend.

In order to prevent this situation, the strategy of reducing the ratio aspect $R=a / b$, proposed in section 3.6.2 will be used. As indicated there, the adequate way to proceed is reducing $a$ while $b$ is kept fixed, since, otherwise, the problem is scaled in frequency. Following this premise, several DCSWW with smaller radius than previous are studied. In Figs. 4.4(b)-4.4(d) it is shown the same information as before ( $\eta$ vs. $t$ ), but for smaller radius, of $a=20 \mu \mathrm{m}, a=5 \mu \mathrm{m}$ and $a=1 \mu \mathrm{m}$, respectively. The effect on $\mathrm{HE}$ and $\mathrm{EH}$ modes of this action is noticeably. As the radius decrease, they require much more coating thickness $t$ to become confined. Note also how the axialsymmetric modes, $\mathrm{TE}_{01}$ and $\mathrm{TM}_{02}$, experience only a small change in their curves, since these modes are practically only influenced by the coat- 
ing thickness. The most important effect is that the degeneracy between $\mathrm{TM}_{01}$ and $\mathrm{HE}_{11}$ modes is broken. Not only the $\mathrm{HE}_{11}$ requires larger values of $t$ to be confined, but the fundamental mode shows an anomalous behavior, becoming confined with lower values of $t$ than with the case of larger radius.

Despite of the fact that $\mathrm{TM}_{01}$ and $\mathrm{HE}_{11}$ modes broken its degeneracy, choosing a $t$ enough small to force the $\mathrm{HE}_{11}$ to its virtual cutoff (see vertical solid pink lines in Figs. 4.4(b)-4.4(d)) may cause the $\eta(\%)$ of the fundamental mode to be not large enough, hence having not the desired confinement to prevent radiation via TIR effect. By this, it seems more reasonable to establish as a monomode criteria that $\eta<10 \%$ for the $\mathrm{HE}_{11}$ (see vertical dashed pink lines in Figs. 4.4(b)-4.4(d)), together with the imposition of the fundamental being quite more confined. In this way, $\eta$ of both modes will differ enough to cause low coupling efficiency. Therefore, from the results in Figs. 4.4(b)-4.4(d), it seems that $a=5 \mu \mathrm{m}$ offers a suitable choice, being that $a=20 \mu \mathrm{m}$ does not offer enough large difference between $\eta$ of $\mathrm{TM}_{01}$ and $\mathrm{HE}_{11}$ modes at the criteria point, and $a=1 \mu \mathrm{m}$, apart of its inherent high attenuation losses, may result very challenging for the manufacturing process. If $a=5 \mu \mathrm{m}$, the thickness satisfying $\eta<10 \%$ for the $\mathrm{HE}_{11}$ mode is $t=40 \mu \mathrm{m}$

The previous information can be approached from other point of view that can provide good insight to understand the concepts. In Fig. 4.5, the percentage of power in the dielectric medium $\eta(\%)$ for $\mathrm{TM}_{01}, \mathrm{HE}_{11}$ and $\mathrm{HE}_{21}$ modes is displayed this time as a function of the radius $a$, showing each plot the results for a particular $t$. The effect of reducing $a$ is the divergence of the $\mathrm{TM}_{01}$ mode and $\mathrm{HE}_{11}$ mode curves, which is much better quantified in these plots. However, to cause enough difference between both curves without requiring very small radius, the thickness must be kept small enough. See how the situation for $t=75 \mu \mathrm{m}$ and $t=100 \mu \mathrm{m}$, Figs. 4.5(c) and 4.5(c), is not suitable since $\eta$ of the $\mathrm{HE}_{11}$ mode cannot be diminished as desired by decreasing $a$ in the considered range, and even the $\mathrm{HE}_{21}$ mode is suitable to couple for some of the radius in the considered range.

On the other hand, in the case of $t=25 \mu \mathrm{m}$, Fig. $4.5(\mathrm{a})$, the confinement of the $\mathrm{HE}_{11}$ mode can be made small, however, the confinement of the fundamental $\mathrm{TM}_{01}$ mode does not reach the desired enough large values. Finally, the case $t=50 \mu \mathrm{m}$, Fig. 4.5(b), provides a good difference between the $\mathrm{TM}_{01}$ mode and $\mathrm{HE}_{11}$ mode curves. Probably, a slight smaller $\eta$ for the $\mathrm{HE}_{11}$ mode would be desirable if the criteria is $\eta<10 \%$ for this mode, thus concluding that interesting values of $t$ are comprised in between of $t=25 \mu \mathrm{m}$ and $t=50 \mu \mathrm{m}$. The previous choice $(a=5 \mu \mathrm{m}$, $t=40 \mu \mathrm{m})$ is in agreement with this.

In order to endorse the decision of choosing $(a=5 \mu \mathrm{m}, t=40 \mu \mathrm{m})$, the propagation characteristics of a DCSWW with these dimensions are compared to those of the cases corresponding to $a=20 \mu \mathrm{m}$ and $a=1 \mu \mathrm{m}$, choosing in each case, the thickness that satisfies the $\eta<10 \%$ criteria for the $\mathrm{HE}_{11}$ mode. First, in Fig. 4.6(a), the attenuation in $\mathrm{dB} / \mathrm{mm}$ is shown in the operating band $[0.5 \mathrm{THz}-1 \mathrm{THz}]$ for the aforementioned cases.As can be observed, with $a=5 \mu \mathrm{m}$, losses keep under acceptable levels. However, in the case of a very small radius, $a=1 \mu \mathrm{m}$, losses raise to such level that the solution for the bend may be counterproductive, becoming the propagation losses more important than the radiation losses.

Second, in Fig. 4.6(b), the normalized velocity group is shown for the three considered DCSWW designs. In all of them the dispersion is quite acceptable thanks 


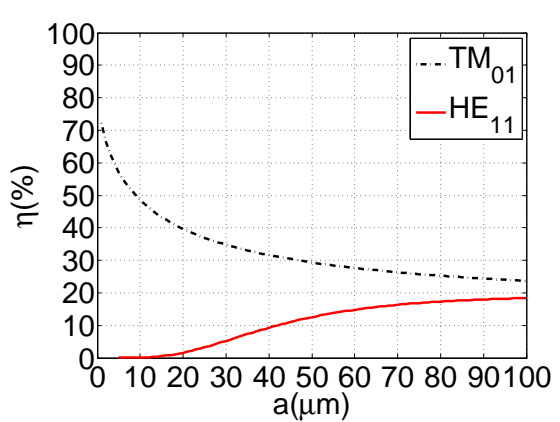

(a)

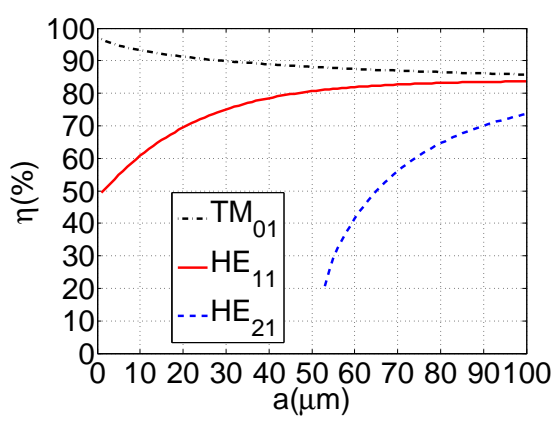

(c)

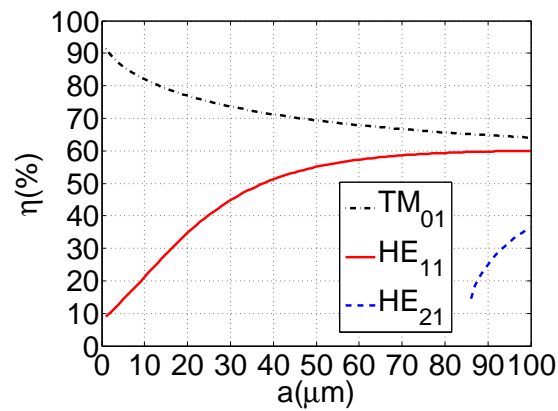

(b)

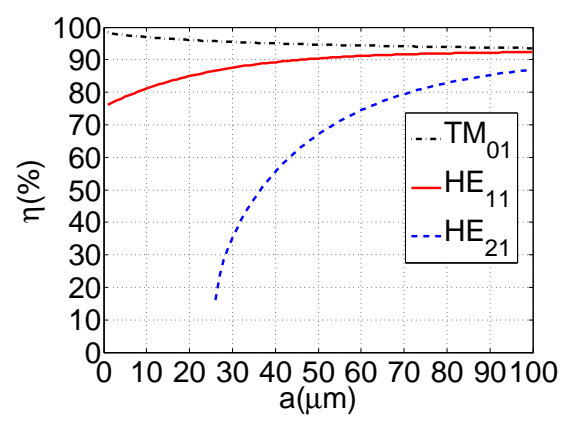

(d)

Figure 4.5: Percentage of power inside the dielectric coating $\eta(\%)$ vs. conductor radius a for the fundamental mode $\left(T M_{01}\right)$, the first higher order mode $\left(H E_{11}\right)$ and, in some cases, the second higher order mode $\left(\mathrm{HE}_{21}\right)$ of a DCSWW operating at $f=1 \mathrm{THz}$. Conductor is copper $\left(\sigma=5.81 \cdot 10^{7} \mathrm{~S} / \mathrm{m}\right)$ and dielectric is $\operatorname{TPX}^{\circledR}\left(\varepsilon_{r}=2.1, \tan \delta=0.001\right)$. a) $\left.t=25 \mu \mathrm{m} ; b\right) t=50 \mu \mathrm{m}$; c) $\left.t=75 \mu \mathrm{m} ; b\right)$ $t=100 \mu \mathrm{m}$.

to the low permittivity choice. Particularly, the $a=5 \mu \mathrm{m}$ case shows a variation under $10 \%$ between the minimum and maximum frequencies velocity group.

From these graphs, $(a=5 \mu \mathrm{m}, t=40 \mu \mathrm{m})$ seems a reasonable choice. Moreover, due to the exponential grow of losses as a decreases, and the foreseeable manufacturing complexity, DCSWW designs with radius between $a=5 \mu \mathrm{m}$ and $a=20 \mu \mathrm{m}$ are preferred. As a data of interest, successful experiments with small radius SWW/DCSWW $(a=10 \mu \mathrm{m}$ to $a=30 \mu \mathrm{m})$ [507], [513], [514], and manufacturing of DCSWW with conductor radius ranging from $a=0.4 \mu \mathrm{m}$ to $a=15 \mu \mathrm{m}$ and coating thickness ranging $t$ from $t=2 \mu \mathrm{m}$ to $t=15 \mu \mathrm{m}$ have been reported in the last years [589].

\subsubsection{Numerical simulations and validation of the models}

Analytical resolution of a curved SWW or DCSWW may be very complex and any further change of the electromagnetic problem, such as inclusion of transitions would imply the model to be not valid anymore. Therefore, to solve the electromagnetic problem of propagation on bends in wire-type structures, numerical resolution 


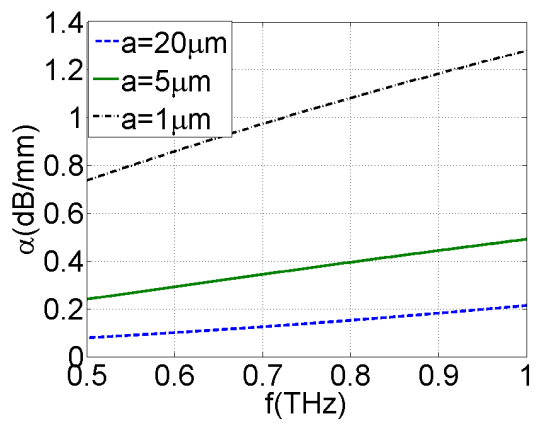

(a)

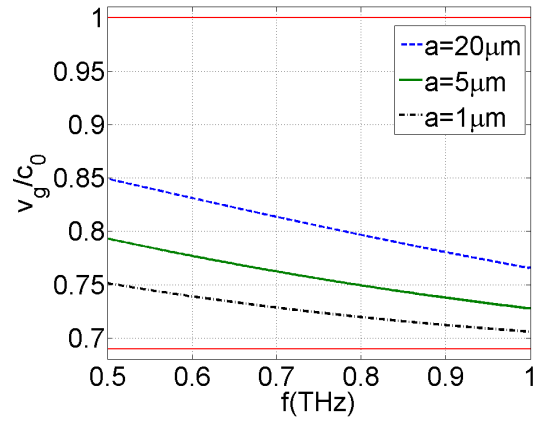

(b)

Figure 4.6: Propagation characteristics of a DCSWW with conductor copper $\left(\sigma=5.81 \cdot 10^{7} \mathrm{~S} / \mathrm{m}\right)$ and dielectric is $\operatorname{TPX}^{\circledR}\left(\varepsilon_{r}=2.1, \tan \delta=0.001\right)$, for several small radius $(a=20 \mu \mathrm{m}, a=5 \mu \mathrm{m}$ and $a=1 \mu \mathrm{m})$ in the considered operation band [0.5 THz-1 THz]. a) Attenuation in $\mathrm{dB} / \mathrm{mm} ; b)$ Normalized velocity group.

through conventional commercial solvers is preferred. In this case, HFSS ${ }^{\circledR}$ [411], which implements the Finite Element Method (FEM) [590], has been chosen.

Whereas in closed structures the use of the FEM technique is obvious, as the space to grid is finite, in the case of open structures the problem must be treated more carefully since the infinite space involved in the problem must be truncated to a finite one and appropriate boundary conditions must be used. The base simulation model for the SWW and DCSWW structures is shown in Fig. 4.7(a). This model consist in a finite length section of waveguide in the axial direction (matched at both ports), and truncated transversely in the external air medium at $r=R$. Radiation boundary conditions are displayed at the boundary described by $r=R$. According to this base model, and the problem description of Fig. 4.2, the model of a bend has been developed as shown in Fig. 4.7(b).

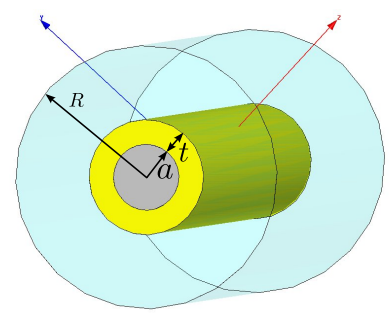

(a)

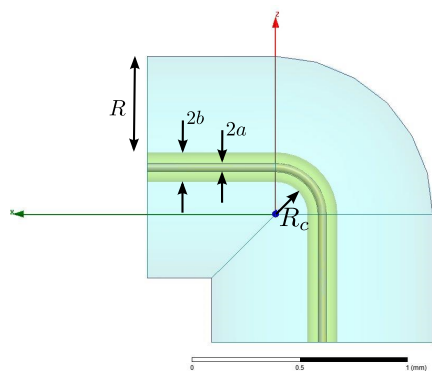

(b)

Figure 4.7: HFSS $^{\circledR}$ model for wire-type structures. External medium is truncated at $r=R$. a) Straight path; b) Bend.

The choice of the external medium truncating radius $R$ results critical inasmuch as it must be large enough to not cause errors by disregarding not negligible fields, but small enough to enable the simulation to be afforded with a reasonable com- 
putation time. Note that the total volume increase with $R^{3}$ and that, the larger $R$, the larger the required straight paths of waveguide in the model of the bend, so that the model elements do not intersect causing errors in the solver. Therefore, a study to properly choose a correct value of $R$ results to be a mandatory step. To do that, the worse situation is considered. As concluded in previous chapter, in SWW and DCSWW structures, the larger extension of fields take place for larger radius. In this study, the maximum radius to be considered will be $a=100 \mu \mathrm{m}$, hence it is chosen for the $R$ test. This test will consist in comparison of the simulation results using the model of Fig. 4.7(a) and the analytical models of previous chapter.

In Fig. 4.8(a) calculations of the propagation constant of the fundamental $\mathrm{TM}_{01}$ mode of a DCSWW, obtained with the analytical model (solid lines) and simulations (empty circles), are compared as a function ${ }^{1}$ of the truncation radius $R$ for several cases of thickness $t$. It is clearly observed that simulation results rapidly converge to those predicted by the analytical model as $R$ increases. Similarly, the attenuation constant provided by both models is compared in Fig. 4.8(b). In this case, differences are more appreciable when $R$ is not large enough. This is very evident for the case of using a thin coating (see the $t=5 \mu \mathrm{m}$ curve, in blue), caused by the fact that the effective transversal area of transmission is larger in such situation. Actually, since the truncation of the transversal area implies some power to not be taken into account, losses are consequently predicted larger in this case. However, reasonable values of $R$ give very good agreement between numerical simulations and analytical results. A suitable choice seems to be $R=0.5 \mathrm{~mm}$, which has been tested also as a truncation radius that allows for not long simulations.

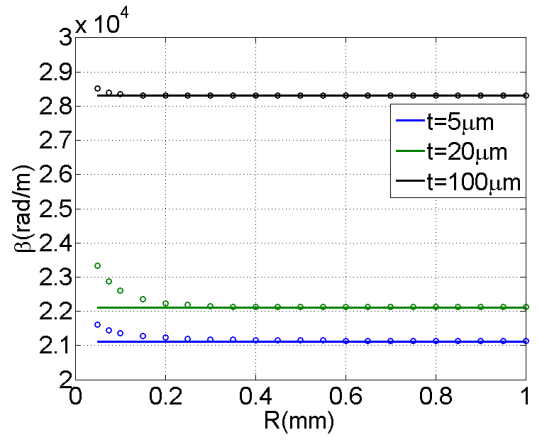

(a)

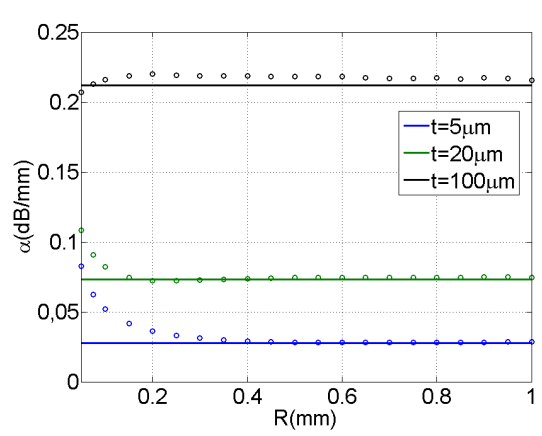

(b)

Figure 4.8: Test of the HFSS ${ }^{\circledR}$ model to determine an adequate value of $R$ to give accurate simulations. Propagation and attenuation constants are calculated by simulation as a function of $R$ for a DCSWW $a=100 \mu \mathrm{m}$, considering several coating thickness values $t$, and compared with those provided by the analytical models developed in chapter 3. Conductor copper $\left(\sigma=5.81 \cdot 10^{7} \mathrm{~S} / \mathrm{m}\right)$ and dielectric is $\operatorname{TPX}^{\circledR}\left(\varepsilon_{r}=2.1, \tan \delta=0.001\right)$. a) $\beta(\mathrm{rad} / \mathrm{m})$ vs. $\left.R(\mathrm{~mm}) ; b\right) \alpha(\mathrm{dB} / \mathrm{mm})$ vs. $R(\mathrm{~mm})$.

Before applying the chosen truncation radius $R$ to the resolution of problems including propagation on bends, it is firstly tested by comparing, for some cases

\footnotetext{
${ }^{1}$ The only curves to which refer $R$ are those corresponding to numerical simulation, hence analytical results are horizontal curves showing the exact value.
} 
of interest, the dispersion diagram of a DCSWW provided by simulations using this value of $R$ with its counterpart provided by the analytical model developed in the previous chapter. Results of this analysis are shown in Fig. 4.9, where the propagation constant of the first modes of a DCSWW is shown as a function of the coating thickness $t$ for the cases of $a=5 \mu \mathrm{m}$, Fig. 4.9(a), and $a=20 \mu \mathrm{m}$, Fig. 4.9(b). There is an observable good agreement between simulations and analytical model results, specially for the fundamental mode, thus confirming that the chosen $R$ provides the desired accuracy. Observe that, since coupling to high-order modes is a recurrent phenomena to observe in this study, the model must be tested not only with the fundamental mode, but must provide reliable information about high-order modes.

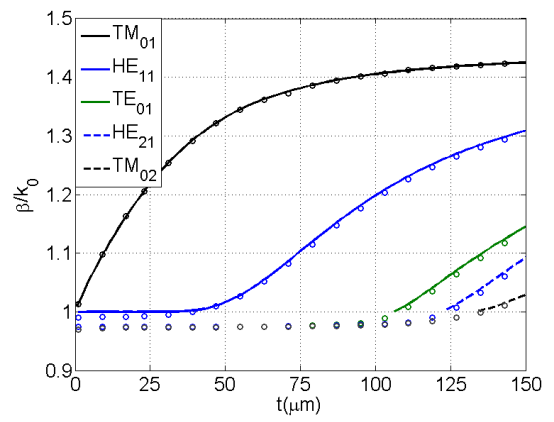

(a)

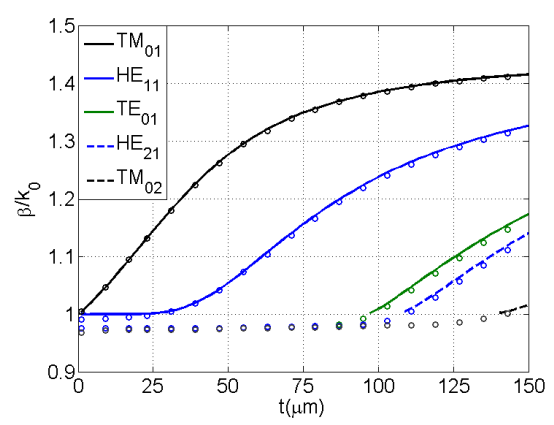

(b)

Figure 4.9: Test of the HFSS ${ }^{\circledR}$ model with the chosen value $R=0.5 \mathrm{~mm}$. The simulated normalized propagation constant $\beta / k_{0}$ of the first modes of a DCSWW is displayed as a function of the coating thickness $t$ and compared with the calculations through the analytical models developed in chapter 3. a) $a=20 \mu \mathrm{m}$; b) $a=5 \mu \mathrm{m}$.

Determined the model, the propagation on a SWW $90^{\circ}$ bend is firstly analyzed. The chosen frequency is the maximum of the considered band ( $f=1 \mathrm{THz}$ ). Two radius are considered: $a=100 \mu \mathrm{m}$ as a large radius case, and $a=5 \mu \mathrm{m}$, as a small radius case. The curvature radius is set to $R_{c}=0.2 \mathrm{~mm}$, which imply a rather compact bend. In the first row of images in Fig. 4.10 it is represented the E-field in the structure, displayed in logarithmic scale. In the case of $a=100 \mu \mathrm{m}$, Fig. 4.10(a), the negative effect of the bend results evident since the amount of power reaching the output port after the bend is negligible. It is observed how the propagating waves, which are guided just attached to the surface, do not follow the change of direction, and continue in the direction they were following, being radiated away from the waveguide.

The effect that a simple reduction of radius has, with even no coating, see the case of $a=5 \mu \mathrm{m}$ in Fig. 4.10(a), is very interesting. As pointed out in section 3.5.2, the reduction of the radius makes the fundamental $\mathrm{TM}_{0}$ mode to interact more with the guiding surface, causing more losses and larger compactness of the effective transmission area. Here, this larger interaction is proved to result interesting in order to keep the propagating surface waves guided by the SWW and not to show an extremely large radiation as the large radius case. There are still some radiation 
losses, that can be qualitatively appreciated by the decrement of E-field intensity at the output port, but, visually, the waveguide is able in this case to force the guided waves to follow a non-straight path as sharp as it is the proposed bend.

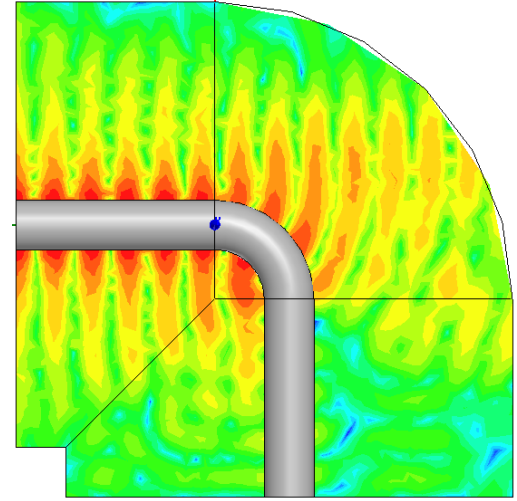

(a)

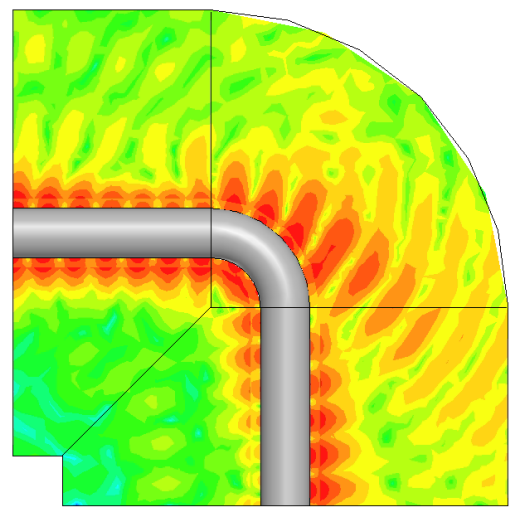

(c)

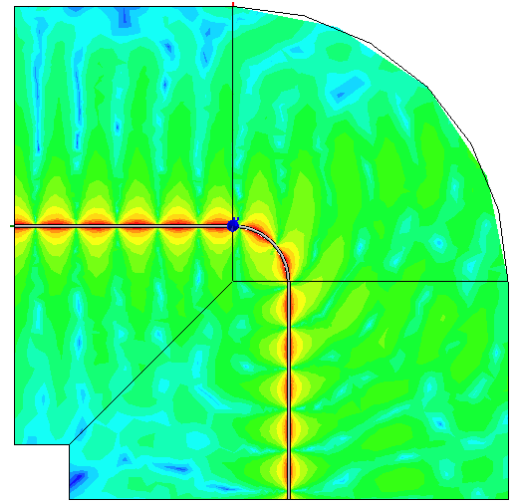

(b)

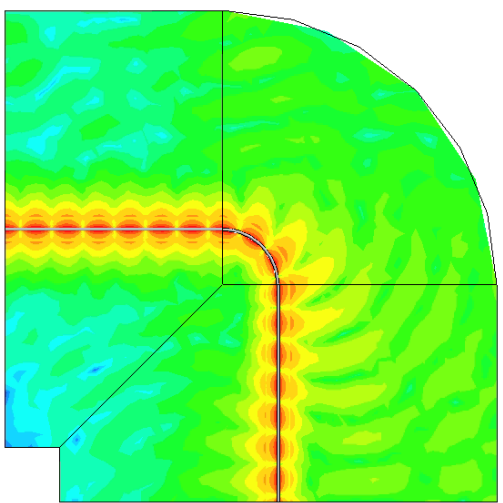

(d)

Figure 4.10: E-field magnitude in logarithmic scale in a 90 degree bend implemented with a bare $S W W$ (first row) and with a DCSWW having a coating of $t=40 \mu \mathrm{m}$ (second row). The curvature radius is $R_{c}=200 \mu \mathrm{m}$. a) $S W W, a=100 \mu \mathrm{m}$; b) $S W W, a=5 \mu \mathrm{m}$; c) DCSWW, $a=100 \mu \mathrm{m}$ and $t=40 \mu \mathrm{m} ;$ d) $D C S W W, a=5 \mu \mathrm{m}$ and $t=40 \mu \mathrm{m}$.

The same bend with an added TPX ${ }^{\circledR}$ coating is considered now. Specifically, a coating thickness of $t=40 \mu \mathrm{m}$ is chosen in as much this thickness provide the strict monomode condition for the case of $a=5 \mu \mathrm{m}$. In the case of $a=100 \mu \mathrm{m}$ this coating (and even a much thinner one) allows some high-order modes to propagate. However, the $\mathrm{TM}_{02}$ is not in that set of modes. In this way, weak and strict monomode conditions proposed in 3.6 are tested to check their validity or necessity for this particular problem.

Results of the simulation are shown in the second row of images of Fig. 4.10. The coating helps to prevent radiation in both cases. In the case of $a=100 \mu \mathrm{m}$ the difference is very noticeable, Fig. 4.10(c). Despite of this fact, for this large 
radius case, the aspect of the E-field magnitude suggests some undesired effects. On the one hand, there is still some power radiated as the fundamental mode is less confined inside the dielectric than in the case of a small radius (see $4.10(\mathrm{~d})$ ). On the other hand, despite a noticeable amount of power reaches the output port, the asymmetry on the field pattern indicates high coupling to the $\mathrm{HE}_{11}$ mode or even higher order $\mathrm{HE}_{n 1}$ modes. Since coupling occurs, the problem of lack of confinement of the fundamental mode cannot successfully solved, being that increasing $t$ would even enhance the undesired coupling. Contrarily, for $a=5 \mu \mathrm{m}$, Fig. $4.10(\mathrm{~d})$, the E-field indicates an improvement respect to the SWW in the desired way.

The previous plots help to visualize the radiation problem on bends and the effect of reducing the wire radius or adding the dielectric coating in a very intuitive way. However, to more specifically quantify the improvement achieved by the previous actions it is more suitable to represent the power that reach the output port of the bend. It is very important in this case to distinguish the mode that transmits that power, being that the power that is not transmitted by the fundamental $\mathrm{TM}_{01}$ is, generally, power to be lost.

In Fig. 4.11, the $S_{21}$ parameter of the analyzed two-port bend structure of Fig. 4.7(b), de-embedeed so that only the curved path losses are obtained, is represented as a function of the coating thickness $t$ for the two considered radius in this study: $a=100 \mu \mathrm{m}$, Fig. 4.11(a), and $a=5 \mu \mathrm{m}$, Fig. 4.11(b). Observe how for the $a=100 \mu \mathrm{m}$ case, the amount of power coupled to the $\mathrm{HE}_{11}$ is considerably high. Even for small values of $t$, the power transmitted by the $\mathrm{HE}_{11}$ mode is even larger than that transmitted by the fundamental $\mathrm{TM}_{01}$ mode, and, although more power reach the output as $t$ increases, this additional power is carried by the $\mathrm{HE}_{11}$ mode so that if the coating was lately removed, this power would be lost. What occurs for this large radius case is that the difference in length between the internal and external paths on the curved section is large enough to produce an appreciable phase shift in the $\mathrm{TM}_{01}$ mode pattern, exciting the $\mathrm{HE}_{11}$ mode. Specifically, the difference between inner and outer path is given by:

$$
\begin{aligned}
\Delta L & =\left(R_{c}+2 a\right) \theta-R_{c} \theta \\
& =2 a \theta
\end{aligned}
$$

where $\theta$ is in radians. Taking into account that for $a=100 \mu \mathrm{m}, \Delta L=200 \mu \mathrm{m}$, and that $\lambda_{0}=300 \mu \mathrm{m}$ ( $\lambda_{g}$ slightly smaller), it is understood that the phase shift, rather close to $\pi$ radians than to $2 \pi$ radians, causes a phase shift in the $\mathrm{TM}_{01}$ mode pattern very suitable for the undesired coupling to the $\mathrm{HE}_{11}$ mode.

Contrarily, in the case of $a=5 \mu \mathrm{m}$, Fig. 4.11(b), the coupling to the $\mathrm{HE}_{11}$ mode results to be small unless $t$ reach quite large values. The power received in the $\mathrm{TM}_{01}$ mode is close to $-2 \mathrm{~dB}$ respect to the input power, being appreciated how it diminishes as the $\mathrm{HE}_{11}$ mode starts to couple more. Note that the maximum received power in the $\mathrm{TM}_{01}$ mode occurs for $t=40 \mu \mathrm{m}$ approximately, indicating that the study of the modal spectrum in terms of the power confinement parameter $\eta$ is a very powerful design tool when the coupling to high-order modes is a problem to give treatment, being that, with the established criteria, any further optimization has been necessary. 


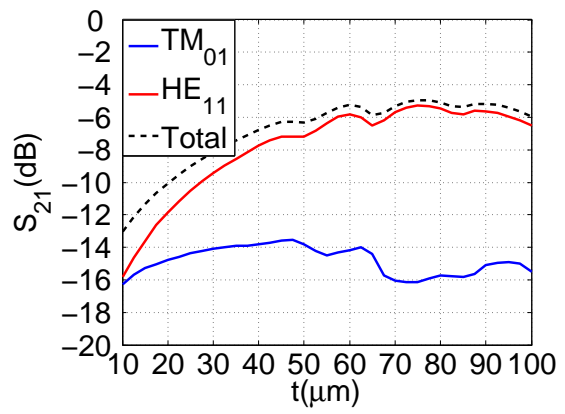

(a)

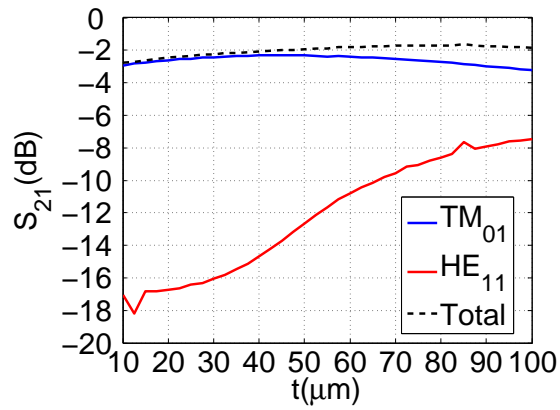

(b)

Figure 4.11: Study of the coupled power to the first higher order mode $\left(H E_{11}\right)$ in a 90 degree bent DCSWW: $S_{21}(d B)$ vs. coating thickness $t$ for the fundamental $T M_{01}$ mode and the $H E_{11}$ mode. a) $a=100 \mu \mathrm{m}$; b) $a=5 \mu \mathrm{m}$.

\subsubsection{Conclusions and important remarks}

By the study carried out in this question, important conclusions are obtained. First, the truncation radius $R$ is a parameter to check, and it must be properly chosen to provide good enough accuracy and low computation time. Good accuracy in the numerical simulation of SWW and DCSWW open structures has been proven to be possible. Second, and with more relevance, it is the fact that the radius $a$ has much more influence than the thickness $t$ in minimizing the negative effects of a bend in wire-type structures. It has been shown that the origin of this fact relies on the large coupling to higher order modes when the radius is large. A proper study of the modal spectrum of the DCSWW in terms of the power distribution has been shown to be a powerful design tool, which allows to minimize the coupling to undesired high-order modes. In the case of bends, it has been shown that the strict monomode condition of having at least $\eta<10 \%$ for the $\mathrm{HE}_{11}$ mode is the right condition in as much $\mathrm{HE}_{n 1}$ modes are very suitable to couple in bends due to the phase shift caused by the different lengths of the interior and exterior bend paths in the mode pattern. In this sense it is pointed out that other perturbations or situations suitable of mode coupling, such as the excitation process, may allow for a weaker condition, such that provided by the $\mathrm{TM}_{02}$ mode, as long as this phase shift effect does not occur.

In this section, a $90^{\circ}$ bend exhibiting only $2 \mathrm{~dB}$ losses at $f=1 \mathrm{THz}$ has been achieved by choosing a DCSWW with $(a=5 \mu \mathrm{m}, t=40 \mu \mathrm{m})$. In this bend, the losses are only slight dependent on $t$, what gives additional manufacturing flexibility. This losses are noticeably smaller than those obtained with the usual large radius SWW and DCSWW, normally beyond $15 \mathrm{~dB}$. However, straight path losses of such design are prohibitively large $(0.5 \mathrm{~dB} / \mathrm{mm})$ for reasonable practical distances. Therefore, it is clear that the success of this solution relies on the possibility of applying it exclusively on the curved path, and this relies on the use of transitions. Several scenarios appear depending on the constrains in the manufacturing process (cost, complexity, etc.) and on the practical application (robustness of the waveguide, required dimensions, etc.). It may be possible a) to modify both, radius $a$ and coating thickness $t$; b) to modify only the coating thickness $t$ (as the case of a SWW 
is provided and the coating is added by some chemical process [507]); c) the most limiting case of being constrained to some strict requirements in both parameters. This last challenging case is treated in the next section named as solution $\mathrm{B}$ for the bend radiation problem, and the former two will be afforded later when transitions are considered.

\subsection{Solution B: Control of the curvature phase shift}

The results in previous sections indicate that, if the restrictions on the SWW or DCSWW parameters do not allow to choose an small radius $a$, then, even optimization is done over the coating thickness $t$, high order modes will be very prone to couple in bends with reasonable angle $\theta$. However, if any other option is possible, it worth to explore what can be done with such critical limitations. The answer resides in using the same mechanism that favors the radiation in a thoughtful way.

The main loss mechanism in a bend takes place by the different phase shift that the fundamental mode experience in its axialsymmetric mode pattern due to the different lengths of the internal and external paths of a curve. This mechanism is illustrated through several images in Fig. 4.12. The schematic of a general DCSWW bend of angle $\theta$ and radius of curvature $R_{c}$ is illustrated in Fig. 4.12(a). In this schematic the internal and external paths ${ }^{2}$ of the curve are highlighted in blue and red, respectively. As pointed out in equations (4.1) and (4.2), the internal path has a length of $L_{1}=R_{c} \theta$, whereas the external path has a length of $L_{2}=\left(R_{c}+2 a\right) \theta$, hence existing a difference between both paths of $\Delta L=L_{2}-L_{1}=2 a \theta$. Note that this difference is totally independent of the curvature radius $R_{c}$, but it is only a function of the angle $\theta$ and the wire radius $a$.

This difference of length $\Delta L$ causes a phase shift, which is given by $\psi=\beta \Delta L$. Therefore, it is relevant to know how large is this length in terms of the guided wavelenght, i.e., the size of $\Delta L / \lambda_{g}$. This parameter offers an approximate idea (since $r=a$ is taken as the reference radial distance at which both paths are described) of how critical is the phase change. The worse case corresponds to $\Delta L / \lambda_{g}=0.5+k$, $k \in \mathbb{N}$, causing internal and external paths to provide opposite phase at their end, whilst the best case correspond to $\Delta L / \lambda_{g}=k$, implying that the internal and external paths not to cause any phase shift. By observing the mode patterns of the fundamental $\mathrm{TM}_{01}$ and the first high-order mode with radial polarization, the $\mathrm{HE}_{11}$ (see Figs. 4.12(c) and 4.12(d)), it is easy to understand that, when the worse case occur, the situation is very suitable for causing the energy lost by the $\mathrm{TM}_{01}$ mode to be coupled to the $\mathrm{HE}_{11}$ mode in order to satisfy the boundary conditions.

The parameter $\Delta L / \lambda_{g}$ is represented as a function of the curvature angle $\theta$ for several cases of radius and thickness in Fig. 4.12(b). In that plot it is appreciated how for small radius, $\Delta L$ is too small so that $\Delta L / \lambda_{g}$ describes a situation that worsen monotonically as $\theta$ increase being that $\Delta L / \lambda_{g}$ starts from 0 and never reaches 0.5 . However, if the radius is enough large, $\Delta L / \lambda_{g}$ reach 0.5 first and then continues to 1 as the angle increases, this indicating that an anomalous behavior may be found since larger $\theta$ may cause a more appropiated phase shift, suggesting lower losses. In

\footnotetext{
${ }^{2}$ The distance $r=a$ is taken as reference. This simplification is like affirming that all the mode is located at $r=a$.
} 
the case $a=100 \mu \mathrm{m}$, a unique cycle takes place, whereas $a=200 \mu \mathrm{m}$ provides two cycles being that the minimum phase shift is reached two times: $\Delta L / \lambda_{g}=1$ and $\Delta L / \lambda_{g}=2$. In this graph it is also appreciated how the presence of the coating reduces the size of the guided wavelength $\lambda_{g}$, therefore, it is important to calculate which is exactly the propagation constant of a particular DCSWW design $(a, t$, $\left.\varepsilon_{r}\right)$ in order to provide a good estimation of the phase shift. Regarding to this question, it is worth to mention that, the model illustrated 4.12(a) simplifies all the mode pattern to be located at $r=a$, i.e, $x= \pm a$, and $\Delta L$ is calculated from this assumption. However, the mode is indeed, distributed. Therefore, the more confined the mode, the more exact the proposed model. This suggest that the phase shift effect will be much more complex to be characterize in a SWW, and the presented model may be a rough approximation in some cases.

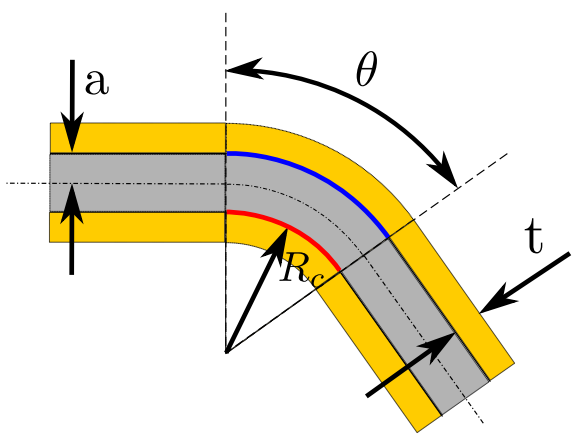

(a)

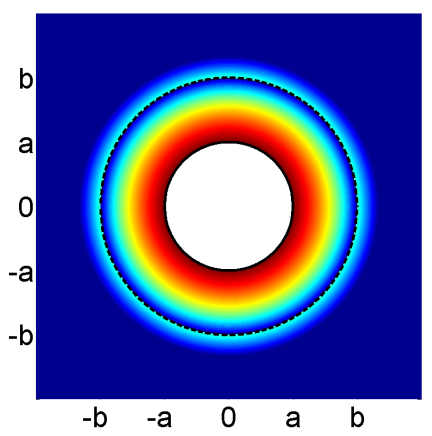

(c)

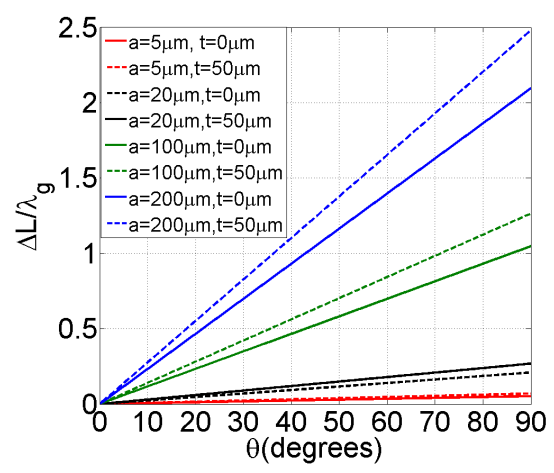

(b)

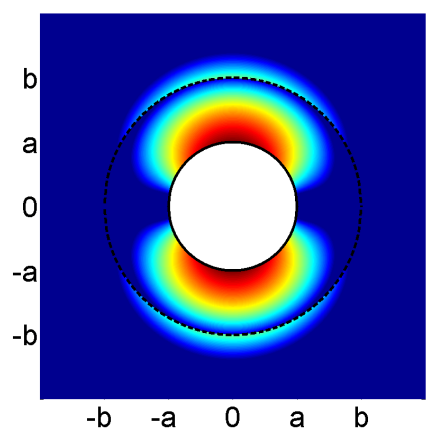

(d)

Figure 4.12: Description of the phase shift problem in bent wire-type waveguides. a) General bend schematic showing the internal (blue) and external (red) paths; b) Difference in length between internal and external paths relative to the guided wavelength $\left.\Delta L / \lambda_{g} c\right) T M_{01}$ mode E-field magnitude mode pattern; d) $H E_{11}$ mode E-field magnitude mode pattern.

To prove the existence of the aforementioned effect, the power received at the output port of a bend is displayed as a function of the curvature angle $\theta$ in Fig. 4.13. In the plots, they have been considered two small radius $(a=5 \mu \mathrm{m}$ and $a=20 \mu \mathrm{m})$, 
and a large radius $(a=100 \mu \mathrm{m})$. For each radius case, three coating cases are considered: no coating $(t=0 \mu \mathrm{m}), t=25 \mu \mathrm{m}$ and $t=50 \mu \mathrm{m}$. As in the previous study, the curvature radius is $R_{c}=200 \mu \mathrm{m}$, the operating single frequency is $f=1$ $\mathrm{THz}$, the chosen conductor is copper $\left(\sigma=5.81 \cdot 10^{7} \mathrm{~S} / \mathrm{m}\right)$ and the dielectric is TPX ${ }^{\circledR}$ $\left(\varepsilon_{r}=2.1, \tan \delta=0.001\right)$. The received contribution of the fundamental mode is shown in Fig. 4.13(a) whereas the received contribution of the $\mathrm{HE}_{11}$ mode is shown in Fig. 4.13(b).

Looking first at the received power of the fundamental mode in Fig. 4.13(a), it is observed a situation that corresponds well from what was predicted through the analysis of $\Delta L / \lambda_{g}$ in Fig. 4.12(b). For small radius, the received power decreases monotonically with $\theta$, and the slope of the curves depends mainly on the size of $a$. However, the case of $a=100 \mu \mathrm{m}$ exhibits an anomalous behavior, exhibiting a local minimum and a local maximum that confirms the existence of a minimum and a maximum phase shift when the angle $\theta$ is varied along the considered range. Note that Fig. $4.12(\mathrm{~b})$ predicts the local minimum at $\theta=40^{\circ}$ and the local maximum at $\theta=70^{\circ}$, what proves the accuracy of the proposed model.

In Fig. 4.13(a), it can also be observed that even a small coating produces a good improvement if $\theta$ is large enough. However, increasing $t$ towards large values produces no further gain. This holds even for the large radius case $(a=100 \mu \mathrm{m})$. In this case, the use of some coating results crucial since, for a bare SWW, losses for a reasonable angle $\theta>45^{\circ}$ have its minimum value in $19 \mathrm{~dB}$, whereas if a $t=50 \mu \mathrm{m}$ this value is reduced to $8 \mathrm{~dB}$, which is pretty good level for such a large radius (remind that in previous section losses for a DCSWW with $a=100 \mu \mathrm{m}$ were over $15 \mathrm{~dB}$, no matter the coating used). It is worth to point that, in this approach, the coating is not as restricted as in the previous section since the design is not limited by the monomode strict condition, but the coupling is minimized via engineering the curvature parameters.

Finally, Fig. 4.13(b) confirms that, as was expected, the power that is lost by the fundamental mode due to the asymmetry caused by the phase shift goes mainly to the first non-axialsymmetric mode, the $\mathrm{HE}_{11}$ mode. As can be observed, there is a good agreement between the local minimum of the $\mathrm{TM}_{01}$ mode curve and the local maximum of the $\mathrm{HE}_{11}$ mode and vice versa.

The same analysis is done in Fig. 4.14 but in this case only large radius are considered ( $a=100 \mu \mathrm{m}$ in first row, $a=200 \mu \mathrm{m}$ in second row), and only one radius is displayed in each plot for clarity purposes. An additional value of thickness $(t=75 \mu \mathrm{m})$ has been also added. In the case of $a=100 \mu \mathrm{m}$, it is observed that the model fits well for the DCSWW and with slightly less agreement for the SWW case $(t=0 \mu \mathrm{m})$, this originated by the aforementioned approximation issue of the model. From the plots, it can be also extracted that continue increasing $t$ beyond $t=50 \mu \mathrm{m}$ does not give additional improvement.

From the plots, it is extracted that the improvement with $t$ decays exponentially: when the coating is small, increasing it causes a great improvement, however, when $t$ reach some level any positive influence is found by increasing it. In this sense, it results rather surprising that using $t=25 \mu \mathrm{m}$, which implies only $\eta=25 \%$ for the fundamental mode according to Fig. 4.4(a), losses are very similar to those obtained with $t=75 \mu \mathrm{m}$, which implies $\eta=85 \%$. The justification of this effect relies again in the mode coupling. The power that is lost by the $\mathrm{TM}_{01}$ due to the perturbation 


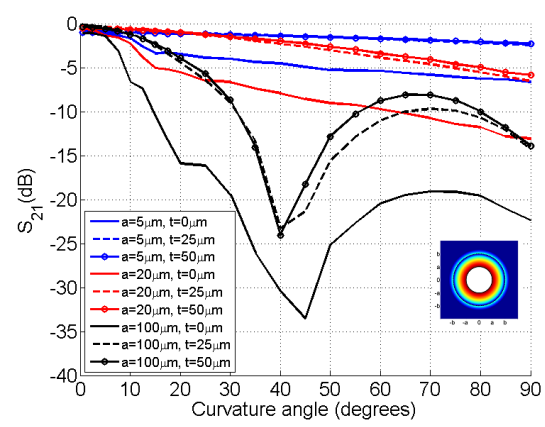

(a)

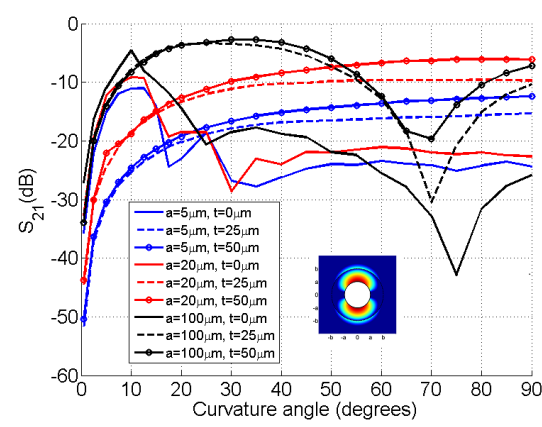

(b)

Figure 4.13: Received power at the output port, $S_{21}(d B)$ vs. curvature angle $\theta$, for several cases cases of $a$ and $t$ in wire-type waveguides. a) Fundamental $T M_{01}$ mode; b) $H E_{11}$ mode.

of the mode pattern during the bend, goes to suitable modes to coupling accordingly to this perturbation ( $\mathrm{HE}_{n 1}$ modes). When $t$ is small, these modes are still radiative, whereas when $t$ is large some of them propagate similarly to the fundamental mode. Therefore, although by increasing $t$, the fundamental mode power distribution shows a more confined pattern, high-order modes also do, therefore being as well or even more suitable to couple than when $t$ is small. The main difference between both situations is that, for large $t$, the power is not lost by radiation but is guided by high-order propagating modes apart of the $\mathrm{HE}_{11}$, which are not displayed in the plots. In other words, although larger $t$ implies lower radiation losses via TIR effect, the additional power reaching the output of the bend results to be coupled to highorder modes, hence being useless power unless any mechanism to couple it back to the $\mathrm{TM}_{01}$ mode is provided.

Regarding to the $a=200 \mu \mathrm{m}$ case, Figs. 4.14(c) and 4.14(d), two cycles and a half can be observed on the plots, i. e., three local minimums and two local maximums in the fundamental mode curves, Fig. 4.14(c). Several differences can be appreciated regarding to the previous case. First, the SWW curve becomes more chaotic, something that can be appreciated in both, Figs. 4.14(c) and 4.14(d). This is caused by the less compact effective transmission area as $a$ is larger, causing the model to be a worse approximation. In this sense, less improvement is achieved with this approach. Note that, in the case that no coating could be used on large radius wires, this approach would result interesting to gain some dBs. As shown these figures, the benefit is reasonable for $a=100 \mu \mathrm{m}$ but less interesting for $a=200 \mu \mathrm{m}$.

Second, the curve for $t=75 \mu \mathrm{m}$ turns more chaotic in the fundamental mode plot, however it does not on the $\mathrm{HE}_{11}$ mode plot. What occurs in this case is that such a thick coating, together with a large radius is prone to coupling an important amount of power to the $\mathrm{HE}_{21}$, not depicted. In this way, the $\mathrm{HE}_{11}$ mode continues receiving power as the model describes, but additional losses are experienced by the fundamental mode due to other sources of coupling, which become relevant. Even the curve for $t=75 \mu \mathrm{m}$ exhibits a more complex behavior, it shows a peak with level $S_{21}=-7.5 \mathrm{~dB}$ for an angle as large as $\theta=60^{\circ}$. The only problem is that this peak is less predictable with the proposed model. Nevertheless, comparing 


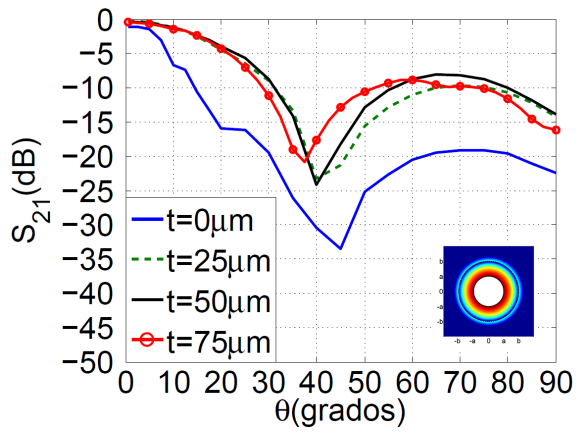

(a)

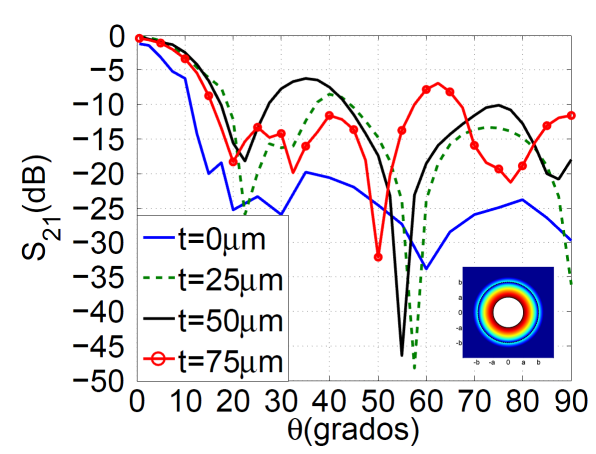

(c)

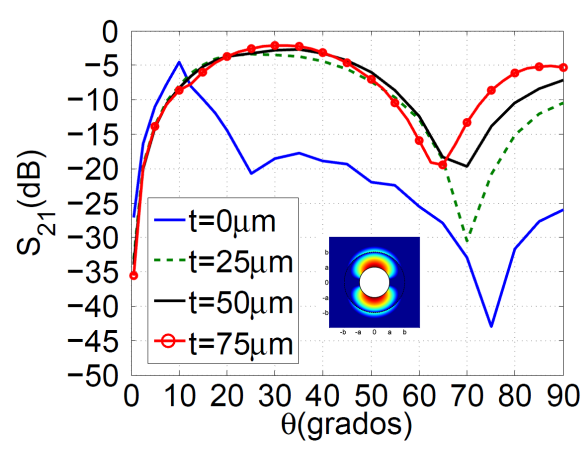

(b)

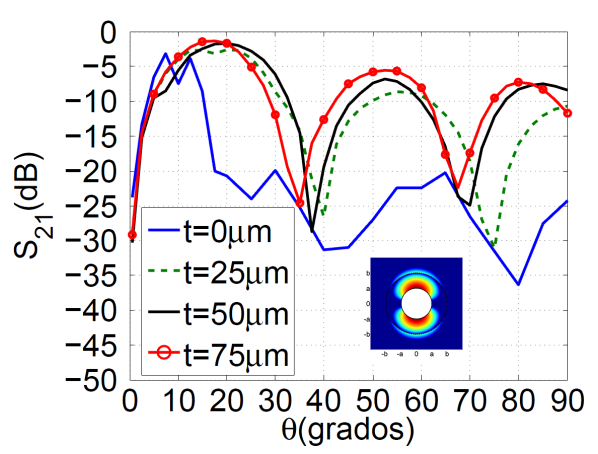

(d)

Figure 4.14: Received power at the output port, $S_{21}(\mathrm{~dB})$ vs. curvature angle $\theta$, for large radius ( $a=100 \mu \mathrm{m}$ in first row, $a=200 \mu \mathrm{m}$ in second row) and different $t$ in wire-type waveguides. a) $a=100 \mu \mathrm{m}, T M_{01}$ mode; b) $a=100 \mu \mathrm{m}, H E_{11}$ mode; c) $a=200 \mu \mathrm{m}, \mathrm{TM}_{01}$ mode; d) $a=200 \mu \mathrm{m}$, $H E_{11}$ mode.

to a bare SWW, the reduction of losses by using a coating and this procedure is very important. Note that by proceeding as described, a conventional $a=200 \mu \mathrm{m}$ may be used with any transition reducing the radius, which is a very flexible case regarding the manufacturing process. The ideal solution for such situation would be the employment of a transition on the coating, similar to that described in the next section. It is noted, however, that the loss level obtained with the design procedure just described suggests to implement only or one of two bends, being prohibitively lossy to include more in the final device.

To end this section, a design is realized based on the observed phenomena. Taking as a restriction that the bend must be of $\theta=90^{\circ}$, and taking $f=1 \mathrm{THz}$ as the operating frequency, it is found that, for intermedium values of coating thickness, $a=70 \mu \mathrm{m}$ offers the optimum solution, providing a maximum in the received power of the $\mathrm{TM}_{01}$ mode. With this radius, two cases of coating thickness, $t=50 \mu \mathrm{m}$ and $t=75 \mu \mathrm{m}$ are studied in the frequency band ranging from $0.7 \mathrm{THz}$ to $1.3 \mathrm{THz}$ $(R B W=60 \%)$. Note that, for the presented procedure, study of the frequency response results particularly necessary being that $\Delta L / \lambda_{g}$ depends directly on $\beta$, hence inherently having some frequency dependence. 
In Fig. 4.15, there is represented the received power by each mode, $\mathrm{TM}_{01}$ and $\mathrm{HE}_{11}$, and the sum of both, at the output port of the bend. In both cases, $t=50 \mu \mathrm{m}$, Fig. 4.15(a), and $t=75 \mu \mathrm{m}$, Fig. 4.15(b), it is observed how, although total received power remains quite constant along the operation band, as frequency moves from the central frequency, the design becomes detuned and more power is coupled from the $\mathrm{TM}_{01}$ mode to the $\mathrm{HE}_{11}$ mode. In the case of $t=75 \mu \mathrm{m}$ (see Fig. $4.15(\mathrm{~b})$ ) this is more pronounced for the highest frequencies of the band, indicating that a thicker coating is facilitating the coupling to the $\mathrm{HE}_{11}$ mode. Therefore, $t=50 \mu \mathrm{m}$ or even thinner coatings seem more adequate. Nevertheless, for the case of $t=50 \mu \mathrm{m}$, minimum losses of only $6.5 \mathrm{~dB}$ and a $-3 \mathrm{~dB}$ bandwidth of $0.5 \mathrm{THz}$ are obtained, what means a $R B W$ of $50 \%$, i. e., a clear wide-band response. This fact is very important since it proves that the described solution is in concordance with the main feature of the SWW, its broadband good behavior. Therefore, although the restriction of having an inferior limit for the radius $a$, or even requiring certain minimum value for $t$ can be quite challenging to the bend performance, the proposed solution has been shown to alleviate quite well the problem.

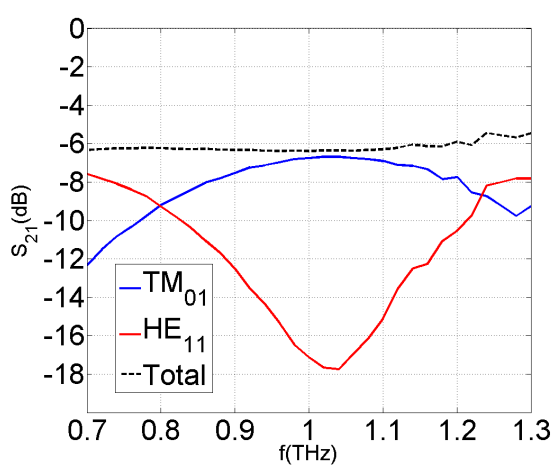

(a)

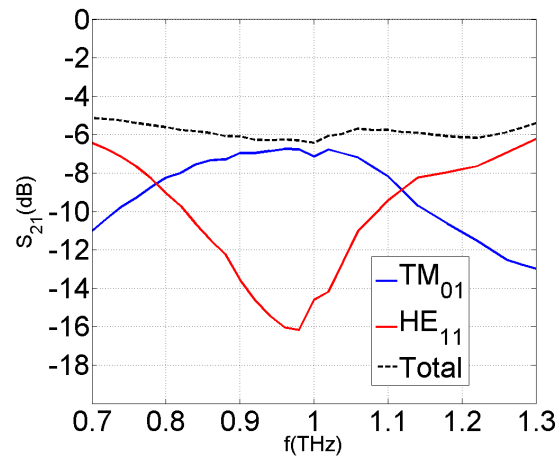

(b)

Figure 4.15: Frequency response in the operating band [0.7 THz-1.3 THz] of the proposed optimal $90 \circ$ bend of $a=70 \mu \mathrm{m}$. a) $t=50 \mu \mathrm{m}$; b) $t=75 \mu \mathrm{m}$.

To end this section, and in order to compare the solution presented in this section (solution B) and that of the previous section (solution A), the frequency response in the same band of some designs based on the solution A are presented in Fig. 4.16. The case of a radius $a=5 \mu \mathrm{m}$ is plotted in Figs. 4.16(a) and 4.16(b), which show, respectively, the cases corresponding to $t=30 \mu \mathrm{m}$ and $t=50 \mu \mathrm{m}$. As expected, the frequency response of the reduced radius solution is noticeably better than that of the phase shift adjustment solution. For such a small radius, even in the case that $t$ is chosen above its optimum, that was $t=40 \mu \mathrm{m}$, the additional loss by coupling only implies the highest frequencies to loss $1 \mathrm{~dB}$ more than the lowest ones (see Fig. $4.16(\mathrm{~b})$, corresponding to $t=50 \mu \mathrm{m}$ ). If the radius is chosen to be $t=30 \mu \mathrm{m}$, the frequency response is practically plane and close to $S_{21}=-2 \mathrm{~dB}$. This indicates that, since the response is quite stable with $t$, in order to provide the best performance also for larger frequencies, a slight smaller $t$ than the optimum calculated for the central frequency $(t=40 \mu \mathrm{m})$ seems more adequate. 
Nevertheless, if the radius is small but no as much as desired, the situation worsen. In Figs. 4.16(c) and 4.16(d), the cases of $(a=20 \mu \mathrm{m}, t=20 \mu \mathrm{m})$ and ( $a=20 \mu \mathrm{m}, t=30 \mu \mathrm{m}$ ) are represented, respectively. In both cases the received power of the fundamental mode ranges approximately between $S_{21}=-5 \mathrm{~dB}$ (lowest frequency) and $S_{21}=-8 \mathrm{~dB}$ (largest frequency). This means that, although in this case the variation is monotone, the received power oscillate in similar values to those of the solution B in Fig. 4.15(a). Therefore, although the response of solution A is better, for the case of $a=20 \mu \mathrm{m}$ the difference is not as noticeable. Note that, in the case of solution A, reaching values as small as $a=5 \mu \mathrm{m}$ is necessary to prevent loss by coupling. In Fig. 4.16(d), it can be appreciated how, even for the case of rather small radius as it is $a=20 \mu \mathrm{m}$, the coating becomes very limited being that increasing it only causes more coupling to the $\mathrm{HE}_{11}$ mode, or even higher order modes.

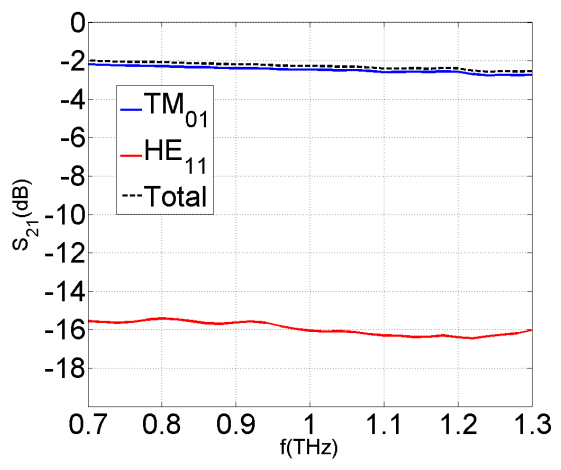

(a)

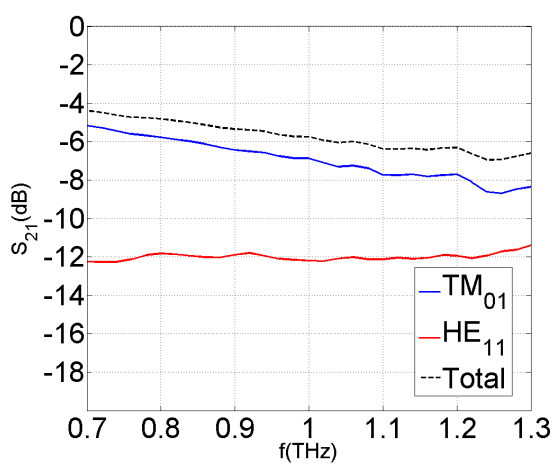

(c)

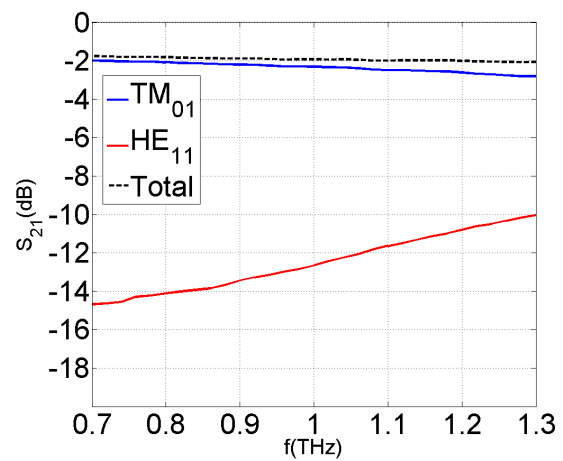

(b)

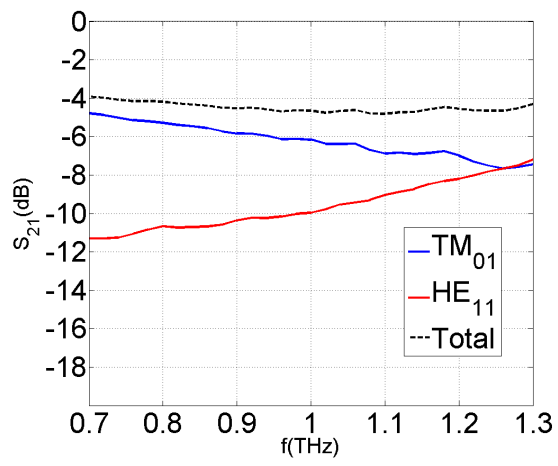

(d)

Figure 4.16: Frequency response in the operating band [0.7 THz-1.3 THz] of a small radius design as those presented in section 4.2 ; a) $a=5 \mu \mathrm{m}, t=30 \mu \mathrm{m}$; b) $a=5 \mu \mathrm{m}, t=50 \mu \mathrm{m}$; a) $a=20 \mu \mathrm{m}$, $t=20 \mu \mathrm{m}$; a) $a=20 \mu \mathrm{m}, t=30 \mu \mathrm{m}$;

The conclusion extracted from the study of solutions A (reducing the wire radius) and $\mathrm{B}$ (control of the curvature phase shift) is that solution A shows more performance. However, this is only true in a large extent whenever the radius $a$ 
can be made very small. The solution B, which may be intuited at first view as a narrow-band one to avoid, shows surprisingly good response, and it may make sense for practical low-cost designs requiring more manufacturing flexibility.

\subsection{SWW-DCSWW transitions}

In the previous section, by means of the criteria A and B, DCSWW optimal designs performing with minimum losses at bends have been achieved. The obtained waveguides, however, provide a performance in terms of propagation losses and frequency dispersion that is far from that of a large radius bare SWW, which is, actually, the outstanding performance that has drawn attention to this waveguide for guided transmission of $\mathrm{THz}$ energy. Therefore, if no further action is done, and the bend design conditions the full circuit design, the overall performance will worsen considerably. This is specially true for the solution A, that uses a DCSWW with very high propagation losses. In such case, it is even possible that not to adopt any solution for the bend may be actually better if the previous and posterior straight paths are very long.

To make these affirmations more quantifiable, in Fig. 4.17 propagation losses in $\mathrm{dB} / \mathrm{mm}$ of a DCSWW are displayed as a function of the radius in the range that it has been considered along these sections, for several values of the coating thickness $t$, including the case of a bare SWW $(t=0 \mu \mathrm{m})$, at $f=1 \mathrm{THz}$. To highlight some figures, it is noted that a SWW with $a=200 \mu \mathrm{m}$ presents an attenuation less than $0.005 \mathrm{~dB} / \mathrm{mm}$, whereas if $a=5 \mu \mathrm{m}$, this value raises up to $0.116 \mathrm{~dB} / \mathrm{mm}$. Observe also that a large radius DCSWW with even a small coating $(a=200 \mu \mathrm{m}, t=25 \mu \mathrm{m})$ reaches $0.075 \mathrm{~dB} / \mathrm{mm}$.

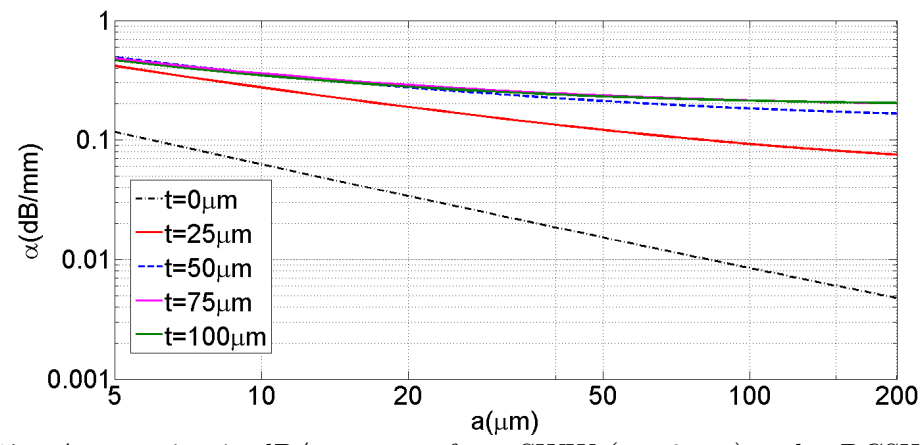

Figure 4.17: Attenuation in $d B / m m$ vs. a for a $S W W(t=0 \mu \mathrm{m})$ and a $D C S W W(t=25 \mu \mathrm{m}$, $t=50 \mu \mathrm{m}, t=75 \mu \mathrm{m}$ and $t=100 \mu \mathrm{m}$.

In this sense, it becomes mandatory to look for an optimal strategy, using a low propagation losses design on straight paths, and a different design on bends, such as proposed before, presenting minimum radiation losses. This can be only possible if adequate low-loss transitions are designed. To provide adequate transition designs, establishing adequate design procedures, is the aim of this section. From what has been reviewed so far, a large radius SWW is very suitable for straight paths, whereas a small radius DCSWW, with $t$ small enough to avoid the coupling to high-order 
modes, is the best option for the curved trajectories. This kind of transition implies a reduction of the radius, together with the addition of a dielectric coating and vice versa in the return to the original SWW. Actually, it is the optimal solution.

However, although experimental tapers with the SWW have been reported [508], [513], [514], may be possible that the restrictions in the manufacturing process, or even in the application, make this impossible. Therefore, since this is a general study, as in the case of the previous section, several solutions are given in such way that, given certain design restrictions, several suitable options are available. In this way, first, the case of modifying only the coating thickness is considered. This option has a easier manufacturing process in as much chemical creation and manipulation of the dielectric material is more flexible than in the case of metals, and the literature is plenty of experimental demonstrations [507], [591], [592]. Then, the full transition allowing variation of both, radius $a$ and coating thickness $t$, is presented.

\subsubsection{Tapered coating}

In this case, any change is made in the radius $a$, which will be the same for the whole path, straight and curved paths included. For those curved paths, adding the coating is considered. Therefore, in this sense, the radius chosen for the bend conditions somehow the straight path or vice versa. If the priority is given to the bend, solution A will be taken, and the radius $a$ will be rather small. If the priority is given to the straight paths performance, solution B must be taken and the radius will be moderate. Note that the solution $B$ with very large radius may results too inefficient for the purpose. In this section, it will be assumed the first case, choosing the optimal design for the bend according to the solution A, and all the results will be given for such case. Nevertheless, it is pointed out that if the choice was the solution $\mathrm{B}$, the procedure and the conclusions would be analogous and, in the following design steps, where some small particularities could be appreciated, remarks will be given.

The proposed design is shown first in a straight path in Fig. 4.18(a). It consist in two transitions, one from SWW to DCSWW and other from DCSWW to SWW. Accordingly to section 4.2 , the radius is chosen to be $a=5 \mu \mathrm{m}$, whereas the coating for the DCSWW, i.e., the maximum value of the coating is chosen to be $t=30 \mu \mathrm{m}$. In this last parameter, it is pointed out that $t=30 \mu \mathrm{m}$ is preferred to $t=40 \mu \mathrm{m}$ since both present very similar performance, but a smaller value guarantees a good extension of the performance to higher frequencies, which will be useful when the design is tested for some operation band centered at $f=1 \mathrm{THz}$.

Apart of the SWW and DCSWW waveguide parameters, the unique parameter that define the transition is $l_{t r a n}$, which describes the length of the path section in which the coating is added progressively from $t=0 \mu \mathrm{m}$ to $t=30 \mu \mathrm{m}$. The increment of the coating thickness is linear, which is the simplest case, regarding in this way to a easier manufacturing process. In order to that the transition presents low losses, $l_{\text {tran }}$ must be optimized to provide good matching between the modal patterns and propagation constants of the SWW and DCSWW at both sides of each transition. To do that, the same structure that Fig. 4.18(a) is simulated inasmuch it is less complex that a full bend including the transitions. 
Results of the optimization of $l_{\text {tran }}$ are shown in Fig. 4.18(b). In this plot, they are shown the total losses of the two transitions (SWW and DCSWW straight path losses are subtracted using analytical results) as a function of $l_{\text {tran }}$. If no transition was place, losses as high as $6 \mathrm{~dB}$ would take place. As expected, increasing $l_{\text {tran }}$ allows for a better matching between waveguides, reducing the losses. However, a minimum of $1.12 \mathrm{~dB}$ losses is found at $l_{\text {tran }}=0.68 \mathrm{~mm}$. Beyond that minimum, losses increase as $l_{\text {tran }}$ increases. This meas that, at that point, the transition is soft enough, and there is not much gain by making it longer, but propagation losses continue increasing. In that case, the overall amount of losses is higher when the transition is longer. This is another prove for the fact that, when dealing with wire-type structures, or in general $\mathrm{THz}$ waveguides operating in confined configuration, propagation losses are critical. Therefore, unlike typical microwave transitions, which are mainly limited by space, size or weight restrictions, in these designs, it is usually found an optimal length such that beyond it, propagation losses become a large contribution.

Nevertheless, total losses of $1.12 \mathrm{~dB} / \mathrm{mm}$ are a very good figure, since this roughly means $0.5 \mathrm{~dB}$ per transition. It is worth to mention that, apart of this minimum, the design is quite stable, presenting losses under $1.5 \mathrm{~dB}$ in the range $0.55 \mathrm{~mm}-0.85$ $\mathrm{mm}$. This indicates that the behavior of the structure is not highly sensitive to variations in the length of the transition around the optimal solution, which is a positive feature in case of fabrication. Since the guided wavelength of the SWW and DCSWW forming the design are, respectively, $\lambda_{g}(S W W) \approx \lambda_{0}=300 \mu \mathrm{m}$ and $\lambda_{g}(D C S W W)=280 \mu \mathrm{m}$, it could be roughly said that good matching of this kind of transitions is produced when $l_{\text {tran }}$ is about 2 wavelengths.

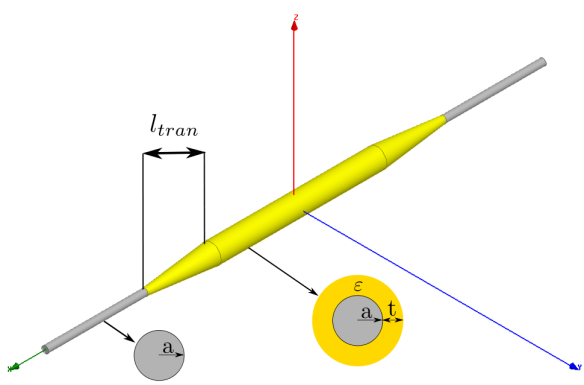

(a)

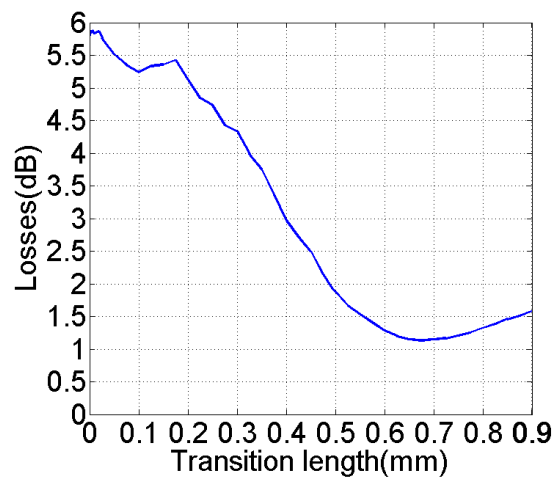

(b)

Figure 4.18: Proposed $S W W-D C S W W$ and DCSWW-SWW transitions varying only the coating thickness $t$, tested over a straight path. a) Schematic; b) Losses vs. $l_{\text {tran }}$.

The effect of the transition and the phenomena in the simulated design are well visualized in Fig. 4.19, where the electric field on the structure has been represented in logarithmic scale of $20 \mathrm{~dB}$ range for two cases of $l_{\text {tran }}$. In Fig. 4.19(a) is is plotted the case $l_{\text {tran }}=0.2 \mathrm{~mm}$, which according to Fig. $4.18(\mathrm{~b})$ implies total losses of $5.1 \mathrm{~dB}$. In this plot it can be observed how, in the input transition, the difference 
between the mode patterns of SWW and DCSWW, provided by the differences in power distribution, causes a power reflection backwards inasmuch as the transition is not soft enough to match that difference. Observe also how, at the output transition, the perturbation caused by such abrupt change on the coating thickness leads to the coupling to some high-order modes, which are radiative on the SWW.

At this point it is worth to mention that transitions optimized for the solution $\mathrm{B}$ will present in general a larger $l_{\text {tran }}$ by two reasons. On the one had, due to the larger radius, the mode pattern difference between the SWW and the DCSWW will be larger. On the other hand, the propagation losses will be lower. Thus, it is reasonable to think that the inflexion point at which the propagation losses become more relevant that the gain in matching will occur for larger $l_{\text {tran }}$.

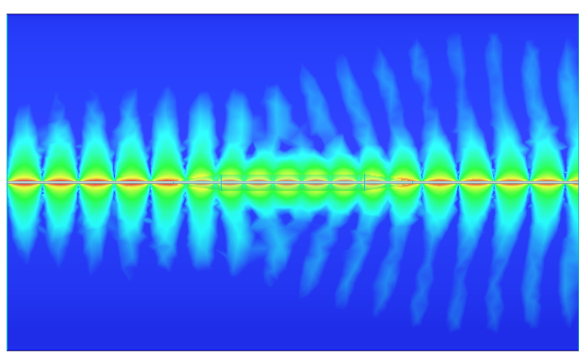

(a)

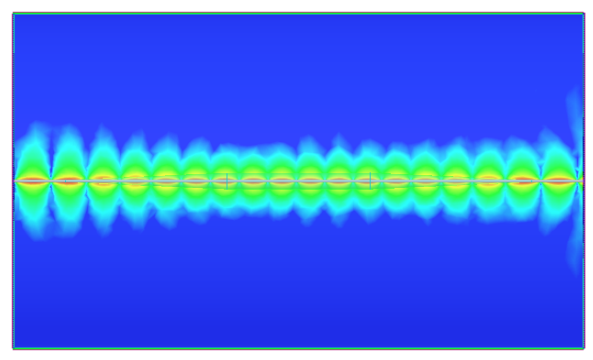

(b)

Figure 4.19: E-field magnitude in logarithmic scale with $20 \mathrm{~dB}$ range in the straigh path with transitions. a) $l_{\text {tran }}=0.2 \mathrm{~mm}$ (unmatched); b) $l_{\text {tran }}=0.68 \mathrm{~mm}$ (optimal).

Adversely, in Fig. 4.19(b), where the optimal transition case is shown $\left(l_{\text {tran }}=\right.$ $0.68 \mathrm{~mm})$, it is possible to observe that the fundamental mode pattern is preserved and there is no coupling to high-order modes. Any radiation is appreciable on the fields at such scale. It is also clearly observed how the presence of the dielectric coating makes the fundamental mode to be mostly confined inside it, this producing a reduction of the extension of the power in the transverse direction.

Once the optimal design for the transitions has been found, an optimal bend implementing them is designed. The $3 \mathrm{D}$ view of the proposed structure is shown in Fig. 4.20(a), and its lateral $2 \mathrm{D}$ view, including the main structure parameters, is shown in Fig. 4.20(b). As in the previous studied bends, $R_{c}=0.2 \mathrm{~mm}$, therefore $l_{\text {bend }}=R_{c}(\pi / 2)=0.31 \mathrm{~mm}$. The total length of the structure, $L=2 \cdot l_{\text {wire }}+2$. $l_{\text {tran }}+l_{\text {bend }}$, is forced to be $2 \mathrm{~mm}$ in order to facilitate the later comparison with the SWW and DCSWW bends, already simulated in the previous section. Accordingly, $l_{\text {wire }}=0.165 \mathrm{~mm}$.

To visualize how the proposed design including coating transitions reduces the radiation on bends, the E-field at $f=1 \mathrm{THz}$ on a $90^{\circ}$ bend is displayed in logarithmic scale of $20 \mathrm{~dB}$ range for both cases: a bare SWW bend, Fig. 4.21(a), and the aforementioned bend with transitions and a DCSWW curved section, Fig. 4.21(b). In the case of a bare SWW, in spite of the fact that the radius is small enough to produce some attachment of the guided waves (some guiding features are observed), the radiation is still evident as appreciable waves continue the input direction path, 


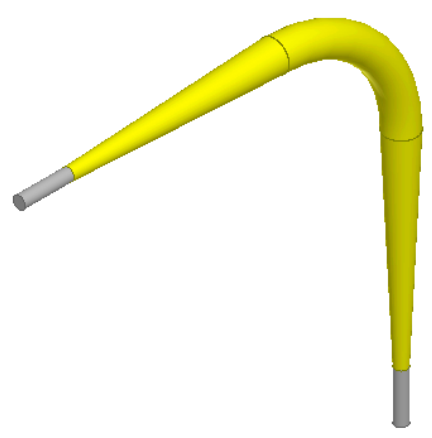

(a)

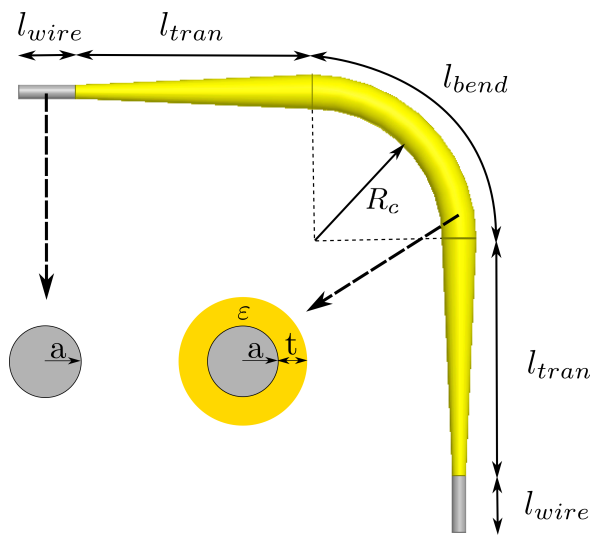

(b)

Figure 4.20: Final bend including the transitions and the optimal coating at the curved path. a) $3 D$ view; b) Lateral view and parameters.

not following the waveguide. Note how the intensity at the output port is visually lower than the intensity at the input port.

Conversely, in the proposed design, it is firstly observed the effect of confinement experienced by the guided waves due to the added coating. Observe how the area inside which the field is concentrated is noticeably lower in this case. Under this conditions, the waves follow well the curved path and the larger intensity at the output port regarding to the SWW case is quite evident.

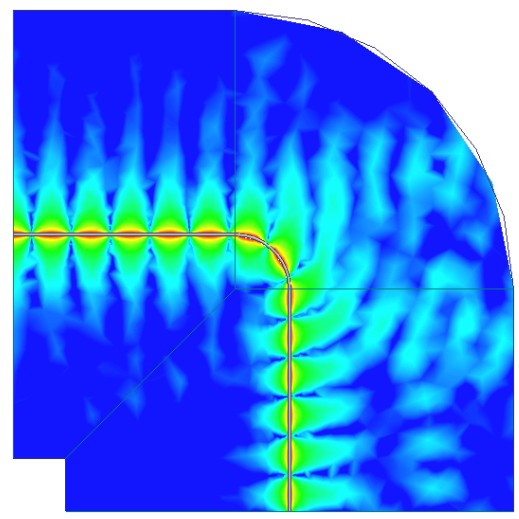

(a)

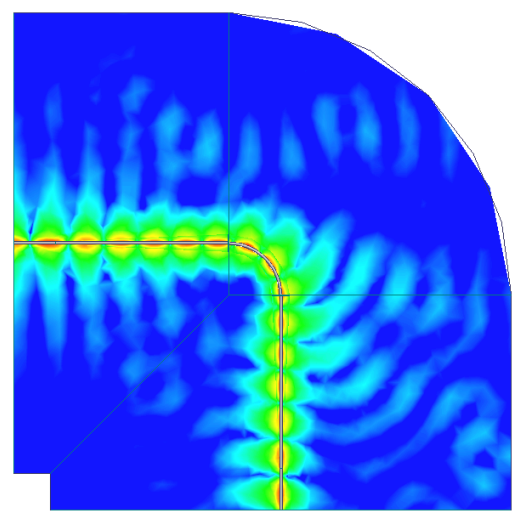

(b)

Figure 4.21: E-field in a bend. Logarithmic scale with $20 \mathrm{~dB}$ range a) $S W W$; b) Proposed design with transitions.

Specifically, the described bend with the proposed design presents $3.25 \mathrm{~dB}$ of total losses, only $0.3 \mathrm{~dB}$ more than the same curve with a DCSWW. This means that the cost of using the coating exclusively on the bend is extremely low compared to the obtained benefit, being that the attenuation of a SWW is much lower than 


\begin{tabular}{lccc}
\hline Design & SWW & DCSWW & Transitions \\
\hline \hline & & & \\
& & & \\
\hline $90^{\circ}$ bend losses & $7.1 \mathrm{~dB}$ & $2.95 \mathrm{~dB}$ & $3.25 \mathrm{~dB}$ \\
Straight path losses & $0.116 \mathrm{~dB} / \mathrm{mm}$ & $0.43 \mathrm{~dB} / \mathrm{mm}$ & $0.116 \mathrm{~dB} / \mathrm{mm}$ \\
\hline \multicolumn{4}{c}{ Table 4.1: } \\
\hline \multicolumn{4}{c}{ Comparison of losses of the studied designs. }
\end{tabular}

that of a DCSWW. To give direct comparison between the three cases that are considered (SWW, DCSWW and proposed design), in table 4.1 they are displayed the straight path losses and the losses for the considered bend for all of them. 3D schematics are added for clarity purposes. Note that, with the proposed design, at the cost of $0.3 \mathrm{~dB}$, they are saved more than $3 \mathrm{~dB} / \mathrm{cm}$ due to the reduction of the propagation losses. Also, if the proposed design is compared with the SWW case, close to $4 \mathrm{~dB}$ are saved each time that a curve appears, whereas both designs imply the same losses along straight paths.

It results important to note that the transitions are as a important as if the design was conditioned by the bend, i.e., the case of having the coating present in the whole waveguiding path, the overall performance would be nefarious. This is well illustrated in Fig. 4.22(a) where losses of a path including a $90^{\circ}$ bend in the middle are calculated as a function of the total length of the path, $L$, for the three considered cases. Realize that in that plot, the straight lines appearing have an initial offset equal to the losses of the bend, and a slope ruled by the propagation losses. In this way, the DCSWW case reach very fast high values, being even more reasonable not to use any coating and afford the bend with a bare SWW if $L>2$ $\mathrm{cm}$, which is a rather small distance. Of course, this becomes less and less true if more bends are present. However, since transitions are shown to be realizable, a great improvement is possible in such a challenging case including several bends and reasonable straight paths.

So far, the typical case of a $90^{\circ}$ bend has been considered along this section. However, other angles may be required in certain applications, hence it results of interest to check how the previous conclusions adapt to such cases. Following this premise, Fig. 4.22(b) shows the losses of a bend having a total length of $2 \mathrm{~mm}$, as a function of its angle $\theta$ for the three considered designs. When the curvature angle is very low, radiation losses on the bend are negligible. Since the total distance is short, propagation losses are small and the reflection losses of the transition become noticeable. This makes the proposed design to behave slightly worse than the SWW and the DCSWW designs. As the angle of curvature increases, the design with transitions rapidly overcomes the SWW, which experiment a remarkable worsening. However, it is important to mention that, when the gain of using transitions is small (see for example the case of $\theta=30^{\circ}$, with only $1.5 \mathrm{~dB}$ of improvement), it might be preferable to use the SWW inasmuch as the structure is simpler and the increment of complexity may not compensate the benefit. 


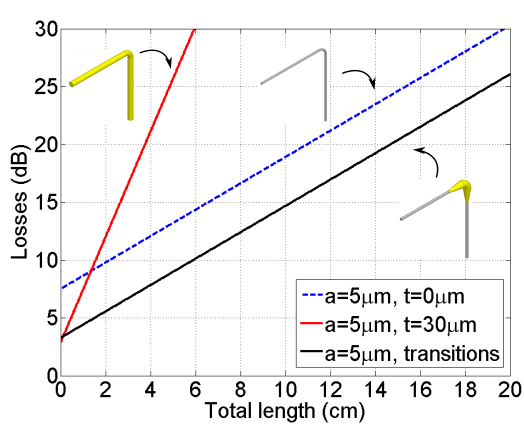

(a)

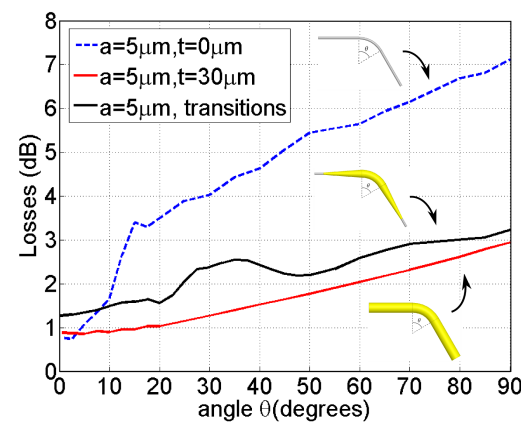

(b)

Figure 4.22: Performance comparison between the $S W W$, the DCSWW and the proposed using both by means of transitions. a) Total losses of a path with a $90^{\circ}$ bend in the middle as a function of its total length $L$; b) Bend losses as a function of the bend angle $\theta$.

In the aforementioned plot, it is also observed that losses of the proposed design remain only slightly higher than those of the DCSWW bend in the whole range. Angles included between $\theta=30^{\circ}$ and $\theta=50^{\circ}$ experiment and additional attenuation. This is probably caused by the combined effect of the transitions and the bend, which produces a slightly worse matching in these cases. Nevertheless, this additional attenuation remains lower than $0.5 \mathrm{~dB}$ in the whole range of $\theta$, hence it is concluded that the behavior of the proposed structure and that of the DCSWW bend is quite similar at any angle.

The proposed structure has shown to cause a large improvement compared to the designs having not coating transitions at the operation frequency, $f=1 \mathrm{THz}$. Therefore, at this point, it results interesting to evaluate the frequency response of such transition. In the frequency analysis carried next, the band $[0.5 \mathrm{THz}-1.5 \mathrm{THz}]$ is considered, which means a relative bandwidth of $100 \%$, hardly to be covered by usual waveguiding structures. To analyze the distortion introduced by the structure, two points must be studied. The first one is the frequency dependence of losses or attenuation dispersion. The second one is the velocity group dispersion or group delay dispersion, which is directly related to the broadening of a temporal broadband pulse.

In a first stage, the propagation characteristics of the SWW and DCSWW conforming the proposed design, are evaluated in Fig. 4.23 in order to gain some physical insight, helpful to understand the origin of the posterior results. In Fig. 4.23(a), the attenuation of both waveguides is displayed. In the case of the DCSWW, losses are broken down in the contributions corresponding to the ohmic losses caused by the conductor $\left(\alpha_{c}\right.$, marked with circles) and the absorption losses caused by the dielectric $\left(\alpha_{d}\right.$, marked with crosses).

In that plot, it is observed that the curve of the SWW is quite flat, even so, the large considered bandwidth causes the losses of the highest frequency to be twice those of the lowest frequency. It cannot be said that this SWW has a low-loss performance, but losses remain on acceptable levels for such a ultra-wide band. The case of the DCSWW shows a quite worse shape. As was already appreciated in 
section 3.6.4.1, ohmic losses contribute to a large degree in the total loss level. This contribution is not only worsening the attenuation level, but also the attenuation dispersion, since, for the DCSWW, the difference in attenuation level between the limits of the band is much more evident than in the SWW case. The increment of the surface resistance of the conductor with the frequency, and the increase of confinement with the frequency join together to produce such undesirable situation. Therefore, apart of the prohibitive losses of the considered small radius DCSWW, its attenuation dispersion suggest strong distortion of the $\mathrm{THz}$ pulsed signals, a great additional reason to consider transitions.

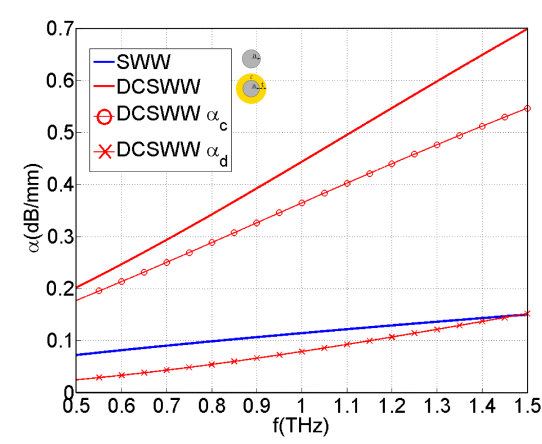

(a)

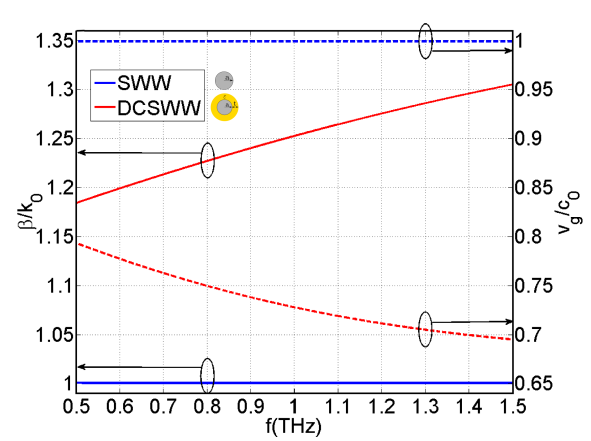

(b)

Figure 4.23: Propagation characteristics of the $S W W$ and DCSWW forming the proposed design with transitions in the operation band $[0.5 \mathrm{THz}-1.5 \mathrm{THz}]$. a) Attenuation in $\mathrm{dB} / \mathrm{mm}$. In the case of the DCSWW $\alpha$ is breakdown in the contribution of the conductor losses $\alpha_{c}$ and the dielectric losses $\alpha_{d}$; b) Dispersion characteristics: normalized propagation constant $\beta / k_{0}$ and normalized group velocity $v_{g} / c_{0}$.

In Fig. 4.23(b), the same waveguides are evaluated in terms of their normalized propagation constant $\left(\beta / k_{0}\right.$, in the left ordinates axis) and the normalized group velocity $\left(v_{g} / c_{0}\right.$, in the right ordinates axis), which determine the usually called frequency dispersion by indicating how a pulse is broadened. The response of the SWW is totally frequency invariant, indicating that any distortion from this kind may be expected for that waveguide. The DCSWW present some dispersion, however, this problem is of a minor order compared to the attenuation one. Nevertheless, it is interesting to observe that the use of transitions restrict the dispersion effects to the curved path section (and slightly in the transitions, when the coating is progressively added). Therefore, being that those sections are short, small differences in the group delay are expected along the operating band.

The presented bend design is tested now on the [0.5 THz-1 THz]. Fig. 4.24(a) shows the losses of the designed $2 \mathrm{~mm}$-long bend including the transitions as a function of the frequency in the mentioned band. Marks are simulated results while the line is a linear regression of them, which provides a correlation coefficient close to 1 . As can be observed, high frequencies present more losses than lower ones. This is not only caused by the larger propagation losses of the DCSWW at higher frequencies, just reviewed, but also because the transitions work worse. Mainly, there is more coupling to high order modes, which radiate after the the coating is retired. More- 
over, though the transitions are electrically larger for higher frequencies, the mode patterns of the fundamental mode on the SWW and the DCSWW differ more due to the greater confinement of the mode inside the dielectric. This slightly increase the reflection losses. Even so, all the total attenuation dispersion caused by all this effects joining together is a difference of only $3 \mathrm{~dB}$ between the minimum and the maximum frequency of the band, which is a quite good results if one remembers that $f_{\max }=3 f_{\min }$.

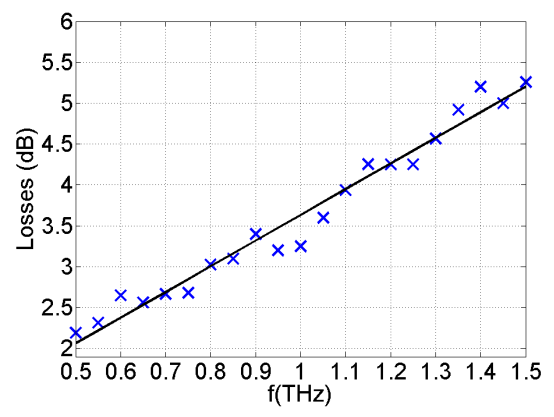

(a)

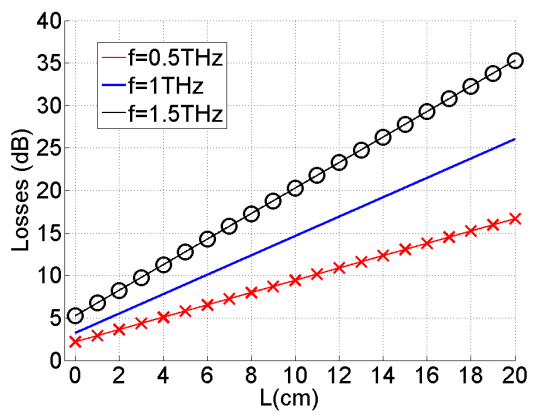

(b)

Figure 4.24: Losses frequency dispersion of the proposed transitions in the operation band [0.5 $\mathrm{THz}-1.5 \mathrm{THz}]$. a) Losses of the transition vs. frequency. Crosses are simulated frequency points whereas the solid line is the linear regression of those points; b) Total losses of a path with a $90^{\circ}$ bend in the middle, implemented with the proposed design, as a function of its total length $L$ for the limiting and central frequencies of the operation band.

Similarly as it was done in Fig. 4.22(a) for the three designs, the total losses of a path with a $90^{\circ}$ bend in the middle as a function of the path length $L$ are represented in Fig. 4.24(b) for the proposed design, but considering now the limiting $(f=0.5$ $\mathrm{THz}$ and $f=1.5 \mathrm{THz})$ and central $(f=1 \mathrm{THz})$ frequencies of the band. For short paths $(L \rightarrow 0)$, the attenuation dispersion is mainly given by the different loss in the bend, just described in Fig. 4.24(a), which are quite affordable. Nevertheless, as $L$ increases the attenuation dispersion of the bare SWW in the straight paths causes the curves to diverge progressively. If $L$ is very large, losses may reach high, critical for some applications.

However, despite of the rather large final absolute levels of attenuation distortion, it must be pointed out that they are mainly caused by the SWW natural attenuation dispersion, which, from what was observed in the state of the art chapter and the wire-type structures analysis chapter, is much lower than that of dielectric waveguides or usual microwave waveguides. Therefore, affording such length paths for transmission of ultra-wide band $\mathrm{THz}$ pulses would be rather a challenging task for other waveguiding structures. It is true, notwithstanding, that reduce the attenuation level during the straight paths would dispose the performance in a superior level. This configuration demand for the use of a larger radius SWW in straight waveguiding sections, and will be achieved in the next section.

Additionally, in Figs. 4.25(a) and 4.25(b) the E-field in logarithmic scale of 20 $\mathrm{dB}$ range is represented in the proposed bend structure for $f=0.5 \mathrm{THz}$ and $f=1.5$ $\mathrm{THz}$, respectively. In those plots they are well visualized the causes of the larger 


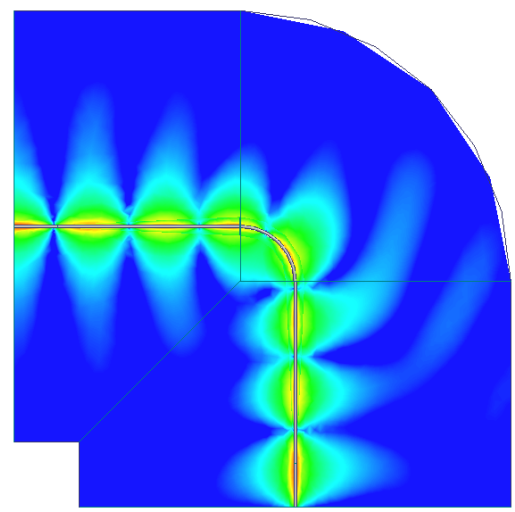

(a)

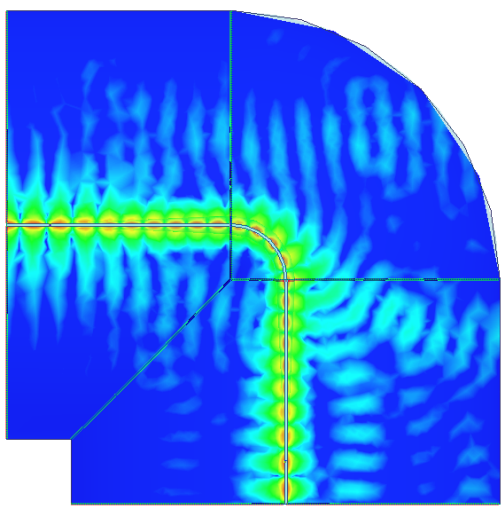

(b)

Figure 4.25: E-field in a bend for the limiting frequencies of the operation band. Logarithmic scale with $20 \mathrm{~dB}$ range a) $f=0.5 \mathrm{THz}$; b) $f=1.5 \mathrm{THz}$.

losses of higher frequencies in the proposed design. Note that, higher frequencies become more confined when the coating is added, what prevents from radiation losses. Nevertheless, the mismatch at the transitions is evident from the reflected and radiated waves observed in the plot. Furthermore, note that at the curved path, the waves are noticeably more damped than in the case of lower frequencies. Therefore, although in principle it could be though that high frequencies will exhibit lower losses due to the larger confinement and lower radiation losses, the other contributions of loss result very determinant and cause the response observed in Fig. 4.24(a).

The group delay response of the structure is studied in Fig. 4.26(a). As before, simulation points are represented with marks, and the solid line is a linear regression fitting well with them. The time delay is expressed respect to the central frequency. It is observable that the higher frequencies of the band travel slower than the lower ones, what is caused by the larger confinement in the dielectric for higher frequencies, so that they experience lower group velocity. Nevertheless, note that the maximum difference between components is only of $0.8 \mathrm{ps}$, therefore, the proposed structure itself is not a very dispersive one.

Now, in Fig. 4.26(b) it is represented the total group delay caused by a path of length $L$ with a $90^{\circ}$ bend in the middle, as a function of $L$, for the limiting frequencies of the band, $f=0.5 \mathrm{THz}$ and $f=1.5 \mathrm{THz}$. In this case, the situation is quite different to the losses case of Fig. 4.24(a). Note that, not only the group delay does not become larger, but it diminishes. This is due to the singular behavior of the SWW fundamental mode, which group velocity is slightly (remember that the SWW is very low dispersive) higher for higher frequencies. This clearly differes from the typical response of a dielectric waveguide, which dispersion would make the curves to diverge noticeably. Since the SWW is very low dispersive, the compensation is not very large, but the point is that the group delay dispersion only goes to better levels as the path increases, thus keeping the low dispersion characteristic.

From the previous results, it seems evident that the proposed design for curved paths is inherently well prepared in terms of group delay frequency dispersion. The 


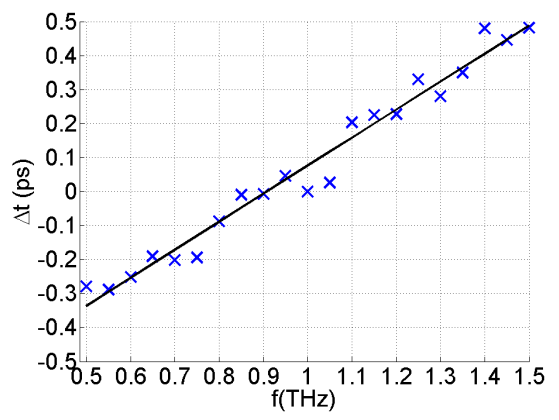

(a)

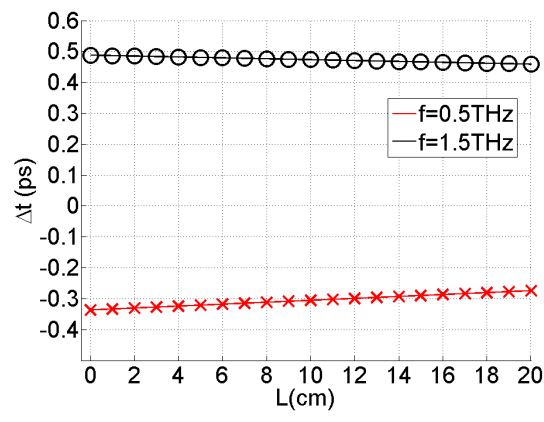

(b)

Figure 4.26: Frequency dispersion of the proposed transition in the operating band [0.5 THz-1.5 $\mathrm{THz}$, in terms of the group delay $(G D)$ respect to the central frequency $f=1 \mathrm{THz}$. a) GD vs. $f$; b) Total GD experience by the minimum a maximum frequencies of the band along path with a $90^{\circ}$ bend in the middle, implemented with the proposed design, as a function of its total path length $L$.

attenuation dispersion is not as good by far. Nevertheless, the possible distortion introduced would be less than for other kind of waveguiding structures. Moreover, it should be mentioned that recovering the attenuation distortion may be easier with proper amplifiers than to compress pulses, what require from more complex structures [593], [594]. In this sense, it is a positive result to found that the proposed solution preserves the main desirable characteristic of wire-type structures of providing good support for wide-band $\mathrm{THz}$ pulse transmission.

\subsubsection{Tapered wire and coating}

In this section the optimal situation is afforded finally. Up to now, bend losses caused by radiation have been shown to be easily mitigated in wire-type structures by means of a proper bend design: a DCSWW following either the solution A of section 4.2 or the solution $\mathrm{B}$ of section 4.3. In addition, transitions allowing the use of the coating exclusively on the curved section have been presented, exhibiting pretty good results. However, despite of the gain in using transitions for the coating, the demanded design for the bend still conditioned the radius size. This is particularly true for the solution A, which is, at the same time, the best option for bends. Therefore, although quite good results were obtained, the possibility of engineering the radius in an optimal way, i. e., allowing large $a \mathrm{SWW}$ for straight waveguiding sections, would raise the SWW performance to a superior level.

To appreciate the above fact, let put the scenario of this section on the view. The optimal bend demands for a SWW with $a=5 \mu \mathrm{m}$, which is small. In Fig. $4.27(\mathrm{a})$, losses in $\mathrm{dB} / \mathrm{mm}$ of a $\mathrm{SWW}$ are displayed as a function of the radius. Note that the $a=5 \mu \mathrm{m}$ point causes $0.116 \mathrm{~dB} / \mathrm{mm}$. If moderate to large radius are considered ( $a=100 \mu \mathrm{m}$ will be the choice in this section, but even larger radius may be afforded) the attenuation reduces drastically. Not in vain, $a=100 \mu \mathrm{m}$ implies only $0.008 \mathrm{~dB} / \mathrm{mm}$. Since the radiation on bends of an $a=100 \mu \mathrm{m}$ SWW (see Fig. $4.27(\mathrm{~b}))$ is extremely large (22 $\mathrm{dB}$ of radiation losses), the benefit of such transitions 


\begin{tabular}{lcc}
\hline Wire radius & $a=5 \mu \mathrm{m}$ & $a=100 \mu \mathrm{m}$ \\
\hline \hline & & \\
& & \\
\hline $90^{\circ}$ bend losses & $7.1 \mathrm{~dB}$ & $22 \mathrm{~dB}$ \\
Straight path losses & $0.116 \mathrm{~dB} / \mathrm{mm}$ & $0.008 \mathrm{~dB} / \mathrm{mm}$ \\
\hline
\end{tabular}

Table 4.2: Duality of propagation and radiation losses in the $S W W$ for the cases of a small and a large radius.

really worth to engineer the design to incorporate them. The table 4.2 summarizes well the duality existent between the propagation losses and bend losses due to radiation. The possibility of combining the best of each design strongly motivates the consideration of transitions to adapt different radius wire-type structures.

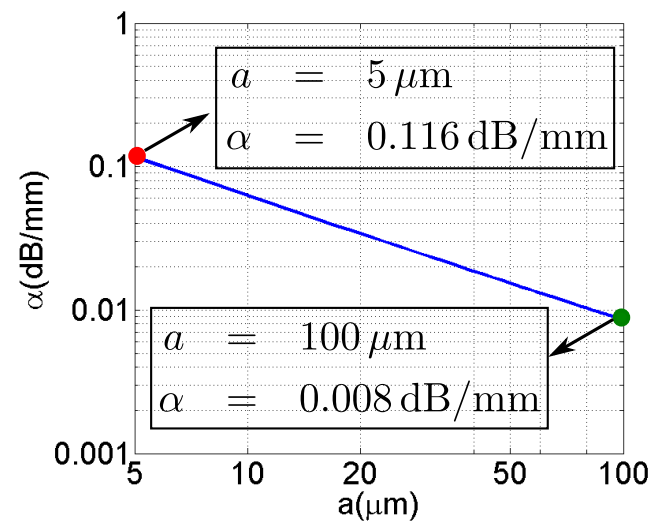

(a)

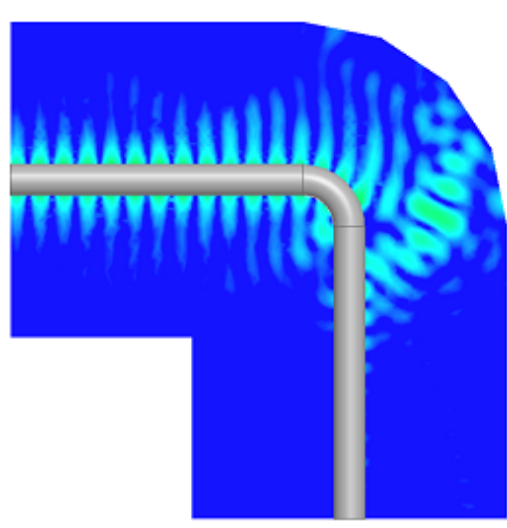

(b)

Figure 4.27: Dilemma of large radius $S W W$. a) Attenuation in $d B / m m$ of a $S W W$ as a function of its radius $a$. The cases of small $(a=5 \mu \mathrm{m})$ and large $(a=100 \mu \mathrm{m})$ radius are highlighted in the graph; b) E-field in logarithmic scale of $20 \mathrm{~dB}$ on a $90^{\circ}$ bend implemented with a $S W W$ of $a=100 \mu \mathrm{m}$, showing strong radiation (losses of $22 \mathrm{~dB}$ ).

The proposed designs incorporating the aforementioned transitions are shown in Figs. 4.28(a) and 4.28(b). Again, to keep generality and consider a great variety of cases, apart of the full transition tapering conductor radius and dielectric coating (that of Fig. 4.28(b)), the possibility of a transition using no coating, and just tapering the conductor radius, see Fig. 4.28(a), is as well considered. This is done not only to cover the possible case of having a manufacturing process that, for some reason, may become alleviated if the coating is avoided, but also because, in this way, the improvement gained with the transitions its breakdown in the separated contributions of tapering the wire and tapering the coating, hence getting additional interesting physical insight. 
The input waveguide is a SWW with radius $a=100 \mu \mathrm{m}$. In the first design, which provide tapering of the conductor radius but does not include coating, Fig. $4.28(\mathrm{a})$, the waveguide at the bend is a SWW with radius $a=5 \mu \mathrm{m}$. The reduction of the radius is done by means of a linear tapper of length $l_{t}$. The transition at the bend output is analogous, but in the reverse direction. In the case that includes the dielectric coating, Fig. 4.28(b), the waveguide at the bend is a DCSWW with radius $a=5 \mu \mathrm{m}$ and coating thickness $t=30 \mu \mathrm{m}$, i.e, $b=35 \mu \mathrm{m}$. The procedure with the conductor is exactly the same. Regarding to the dielectric coating, the proposed procedure consist in adding some coating of thickness $t$ from the point that $a$ becomes smaller than the final external radius of the DCSWW $b=a+t$. This means that, assuming $\hat{z}$ as propagation direction, the dielectric coating is present from $z$ such that $a(z)<35 \mu \mathrm{m}$. The coating at each point is such that $a(z)+t(z)=35 \mu \mathrm{m}$. Therefore, when the bend approaches, the radius is reduced, and, from the point that it becomes smaller than the total radius of the final DCSWW, some coating is added so that the minimum total radius appreciable is $b=a+t=35 \mu \mathrm{m}$, see Fig. $4.28(\mathrm{~b})$.

To visualize the actual effect of the transition on the guided waves, in Figs. $4.28(\mathrm{c})$ and $4.28(\mathrm{~d})$ it is represented the E-field in logarithmic scale of $20 \mathrm{~dB}$ range, in a $90^{\circ}$ bend implemented with the radius transition without coating and the radius transition with coating, respectively. The transition length for this simulation has been chosen to be $l_{t}=1.6 \mathrm{~mm}$ (lately proved as an optimal one). If one compares these plots with that of Fig. 4.27(b), the difference is notorious, and it must be highlighted that the input and output waveguides are the same for all three cases: an $a=100 \mu \mathrm{m}$ SWW.

In field plots corresponding to the two proposed transitions, it is appreciated how the reduction of the radius causes confinement of the fields, transmitted in a more compact area. In the case of using coating, Fig. 4.28(d), the field is clearly more confined in the curved path, and note how there is found more field intensity at the end of that curved path. In this plot, radiation is appreciable around the curve, even with more evidence than in the case without coating. However, this is caused by the additional reflections that the presence of the coating is producing. As more power reaches the end of the curve, this posterior radiations seems to be causing that visual enhancement. Nevertheless, note that intensity of the E-field at the very output of the structure is larger for the case including coating, indicating that this structure performs better. In fact, for the structure without coating $S_{21}=-9.5 \mathrm{~dB}$, whilst for the structure with coating $S_{21}=-5.5 \mathrm{~dB}$.

The optimization of the transition length $l_{t}$ for both structures is shown in Fig. $4.29(\mathrm{a})$. In the case of the transition with coating (blue solid curve), minimum losses of $5.5 \mathrm{~dB}$ are found for an optimal length of $l_{t}=1.6 \mathrm{~mm}$. From that value, increasing the $l_{t}$ do not produce any appreciable gain but propagation losses cause the performance to worsen. This optimal length is quite larger (more than twice) than in the coating transition of the previous section. In the present case, the difference between the modal patterns is considerably larger. Observe for instance that with no transition losses as high as $16 \mathrm{~dB}$ would take place, and that there is an important gain in the range $l_{t} \in[0 \mathrm{~mm}-0.6 \mathrm{~mm}]$ (see the slope of the curve), this indicating how the increasing the $l_{t}$ produces a remarkable effect in the matching. Moreover, in this case, the transition section starts to produce considerable propagation losses 


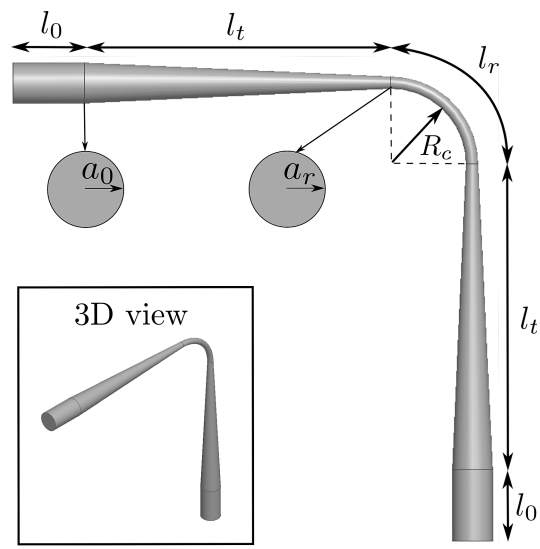

(a)

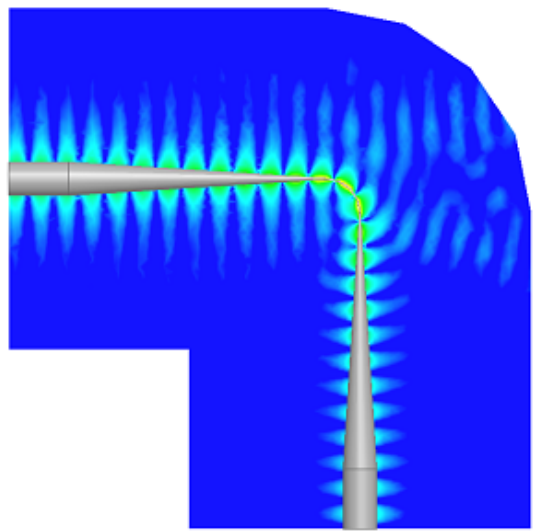

(c)

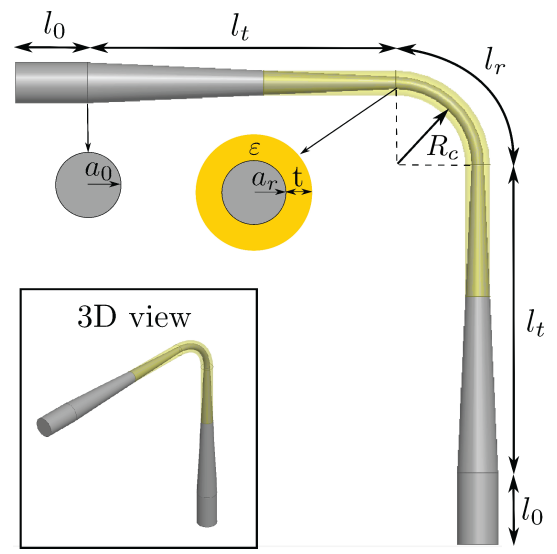

(b)

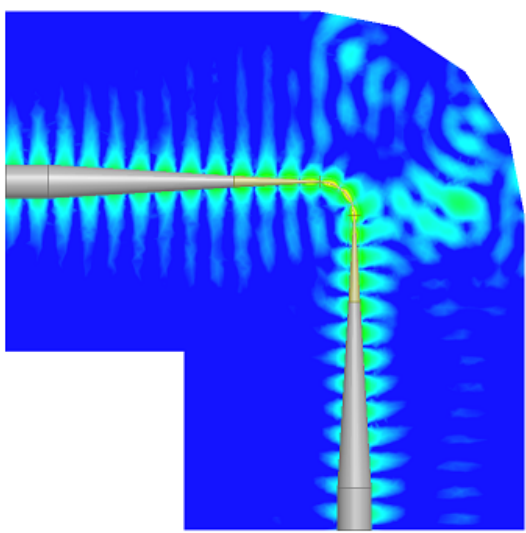

(d)

Figure 4.28: Proposed transitions, tapering the conductor radius $a$. $2 D$ schematics with the main transition parameters are shown in the first row images. a) Without coating; b) With coating; Efield in logarithmic scale of $20 \mathrm{~dB}$ on a $90^{\circ}$ bend. c) Without coating; d) With coating.

only when the radius reach small values, thus most of the transition section does not produce significant propagation losses. This also causes the inflexion point to be much less evident. Actually, the minimum is very smoothly marked. In fact, if transition size would be a problem, $l_{t}=0.6 \mathrm{~mm}$ performs quite well, exhibiting only additional losses of $1.5 \mathrm{~dB}$ regarding to the optimum design.

In the case without coating the last effect is much more evident. In this case, although as $l_{t}$ becomes large the gain in matching becomes smaller and smaller, the curve rather saturates to a limit than exhibiting a minimum. In fact, this minimum occurs for very large $l_{t}$, outside of the visualized range, and it is very smooth. This is because, in this case, the additional propagation losses caused by larger $l_{t}$ are even smaller. Note also that this case is lower bounded below by the $7.1 \mathrm{~dB}$ imposed by the losses of the curved path itself. Therefore, $9.5 \mathrm{~dB}$ is a pretty good value in as much roughly $2.5 \mathrm{~dB}$ are lost to gain later more than $1 \mathrm{~dB} / \mathrm{cm}$ in the actual 
propagating straight paths (see table 4.2). Therefore, if possible, introducing the transition is an action to take without doubt.

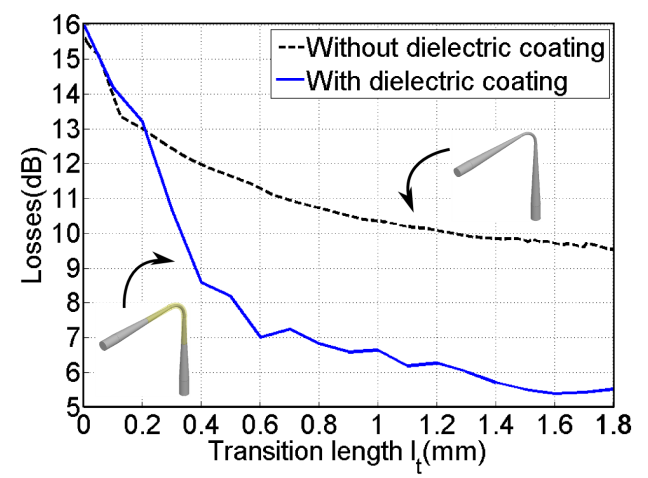

(a)

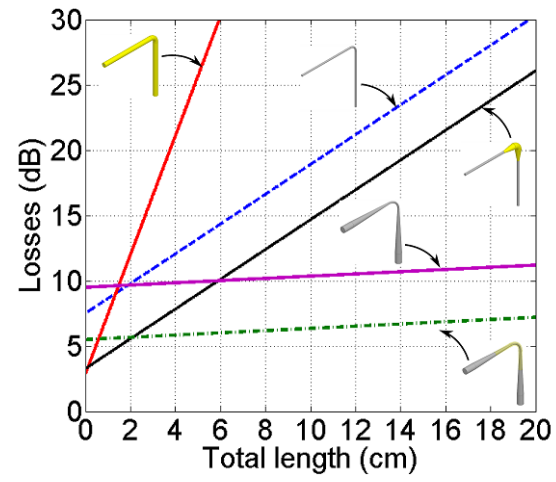

(b)

Figure 4.29: Optimization of transitions and final results. a) Total losses on a $90^{\circ}$ bend of the proposed bend designs including transitions as a function of the transition length $l_{t}$; b) Comparative between conventional $S W W$ and DCSWW designs and all designs proposed in this section (transition in coating, transition in radius, and transition in both, radius and coating). Total losses in a path of length $L$ including a $90^{\circ}$ bend, as a function of $L$.

To conclude this chapter, a general view of the improvement introduced by the proposals of this thesis, all the bend designs considered along the chapter (fixed SWW, fixed DCSWW, varying only $t$, varying only $a$, and varying both $a$ and $t$ at the same time) are compared in Fig. 4.29(b). As usual, the way to do it has been calculating the total losses of a waveguiding path with a $90^{\circ}$ bend in the middle, as a function of the total length of the path, $L$. The offset of the straight lines is given by the bend losses of each design, and the slope is given by the propagation losses of the straight path waveguide in each design.

It results evident that the use of transitions varying the radius causes the performance to be in a superior level. The possibility of using a large radius SWW causes the total loss curves to be practically horizontal. Note that, indeed, the attenuation of SWW with $a=100 \mu \mathrm{m}$ is, considering now large distances, $\alpha=8 \mathrm{~dB} / \mathrm{m}$, which is a very low value. The case of the full transition varying both, radius and coating is specially interesting. Observe that the losses of this design are only $2.25 \mathrm{~dB}$ higher than those of the coating transition of fixed $a=5 \mu \mathrm{m}$ of the previous section. This small additional loss gives an appreciable gain over a structure (the fixed $a=5 \mu \mathrm{m}$ transition) which already exhibits a good performance compared with the conventional designs. Note also that even the case of a transition varying only the radius results rapidly advantageous as the total length of the path increases, despite of the bend losses are close to $10 \mathrm{~dB}$. The only problem foreseen for such transition is that, in case of having additional bends, total losses may reach high levels

A summarized comparison of losses between the three proposed designs in this chapter is shown in table. 4.3. The low propagation losses of the transitions varying the conductor radius are highlighted to give evidence that the gain in this feature is more important than the worsening in bend losses. In fact, note that the environ- 


\begin{tabular}{lccc}
\hline Design & Coating & Radius & Radius \& Coating \\
\hline \hline & & & \\
& & $9.5 \mathrm{~dB}$ & $5.5 \mathrm{~dB}$ \\
& & & \\
\hline $90^{\circ}$ bend losses & $3.25 \mathrm{~dB}$ & $\mathbf{0 . 0 0 8} \mathbf{~ d B} / \mathbf{m m}$ & $\mathbf{0 . 0 0 8} \mathbf{~ d B} / \mathbf{m m}$ \\
\hline Straight path losses & $0.116 \mathrm{~dB} / \mathrm{mm}$ & $\mathbf{0 . 0 0 8}$ \\
\hline
\end{tabular}

Table 4.3: Comparison of losses between three proposed transition designs.

ments in which wire-type are though to operate do not contemplate a large number of bends, as much two or three. Consider for instance the case of scanning some inner tissues of the human body in order to get its spectral response (examples similar to those exposed in [60]-[68]). It could be foreseen wire-type structures prepared to be bent in such sections implementing the proposed designs ${ }^{3}$, and it seems apparently enough to provide two and as maximum three changes of direction if the access to the tissue is well provided. In this way, a good figure to keep in mind is that with the best design provided in this thesis, a 1 meter long wire-type cable including 3 bends would allow to obtain only $24.5 \mathrm{~dB}$ of total losses. This quantity is easily recoverable at reception and even gives margin for the additional losses caused by the real, non-ideal situation (manufacturing imperfections, non-perfect use by the operator, challenging environment, etc.).

Therefore, it is concluded that, although the idea of using impenetrable surface waveguides is sometimes criticized arguing a difficult practical implementation due to problems related with the excitation efficiency and the radiation losses in bends, or similar perturbations, a proper study of the problem drives to good performance solutions quite successfully. Moreover, the work carried out in this part of the thesis may be regarded as a first approach. More complex modifications, or further thoughtful solutions might come in the next years by proper dedicated research. In this way, it seems possible that this kind of surface waveguides could become for broadband $\mathrm{THz}$ pulses waveguiding what hollow metallic waveguides have been for low-loss microwave waveguiding, or the silica derivatives fibers for low-loss long distance optical signals transmission. Several possibilities have been given, considering as many as possible scenarios of design, where some restriction may appear regarding the nature of the application. Certain amount of work is still to be done, but at least, it is clear that good engineering on wire-type designs provides a powerful tool in the $\mathrm{THz}$ technology environment.

\footnotetext{
${ }^{3}$ The idea is a wire-type structure with some sections prepared to be bent. In those sections the designs proposed in this chapter would take place.
} 


\section{Chapter 5}

\section{Experimental results}

Chapter 3 has provided rigorous analysis tools for wire-type structures as well as interesting remarks and conclusions, result of a deep study of such structures that have been successfully applied in Chapter 4 to design optimized bends and transitions. In this way, important information regarding wire-type structures behavior and some interesting solutions for practical designs concerning bends have been exposed in detail with the aim of giving a useful, complete text for the $\mathrm{THz}$ waveguide researcher. This conforms the main set of objectives in this part of the thesis and it has completeness itself. Nevertheless, experimental validation is always recommendable as an additional substantiation for the treated concepts. In the case of wire-type structures at $\mathrm{THz}$, the required prototypes and experimental setups are rather complex and/or expensive in general (see [492], [506] or [514] for instance). However, in the scope of this part of the thesis, experimental results are required just to give evidence in a real environment of some of the observed effects in the previous chapters, without the intention of designing very sophisticated prototypes satisfying certain specifications. Therefore, in this chapter, the objective of given experimental proof to the reviewed theoretical concepts and synthesis procedures is straightforwardly pursued with affordable experimental setups, in concordance with available manufacturing and testing resources.

The work carried out in this chapter consists of two parts. In a first approach, experimental results are obtained at mm-wave frequencies, particularly, in the Kaband. In this way, possible manufacturing tolerances effects is alleviated, insomuch the wavelength is one or two orders of magnitude larger than at $\mathrm{THz}$ frequencies. In addition, assemblage, excitation, and measurement process, are quite simpler than at $\mathrm{THz}$ frequencies. For instance, excitation schemes such as that presented in [497] make sense. In this way, facilities of our research group, prepared for microwave frequencies, are used right away. In a second approach, an experimental setup prepared to work at actual $\mathrm{THz}$ frequencies is assembled taking advantage of some facilities in the Polytechnique University of Catalunya / Universitat Politècnica de Catalunya (UPV). 


\subsection{Ka-band prototypes results}

The first experimental study is carried out in the $[38 \mathrm{GHz}-47 \mathrm{GHz}]$ band, being $f=45 \mathrm{GHz}$ the reference frequency. The aim of the experiments is to evidence the differences between SWW and DCSWW in terms of propagation losses in straight and curved paths. The most relevant objective is to give proof of that a SWW incorporating a coating exclusively in the curved path noticeably improves the behavior of a standard SWW, being much better solution than using coating along all the path, i. e., to use a DCSWW. In this way, a simplified version of the first transition of chapter 4 (tapered coating) is experimentally implemented. First, some previous considerations are given, including some simulated results related with the posterior experiments so that an estimation of the experimental results to be obtained is provided. Then, experimental evaluation of the excitation and the behavior of wire-type structures in straight propagation sections is performed. Later, a first configuration to evaluate the performance of such waveguides in curved propagation sections is presented. In this configuration the bend is created by means of two fasting elements. After this, a second configuration using only one fasting element, more suitable, is presented. Differences between SWW, SWW with coating exclusively on curved sections, and DCSWW are experimentally evidenced.

\subsubsection{Previous considerations}

Excitation of wire-type waveguides is based on the coaxial cable excitation proposed by Goubau [497]. Conceptually, the way to excite a DCSWW with good efficiency by using a coaxial cable, Fig. 5.1(a), consists in a progressive opening of its outer conductor, creating a horn, i. e., tapering the external conductor radius, see Fig. 5.1(b). From this point, power can be transferred to a SWW by just removing the dielectric coating with a taper, see Fig. 5.1(c). From the conclusions of previous chapters, it seems clear that removing the coating outside of the horn area is better than inside of it. Note that, when the coating is removed, field spreads laterally, what could enhance losses due to the interaction with the horn if the transition takes places inside of it. In addition, a larger mismatch would appear at the discontinuity observed at the horn aperture. Note that when the coating is present it acts as a guiding/confining element so that the effect of the external coaxial conductor on the fundamental mode is weaker than for a non-coated wire. If the coating is retired with the external conductor present, it is formed a large area coaxial, that has a worse matching with free space.

The employed coaxial cable in the experiments is a UT-085 from Microcoax ${ }^{\circledR}$ [595] with a Silver Plated Copper (SPC) inner conductor of radius of $a=255 \mu \mathrm{m}$ and and an outer copper conductor of radius of $b=838 \mu \mathrm{m}$. In both cases conductivity is assumed to be $\sigma=5.8 \cdot 10^{7} \mathrm{~S} / \mathrm{m}$. The dielectric is Teflon $\left(\varepsilon_{r}=2.04, \tan \delta=2 \cdot 10^{4}\right.$ for $f<100 \mathrm{GHz}[21])$. This provides that the first high-order mode propagates at $f_{c_{1}}=61 \mathrm{GHz}$, outside of the operation band. The input and output horns have a maximum aperture of radius $b^{\prime}=10 \mathrm{~mm}$, and a total length of $L=30 \mathrm{~mm}$, what implies an aperture angle of $\phi=19.5^{\circ}$. The described system has been simulated with $\operatorname{HFSS}^{\circledR}$ [411] for several lengths of the in-middle waveguide, considering SSW 


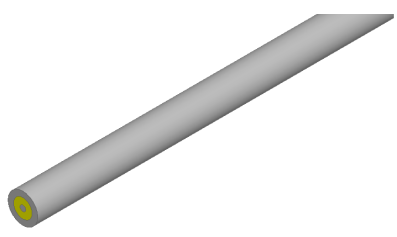

(a)

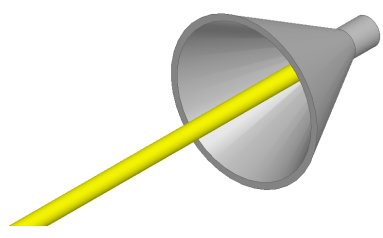

(b)

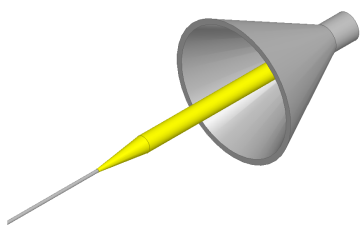

(c)

Figure 5.1: Schematics of wire-type waveguides excitation at mm-wave frequencies by means of a coaxial cable. a) Initial coaxial cable; b) Excitation of a DCSWW: the outer conductor of a coaxial cable is opened with a taper to create a horn providing good matching; c) Excitation of a $S W W$ : after the excitation of the DCSWW, the dielectric coating is removed with a taper.

and DCSWW. The behavior of a simple coaxial cable without any taper has been also studied for comparison.

Simulation results at $f=45 \mathrm{GHz}$ are shown in Fig. 5.2(a), where received power is displayed as a function of the waveguiding section length. In the plot, simulated results are represented with solid lines. Note that simulations have been carried out only up to $L=0.95 \mathrm{~m}$ since otherwise the volume to discretize leads to unaffordable simulations. By the same reason, the external air region surrounding DCSWW and SWW has been truncated to $R=20 \mathrm{~mm}$. This has no influence in the DCSWW case, however, in the SWW case, part of the transmitted power is neglected. Theoretical calculations indicate that the considered volume takes into account at least $80 \%$ of the transmitted power. In this way, some trade-off between accuracy and amount of results/data has been necessary in the SSW case. Fig. $5.2(\mathrm{~b})$, where the E-field magnitude in an axial plane is represented, qualitatively shows the effect of the aforementioned truncation on the SWW. Whereas in the DCSWW case the considered air volume surrounding the waveguide seems large enough, for the SWW it is clear that part of the involved fields is missed.

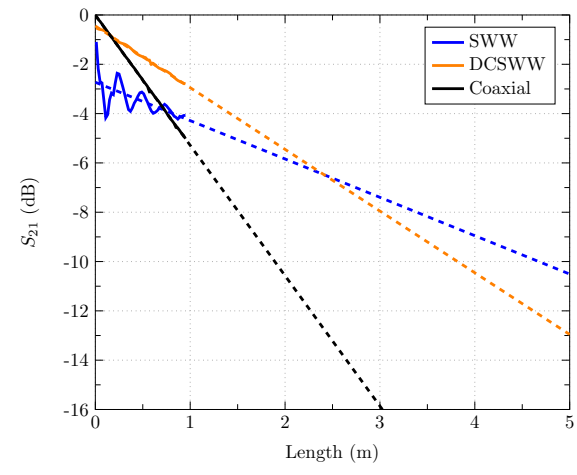

(a)

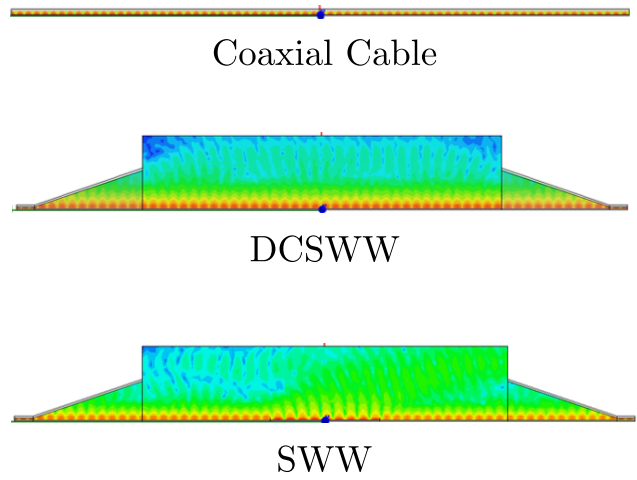

(b)

Figure 5.2: Simulation results at $f=45 \mathrm{GHz}$ with the three considered structures: coaxial, $D C S W W$ and $S W W$. a) Estimation of waveguide attenuation $\alpha(d B / m): S_{21}(d B)$ vs. length. Solid lines are simulated data whereas dashed lines are extrapolated linear regressions of the simulated data; b) E-field magnitude in the simulation domain. 
The truncation effect added to the transitions mismatch (transitions have not been optimized since in the experimental setup they are build manually) cause certain ripple on the results for the SWW. Dashed lines are a linear regression of the obtained data, which are extrapolated up to $L=3 \mathrm{~m}$ to inference what occurs as the propagation length increase. Notice how, from certain length, propagation losses become more relevant than mismatch and transition losses. This regression is specially recommendable to process SWW data, which are affected by the aforementioned undesired effects. The slope of such regression lines are an estimation of the propagation losses of each waveguide. The estimated losses are displayed in Table 5.1 for clarity. Note how, even though the employed frequency is low (taking the $\mathrm{THz}$ band as reference), the DCSWW already introduces an important improvement regarding to the coaxial cable. Theoretical calculations indicate an attenuation of $\alpha=2.36$ $\mathrm{dB} / \mathrm{m}\left(\alpha_{c}=1.86 \mathrm{~dB} / \mathrm{m}\right.$ and $\left.\alpha_{d}=0.5 \mathrm{~dB} / \mathrm{m}\right)$, hence the estimation through simulations shows good agreement. The SWW presents close to $40 \%$ lower propagation losses than the DCSWW, which is an important difference, but not as evident as at $\mathrm{THz}$ frequencies. Note that the percentage of power inside the dielectric in the DCSWW is $\eta=48 \%$ and the dielectric is quite good in terms of losses. Theoretical calculations predict losses of $\alpha=1.21 \mathrm{~dB} / \mathrm{m}$ for the SWW, slightly lower than those obtained by the presented simulation due to the truncation of the surrounding air medium. Note that despite of the undesired ripple, the regression line gives a reliable enough estimation of SWW propagation losses for the purposes of the study.

\begin{tabular}{cccc} 
Waveguide & Coaxial & DCSWW & SWW \\
\hline Estimated $\alpha(\mathrm{dB} / \mathrm{m})$ & 5.28 & 2.50 & 1.56
\end{tabular}

Table 5.1: Estimated attenuation through simulation results at $f=45 \mathrm{GHz}$.

Previous results indicate that for reasonable lengths, wire-type structures become more convenient than the classical coaxial cable in terms of losses. The SWW becomes more convenient than the DCSWW from $L=2.4 \mathrm{~m}$. This length scaled to $f=1 \mathrm{THz}$ means only $L=10.8 \mathrm{~cm}$, and it must be noted that direct scaling is not a totally fair comparison since ohmic and dielectric losses in a DCSWW would worsen much more than those of a SWW as the frequency increases. In order to take advantage of the lower propagation losses of SWW in straight paths, a SWW prototype with coating exclusively placed on the bend will be considered. The previous simulation results are kept as a reference for the next presented experimental results.

\subsubsection{Measurements of straight path losses}

The initial experimental setup is conceived to measure straight path losses of the different waveguides under study as well as to quantify the effect of the horn, i. e., the improvement caused by the better matching provided by a tapering of the outer conductor radius instead of a direct transition from coaxial to DCSWW. The complete experimental setup is shown in Fig. 5.3(a). The system is formed by transmitter and receiver coaxial horns, the wire-type waveguide under study and the mechanical alignment system. The coaxial employed to create a DCSWW and a SWW is shown in Fig. 5.3(b). In Fig. 5.3(c) it can be appreciated how this 
coaxial cable is inserted in the horn structure to implement in practice the previously commented transition from coaxial to DCSWW. Fig. 5.3(d) shows how the DCSWW arises from one of the two employed horns. Finally, Figs. 5.4(a) and 5.4(b) show the excitation schemes for SWW and DCSWW, respectively. In Fig. 5.4(b) it can be appreciated how, for the case of a SWW, the dielectric is removed few centimeters after the horn aperture by means of a manually created transition.

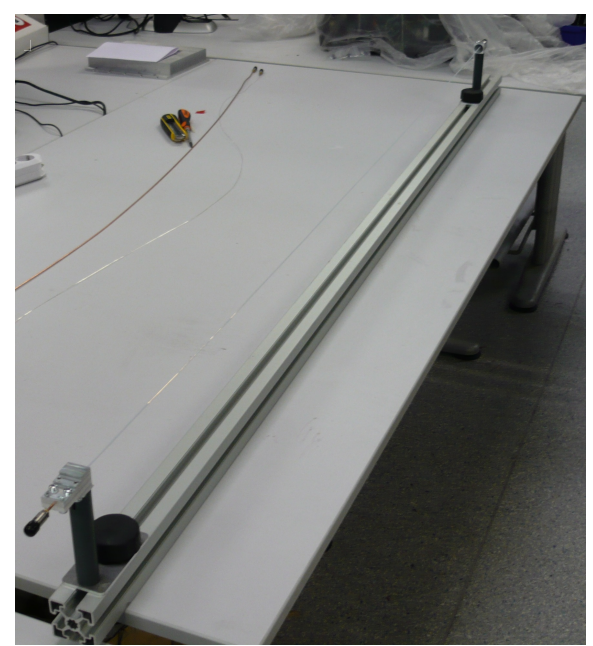

(a)

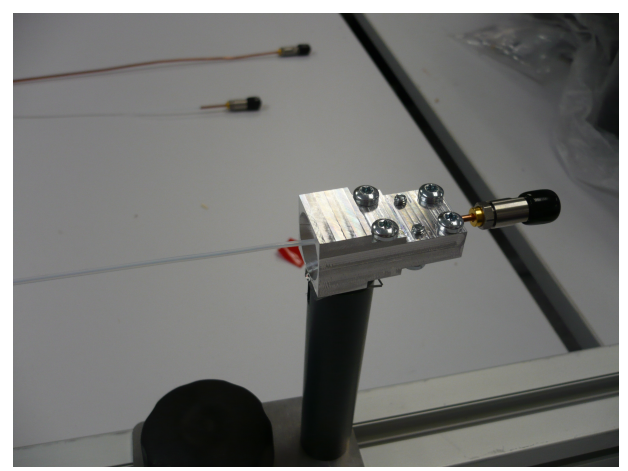

(c)

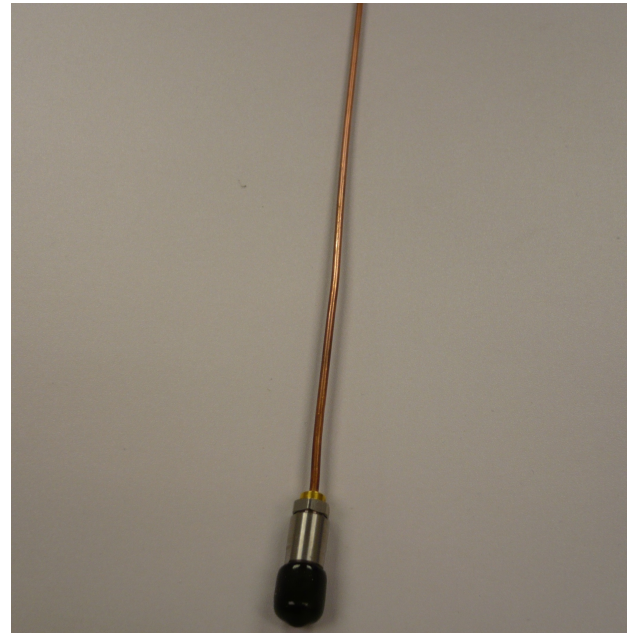

(b)

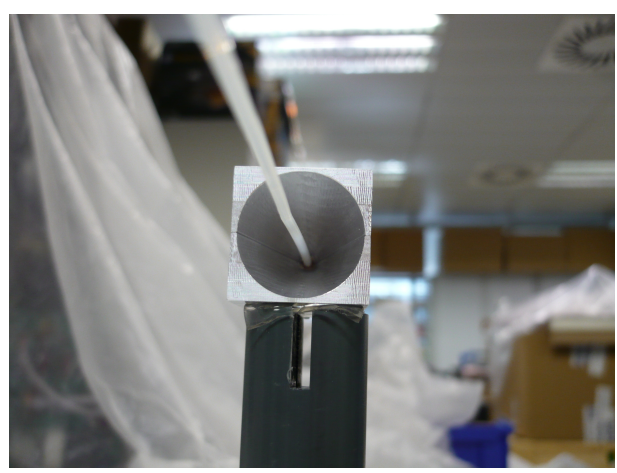

(d)

Figure 5.3: Wire-type waveguides experimental setup. a) Complete system including transmitter and receiver coaxial horns, wire-type waveguide under study and mechanical alignment system; $b$ ) Employed coaxial cable; c) Coaxial inserted in the horn structure with progressively aperture of its outer conductor in order to excite a DCSWW; d) Detail of a DCSWW arising from the horn.

Experimental results with the described system are shown in Fig. 5.5. A straight path of $L=1.8 \mathrm{~m}$ has been considered for the evaluated waveguides. First, the effect of the horn is determined. Fig. 5.5(a) shows the received power vs. frequency for SWW and DCSWW considering the cases of direct transition from coaxial and employment of the horn of Figs. 5.4(a) and 5.4(b). Solid lines are measurement points whereas dashed lines are linear regressions of the data. It can be appreciated 


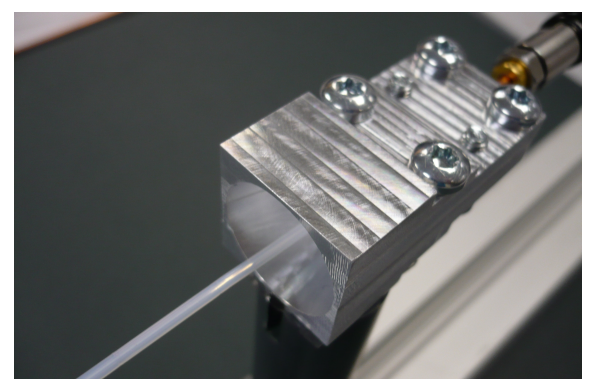

(a)

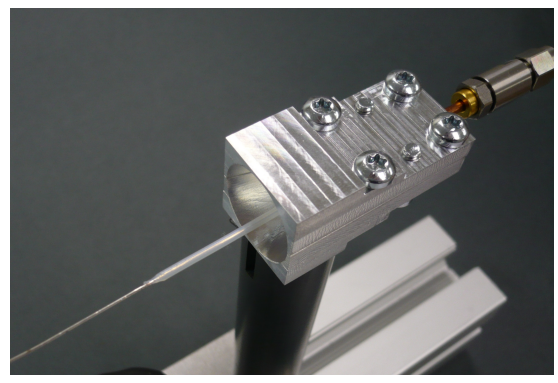

(b)

Figure 5.4: Practical excitation of wire-type waveguide. a) $D C S W W$; b) $S W W$.

how the horns cause a total improvement of approximately $4 \mathrm{~dB}$ in the received power, what means $2 \mathrm{~dB}$ per horn transition. Therefore, horns are a quite necessary in the system.

Fig. 5.5(b) compares the received power vs. frequency for SWW and DCSWW employing horns, and the original coaxial cable. Again, solid lines are measurement points whereas dashed lines are linear regressions. These measurements are compared with the simulations of Fig. 5.2(a) in Table 5.2. There is reasonable good agreement between simulations and measurements given that several effects are not contemplated in the simulation model. The most evident one are the losses introduced by the transitions from DCSWW to SWW, which, since are created manually, perform worse than what was estimated in the simulation. The excessive DCSWWSWW transition losses together with the relatively short waveguiding path cause the DCSWW to introduce less total losses than the SWW. By comparing simulated and measured results of SWW and DCSWW it could be affirmed that proper manufactured DCSWW-SWW transitions may improve the received power close to $2 \mathrm{~dB}$. In the case of the coaxial cable, received power is slightly larger than predicted by simulations. This is probably caused by the larger conductivity of the SPC of the inner conductor which has been modeled in simulations as copper.

\begin{tabular}{cccc} 
Waveguide & Coaxial & DCSWW & SWW \\
\hline Simulated & $-10.4 \mathrm{~dB}$ & $-5 \mathrm{~dB}$ & $-5.6 \mathrm{~dB}$ \\
\hline Measured & $-9.6 \mathrm{~dB}$ & $-5.7 \mathrm{~dB}$ & $-8.2 \mathrm{~dB}$
\end{tabular}

Table 5.2: Comparison between simulation and experimental results regarding the received power after a straight path of $L=1.8 \mathrm{~m}$. In the case of $S W W$ and DCSWW horns are employed in the excitation.

By the above, it can be affirmed that the first set of experimental measurements provide reasonable results according to simulations. The sources of the slight disagreement with simulations have been clearly identified taking into account the gained knowledge from results of previous chapters. Therefore, the measurement system has been successfully tested to be reliable for more complex setups, including curves, which are presented in the following sections. Regarding the results, it seems clear that, given the reasonable agreement between simulations and mea- 


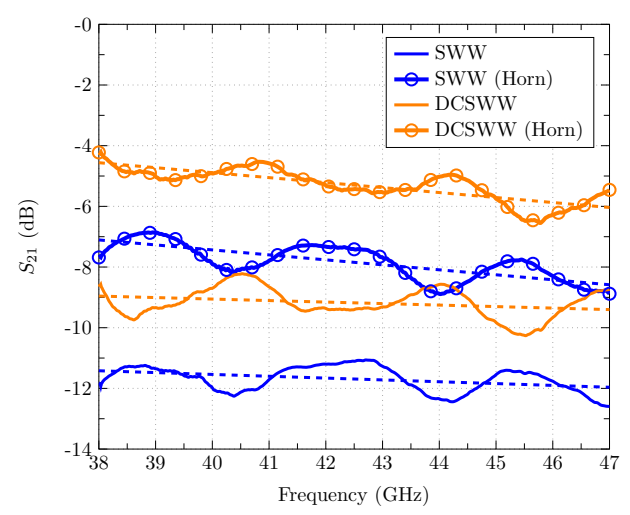

(a)

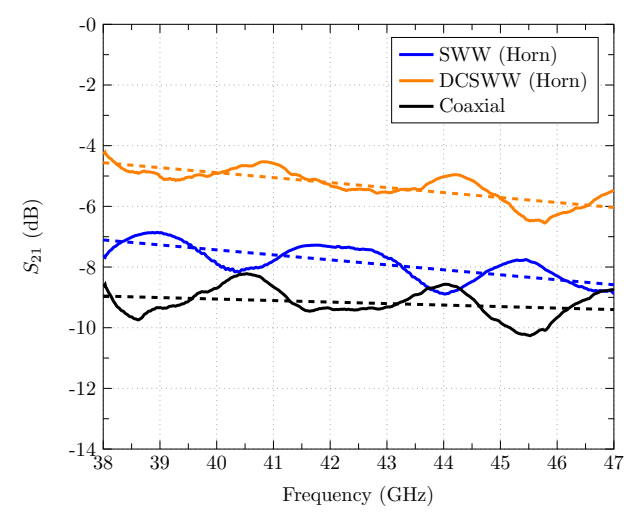

(b)

Figure 5.5: Measurement of straight path losses. Photographs of the experimental excitation scheme using horns for the matching with the input coaxial cable: a) DCSWW; b) $S W W$. Results for a path length of $L=1.8 \mathrm{~m}$ : c) Received power $S_{21}(\mathrm{~dB})$ vs. frequency of a $S W W$ and a $D C S W W$ with and without matching horn. Dashed lines are a linear regression of the measured points; d) Comparison of Losses vs. frequency of wire-type waveguides using matching horn and the initial coaxial cable. Dashed lines are a linear regression of the measured points.

surements, predictions of Fig. 5.2(a) would be satisfied in practice if enough large wires were used. Nevertheless, it must be highlighted that proper SWW-DCSWW transitions are essential in order reduce the length from which the SWW is more convenient than the DCSWW.

\subsubsection{First curved path measurements}

The first experimental setup employed to evaluate the performance of SWW and DCSWW on curves is shown in Fig. 5.6(a). In the image it can be appreciated how, in the system, the two alignment arms where transmitter and receiver are positioned are attached to a metallic disc which acts as an angular positioner that adjusts the curvature angle. At the end of each alignment arm a fasting element made of Rohacell ${ }^{\circledR}$ is placed so that a curve is created with the wire-type waveguide under test being fixed at the two edges of such curve, see Fig. 5.6(b). Detail of one of the Rohacell ${ }^{\circledR}$ fasting elements is shown in Fig. 5.6(c). This is a simple way to have precise control of the curvature angle, that implies a moderate-large curvature radius.

Results obtained with the previous experimental setup for SWW and DCSWW are shown in Figs. 5.7(a) and 5.7(b). The plots show the received power in the operation band for several curvature angles. A detail of the employed angle positioner indicating the different analyzed angles is shown in Fig. 5.7(c). In Fig. 5.7(a) it can be appreciated how the received power with the SWW rapidly drops as the curvature angle $\theta$ increases. A $90^{\circ}$ angle is causes radiation losses of approximately $13 \mathrm{~dB}$. Separation between curves corresponding to the different analyzed angles indicate that, roughly, radiation losses increase a rate of $1 \mathrm{~dB} / 10^{\circ}$. The larger difference between the straight path line and the curve corresponding to $\theta=30^{\circ}$ indicates 


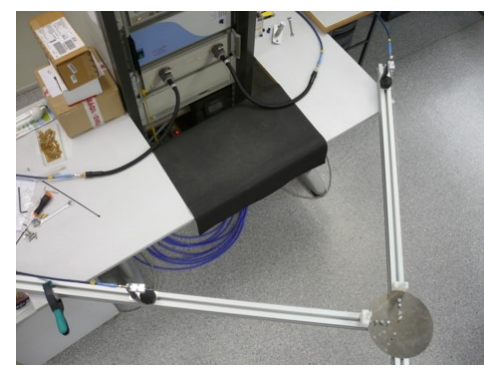

(a)

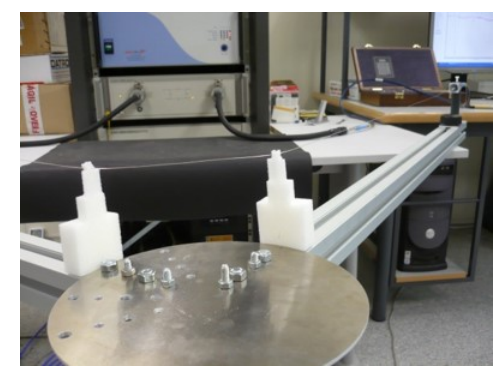

(b)

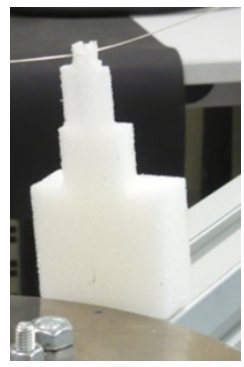

(c)

Figure 5.6: First experimental setup for measurement of bend losses in wire-type structures. a) Top view of the complete system including transmitter and receiver coaxial horns, waveguide under study, alignment arms, fastening elements for the bend, and angle positioner piece; b) Detail of the bend where the two fasting elements can be appreciated; c) Detail of one of the fasting elements.

that the mere fact of introducing some curvature causes a strong perturbation. This perturbation is enhanced with a lower rate when the curvature angle is increased from an existing initial, non-zero angle. This fact was already described by the simulation results of Fig. 4.22(b), when advantages of using coating transitions were evaluated. Contrarily, in the DCSWW case the received power remains totally unaffected by the curvature angle, see Fig. 5.7(b). Fig. 5.7(d) displays the received power vs. curvature angle for $f=45 \mathrm{GHz}$, so that comparison between different curvature angles can be carried more easily. The previously commented effects are quite evident in the plot.

From this first set of experimental results evaluating losses in curved wire-type structures, it seems clear that using some dielectric coating to prevent radiation losses is extremely necessary. The received power after the waveguiding section has been much larger by using a DCSWW. However, it must be affirmed that, below $\mathrm{THz}$ frequencies, the DCSWW has still acceptable propagation losses. Similar results would be obtained at $\mathrm{THz}$ frequencies only for very short propagation paths including a bend (for $L<1 \mathrm{~cm}$ according to results of previous chapter). Other aspect that can surprise from previous results is that the curvature angle has not any effect on the DCSWW, i. e., a total independence with the curvature angle is observed. Note that even though the radius is quite small $\left(a=\lambda_{0} / 25\right.$, equivalent to $a=12 \mu \mathrm{m}$ at $f=1$ $\mathrm{THz}$ ), at least slight differences between curves should be expected according to results of previous chapter, see 4.13(a) for instance. This fact seems to be originated by the way in how the bend is created. Since the wire is fasted at the edges of the curve, some tension is created so that the bend is somehow chamfered. Strictly speaking, this structure rather implies two bends of lower curvature angle than a single one of the desired curvature angle. Under such situation, differences between the studied curvature angles are alleviated insomuch as all are formed by two soft angles, which do almost not perturb the DCSWW fundamental mode. Regarding to these commentaries, it must be observed that the fact that the radius is quite small does not prevent radiation in the SWW as it could be expected. Note that, at the operating frequencies, the impenetrability of the metallic surface is quite higher than at $\mathrm{THz}$ frequencies, what causes a more expanded profile of the fundamental 


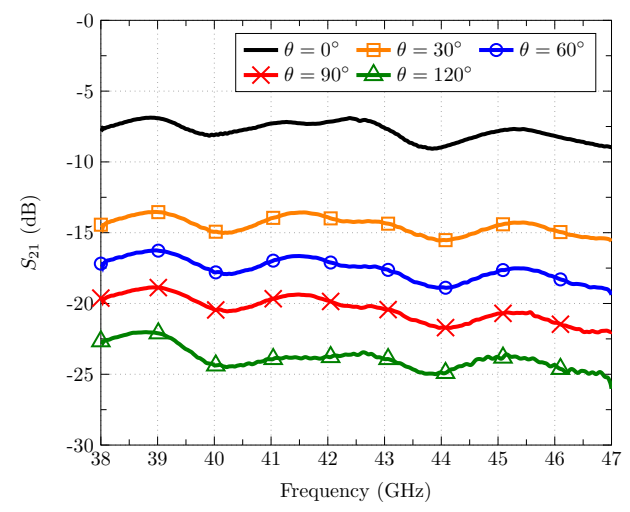

(a)

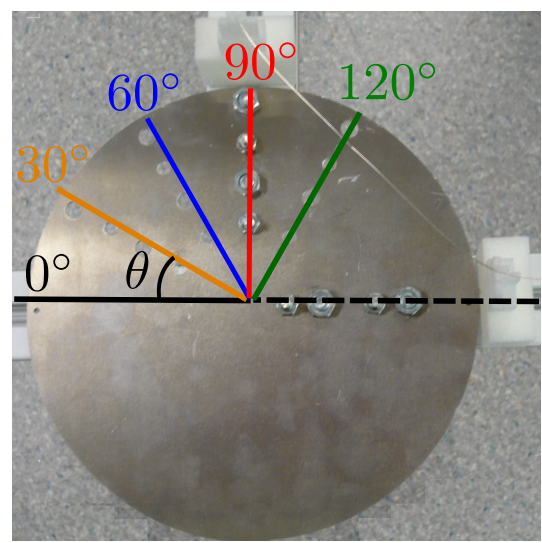

(c)

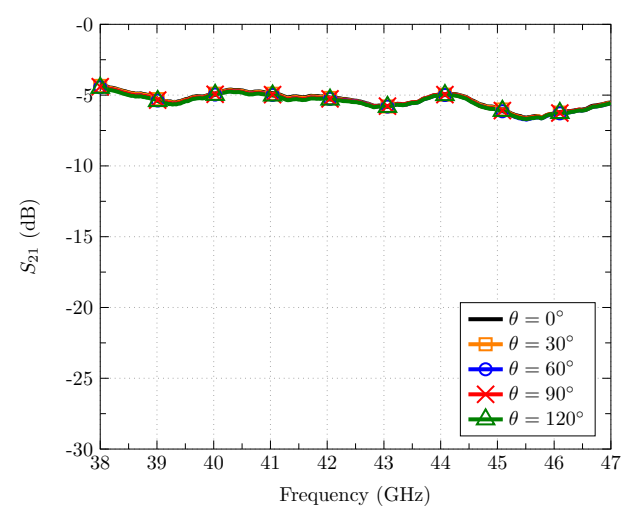

(b)

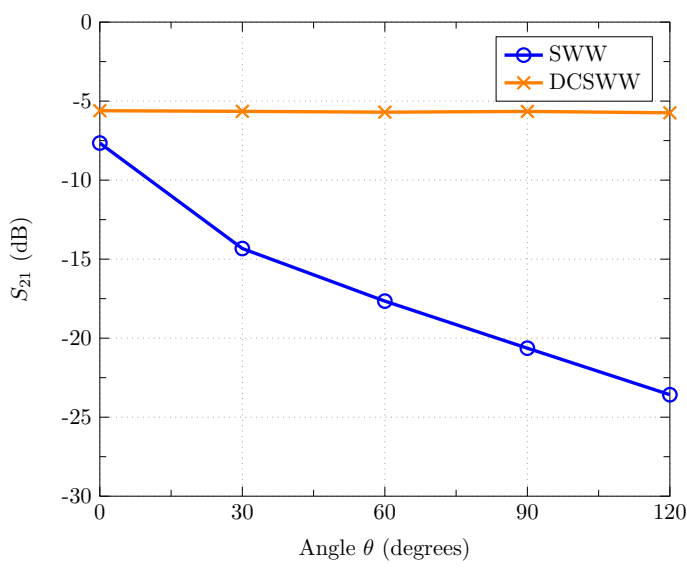

(d)

Figure 5.7: Losses of wire-type waveguide in bends obtained with the first experimental setup. The employed $S W W$ and $D C S W W$ have a length of $L=1.8 \mathrm{~m}$. a) $S W W: S_{21}$ (dB) vs. frequency for several angles of curvature; b) DCSWW: $S_{21}(d B)$ vs. frequency for several angles of curvature. c) Angle positioner; d) $S_{21}(\mathrm{~dB})$ vs. curvature angle $\theta$ at $f=45 \mathrm{GHz}$.

mode in the external medium, hence a mode pattern more affected by the curvature. In other words, the lower penetration of the metal compensates attachment effects caused by the small radius of the wire.

\subsubsection{Second curved path measurements}

A second, improved measured setup is proposed in order to exactly characterize the losses of a single bend with the usual topology. To do so, fabric yarn is used to tense the wire-type waveguide under study, creating a sharp bend, as it can be observed in Figs. 5.8(a) and 5.8(b). Particularly, in Fig. 5.8(b) it can be observed how the curve takes the desired topological shape with this configuration. The curvature angle is controlled by an angle measurement instrument and the position of the transmitter and receiver at the alignment arms. 


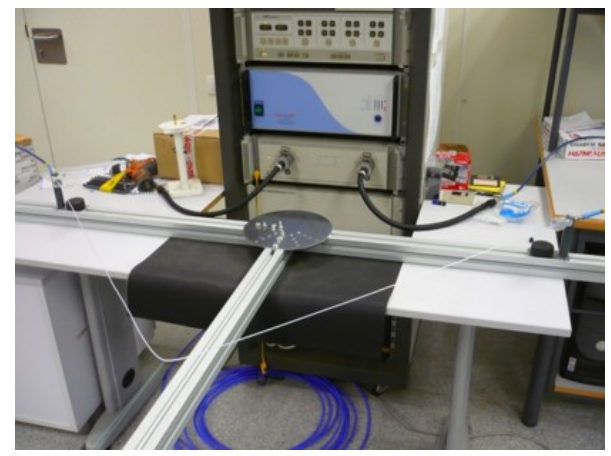

(a)

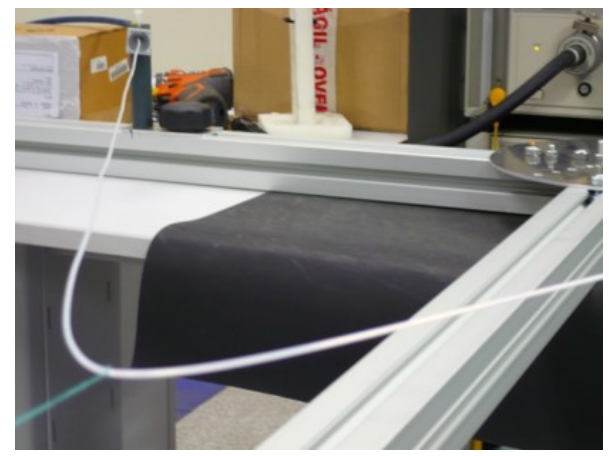

(b)

Figure 5.8: Second experimental setup for measurement of bend losses in wire-type structures. a) Complete system including transmitter and receiver coaxial horns, waveguide under study, alignment arms, fabric yarn as tensor to create the bend, and angle positioner piece; b) Detail of the fabric yarn tensing the wire-type waveguide to create a bend.

Experimental results with this configuration are shown in Figs. 5.9(a) and 5.9(b). A detail of how the different analyzed angles are obtained with the system is shown in Fig. 5.9(c). The aspect of the plot corresponding to the SWW, Fig. 5.7(a), is quite similar to that obtained with the first experimental setup. Again, a rate of $1 \mathrm{~dB} / 10^{\circ}$ is obtained regarding the separation of the curves corresponding to different curvature angles. However, it is found that losses caused by the bend are slightly lower, and the differences between the curves corresponding to $\theta=0^{\circ}$ and $\theta=30^{\circ}$ are slightly smaller. It seems that, since small curvatures already affect the SWW, having two curves of angle $\theta / 2$ instead of only one of angle $\theta$ causes larger additional losses. Differences between both configurations are even more appreciated by observing DCSWW results in Fig. 5.9(b). For this configuration some difference between curves is noticed. The inset in the plot highlights such difference. Note how, actually, main differences are observed beyond $\theta=60^{\circ}$. Received power vs. curvature angle $\theta$ is displayed in Fig. $5.9(\mathrm{~d})$ for $f=45 \mathrm{GHz}$. Note in this plot how the DCSWW experiences a slight fading as the angle increases. As commented before, the decrement is not linear, something already observed in Fig. 4.13(a) for moderate-large radius ( $a=20 \mu \mathrm{m}$ or larger). Although the phase shift follows a linear law with the curvature angle, since the fundamental mode pattern is not exactly concentrated at $r=a$ (it is exponentially distributed towards larger $r$ ), the phase shift does not infer a linear perturbation on the pattern, hence radiation does not follow a linear law. The effect is enhanced by the lower prevalence of TIR effect for more pronounced angles.

At this point, it has been experimentally observed that using dielectric coating on bends is essential. On the other hand, evaluation of simulation and experimental results also predicts advantage of using SWW instead of DCSWW for straight paths of moderate length (so that losses from the excitation transitions are compensated). Therefore, it is the moment to test the performance of a SWW using dielectric coating exclusively in the curved paths. To do so transitions emulating the coating tapering proposed in section 4.4.1 are employed. Actually, these transitions are 


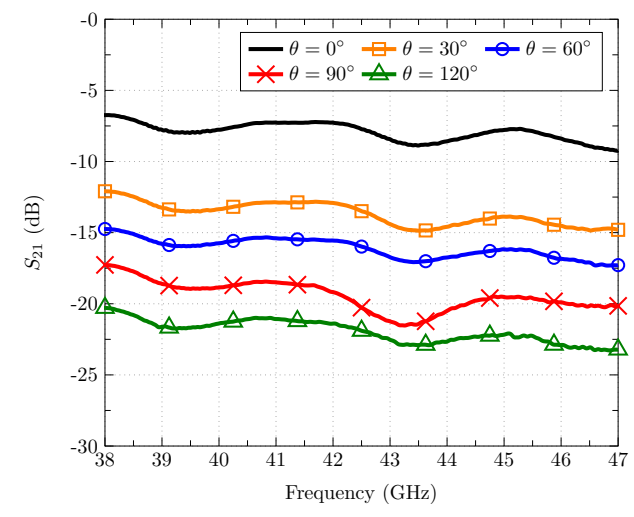

(a)

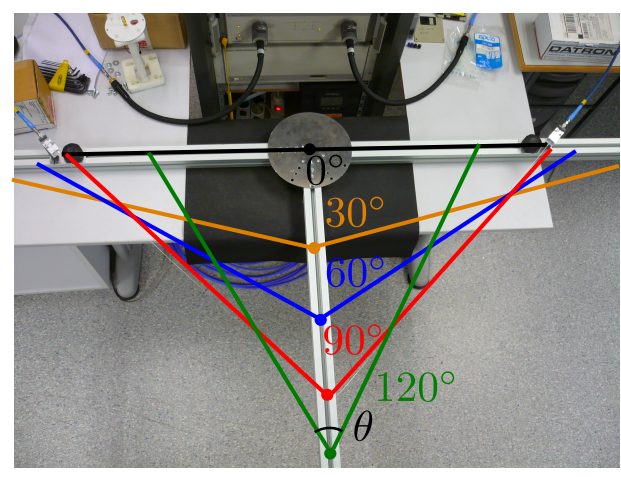

(c)

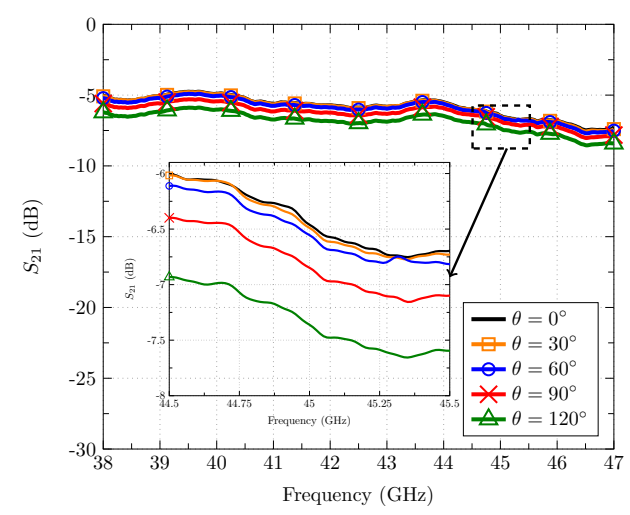

(b)

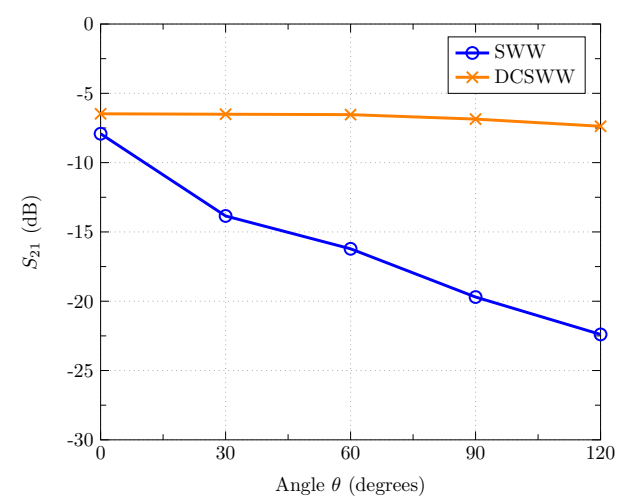

(d)

Figure 5.9: Experimental results of wire-type waveguide losses in bends for the second experimental setup. a) $S W W: S_{21}(d B)$ vs. frequency for several angles of curvature; b) DCSWW: $S_{21}$ $(d B)$ vs. frequency for several angles of curvature. c) Angle positioner; d) Losses vs. curvature angle $\theta$.

manually created in the same way as the DCSWW-SWW transitions used to excite the SWW after the horn structure. The aspect of the manufactured Single Wire Waveguide with Coating on Bend (SWW-CoB) can be appreciated in Fig. 5.10. Fig. 5.10(a) shows the SWW-CoB in the system. As it can be observed, the second proposed configuration to create bends, using a tensor fabric yarn, is used since a single bend with the desired curvature angle is obtained. Fig. 5.10(b) shows a detail of the curved section, where it is clearly appreciated that the coating is exclusively placed on the bend.

In Fig. 5.11, experimental results with a conventional SWW, Fig. 5.11(a), are compared with the proposed SWW-CoB, Fig. 5.11(b). The difference between both cases is quite evident. There is a noticeably reduction of losses, driven by the lower radiation losses, which are of only of $3 \mathrm{~dB}$ for a $90^{\circ}$ in a $\mathrm{SWW}-\mathrm{CoB}$. On the other hand, total losses in a SWW-CoB are increased by the contribution of the two additional SWW-DCSWW transitions, which approximately introduce a total of 


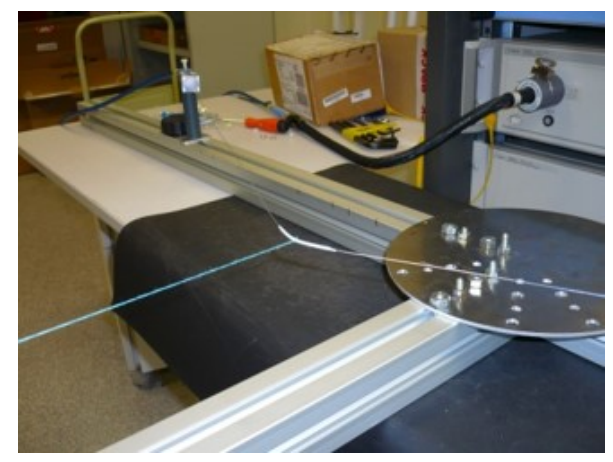

(a)

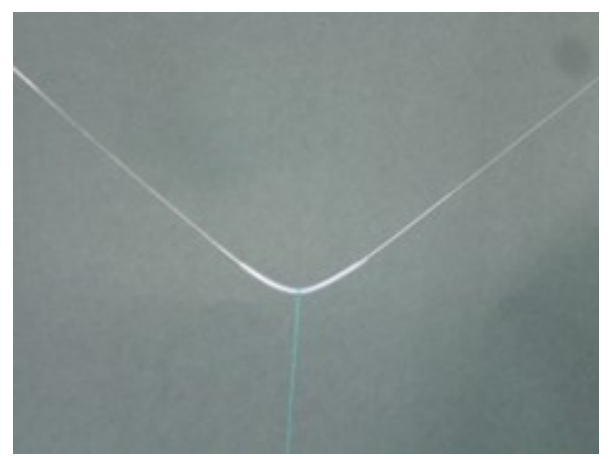

(b)

Figure 5.10: $S W W$ prototype with coating exclusively placed on the curved section. a) Prototype in the measurement system; b) Detail of the curved section with the coating and the fabric yarn acting as a tensor element.

1.3-1.8 dB additional losses. In the case of a $\mathrm{SWW}-\mathrm{CoB}$, since coating is present on the bend, the effect of the curvature angle is strongly reduced. However, comparing Fig. 5.11(b) with Fig. 5.9(b) (DCSWW case), it is found that the effect of the curvature angle is more evident in the case of a SWW-CoB. This is probably caused due to the fact that smaller curvature angles allow to recover residual part of the radiation caused by the first transition. This is, since input and output SWWs are more aligned, part of the power radiated at the initial transition is coupled again at the output of the bend. However, since this coupling is residual, differences between SWW-CoB and DCSWW are quite small.

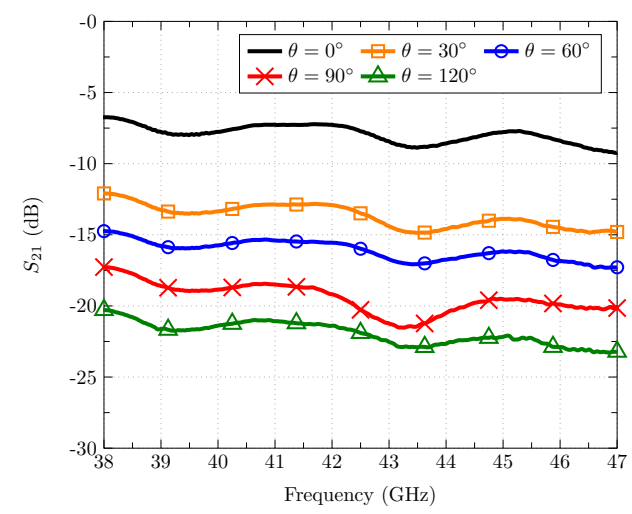

(a)

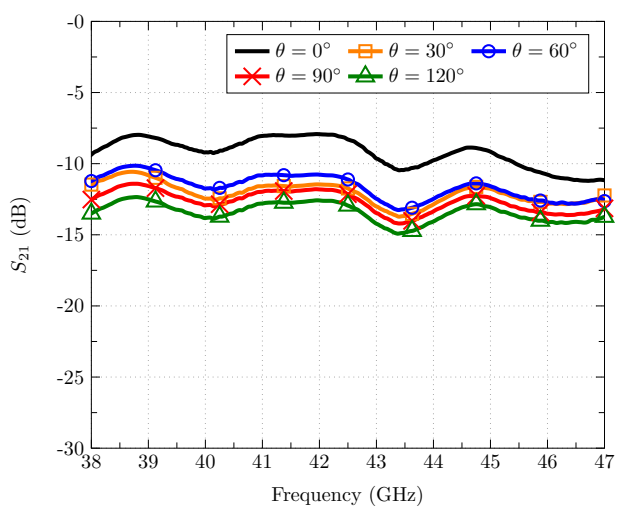

(b)

Figure 5.11: Comparison of the measured results of the $S W W$ prototype with and without coating in the curved section. a) $S W W: S_{21}(d B)$ vs. frequency for several curvature angles; b) $S W W-C o B$ : $S_{21}(d B)$ vs. frequency for several curvature angles.

Fig. 5.12(a) shows the received power after the section of $L=1.8 \mathrm{~m}$ with a bend in the middle as a function of the curvature angle of the bend for the three considered cases: SWW, SWW-CoB, and DCSWW. Note how, if any curvature is 
present, the SWW-CoB is penalized with the transition losses. However, it presents a dependence with the curvature angle very similar to that of a DCSWW. Main observable difference between these DCSWW and SWW-CoB cases in this plot takes place between $\theta=0^{\circ}$ and $\theta=30^{\circ}$ (see the slope difference between both curves), what is caused due to the aforementioned residual coupling, which is quite evident if input and output SWWs are aligned $\left(\theta=0^{\circ}\right)$. The plot clearly shows a great advantage in using coating on the bend from using a conventional SWW. Note also that the level of the SWW-CoB curve is affected by four SWW-DCSWW transitions that have been roughly manufactured. Improvement of such transitions performance would lead to a gain of $2-3 \mathrm{~dB}$.

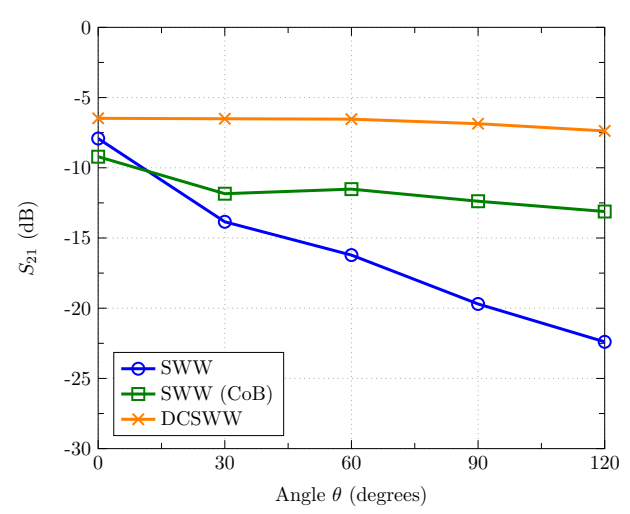

(a)

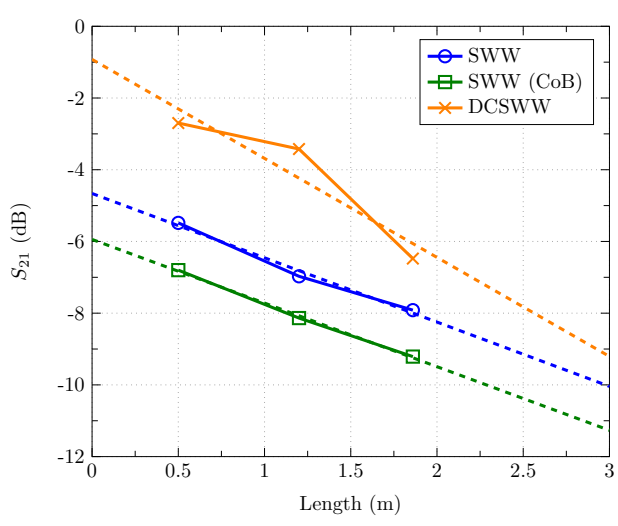

(b)

Figure 5.12: Comparison of the measured results of the three manufactured wire-type waveguide prototypes: $S W W, S W W-C o B$, and DCSWW. a) $S_{21}(\mathrm{~dB})$ vs. curvature angle $\theta$; b) Estimation of the attenuation $\alpha(\mathrm{dB} / \mathrm{m}): S_{21}(\mathrm{~dB})$ for straight paths of $L=0.5 \mathrm{~m}, L=1.2 \mathrm{~m}$, and $L=1.8 \mathrm{~m}$.

In order to have an estimation of propagation losses in straight path sections, several lengths have been considered for the three evaluated wire-type waveguides. Besides the already evaluated $L=1.8 \mathrm{~m}$, waveguiding section lengths of $L=0.5 \mathrm{~m}$ and $L=1.2 \mathrm{~m}$ have been manufactured and experimentally tested at $f=45 \mathrm{GHz}$. Fig. 5.12(b) shows the received power as a function of the waveguiding section length for the three cases: SWW, SWW-CoB and DCSWW. Marks indicate the measurement results, which are united by solid lines. Dashed lines are linear regressions of the measurement points. The slope of this curves is an estimation of the straight path losses of the waveguides. Results of this estimation are shown in Table 5.3, where analytical and simulated results are added for comparison. It is found good agreement between analytical calculations, simulations and measurements, specially in the case of the DCSWW. Measured attenuation in the SWW case is slightly larger than expected (note that simulated value is already larger than real due to truncation effects). This is probably caused by the larger sensitivity of SWW to imperfections and the surrounding environment. Straight path losses of SWW and SWW-CoB are very similar as it is expected. The difference between the curves corresponding to these waveguides is exclusively caused by the losses introduced by the 
two SWW-DCSWW transitions located in the middle of the section. Measurements indicate that these transitions are adding approximately $1.5 \mathrm{~dB}$ losses.

\begin{tabular}{cccc} 
Waveguide & SWW & SWW-CoB & DCSWW \\
\hline Analytical $\alpha(\mathrm{dB} / \mathrm{m})$ & 1.21 & - & 2.36 \\
\hline Simulated $\alpha(\mathrm{dB} / \mathrm{m})$ & 1.56 & - & 2.50 \\
\hline Measured $\alpha(\mathrm{dB} / \mathrm{m})$ & 1.79 & 1.74 & 2.76
\end{tabular}

Table 5.3: Comparison of the estimated attenuation through analytical calculation, simulations, and measured results.

Extrapolation of curves indicate that removing the coating in straight path sections is advantageous for moderate propagation lengths. Due to transitions losses, the length from which the SWW overcomes the DCSWW is beyond the maximum length considered in the plot. However, results show experimental evidence of the convenience of using a SWW. At THz, the distances are considerably shorter, and the attenuation (slope) corresponding to a DCSWW more pronounced. Since a SWW with coating exclusively on the bend has experimentally shown both, low radiation losses and low propagation losses, it can be postulated as the optimum solution. In this way, experiments in this section have demonstrated that concepts presented in previous chapters hold in practice. Therefore, it can be affirmed that better optimized structures, manufacturing processes and measurement setups may drive to practice more advanced structures such a SWW incorporating transitions of radius and coating. Results in this section satisfactorily achieve the objective of giving experimental evidence the conclusions obtained in previous chapters.

\section{$5.2 \mathrm{THz}$ prototypes results}

The second experimental study is carried out with a $\mathrm{THz}$ measurement setup, whose nature clearly differs from the previous one operating at mm-wave frequencies. Although the employed $\mathrm{THz}$ source provides power up to $f=0.8 \mathrm{THz}$, close to this maximum frequency, the generated power is close to the noise level of the system. Actually, the spectrum provided by the source exhibits noticeably higher power levels at lower frequencies. Since the aim of the experiments of this section is to evaluate the performance of wire-type waveguides in straight path and bends at the $\mathrm{THz}$ regime, it is necessary to have a large enough margin from the noise level in order to properly recover the received signal in those cases in which propagation and radiation losses are high. By this reason, $f=0.2 \mathrm{THz}$ has been chosen as the reference frequency. Since the employed wire-type waveguides are the same as previous section, their radius will be electrically larger in the next experiments. Therefore, this section will give evidence of the observed effects in chapter 4 for large radius wires, not observable in the mm-wave experiments. In addition, this section will lead to appreciate practical differences between conventional radiofrequency setups and $\mathrm{THz}$ setups dealing with wire-type waveguides. 


\subsubsection{Measurement setup}

The employed setup to carry out measurements of losses of wire-type waveguides at $\mathrm{THz}$ frequencies is shown in Fig. 5.13. In order to generate the input $\mathrm{THz}$ signal two tunable diode lasers DL 100 from Toptica Photonics AG [596] are employed, Fig. 5.13(a). Each one of these laser provides output power at a certain optical frequency that can be adjusted by temperature. A $\mathrm{THz}$ carrier signal is generated by photomixing from the two input optical signals as the difference $\left(f_{T H z}=f_{1}-f_{2}\right)$ at a $2 \times 2$ fiber splitter from Toptica Photonics AG, Fig. 5.13(b). The complete system can be appreciated in Fig. 5.13(c). The THz carriers arrive to transmitter and receiver through the blue cables observable in the image. In both, transmitter and receiver, the $\mathrm{THz}$ signal is modulated in amplitude with a $\mathrm{RF}$ signal coming from the black coaxial cable. The final $\mathrm{THz}$ modulated signal is launched (received) to (from) free space by means of horns with an incorporated dielectric lens, located at the transmitter (receiver). Fig. 5.13(d) shows a zoom of one of the two horns with an incorporated dielectric lens.

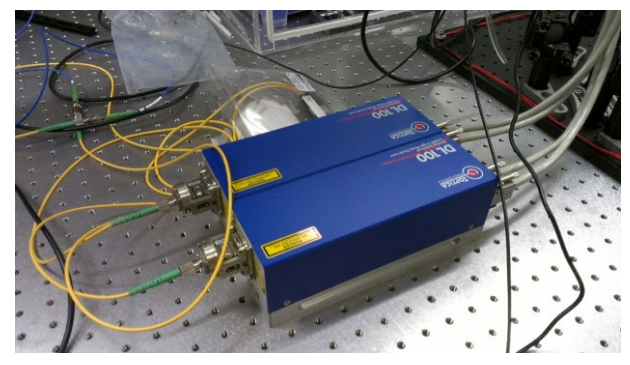

(a)

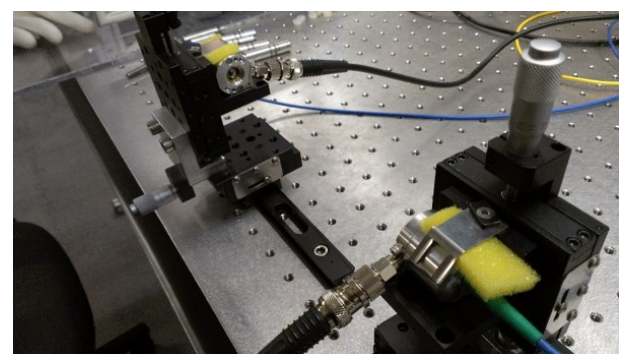

(c)

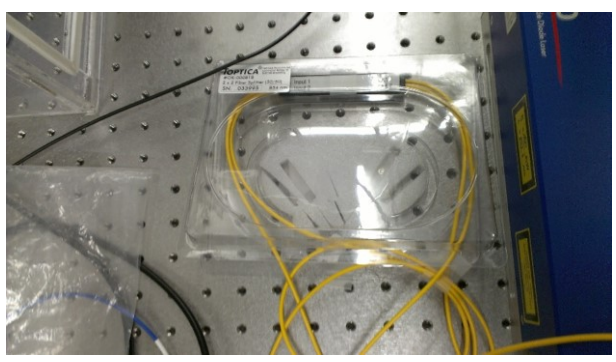

(b)

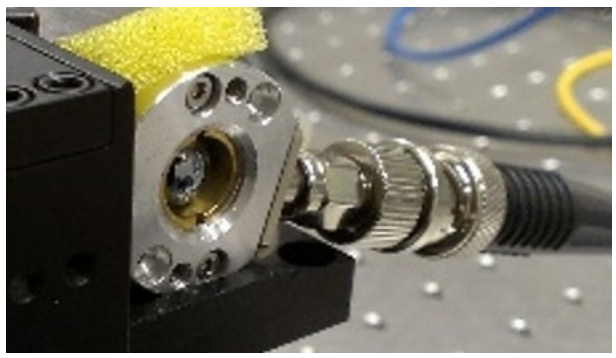

(d)

Figure 5.13: Experimental setup to carry out measurements of losses of wire-type waveguides at $\mathrm{THz}$ frequencies. a) Two tunable diode lasers DL 100 from Toptica Photonics AG; b) $2 \mathrm{x}$ 2 fiber splitter from Toptica Photonics AG. A THz carrier signal is generated by photomixing from the two input optical signals generated by the lasers; c) Complete system. The THz carriers arrive to transmitter and receiver through the blue cables. In both, transmitter and receiver, the $\mathrm{THz}$ signal is amplitude modulated with a RF signal coming from the black coaxial cable. The final $\mathrm{THz}$ modulated signal is launched (received) to (from) free space by means of horn with an incorporated dielectric lens, located at the transmitter (receiver); d) Detail of one of the two horns with an incorporated dielectric lens. 


\subsubsection{Excitation system}

As mentioned before, the same coaxial cable UT-085 from Microcoax ${ }^{\circledR}$ [595] with inner conductor of radius of $a=255 \mu \mathrm{m}$ and an outer conductor radius of $b=$ $838 \mu \mathrm{m}$ is employed to create SWW and DCSWW structures. This implies that excitation by means of tapering the outer conductor radius is not possible, since this cable is not prepared to operate at $\mathrm{THz}$ frequencies. Whereas at Ka-band frequencies, the coaxial cable was the direct output transmission line from the source, at $\mathrm{THz}$ frequencies, the optically generated $\mathrm{THz}$ power would be hardly coupled to it. In addition, at $f=0.2 \mathrm{THz}$, the employed coaxial cable is clearly overmoded. Therefore, alternative schemes must be considered. For this work, the excitation scheme employed in [492] has been applied for simplicity. Whereas it is not the most efficient one (see alternatives such as those proposed in [506] or [507] for instance), it does not require additional high-precision elements, the assembly is straightforward, and the performance is good enough for the purpose of the experiments, which does not pretend to be too deep or accurate but to give certain experimental evidence of some effects described in previous chapters.

The employed excitation scheme works as follows (see [492] for details). The transmitter launches to free space a signal that is partially coupled to the corresponding wire-type structure (thanks to their open nature). Due to its intrinsic radiative nature, this excitation system presents low efficiency and important part of the radiated power by the transmitter does not couple to the wire-type waveguide to be excited. This schemes clearly differs from that employed at the Ka-band system, where excitation is done by means of a waveguiding element (coaxial cable) so that small residual radiation is present only on transitions when they are present. Therefore, in the $\mathrm{THz}$ excitation scheme it results crucial to avoid line of sight between of transmitter and receiver, i. e., that power from the transmitter couple directly to the receiver. If this occur, larger received power than the real one delivered by the waveguide under test would be observed.

In order to avoid the previous source of error, a mandatory test is the following one. As it shows Fig. 5.14(a), the system is disposed without any wire-type waveguide between transmitter and receiver, i. e., free space transmission. Then, the angle between transmitter and receiver is varied (see color lines of Fig. 5.14(a)) up to the received power is of the order of the noise level. Results of the experiment are shown in Fig. 5.14(b). The plot shows the received power at the different samples of the measurement system. Solid lines are the real obtained data whereas dashed lines are the cumulative mean up to the corresponding sample number, which allows suppression of random noise. In this kind of plots, the most reliable data is the cumulative mean at the last sampling point. It is observed how, actually, a quite large amount of power is recovered at the receiver if there is direct line of sight or small angle offset (cases $\theta=0^{\circ}$ and $\theta=10^{\circ}$ ). Power rapidly drops as the angle increases, however, it is not up to $\theta=45^{\circ}$, when it is reached the desired uncoupling between transmitter and receiver. Note how the curve corresponding to $\theta=45^{\circ}$ is clearly affected by noise oscillations since the received power is at the noise level. These results lead to the use of this angle offset in the later experiments.

The main drawback of the employed excitation scheme is its low efficiency, since there is a great mismatch between the fundamental mode of the wire-type waveguide 


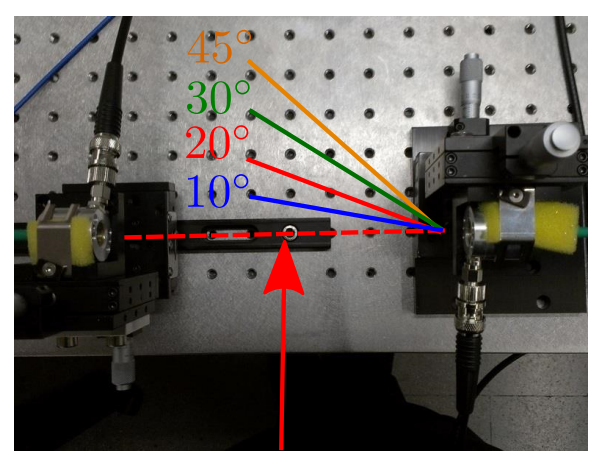

Tx-Rx Line of Sight

(a)

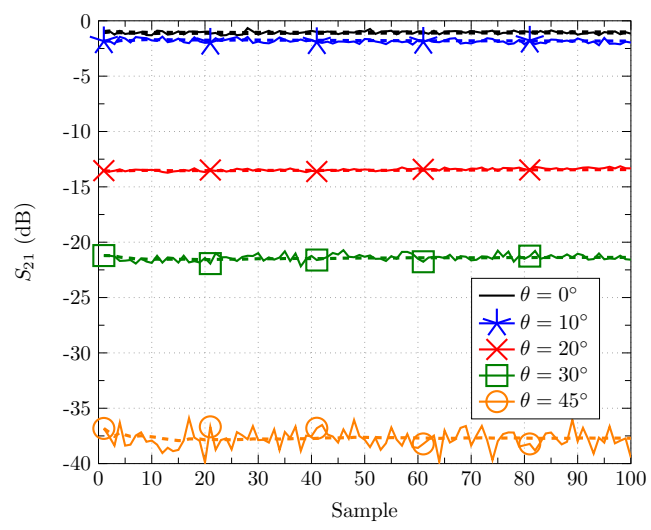

(b)

Figure 5.14: Evaluation of the received free-space radiation generated by the system itself (no waveguide). a) Top view of the system with the receiver in line of sight with the transmitter; b) Received power vs. measurement sample number $\theta$ of the transmitter radiating direction respect to the line of sight of the receiver. Solid lines link the sampled points whereas dashed lines are the cumulative mean of such sampled points.

under question and the incoming plane wave with oblique incidence. As suggested in [492], a perpendicular auxiliary wire is placed close to wire-type waveguide to be excited in order to enhance efficiency. Fig. 5.15(a) shows a schematic of such excitation scheme, with the yellow wire as the main waveguide and the purple wire as the auxiliary excitation element. It results important to locate the auxiliary wire at an optimum position in order to maximize the coupling enhancement effect. The distance $s$ between main and auxiliary wires, see Fig. 5.15(b), must be adjusted properly.

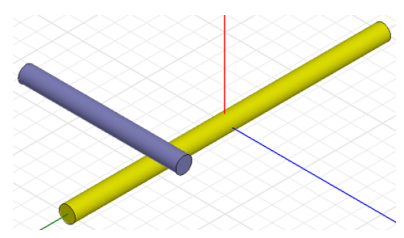

(a)

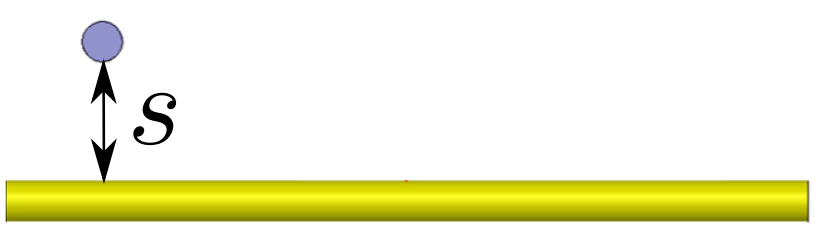

(b)

Figure 5.15: Schematics of the employed excitation method based on the scheme presented in [492]. An auxiliary $S W W$ (purple wire) is placed perpendicularly to the wire-type waveguide under test (yellow wire) to enhance power coupling from the incoming radiation from the transmitter. a) $3 D$ view; b) Side view.

According to the above, several simulations have been carried out, considering different values of the separation $s$ between wires. Results are shown in Fig. 5.16, where it is represented the E-field magnitude at the plane that is transversal to the auxiliary wire and contains a diameter of the main wire. The incoming radiation from the transmitter has an angle of incidence of $\theta=45^{\circ}$. Results reveal that small values of $s$ provide more efficient coupling, a fact clearly appreciable by observing 
the symmetric surface waves at the output of the main wire, corresponding to the fundamental mode. Plots also suggest that, despite of the improvement introduced by the auxiliary wire, important amount of power is still lost, either scattered by the auxiliary wire or being not coupled, continuing with its incidence direction. Since auxiliary and main wires must never touch, $s=0.5 \mathrm{~mm}$ has been chosen as a small enough distance with affordable implementation.

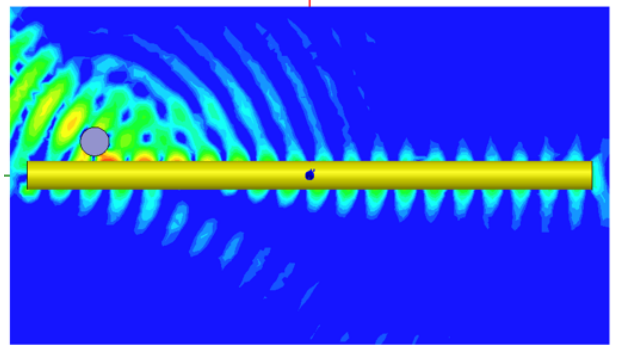

(a)

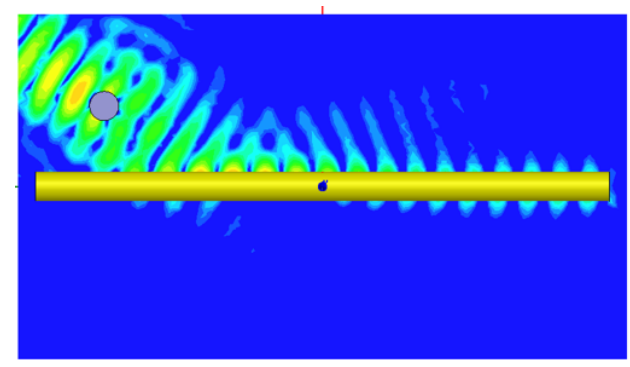

(c)

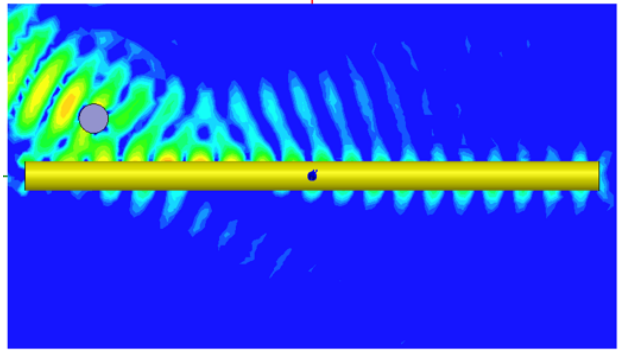

(b)

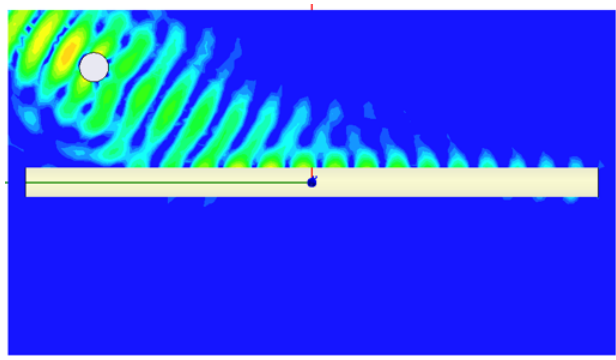

(d)

Figure 5.16: Simulated E-field magnitude in the excitation system composed by a $S W W$ and an auxiliary $S W W$ for several cases of separation $s$ between both $S W W$ s. The incoming radiation from the transmitter has an angle of incidence of $\theta=45^{\circ}$. a) $s=0.5 \mathrm{~mm}$; b) $s=1 \mathrm{~mm}$; $) \mathrm{s}=1.5$ $\mathrm{mm}$; d) $s=2 \mathrm{~mm}$;

Images of the practical implementation of the previously described excitation system are shown in Fig. 5.17 for the case of exciting a SWW. In all the experiments in this section, the auxiliary wire-type waveguide is a SWW. Fig. 5.17(a) shows a detail of the excitation scheme, where main and auxiliary SWWs are appreciated. In the image, it can be also observed the transmitter, tilted $\theta=45^{\circ}$ regarding to the main SWW, and pointing to the intersection of both SWWs. The main SWW lies horizontally over a Rohacell ${ }^{\circledR}$ block whereas the coupling SWW is vertically inserted in another Rohacell ${ }^{\circledR}$ block. The properties of Rohacell ${ }^{\circledR}$ are close enough to free space [597] so that the introduced extra losses are residual. Fig. 5.17(b) shows the excitation scheme view from the plane orthogonal to the line of sight of the receiver. Fig. 5.17(c) shows the top view of the excitation scheme. The coupling area is marked with a circle, where it can be appreciated that main and auxiliary SWWs are close but not touching. Incidence angle and focusing of the transmitter are also well appreciated in the image. Finally, Fig. 5.17(d) shows an alternative configuration with improved robustness, in which an additional Rohacell ${ }^{\circledR}$ block is 
placed over the SWW under test to sandwich it, hence avoiding possible movements. Note that the systems is quite sensitive to SWW movements, which can make vary the distance $s$ or to produce misalignment with the receiver. It must be mentioned that for signal reception the SWW is directly aligned with the receiver horn since this is more efficient than the coupling scheme employed to excite the SWW under test. However, direct coupling cannot be used at both horns since, in such case, free-space radiation may lead to wrong measurements, as it is clearly deduced from the study of Fig. 5.14.

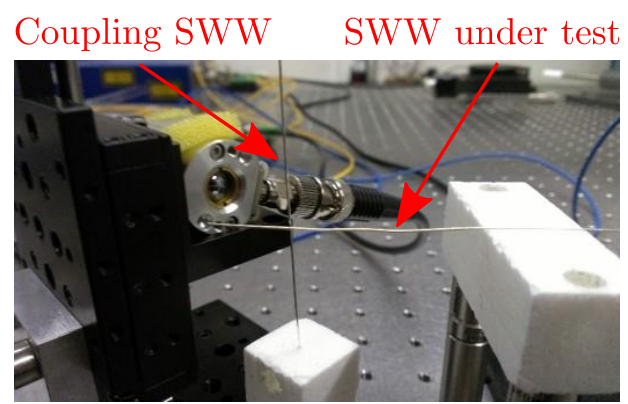

(a)

Coupling area

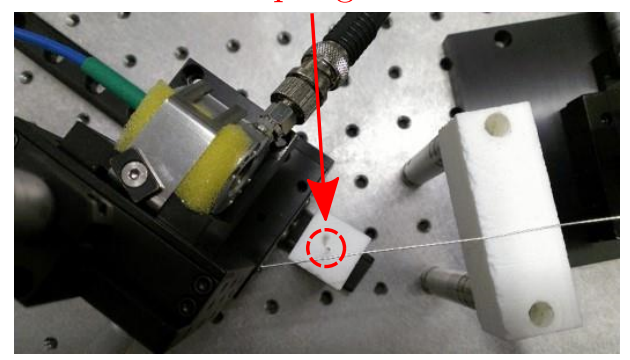

(c)
Coupling SWW SWW under test

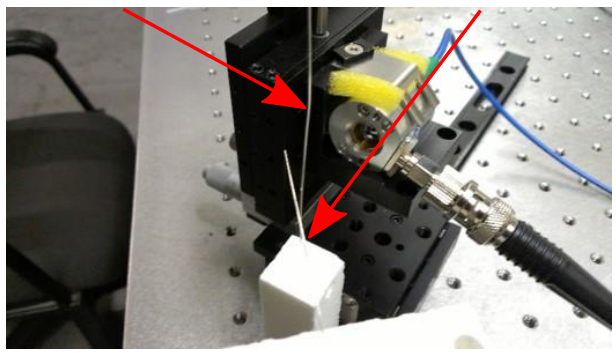

(b)

Rohacell fasting block

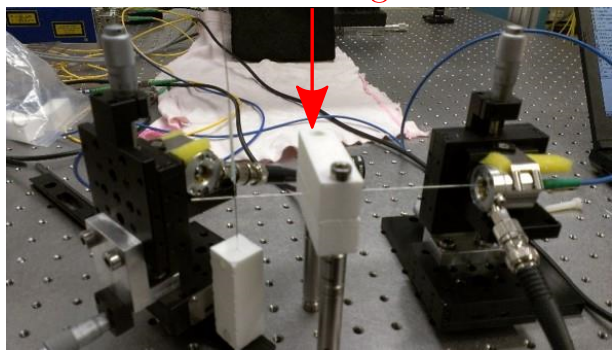

(d)

Figure 5.17: Photographs of the excitation system implemented in the measurement setup. a) Detail of the $S W W$ under test and the coupling $S W W$. The $S W W$ under test lies horizontally over a Rohacell ${ }^{\circledR}$ block whereas the coupling $S W W$ is vertically inserted in another Rohacell ${ }^{\circledR}$ block; b) View from the plane orthogonal to the line of sight of the receiver; c) Top view, where it can be appreciated the transmitter, rotated $\theta=45^{\circ}$ degrees. The coupling area where the two $S W W \mathrm{~s}$ interact with the incoming radiation and excitation of the $S W W$ occurs is marked with a circle; d) Alternative system with improved robustness: an additional Rohacell ${ }^{\circledR}$ block is placed over the SWW under test to sandwich it, hence avoiding possible movements.

Once the excitation scheme has been defined, it has been considered interesting to test the effect of the different elements of the system on the final received power. Fig. 5.18(a) shows the experimental setup with transmitter and receiver as fixed elements, marked in blue, and the main and auxiliary SWWs as additional elements, marked in red. The test considers four configurations: nothing (neither main SWW nor auxiliary SWW), only coupling SWW (no main SWW), only main SWW (no coupling SWW), and the complete system with both SWWs. Results are shown in Fig. 5.18(b). As expected, without any element, only noise arrives the receiver. If 
the coupling SWW is disposed, a slightly larger amount of power reaches the receiver thanks to the scattered radiation. Surprisingly, a very similar received power, only slightly larger, is received if the main SWW is present but not the coupling SWW. This means that, without auxiliary SWW, coupling results extremely inefficient. The main SWW results almost invisible for the incident wave in this case. Finally, if both, main and coupling SWWs, are used, the received power results noticeably enhanced. Almost $10 \mathrm{~dB}$ are gained by disposing the auxiliary SWW in the system. These results give experimental evidence of how important results this auxiliary element for this type of configuration.

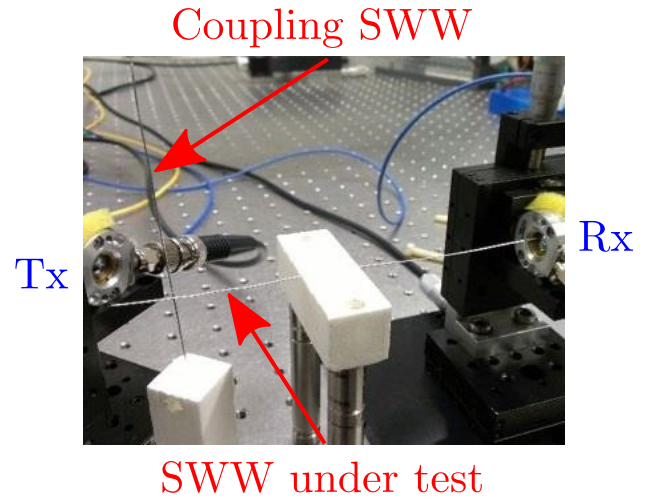

(a)

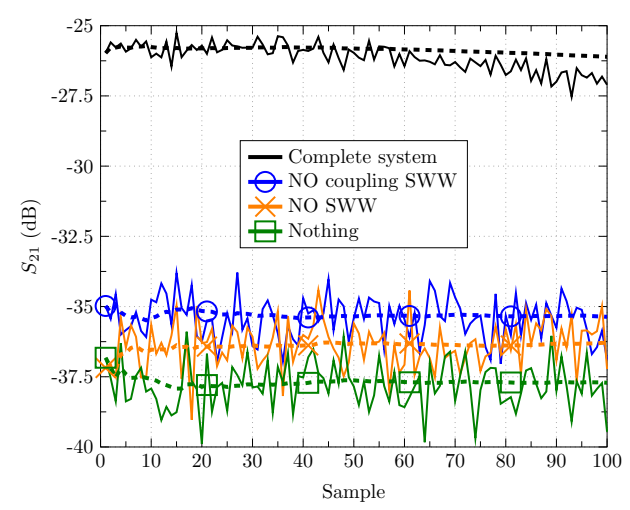

(b)

Figure 5.18: Excitation system performance test. a) Excitation system and involved elements; b) Comparison between the received power when the system is complete and several cases in which one or more elements are missing.

\subsubsection{SWW measurements}

Once the system has been presented and several aspects have been reviewed and evaluated, actual measurements involving wire-type waveguides are presented next. First, a SWW of radius $a=255 \mu \mathrm{m}$ is measured in the system. Fig. 5.19(a) shows the SWW under test in the configuration used to measure curved path losses. Fig. 5.19(b) shows a top view of the waveguiding path with a bend, where the curvature can be clearly appreciated, being it sketched in the image. Note how, in this setup, holding the desired curvature for the SWW under test does not require extra auxiliary elements as in the mm-wave case since, due to the employed shorter distances, the semi-rigid property of the whole wire is better preserved, and the section under test results more easily tractable.

First, attenuation of the SWW under test is derived by measuring losses introduced by two SWW of different lengths: $L=12 \mathrm{~cm}$ and $L=15 \mathrm{~cm}$. Fig. 5.20 shows the received power samples after the signal is propagated along the SWW for each one of the two cases. Again, solid lines are the measured value for each sample whereas dashed lines represent the cumulative mean. Therefore, the attenuation constant can be estimated by observing the difference between the cumulative mean of both cases at the last sample. This calculation provides a measured value 


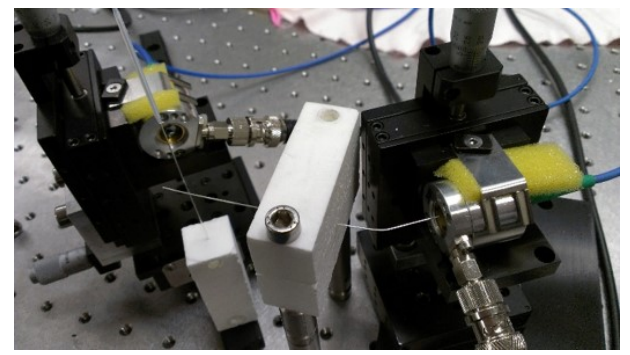

(a)

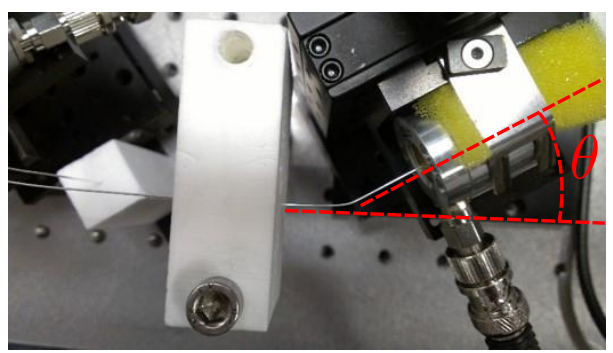

(b)

Figure 5.19: Measurement system for the evaluation of straight and curved path losses of a $S W W$. a) $3 D$ view the system with a curved $S W W$ under test; b) Top view with the curvature angle sketched.

of $\alpha=0.003 \mathrm{~dB} / \mathrm{mm}$. On the other hand, theoretical calculations with the developed analytical models predict an attenuation of $\alpha=0.004 \mathrm{~dB} / \mathrm{mm}$. Although both values are quite similar, generally, larger attenuation is expected in experimental results since there are several undesired effects that often appear in practice which are not taken into account by theoretical models.

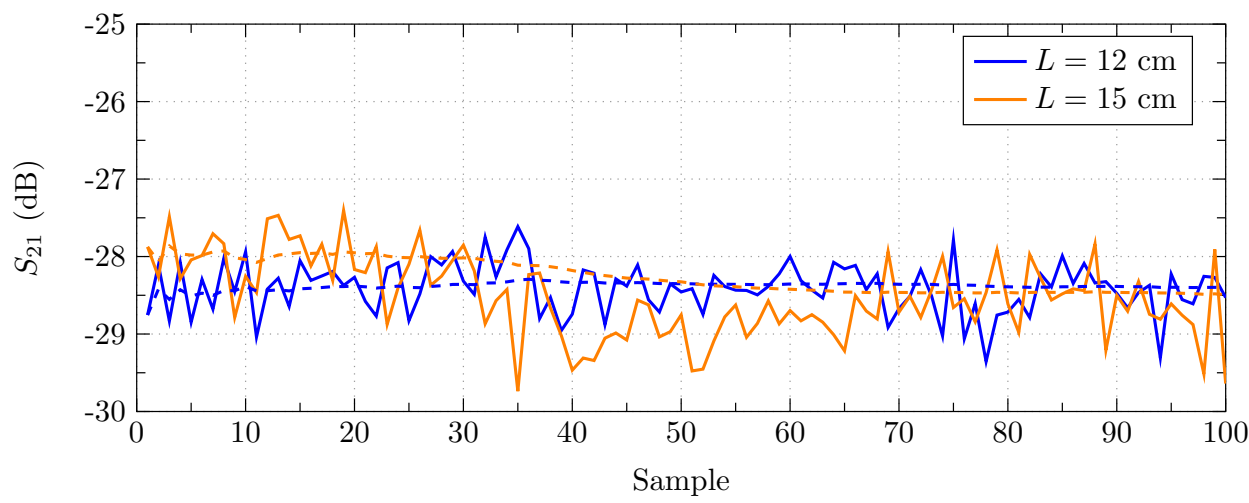

Figure 5.20: Samples of the received power for two identical SWWs of different length. Solid lines link the sampled points whereas dashed lines are the cumulative mean. The attenuation of the considered $S W W$ can be estimated by calculating the difference on the cumulative mean at the last sampling point between the two considered cases.

The low measured attenuation value is partially originated by the influence of some error in the first samples, for which higher received power is obtained with the $L=15 \mathrm{~cm}$ SWW than with the $L=12 \mathrm{~cm} \mathrm{SWW}$, what makes no sense. By avoiding the first 30 samples, where this error seems to concentrate, a value of $\alpha=0.009$ $\mathrm{dB} / \mathrm{mm}$ is obtained. Even though this value differs more the theoretical one, it seems to be more representative of the real experienced attenuation. Nevertheless, note that the attenuation of the SWW under test is excessively small to be properly measured with the employed setup, better prepared to evaluate larger differences in losses, as those occurring in curves. In order to obtain more accurate results, larger propagation distances are required. However, results experimentally evidence that the SWW presents very low attenuation. 
Now, performance at curves of the SWW under test is evaluated. To do so, losses of a $L=15 \mathrm{~cm}$ SWW with a bend in the middle of the waveguiding path are measured for several curvature angles $\theta$. Measurement results are shown in Fig. 5.21(a), where the received power is displayed as a function of the measurement sample. Additionally, Fig. 5.21(b) shows the cumulative mean at the last sampling point (mean received power) as a function of the curvature angle so that differences between cases can be more easily extracted. As it can be appreciated, even a small curvature angle such as $\theta=10^{\circ}$, causes large losses (12 dB approximately). An additional increase of curvature up to $\theta=22.5^{\circ}$ causes, in principle, extra $6 \mathrm{~dB}$ loss, what would indicate that radiation losses increase, but at a lower rate than for lower angles. However, for this last case, the received power is quite low, what means that the actual value may have fallen under the noise level. Assuming this, it is very probable that the curve would have continue decreasing at a similar rate (if not more), what would be more in concordance with results of previous chapter. The previous argumentation is reinforced by the fact that for $\theta=45^{\circ}$ similar received power is obtained. This suggests that the noise level of the measurement is approximately located at $-43 \mathrm{~dB}$, where both curves saturate. As it was expected from previous chapter conclusions, since the SWW radius is not small, the waveguide is strongly affected by radiation losses at bends.

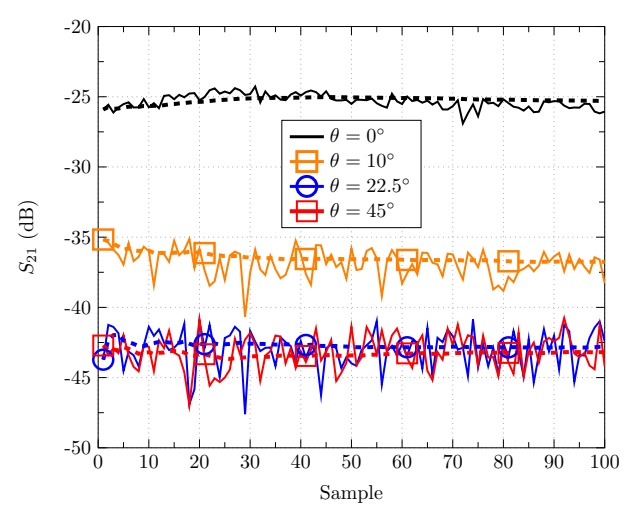

(a)

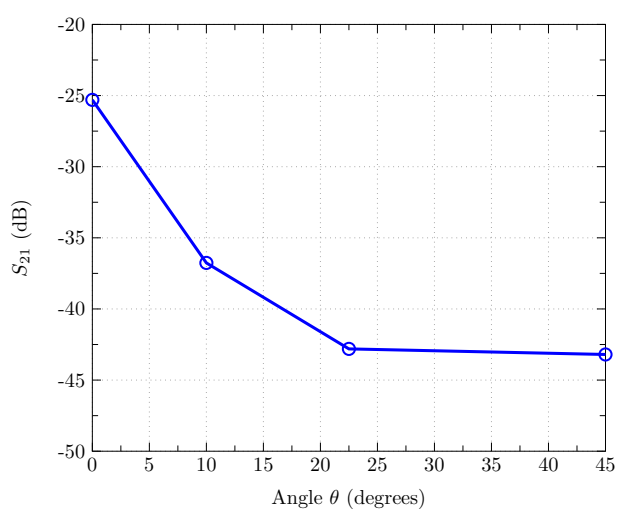

(b)

Figure 5.21: Evaluation of losses in a curved $S W W$ of total length of $L=15 \mathrm{~cm}$. a) Samples of the received power for several cases of the curvature angle $\theta$. Solid lines link the sampled points whereas dashed lines are the cumulative mean; b) Mean received power vs. curvature angle $\theta$.

\subsubsection{DCSWW measurements}

The same evaluation is performed for a DCSWW now. Figs. 5.22(a) and 5.22(b) show the measurement system for the evaluation of straight and curved path losses with a DCSWW under test. As it can be appreciated, the same excitation scheme using an auxiliary SWW to enhance coupling efficiency is employed.

As before, first, the attenuation of the waveguide is estimated. To do so, losses introduced by two straight DCSWW sections of lengths $L=11.5 \mathrm{~cm}$ and $L=15$ 


\section{Coupling SWW}

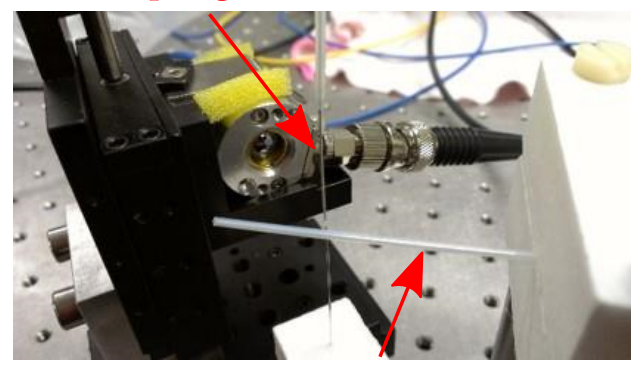

DCSWW under test

(a)

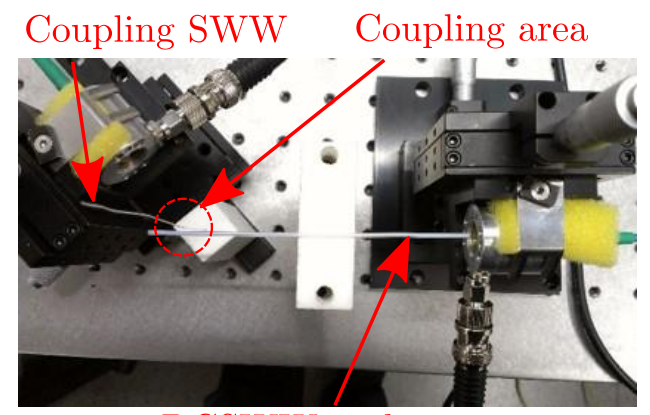

DCSWW under test

(b)

Figure 5.22: Measurement system for the evaluation of straight and curved path losses of a DCSWW. a) Lateral view; b) Top view.

$\mathrm{cm}$ have been measured. Fig. 5.23 shows the received power in each sampling (solid lines) as well as the cumulative mean (dashed lines), for the different considered waveguiding lengths. By using the measured mean received power, attenuation is estimated to be $\alpha=0.106 \mathrm{~dB} / \mathrm{mm}$. Note that for the DCSWW, since the attenuation is larger, noise influence is less relevant, and more reliable results can be obtained. Theoretical calculations give $\alpha=0.071 \mathrm{~dB} / \mathrm{m}$ if $\tan \delta=0.002$ is assumed for Teflon at $f=0.2 \mathrm{THz}$, what indicates reasonable agreement between theoretical and experimental results. At THz frequencies, the employed DCSWW presents noticeably greater confinement inside of the dielectric coating than when the operating frequency was in the Ka-band $(\eta=96 \%$ front $\eta=48 \%)$. This implies, on the one hand, enhanced difference in attenuation between SWW and DCSWW (even more accentuated by the higher operation frequency) and, on the other hand, larger influence of the waveguide material parameters $(\sigma$ and $\tan \delta)$ on the propagation losses. Probably, these parameters are taken into account in the theoretical model better than they are in reality, what may be causing the theoretically predicted value $\alpha$ to be lower than the measured one. For instance, the measured attenuation value is recovered if $\tan \delta=3.5 \cdot 10^{-3}$ is used instead. Despite the limited accuracy of the measured system, it has been clearly observed that the a SWW presents much lower attenuation than a DCSWW for moderate-large radius (in the presented experiments $a \approx \lambda / 6$, e. g, what occurs at $f=1 \mathrm{THz}$ with $a=50 \mu \mathrm{m}$ should be expected).

Performance of the DCSWW under test when bends are present is described now. Losses of a $L=15 \mathrm{~cm}$ DCSWW section incorporating a bend have been measured for several values of the curvature angle $\theta$. Fig. 5.24(a) shows the sampled received power (solid lines) and the corresponding cumulative mean (dashed lines), whereas Fig. 5.24(b) shows the mean received power as a function of the curvature angle. It is observed that, in a similar way than it occurs with the studied SWW, even small curves cause an important radiation. With $\theta=10^{\circ}$, there is an increment of at least $10 \mathrm{~dB}$ losses respect to the straight line case (note that the received power it is probably falling under the noise level). 


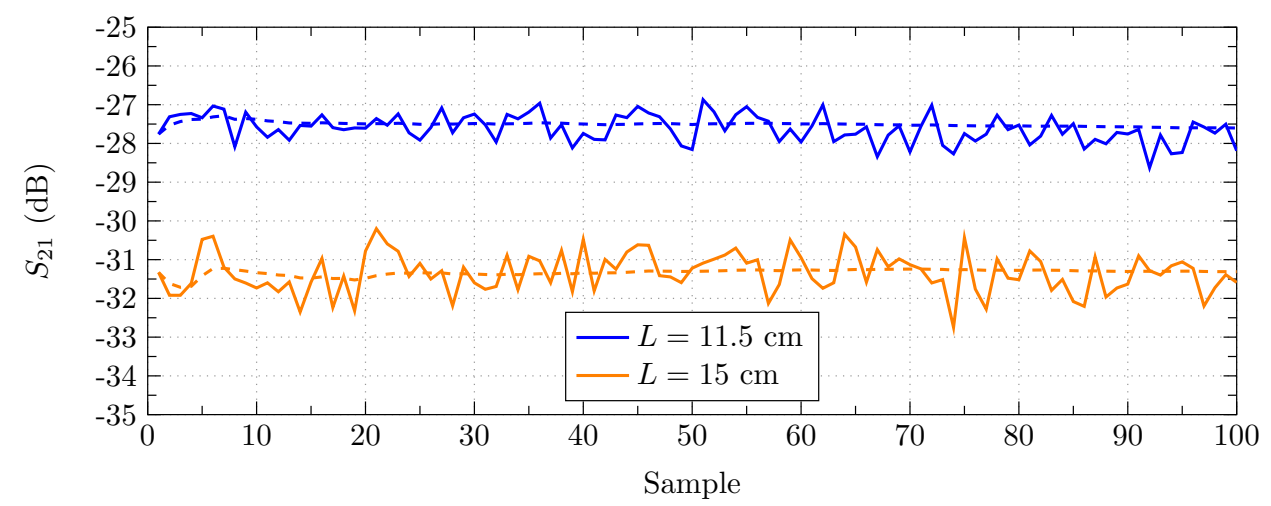

Figure 5.23: Samples of the received power for two identical DCSWWs of different length. Solid lines link the sampled points whereas dashed lines are the cumulative mean. The attenuation of the DCSWW under test can be estimated by calculating the difference on the cumulative mean at the last sampling point between the two considered cases.

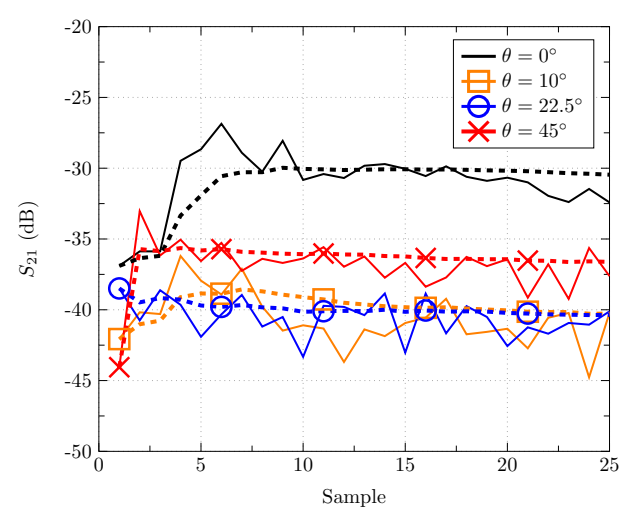

(a)

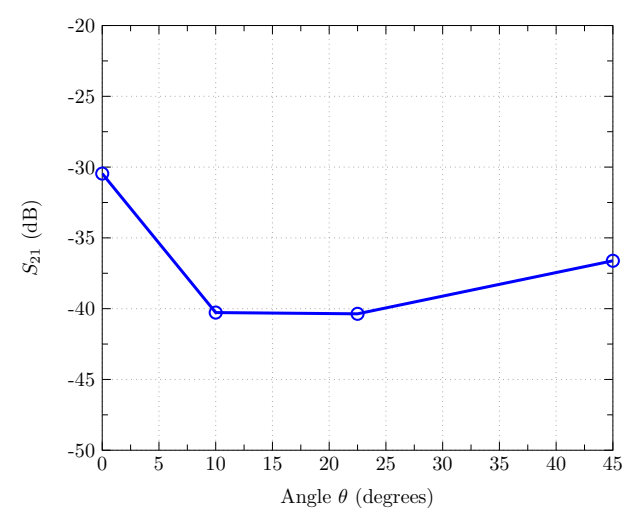

(b)

Figure 5.24: Evaluation of losses in a curved DCSWW of total length of $L=15 \mathrm{~cm}$. a) Samples of the received power for several cases of the curvature angle $\theta$. Solid lines link the sampled points whereas dashed lines are the cumulative mean; b) Mean received power vs. curvature angle $\theta$.

However, an important difference is appreciated now. Whereas for $\theta=22.5^{\circ}$ the received power seems to be lower than the noise level (hence both points, $\theta=10^{\circ}$ and $\theta=22.5^{\circ}$ saturate at the lowest limit), an increment of received power is appreciated for a larger curvature angle, see the case of $\theta=45^{\circ}$. Since a large radius DCSWW is used, important phase shifts perturbing the mode pattern take place. Furthermore, note that the coating is quite thick $(t=583 \mu \mathrm{m})$, this involving a more expanded mode pattern. This implies that every point of the mode front experiences an increment of length between internal and external path that is between $\Delta L=2 a \theta$ and $\Delta L=2 b \theta$, which can be quite larger than $\Delta L=2 a \theta$. Note also that, since the mode pattern is not concentrated radially, the phase shift modeling presented in previous chapter does not hold any more. A more complex distributed phase shift occurs. Nevertheless, the use of this model together with power distribution 
information of the mode allows to explain quite well the observed phenomena. What actually occurs is that for $\theta=45^{\circ}$ the increment of length is close enough to $\lambda_{g}$ in average so that a smaller phase shift (hence mode pattern perturbation) takes place than when $\theta$ is smaller. Therefore, despite of the fact that the curve is more pronounced, more power is received since less coupling to the first high-order mode $\left(\mathrm{HE}_{11}\right)$ occurs. Indeed, less than $7 \mathrm{~dB}$ are lost when $\theta=45^{\circ}$, which is a quite good result. Therefore, experimental results confirm predictions of section 4.3, where control of the curvature phase shift was presented as solution B to radiation problems of wire-type waveguides on bends.

Although the previous $\mathrm{THz}$ experiments are quite limited in terms of received power, hence demanding from improved excitation schemes and/or equipment, they complete the objective of giving basic experimental evidence at $\mathrm{THz}$ frequencies of some of the concepts deeply treated in previous chapters. First, it has been observed how the SWW acts as a waveguide when radiated power is coupled to it. Second, it has been checked that a SWW presents much lower attenuation than a DCSWW. Third, the phenomenon of no monotonically increment of losses with the curvature angle, caused by the perturbation on the mode pattern due to the different lengths experienced by inner and outer waveguiding paths of a curve, has been appreciated on the results. The presented preliminary $\mathrm{THz}$ setup lefts great margin of improvement for future experiments. In the same way, accurately manufactured prototypes having optimized dimensions are as well demanded. This premises conform the future work lines regarding the work presented in this chapter. If carried out properly, most of the conclusions predicted in this part of the thesis could be experimentally checked, and outstanding results at $\mathrm{THz}$ frequencies could be achieved, gaining the attention of the research community. 
Chapter 5. Experimental results 


\section{Chapter 6}

\section{Conclusions and future Work}

The conclusions extracted along this part of the thesis are compiled in this chapter for clarity and general view purposes. Possible future work lines, directly foreseen, either from encountered issues through the developed work or the conclusions, are as well proposed.

\subsection{Conclusions}

All the imposed objectives in the scope of this part of the thesis have been tackled and worked deeply until they have been accomplished with the expected grade of success. During the followed procedure, several concepts and remarks with special importance have appeared. From the perspective obtained at this point of the thesis, these elements are able to generate well-founded conclusions that are given next classified by chapters.

In Chapter 2, the state of the art involving the $\mathrm{THz}$ band technology and applications was deeply studied. From this comprehensive work it can be affirmed that:

- The $\mathrm{THz}$ band has a great potential and a unique transversality that can be hardly found in other bands of the electromagnetic spectrum. Whereas the technology of microwave and optic bands found its main application in the communications area (of course, other areas benefit from them, but with less extent), the $\mathrm{THz}$ frequencies have been shown to be provide unique characteristics, very suitable for a large variety of areas such as astronomy, chemistry, biology, medicine, pharmacology, industrial processes, communications, security, art, or even cultural heritage. There is still a large amount of work to be done, and this task will demand from new professional profiles, more transversal, in order to exploit all the potentiality of this part of the spectrum. In this sense, all the amount of information produced in the last two decades must be rapidly incorporated to the formation programs of the new generation professionals. New structures, mathematical models, manufacturing processes, materials and strategies to follow will continuing being proposed in the next 
years. The activity in this area is and will be intense. Those researchers entering in the $\mathrm{THz}$ world must be aware of such constant change in this topic.

- The THz technology has suffered an important advance from the starting of this century. The technology is still not mature at this point, but is not as well a rare area involving only a few researchers. Actually, the $\mathrm{THz}$ technology is in its way to become consolidated and widely used. $\mathrm{THz}$ sensors have lead the experienced progress, and, according to the recent research, it seems they will continue advancing fast and driving the $\mathrm{THz}$ technology headway. Sources are more limited physically, but tailored structures such as Quantum Cascade Lasers, and the parallel research in new materials, promise an optimistic future.

- The exhaustive analysis of waveguiding structures is starting to give its fruits, and some designs begin to foreground over others, defining a more suitable design environment. The large variety of possible waveguiding scenarios, provided by the large amount of possible $\mathrm{THz}$ applications, suggests the necessity of a chart of waveguides with different characteristics, each one performing the best for the corresponding scenario. Despite of the appreciable advances, at this stage, it is still necessary much work ahead in characterization, optimization and manufacturing techniques. Regarding to passive components, a great variety of $\mathrm{THz}$ antennas designs are found in the literature thanks to that it is a quite direct component to engineer, notwithstanding, still much work must be done to reach the performance obtained at microwave frequencies. Other structures such couplers, filters, etc., more depending on waveguide structure remain much less treated since the advances in waveguides have still not reach the desired consolidation level. This is an important area to promote inside the $\mathrm{THz}$ technology in the next years.

- Metallic waveguides coming from the microwave regime dominate the scenario up to $1 \mathrm{THz}$. New manufacturing techniques have recently alleviated the problem of manufacturing complexity due to the small required dimensions. If the production cost of this methods is diminished to affordable levels, these family of waveguides will have their role at $\mathrm{THz}$ frequencies. Nevertheless, bulk designs are inherently limited in bandwidth and in less extent, in losses, whereas planar designs are very lossy. Therefore, some innovative designs including modifications on the original structures must come in order to provide the desired performance. Overmoded hollow waveguides are an example of such strategy.

- Dielectric waveguides must be used in a different way than in the optical regime: the design must maximize the fraction of power transmitted in the air regions of the waveguide. The best option is to provide internal air regions, thus having low propagation losses and, at the same time, low radiation losses. Some results in the literature are startling. However, careful reading of those papers outcrops important disadvantages. These waveguides are inherently associated to strong attenuation and propagation dispersion and are, generally, very narrow-band. Some designs are quite complex, and the manufacturing techniques are still far from the required accuracy to afford the proposed designs. Nevertheless, the development of new materials and the rapid progress 
in $3 \mathrm{D}$ printers may dispose are more suitable scenario in few years. In such case, if a good work is carried out in developing analysis and optimization tools for those geometrically complex structures, they may play a role in $\mathrm{THz}$ waveguiding for narrow-band applications.

- Periodic structures offer an innovative and alternative approach that allows to be tailored for $\mathrm{THz}$ frequencies. In these structures, the geometry alleviates in a thoughtful way the dependence of the performance on the employed materials that conform the waveguide. Nevertheless, the nature of these waveguides cause them to be inherently narrow-band. With such restriction, they are an option to take into account.

- Surface and plasmonic waveguides are a old theoretical solution that results innovative in practical terms, inasmuch as it is a $\mathrm{THz}$ were they have been seriously considered as a waveguiding support for practical purposes. Their special features involving a weak waveguiding (rather railing the waves than guiding them) disposes a challenging scenario in order to provide practical designs of important complexity. On the other hand, their simultaneously provided low-loss and low dispersion (in propagation and attenuation), specially in the case of metallic (plasmonic) surfaces, are unique. Furthermore, these structures have simple geometries, and their optimization possibilities remain rather unexplored, hence having some improvement margin. They are called to dominate ultra-wide band scenarios or those involving the highest frequencies of the $\mathrm{THz}$ band. Their special propagation features, differing from the other kind of waveguides, demand from the careful study of the phenomena involved in them and the development of new software packages as a necessary design tools.

- Among other surface waveguides, wire-type structures (SWW and DCSWW) have catch most of the attention. The results in the literature are promising and have driven to a significant activity in this area of research. However, most of the published work is very practical, and sometimes lack of proper theoretical foundations. The proposed structures are not optimized, causing that great part of the potential of these waveguides is still to be discovered. There is a lack of modern theory basis in the literature that this thesis aims to cover to some extent, and that may provide the necessary basis for reliable design tools, indispensable to carry out high-performance designs. With this amends and a proper dedicated research on manufacturing techniques, wire-type structures may be implementing soon advanced designs with unique performance, able to make reality applications that are unthinkable today.

In Chapter 3 the complete modal analysis of the SWW and DCSWW was carried out. To do that, the theoretical bases of a surface waveguide problem were first established, and the properties of metals were reviewed under the scope of the $\mathrm{THz}$ band. In the analysis carried out, the fundamental mode of each waveguide received special attention. The obtained conclusions are the following:

- Surface waveguiding share some characteristics with conventional waveguiding at microwaves, however, important differences are as well found. Those differ- 
ences impose that a particular treatment of Maxwell equations and involved boundary conditions must be given when a surface waveguiding problem is posed. Nevertheless, it must be taken advantage of the similitude with conventional waveguiding since every found parallelism is a bridge allowing the use of the well-established, powerful theory and techniques developed for conventional microwave waveguides in the last decades.

- The characterization of metals as dielectrics is well afforded at microwaves with the simple skin-effect model. Conversely, the infrared region demands mandatorily from the use of the Drude model, more sophisticated. In between, at $\mathrm{THz}$ frequencies, both models show discrepancies that may be or not relevant depending on the context in which the model is applied. For instance, in this thesis both cases appear. The SWW is very sensitive to the metal properties, hence the Drude Model is mandatory. Conversely, in the DCSWW the presence of the dielectric allows for simpler models for the metallic conductor. Since the variety of structures in which metals are involved is huge, this question must be always formulated. The key point underlying the differences of both models resides in the real part of the permittivity, which is not present in the skin-effect model. It has been also found that the Drude Model dispose a new scenario in which the concept of good or bad conductor becomes more diffuse. The specific empirical model of each metal must be revised being that a different outlook than that of the microwave regime, regarding the compared characteristics between materials, takes place. From the scope of this thesis it is found that there is certain lack of information, hence new studies characterizing metals at $\mathrm{THz}$ in a more dedicated way would be advisable. This task encompasses the characterization of metals over different geometries (not to restrict the study to bulky pieces of material) and the development of specific models for $\mathrm{THz}$ frequencies based on experimental results focused in this band. This information must be incorporated on commercial solvers, nowadays providing results that may fall into question. At this moment, widely used simulation tools may be using excessively simple models, depending on the case, and providing results with certain grade of error. This issue is of extreme importance being that high reliability on the available analysis tools is a mandatory fact to provide designs meeting the expectations in practice.

- The dielectric cylinder with real $\varepsilon_{r}$, despite of being the simplest open waveguiding problem of two dimensions, provides a great variety of cases depending on $\varepsilon_{r}$. This is originated on the boundary, that unlike the Perfect Electric/Magnetic Conductor conditions provides a variety of cases depending on the contrast between the two mediums defining the interface. Large $\varepsilon_{r}$ origins complex phenomena and less habitual situations that must be reviewed. The observed phenomena are suitable to be observed in any structure involving dielectric-dielectric interfaces. It is worth to highlight that a large contrast between dielectric media origins segregation between modes: in terms of the E-field polarization, (TM/HE) are totally/predominantly radial whereas (TE/EH) are totally/predominantly azimuthal. Those modes with predominant radial polarization are more affected by the surface and they exhibit a surface wave mode behavior for certain range of frequencies. 
- The consideration of the lossless cylindrical dielectric waveguide problem under the assumption of a complex propagation constant $\gamma$ reveals that, for open waveguides, they must be considered two cutoff conditions. First, when they are satisfied simultaneously $\alpha=0$ and $\beta<k_{0}$, the mode is not guided any more by the waveguide, but it becomes radiative (leaky). Second, when $\beta=0$, there is not any kind of propagation (neither guided, nor radiative), but the mode becomes evanescent. The gained information by considering $\gamma$ instead of $\beta$ suggest this procedure as a very interesting one to properly apply modal methods to open waveguides.

- When complex permittivities are considered to account for losses, the imaginary part of $\varepsilon_{r}=\varepsilon_{r}^{\prime}-j \varepsilon_{r}^{\prime \prime}$ causes that the obtained modes have not a pure character any more. This means, they cannot classified as purely evanescent, purely radiative or purely propagative. Regarding to the case of $\varepsilon_{r}$ pertaining the $4^{\text {th }}$ quadrant of the complex plane (lossy cylinders), it has been found that a small $\varepsilon_{r}^{\prime \prime}$ still allows for a loose classification, however, large $\varepsilon_{r}^{\prime \prime}$ dispose that the nature of each mode is a very mixed one that depends on the exact location of the solution $\gamma$ in the complex plane. The presence of losses allow to better observe a relevant fact: there is always a surface mode and a set of bulk modes, however, the surface mode only exists for radial dominant E-field polarization modes $(\mathrm{TM} / \mathrm{HE})$. The surface mode is in the vicinity of the solution for the equivalent planar interface (surface mode zone), whose location depends on $\varepsilon_{r}$. This mode presents a field pattern concentrated on the external side of the interface and exhibits exponential decay in the outer region. The surface mode is radiative in this case $\left(\varepsilon_{r}\right.$ in the $4^{\text {th }}$ quadrant), hence not contemplated by the usual analysis found in the literature. The surface mode zone and the bulk mode zone are connected and a complex propagation phenomena occur: there is not an independent surface mode but the surface mode is a transitory state that a particular mode may experience or not depending on its order and the value of $\varepsilon_{r}$. This complex phenomena is not found in the literature and may open the door for the study of lossy dielectric mediums.

- If $\varepsilon_{r}$ is real and negative, it occurs that surface mode zone and bulk modes zone become disjointed and practically independent. In this case, the surface mode is fixed mode itself (again, only found for $(\mathrm{TM} / \mathrm{HE})$ modes). It has a propagation constant close to that of a plane wave. Except in the case $n=0$ (fundamental mode, $\mathrm{TM}_{0}$ ), the rest of surface modes exhibit a cutoff in the sense of becoming radiative $\left(\beta<k_{0}\right)$ as $\alpha=0$ is always satisfied since the problem is lossless. The fundamental mode exhibits an anomalous behavior: instead of going towards cutoff when the electrical size of the waveguide is diminished, it experience an increment of both, attenuation and propagation constants. It is found that, for surface modes, the waveguide is electrically larger as the closer is $\varepsilon_{r}$ to -1 , this originated by the expression of the propagation constant of a surface (plasmonic) mode on a planar interface, which becomes singular for $\varepsilon_{r}=-1$. Bulk modes are evanescent.

- When $\varepsilon_{r}$ is in the $3^{r d}$ quadrant of the complex plane (dielectric equivalent for metals by means of the Drude model), i. e., losses are added to the $\varepsilon_{r}^{\prime}<0$ 
case, the surface modes experiences some attenuation. However, the increase of the surface mode attenuation is less than that predicted by the losses of the dielectric material. Also, the larger the losses the larger the confinement of the surface mode, which require a smaller effective area of transmission in the external region. When losses are present, if the electrical size of the waveguide is diminished, the high-order surface modes reach first the radiative region $\left(\beta<k_{0}\right)$ to later disappear when the condition $\alpha=0$ is finally satisfied. Bulk modes exhibit some propagation features but are mainly evanescent, as they propagate mostly confined in a medium were natural propagation of plane waves is evanescent as it models a metallic material.

- All the previous conclusions conform the basis for the following set of conclusions that is one of the most relevant results in this thesis. From the complete modal study of the SWW it is derived that:

> The complete modal spectrum of the SWW has been determined for the $\mathrm{THz}$ band and proper nomenclature has been established. The SWW has two sets of modes presenting quasi-independence due to their differences in terms of complex propagation constant $(\gamma)$ and power distribution. If $n=0$, it appears the $\mathrm{TM}_{0}$ surface mode, and a set of bulk $\mathrm{TM}_{0 m}$ and $\mathrm{TE}_{0 m}$ modes. If $n>0$, it appears the $\mathrm{HE}_{n}$ surface mode, and a set of bulk $\mathrm{HE}_{n m}$ and $\mathrm{EH}_{n m}$ modes. Since for each $n$ only one surface mode appear, any radial index $m$ is required in the nomenclature.

> The surface modes $\left(\mathrm{TM}_{0}\right.$ and $\left.\mathrm{HE}_{n}\right)$ show radial polarization, i. e., $\mathrm{HE}_{n}$ are actually quasi- $\mathrm{TM}_{n}$ modes. When the electrical size of the waveguide increases the propagation of all these modes tend towards to the propagation dictated by the planar surface limit. When the electrical size of the waveguide diminishes, all the $\mathrm{HE}_{n}$ become first radiative to later disappear. Conversely, the fundamental $\mathrm{TM}_{0}$ shows an anomalous behavior, increasing its attenuation and propagation constants. Nevertheless, since at $\mathrm{THz}$ frequencies metals are modeled with a permittivity exhibiting large magnitude terms, the planar surface limit propagation is extremely close to the propagation of a plane wave in the external air medium. Therefore, except of the fundamental mode (if an electrically small enough SWW is considered to make arise the anomalous behavior of this mode), the rest of the modes exhibit an extremely large effective area of propagation and suppose a totally unpractical support, hence the power coupled to them can be assumed as automatically lost.

> The works found at the literature treating high-order modes pertain to other epoch. At that time, the skin-depth effect model was considered to model the metallic conductor of a SWW. Under this condition the planar surface limit of propagation is in the radiative region. Although, under in this conditions, high-order $\mathrm{HE}_{n}$ modes exhibit propagation for certain electrical sizes of the waveguide, at the time of those works the numerical tools were not capable to capture this behavior. Therefore, high-order surface modes were totally missed. The more exact Drude model provides 
that $\mathrm{HE}_{n}$ modes are even more present than it is predicted by classic skindepth model and they have a relevant role in the SWW. Recent works dealing with the SWW still assume as valid the conclusions of those longstanding works, which are partially true, but do not show the complete reality. Taken into account high-order surface modes becomes necessary to provide optimal designs and to proper model local effects by applying modal methods.

- It is possible to achieve the monomode condition with the use of the fundamental mode as a practical support for $\mathrm{THz}$ waveguiding. By choosing the electrical size small enough, all high-order $\mathrm{HE}_{n}$ modes are below cutoff and the fundamental mode exhibits a manageable effective area of transmission. The analysis reveals that, under ideal conditions of metal smoothness, $\mathrm{HE}_{n}$ modes are only a threat for the highest frequencies of the band or if extremely large radius are used. Nevertheless, the conductivity of the metal has shown to have much more influence (roughly 3 times) than the frequency. Hence, strategies to prevent propagation of high-order $\mathrm{HE}_{n}$ must be definitively considered, being that practical implementations of the SWW may easily present some surface roughness or other sources of imperfection that facilitate the propagation of these undesired modes.

- A careful analysis of the fundamental mode $\left(\mathrm{TM}_{0}\right)$ of the SWW has given the following important set of conclusions:

- The fundamental $\mathrm{TM}_{0}$ mode of a SWW shows practically zero dispersion at $\mathrm{THz}$ frequencies. This is caused by the high impenetrability offered by the metallic surface of the wire. The mode is propagated almost in its totality in the external air medium, hence experiencing quasi-TEM features of a plane wave propagating in such medium. It shows pure radial polarization and the power distribution exhibits and exponential decay of intensity from the waveguide surface to the infinity.

> The larger the radius, the lower the attenuation, being the product $a \cdot \alpha$ quite constant. The attenuation dispersion with the radius parameter is, therefore, moderate, and noticeably lower than that of other kind of waveguides (closed metallic, dielectric, etc.). Inside a range of reasonable values for the radius it is possible to obtain low-loss features with the largest ones and, at the same time, rather high values of loss for the smallest ones. An intermedium reference is postulated to be $a=100 \mu \mathrm{m}$ at $f=1 \mathrm{THz}$.

- The larger the radius, the larger the radial extension of the fields, or the effective area of transmission. The variation of this parameter with the radius is less pronounced than the variation of the losses. Similar conclusions to those for the attenuation are obtained by considering a range of reasonable radius.

- The main challenge when designing a SWW comes from the existent trade-off between losses and effective area of transmission: the lower the 
attenuation, the larger the effective area of transmission. Therefore, although extremely low-loss SWW can be designed, they may result impractical due to an excesive transversal extension of the fields.

$>$ The larger the frequency, the larger the attenuation, and the smaller the effective area of transmission. This is caused by the reduction of the impenetrability of the metallic surface as Drude model describes, which even compensates the fact that the waveguide becomes electrically larger at higher frequencies. Nevertheless, this anomalous effect is the origin of the small attenuation dispersion in frequency of the fundamental $\mathrm{TM}_{0}$ mode of the SWW.

- A positive aspect, inherent to SWW implementations, is that the attenuation dispersion caused by the propagation losses and the attenuation dispersion caused by possible losses during the excitation are predicted to contribute in a compensatory way: lower frequencies exhibit larger area of transmission, hence are more inefficiently excited, however, these frequencies present also lower propagation losses.

- The better the conductor, the lower the losses, and the larger the effective area of transmission, this caused by the larger impenetrability of the conductor surface. However, the influence of the conductor characteristics is much lower than the influence of radius or the frequency. For large radius or low frequencies, the used material (assuming only good metals are considered) is irrelevant. For small radius or high frequencies, i. e., when the mode collapses on the wire surface, there is more confinement for worse metals. This fact may be used for excitation purposes. However, in such cases, the characteristics of the SWW present also a slightly higher dependence with the frequency.

> Regarding to the excitation or coupling actions with the SWW, it must be highlighted that small SWW provide more compact patterns with circular shape, whereas large SWW provide larger extent patterns with ring shape, this last caused by the appreciable empty-of-fields area occupied by the wire.

- Compared to closed or semi-closed metallic waveguides, the same tendency with the waveguide size is found: the larger the waveguide, the lower the losses. However, the difference is that in closed waveguides, monomode condition is very sensitive to the waveguide size, and they present more attenuation dispersion.

> Compared to dielectric waveguides, it is found that, whereas both present an increment of losses with the frequency, dielectric waveguides also show an increment of losses with the waveguide electrical size as they present very penetrable surfaces. Therefore, in dielectric waveguides both effects do not compensate each, causing the response to be very frequency dependent. Furthermore, the high penetrability of the dielectric waveguides involved surfaces implies the group velocity dispersion to be high in them.

- Similarly, the analysis of the modal spectrum of the DCSWW has also provided a set of fundamental conclusions: 
> The coating in the DCSWW provides waveguiding capacity itself, which, even for small coatings, assumes an important role on the waveguiding features. Therefore, it has been found that the influence of the metallic material conforming the wire is of second order. This has allowed the use of simplified model assuming the conductor as PEC, avoiding in this way a challenging problem of three regions and complex propagation constant.

- When dealing with DCSWW, a large variety of cases are possible depending on the value of the ratio $R=a / b$. When $R \rightarrow 1$ the problem tends to the SWW case. In this situation, the modes degenerate by groups ordered according the radial index $m$. For each $m$ there is a first group composed by the $\left(\mathrm{TM}_{0 m} / \mathrm{HE}_{n m}\right)$ modes, and a second group composed by the $\left(\mathrm{TE}_{0 m} / \mathrm{EH}_{n m}\right)$ modes. In the limit, only the very first group $\left(\mathrm{TM}_{01} / \mathrm{HE}_{n 1}\right)$ survives, founding that these modes correspond to the $\left(\mathrm{TM}_{0} / \mathrm{HE}_{n}\right)$ surface modes of the SWW. Contrarily, as $R \rightarrow 0$ the problem tends to the dielectric cylinder case. However, a particularity in this case is that although the radius $a$ become infinitesimal, the PEC condition is always influencing the electromagnetic fields, hence it is not possible to find direct correspondence between the dielectric cylinder problem and a $R \rightarrow 0$ DCSWW. Under the small $R$ situation, the degeneracy between modes is broken. The most relevant effect is that the fundamental mode exhibits an anomalous behavior in this case, becoming more confined, whereas the first high-order mode $\left(\mathrm{HE}_{11}\right)$ becomes less confined. Therefore, fixing $b$, according to the operation frequency, and then reducing $a$, is found to be a good strategy to minimize mode coupling between these modes.

> It can be affirmed that $\mathrm{HE}_{n m}$ are quasi-TM modes, and that $\mathrm{EH}_{n m}$ are quasi-TE modes.

> According to the polarization and power distribution characteristics, it has been found a first condition to provide monomode propagation in the DCSWW. This condition assumes that axialsymmetry can be maintained in such way that $\mathrm{HE}_{n m}$ modes are avoided, and the first mode suitable to couple results to be the $\mathrm{TM}_{02}$ mode. Due to this fact, the condition has been named as weak condition. The propagation of the $\mathrm{TM}_{02}$ mode is almost totally determined by the coating thickness $t$. It has been found that the condition to avoid this mode in the case of the planar version of the DCSWW $\left(t_{\max }=\lambda_{0} / 2 \sqrt{\varepsilon_{r}-1}\right)$ fits very well in practice.

> Losses of the fundamental $\mathrm{TM}_{01}$ mode are mainly influenced by the coating for reasonable values of $t$. In such conditions the radius $a$ plays a minor role, and the coating itself is providing the main waveguiding mechanism. When this occurs, the fundamental propagates confined inside the coating and the propagation losses are high.

> The effect of the coating on the fundamental $\mathrm{TM}_{01}$ mode has been more deeply studied respecting the weak monomode condition. High permittivities must be avoided chiefly because they imply large losses. This is mainly caused by the high ohmic losses, originated by the high concentration of electromagnetic fields inside of the small volume provided by the 
coating, which has to be thin due to the use of a high permittivity. The maximum thickness provided by the weak monomode condition must be used being that it provides the maximum confinement, avoids the local maximum of losses for intermedium values of the thickness, and avoids dispersion.

In Chapter 4, the problem of radiation on bends affecting the SWW has been faced and solved through several design proposals. First, a proper criteria to avoid radiation on bends have been established, mainly based in minimizing mode coupling through exploiting the SWW and DCSWW modal spectrum characteristics. Then, transitions have been designed in order to keep independence between the bend design and the rest of the waveguiding sections. The following have been found to be important conclusions along all the steps carried out:

- The surface nature of the fundamental $\mathrm{TM}_{0}$ mode of a SWW implies that, for conventional radius, the change of direction provided by a bend causes radiation being that the mode do not follow the path described by the waveguide.

- A dielectric coating (to convert a SWW in a DCSWW) is as an effective element to prevent radiation on bends, however, coupling to high-order modes may occur if it is not prevented. An important observed fact is that bends are prone to cause power loss in the fundamental mode that goes to the axial asymmetric high-order modes (that may be radiative in some cases) due to the different lengths of the internal and external paths of the bend, what cause a phase shift in the fundamental modal pattern. Since the fundamental mode has a axialsymmetric mode pattern, this phase shift is very influential. Since the coating is thought to be present only when necessary, regarding to the previous effect, it is worth to mention that all the power coupled to high-order modes must be supposed as lost, being that these modes become radiative when the coating is removed and the DCSWW becomes a SWW. Therefore, under this situation, although more power reach the output of a bend by using the power, it is, in principle, useless power.

- A small radius SWW exhibits less radiation on bends since, apart of the larger interaction of the mode with the wire surface, it provides a smaller difference of length between the internal and external paths of the bend.

- Due to the fact that bends are very prone to excite axialsymmetric modes, the weak monomode condition results too permissive and a strict one has been establish as the adequate for this problem. The strict condition considers the very first high-order mode $\left(\mathrm{HE}_{11}\right)$ as the most suitable mode to couple to the fundamental mode. To satisfy this condition, both parameters, $a$ and $t$, are influential, but $a$ is the most determinant. By reducing $a$, power confinement of the $\mathrm{TM}_{01} \mathrm{HE}_{11}$ modes differs more due to the reduction of degeneracy. In such situation it is possible to find a $t$ providing at the same time that the $\mathrm{TM}_{01}$ mode is transmitted mainly inside of the coating, and that the $\mathrm{HE}_{11}$ mode is transmitted mainly outside of the coating, in the external air medium. Therefore, coupling efficiency between both modes diminishes in such case. 
- Analysis of curved SWW and DCSWW requires from the use of numerical tools, well provided by commercial solvers. However, since the region of the problem is infinite, it must be truncated. The simulated volume must be large enough to provide accuracy, but small enough to provide reasonable computation times. Size parametrization of the additional volume considered in the external air medium and comparison of the results with the developed analytical models has been show a very effective practice to find the correct simulation model. It has been found possible to find a good trade-off between accuracy and computation time.

- They have been found two suitable alternative solutions, A and B, to minimize the losses at bends in wire-type structures, each one attending to specific restrictions on the design:

> Solution A consists in minimizing the coupling to the $\mathrm{HE}_{11}$ mode by means of the strict monomode condition. A small radius is firstly chosen and then a proper coating is engineered to provide small radiation losses of the fundamental mode as well as low coupling. This solution presents very low radiation losses and very good performance in terms of bandwidth, being that if it is properly applied, it is almost frequency independent.

> Solution B is proposed for those cases in which the manufacturing process or the application do not permit the use of the small radius demanded by the solution $\mathrm{A}$. In this case, coupling to the $\mathrm{HE}_{11}$ mode (and high-order $\mathrm{HE}_{n 1}$ modes if $a$ is large) is minimized by adjusting the phase shift caused by the difference between the lengths of the internal and external paths of the bend in order to cause the minimum distortion to the fundamental mode field pattern. This solution provides improvement even in the case of no having not coating, however, the use of a coating has been shown to provide much better results. Nevertheless, thick coatings do not improve the result of thin ones. It has been found that this is caused because the additional power that thick coatings prevent from radiation is coupled to high-order modes. Despite of the fact that this solution is inherently frequency dependent, a surprisingly good frequency response is obtained.

> Solution A offers better performance in terms of losses and bandwidth than the solution B. However, if the radius of solution A is not chosen small enough, the performance of both solutions become comparable.

- The propagation characteristics of the optimum designs for straight path sections and curved sections in wire-type structures are antagonistic. In straights paths, a large radius SWW provides low losses and almost zero dispersion whereas, in bends, a small radius DCSWW (as suggested by solution A) provides the lowest radiation losses. However, a small radius DCSWW is a lossy, high dispersive waveguide. Therefore, if the bend design conditions all the waveguiding sections, the interesting properties of the SWW are lost and the general behavior of the whole waveguiding system may be even worsened by this conditioning. In this sense, it is found that the use of transitions is very recommendable. Three kinds of transitions, based on linear tapers, has been 
proposed in order to cover different types of scenarios attending to the design restrictions. The use of such transitions shows a successful and important improvement over conventional uniform designs. The main remarks attending to each design are:

> The first transition consist in tapering exclusively the coating thickness. This proposal is suitable for such scenarios where the radius cannot be tapered by some reason. By using this transition, the propagation losses in straight paths are considerably alleviated since a SWW can be used instead of a DCSWW. However, the main disadvantage is that the bend still conditions the size of the radius, and a small SWW presents acceptable, but not small losses, much higher than those of a large radius SWW. Regarding to the case of using a small radius bare SWW, without any coating at bends, this transition gives important advantages if several bends are present.

- The second transition consists in tapering exclusively the conductor radius. With this design, propagation losses result to be small, inasmuch as the transition allows for a large radius in straight paths. However, despite that the bend is faced with a small radius SWW (which cause less radiation losses than a large radius one), the lack of coating causes the radiation losses to be still larger than desired. Therefore, although large straight paths section cause low total losses in this design, the number of bends included on the path results critical. Nevertheless, the performance of this second transition is quite superior than that of the first transition.

> The third transition consists in tapering simultaneously conductor radius and coating thickness. This design improves the second transition proposal alleviating the bend losses with the use of the coating, hence achieving the optimal response. The improvement regarding to conventional designs is appreciable. Calculated losses for this transition indicate that it may provide the required high performance of real systems.

In Chapter 5 it has given experimental validation to several analysis tools and concepts arising from synthesis procedures developed along previous chapters. The results have come from two different experimental setups using the same prototypes. In the first one, operating at Ka-band frequencies, the employed wire-type structures have an electrically small radius, whilst, in the second one, operating at $\mathrm{THz}$ frequencies, they present an electrically large radius. Evaluation of the procedure and analysis of all the obtained results provide the following list of conclusions:

- Working at Ka-band frequencies allows to use simpler experimental setups with conventional microwave devices and techniques at the cost of do not having actual $\mathrm{THz}$ information. On the one hand, the further the frequency from the $\mathrm{THz}$ regime, the more different is the operating environment from the actual one, what implies that the recovered information is not exactly the desired one. However, on the other hand, excitation efficiency at Ka-band frequencies is much higher than that obtained at $\mathrm{THz}$ frequencies, and the system is more robust. In addition, manufacturing errors are less influential 
on the final results. This allows to better observe some effects and to extract more solid and elaborated conclusions. These conclusions are listed next:

> Advantages of wire-type structures from its equivalent closed waveguiding structure, the coaxial cable, is already evident at Ka-band frequencies. Nevertheless, advantages of the SWW front the DCSWW are less evident at these frequencies. The main reason for this last fact is the better performance of materials (metals and dielectrics) at microwave frequencies. In general, losses are quite lower at the Ka-band than at the $\mathrm{THz}$ band due to both, frequency scaling and material performance. This implies the use of considerable waveguiding lengths, beyond $L=1 \mathrm{~m}$, to produce appreciable losses in straight waveguiding paths.

- Excitation of wire-type waveguides by means of a coaxial with tapering on the external conductor (creating a horn) is very convenient at these frequencies. Transition from coaxial to DCSWW presents insignificant losses. It must highlighted that if any launching horns is present (direct transition from coaxial to DCSWW), an important amount of power is lost.

- Using optimized transitions results essential insomuch as several dBs could be gained. The lower excitation efficiency in the case of the SWW, as well as the appreciable penalty of adding coating exclusively on bends is mainly caused by the losses introduced by the required DCSWW-SWW transitions, which, since have not been accurately designed and manufactured, produce an important mismatch.

- A first implementation of bends for curved paths loss measurements has consisted in creating a soft bend with large curvature radius by means of two supports at the edges of the curved path. In practice, nevertheless, it has been realized that this rather provides chamfered bends, i. e., two bends of half of the curvature angle. Under this conditions it has been found that:

* The SWW experiences noticeably larger radiation losses than the DCSWW, however, this losses are still moderate taken into account that the radius is electrically small. It must be highlighted that they are prevented to be smaller due to the high impenetrability of the metallic surface of the SWW at microwave frequencies: the mode is more expanded, thus the mode pattern is more perturbed by the difference in length between inner and outer paths of the bend. It has been also found that increments of the curvature angle affect slightly more when the curvature angle is lower, i. e., the relationship (losses vs. curvature angle) is less than linear.

* The DCSWW remains invariant to the curvature angle. This occurs mainly due to the fact that the employed radius is very small in terms of the operating wavelength. In addition, the presence of the coating causes reduces the impenetrability of the waveguiding surface, hence confining the mode. Therefore, difference between inner and outer 
paths lengths in the curve is very small, so that the mode pattern is practically unperturbed.

- A second implementation of bends for curved paths loss measurements has consisted in creating a sharp bend with small curvature radius by means of a fabric yarn executing tension in the middle of the curved path. In general terms, the obtained results have been found quite similar to those with the first bend design. Nevertheless, the appreciated small differences have allowed to identify some phenomena that are related with the concepts motivating the experiment:

* The SWW presents slightly lower losses. Since the relationship (lossescurvature angle) is less than linear, a curvature of angle $\theta$ causes less radiation losses than two angles of $\theta / 2$.

* In the case of DCSWW, a slight, very small increment of radiation losses with the curvature angle is appreciated. This increment progressively grows as the curvature angle increases, i. e., the contrary tendency than for a SWW. This effect seems to be caused by the fact that the mode pattern perturbation does not follow a linear law with the curvature angle, i. e., initial increments of the curvature angle cause lower perturbations than posterior ones. This provided by the large radial expansion of the mode provided by the thick coating. Additionally, TIR effect is progressively lost as the curvature angle increases. Differences respect to the first curved path studies are also originated by these phenomena. In the case of a DCSWW, a angle $\theta$ causes more radiation losses than two angles of $\theta / 2$.

- A SWW with coating exclusively placed on bends (SWW-CoB) achieves optimum results in practice. Measurements confirm that a SWW-CoB experiences same straight path losses than a SWW and same losses at bends than a DCSWW. Critical point are, nevertheless, SWW-DCSWW transitions, which introduce a loss penalty that could significantly avoidable if this element were properly designed. Promising results are expected if more accurate prototypes are manufactured.

- Results obtained with Ka-band experimental setups are consistent and provide reasonable good agreement with both simulations and theoretical calculations. In addition effects originating differences between the calculations and measurements are easily identified, sometimes helping to give experimental evidence some of the evaluated concepts. It can be concluded that Ka-band experiments have resulted very satisfactory since a large quantity of predicted results and effects have been experimentally observed either as a primary or as a secondary result of certain experiment.

- Dealing with $\mathrm{THz}$ experimental setups results must be done carefully inasmuch as they are more sensible, more influenced by undesired effects, and the equipment performance is lower than at microwave frequencies. On the other hand, prototypes are evaluated in a more real environment regarding projected applications, hence experimental results link much better with the concepts treated 
previously. In addition, several effects, not observable at lower frequencies due to the excessive wavelength size, arise very evidently. From the set of experiments at $\mathrm{THz}$ frequencies the following conclusions are extracted:

- When measuring losses of certain magnitude, as those caused by bends, dynamic margin results crucial. In this sense it is important to check the power spectrum provided by the source and to choose an adequate frequency for the experiment purposes.

> Since at $\mathrm{THz}$ frequencies, transmitter and receiver elements of the measurement system are generally antennas, it is essential to check that there is not direct coupling from transmitter to receiver. If this occur, higher received power than real is recovered.

- Careful supporting and sandwiching elements using an almost transparent material such as it is Rohacell ${ }^{\circledR}$ has been found necessary in many cases. It is highlighted that it has been experienced high sensibility of the system to movements so that these elements are crucial in order to keep the required robustness and to ensure repeatability.

> Excitation of wire-type by means of direct coupling of oblique incident radiation has been implemented straightforwardly. However, it is found that the efficiency is quite low. It has been observed that the use of an auxiliary SWW, orthogonal to the wire to be excited in the area where the incident radiation is focused, results indispensable. Otherwise, almost no power is coupled to the wire-type waveguide under test. In addition, it has been checked that the auxiliary SWW must be as close as possible to the wire-type waveguide to be excited.

> Experiments clearly show that a SWW presents considerably lower loss than a DCSWW at THz frequencies. In fact, the attenuation of the evaluated SWW is as low that to measure it with accuracy results challenging given the small waveguiding lengths of the system and the influence of noise.

> Measurements confirm that a large radius SWW experiences more losses at bends than a small radius one (test at Ka-band frequencies). Comparison with Ka-band experiments also shows that the radius has much more influence in the radiation losses than it has the penetrability of the metal, this last varying with the frequency. The experiments at $\mathrm{THz}$ frequencies reveal that even small angles cause a noticeably large radiation. This makes evidence of the mode pattern perturbation effect on curves for large radius cases, which is the foundation of the solution $\mathrm{B}$ of chapter 4 for radiation on bends.

- Due to the large radius employed, high losses are as well appreciated for the studied DCSWW despite of the fact that almost all power propagates confined inside the dielectric coating region. The received power rapidly drops as the curvature angle increases. This fact reveals high coupling to the first high-order mode, the $\mathrm{HE}_{11}$. As in the case of the SWW, this effect is provided by the mode pattern perturbation originated by a large 
difference in length between internal and external paths of the bend due to the large radius. This effect is further confirmed in a more direct way by the fact that from a certain curvature angle, the received power grows, being observable a non-monotone behavior of the received power with the curvature angle. Therefore, the potential of controlling the curvature phase shift to reduce radiation losses for certain range of curvature angles has been clearly evidenced with actual $\mathrm{THz}$ measurements.

> Measurements of curved path losses have been significantly limited by noise since the received signal was under the noise level in many cases, what has obscured interesting information. Larger available power, more efficient excitation schemes, improved receiver sensitivity, as well as a more exhaustive measurement plan considering more cases could lead to access to information revealing deeper detail of the observable phenomena.

- It can be concluded that experimental results at $\mathrm{THz}$ frequencies have resulted satisfactory despite of the system and waveguide prototypes limitations. Reasonable good agreement with theoretical calculations is found, and several interesting phenomena, hardly or not observable from Kaband measurements, have been clearly observed. The objective of giving experimental evidence to predicted effects and show the potential application of some of the presented design procedures has been successfully achieved with the additional merit, in this case, of doing it at $\mathrm{THz}$ frequencies.

\subsection{Future Lines}

Despite the solutions proposed in this part of thesis have provided significant knowledge and progress under the demands of the state of the art, as usual, there is still much room for further research. Along the previous chapters several future work lines have been pointed, some of them coming from present research necessities, lacking in the literature. Other future work lines come directly as a natural continuation of this thesis. All of them are listed next:

- As a general work line, it becomes necessary in the future to unify criteria, nomenclature and procedures coming from microwave and optical researchers. This task of standardization would enhance the communication and links between researches and research groups, thus the achieved advances could contribute jointly in a more efficient way.

- An interesting foreseen work line would be the development of suitable modal methods for open structures based on the knowledge of the phenomena taking place in the simplest structures, such as the dielectric cylinder. The idea of being able to use a similar vast amount of modal numerical techniques such that successfully applied for closed metallic structures is an amazing one. Although conceptual and numerical complexity are tough, present computational tools provide a suitable scenario to embark on this challenge. 
- It results almost a mandatory future work line to establish the limits of use of simple models for metals such as the obvious PEC condition, or the skin-effect model. Since this is not a very well determined question (being that is very problem dependent), at least, a set of conclusions or a standardized procedure should be investigated. Metals should be characterized in different geometries at $\mathrm{THz}$ frequencies through appropriated models such the Drude model (or even new tailored models) to provide reliable information for $\mathrm{THz}$ researchers. Agreement between simulation and practical implementations relies strongly on the modeling, hence an effort is this sense would be appreciated.

- As a direct consequence of the gained knowledge about the modal spectra of the SWW and DCSWW, it would be a good line to work in, the development of new excitation/reception schemes exploiting all this knowledge in a similar way that bends or transitions have been designed in this thesis. Actually, this interesting work line could perfectly have been in the scope of this thesis if time allowed for it.

- A very concrete future work line pointed by this thesis is the analysis of the DCSWW modeling the conductor through Drude Model. Starting from the results obtained from this thesis, the resolution of this problem is perfectly affordable. In this way, mathematical continuity between SWW and DCSWW would be provided. This would allow to perfectly understand the transition between both, an obscured point that has been just perceived in this thesis with the aid of the gained knowledge. Furthermore, modal methods using this information may allow for the analysis of more complex structures. Imagine for instance a filter implemented with intertwined sections of SWW and DCSWW. Moreover, under this exact, complex propagation constant approach, very lossy coating DCSWW could be perfectly characterized. This would open the door to the modeling of a DCSWW acting as a sensor of conventional materials, usually presenting high losses.

- In addition, the provided analysis of the SWW and DCSWW structures, open directly the door to study similar problems with: a) different geometries; b) different constitutive parameters. Regarding to the geometry, the methodology used in this part of the thesis would apply directly to elliptical SWW/DCSWW (modeling some imperfections of the wire) or, by using straightforward numerical techniques, to rectangular SWW/DCSWW, this providing very suitable models for the planar version of these waveguides or for more bulk consistent square-shape SWW/DCSWW or similar modifications. In the same way, multilayer structures may as well be directly faced by following the presented procedures. Regarding to the materials, it is obvious that all the observed phenomena exploring the dielectric permittivity have its dual counterpart if the same exploration is done with the magnetic permeability. Even more, if both constitutive parameters adopt their more general form, interesting phenomena may appear, which will, for sure, better understood starting from the conclusions obtained here. This work line would be very welcome on the world of metamaterials, plenty of activity these days. 
- Another work line arising from the results and conclusions of this thesis is the incorporation of adequate packages to the commercial solvers routines in order to provide more suitable analysis tools for surface waveguiding structures. Since commercial solvers are mostly focused to conventional structures lacking of some phenomena appreciated in this thesis, it would be interesting to add some packages including algorithms to enhance the performance of the simulations in terms of accuracy and efficiency. The results from this thesis may inspire those implementations, and may help to understand the obtained results, facilitating the user task.

- A desirable future work line is to carry out further experimental tests with more accurate systems. The preliminary experiments provided in this part of the thesis have superficially shown the potential of some ideas proposed in this part of the thesis. Future work must pursue higher operating frequencies and bandwidths, i. e., the operating conditions under which wire-type waveguides result noticeably more suitable than other alternatives. In addition, prototypes with proper dimensions, implementing optimized designs with transitions must be manufactured with precision in order to achieve actual outstanding performance. As calculations from chapter 4 indicate, great improvement is expected. Such experimental work would be a very motivating one, endorsing well all the effort put in this part of the thesis. In an optimal research scenario, those prototypes would be tested under very similar conditions than those founds in real environments of application.

- Finally, it must be cited the direct future work line of developing SWW/DCSWW advanced components. Once the waveguiding structures are well known, the direct step to follow is to face the design of more complicated components, such as antennas, couplers, dividers or filters. It would be very interesting to merge design concepts applied to other technologies with those concepts obtained through the work in this part of the thesis to create high-reliability components that would dispose high functionality circuits based on wire-type structures. For instance, the open nature of the wire-type structures suggest many possibilities arising form coupled transmission line theory. 


\section{Part II}

\section{mm-wave band components}





\section{Chapter 7}

\section{Introduction}

\subsection{Motivation}

With microwave technology well established in its maturity stage and a progressively more saturated spectrum in such band, radiofrequency technology market points towards the new arising niche provided by mm-wave frequencies. Without doubt, mm-wave band is called to allocate new generation communication services as a supplementary spectrum to the microwave band, which has become small for the incoming necessities. In addition, taking advantages of the small, millimeter wavelength, the use of mm-wave frequencies for automotive radars or imaging for security has the inertia to become soon universal. Nevertheless, in order to reach the desired high-quality user experience and comfort, as well as the required reliability in critical applications, mm-wave technology must reach a maturity level that it does not have yet nowadays.

Several facts prevent these days mm-wave technology from achieving the desired performance level, being the large degradation that passive components experiences at mm-wave frequencies an important one among of them. Conventional microwave waveguides and transmission lines seem to be forced beyond its possibilities when they are scaled to work at mm-wave frequencies. Dimensional problems arising from the use of such small wavelengths together with the larger dielectric absorption that dielectrics experiences at mm-wave frequencies complicate the design scenario of passive components. For instance, hollow metallic waveguides suffer from bad metal contact and alignment problems between parts, which causes strong response degradation. Similarly, conventional transmission lines such microstrip or CPW experience a noticeable increase of losses caused by large dielectric losses that together with the natural increase of ohmic losses and the enhancement of some negative effects such radiation and parasitic modes leads to reach unacceptable attenuation levels. Given that important advances in dielectric materials and manufacturing techniques are rarely expected in short-therm, this complicated design scenario forced the consideration of new topologies. Among several alternatives, Gap Waveguides seem to be very suitable, being that these waveguides are specially tailored to avoid the aforementioned problems affecting conventional waveguiding structures. 
Gap Waveguide (GW) are based on a periodic structure that allows to create waveguiding structures without requirement of metal contact between manufactured parts. In addition, dielectric losses are either totally avoided or become a residual loss depending on the specific GW design. Despite of the fact that these features suggest remarkable potential for their use at mm-wave frequencies, are not few the issues to address in order to GW technology become consolidated and commercially used. On the one hand, full-wave simulation of GW components is time-costly insomuch as the periodic structure present in them is formed by a large number of small elements to be discretized. Furthermore, there are not neither efficient analysis methods nor adequate models performing properly enough to facilitate the synthesis process, which becomes complex in general, and specially discouraging for the designer without any or with merely superficial knowledge about GWs. For instance, transmission line theory is hardly applicable to GW transmission lines due to the lack of proper impedance models or analysis methods. Similarly, powerful modal techniques such those used in rectangular waveguide designs are today inconceivable for the GW counterparts.

On the other hand, it is the technological aspect. First, there is still not work enough proving that, effectively, GW technology shows clear advantages front conventional technology, what, to the moment, has been only partially proved. This issue demands from extensive work experimentally comparing the performance of GW and conventional components in a real scenario context, i. e., at frequencies high enough and with components providing advanced responses. Second, and maybe more close to be reality these days, it is necessary to provide a large variety of different high-performance component designs, with good interoperability, that prove GW technology to be consistent to implement complete systems of high reliability.

The work in this part of the thesis is motivated by the conviction that Gap Waveguides can truly improve mm-wave technology in benefit of the human society. To this occur, GW technology must become and be shown as a solid and consistent technology to the eyes of research and industrial worlds. It must be taken into account that the only way for a new technology to replace or supply an old one is to show not only slight improvement, but an edge. In this sense, it must be highlighted that general advantage is not only composed of performance advantages, but ease of analysis, design and manufacturing processes play a non-trivial role. This part of the thesis purses to cover the current lack of work regarding these issues, by means of new-developed efficient analysis and design techniques, implementation of GW devices showing high-performance, adequate comparatives with conventional structures, and procedures to alleviate manufacturing processes errors. In addition, the work in this part of the thesis is as well motivated by the possibility of providing comprehensive, updated information about GW technology in order to both, to give proof of the current technological consistence of GWs and to facilitate the work to future GW researcher. 


\section{$7.2 \quad$ Objectives}

An important number of objectives is pursued in this part of the thesis. Classified by sets, they describe the later structure of the thesis. These objectives are:

1. Related with general mm-wave technology and Gap Waveguide technology scopes:

- To get a general and deep view of mm-wave technology and applications. In order to contribute in an optimal way to certain technological area it is necessary to know well the minors of it. In the case of a semi-mature technology, as it is the mm-wave band one, it is recommendable to review the history in order to understand the reasons that motivate its development as well as the research inertia that describes current challenges and necessities. In the same way, it results very important to be aware of current regulation, specification and environment of use of what has to be developed either analysis techniques or components. The achievement of this objective is a good guarantee follow the best approach during the research work, even if such work is focused in the very low level from the application point of view, as it is the case of this thesis, that deal with analysis and design techniques as well with basic hardware elements.

- To clearly identify main technological problems affecting mmwave passive components. Since the thesis is focused in passive components, it results of primary importance to give special attention in the mm-wave technology review to those problems affecting this kind of component. Main technological problems of conventional structures are needed to be well understood by means of a wide research on the literature, avoiding commonly found biased opinions and lack of information, in order to enhance Gap Waveguides properties with appropriate improvements, adding value to current mm-wave technology. This also involves to inquire which components are more affected by mm-wave technological problems in order to focus the research on them.

- To carry out an extensive review of Gap Waveguide technology. This objective is motivated by two main reasons. On the one hand, since this part of the thesis deals with Gap Waveguide techniques and components, it is obvious that to contextualize the work to be done is essential. To know well the employed techniques and topologies in the past, advantages and drawbacks of each proposal, and current challenges is mandatory to introduce improvements and to generate new ideas of high quality. On the other hand, it is observed that, although there is already certain amount of good work dealing with Gap Waveguides, it remains dispersed, specially taking into account recent research. In this way it is pretended to carry out an exhaustive information recollection that allow not only to fine contextualization of the work in this part of the thesis, but also to help future researches in the area, thus contributing to the consolidation of Gap Waveguide technology. 


\section{Related with the Suspended-Strip Gap Waveguide (SSGW):}

- To develop an analysis method to obtain the characteristic impedance of a SSGW. This objective pretends, on a first stage, to solve the current uncertainty regarding the definition of characteristic impedance in Gap Waveguides in a practical way, leading to an scenario of straightforward treatment of Gap Waveguides design using transmission line theory. On a second stage, it is desired to establish an analysis method with rigorous foundations, able to recover the impedance of a Gap Waveguide transmission line with controlled accuracy, with particular interest in the analysis of the SSGW. The idea is to characterize the SSGW in terms of its impedance in order to facilitate the design of advanced components by means of conventional transmission line theory techniques, hence alleviating the design process from some unnecessary, costly fullwave simulations.

- To solve the problem of the high dependence of the SSGW characteristic impedance on the relative position of the strip respect to the pin structure. Up to date, proposed SSGW circuits present a characteristic impedance that has a strong dependence on the relative position of the conducting strip regarding the underlying pin structure. This behavior noticeably hinders the design process with the SSGW and prevents this transmission line to implement high performance, robust circuits and to be adopted for general commercial use. Accordingly, a design procedure leading to very stable impedance SSGWs is pursued in the scope of this objective. In addition, it is desirable to understand well the characteristics of the SSGW under such configuration, identifying similarities and differences with conventional transmission lines.

- To study the properties of SSGW coupled line structures and to provide analysis and design procedures for them. The intention with this objective is to facilitate the design of more advanced SSGW components, being coupled transmission line theory a very useful tool. In order to achieve this, first, it must be check that coupled SSGW lines act in the same way as conventional coupled transmission lines. In this sense, this objective also involves to determine, for the first time, the properties of SSGW coupled line from a transmission line perspective. Second, an analysis tool must be provided in order to generate the necessary information to carry out straightforward, transmission-line based design processes. Finally, it is intended to describe clear synthesis procedures and to provide useful tools for the design process in order to make it simple for any radiofrequency engineer, not necessarily aware of the minors of the structure. This objective is the natural extension of the previous one, clearly conditioned by the success of it.

- To design a simple component based on SSGW. This objective is the final one of this set of objectives related with the SSGW, and concerns the correct satisfaction of all previous ones. The aim is to describe in a clear way, from a practical perspective, how all the developed analysis and 
synthesis procedures must be applied in a real design. In addition, it is pretended to show the potential of the SSGW front similar alternatives to implement challenging high-performance passive devices in the mm-wave band.

\section{Related with the Groove Gap Waveguide (GGW):}

- To study the propagation characteristics of the Groove Gap Waveguide and to develop an efficient analysis method. To date, only basic information about Groove Gap Waveguide propagation characteristics is known. The first stage of this objective involves the study of this waveguide under a wide range of design cases, involving different geometrical parameters and operation regime. For instance, the possibility of operation below cutoff, which has not been considered up to now, must be analyzed. All this information must be used to develop an efficient analysis method that can provide practical information to the GGW device designer. In addition, it would be desirable to find out an accurate equivalence between GGW and rectangular waveguide since this would greatly facilitate the comprehension of GGW properties and would drive to an easy design process. Definitely, such an equivalent would endorse in a great extent the adoption of GGW by the mm-wave band community, hence becoming a relevant and challenging objective of this part of the thesis.

- To implement a narrow-band filter prototype fully satisfying the demanded specifications and with very competitive response. Design of narrow-band filters satisfying specifications is currently one of the most faced challenges of mm-wave technology. Rectangular waveguide exhibits appreciable weakness for such implementations at mm-wave frequencies. The objective is to consider a filter topology on a simple basis such the all-pole configuration and work on it, introducing the improvements and applying the necessary procedures in order to implement a narrow-band filter prototype operating at mm-wave frequencies that fully satisfy a set of previously established specifications. The very final goal after this objective is to give evidence of the suitability of GGW as an optimum candidate to implement narrow-band filters and other components suffering from similar technological problems at mm-wave frequencies.

- To contextualize the implemented narrow-band filter designs in the state of the art. As an auxiliary objective, complementing the involved goals of the previous one, a wide review of the recent literature involving mm-wave band narrow-band filters must be carried out in order to understand the usual encountered problems when dealing with the design this component. This may not only provide useful information to enhance the design process, but it is also necessary to give a proper assessment of the designed prototypes once implemented, and quantify how competitive they are. 
- To show experimentally, in a clear and evident way, that GGW topologies are more convenient than rectangular waveguide topologies for certain design scenarios. Several works argue advantages of GGW front the rectangular waveguide, however, a clear and fair comparison still lacks. It is desired to provide an experimental comparison between GGW and rectangular waveguide prototypes implementing the same component and specifications. In this sense, the consideration of a narrow-band filter is very adequate since, in this way, this objective relates with the previous one. Several test must be carried out giving conclusions evident enough in order to affirm that GGW topologies must be preferentially chosen instead of rectangular waveguide topologies in certain design scenarios.

\subsection{Methodology}

Following the same premises of the first part of this thesis, a strict methodology has been followed as a necessary basis for success. Again, different methodologies are followed depending on the task type and data involved, with the aim of proceeding in the most correct way. Similarly, systematic procedure for homogeneous data and tasks has been followed rigorously in order to facilitate conceptual comprehension.

Bibliographic research has been a fundamental pillar in this part of the thesis. A large number of scientific works have been reviewed. In this sense, Google Scholar ${ }^{\circledR}$ as a source to consult has been the main tool to find specific papers giving answer or basic information for the posed issues. Similarly, qualified books, available in the resources of the universities in which this thesis has been realized, have been consulted frequently. Front such vast quantity of information, a procedure to establish information priorities has been carried out. The reading of scientific papers has been structured by their importance related to this part of the thesis necessities, and the depth and quantity of notes taken from them has been adapted accordingly. When reviewing more specific papers dealing with GW components and techniques or with mm-wave filters, some additional calculations have been carried out with the aim of dispose of quantitative information to provide a clear and fair comparison between different works.

The development of analysis methods for Gap Waveguides is of great relevance in this part of the thesis. When carrying out this task, a major premise has been to keep present that conceptual simplicity and ease of use are always recommendable characteristics of an analysis method since, in this way, future designers using the analysis method do not require a deep knowledge of what is analyzed. This facilitates the adoption of Gap Waveguide technology what is one of the general objectives pretended for this thesis. Transmission line theory has been found a perfect basis to accomplish that premise since its foundations are well-known by most engineers, not only in the radiofrequency field. Several reference books and papers have been consulted and posterior rigorous work on paper has led to the adopted strategies and consequent mathematical expressions. The employed approach has driven to hybrid methods that have both analytical and numerical, full-wave procedures. To keep efficiency has been another important premise. Therefore, it has been tried to 
reduce at maximum the full-wave simulations load on the procedures in preference of the fast use of analytical expressions. Robustness has been also a pursued feature of the developed methods, which have been conceived and tested for a wide range of cases.

Numerical evaluation of the involved analytical expressions has been easily afforded by the aid of $M A T L A B^{\circledR}$, which thanks to its versatility and user-friendly interface has allowed to proceed straightforward and correctly optimize the developed analysis methods. In this part of the thesis, the involved expressions are quite simple, and the cost of the analytical part of the developed analysis methods is practically negligible. Therefore, in terms of speed, any more efficient coding is required by far, being preferable to use the suitable libraries and visualization tools of MATLAB ${ }^{\circledR}$.

On the other hand, the required full-wave simulations have been carried out with the two of the most commonly used general purpose software tools: HFSS ${ }^{\circledR}$ and $C S T^{\circledR}$. In both cases Finite Element Method (FEM) has been the underlying numerical method, being that it has been found more accurate than other possibilities such Finite Differences in Time Domain (FDTD), implementable with $C S T^{\circledR}$, for instance.

Synthesis of components has also a major role in this part of the thesis. In this sense, several full-wave simulations and optimization processes have been required. Again, HFSS ${ }^{\circledR}$ and $C S T^{\circledR}$ have been the mainly employed tools. The availability of two software tools allows to select for each design the one that seems to be more suitable or to perform better, and, in addition, to realize a double check in case of doubt in front certain result. For instance HFSS ${ }^{\circledR}$ has been preferred for GGW filters whereas $C S T^{\circledR}$ has been found preferable for large SSGW components. In addition Full-wave Electromagnetic Simulation Tool (FEST3D $\left.{ }^{\circledR}\right)$, implementing the efficient Boundary Integral-Resonant Mode Expansion (BI-RME) method has been employed to provide fast simulation of some structures when it has been possible.

Optimization has been a essential task, requiring in some cases advances strategies in order to achieve convergence and to do it efficiently. Therefore, the incorporated optimization strategies of $H F S S^{\circledR}$ and $C S T^{\circledR}$ have been manually intervened up to a great extent. To face such a delicate problem with no clear solution, several books and references have been consulted in order to proceed with good criteria. Although this underlying work is not detailed in the thesis due to space reasons, it has become an important part of the procedure.

Beyond full-wave simulations, experimental validation has been a must for most of the designs in this part of the thesis. The operating frequency has allowed to use own in-house facilities to manufacture and measure Gap Waveguide mm-wave components. Along the work in this part of the thesis, both, design and manufacture have been kept in mind in order to restrict the design to easily manufacturable components. Similarly, the manufacturing process has been strategically faced and supervised with the intention of preserving the desired characteristics of the component once implemented in practice. This way to proceed is essential, design and implementation must be conceived together in order to produce engineering research of quality. Therefore, even in those cases in which final prototypes have not been manufactured, topologies and dimensions have been previously ensured to be affordable so that the corresponding component can be implemented in a future work. 
Finally, figures have been created in the usual way, following similar premises than in part I of the thesis. Base figures have been obtained by the employed software, either $M A T L A B^{\circledR}, A W R M O^{\circledR}, H F S S^{\circledR}, C S T^{\circledR}$. This last software tool has been found specially useful to create illustrative 3D models. When necessary, additional features have been added or edited in such figures by means of Inskape ${ }^{\circledR}$. This software drawing tool has been also used to create very specific schematics starting off without any base image. Additionally, $P G F / T i k Z$ plots have been created from $M A T L A B^{\circledR}$ data in such cases requiring special detail or quality on the figure.

\section{$7.4 \quad$ Structure}

This thesis is organized as follows:

Chapter 8 mainly reviews definition, characteristics, history and current applications scenario of the mm-wave band. Then technological problems in this band affecting passive components are detailed. Finally, this contextualization chapter ends with an extensive review of the Gap Waveguide technology state of the art, with special interest in passive structures and analysis methods.

Chapter 9 faces analysis and design of Suspended-Strip Gap Waveguide (SSGW) components. First, essential transmission line theory foundations are described. They conform the basis of the developed analysis method, which is presented next and includes a rigorous error analysis. After the validation of the method, it is faced the objective of designing a SSGW with very stable impedance regarding the relative strip position respect to the pin structure. As a second part of the chapter, SSGW coupled lines are studied. First, transmission line theory concepts regarding coupled lines are described. These concepts are used next together with the previously developed analysis tool to implement an analysis procedure for SSGW coupled lines. A design chart for the Ka-band is created by extensive use of such analysis tool and finally, a simple SSGW coupled line filter is designed as proof of the utility of the developed tools and procedures. The chapter closes with a brief comparative of losses between Ridge Gap Waveguide (RGW), SSGW and Substrate Integrated Waveguide (SIW).

Chapter 10 faces analysis and design of Groove Gap Waveguide (GGW) components. In a first stage, propagation characteristics of the GGW are analyzed both, below and above cutoff. On the conclusions of this study, an analysis method is developed, providing an accurate equivalent with the rectangular waveguide. The model is validated with full-wave simulations and experimentally. In a second stage, GGW narrow band filter design at mm-wave frequencies is faced. Three designs are carried out, including experimental validation: 1) a fourth-order filter operating in the Ka-band; 2) a fifth-order filter operating in the V-band; 3) a fifth-order filter operating in the $\mathrm{V}$-band with post-manufacturing tuning elements. Finally, taken Design 2 as reference, a comparative between GGW and rectangular waveguide is carried out on a experimental basis.

Chapter 11 closes this part of the thesis, summarizing up those information and conclusions derived from the work procedure and results that are considered specially relevant. Moreover, since the work carried out easily leads to further research, several suitable future work lines are described. 


\section{Chapter 8}

\section{State of the art}

\subsection{The mm-wave band}

\subsubsection{Definition}

The millimeter-wave (mm-wave) band takes its name regarding to the wavelength size, which is $\lambda \in[1 \mathrm{~mm}-10 \mathrm{~mm}]$, giving in this way special emphasis to the involved sizes when dealing with this electromagnetic spectrum band. Accordingly, the frequency range of the mm-wave band is $f \in[30 \mathrm{GHz}-300 \mathrm{GHz}]$, see Fig. 8.1. Therefore, the mm-wave starts at the highest frequencies of the technologically mature microwave band to be the antechamber of the pioneer and challenging $\mathrm{THz}$ band. Both limits suggests good information about what can be encountered in the mm-wave band in terms of suitable applications and technology. Some years ago, in the $80 \mathrm{~s}$, when the mm-wave scenario had not the present technological maturity suggesting an imminent commercial use of this band, microwave and optical techniques were both considered to conquer the realm of mm-wave frequencies [49], [598], curiously, in a similar manner than $\mathrm{THz}$ frequencies are treated now. Over time it has been total imposition of microwave techniques, which have evolved very well up in frequency these last three decades. The applications scenario remains similar, although the particular evolution of each application area has been different. Roughly, four areas of applications can be found nowadays, all them summarized in Fig. 8.2: imaging, radar, communications and scientific radiometry.

Treating deeply the mm-wave definition, it is true that the International Telecommunication Union (ITU) [599] definition is in concordance with the previously given one, however, some remarks are commonly done in the literature. Similarly as the definition of the $\mathrm{THz}$ band was loosely defined sometimes due to scientific reasons (dominant microwave-band or optical background of the involved researchers), the mm-wave suffers as well from some particular re-definitions, but in this case coming from commercial point of view of the involved applications and technology. In the good overview of Olver [598], the band is suggested to be extended downwards sometimes to $20 \mathrm{GHz}$ in order to encompass frequencies of the microwave regime that are (maybe not any more) as well technologically challenging. Other authors coming from the industry (SAMSUNG $\left.{ }^{\circledR}\right)$ [600], quite focused in the mobile com- 


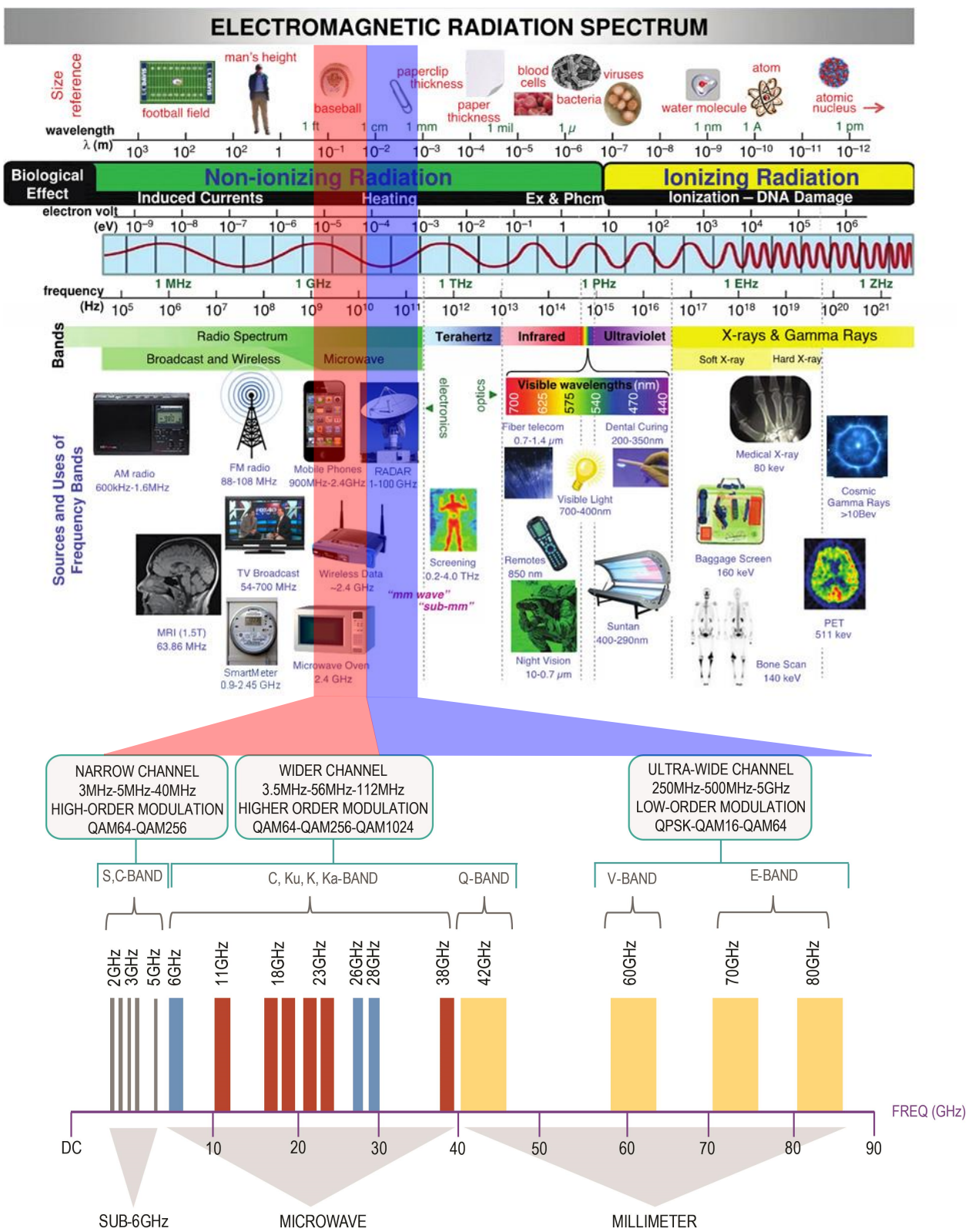

Figure 8.1: Electromagnetic spectrum with the microwave (red) and mm-wave (blue) bands highlighted. The bottom figure details the most relevant microwave and mm-wave sub-bands employed in communications.

munications area, explicitly extend it down even further, giving a lower limit of $f=3 \mathrm{GHz}$, arguing that similar propagation characteristics can be appreciated, and to distinct it from the conventionally used spectrum. The use of $f=28 \mathrm{GHz}$ 
as lower limit is as well quite habitual in communication applications [601]-[603], being that this frequency is the carrier of one of the potentially useful bands for such applications. The same can be applied to $f=24 \mathrm{GHz}$ when talking about radar applications [604]-[607]. Other works [608] proceed contrarily and incise in extending the lower limit up to $f=55 \mathrm{GHz}$ in its definition, being this justified by that the frequencies below have already technological and regulatory maturity. What the study of the mm-wave band literature suggest with this variety of definitions is that the term $m m$-wave goes associated to those frequencies at which some element (technological, application, regulatory) is not well established and demands from a different research/development than that applied to the mature microwave band. In the scope of this part of the thesis, regarding passive components, designs will be focused beyond $f=40 \mathrm{GHz}$, since this limit seems adequate in the context of the presented work to mark the frontier between what is consolidated and what still needs development.

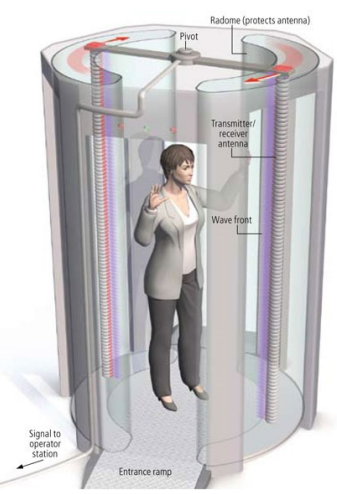

(a)

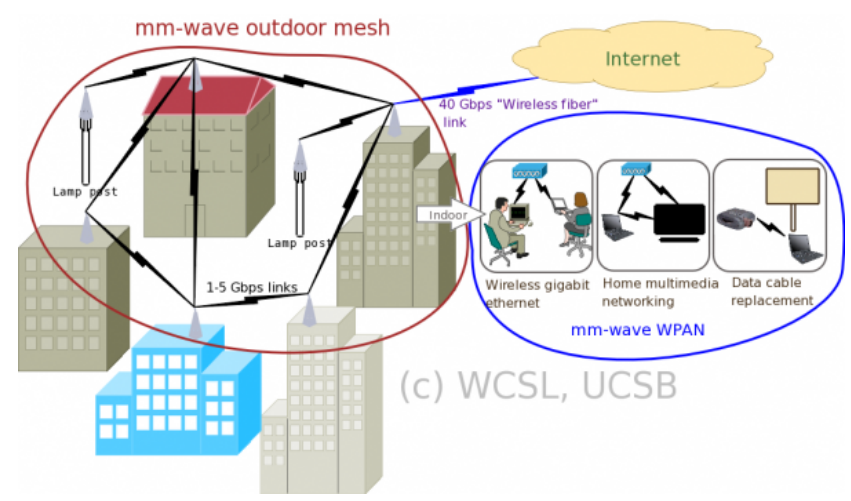

(b)

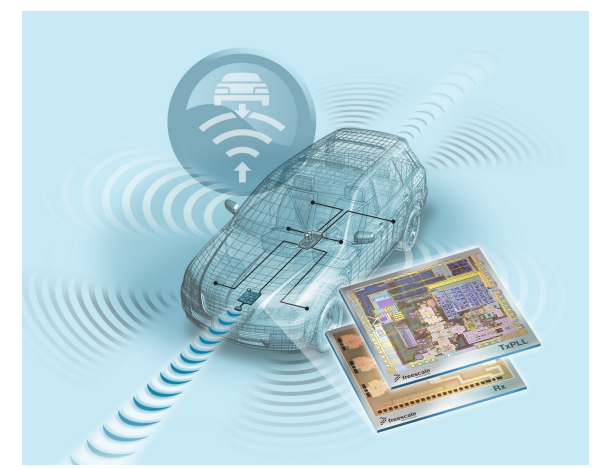

(c)

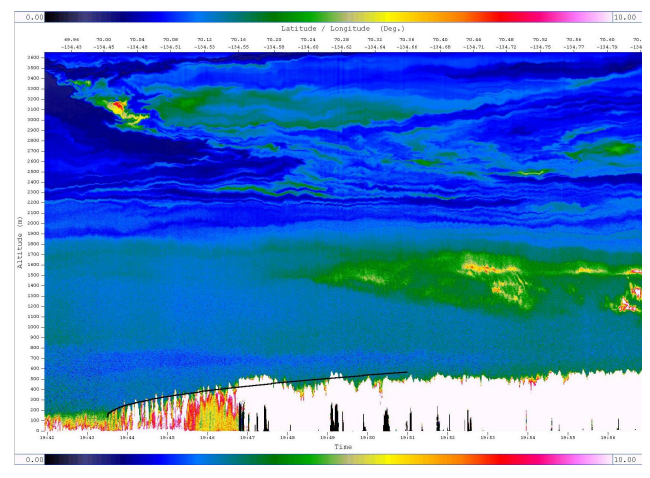

(d)

Figure 8.2: Most popular mm-wave applications. a) Imaging. Example of use for security purposes at airport security controls; b) Communications. Several kind of possible usage of mmwave radio [609]; c) Automotive radar. Schematization of an scenario with multiple radar systems incorporated in a vehicle to sense its environment; d) Radiometry; Formation of a cloud over the polynya that is advected downwind [610]. 


\subsubsection{Historical background}

It is rather surprising and probably only known by a few that the interest on mmwave is not novelty of our days, but it is the third time in the history that this band relies on the worldwide scientific focus. In the following, a brief historical background in mm-wave is given.

The first treatment of mm-waves started only few years after the discovery of electromagnetic waves by Heinrich Hertz. Even more, it was the intense research carried by Hertz the actual trigger for the consideration of such high frequencies [598]. Hertz was trying to prove experimentally that electromagnetic waves and light were the same concept. If this was true, electromagnetic waves should exhibit the same finite velocity than light $c=3 \cdot 10^{8} \mathrm{~m} / \mathrm{s}[611]$. Unfortunately, he found an important problem in the undesired reflections from the walls of the lecture room where he conducted his experiments. He realized that to overcome this problem, at higher operation frequency was required. He was operating mainly at $f=450 \mathrm{MHz}$.

Hertz's discoveries and conclusions inspired some later researches who could push up the frequency. For instance, Righi [612] worked between $3 \mathrm{GHz}$ and $10 \mathrm{GHz}$ using parabolic cylinder reflectors. Others such Lodge [613] and Flemming [614] worked with similar frequencies, developing what later would become the rectangular and circular waveguides. The race up in frequency was in good progress.

This progress lead to the extension of the operation frequency until reaching finally the mm-wave band. Of major relevance is the work of Prof. Bose [615]. Before of the end of the XIX ${ }^{t h}$ century he was able to demonstrate a complete mm-wave waveguide bench at the Royal Institution in Calcutta, India. He argued that for such experimental investigations a narrow pencil radiation beam was necessary, which he found difficult to obtain unless very short wavelengths were used (as radiating elements of fixed size become electrically larger).

Therefore, in only one decade from the discovering of radio waves, the microwave and mm-wave region had already a role, an important one actually. Such fast progression suggested the incoming use of these bands. However, historical and commercial facts prevented this to occur. The new century started with great interest in lower frequencies, impulsed by the proposal of Marconi [616], who suggest exploiting them for communication purposes, very required at that time. The radio revolution at such low frequencies left in oblivion microwaves and mm-wave bands during close to 30 years (excluding some scientific work in spectrometry, characterizing solids, liquids and gases [49]). A good historical fact revealing the importance of the necessities and wills of the market.

In the 30s extensive work came up again with developments in waveguides and sources. The radar was developed initially at Very High Frequency (VHF) band (30 $\mathrm{MHz}-300 \mathrm{MHz}$ ) [617], however, the invention of the magnetron made the microwave band to be reconsidered. The provided narrow beams were shown to be much more advantageous, and at the end of the Second World War, radar systems were operating between $20 \mathrm{GHz}$ and $30 \mathrm{GHz}$ [49], [598]. Microwave radio relay communication systems were also developed during the $30 \mathrm{~s}$ and the $40 \mathrm{~s}$. All this originated an important revolution in the microwave band, whereas the mm-wave band was left at one side. The strong potential of the microwave band, with much empty room 
to be filled, and the difficulty of developing a mm-wave klystron, the only possible source, justified this tendency clearly.

With a timid progression from that point, the mm-wave band was mainly driven by the usual scientific applications: spectroscopic measurements and plasma diagnostic. However, at the $60 \mathrm{~s}$, a second gold era for mm-wave came from the hand of a big project: The millimeter low-loss waveguide [598]. The idea supporting this project was the development of a high capacity trunk telecommunication system with a bandwidth of up to $80 \mathrm{GHz}$, thus being capable of carrying nearly half a million two-way telephone channels or 250 two-way television channels. To do that, an overmoded circular waveguide, operating with the low-loss $\mathrm{TE}_{01}$ mode was proposed. During the $60 \mathrm{~s}$ and $70 \mathrm{~s}$, big amounts of money came from telecommunication industries of France, Italy, Japan, UK, USA, USSR and West Germany.

The problem of the possible mode conversion under this configuration during curved sections was technically overcome with the employment of two specific designs for the bend: the use of a helix waveguide, which acts as a mode filter and prevents currents from flowing longitudinally along the waveguide, or by using a solid circular waveguide, lined with a thin layer of dielectric, which breaks the degeneracy between modes. Curiously, this way of proceed shares many aspects with the proposals of chapter 4 of this thesis, regarding enhanced monomode use of wire-type waveguides. The project was successfully accomplishing objectives, providing experimental results from $30 \mathrm{GHz}$ to $110 \mathrm{GHz}$ by using CWGs between $50 \mathrm{~mm}$ and $70 \mathrm{~mm}$ diameter. In fact, the measured attenuation was of the order of $2 \mathrm{~dB} / \mathrm{km}$, reaching even $0.5 \mathrm{~dB} / \mathrm{km}$ in some cases [598]. The system, projected to have repeaters spacing of about $50 \mathrm{~km}$, was thought totally viable.

However, optical fibers appeared contemporarily. With an attenuation as low as $0.2 \mathrm{~dB} / \mathrm{km}$, their extremely high capacity by their obviously larger bandwidth, and their simplicity compared with those bulky, heavy metallic tubes, totally killed the project. In addition, with second order influence, satellite communications helped to relegate this long distance mm-wave guided transmission proposal [49]. Actually, at the time this happened, the grow in telecommunication traffic demand slowed down, so such an ultra-high trunk waveguide could not be justified. Inasmuch as the optical fiber and this low-loss mm-wave band waveguide were regarded as technological rivals, any commercial niche left for the later. It was the second time that market wills displaced mm-wave technology. Although in 1989 Olver [598] contemplated that, with a little more demand, coexistence of both technologies was possible, the impressive capacities of optical communications nowadays suggest it would have been a wrong decision. Nevertheless, a good positive impact of the low-loss waveguide project was the spur it gave to the development of mm-wave solid-state devices, which are pointed as the necessary trigger for a commercial acceptance of a technology [598]. They were for microwave some decades ago, and they are being now for the mm-wave band [609].

Time passed, and the activity in commercial areas was intense at the microwave regime whereas only military applications were found for the mm-wave band [618][620]. Microwave technology was acquiring its maturity, and the spectrum began to be more and more used in this band, suggesting that someday would be insufficient to support such increasing demand. At the 90s, that situation triggered the beginning of the third era of the mm-wave. Several reviews clarified the technological and 
applications picture and traced the directions to follow [598], [621]-[622]. Vehicular communications and control were seriously considered [623]. Some scientific papers in this area, coming from the industry, are a good prove of the leadership of Japan in the mm-wave technology from that time to our days [624]-[625]. Actually, in spite of the fact that communications were in a secondary scene at that time (due to the strong activity under $3 \mathrm{GHz}$ ), Japan was leading serious research on the topic [626]-[628]. This country has been always one step further than the rest of the world when regarding commercial application of mm-wave technology [604].

It could be said that, at the $90 \mathrm{~s}$, the mm-wave band was in a similar situation (maybe slightly more developed) than the $\mathrm{THz}$ band today. Therefore, a decade of intense research activity drove mm-wave technology to the commercial revolution in the 2000s. Several works showed automotive radars systems ready to perform in practical environments [604], [625], [629]. By 2003, most of the car houses offered this kind of radars in their higher classes [605], and from the recent activity [605], [607], [630], [631], the incoming universal use of these systems is, simply, inevitable. However, the real present driver of the mm-wave technology are communications. Their role in our days society has put a lot of money on the table, triggering an explosion of work to satisfy new created service necessities [600]-[603], [608]-[609], [632]-[636]. The literature in the topic is tremendously vast, and the commercial race, phrenetic. At the shadow of the mm-wave leadership communications, mm-wave imaging is also becoming good market impulsing well-founded research [637]-[644]. State of the art in those areas will be reviewed later, after the main characteristics that cause the differences between the mm-wave band and the microwave band are exposed.

\subsubsection{Characteristics}

In order to understand why the mm-wave band deserves to be distinguished from the microwave band, to know the technological design scenario, and to realize why the aforementioned applications are suggested for this band, the main characteristics of mm-waves are next described. Following a similar scheme of that well-suited one in [598], three points are treated: 1) antenna beamwidth; 2) propagation characteristics of the atmosphere; 3 ) frequency bandwidth and regulation. In an implicit manner, concepts belonging to these three points are referenced somehow in every work in the literature.

\subsubsection{Antenna beamwidth}

The antenna is a passive element whose performance in terms of directivity has inherently a strong dependence with its size [645]. It is well-known that the angular beamwidth of an aperture or an array antenna is inversely proportional to the electrical diameter of the antenna. From the transmission perspective, the radiated energy is concentrated into a pencil beam, which narrows as the aperture increases in electrical size. Therefore, giving a fixed size, increasing the frequency narrows the beam. Consequently, beyond the obvious frequency scaling, with moderate diameters having practical sizes regarding the human environment and activities, it is possible to obtain high angular resolution at mm-waves. As a good example, in 
[598] is mentioned that a $10 \mathrm{~cm}$ diameter antenna provides a beamwidth of only 2.1 degrees at $f=100 \mathrm{GHz}$. In a more visual way, Fig. 8.3(a) shows the parabolic antenna sizes to to produce a $1^{\circ}$ beamwidth, which implies a gain of $G=44 \mathrm{~dB}$ if the antenna efficiency is $\eta=50 \%$ [646]. Think for instance in the reduced complexity of a mm-wave radio station allocating such a high gain parabolic reflectors in the $\mathrm{cm}$ range.

As [632] and [634] indicate, at $f=60 \mathrm{GHz}$, antennas are in the centimeter or even sub-centimeter size, and they achieve $10 \mathrm{dBi}$ to $20 \mathrm{dBi}$ without pushing too much the design, which encourages the use of directive antennas since a high antenna gain, i. e., a narrow antenna pattern or high directivity, is desired to improve the Signal to Noise Ratio (SNR) and to reduce the undesired inter-user interferences. Regarding to this fact, it is important to prevent low reception levels in scenarios without Line of Sight (LOS), in which Non Line of Sight (NLOS) contributions, coming from reflections could help to recover the transmitted signal. In this way, multi-beam steering method, switching/selection beam methods and phased array antenna enter as antenna solutions into the mm-wave scene. The compactness of the antennas allows the integration of several transceivers and antenna arrays on a given device. In the case of portable mobile devices, the mm-wave short wavelengths open the door to directive antennas of practical size and weight that would be unthinkable at microwave frequencies. See for instance the size of the chip integrated phase array presented to operate at $f=94 \mathrm{GHz}$, presented in 2013 by $\operatorname{IBM}^{\circledR}[647]$.

Notice that the fact of having a high gain, narrow-beam scenario provided by these small, millimetric wavelengths, implies the use of new strategies, adequate to the new available possibilities. In this sense, exploiting the polarization and spatial processing techniques provided by the recently developed massive Multiple Input Multiple Output (MIMO) and adaptive beamforming results very promising [632], [648]. Therefore, space multiplexing is added to the today's conventional time, frequency and even code multiplexing. To be more specific, this fact disposes a mobile communications scenario where operators are allowed to reduce cell coverage areas, the cost per station drops as they become more plentiful and more densely distributed, hence gaining the essential flexibility, quick development and reduction of ongoing operation cost.

\subsubsection{Propagation characteristics of usual channels}

\section{Atmosphere characteristics:}

The phenomena of emission and absorption of electromagnetic radiation is caused due to the fact that, when an electromagnetic wave of a particular frequency is incident on a gas molecule, the energy level is shifted by resonance. Later, when the molecule returns to its original state, it radiates at the same frequency but in a random fashion. The effect causes attenuation of the incident wave and generation of an incoherent noise at the same frequency [598]. It is well-known that the Earth's atmosphere emits and absorbs radiation at all frequencies. However, at microwave frequencies and below, the magnitude of this phenomena it is not significant. On the other hand, it has been seen in section 2.2.1.2 that at $\mathrm{THz}$ frequencies these molecular phenomena are specially strong. At the mm-wave band, they occur both 


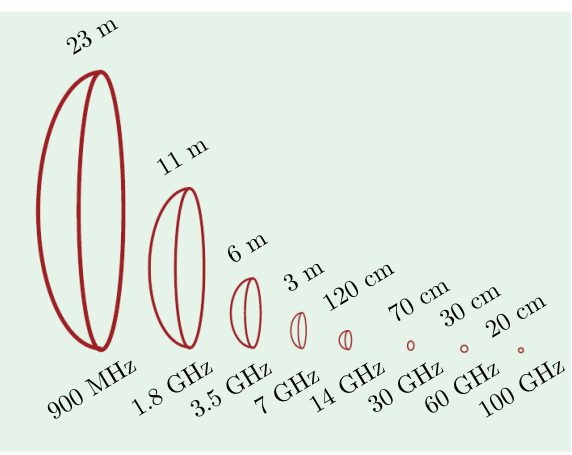

(a)

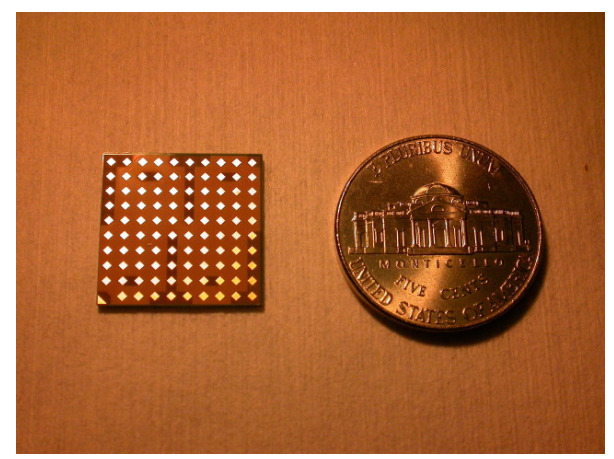

(b)

Figure 8.3: Examples of how the antenna characteristics at mm-wave change the technological and application scenarios. a) Antenna size needed to produce a $1^{\circ}$ beamwidth (gain of $G=44 \mathrm{~dB}$ if the efficiency is $\eta=50 \%$ ). Example adapted from [646]; b) Integrated Circuit (IC) phased array operating at $f=94 \mathrm{GHz}$, proposed by $I B M^{\circledR}[647]$.

cases, for some sub-bands the absorption is large, and for others rather low, see. Fig. 8.4.

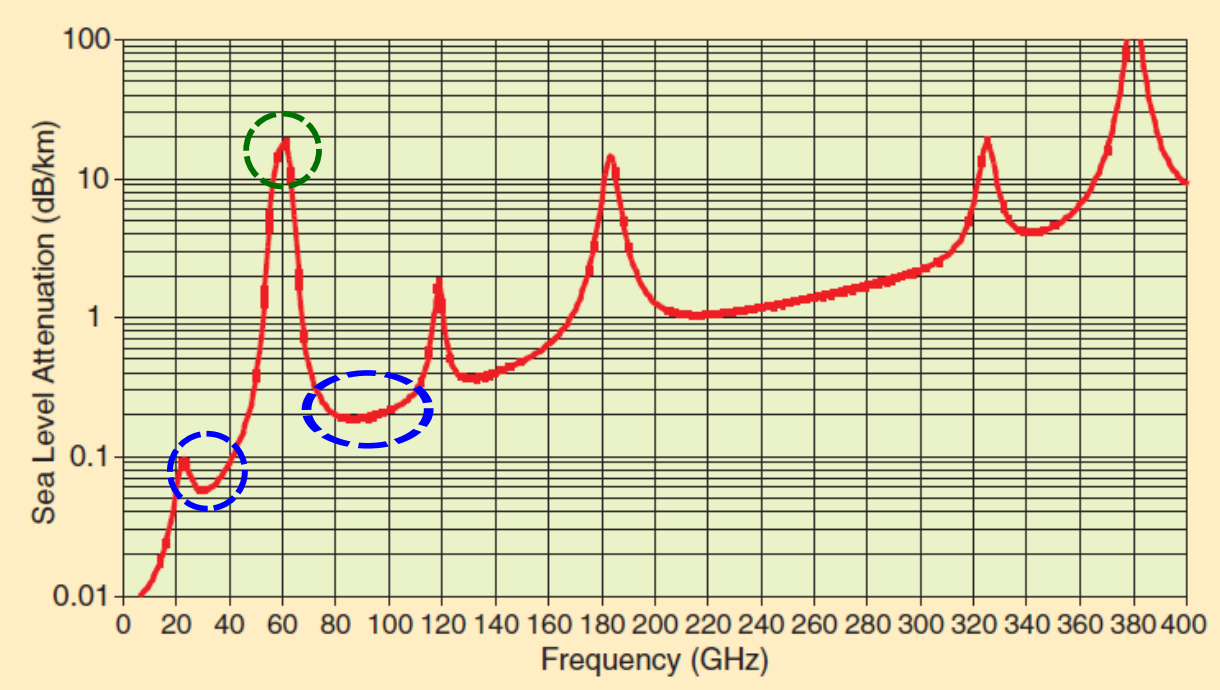

Figure 8.4: Average millimeter-wave atmospheric absorption [608]. The main sub-bands under the focus for medium-large range are highlighted with blue circles. The main sub-band for short range transmission (around $f=60 \mathrm{GHz}$ ) is highlighted with a green circle.

The observed peak at $f=60 \mathrm{GHz}$ is caused by the more than 40 resonant lines around this frequency of the Oxygen magnetic dipole moment. This peak results very relevant since it imposes how the presently projected application must allocate in the mm-wave band spectrum. In Fig. 8.4, the bands highlighted with blue circles, $[28 \mathrm{GHz}-38 \mathrm{GHz}]$ and $[70 \mathrm{GHz}-110 \mathrm{GHz}]$, are those which present the lowest atmospheric propagation losses. Consequently they are projected for outdoor ap- 
plications, including mobile communications [601]-[602], Point-to-Point (P2P) highdata-rate links [608], [646], vehicular radars (mainly planned at $f=77 \mathrm{GHz}$ ) [605], [629], [649], radiometry (often using the minimum of absorption at $f=94 \mathrm{GHz}$ ) [650], [651], or imaging (also taking advantage of the atmospheric properties at $f=94 \mathrm{GHz}$ ) [638], [640]. On the other hand, the frequencies surrounding $f=60$ $\mathrm{GHz}$, marked with a green circle, present atmospheric attenuation as high as 20 $\mathrm{dB} / \mathrm{km}$. Nevertheless, they are very suitable for a short-range scenario in which it can be taken advantage of the isolation from sources of interference. Consequently, there is a huge interest in these frequencies, generally for short-range high data rate communications [632], [609]. There are as well important peaks at $f=118.75$ $\mathrm{GHz}$ (Oxygen single-line dipole moment) and at $f=183 \mathrm{GHz}$ (water vapor electric dipole moment), which also define high/low absorption sub-bands [598]. However, since commercial applications are imminent below $f=100 \mathrm{GHz}$, the attention to portions of the mm-wave band spectrum beyond this frequency is considerably lower. Generally speaking, in the context of mm-wave commercial applications (some $\mathrm{THz}$ applications previously described may cause exceptions), what can be expected for such sub-bands is a re-allocation of some of the aforementioned applications when the lowest mm-wave spectrum start to saturate some day.

It is worth to mention that rain conditions may noticeably change the above described situation. Note that rain drops are in the order of the wavelength size at mm-wave frequencies. According to the ITU data about rain attenuation [652], as it is pointed out in several works, high rains can cause total fading of mm-wave signals [598], [600]-[601], [603], [608], [634], [646]. To better quantify the rain impact on the propagation channel, Fig. 8.5(a) [600] shows the attenuation in $\mathrm{dB} / \mathrm{km}$ as a function of the frequency for different rain rates. A light rain $(2.5 \mathrm{~mm} / \mathrm{h})$ may only barely affect the total performance of the system, which can be prepared for that case (note that the projected links do not extent more than 2-5 km). However, if the rain is heavy $(25 \mathrm{~mm} / \mathrm{h}$ and beyond), the mm-wave band signals may suffer an attenuation so large that the signal to be received is lost.

Fortunately, most of the countries rarely present such high rain rates, and in those countries where these rains occur, they do not occur often. Therefore, the correct performance of the system can be ensured except of few minutes or as maximum several hours a year. For instance, currently available equipment at 70/80 $\mathrm{GHz}$ can achieve 1 Gbps connectivity with $99.999 \%$ (the usual five nines term of carrier class performance, equivalent to only $5 \mathrm{~min}$ of system fail caused by weather per year) over distances of 2-3 km throughout most of Europe and United States. Considering $99.9 \%$ (three nines availability, equivalent to 8 hours of system fail per year) the distance can be increased to $5 \mathrm{~km}$. Ring configurations may even increase the performance due to the clustering nature of heavy rains [608], [646], [634]. Most of the experts indicate that, although the influence of the rain on the system is not as benign as for lower frequencies, the projected uses of mm-wave frequencies still allow to prepare the system against this problem. For example, when talking about communications, if the link is planned to be short, small dependence on the rain is expected. However, this planning is very necessary and radiofrequency engineers must study the climatology of the area where the system is thought to operate. Actually, rain is the main fading threat at mm-wave frequencies [646]. 


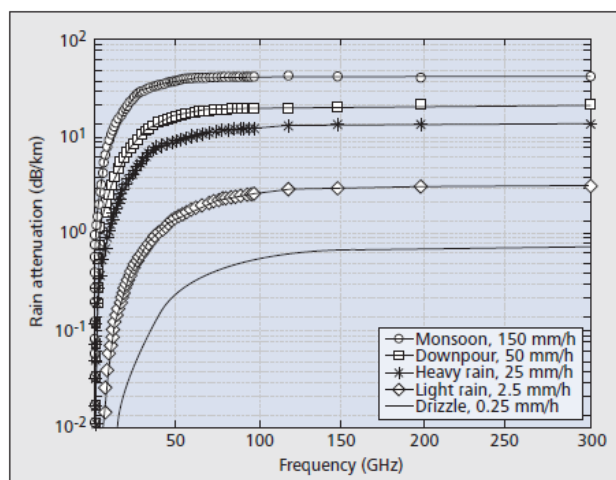

(a)

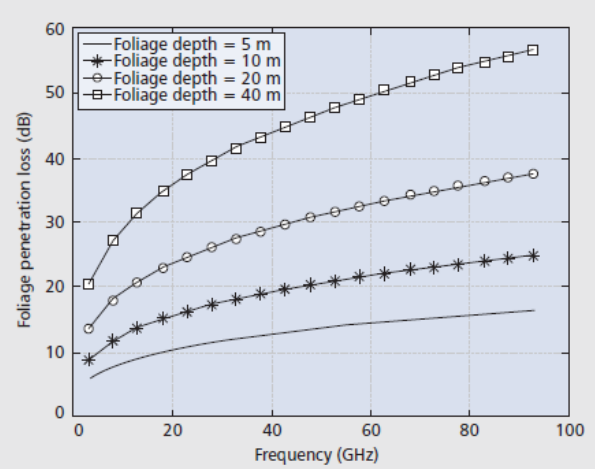

(b)

Figure 8.5: Attenuation caused by rain (a) and foliage (b) at mm-wave frequencies [600].

It must be also indicated that higher altitudes in the atmosphere present higher attenuation. For high altitudes, the marked valleys forming low-loss transmission sub-bands in Fig. 8.5 become much more smooth. For common communication links or other terrestrial applications this fact is not a problem, however it must be taken into account to plan satellite communications or atmospheric radiometry experiments [646], [653]-[655]. For instance, in [655], a lossy dielectric plate is inserted to compensate the difference in losses of the high-angle and low-angle sky spectra. Similarly, in hot and humid conditions, the atmospheric attenuation increases about $1.5-2 \mathrm{~dB} / \mathrm{km}$ at the sea level [646].

On the other hand, smoke, dust, fog, snow, haze and blowing sand present low absorption characteristics at mm-wave frequencies, this giving strong advantage in these conditions to the mm-wave band front higher bands such as the infrared or optics. As a matter of fact, a $14 \mathrm{~km} \mathrm{mm-wave} \mathrm{link} \mathrm{operating} \mathrm{in} \mathrm{the} \mathrm{Middle} \mathrm{East,}$ operated error-free during the most severe sand storm experienced in the recent history [646].

\section{Forestal/vegetal, urban and indoor scenarios characteristics:}

Finally, beyond the usually commented atmospheric losses, specific highlights about other source of usual losses than can be encountered in a some mm-wave channels must be given. This is the case of foliage losses, which are significant and can limit the propagation under certain conditions [600], [634], [635]. Fig. 8.5(b), shows the foliage losses as a function of the frequency for several foliage depths [600]. It is noted that, at $f=80 \mathrm{GHz}, 10 \mathrm{~m}$ foliage penetration impose a propagation loss of $23.5 \mathrm{~dB}, 15 \mathrm{~dB}$ higher than the propagation loss at $f=3 \mathrm{GHz}$. The account for this losses is explicitly given in many plans for outdoor communication systems, specially for military applications [656].

The elements conforming urban and indoor scenarios present as well considerable different response in mm-wave when comparing to microwaves. For example, the attenuation in $\mathrm{dB} / \mathrm{cm}$ of concrete is one order of magnitude larger at $f=40 \mathrm{GHz}$ than at $f=3 \mathrm{GHz}$. Actually, few centimeters of this material totally damp the signal. Similarly, an office whiteboard is almost transparent at microwaves, whereas 
at mm-wave band frequencies it imposes an attenuation of $10 \mathrm{~dB}$. On the other hand (and fortunately), drywalls, mesh glasses or wood do not show significant increase at mm-wave frequencies [600]. Again, the radio-frequency engineer to plan the system must be aware of such a new scenario. A good work characterizing this kind of scenarios, but below $f=40 \mathrm{GHz}$, is found in [601].

\subsubsection{Frequency bandwidth and regulation:}

From the starting of the mm-wave band true progression, the large available bandwidth at the mm-wave band was a very attractive good [598], mainly for communications applications. At the $90 \mathrm{~s}$, the spectrum below $1 \mathrm{GHz}$ was already saturated, a bandwidth that is only $1 \%$ at $100 \mathrm{GHz}$. The situation is not very different nowadays, where most of the applications operate below $f=3 \mathrm{GHz}$. The true potential of this new available bandwidth provided by $\mathrm{mm}$-wave frequencies is not in replacing services allocated at lower frequencies, but in offering new ones requiring larger bandwidth, or in giving extra support to those existent. An example of the first case is the transmission of heavy files such as high-quality video by means of high-datarate traffic wireless systems [608], [609], whereas an example of the second one can be the new generation of mobile networks [601], [635].

In order to transmit at considerably high data rates employing a wireless system, the regulated transmission channels must be wide enough to allow for the required bandwidth for such high rates. Up to $40 \mathrm{GHz}$, regulators deliberately slice the available spectrum into many narrow channels to encourage competition and permit users to enjoy the services without interference. This means that only at the higher mm-wave band frequencies $(f>40 \mathrm{GHz})$ channels are large enough to support the highest data rates. For instance, the most popular wireless standards in the sub-10 $\mathrm{GHz}$ region (cellular, WiFi, WiMAX, etc.) support data rates of up to several tens of megabit per second. If the frequency is extended up to $40 \mathrm{GHz}$ (the case of long distance transmission, cellular backhaul ${ }^{1}$, etc.), data rates may grow to hundreds of megabits. However, to reach the barrier of 1 Gigabit(s) per second (Gbps), the use of the bands at $60 \mathrm{GHz}, 70 \mathrm{GHz}$ or $80 \mathrm{GHz}$, in which wider channels are available, is mandatory. If the data rate is desired to be $10 \mathrm{Gbps}$ or higher (maybe for the next decade developing standards), the bands around and beyond $100 \mathrm{GHz}$ will be required [608]. In addition, it must be commented that power regulations will play a decisive role in the achievement of such rates [634].

As it was pointed out when the propagation characteristics of the atmosphere were reviewed, not all transmission windows (valleys in the graph of Fig. 8.4) are available for commercial services. In fact, whereas when the band was virgin, as it occurs with the $\mathrm{THz}$ band these days, atmospherical windows posed the scenario [598], when the commercial use is imminent and regulation ${ }^{2}$ enters in scene, the objectives and uses are much more specific [608]. Actually, extensive review of the literature indicates that there are three main attractive bands in general. First,

\footnotetext{
${ }^{1}$ The backhaul network is the portion of the network provide the intermediate links to connect the backbone or core network with the small sub-networks located at the edge of the entire hierarchical network [657].

${ }^{2}$ Communications applications, which are dominant, determine the main regulation. The use of other kind of applications such as radar or imaging is usually adapted to that regulation.
} 
one has the spectrum around $60 \mathrm{GHz}$, the $[57 \mathrm{GHz}-64 \mathrm{GHz}]$ band, the so-called the V-band. Second, the defined bands in the $70 / 80 \mathrm{GHz}$ are $[71 \mathrm{GHz}-76 \mathrm{GHz}]$ and [81 GHz- $86 \mathrm{GHz}]$, the so-called E-band. Both are under the focus due to the large available bandwidth to support the aforementioned Gbps transmission. Third, the bands around $90 \mathrm{GHz}$ are $[92 \mathrm{GHz}-94 \mathrm{GHz}]$ and $[94.1 \mathrm{GHz}-95 \mathrm{GHz}]$. Note that $[94$ GHz-94.1 GHz] is reserved for military applications. Therefore, in this last case, although there is significant bandwidth to exploit, its uneven allocation and the proximity to the military bands may suppose a handicap in the future use. In Fig. 8.6(a) [608], these bands are shown together with the most important microwave and cellular bands allocated in the regulation.

If the mm-wave band is, from the commercial point of view, already restricted to much more specific frequencies, the regulation per regions may tighten the noose even more. In Fig. 8.6(b), also from [608], the regulation of the three aforementioned bands $(60 \mathrm{GHz}, 70 / 80 \mathrm{GHz}, 90 \mathrm{GHz})$ per regions in 2009 is shown. The most open case is USA, where all three bands are used. However, most of the countries only pose V-band on the scen, and in some cases, managing it with additional restrictions. This is the case of Europe. Here, the V-band is split in two relatively small allocations, hence reducing its potential for high data rate transmissions compared to the $7 \mathrm{GHz}$ wide band allocation available in USA. Although in [608] was cited the imminent reconsideration for this decision by European regulators, current data from ITU [599] indicate that this issue is still under review. Despite of the welcome from the industry to the idea of employing a $7 \mathrm{GHz}$ unsegregated bandwidth, some countries place the $[59 \mathrm{GHz}-61 \mathrm{GHz}]$ band for $\mathrm{NATO}^{3} /$ military applications and the $[61 \mathrm{GHz}-$ $61.5 \mathrm{GHz}$ ] for Industrial, Scientific and Medical (ISM) use only. In this issue, Europe present nowadays a quite heterogeneous status per country. Regarding to the current situation of the E-band, despite Brasil, and some countries in the Middle East that have opened this band, in general, South America and Asia are, currently, still one step behind.

Additionally, but with less relevance, it is found the spectrum in the band [28 GHz-30 GHz], mainly considered for Local Multipoint Distribution Service (LMDS), conceived for the last mile. In USA, the Federal Communications Comission (FCC) auctioned two LMDS licenses per market. The A license includes a total of 1.15 $\mathrm{GHz}$ bandwidth split in the bands [27.5 GHz-28.35 GHz], [29.1 GHz-29.25 GHz] and $[31.075 \mathrm{GHz}-31.225 \mathrm{GHz}]$. The $\mathrm{B}$ license is only $150 \mathrm{MHz}$ wide, split in [31 GHz-31.075 GHz] and [31.225 GHz-31.3 GHz] bands [600]. As can be understood, the scenario for this spectrum resembles much more to a cellular one than to those, rather wide-band, aforementioned in the previous paragraph. The situation around $38 \mathrm{GHz}$ is quite similar. Both spectra are considered as a possible allocation for future mobile communications [601], [602]. For completeness, it is mentioned that the band [3.1 GHz-10.6 GHz], so-called microwave Ultra-Wideband (UWB) impulse radio, was regarded as an attractive allocation with interesting bandwidth and propagation properties. However, the lack of international coordination (that, contrarily, has worked well for higher frequencies) and the existent of other unlicensed bands in Europe and Japan, such as the $[2.4 \mathrm{GHz}-5 \mathrm{GHz}]$ band, suggesting interference problems, have capriciously relegated this part of the high microwave spectrum [632].

\footnotetext{
${ }^{3}$ North Atlantic Treaty Organization [658].
} 


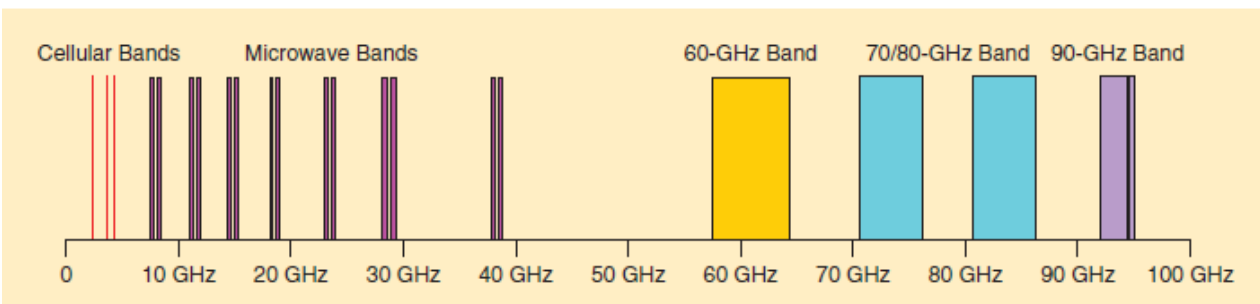

(a)

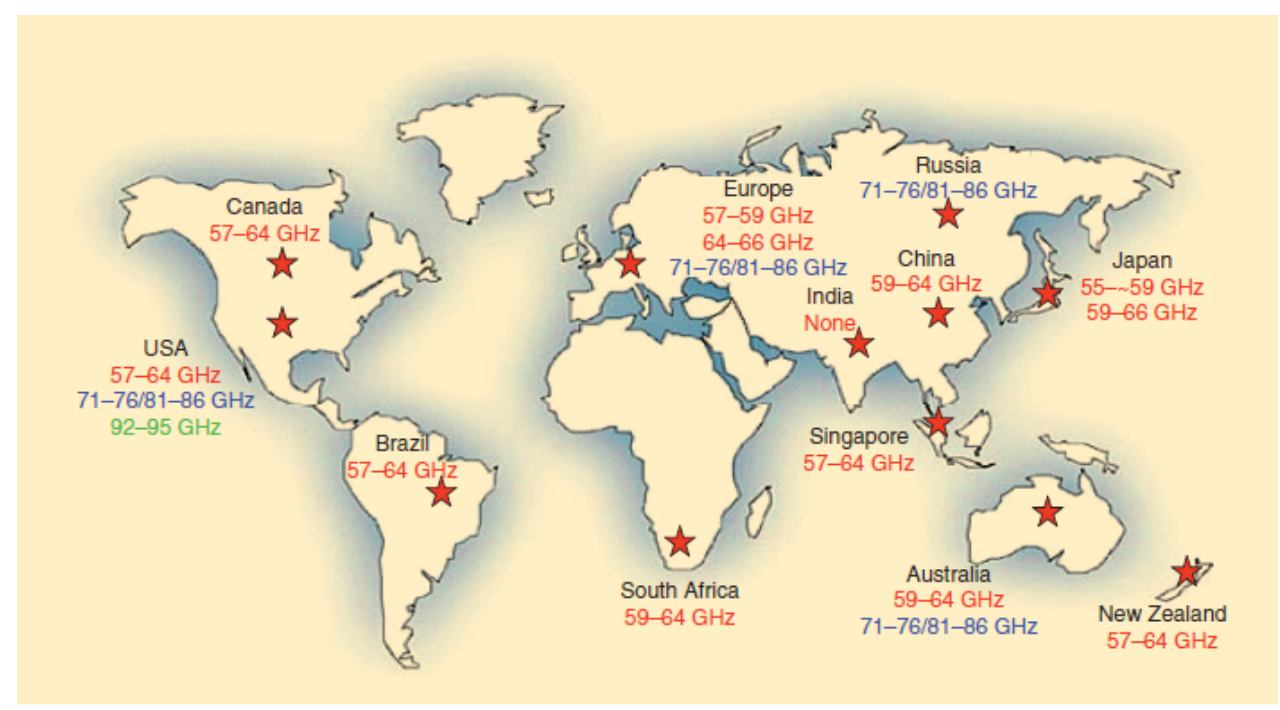

(b)

Figure 8.6: Millimeter-wave sub-bands and regulation [608]; a) Main microwave and mm-wave sub-bands; b) mm-wave sub-bands availability and allocations in the most important global markets in November 2008.

To finish this apart, it must be highlighted that, since communications regulatory organisms fix the employable bands, other kind of applications are consequently allocated in free-used bands or unlicenced spectrum. Radar has been mainly focused around $77 \mathrm{GHz}$, being the available bands [76 GHz-77 GHz] and [79 GHz-81 GHz], [604]-[606]. Alternatively, some proposals are found around $24 \mathrm{GHz}$ and $94 \mathrm{GHz}$, this last more dedicated to military and scientific uses. For the former frequency there is regulation on the band [21.65 GHz-26.65 GHz] [604]. Other suggested frequencies for are $35 \mathrm{GHz}, 47 \mathrm{GHz}$ and $60 \mathrm{GHz}$, however, the demanded standardization in other commercial areas $(60 \mathrm{GHz}$ in communications, for instance) will probably relegate them. When talking about radar, the projected required bandwidth for the necessary resolution, provided by the common traffic scenarios is $1-2 \mathrm{GHz}[659]$, that some authors increase up to $5 \mathrm{GHz}$ for the more challenging short-range urban scenario [606]. This fact justifies the preference of higher frequency spectrum spots. Regarding imaging, $f=94 \mathrm{GHz}$ is the most common frequency [640], [641], [644]. Most of the imaging systems demand for narrow-band operation, although excep- 
tions are found. For example, in [660], a $30 \mathrm{GHz}$ bandwidth ([10 GHz-40 GHz]) is proposed to gain depth resolution up to $5 \mathrm{~mm}$.

\subsubsection{Applications}

Next the main application areas for the mm-wave will be reviewed. The number of areas is more reduced than that for $\mathrm{THz}$, and in most cases, the experimental stage has left to become a reality that is already commercially available or it will be very soon. As described in Fig. 8.2, these areas are radar, communications, imaging and scientific radiometry.

\subsubsection{Radar}

Radar applications were the initial trigger for this ongoing mm-wave revolution. Considerable effort was put at the end of the $80 \mathrm{~s}$ and starting of the $90 \mathrm{~s}$ in producing reliable, low cost, high performance radars. The development of such area was not comparable with any other at that time [598]. The improved resolution due to the employed short wavelengths, the penetration through fog, clouds and humid atmosphere, and the wide frequency space available allowing for free choice of modulation methods, resulted and result very attractive for this kind of applications. Additionally, narrow beams, easily obtained mm-wave frequencies, provide high angular resolution, which diminishes mutual interference between systems, and improves detection security. Furthermore, as the target becomes comparable with the wavelength, polarization information becomes available, a further contribution to position and velocity information. Other technologies, such as image sensors or laser radars are weak to rain, dirt or light conditions, whereas ultrasonic sensors are short-range and very affected by the wind [624]

As usual, military applications impulsed first the development of the technology. Military radars have interest in: a) surveillance and target acquisition of small size targets demanding from high resolution and low weight equipment; b) fire control and tracking at short range, where high spacial accuracy is required; c) guidance and seekers (the most active area due to the advantages in size and weight); d) instrumentation and measurements (due to the requirement or large databases of the target signatures and the clutter reflectivity characteristics). Of particular interest is the possible use of infrared and mm-wave systems working together. Whereas the first provide good spatial resolution and good target discrimination over a long range in clear weather, the second can operate in harder weather conditions, detecting moving targets easily, and providing accurate range information [598]. Nowadays, the mm-wave band is strongly consolidated in the well-founded military environments (it is time to civil applications now [607]). Current designs operating at $f=94 \mathrm{GHz}$ show very good performance. For instance, in [661], a radar to be incorporated in aircrafts manages an output power of $100 \mathrm{~mW}$ over a bandwidth of $1 \mathrm{GHz}$, giving a resolution of $15 \mathrm{~cm}$ and a dynamic range of $60 \mathrm{~dB}$. Similar figures are presented in [662] with the intention of incorporating it to unmanned aerial vehicles. Therefore, pioneer research in military areas is focused now around $220 \mathrm{GHz}$, where other minimum of atmospheric attenuation is found (see Fig. 8.4) [663]-[665]. 
However, the intense activity in radar applications belongs by far to the automotive industry [604]-[606], [624]-[625], [629]-[630], [649]. A good review on the evolution of this area is carried in [659] by Meinel, a relevant figure on the field, [621]-[623], [666]. Already in the 70s, first movements in the sector appeared by the hand of radars operating at $35 \mathrm{GHz}$. Fig. 8.7(a) shows a $35 \mathrm{GHz}$ automotive radar system built by AEG-Telefunken in 1974, the car being driven by the same Meinel. At the 90s, technology at $f=77 \mathrm{GHz}$ became available, and, initiated by Germany (Mercedes-Benz(B) was very involved), radars incorporated in premium cars moved to that frequency. More recently, in the last decade, combination of 24 $\mathrm{GHz}$ and $77 \mathrm{GHz}$ radars was though appropriated for urban traffic scenarios. This scheme is illustrated in Fig. 8.7(b) [659], where the radar at $24 \mathrm{GHz}$ (green) provides short-range vision, whereas the radar at $77 \mathrm{GHz}$ (yellow) provides long-range vision.

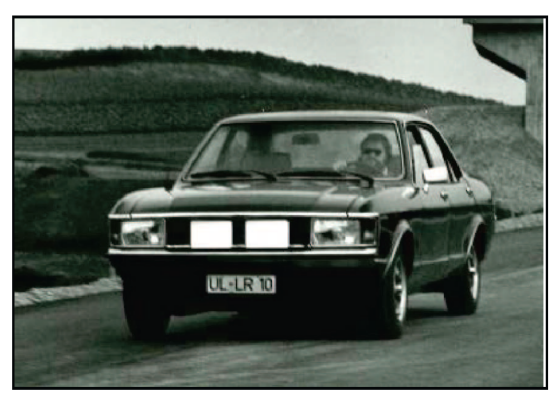

(a)

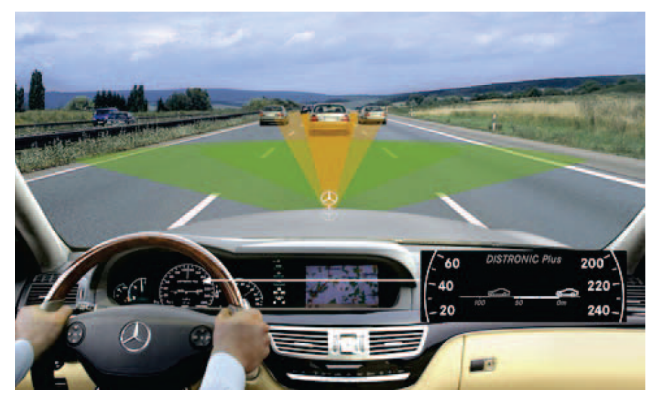

(b)

Figure 8.7: Automotive radars. a) $35 \mathrm{GHz}$ automotive radar system built by AEG-Telefunken in 1974 [659] (driven by Holger H. Meinel, the author of the paper); b) Combined used of $24 \mathrm{GHz}$ (green) and $77 \mathrm{GHz}$ (yellow) radars for short/large region vision. [659].

Nowadays, radar systems are entering in the medium-class cars. Furthermore, whereas in an early stage automotive radars were exclusively used for comfort purposes, e.g., automatic speed control by keeping a safe distance to the preceding vehicle, in these days, advanced safety functions are demanded [606]. More specifically, according to [605] the following four services, with increasing grade of complexity are visioned:

- Passive Comfort: Only additional information is given to the driver. Example: parking aid.

- Passive Safety: Actions are taken in order to preserve the passenger security when some safety risk is detected. Example: airbag.

- Active Comfort: The vehicle takes control over monotone task to increase the comfort of the driver. Example: Adaptive Cruise Control (ACC).

- Active Safety: The vehicle takes control on emergency situations. Example: Sharply deceleration in case of a obstacle on the road.

All this services imply a large variety of targets to be detected, hence different radar requirements. Sensors will require to look in all directions around the vehicle 
with differently defined scanning and distance relations, see Figs. 8.8-1 and 8.8-2, from [605]. Rear traffic crossing alert and blind spot detection operate in the near range and simply give information about whether one or more targets in a certain area are present, whereas ACC and forward collision prevention may have to scan the whole traffic scheme in near, mid and far ranges, depending on the velocity and the environment of the car [606]. Fig. 8.8-3 shows a typical monostatic Radar Cross Section (RCS) of a car [605]. The laterals of the car shown an important peak that can be advantageous to prevent accidents during lateral movement actions. The issue of defining more specifically the near, mid and far ranges, generally diffuse in the literature, is quite well explained in [605]. Data of that work are summarized in the following list:

- Long Range Radar (LRR): The beam is narrow and points forward. Used in ACC, see Fig. 8.8-1 or Fig. 8.7(b)(yellow). Distances range between $10 \mathrm{~m}$ and $250 \mathrm{~m}$. Distance resolution of $\Delta R=0.5 \mathrm{~m}$ and angular resolution of $0.1^{\circ}$ are demanded (see Fig. 8.8-2). Implementable with $600 \mathrm{MHz}$ bandwidth.

- Medium Range Radar (MRR): Intended for applications with an intermedium distance and speed profile. Good examples are the Cross Traffic Alert (CTA), watching for traffic when starting overtaking action, or the Lane Change Assistant (LCA), giving alert of the approaching vehicles at a junction, see Fig. 8.8-1. Distances range between $1 \mathrm{~m}$ and $100 \mathrm{~m}$. Distance resolution of $\Delta R=0.5 \mathrm{~m}$ and angular resolution of $0.5^{\circ}$ are demanded (see Fig. 8.8-2). Implementable as well with $600 \mathrm{MHz}$ bandwidth. Note that, since in some implementations this radar points laterally, valuable information of the environment, complementary to the forward-looking radar is obtained (think in curved trajectories for instance).

- Short Range Radar (SRR): This kind of radar is though for applications sensing in direct proximity of the vehicle like obstacle detection and parking aid. This kind of service is quite universally incorporated nowadays. Distances range between $15 \mathrm{~cm}$ and $30 \mathrm{~m}$. Distance resolution of $\Delta R=10 \mathrm{~cm}$, distance accuracy of only $2 \mathrm{~cm}$, and angular resolution of $1^{\circ}$, are demanded (see Fig. 8.82 ). In this case, the accuracy demanded for the depth suggest the employment of up to $4 \mathrm{GHz}$ for the new generation radars.

In general, radars at $24 \mathrm{GHz}$ have been preferred when long range is not required. The components for this microwave frequency have high availability and performance, brought from the telecommunications industry. This frequency offers the best compromise between current component cost and sensor size. Since short range sensors do not measure the angle of detected objects, single antenna elements are sufficient. The antenna beams are only vertically directed in order to increase the antenna gain and minimize clutter effects from the road surface. To measure not only the target distance, but also its angular position, several adjacent sensors can be implemented [604].

Nevertheless, radars at $77 \mathrm{GHz}$, in the recent past reserved for LRR applications in which the use of higher frequency was clearly necessary, is gaining terrain these days. It is expected that every kind of radar, SRR, MRR, LRR will be soon 

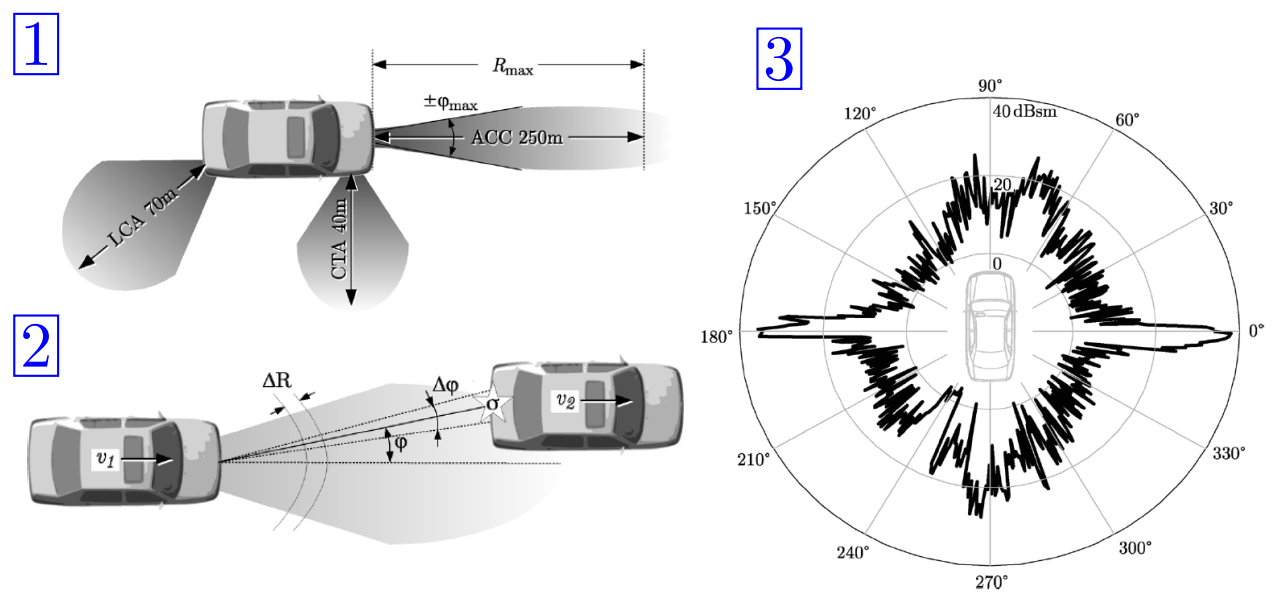

Figure 8.8: Automotive radars main parameters and functions [605]. 1) Field of view and distance range for three exemplar functions: Lane Change Assistant (LCA), Cross Traffic Alert (CTA) and Adaptive Cruise Control (ACC); 2) Range resolution and angular resolution; 3) Typical monostatic RCS of a vehicle.

implemented exclusively in the $77 \mathrm{GHz}$ band. As Meinel [659] indicates, there are several reasons to believe in the previous affirmation. First, there is an increase in the number of services and complexity of them. The accuracy in space and time is demanded to be superior, and $77 \mathrm{GHz}$ radars have much empty room to provide this thanks to the shorter wavelength and available bandwidth than the more inherently limited $24 \mathrm{GHz}$ radars. Second, vehicle integration and sensor packaging demands minimization, while enhancing sensor performances. Again the shorter wavelength at $77 \mathrm{GHz}$ will facilitate the achievement of this demand. As an example, this year, in [630], a radar system was fit in only $2.5 \mathrm{~cm} \times 2.5 \mathrm{~cm} \times 1 \mathrm{~cm}$, see Fig. 8.9. Third, there will be a cost reduction caused by the well-known effect of economies of scale. This will not only be provided by the massive development of all kind of radar types (SRR, MRR and LRR) as points [659], but the development to come from the telecommunications industry has much to say [600]-[603], [608]-[609], [632]-[636]. Regarding to this unavoidable relationship between both areas, a further reason can be added in this point. Note that the big amount of data provided by the radars must be managed and transmitted. Operate on the future high rate data channels at similar frequencies may allow for better integration of the total systems. Finally, the interoperability requirements. Advert that due to the expected massive use of automotive radars, mitigation of interferences will be required. Therefore, the use of a large bandwidth in order to enable frequency hopping or other similar modulation techniques, reducing incoming noise of adjacent channels, is clearly necessary. Worldwide, this wide-band channel requirement is only provided by the $[76 \mathrm{GHz}-81$ $\mathrm{GHz}]$ band these days.

The future of automotive radars is promising according to the market, and suitable (if not necessary) according to the motoring traffic statistics. The traffic saturation in populated cities, and the fast development in the BRIC ${ }^{4}$ States during

\footnotetext{
${ }^{4}$ Brazil, Russia, India and China
} 


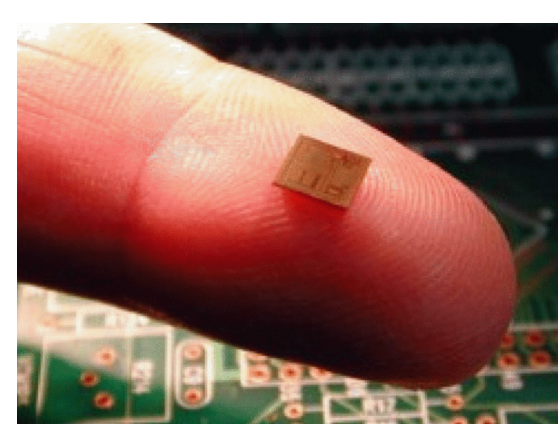

(a)

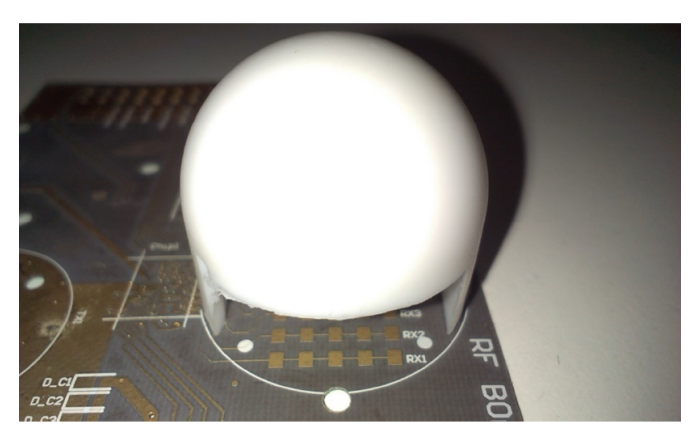

(b)

Figure 8.9: Single-chip mm-wave radar operating at [76 GHz-77 GHz] of [630]. a) Single-chip radar system based on SiGe Bipolar Complementary Metal-Oxide-Semiconductor (BiCMOS); b) Microstrip series fed array patch with an incorporated lens.

the last years, dispose an scenario with increasing risk of accident, demanding for safety and prevention elements. The social-economic cost of road traffic accidents is Spain implies annually about $0.04 \%$ of the total Gross Domestic Product (GDP) [667], apart of the invaluable human cost. Experimental essays in 1992 showed that a collision warning system based on a radar operating at $f=24 \mathrm{GHz}$, which was implemented in more 4000 US buses and tracks, caused an accident reduction of more than $50 \%$. Even more, the severity of accidents that still occurred was noticeably reduced [659].

\subsubsection{Communications}

Two/three decades ago, technology supporting mm-wave communications was much less advanced than mm-wave radars. There was too much work to do in developing the first generation of mobile networks, and it was the moment to deploy optical fiber networks. Satellite technology was also relying in the microwave regime [598]. Despite of this fact, it was visioned that some day the mm-wave band would gain an important role, and some marginal (compared to present levels) research was carried out. Point-to-point links were the most obvious scenario (as deploy fiber networks is sometimes not economically justified in unpopulated areas), however a lack of bandwidth regulation is highly appreciated in the literature in the $80 \mathrm{~s}$ and $90 \mathrm{~s}$, and, inasmuch as modulation techniques and signal processing were not as advanced as today, data rates were not, by far, current ones. For instance, in [598] it is mentioned a system operating at $f=29 \mathrm{GHz}$, merely giving 2 Mbps. In [666] and [622] similar examples (data rates of several Mbps) were reported, most of them including systems operating at $f=50 \mathrm{GHz}$. In those works, other scenarios such as mobile or indoor communications were, it could be say, well foreseen. However, only general ideas were given rather than concrete implementable systems, hence the treatment of such concepts was merely qualitative. Satellite $\mathrm{mm}$-wave communications were also predicted to have their role if some disadvantages were overcome [598], [622], [653]. Compactness and weight reduction were pointed as good advantages inasmuch as launch cost can be noticeably diminished. On the other hand, the problem of larger 
atmospheric attenuation was regarded as a critical one, only avoidable by enhancing the use of directive antennas, good tracking systems and robust modulations.

Nowadays it can be affirmed that, although radar applications have been a good driver of mm-wave technology, the true flagship role correspond to communication applications. At least, current service demand and economical weight of this area are major reasons to drive a successful achievement of the technological maturity status in this band in the next years [354], [634]. Backhaul networks by means of point-to-point links continues being the main role of mm-wave in communications, with many systems already implemented, and others easily foreseen [608]-[609], [634], [646]. However, mobile and high data rate indoor communications are quite close, very involved in the current landscape [600]-[603], [609], [632], [635]. These inertia of increasing the operation frequency affects as well satellite communications, and some work is ongoing [668]-[669], however, the activity is not comparable with the terrestrial area. Finally, the incoming big data scenario in vehicles due to the quantity of sensors that new generation of cars will incorporate, has make arise vehicle-vehicle and vehicle-infrastructure communications on the mm-wave band [631]. Most relevant aspects and innovating works in each category are reviewed next.

\subsection{Point-to-point links to support backhaul and backbone:}

The realm of backbone networks, i. e., the true core supporting the large aggregated data of all users is well dominated by optical fibers. Optical systems operate nowadays with 50-100 channels per fiber and with 50-100 fibers per communications bundle. Each wavelength channel operates between 10 Gbps and 40 Gbps. Furthermore, the cost for hardware is decreasing fast, specially for optical equipment providing Ethernet links [646]. In addition, the transmission of radio signals is being implemented over free space optics links [670]-[671] and over fiber optics [672]-[673], this last the so-called Radio over Fiber (RoF). Advances in microwave photonics also contribute to this optical domination [593].

Despite of these facts, fiber communications links have not been deployed in may USA and Europe areas (Asia achieves more fiber penetration), not only those with lack of high data rate demand, but in congested areas requiring evident bandwidth needs. The fiber is not everywhere, but the fibers rings do not stop at every building and this creates a broadband last-mile problem. For instance, in USA, only $19.1 \%$ of the business locations with more than 20 people had fiber access in 2008. The key point causing this scenario, surprising at first view, is the cost of deploying fiber networks. Whereas the optical fiber equipment is about US\$10,000, the cost of the installation is estimated to be between US $\$ 200,000$ and US $\$ 450,000$, which gives a ridicule percentage weight to the technological cost. Roof rights, trenching and pole construction are quite responsible of this [646], and the cost of leasing high-speed fibers come as a second order reason [608]. As [646] informally indicates "Backhoes don't follow Moore's law".

Contrarily, the cost of a mm-wave radio installation is in the range of US\$50,000. Therefore, the larger flexibility and low-cost of mm-wave systems, that nowadays can afford perfectly the Gbps demanded rates, make them a very suitable alternative. Furthermore, the deployment is much faster and causes less environmental impact than a optical fiber deployment [608], [646]. Note that even provisional radio towers can be employed, thus providing a dynamic network configuration, which can be 
easily adapted to the demand bursts 8.10(a). To give some figures, $60 \mathrm{GHz}$ band standards are available to yield up to 4 Gbps speeds, whereas the $70 / 80 \mathrm{GHz}$ equipment is already available, providing $1.25 \mathrm{Gbps}$. For this last case, the connectivity is achieved with a $99.999 \%$ weather availability, the famous five nines or carrier class performance, over $2-3 \mathrm{~km}$ distances in most of the USA, which is a country presenting a great variety of weather conditions. In addition, if the network is more permissive, i. e., allowing a performance slightly lower than five nines $(99.99 \%$ or $99.9 \%$ ), coverage is increased and the cost is reduced even more. In addition, proper network stations configuration strategies can thoughtfully minimize weather influence. This fact throws away the idea of that mm-wave networks are wrecked by rain.

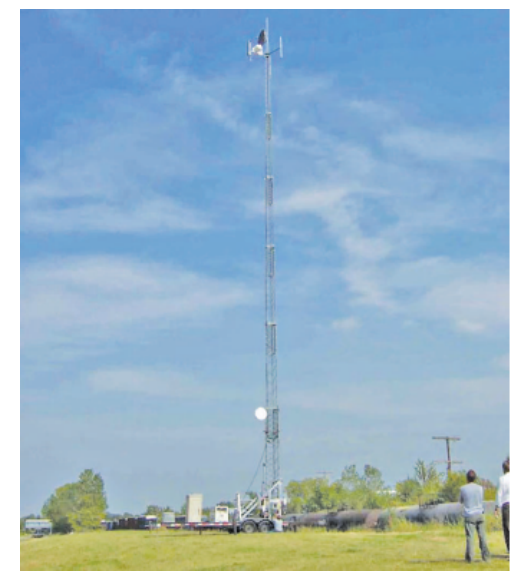

(a)

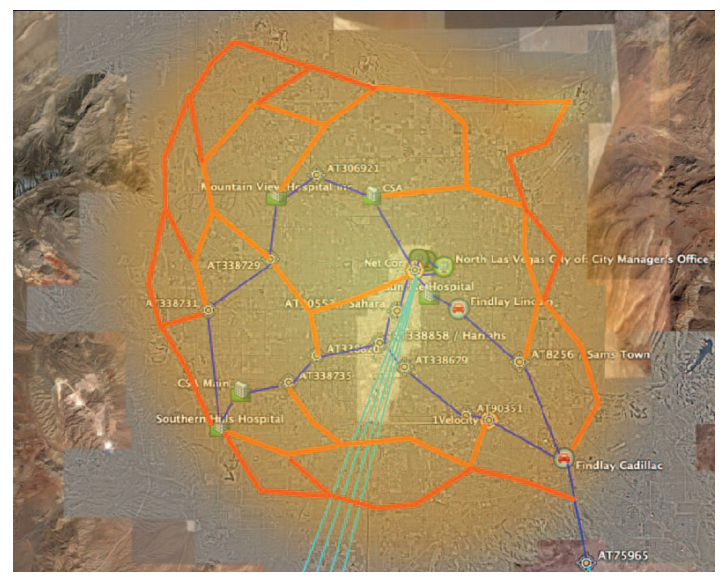

(b)

Figure 8.10: Point-to-point links for backhauling at mm-wave frequencies [646]. a) Millimeterwave radio backbone (orange lines) deployed by 1 Velocity in Las Vegas, Nevada; b) Intelco tower Systems SR 106 portable tower at full extension of $32 \mathrm{~m}$, with $\mathrm{mm}$-wave radio systems installed at $3 \mathrm{~m}$ and $6 \mathrm{~m}$ elevation.

Moreover, if one considers the optical alternative competitor, Free Space Optics (FSO) [670]-[671], some disadvantages are found. For instance, fog conditions seriously affect the link: whereas a mm-wave link would experience $0.4 \mathrm{~dB} / \mathrm{km}$ in fog conditions, the attenuation of a FSO link is as high as $225 \mathrm{~dB} / \mathrm{km}$. Since fog occurrences are hard to predict, and they extend for many hours when appear, FSO links experience significant disconnections. In addition, many elements can block the ray path. This is the case of birds, snow, sand, dust, flying debris or agricultural burning residue. In addition, the required precised alignment is challenged by the tower sway or movements of solid buildings as they naturally heat up and cool down during the day. Longer east or west facing optical links can be also affected by diurnal sunlight effects. Thus, even FSO is a promising technology which provided very good performance under optimum condition, mm-wave links are much more robust [608].

In this way, the primary applications in this area comprise Gigabit Ethernet format of voice, video and data traffics, applied for campus connectivity, Local Area 
Network (LAN) bridges, last mile access to Internet and metro Ethernet backbones. Equipment is already installed in several business, schools, universities, medical institutions, government buildings and entertainment companies. The examples of specific benefit of the enhanced high data rate provided by the mm-wave backhaul and backbone networks given in [646] result quite interesting: X-ray and Magnetic Resonant Imaging may be delivered close to real time at medical campus or between several of them, or high definition video material may be almost instantaneously delivered between a sound stage and a producer location in Hollywood. Nevertheless, the most significant example given in that work is the 65-link metro Ethernet backbone in Las Vegas, Nevada, developed by 1Velocity [674], see Fig. 8.10(b). The backbone network footprint is approximately $32 \mathrm{~km} \times 48 \mathrm{~km}$, covering the entire metropolitan area. Each Gigabit node provides 10-100 Mbps to individual customers through lower-frequency radio channels. It is interesting to note that the network offers less than $0.5 \mathrm{~ms}$ of latency across the entire network footprint. In addition, a single ring element failover is recovered in less than $0.7 \mathrm{~ms}$. These figures are representative of very good performance in a network of that size.

\subsection{Indoor communications:}

Indoor wireless communications define small, short-range networks inside a building, house, office or even a room, in which few devices are connected (quite often on the device-device configuration). The bands around $60 \mathrm{GHz}$ provide the perfect support for such scenario inasmuch as they provide wide bandwidth and suppose an alternative spectrum to the $70 / 80 \mathrm{GHz}$ bands, more suitable for point-to-point links due to their lower atmospheric attenuation. A good classification of indoor communications is that given in [632]: a) high-definition video streaming; b) file transfer; c) wireless Gigabit Ethernet; d) wireless docking station and desktop point to multi-point connections; e) wireless ad hoc networks. The most relevant in the current land scape are a)-c) due to their universal impact to the user. The aforementioned categories can be even subdivided by cases depending on 1) whether they are used in residential areas or offices; 2) distance between transmitter and receivers; 3) LOS or NLOS connection; 4) position of the transceivers, and 5) mobility of the devices. Information about such categorization can be found in [632] and the regulatory document [675].

High-Definition (HD) video streaming is the nowadays Internet revolutionary. In the same way that MP3 did with the music industry in the 2000s, HD video is doing at present through popular web platforms such as Youtoube ${ }^{\circledR}$. Front classical $\mathrm{TV}$, Internet offers wider content by far and the possibility of moving it from one device to another easily. This scenario urgently demands for an obvious link that lacks today (at least in the required data rates): the Device-to-device (D2D) link [609]. The $60 \mathrm{GHz}$ wide bandwidth channels open the door to uncompressed video streaming for residential use, which is very interesting, not only for comfort, but because alleviates the receiver device complexity, which will not need to incorporate compression/decompression functions. Uncompressed High-Definition Television (HDTV) may be sent from the reader device (DVD, computer, PDA, etc.) to the receiver with typical distances of $5 \mathrm{~m}$ to $10 \mathrm{~m}$. Another interesting scenario considering uncompressed video streaming are conference rooms where people can share the same projector and easily connect to the projector without switching cables. 
LOS and NLOS connections must be considered [632]. The use of High-Definition Multi-media Interface (HDMI) cables to share video formats between devices may disappear in few years to the more comfortable and flexible wireless possibility [609]. The consideration of LOS results important since the direct propagation path may be temporarily blocked by human bodies or objects, and video streaming is a realtime application generally. Since transmitter and receiver are quite static in these kind of applications, strategies to enhance LOS connections when necessary must be considered.

File transfer has even more uses. In offices and residential areas a very usual connection is that between a PC and its peripherals, including printers, digital cameras, mobile phones, camcorders, etc [632]. Data is increasing and the user wants it in all its devices, instantly and at any location. A good example of how a mature $\mathrm{mm}$-wave indoor communications network may change the future is the possibility of substituting the usual USB use to share files between computers [609]. Another one, suggested in [632], is the possibility of acquiring digital content on demand from kiosks, stores or similar in public spaces. Life is becoming fast, and the user will demand services providing the required files in just few seconds.

Finally, Wireless Gigabit Ethernet links have the function of being a media converter from a fiber link to a duplex wireless link giving data rates about 1 Gbps or beyond. Therefore, it can be regarded as the extension of the optical fiber (or mm-wave radio based) backhaul to the user, with the aim of no losing data rate performance [676]. Note that, no mater how fast files are derived to the building or house node, if the final wireless section is not fast enough, the user will not experience high data rates, i. e., the classical bottleneck problem. In this way, mm-wave indoor communications may give the essential support to enhance considerably the user experience without any major changes in the core of the network, thus being a very efficient investment.

\subsection{Mobile communications:}

Mobile communications is, probably, the most relevant category inside communications for obvious reasons. The universal use of mobile phones, most of them smart nowadays, is an undeniable reality. The demand of higher data rates experiments a considerable annual grow that is saturating the network [677]. Aside from the backhaul network saturation, the cellular network providing connection to the user in the last section is in its limit these days. Cellular providers currently face a situation in which they have to deliver high quality, low latency video and multimedia applications to wireless devices with a spectrum ranging between $700 \mathrm{MHz}$ and $2.6 \mathrm{GHz}$ (each major provider has about $200 \mathrm{MHz}$ across all the different cellular bands of the available spectrum). Efficient modulation techniques, new division multiple access techniques including time, frequency, code, and now space, have been proposed [678] so the throughput provided by each portion of the spectrum is maximized. However, despite the spectral efficiency is pushed up, the theoretical limit does not give much more room [600]. If this was not enough, providers must service users with older inefficient cellphones as well as customers with newer smartphones, so they must manage their resources to allocate multiple technologies and standards in the per se saturated spectrum [601]. Therefore, enabling new bands to give cellular services would alleviate the situation considerably. 
In [600], a standalone mm-wave broadband (MMB) network is proposed to operate in the E-band, see Fig. 8.11(a). This network consist in multiple MMB base stations that cover a geographic area. The good coverage is ensured by a network of MMB base stations which are deployed with higher density than macrocellular base stations. As a rule of thumb, the given recommendation is to provide the same site-to-site distance than microcell or picocell deployment in urban environments. Since $\mathrm{mm}$-waves are employed, transmission and reception are based on narrow beams, hence the interferences from neighboring MMB base stations are practically suppressed. This permits overlapping of coverage among neighbor base stations. Whereas in conventional cellular systems the area is partitioned and each cell is served by one or only few base stations, the MMB base station is thought to form a grid with a large number of nodes to which any MMB mobile station can attach. This strategy is very suitable to eliminate the problem of poor link quality at the cell edge, which is inherent to cellular systems, so that high-quality Equal Grade of Service (EGOS) will be given in MMB networks regardless of the mobile location. In addition, as [601] points, aside EGOS enhancement by the geographical distribution of the stations, mm-wave considered bands are much more uniform regarding the channel (note for instance that $2.6 \mathrm{GHz}$ is more than 3 times $700 \mathrm{MHz}$ ).

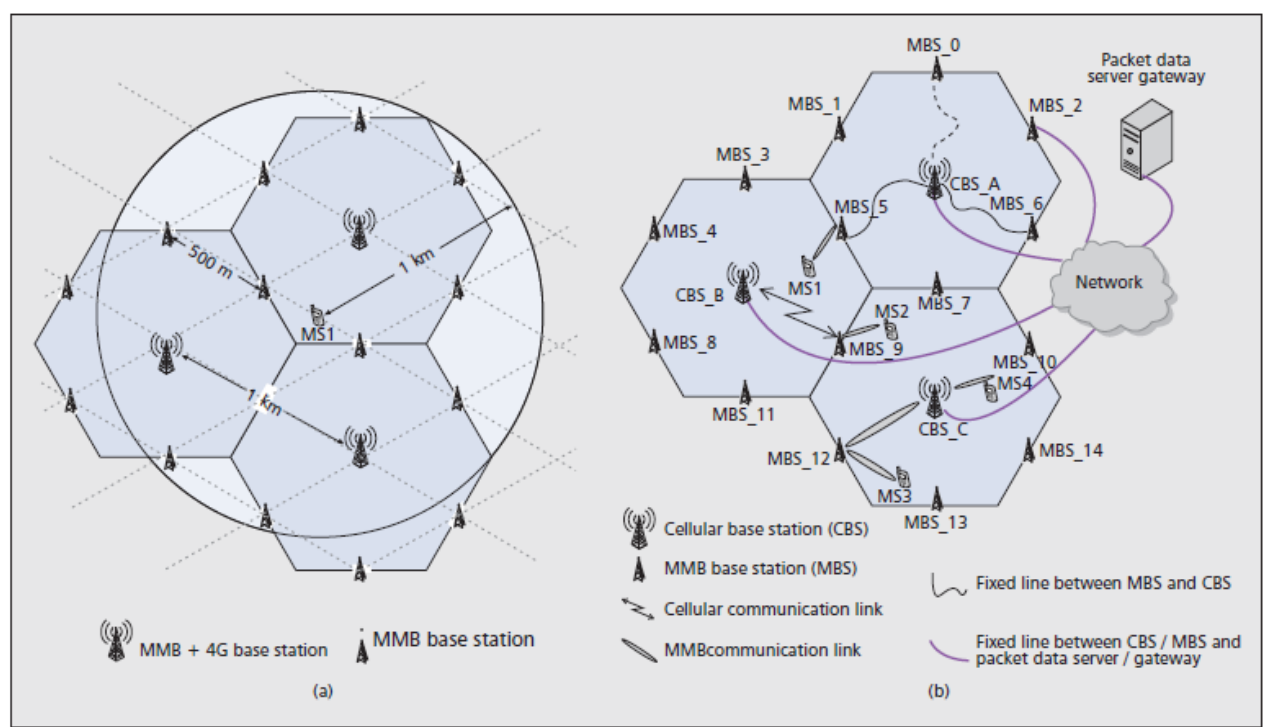

Figure 8.11: mm-wave Broadband (MMB) network proposed in [600]. a) Final MMB architecture overlapped with the conventional $4 G$ network; b) Intermedium architecture based on a hybrid $M M B+4 G$ system.

Since the investment to arrive to the scheme of Fig. 8.11(a) may be challenging for the provider, intermedium schemes are possible thanks to flexibility of radio links. In [600] it is posed a hybrid $\mathrm{MMB}+4 \mathrm{G}$ system as a solution to be implemented in the immediate future, see Fig. 8.11(b). Possible initial coverage holes in areas of low MMB base stations density should not affect considerably the user experience inasmuch as $4 \mathrm{G}$ networks are though to give good coverage when the MMB begin to deploy. In the hybrid system, the mm-wave spectrum will be dedicated exclusively 
to data communication, being the control channel and feedback supported by the old $4 \mathrm{G}$ system. Note that frequencies below $3 \mathrm{GHz}$ provide better penetration through obstacles and they are less sensitive to LOS, rain and other undesired situations, hence it seems appropriate to transmit important control channels in the microwave spectrum, whilst mm-wave frequencies are reserved for high data rate transmission.

Similar proposals to that of [600] can be found at the $28 \mathrm{GHz}$ and $38 \mathrm{GHz}$ bands [601], [602]. There are also cases proposed for the $60 \mathrm{GHz}$ bands despite of the undesired atmospheric attenuation. In [634], a simulation of a $60 \mathrm{GHz}$ based mm-wave cellular network gives an increment of the throughput by 30 times when compared to the best case operating at $f=3.5 \mathrm{GHz}$. All the schemes are based in networks with larger number of elements and with increased collaborative features provided by both, terminals and base stations. The cost of the technology and the consolidation of MIMO and beamforming techniques are the main current issues, which solution is hoped by providers to proceed with the network deployment [600], [609], [632], [648].

\subsection{Satellite communications:}

The allocated spectrum for satellite communications is as well becoming congested these days [668]-[669]. The situation maybe not as evident as the case of mobile communications, however, as high data rates increase and the transmitted elements demand from higher throughput in every kind of network, new generation of satellites to be operative in the next decade must incorporate suitable equipment to face the challenging demands of the market. The necessary technology for the use of this kind of communications over the mm-wave spectrum has been already developed and is ready for practical implementations [668], [679].

Due to the atmospheric absorption, current state of the art points to the use of $[37 \mathrm{GHz}-42 \mathrm{GHz}]$ inside the Q-band, and [71 GHz-76 GHz] inside the E-band [669], although some previous commercial prospects considered as well [46 GHz-56 $\mathrm{GHz}]$ in the V-band. Serious commercial proposals indicate as possible geostationary orbits, medium earth orbit, low earth orbit, and Molniya orbit, or in some cases combinations of two of these orbits. In general, the Geostationary Orbit (GEO) is preferred since it does not require tracking by earth station antennas. Nevertheless, the Low-Earth Orbit (LEO) has gained attention since half-second is gained over GEO orbits, a rather important temporal delay, since it can limit the speed of access to the Internet and degrade the quality of real-time applications such as teleconferencing. Systems are called to incorporate multiple narrow spot-beam antennas, onboard demodulation and routing of traffic between beams, inter-satellite links, and in some cases scanning beams in order to illuminate the service area continuously. In general, advanced antenna configurations will be required to face the propagation impairments, caused by a challenging channel, very affected by the atmospheric attenuation. In addition, the cost of solid-state power devices still remains higher than desired [653]. Several efforts are ongoing in the development of smart antennas able to provide beam-switching or adaptively beam-steering [668]-[669], [680], and to mature solid-state devices for satellite applications [681]-[682]. 


\subsection{Vehicular communications:}

The automation of vehicles is a reality. The next generation of cars will be equipped with an enormous quantity of sensors, generating important data rates. For instance, several radars such as described in this chapter will be massively incorporated for object detection. In addition, several visual cameras will act as virtual mirrors, and LIght Detection and Ranging (LIDAR) for generation of high resolution depth associated range maps will be as well incorporated, see Fig. 8.12(a) [631]. The sensor intelligence inside a car will have such level of complexity that even the interior of cars is being these days under study for channel modeling [683]. Under this situation, it seems very attractive to exploit all the obtained information, not only for comfort and safety inside the car that is obtaining the data, but for generating a collaborative network between cars or infrastructure elements, see Fig. 8.12(b). In this way, possible limits caused by LOS situations may be easily overcome if the information that each car knows is shared on the network.

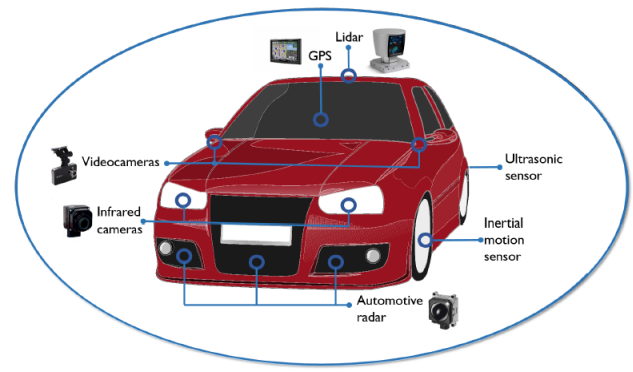

(a)

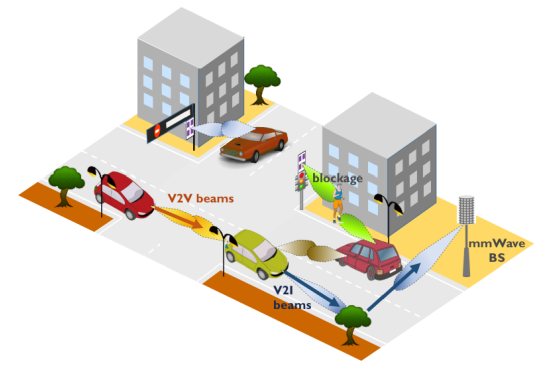

(b)

Figure 8.12: Vehicular communications [631]. a) A car with several automotive sensors to enhance safety and performance; b) Conceptual scenario of vehicular communications at mm-wave frequencies. Multiple mm-wave transceivers are placed on the vehicle in order to simultaneously establish vehicle to vehicle $(\mathrm{V} 2 \mathrm{~V})$ and vehicle to infrastructure (V2I) communication links.

Conventional technologies such as Dedicated Short-Range Communication (DSRC) and $4 \mathrm{G}$ cellular communication cannot support the Gbps data rates that are required for uncompressed sensor data exchange between vehicles. For instance, note that DSRC allows vehicles to exchange messages with basic sensor information with a range up to $1000 \mathrm{~m}$ in theory, however, the maximum data rate in practice only reaches 2-6 Mbps. Alternatively, 4G cellular systems may be used in D2D mode in order to provide V2X (Vehicle-to-Vehicle -V2V- or Vehicle-to-Infrastructure -V2I) communication systems. Nevertheless, although these systems are limited to 100 Mbps in theory, the data rate is noticeably reduced when high mobility enters in scene. These technologies are definitely not prepared to handle the data rates in the order of terabytes per hour that those automotive sensors will generate in an scenario inherently presenting important mobility [631], [684].

The contemplated mm-wave alternatives are: to support the architecture over the $5 \mathrm{G}$ mobile network standard possibilities, to include a modification of the IEEE 802.11ad (a standard defining the use of $60 \mathrm{GHz}$ bands under the OrthogonalFrequency-Division-Multiplexing -OFDM-, see [685]), or, if the demand for this service reach high levels, it may be even convenient to develop an specific new standard. 
The $60 \mathrm{GHz}$ band, provided by the IEEE 802.11ad may allow to transmissions of up to $7 \mathrm{Gbps}$, but other regions, such the spectrum around $28 \mathrm{GHz}, 37 \mathrm{GHz}$ or 39 $\mathrm{GHz}$, are also considered.

Also in this kind of communication service, a large number of antennas at both, transmitter and receiver, will be present, thanks to the small antenna size at mmwave frequencies. Since a car can easily allocate several antennas due to the available physical space, and the power supply will not be a problem, this configuration is particularly affordable in vehicular communications. In addition, it is very necessary inasmuch as that nearby vehicles and pedestrians will be continuously blocking some paths. This blockage can be mitigated by using the gap under vehicles as a waveguard. Nevertheless, the blockage effect is contemplated in [631] as a positive feature rather than a negative one. The justification resides in the reduction of inter-vehicle interference, and the fact that only vehicles in proximity would share their uncompressed sensor data while the basic safety information will be broadcasted using other channels.

Despite of the fact that this emerging service is very promising, it must be said that several issues must be treated. Due to the novelty in the field, few studies have treated properly the modeling of the vehicular channel at mm-wave frequencies. Also, the true final performance of the systems as conceived and described here would be only achieved once the percentage of vehicles incorporating the required equipment reach a reasonable penetration level. Regarding to this aspect, note that, car renovation rate is much lower than for other goods, e. g., mobile terminals, since they are noticeably more expensive and with a larger life cycle. Therefore, evolution up to reach the described future mm-wave scenario is inherently slower than in other areas such as mobile communications. Finally, there is a lack of simple mm-wave beam alignment algorithms, non-fast enough to allow for vehicular communications [631], [684].

\subsubsection{Imaging}

Millimeter-wave imaging shares many aspects with $\mathrm{THz}$ imaging. Both represent a suitable increase of resolution regarding to the microwave regime, and, in the case of $\mathrm{THz}$ frequencies, special sensitivity to molecular reactions. Refining more, if mmwave and $\mathrm{THz}$ are compared, as it was pointed out in section 2.2.3, the larger the frequency, the larger the resolution, but also the lower the penetration in fabrics an other elements that are desired to be rather transparent (think in security applications, for instance). Therefore, inasmuch as resolution is a suitable feature, but penetration may result mandatory, leaving apart the friendly environments of molecular characterization applications, nowadays technology suggest as more suitable to employ, for the moment, the lower frequencies offered by the mm-wave band. A good example of this comparison is found in [118] (consult again Fig. 2.13(b)). A proof that mm-wave imaging is ready for commercial purposes is the large number of applicable system concepts patented these last years (see for instance [686]-[688])

At any scenario, objects emit and reflect radiation in the mm-wave regime. The amount that each object emits or reflects is characterized by the object emissivity $\rho$. This parameter is a function of the dielectric properties, roughness of its surface, and the observation angle. A perfect radiator, sometimes called blackbody, presents 
$\rho=1$. A perfect reflector presents $\rho=0$. At mm-waves, the skin of the human body shows an emissivity of roughly $\rho=0.9$, i. e, it has some reflectivity in this frequency band [640]. The key point to discriminate the metallic elements resides in what radiation are they reflecting, inasmuch as ideally, the emission from metallic parts is low, however, sources or radiation and reflected contributions may cause to perception of metallic elements with higher emissivities, thus reducing the contrast with the human body in passive imaging. Contrarily, active imaging, will enhance those metallic elements in the received radiation.

When talking about mm-waves, imaging applications literature and current commercial use become restricted to much less areas if compared with those of $\mathrm{THz}$ frequencies, being security the most popular (something that can be well understood from the aforementioned emissivity properties of the human body and metals) [637][644]. However, some new proposals such as concrete walls crack detectors [690] or recycled paper classification [691], are also found. The advances in mm-wave Monolithic Microwave Integrated Circuits (MMIC) together with the improved designs combining mechanically scanned optics have led to an scenario in which mm-wave security cameras are cost effective, at least in some areas. The use of this cameras is a reality, mainly in the USA [692].

Already in 2006, mm-wave imaging systems presented good features [693]: a) image uniformity was achieved by panning a vertical line scan, hence avoiding the loss quality introduced by previous scanning systems, which needed to reconstruct the picture to form an image; b) high efficiency of the optic system, that allowed to use up to $95 \%$ of the incoming signal reaching the receiver; c) frequency independence, inasmuch as the system was prepared for interchangeability between $77 \mathrm{GHz}, 94$ $\mathrm{GHz}$ and even $140 \mathrm{GHz}$ receivers; d) versatility to operate in passive or active mode; e) high refresh rates thanks to the use of arrays of receivers of typically 4 to 32 . With 32 receivers, real-time video refresh rates were achieved.

Also in 2006, Yujiri [640] presented interesting images taken with a NGC 1040element Focal Plane Array (FPA) Passive Millimeter Waver (PMMWC) video camera, shown in Fig. 8.13-1. The camera, operating at $94 \mathrm{GHz}$, used a $71 \mathrm{~cm}$ diameter plastic lens to collect and quasi-optically focus the radiation. It was able to generate 17 frames per second with a Maximum Resolution Temperature (MRT) of 2 K. Fig. 8.13-2 shows an example of use in indoor environment. As it is observed in the right image, the subject has a plastic bag with shrapnel on his torso. The subject is able to cover that threatening security target by using a sweatshirt as observed in the left image. The central image shows the mm-wave image captured by the camera, revealing metallic elements quite precisely (darker shades represent warmer temperatures). Note that in this indoor image, the shrapnel is reflecting the room radiation, hence appearing with the same color. Therefore, the dynamic range in the scene is of only $15 \mathrm{~K}$, something usual in indoor images. With a similar format, Figs. 8.13-3 and 8.13-4 show images taken outdoors, where a handgun and a bag with shrapnel are, respectively, the security threats. In those images, the dark tones represent cooler temperatures. It is observed larger contrast in these mm-wave images (central in each set) than in the indoors one. In fact, the dynamic range is of $250 \mathrm{~K}$, thanks to that outdoors, the main source of metallic parts reflection is the cold sky, at only $T=60 \mathrm{~K}$, much less than the warm peaks at $T=310 \mathrm{~K}$, radiated by the human body through the fabrics. 

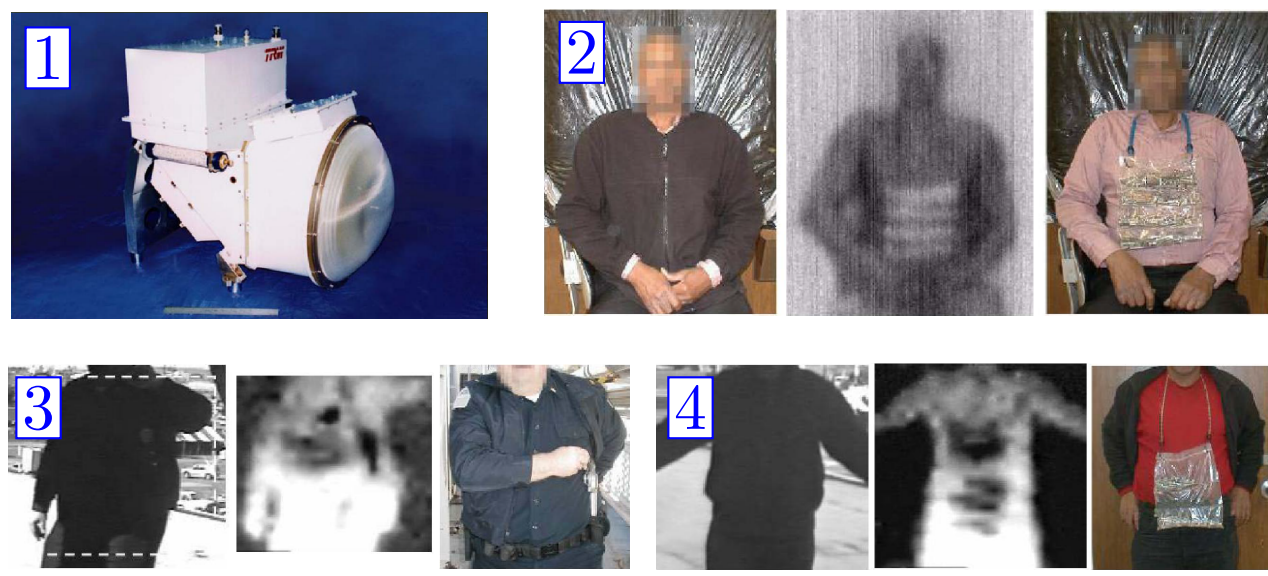

Figure 8.13: Millimeter wave imaging in security applications using a PMMWC camera operating at $f=94 \mathrm{GHz}$ [640]. 1) The NGC 1040-element Focal Plane Array (FPA) Passive Millimeter Wave (PMMW) video camera; 2) Indoor imaging for metal weapon detection (the target is a plastic bag with shrapnel). From left to right: visible image of the subject wearing a sweatshirt covering the target, mm-wave imaging revealing a metallic elements, and visible image of the subject without the sweatshirt in which the target is appreciated. Darker shades indicate warmer temperatures; 3) Outdoor imaging for weapon detection (the target is a handgun). From left to right: visible image of the subject covering the handgun with a coat, $\mathrm{mm}$-wave imaging revealing the target and other metallic elements, and visible image with the subject showing the handgun. Darker shades indicate cooler temperatures; 4) Outdoor imaging for weapon detection (the target is a plastic bag with shrapnel). From left to right, the subject covering the bag with shrapnel with a coat, mm-wave image revealing the shrapnel together with other reflective elements (false positive caused by a small metalized bag containing a moist towelette), and visible image with the subject exhibiting the bag with shrapnel. Darker shades indicate cooler temperatures.

The most common frequency used for mm-wave imaging is $f=94 \mathrm{GHz}$, followed by $f=77 \mathrm{GHz} . \quad f=35 \mathrm{GHz}$ is as well possible, however it is resolution-limited. On the other hand, $f=140 \mathrm{GHz}$ and $f=220 \mathrm{GHz}$ are also suitable for imaging purposes due to the atmospheric windows around them, however, beyond the $\mathrm{W}$ band $(75 \mathrm{GHz}-110 \mathrm{GHz})$, MMIC products are not still mature, the performance is lower and the prizes soar [640], [642], [693]. It is foreseeable a migration of the systems towards these frequencies once low-cost/mass-production technology is able to support them. Passive imaging is more common inasmuch as the system is alleviated from some complexity [640], [691], [693], however, some active designs are as well found [643], [690]. Gunn diode oscillators are the preferred option for these active cameras. Although larger contrast is obtained with active systems, interference and multi-pah problem are challenging, as well as managing coherent illumination and providing a uniform amplitude and phase distribution along the receiver array. The usually handled distances range from $1 \mathrm{~m}$ to $5 \mathrm{~m}$, hence being in the far distance region (note that $10 \lambda$ at $f=94 \mathrm{GHz}$ are roughly $30 \mathrm{~mm}$ ). The inherent problem to this practical fact is that degradation of resolution and diffusive attenuation occur. The usual solution is to incorporate a focal dielectric lens. These lens, usually made of Teflon, can weight several kilograms [642], [690], a handicap in the case of portable systems. 
Recent proposals have tried to bring the mm-wave camera concept to the low-cost market. This is the case of the work in [644], therein the feasibility of using a lowcost commercial plasma display as mm-wave imaging device is demonstrated. The obtained performance does not reach the level of mm-wave cameras implemented with individual glow (gas) discharge detectors, but it allows to obtain short range imaging with good results. A cheap, commercially available monochrome plasma display with 4096 (128 x 32) individually addressable cells is used as a focal plane array. While each element is on and illuminated, the voltage across the electrodes is monitored and any changes will be proportional to the incident radiation. The cost of the display is US $\$ 300$ what implies a cost of several cents for each cell. The authors suggest this type of solution as a possible paradigma shift due to the expected impact of such high density focal plane arrays with easy commercial applicability. Extension to 3D mm-wave imaging systems working beyond 10 frames per second is foreseen by using this plasma system whenever some issues regarding the still high Noise Equivalent Power (NEP) are minimized.

Finally, a very recent proposal is reviewed. Imaging at mm-wave has been considered for recycled paper discrimination in [691]. Despite of being less famed, paper production and recycling industry has very significant activity in many countries. According to that reference, global production of recycled paper and paperboard amounted to 400 million tons in 2012 , this having a total cost of $\$ 108$ billion. Waste paper represents, by weight, the $40 \%$ of the total solid waste generated in the world. Therefore, recycling waste paper is a relevant task to be faced properly.

Nowadays the classification process applied to separate the waste paper by categories is still done manually, what increases the cost noticeably the cost. Some automatic paper sorting machines incorporating optical sensors have been proposed, but their efficiency is low inasmuch as the information that they provide results no discriminative most of the time. In [691], it is used a radiometer operating in the band $[94 \mathrm{GHz}-96 \mathrm{GHz}]$ with a temperature sensitivity of only $0.2 \mathrm{~K}$. Fig. 8.14 shows the results of the of the experiment. A journal magazine of 50 pages is used as reference and it is compared with other possible paper elements. In Fig. 8.14(a) a piece of corrugated card board is evaluated. The loss factor of the piece is 2.5 $\mathrm{dB}$ front the $6 \mathrm{~dB}$ of the reference, what gives good contrast to discriminate both pieces. Similarly, in Fig. 8.14(b), a newspaper is evaluated, giving a loss factor of only $0.7 \mathrm{~dB}$, allowing to an even better discrimination. Finally, Fig. 8.14(c) shows a discrimination test between two magazines. Despite of the similarity of the objects, a contrast of $2 \mathrm{~dB}$ is obtained. Availability of proper data bases, and improvement of object boundary location by means of optical systems may enhance even more the performance.

From the review in mm-wave imaging it seems that the immediate future points to mm-wave systems operating at $f=94 \mathrm{GHz}$, gaining social penetration in the next decade. Then, it is probable that solid-state technology allow the use of bands around $f=220 \mathrm{GHz}$. Active imaging will gain terrain as well to passive imaging. In this way, $\mathrm{THz}$ imaging will be considered only in its specific areas, in which it provides considerable advantage from mm-waves (THz-TDSI, molecular characteristics, etc.). 

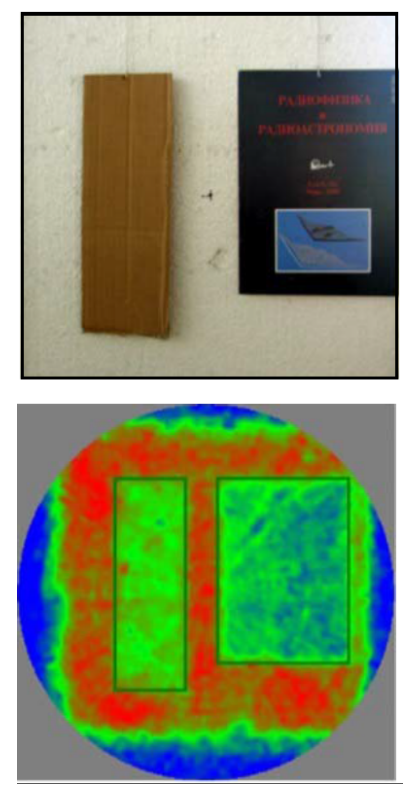

(a)
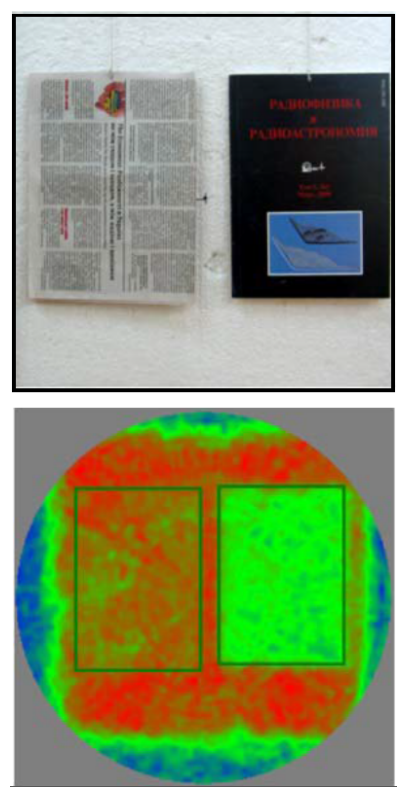

(b)
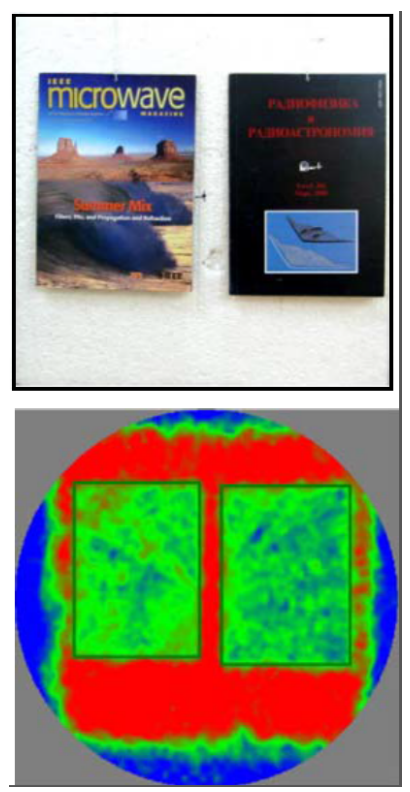

(c)

Figure 8.14: Millimeter wave imaging for recycled paper classification using a radiometric apparatus operating at $f=95 \mathrm{GHz}$ with a bandwidth of $2 \mathrm{GHz}$ [691]. The top row shows visible images whereas the bottom row show mm-wave images. The reference object is a journal (black cover) of 50 pages. a) Discrimination from corrugated carton; b) Discrimination from a newspaper; c) Discrimination from another journal of 50 pages.

\subsubsection{Radiometry}

The term radiometry defines the detection of electromagnetic energy radiated by natural sources. It differs from radar and communications in several ways. In radiometry, a sensitive receiver is used, but there is not transmitter in the system [598]. In this way, radiometry is passive imaging. However, as imaging commercial applications have enough entity per se so that a dedicated section has been reserved to them, this section will focus in scientific radiometry uses. The two main areas to be distinguished are atmospheric characterization and meteorology [650], [655], [694]-[695], and remote sensing of sea or ice [610], [696]-[698]. In this way, it must be pointed out a difference respect to the $\mathrm{THz}$ band: whereas $\mathrm{THz}$ frequencies result more suitable for space sensing (some mm-wave works can be also found [699], but are less abundant), the mm-wave band seems more suitable to sense the surroundings of the Earth. The literature in those scientific areas is quite denser and deeper than that of the previous sections, something that can be appreciated in the grade of detail of the cited works, hence only brief remarks will be given here.

The use of mm-wave radiometry for meteorologic purposes is quite directly related with the exploitation of information given by the resonant lines of oxygen, water vapor and other gases. Oxygen lines around $f=60 \mathrm{GHz}$ and $f=118 \mathrm{GHz}$ give good temperature estimation, being them almost undisturbed by the presence of clouds. The water vapor line at $183 \mathrm{GHz}$ is a good tool for humidity sounding. 
Measurements using ground-based, aircraft and balloon radiometers have been used for decades, rapidly followed by the use of dedicated satellites [598], giving very good performance these days [700]-[701]. Although radiometric systems for these purposes employ last generation equipment, quite more advanced than that employed in commercial environments (military and radiometric scientific applications have been always driving technological advances that later have penetrated into civil society [1], [598]), giving accurate characterization of the involved phenomena is extremely complex. In fact, extensive work can be found only in the area of studying the atmospheric noise and path delay errors perturbing the required measurements [697], [702]-[703].

Ice clouds also play an important role that is not well quantified nowadays [695]. Further improvements in this area require estimating ice water path (vertically integrated ice mass) and particle size. Except in the case of thin cirrus clouds, optical and infrared frequencies are more suitable to observe the cloud top, whereas mmwave frequencies are have a more equally distributed influence in the whole ice cloud. Therefore, all bands are integrated in the system. The infrared and optical frequencies give information about the second moment of the size distribution, whereas the larger mm-wave wavelengths are sensitive to higher moments of the particle size distribution and hence to larger ice particles. In this way, mm-wave frequencies are an indispensable complement to this systems, yesteryear based in shorter wavelengths.

A lot of work can be also found in the area of characterizing the ozone chemistry, with special emphasis on the stratosphere. There are a large number of ozone lines in the mm-wave spectrum $(f=110.830 \mathrm{GHz}$ is a strong one [655]), what helps to quantify the ozone destruction process. During this process, a chlorine oxide radical is formed as an intermediate process. This compound resonates at $f=204 \mathrm{GHz}$, frequency at which good discrimination is obtained by the employed radiometric equipment. For this purpose, horizontal viewing radiometers mounted on satellites are preferred in order to allow for a larger range of altitudes [598], [655], [694]. The task is challenging inasmuch as ozone shows a high variability in both, space and time, and in many cases modeling must be enhanced by using additional radiometric and climatological collateral data [694].

Finally, characterization of coastal zone patterns, hydrology and ice has a relevant role these days due to the valuable information regarding global warming or natural disaster threats [610], [696]-[698]. Although these radiometric systems usually incorporate multi-band equipment (mm-wave, infrared and optical), mm-wave frequencies emissivity is specially sensitive to changes in frequency, polarization and angle of incidence [696]. A representative mission in this area was the First IS$\mathrm{CCP}^{5}$ Regional Experiment (FIRE) Artic Cloud Experiment [610]. The scientific objectives of the FIRE were to study impact of Arctic clouds on radiation exchange between surface, atmosphere, and space, and the influence of surface characteristics of sea ice, leads, and ice melt ponds on these clouds. The intention of this work was to document, understand, and predict the Arctic cloud-radiation feedbacks, including changes in cloud fraction and vertical distribution, water vapor cloud content, cloud particle concentration and size, cloud phase, and atmospheric temperature and chemical composition change. Fig. 8.15 from [610], shows a complete composite

\footnotetext{
${ }^{5}$ International Satellite Cloud Climatology Project (ISCCP).
} 
set of images in the context of this experiment. From left to right, they are shown images taken at $f=455 \mathrm{THz}, f=185 \mathrm{THZ}, f=37 \mathrm{GHz}, f=220 \mathrm{GHz}$, and from a LIDAR system. The swath includes tundra covered by snow (and cloud), open water near the coast, and sea ice floes off shore. Note how clouds are well observed at $f=185 \mathrm{THz}$, as they are quite reflective at this frequency, in contrast to open water and sea ice, dark at $f=185 \mathrm{THz}$. The visible image at $f=455 \mathrm{THz}$ is helpful to observe that clouds are optically thick over the tundra but they thin and become transparent over open water. However, this fact requires from the mm-wave radiometric image at $f=37 \mathrm{GHz}$ to be confirmed. As the atmospheric attenuation is low at this frequency, the surface features are perfectly appreciated: open water has low emissivity and hence low brightness temperature at the $f=37 \mathrm{GHz}$, while snow and ice have high emissivity at this frequency, hence they appear bright. The image at $f=220 \mathrm{GHz}$ gives further information about the see water inasmuch as at this frequency the water brightness is higher. Over sea ice, the low brightness temperature at $f=220 \mathrm{GHz}$ (but not at $f=37 \mathrm{GHz}$ ) indicates that there is some snow cover on the sea ice, which the $f=220 \mathrm{GHz}$ radiometer sees because of its shallower penetration depth. Finally, the LIDAR system determines that the clouds are at $600 \mathrm{~m}$ altitude.
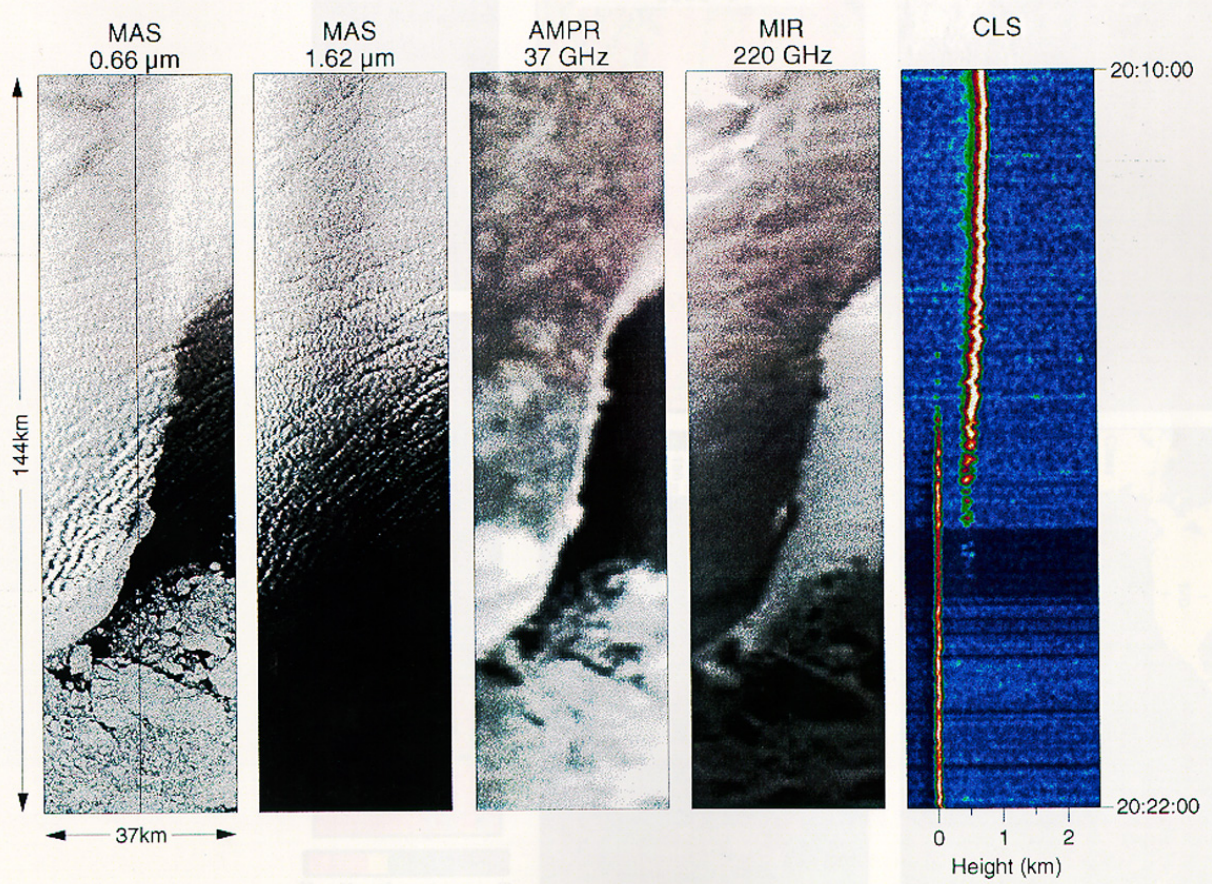

Figure 8.15: Reflection function of see ice, composite of observations obtained from the NASA ER-2 aircraft on 20 May, 1998 in the vicinity of the coast off Barrow [610]. The swath includes tundra covered by snow (and cloud), open water near the coast, and sea ice floes off shore. From left to right, they are shown images taken at $f=455 \mathrm{THz}, f=185 \mathrm{THz}, f=37 \mathrm{GHz}, f=220$ $\mathrm{GHz}$, and from a LIDAR system. 


\subsubsection{Technological problems}

The mm-wave technology has already allowed the implementation of systems to be used in certain commercial applications with enough performance, or, in others, it has given valuable proof of concept of the most innovative systems, at least. In this way, the mm-wave band currently shows features in between the mature microwave band and the very experimental $\mathrm{THz}$ band. In many cases, the required last endorsement lacks due to remaining technological problems, some of them well pointed by application-focused papers, others remaining rather hidden. Several parts intervene in a system, so that even a small improvement in each of them can contribute to an appreciable enhancement of the final observable performance (why if not there is still research in the microwave band these days). This thesis is focused in passive elements, and the improvements raise from the most basic element, the waveguide. Therefore, in this section, several technological problems to be addressed in the mm-wave band, related with waveguiding elements, are commented.

\subsubsection{Hollow metallic waveguides}

In the microwave regime, it has been a common and reasonable practice to choose hollow metallic waveguides when high performance components have been required. The Rectangular Waveguide (RWG) offers larger bandwidth and inherent structural and geometrical flexibility, natural of rectangular geometries, whereas the circular waveguide (CWG) offers special low-loss features [376]. Figs. 8.16 and 8.17 show typical passive components implemented with rectangular and circular waveguides, respectively. Nevertheless, these components suffer from performance degradation as the frequency increase since smaller holes defining the propagation channel must be achieved, and ensuring the required metal contact and alignment between pieces becomes a problem. As a matter of fact, in [704] it is generally affirmed that hollow metallic waveguide cavities reach only $65 \%-80 \%$ of the simulated values due to imperfections, this including RWG and CWG, and H-plane and E-plane manufacturing techniques.

The problem of ensuring metal contact between pieces in H-plane RWG designs is found repeatedly cited in the literature either explicitly [338], [704],[708]-[712], or implicitly on the results [337], [353], [713]. Under this configuration, continuity of the currents becomes threaten at the joint between the body and the top metal plate. See for instance the H-plane manufactured diplexer composed of two inductive filters in Fig. 8.16(b) [706]. In some designs, this problem is avoided by drawing upon the E-plane configuration at the expense of a more complex manufacturing process (note that two bodies must be manufactured in that case) [714]-[715]. However, there are several cases in which this solution not suitable by geometrical/structural reasons, or it is even not possible. See for example the coaxial diplexer with combline resonators of Fig. 8.16(c) [706]. Other well-known case are the hollow waveguide slot array antennas [716]-[718], being usual the consideration of alternatives when the frequency expands in the mm-wave band [719]- [721].

Even the E-plane configuration is possible in some RWG designs, despite of the effort in keeping the split parts aligned with several screws [706]-[707], that these components suffer from alignment problems is another extensively found evidence 


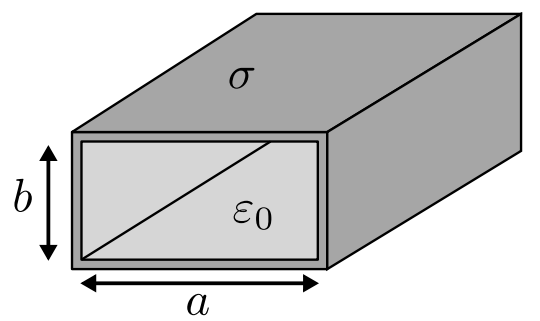

(a)

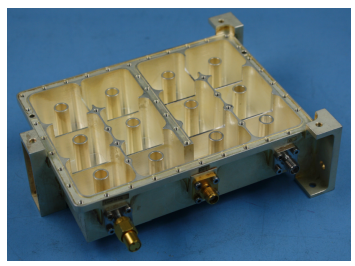

(c)

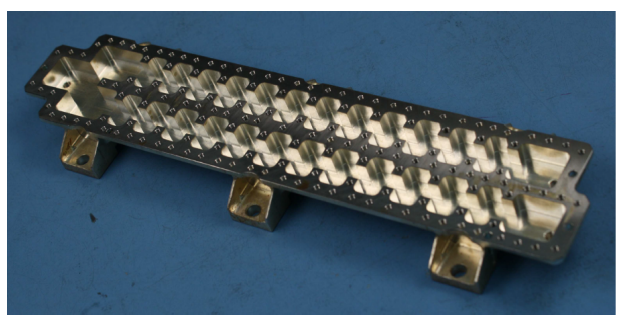

(b)

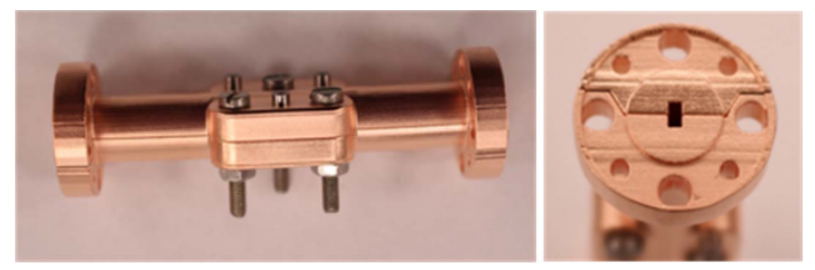

(d)

Figure 8.16: Rectangular waveguide practical implementations. a) Rectangular waveguide schematic; b) Body of a H-plane manufactured diplexer composed of two inductive filters with rounded corners (Thales Alenia Space Spain [705]) [706]; c) H-plane manufactured coaxial diplexer with combline resonators coupled by small lateral apertures (Thales Alenia Space Spain [705]) [706]; d) 3-D printed and copper-plated WR-10 thru line after assembly of the E-plane manufactured parts, side-view (left) and end-view (right) showing the self-aligned flange [707].

[349], [707], [722]-[723]. See for instance the E-plane manufactured waveguide of Fig. 8.16(d) [707]. The filter is built by means of a novel 3-D printer technique plus copper plating. Observe the structure size and required mechanical robustness to properly allocate the alignment screws and assembly the piece. Despite of the good performance of the proposed technique in manufacturing the two waveguide blocks, alignment errors are present. Note that a normally missed threat is the possible uneven pressure of the screws. Even a pressure precision machine is used to tight them, if the structure is not perfectly symmetric, deformations will be present. Although this may not affect insertion loss with the H-plane manufacture severity, it threats for sure the performance of dimension-sensitive components, such as narrowband filters. Additionally, round corners at both external sizes of the component are unavoidable by this manufacturing process. This corners may become a source of degradation in small mm-wave components [724].

The CWG case may be less obvious at first view, but paying attention the conventional techniques to assembly CWG components gives as well several sources of degradation. In general, several pieces are milled separately and jointed later axially [706], [725]-[726]. Proper alignment of this pieces, which is critical, is difficult to be ensured up to the desired level. Therefore, degradation effects are appreciated. The same arguments given for the screw alignment applied to E-plane RWGs fit here. In addition, in CWG designs, usually very sensitive to manufacturing errors [706], [727]-[729], the roughness caused by the milling process (even when using current high-performance equipment) makes arise some response degradation [730]. To the aforementioned problems, they must be added those occurring during calibra- 


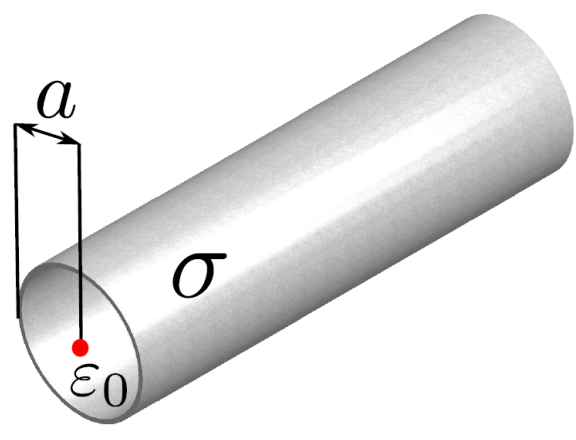

(a)

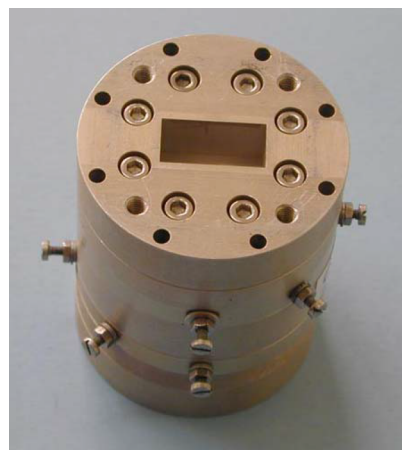

(b)

Figure 8.17: Circular waveguide practical implementation. a) Circular waveguide schematic; b) Assembled fourth-order dual-mode circular waveguide filter [706].

tion and measurement processes in which these waveguides are used [731], again by manufacturing process irregularities and alignment with flanges. Similar effects are found in transitions involving these waveguides [726], [732].

Also, it is worth to make special emphasis in those effects that are less obvious since more complex phenomena are involved. This is the case of Passive Intermodulation (PIM) [710] and Multipactor effects [733]-[735]. Small gaps between metallic parts are very prone to induce both effects, which are common in high-power applications such satellite communications up to the point that they represent one of the main issues to address in future radiofrequency satellite missions [736]-[737].

Finally, notice that even novel precision techniques, giving noticeably higher accuracy than conventional milling techniques, have been proposed, they do not fit well for mm-wave frequencies. This is the case of the SU-8 photoresist material process [346], DRIE [365] or LIGA [350], [366], techniques, already commented when hollow waveguides were reviewed for their used at $\mathrm{THz}$ frequencies in section 2.3.3.1. Unlike conventional milling techniques, in these photolitographic and chemical techniques, depth and size are preferred to be small. In fact, achieving large dimensions is often a challenge [337]-[338], [346], [350], [365]-[366]. Therefore, whereas these techniques are mandatory at optical and infrared frequencies when dealing with dielectric, Sibased waveguides [465], and preferred at reasonably high $\mathrm{THz}$ frequencies for RWG designs [351], it makes little sense in forcing them down in frequency. On the one hand, significant technological advances are required regarding these techniques in order to provide a scenario in which they overcome the precission of conventional milling techniques at mm-wave frequencies. On the other hand, the cost would be prohibitive. $\mathrm{THz}$ circuits budged may afford the cost, however, the mm-wave band requires both high performance and low-cost at the present moment. Although research in developing manufacturing techniques is always ongoing (and results are welcome), this including advances in conventional milling techniques, there is much more empty room to improve the performance by alleviating the manufacturing process through modified geometries. In this part of the thesis, the aforementioned manufacturing issues are approached from this point of view, being that Gap Waveguide $(\mathrm{GW})$ technology gives good solution for some configurations. 


\subsubsection{Planar transmission lines}

Planar transmission lines adopt a specially relevant role at mm-wave frequencies. Note that beyond the manufacturing issues of hollow metallic waveguides, those topologies show a high grade of incompatibility with MMIC circuits, and, since high-performance simple transitions lack, integrated circuits employing planar lines are preferred for several types of design [738], [739].

Since the last mm-wave progression beginnings, the question about which transmission line is more adequate has remained open [598], [740]-[743]. With the pass of the years, microwave planar transmission lines have imposed to dielectric designs since they result more practical, and novel proposals, treated later, have appeared. The four most relevant planar transmission lines coming from microwave technology are the the stripline, Fig. 8.18(a), the microstrip, Fig. 8.18(b), the slotline (SL), Fig. 8.18(c), and the coplanar waveguide (CPW), Fig. 8.18(d), [337], [400].

The microstrip line dominates the landscape in conventional designs since it presents very suitable robustness, low cost and easy integration with active components. [743]-[744]. This solution is much more convenient than the stripline, which despite of providing similar propagation characteristics (with the additional advantage of almost zero dispersion), imposes symmetry requirements which at the small mm-wave band wavelengths difficult the circuit design and dispose several structural barriers to the incorporation of active elements or the bias circuitry. At mm-wave frequencies, designs with microstrip lines fall into two groups: classical microstrip lines patterned on an electrically thick substrate carrier, and thin microstrip lines having a substrate with similar thickness to that of the metallic strip [745].

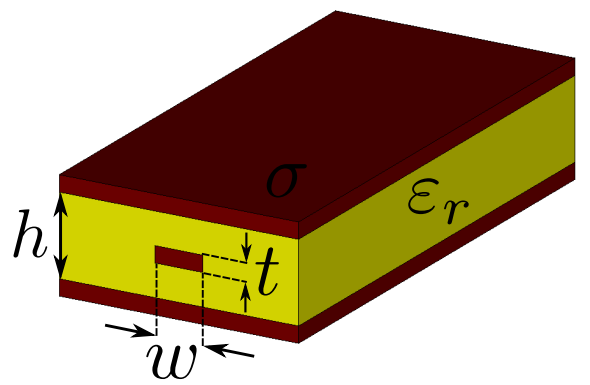

(a)

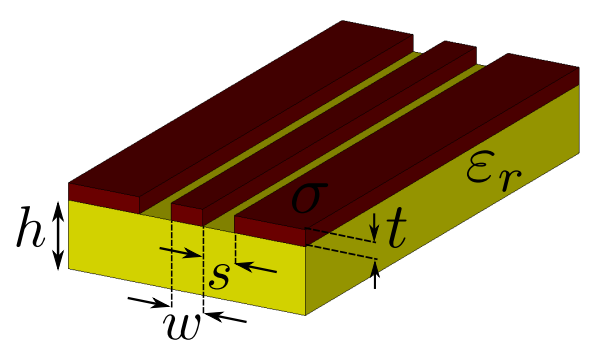

(c)

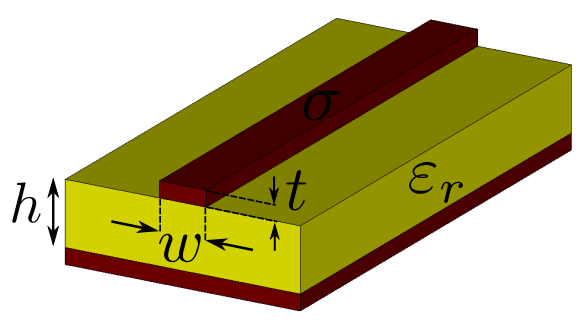

(b)

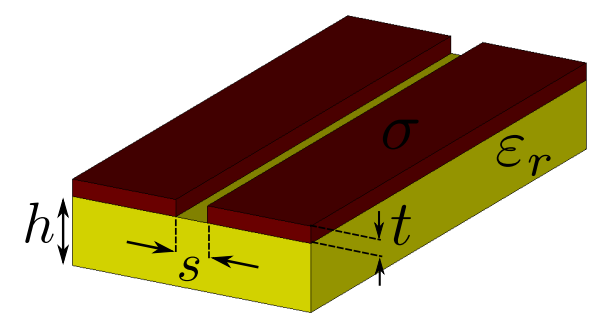

(d)

Figure 8.18: Planar waveguides at $\mathrm{THz}$ frequencies 1. a) Stripline; b) Microstrip; c) Coplanar Waveguide $(C P W) ;$ d) Slotline (SL). 
The first group is associated to the usual and well-known traditional single-layer manufacturing technologies, based on a ceramic or a plastic dielectric substrate with thickness beyond $0.1 \mathrm{~mm}$. The characteristic drawbacks of those designs are the inherent strong coupling effects to substrate modes, resulting in highly dispersive and lossy non-quasi-TEM transmission characteristics with poor intercircuit isolation. Another important drawback in this configuration is the electrically large line width, which causes excessive parasitic reactances, radiation and high phase shifts at meander or junction discontinuities. In addition, grounding paths show high shortcircuit inductance values due to the usually long grounding vias. By all this issues, this configuration has been quite avoided at mm-wave frequencies.

The second group comprises the microstrip interconnect structures found in numerous integrated circuits such as CMOS or BiCMOS. Although many problems associated to the excessive electrical size of some structure dimensions due to the reduction in wavelength at mm-wave frequencies are avoided in this configuration, it results obvious that excessive conductor losses arise. Additionally, strong lowfrequency dispersion appears [745].

By the above, it has been common choice to take advantage from the new hybrid interconnect technologies and to assemble multilayer substrate microstrip lines. It is possible to use a great variety of materials stacked in several layers which thickness range from a few micrometers to one or two millimeters [745]-[746]. This design flexibility allows to create microstrip lines in between of both described group cases. However, although particular disadvantages of each of both configurations are partially alleviated, it is not found the desired grade of performance. Losses and other undesired effects are inherently associated to this structure at mm-wave band frequencies.

The CPW is a suitable alternative to the microstrip line when it is possible to take advantage of some of its particular structural features. Note that, unlike the microstrip line, the CPW does not require grounding vias to be used when shortcircuit based elements are demanded. In addition, the distance from the center strip to the ground strips is typically lower than that for classical microstrip lines, hence lower high-frequency dispersion is obtained. However, it must be said that for very low values of this spacing, the low-frequency dispersion, very pronounced in thin microstrip lines, is also found in CPWs. Nevertheless, CPWs are less sensitive to the substrate thickness tolerances when compared with microstrip lines, unless the substrate is very thin, a case that implies high losses. Furthermore, the CPW present lower dielectric losses inasmuch the power distribution is such that an equal distribution between dielectric at air takes place, whereas in the microstrip line the propagation inside the dielectric medium is more important. Moreover, the CPW allows more flexibility to engineer a specific characteristic impedance value from its structural parameters.

On the other hand, it must be highlighted that the CPW has also some important drawbacks at mm-wave frequencies. Notice that as the lower confinement inside the dielectric gives lower dielectric losses, it also causes high radiation losses, which is an important problem [401], [415]-[409], [747], [748]. To reduce this losses, it is possible to adopt a solution with more proximity between conductor and ground strips, but this generates higher ohmic losses. Therefore, it is difficult to achieve a good overall low-loss behavior with the CPW. Other inherent problem in CPWs is the well-known 
slotline mode, which is considered a parasitic effect that affects the performance and which must be always prevented (complicating the design and this originating new objections [745], [749]).

In addition, from the practical implementation point of view, it must be taken into account that hybrid interconnect technologies with a very limited metal pattern resolution may not afford the CPW design requirements [745] (however, if high resolution is available, MMIC circuits with CPW provide high integration [750], and low inductance short-circuit elements are easily feasible). In general, to practically implement the CPW concept (note that an ideal CPW is not implementable due to its infinite lateral ground strips), they are encountered much more barriers than for the microstrip line. Additional elements, such as the conductor backing, originates undesired modes [409], [745]. Also, CPW designs suffer generally from the problem of having large cross section. This implies for instance problems at synthesizing distributed passive components. Note that high phase shifts (40-50 degrees at 60 GHz-70 GHz) are generated by the compensated bends or junctions that show a very reactive behavior due to the large cross-sections. An example of this problem is reported in [751], were the design of a Wilkinson divider at $f=50 \mathrm{GHz}$ was carried out. Observe in Fig. 8.19(a) the complicated design, including several small air bridges (AB), proposed in that work. Similarly, design high selectivity elements results challenging and unpractical. The required high separation between coupled-line sections forces the use of even larger ground to ground spaces, this causing that the overall structure may become wider than larger, hence providing a frequency characteristic practically dominated by the electrically large discontinuities. Even high metal resolution MMIC circuits reach its limits in the realization of narrowband coplanar coupled-line filters in the W-band [752]. In Fig. 8.19(b) the challenging required $\mathrm{AB}$ employed in this work are shown. Finally, intercircuit isolation is low due to the large cross-section dimensions of the involved transmission lines, having discontinuities placed on nonnegligibly thick substrates. The inherent radiation problem of the $\mathrm{CPW}$ enhances leaky waves, surface waves and parasitic mode coupling.

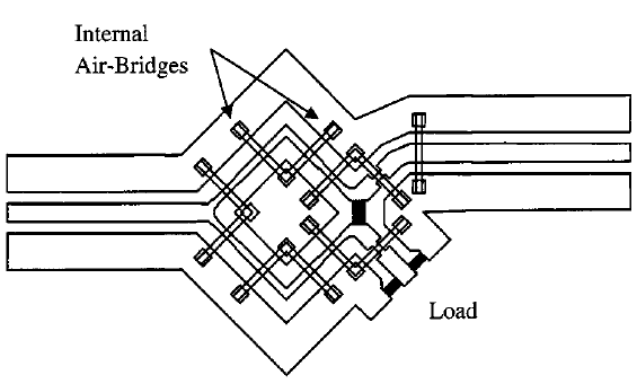

(a)

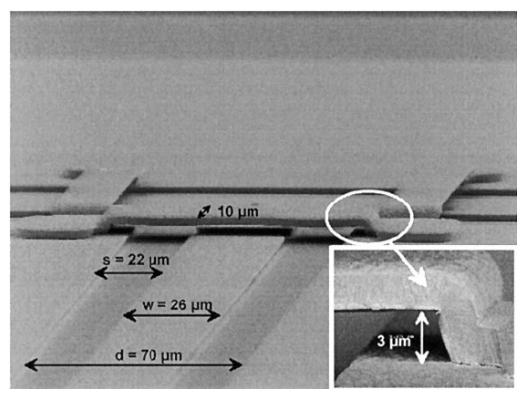

(b)

Figure 8.19: $C P W$ practical problem at mm-wave frequencies. a) CPW Wilkinson divider with several air bridges, showing a complicated implementation [751]; b) Air bridges on a $Z_{0}=50 \Omega$ CPW T-junction [752]. 
By the above, the main conclusion is that even the more suited planar lines (microstrip line and CPW) become forced when they are designed to operate with $\mathrm{mm}$-wave frequencies. Note that, maybe not in the same way as at $\mathrm{THz}$ frequencies, but dielectrics are not as good as at microwave frequencies, and the required small sizes provide that total losses, ohmic, dielectric plus radiation, reach levels that dispose a challenging design scenario for an engineer facing a commercial design, demanding from high reliability, robustness and usually very restricted in cost. The structural problems of such planar designs arise when the dimensions are small, and advantages that they offer at the low frequencies of the microwave band result to disappear in the mm-wave band. In this way, it makes sense to leave even proposed modifications of this structures and explore new geometries (something hard and that takes time to the worldwide research community, specially to adopt them universally).

A good example is the Substrate Integrated Waveguide (SIW), Fig. 8.20(a), proposed by Ke Wu in 2001 [739]. As it shows Fig. 8.20(a), the SIW is created by means of a conventional dielectric substrate of height $h$ which is metalized at both vertical sides. Then, several metalized via holes determine the waveguide in its horizontal dimension. Diameter $d$ and separation $s$ of the via holes must constitute an enough dense pattern in order to create the required confinement of the fundamental mode, see Fig. 8.20(b). Under this conditions, the SIW acts as a rectangular waveguide with a small $b / a$ ratio. By its structural characteristics the SIW is not only a planar line with easy implementation, but offers a performance that lie in between conventional hollow metallic waveguides and planar transmission lines, thus filling an operational gap with very promising possibilities at mm-wave frequencies.

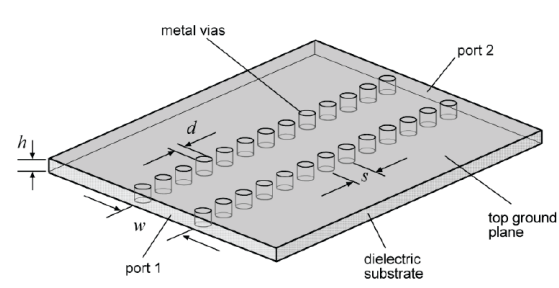

(a)

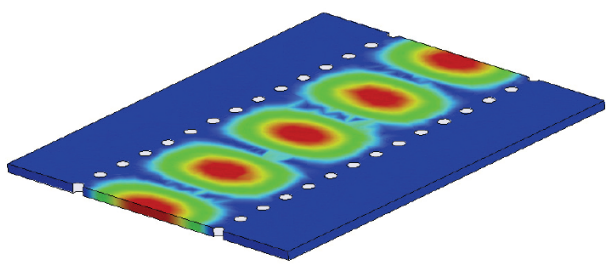

(b)

Figure 8.20: Substrate Integrated Waveguide (SIW). a) Structure and parameters; b) E-field magnitude of the fundamental mode.

The aforementioned features have justified an extensive study of the possibilities of SIW structures. General Substrate Integrate Circuits (SIC) also include modifications of the original structure such as the Substrate Integrated Slab Waveguide (SISW) [753], the Substrate Integrated Non-Radiating Dielectric Guide (SINRD) [754], or the Half-Mode Substrate Integrated Waveguide (HMSIW) [755], to mention a few. This variety of proposals is a good proof of the necessity in exploring new geometries to find appropriated mm-wave transmission lines.

As it is pointed out in [756], the original SIW structure has become the most popular among all its variants due to its similarities with the RWG, which allow to an straight-forward applications of the well developed design techniques for the RWG. In this sense, it must be highlighted that the prolific work with SIW structures has 
been only possible by means of proper SIW models and equivalents with the RWG [757]-[759] and an extensive work in ad hoc analysis techniques for SIW structures [760]-[763]. Thanks to this, SIW related literature is plenty of designs including antennas [764]-[766], filters [767]-[771] (a good review of SIW filters can be found in [772]), oscillators and mixers [773]-[774], and appropriate transitions to enable connectivity with other planar structures [775]-[777]. Therefore, whenever a new structure is introduced in the engineering scientific community, its success not only depends on its potential performance, but also in its easiness to carry out synthesis task, this last provided by appropriate analysis methods, and its consistency as a technology (availability of different types of components and elements based on or directly compatible with the technology). Since SIW technology is a good reference point as a technology that has successfully achieved a good penetration grade still it was proposed quite recently, it is a good practice to develop GW technology with a similar procedure, so that mm-wave community can benefit of the advantages of this technology when it is adopted. This clearly justifies several objectives regarding this part of the thesis such as tackling the issue regarding analysis methods, or developing high-performance passive components to consolidate the Gap Waveguide offered technology chart.

Even though SIW technology have proof some suitable characteristics in the X, $\mathrm{Ka}, \mathrm{K}$ and $\mathrm{Ku}$ bands, dielectric losses still remain as a problem that should be addressed. An interesting proposal can be found in [756], therein described an integrated line named as Empty Substrate Integrated Waveguide (ESIW), which can be understood as planar version of the RWG, hence offering similar propagation characteristics than that of the SIW, but totally avoiding the dielectric losses problem since the ESIW is thoughtfully filled by air by construction, see Fig. 8.21(a). Fig. 8.21(b), from [756], shows a ESIW filter centered at $f_{0}=19 \mathrm{GHz}$ before assembling. The quality factor $Q$ of such filter was reported to be 4.5 times than that of the same design with a SIW, which is a considerably reduction of losses. Note, however, that the ESIW structure is conceived to provide good integration properties and planar profile, but the structure demands from perfect metal contact between parts (see the three pieces of the filter of Fig. 8.21(b)). Therefore, this topology will probably suffer from the same problems than the RWG at the high frequencies of the V-band and E-band. In addition, the SIW based structures suffer from the inherent dispersion that a RWG structures presents due to their similarities.

By all the above, regarding to the planar transmission lines technological problems, this part of the thesis focuses on solutions that provide ease of integration with active devices, quasi-TEM (low-dispersion) properties and avoid the use of dielectrics. Gap Waveguides will result attractive under some particular configurations to achieve this goal. Furthermore, packaging, an additional problem of planar transmission lines when they a practically implemented [778]-[780], has been proved to naturally solved in such GW structures [750], [781].

\subsubsection{Narrow-band filters}

The two previous points have described how both, bulk and planar conventional microwave band designs, are not as suitable as desired for mm-wave frequencies, and furthermore, interconnection between them is complicated, lacking a design showing 


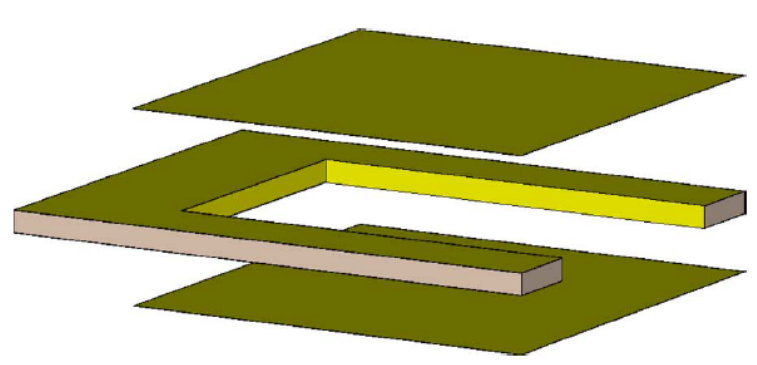

(a)

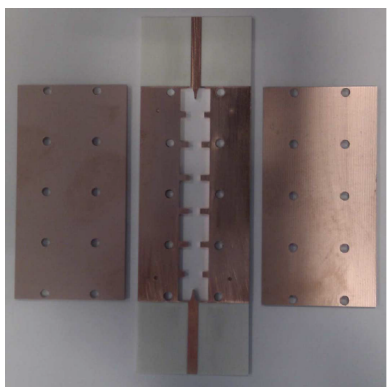

(b)

Figure 8.21: Empty Substrate Integrated Waveguide (ESIW). a) ESIW layout; b) ESIW filter with central frequency $f_{0}=19 \mathrm{GHz}$ before assembling.

intermediate properties (SIW may cover this gap to some extent). Therefore, it is obvious that all passive components at mm-wave band directly inherit the consequences of performance degradation of the employed waveguides and transmission lines that conform them when they are scaled to work at higher frequencies. Nevertheless, among other components, Narrow-Band Filterss (NBFs) are very sensitive, and they must received special attention by three important reasons.

First, note that insertion losses of a filter (in $\mathrm{dB}$ ) are, on the one hand, inversely proportional to the quality factor of the resonators (hence to the propagation losses of the waveguide which conform the resonators), and, on the other hand, inversely proportional to the RBW of the filter [782]. This is, insertion losses are inversely proportional to the product $Q \cdot R B W$. Obviously no matter the RBW of a filter, it is always desirable that resonators provide a high quality factor. However, whereas for a wide-band filter the degradation caused by having low Q resonators may be assumed, in narrow-band filters is critical. Not in vain, CWG is preferred to RWG to implement very narrow-band filters inasmuch as the improvement in the $Q$ of resonators is practically mandatory to reach acceptable levels of insertion losses [706].

Second, narrow-band filters are extremely sensitive to the layout dimensions, and suffer easily from noticeably frequency deviation due to tolerances in the manufacturing process [729]. Note that even this deviation may not be so large in relative terms to the central frequency, the narrow-band feature of the component makes the difference to be quite significant so that the specifications are totally missed even with small manufacturing errors. Since the implied wavelengths are small at the mm-wave band, this fact becomes even more relevant. In this sense, it results challenging to provide a good frequency response with structures that require a complicate alignment or that can be easily deformed, either by mechanical pressure to ensure contact or by temperature, which is the case of hollow metallic waveguides, or with structures that imply complicated layouts and are prone to exhibit parasitic effects, which is the case of microstrip and CPW transmission lines. In addition, note that even a structure showing inherent robustness to the manufacturing process, i. e., final dimensions are quite close to the desired ones, may suffer from small deviations that become appreciable at the small mm-wave band wavelengths. Therefore, 
tuning elements are generally indispensable in a narrow-band filter designs. Since conventional tuning screws or other bulky elements are costly implementable at the mm-wave band due to their large size, which does not fit wells for the small wavelenghts of operation, other solutions such as fixed metallic insertions on removable pieces have been explored, and structures supporting them are preferred (see [729] for instance).

Third, from the application approach, note that, in general, filters and diplexers are critical in most of the systems, being the most expensive element [750]. In those applications requiring subdivision of the spectrum in channels, selective filters, very restricted to the specifications, must be designed. Therefore, progress in narrowband filters has a lot of influence in the progress of the overall system. Notice that the main problems in a wireless receiver are noise and non-linearity, which are mainly originated by dissipation losses, a parameter very related with the filter quality.

The assessments treated along this section related with passive elements technological problems can be summarized in a compact way, affirming that mm-wave frequencies demand from specialized transmission lines that avoid requirement of perfect metal contact between pieces and dielectric losses, and that provide ease of integration and packaging. It must be pointed out that the search for new structures is much more bounded than in the $\mathrm{THz}$ range inasmuch as microwave band solution are much more suitable than optical band ones, i.e., closed metallic structures are preferred. In addition, it must be highlighted that the research in this line must include both, proper designs and proper analysis tools as an indivisible pack in order to give a successful solution to nowadays problems.

\subsection{Gap Waveguide Technology}

The recurrent technological problems affecting transmission lines at the mm-wave band drove in 2009 Prof. Kildal to consider a family of structures [360] based on the Electromagnetic Bandgap (EBG) effect [468]-[469], according to his background with soft and hard surfaces [783]-[785]. They were called Gap Waveguides (GWs). This section reviews the emergence of GW proposals and intense recent research, comprising designs of a variety of devices, its characterization and modeling, and test of their use as a complementary packaging structures. However, before carrying out to this overview it seems adequate to introduce the different GW topologies, and comment their main parameters and characteristics. In this way the overview will be better followed.

\subsubsection{Gap waveguides}

All the different GWs are based on a EBG structure whose operation principle is explained in Fig. 8.22. In general, a periodic lattice of square metal pins, see Fig. $8.22(\mathrm{a})$, is used to conform the EBG (other similar options will be commented later). This periodic lattice is described by the pins width $w_{p}$, the pins height $h_{p}$, and the period $p$. Following the principles described in [783], in a first approximation, it is found that, at the frequency $f_{0}$ at which $h_{p}=\lambda_{0} / 4$, a condition of Perfect Magnetic Conductor (PMC) is created at the plane over the pins, see Fig. 8.22(a). In this 
situation, if a Perfect Electric Conductor (PEC) (implemented by a common metallic plate) is placed at a distance $h_{a}$ less than $\lambda_{0} / 4$, a EBG is created and no wave can propagate transversely inside the structure, see Fig. 8.22(b). The EBG condition occurs not only for the central frequency $f_{0}$ but for certain stopband (that can be surprisingly wide in some cases) which depend on the periodic lattice parameters. This stopband is determined by the undesired propagation of the Parallel-Plate cavity modes. Given this structure that does not allow propagation inside certain stopband, some modifications can be applied in order to create waveguiding channels. The way in that these modifications are done drives to the different Gap Waveguides, described next.

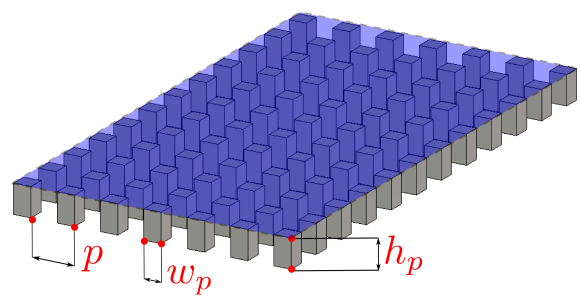

(a)

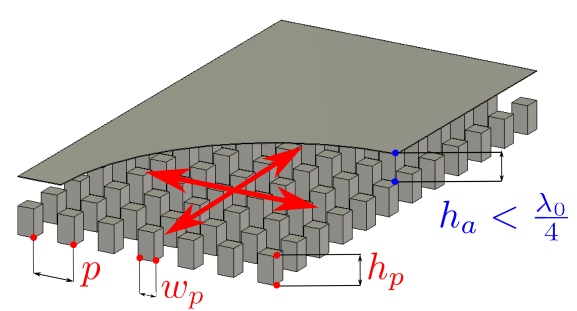

(b)

Figure 8.22: Operation principle of the Electromagnetic BandGap (EBG) structure in Gap Waveguides (GWs). a) A Perfect Magnetic Conductor (PMC) condition is created at the plane over the pins when their height is $h_{p}=\lambda_{0} / 4$; b) If a Perfect Electric Conductor (PEC) (metallic plate) is placed at a distance $h_{a}$ less than $\lambda_{0} / 4$, no wave can propagate inside the structure. This is achieved not only at the central frequency of design $f_{0}$, but for certain stopband that depends on the design parameters.

\subsubsection{Ridge Gap Waveguide (RGW)}

The first proposal to modify the EBG structure of Fig. 8.22(b) to create a waveguide was to place a metallic ridge of width $w$ on the same plane of the pins, see Fig. 8.23. This structure is called Ridge Gap Waveguide (RGW). When the ridge is placed, a propagation channel is created between the ridge top and the top metal plate, see green zone of Fig. 8.23(a). At the sides, the periodic structure does not allow the waves to spread laterally (red zones of forbidden propagation in Fig. 8.23(a)), being the waves confined mainly over the ridge. This can be appreciated in Fig. 8.23(c), where the E-field magnitude of the fundamental mode of the RGW is shown. Note that confined propagation is achieved despite of the fact that any contact is present between the two pieces forming the structure inasmuch as the bottom piece with the pins and the top metal plate are separated a distance $h_{a}$.

Although some modifications can be done, generally, the ridge height has the same height as the pins, i. e., $h_{p}$. Under this condition, following an image theory approach, the PMC plane causes the structure to be equivalent to an stripline filled by air. Therefore, the RGW inherits the stripline quasi-TEM features. However, this is done without the use of any dielectric and without the requirement of any metallic contact between pieces. Furthermore, note that any spurious surface mode or resonance cavity mode can appear in the structure inside the stopband. Therefore, 


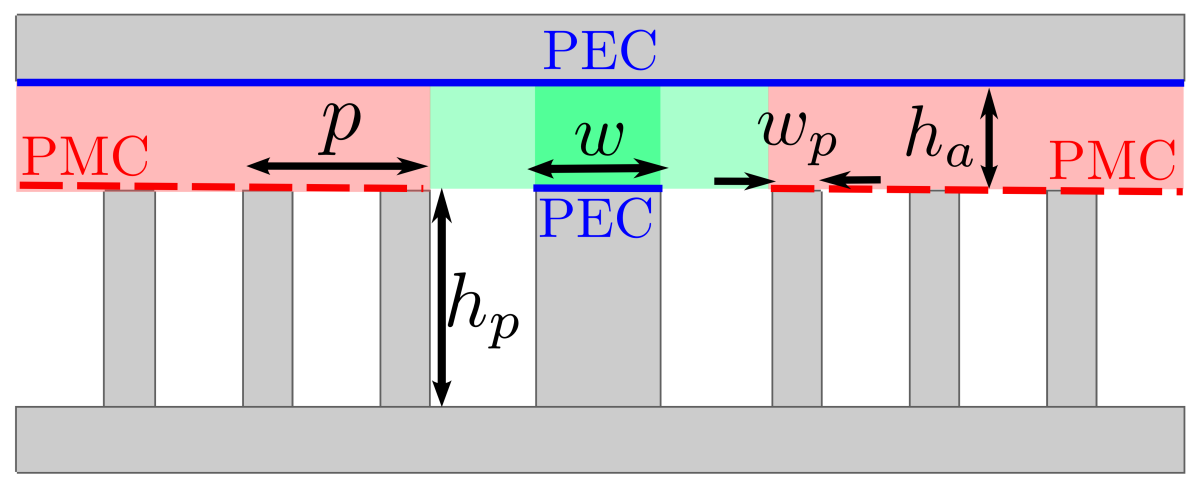

(a)

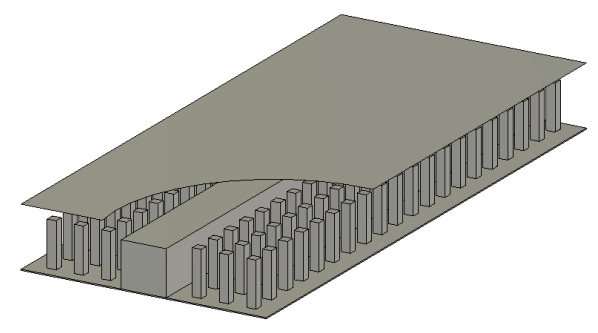

(b)

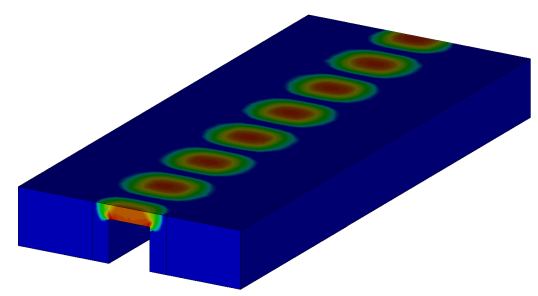

(c)

Figure 8.23: Ridge Gap Waveguide $(R G W)$. a) Transversal view and main parameters. The specific parameter of this $G W$ is its ridge width $w$. Generally the ridge height is the same as pins height $h_{p}$; b) $3 D$ view; c) E-field inside the structure.

it is already observed with this structure how GW topology faces all-in-one the main problems affecting mm-wave waveguides.

An alternative proposal to the RGW is the so-called Microstrip Ridge Gap Waveguide (MRGW), shown in Fig. 8.24. As commented before, the periodic structure is not necessarily formed by square pin elements, but other topologies can be used. One important case are mushroom-type structures, proposed by Sievenpiper [786], and that can be appreciated in Fig. 8.24(a). The microstrip RGW employ these elements, consisting on a patch over a dielectric substrate, connected to the ground by means of a metallic via, instead of square metal pins. The ridge is created in a similar way: a metallic strip is grounded by means of metallic vias. This allows to reduce the waveguide thickness and to make it compact. In addition, the structure demands for totally different manufacturing techniques than those required by the conventional RGW. On the other hand, dielectric is employed and, although propagation takes place mainly in the air region, the small field leakage induce some additional losses which are not negligible. Therefore, the MRGW is a more compact version of the RGW, presenting additional losses, and demanding from Printed Circuit Board (PCB) manufacturing techniques, thus covering different application requirements than those of the RGW. 


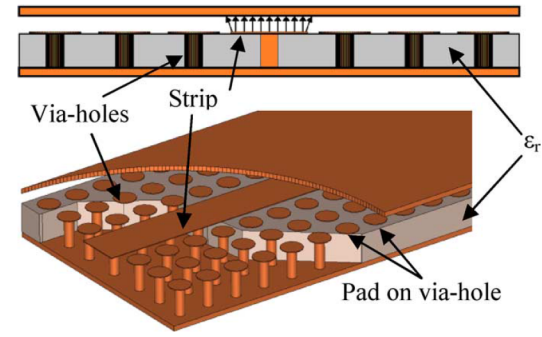

(a)

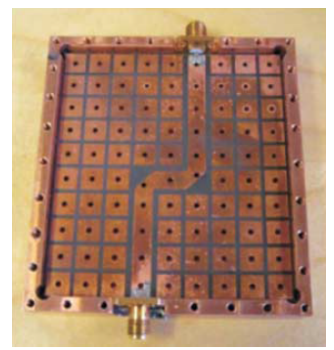

(b)

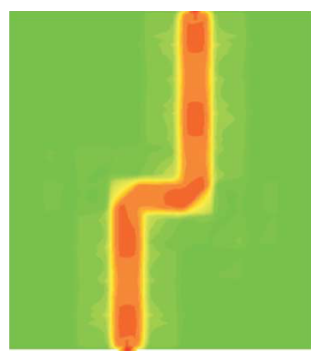

Figure 8.24: Microstrip Ridge Gap Waveguide (MRGW). a) $3 D$ and transversal views [787]; b) Top view from a prototype and E-field in the structure [788].

\subsubsection{Suspended-Strip Gap Waveguide (SSGW)}

Complex circuit layouts in RGW require from a redistribution of the pins, which may complicate the design in some cases. A solution for this situation is given by the Suspended-Strip Gap Waveguide (SSGW), Fig. 8.25. In the SSGW, a fixed periodic lattice is used. Over it, a substrate with a printed metallic strip is placed, allowing in this way propagation between the strip and the top metal plate (see green zone of Fig. 8.25(a)). Consequently, propagation principles of the SSGW are very similar to those of the RGW, hence similar characteristics are found (airfilled stripline behavior). See for instance Fig. $8.25(\mathrm{c})$, where the magnitude of the fundamental mode E-field is shown in the structure. As it can be appreciated the plot is very similar to that of Fig. 8.23(c). However, in the SSGW configuration, there is a slight field leakage under the strip (unlike in the bulky ridge case). This causes some increment of losses due to the interaction of the EM fields with the underlying pins and dielectric substrate. In addition, by obvious reasons, the RGW is a more robust structure. The specific parameters of the SSGW are the strip width $w$, the strip thickness $t$, the substrate thickness $t_{d}$, and the substrate properties $\left(\varepsilon_{r}\right.$, $\tan \delta$ ). It is also common in the case of the SSGW to find some designs employing other suitable periodic elements such as mushroom-type ones.

\subsubsection{Groove Gap Waveguide (GGW)}

A different option to enable propagation from the original EBG structure is to remove some pin rows to create a groove, as it is shown in Fig. 8.26. This topology is called Groove Gap Waveguide (GGW). Once the rectangular groove channel is created (green zone of Fig. 8.26(a)), the forbidden propagation zones (red zones of Fig. 8.26(a)) force the waves to propagate along this channel. Consequently, the GGW acts in a similar way as a rectangular waveguide. This can be appreciated in Fig. 8.26(c), where the E-field magnitude of the fundamental mode of this waveguide is shown. However, note the key difference: the GGW is formed by two independent pieces that do not require metal contact to guide the waves confined inside the propagation channel without leakage. The main specific parameter of the GGW is, as it is in the rectangular waveguide, the waveguide channel width $a$. The height of the waveguide is determined as $b=h_{p}+h_{a}$. 


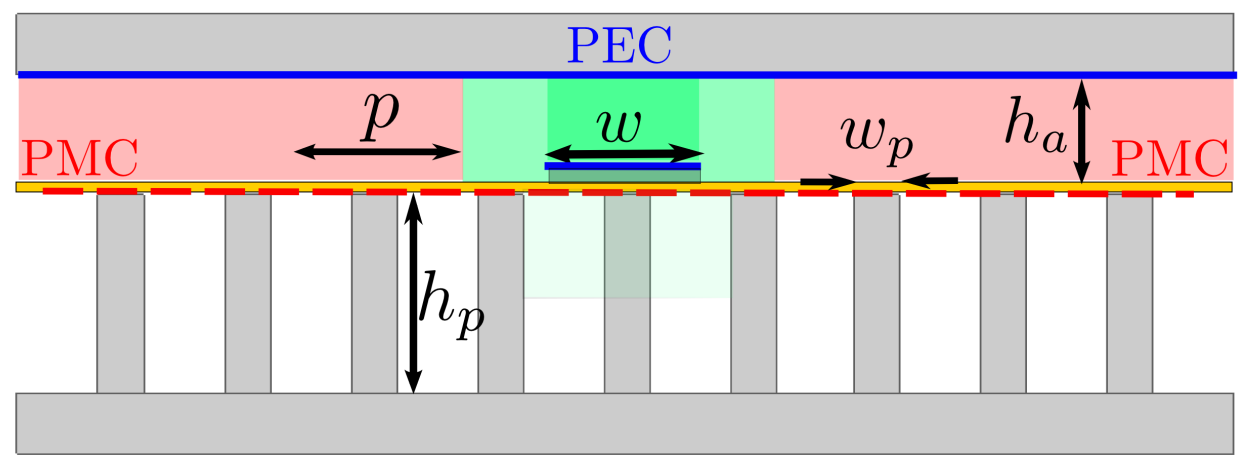

(a)

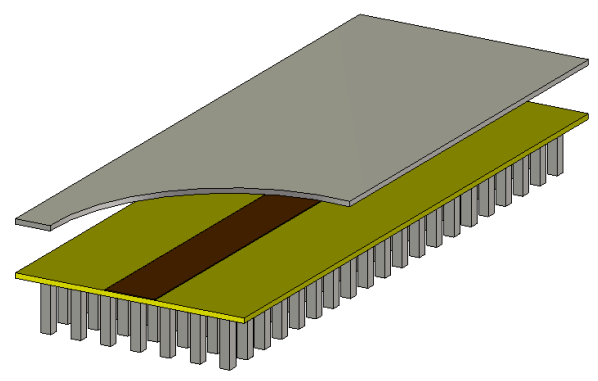

(b)

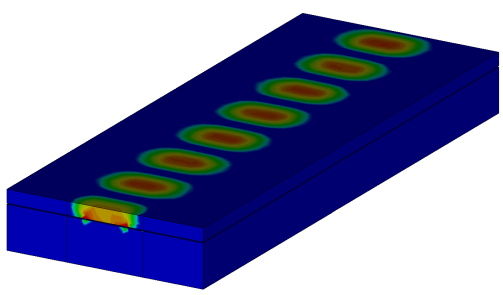

(c)

Figure 8.25: Suspended-Strip Gap Waveguide (SSGW). a) Transversal view and main parameters. The specific parameters of this $G W$ are the strip width $w$, the strip thickness $t$, the substrate thickness $t_{d}$, and the substrate properties $\left.\left(\varepsilon_{r}, \tan \delta\right) ; b\right) 3 D$ view; c) E-field inside the structure.

With a similar principle, the versatility of the EBG structure allows to create an alternative GGW. This is the Groove Gap Waveguide Horizontal Polarization $(\mathrm{GGW}-\mathrm{HP})^{6}$, shown in Fig. 8.27. In the GGW-HP, the rectangular propagation channel is created in such way that is larger in the vertical dimension. Since the electromagnetic fields are forced to to propagate in this channel (see orange zone of Fig. 8.27(b)) due to the surrounding EGB structure zones, one has an equivalent rectangular waveguide, but rotated 90 degrees respect to the conventional GGW (GGW-VP). This causes the E-field polarization to be horizontal, hence the name of the waveguide. As in the case of the GGW-VP, the GGW-HP acts as a rectangular waveguide with the advantage of not requiring any metal contact between the two pieces forming the waveguide. In Fig. 8.27(c), the fundamental mode E-field magnitude is shown inside a GGW-HP. A cut has been applied in the vertical symmetry plane of the structure to appreciate the E-field pattern. As it can be observed, this pattern is very similar to the classical pattern of a rectangular waveguide.

Nevertheless, some differences respect to the GGW-VP case are observed. Due to the horizontal E-field polarization, some local effects take place close to the pin

\footnotetext{
${ }^{6}$ In some text it is common to found the original GGW as Groove Gap Waveguide Vertical Polarization (GGW-VP). Generally, once adopted the configuration, either VP or HP, the waveguide is referred simply as GGW.
} 


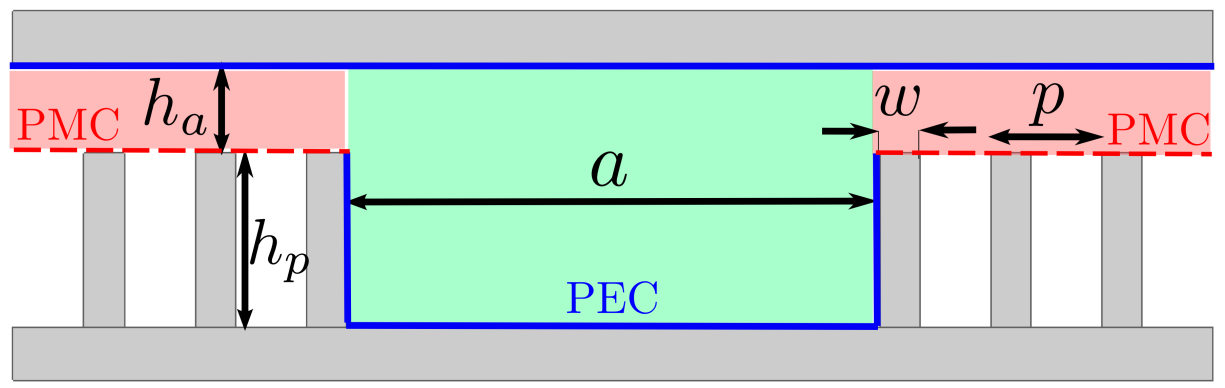

(a)

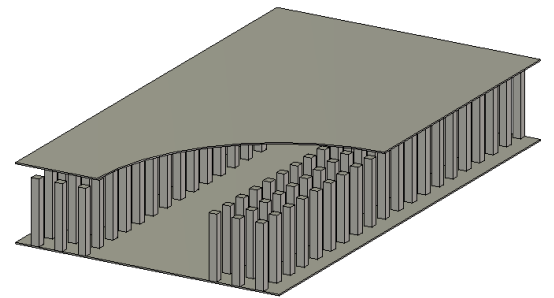

(b)

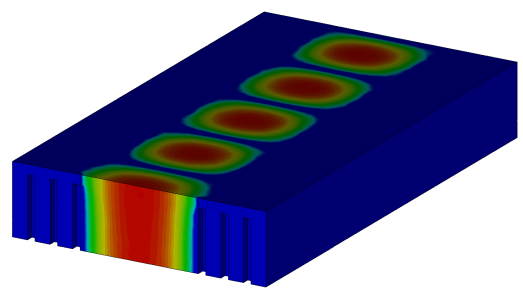

(c)

Figure 8.26: Groove Gap Waveguide $(G G W)$. a) Transversal view and main parameters. The specific parameter of this $G W$ is its propagation channel (in green) width $a$; b) $3 D$ view; c) E-field inside the structure.

surface, where the polarization is rotated to be vertical (E-field lines going from the pins top to the top plate), so that the GGW-HP is less similar to the rectangular waveguide than it is the GGW-VP. The specific parameters of this waveguide are its width $a$ and its height $b$. Note that in the GGW-HP the width is controlled by the groove depth, which makes the groove go under the pin plane level. In the case of the GGW-HP, the parameter $b$, adjusted by the separation between the two lateral pin zones, has an important behaviour on the waveguide characteristics.

\subsubsection{Overview of Gap Waveguide Technology}

Once the different Gap Waveguide topologies have been introduced, the overview of Gap Waveguide Technology proposals and state of the art can be carried out appropriately. The overview starts with the early general papers, and with those in which the different GW topologies were presented for the first time, opening the scenario for more advanced designs. After these set of papers, the number of GW related papers has increased exponentially up to this moment. Since the number of proposals to be commented is rather large, they are classified by groups and treated in a proper order in such way that motivation, properties, uses, and conceptual and technological aspects of GW technology are understood in a proper way. First, some works dealing with the evaluation of GWs losses are commented in order to quantify the improvement provided by GWs respect to conventional technologies. Then, a 


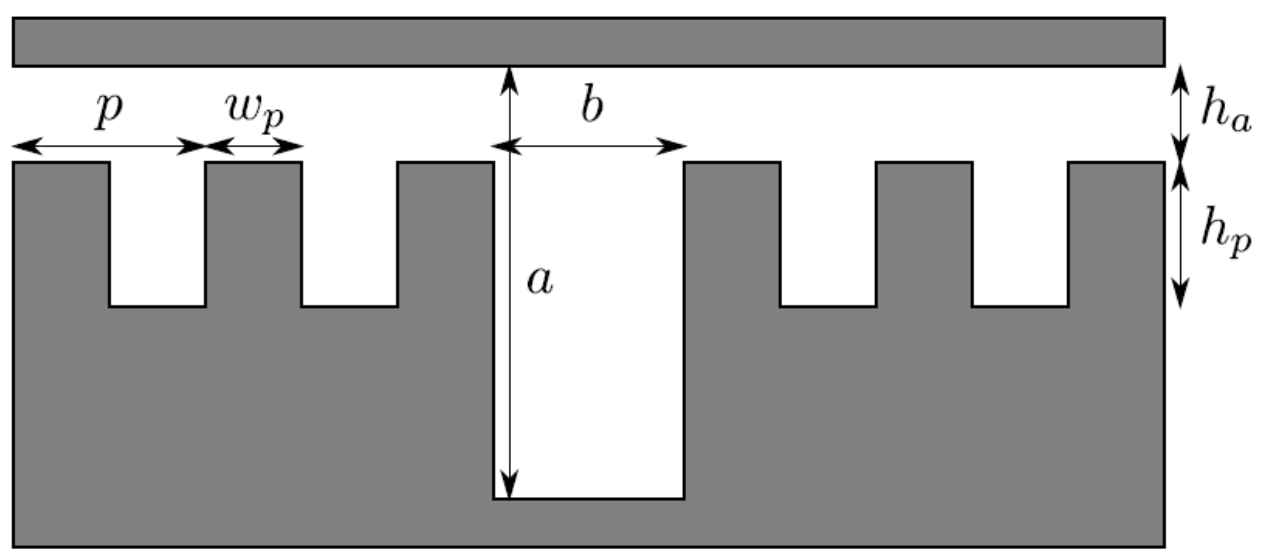

(a)

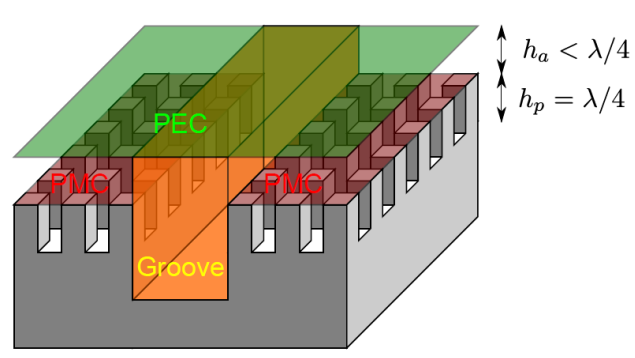

(b)

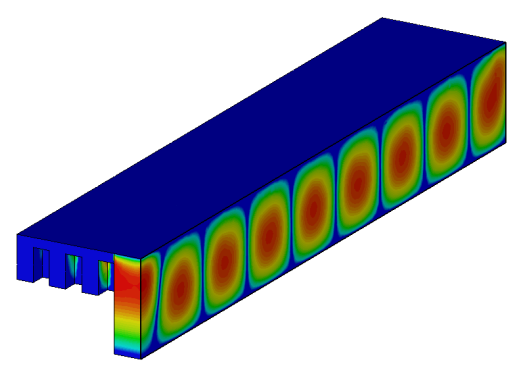

(c)

Figure 8.27: Groove Gap Waveguide Horizontal Polarization (GGW-HP). a) Transversal view and main parameters. The specific parameter of this $G W$ are its propagation channel width $a$ and height $b$; b) $3 D$ view; c) E-field inside the structure (the structure is cut at its symmetry plane to show the field inside the propagation channel).

bulk part of the matter is given through the review of several device proposals, which mainly consist in antennas and filters. After that, transition and flanges works are mentioned, as an important demanding element from GW technology due to compatibility reasons. To finish with review of GW components, special treatment is given to those designs involving very high frequencies (beyond $f=100$ $\mathrm{GHz}$ ) inasmuch as different manufacturing approach and working environment is found. Then, after the different GW devices topologies are understood, a key topic is afforded: the modeling of GW structures through appropriate analysis methods. Finally, although with less relevance in this thesis, some packaging related works are commented to give completeness to this study with this additional (and quite relevant) use of GW technology.

As commented before, it was in 2009 when Prof. Kildal, following his background in soft and hard surfaces [783]-[784], and due to the recently obtained good results in 2008 with hard waveguides [785], conceived and proposed the idea of Gap Waveguides. Firstly, the RGW was proposed in [712] as a planar transmission line, contactless, avoiding dielectrics, and giving inherent good packaging features. In 
this way, the proposal was foreseen to overcome SIW waveguides, conceived to fill the gap between hollow waveguides and classical planar lines. In the paper ([712]) it is highlighted an important difference between GWs and other waveguides including periodic structures, e. g., metamaterial waveguides. Whereas in these last propagation takes place inside the periodic structure, in GWs the periodic structure just encloses the waves to propagate in suitable low-loss channels. It must be noted, however, that the period in GWs is usually comparable with the wavelength, hence GWs are not, indeed, metamaterial structures. At first, equivalence between the RGW and an inverted microstrip was suggested. Quasi-TEM behaviour was observed inside an stopband ranging from $10 \mathrm{GHz}$ and $19 \mathrm{GHz}$, with the fields falling off away from the ridge at a rate of $10 \mathrm{~dB}-20 \mathrm{~dB}$ per row.

Very soon, in the same year, different topologies were conceived. In [360], Kildal added the MRGW and the GGW structures to the already presented RGW. In this work it was observed that conventional pin structures achieve roughly 2:1 bandwidth stopbands whereas Sievenpiper mushrooms may reach up to 4:1 bandwidth stopbands. Also, the GGW (called then as Ridge GGW) was presented as a dispersive alternative to the RGW, surprisingly referring to it as a less interesting alternative, something that changed later. After the proposal, some initial general characterization papers came out. In 2009, numerical characterization of a RGW was afforded by assuming a PMC condition over the pins in [789]. In this paper, a method to determine the operating bandwidth, based on the analysis of fields propagating inside the structure was presented. However, it was found that the PMC approximation results rough.

A year later, in 2010, design and experimental verification of RGW was provided in a quite complete paper [790]. In that paper, an stopband from $10 \mathrm{GHz}$ to $23 \mathrm{GHz}$ was designed, and an experimental device to give proof of concept was manufactured. The device consisted in a RGW line with two $90^{\circ}$ bends, excited by means of coaxial cables. A Thru-Reflect-Line (TRL) [791] calibration kit was designed to extract the actual S-parameters. In this work is found the famous E-field simulation showing how the waves follow the ridge despite of the fact that there are now walls at its sides (see Fig. 8.28). Notice how outside the stopband the fields spread laterally in the PPW cavity. The measured result gave validation to such simulation, hence confirming the GW technology potential.

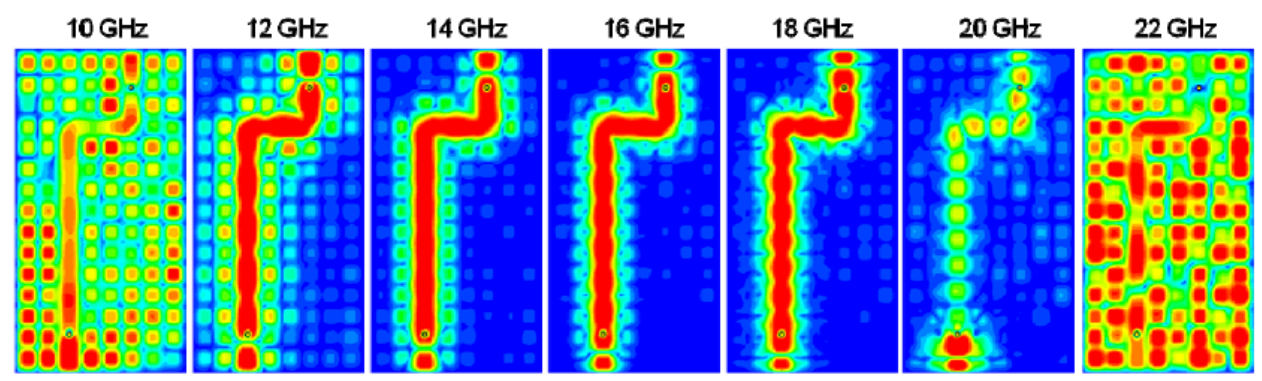

Figure 8.28: Computed vertical E-field distribution at the surface of the top metal plate of the demonstrator used in [790], which consists on a RGW waveguiding path including two $90^{\circ}$ bends. 
The first relevant parametric study regarding GWs was carried out by Rajo in [792]. Following the work in [793], dealing with EBG structures, the influence of the periodic structure parameters on the stopband bandwidth was deeply analyzed. The pin height was studied founding that the condition $\lambda_{0} / 4<h_{p}<\lambda_{0} / 2$ must be satisfied, with the previous limits given by the change of the pin surface plane impedance properties from inductive to capacitive and vice versa. The most influential parameter regarding the stopband bandwidth was found to be $h_{a}$ : the smaller $h_{a}$, the wider the stopband. In addition, given $h_{a}$ there is an optimum $w_{p} / p$ ratio. It is worth also to highlight that this work shows that the same stopband can be achieved with different periodic structure parameters. For instance, small pins and small periods $\left(p \approx 0.05 \lambda_{0}\right)$ and large pins with moderate periods $\left(p \in\left[0.15 \lambda_{0}-0.2 \lambda_{0}\right]\right)$ achieve a similar stopband with large bandwidth if the rest of parameters are chosen accordingly. It is highlighted that, in order to maximize the stopband, the period of periodic structure must be comparable with the waveglength, i. e., much larger period than that of metamaterial designs. Pins of circular shape were also studied, founding that the same behavior regarding to the square pin case is obtained whenever the radius is $r=a / 2$ (in general, pins with similar area are expected to work very similar, however, rectangular shape facilitates the manufacturing by means of conventional milling process, and, in general, for most of alternative manufacturing techniques). Regarding to the periodic lattice, it was not found appreciable differences in using other lattices such as a triangular one, resulting in that the original rectangular lattice is more convenient to alleviate the manufacturing process complexity. It is also determined that, since mushroom-type periodic structures give compactness and require dielectrics, they are mainly restricted to lower frequencies.

The first dedicated analysis to the GGW was done also by Rajo in [794]. The periodic structure designed in that work provided an stopband from $10 \mathrm{GHz}$ to 20 $\mathrm{GHz}$. It was found that the propagation constant of a GGW is quite similar to that of the rectangular waveguide, however, the larger differences in the case of a GGW$\mathrm{HP}$ are evidenced and highlighted. It was also found larger confinement (less fields leakage to the pin structures at the lateral sides) for the GGW-HP, justified by the larger exposed metallic area in the practiced groove when using this configuration. The combination of both geometries, GGW-VP and GGW-HP was also conceived as a great opportunity to implement dual-polarized horn antennas.

Similarly, the first serious consideration of the SSGW is found mainly in [795], but also coeval in [796]. These works come after it was found that, as a RGW circuit layout becomes more complicated, the location of the pins, conditioned by such layout, increases the complexity of both, design and manufacturing processes, see Fig. 8.29(a) [795]. The SSGW is presented as a simpler and cheaper alternative with less performance than the RWG. In [795], it is mainly highlighted that the SSGW may be used in the prototype stage when carrying out designs with the RGW. However, the posterior works have shown that differences between both waveguides may suggest a direct design in the RGW topology. In [796], it is highlighted the similarity of the SSGW with the microstrip (more evident than the similarity of a microstrip line with a RGW). In this work, the SSGW is endorsed as a solution to the difficult feeding of slot arrays from microstrip lines, very affected by the packaging resonances. 
Nevertheless, it can be also affirmed that the SSGW may find its niche as a low-cost structure for mass-production circuits. Note that, in principle, only the substrate with the printed layout is required to be designed, and a universal pin lattice could be applied, which would noticeably diminish the total circuit cost. See for instance in Fig. 8.29(b) the aspect of the SSGW corporate feed designed in [795]. The designed stopband in that work was $[10 \mathrm{GHz}-20 \mathrm{GHz}]$. The authors highlight that the carried out analysis indicates that unless the strip width $w$ is very large, the air-filled stripline model, assuming a PMC condition over the pins, results very rough. This can be appreciated in Fig. 8.29(c), which shows the characteristic impedance $Z_{0}$ vs. the strip/ridge width $w$ for a RGW, a SSGW, and an ideal transmission line model based on a stripline. In addition, an important drawback of the SSGW is observed in this study: as the strip width is reduced, there is an inflexion point from which $Z_{0}$ does not increase any more, but falls down, preventing the SSGW to achieve impedance values beyond $60 \Omega$. This occurs because when the strip becomes narrower than the pins, it actually sees them, and this interaction makes the strip to be effectively wider.

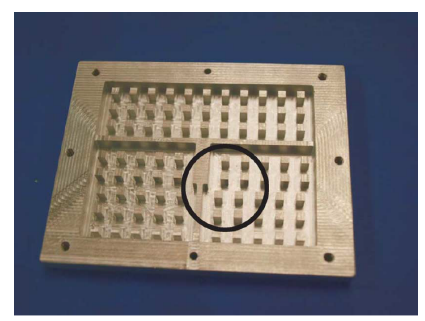

(a)

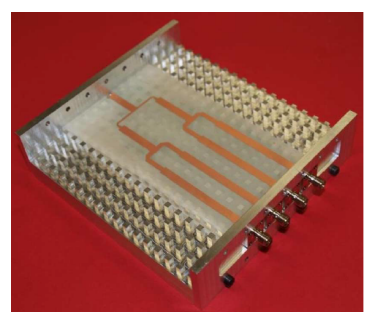

(b)

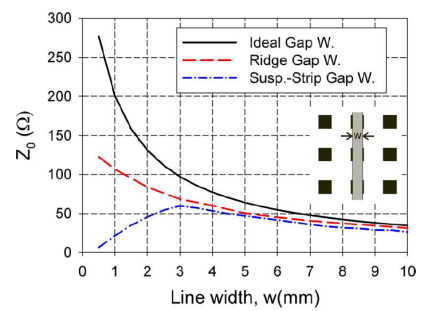

(c)

Figure 8.29: Suspended-Strip Gap Waveguide proposal in [795]. a) Motivation of the SSGW: required ad hoc pin distributions according to the circuit layout in $R G W$; b) Corporate-feed network made using SSGW lines. The top plate is removed to show the circuit; c) Characteristic impedance $Z_{0}$ vs. line width at $f=15 \mathrm{GHz}$. Comparison between $R G W$ and SSGW. The characteristic impedance of an ideal transmission line model assuming a PMC layer over the pins (stripline model) is added for comparison.

In addition, the impedance was found to have a large value excursion depending on the strip position relative to the pins. Therefore, the circuit layout was conditioned by the position of the underlying pins, complicating the process. For instance, $3 \lambda / 4$ impedance transformers are used instead of the common $\lambda / 4$ transformers in order to allow enough pin periods to be seen by the transformers, hence having the desired impedance effect. It is foreseen that denser pin lattices are required, a solution treated later in this part of the thesis, and that the literature has confirmed recently. In addition, the lack adequate transitions for GWs was found to be an important technological issue to address.

For the SSGW, an innovative alternative design using dielectric pins instead of metallic ones is found in [797]. Fig. 8.30(b) shows the manufactured prototype (top metal plate not present) with a periodic structure of $16 \times 16$ dielectric pins over a dielectric substrate, embedded in a metallic groove. The possibility of using dielectric pins is explored in this work since it may alleviate the manufacturing process and allow for easy integration of active devices. It is highlighted that the main problem 
for active device integration is the device height, generally larger than $h_{a}$. With the use of dielectric pins, the active device may be located between pins without the risk of producing a short-circuit.

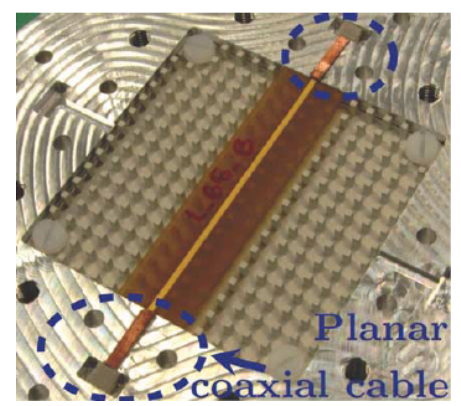

(a)

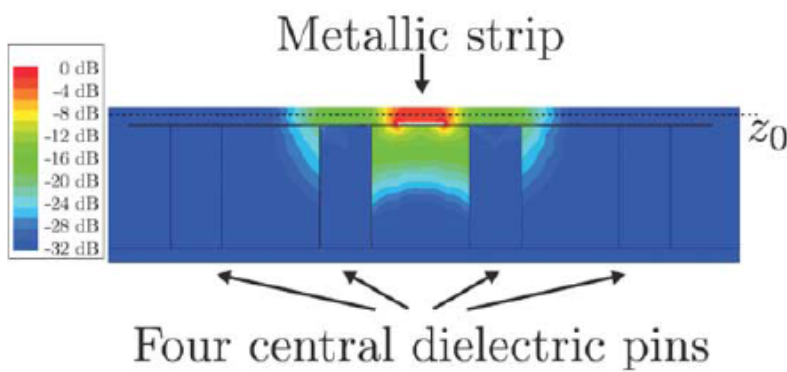

(b)

Figure 8.30: Suspended-Strip Gap Waveguide with dielectric pins proposed in [797]. a) Manufactured prototype (top plate not present); b) Total electric field on a transverse plane passing through a pin row at $f=37 \mathrm{GHz}$.

Best results are found for high permittivity dielectrics. Particularly, using Rogers $\mathrm{TMM}^{\circledR} 10 \mathrm{i}\left(\varepsilon_{r}=9.9, \tan \delta=0.08\right.$ in the Ka-band), a periodic structure with parameters $p=3.07 \mathrm{~mm}, w_{p}=1.05 \mathrm{~mm}, h_{p}=2.56 \mathrm{~mm}$, and $h_{a}=0.4 \mathrm{~mm}$ provides a stopband of $R B W=17.34 \%$ around $f=36.6 \mathrm{GHz}$. This stopband is much narrower than the metallic pin structure one $(R B W=71.84 \%)$, however, this will not result a limitation for almost any designs, being that there are components in the device that usually present a narrower bandwidth response, a fact that will be appreciated along this section.

The SSGW is formed using a gold-plated copper metallic strip with a thickness $t=35 \mu \mathrm{m}$ and a width $w=0.95 \mathrm{~mm}$, suspended on Pyralux AP-912R of DuPont $\left(\varepsilon_{r}=3.6\right.$ and $\left.\tan \delta=0.06\right)$, with thickness $t_{d}=50.8 \mu \mathrm{m}$. Losses of the presented SSGW are $0.44 \mathrm{~dB} / \mathrm{cm}$, slightly higher than those of the conventional SSGW, of $0.34 \mathrm{~dB} / \mathrm{cm}$. This indicates that the leaky field around the strip has a more lossy interaction with dielectric pins than for metallic ones. Fig. 8.30(b) shows the total electric field on a transverse plane passing through a pin row at $f=37 \mathrm{GHz}$. Although the E-field intensity is quite low inside the dielectric pins, it is enough to cause certain extra loss. Finally, the authors highlight that other structures such as metalized dielectric RGW are as well possible with similar technology. These kind of structures may take soon advantage of 3D dielectric printers technology. The light way of such structures may also result very suitable for applications with weight restrictions such as satellite communications. Although other basic structures and functionalities must mature first in GW technology, an eye must be kept in this possibility for future advanced devices including active elements.

Finally, the first dedicated work with the MRGW structure is found in [788]. The proposed topology characteristics and performance lie in between of those given by the RGW and the SSGW. Due to the similarities with the microstrip line, the MRGW seems as a good alternative to it, more suitable than the conventional (some alternatives will be presented later in this thesis) SSGW due to the aforementioned 
problems with the impedance. In the work of [788] it is mainly highlighted the elimination of the problem of packaging resonances and surface modes. The stopband is designed to be $[5 \mathrm{GHz}-11.5 \mathrm{GHz}]$. It is noted that at $f=10 \mathrm{GHz}$ the required strip width to achieve $Z_{0}=50 \Omega$ is $w=5 \mathrm{~mm}$, a much wider strip than in microstrip designs, which translates in lower ohmic losses. Simulations show that packaging with MRGW is noticeably better than in the case of a conventional microstrip circuit, strongly affect by the undesired resonances.

\subsubsection{Study of losses}

Study of losses in GW structures has been deeply afforded by Pucci in [796]-[800]. The motivation of her work is found to be the lack of precision of previous loss measurements, carried out with the TRL calibration kit presented in [801]. Since waveguide losses are very small for short propagation distances, another approach seems necessary. The proposed method is based on evaluating the quality factor $Q$ of resonators created with the different GWs, being that this parameter is directly related with the waveguide loss.

In [796], a [7 GHz-14 GHz] stopband is designed and a comparison of losses is done by means of the quality factor method for three similar GW structures: a conventional SSGW $(Q=2197)$, a mushroom-type SSGW $(Q=1357)$, and a MRGW $(Q=1709)$, thus giving valuable information in order to chose between these low-cost quasi-TEM GW options given certain application environment. It is highlighted that all of them overcome by far microstrip losses $(Q=602)$.

In [798], a $[12 \mathrm{GHz}-17 \mathrm{GHz}]$ stopband is designed, insomuch as the main frequency is considered to be $f_{0}=13.5 \mathrm{GHz}$. Simulations indicate that a RGW presents a quality factor of $Q=4713$ at this frequency, much higher than a conventional microstrip line, which reaches only $Q=179$ due to both, larger ohmic and dielectric losses. On the other hand, the RGW cannot reach the rectangular waveguide levels, located at $Q=8463$. It is found, however, that increasing $h_{a}$ at the cost some stopband bandwidth sacrifice, may drive the RGW to achieve $Q=5500$, a reasonable good value.

In [799], the analysis is focused on the MRGW in the context of the consideration of this waveguide as a compact low-cost solution with simple manufacture for printed slot array antennas. It is pointed out that grounding the ridge strip with vias is essential to reduce losses, being that if this is not done, the EM fields extend under the strip, into the substrate, and spread more laterally (see Fig. 8.31(a)), hence increasing both, ohmic and dielectric losses. To calculate MRGW losses, the resonator of Fig. 8.31(b) is implemented. The ridge strip is totally surrounded by the mushroom periodic lattice, forming in this way an open-circuit ended resonator. At $f=10 \mathrm{GHz}$, the quality factor of this resonator is found to be $Q=974$, a performance noticeably lower than that of the RGW, but as well noticeably higher than that of the conventional microstrip, which is the reference structure.

Finally, in [800], the work of [798] is extended to the GGW, giving a variety of measurements for both topologies, GGW and RGW, and providing an interesting and necessary comparison with the rectangular waveguide, in order to determine differences of such conventional technology with GW technology. Calculation of losses is done by means of different resonators, shown in Fig. 8.32. For each topology, 


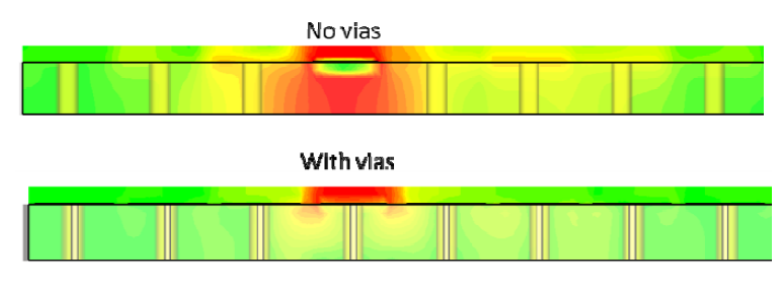

(a)
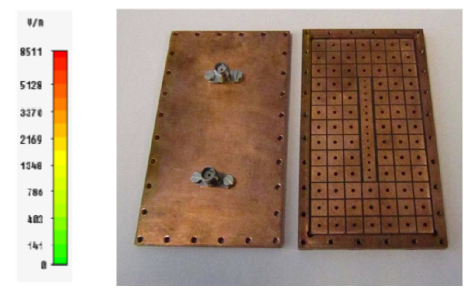

(b)

Figure 8.31: Study of MRGW losses in [799]. a) Comparison of E-field magnitude distribution in the MRGW for the cases of a strip without grounding vias (up) and a strip with grounding vias (bottom); b) Manufactured prototype of a MRGW resonator for the calculation of losses through the quality factor method. The resonator is totally surrounded by the periodic structure, thus being an open-circuit ended resonator.

RGW and GGW, short-circuit ended resonators (see Figs. 8.32(a) and 8.32(b)) and open-circuit ended resonators (see Figs. $8.32(\mathrm{c})$ and $8.32(\mathrm{~d})$ ) are considered. Thanks to this approach it is determined that open-circuit resonators, i. e., when the cavity is totally surrounded by the periodic lattice, give better performance. Specifically, at $f=13 \mathrm{GHz}$, the open-circuit RGW resonator gives $Q=4130$, whereas the shortcircuit one gives $Q=2255$, roughly a half. In the case of the GGW, open-circuit resonator gives $Q=5883$, whereas the short-circuit one gives $Q=5200$.

The most relevant achievements of the work in [800] are, first, that the GGW present lower losses than the RGW, thus motivating the interest in this waveguide, less considered than the RGW until that moment. Second, it is found that the rectangular waveguide topology is less robust to the manufacturing process than it is the GGW. Although the rectangular waveguide presents higher quality factor than the GGW in theory, the manufactured rectangular waveguide cavity gives $Q=5400$, only $65 \%$ of the predicted value. The manufactured GGW resonator achieves $96 \%$ of the predicted value in simulations. It must be commented that, probably, the employed milling manufacturing techniques are not the best-performance according to the state of the art, being that the frequency is low for the rectangular waveguide present such degradation. However, it is undeniable that the GGW topology is more robust to the manufacturing process. It is recovered that hollow waveguide cavities reach in practice as much as $80 \%$ of their predicted value [704].

More recently, in [787], characterization and study of MRGW losses is afforded at the more challenging operation frequencies of $\mathrm{V}$-band: the designed mushroom structure creates a stopband from $45 \mathrm{GHz}$ to $72 \mathrm{GHz}$. In this work, long lines, including bends are used to obtain the losses in the usual way, instead of resonators. This work treats deeply the influence of the surrounding mushroom elements, founding that they must be carefully displayed according to the shape of the circuit. As a circuit example, a 90 degrees bend is studied. The performance degradation caused by the modification of the periodic elements surrounding the circuit is noticeable (the closer the element to the discontinuity the larger the influence), thus arising that the MRGW suffers from the same design and implementation drawback as the RGW, i. e., the circuit layout strongly conditions the periodic structure layout. Strip horizontal shifts on straight paths are not very influential, but it is highlighted that 


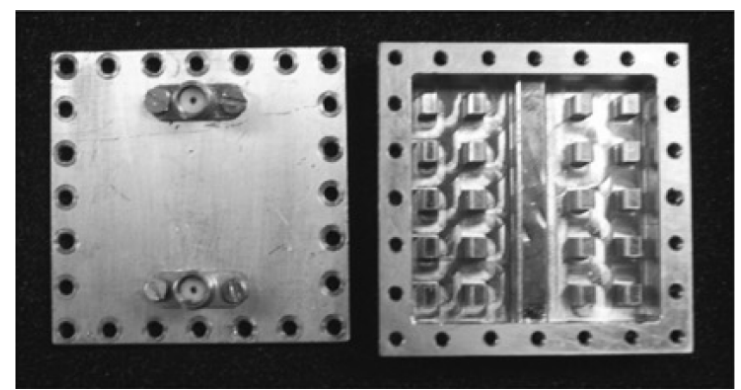

(a)

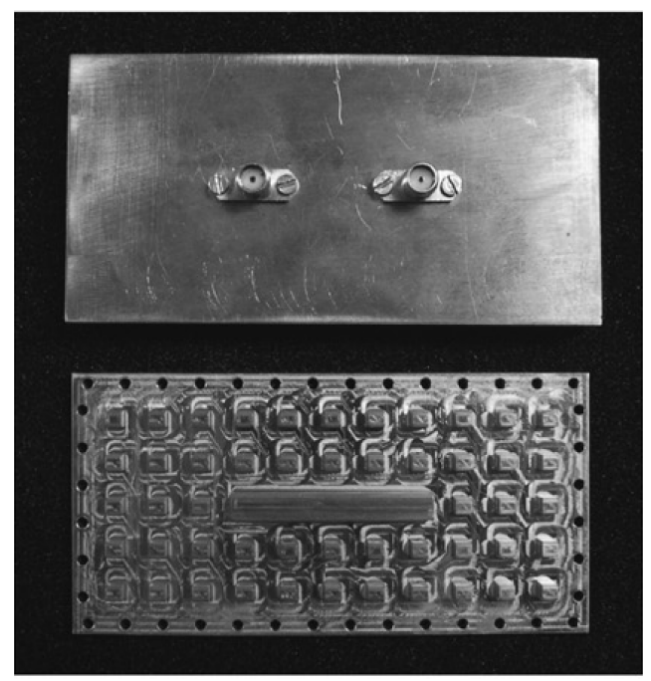

(c)

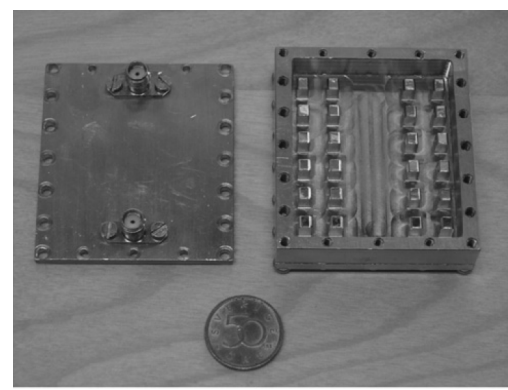

(b)

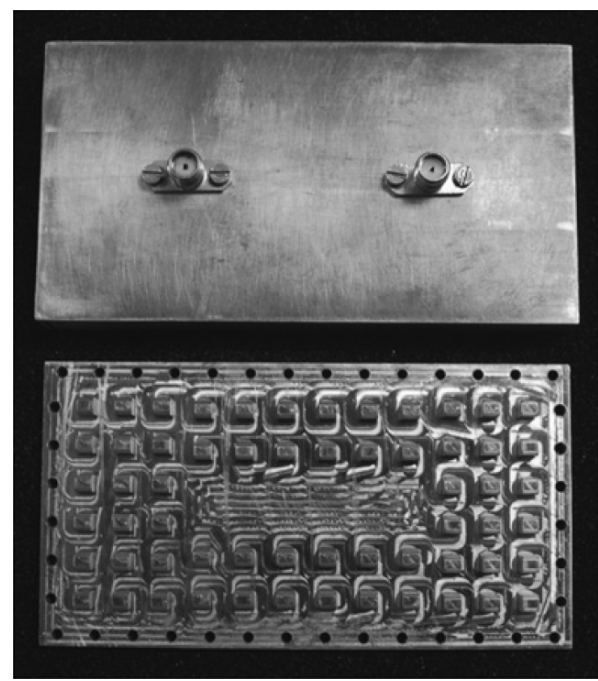

(d)

Figure 8.32: Different resonators employed for the study of losses in RGW and GGW by means of the quality factor method in [800]. a) Short-circuit ended RGW resonator; b) Short-circuit ended $G G W$ resonator; c) Open-circuit ended $R G W$ resonator; d) Open-circuit ended $G G W$ resonator.

the contact of the strip with the surrounding elements must be necessarily avoided, inasmuch as the performance of the circuit is totally lost when this occurs .

It is as well found that Electroless Nickel Immersion (ENIG) is not suitable for this topology, whereas silver-platted copper gives acceptable results. Losses of the manufactured MRGW are about $0.18 \mathrm{~dB} / \mathrm{cm}$, twice than simulated ones. Although this attenuation clearly overcomes microstrip line or inverted microstrip line performance, it is very similar to that obtained with SIW structures $(0.15 \mathrm{~dB} / \mathrm{cm})$. Since these last are very easy to manufacture and well adopted by the research community, the applicability of the MRGW will depend on the use of proper manufacturing techniques exploiting all its potential. Other remarkable points in the work are the designed WR-15 to MRGW transition (the WR-15 enters from the bottom plate and the E-field is twisted, providing matching with a stub implemented with the strip), 
with only $0.2 \mathrm{~dB}$ of insertion losses, and the implemented bend, presenting only 0.04 $\mathrm{dB}$ of losses.

\subsubsection{Antennas}

By far, the most prolific area in the GW technology are antennas, this probably caused due to the fact that GW technology originates from soft and hard surfaces concepts, coming from area of antennas in electromagnetics. The most pursued designs have been those related with Slot Array Antennas (SAAs) [796], [802]-[820], some of them implementing advanced phased array configurations [802]. However, other topologies such as horns [821], RGW fed microstrip patch antennas [822], leaky-wave antennas in different configurations [823]-[824], or Rotman lenses [825][826] are as well found in the literature.

The SAAs receive great attention in GW technology since those antennas are an interesting option for mm-wave applications, inasmuch as whenever a low-loss waveguide is used for their implementation, high efficiency levels are achieved, and their planar profile results very suitable for many applications. The usual challenge with this kind of antennas is the difficult design and manufacture of the corporate fed. Although SAAs with series distribution are simple, they are narrow-band $(R B W<$ $5 \%$ ). This last is caused by the feeding line length, which is also the cause of undesired beam tilting [827]-[828]. On the other hand, 2D arrays provide reasonable bandwidth, however, in the case of hollow waveguides, ensuring good metallic contact of the required internal walls makes expensive the manufacturing cost, otherwise cheaper than that of other kind of antennas. In the case of using the microstrip line as a waveguiding structure, packaging resonances and surface modes also difficult the design [796]. In addition, ensuring the required small distance between elements to avoid grating lobes results noticeably challenging unless multilayer structures are used. However, multilayer structures must have good assembling properties, arising again the metal contact issue. The aforementioned weak points are well addressed by GW antennas in more or less extent as describes the next literature review.

One of the first works, with a remarkable quality and completeness (a mandatory reference for the GW researcher) is that of Kirino [802], in which a $76 \mathrm{GHz}$ multilayered Phased Array Antenna (PAA) is presented, based on RGW ${ }^{7}$. Fig. 8.33(a) shows an schematic of the whole structure separated by layers. The path followed by the signal in order to implement the different required functions is depicted in the schematic. Fig. 8.33(b) shows the side view of the layered structure and Fig. 8.33(c) shows the GW layout of the different layers and photographs of the actual manufactured pieces and assembled prototype.

The authors meticulously describe the design of the required phase shifters, radiators and feeding network. Specifically, phase shifters are implemented by using an intermedium movable piece which determines the length of the path followed by the signal. Therefore, the contactless feature of the RGW results to be very advantageous in this design. Insertion losses are under $1.5 \mathrm{~dB}$. The radiators are based on a resonant RWG transmission line ended with open circuits, which creates a standing wave. Resonant slots, inclined $45^{\circ}$ at the maximum current positions are used. It is

\footnotetext{
${ }^{7}$ The authors were already working with a structures similar to the RGW, under the name of Waffle-Iron Ridge Waveguide (WIRWG) [829]-[830]
} 
highlighted that, unlike conventional structures using traveling waves (which suffer from beam tilting due to the fact that the current is not the same in all positions of the line), the beam is perfectly directed toward the bore-site direction. A dielectric rod is used to avoid Grating Lobes (GLs). The antenna is composed by a total of 16 radiators, providing a gain of $G=32 \mathrm{~dB}$. It is possible to tilt the radiation angle $\pm 18^{\circ}$ and the Side Lobe Level (SLL) level is $-13 \mathrm{~dB}$. The dimensions are only $62 \mathrm{~mm}$ $\times 62 \mathrm{~mm} \times 25 \mathrm{~mm}$. The authors highlight the suitability of the presented design for automotive radars since it is tolerant to high temperature contrasts and long-term vibrations. On the other hand, they are still found some deviations in the amplitude ( $7 \mathrm{~dB}$ max. $)$ and the phase $\left(70^{\circ}\right.$ max. $)$ of the radiating elements feeding, what is left as future work. As additional valuable information, this paper clearly shows how tuning the height of the ridge allows interesting dispersion engineering of the RWG. It is also found that the first row of pins provides $15 \mathrm{~dB}$ of lateral attenuation, and $10 \mathrm{~dB}$ are provided per each additional row.

Some problems of the previous work are addressed by the same authors in [803]. As it is well-known, GLs are product of the relationship between the array distance and the wavelength in free space, whereas the Side Lobes (SLs) are product of the excitation illumination of the array. In the structure of [802], GLs appear at lower frequencies and there is no method to control SLs. The authors propose an interesting solution: practice periodic grooves on the ridge to create a slowwave structure. A good parametrization, giving useful information for dispersion engineering through the guided wavelength $\lambda_{g}$, is provided. The slow wave RGW implies a reduction of the GLs of the proposed array antenna, which is fed by a standwave structure. In addition, it is achieved arbitrary excitation illumination while keeping the element space and excitation phase since $\lambda_{g}$ can be widely tailored in the standwave structure. The SLL is reduced to $-26 \mathrm{~dB}$.

In [804], our research group from Universitat Politècnica de València proposes a design which avoids the cross polarization problem of linear slot antennas, caused by the necessary slot tilting to provide radiation. It is proposed to use a tilted dipole etched on a dielectric substrate underlying each slot, as it is shown in Fig. 8.34(a) for the case of a rectangular waveguide. The GGW-HP ${ }^{8}$ results very suitable for the implementation since the cover layer can be manufactured independently to be later assembled, obtaining good results insomuch as contact with the piece with the pins is not critical. In this way, it is possible to manufacture a copper cladded cover with the slots on the one side and the dipoles on the other side. It is highlighted that this configuration allows the dipoles to be rigidly arranged in the same piece. In addition, since the GGW has not side walls (it has periodic structures of pins instead), slots can be made longer, which is found convenient to achieve good radiation for a wider range of dipole tilting angles. The tilt angle is responsible for the amount of coupled radiated power by the slot. The paper shows that proper optimization provides that up to $50 \%$ the input power can be radiated. An 8 element array is designed at $f=37.5 \mathrm{GHz}$, providing a directivity of $D=16.2 \mathrm{~dB}$ and a SLL of $-11.8 \mathrm{~dB}$.

On the other hand, compensation of strong mutual coupling seems necessary in the previous antenna, this task demanding from optimization techniques with efficient analysis tools. In [805], the work in [804] is extended in a well structured

\footnotetext{
${ }^{8}$ Slots on the narrow wall of the equivalent rectangular waveguide.
} 


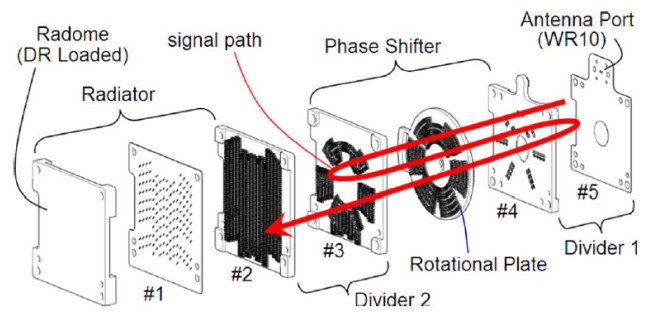

(a)

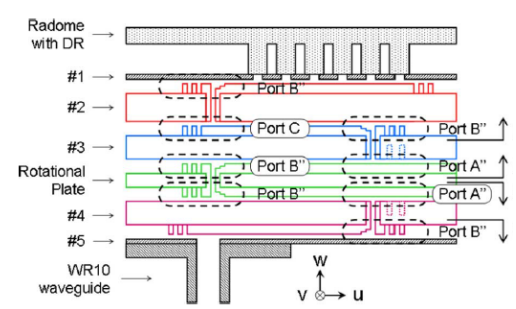

(b)
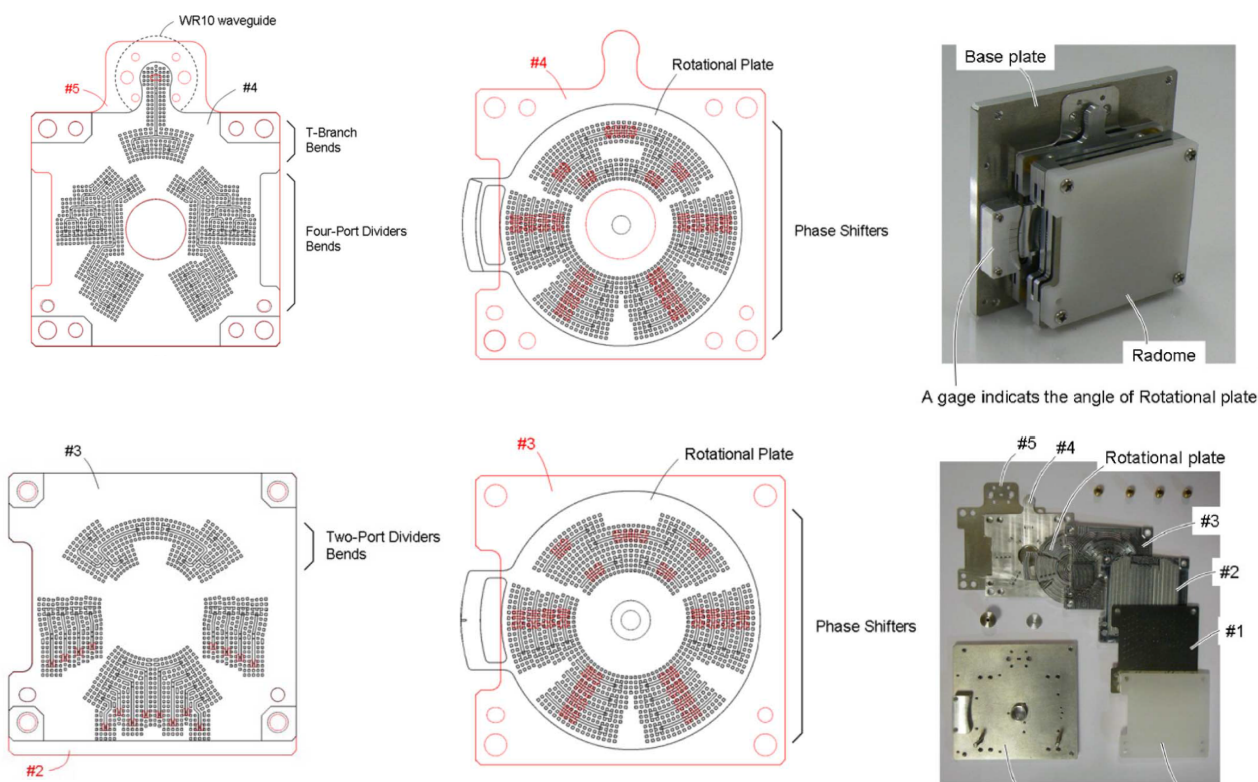

(c)

Figure 8.33: $76 \mathrm{GHz}$ multi-layered phased array antenna of [802]. a) Different layers of the structure and path followed by the signal; b) Side view of the layer structure; c) GW Layout of the different layers and photographs of the different pieces and the final assembled prototype.

paper, facing the mutual coupling effects, which are internal and external, being that the distance between elements is $d=0.68 \lambda$. To do that, the method developed in [831], later described in the modeling apart, is used, since analytical expressions for the GGW modes are not available. It is highlighted that, even though dipole parameters exhibit a symmetrical pattern with respect to the array center, the calculated optimum voltages do not. This is caused by the inhomogeneity of the GGW in the propagation direction, a fact to take account in the design process. The work presents 8-element prototype with Taylor distribution, working at $f=37.5 \mathrm{GHz}$. The SLL is below $-20 \mathrm{~dB}$.

The activity of Chalmers University of Technology (CUT) group has been particularly intense. In [806], a T-junction for corporate feed in RGW is designed by means of a $\lambda / 4$ transformer and 3 sections of $Z_{0}=50 \Omega$. Also it is studied the excitation of 


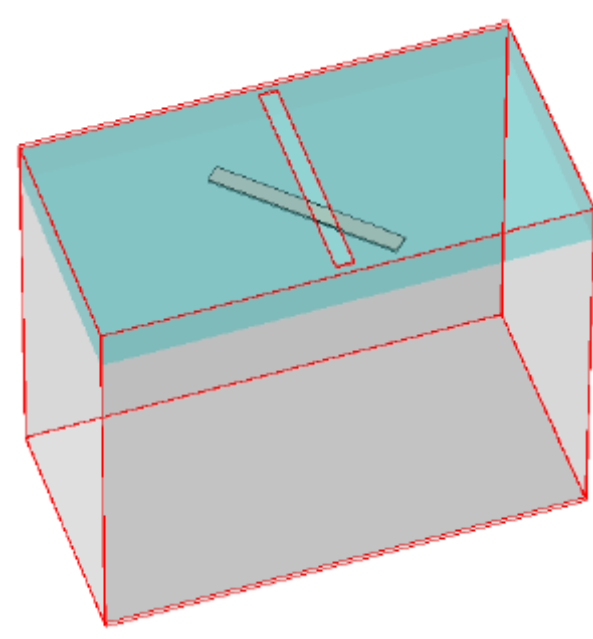

(a)

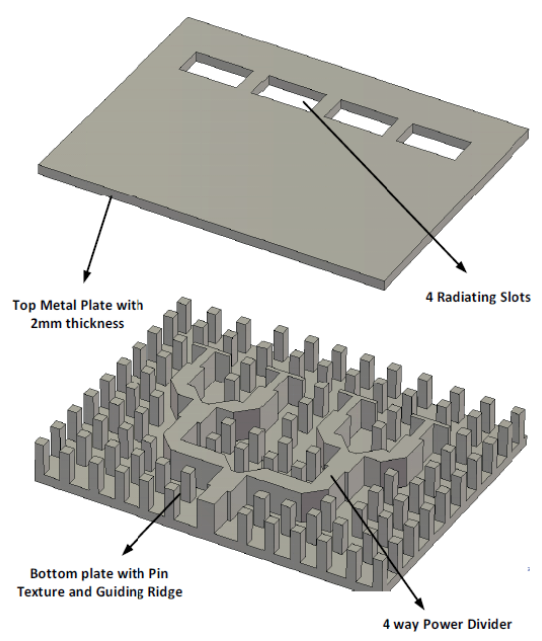

(b)

Figure 8.34: GW slot array antenna designs. a) Untilted slot in narrow wall of a rectangular waveguide [804]; b) Linear array in $R G W$ [807].

a single slot element to operate from $12 \mathrm{GHz}$ to $14 \mathrm{GHz}(R B W=15 \%)$ by means of T-shaped stub. This line work is continued in [807] where a 4-element linear SAA is implemented, see Fig. 8.34(b). The antenna presents $R B W=15 \%$ around $f_{0}=13$ $\mathrm{GHz}$ in terms of matching, i. e., return losses (RL), which satisfy $R L<10 \mathrm{~dB}$., with a directivity of $D=12.4 \mathrm{~dB}$ and an efficiency of $\eta=75 \%$. It results interesting that, despite of the fact that there is only one row of pins placed between the slots feeding lines, the isolation is over $20 \mathrm{~dB}$. The work is further extended in [808], where the T-shaped stub exciting the slots is carefully optimized. The bandwidth of the 4-element linear is improved up to $20 \%$, and a $2 \times 2$ planar array antenna with $R B W=21 \%$ is presented. Prototypes of both designs are shown in Fig. 8.35. The $2 \times 2$ array presents a gain of $G=12 \mathrm{~dB}$, and GLs of $-12.5 \mathrm{~dB}$ and $-10 \mathrm{~dB}$ in the H-plane and E-plane cuts, respectively. It is also the most compact option, presenting a separation $s=0.75 \lambda$ between elements, and a total area of $35 \times 35$ $\mathrm{mm}^{2}$. The $2 \times 2$ array is excited by a standard $\mathrm{Ku}$-band rectangular waveguide by means of a proper transition. In the case of the $4 \times 1$ array, the feeding is done by means of a SMA connector exciting a GGW and Chebyshev stepped transition from GGW to RGW. Although the performance of this last transition is quite good it seems that higher frequencies can be better launched with the former approach.

A standing-wave configuration is presented in [809]. In this work a slot array antenna is implemented by means of two sub-arrays of 8-element each one, fed by two RGW standing-wave sections, adjacent and isolated, which are fed by a bottom feeding layer implemented also with RGW. This bottom layer splits the input signal, coming from a coaxial cable. The antenna is designed to operate in the band $[13.6$ $\mathrm{GHz}-15.7 \mathrm{GHz}](R B W=14.5 \%)$. The gain is $G=13.9 \mathrm{~dB}$, the SLL is under -11 $\mathrm{dB}$ and cross polarization is under $-34 \mathrm{~dB}$. The simulated efficiency is $\eta=95 \%$, however, there is not prototype to confirm such a good performance. 


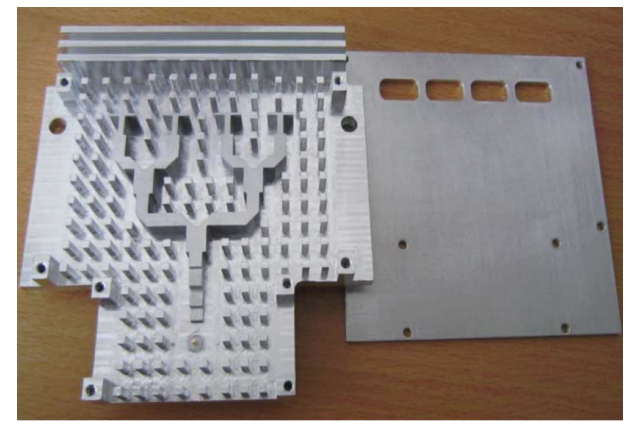

(a)

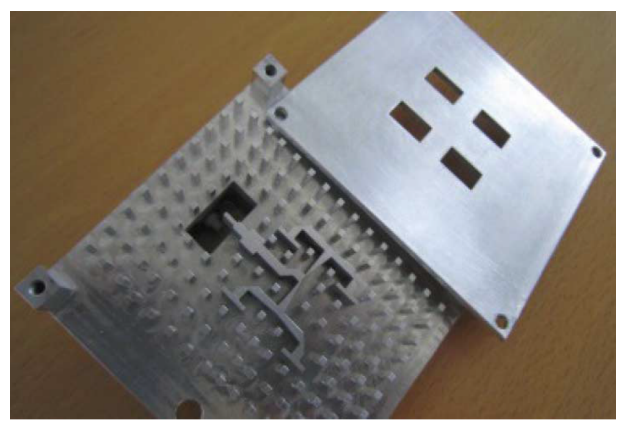

(b)

Figure 8.35: $R G W$ slot array antenna design of [808]. a) Manufactured prototype of $4 \times 1$ linear array; b) Manufactured prototype of $2 \times 2$ planar array.

In more recent works from 2015, the frequency is increased to reach the V-band, where the GW technology can better deserve its niche. For example, in [810] fourport Butler matrix is designed on SSGW to operate around $f=60 \mathrm{GHz}$, see Fig. 8.36(a). In this work, a hybrid and a $45^{\circ}$ phase shifter are designed, being pointed out that the simulation process is complicated, and suggesting to use the PMC approximation in a first stage, a prove that new GW dedicated analysis tools are required. Although the obtained amplitude values are acceptable, the phase must be optimized. However, this task seems complicated unless a denser lattice is used inasmuch as strip widths are comparable or even small than the pin period.

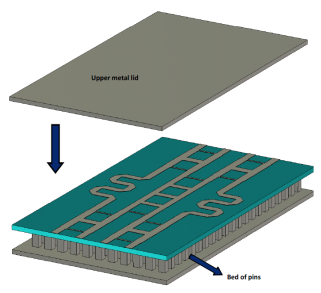

(a)

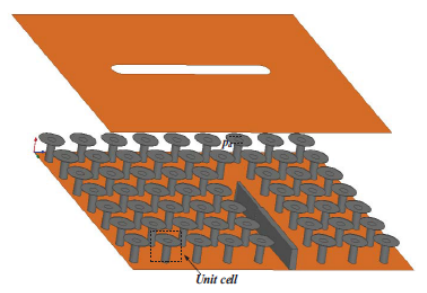

(b)

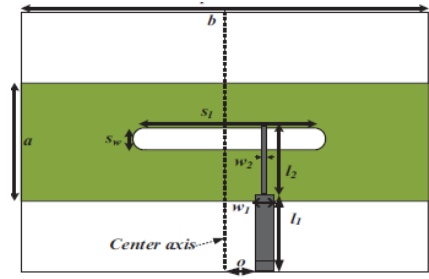

(c)

Figure 8.36: V-band SAA designs. a) Four-port Butler matrix in SSGW [810]; b) Schematic of the slot antenna based on RGW with mushroom-type EBG, proposed in [811]. c) Detail of the slot excitation of the antenna in $b$ ).

In [811], a mushroom-type RGW is proposed to excite a slot element, see Figs. 8.36(b) and 8.36(c). Operation is considered at $f=68.7 \mathrm{GHz}$ with a bandwidth of $900 \mathrm{MHz}(R B W=1.3 \%)$. The simulated gain is $G=7 \mathrm{~dB}$ and the return loss is roughly $R L=20 \mathrm{~dB}$. Through this design the authors confirm that RGW overcomes SIW performance, affirming that the designed antenna exhibits better performance than a similar one presented in SIW [832]. Nevertheless, a review of the results in [832] indicates that the bandwidth should be improved to confirm this. Practical implementation of the excitation (done by means of a coaxial cable) is an issue to address in this sense. 
Other interesting type of proposals are those implementing multilayer SAAs excited by means of cavities, [812]-[814]. The radiating slots are grouped in $2 \times 2$ elements, excited by a underlying cavity layer. Cavities are excited by the bottom layer of the structure, containing the corporate feed network. This structure is illustrated in Fig. 8.37(a), where different options for the feeding layer are shown: RGW (left), GGW (middle), SSGW (right). In this way, these GW antennas export the concept applied for hollow waveguides in [716] to GW technology, much more suitable for multilayer assembled structures. This configuration provides wideband matching and uniform aperture field distribution.

More specifically, the design in [812] consist in a $8 \times 8$ array with RGW corporate feed. The main feeding is done by means of a WR-15 and a properly designed transition to RGW. The antenna is designed to work around $f=60 \mathrm{GHz}$ with $R B W=14 \%(R L<10 \mathrm{~dB})$. A manufactured prototype by means of CNC milling, Fig. 8.37(b), exhibits an aperture efficiency $\eta>65 \%$, being the gain $G=25 \mathrm{~dB}$.

Similarly, [813] implements a $16 \times 16$ array, Fig. 8.37(c). The cavity configuration is slightly different than that of [812], giving some improvement in the feeding. Simulations show operation between $57 \mathrm{GHz}$ and $66 \mathrm{GHz}(R B W=16 \%)$, a gain $G=32.5 \mathrm{~dB}$. Side lobes are at $-13 \mathrm{~dB}$ in the $\mathrm{E} / \mathrm{H}$ planes and at $-25 \mathrm{~dB}$ in the $45^{\circ}$ plane, originated by the fact that element spacing is larger than the wavelength.

The work in [814] extends [813] to other topologies for the feeding network: GGW and mushroom-type SSGW. Results for the different topologies are quite similar, however, it lacks a proper comparison. In addition, prototyping is also missed. It would be interesting to compare all three options experimentally since the antenna concept and theoretical performance are interesting. In this sense, it is important to determine advantages and disadvantages of the different implementable options.

Other interesting new proposal impulsed by several groups are hybrid slot array antennas in which the corporate feed is implemented in MRGW, whereas the slot excitation is done by means of SIW cavities [815]-[817], exporting also in these designs the thoughtful proposal for the slot excitation of the group of M. Ando, done for rectangular waveguide in [716]. The main motivation of these works are the integration problems of hollow waveguides and the excessive dielectric loss of SIW technology, this driving to the consideration of GW technology. The different proposed designs are shown in Fig. 8.38.

Particularly, in [815], it is presented a $2 \times 2$ subarray with a MRGW-based corporate feed and SIW cavities in the conventional way as the previously commented SAA based on the work in [716]. MRGW is chosen instead of the SSGW due to the easy feeding with a WR-15 from the back side. The final idea of the authors is to design a $32 \times 32$ SAA. Reduce the undesired coupling between the $2 \times 2$ elements in the E-plane is provided by means of a corrugation in the SIW plane, implementing a soft surface. In this way, the GLs are reduced, and the distance between elements can be larger, increasing the directivity. Concept validation is done by means of a 4 $\times 4$ array, adding dummy elements to reduce edge effects. The designed stopband is [44.5-80 GHz] and antenna operates from $55.68 \mathrm{GHz}$ to $65.17 \mathrm{GHz}(R B W=17 \%)$, presenting a directivity of $D=20 \mathrm{~dB}$. It must be highlighted to good correspondence between simulation and results, what results promising for the conceived $32 \times 32$ array. Directivity greater than $40 \mathrm{~dB}$ is expected, overcoming the results of [716]. 


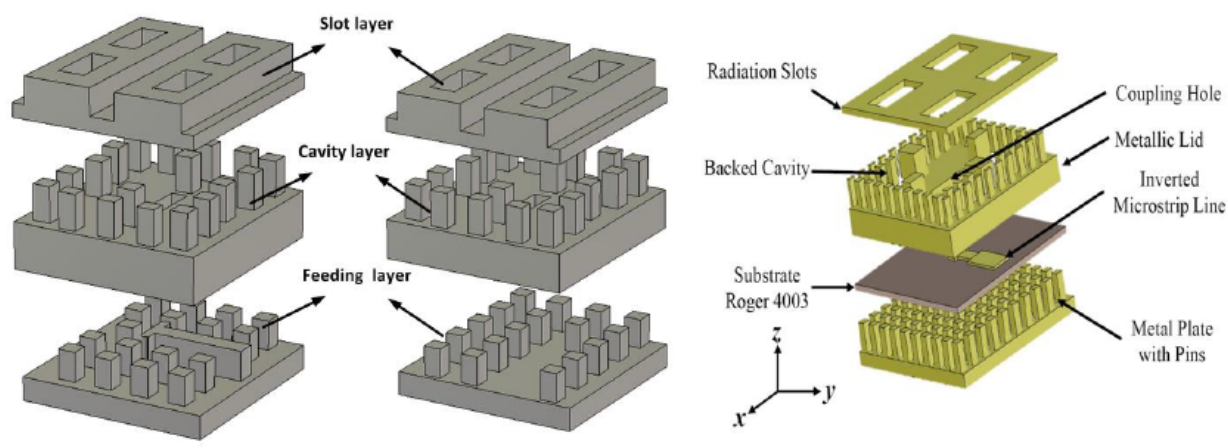

(a)

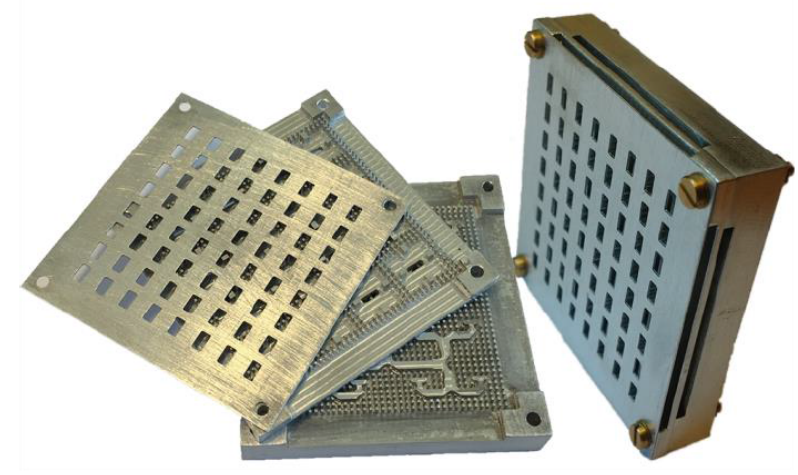

(b)

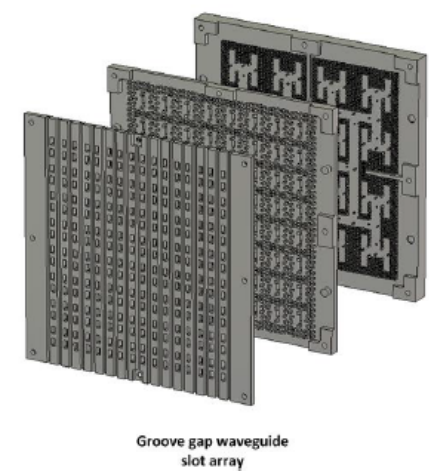

(c)

Figure 8.37: Slot array antennas based on $2 \times 2$ subarrays fed by backed cavities [812]-[814]. a) $2 \times 2$ subarray schematic of [814] considering different options for the feeding layer: $R G W$ (left), GGW (middle), SSGW (right) [814]; b) Manufactured prototype of the $8 \times 8$ array fed with $R G W$ in [812]; c) $16 \times 16$ array fed with $G G W$ in [814].

In [816], the SIW cavities include an L-probe and the whole structure is manufactured with Low Temperature Co-fired Ceramic (LTCC) technology [833]. In Fig. $8.38(\mathrm{~b})$, the different layers of the proposed $2 \times 2$ subarray are shown, being also indicated the number of LTCC layers forming each element. The authors highlight that SIW topology is a good solution to implement the cavities, since surface modes are avoided and they do not require complicated thick air cavities, shown to be difficult in LTCC technology [834]. A good point of the work is that a comparison between SIW and MRGW for the feeding network is carried out. The MRGW provides higher efficiency and gain, better transition from the feeding rectangular waveguide, and wider band impedance matching. The presented $8 \times 8$ elements prototype has an stopband of [75 GHz-119 GHz], operates at $f=94 \mathrm{GHz}$ with a $R B W=15 \%$, an efficiency of $\eta=42.3 \%$, and a gain of $G=23.8 \mathrm{~dB}$. The low efficiency is originated by dielectric losses, which must be improved somehow in future designs. 


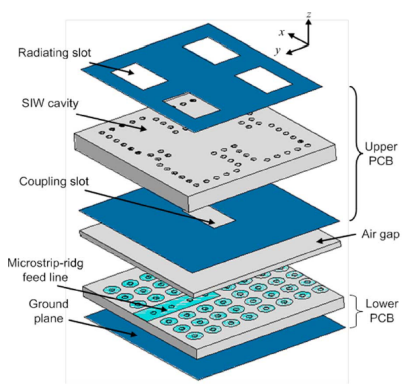

(a)

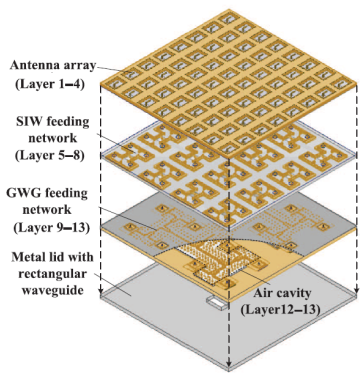

(b)

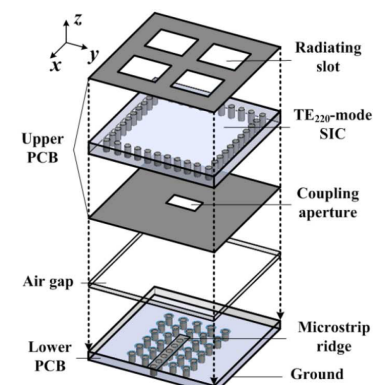

(c)
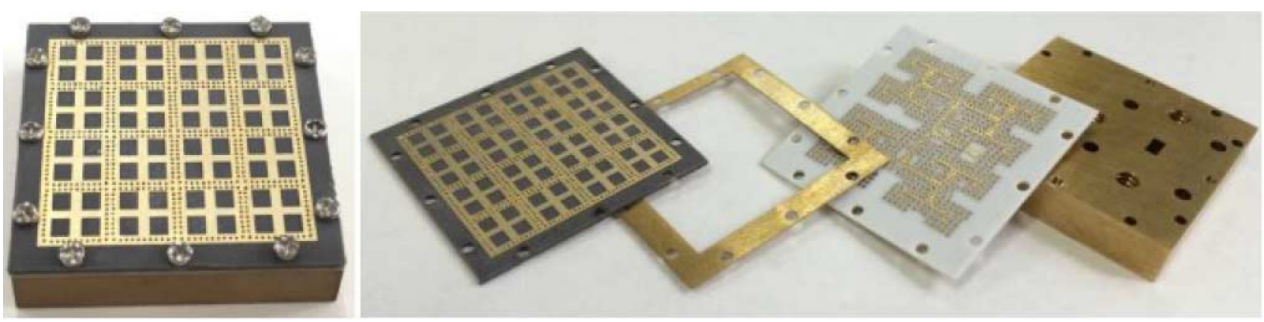

(d)

Figure 8.38: Hybrid GW-SIW slot array antennas. a) Layer structure of the $2 \times 2$ subarray proposed in [815] with a MRGW-based corporate feed and SIW cavities; b) Layer structure of the 2x2 subarray proposed in [816] with a MRGW-based corporate feed, SIW cavities, and with each slot incorporating an L-probe. The structure is designed to be manufactured with Low Temperature Co-fired Ceramic (LTCC); c) Layer structure of the $2 \times 2$ subarray proposed in [817] with a MRGW-based corporate feed and SIW cavities working with the TE 220 mode. d) Photograph of the fabricated PCB-based $8 \times 8$ antenna of [817]: assembled prototype (left), and the different layers composing it, including the radiating $P C B$ with the $S I W$ cavities, a metal spacer defining the air gap, the MRGW corporate feed network, and a thick metal support plate with a WR-10 flange in it.

Finally, in [817], performance is improved by using SIW cavities working with the $\mathrm{TE}_{220}$ mode, which provides constant phase to the slots, hence avoiding the use of SIW dividers, see Fig. 8.38(c). The manufactured $8 \times 8$ elements prototype is shown in Fig. $8.38(\mathrm{~d})$. A $[75 \mathrm{GHz}-110 \mathrm{GHz}]$ stopband is designed, and the antenna operates from $82 \mathrm{GHz}$ to $102 \mathrm{GHz}(R B W=22 \%)$, with a gain of $G=25$ $\mathrm{dB}$. The cross polarization is under $-30 \mathrm{~dB}$, thanks to that E-field components at the $\mathrm{x}$-axis direction are the same for the four slots of the $2 \times 2$ elements, hence the opposite field distribution makes the E-field components to mutually offset at the y-axis direction. The authors show that the proposed design has a bandwidth twice or even three times than other proposals with similar gains, implemented in technologies such as double-layer hollow waveguides or SIW patch antennas, that are even more complicated to manufacture. The participation of the Bohai Microsystem Company, Ltd. in the work endorses the interest of the industry in this technology.

Also from our group in UPV, they come proposals based in GGW [818] (VP), [819]-[820] (HP), more focused to provide an alternative to rectangular waveguide and the recently proposed diffusion bounding, quite expensive. In [818], a 4-way 
corporate feed network composed by E-plane splitters is used. The central frequency of the antenna is $f=20 \mathrm{GHz}$, being a narrow-band design $(R B W=5.5 \%)$, with a good SLL (less than $-15 \mathrm{~dB}$ ). Increasing the bandwidth is left as future work. It is pointed out that a rectangular waveguide equivalent is used for the optimization, but better models are demanded.

The work in [819]-[820] results particularly interesting. The corporate feed network is based on E-plane splitters and is backed by coaxial cavities. These coaxial cavities are integrated on the bed of nails, being that these cavities are implemented by merely lowering the height of a particular pin with respect to the surrounding ones, see Fig. 8.39(a). The shortened pin has a filtering effect, causing a transmission zero at a certain frequency that depends on its height $h_{p}^{\prime}$. In Fig. 8.39(b) it is shown the fabricated $4 \times 4$ prototype. The top image shows the complete assembly, whereas the bottom shows an image in which the cover has been removed to show the feeding network. The antenna operates at $f=37.5 \mathrm{GHz}$, exhibiting $R B W=6.56 \%$. The directivity is $D=20 \mathrm{~dB}$ and the efficiency is $\eta=80 \%$. There is good agreement between simulations and measurements, hence the structure, which is formed exclusively by one layer, is easy to manufacture. A possible drawback is the internal coupling between the feeding network and the cavities since it is not provided good isolation, which usually requires two or three rows of pins. It is also suggested the possibility of exploiting the concept to design filters in the future.

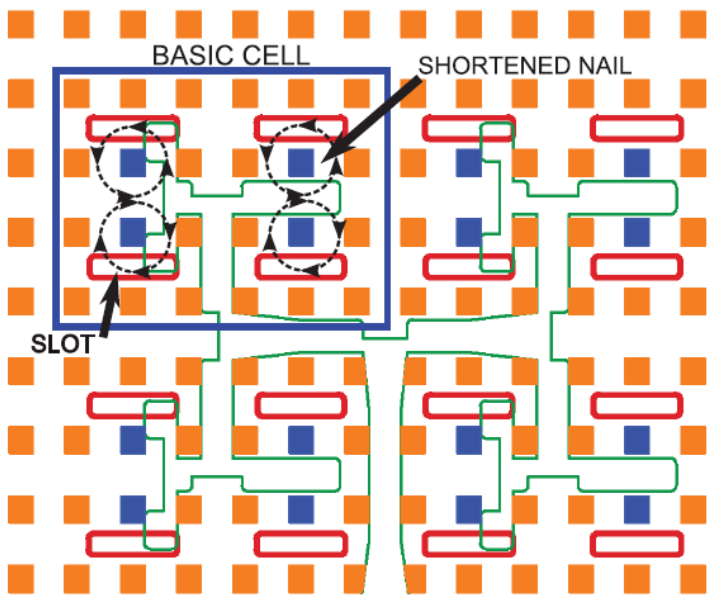

(a)
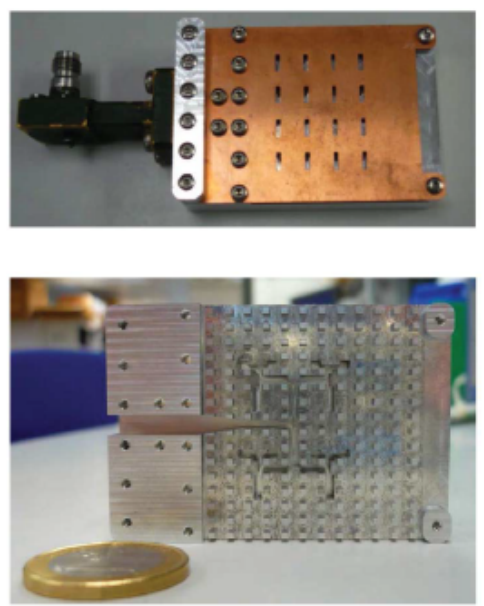

(b)

Figure 8.39: $4 \times 4$ slot array antenna implemented by means of coaxial cavities created by shortening certain pins [820]. a) Schematic showing the feeding network and the location of the shortened pins and the slots; Manufactured prototype. Upper image shows the complete assembled antenna, whereas at the bottom image the top cover has been removed to show the feeding network.

Leaving back SAA proposals, few different configurations are commented now. In [821] it is presented a planar dual-mode horn planar array $(4 \times 4)$ with corporate feed network based on a SSGW structure. Fig. 8.40(a) showns the H-plane and E-plane views of a dual-mode horn element fed by a SSGW, Fig. 8.40(b) shows the manufactured horn layer with a $4 \times 4$ distribution, and Fig. 8.40(c) shows the 
SSGW corporate feed network (the foam layer that is located above the network to keep space with horn layer is not shown). In this work, the SSGW is suggested as a good solution avoiding surface wave modes, better than SIW (leakage and losses) and hollow waveguides (complex). A proper design of the horns allows to overcome the effects of the large slot separation $(s=2 \lambda)$. The goal is to design antennas with a nearly uniform aperture distribution so that the first GL ideally fall in the first null of the individual element radiation patter. The SSGW stopband is $[8.5$ $\mathrm{GHz}-13 \mathrm{GHz}]$ and the manufactured prototype radiates from $10 \mathrm{GHz}$ to $11.2 \mathrm{GHz}$ $(R B W=11.3 \%)$ with an efficiency of $\eta=60 \%$ and providing a gain of $G=25 \mathrm{~dB}$. The use of foam instead air is responsible of a half of the total conduction losses of the antenna. It is pointed out that optimization of the structure results complex due to the lack of proper analysis tools, being necessary to use the PMC approximation, which is quite rough. The necessity of good connectors, flanges and transitions, suitable for GW technology is also highlighted.

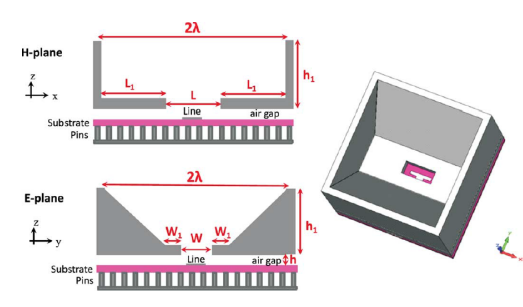

(a)

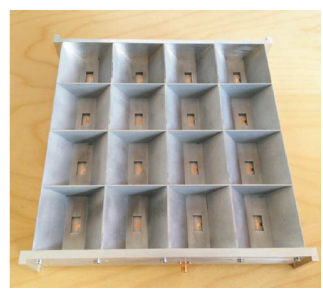

(b)

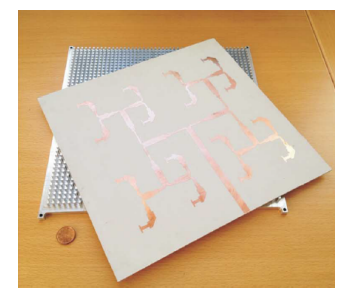

(c)

Figure 8.40: Dual-mode horn array antenna with corporate feed network in SSGW from [821]. a) H-plane, E-plane, and 3D views of a dual-mode horn element fed by a SSGW; b) Manufactured horn layer with a $4 \times 4$ distribution; c) SSGW corporate feed network. The foam layer above the feed network, used to provide mechanical stability is not shown.

Another topology is the $2 \times 2$ microstrip patch subarray with a RGW feeding structure proposed in [822]. This radiating structure works from $56 \mathrm{GHz}$ to $66 \mathrm{GHz}$ $(R B W=15 \%)$ for $R L<12 \mathrm{~dB}$. Simulated directivity for a $16 \times 16$ array is found to be $D=28.7 \mathrm{~dB}$. The authors highlight the lack of any substrate in the feeding layer, what reduces the losses, and the reduced size of the $2 \times 2$ unit cell. Nevertheless, the dielectric of the patch layer suggest additional losses and surface mode problems, not appearing in the previously commented SAA designs.

Finally, to end this antenna proposals section, they are reviewed totally different designs, implementing Leaky-Wave Antennas (LWAs), proposed in [824] and [823], see Fig. 8.41. The authors indicate that an important drawback of SAAs not found in LWAs is that it is hard to ensure small distances between the radiating slots and that optimization is very time consuming.

More specifically, in [824], an H-polarized LWA based on a Metal Strip Grating (MSG) and a quasi-TEM horn implemented with RGW (see Fig. 8.41(a)), which implements the required uniform field distribution to excite the LWA (see Fig. 8.41(b)). The authors highlight that this kind of MSG-LWA is very suitable for beam scanning in the mm-wave band, with the advantages of eliminating the complexity of the feed network in microstrip phased arrays, which causes high cross-polarized field, and providing a more compact, low profile. In addition, expensive phase shifters are 
not required. The RGW is chosen as good candidate for the horn, with the air gap height $h_{a}$ designed in such way that the MSG substrate is inserted in it. This solution is better than SIW (dielectric losses) and conventional rectangular waveguide (too bulky due to its height, causing mismatch between the horn and the MSG and radiation leakage from the horn aperture). The RGW is pointed by the authors as a promising structure for this type of horns, due to its versatility in size, propagation characteristics (quasi-TEM) and low losses. The LWA antenna works with $R L<10$ $\mathrm{dB}$ from $27.5 \mathrm{GHz}$ to $29.3 \mathrm{GHz}(R B W=6.33 \%)$, scanning angles from $\theta=-25^{\circ}$ to $\theta=-16^{\circ}$. The efficiency is $\eta=90 \%$ and the gain $G=24 \mathrm{~dB}$. GLs are very low, finding however a $-15 \mathrm{~dB}$ peak at the symmetric angle of radiation due to the reflexions at the end of the MSG structure.

In [823], the design is based on a GGW having only one row of pins at one of the lateral sides to provide leakage, see Figs. 8.41(c) and 8.41(d). As it can be appreciated in the figures, in this LWA the feeding is integrated with the same LWA, and advantage regarding to the design of [824]. An important point to highlight from this work in the context of this thesis is that the authors successfully use the analysis method for the GGW presented in [361], later detailed in section 10.2.1, proving its efficiency for design task in devices implemented with this waveguide. By using this analysis tool, the GGW width is optimized to provide the desired leakage by means of choosing the required propagation constant. The manufactured prototype exhibits good correspondence with the simulations. The LWA operates from $9 \mathrm{GHz}$ to $11.5 \mathrm{GHz}(R B W=25 \%)$, scanning angles from $\theta=35^{\circ}$ to $\theta=50^{\circ}$, with a gain of $G=20 \mathrm{~dB}$ and keeping the SLL under $-17 \mathrm{~dB}$.

\subsubsection{Filters and diplexers}

In the last years there has been also appreciable activity in the research area of GW filters and diplexers [835]-[849]. As it occurs with the antennas, there is a favorite topology or objective, but other proposals are also found. In the case of the filters, to achieve narrow-band designs exhibiting low insertion losses has been the main objective. To do that, the GGW has been chosen to implement cavity resonators inasmuch as it provides the highest quality factor among GWs. The GGW cavities are formed in the same way as in rectangular waveguide cavity filters, using the pins as walls. In Fig. 8.42(a), this kind of resonator and the E-field inside it is shown [835]. Coupling is done through inductive windows implemented by means of different topologies, see Figs. 8.42(b)-8.42(d) [835]-[838]. To date, only the conventional all-pole Chebyshev responses have been afforded. The coupling has been classically horizontal [835]-[842], but also vertical topologies have been recently proposed [845]. Alternatively, but with less work published, notch filters by using single and dual mode cavities [846], or SSGW filters are also found [847]-[849].

The first GGW filter proposals are found in [835]. In this work, it is shown that GGW offers a $20 \%$ quality factor than the RGW. A $3^{r d}$-order and a $5^{\text {th }}$-order Chebyshev filters with equal ripple $R=0.1 \mathrm{~dB}$, are designed to work in the Ku-band. In Fig. $8.42(\mathrm{~b})$ it is shown the layout of the $N=3$ filter. As can be observed, the coupling between cavities is controlled with the separation $s$ of the two rows that come between each two cavities, and the feeding is done by coaxial cables, located on the top plate. The central frequency is $f=14 \mathrm{GHz}$ and the relative bandwidth 


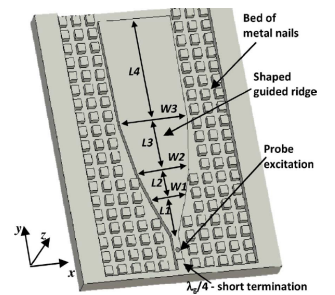

(a)

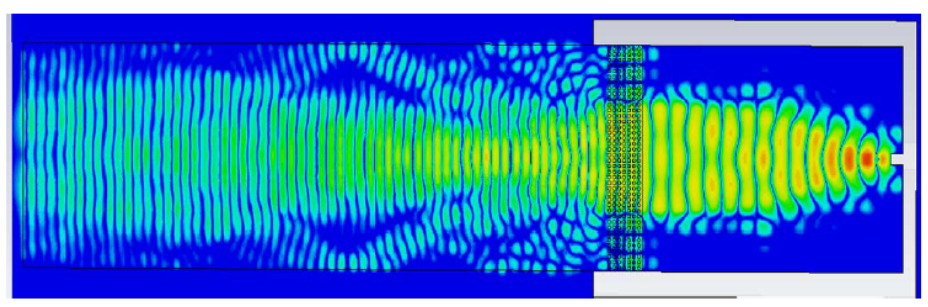

(b)
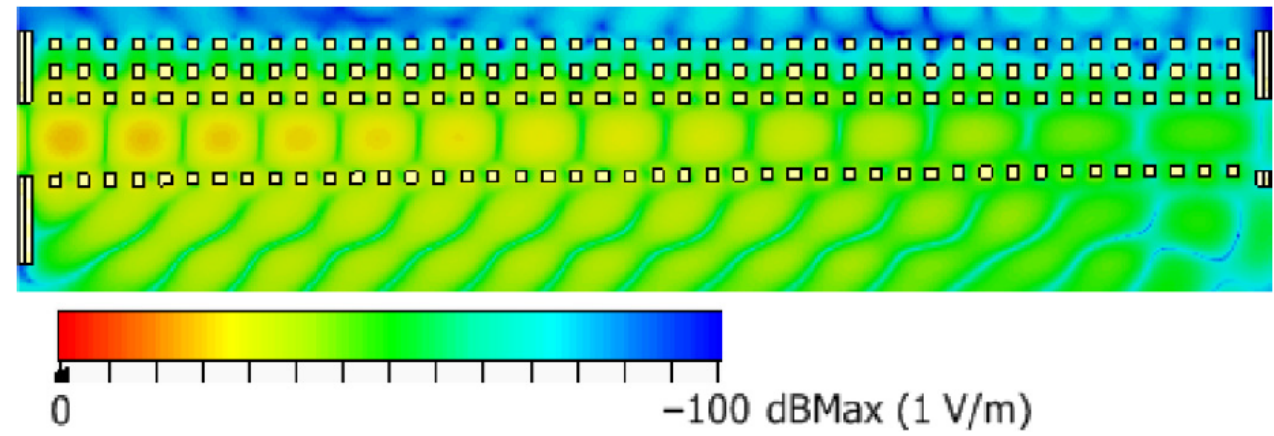

(c)

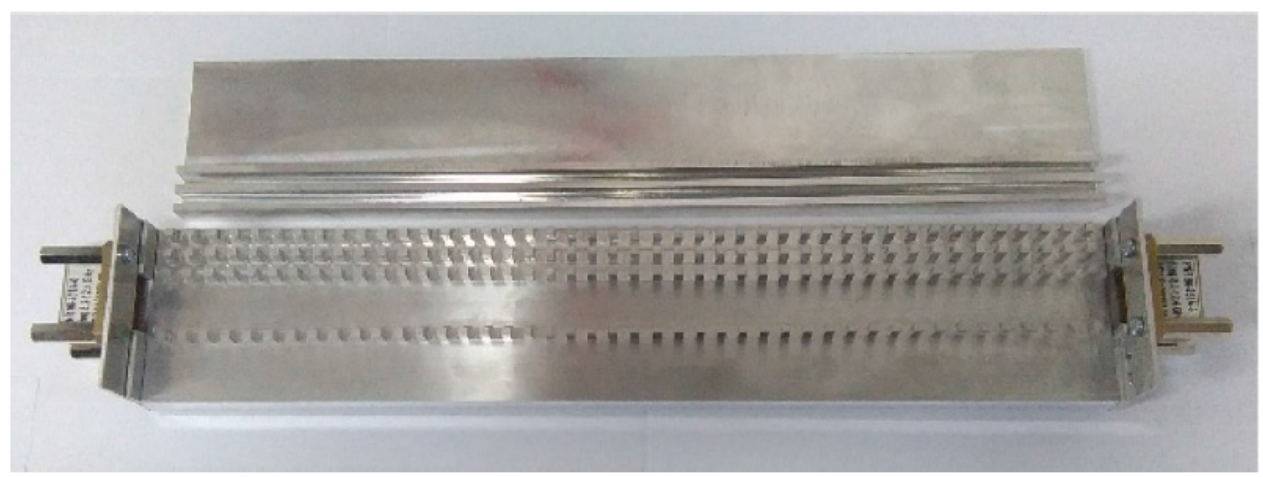

(d)

Figure 8.41: GW Leaky-Wave Antennas (LWAs) [824]-[823]. a) Schematic of the bottom part of the RGW horn launcher used in [824]; b) Near field distribution of the co-polarized E-field at the central frequency $(f=28.5 \mathrm{GHz})$ on a plane passing by the air gap of the launcher and the substrate with the Metal Strip Grating (MSG) in the LWA of [824]; c) Snapshot of the E-field distribution at $f=9.5 \mathrm{GHz}$ in the GGW LWA proposed in [823]; d) Manufactured LWA in [823].

is only $R B W=1 \%$. Experimental results for the $5^{\text {th }}$-order designs exhibit insertion losses between $1 \mathrm{~dB}$ and $1.76 \mathrm{~dB}$ (minimum equivalent $Q$ of 1713), and a slight frequency shift $(0.16 \%)$, i. e., $1 / 6^{\text {th }}$ of the bandwidth. The results are acceptable but higher quality is expected at this low frequencies. The authors suggest silver plating for future designs. Valuable points of the work is that it is shown how results 


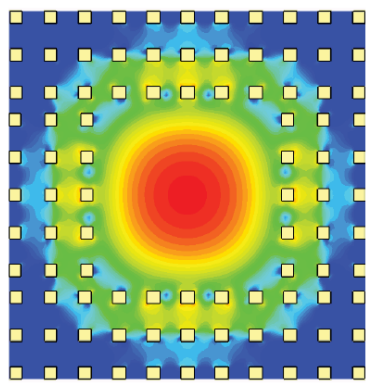

(a)

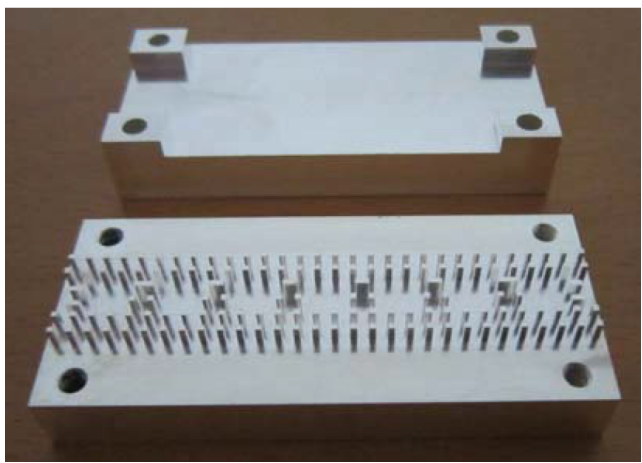

(c)

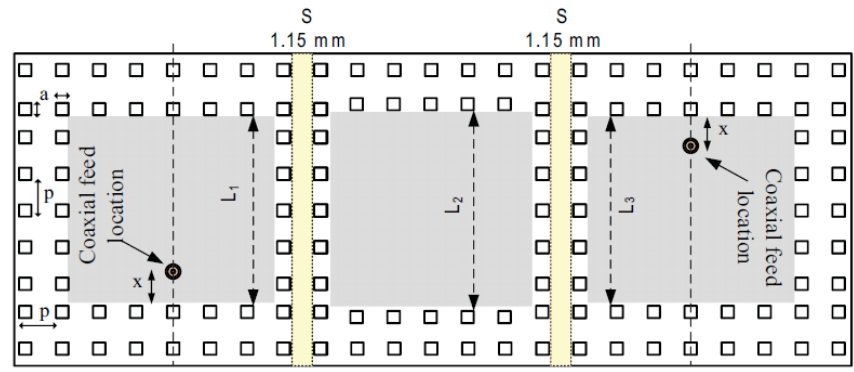

(b)

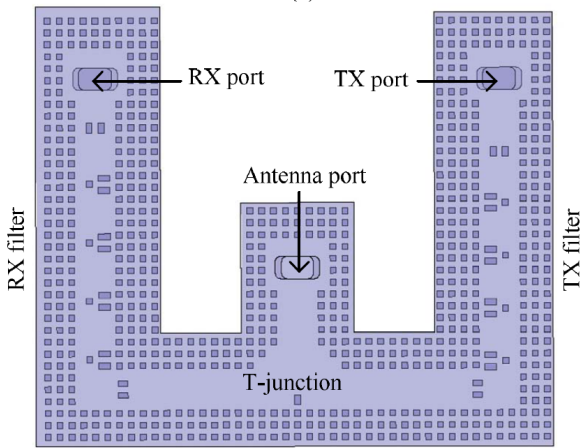

(d)

Figure 8.42: Narrow-band filters implemented with GGW by using rectangular cavities and horizontal inductive coupling. a) GGW cavity resonator and E-field magnitude distribution [835]; b) $3^{\text {rd }}$ order Chebyshev-type filter of [835] in which the inductive coupling is controlled by the separation of the two rows of pins separating the resonators; c) Manufactured prototype of the $5^{\text {th }}$ order filter of [836]; d) GGW diplexer formed by two $5^{\text {th }}$ order Chebyshev filters and a T-junction [839].

are independent on the presence of side walls with GGW topology, and that spurious are totally avoid at the stopband $[11 \mathrm{GHz}-20 \mathrm{GHz}]$.

In [836] a similar topology is proposed for the Ka-band. The central frequency is $f=37.37 \mathrm{GHz}$, and the bandwidth $B W=560 \mathrm{MHz}(R B W=1.5 \%)$. In this design, in order to reach higher coupling factors $(k)$, two pins of the set of pins separating the cavities is replaced by a ridge, which height and width are adjusted. The feeding is provided by a WR-28 and two ridges are properly designed to adjust properly the external cavity factor $Q_{\text {ext }}$. In this work it is found that increasing the air gap height $h_{a}$ causes $Q$ to increase up to $15 \%$, however, this decreases the stopband, designed in principle as $[25 \mathrm{GHz}-50 \mathrm{GHz}]$. Insertion losses (IL) are $I L=1.3 \mathrm{~dB}$ and return losses are $R>17 \mathrm{~dB}$. A slight frequency shift of $60 \mathrm{MHz}$ is found, caused by the used silver plating. Tuning screws are shown to be useful, however $I L$ increase $0.25 \mathrm{~dB}$ and $R L$ suffers certain degradation. A rectangular waveguide prototype is compared with the proposed design, exhibiting the same insertion loss despite of the fact that the rectangular waveguide filter response has a slightly larger bandwidth ${ }^{9}$.

\footnotetext{
${ }^{9}$ Note that $I L$ are inversely proportional to the response bandwidth [782].
} 
A temperature test also shows that the GGW filter suffers less response deviation. Although the comparison gives interesting information, it would be desirable to provide a comparison of two filters with the same type of response (the rectangular waveguide filter shows one transmission zero).

To overcome some CNC milling process challenges at high frequencies, in [837] it is proposed a GGW topology made by means of via holes, this giving higher density than mushrooms. In order to keep a high quality factor for the cavities, the substrate is removed in them, a similar concept to that proposed for SIW topologies in [756]. Feeding is done by coaxial cables, and the coupling is done by asymmetric windows, described by the via holes. The designed $5^{\text {th }}$-order Chebyshev filter has a central frequency of $f=30.03 \mathrm{GHz}$, and a relative bandwidth of $R B W=1.03 \%$. Simulations give $I L=1.4 \mathrm{~dB}$ and good return losses. However, experimental results are required to confirm that this topology provides a robust manufacturing process.

More recently, the group of Chalmers has faced V-band designs. In [838] a $5^{t h}$ order filter with $f_{0}=59.5 \mathrm{GHz}$ and $B W=1 \mathrm{GHz}(R B W=1.68 \%)$ is proposed. The coupling between cavities follow an scheme very similar to that of [836], whereas the feeding is done by means of two WR-15 coming from the bottom plate. WR-15 to GGW transitions are designed, showing $R L<20 \mathrm{~dB}$ over a relative bandwidth of $R B W=7.5 \%$, enough for the design. Experimental results show a best insertion loss value of $I L=1.7 \mathrm{~dB}$, which is a quite good result. However, a degraded response with $R L=9 \mathrm{~dB}$ and an appreciable frequency shift are also found. The use of tuning screws is proposed but the response remains still degraded and far from the specifications. In the work, a design with the feeding in the top plate is also affording, revealing that alignment results critic in such case, this caused by the high frequency of operation.

The previous work is extended in [839], where a diplexer based on the previous filter design is proposed. The central frequencies are $f_{1}=59.5 \mathrm{GHz}$ and $f_{2}=62.5$ $\mathrm{GHz}$, the transmitter and receiver are located at the bottom plate, and the antenna is located on the top plate. A T-junction showing $R L>20 \mathrm{~dB}$ in the band of interest is designed. The simulations give $I L=1 \mathrm{~dB}$ and $R L>10 \mathrm{~dB}$ for the filters, and $80 \mathrm{~dB}$ of isolation for the two channels, however, results in [838] suggest that experimental results may suffer appreciable degradation.

Part of the work carried out in this part of the thesis, published in [840]-[843], fits in the previous works context. Since later this work is detailed, only few remarks are given at this point in order to better contextualize it. First, in [840], a $4^{\text {th }}$-order filter centered at $f_{0}=40 \mathrm{GHz}$ and with $R B W=2.5 \%$ is designed and manufactured, exhibiting $I L=1 \mathrm{~dB}$ and $R L$ close to $20 \mathrm{~dB}$. However, the obtained frequency shift was found a difficult problem to face.

Then, in [841] a similar design, but with order $N=5$, is proposed for the $\mathrm{V}$ band $\left(f_{0}=61 \mathrm{GHz}\right)$. A novelty in the design is the incorporation of the coaxial feeding in the bottom plate, thus alleviating the alignment of the top metal plate, which becomes a very simple piece. The experimental results show $I L=1.6 \mathrm{~dB}$ and $R L<13 \mathrm{~dB}$. In addition, the response is not degraded, fitting well in the desired bandwidth, something not found in other contemporary designs. However, despite of the good tolerances of the milling process, the frequency shift is observed as a problem to address. This design is experimentally compared with an identical Hplane manufactured rectangular waveguide filter in [842], revealing that the GGW 
clearly overcomes the rectangular waveguide, being that the rectangular waveguide filter presents a degraded response, with even more frequency shift. In addition, an assembly test reveals that the GGW filter response is totally invariant after reassembling, whereas the rectangular waveguide filter clearly worsens its response.

This work is concluded in [843], where a novel tuning solution to correct the frequency shift is proposed and successfully demonstrated. It consist in displaying metal insertions on the top metal plate that are able to modify the resonance frequency of the cavities, the coupling between them, and the external quality factor. As this insertions are fixed, an iterative process is necessary. Nevertheless, extremely fast convergence is proven through the Aggressive Space Mapping (ASM) algorithm [850]-[851]. The results in this paper open the door to challenging high frequency device response correction in order to satisfy the required specifications in practical applications.

Other interesting proposal is the work in [845], in which the coupling is not horizontally done, but vertically, and both, GGW and RGW topologies are considered, see Fig. 8.43. The filters specifications are $f_{0}=35 \mathrm{GHz}, B W=350 \mathrm{MHz}$ $(R B W=1 \%), I L<1 \mathrm{~dB}$ and $R L>20 \mathrm{~dB}$. The feeding is done with rectangular waveguide, controlling the external quality factor by means of ridge, whereas the coupling between cavities is provided with irises. It must be highlighted that the design process followed in this work, found in [852], seems to be very interesting for GW structures, which generally demand from time consuming simulations. This method is similar to the ASM, and relates the physical parameters of the structure with the response through a simplified circuital model. The experimental results show $I L \approx 1 \mathrm{~dB}$ for both filters and $R L>10 \mathrm{~dB}$, without any frequency shift. Surprisingly, results for the RGW are better than those for the GGW. The good results suggest to test this kind of topology at higher frequencies.

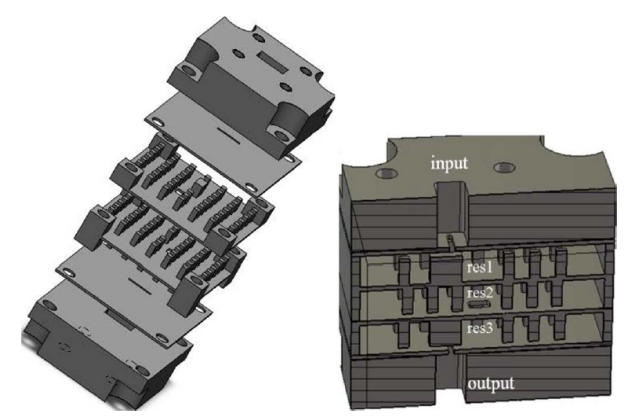

(a)

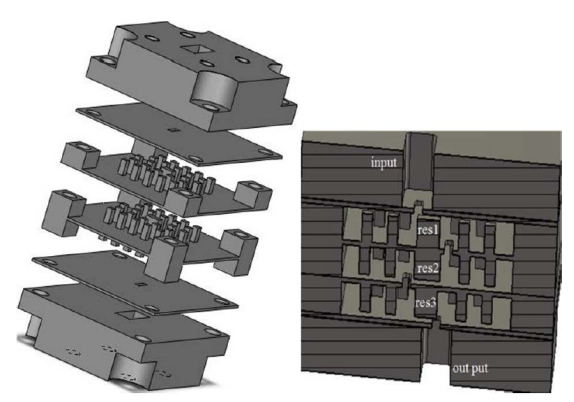

(b)

Figure 8.43: Vertical coupled resonator filters [845]. a) GGW based filter; b) $R G W$ based filter.

Finally, some alternative topologies are reviewed. In [846], single and dual mode cavities are created by defecting the pin structure of a RGW. The single mode cavity is created by placing a dielectric pin instead of a metallic one at a certain position of the first row, whereas the dual mode cavity is created by removing two adjacent pins inside of the periodic structure. By using these cavities a notch RGW filter is designed, see Fig. 8.44(a). 


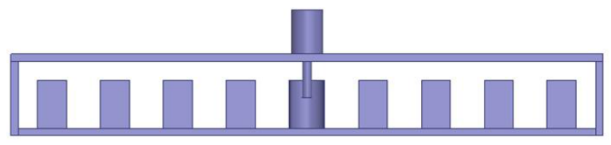

(a)

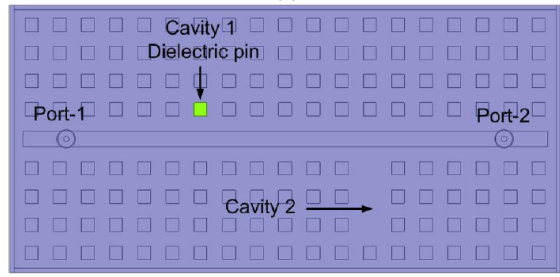

(a)

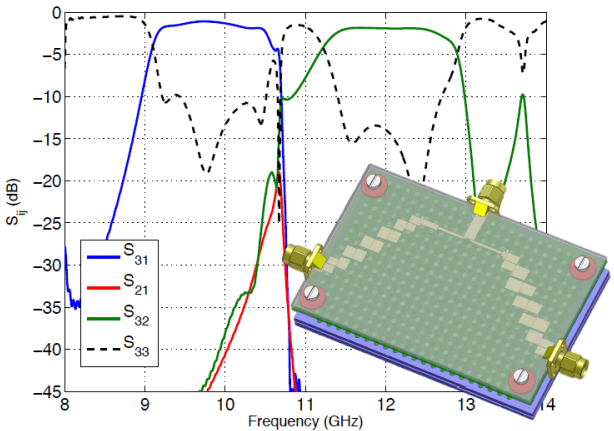

(b)

Figure 8.44: Other filter topologies. a) Notch filter implemented in [846] by means of cavities created by defecting the pin structure of a $R G W$; b) Diplexer implemented by means of two SSGW coupled-line filters [847].

In [847], the SSGW topology is used to implement a diplexer with $f_{1}=10 \mathrm{GHz}$ and $f_{2}=12 \mathrm{GHz}$. The SSGW is built using a periodic structure of screws, and the effect of its density on the filter response is studied, observing that higher densities lead to a situation closer to the ideal PMC condition. The designed filters are $5^{\text {th }}$ order with $R B W=10 \%$, providing in the diplexer $I L \approx 1.5 \mathrm{~dB}$ and $R L>20 \mathrm{~dB}$, with isolation higher than $40 \mathrm{~dB}$. Fig. 8.44(b) shows the diplexer and its response. A slot fed with a T-shape line is added to the diplexer to implement a self-diplexed antenna obtaining good matching.

The SSGW is used also in [849] to create and end-coupled band-pass filter. The filter is $4^{t h}$-order, Chebyshev-type with $R=0.1 \mathrm{~dB}$, with a central frequency $f_{0}=60$ $\mathrm{GHz}$ and a bandwidth of $B W=2 \mathrm{GHz}(R B W=3.33 \%)$. The prototype is embedded in a $10 \mathrm{~cm}$ SSGW which incorporates transitions to rectangular waveguide. Experimental results show a response that shifts to higher frequencies since the manufactured $h_{a}$ is smaller. However, corrected simulations show noticeable good correspondence and remarkable good results: $I L=1.4 \mathrm{~dB}$ and $R L>12.5 \mathrm{~dB}$. It is worth to mention that the insertion losses are only a half of a similar $3^{\text {rd }}$-order design with SIW [853].

Finally, in the context of SSGW filters, a simple $N=2$ Chebyshev filter is derived as part of the work of this thesis, as a demonstrator of some of the treated concepts in chapter 9 . The filter is implemented by means of coupled-line sections. The central frequency is $f_{0}=35 \mathrm{GHz}$ with $R B W=5 \%$. Simulations show promising $I L<0.4 \mathrm{~dB}$ and $R L>15 \mathrm{~dB}$. A good point to highlight is that the response is almost independent to the position of the substrate with the printed filter layout thanks to the use of a very dense pin lattice.

\subsubsection{Transition and flanges}

Transition and flanges are also devices that have received attention since GW were proposed [825]-[826], [854]-[864]. Notice that good interconnection with conventional technologies is critical in order to prove the validity of the designs, something claimed 
in most of the previous antenna and filter papers, that even usually incorporate a section dedicated to the design of the required transition/s. In addition, consolidation of GW technology require as well from transitions interconnecting the different GW topologies. In this area, most of the work has been carried out by the research groups of Chalmers University and the Polytechnic University of Valencia, but other groups have also joined recently this research line.

The first approach is found in [855], from 2009, where a coaxial cable to RGW transition is studied. The design is very basic, achieving $R L>10$ in the $[12 \mathrm{GHz}-$ $15.75 \mathrm{GHz}]$ band $(R B W \approx 20 \%)$, and it was used in the first GW designs. Three years later, Algaba [856] designs a transition between a CPW and a RGW through capacitive coupling with the aim of providing integration of active devices in RGW and facilitate measurements around $f=100 \mathrm{GHz}$. The transition consist in a CPW rectangular patch that couples to a RGW which has special end sections, adjacent to the ridge, in order to optimize the coupling. To avoid radiation loss to the back side, an additional bed on nails is placed under the CPW circuit, avoiding also in this way resonances and response degradation, and giving additional mechanical support. Simulations predict $R L>15 \mathrm{~dB}$ over a $R B W=10 \%$. Insertion loss of a single transition is found to be only $I L=0.5 \mathrm{~dB}$ in the frequency range from 100 $\mathrm{GHz}$ to $108 \mathrm{GHz}$.

Similarly, also Algaba in [857], designs a microstrip to RGW transition, proposed with the aim of giving the required compatibility with probe stations to RGW structures. Again a patch is used as a coupling element. Frontal and perspective views of this transitions are represented in Fig. 8.45(a). In this work, the microstrip line is preferred against the CPW, being that problems such as excitation of high-order modes, or the undesired coupling between even and odd modes in the CPW are not present in the microstrip line. Actually, the design gets an improved performance, extending the bandwidth up to $R B W=25 \%$ and reducing the insertion loss to $I L=0.425 \mathrm{~dB}$. Furthermore, it is highlighted that the optimization process results easier with the microstrip line as the aforementioned undesired effects are not present.

On the other hand, the proposal of Zaman in [858] consists in a microstrip line to RGW transition that directly transforms the EM fields between both lines, given its similarities. To do that, the air-gap between the ridge and the top metal plate is set equal to the microstrip substrate height. A ridge affix makes pressure over the part of microstrip line at the other side of the substrate. This solution is preferred than soldering since it provides flexibility an easy replacing. In addition, the affix acts as a matching element (narrow ridge) to provide a softer transition to the EM field mode pattern. It is highlighted that better matching would be obtained by the use of Chebyshev transitions, however, the authors prefer to provide larger simplicity and compactness. The work is focused to the microstrip line inasmuch as it is the common input line for MMIC active devices, and to achieve a large bandwidth is the main objective in this work. In fact, the design has a stopband from $22 \mathrm{GHz}$ to $46 \mathrm{GHz}$, and the measurements show a matching of $R L>14 \mathrm{~dB}$ in the $[23 \mathrm{GHz}-43$ $\mathrm{GHz}]$ band $(R B W=60 \%)$, with only $I L=0.16 \mathrm{~dB}$, which is an outstanding result.

Contemporary to these transitions it appeared a promising flange design proposal from Pucci [854]. In this work, one of the big problems of the mm-wave hollow waveguide technology are faced: the leakage in flanges due to the bad metal contact 
and difficult alignment, well documented in [731]. First, an stopband from $180 \mathrm{GHz}$ to $400 \mathrm{GHz}$ is designed to amply cover the WR-3 band $(220 \mathrm{GHz}$ to $325 \mathrm{GHz})$. Then, GW flanges are implemented by modifying a WR-3 waveguide flange: two rows of pins are added around the waveguide and a smooth metal surface of the pins height is added on each side of the waveguide cross-section before the first pin row. Fig. 8.45(b) shows a design with circular walls around the rectangular waveguide aperture, which provides the best matching, and the simulated S-parameters. As it can be observed, the designed flange provides a matching with $R L>20 \mathrm{~dB}$ over the [180 GHz-320 GHz] band. In this work it is also reported that choke flanges present similar performance. However, the presented design adds degrees of freedom in the choice of the position and range of applications. In fact, it is suggested the possibility of connecting two waveguides, inserting them on one of the flanges, or combining two waveguide pieces along their longitudinal walls, avoiding flow of currents at the joints and leakage when a gap is present.
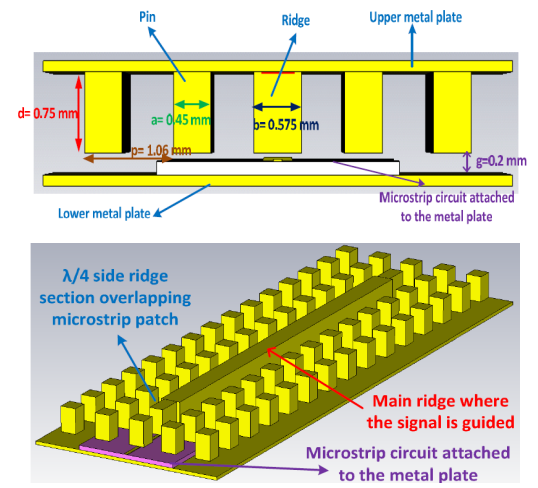

(a)

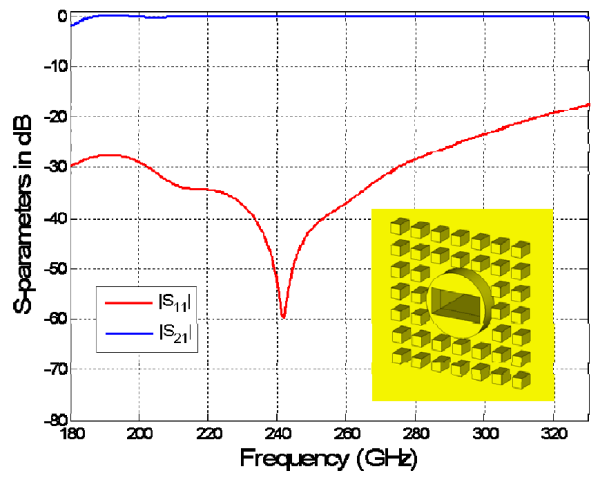

(b)

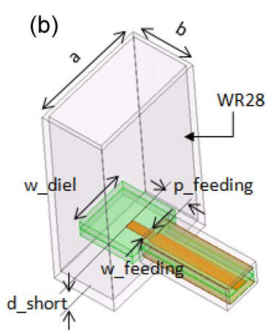

(c)

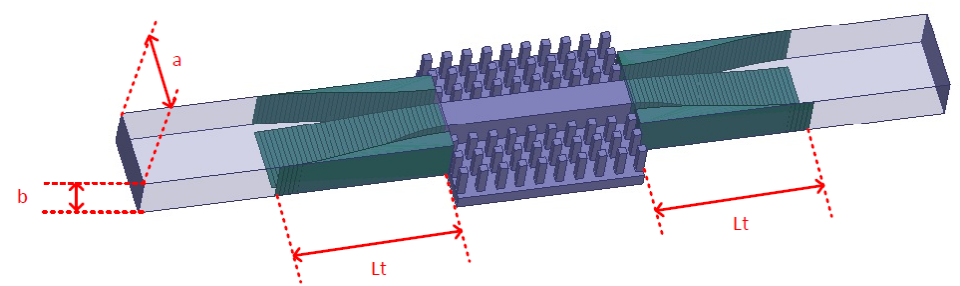

(d)

Figure 8.45: Transitions and flanges in GW technology 1. a) Front and perspective views of a microstrip to RGW transition to operate around $f=100 \mathrm{GHz}$ [857]; b) $180 \mathrm{GHz}-320 \mathrm{GHz}$ rectangular waveguide GW flange with circular walls presented in [854] and its simulated S-parameters; c) Test-fixture for a SSGW to operate in the Ka-band with a WR-28 as the output; d) Rectangular waveguide to $R G W$ back-to-back transition [825].

From UPV, Gahete [859] proposes a test-fixture for SSGW, to give transition to a WR-28 for operation in the Ka-band. A schematic of the proposed device is shown in Fig. $8.45(\mathrm{c})$. As it can be appreciated, the WR-28 is coupled by a 
rectangular coaxial which is formed sandwiching the strip of the SSGW. The work includes the design and manufacture of a TRL calibration kit to de-embed the effect of the coaxial to WR-28 transition. The S-parameters of a SSGW are measured, and the repeatability of the process is validated. As final experimental result, a 3-way power divider prototype is presented, showing good agreement between simulated and measured results. This transition is as well successfully used in the feeding of a the dielectric pin structure SSGW of [797], previously commented in this section.

Also from UPV, Carrera [825] presents two transitions in the context of a Rotman Lens [865]-[866] design. The authors indicate the interest of Rotman lenses for communications and radar mm-wave applications, front other proposed options to point the beam in specific directions or to sweep the beam in a given area such as electronic controlled switches, mechanical scanning systems, phase shifters or integrated lens antennas. A first transition from a PPW cavity to RWG is designed, exhibiting $R L>20 \mathrm{~dB}$ from $26 \mathrm{GHz}$ to $40 \mathrm{GHz}$ (almost coinciding with the designed stopband). The second transition is from a WR-28 to a RGW (see Fig. 8.45(d). The matching is similar, and insertion losses are of only $I L=0.15 \mathrm{~dB}$. In both cases a $3^{r d}$-order polynomial models the up and bottom, and side walls. Regarding to the isolation, the effect of adjacent ports is under $-15 \mathrm{~dB}$. The study of absorption conditions for the periphery is left as future work to study.

More recently, during 2015 and 2016, from Chalmers, Algaba has presented several advanced transitions [860]-[862]. In [860], a complete work, including design methodology, experimental validation and practical considerations is presented. Two transitions are designed: SSGW to WR-15, and microstrip packaged with GW technology (later explained in section 8.2.2.7) to WR-15. Regarding to the SSGW to WR-15 transition, it is highlighted that the test-fixture of [859] presents the problem of having the rectangular waveguide extending vertically upwards from the top side of the PCB, whereas for antenna applications it is more convenient extend it downwards, as it is done in [787], for instance. The work improves a previous design [867] that uses a patch (as in or [856] or [857]), for the excitation of a rectangular waveguide. In the new design (see Fig. 8.46(a)), the rectangular waveguide is simply embedded in the bottom plate, with the aperture surrounded by pins, whereas the strip is extended as a probe with an impedance matching circuit. A cavity backshort is located over the transition. Experimental results shown $R L>10 \mathrm{~dB}$ (simulated $15 \mathrm{~dB})$ in the $[55 \mathrm{GHz}-70 \mathrm{GHz}]$ band $(\mathrm{RBW}=24.7 \%)$. The insertion loss is $I L \approx 0.5$ $\mathrm{dB}$. However, it must be reported that some response degradation is found, with an important frequency shift. The authors indicate that this is probably caused by substrate curvature over the pins, and solutions such as employing rigid substrates, or introducing pressure over it, must be studied. A similar concept is developed as well for the packaged microstrip. The work also concludes in that SSGW overcomes SIW technology in terms of losses if the same substrate is used, and in that a packaged microstrip line present twice as much losses as a SSGW line, whereas an unpackaged microstrip line presents four times as much losses as a SSGW line.

In [861], the previous concept is extended to a design that does not require a cavity backshort. First, this element is replaced by SIW cavity in the radiating layer to provide a more compact design. A slot, which dimensions are finely tuned, is placed below the SIW cavity in order to provide good matching and to avoid high-order modes. The design, conceived for the V-band, presents simulation results 


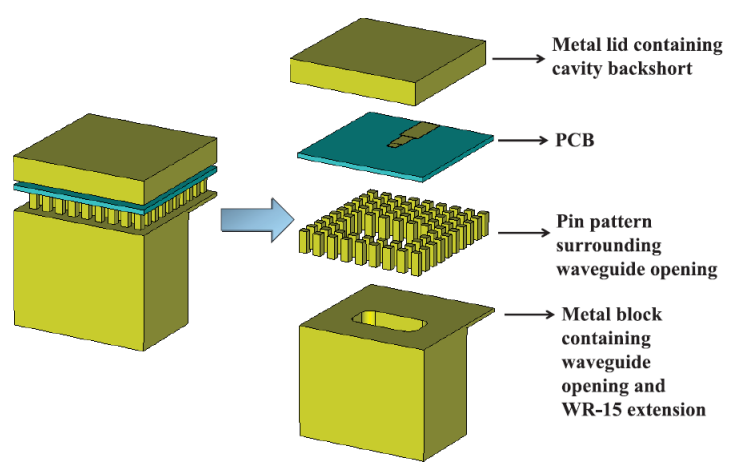

(a)
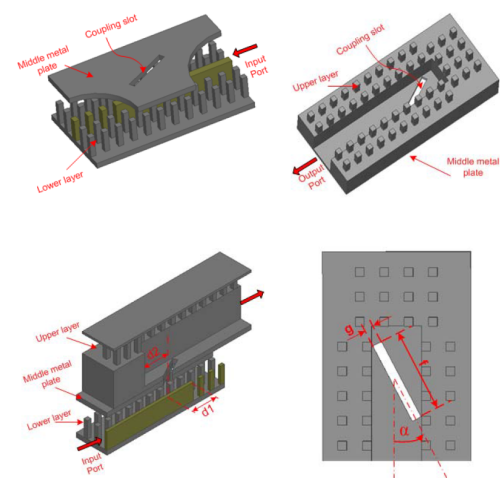

(b)

Figure 8.46: Transitions and flanges in GW technology 2. a) SSGW to WR-15 transition with distinction of all different components of the structure; b) Details of the slot-coupled transition between $R G W$ and GGW proposed in [826]. From left to right, up to down: lower layer (RGW), upper layer (GGW), longitudinal cut, and coupling slot.

of $R L>10 \mathrm{~dB}$ from $55.3 \mathrm{GHz}$ to $67.6 \mathrm{GHz}(R B W=22.4 \%)$, and $I L \approx 0.5 \mathrm{~dB}$. A second design in which an air-filled GGW cavity instead of the SIW cavity is proposed. The bandwidth is improved, being that $R L>10 \mathrm{~dB}$ is satisfied in $[52.15$ $\mathrm{GHz}-72 \mathrm{GHz}](R B W=38 \%)$ and $R L>15 \mathrm{~dB}$ is satisfied in $[55 \mathrm{GHz}-70 \mathrm{GHz}]$ $(R B W=24 \%)$, but the insertion loss slightly worsen $(I L \approx 0.7 \mathrm{~dB})$.

In [862], a F-band (90 GHz-140 GHz) transition from microstrip line to RGW is carefully studied, providing a Monte Carlo tolerance analysis [868] for the assembly. The work is motivated by the poor performance of the coaxial solution of [855] at high frequencies. It is also highlighted that, despite of the fact that in [854] $\mathrm{RGW}$ to rectangular waveguide transitions are investigated up to $f=300 \mathrm{GHz}$, the alignment of the RGW and the flange by means of inline step transitions produces certain degradation, being more recommendable an approach closer to those of [856] or [857]. The adopted solution consists of a microstrip to RGW including a patch as it is done in [857]. In the new proposal, several sections are added to the ridge in order to provide better matching. A CPW to microstrip transition is also included since measurements are done using a Ground-Signal-Ground wafer probe. The simulations give good results $(R L>15 \mathrm{~dB}$ over a $R B W=23.6 \%$, and $I L=0.65 \mathrm{~dB})$, however, the degradation of the response is appreciable in the measurements, despite of the fact that a precise CNC milling process with $\pm 1 \mu \mathrm{m}$ tolerance is reported. The problem seems to come from the difficulty of keeping the air-gap distance constant due to the soldering liquid, which add up to several tens of micrometers of thickness below the PCB, hence lifting the board in a uneven way. This fact justifies the inclusion of a Monte Carlo analysis to identify and quantify the most critical misalignment. Vertical and horizontal uneven offsets as well as rotations are included in this analysis. In general it is found that the structure is quite sensitive. The critical values for each source of misalignment are: $10 \mu \mathrm{m}$ (vertical), $20 \mu \mathrm{m}$ (horizontal) and $0.5^{\circ}$ (rotation). Therefore, at such high operation frequencies, elements to ensure a proper alignment become, perforce, necessary. 
Beyond the study of transitions connecting GW technology with the conventional waveguides and transmissions lines, it results relevant the work of Carrera in the study of transitions between GWs [826], [863], being that it is the required next step for the technology adoption. In [863], a vertical transition between RGW and GGW$\mathrm{HP}$ is proposed as a suitable element for compact multilayer SAAs. The design is a twist transition being that the fields experience a rotation (E-field is vertical in the RGW and horizontal in the GGW-HP). To do that a rotated (to cut the currents of the GGW-HP) slot is used as a coupling element, located at a distance $d=\lambda_{g} / 4$ of the ends of both waveguides, established by a wall of pins, see Fig. 8.46(b). This transition presents $R L>20 \mathrm{~dB}$ from $37.3 \mathrm{GHz}$ to $38.3 \mathrm{GHz}(R B W=2.6 \%)$, hence being narrow-band. The insertion loss is only $I L=0.4 \mathrm{~dB}$. It is successfully applied to the design of [804], obtaining good simulation results.

The previous work is extended in [826], in the application context of a Rotman Lens for PAAs, following [825]. Besides the previous transition, a vertical transition between two RGW, using similar concepts to that proposed by Kirino [802], is designed. An H-type slot is used for coupling, with the orthogonal part cutting the RGW currents and the parallel arms contributing with additional matching. For this transition, the bandwidth of operation is $[36.4 \mathrm{GHz}-40.6 \mathrm{GHz}]$, what implies $R B W=11 \%$. In addition, the insertion loss is reduced to $I L=0.3 \mathrm{~dB}$.

Finally, in [864] it is found a novel wideband design to provide connection between a microstrip and a RGW using a defected ground slot. The authors argue their motivation in that the coaxial to RGW transition of [855] provides a non-flat response from $12 \mathrm{GHz}$ to $15.75 \mathrm{GHz}$ and that the work in [856]-[858], although valuable, still presents some drawbacks. For instance, despite of the remarkable performance of the microstrip to RGW transition of [858] (sandwiched microstrip between ridge and upper plate), it results quite sensitive to accurate joining of the microstrip line to the center of the main ridge, and the packaging for such transition is foreseen as challenging. In addition, it imposes the restriction of the air gap of equal height as the substrate $\left(h_{a}=h_{\text {sus }}\right)$. The proposed design uses a T-shaped microstrip with a wide orthogonal end, and with the corresponding slot in the ground plane (defected ground). The RGW is matched with a step matching line in the coupling zone. In the experimental setup the microstrip is fed by a coaxial line and a rectangular box is used to embed the microstrip circuit that prevents the radiation of the microstrip line and reduces the transmission loss, being very suitable as well for shielding MMICbased RF circuits integrated with the microstrip line. The prototype exhibits $R L>$ $10 \mathrm{~dB}$ from $11.8 \mathrm{GHz}$ to $18.8 \mathrm{GHz}(R B W=45 \%)$ and $I L=0.25 \mathrm{~dB}$. The proposed transition is high-performance and practical, however, it must be said that it does not reach the performance of the transition presented in [858]).

\subsubsection{High frequency designs and manufacturing techniques}

Most of the previous designs are manufactured either by conventional CNC milling techniques, or by conventional PCB techniques, similar to those employed with other technologies such as SIW. Nevertheless, GWs features suggest that these waveguides not only are able to introduce performance enhancement at the lower mm-wave frequencies, but this improvement maybe even more appreciable at higher frequencies inasmuch as the undesired effects caused by bad metal contact between joints, or 
dielectric losses, increase with the frequency. Therefore, although most of the research is nowadays focused on the Ka and $\mathrm{V}$ bands, some works have recently start to prove the suitability of GW technology at higher frequencies in the mm-wave band [869]-[875].

The pioneer work in this area appeared in 2012. Rahiminejad [870] developed a micromachined RGW line including two $90^{\circ}$ bends, and a resonator, considering the operation band described by the WR-3 $(220 \mathrm{GHz}-325 \mathrm{GHz})$. It is highlighted that, despite of the advantages of GWs front other structures such as hollow waveguides (robustness to the manufacturing process at high frequencies), when the frequency goes beyond $f=100 \mathrm{GHz}$, manufacturing the pin structure by conventional $\mathrm{CNC}$ milling techniques becomes quite challenging. Therefore, it is proposed the use of Microelectromechanial Systems (MEMS) manufacturing techniques [876]. The process is schematized in Fig. 8.47(a): 1) A $0.5 \mu \mathrm{m}$ layer of aluminum is sputtered; 2) a thin photoresist layer is displayed on the top; 3) the photoresist is developed and the exposed aluminum is etched; 4) DRIE is used to etch the pillars, after the aluminum and remaining photoresist is stripped; 5) $1 \mu \mathrm{m}$ gold is sputtered (seed layer) and electroplated.

The manufactured prototypes have pins of only $h_{p}=277 \mu \mathrm{m}$, providing an stopband from $210 \mathrm{GHz}$ to $340 \mathrm{GHz}$. The resonator works with only two rows at the sides and three at the ends, providing a quality factor of $Q=282$ at $f=282 \mathrm{GHz}$. Losses of the manufactured RGW are found to be only $0.049 \mathrm{~dB} / \mathrm{mm}$ (a rectangular waveguide and a finline with similar range of operation experience losses of 0.02 $\mathrm{dB} / \mathrm{mm}$ and $0.6 \mathrm{~dB} / \mathrm{mm}$, respectively). The authors highlight the problems suffered during the measurement process due to the leakage between waveguide flanges and resonator.

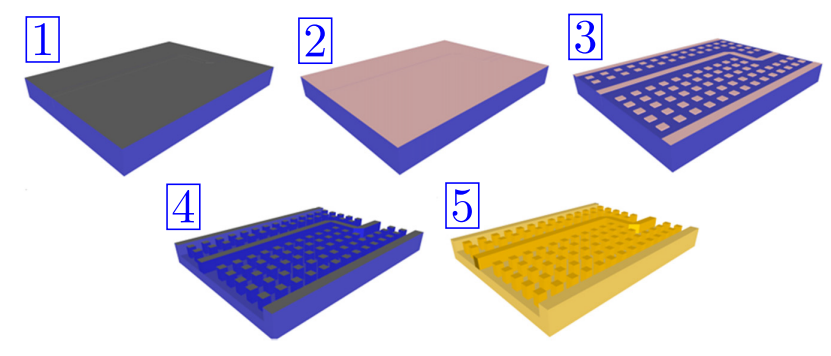

(a)

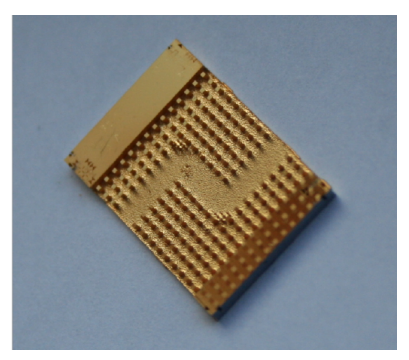

(b)

Figure 8.47: Micromachined high frequency GWs. a) Manufacturing process in [869] and [870]: 1) A $0.5 \mu \mathrm{m}$ layer of aluminum is sputtered; 2) A thin photoresist layer is displayed on the top; 3) The photoresist is developed and the exposed aluminum is etched; 4) DRIE is used to etch the pillars, after the aluminium and remaining photoresist is stripped; 5) $1 \mu \mathrm{m}$ gold is sputtered (seed layer) and electroplated; b) Micromachined GGW with two $90^{\circ}$ bends designed in [870] to operate at $f=100 \mathrm{GHz}$.

Similarly, the same authors manufacture micromachined GGW prototypes in [870]. A resonator and a line with two $90^{\circ}$ bends are again chosen as demonstrators. This last is shown in Fig. 8.47(b). In this case, the operation frequency is $f=100$ GHz. Accordingly, the manufactured pins are $h_{p}=750 \mu \mathrm{m}$ and $w_{p}=450 \mu \mathrm{m}$, providing a stopband from $90 \mathrm{GHz}$ to $140 \mathrm{GHz}$. The reported tolerance is of only 
$\pm 1 \mu \mathrm{m}$, however, the manufactured pins result to be slightly smaller at the bottom than at the top, this causing certain frequency shift of the cavity resonances. The manufactured bends work properly in terms of confinement, but some undesired resonances are reported. The quality factor of the cavity is found to be $Q=1200$. Measuring the losses on the straight line gives $\alpha=0.048 \mathrm{~dB} / \mathrm{mm}$, however, the applying the quality factor method to the resonator gives $\alpha=0.009 \mathrm{~dB} / \mathrm{mm}$, a quite good result. Again problems with the connection to the measurement equipment are found.

This work line has driven to the work [875] in 2016, where it is presented a 4 element linear slot array with planar distribution network, working around $f_{0}=100$ $\mathrm{GHz}$, manufactured with the same process as that employed in [869] and [870]. A WR-10 to RGW transition, similar to that of [813] provides the device feeding. The signal distribution is made by the usual T-junctions. The 4 slots are excited by means of T-sections at the end of the RGWs. Some corrugations are added beyond the slots to prevent back radiation. In this work, the measurement process is improved by using the flanges proposed in [854], shown in Fig. 8.48(a). The manufactured feeding network is shown in Fig. 8.48(b), and the top plate with the linear array of 4 slots is shown in Fig. 8.48(c). The total area of the device is 20 $\mathrm{mm} \times 20.8 \mathrm{~mm}$. Notice that only low-pressure screws and guiding pins are used to ensure alignment. It is pointed out that increasing the pressure on the structure to ensure contact with a large set of screws is very prone to cause deformations in this Si-based manufactured pieces. This is an additional reason to affirm GW technology results more suitable than conventional hollow waveguide technology. The proposed antenna shows a gain of $G=10 \mathrm{~dB}$ on a $R B W=15.5 \%$, with a radiation pattern having GLs under $-12 \mathrm{~dB}$. Moreover, the obtained pattern is shown to be very stable with the frequency.

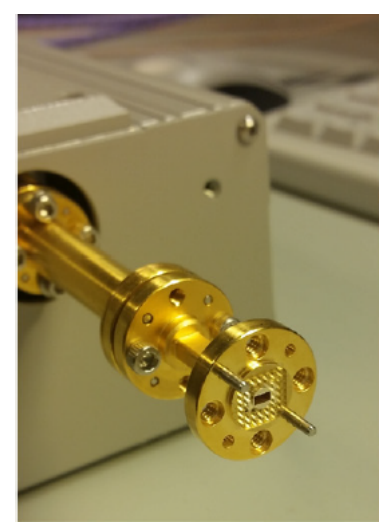

(a)

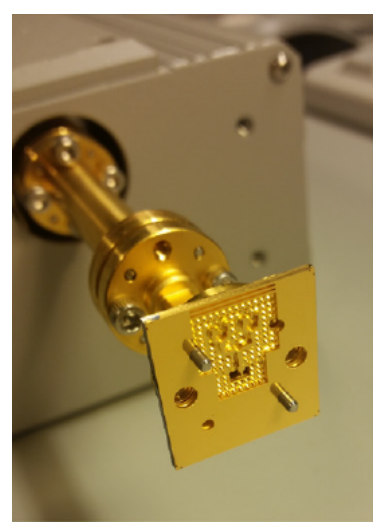

(b)

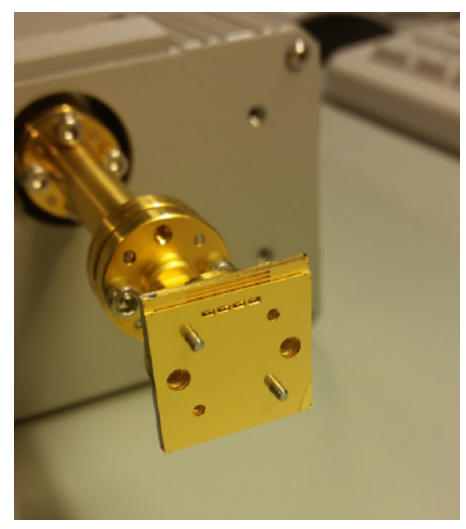

(c)

Figure 8.48: Experimental setup of [875]. a) Rectangular waveguide flange with a pin structure and circular smooth walls around around it [854]; b) RGW feed network fitted onto the guiding pins of the waveguide flange; c) Top metal plate with the radiating slots.

To reach even more high frequencies, beyond $f=200 \mathrm{GHz}$, an alternative manufacturing process is proposed in [874]. The proposal is based on Carbon Nanotubes 
(CNT) [877], which are allotropic ${ }^{10}$ forms of carbon with extraordinary electrical, thermal and mechanical properties. The authors (which are the same as in [869][870], [875]) indicate that, despite of some benefits of Si microfabrication, it results time consuming when etching high ratio structures. Conversely, CNT can grow hundreds of micrometers within minutes. For instance, to the authors experience, CNT devices are manufactured 50 times faster than DRIE ones. Also, the designs in [869] were manufactured using SU-8 in [879] with a total time process of 5 hours front the 2 hours provided by the CNT manufacturing process. A RGW resonator to work in the WR-3 operation band $(220 \mathrm{GHz}-325 \mathrm{GHz})$ is designed. Fig. 8.49(a) shows a Scanning Electron Microscope (SEM) image of the CNT manufactured device, whereas Fig. 8.49(b) shows a detail of the pins, with dimensions $h_{p}=277 \mu \mathrm{m}$ and $w_{p}=167 \mu \mathrm{m}$. Regarding to the first resonance at $f=237.8 \mathrm{GHz}$, results are not very promising for $\mathrm{CNT}$, being that the quality factor is $Q=240$, front $Q=642$ of the Si-based designs. This is probably caused by the larger roughness in the CNT device. However, by applying the quality factor method to the second resonance it is obtained that losses for the CNT RGW are $\alpha=0.051 \mathrm{~dB} / \mathrm{mm}$, close to $\alpha=0.044$ $\mathrm{dB} / \mathrm{mm}$ of the Si-based resonator. As indicated in section 2.3.3.1, the state of the art at these frequencies in rectangular waveguide is $\alpha=0.021 \mathrm{~dB} / \mathrm{mm}$ [343]. Therefore, it would be interesting to explore the possibilities of those novel manufacturing techniques in the GGW topology, which presents lower losses than the RGW.

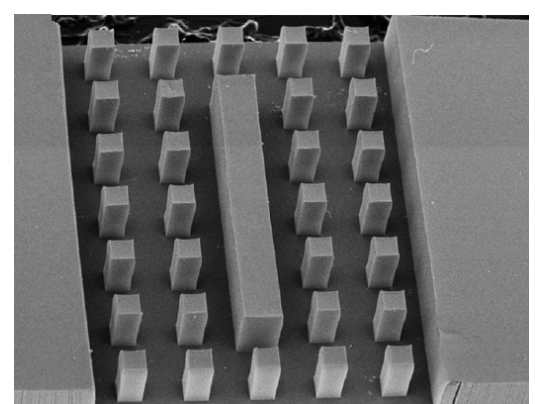

(a)

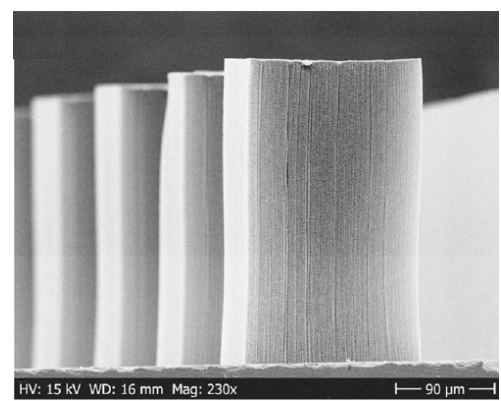

(b)

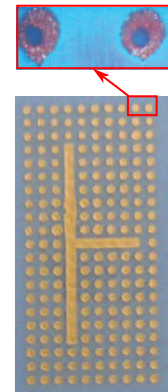

(c)

Figure 8.49: $G W$ devices conceived for high-frequency operation. a) Scanning Electron Microscope (SEM) image of a RGW resonator made by Carbon Nanotubes (CNTs) [874]; b) Detail SEM image of the $R G W$ resonator pins [874]; c) LTCC prototype of a $S S G W$ incorporating a resonant stub for microfluidic sensing applications.

The MRGW topology has been also explored at high frequencies in the mm-wave band. In [871], it is studied the possibility of manufacturing this transmission line using a LTCC process. In addition, the work provides an interesting comparison with SIW in terms of performance and manufacturing challenges. A period $p=250 \mu \mathrm{m}$ is chosen to create a stopband from $154.6 \mathrm{GHz}$ to $192.02 \mathrm{GHz}$. To provide similar confinement at $f=170 \mathrm{GHz}$, a SIW structure is shown to require a via hole period of $p=100 \mu \mathrm{m}$ (2.5 times smaller). In addition, for the MRGW, the period can be increased up to $p=400 \mu \mathrm{m}$ without loss of performance, whereas in the case of the

\footnotetext{
${ }^{10}$ Allotropy or allotropism is the property of some chemical elements to exist in two or more different forms in the same physical state [878].
} 
SIW the leakage totally destroys the performance. The larger period of via holes in GW structures is an advantage for the LTCC manufacturing process. Simulations show that, with small periods, a SIW presents twice loss as much it presents a MRGW, but this difference increases up to four times when the period is increased. Actually, the MRGW losses remain almost constant with the period whilst the SIW losses increase considerably due to leakage. This work presents also a transition from microstrip line with a $\mathrm{H}$-shaped slot defected ground, with the top plate above the ridge acting as the microstrip ground. Return losses are under $10 \mathrm{~dB}$ around $f_{0}=167.5 \mathrm{GHz}$ with $R B W=19.2 \%$, which is a good result. However, the best insertion loss value is $I L \approx 2 \mathrm{~dB}$, a high value probably caused by the large ohmic and dielectric losses at such high operation frequencies.

As a continuation of the previous work, in [872] and [873], MRGW microfluidic sensors are manufactured with LTCC and tested to give proof of concept, however, at lower frequencies. In [872], a resonator is designed by means of MRGW transmission line incorporating a $l=3 \lambda_{g} / 4$ resonating stub to operate at $f=59 \mathrm{GHz}$. The manufactured prototype is shown in Fig. 8.49(c), beside a detail of the via holes. The resonance frequency shifts when some liquid is present along a created microfluidic channel. The designed stopband of the structure is [ $45 \mathrm{GHz}-75 \mathrm{GHz}$ ], quite wide to afford this shift. It is shown that when water is present in the channel, the resonance shifts to $f_{\text {water }}=49 \mathrm{GHz}$, and when this is done with methanol, the frequency shifts to $f_{\text {methanol }}=52 \mathrm{GHz}$. The large difference between resonant frequencies proves the high sensitivity of these frequencies for this kind of applications, to date relegated to lower frequencies, less sensitive. Additionally, in [873], results are compared with a similar structure implemented with SIW technology. Surprisingly, the sensitivity with a SIW device is much lower, being $f_{\text {water }}=57.5 \mathrm{GHz}$ and $f_{\text {methanol }}=58.15$ $\mathrm{GHz}$. Therefore, this set of works give good proof of the suitability of GW technology front the more adopted SIW technology for this kind of applications.

\subsubsection{Modeling and analysis methods}

Up to now, device designs and manufacturing processes have been reviewed. This apart faces a total different area: the mathematical modeling of GW structures and the considered analysis methods. In the same way as transitions and flanges were mentioned as essential in order to GW technology become adopted in practical environments, simple and efficient analysis methods are essential in order to GW technology become accepted and used by those engineers who develop new generation systems. No matter how promising technology can be, if there is a lack of analysis tools that causes the design process to be excessively cumbersome, it is condemned to fall into oblivion. GW topologies present several practical advantages, however, they are inherently very difficult to analyze by means of full-wave methods since a periodic structure with a large number of small elements must be discretized. In addition, the well-developed analysis methods for conventional structures make easier the design process for such structures, what implies that advances in GW analysis tools are very required these days in order to GW topologies gains terrain to their conventional counterparts. A noticeable part of the effort in this part of the thesis faces this issue. Since the first GW proposals came out, the activity in this area has been intense. One important objective has been the calculation of the stopband 
and the dispersion curves. Homogenization of the pin structure has been a common practice [880]-[887], however, its applicability restrictions have recently motivated other methods considering the real dimensions of the pins [888], sometimes only in part [831], or using semi-empirical models [361]. A second important objective has been the determination of the characteristic impedance of quasi-TEM GW, with specially dedicated papers [790], [848], [889]-[894]. Nevertheless, a remarkable part of the aforementioned works face both objectives.

When talking about GWs modeling, a reference work is that published in 2008 by Silveirinha [880]. In that work, the basis for the homogenization of a periodic pin structure are rigorously established. The authors find an equivalent anisotropic dielectric of a metal plate supporting a periodic structure of cylindrical metal pins. It is the axial $(\hat{z})$ component of the dielectric constant dyadic $\overline{\overline{\varepsilon_{r}}}$ which gives relationship with the properties of the periodic lattice, i. e., period $p$ and height $h_{p}$. The pins are assumed electrically small so that the model does not take into account their size. The method results quite advanced, being that spatial dispersion effects are taken into account, and additional boundary conditions are considered to remove the extra degrees of freedom generated by the proposed approach. The authors highlight that it allows an easy engineering of almost any impedance with a periodic structure of pins, only limited by the skin depth of the metal pins. However, the model does not fit well for periodic structures with low density. The periodic structures used in GW are usually neither dense, nor with a electrically small period (except some exceptional works with $p \in[0.08 \lambda-0.12 \lambda]$, which is in the limit to be considered a small period), in order to provide a wide stopband and an easier fabrication process. In general, larger periods are demanded [792], close to $0.35 \lambda$ (see [808] or [845] for instance). Therefore, despite of the fact that this work presents a very interesting tool that has been widely used in the GW literature, it can be said that it usually results excessively rough for GW topologies. Nevertheless, a great part of the following works are based on it.

One of the first analysis methods using the model in [880] is developed by Bosiljevac in [881]. In this work it is proposed the use of Spectral Domain (SD) Green's functions considering the dielectric function given by the homogenization of Silveirinha's model. The authors highlight the efficiency of this method when compared with the straightforward use of 3D general codes of a finite structure, or any combination of Floquet's mode expansion [895] with general approach numerical methods such as Method of Moments (MoM), Finite Element Method (FEM) or Finite Differences Time Domain (FDTD) (see [590] for information about these methods). The idea is to give treatment to GW problems with the SD approach in a similar way that it is done for microstrip circuits and antennas, at least, giving a first approximation to alleviate posterior calculations or time-consuming optimization processes. Mushroom-type pins are also considered by using the homogenization proposed in [896], with a simpler formulation than that for the pin structure case. Despite of the rigour of the approach, the assumed homogenization limits its validity. Small circular pins (diameter $d=\lambda / 30$ ) in sparse periodic structures are considered (ratio $p / d=7$ ), whereas usual designs use $p / w_{p} \in[2,3.5]$ (see for instance [812], [841] or [845]). The stopband of pin and mushroom-type periodic structures are calculated. Some discrepancies are found for those high frequencies at which the wavelength becomes comparable with the period, specially in the case of the mushroom-type 
element. A better characterization of the Asymptotic Boundary Conditions (ABC) imposed by the mushroom elements is proposed in [884], providing the SD approach similar accuracy for both types of periodic cell in that work.

In [883], the previous work is extended and posed in a detailed and organized way. In this case, not only the periodic structure is considered, but the fundamental mode propagating in a RGW is also calculated through the SD approach. Despite of the fact that the currents of the ridge are approximated as purely longitudinal, there is good agreement between the proposed analysis method and the full-wave simulation in the calculated propagation constant and EM fields on the ridge. Fig. 8.50(a) shows the calculated dispersion diagram of the pin structure (to calculate the stopband), including full-wave simulation with $\mathrm{CST}^{\circledR}[412]$ for comparison. As it can be appreciated, disagreement only occurs for high frequencies in certain directions. The proposed Green functions open the door to further studies of currents in GW structures. However, the authors indicate as well that, in those cases in which $p$ is not small, some differences are found with numerical stopband calculations such as those provided in [792].

Contemporary, a different approach is presented by Polemi in [897], where, by applying the homogenization of [880], a three homogeneous region problem is posed, forcing the modes to be matched by continuity of tangential fields a the top plate. In each one of the lateral regions, where the pins are located, one $\mathrm{TM}_{y}$ mode and $\mathrm{TE}_{y}$ mode are considered to be propagating in the axial direction $(\hat{z})$, evanescent in the lateral direction $(\hat{z})$, i. e., modeling the attenuation caused by the EBG condition, and stationary in the vertical direction $(\hat{y})$. In the ridge region, a single mode is adopted to represent the field: a plane wave bouncing between the two region boundaries placed at ridge zone edges, having almost grazing incidence. These three modes are an approximation of the global quasi- $\mathrm{TEM}_{z}$ mode supported by the RGW.

The previous approach is extended and posed with detail in [882] and [898]. The focus in [882] is put on the propagation of the first high-order mode supported by the ridge and on the impedance definition and calculation. It is highlighted that, for wide ridges, the operation band is limited by the cutoff of this high-order mode. Since the approach is analytical, the authors are able to derive a closed expression to give the maximum ridge width $\left(w_{\max }\right)$ for monomode operation. The impedance study in this work results also complete and interesting. The basic TEM mode approximation and the stripline quasi-TEM approximation are observed to be excessively rough due to the frequency dispersion of this parameter, being that these approximations are frequency independent. In addition, the three usual impedance formulations (voltage/power -VP-, voltage/current -VI- and power/current -PI-) are studied. Since the waveguide is not a TEM waveguide, the voltage is not uniquely defined, hence causing disagreement between the three formulations. The PI formulation is suggested by the authors as the most adequate being that power and current are uniquely defined. Fig. 8.50(b) shows the presented comparison of the 5 commented approaches to calculate the impedance of a RGW in that work. All curves are totally analytical. Full-wave simulations with $\mathrm{CST}^{\circledR}$ using the formulation PI (integrating the simulated EM fields) are displayed as black dots for comparison. Although the analytical curve follows the frequency variation, there is certain disagreement with the full-wave simulations. In addition, the uncertainly of which impedance definition must be used remains open. On the other hand, in [898] it is 


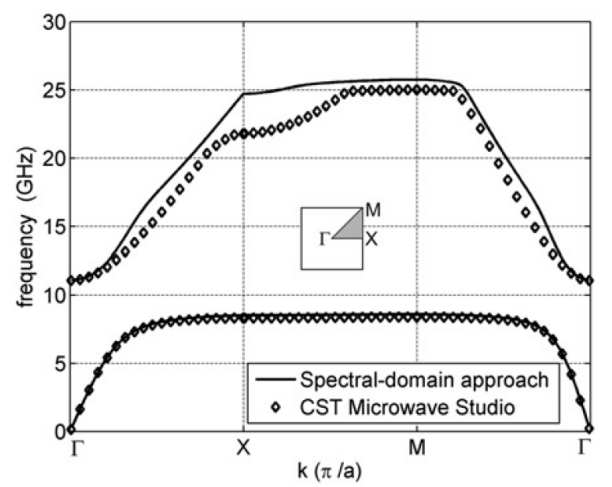

(a)

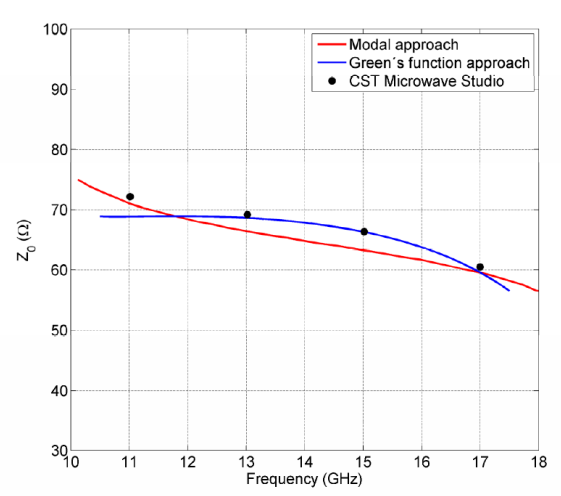

(c)

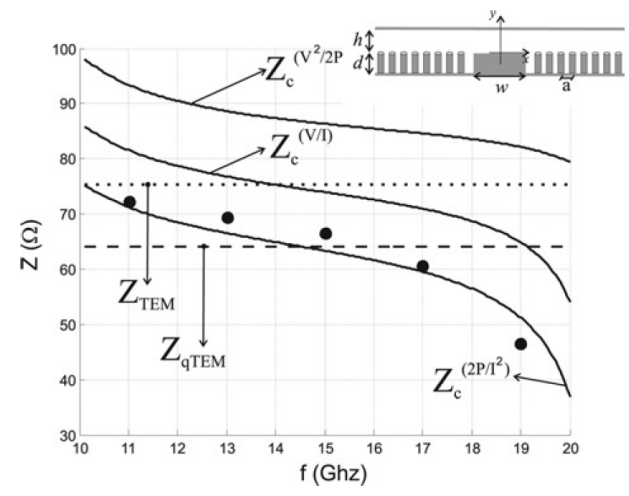

(b)

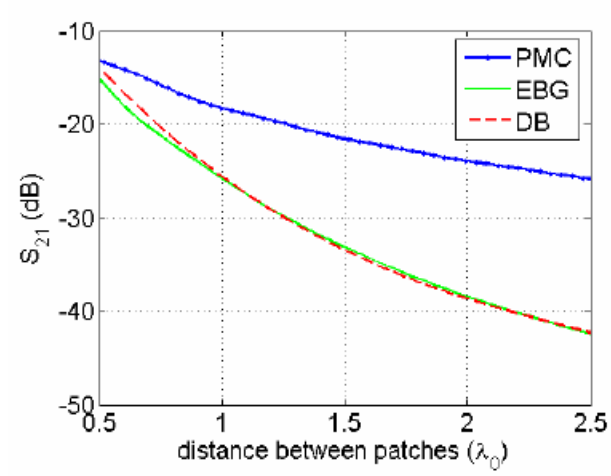

(d)

Figure 8.50: $G W$ analysis methods. 1) Calculation of the dispersion diagram (stopband) of a periodic pin structure. Comparison between full-wave (CST ${ }^{\circledR}$ [412]) and the proposed Spectraldomain (SD) approach in [883]; b) Calculation of a $R G W$ characteristic impedance $\left(Z_{0}\right)$ vs. frequency [882]. The curves relevant to the three definitions given in the work are compared with the ideal TEM mode impedance and with a quasi-TEM simplified model based on a stripline equivalent. The $Z_{c}^{(2 P / I)^{2}}$ case (PI formulation) is also computed through a CST ${ }^{\circledR}$ simulation (black dots); c) Comparison between the methods in [883] and [882], and full-wave simulation through $C S T^{\circledR}$ for the calculation of a $R G W$ impedance; d) $S_{21}$ parameter as a function of the distance between two $L=10 \mathrm{~mm}$ dipoles located above the observed surfaces [887]. The curve of the actual EBG structure made by means of mushroom-type element (green solid line) is compared with the approximations using the SD approach with PMC condition (blue solid line) and the DB-amended proposed condition (red dashed line).

given a circuital approach to the homogenization of Silveirinha and focus is put on the field patterns on the transversal cut of the waveguide. As expected, local effects of the field are not captured by the method. However, the proposed approach gives a good idea of the field decay to the lateral regions: $\alpha_{\text {lat }} \approx 100 \mathrm{~dB} / \lambda$.

Later, in [899] the previous mode-matching approach is compared with the SD approach of [881]-[883], highlighting the suitable physical insight provided by these methods front direct full-wave simulations. Two RGW with different pin structures 
and, for the first time, a GGW, are considered in the study. Nevertheless, it must be pointed out that all the considered periodic structures present small pins (although period comparable with $\lambda$ in some cases) to allow for an accurate homogenization. As a reference, the authors take full-wave simulations with CST $^{\circledR}$. In the stopband diagram calculation both methods practically present the same results, with good agreement. However, for impedance calculation, the SD approach seems to provide better results (see Fig. 8.50(c)). In addition, when calculating the propagation constant of the RGW, whilst the SD approach gives very good agreement, the modal approach gives errors larger than $5 \%$. In the case of the GGW propagation constant both methods result rough, being the SD curve which gives better fitting. It is important to highlight that these results are an indicator of the additional complexity in characterizing the GGW, demanding from more exact methods, a fact that motivates the later proposal in the scope of this thesis in section 10.2.

A further improvement for the SD approach is reported by Sipus in [887], where a correction to the PMC boundary condition is given. The authors highlight that, when a EGB surface is modeled as an isotropic soft surface, the usual DB conditions are used, i.e., the nullity of the normal components of the vectors $\vec{D}$ and $\vec{B}$ over the boundary. Therefore, since the case of normal incidence is not restricted by them (boundary conditions are automatically satisfied in such case), under that situation, important errors are obtained in the calculations. The presented amendedDB boundary conditions are used to calculate the far-field radiation pattern of a horizontal dipole over a EBG structure, showing good agreement with CST ${ }^{\circledR}$ fullwave simulations. In Fig. 8.50(d), PMC and DB-amended boundary conditions are compared with full-wave simulations in the calculation of the $S_{21}$ parameter as a function of the distance between two $L=10 \mathrm{~mm}$ dipoles located above the observed surfaces. The good agreement of DB-amended boundary conditions with the fullwave simulation indicates its suitability for first-stage modeling of EBG problems, with noticeably better results than assuming PMC conditions.

Sipus and Bosiljevac extend the spectral domain approach to the characterization of cross-coupling between RGWs for the first time in [885] and [886]. The usual even-odd [22] mode analysis for coupled-lines is applied, using the PI formulation to calculate the characteristic impedance of the involved lines. A prototype is presented to give experimental validation to the proposed SD approach. A periodic structure with $h_{p}=10 \mathrm{~mm}, w=3.5 \mathrm{~mm}$ and $p=7 \mathrm{~mm}(p / \lambda=5.35$, i.e, period comparable with $\lambda$ ) is used to provide a stopband from $6.6 \mathrm{GHz}$ to $10.66 \mathrm{GHz}$ according to fullwave simulations. The manufactured RGW prototype actually consists on a SSGW with no substrate, in which the strips are forced to float over the pins by anchoring them at the ends. The measured stopband is $[6.4 \mathrm{GHz}-10.4 \mathrm{GHz}]$, which is close to the full-wave simulation, however, showing some disagreement with the SD approach calculated one, which is roughly [7.1 GHz- $11.5 \mathrm{GHz}]$. The authors highlight that this disagreement is caused due to the fact that Silveirinha homogenization is used on a large period structure. However, the results seem valid enough for a first approach in those periodic structures with non-small period, which is, actually, the usual case. Experimental results on the cross-coupling are shown in Fig. 8.51(b), where it is represented the cross coupling between neighboring lines of length $L=15 \mathrm{~cm}$ as a function of their separation, using the air gap height $h_{a}\left(h_{w}\right.$ in the graph) as a parameter. The operation frequency is $f=7.5 \mathrm{GHz}$. Again, results show some 
disagreement, but still provide useful information for the initial stages of the design process. As a practical result, the lateral attenuation is found to be $10 \mathrm{~dB}$ per row of pins or $50 \mathrm{~dB}$ per wavelength.

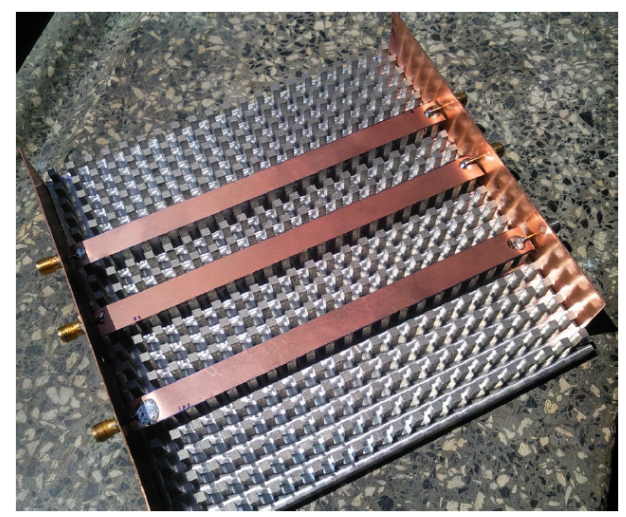

(a)

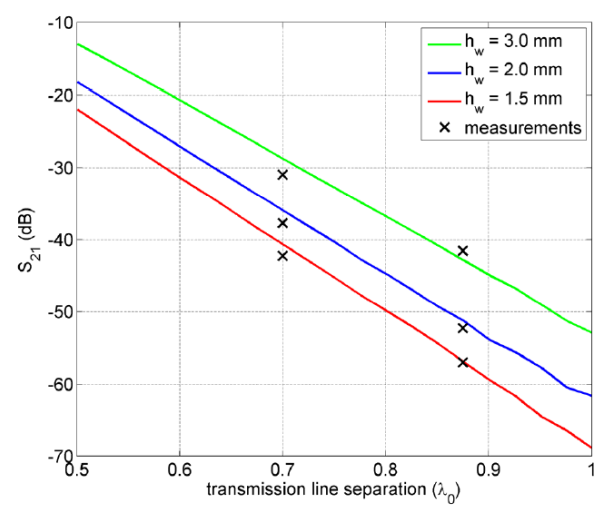

(b)

Figure 8.51: $S D$ approach for the evaluation of cross-coupling inside GWs. a) Manufactured SSGW prototype used for validation in [886]. Three parallel strips are disposed over a periodic pin structure $\left(h_{p}=10 \mathrm{~mm}, w=3.5 \mathrm{~mm}\right.$ and $\left.p=7 \mathrm{~mm}\right)$, anchored at their ends, hence forming SSGW lines with no substrate; b) Cross coupling between neighboring lines as a function of their separation. The working frequency is $f=7.5 \mathrm{GHz}$, the transmission lines length is $L=15 \mathrm{~cm}$, and the air gap height $h_{a}\left(h_{w}\right.$ in the graph) is the parameter.

A new approach has been also proposed by Sipus' group in [888]. In such work a MoM/mode-matching approach is applied to determine the scattering of each element in the grid, assumed as a circular metal pin. This is done by determining the currents on the elements by means of the Green's function of a single element in a PPW. First, it is found the current distribution in the cylinder considering an incident plane wave. Then, the contribution of all the cylinders of a row is considered, and by Bloch's Theorem, the contribution of the rest of the cylinder rows. This new approach is able to capture the pin features, thus avoiding inaccuracy of ABC following the homogenization of [880]. The stopband of a periodic structure with $p=0.125 \lambda$ is calculated with this method and also by applying the SD approach with ABC. Both results are compared with full-wave simulations, showing that, even though the period is rather small, there are differences between both methods, observing that the agreement of the MoM/mode-matching approach method with full-wave simulations is larger than that provided by the ABC SD approach.

Other totally new approach pertains to the work in this thesis, presented in [361], and detailed later. Basically, the analysis method finds an equivalence between the GGW and the rectangular waveguide, given a certain periodic structure. A fast full-wave simulation of a row of pins is carried out to find out the impedance that the lateral regions of pins impose at the sides of the propagation channel when propagation is considered to the transversal direction. Since this impedance varies with the frequency, by transmission theory, it is found a frequency-dependent equivalence between the GGW and the rectangular waveguide. Once this equivalence is found, analytical expressions for the rectangular waveguide are easily adapted to the prob- 
lem, recovering the dispersion curves for the GGW with very good agreement with a computation time two orders of magnitude less than with full-time simulations. Therefore, this new semi-empirical approach is, one the one hand, efficient, and, on the other hand, gives good physical insight by providing an equivalence with a well-known structure as it is the rectangular waveguide, allowing to employ or easily adapt analysis tools developed for it. The method has been already tested for the design of a leaky-wave antenna [823], and everything indicates that it might dispose a much more suitable GW device design scenario, thus becoming a paradigm for new GW analysis methods.

As mentioned in the introduction of this apart, definition and calculation of the characteristic impedance of GW transmission lines has received special attention, being the main motivation of some published works. Notice that, in the case of the characteristic impedance, to develop simple expressions or procedures that lead to advanced circuit designs in practice is essential, as the engineer who face such designs may be aware of transmission line theory, but not to necessarily know the propagation minors in GW structures. In a very suitable future scenario it would be desirable to have GW transmission line design tools and expressions of comparable complexity to that of conventional transmission lines such as the stripline, microstrip or CPW, found elsewhere in the radiofrequency engineering literature [22], [376], [895], [900].

At the initial GW technology stage, the stripline equivalent was used in [889] to characterize the impedance of a RWG. If the condition over the pins is assumed to be PMC, by image theory it is obtained that $Z_{0}^{(R G W)}=2 Z_{0}^{(\text {stripline })}$. Notice that, roughly explained, this is the origin of the fact that GW transmission lines require wider strips than conventional transmission lines to achieve a given impedance. This stripline model works well if a true PMC was used, what means that stripline expressions (see [22] for instance) could be successfully used in circuit design. However, as reported in this first work ([889]), when dealing with the RGW, the impedance condition over the pins changes with the frequency (as expected inasmuch as their electrical height does). Therefore, only very rough first stages designs can be obtained with this procedure.

The first work dealing deeper with the impedance concept is found in [790]. In the apart dedicated to the characteristic impedance is first established that the definition of characteristic impedance is not unique. Said this, three ways to calculate the characteristic impedance are proposed: Voltage-Current (VI) model, Power-Current (PI) model, and waveguide port. The first two, already commented when describing the work of Polemi [882], are based on the integration of the EM fields inside the structure (voltage is not uniquely defined and an integration path must be specified). The last one consists in a different approach that has led to a class of impedance analysis methods. The waveguide port method assumes that the characteristic impedance of a certain GW is given by the equivalent waveguide that describes the propagation channel. In the case of a RGW this is the classical ridge waveguide [901]-[902]. Note that choosing a conventional ridge waveguide port in a numerical full-wave solver is a common sense solution. In this way, the same commercial solver facilitates directly a characteristic impedance. In addition, established the equivalent, further investigations on the definition and calculation of the impedance can be carried out by 
the advanced designer by making use of some interesting work done for the classical ridge waveguide [903]. The problem of ignoring the effect of the pins is that the frequency-dependence that they introduce is as well ignored. Therefore, this procedure gives a rather constant impedance, more accurate but similar to that provided by the stripline method. In [790], the classical VI is chosen as the most accurate way to calculate the impedance. They are found differences as high as $30 \%$ when comparing the stripline model with the VI formulation. The conclusions of the work of Polemi [882], previously commented in this section, could be understood as an extension of those in [790]. The PI formulation is preferred in [882] due to lack of uniqueness in the voltage definition, however, there are not provided advances in the simplification of the design procedure.

A different approach is firstly disposed in [890]. The work, motivated also by the lack of numerical ports for GW (as it is obvious since there are not proper transmission line equivalents), extends the waveguide port approach of [790]. First, the port definition is faced, and optimization of numerical ports is carried out for the two usual full-wave commercial solvers, HFSS ${ }^{\circledR}$ and CST $^{\circledR}$. As it can be appreciated in Fig. 8.52(a), there are several parameters involved in the port definition: the axial distance $k_{1}$ to the first row of pins, the horizontal distance $k_{2}$ from each side of the ridge to the port edge, and the port height $h_{\text {port }}$ and location (note that given the port height, the vertical offset of the port can be as well chosen). The reflection coefficient obtained by using a given numerical port is the quality parameter. In general, return losses are more than $25 \mathrm{~dB}$, except at the edges of the designed stopband [11 GHz-22 GHz], at which is difficult to provide good matching. However, proper optimization of the port gives $R L>40 \mathrm{~dB}$. In this way, the waveguide port approach can be applied in the best way. In both cases, RGW and GGW ${ }^{11}$, the best matching is obtained by using a port with the same dimensions as the propagation channel and $k_{1}=p / 2$, i. e., the port is exactly located between two pin rows.

However, the proposed method goes beyond this optimization, and it is able to correct the waveguide port impedance to capture the effect of the pins, varying with the frequency. Basically, the proposed method analyzes the $S_{11}$ parameter, which indicates the matching between the employed numerical port and the waveguide, treating this last as a black box, i. e., what the method does is to calculate the impedance offered by a single-port structure no matter its nature or functionality, in this particular case, a transmission line. By this reason, it seems a very suitable approach from a practical design point of view, being that it characterizes the corresponding transmission line as a circuital element. In Fig. 8.52(b), the characteristic impedance of RGWs of several heights, calculated with the aforementioned method, is displayed together with the waveguide port impedance. As can be observed, differences between the waveguide port impedance and the corrected impedance are appreciated for shorter ridges (the same could be affirmed, therefore, for larger values of the air gap $h_{a}$ ), and for lower frequencies, close to the lower stopband limit. In those cases, larger values of the $S_{11}$ are found, providing the required correction. In this way, the method is more accurate than the simple waveguide port equiva-

\footnotetext{
${ }^{11}$ Note that in the case of the GGW it makes only sense to talk about wave impedance as it is done for the rectangular waveguide since the fundamental mode is not TEM or quasi-TEM. However, the wave impedance is totally defined by the propagation constant [22], generally easier to compute (think in applying the method in [361] for instance).
} 
lent suggested in [790]. Nevertheless, for usual RGW designs, the correction is small (around 2\%) unless all the stopband is used, which is not usually the case. This indicates that the waveguide port could be, despite of ignoring pin effects, an acceptable choice for most RGW designs. However, the correction taking account the pin side structures dispersive effects seems to be unavoidable for some pin configurations which give strong dispersion characteristics [361].

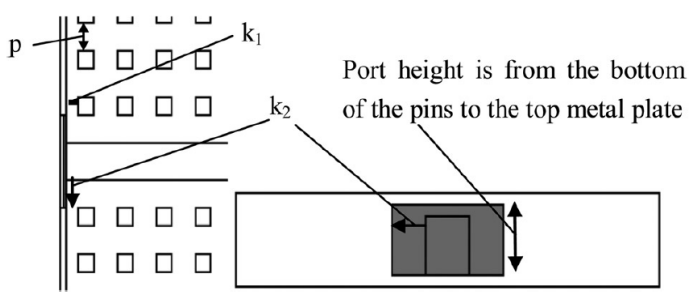

(a)

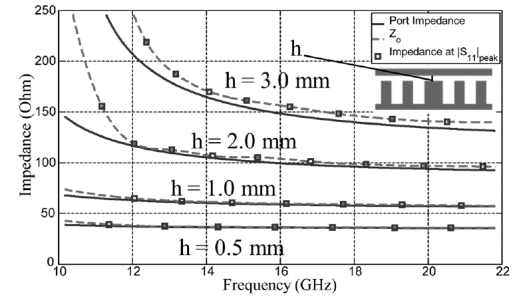

(b)

Figure 8.52: Waveguide port approach for characteristic impedance calculation. a) Numerical port parameters is used in [890] for the optimization of such ports in HFSS ${ }^{\circledR}$ and CST® full-wave commercial solvers; b) Comparison between the impedance given by a numerical port and the corrected impedance through the method proposed in [890] for a $R G W$ of different ridge heights.

In fact, the aforementioned method shows better its potential when the SSGW is treated, as proposed in [891]. Note that for the SSGW there is not an obvious equivalent waveguide port since the the substrate with the strip is located over an homogeneous perfect periodic structure of pins, this providing many possible situations for the relative position of the strip relative to the pins (over a row of pins, in between two rows, with one side starting at the end of the row, etc.), a number of situations that even extend if the strip width is parametrized. Therefore, for the SSGW, the port definition is not obvious. The proposal in [891] is to use a rectangular port with PEC conditions at the top and bottom sides and PMC conditions at the lateral sides. Optimization of this numerical port founds the best situation in leaving a horizontal distance of two periods at each side of the strip, an axial distance of $p / 4$ from the first row of pins, and locating the bottom PMC wall at a height corresponding to $h_{p} / 2$. However, unlike the RGW and GGW, the optimal numerical port may considerably differ from one design to another. This implies that every design may require a previous study, or, more desirably in a future, a study with empirical guidelines must establish the basis of the port choice (some attempts in this line are appearing these days [904]). The results in [891] indicate that the SSGW requires from noticeable corrections given the waveguide port impedance, and that the inverted microstrip [905] equivalent provides coincidence only for very specific situations. An important conclusion of this work is that it confirms the problem of the dependence of the impedance on the relative position of the strip over the pin structure, as firstly observed in [795]. This effect results very negative for circuit design, inasmuch as it is not possible to know a priori which will be the impedance of the line given its width. A denser grid (the modified period is made a half of the original period) with the strip rotated 45 degrees, i. e., making this angle with the pin grid lines, is proposed as solution. Nevertheless, rotating the strip 45 degrees makes only sense for simple circuits in which lines are predominately 
orientated in one direction. A separate study of each modifications seems to be appropriate in order to extract the isolated effects of each one.

Although the previous works dealing with the waveguide port impedance correction give a very interesting approach, some issues still remain to be solved/improved, motivating part of the work in this thesis, later described in chapter 9 , and which is recompiled in [892]-[894]. First, in [892], it is shown how some approximations in [891] lead to wrong results in the calculation of the SSGW impedance. It is found that only the magnitude of the $S_{11}$ parameter is used in [891], but not the phase, this forcing that $Z_{0}>Z_{p}$, which is not necessarily true. This is, indeed, the case of the presented SSGW design, which presents $Z_{0}<Z_{p}$. By this reason, the impedance calculation approach is rigorously posed in [892] and [893], giving, furthermore, error bounds for the analysis method. In addition, solution to the impedance dispersion with the strip position is given in a more definitive way. The work is extended in [848] and [894] to the calculation of the impedance of coupled SSGW lines for the first time. A simple coupled-line filter design gives proof of the utility of the described procedures. In addition, the filter response is shown to have very small sensitivity to the circuit position relative to the underlying pin structure. Therefore, the guidelines given in these works lead to a straightforward design process and robust designs, two desired features when talking about SSGW synthesis procedures.

\subsubsection{Packaging}

The overview of the GW technological proposals ends with an application of the GW periodic structure of metal pins that is totally different from what has been reviewed up to now. Previous designs modify the periodic structure to enable propagation channels, creating RGW, MRGW, SSGW, or GGW structures to conform devices such as waveguides, transitions, dividers, couplers, antennas or filters. However, the periodic structure can be used for packaging purposes in conventional transmission line circuits based on microstrip lines or CPWs. In that case, the top metal plate of the typical packaging box is replaced by a plate including the pins facing down, towards the circuit to be packaged. In this way, the circuit is isolated with the great advantage of avoiding any undesired resonance inside the stopband provided by the periodic structure, a degradation effect that usually threats conventional packaging boxes. Furthermore, the use of periodic distributions of pins has been shown useful to provide even in-circuit isolation between active element chips. The packaging problem is specially relevant at mm-wave frequencies inasmuch as undesired cavity resonances appear even for small packaging boxes. Although this use of GW technology is probably the furthest away from the scope of this thesis, the review some of the published work in [788], [906]-[913] is necessary to give completeness to this overview and transfer to the reader a correct global vision of GW technology potential applications.

The first proposal of using a periodic structure of pins for packaging purposes is found in the work of Rajo [906], dating from the starting of 2010, only few months later of the introduction of GWs the research community. In this work, a periodic structure providing a stopband from $8 \mathrm{GHz}$ to $21 \mathrm{GHz}(R B W=90 \%)$ is used to package a microstrip circuit consisting on a waveguiding path with two bends of 90 
degrees, see Fig. 8.53(a). The improvement introduced by the periodic structure is noticeable. Fig. 8.53(b) shows the simulated near field inside the structure, comparing the case of a conventional packaging box with a smooth metal plate (top row of images) and the case of GW packaging (bottom row of images), at 5 frequencies in the range $[10 \mathrm{GHz}-20 \mathrm{GHz}]$. It is appreciated that, whereas in the $\mathrm{GW}$ packaging case the E-field is restricted to the microstrip line, in the conventional packaging case there is an important spreading effect, and resonances are appreciated. Due to the reduced radiation, the circuit using a GW packaging works even better than the case of leaving the circuit open, without any packaging, which is, actually, a case with total lack of practical use. When compared with usual packaging solutions, either the shielded packaging with a smooth metal plate, or the open packaging with an open rim, the GW packaging performance is totally superior, being that for the conventional packagings strong resonance peaks, coming from the cavity modes, and larger insertion losses are found. The presented structure is similar to that used in [788], where cavity resonances are shown to strongly affect the microstrip circuit. In that work the MRGW is presented as solution. Therefore, it could be said that if the main problem comes from the packaging and not from the losses (this may occur at low frequencies), the use of conventional microstrip circuits packaged with GW technology is a simple, direct solution, whereas the use of a MRGW to implement the circuit is an advanced, more complex solution, deserved only by special cases.

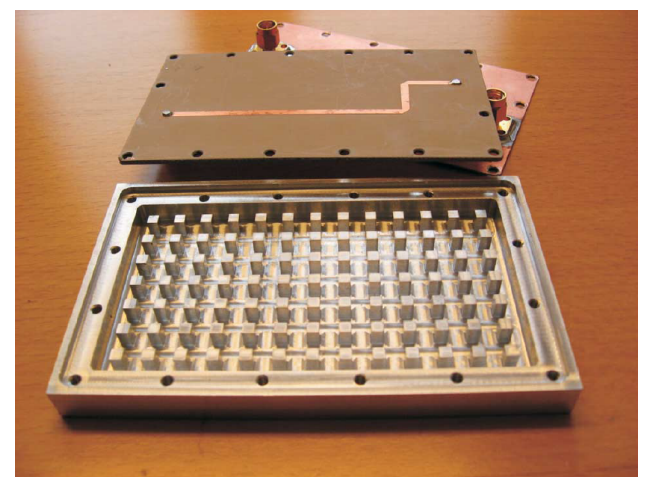

(a)
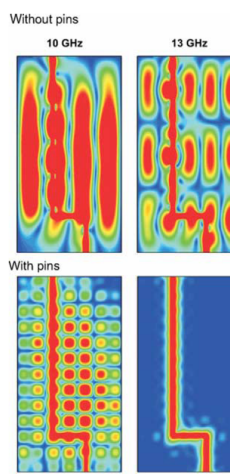
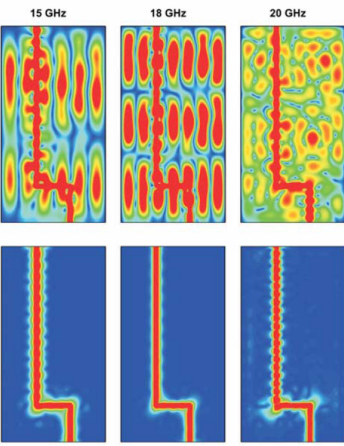

(b)

Figure 8.53: Experimental demonstration of microstrip circuits packaging using a $G W$ periodic structure [906]. a) Manufactured prototype: waveguiding path including two bends of 90 degrees, packaged inside a box including a periodic structure of pins in the top plate; b) Comparison of the simulated near field in the structure between common packaging with a smooth top metal plate (top row of images) and packaging with a top metal plate with a periodic structure of pins (bottom row of images) for five frequencies.

Similarly, in [907] a microstrip coupled-line filter of $3^{\text {rd }}$ order with Chebyshev response (constant ripple $R=0.5 \mathrm{~dB}$ ), centered at $f_{0}=15 \mathrm{GHz}$ with $R B W=10 \%$, is designed considering its packaging. The periodic structure is designed to provide a [11 GHz-20 GHz] stopband, inside of which any spurious corresponding to frequencies outside the filter passband can be found. The simulation model of the filter packaged with the GW structure is shown in Fig. 8.54(a). The box is $20 \mathrm{~mm} \times 19.26 \mathrm{~mm} \times$ $7.2 \mathrm{~mm}$, which is roughly $\lambda \times \lambda \times \lambda / 2$. Several cases are compared, including no 
packaging, conventional packaging with a smooth metal plate, GW packaging, and ideal PMC packaging. The $S_{21}$ parameter of the filter for those packaging options is shown in Fig. 8.54(b). The results indicate that either no packaging (what is not possible in practical implementations), or conventional packaging, imply a degraded response. An additional important conclusion from the plot is that not only the GW packaging works well (flat response with only $I L=0.87 \mathrm{~dB}$ ), but the response is quite close to that of an ideal PMC top plate packaging, i. e., assuming a PMC condition on the plane described by the pin heads. This observation is very advantageous in computation time terms. Notice that optimizing the proposed filter with the GW packaging require a quite larger number of discretization cells than a conventional packaging, however, a PMC packaging implies a structure with simulation complexity comparable with conventional packagings (note than the only change is replacing the top plate PEC condition by a PMC condition).

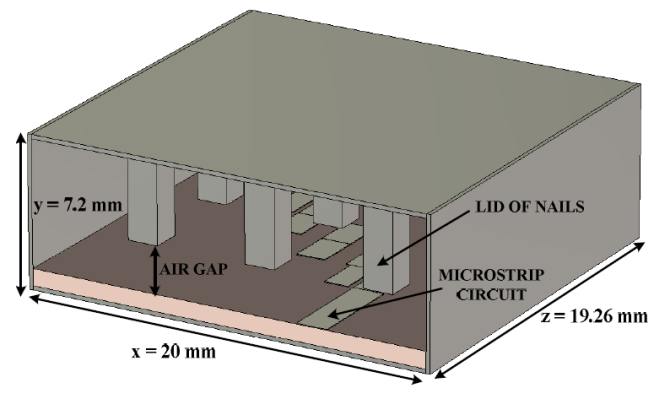

(a)

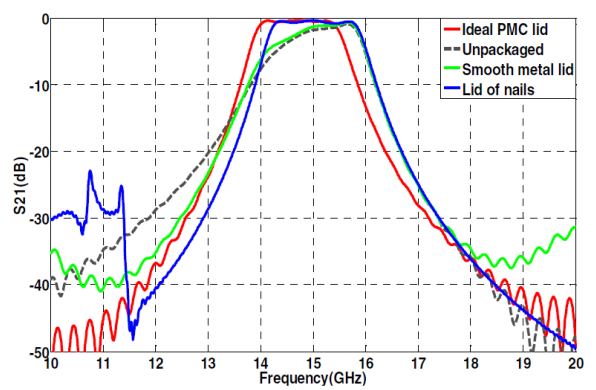

(b)

Figure 8.54: Use of the GW periodic structure for packaging microstrip coupled-line filters [907]. a) Packaged coupled-line microstrip filter; b) Comparison of the simulated $S_{21}$ of the filter for different packaging options.

The computation time issue is further investigated by Kishk in [909]. This work, is totally focused on the simulation issues in the optimization of packaged structures. It is highlighted that commonly used volume-based methods such as FEM or FDTD experience an increment of complexity when GW packagings are simulated inasmuch as there are many additional elements (pins) to mesh and, sometimes, a larger volume. Contrarily, when simulations are performed with PMC, they are even faster than with PEC, being that volume is even smaller (note that the PMC layer is placed at the end of the pins, close to the microstrip circuit). It is highlighted that unlike a PEC layer, a PMC layer can be located quite close to the circuit without affecting it. Nevertheless, it is recommended to keep a distance of $3 t-4 t$ between the PMC and the microstrip circuit, with $t$ the thickness of the microstrip substrate, in order to avoid perturbations on the characteristic impedance, which maybe caused if the E-field becomes more confined to the strip in such way that its effective width gets reduced. Simulations of packaged circuits show how the GW packaging reduces radiation as already observed in [906], what implies lower insertion losses. Nevertheless, it is pointed out that practical realizations of the PMC with pins must be done in such way that insertion losses are not affected. Simulations give good correspondence between the PMC packaging and the actual 
GW packaging, at least, better than that observed when designing GW circuits using PMC approximations. Simulation times confirm that GW packaged circuits require more than twice time than conventional packaged circuits, whereas PMC packaged circuits are simulated almost $20 \%$ faster than conventional packaged circuits.

Another interesting work treating GW packaging is found in [908]. In this work a microstrip power divider following Klopfenstein design [22] is packaged. The challenge in this work comes from the fact that the proposed Klopfenstein power divider is ultra-wide band, offering a bandwidths of 10:1, whereas GW stopbands are usually $2: 1,3: 1$ as maximum. The proposed solution consist on a packaging box that is made smallest as possible, thus avoiding low frequency resonances. The periodic structure is responsible to protect only part (higher frequencies) of the band. The power divider presents $R L<-10 \mathrm{~dB}$ over the band [ $1 \mathrm{GHz}-13.5 \mathrm{GHz}$ ]. Accordingly, the stopband is designed to be $[5 \mathrm{GHz}-15 \mathrm{GHz}]$. Since the box is small enough any resonance is found outside the stopband (from $1 \mathrm{GHz}$ to $4 \mathrm{GHz}$ ), and the improvement respect to the conventional packaging, which response presents a strong spike at $f=10 \mathrm{GHz}$, is noticeable. The agreement between simulations and measurements is acceptable, with slight differences in the ohmic losses, which are not related with the packaging. Therefore, the proposal shows great effectiveness.

Probably, the most complete and deep investigation in GW packaging is carried out by Algaba in [910]. This work extends [907], including the experimental packaging of two coupled-line microstrip filters. One of them is that designed in [907], and the other has the same topology but the order is $N=5$ and the ripple of the Chebyshev response is set as $R=0.1 \mathrm{~dB}$. The optimization process of both filters is alleviated by proper use of PMC condition instead of the actual structure with pins. Nevertheless, it is highlighted that when applying the actual structure with pins, the input impedance shifts from $Z_{i}=50 \Omega$ to $Z_{i}=47 \Omega$, and a frequency shift of $\Delta f=290 \mathrm{MHz}$ towards higher frequencies occurs. This effect is deeply studied and it is found that what occurs is that the effective permittivity of the microstrip line is slightly affected by the presence of pins, this influencing the line impedance and the resonance frequency of the filter resonators. However, it is found that the described procedure using a PMC model drives to good final results, which always can be further optimized with the real structure with pins, being that the response is close to the specifications, i. e., a good starting point for the optimization is achieved.

The two manufactured prototypes of the filters are shown in Figs. 8.55(a) and 8.55(b). Along the paper, the response of the filters is compared for several packagings including the cases of no packaging, conventional packaging with a smooth metal plate, GW packaging with different number of pins, and, for the first time, conventional packaging including dielectric absorber [779]. As an example, Fig. 8.55(c) shows a detail of the insertion loss of the $N=5$ filter. In the plot it is appreciated how radiation losses degrade the response of the unpackaged microstrip circuit, and how a strong spike at $f=14.8 \mathrm{GHz}$ totally destroys the, a priori good, performance of the conventionally packaged filter. In the case of the absorber, the response is even worse due to the introduced dielectric losses, a clear indicator that this kind of packaging must be avoided at mm-wave frequencies. Conversely, both GW packaged filters show good response, with $I L<1.7 \mathrm{~dB}$ over a flat passband. Notice that such a good packaging is achieved with only few pins (9 or 15, see again Figs. 8.55(a) and 
8.55(b)). Another interesting point treated in this paper is the study of the effect of the parameters of the periodic structure on the final filter response, being that in previous works any consideration about how different periodic structures act on the device final response is given. It is found that decreasing the pin width $w_{p}$ causes the outband spurious move close to the passband from the right, but decreasing their level. Similarly, decreasing the period $p$ cause the same effect, but the spurious increase their level, which is a worse situation. Additionally, return losses are slightly affected. Decreasing the air gap $h_{a}$ produces a noticeable increment of the stopband bandwidth at the price of a slight degradation of the return losses. Finally, to shift the plate with the pins (to cause an offset with the microstrip circuit) has almost no influence.

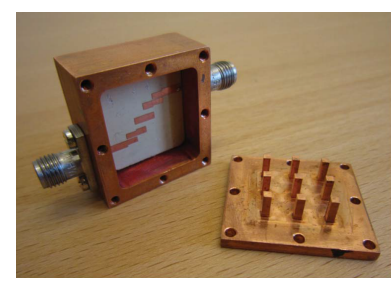

(a)

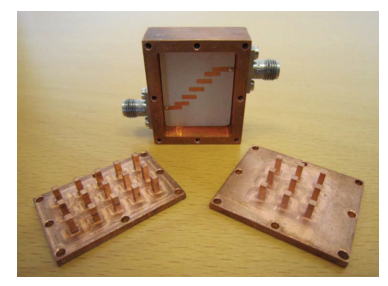

(b)

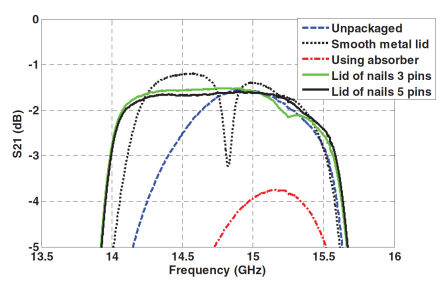

(c)

Figure 8.55: Microstrip coupled-line filters packaged with $G W$ periodic structures [910]. a) Manufactured $N=3$ filter; b) Manufactured $N=5$ filter; c) Measured insertion loss of the $N=5$ filter, comparing several packaging options.

In [911], Zaman presents an interesting alternative use of GW packagings, to provide isolation in circuits hosting high-gain amplifier chains. Two isolation scenarios are tested in this work. The first one is an amplifier chain which gives a gain of $G=65 \mathrm{~dB}$, and the second one consist in two parallel amplifier chains demanding from high isolation. Fig. 8.56(a) shows the first scenario for the both cases, GW packaging (top) and conventional packaging with metallic walls and Eccosorb absorbing material (bottom). Similarly, Fig. 8.56(b) show the second scenario for GW packaging (top) and conventional packaging (bottom). It is highlighted that in conventional packagings, shielding cause cavity resonances which are damped out by using dielectric absorbers. However, dielectric losses are an undesired negative effect, hence this absorbers must be only considered for low-gain amplifiers. Furthermore, vias are required near the hot spots to stop energy coupling through the substrate leaky-wave modes. Several solutions are found in the literature for such problem, however, their effectiveness is highly committed as the frequency increase. GW packaging is presented as a high frequency complete and simple solution for those cases with a large set of advantages which are listed next as they give a good overall idea of the introduced improvement:

1. It works within a desired frequency band and is totally independent of the cavity dimension.

2. It is easily scalable to different frequency bands. 
3. It is very efficient in terms of suppressing both, coupling over the air volume and coupling via the substrate modes within the band of the interest, which can be as wide as the stopband.

4. It suppresses the cavity modes within the desired band.

5. It improves overall insertion loss of the complete microwave circuit since leakage to unwanted modes are suppressed.

6. It reduces the need for trial and error processes with absorber placements for removing the cavity modes after the unpackaged design has been completed.

7. It removes the need for good metal contact between the circuit board and the metal enclosure. There is no need of proper grounding or any conductive adhesive or gasket material used for multi-compartment microwave modules.

8. Running signal lines from one compartment to another compartment within the microwave module is also done without degrading the isolation performance.

9. The isolation performance does not depend on mechanical issues such as stress and corrosion, joint reliability, surface properties, and deformations and apertures caused by thermal expansions and contractions.

Several computations and experimental validation of the proposal are carried out for a GW packaging which provides a stopband ranging from $26 \mathrm{GHz}$ to $46 \mathrm{GHz}$. In the case of the single amplifier chain, GW packaging keeps the gain quite stable at $G=65 \mathrm{~dB}$, whereas the conventional packaging reduces it to $G=40 \mathrm{~dB}$. In the case of the two parallel chains, the isolation provided by the GW packaging is as high as $78 \mathrm{~dB}$, whereas the conventional packaging gives $64 \mathrm{~dB}$ of isolation. In general, results show how GW packaging improve the gain, however, some practical issues are found. For instance, when providing isolation between adjacent chips, pins height must be reduced at the location of the chips. This non-uniformity reduces the isolation and degrades the response. The presence of bias, not taken into account in this work, is also pointed as a source of undesired effects. Nevertheless, it must be mentioned that GW packaging is shown to give noticeably better results than using absorbers. Also, to provide good isolation between parallel amplifier chains, up to 8 pin rows are necessary, being that using less rows (something that can be necessary by space) cause an undesired decrement of the isolation. Further work seems necessary in this line to better check the use of GW packaging in more real environments, but the presented results are promising.

GW packaging has even been considered for SIW structures. An interesting work, signed by Ke Wu, has been published recently in [913]. The work arises from a deep study of the authors on the GW stopband properties and its application for grounded CPW circuits [914]. The authors highlight that, since SIW structures are under the focus due to their better properties for mm-waves, to study the optimal way to package them is a mandatory research line these days. In this work, a GW periodic structure providing a $[10 \mathrm{GHz}-24 \mathrm{GHz}]$ stopband is used. It is checked that in a simple SIW straight line, GW packaging provides better results than leaving the 

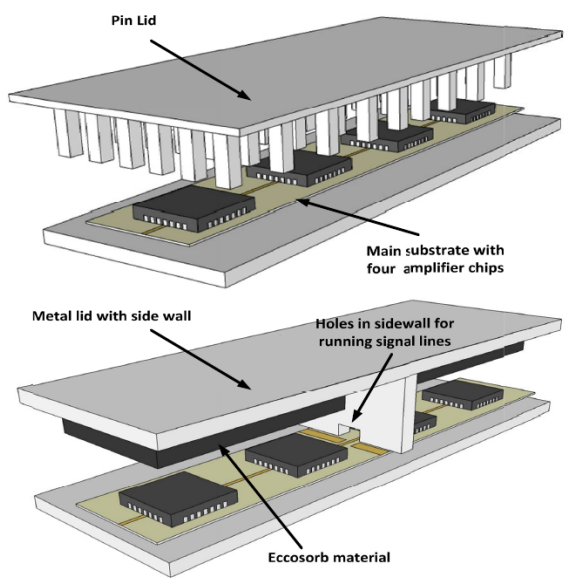

(a)

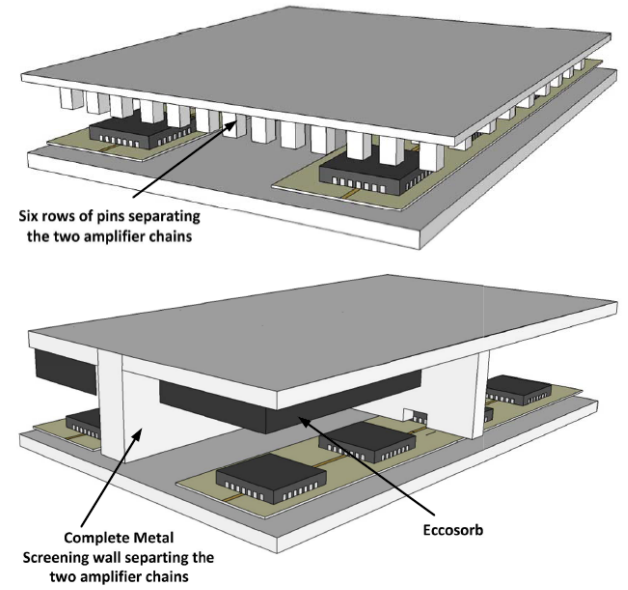

(b)

Figure 8.56: Use of the GW periodic structure for packaging high-gain amplifier chains microwave circuits [911]. a) Example use of $G W$ (top) and common (botton) packaging to provide isolation between amplifiers inside a single amplifier chain; b) Example use of $G W$ (top) and common (bottom) packaging to isolate two parallel amplifier chains.

structure open. Further validations are made with a SIW cavity filter centered at $f=14.25 \mathrm{GHz}$ with $R B W=3.5 \%$, implementing $N=5$ Chebyshev response with a ripple of $R=0.1 \mathrm{~dB}$. The SIW filter is packaged using a metal box, considering four different options for the upper lid: smooth metal lid, modified smooth lid (filling more volume of the box with a metal block over the circuit), GW lid, and simplified GW lid (pins are placed elsewhere, but not above the SIW circuit). The manufactured prototype is shown in Fig. 8.57(a), whereas the measured $S_{21}$ response for the aforementioned four options and the additional case of no packaging is shown in Fig. 8.57(b). The improvement with the GW packaging is noticeable: conventional smooth lid packaging destroys the response and the option of no packaging, besides it is not practical, gives $0.3 \mathrm{~dB}$ additional losses respect to the $\mathrm{GW}$ packaging. The simplified GW packaging with no pins over the SIW circuit, proposed by the authors, is suggested as the optimal option, being that some SIW circuits may not support the full GW packaging as the upper part of the SIW circuit may be in risk of being touched by the pins. The filter performance $(R L<12 \mathrm{~dB}$ and $I L=3.5$ $\mathrm{dB})$ is competitive with the SIW filter state of the art [772], hence this work may be a trendsetter for future SIW packaging solutions using GW technology. The use of additional lateral pins to avoid any kind of leakage of the SIW circuit could be an interesting future research line in this sense.

Finally, in [912], the packaging of a Zero-Order Resonator (ZOR) [915] circuit implemented with microstrip lines with PMC conditions is considered. This kind of resonator exhibits EM field patterns with spatial uniform phase and amplitude along the line, which leads to applications of the resonance to antennas. This kind of resonators are mainly conceived for size reduction in planar designs. Therefore, a bulky packaging box as it can be that provided by a GW packaging with pins 


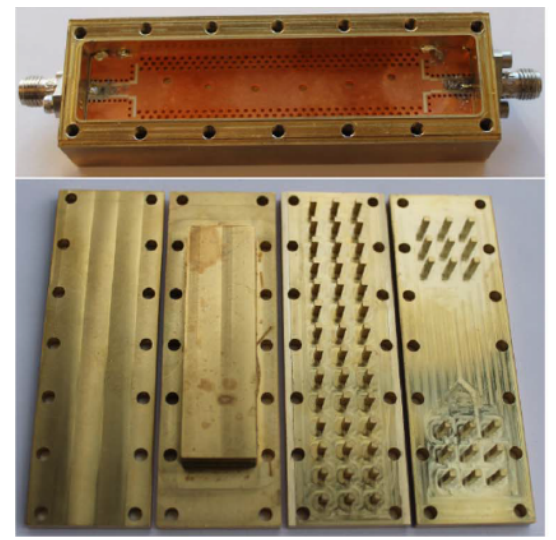

(a)

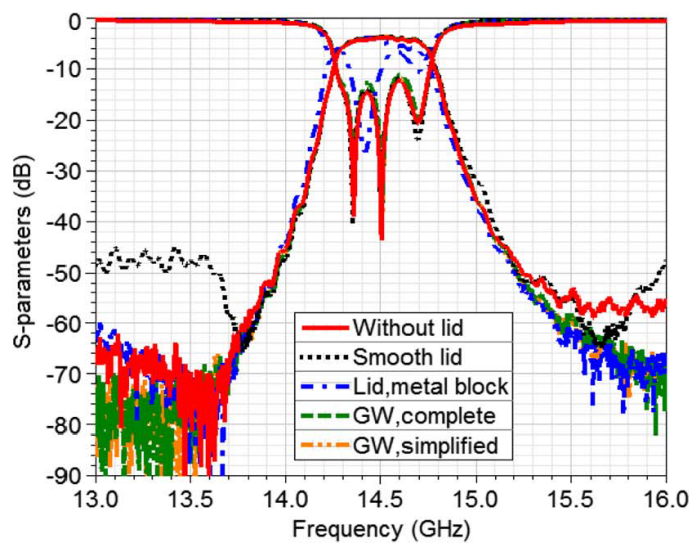

(b)

Figure 8.57: GW packaging for SIW circuits [913]. a) Manufactured SIW cavity filter with four different packaging lids: smooth metal lid, modified smooth lid (filling more volume of the box with a metal block over the circuit), GW lid, and simplified $G W$ lid (pins are not placed above the circuit); b) Measured S-parameters comparing the previous four packaging solutions. The case of no packaging is as well included.

would imply to loss, in part, the planar profile of the device. The authors propose the use of spiral nails in order to reduce the height. The designed ZOR operates at $f=10.5 \mathrm{GHz}$, hence the stopband is designed around this frequency. Whereas the designed stopband is achieved with conventional pins of $h_{p}=7 \mathrm{~mm}$, spiral pins require only $h_{p}=4 \mathrm{~mm}$, a reduction by a factor of 1.75 . Simulations show that this GW packaging improves the quality of the resonator respect to the case without packaging, and any spike is found in the response. Although there is some lack of detail in the work, and more simulations or experimental test would be desirable, the idea is interesting and it highlights the important issue of keeping a planar profile feature when planar circuits are packaged. To study low-profile GW packaging alternatives using other type of periodic elements could be a fruitful research topic. 


\section{Chapter 9}

\section{Suspended Strip Gap Waveguide (SSGW) components}

The overview of GW technology state of the art indicates that the SSGW results interesting in the context of low cost circuits, since the periodic pin lattice is fixed for this GW, and the bulk of the design task resides in a printed substrate with the circuit layout. In this way, the SSGW is a serious alternative to classical printed transmission lines in the high frequency microwave regime and the mm-wave band. This chapter faces the main analysis and design issues of SSGW structures. A first section is dedicated to the analysis task. This section deals with the impedance issue, previously introduced in the GW overview. First, the necessary theoretical tools in the context of basic transmission line theory are given since they are essential to rigorously pose the proposed analysis method. Then, such analysis method is presented and its advantages, disadvantages, limitations and practical aspects are reviewed. This method is applied in the second section to the design of a practical SSGW presenting constant impedance with the strip position. The last section gives treatment to the SSGW coupled-line problem. In a first part, coupled-line theory is exposed. Then the developed impedance analysis method is extended to be applied in the context of coupled lines by applying the previous theory. The section concludes with a practical synthesis example: a coupled-line filter.

\subsection{Impedance Analysis}

\subsubsection{Transmission line theory}

According to [22], transmission line theory fills the conceptual gap between the complex full-wave field analysis, many times unavoidable at microwave frequencies, and the basic circuit theory used at lower frequencies and DC. Therefore, wave propagation on transmission lines is either approached as an extension of circuit theory or a specialization of Maxwell's equations. In this way, transmission line 
theory becomes a very suitable tool to carry out the synthesis of microwave networks otherwise cumbersome to analyze.

From the circuit theory point of view, the key difference when dealing with transmission lines is the electrical size. Whereas in conventional circuit analysis the physical dimensions of a network are much smaller than the electrical length, transmission lines may be a considerable fraction of the wavelength, or even many wavelengths, in size. Thus, a transmission line is a distributed-parameter network, where voltages and currents can vary in magnitude and phase over its length.

Conventionally, a transmission line is represented as in Fig. 9.1(a), i. e., it is considered that the transmission line has two conductors so that waves are guided by a TEM fundamental mode. The short piece of line of length $\Delta z$ of Fig. 9.1(a) can be modeled as a lumped-element circuit, as shown in Fig. 9.1(b), where $(R, L, G, C)$ are, per unit length, the quantities defined as follows:

- $R$ : Series resistance per unit length for both conductors $(\Omega / \mathrm{m})$.

- $L$ : Series inductance per unit length for both conductors $(H / \mathrm{m})$.

- $G$ : Shunt conductance per unit length $(H / \mathrm{m})$.

- $C$ : Shunt capacitance per unit length $(L / \mathrm{m})$.

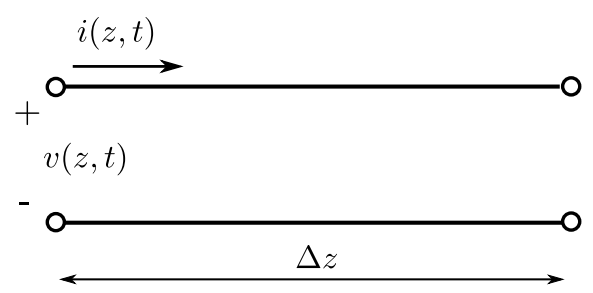

(a)

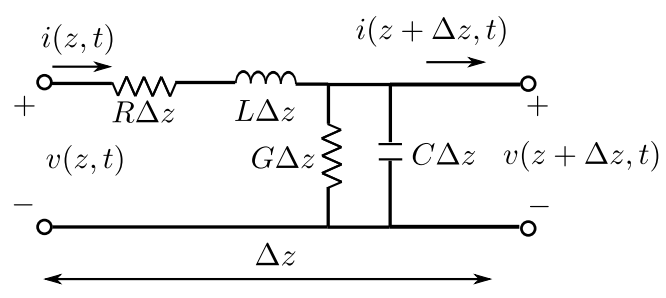

(b)

Figure 9.1: Mathematical modeling of a transmission line. a) Voltage and current definitions; b) Lumped-element equivalent circuit.

The series inductance $L$ represents the total self-inductance of the two conductors, and the shunt capacitance $C$ is due to the close proximity of the two conductors. The series resistance $R$ represents the resistance due to the finite conductivity of the conductors, and the shunt conductance $G$ is due to dielectric loss in the material between the conductors. Therefore, $R$ and $G$ are loss terms, representatives of the ohmic and dielectric losses, respectively. A finite length of transmission line can be viewed as a cascade of sections of the form of Fig. 9.1(b).

Now, if Kirchhoff's voltage law is applied to the circuit of Fig. 9.1(b):

$$
v(z, t)-R \Delta z i(z, t)-L \Delta z \frac{\partial i(z, t)}{\partial t}-v(z+\Delta z, t)=0,
$$

whereas Kirchhoff's current law leads to

$$
i(z, t)-G \Delta z v(z, t)-C \Delta z \frac{\partial v(z, t)}{\partial t}-i(z+\Delta z, t)=0,
$$


Dividing (9.1) by $\Delta z$ and (9.2) and taking the limit as $\Delta z \rightarrow 0$ (differential section of a line) gives the following differential equations:

$$
\begin{aligned}
& \frac{\partial v(z, t)}{\partial z}=-\operatorname{Ri}(z, t)-L \frac{\partial i(z, t)}{\partial t} \\
& \frac{\partial i(z, t)}{\partial z}=-G v(z, t)-C \frac{\partial v(z, t)}{\partial t}
\end{aligned}
$$

These equations, the so-called the telegrapher equations represent the time-domain form of the transmission line. In the case of sinusoidal steady-state condition with cosine-based phasors, they simplify to:

$$
\begin{aligned}
\frac{\mathrm{d} V(z)}{\mathrm{d} z} & =-(R+j \omega L) I(z) \\
\frac{\mathrm{d} I(z)}{\mathrm{d} z} & =-(G+j \omega C) V(z)
\end{aligned}
$$

Now, the coupled system formed by (9.5) and (9.6) can be uncoupled by substitution to be expressed in such way that the following two wave equations for $V(z)$ and $I(z)$ are obtained:

$$
\begin{aligned}
\frac{\mathrm{d}^{2} V(z)}{\mathrm{d} z^{2}}-\gamma^{2} V(z) & =0 \\
\frac{\mathrm{d}^{2} I(z)}{\mathrm{d} z^{2}}-\gamma^{2} I(z) & =0
\end{aligned}
$$

where

$$
\gamma=\alpha+j \beta=\sqrt{(R+j \omega L)(G+j \omega C)}
$$

is the complex propagation constant, the same that was defined in chapter 3 in the context of full-wave analysis of propagation in dielectric waveguides, and that depends on the frequency. Traveling wave solutions to (9.7) and (9.8) are:

$$
\begin{aligned}
V(z) & =V_{0}^{+} e^{-\gamma z}+V_{0}^{-} e^{-\gamma z} \\
I(z) & =I_{0}^{+} e^{-\gamma z}+I_{0}^{-} e^{-\gamma z}
\end{aligned}
$$

where the $e^{-\gamma z}$ term represents wave propagation in the $+\hat{z}$ direction, and the $e^{\gamma z}$ term represents wave propagation in the $-\hat{z}$ direction.

Now, applying (9.5) to the voltage equation (9.10) gives the current on the line as:

$$
I(z)=\frac{\gamma}{R+j \omega L}\left[V_{0}^{+} e^{-\gamma z}+V_{0}^{-} e^{-\gamma z}\right]
$$

Comparison of the previous equation with (9.11) shows that a characteristic impedance $Z_{0}$ can be defined as 


$$
Z_{0}=\frac{R+j \Omega L}{\gamma}=\sqrt{\frac{R+j \omega L}{G+j \omega C}}
$$

to relate the voltage and current on the line as

$$
Z_{0}=\frac{V_{0}^{+}}{I_{0}^{+}}=-\frac{-V_{0}^{-}}{I_{0}^{-}}
$$

Then, (9.11) can be rewritten as

$$
I(z)=\frac{V_{0}^{+}}{Z_{0}} e^{-\gamma z}-\frac{V_{0}^{-}}{Z_{0}} e^{\gamma z}
$$

Converting back to the time domain, the voltage waveform can be expressed as

$$
v(z, t)=\left|V_{0}^{+}\right| \cos \left(\omega t-\beta z+\phi^{+}\right) e^{-\alpha z}+\left|V_{0}^{-}\right| \cos \left(\omega t-\beta z+\phi^{-}\right) e^{-\alpha z}
$$

where $\phi^{ \pm}$is the phase angle of the complex voltage $V_{0}^{ \pm}$. From the previous expression they are easily derived the guided wavelength

$$
\lambda_{g}=\frac{2 \pi}{\beta}
$$

and the phase velocity

$$
v_{p}=\frac{\omega}{\beta}=\lambda_{g} f
$$

\subsubsection{The lossless transmission line}

Once the general solution has been posed, as usual, it is convenient to consider the lossless case, which is often a suitable approximation that simplifies the resolution of the problem without introducing excessive error since, in general, transmission line losses are so small that they can be neglected in a first analysis stage dealing with propagation characteristics. Therefore, if $R=0$ and $G=0$ are assumed in (9.9) it is found that

$$
\gamma=\alpha+j \beta=j \omega \sqrt{L C}
$$

or

$$
\begin{aligned}
& \alpha=0 \\
& \beta=\omega \sqrt{L C}
\end{aligned}
$$

Then, the characteristic impedance reduces to

$$
Z_{0}=\sqrt{\frac{L}{C}}
$$


which is now a real number. The general solutions for voltage and current on a lossless transmission line can be accordingly written as

$$
\begin{aligned}
V(z) & =V_{0}^{+} e^{-j \beta z}+V_{0}^{-} e^{j \beta z} \\
I(z) & =\frac{V_{0}^{+}}{Z_{0}} e^{-j \beta z}-\frac{V_{0}^{-}}{Z_{0}} e^{j \beta z}
\end{aligned}
$$

being now the guided wavelength

$$
\lambda_{g}=\frac{2 \pi}{\beta}=\frac{2 \pi}{\omega \sqrt{L C}}
$$

and the phase velocity

$$
v_{p}=\frac{\omega}{\beta}=\frac{1}{\sqrt{L C}}
$$

\subsubsection{The terminated lossless transmission line}

The problem of a terminated lossless transmission line, which will be essential for the rest of this thesis, is posed now with the aid of the circuit schematic description of Fig. 9.2. As the circuit schematic shows, a lossless line described by its characteristic impedance and propagation constant $\left(Z_{0}, \beta\right)$ is terminated by an arbitrary impedance $Z_{l}$. This will cause, in general, reflections, which is a fundamental property of distributed systems that leads to an important concept: the impedance viewed from a reference plane in a transmission line.

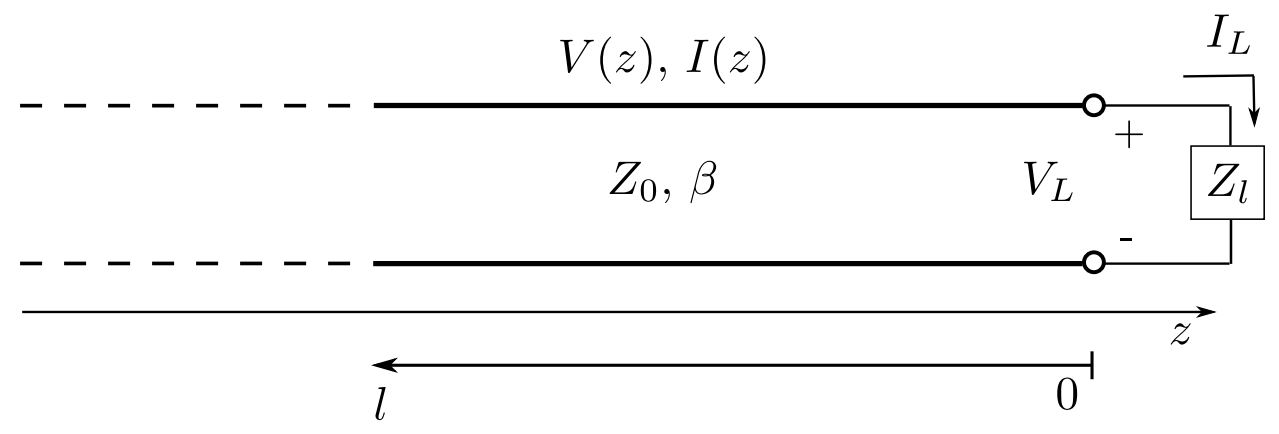

Figure 9.2: Transmission line terminated with a load impedance $Z_{l}$.

Assume that an incident wave of the form $V_{0}^{+} e^{-j \beta z}$ is generated from a source at $z<0$. It has been previously shown that the ratio of voltage to current for such a traveling wave is $Z_{0}$, the characteristic impedance of the transmission line. However, since the line is terminated in an arbitrary load $Z_{L} \neq Z_{0}$, the ratio of voltage to current at the load must be $Z_{L}$. Therefore, a reflected wave must be excited with the appropriate amplitude to satisfy such condition. The total voltage on the line can then be written as (9.23), i. e., as a sum of incident and reflected waves. Similarly, 
the total current on the line is described by (9.24). Therefore, at the load $(z=0)$ the total voltage and current are related by the load impedance so that

$$
Z_{L}=\frac{V(0)}{I(0)}=\frac{V_{0}^{+}+V_{0}^{-}}{V_{0}^{+}-V_{0}^{-}} Z_{0}
$$

expression that solved for $V_{0}^{-}$gives

$$
V_{0}^{-}=\frac{Z_{L}-Z_{0}}{Z_{L}+Z_{0}} V_{0}^{+}
$$

The amplitude of the reflected voltage wave normalized to the amplitude of the incident voltage wave is known as the voltage reflection coefficient, defined as:

$$
\Gamma=\frac{V_{0}^{-}}{V_{0}^{+}}=\frac{Z_{L}-Z_{0}}{Z_{L}+Z_{0}}
$$

A current reflection coefficient, which gives the normalized amplitude of the reflected current wave, can also be defined. However, since such a current reflection coefficient is just the negative of the voltage reflection coefficient, something that can be seen by comparing (9.23) and (9.24), is a common practice to avoid it and to work exclusively with the voltage reflection coefficient.

By the above, the total voltage and current waves on the line can then be written as

$$
\begin{aligned}
V(z) & =V_{0}^{+}\left[e^{-j \beta z}+\Gamma e^{-j \beta z}\right] \\
I(z) & =\frac{V_{0}^{+}}{Z_{0}}\left[e^{-j \beta z}-\Gamma e^{-j \beta z}\right]
\end{aligned}
$$

From these equations it is observed that the voltage and current on the line consist of a superposition of an incident and reflected wave, what it is usually called standing waves. Only in the particular case of $\Gamma=0$ is there no reflected wave. This occurs when the load impedance is equal to the characteristic impedance, i. e., $Z_{L}=Z_{0}$ of the transmission line, something that can be appreciated by observing 9.29. In this situation it is said that the load is matched to the line, since there is no reflection of the incident wave.

Now, the time-average power flow along the line at the point $z$ is considered:

$$
P=\frac{1}{2} \operatorname{Re}\left[V(z) I(z)^{*}\right]=\frac{1}{2} \frac{V_{0}^{+}}{Z_{0}} \operatorname{Re}\left\{1-\Gamma^{*} e^{-2 j \beta z}+\Gamma e^{2 j \beta z}-|\Gamma|^{2}\right\}
$$

where (9.30) and (9.31) have been used. If it is defined $A=\Gamma^{*} e^{-2 j \beta z}$, it is found that the two middle terms of (9.32) are $A-A^{*}=2 j \operatorname{Im}(A)$, hence they are purely imaginary. This simplifies the result to

$$
P=\frac{1}{2} \frac{\left|V_{0}^{+}\right|^{2}}{Z_{0}}\left(1-|\Gamma|^{2}\right)
$$


which shows that the average power flow is constant at any point on the line, and that the total power delivered to the load $P$ is equal to the incident power $\left(\left|V_{0}^{+}\right|^{2} / Z_{0}\right)$ minus the reflected power $\left(\left|V_{0}\right|^{2}|\Gamma|^{2} / 2 Z_{0}\right)$. Under matching conditions $(\Gamma=0)$, maximum power is delivered to the load, while no power is delivered when $\Gamma=1$, situation that occurs either when $Z_{L}=0$ (short circuit) or $Z_{L}=\infty$ (open circuit), according to (9.29). It is worth to note that in the described procedure it is always assumed that the generator is matched, so that there is no reflection of the reflected wave from $z<0$.

When the load is mismatched, then, not all the available power from the generator is delivered to the load. This loss is the so-called Return Loss (RL), recurrently mentioned along this thesis. It is defined as

$$
R L=-20 \log |\Gamma|(\mathrm{dB})
$$

From a network point of view, if the the device under consideration has its input in the access $i, S_{i i}=R L$. The previous definition gives that under matching conditions $\Gamma=0$, hence $R L=\infty \mathrm{dB}$ (no reflected power), whereas in total reflection conditions $\Gamma=1$, hence $R L=0 \mathrm{~dB}$ (all incident power is reflected).

If the load is matched to the line, $\Gamma=0$, and the magnitude of the voltage on the line is $|V(z)|=\left|V_{0}^{+}\right|$, which is a constant. However, when the load is mismatched, the presence of a reflected wave leads to standing waves where the magnitude of the voltage on the line is not constant. Thus, from (9.30):

$$
\begin{aligned}
|V(z)| & =\left|V_{0}^{+} \| 1+\Gamma e^{2 j \beta z}\right| \\
& =\left|V_{0}^{+} \| 1+\Gamma e^{-2 j \beta l}\right| \\
& =\left|V_{0}^{+}\right||1+| \Gamma\left|e^{\theta-2 j \beta l}\right|
\end{aligned}
$$

where $l=-z$ is a change of variable to take into account the distance measured from the load $z=0$ as positive, and $\theta$ is the phase of the complex ${ }^{1}$ reflection coefficient $\left(\Gamma=|\Gamma| e^{j} \theta\right)$. The previous expressions show that voltage magnitude oscillates with the position $z$ along the line. The maximum value occurs when the phase term $e^{(\theta-2 \beta l)}=1$, and is given by

$$
V_{\max }=\left|V_{0}^{+}\right|(1+|\Gamma|)
$$

whereas the minimum value occurs when the phase term $e^{(\theta-2 \beta l)}=-1$, and is given by

$$
V_{\text {min }}=\left|V_{0}^{+}\right|(1-|\Gamma|)
$$

As $|\Gamma|$ increases, the ratio of $V_{\max }$ to $V_{\min }$ increases. In this context it is usual to employ the Standing Wave Ratio (SWR) defined as

$$
S W R=\frac{V_{\max }}{V_{\min }}=\frac{1+|\Gamma|}{1-|\Gamma|}
$$

which satisfies $1 \leq S W R \leq \infty$.

\footnotetext{
${ }^{1}$ Note that $Z_{L}$ is arbitrary and may be complex.
} 
Following (9.37), it is also deduced that the distance between two successive voltage maxima (or minima) is $d=2 \pi / 2 \beta=\lambda / 2$, whilst the distance between a maximum and a minimum is $d=\pi / 2 \beta=\lambda / 4$, where $\lambda$ is the wavelength on the transmission line.

Previously, the reflection coefficient has been defined in (9.29) as the ratio of the reflected to the incident voltage wave amplitudes at the load $(l=0)$, but this quantity can be generalized to any point $l$ on the line as follows. If $z=-l$ in (9.23) and $(9.24)$, it can be derived the ratio of the reflected component to the incident component at that point as:

$$
\Gamma(l)=\frac{V_{0}^{-} e^{-j \beta l}}{V_{0}^{+} e^{j \beta l}}=\Gamma(0) e^{-2 j \beta l}
$$

where $\Gamma(0)$ is the reflection coefficient at $z=0$ given in (9.29). This form is useful when transforming the effect of a load mismatch down the line, a procedure that is, actually, the backbound of the methods presented in section 9.1.2 and section 10.2.1.

It has been shown how the real power flow on the transmission line is a constant but that the voltage amplitude, at least for a mismatched line, is oscillatory with position on the line. Therefore, by appreciating the change of sign on the expressions (9.23) to (9.23) it is easy to conclude that the impedance observed from a certain reference plane on the transmission line will, indeed, depend on the position. At a distance $l=-z$ from the load, the input impedance seen looking toward the load is

$$
Z_{i n}=\frac{V(-l)}{I(-l)}
$$

which through (9.30) and (9.31) converts in

$$
Z_{i n}=\frac{V_{0}^{+}\left[e^{j \beta l}+\Gamma e^{-j \beta l}\right]}{V_{0}^{+}\left[e^{j \beta l}-\Gamma e^{-j \beta l}\right]} Z_{0}
$$

that finally simplifies in

$$
Z_{i n} \frac{1+\Gamma e^{-2 j \beta l}}{1-\Gamma e^{-2 j \beta l}}
$$

The previous expressions results more suitable in terms of the design parameters $Z_{0}$ and $Z_{L}$, what can be achieved by applying the expression (9.29) of $\Gamma$ :

$$
Z_{i n}=Z_{0} \frac{\left(Z_{L}+Z_{0}\right) e^{j \beta l}+\left(Z_{L}-Z_{0}\right) e^{-j \beta l}}{\left(Z_{L}+Z_{0}\right) e^{j \beta l}-\left(Z_{L}-Z_{0}\right) e^{-j \beta l}}
$$

Finally, applying the well-known relationships of exponential and trigonometric functions [542]:

$$
Z_{i n}=Z_{0} \frac{Z_{L} \cos (\beta l)+j Z_{0} \sin (\beta l)}{Z_{0} \cos (\beta l)+j Z_{L} \sin (\beta l)}
$$

or

$$
Z_{\text {in }}=Z_{0} \frac{Z_{L}+j Z_{0} \tan (\beta l)}{Z_{0}+j Z_{L} \tan (\beta l)}
$$


The previous result (usually called transmission line impedance equation) is very relevant insomuch as it gives the input impedance of a length of transmission line with an arbitrary load impedance. Note that $\tan (x)$ is a periodic function of period $2 \pi$ that monotonically grows from $-\infty$ to $\infty$ inside each period. By taking limits in (9.47) it is easily observable that when $\tan (\beta l) \rightarrow \pm \infty, Z_{i}=1 / Z_{L}$, whereas when $\tan (\beta l)=0 Z_{i}=Z_{L}$, which often are useful particular values.

\subsubsection{Short circuit terminated line}

A particular case of interest in the scope of this thesis is when the load is a short circuit, i. e, $Z_{L}=0$. From (9.29) it is easily seen that such situation implies $\Gamma=-1$, what gives a $S W R=1$, and implies that the voltage and current equation become

$$
\begin{aligned}
V(z) & =V_{0}^{+}\left[e^{-j \beta z}-e^{-j \beta z}\right] \\
I(z) & =\frac{V_{0}^{+}}{Z_{0}}\left[e^{-j \beta z}+e^{-j \beta z}\right]
\end{aligned}
$$

or equivalently

$$
\begin{aligned}
V(z) & =-2 j V_{0}^{+} \sin (\beta z) \\
I(z) & =\frac{2 V_{0}^{+}}{Z_{0}} \cos (\beta z)
\end{aligned}
$$

Therefore, the input impedance at $z=-l$, defined as the ratio $V(-l) / I(-l)$ is:

$$
Z_{i n}=j Z_{0} \tan (\beta l)
$$

which is, perforce, purely imaginary for any length $l$, and takes all values between $Z_{i n}=-j \infty$ and $Z_{i n}=+j \infty$, as the function $\tan (\beta l)$ determines. The previous expression also indicates that the impedance is periodic in $l$, repeating for multiples of $\lambda / 2$.

\subsubsection{Proposed analysis method}

According to the state of the art in GW analysis methods, the impedance definition and calculation is a major issue to address, which must be solved in order to GW technology truly offer a commercial alternative to classical microwave circuits adapted for mm-wave frequencies. The intrinsic periodic character of $\mathrm{GW}$ waveguides complicates the analysis not only in the calculations, but also in a conceptual way. This drawback imposes a critical limit in circuit design, since a precise knowledge and handling of the impedance concept and practical expressions are fundamental when more elaborated components such as directional couplers, filters or antennas have to be synthesized [22]. In this section, it is presented a rigorous method to obtain both, the propagation constant and the impedance of an unknown transmission line from a scattering parameter analysis of an auxiliary network containing the transmission line under study. The method is based on the waveguide 
port correction method of [890], however, some issues are addressed and it is posed as rigorous as possible, giving, furthermore, an error analysis in order to quantify or to bound the miscalculations originated by this numerical method as a function of the involved full-wave analysis error parameters. This way to calculate the impedance is, conceptually, more adequate for circuit synthesis since it obtains the impedance of a line as a circuit element that later will be embedded on a network. From the engineering point of view the approach is closer to practical scenario designs than observing locally the full EM fields of the structure. In addition, the method inherently links conventional well-known structures, which conform the waveguide ports, with the structures under study.

\subsubsection{Basis of the method}

The procedure is as follows. Consider the circuit of Fig. 9.3(a). It consist of a two ports network with three transmission lines in cascade connection. The first transmission line is characterized by known characteristic impedance and propagation constant $\left(Z_{p}, \beta_{p}\right)$, and has length $l_{p}$; the second transmission line is characterized by unknown $\left(Z_{0}, \beta\right)$ and has length $l$, and the third transmission line has the same characteristics as the first. This is the usual situation when a commercial solver such as $\mathrm{HFSS}^{\circledR}$ or $\mathrm{CST}^{\circledR}$ is used, since the waveguide ports must have a finite length when some flexibility on the boundary conditions is desired. Fig. 9.3(b) shows the HFSS ${ }^{\circledR}$ simulation model of the SSGW implementation of the circuit of Fig. 9.3(a).

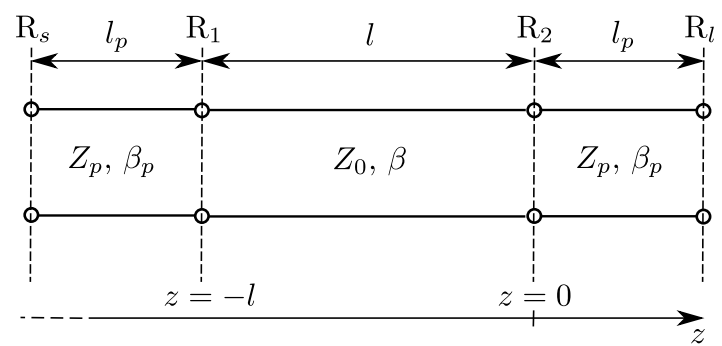

(a)

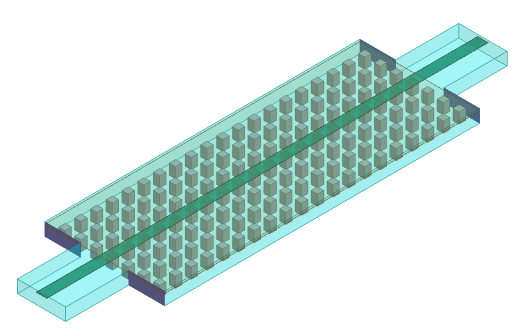

(b)

Figure 9.3: Analyzed network in the proposed method. a) Transmission line schematic; b) Actual HFSS ${ }^{\circledR}$ simulation model.

In the network of Fig. 9.3(a) the voltage and the current along the second transmission line are, according to section 9.1.1.2:

$$
\begin{aligned}
V(z) & =V^{+}\left(e^{-j \beta z}+\Gamma e^{j \beta z}\right) \\
I(z) & =\frac{V^{+}}{Z_{0}}\left(e^{-j \beta z}-\Gamma e^{j \beta z}\right)
\end{aligned}
$$

where

$$
\Gamma=\frac{Z_{p}-Z_{0}}{Z_{p}+Z_{0}}
$$


The impedance at the reference plane $R_{1}$, placed at $z=-l$, is $Z_{1}=V(-l) / I(-l)$, thus:

$$
Z_{1}=Z_{0} \frac{e^{j \beta l}+\Gamma e^{-j \beta l}}{e^{j \beta l}-\Gamma e^{-j \beta l}}
$$

Considering the scattering parameters referred to the port impedance $Z_{p}$ at the reference planes $R_{1}$ and $R_{2}$, it is satisfied

$$
S_{11}=\left(Z_{1}-Z_{p}\right) /\left(Z_{1}+Z_{p}\right)
$$

so that after some algebraic manipulations it is obtained

$$
S_{11}=j \frac{\left(Z_{0}^{2}-Z_{p}^{2}\right) \tan (\beta l)}{2 Z_{0} Z_{p}+j\left(Z_{0}^{2}+Z_{p}^{2}\right) \tan (\beta l)}
$$

Then, since

$$
S_{21}=V^{+}(z=0) / V^{+}(z=-l)
$$

or equivalently,

$$
S_{21}=\left(1+S_{11}\right) V(z=0) / V(z=-l)
$$

one has that

$$
S_{21}=\frac{2 Z_{0} Z_{p}}{2 Z_{0} Z_{p} \cos (\beta l)+j\left(Z_{0}^{2}+Z_{p}^{2}\right) \sin (\beta l)}
$$

Now, the equation (9.58) can be manipulated to give

$$
\tan (\beta l)=-j \frac{2 Z_{p} Z_{0} S_{11}}{Z_{0}^{2}\left(1-S_{11}\right)-Z_{p}^{2}\left(1+S_{11}\right)}
$$

If the previous expression is used in $(9.61)^{2}$, one has the $S_{21}$ parameter as a function of only $Z_{0}$.

Since the scattering parameters of the network can be obtained with the simulation model at the planes planes $R_{s}$ and $R_{l}$, they can be trivially translated to the planes planes $R_{1}$ and $R_{2}$ multiplying by the factor $e^{j 2 \beta_{p} l_{p}}$ in order to equate the derived expressions to an empirical value. Therefore, the unknown impedance $Z_{0}$ can be easily found through any zero-finding routine [528].

Once $Z_{0}$ is known, the value of $\tan (\beta l)$ is calculated from (9.62). Since the function $f(x)=\tan ^{-1}(x)$ is not single-valued, there is an infinite number of possible solutions for $\beta l$, each of them separated by $n \pi$. However, if an estimation of this value is available, this is not truly a problem to derive the correct solution.

Therefore, it is possible to characterize the waveguide under study by just obtaining the S-parameters of the network of Fig. 9.3(a). Nevertheless, it is important to remark that the proposed formulation gives correct results provided that the $S_{11}$ parameter is not in a minimum, i.e., $\beta l \neq n \pi$, since in that case no information

\footnotetext{
${ }^{2}$ Notice the following trigonometric relationships: $\sin (x)=\tan (x) / \sqrt{1+\tan ^{2}(x)}, \cos (x)=$ $1 / \sqrt{1+\tan ^{2}(x)}$.
} 
about the impedance is obtained. Thus, the procedure must be done for several lengths $l$ or for several frequencies. Figs. 9.4(a)-9.4(c) show simulation models of several lengths, which are achieved by varying the number of periods of the SSGW under study. However, it will be generally preferred to analyze several frequencies since this allows to obtain the impedance in a whole frequency band: when the $S_{11}$ parameter is known in the analyzed frequency band, frequency points far from the local minimums must be chosen to obtain the transmission line parameters $\left(\beta, Z_{0}\right)$. Then, by interpolation and extrapolation these parameters are obtained for all the band. Fig. 9.4(d) shows a typical $\left|S_{11}(\mathrm{~dB})\right|$ response in this kind of analysis. Note that the periodicity of the $S_{11}$ parameter comes from the term $\tan (\beta l)$ in (9.58). Therefore, the larger $l$, the larger the number of peaks in the function $\left|S_{11}(f)\right|$, what is interesting since the peaks are the points with less relative error, thus having the maximum information. Note as well, however, that larger values of $l$ implies as well larger computation times, hence, as usual, a trade-off must be found depending on the problem context.

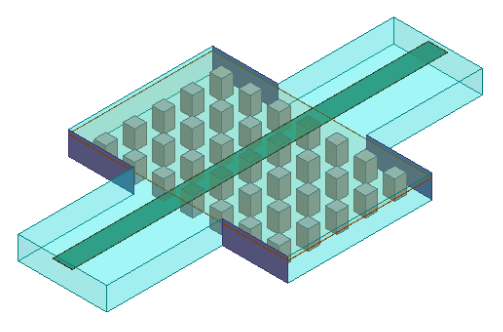

(a)

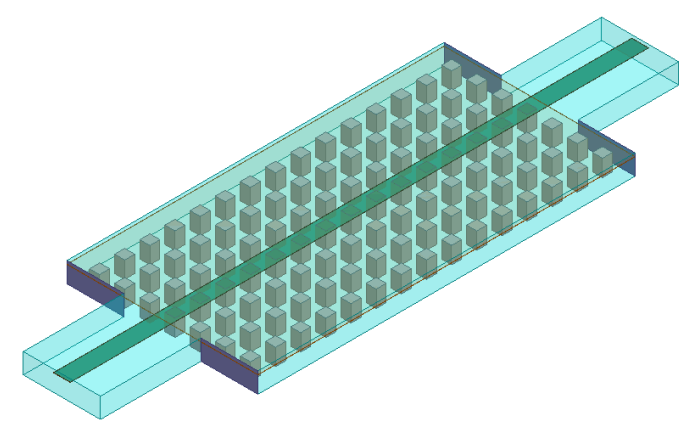

(c)

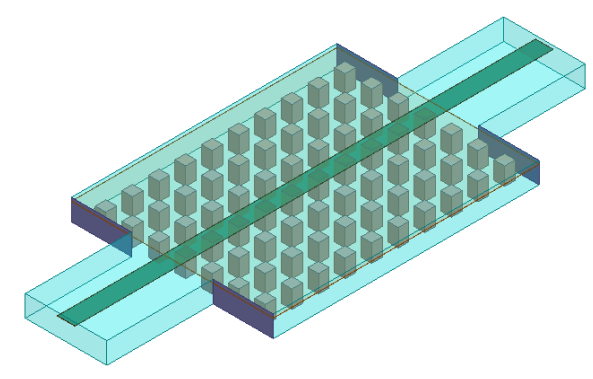

(b)

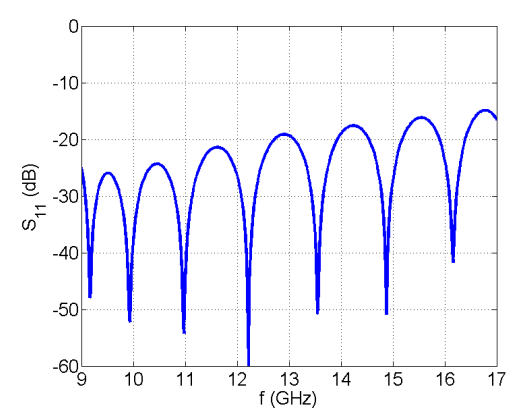

(d)

Figure 9.4: Simulation models with different lengths $l$ (varying the number of periods $N_{p}$ ) and typical $\left|S_{11}(d B)\right|$ parameter frequency response of the network under analysis. a) $N_{p}=5$; b) $N_{p}=10$; c) $N_{p}=15 ;$ d) $\left|S_{11}(d B)\right|$ frequency response exhibiting several peaks. The larger the length $l$, the larger the number of peaks in a given frequency band.

If fact, the previous procedure leads to an important simplification, as observed in [890]. At the frequency points where the $S_{11}$ parameter is maximum, it is satisfied that $\beta l=\pi / 2+n \pi$, therefore one has 


$$
S_{11}=\frac{Z_{0}^{2}-Z_{p}^{2}}{Z_{0}^{2}+Z_{p}^{2}}
$$

or

$$
Z_{0}=Z_{p}\left(\frac{1+S_{11}}{1-S_{11}}\right)^{\frac{1}{2}}
$$

where the unknown impedance is trivially obtained. This option will be used. Note that the propagation constant can be also found just by knowing the value of $n$ in $\beta l=\pi / 2+n \pi$ through the initial estimation for $\beta l$.

Finally, one more simplification can be introduced if $Z_{p} \approx Z_{0}$. Under this condition, the previous equation for $Z_{0}$ can be approximated by ${ }^{3}$

$$
Z_{0}=Z_{p} \frac{2+S_{11}}{2-S_{11}}
$$

which is the expression used in [890] and [891]. However, since (9.64) is analytical, this approximation seems to be unnecessary and limits the method to cases in which $Z_{p} \approx Z_{0}$ is satisfied. In Fig. 9.5(a), it is shown the percent error of the approximation as a function of the quotient $Z_{0} / Z_{p}$. The proposed method in this thesis, which uses (9.64), can be applied to the case of a large difference between $Z_{0}$ and $Z_{p}$ with the only requirement of avoiding the excitation of high-order modes. By this, it makes no sense any further simplification.

At this point, it is worth to mention one mistake that could lead to important errors. In the equations (9.64) and (9.65), since $Z_{0}$ should be real, and the $S_{11}$ parameter is observed in its module peaks, one can forget that this not implies the related $S_{11}$ to be positive, and the temptation of using the absolute value of the $S_{11}$ parameter must be avoided. In that case, a greater mismatch, i. e., greater values of $\left|S_{11}\right|$, would lead only to greater values of $Z_{0}$. This seems to be the case in [891], where a characteristic impedance satisfying $Z_{0}>Z_{p}$ is obtained for all cases of the position of the strip, whereas as it is shown in [795], and as will be explained in the next section, what actually occurs is just the contrary $\left(Z_{0}<Z_{p}\right)$. It is easy to foresee that with such a wrong way of proceeding large errors may be obtained, being that the corresponding correction is applied in the contrary sense.

\subsubsection{Error analysis}

Explained the method, it would be interesting to quantify the error in the calculated impedance. Instead of the exact $S_{11}{ }^{4}$, generally, the quantity $S_{11}+\varepsilon$ is obtained, where $\varepsilon \in \mathbb{C}$. Of course, since the exact solution is unknown, the value $\varepsilon$ must be approximated, for example, by the difference between the solution of two iterations in the simulation process. This is, if the three-section simulation model under study, such as those shown in Figs. 9.4(a)-9.4(c), is solved by the FEM (incorporated in

\footnotetext{
${ }^{3}$ Notice that the expansion at $x=0$ of $f(x)=\sqrt{1+a x}$ is $f(x \approx 0)=1+\frac{a x}{2}$.

${ }^{4}$ The parameters will be obtained referred to the planes $R_{s}$ and $R_{l}$ and it is supposed that the error on $\beta_{p}$ is insignificant so that the trivial convection to the planes $R_{1}$ and $R_{2}$ do not introduce an extra error.
} 
HFSS $^{\circledR}$ or CST, for example), $\varepsilon$ is assumed as the difference on the $S_{11}$ caused by the refinement of the mesh from one iteration to the next.

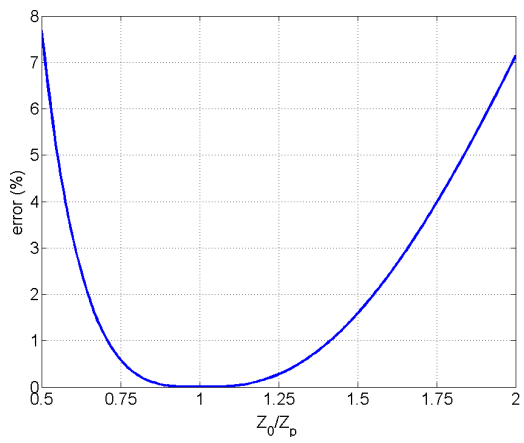

(a)

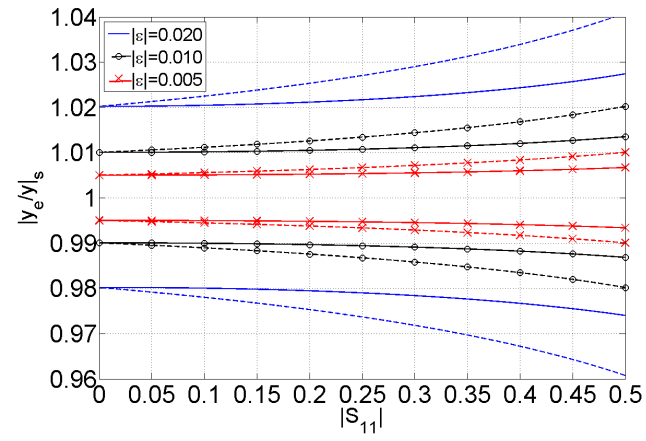

(b)

Figure 9.5: Error analysis. a) Percentage error related to the term $Z_{0} / Z_{p}$ due to the use of the approximated expression (9.65) instead of the exact (9.64); b) Bounds for the term $\left|y_{e} / y\right|$. Dashed lines correspond to the first described cotes, in equation (9.72), whereas solid lines correspond to the second described cotes, in equations (9.75) and (9.76). Each curve has two branches corresponding to the lower and upper limits, respectively.

Let be

$$
\begin{aligned}
y & =\left(\frac{Z_{0}}{Z_{p}}\right)=\left(\frac{1+S_{11}}{1-S_{11}}\right)^{\frac{1}{2}} \\
y_{e} & =\left(\frac{Z_{0}}{Z_{p}}\right)_{e}=\left(\frac{1+S_{11}+\varepsilon}{1-S_{11}-\varepsilon}\right)^{\frac{1}{2}}
\end{aligned}
$$

so that

$$
\left|\frac{y_{e}^{2}}{y^{2}}\right|=\left|\frac{1-S_{11}}{1+S_{11}}\right|\left|\frac{1+S_{11}+\varepsilon}{1-S_{11}-\varepsilon}\right|
$$

By applying the triangular inequality ${ }^{5}$

$$
\begin{aligned}
\left|\frac{y_{e}^{2}}{y^{2}}\right| & \leq\left|\frac{1-S_{11}}{1+S_{11}}\right| \frac{\left|1+S_{11}\right|+|\varepsilon|}{\left|1-S_{11}\right|-|\varepsilon|} \\
& =\frac{1+\frac{|\varepsilon|}{\left|1+S_{11}\right|}}{1-\frac{|\varepsilon|}{\left|1-S_{11}\right|}} \leq \frac{1+\frac{|\varepsilon|}{1-\left|S_{11}\right|}}{1-\frac{|\varepsilon|}{1-\left|S_{11}\right|}}
\end{aligned}
$$

and

\footnotetext{
${ }^{5}$ The triangular inequality (sometimes called Minkowski inequality) establishes that $|a+b| \leq$ $|a|+|b|$ for any real numbers $a$ and $b[916]$.
} 


$$
\begin{aligned}
\left|\frac{y_{e}^{2}}{y^{2}}\right| & \geq\left|\frac{1-S_{11}}{1+S_{11}}\right| \frac{\left|1+S_{11}\right|-|\varepsilon|}{\left|1-S_{11}\right|+|\varepsilon|} \\
& =\frac{1-\frac{|\varepsilon|}{\left|1+S_{11}\right|}}{1+\frac{|\varepsilon|}{\left|1-S_{11}\right|}} \geq \frac{1-\frac{|\varepsilon|}{1-\left|S_{11}\right|}}{1+\frac{|\varepsilon|}{1-\left|S_{11}\right|}}
\end{aligned}
$$

This means

$$
\left(\frac{1-f\left(\left|S_{11}\right|,|\varepsilon|\right)}{1+f\left(\left|S_{11}\right|,|\varepsilon|\right)}\right)^{\frac{1}{2}} \leq\left|\frac{y_{e}}{y}\right| \leq\left(\frac{1+f\left(\left|S_{11}\right|,|\varepsilon|\right)}{1-f\left(\left|S_{11}\right|,|\varepsilon|\right)}\right)^{\frac{1}{2}}
$$

where

$$
f\left(\left|S_{11}\right|,|\varepsilon|\right)=\frac{|\varepsilon|}{1-\left|S_{11}\right|}
$$

Therefore, we can control the error in $Z_{0}$ by limiting the absolute value of the error $\varepsilon$. In most of cases, the bounds described by (9.71) will be quite far from the real error, so more adjusted bounds could be applied regarding to the following. Let consider the functions

$$
\begin{aligned}
g_{u}\left(\left|S_{11}\right|,|\varepsilon|\right)= & \frac{1+\frac{|\varepsilon|}{\left|1+S_{11}\right|}}{1-\frac{|\varepsilon|}{\left|1-S_{11}\right|}} \\
g_{l}\left(\left|S_{11}\right|,|\varepsilon|\right) & =\frac{1-\frac{|\varepsilon|}{\left|1+S_{11}\right|}}{1+\frac{|\varepsilon|}{\left|1-S_{11}\right|}}
\end{aligned}
$$

which from (9.69) and (9.70) satisfy $g_{l} \leq\left|y_{e}^{2} / y^{2}\right| \leq g_{u}$

If the term $|\varepsilon| /\left|1-S_{11}\right|$ is lower than unity, which always will be the case, the upper limit function $g_{u}$ obtains its maximum (and the function $g_{i}$ its minimum) value for lower values of the term $\left|1-S_{11}\right|$. For a given maximum value of $\left|S_{11}\right|$ this occurs when $S_{11}=\left|S_{11}\right|$, so that calling

$$
h_{u}\left(\left|S_{11}\right|,|\varepsilon|\right)=\left(\frac{1+\frac{|\varepsilon|}{1+\left|S_{11}\right|}}{1-\frac{|\varepsilon|}{1-\left|S_{11}\right|}}\right)^{\frac{1}{2}}
$$

and 


$$
h_{l}\left(\left|S_{11}\right|,|\varepsilon|\right)=\left(\frac{1-\frac{|\varepsilon|}{1+\left|S_{11}\right|}}{1+\frac{|\varepsilon|}{1-\left|S_{11}\right|}}\right)^{\frac{1}{2}}
$$

it can be said that $h_{l} \leq\left|y_{e} / y\right| \leq h_{u}$. To observe how the error is, the lower and upper bounds for the two described criteria are represented in Fig. 9.5(b). The second criteria gives a quite more adjusted bound and note that, if the $\left|S_{11}\right|$ is not too large, the relative error in $y_{e}=\left(Z_{0} / Z_{p}\right)_{e}$ is very near from the value of $|\varepsilon|$, which is a really good estimation for cases where $Z_{0} \approx Z_{p}$. The graphic also indicates that a better matched port, i. e., the waveguide under study is more similar to the waveguide port introduce lower errors. Nevertheless, the effect of reducing $|\varepsilon|$ is much more significant, and gives the real control on the error, being that it can be chosen, whereas the impedance of the waveguide under study is rather arbitrary.

\subsubsection{Validation of the method}

The proposed method has been presented in its more rigorous and general form. Now, in this section, it will be validated using some published previous results as reference. The SSGW design of [795] is chosen for this task. In that work a SSGW is defined with the following parameters (see Fig. 9.6(a)): $w=5 \mathrm{~mm}, p=7$ $\mathrm{mm}, w_{p}=3 \mathrm{~mm}, h_{p}=5 \mathrm{~mm}, h_{a}=1 \mathrm{~mm}, t=50 \mu \mathrm{m}, t_{d}=50 \mu \mathrm{m}$, and substrate polymide $\left(\varepsilon_{r}=3.4\right)$. Such geometrical configuration provides a stopband between 10 $\mathrm{GHz}$ and $19 \mathrm{GHz}$, being that the central operation frequency chosen by the authors is $f=15 \mathrm{GHz}$. At that frequency, it is calculated the impedance of the proposed SSGW by integration of the full-wave calculated fields and application of the P-I formulation. The authors perform an study of the impedance value depending on the strip position, defining the offset $\Delta x$ as the displacement distance respect to the original position. This original position corresponds to that in which the center of the strip is aligned with the center of a row of pins. Note that the maximum different geometrical variation respect to that case corresponds to $\Delta x=p / 2$. Therefore, the authors parametrize $\Delta x$ from $-p / 2$ to $p / 2$ and symmetry around $\Delta x=0$ is expected. The results of this study correspond to the red curve in Fig. 9.6(b). The same calculation using the proposed method in this thesis, with an estimated error of $\varepsilon \approx 1 \%$, is displayed in blue for comparison and validation.

As it can be appreciated, the impedance calculated with the proposed method is slightly lower than the impedance calculated in [795], but the behavior is the same. Therefore, the method captures well the phenomena of the impedance dispersion with the displacement of the strip, what gives validation. Note that, as it was commented at the beginning of section 9.1.2, the proposed method results to be more adequate in the context of device design processes, being that it makes sense to think that the employed impedance values observed at some points of the circuit under design will be closer to those given by the proposed method, which consider the transmission line sections as a circuital element, than those offered by local field calculations. By this reason, beyond the previous validation, the proposed method is 


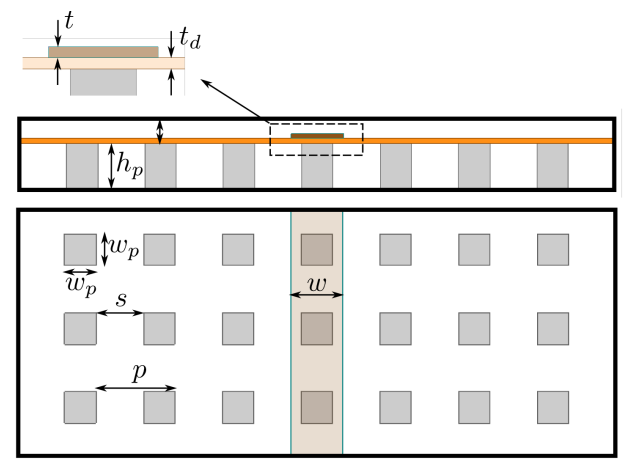

(a)

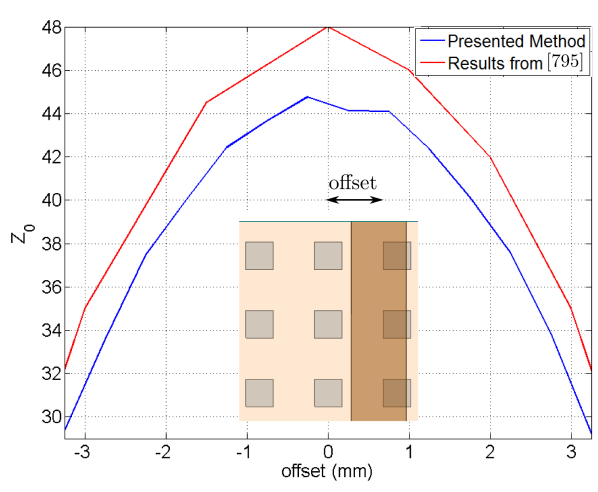

(b)

Figure 9.6: Validation of the proposed method for calculating SSGWs characteristic impedance. a) Frontal and upper view of the SSGW with its main geometrical parameters; b) Comparison of the presented method (correction of waveguide port impedance) with the results in [795] (impedance calculated from the EM fields through the P-I formulation) in the calculation of the impedance of a $S S G W$ as a function of the strip position. $w=5 \mathrm{~mm}, p=7 \mathrm{~mm}, w_{p}=3 \mathrm{~mm}, h_{p}=5 \mathrm{~mm}$, $h_{a}=1 \mathrm{~mm}, t=50 \mu \mathrm{m}, t_{d}=50 \mu \mathrm{m}$, and the substrate is polymide with $\varepsilon_{r}=3.4$.

used as the reference one in the following sections, where some ideas and guidelines to improve the SSGW performance and design possibilities are given.

\subsection{Constant Impedance SSGWs}

The results in Fig. 9.6(b) show an evidence: the impedance of a SSGW has a strong dependence on the position of the strip relative to the pins. Since the strip width is smaller than the pin lattice period, several different geometrical situations appear, so that the impedance value presents a large excursion. In the case of Fig. 9.6(b), the value of the impedance decreases up to $33 \%$ respect to the original position value, what is an aberration for design purposes. Fig. 9.7 illustrates this fact. The original or conventional situation is to consider the center of the strip aligned with the center of a pin row, Fig. 9.7(a). From such original position, the strip can be horizontally displaced, causing different geometrical situations that will obviously provide different propagation characteristics, this including a different characteristic impedance value. As commented before, the maximum difference takes place when $\Delta x=p / 2$, Fig. 9.7(c). Note how appreciable is the difference between Fig. 9.7(a) and Fig. 9.7(c). Nevertheless, this is only caused by using a period such that the strip observes an inhomogeneous underlying medium. This is the issue to be solved next.

The design of [890] is taken as starting point. The SSGW parameters in such design are: $w=2.2 \mathrm{~mm}, p=6.5 \mathrm{~mm}, w_{p}=3 \mathrm{~mm}, h_{p}=5 \mathrm{~mm}, h_{a}=0.5 \mathrm{~mm}$, $t=25 \mu \mathrm{m}, t_{d}=762 \mu \mathrm{m}$, and $\varepsilon_{r}=3.27$. Fig. 9.8(a) shows the aspect of this SSGW. The previous parameters provide a $[9 \mathrm{GHz}-18 \mathrm{GHz}]$ stopband. By applying the proposed impedance calculation method, the characteristic impedance of that 


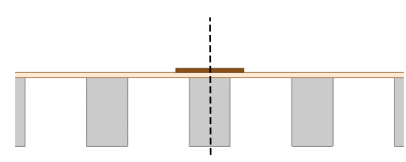

(a)

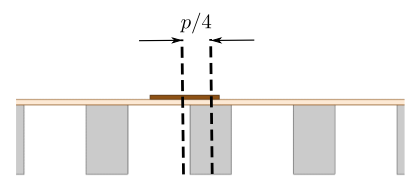

(b)

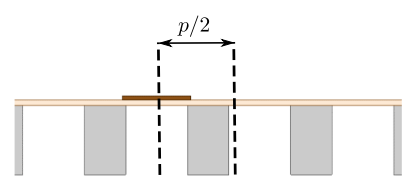

(c)

Figure 9.7: Variation of the topology in the SSGW depending on the strip position. a) Conventional case with the center of the strip aligned with the center of a pin row; b) Offset of $\Delta x=p / 4$; c) Offset of $\Delta x=p / 2$ (maximum possible).

SSGW has been calculated as a function of the relative position of the strip for several equally distributed frequencies in the stopband. Results are shown in Fig. 9.8(b), where it can be appreciated that the impedance presents an important variation with the relative position of the strip. Again, as the strip is displaced from its original position, the impedance decreases, what confirms this behavior in SSGWs, which is contrary to the results in [890] where the correction of the waveguide port impedance is done with the contrary sign due to missing the phase of the $S_{11}$ parameter. For the aforementioned set of parameters, obtained results exhibit a local minimum of impedance for an offset of $\Delta x=2.25$. It is also observed that the impedance dispersion is specially strong at the upper limit of the stopband, this probably caused by the spreading of the fields at such stopband limiting frequency.

To face this issue, in [890] it is proposed an alternative periodic lattice with a smaller period and a rotated strip, 45 degrees respect to the original case (strip parallel to the pin rows). Both modifications (denser lattice and rotated strip) improve the homogeneity. Note that given a periodic lattice of period $p$, if the strip is parallel to the pin rows, the observed period is $p$, Fig. 9.9(a). However, if the strip is rotated 45 degrees respect to the pin rows, the effective period that the strip observes is $p / \sqrt{2}$ (see Fig. $9.9(\mathrm{~b})$ ), i. e., the strip observers a more homogeneous medium without modifying the period of the pin lattice. The result can be easily obtained by applying basic trigonometry in Fig. 9.9(b) to the blue triangle first, and then to the red triangle.

In order to quantify the isolated improvement caused by this modification, the same SSGW is considered, but now with the strip rotated 45 degrees. Fig. 9.8(c) shows the $3 \mathrm{D}$ view of such SSGW structure. The same calculations as for the original orientation are carried out and displayed in Fig. 9.8(d). In the plot it is observed how the reduction of the observed inhomogeneity implies an impedance which is more constant than in the original case. However, the excursion of the impedance value is still excessive in order to carry out a straightforward circuit design. In addition, rotating the strip 45 degrees is a solution that only makes sense in circuits in which line sections are predominantly oriented in one direction. Otherwise, different orientations will take place at the circuit, being the involved SSGW transmission line sections affected in different grades by the impedance dispersion problem.

By the above, beyond locating the circuit so that most of the lines in it are rotated 45 degrees with respect to the pins, it seems necessary the use of a periodic lattice with more density. It is proposed a periodic pin structure with the following geometrical parameters: $p=1.2 \mathrm{~mm}, w_{p}=0.56 \mathrm{~mm}, h_{p}=7 \mathrm{~mm}$ and $h_{a}=0.6$ 


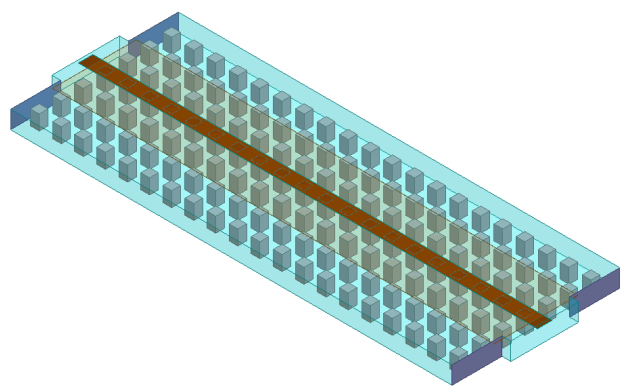

(a)

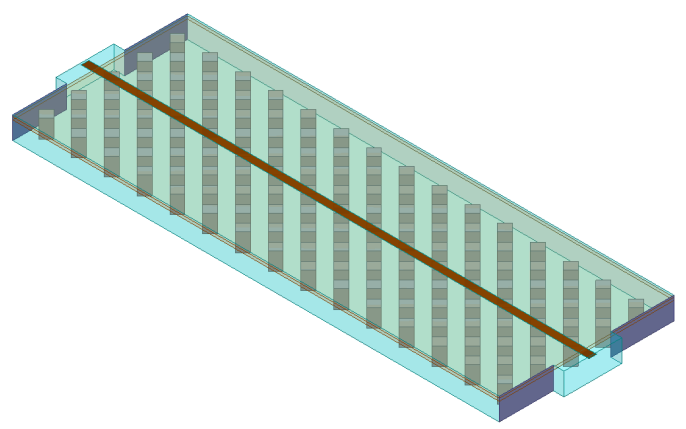

(c)

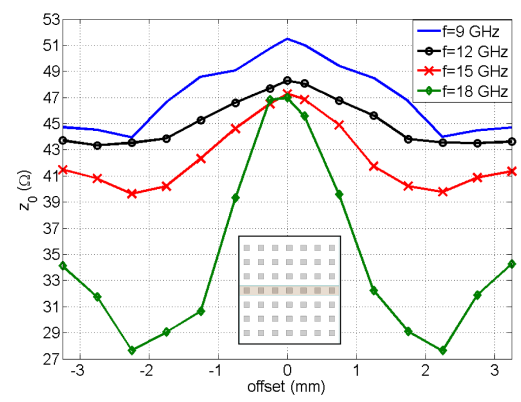

(b)

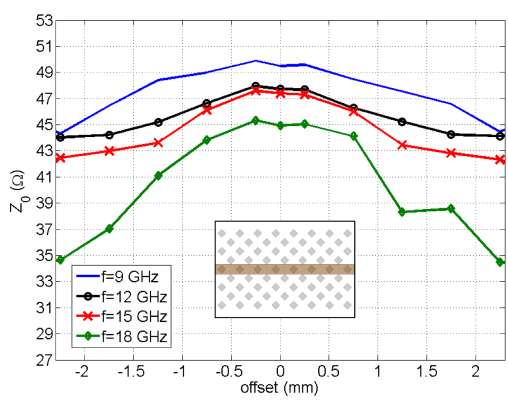

(d)

Figure 9.8: Impedance of a $K u$-band $S S G W$ as a function on the strip position for the cases of strip parallel to the pin rows and strip rotated 45 degrees respect to the pin rows. The SSGW parameters are: $w=2.2 \mathrm{~mm}, p=6.5 \mathrm{~mm}, w_{p}=3 \mathrm{~mm}, h_{p}=5 \mathrm{~mm}, h_{a}=0.5 \mathrm{~mm}, t=25 \mu \mathrm{m}$, $t_{d}=762 \mu \mathrm{m}$, and $\varepsilon_{r}=3.27$. a) $3 D$ view of a $S S G W$ with the strip parallel to the pin rows; b) $Z_{0}$ vs. $\Delta x$ (offset) SSGW with the strip parallel to the pin rows; c) $3 D$ view of a $S S G W$ with the strip rotated 45 degrees respect to the pin rows; d) $Z_{0}$ vs. $\Delta x$ (offset) SSGW with the strip rotated 45 degrees respect to the pin rows.

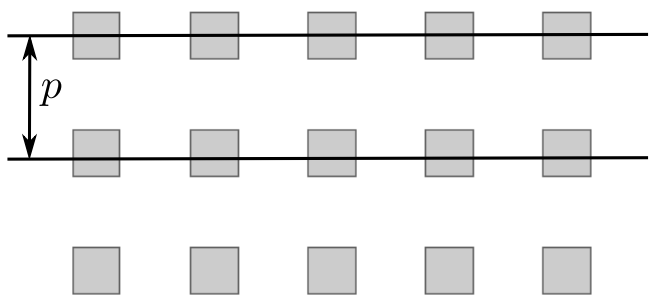

(a)

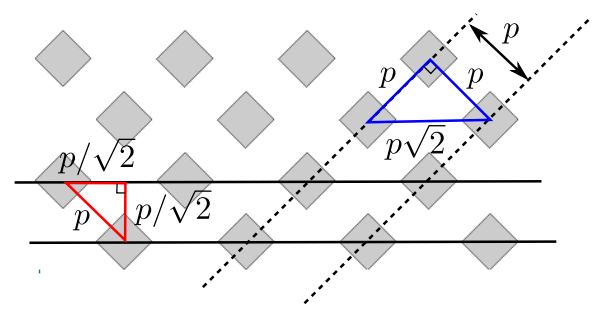

(b)

Figure 9.9: Effecitive period observed by the strip. a) Original orientation with the strip parallel to the pin rows; b) Strip rotated 45 degrees respect to the pin rows.

$\mathrm{m}$, optimized to produce a stop band from $10 \mathrm{GHz}$ to $17 \mathrm{GHz}(52 \% \mathrm{RBW})$. Table 9.1 list the set of parameters of both cases, normal and dense periodic structure, for comparison purposes. The comparison can also be done visually by comparing 


\begin{tabular}{lccccc} 
Periodic structure & $p$ & $w_{p}$ & $h_{p}$ & $h_{a}$ & Stopband \\
\hline Normal [890] & $6.5 \mathrm{~mm}$ & $3 \mathrm{~mm}$ & $5 \mathrm{~mm}$ & $0.5 \mathrm{~mm}$ & $9 \mathrm{GHz}-18 \mathrm{GHz}$ \\
Dense & $1.2 \mathrm{~mm}$ & $0.56 \mathrm{~mm}$ & $7 \mathrm{~mm}$ & $0.6 \mathrm{~mm}$ & $10 \mathrm{GHz}-17 \mathrm{GHz}$ \\
\hline
\end{tabular}

Table 9.1: Comparison between the geometrical parameters and stopband of a normal GW periodic structure (design of [890]) and the dense GW periodic structure proposed in this thesis.

the $3 \mathrm{D}$ views of both designs. The SSGW with normal or conventional periodic pin lattice used in [890] is shown in Fig. 9.8(a), whereas the proposed SSGW with a dense pin lattice is shown in Fig. 9.10(a). Even though both structures differ noticeably (in the dense periodic lattice the period is close to 6 times smaller), a similar stopband has been easily engineered following the conclusions of [792]. In addition, the proposed structure has an even larger air gap height $h_{a}$, which implies less losses.

Now, by using the same substrate and strip as in [795], but setting the strip width to $w=2 \mathrm{~mm}$, a transmission line with very stable impedance, close to $Z_{0}=50 \Omega$, is obtained. This can be appreciated in Fig. 9.10(b), which shows the characteristic impedance as a function of the horizontal offset $\Delta x$ for the proposed SSGW with dense pin lattice. The excursion of the impedance values for each frequency is less than $1 \Omega$ for most of the frequencies of the stopband, and less than $2 \Omega$ close to the stopband limits, which is a very good result for circuit design purposes. Since this occurs for each one of the evaluated frequencies, the same designed periodic structure can be used to allocate different sub-bands circuits with high reliability. Note, furthermore, that it has been considered the worst possible case, which is a strip parallel to the pin rows according to the conclusions derived in this section. This means that in a complex layout circuit, the impedance dispersion of the lines will be, at most, that reported in Fig. 9.10(b). The frequency dispersion, given by the separation of the lines corresponding to the different frequencies in Fig. 9.10(b) is a minor issue, inherent of GW structures as already observed in the GW technology overview, and that will only difficult ultra-wide band designs.

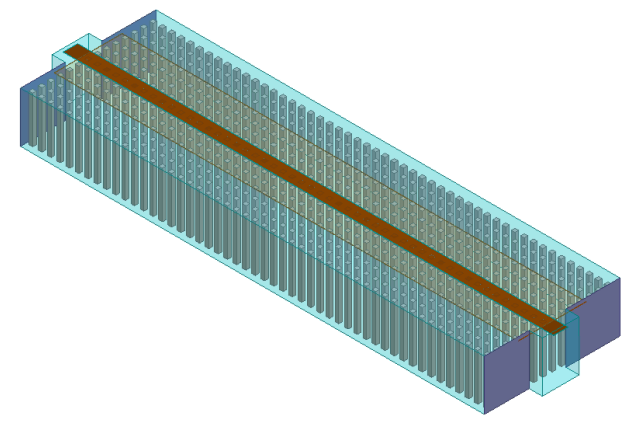

(a)

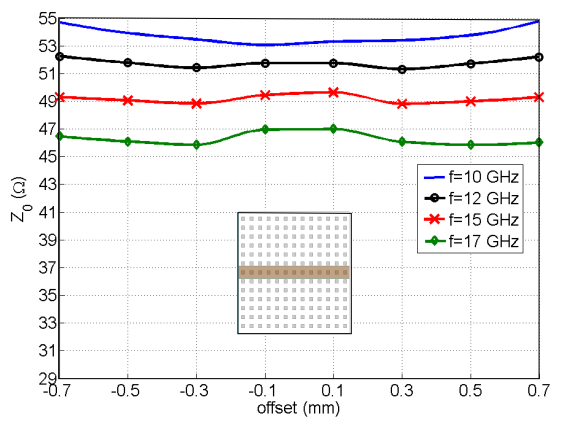

(b)

Figure 9.10: Impedance of a $\mathrm{Ku}$-band $S S G W$ as a function on the strip position in the case of using a dense periodic lattice of pins. $w=2.2 \mathrm{~mm}, p=1.2 \mathrm{~mm}, w_{p}=0.56 \mathrm{~mm}, h_{p}=7 \mathrm{~mm}$, $h_{a}=0.6 \mathrm{~mm}, t=25 \mu \mathrm{m}, t_{d}=762 \mu \mathrm{m}$, and $\varepsilon_{r}=3.27$. a) $3 D$ view; b) $Z_{0}$ vs. $\Delta x$ (offset). 


\begin{tabular}{lccccc} 
Periodic structure & $p$ & $w_{p}$ & $h_{p}$ & $h_{a}$ & Stopband \\
\hline Normal [859] & $3 \mathrm{~mm}$ & $1.2 \mathrm{~mm}$ & $2 \mathrm{~mm}$ & $0.4 \mathrm{~mm}$ & $23 \mathrm{GHz}-49 \mathrm{GHz}$ \\
Dense & $0.5 \mathrm{~mm}$ & $0.19 \mathrm{~mm}$ & $2.5 \mathrm{~mm}$ & $0.5 \mathrm{~mm}$ & $25.8 \mathrm{GHz}-44.5 \mathrm{GHz}$ \\
\hline
\end{tabular}

Table 9.2: Comparison between the geometrical parameters and stopband of a normal GW periodic structure (design of [890]) and the dense GW periodic structure proposed in this thesis.

To conclude this section dedicated to postulate the guidelines to design SSGW with an impedance independent on the strip relative position, the Ka-band design proposed in [859] is studied and improved. The SSGW periodic structure parameters used in that work are listed in the first row of table 9.3. In addition, the dielectric substrate has a thickness $t_{d}=50.8 \mu \mathrm{m}$, and the strip has a thickness $t=50 \mu \mathrm{m}$. This SSGW and its dispersion diagram describing the stopband are represented in Figs. 9.11(a) and 9.11(c), respectively. The achieved stopband is [23 GHz-49 GHz] $(R B W=72 \%)$. In [859], a three-way power divider is designed with such SSGW. For this simple circuit, the layout fits well with the periodic lattice so that the center of the strip is always aligned with the center of a row of pins. However, if the impedance of such SSGW is studied considering different relative positions, dispersion is found, see Fig. 9.12(a). In the plot, where the impedance is calculated for several frequencies in the stopband, they can be observed impedance variations ranging from $7 \Omega$ to $10 \Omega$, which definitively limits synthesis processes of moderate complexity with this SSGW.

As in the case of the Ku-band design, a dense periodic structure is the suitable alternative to avoid the previous issue. If the pin lattice parameters are set to those listed in the second row of the table 9.3, a six times denser lattice than in [859] is obtained, with a similar stopband: $[25.8 \mathrm{GHz}-44.5 \mathrm{GHz}](R B W=67 \%)$. Note that the stopband is only slightly narrower ( $5 \%$ less), however, it is wide enough to amply cover the whole Ka-band $(26.5 \mathrm{GHz}-40 \mathrm{GHz})$. The proposed structure and its dispersion diagram describing its stopband are shown in Figs. 9.11(b) and 9.11(d), respectively. The characteristic impedance $Z_{0}$ of this SSGW as a function of the strip horizontal offset $\Delta x$ is represented in Fig. 9.12(a). A strip width of $w=1.8 \mathrm{~mm}$ has been chosen so that the impedance is almost constant with the relative position of the strip with values around $Z_{0}=50 \Omega$. Furthermore, the proposed design presents an appreciable lower frequency dispersion being that the curves corresponding to the different frequencies are very close to each other. This means that wide band desings (in this case at least from $28 \mathrm{GHz}$ to $40 \mathrm{GHz}, R B W=35 \%$ ) would be affordable, at least, from the impedance point of view.

The previous approach can be summarized in the following guidelines which are the essence of this section:

- The periodic structure underlying the printed substrate of a SSGW must be designed with the premise of having a period small enough so that the strip cannot appreciate a noticeable difference in the local geometry under it.

- With the previous restriction on the pin period, the parameters of the periodic structure must be engineered to guarantee the required stopband. 


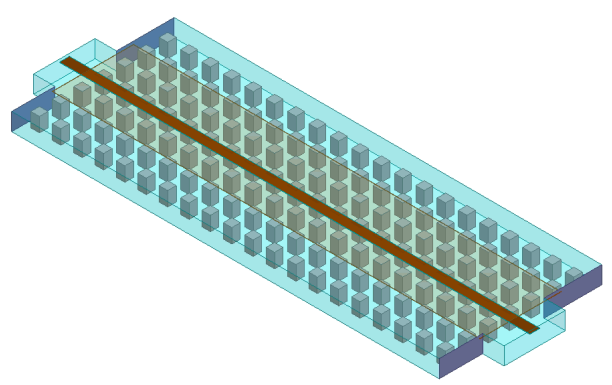

(a)

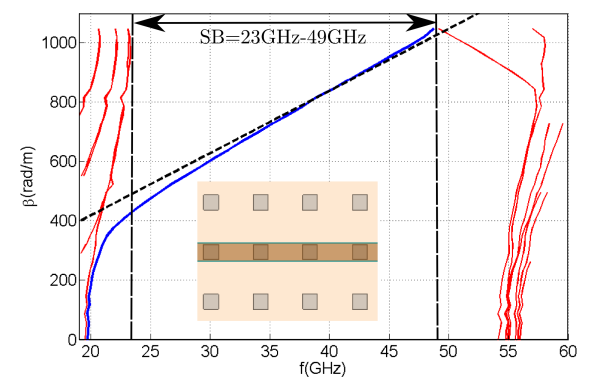

(c)

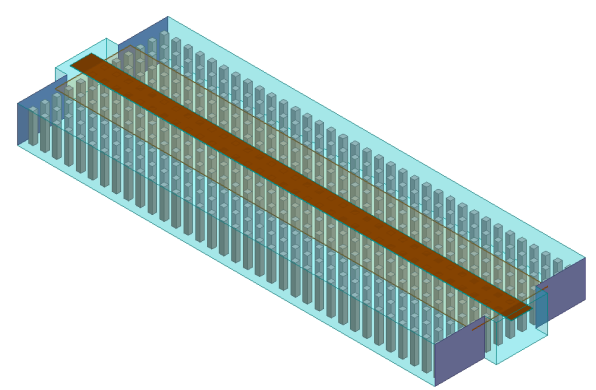

(b)

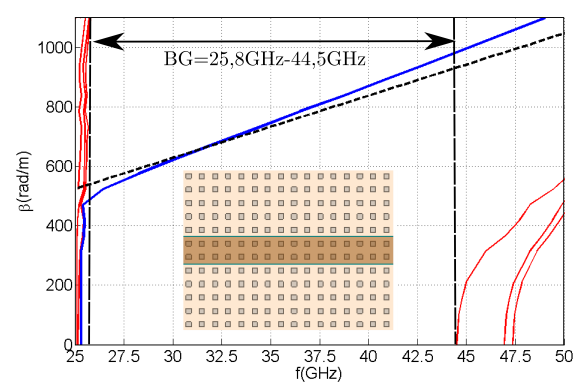

(d)

Figure 9.11: Comparison between the conventional SSGW proposed in [859] and the dense periodic lattice $S S G W$ proposed in this thesis (see 9.3). a) $3 D$ view of the $S S G W$ in [859]; b) $3 D$ view of the dense SSGW; c) Stopband of the SSGW in [859]; d) Stopband of the dense SSGW.

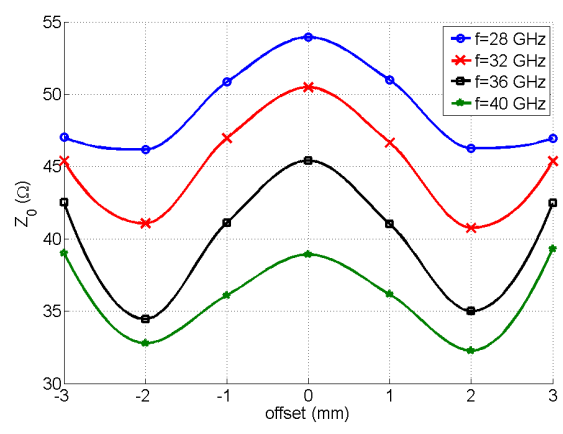

(a)

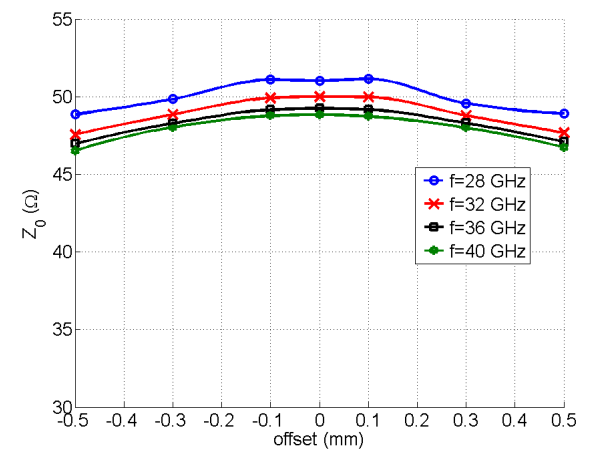

(b)

Figure 9.12: $Z_{0}$ vs. $\Delta x$, comparison between the conventional SSGW proposed in [859] and the dense periodic lattice SSGW proposed in this thesis. a) SSGW of [859]; b) Dense periodic lattice $S S G W$.

If the first rule is guarantee, conventional transmission line design techniques can be easily adapted to the SSGW and, in a suitable future design scenario, even closed expressions will be available for the design of SSGW components. This is, indeed, a 
future line of this work line. Otherwise, SSGW devices will demand, except for very simple circuits, from full-wave techniques and complex optimization processes. Such a complex design process would withdraw, for sure, any interest in this transmission line. It has been shown that a very stable impedance can be achieved. Moreover, reduction of the pin lattice period not only provides a proper design scenario for a single frequency, but also diminishes the frequency dispersion, inherent of non-pure TEM transmission lines, and that, in the case of GWs, is specially evident. The second principle has been also shown to be easily affordable just by following the guidelines given in [792]. The large number of parameters of the periodic structure in GWs implies several degrees of freedom so that a specified stopband can be achieved by quite different sets of parameters.

The section concludes with some important reflections that may give a necessary further clarification of the advantages and disadvantages of the proposed approach. The advantages have been detailed already. As main disadvantage, it must be said that the structure complexity increase noticeably due the larger number of pins, which, furthermore, are smaller. This has two implications. On the one hand, it can be easily understood that the manufacturing process complexity may be obviously affected. However, note a particular detail in the case of the SSGW regarding other GWs. In the SSGW, the function of the periodic structure is to merely provide a high impedance condition to be observed by the strip. This means that any structure achieving this demand is valid. For instance, roughly shaped pins, or other kind of periodic structures cheaply manufactured, i. e., not carefully shaped, can be as valid as perfect square metal pins. This is not the case of RGWs, and specially, GGWs. In this last, for instance, the shape of the pins delimits the propagation channel width, which is the essential parameter of design. Conversely, the manufacturing precision in the SSGW resides in the printed substrate shaping, specially in the strip, as occur with conventional printed substrate transmission lines (microstrip line and CPW, for instance). Therefore, it is foreseeable that the manufacturing industry will be able to provide a low-cost dense lattice solution easily (this include low-profile lattices that may provide a rather planar characteristic to the SSGW, something desirable to compete with conventional planar transmission lines). Observe that, according to this section, better results will be obtained using roughly shaped dense lattices than using well shaped sparse lattices.

On the other hand, full-wave analysis is obviously more complex given a periodic structure if a large number of small elements compose it. In this section, time-consuming full-wave simulations have been carried out to provide proper comparison with conventional designs. Nevertheless, as the required conclusions has been established, further steps may totally solve this problem. Note that in the case of small period SSGW, homogenization is rather licit, and Silverinha model [880] could be applied with small error. This will turn the situation, being faster to simulate dense lattice SSGW circuits. In addition, as commented before, to obtain closed-form expressions defining the impedance and propagation constant of a SSGW given their parameters of design is a reachable goal whenever the SSGW present a stable impedance, which is the case of the proposals in this part of the thesis.

The other important reflection addresses the stopband issue. In the previous designs, a large stopband (close to $70 \%$ ) has been achieved even in very dense 
lattices. This might sometimes force some of the parameters to rather extreme values, or, at least, to values implying a more difficult manufacturing process. In general, in the GW literature, maximizing the stopband has been a non-debatable premise. However, it falls into question, how practical can be such approach. Notice that most of the designs exhibit $R B W<20 \%$ since some parts of the device are very prone to become a bandwidth bottleneck (think, for instance, in transitions). Others, are inherently narrow-band, as it its the case of narrow-band filters. Therefore, it makes sense to investigate narrower stopband periodic structures in the future, such that they satisfy the required specifications, but impose less restrictions to the design. For example, it is observed that use a larger $h_{a}$ reduces noticeably the stopband bandwidth, however, losses are as well noticeably decreased [792], [836]. Both reflections indicate that the foreseen disadvantages of using dense lattices in SSGW may not be as severe as they can be though on a first view.

\subsection{SSGW coupled lines}

\subsubsection{Coupled transmission lines theory}

When two unshielded transmission lines are close together, power can be coupled between the lines due to the interaction of the electromagnetic fields of each line. Such lines are referred to as coupled transmission lines, and usually consist of three conductors in close proximity, although more conductors can be used. Coupled transmission lines are usually assumed to operate in the TEM mode, which is rigorously valid for stripline structures, and approximately valid for quasi-TEM transmission lines such as the microstrip line, or, in the context of this thesis, the SSGW.

Coupled transmission lines theory facilitates the analysis of those problems in which coupled-lines appear, posing a more complex scenario than that of isolated transmission lines. In general, a three-wire line such as that schematized in Fig. 9.13 support two distinct propagating TEM (or quasi-TEM) modes. This feature can be used to implement directional couplers, hybrids and filters. In addition, by carefully analyzing those modes, physical insight is gained and the design process complexity can be easily reduced to that of isolated lines. Note that if a TEM-type propagation is assumed, then the electrical characteristics of the coupled lines can be completely determined from the effective capacitances between the lines and the propagation velocity on the line. In Fig. 9.13, it is also shown the equivalent capacitance network of a pair of coupled transmission lines. Specifically, $C_{12}$ represents the capacitance between the two strip conductors in the absence of the ground conductor, whereas $C_{11}$ and $C_{22}$ represent the capacitance between each strip conductor and the ground plane in the absence of the other strip conductor. It is worth to mention that the designation of which conductor assumes the role of ground is arbitrary along the procedure, however, it is usual to find one among the three conductors that clearly has the ground role. In the case of the SSGW the top plate takes this role.

Now, let consider two special types of excitation for the coupled line: the even mode, where the currents in the strip conductors are equal in amplitude and in the same direction, and the odd mode, where the currents in the strip conductors are equal in amplitude but in the opposite directions. The schematic of such cases, 


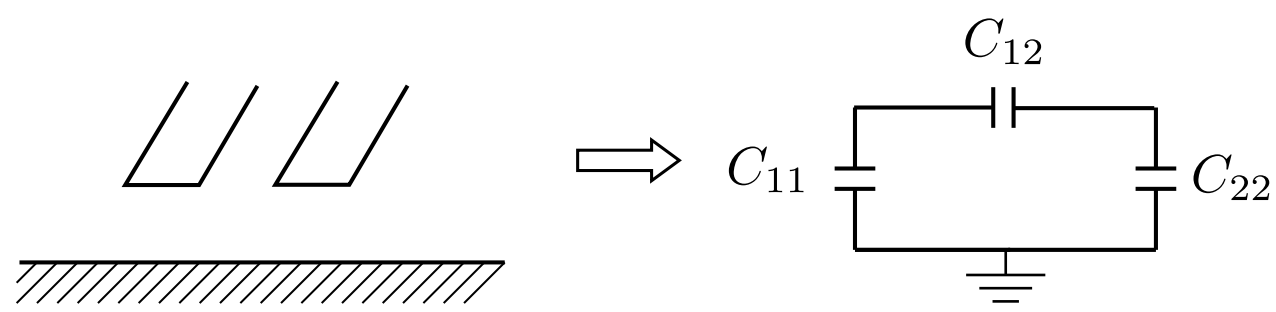

Figure 9.13: Coupled transmission lines consisting in a three-conductor structure formed by the two coupled lines and the ground conductor, and its equivalent capacitance network.

including E-field lines and the equivalent capacitance networks are shown in Fig. 9.14. For the even mode, Fig. 9.14(a), the electric field has even symmetry about the center line, and no current flows between the two strip conductors. This case is equivalent to have a Perfect Magnetic Conductor (PMC) wall in the symmetry plane of the coupled-line circuit, shown in red in Fig. 9.14(a). This leads to an equivalent circuit in which $C_{12}$ is effectively open-circuited. Then the resulting capacitance of either line to the ground plane is:

$$
C_{e}=C_{11}=C_{22}
$$
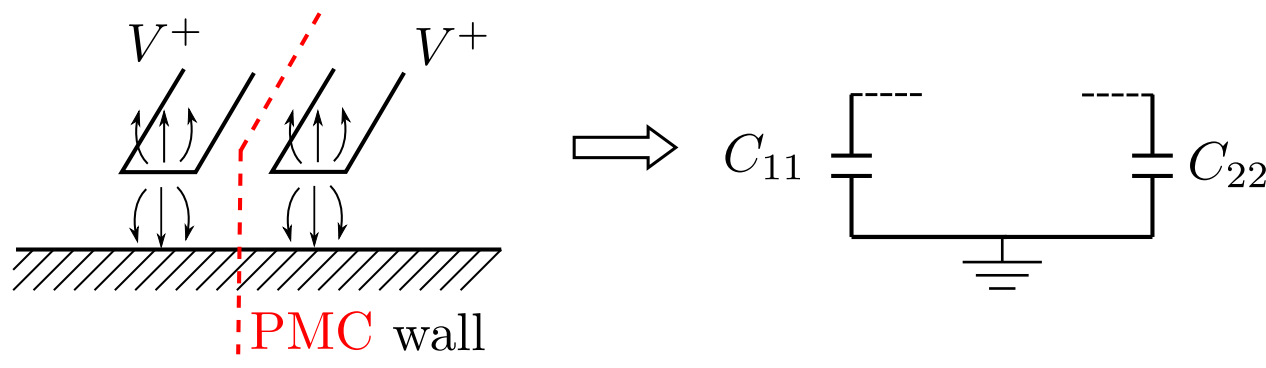

(a)
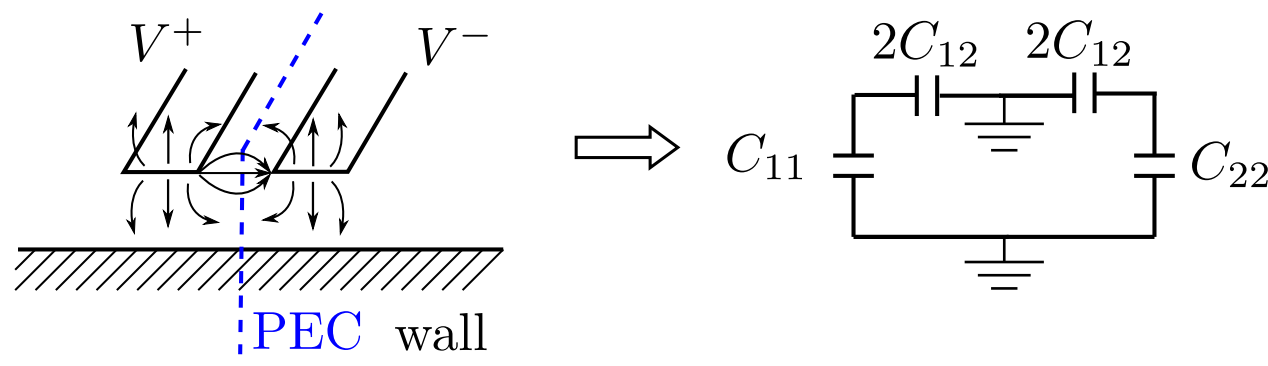

(b)

Figure 9.14: Even- and odd-mode excitation for a pair of coupled transmission lines, and the resulting equivalent capacitance networks. PEC and PMC conditions are indicated an E-field lines are included in the schematic. a) Even-mode excitation; b) Odd-mode excitation 
assuming that the two strip conductors are identical in size and location, which it will be always the case in the scope of this thesis. Then, the characteristic impedance for the even mode is, according to (9.22),

$$
Z_{0}^{e}=\frac{L}{C_{e}}=\frac{\sqrt{L C_{e}}}{C_{e}}=\frac{1}{v_{g} C_{e}}
$$

where $v_{g}$ is the propagation velocity on the line, the group velocity.

Conversely, for the odd mode, the electric field lines have an odd symmetry about the center line, and a voltage null exist between the two strip conductors. This case is equivalent to have a Perfect Electric Conductor (PEC) wall in the symmetry plane of the coupled-line circuit, shown in blue in Fig. 9.14(b). Since this PEC plane passes through the middle of $C_{12}$, this capacitance can be split in two series $2 C_{12}$ in such way that the common point of them is connected to the ground, as it shows Fig. 9.14(b). Therefore, the effective capacitance in this case is

$$
C_{o}=C_{11}+2 C_{12}=C_{22}+2 C_{12}
$$

and consequently the odd mode impedance is

$$
Z_{0}^{o}=\frac{L}{C_{o}}=\frac{\sqrt{L C_{o}}}{C_{o}}=\frac{1}{v_{g} C_{o}}
$$

The above procedure describes how $Z_{0}^{e}\left(Z_{0}^{o}\right)$ is the characteristic impedance of one of the strip conductors relative to the ground conductor when the coupled line is operated in the even (odd) mode. The valuable key point in the analysis of coupledlines is that an arbitrary excitation of a coupled line can always be treated as a superposition of appropriate amplitudes of even and odd modes, hence the problem can be always posed in terms of superposition of isolated line problems consisting either on the even-mode or on the odd-mode problem.

In the basic case of purely TEM transmission lines such as the coaxial cable, the PPW, or the stripline, analytical techniques such as conformal mapping [528] can be used to evaluate the capacitances per unit length of the line under study, so that even- and odd-mode characteristic impedances can be determined. For quasiTEM lines, such as the microstrip line, these results can be obtained numerically or by approximated quasi-static techniques otherwise. As usual, for well-adopted transmission lines, as it is the case of the microstrip line, is even easy to find works that focus on extract closed form expressions for the even- and odd-mode problems [917]. However, in a more general case, as it is the SSGW case, a topology for which any general closed form expression is available to obtain the impedance of even an isolated line, the same procedure as that employed to calculate the SSGW impedance must be applied to the calculation of the even- and odd-mode impedance by applying the proper boundary conditions. Therefore, full-wave simulations using the corrected port impedance, similar to those of section 9.1.2, must be adapted for the new problem. 


\begin{tabular}{lccccc} 
Periodic structure & $p$ & $w_{p}$ & $h_{p}$ & $h_{a}$ & Stopband \\
\hline Ka-band & $0.5 \mathrm{~mm}$ & $0.19 \mathrm{~mm}$ & $2.5 \mathrm{~mm}$ & $0.5 \mathrm{~mm}$ & $26.5 \mathrm{GHz}-45.7 \mathrm{GHz}$ \\
\hline
\end{tabular}

Table 9.3: Geometrical parameters to provide a stopband covering the Ka-band with the restriction of having a small period $p$ to provide an enough dense peridoic lattice.

\subsubsection{Analysis}

Previously, it has been rigorously presented a semi-empirical method to compute the impedance of arbitrary transmission lines from a practical point of view regarding to the design process. This method, applied to the SSGW has derived in several necessary premises to provide a straightforward design process with this transmission line, being the most important one to use dense pin lattices in order to keep a stable impedance, independent on the strip location. In this section, the design possibilities with the SSGW are extended one step further by studying the problem of SSGW coupled lines, Figs. 9.15(a) and 9.15(b). In Fig. 9.15(b), the main parameters determining the structure are represented: the strip width $w$ and the separation between strips $s$. According to the previous theory section, properties of this configuration can be easily extracted by the analysis of the even- and oddmode of the SSGW coupled-line structure, whose E-field patterns are represented in Figs. 9.15(c). As section 9.3.1 establishes, main information for design purposes regarding coupled lines is related with even- and odd-mode impedances $Z_{0}^{e}$ and $Z_{0}^{o}$. This impedances will be calculated with the same procedure as for single SSGW, by applying the presented impedance calculation method.

Although some works have studied basic coupling phenomena in GWs (see [885] or [886] for instance), the use of coupled transmission line theory applied to GWs for device design has remained a work line to face, becoming an objective of this part the thesis. The aim of this section is to face this objective from a practical point of view, so that posterior researches can easily apply the later given information. In fact, coupled SSGW lines to operate in the Ka-band will be designed, and a useful design chart will be provided in such a way that coupled-line devices with a SSGW can be designed without requiring detailed knowledge of this waveguide, but only basic coupled transmission line knowledge. Next, a quite complete process is carried out given basic guidelines and general process overview. First, the stopband is designed and bandwidth issues are studied. Then, single SSGW impedance is calculated in order to gain quantitative vision of the problem, and determine the frequency dispersion and the range of values of interest for the strip width $w$. Then, even- and odd-mode impedances of SSGW coupled-lines are calculated, and, finally, by a complete parametrization of the strip width $w$ and the strip separation $s$, data for generation of a design chart are obtained.

In order to adjust the stopband coverage to the Ka-band, the periodic structure parameters listed in table 9.3 are used. The SSGW is additionally composed by a layer of substrate Pyralux AP9121R $\left(\varepsilon_{r}=3.6\right.$ and $\left.\tan \delta=0.06 @ 36.85 \mathrm{GHz}[797]\right)$ with thickness $t_{d}=50.8 \mu \mathrm{m}$ is used. With this design, a stopband from $26.5 \mathrm{GHz}$ to 45.7 GHz (53\% RBW) is achieved, Fig. 9.16(a). A metallic strip over the substrate, allows the propagation of the desired quasi-TEM mode. Several cases for the strip width $w$ are analyzed. The strip thickness has been set to $t=50 \mu \mathrm{m}$. 


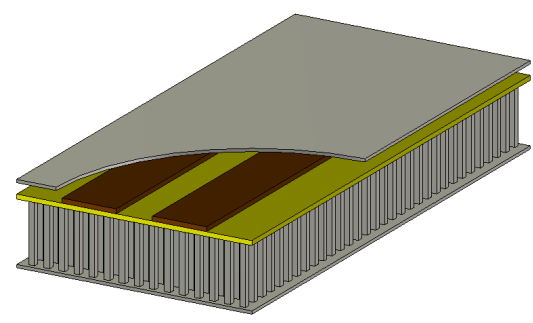

(a)

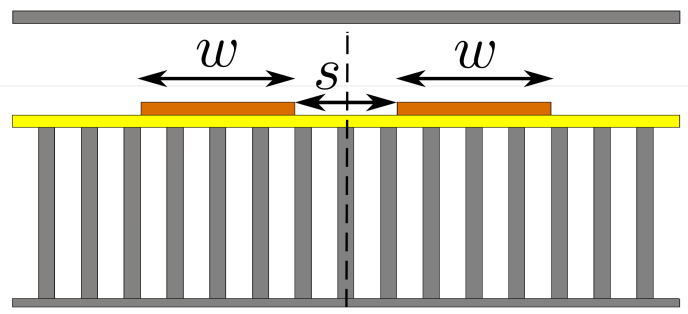

(b)
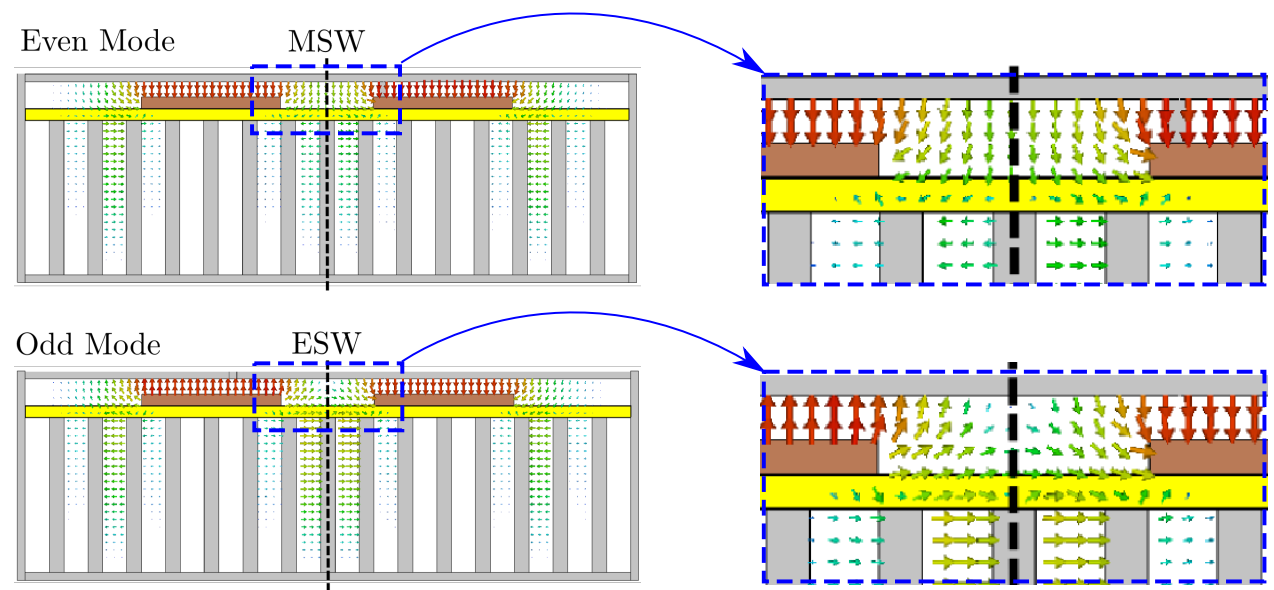

(c)

Figure 9.15: Coupled SSGW lines. a) $3 D$ view; b) $2 D$ view and main SSGW coupled-line parameteres: strip width $w$ and strip separation $s$; c) Even and odd mode E-field patterns. Right images are a detail of the E-field in the main coupling area.

As it occurs in conventional quasi-TEM transmission lines, narrower strips imply the propagation constant to differ more from the space wave number $k$, i. e., from the pure TEM propagation. In the case of the SSGW, since the the propagation takes place mainly through an air region, $\beta$ is close to $k=k_{0}$. Note, however, that the previously mentioned effect in narrow strips is not so severe, being that even in that case the curves show low dispersive features (see $w=0.5 \mathrm{~mm}$ for instance). Nevertheless, what seems more relevant is the fact that wider lines can allow highorder modes propagation, thus narrowing the effective available stopband. This fact should be taken into account when low-impedance lines operating at the high frequencies of the stopband are demanded by a particular design. In such cases, a re-design of the periodic structure may be necessary. For example, a reduction of the distance $h_{a}$ may be a good solution since the high-order modes cutoff frequency would be increased, and the stopband would be widened [792], at the expense of larger propagation losses [836]. 


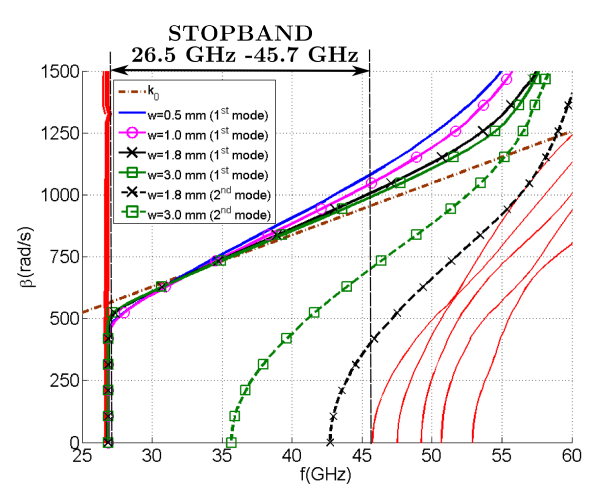

(a)

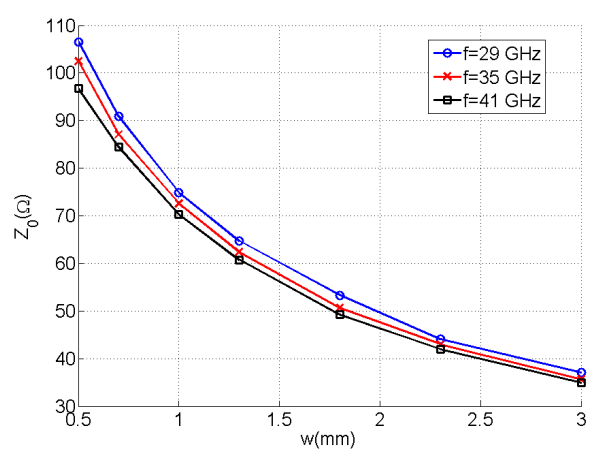

(b)

Figure 9.16: Proposed SSGW Ka-band design. The periodic structure parameters are: $p=0.5$ $\mathrm{mm}, w_{p}=0.19 \mathrm{~mm}, h_{p}=2.5 \mathrm{~mm}$ and $h_{a}=0.5 \mathrm{~mm}$. The substrate is Pyralux AP9121R $\left(\varepsilon_{r}=3.6\right.$ and $\tan \delta=0.06 @ 36.85 \mathrm{GHz}$ [797]) with thickness $t_{d}=50.8 \mu \mathrm{m}$. The strip thickness is $t=50 \mu \mathrm{m}$. a) Dispersion diagram including the propagation constant of undesired PPW modes (red lines), which describe the stopband, and the propagation constant of the fundamental and the first highorder mode on the strip for several strip widths; b) $Z_{0}$ vs. $w$ for several frequencies in the Ka-band.

The single SSGW characteristic impedance is calculated for a wide range of strip width values $(w)$ with the method of section 9.1 .2 with an estimated error of $\varepsilon=1 \%$, for several frequencies. Results of this parametric study are shown in Fig. 9.16(b), where values between $Z_{0}=30 \Omega \mathrm{m}$ and $Z_{0}=110 \Omega$ are observed for $Z_{0}$. The use of a dense structure not only prevents the impedance to be dependent on the strip location, but also makes possible the achievement of a wide range of impedances, a problem that remains hidden at first view, when the usual $Z_{0}=50 \Omega$ is pursued. Note, for example, that in [795], since the width is smaller than the period $p$ and comparable with the pin width $w_{p}$, small values of $w$ imply the strip to be narrower than the underlying row of pins. This causes a strong local effect inasmuch as the main mode fields interact with those pins, causing a growth of the effective width. As a result, in that work there is a local maximum of $Z_{0}=60 \Omega$, obtained for a certain strip width, i. e., it is not possible to design SSGW lines with $Z_{0}>60 \Omega$. Narrower or wider widths cause lower impedance, what, as it can be easily intuited, strongly limits the design possibilities. This fact is another strong reason that justifies the use of dense lattices.

By observing the curves corresponding to the different frequencies, certain dispersive features are observed, although quite moderate (the different curves are quite close to each other despite of the relative bandwidth in $[29 \mathrm{GHz}-41 \mathrm{GHz}]$ is $R B W=34 \%$ ). Notice that, due to the periodic structure, it is obvious that the impedance dispersion of the structure must be higher than other quasi-TEM transmission lines, but it is low enough for moderate-wide bandwidth applications. $\mathrm{Cu}-$ riously, high frequencies present lower impedance. This differs from the microstrip line, which presents a slight increment of $Z_{0}$ with the frequency [22]. This can be explained by looking at the surface impedance over the pins. The function of the pins in the structure is to generate a virtual PMC condition, in order to provide stopband conditions when the top plate is located at a distance $h_{a}<\lambda / 4$. At high 
frequencies, this PMC condition is generated at a lower height, being the distance between the PEC plate and the PMC surface larger. This implies the field to spread more laterally, which is equivalent to have a larger effective width of the strip and, hence, lower impedances are achieved. Observation of this fact makes to arise an interesting question. It could be interesting to characterize the impedance on the plane over the pins observed downwards in order to provide a simple and accurate modeling of the SSGW in a similar way that it will be done for the GGW in the section 10.2.1. It is foreseen as quite affordable future work line objective.

Now, two coupled SSGW lines are considered under the even- and odd-mode approach, according to the symmetry of the structure in the plane described by the vertical dashed line in Fig. 9.15(b). According to section 9.3, in the case of the even-mode, the symmetry plane imposes a PMC condition, whereas in the case of the odd-mode, the symmetry plane imposes a PEC condition. Therefore, what is done is to introduce planes with these conditions in the simulation model of the full-wave commercial solver, $\mathrm{CST}^{\circledR}$ in this case. The full-wave simulation models to calculate the even- and odd- mode impedances by means of the presented method are shown in Fig. 9.17(a). Note that only one of the symmetric parts of the coupledline structure is discretized and solved, what can be even faster than simulation of an isolated SSGW since the presence of a neighboring PEC/PMC plane cause confinement of the EM fields. In the models of Fig. 9.17(a) the solver only considers the region at the left of the plane.
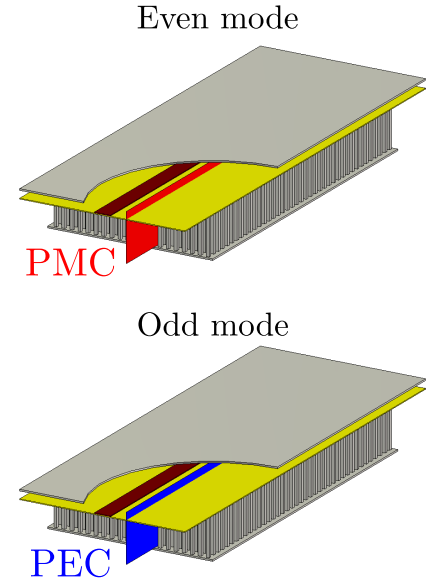

(a)

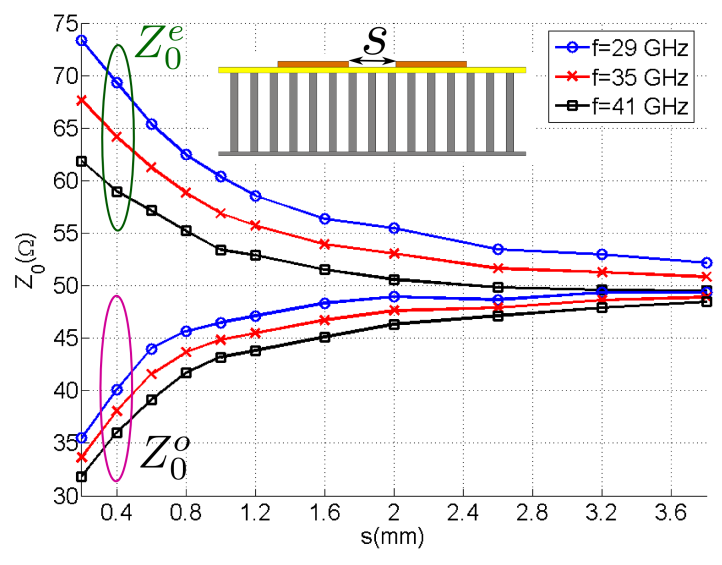

(b)

Figure 9.17: Calculation of SSGW even- and odd-mode impedances. a) Simulation models with CST ${ }^{\circledR}$. A magnetic (electric) wall is placed on the symmetry plane between the two strips to calculate even- (odd-) mode impedances, $Z_{0}^{e}$ and $Z_{0}^{o}$. The main parameters of the model as those described in Fig. 9.15(b): strip width $w$ and strip separation $s$ (note that the distance to the $P M C / P E C$ plane is $s / 2 ; b)$ Calculated $Z_{0}^{e}$ and $Z_{0}^{o}$ vs. $s$ for several frequencies in the Ka-band.

The width $w=1.8 \mathrm{~mm}$, which provides $Z_{0}=50 \Omega$ according to Fig. 9.16(b), is taken as a reference for a first study. For this width, even- and odd-mode impedance are calculated as a function of the edge-coupled strips separation $s$. Results are shown in Fig. 9.18(a), where upper curves correspond to the even-mode impedance 
$\left(Z_{0}^{e}\right)$ and the lower curves correspond to the odd-mode impedance $\left(Z_{0}^{e}\right)$. These results are totally consistent with coupled-line theory applied to conventional waveguides [22], being found that even-mode impedance is always larger then the isolated line impedance, whereas odd-mode impedance is always smaller than the isolated line impedance. As it is also obviously expected, as the distance $s$ increase, both, $Z_{0}^{e}$ and $Z_{0}^{o}$ converge to $Z_{0}$, in this case, $Z_{0}=50 \Omega$. Therefore, it can be said that the proposed method has been successfully applied to the calculation of even- and odd-mode impedance, giving very reasonable values. Since several frequencies have been considered, the frequency dispersion of $Z_{0}^{e}$ and $Z_{0}^{o}$ is also reflected in the plot. Again, although it is found more dispersion than in the case of conventional transmission lines, this is low enough to provide straightforward component design in the case to moderate bandwidths in the specification. Notice that interpolation of the plot data of Fig. 9.17(b) suggest a variation of the impedance of roughly $2 \%$ if $R B W=5 \%$. The successful design of a filter with this bandwidth in section 9.3.3 will give a satisfactory proof for this affirmation.

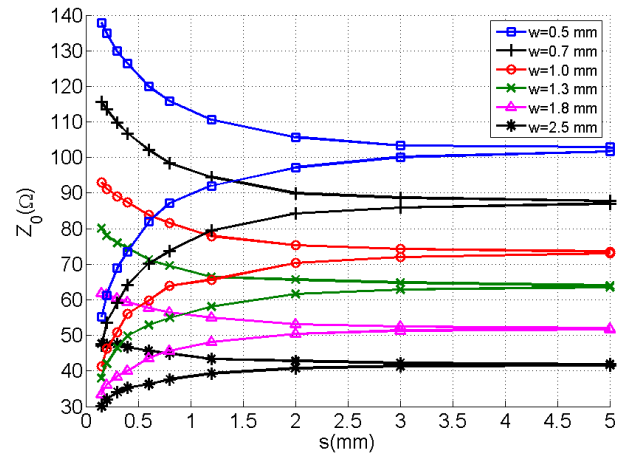

(a)

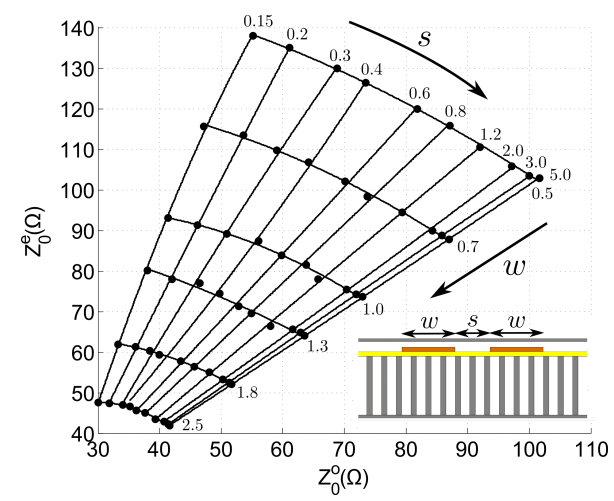

(b)

Figure 9.18: Parametrization of coupled SSGW lines main parameters, strip width $w$ and strip separation $s$ for the generation of a design chart. a) Even-mode ( $Z_{0}^{e}$, upper branches) and odd-mode ( $Z_{0}^{o}$, lower branches) impedance vs. $s$ for several strip widths $\left.w ; b\right)$ Same data as in a) displayed in the usual design chart way [22].

Repetitive calculation of curves such as those presented in Fig. 9.17(b) in the context of a full parametrization of the coupled-line structure provide a large quantity of data that can be displayed as a design chart for coupled-line components such as hybrids, couplers, filters, etc. This has been done for $f=35 \mathrm{GHz}$. Fig. 9.18 (a) shows the even- and odd-mode impedances as a function of the separation between lines $s$, for several widths $w$. Note that $Z_{0}^{e}$ and $Z_{0}^{o}$ depend both on $w$ and $s$, being possible by proper non-linear curve fitting [528] to find numerical functions $Z_{0}^{e}=f_{e}(w, s)$ and $Z_{0}^{o}=f_{o}(w, s)$ that predict the value of even- and odd-mode impedances for any given pair $(w, s)$.

These data can be also displayed in the usual coupled-line design chart form, i. e., displaying $Z_{0}^{e}$ as a function of $Z_{0}^{o}$ (or vice versa), in the same way as Fig. 9.18(b) shows. This plot results very helpful to understand how the coupled-line structure behaves in terms of impedances according to the coupled-line parameters. Since 
a lot of information is condensed in simple way, a good overall view is obtained. Generally, the design of a coupled-line component will demand from a section of coupled lines offering a certain pair $\left(Z_{0}^{e}, Z_{0}^{o}\right)$. With the current computation tools performance, is almost no time consuming to consider $f_{e}(w, s)$ and $f_{o}(w, s)$ and to solve numerically a 2 -order non-linear equation system to obtain the exact values. Nevertheless, note that the transmission line approach does not take into account second order effects in real circuits, only captured by full-wave simulations, the $\left(Z_{0}^{e}, Z_{0}^{o}\right)$ values involved in the design only provide estimated geometrical values, which are used as a starting point of a full-wave optimization. Therefore, visual determination of the pair $\left(Z_{0}^{e}, Z_{0}^{o}\right)$ on the chart of Fig. 9.18(b) is generally valid, and may result more user friendly from a high-level design perspective for those engineers building SSGW devices.

\subsubsection{Practical example: SSGW coupled-line filter}

In order to validate previous section results, the task of designing a coupled.line component is addressed now. A coupled-line filter has been chosen since filters are an essential part of communication systems [734] that usually present an elaborate synthesis and optimization [782], and whose design complexity and performance can be increased by choosing a larger filter order. For a first validation, a low order filter has been preferred.

\subsubsection{Filter design}

The specifications of the filter are the following: order $N=2$ Chebyshev-type with $0.1 \mathrm{~dB}$ passband ripple, centered at $f_{0}=35 \mathrm{GHz}$ with $R B W=5 \%$, i.e. passband from $34.125 \mathrm{GHz}$ to $35.875 \mathrm{GHz}$. Using the expressions for Chebyshevtype responses, the parameters $g_{i}$ are obtained: $g_{0}=1, g_{1}=0.843, g_{2}=0.622$ and $g_{3}=1.3554$. With these data, the inverter constants can be solved [22]:

$$
\begin{aligned}
Z_{0} J_{1} & =\sqrt{\frac{\pi \Delta}{2 g_{1}}} \\
Z_{0} J_{n} & =\frac{\pi \Delta}{2 \sqrt{g_{n-1} g_{n}}} \\
Z_{0} J_{N+1} & =\sqrt{\frac{\pi \Delta}{g_{N} g_{N+1}}}
\end{aligned}
$$

Finally, even- and odd-mode impedances are obtained from

$$
\begin{aligned}
& Z_{0}^{e}=Z_{0}\left[1+J Z_{0}+\left(J Z_{0}\right)^{2}\right] \\
& Z_{0}^{o}=Z_{0}\left[1-J Z_{0}+\left(J Z_{0}\right)^{2}\right]
\end{aligned}
$$

The geometry of the filter and the involved geometrical parameters can be seen in Fig. 9.19(a) and 9.19(b). When designing coupled-line filters, it is adopted usually 


\begin{tabular}{cccccc}
\hline $\mathrm{n}$ & $Z_{0} J_{n}$ & $Z_{0}^{e}(\Omega)$ & $Z_{0}^{e}(\Omega)$ & $w_{n}(\mathrm{~mm})$ & $s_{n}(\mathrm{~mm})$ \\
\hline 1 & 0.3052 & 104.88 & 59.10 & 0.7 & 0.4 \\
2 & 0.1085 & 84.02 & 67.75 & 0.8 & 0.8 \\
3 & 0.3052 & 104.88 & 59.10 & 0.7 & 0.4
\end{tabular}

Table 9.4: Design calculated starting point for the proposed specifications.

\begin{tabular}{lccccccc}
\hline Parameter & $l$ & $w_{1}$ & $s_{1}$ & $w_{2}$ & $s_{2}$ & $w_{3}$ & $s_{3}$ \\
\hline Value $(\mathrm{mm})$ & 1.73 & 0.66 & 0.30 & 0.85 & 0.77 & 0.66 & 0.30
\end{tabular}

Table 9.5: Optimum filter geometric parameters.

$l=\lambda_{g} / 4$, which simplifies the design expressions. Since $\lambda_{g} \approx \lambda_{0}=2.15 \mathrm{~mm}$, and $w_{50 \Omega}=1.8 \mathrm{~mm}$, it has been chosen $Z_{0}=75 \Omega$ which is achieved with $w_{0}=0.95$ $\mathrm{mm}$ in order to avoid a larger width than length, which could produce improper resonance problems. This leads to the parameters shown in the table 9.4.

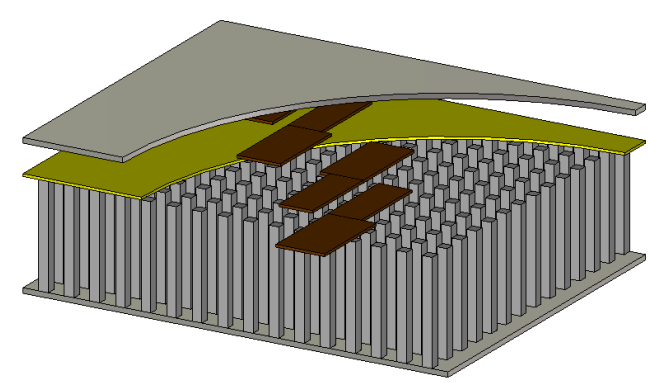

(a)

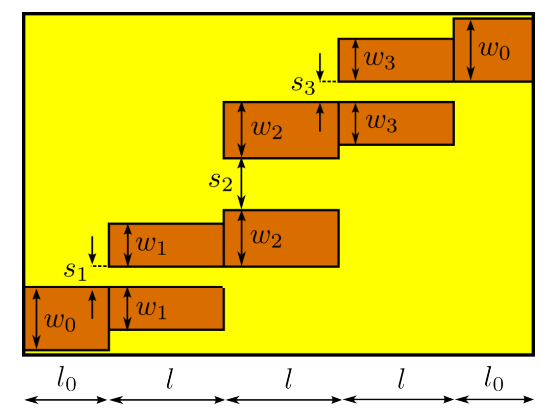

(b)

Figure 9.19: $S S G W$ coupled-line filter of order $N=2$. a) $3 D$ view; b) Circuit layout and main design parameters.

\subsubsection{Results}

The previous procedure based on coupled transmission line theory uses some approximations and full-wave optimization is required to satisfy the desired specifications. However, a good starting point has been obtained, which facilitates the optimization process. The optimum filter dimensions are shown in table 9.5, in which it can be seen that the filter symmetry has been preserved.

The optimized structure response is shown in Fig. 9.20(a) together with the ideal response of a filter with the established specifications. As it can be seen, a good response is obtained. Particularly, insertion losses (IL) of only 0.3-0.4 dB are achieved, which is a good result for the Ka-band. Recovering that IL (dB) are inversely proportional to the $Q_{u}$ of the resonators [782], a microstrip line, for example, would present IL 5-6 times higher than the SSGW [840].

Now, the response dependence on the strip position is afforded. Although a dense structure has been used, some geometrical parameters of the filter are comparable 


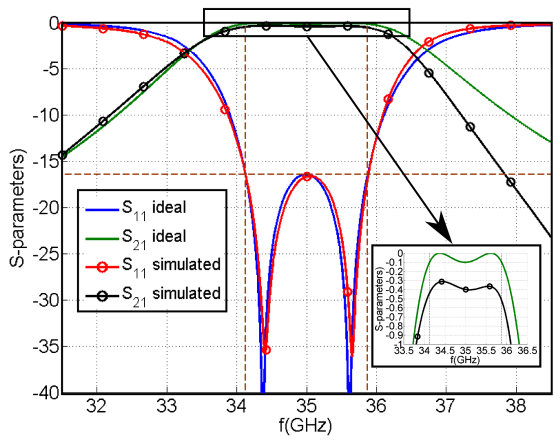

(a)

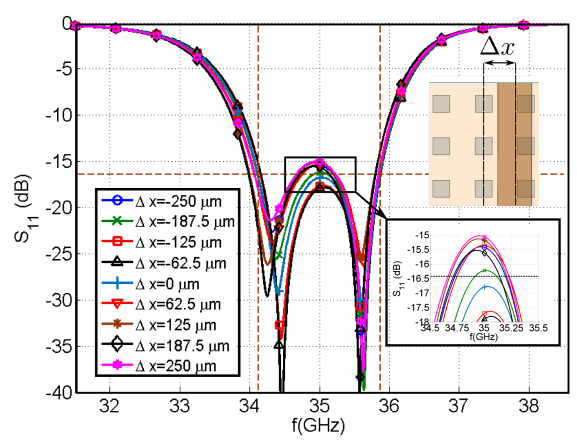

(b)

Figure 9.20: Simulated filter response. a) Ideal response and simulated optimum response; b) Effect of the strip position on the filter response.

with the period, and the response might change. A horizontal displacement of the strip in $\hat{x}, \Delta x$ is considered. The considered range of this displacement is one period, specifically $\Delta x$ goes from $-p / 2$ to $p / 2$, since any case is included in this range due to the periodic nature of the structure.

Results of the strip-positioning study are shown in Fig. 9.20(b), where the $S_{11}$ (dB) curve is displayed. It can be seen that, although there is a change in the response, the curves keep close to the specification. The worse case is found for $\Delta x=p / 2=250 \mu \mathrm{m}$, which present a peak in the return losses of $15 \mathrm{~dB}(1.42 \mathrm{~dB}$ lower than the specification). This value translates in a ripple of $0.18 \mathrm{~dB}$ instead of $0.1 \mathrm{~dB}$, which is quite acceptable. The observed low dependence with the strip position observed could be unreachable with the non-dense pin lattices proposed in [795] or [859], where variations of the characteristic impedance up to $\pm 30 \%$ are observed.

Finally, to better quantify the potential of the SSGW topology to implement low insertion loss filters according to the current technological possibilities, a simple comparison with SIW technology is done by studying the resonator quality of both topologies for an operation frequency of $f=35 \mathrm{GHz}$. The RGW topology is also studied to give additional information. For the RGW and SSGW resonators, the periodic structure parameters are the same as those used previously in this section. The ridge/strip width is set to $w=0.95 \mathrm{~mm}$ as well. The SIW vias are designed with diameter $d=0.3 \mathrm{~mm}$ and separation $s=0.6 \mathrm{~mm}$, dimensions that have been chosen according to the Ka-band SIW literature [918]-[920] and with the aim to enhance the quality of the SIW resonator. The substrates permittivity is set to $\varepsilon_{r}=2.25$, taken from the Nelco NY922 substrate, a good quality reference one [921]. The loss tangent is parametrized to observe the influence of the dielectric quality on the losses. In the case of the SSGW, the substrate thickness is set to $t_{d}=127 \mu \mathrm{m}$ which is a commercial standard value [921], whereas the SIW substrate is set to $t_{d}=508 \mu \mathrm{m}$ a standardized common value for the frequencies of interest [918]-[920].

Fig. 9.21(a) show the 3D view of the studied resonators. As usual, the RGW and SSGW resonators are implemented open circuit, whereas the SIW resonator is 

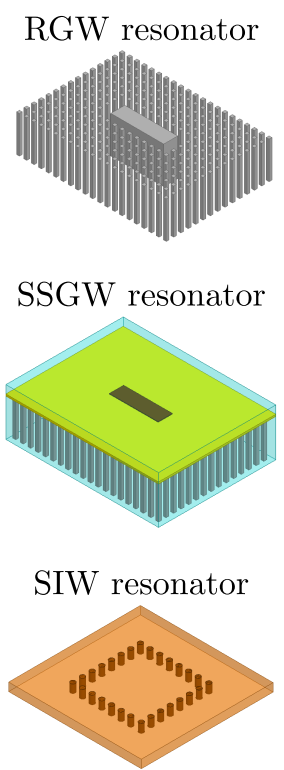

(a)

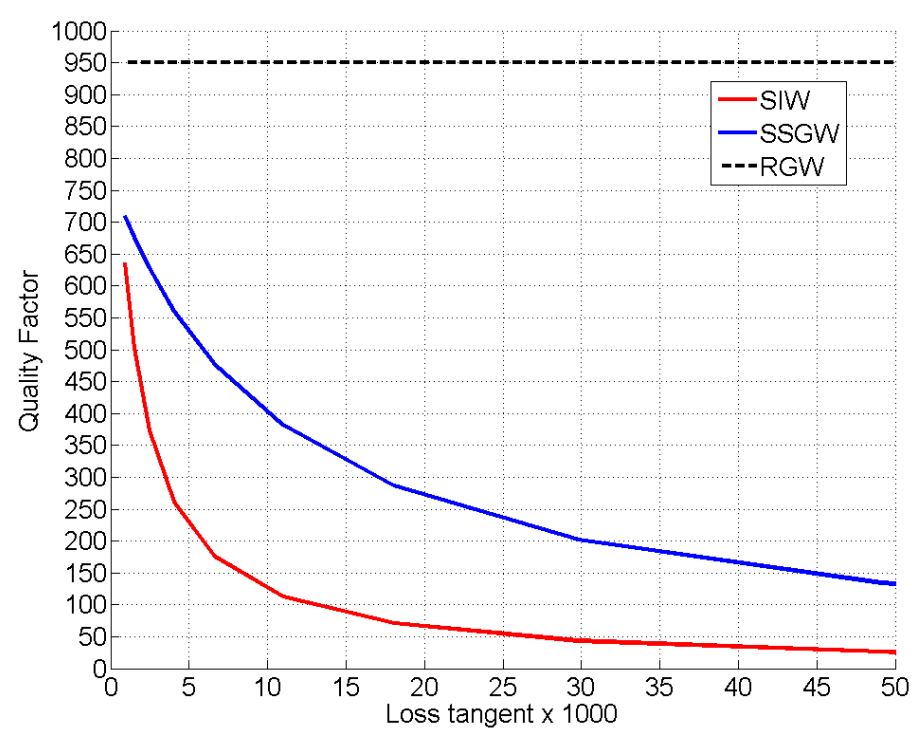

(b)

Figure 9.21: Resonator quality comparison between RGW, SSGW and SIW technologies. For the RGW and SSGW resonators the periodic structure parameters are $p=0.5 \mathrm{~mm}, w_{p}=0.19 \mathrm{~mm}$, $h_{p}=2.5 \mathrm{~mm}$ and $h_{a}=0.5 \mathrm{~mm}$. The ridge/strip width is set to $w=0.95 \mathrm{~mm}$. SIW vias are of diameter $d=0.3 \mathrm{~mm}$ and separation $s=0.6 \mathrm{~mm}$. The reference dielectric permittivity is $\varepsilon_{r}=2.25$ and $\tan \delta$ is parametrized. In the SSGW resonator the dielectric thickness is $t_{d}=127 \mu \mathrm{m}$, whereaas in the $S I W$ resonator $t_{d}=508 \mu \mathrm{m}$. a) $3 D$ view of the studied resonators; $\left.b\right)$ Quality of the $f=35$ $\mathrm{GHz}$ resonators vs. $\tan \delta$.

a short-circuit cavity resonator. The quality of these resonators is calculated taking $\tan \delta$ as a parameter, which obviously do not affect the RGW resonator since any dielectric is present. For the usual values of dielectric losses $(\tan \delta \in[0.002-0.02])$, it can be said that the quality of the SSGW resonator is roughly 3 times that of the SIW resonator. This is a remarkable difference. Notice that the state of the art in SIW filters reports acceptable results in terms of insertion losses [772]. Therefore, the potential of the SSGW technology is clearly foreseen. In practical applications, a high-order filter is required, since steep skirts in the response are usually necessary. In these cases, in order to maintain low insertion losses, a good $Q_{u}$ is demanded, and the SSGW seems very suitable as it is less affected by the high dielectric losses at mmwave frequencies than other topologies such as SIW or the conventional transmission lines.

Note also that for good dielectrics (acceptable values of $\tan \delta$ in this sense have been measured at mm-wave frequencies [921]), the SSGW present competitive values of $Q$. Nevertheless, if the application is very demanding, a SSGW design can be easily mapped to a RGW design, which offers an outstanding performance since any dielectric loss is present. For the studied case, $Q$ is close to 1000, an unreachable value for any other practically implementable quasi-TEM structure. Obviously, the design complexity and the cost increase in such case. 
Along this chapter it has been ascertained that the impedance calculation by means of the presented method, which gives a correction to a known reference impedance port by using transmission line theory, results suitable for the SSGW in the context of circuit design, as the line is characterized as a circuital element. Furthermore, an error analysis guarantees control of the method accuracy. In a further step, using the presented method as an analysis tools, they have been observed two main problems in common SSGW structures: 1) Strong dependence of the impedance on the strip relative position to the pins; 2) impossibility of designing high-value impedances. To overcome these problems, a design procedure has been described. It has been shown how denser periodic lattices provide the required homogenization and they can keep wide the stopband bandwidth if desired. Very stable impedances, independent of the strip position have been obtained. In addition, it has been justified that the manufacturing process should not, in principle, to get involved, being possible to use roughly manufactured pins, since their only mission is to provide a high impedance surface. Moreover, analysis methods based on homogenization of the pin structure, otherwise inaccurate, appear to be suitable for such dense lattice designs. Frequency dispersion has been observed to be enough low for moderate bandwidth devices as well for such design.

Coupled transmission line theory has been also shown to fit well with the proposed dense lattice SSGW topology, and, for the first time, they have been calculated even- and odd-impedances for the SSGW, obtaining coherent results. A design chart for the Ka-band has been built, and a simple SSGW coupled-line filter has been easily designed. The filter response exhibits low insertion losses and negligible dependence on the strip position, what indicates the effectiveness of the design procedure using dense lattices. Furthermore, a simple comparative study analyzing isolated resonators has shown the advantages in terms of performance of SSGW technology front SIW technology, which is already a well-adopted technology at mm-wave frequencies.

Further work involves the use of faster analysis methods taking advantage of the homogenization of the dense periodic lattice. An extensive work either analytic using expressions of known transmission lines, or empirical, based on large amounts of data is demanded in order to arrive to closed form expressions for the SSGW. This is necessary for the acceptance of the technology. Development of dedicated cheap manufactured techniques is as well a very necessary task. In such future scenario, advanced device design will come naturally. The observed SSGW potential for mmwave frequencies indicates that high performance devices could be easily designed once those goals are achieved. 


\section{Chapter 10}

\section{Groove Gap Waveguide (GGW) components}

Despite of the fact that the first GW technology proposals, at the introduction stage of the technology, were mostly based on the RGW topology, literature of the subsequent years, at the adoption stage, shows tendency to choose GGW topology due to its lower losses and similarities with the rectangular waveguide. Current research points towards a maturity stage with GGW topology as the flagship of GW technology. This topology is the GW candidate to be firstly adopted by the commercial applications industry.

Nevertheless, there are two key issues that must be solved to this occur. First, the advantages of the GGW front the classical rectangular waveguide, which are suitable to be appreciated mainly at the V-band and beyond, must be proved with truthfulness. Although the good performance of GGW devices is well reported in several works, and a few comparisons with the rectangular waveguide can be found (see [800] or [836] for instance), it still lacks a proper, fair and determinant comparison. For example, in [800], GGW and rectangular waveguide resonators are compared at the Ku-band, i. e., excessively low frequencies to really appreciate the advantages of GGW front the well-established rectangular waveguide, at least with current industry manufacturing possibilities. In [836], the operation frequency lies in the Ka-band, which although not so high, at least imposes some challenge for the rectangular waveguide. However, a GGW filter is compared with a rectangular waveguide filter with different specifications, hence the conclusions are not rigorously sustained by the experiment. Therefore, it seems evident that to give proof of the necessity of considering GGW as an alternative for rectangular waveguide, a proper comparison is required: it must be chosen a device that clearly demands from rectangular waveguide characteristics, and an operation frequency that makes the rectangular waveguide device response to be prone to degrade due to manufacturing imperfections, so that it make sense to look for alternatives. Notice that the rectangular waveguide has been a very suitable topology for low-loss microwave waveguiding. Hence, it is not only necessary to prove that the GGW performance is 
close to or similar to that of the rectangular waveguide, but clear advantages must be appreciated.

Second, good analysis methods must be developed for the GGW topology in order to have affordable design process for engineers that do not necessarily have the know-how of this waveguide. In relationship with the former point, it must be said that, in order to consider the GGW as an alternative to the rectangular waveguide, apart from better performance, it is necessary to provide analysis methods not much more complex than those used for rectangular waveguide devices design in order to prevent the designers desist from the GGW topology due to avoid a cumbersome task. Notice that the rectangular waveguide topology is extremely simple for analysis purposes as it is a trivial canonical case. The literature on such topic is vast, modal methods are a good example. On the other hand, the GGW is a complex structure, not homogeneous in any direction, an that is very prone to large computation times in standard full-wave simulations. Despite of the fact that the issue of finding a proper analysis method for the GGW topology has been treated in the literature, the followed approaches are not suitable for the demanded purpose. Note that, for a wide adoption, an analysis method must be efficient, general and simplest as possible. For instance, in the case of SIW technology, the most adopted analysis methods are based on giving and equivalence between SIW and rectangular waveguide topologies, hence establishing a conceptual bridge between them that allow to an easy comprehension by those engineers familiarized with the classical rectangular waveguide but just starting to work with SIW technology.

These two issues are deeply treated and solved in the present chapter. First, in section 10.1 a complete analysis of the GGW propagation characteristics is carried out. The derived information is thoughtfully applied to develop an efficient analysis method in section 10.2. This analysis method provides, furthermore, an accurate, direct equivalence between GGW and rectangular waveguide. Later, in section 10.3, several GGW narrow-band filters are designed and experimentally validated, and comparison with the rectangular waveguide topology is given. Therefore, this chapter faces most of the objectives of this part of the thesis.

\subsection{Analysis}

The difficulty in characterizing gap waveguides arises from the presence of the periodic pin structure, which leads to a waveguide which is not homogeneous in the transversal direction, is periodic in the propagation direction, and has many design parameters. The state of the art review reveals that, in fact, the propagation characteristics of GGW have not been analyzed in great detail. Usually, it is assumed that GGW behaves like a rectangular waveguide with the same propagation channel dimensions. However, important differences between GGW and rectangular waveguide will be shown next, hence indicating that such assumption results quite rough. In addition, as a part of such GGW propagation characterization, operation below cutoff is explored for the first time. Notice that there are many practical applications using below-cutoff waveguides, since evanescent-mode components can be very compact, and are very appropriate to exhibit spurious free response [922]-[924]. GW technology has still not explored this interesting possibility. Once GGW propaga- 


\begin{tabular}{lccccc} 
Parameter & $p$ & $w_{p}$ & $h_{p}$ & $h_{a}$ & $a$ \\
\hline Value $(\mathrm{mm})$ & 0.9 & 0.3 & 2.4 & 0.375 & 4.7 \\
\hline
\end{tabular}

Table 10.1: Geometrical parameters of the reference GGW design [840].

tion characteristics are determined, the developed analysis method in the scope of this thesis will be presented. By means of basic transmission line theory, exposed in section 9.1.1, and transversal analysis of the GGW, the method obtains a rectangular waveguide equivalent very suitable to extract the influence of the different waveguide parameters in the dispersion diagram and for parametric analysis and optimization, avoiding the usual time-consuming full-wave EM simulations.

\subsubsection{Groove Gap Waveguide Propagation characteristics}

\subsubsection{Operation above cutoff}

Conventional operation, i. e., above cutoff is analyzed first. To do so, the dimensions of a standard GGW design are chosen. Specifically, the Ka-band design of [840], treated later in 10.3.6, is taken as reference. These dimensions are listed in Table 10.1. Full-wave simulations are carried out with $\mathrm{CST}^{\circledR}$. The simulated model includes three rows of pins at each lateral side, following the usual design choice in the literature. Although for some designs using even more rows may be not critical for reasons of space, weight or complexity, three rows have been checked to be enough to achieve the desired effect of forbidden propagation in the lateral regions. PEC walls are placed as lateral boundary condition at a distance $s=p-w_{p}$ from the third row on each side, i. e., where the the fourth row of pins at each side should be located. It has been checked that, although no difference is obtained between using a PEC wall of leaving the structure open (the field is noticeably attenuated after the third row of pins), the employed model is more suitable in terms of computation time.

Despite of the similarity of the GGW geometry and fundamental mode field pattern, see Fig. 10.1(a), appreciable differences can be found if the dispersion diagram of both waveguides is inspected in its whole domain. Fig. 10.1(b) shows the propagation constant of the first modes of this GGW reference structure. The black dashed curve is the propagation constant of a plane wave, blue curves correspond to unwanted modes of the parallel plate modes, and the green curve with square marks is the desired mode. The propagation constant of a rectangular waveguide with same dimensions of the propagation channel of the GGW (i.e. $a_{R W}=4.72$ $\mathrm{mm}, b_{R W}=h_{a, G G W}+h_{p, G G W}=2.775 \mathrm{~mm}$ ) is displayed for comparison in orange. It can be seen that the band $[28.1 \mathrm{GHz}-52.9 \mathrm{GHz}]$, delimited by the propagation of unwanted parallel-plate modes, represents the stopband of the periodic structure: in such frequency range only the desired mode is propagating in the GGW.

When comparing the curves of the GGW with those of the rectangular waveguide (see Fig. 10.1(b)) it can be seen that in the upper half of the stopband both curves are similar. However, this is not true near cutoff. In fact, both waveguides present a different cutoff frequency, and even for frequencies where they are similar, a greater dispersive behavior is observed for the GGW structure. Therefore, the direct equiv- 

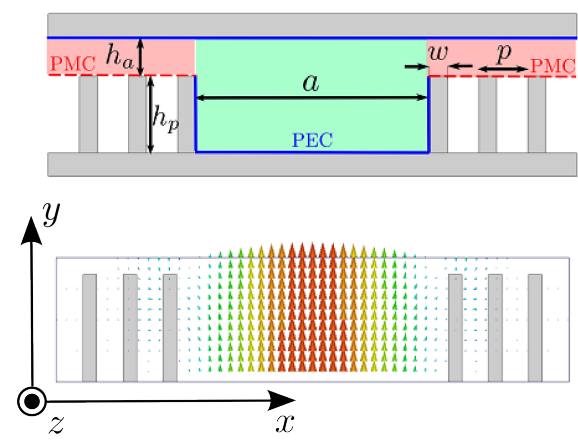

(a)

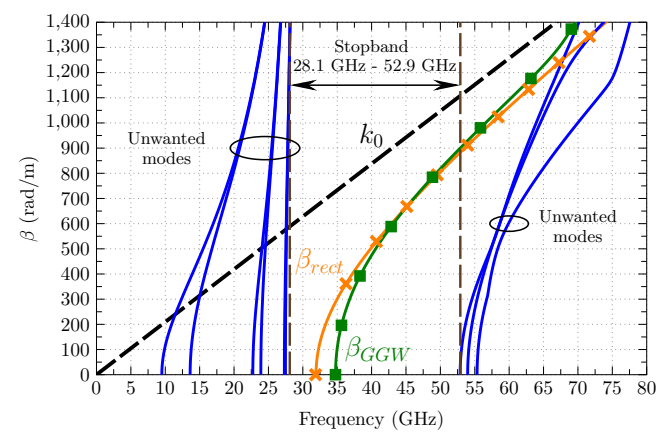

(b)

Figure 10.1: Groove Gap Waveguide propagation characteristics; a) Cross section and E-field distribution of the fundamental mode; b) Dispersion diagram for a GGW with parameters $h_{p}=$ $2.4 \mathrm{~mm} w=0.3 \mathrm{~mm}, p=0.9 \mathrm{~mm}, h_{a}=0.375 \mathrm{~mm}$ and width $a=4.7 \mathrm{~mm}$. Blue solid lines are the unwanted parallel-plate modes, which delimit the stopband of the structure, delimited with vertical dashed brown lines. The green solid curve with square marks correspond to the GGW fundamental mode. The propagation constant of a rectangular waveguide of $a=4.7 \mathrm{~mm}$ is represented with an orange curve with cross marks for comparison purposes. The propagation constant of a plane wave $\left(k_{0}\right)$ is shown with a black dashed line for reference.

alence between both waveguides occurs in very narrow band, which, moreover is not known a priori.

To further characterize the propagation properties of the GGW, the width of the propagation channel $a$ is parametrized. To cover the possible cases of having cutoff going from near the minimum frequency of the stopband to the maximum frequency of the stopband, six values of $a$, equally distributed between $a=2.8 \mathrm{~mm}$ and $a=6$ $\mathrm{mm}$. The reference GGW design is represented by the curve $a=4.72 \mathrm{~mm}$.

The results of this parametrization are shown in Fig. 10.2. Here, in most cases, the GGW presents a higher cutoff frequency, than its rectangular waveguide counterpart, especially as $a$ increases. This means that GGW is effectively smaller in terms of propagating aperture than the equivalent rectangular waveguide assumed for each case (same physical aperture, i.e, $a_{R W}=a_{G G W}$ ). The contrary could be expected since in GGW the fields are not strictly transmitted in the channel but spread evanescently through the lateral pin regions. As a decreases, this difference becomes smaller and, if $a$ is small enough $(a=2.8 \mathrm{~mm})$, the GGW has lower cutoff frequency than the rectangular waveguide, and becomes effectively larger than the equivalent rectangular waveguide. This heterogeneous behavior makes impossible to obtain an equivalent consisting on a simple scaling factor.

In general, simulation reveals GGW to exhibit a greater dispersive behavior when compared with the equivalent rectangular waveguide. For the cases of larger $a$ values, the GGW curve grows faster with frequency and reaches the rectangular waveguide curve, and, although $\beta_{G G W}$ surpasses $\beta_{\text {rect }}$, both curves are quite similar from that point. For the cases of smaller $a$ values, this difference is greater and both curves diverge, having only a very narrow band of coincidence, or even no coincidence (e. g., at $a=2.8 \mathrm{~mm}$, where a very dispersive curve is observed). This behavior will be understood through the analysis of section 10.2. Since a complete exploration of 


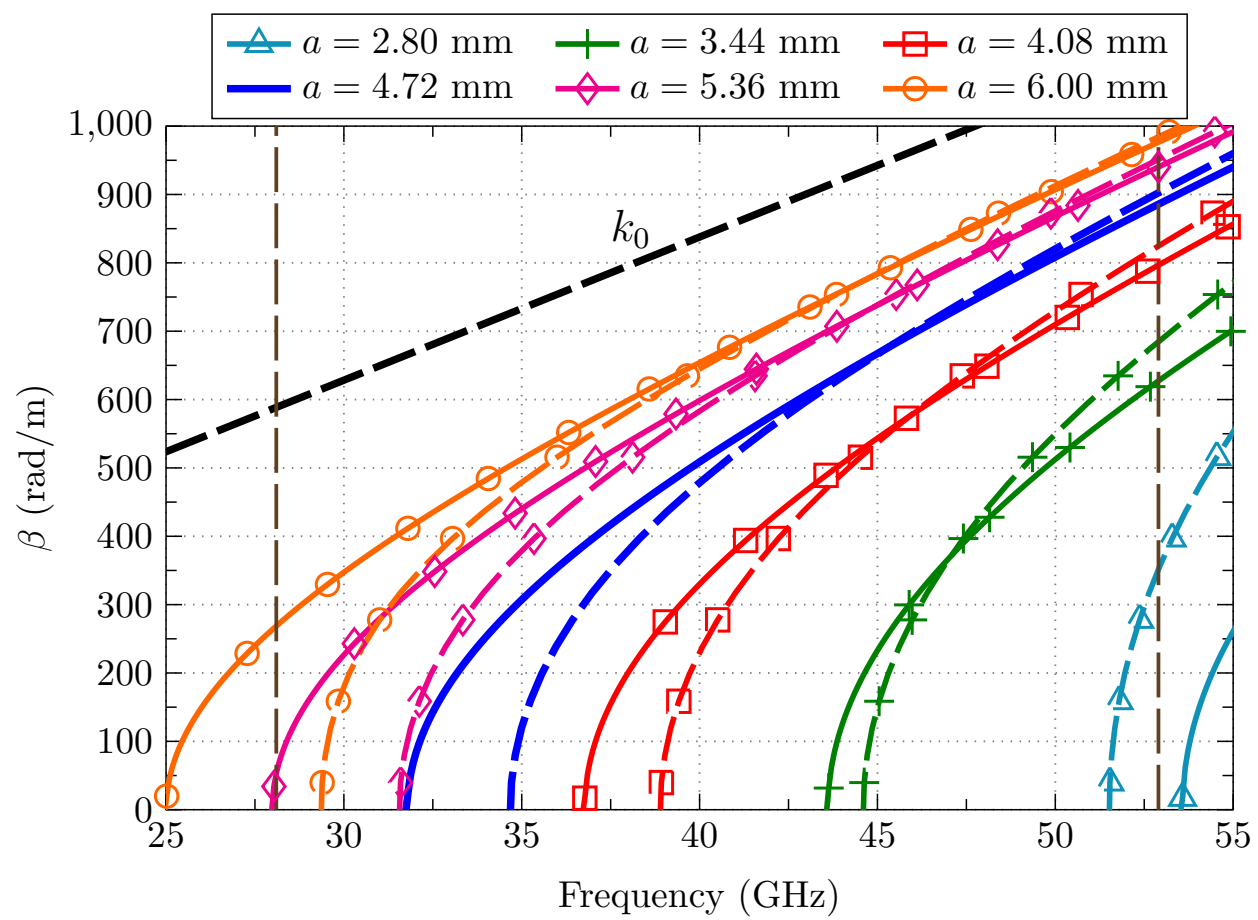

Figure 10.2: Propagation constant of the GGW and of the rectangular waveguide for different values of $a$. Solid lines correspond to the rectangular waveguide and dashed lines correspond to the GGW.

the width parameter possibilities such as done in Fig. 10.2 has not been carried out previously in the literature, the magnitude of difference between both waveguides has remained partly hidden. By the results it can be concluded that the standard assumption of equivalence between rectangular waveguide and GGW is only valid in certain bandwidth, which is determined by the specific value of $a$.

\subsubsection{Operation below cutoff}

In a below cutoff rectangular waveguide the lateral condition are electric walls, and evanescent energy is delivered along the axial direction only. In the GGW, the condition of forbidden propagation into the pins regions also permits exponential decay as a lateral condition. Actually, this phenomena is explicitly introduced in the Polemi's analysis method, based on homogenization of the pin structure [897], [898]. Therefore, when the operation takes place below cutoff, it is found that the same type of condition (exponential decay) is imposed to the guided waves in both, transversal and longitudinal directions. Therefore, it is not possible, a priori, to know if such waves will be forced to clearly propagate evanescently along the axial direction. In fact, in the most general case, evanescent propagation will take place in both directions, transversal and axial, and the predominance of one or another will be determined by the corresponding attenuation constants in such directions. Obviously, to obtain the desired evanescent operation behavior, the lateral attenua- 
tion must be clearly stronger than the axial one. The first objective in this section is to check that this actually occurs so that evanescent mode components are suitable to be implemented with GGW.

Considering the reference case $(a=4.72 \mathrm{~mm})$ EM fields are obtained by means of full-wave analysis of a whole GGW structure of certain length, Fig. 10.3(a). This GGW has a cutoff of $f_{c}=34.68 \mathrm{GHz}$. Since the stopband of the GGW structure starts at $f=28.1 \mathrm{GHz}$, three frequencies are analyzed to give the proper approach to the posed question: $f=28 \mathrm{GHz}$ (outside the stopband, mode below cutoff), $f=29 \mathrm{GHz}$ (inside the stopband, mode below cutoff), and $f=40 \mathrm{GHz}$ (inside the stopband, mode under usual operation above cutoff). The results of this comparison are shown in Figs. 10.3(b)-10.3(d). As can be seen, outside the stopband, the field spreads into the pin structure, whereas inside the stopband energy is delivered along the axial direction in a similar manner to the rectangular waveguide in both cases, below and above cutoff. It is observed, however, that the field spreads more in the lateral directions when the mode is below cutoff. Following the previous argument, it can be understood that, since axial attenuation appears below cutoff, the residual evanescent propagation towards the lateral sides increases inasmuch as the waves encounter a larger restriction to propagate in the axial direction.

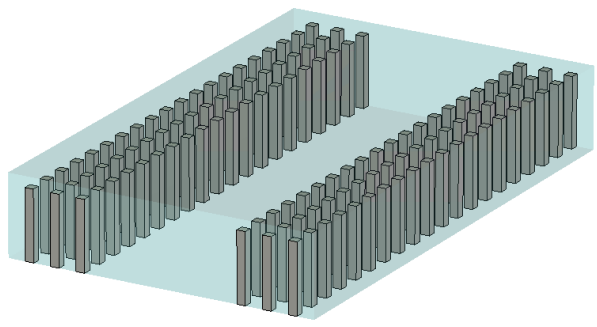

(a)

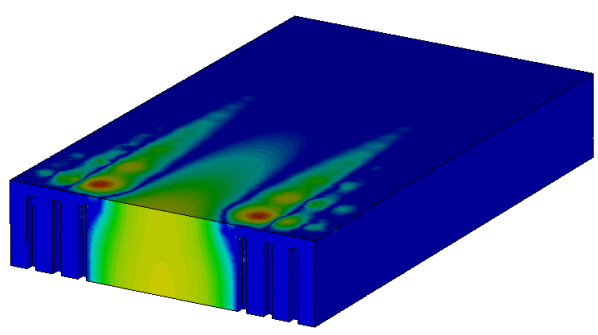

(c)

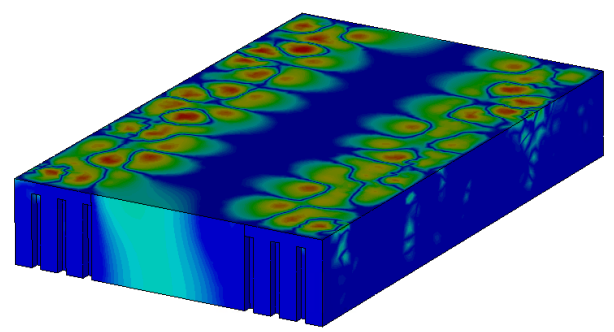

(b)

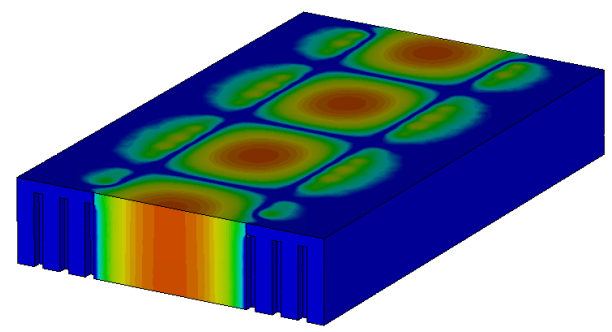

(d)

Figure 10.3: $E_{y}$ field inside of the reference $G G W$ with $a=4.72 \mathrm{~mm}$ for different cases of propagation. a) $3 \mathrm{D}$ view of the structure; b) $f=28 \mathrm{GHz}$ (outside the stopband); c) $f=29 \mathrm{GHz}$ (below cutoff inside the stopband); d) $f=40 \mathrm{GHz}$ (above cutoff inside the stopband).

The analysis of the transversal field pattern inside the structure completes the study of the GGW propagation characteristics. In Fig. 10.4, the $E_{y}$ component is plotted as a function of $x$ on two transverse planes: $z=z_{1}$ (transversal plane cutting the pins at the middle) and $z=z_{2}$ (transversal plane located between two rows of 
pins) for different heights. The frequencies considered are $f=29 \mathrm{GHz}$ (far below cutoff), $f=34 \mathrm{GHz}$ (near below cutoff), and $f=52 \mathrm{GHz}$ (far above cutoff). These graphs allow to quantify better the propagation differences in terms of the operating frequency. In the field plots of Fig. 10.4, each row corresponds to a frequency, with increasing frequencies as the row number increases. The first column corresponds to $z=z_{1}$ whereas the second column corresponds to $z=z_{2}$.

It is seen that the field is better confined in the propagation channel as frequency increases. As it is well known, under below cutoff operation, the axial attenuation is higher when the operating frequency is further from the cutoff (lower frequencies). At those frequencies, relative to the axial attenuation, the lateral attenuation imposed by the pins becomes comparable (i. e., the mode is not clear forced to follow the axial direction as it occurs in other cases where the axial attenuation is lower). This explains why the field spreads more laterally.

Above cutoff lower interaction with the GGW lateral walls occur since the mode is not longer evanescent. The higher the frequency, the lower the interaction with the lateral walls imposed by the pins is, what can be explained if one thinks in the usual two plane wave decomposition of the fundamental mode of a rectangular waveguide: the closer $\beta$ to $k_{0}$, the lower the angle of incidence on the walls is. This fact explains why higher frequencies experience a large confinement (see Figs. 10.4(i) and $10.4(\mathrm{j}))$. The propagation is predominantly axial so field leakage towards the lateral sides is negligible.

It is worth mentioning that in most of the structure (from $y=0$ to $y=h_{p}$ ) the field correspond approximately to the red solid curve with square marks, labeled as $y_{1}$. Therefore, the previous results suggest that the GGW can operate below cutoff in an analogous manner to the rectangular waveguide. However, the differences between both waveguides are accentuated and local effects near or above the first row of pins must be taken into account. In fact, the influence of the pins is clearly appreciated in Fig. 10.4, comparing the two positions in $z$ considered (compare the two rows of images in Fig. 10.4). Concentration of the $E_{y}$ field is observed near the pins, especially for lower frequencies. This should be taken into account if elements such as irises or radiating slots are disposed close to the pins.

The dispersion diagram of the structure under below cutoff operation is finally studied now. Above cutoff, it is sufficient to calculate the propagation constant of the GGW fundamental mode following the procedure firstly described in [790] and widely used in the GGW literature, i. e., solving the corresponding eigenvalue problem. However, commercial solvers are not prepared to recover the evanescent solutions of the problem, and to generate an ad hoc FEM code for such purpose could be an excessively cumbersome task given the purpose. Instead, a whole GGW propagating section, such as that shown in Fig. 10.3(a) can be simulated to obtain the fields inside and, through them, the attenuation constant. For such simulation, rectangular waveguide ports with the dimensions of the GGW propagation channel are employed as suggested in [890]. Once the structure is solved, the following expression is evaluated:

$$
\alpha(\mathrm{Np} / \mathrm{m})=\frac{\ln \left(\frac{E_{y}\left(z_{1}\right)}{E_{y}\left(z_{2}\right)}\right)}{z_{1}-z_{2}}
$$




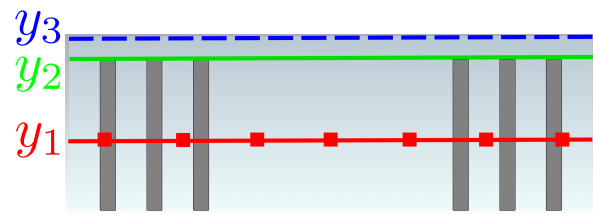

(a)

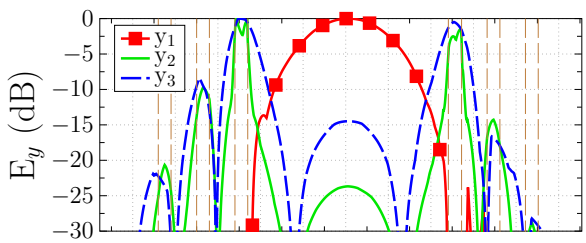

(c)

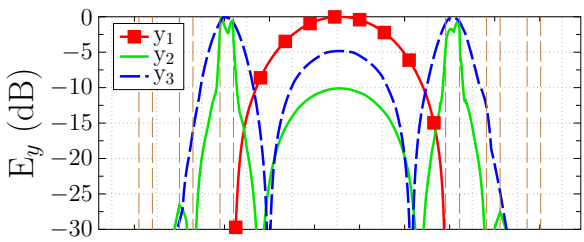

(e)

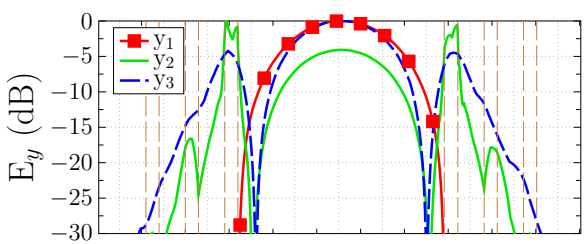

(g)

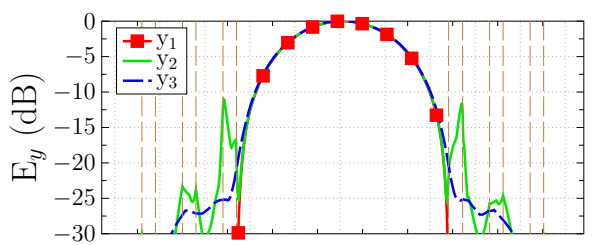

(i)

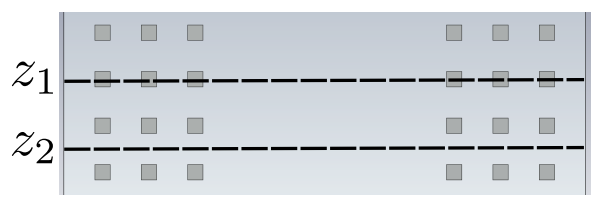

(b)

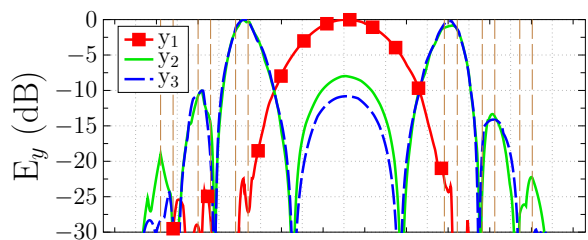

(d)

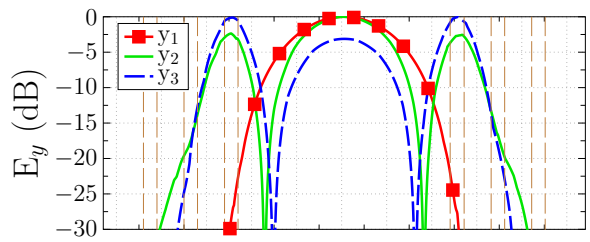

(f)

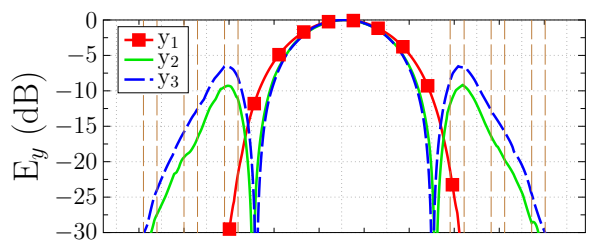

(h)

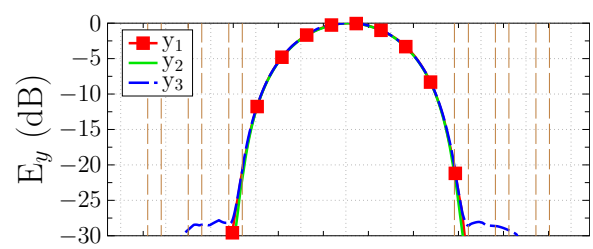

(j)

Figure 10.4: $E_{y}(x) G G W$ with $a=4.72 \mathrm{~mm}$ on a transversal plane at three different heights $y_{i}$ and at two different positions in $z$. Solid line with square marks is $y_{1}=h_{p} / 2$, solid line is $y_{2}=h_{p}$, and dashed line is $y_{3}=h_{p}+h_{a}$. Left column of graphs correspond to position $z=z_{1}$ (transversal plane cutting the pins at the middle) and the right column of graphs correspond to position $z=z_{2}$ (transversal plane located between two rows of pins). Vertical dashed lines are included to indicate the position of the pins in $x$ for clarity purposes. a) Considered heights in $y$; b) Considered positions in $z$; c) $f=29 \mathrm{GHz}, z=z_{1}$; d) $f=29 \mathrm{GHz}, z=z_{2}$; e) $f=34 \mathrm{GHz}$, $z=z_{1}$;f) $f=34 \mathrm{GHz}, z=z_{2}$; g) $f=40 \mathrm{GHz}, z=z_{1}$; h) $f=40 \mathrm{GHz}, z=z_{2}$; i) $f=52 \mathrm{GHz}$, $\left.z=z_{1} ; j\right) f=52 \mathrm{GHz}, z=z_{2}$. 
where $z_{1}>z_{2}$ and $E_{y}\left(z_{i}\right)$ is the amplitude of the $E_{y}$ field component in the center of the waveguide $(x=a / 2)$ at the corresponding $z$-position. With a similar procedure, the phase of the fields can be analyzed for operation frequencies above cutoff to recover the propagation constant.

Fig. 10.5 shows the results of this study based on the full-wave analysis of a whole GGW section (named as Field, circle marks), together with the previous eigenvalue calculations above cutoff (named as Eigenvalue, orange solid line), and the dispersion diagram of a rectangular waveguide with same channel width. The coincidence in the propagation constant above cutoff between the results obtained by means of the eigenvalue problem and those obtained by analysis of the fields in the whole GGW structure gives reliability to those obtained below cutoff in this last method.

The plot in Fig. 10.5 indicates that the difference between the GGW and the rectangular waveguide continue increasing when the frequency goes below cutoff. It is seen that as the frequency decreases, the attenuation in the GGW grows faster than in the rectangular waveguide. Furthermore, the GGW exhibits growth as the stopband limit approaches instead of the expected slope decrease (as it happens with the rectangular waveguide). Similar results have been observed for other values of $a$, indicating that rectangular waveguide and GGW behave noticeably different below cutoff. Actually, the poor coincidence between the curves of these waveguides below cutoff suggest that it makes no sense to assume a rectangular waveguide with the same propagating channel as an equivalent for the GGW.

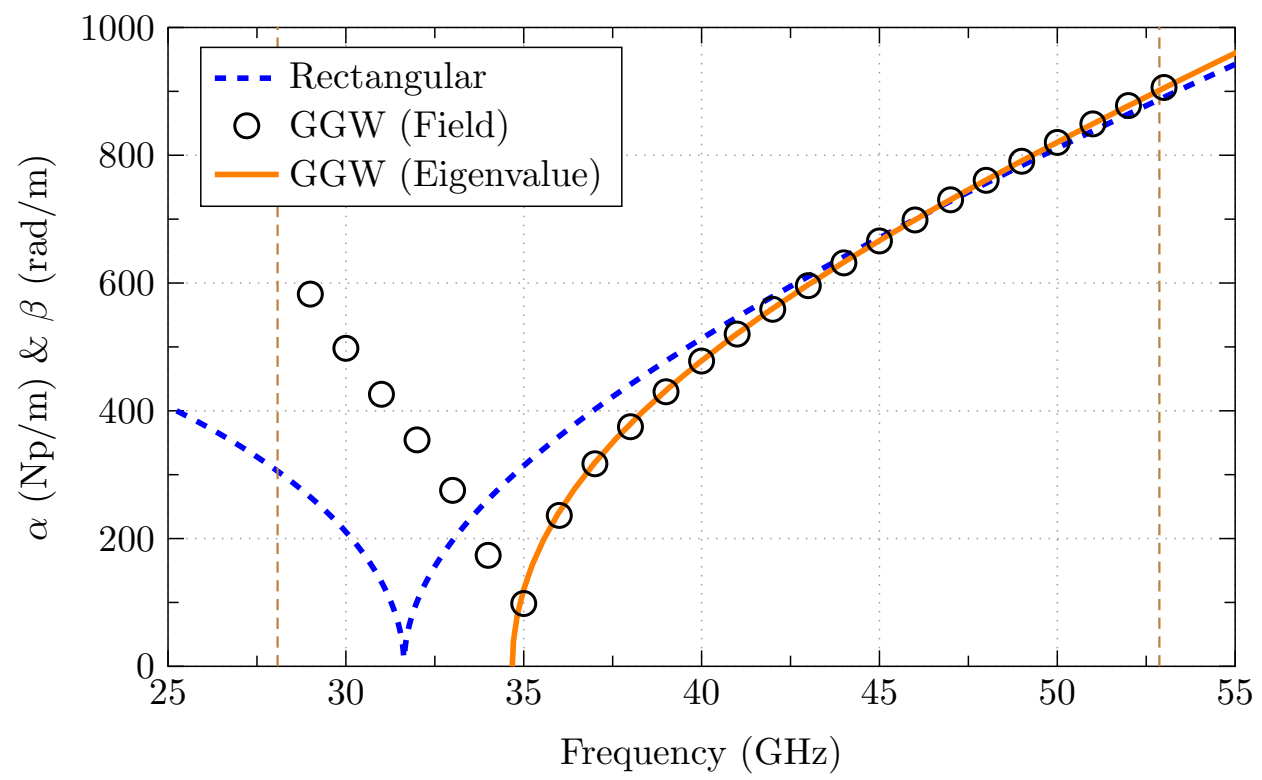

Figure 10.5: Propagation and attenuation constant for a rectangular waveguide and a $G G W$ of $a=4.72 \mathrm{~mm}$. 


\subsection{Proposed Analysis Model}

From the previous study, it is clear that the assumption of an equivalent behavior in a GGW and a rectangular waveguide with identical width $a$ implies errors unless $f_{c}$ is near to the low stopband limit and the operation frequency is far enough from cutoff. Moreover, modeling this behavior is not possible by scaling the rectangular waveguide by a constant factor depending on the geometry (as it occurs with SIW [757]-[758], [769]), since GGW and rectangular waveguide posses different dispersion characteristics. The shape of the obtained curves indicates that a mechanism is occurring within the structure, which involves the presence of a reactance due to the periodic lateral lattice. This reactance modifies the effective width of the GGW with the frequency.

\subsubsection{Analysis Method}

Consider the GGW, but now with regard to propagation in the lateral direction $\hat{x}$. The most interesting parameter in this configuration is the impedance $Z_{l}$ that is seen when looking into the first row of pins (as it is shown in Fig. 10.6(a)). where $Z_{l}$ is the impedance of the waves incident on the sidewall.

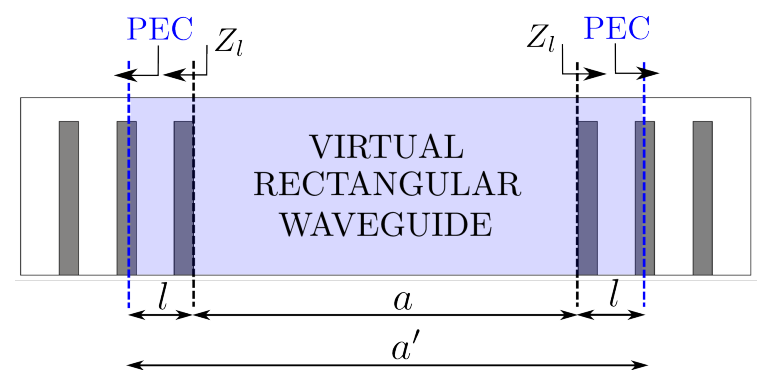

(a)

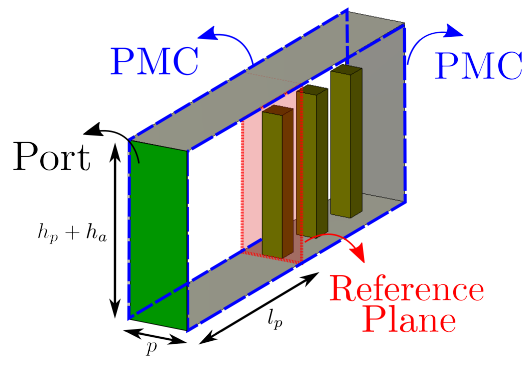

(b)

Figure 10.6: Approach of the proposed analysis method. a) Conceptual schematic; b) CST schematic of the structure solved with CST to obtain $Z_{l}$.

The reference plane is placed on the first pin with the rest of the arrangement terminated with a PEC, located at a distance of value $(p-w)$ with regard to the third pin. Periodicity is considered and only one period is analyzed, using PMC boundaries at the laterals (i.e., at $z=z_{0}$ and $z=z_{0}+p$ ). Difference in the results has not been appreciated when using more pins or adding more space after the third pin, and only a slight difference when using one pin less. Again, in terms of accuracy and time, three rows seems to be the optimum choice.

Referred to this port, the normalized input impedance of the structure is

$$
Z_{l n}=\frac{1+S_{11}}{1-S_{11}}
$$

In order to obtain the required $S_{11}$ parameter, the structure depicted in Fig. 10.6(b) has been simulated. In such schematic, it can be seen the PMC boundary planes at the lateral sides of the structure (providing periodicity by means of image 
theory), the unique port of the structure, and the reference plane at the first pin row, which is, actually, the lateral side of the propagation channel in a GGW. The $S_{11}$ is easily obtained at such reference plane by de-embedding, and using (10.2), the normalized impedance $Z_{l n}$ is calculated.

With the model of Fig. 10.6(b), only a lateral row of three pins and the vacuum volume of the auxiliar feeding parallel-plate $l_{p} \times p \times\left(h_{p}+h_{a}\right)$ are discretized and solved to determine its scattering parameters. Note that $l_{p}$ can be made as small as desired and the discretizing cost of the auxiliary waveguide is negligible. For canonical shapes further reductions in computing time are possible using the methods proposed in [544] or [925] for instance. However, with current computation capacities, fullwave simulation of such small single-port structure is noticeably fast: $24 \mathrm{~s}$. The resulting normalized impedance obtained with this approach is shown in Fig. 10.7(a). It can be appreciated that the periodic structure presents a reactance that exhibits capacitive behavior at the beginning of the stopband, changing to inductive behavior at the end of the stopband and crossing zero at $f=47.94 \mathrm{GHz}$.

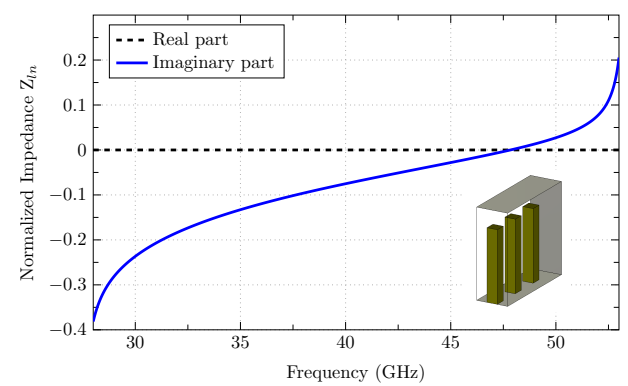

(a)

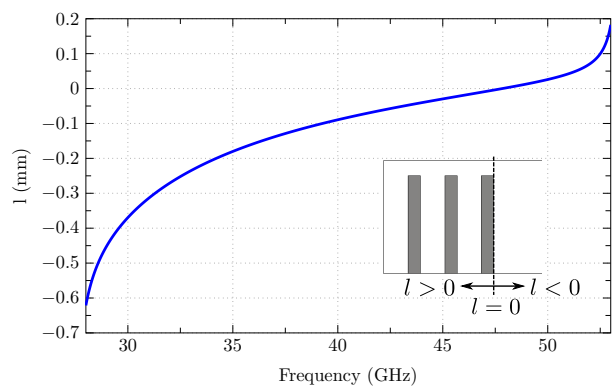

(b)

Figure 10.7: Results for the reference case. a) Lateral impedance $Z_{l}$ viewed at the first pin row; b) Distance $l$ at which a PEC wall would produce the same impedance as the one of the periodic structure.

Let now recover the expression (9.47) describing the normalized input impedance of the transmission line of characteristic impedance $Z_{0}$ loaded with an impedance $Z_{l}$ :

$$
Z_{\text {in }}=Z_{0} \frac{Z_{L}+j Z_{0} \tan (\beta l)}{Z_{0}+j Z_{L} \tan (\beta l)}
$$

If the transmission line is terminated with a short-circuit $\left(Z_{L}=0\right)$, the input impedance becomes

$$
Z_{\text {ln }}=j \tan \left(\beta_{p} l\right)
$$

hence

$$
l=-\frac{1}{\beta_{p}} \tan ^{-1}\left(j Z_{l n}\right)
$$

where $\beta_{p}$ is the propagation constant of the lateral parallel-plate waveguide feeding the structure with three pins. Therefore, at a distance $l$ from the reference plane, 
short-circuit conditions take place. As a consequence, the GGW is equivalent to a rectangular waveguide having lateral walls positioned at a distance that depends on $Z_{l n}$ (and therefore, on the frequency value). Such virtual rectangular waveguide has a width $a^{\prime}=a+2 l$, see again Fig. 10.6(a).

Since for small arguments the function $\tan ^{-1}(x)$ is almost linear and the $\beta_{p}$ variation with frequency is small compared with the $Z_{l}$ frequency variation, the behavior of both $Z_{l}$ and $l$ is quite similar. Depending on the sign of $l$, the GGW will be equivalent to a smaller $(l<0)$ or a larger $(l>0)$ rectangular waveguide, as shown in the inset of Fig. 10.7(b)

\subsubsection{Results}

\subsubsection{Cutoff frequency}

Results obtained by applying the previously described method start with the calculation of the cutoff frequency of the fundamental mode of a GGW. In the case of a rectangular waveguide, this cutoff frequency is described by

$$
f_{c}=\frac{c}{2 a}
$$

Since in the proposed model for the GGW the equivalent width $a^{\prime}$ depends on the frequency, the term $f_{c}$ will also have this dependence. For a given frequency $f_{0}$, one has $a^{\prime}\left(f_{0}\right)$ and, from (10.6), $f_{c}\left(f_{0}\right)$. In order to obtain the cutoff frequency for the GGW, a zero-finding routine is applied to $y(f)=f_{c}(f)-f$. The zero of $y(f)$ gives the cutoff frequency of the GGW being that it is found the frequency $f^{*}$ at which the GGW is equivalent to a rectangular waveguide which cutoff frequency is $f_{c}=f^{*}$, i. e., at which the GGW is equivalent to a rectangular waveguide that is exactly at cutoff. By applying this procedure, the cutoff of the GGW has been calculated as a function of the propagation channel width $a$. Results are displayed in Fig. 10.8, and compared with the GGW cutoff frequency calculated by full-wave solving the eigenvalue problem. The cutoff frequency of a rectangular waveguide is added to the plot as well for comparison purposes. It can be appreciated very good agreement between the proposed method and full-wave solving of the eigenvalue problem. Notice that for each width one eigenvalue problem must be solved. Contrarily, the proposed method results fast inasmuch as the simulation to calculate $Z_{l}$ (then $l$ ) is very short, and the cost of the zero-finding routine is negligible. Alternatively, assume direct equivalence between GGW and rectangular waveguide cause important errors on the calculated cutoff frequency, specially in the case of large widths, for which $|l|$ is considerable.

\subsubsection{Dispersion diagram}

The complete dispersion diagram of the GGW is obtained now. From the previous discussion it is deduced that propagation and attenuation constants of a GGW can be obtained through the standard rectangular waveguide formulas: 


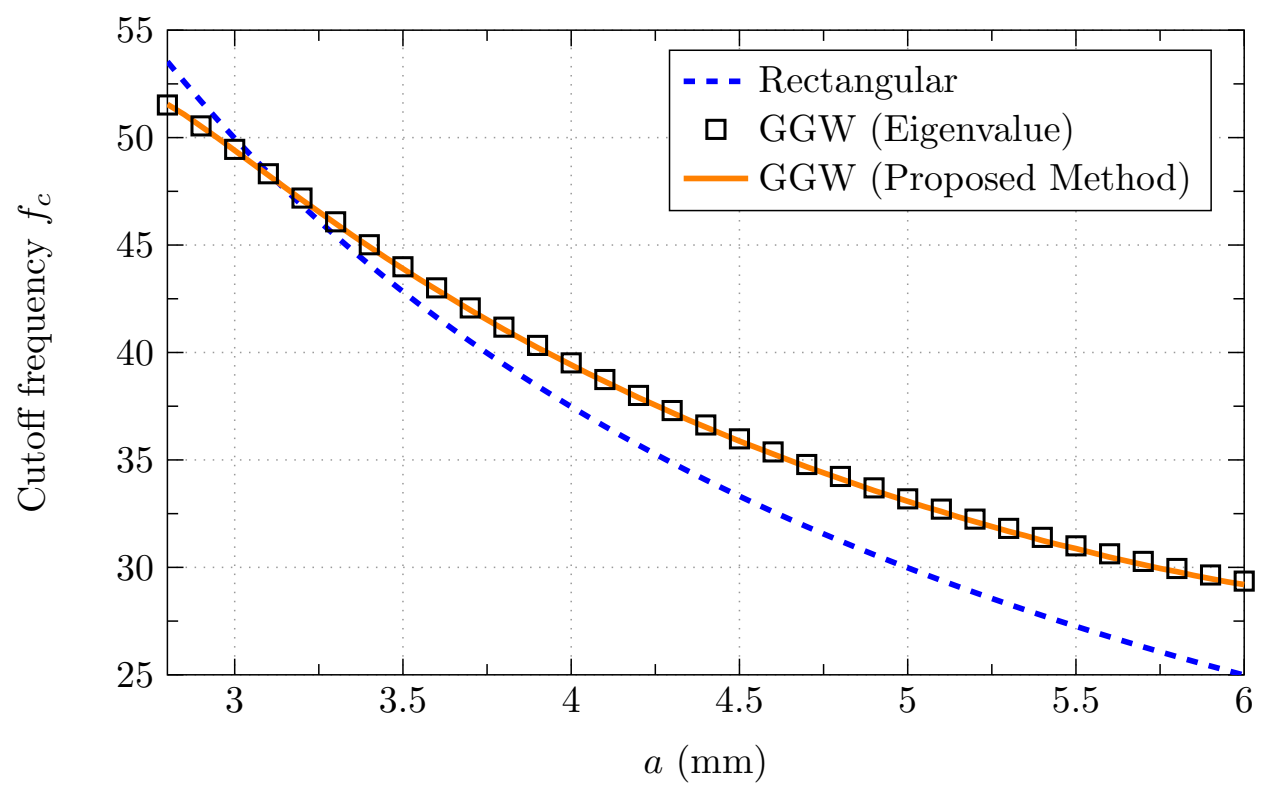

Figure 10.8: Cutoff frequency $f_{c}$ vs. waveguide width $a$.

$$
\begin{aligned}
& \beta=\sqrt{k^{2}-\left(\frac{\pi}{a^{\prime}}\right)^{2}} \quad f \geq f_{c} \\
& \alpha=\sqrt{\left(\frac{\pi}{a^{\prime}}\right)^{2}-k^{2}} \quad f<f_{c}
\end{aligned}
$$

By applying the above expressions, the propagation and attenuation constant has been calculated for the six widths considered in Fig. 10.2. Results are displayed in Fig. 10.9 together with those obtained by using other techniques based on intensive full-wave simulations (i.e., the one based on eigenvalues and the one using field evaluation). Rectangular waveguide curves are added as well for comparison. It can be observed how the presented method shows good agreement with full-wave simulations. Only slight difference are observed in the attenuation constant for the smallest $a$ case (Fig. 10.9(a)) when the operation frequency is far from cutoff. The lower attenuation constant provided by analysis of the field in full-wave simulations is probably caused by the reflections on the sides that have relative influence for such high attenuation rates $(600 \mathrm{~Np} / \mathrm{m})$ and beyond. Therefore, it could be even said that the presented method results, indeed, more reliable to calculate the global attenuation constant of the structure, being that the difference is caused by rather local effects. Nevertheless, note that, furthermore, such region of the dispersion diagram in the evanescent regime is useless for evanescent components design. In fact, the design region will always be much closer to the cutoff frequency, where reasonable small values of the attenuation allow for effective reactive energy coupling in such devices. 
In addition, the presented model gives an interesting physical insight, being that it is possible to understand the shape of the GGW dispersion curves from the rectangular waveguide ones. Above cutoff, as $a^{\prime}$ grows with the frequency, (10.7)implies for the GGW that $\beta$ grows with the frequency faster than for the rectangular waveguide case. When the term $k^{2}$ is large compared with $(\pi / a)^{2}$, the variation of $a^{\prime}$ is less significant, and the propagation behavior is similar to that of a standard rectangular waveguide. This occurs for large values of $a$ and high frequencies.

Below cutoff, as frequency is reduced, the term $k^{2}$ becomes small compared with $\left(\pi / a^{\prime}\right)^{2}$. Thus, with regard to (10.8), $\alpha$ exhibits growth with the decrease of $a^{\prime}$ with the frequency. This effect explains why the $\alpha$ curve of the GGW does not exhibit a reduction of its slope, as it occurs with the rectangular when the frequency decreases. Therefore, below cutoff, the error introduced by the assumption of the rectangular waveguide as an equivalent GGW with the same propagation channel dimensions is caused by the fact that the frequency variation of the reactance imposed by the periodic structure has a larger influence in relative terms.

Finally, it is worth noting that, due to the Foster reactance theorem [926], the lateral reactance will always monotonically increase with the frequency. This, translated through (10.4) and (10.5), gives that the GGW will always exhibit greater or equal dispersion than the equivalent rectangular waveguide. Nevertheless, the topic of non-Foster active metamaterials is widely treated these days [927]-[929]. The idea of creating a waveguide with similar loss than the rectangular waveguide but less dispersive is tentative, and an interesting future work line may be foreseen from merging the results in here with such research topic.

\subsubsection{Extreme GGW geometries}

So far, the proposed method has shown good accuracy for the several cases of $a$ considered, with the fixed periodic structure of the reference case, see Table 10.1. However, it is desirable to check its performance for different geometries of the periodic structure. In this sense, the method has been applied to several geometries, obtaining good results, which proves its robustness front the periodic structure geometry. Here, the results of two extreme cases, A and B, are reported as a proof of this fact. The periodic structure parameters of such cases are listed in Table 10.2. Fig. 10.10 shows the attenuation and dispersion curves for these cases, comparing the results of the proposed method with those of the eigenvalue and whole GGW section full-wave simulations for a fixed width $a=4.72 \mathrm{~mm}$. The rectangular waveguide curves and the reference case curves are added for comparison. In addition, the plot include insets showing the analyzed geometries. As can be observed, the case A consists of a structure with thin pins, sparsely distributed, and with the top plate located far from the pins top. The case B consists of a structure with thick pins and with the top plate located very close to the pins top. It is noted that the distance $h_{a}$ between the pins top and the top plate is the most influential parameter in the periodic structure reactance characteristics.

The case A implies a narrow stopband ([34 GHz-39.4 GHz], $R B W=14.7 \%$ ). As a consequence, the waveguide turns very dispersive for such design. To understand this behaviour, notice that $Z_{l n}$ start to diverge at the limiting frequencies of the stopband (towards $-j \infty$ at the lower limit and towars $+j \infty$ at the upper limit). 


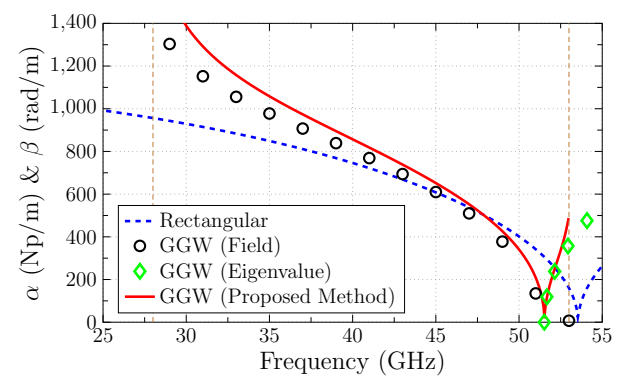

(a)

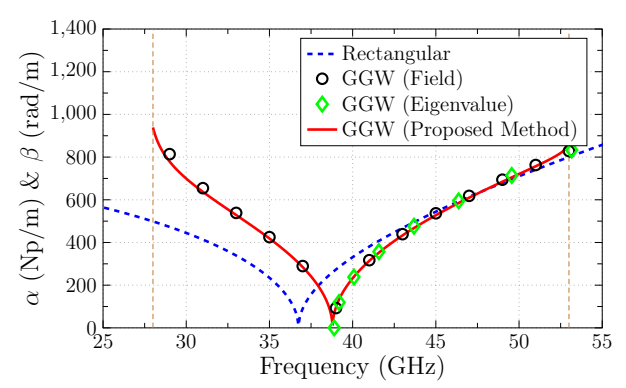

(c)

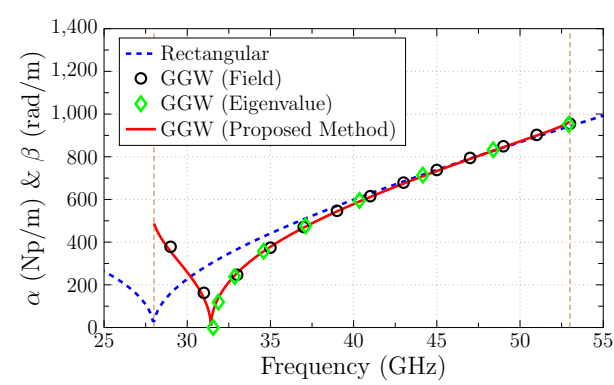

(e)

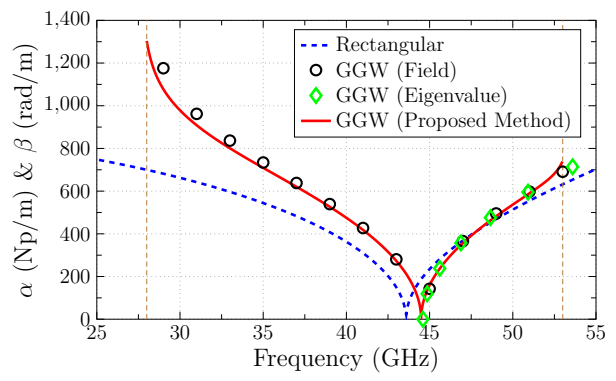

(b)

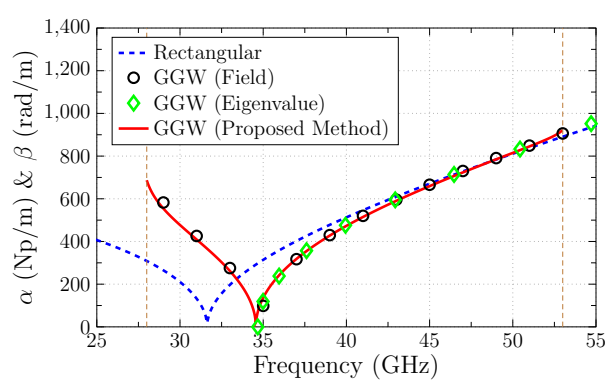

(d)

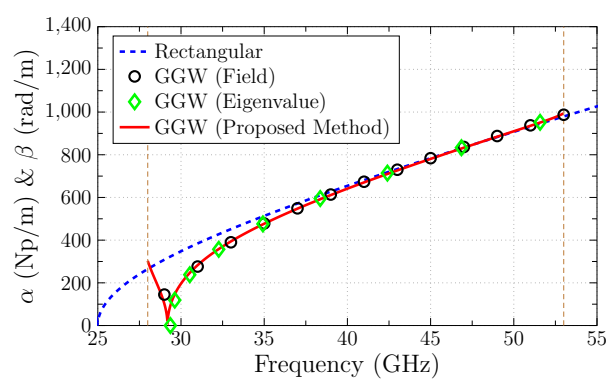

(f)

Figure 10.9: Propagation and attenuation constant of the rectangular waveguide and the GGW, comparing several GGW analysis methods: inspection of the field in full-wave simulations of a whole GGW structure, eigenvalue analysis applied to a period, and the proposed method in this thesis. a) $a=2.80 \mathrm{~mm}$; b) $a=3.44 \mathrm{~mm}$; c) $a=4.08 \mathrm{~mm}$; d) $a=4.72 \mathrm{~mm}$; e) $a=5.36 \mathrm{~mm}$; f) $a=6.00 \mathrm{~mm}$.

Therefore, the excursion of $Z_{l n}$ (hence the excursion of $l$ ) is compressed to a narrower bandwidth, what enhances the dispersive effects introduced by the periodic structure, and which have been explained previously by analyzing (10.7) and (10.8). Despite of this fact, the proposed model perfectly recovers the dispersion curves of the GGW starting from the rectangular waveguide ones. Notice that this has been possible even though the difference between the curves of both waveguides is noticeably large in this case. 


\begin{tabular}{lccccc} 
Parameter & $p$ & $w_{p}$ & $h_{p}$ & $h_{a}$ & STOPBAND \\
\hline Reference & $0.9 \mathrm{~mm}$ & $0.3 \mathrm{~mm}$ & $2.4 \mathrm{~mm}$ & $0.375 \mathrm{~mm}$ & $28.1 \mathrm{GHz}-52.9 \mathrm{GHz}$ \\
\hline Case A & $1.3 \mathrm{~mm}$ & $0.1 \mathrm{~mm}$ & $2 \mathrm{~mm}$ & $1.8 \mathrm{~mm}$ & $34 \mathrm{GHz}-39.4 \mathrm{GHz}$ \\
\hline Case B & $3 \mathrm{~mm}$ & $1.15 \mathrm{~mm}$ & $2.6 \mathrm{~mm}$ & $0.1 \mathrm{~mm}$ & $15 \mathrm{GHz}-54.4 \mathrm{GHz}$ \\
\hline
\end{tabular}

Table 10.2: Analyzed alternative periodic structures.

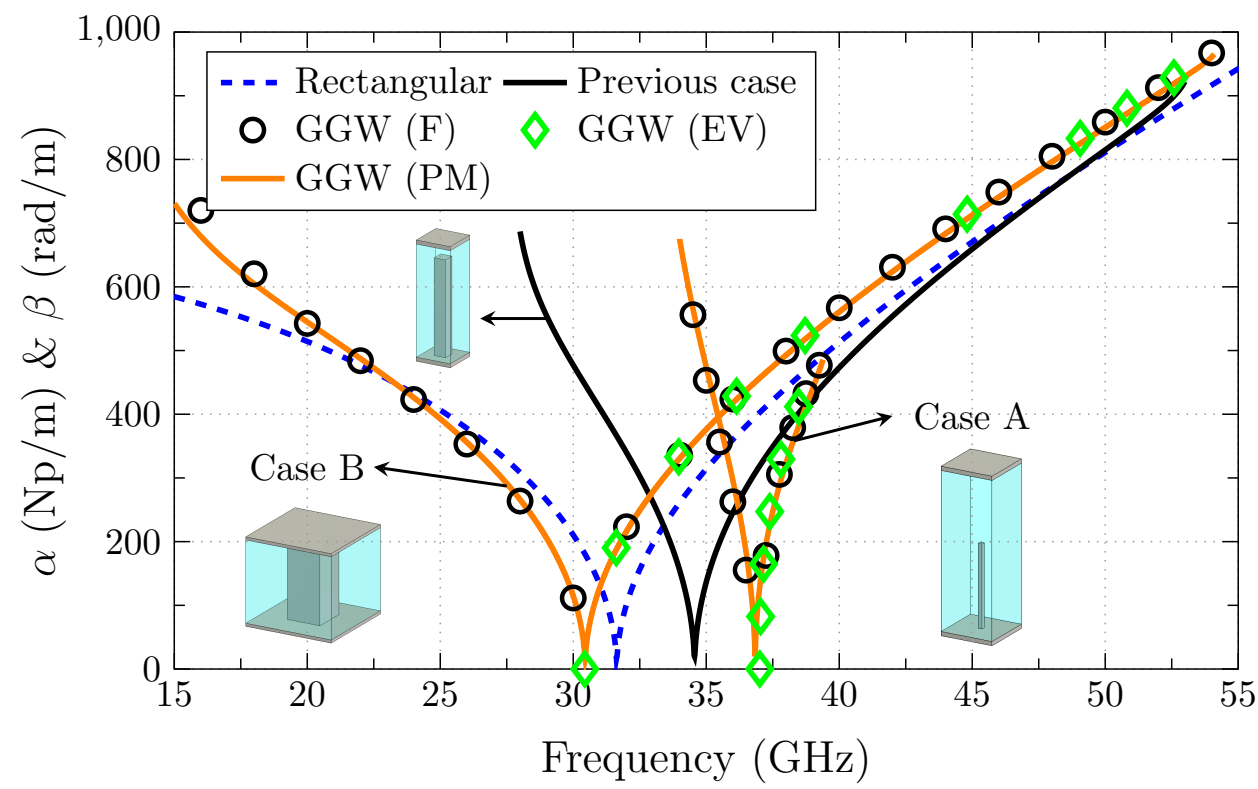

Figure 10.10: Propagation and attenuation constants of a $G G W$ with $a=4.72 \mathrm{~mm}$ for two different cases $(A$ and $B)$ of the pin structure, comparing several calculation methods (field -F-, eigenvalue $-E V$-, proposed method -PM-). Case $A: w=0.1 \mathrm{~mm}, p=1.3 \mathrm{~mm}, h_{p}=2 \mathrm{~mm}$, and $h_{a}=1.8 \mathrm{~mm}$. Case B: $w=1.15 \mathrm{~mm}, p=3 \mathrm{~mm}, h_{p}=2.6 \mathrm{~mm}$, and $h_{a}=0.1 \mathrm{~mm}$. Rectangular waveguide curves and the reference GGW curves have been added for comparison. Also, images of the unit cells of the different geometries are included. GGW curves are only depicted inside the corresponding stopband for each case.

Contrarily, the case B implies a wide stopband ([15 GHz-54.4 GHz], $R B W=$ $88.1 \%)$. Therefore, the dispersive effects introduced by the pins at the sides of the waveguiding channel are alleviated, and the GGW has dispersion characteristics close to those of the rectangular waveguide. However, this geometry infers an inductive behavior on $Z_{l n}$, which causes the GGW to be larger than the rectangular waveguide in terms of effective area, i. e., $l$ is positive for most of the stopband (notice how the curve of case B intersects the rectangular waveguide curve far below cutoff. As a result, also in this case, the rectangular waveguide is not a good approximation, especially in the propagation region, whereas the proposed method keeps its good accuracy.

The presented method has shown good performance even for the previous extreme cases, indicating that the proposed model and procedure are not only fast and accurate, but also robust. Thus, it can be used for pin structure optimiza- 


\begin{tabular}{lcc} 
& Computation time & Recalculation for different $a$ \\
\hline Field (full structure) & $1380 \mathrm{~s}$ & YES \\
Eigenvalue (only $\beta$ ) & $4230 \mathrm{~s}$ & YES \\
Proposed Method & $24 \mathrm{~s}$ & NO \\
\hline
\end{tabular}

Table 10.3: Comparison of CPU times.

tion purposes in a dispersion synthesis task, considering a wide range of geometries. Furthermore, in this section, as part of the validation of the proposed model, extreme geometries for the GGW has been considered for the first time, revealing undiscovered dispersion engineering possibilities of this waveguide. In this sense, an interesting future work line is foreseen which, furthermore, may strongly benefit from the efficiency of the model presented here.

\subsubsection{Computation efficiency evaluation}

To end this section, a computation efficiency evaluation is carried out to give quantification of the advances of the method front conventional full-wave simulations provided by general purpose commercial solvers. The used computing machine incorporates an Intel Xenon CPU E3-1245 @ $3.40 \mathrm{GHz}$ and 16 GB of RAM memory. Results shown in Table 10.3 correspond to the computation time given by CST ${ }^{\circledR}$ for each case. The efficiency of the proposed method with regard to the other cases is clear. The simulation of a whole section of the GGW structure to later analyze the fields inside results in a heavy task because of the structure size. In addition, a thoughful post-processing is required, especially in the calculations involving the field at different frequencies (this additional effort has not been included in the comparison table).

On the other hand, the solution of an eigenvalue problem is cumbersome by nature [916]. Note that, in order to recover the requested data properly, several phase shifts and additional modes must be computed. Moreover, there is not possibility of solving the structure below cutoff with an eigenvalue simulation. Actually, the computation time reflected in the table corresponds exclusively to the calculation of the propagation constant.

Finally, the second column of Table 10.3 highlights a useful feature of this method front conventional analysis techniques. Note that, since the lateral impedance is independent of $a$, the same calculated values can be applied for different waveguide widths. This means that, for instance, the cost of obtaining the dispersion data of all the plots of Fig. 10.9 is the same as the one requested for a single plot. Contrarily, either in the eigenvalue problem or the whole GGW section analysis, a new simulation must be carried every time that $a$ changes, as the analyzed structure changes. This feature results specially interesting for the design of more advanced components, e. g., filters, where GGWs of different widths are involved, since the lateral impedance data can be incorporated into a commercial solver in order to carry out the design using equivalent rectangular waveguide geometries. The computation effort will be noticeably reduced by such procedure, opening the door to the design of more complex GGW structures, today difficult to optimize due to the prohibitive computational times. 


\subsubsection{Experimental validation}

So far the proposed model has been validated by means of full-wave simulations. In this section, experimental validation of the results will be presented. To do so, two TRL [791] calibration kits corresponding to the GGW widths $a=4.08 \mathrm{~mm}$ and $a=5.36 \mathrm{~mm}$ have been manufactured through an in-house process using a DATRON M25 milling system. Figs. 10.11(a) and 10.11(b) show the bottom and top pieces, respectively, for one of the two fabricated calibration kits. A perspective view of the bottom piece, where the metal pins can be clearly appreciated, is shown in Fig. 10.11(c). The use of the two different considered widths allows to further check that the presented method is properly obtaining the propagation constant for different values of the width $a$, and in both operating regions, below and above cutoff.

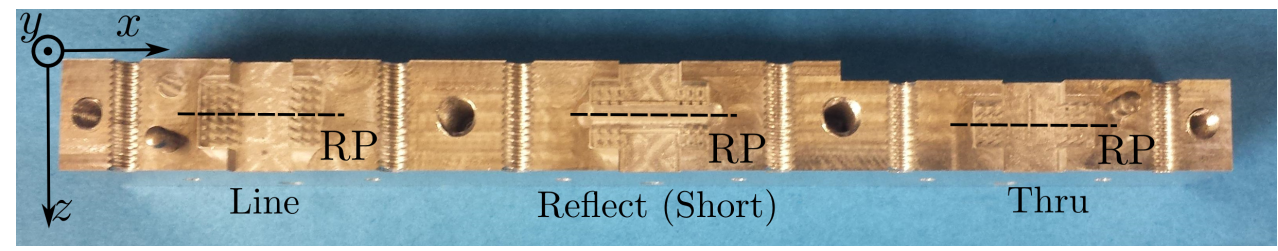

(a)

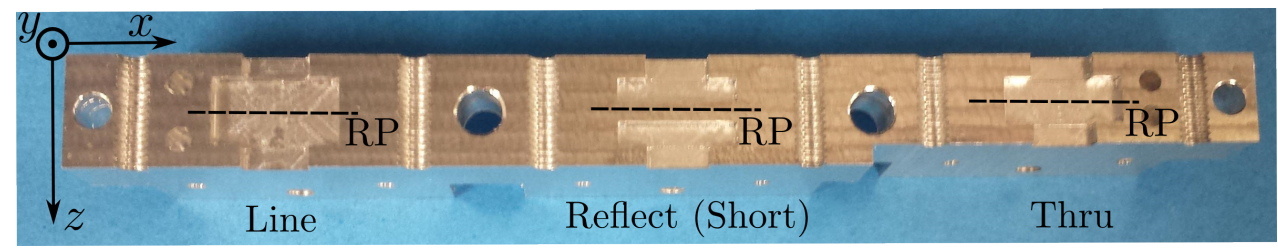

(b)

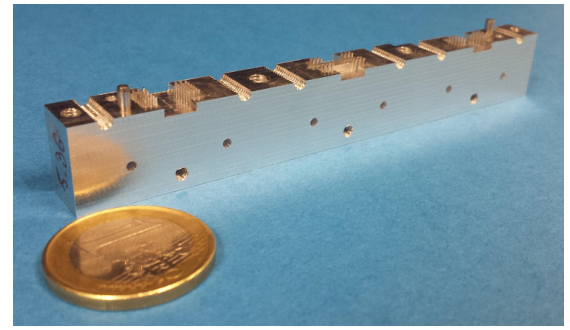

(c)

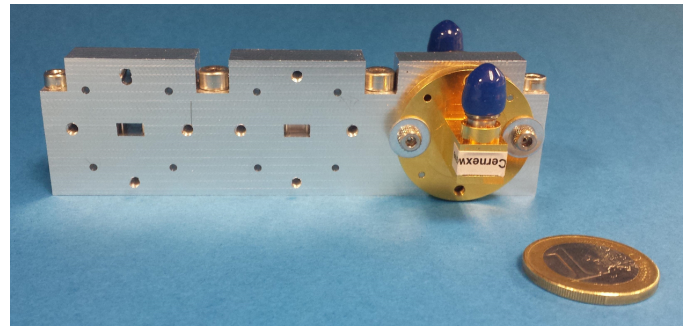

(d)

Figure 10.11: One of the two manufactured TRL calibration kits. a) Bottom piece containing the pins; b) Top piece; c) Perspective view of the bottom piece. d) Assembled complete piece including the feeding by means of a WR-22 rectangular to $2.44 \mathrm{~mm}$ coaxial transition.

The corresponding measurements have been performed using a Keysight PNA N5227A network analyzer. The measurement setup is shown in Fig. 10.12. Note that the TRL calibration algorithm due to itself procedure allows to recover the complex propagation constant $\gamma=\alpha+j \beta$ of each waveguide as a part of the calibration process (see [791] for details). Therefore, the TRL calibration kits allow to recover the propagation constant (or attenuation constant of if the waveguide 
operates below cutoff) for the cases $a=4.08 \mathrm{~mm}$ and $a=5.36 \mathrm{~mm}$. Comparisons between measured data and previous results (obtained with different analysis methods: eigenvalue problem, analysis of fields in a whole GGW section, and the proposed method) are shown in Figs. 10.13(a) and 10.13(b) for each case ( $a$ value). A good agreement is observed between the results obtained with the proposed method and the experimental curve. The slight frequency shift between both curves is justified by the precision of the in-house process, which is estimated to be about $\pm 10 \mu \mathrm{m}$ in the horizontal plane and $\pm 30 \mu \mathrm{m}$ in the vertical plane. Thus, the experimental results in this section confirms that the novel proposed model and procedure provides a very accurate modeling of the GGWs.

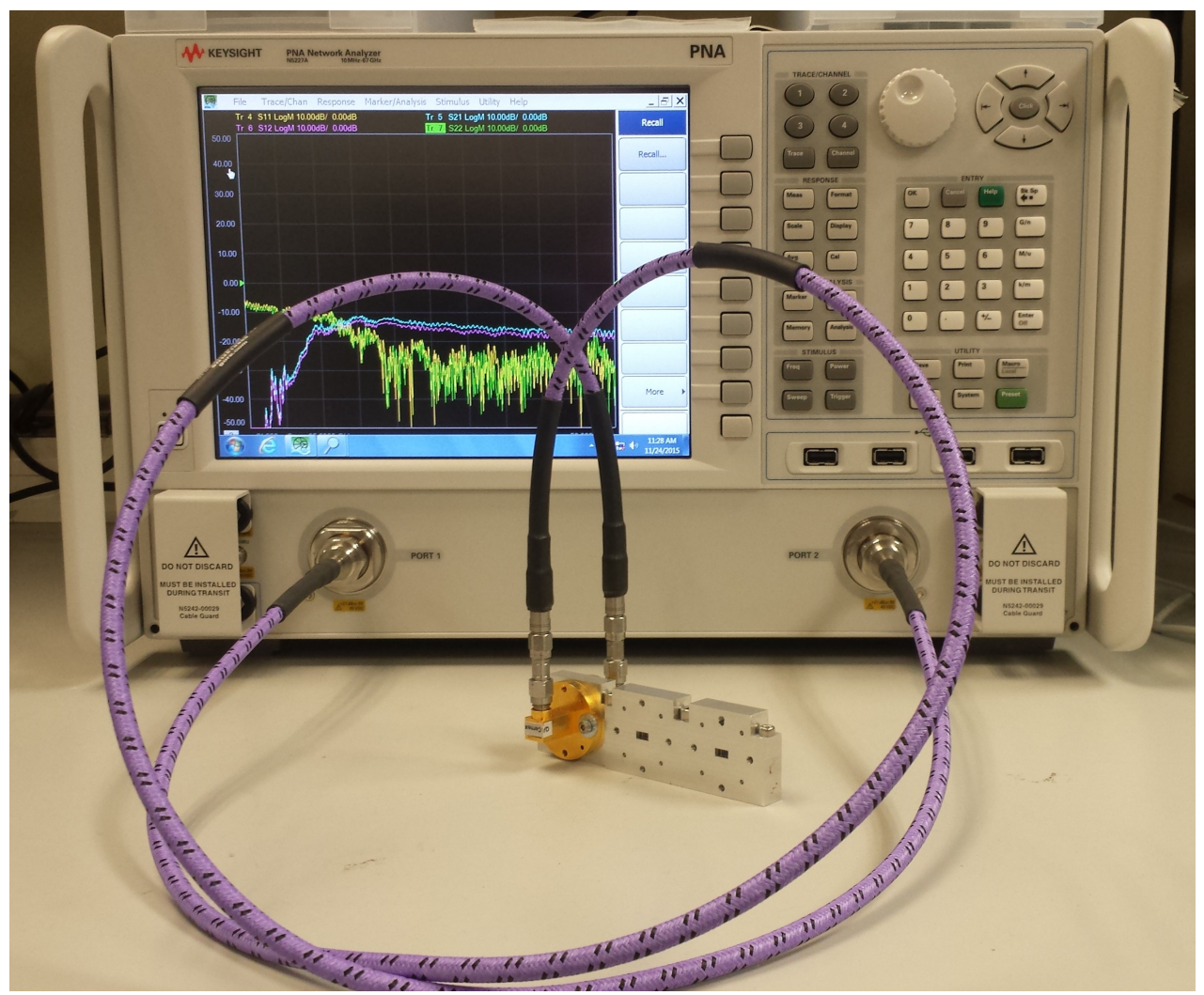

Figure 10.12: Measurement setup.

In this first analysis section of the chapter dedicated to the GGW it has been observed that the direct equivalent correspondence normally assumed between GGW and rectangular waveguide is a rough approximation providing accurate results only for some specific cases. The usual design procedure at the first stages of the design, generally followed in the literature must be rethought. Exploration of several width cases has revealed a frequency-dependent behavior of the GGW that has been missed up to now. It has been also proved that the GGW is able to operate below cutoff in a similar manner than the rectangular waveguide does. Nonetheless, it has been confirmed that, in this region, differences between both waveguides are accentuated. 


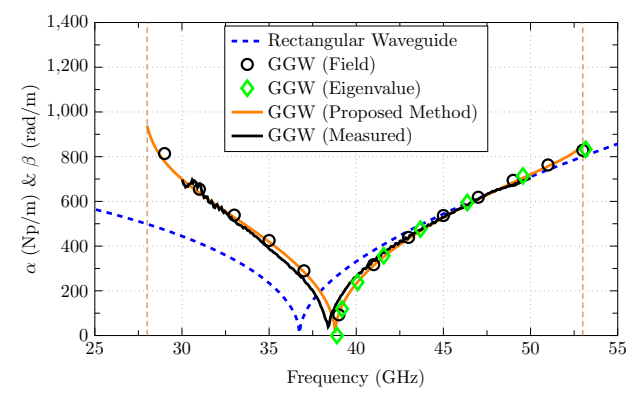

(a)

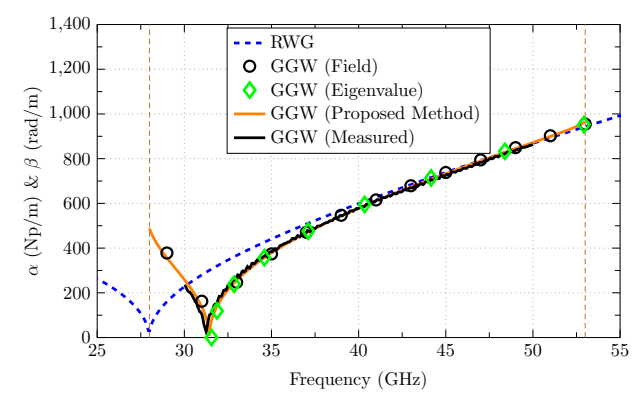

(b)

Figure 10.13: Comparison between simulated and measured results. a) $a=4.08 \mathrm{~mm}$; $b$ ) $a=5.36$ $\mathrm{mm}$.

Future work must explore the GGW possibilities in below cutoff operation, and carry out evanescent component design.

The proposed method focus the origin of the difference between both waveguides: the periodic structure. Through and independent analysis of the periodic structure, the global influence of it is translated to a one each plane at both sides of the propagation channel, hence giving an equivalence with the rectangular waveguide that allows to recover the propagation (attenuation below cutoff) constant of the GGW. Local effects of the field are not taken into account by the model, which can be improved if this information is incorporated. This is a interesting future work line that can drive to an even more powerful analysis tool.

As a part of the validation of the proposed model, the possibilities of the periodic structure geometry have been extensively explored, revealing GGW features hidden up to now. The large number of degrees of freedom that the periodic structure provides turns the GGW in a rectangular waveguide with incredible dispersion engineering possibilities. Without varying the propagation channel width $a$, a wide range of dispersion curves can be achieved just adjusting the periodic structure parameters. Inclusion of active elements may even allow for unconventional features (less dispersion than a rectangular waveguide). Therefore, the work in here opens the door to a new research area exploring all this GGW possibilities. Notice that, without an efficient analysis method such as that provided in this section, dispersion engineering with the GGW could be prohibitive costly in computational terms. Moreover, fixed the periodic structure parameters, optimization of the width can be carried out with the rectangular waveguide equivalent provided by the proposed model, considerably reducing the simulation times. Therefore, complex designs such large array antennas or high-order filters are to come with design procedures of no much more complexity than those required for rectangular waveguide topologies.

By all of the above the presented equivalent rectangular waveguide model for the GGW not only improves the design procedure, up to now cumbersome and timewasting, but provides a useful physical insight on the GGW characteristics. Several possibilities have been revealed for this waveguide, opening the door to several future work lines. The GGW must not be longer only regarded as an alternative to the 
rectangular waveguide to avoid metallic contact problems, but as a very versatile waveguide due to the large degrees of freedom in the design.

\subsection{Groove Gap Waveguide filters}

In section 8.1.5.3, the challenges involved in the design process of narrow-band filters were explained. Narrow-band filters are a critical (and expensive element) of any communication system. The rectangular waveguide has been the common choice as it provides the required low-loss feature to achieve high quality factor resonators, able to work with high power levels and flexible coupling possibilities. When the frequency increases undesired effects appear due to the bad metal contact effects (e.g., leakage and PIM), manufacturing precision, and mechanical stability of the required smaller pieces (as the wavelength is smaller). This leads to consider if any alternative to the, otherwise well-suited, rectangular waveguide is available. At this point, the GW technology enters from the hand of the GGW, which in the previous section has been shown to have essentially the same basic propagation characteristics as the rectangular waveguide (although in the previous section differences between both waveguides are highlighted, it is obvious that they present the same kind of basic features and it has been shown to be always possible to design a GGW with very similar propagation characteristics to certain rectangular waveguide), however provided by a different topology, which is exactly though to overcome the aforementioned problems.

In this section, the design of several GGW narrow-band filters and a performance comparison with the rectangular waveguide topology is carried out. Related with the previous arguments, the aim of the presented work is to show the potential of GGW structures front the rectangular waveguide in a reliable way, so that it can be stated with confidence and rightful reasons that, in some contexts, GGW topology is the best option. Narrow-band filter design is a very suitable scenario for this task. First, basics of narrow-band filter design theory are exposed in order to clarify the lately followed procedures. Then, three designs are carried out. First, a Ka-band $4^{\text {th }}$-order filter, centered at $f=40 \mathrm{GHz}$ is presented. Following the conclusions of this first design, a more complex filter, operating at the V-band, rather designchallenging according to the literature, is presented. The second filter is a $5^{\text {th }}$-order filter centered at $f=60 \mathrm{GHz}$. The third design is a $5^{\text {th }}$-order filter centered at $f=61$ $\mathrm{GHz}$ that incorporates tuning elements to overcome the main observed problems of the second design. Finally, a detailed comparison with the rectangular waveguide is carried out taking the second design as reference. The same filter is designed with the rectangular waveguide in H-plane, and both technologies are compared in several aspects of their performance. In this way, the suitability of GGW technology is not only proved from its good performance ranging from a first basic design at moderate frequencies to a more advanced high-frequency design with tuning capabilities, but the differences with the rectangular waveguide are quantified and main advantages of GGW are clearly explained and justified. 


\subsubsection{Narrow-band filter design basic theory and procedure}

According to the good tutorial in narrow-band filter design of Swanson [782], the term refers narrow-band to those filters with relative bandwidth $R B W$ below $10 \%$, although sometimes the concept can be lawfully extended up to $20 \%$. The designs in this part of the thesis are conceived to provide a bandpass of only $R B W=$ $2.5 \%$, hence the theory and procedures detailed next are well-suited for the posterior presented designs.

In 1951, Dishal published a reference work for the microwave filter community, [930], where it was established that any narrow-band bandpass filter, either based on lumped or distributed elements can be described by three fundamental variables: the synchronous tuning frequency of each resonator $f_{0}$, the coupling coefficient between adjacent resonators, $k_{i, i+1}$, and the singly loaded or external quality factor of the first and last resonators $Q_{e x}$. In that work, it was also demonstrated that filter hardware could be tuned or aligned using knowledge of these fundamental parameters, i.e., by proceeding with such parameters, physical insight is gained, and the filter response is more easily controlled by the design engineer.

\subsubsection{Chebyshev filters}

At the time of Dishal's work it was usual to use measurements ad hoc designed experimental hardware to generate design curves for each of the aformentioned parameters. Since the emergence of EM solvers, this procedure is done by simulation and, due today's computational performance, it is a very simple and fast task. Several good general reviews and tutorials can be found elsewhere in the literature [782], [931]-[932]. Here, the concepts and design flow procedure are related to the particular case of all-pole Chebyshev response filters [933]. This type of response is chosen for the posterior designs since it is the most selective among the basic ones (ladder network synthesis is always possible) and it is quite habitual in practical implementations with not excessively high-performance specifications demand. Transmission zeros should be included in the response otherwise, leading to more advanced designs.

Generally, a bandpass filter specification includes the desired center frequency $f_{0}$, the relative bandwidth $R B W$ (or alternatively the bandpass is explicitly specified), the maximum insertion loss in the pass band and several required rejection levels in the passband. It is very usual to find as well specification on the minimum return loss in the passband which is directly related to the insertion loss ripple. In the case of Chebyshev filters, the bandpass response is characterized by a constant, specified ripple. From a system level design point of view, at least $R L=15 \mathrm{~dB}$ is demanded to avoid mismatch ripple when components are cascaded. Once the passband ripple level is determined, the filter order $N$ can be estimated based on the desired stopband rejection. The well-known graphs and equations in the book of Matthaei and Jones [934] result useful for this task. Nevertheless, such graphs and equations assume ideal symmetrical Chebyshev response, which is not the case of most of real microwave filters, usually presenting some asymmetry. Therefore, the required order may be generally higher (sometimes lower [782]) than the originally estimated. Finally, once the order $N$ is determined, either by tables or equations, 
the normalized lowpass prototype values can be found. A good design table for a ripple $R=0.036 \mathrm{~dB}(R L=20.8 \mathrm{~dB})$ can be found in in [782]). The exact equations and procedure for the general case are listed next.

Given the ripple $R$ the following quantities are sequentially derived:

$$
\begin{aligned}
\varepsilon & =\frac{1}{\sqrt{10^{(R L / 10)}-1}} \\
s & =\sqrt{1+\varepsilon^{2}} \\
\beta & =\ln \left(\frac{s+1}{s-1}\right) \\
\gamma & =\sinh \left(\frac{\beta}{2 N}\right)
\end{aligned}
$$

Then, the filter coefficients $a_{k}$ and $b_{k}$ are determined as:

$$
\begin{array}{ll}
a_{k}=\sin \left(\frac{2 k-1}{2 N} \pi\right) & k=1, \ldots, N \\
b_{k}=\gamma^{2}+\sin ^{2}\left(\frac{k \pi}{N}\right) & k=1, \ldots, N
\end{array}
$$

so that the normalized element values of the ladder network configuration can be finally determined as:

$$
\begin{aligned}
g_{0} & =1 \\
g_{1} & =\frac{2 a_{1}}{\gamma} \\
g_{k} & =\frac{4 a_{k-1} a_{k}}{b_{k-1} g_{k-1}} \\
g_{N+1} & =\left\{\begin{array}{lll}
1 & N & \text { odd } \\
(\varepsilon+s)^{2} & N & \text { even }
\end{array}\right.
\end{aligned}
$$

The normalized elements $g_{i}$ indicate the required coupling coefficients between adjacent resonators and the external quality factors of the first and last resonators:

$$
\begin{aligned}
k_{i, i+1} & =\frac{R B W}{\sqrt{g_{i} \cdot g_{i+1}}} \quad i=1, \ldots,(N-1) \\
Q_{e x_{1}} & =\frac{g_{0} g_{1}}{R B W} \\
Q_{e x_{N}} & =\frac{g_{N} g_{N+1}}{R B W}
\end{aligned}
$$


The design procedure consists in adjusting the filter dimensions to achieve the previous values together with the resonance frequency of the resonators. Therefore, auxiliary simpler subcircuits are analyzed to determine the relationship between the physical geometric parameters and the main variables describing the filter: $\left(f_{r e s}, k_{i, i+1}, Q_{e x}\right)$. By this, a first design is obtained. Due to the approximated approach of the procedure it is usual that such design do not satisfy the specifications, but it is close enough to alleviate the optimization process.

\subsubsection{Resonance frequency}

The first step is to adjust the resonance frequency $f_{\text {res }}$ of the different resonators forming the filter so that all them synchronous resonate, in principle, at the central frequency of the passband. This procedure is illustrated in Fig. 10.14(a). Generally, every geometrical parameter is fixed except one which is parametrized to tune $f_{\text {res }}$ until it is set to $f_{0}$. Nevertheless, it is worth to mention that the other parameters can influence on the resonance sharpness, i.e., in the unloaded quality factor of the resonator $Q_{u}$. The quality factor is quite relevant being that the insertion losses are directly related to it by the following expression:

$$
I_{L}(\mathrm{~dB})=\frac{4.343 f_{0}}{\Delta f Q_{u}} \sum_{i=1}^{N} g_{i}=\frac{4.343}{R B W Q_{u}} \sum_{i=1}^{N} g_{i}
$$

Therefore, since insertion losses are inversely proportional to $R B W$ and $Q_{u}$, and they increase with the order of the filter, for high-order narrow-band filters, it is recommendable to look for low-loss structures, and given a chosen topology, try to maximize $Q_{u}$ by tuning the involved geometrical parameters.

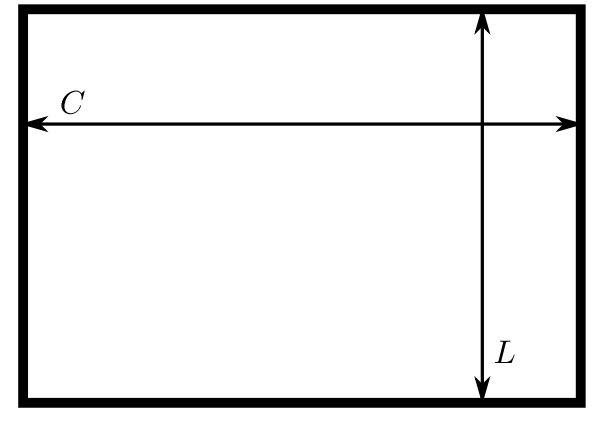

(a)

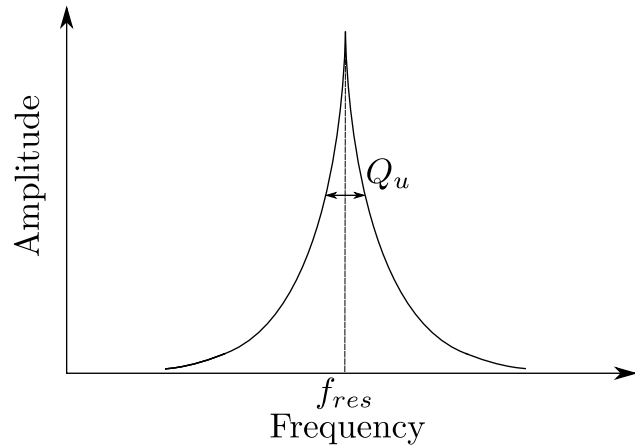

(b)

Figure 10.14: Design of the filter resonators. The resonator geometrical dimensions are adjusted to provide the desired resonance frequency $f_{r e s}$.

\subsubsection{Coupling coefficient}

The coupling between adjacent resonators is controlled by a particular coupling element which geometrical parameters are properly chosen. As before, for simplicity, 
it is usual to work with only one geometrical parameter. The upper part of Fig. 10.15 shows an schematic representing the coupling between two resonators, controlled by the parameter $w$. To calculate the coupling two alternative procedures are possible.

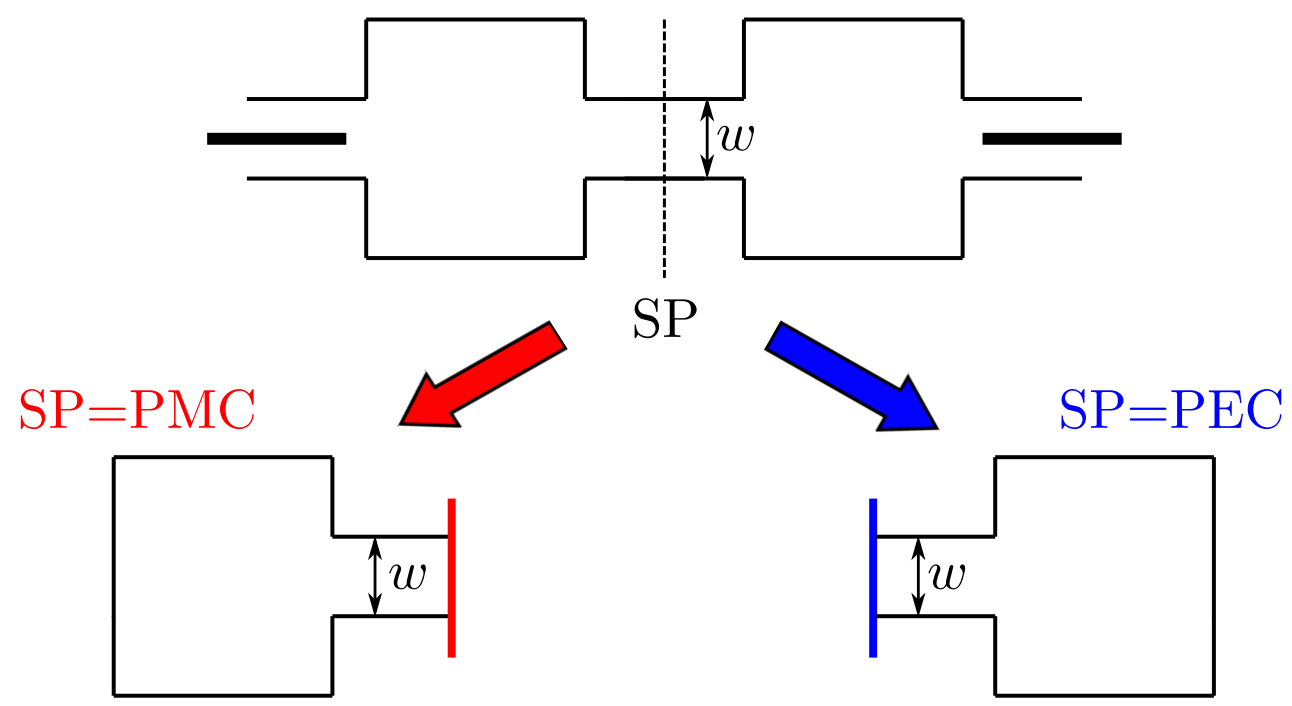

Figure 10.15: Design of the coupling elements.

The first possible procedure is illustrated in Fig. 10.15. It consists in considering the symmetry plane (SP) between the coupled resonators and study two cases: the symmetry plane as a PMC, and the symmetry plane as a PEC. For each case, the resonance frequency of an unloaded resonator including one halve of the coupling element terminated with the corresponding boundary (PEC or PMC). These resonance frequencies are named as $f_{m}$ and $f_{e}$ for the cases of PMC and PEC, respectively. The coupling coefficient is then calculated with the following expression [782]:

$$
k=\frac{f_{m}^{2}-f_{e}^{2}}{f_{m}^{2}+f_{e}^{2}}
$$

Alternatively, a second way to obtain the coupling coefficient by analyzing the transmission response of a two port network that includes the two coupled resonators, Fig. 10.16(a). It is important to weakly feed the structure at both ports to guarantee loose coupling between them. The $S_{21}$ (alternatively $S_{12}$ by reciprocity) of the structure presents two peaks and a valley between them. A good practice is to provide a feeding weak enough so that the valley should be below $-30 \mathrm{~dB}$ [782]. Once the two resonance frequencies $f_{1}$ and $f_{2}$ are found, the coupling coefficient is calculated with the following expression:

$$
k=\frac{f_{2}^{2}-f_{1}^{2}}{f_{2}^{2}+f_{1}^{2}}
$$

In fact, it should be satisfied that $f_{1}=f_{e}$ and $f_{2}=f_{m}$. The use of one of other procedure depends on the structure analysis features. Originally, when the design 


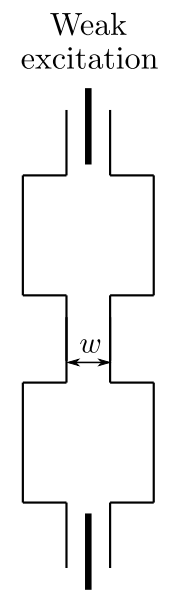

(a)

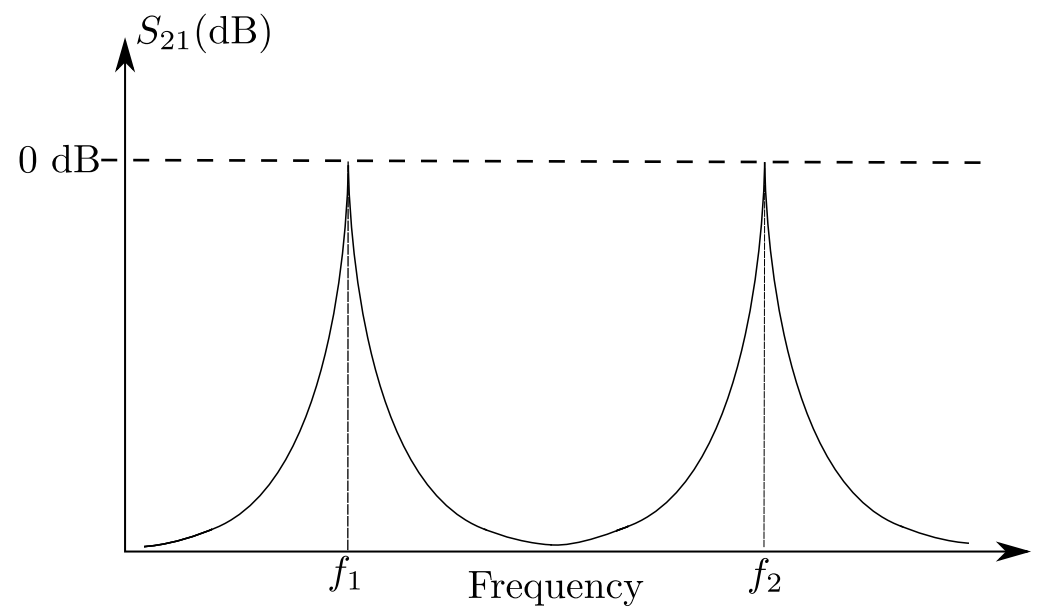

(b)

Figure 10.16: Design of the coupling elements (alternative).

curves were obtained experimentally, the first procedure sometimes presented drawbacks due to the difficulty of experimentally implement PMC and PEC conditions. With the current EM solvers, the main difference between the two techniques is that the first one demands from two independent eigenvalue simulations, whereas the second one requires a conventional simulation to obtain the S-parameters of a structure that is double larger than those of the eigenvalue simulation and includes input and output feeding. In general, both options are implementable and quite fast solved.

\subsubsection{External quality factor}

The external quality factor $Q_{e x}$ measures how the external feeding perturbs the ideal operation of an isolated resonator, Fig. 10.17(a). The dimensions and the geometrical location of the feeding structure determines the strength of the perturbation. As before, several parameters influence $Q_{e x}$, being recommendable to fix all of them except one to be tuned. When one resonator is connected to an external excitation source, the $S_{11}$ of such single-port structure is:

$$
S_{11}=\frac{1-j Q_{e x}\left(2 \Delta f / f_{0}\right)}{1-j Q_{e x}\left(2 \Delta f / f_{0}\right)}
$$

where $\Delta f$ is the frequency displacement caused by loading the resonator. Since losses are not considered, it is always satisfied that $\left|S_{11}\right|=1$. However, according to (10.28), it is found that the $S_{11}$ phase changes depending on the frequency displacement $\Delta f$, see Fig. $10.17(\mathrm{~b})$. Note now that if $\Delta f= \pm f_{0} / 2 Q_{e x}$ in (10.28), the $S_{11}$ phase is equal to 90 degrees. This implies that

$$
Q_{e x}=\frac{f_{0}}{\Delta f_{ \pm 90^{\circ}}}
$$




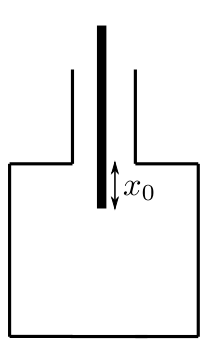

(a)

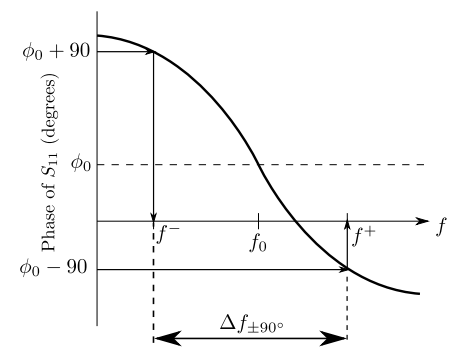

(b)

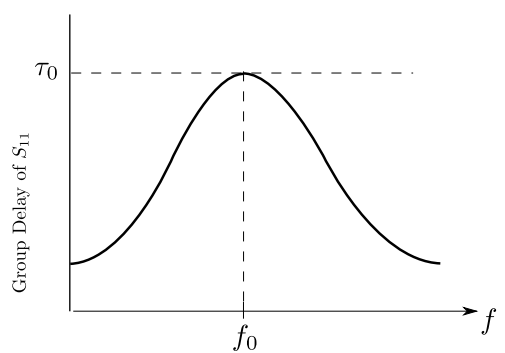

(c)

Figure 10.17: Design of the external coupling.

Therefore, as Fig. 10.17(b) illustrates, the procedure consists in simulating the loaded resonator and look for the $S_{11}$ phase at the central frequency $f_{0}$. This phase is not necessarily zero, but certain value $\phi_{0}$. Then, they are searched the frequencies $f^{-}$and $f^{+}$at which the phase of the $S_{11}$ is $\phi_{0}+90^{\circ}$ and $\phi_{0}-90^{\circ}$, respectively. Finally, $\Delta f_{ \pm 90^{\circ}}=f^{+}-f^{-}$and (10.29) can be applied.

Alternatively, $Q_{\text {ext }}$ can be extracted from the $S_{11}$ group delay at the resonance frequency $f_{0}$. Note that the group delay is defined as:

$$
\tau_{S_{11}}=-\frac{1}{2 \pi} \frac{\partial \phi_{S_{11}}}{\partial f}
$$

where $\phi_{S_{11}}$ is the $S_{11}$ phase. Notice that, according to $(10.28), \phi_{S_{11}}$ of a loaded resonator is:

$$
\phi_{S_{11}}=-2 \tan ^{-1}\left(\frac{2 Q_{e} \Delta(f)}{f_{0}}\right)
$$

expression, that applied to (10.30) gives

$$
\tau_{S_{11}}(f)=\frac{2 Q_{e x}}{\pi f_{0}} \frac{1}{1+\left(2 Q_{e x} \Delta f / f_{0}\right)^{2}}
$$

By inspection of the previous expression it is evident that the maximum group delay is found when $\Delta f=0$, i.e, at the resonance frequency $\left(f=f_{0}\right)$. Therefore,

$$
\tau_{S_{11}}\left(f_{0}\right)=\frac{2 Q_{e x}}{\pi f_{0}}
$$

hence

$$
Q_{e x}=\frac{\pi f \tau}{2}
$$

Fig. $10.17\left(\right.$ c) illustrates the typical $S_{11}$ group delay response where it must be found the value $\tau_{0}=\tau\left(f_{0}\right)$ to calculate $Q_{e x}$. Although both alternatives imply the same analysis, and, usually, the required data for both of them are provided as a result of the same simulation, the last one using the group delay is preferred for practical reasons. The post-processing of the group delay data is easier and more 
automatic than that of the phase data. For instance, it is not necessary to deal with $\pm 180^{\circ}$ phase jumps, and the location the peak of the curve is usually precise and simple. It is noted that as the perturbation of the cavity is varied, both, the peak level $\tau_{0}$ and the peak location $f_{0}$, change. This implies that the cavity dimensions must be modified to satisfy $f_{0}=f_{\text {res }}$ for every considered case of the perturbation in the derivation of the $Q_{e x}$ design chart.

\subsubsection{Optimization process}

The described procedure gives a design that is close but most probably will not satisfy the specifications. Further optimization is generally required from the obtained starting point. Optimization is a cumbersome topic, with large amount of research already carried out and a lot more to come since there is not a general procedure suitable for all kind of problems. For instance, the famous "no free lunch" theorem [935] establishes that any two different algorithms perform equivalent when their performance is averaged across all possible problems. In fact, for most cases, absolute guidelines cannot be assumed even when the type of problem is quite definite. In general, know-how of the problem and a good grade of algorithm tailoring is required. Basic, general development of the topic can be found in [528], [936]-[937]. Here, only few, brief observations are pointed out.

Electromagnetic problems are in general highly non-linear and demanding from global optimization due to the large number of local minima [938]. This is specially true in filter design for filters of certain order due to the large number of parameters, i. e., optimization space dimension [939]-[940]. Since principles of convexity, differentiability and accuracy (how accurately is the error function calculated) are clearly violated in electromagnetic global optimization, it is generally not adequate to use classical local optimization algorithms such as conjugate gradient variants [941]-[942], or quasi-Newton variants [943]-[944], except for refining the solution (if it makes sense according to the manufacturing precision). Nevertheless, it must be mentioned that the Nelder-Mead simplex method [945], which is a local optimization algorithm works quite well in the described context. While the gradient-based or quasi-Newton based methods pursue obstinately the best direction, rushing to the optimum, contrarily, the simplex method avoids the worst direction. Thanks to this strategy the simplex algorithm becomes quite robust front ill-posed global optimization problems at the cost of convergence velocity, being this last a useless feature if convergence to the right solution is very difficult to ensure.

On the other hand, a huge number of global optimization algorithms have been considered in the recent years [946]-[947], mainly inspired in biological, physical and geographical processes. In electromagnetic synthesis problems and, in filter design in particular, Genetic Algorithms (GAs) [948]-[950], and Particle Swarm Optimization (PSO) [951]-[953] have been reported as interesting optimization strategies. A good point of these techniques is that they present apprecible robustness and can be tailored with a good number of parameters, becoming adjusted to the problem to solve. However, despite of the fact that GA optimization has attracted lot of attention, it must highlighted that it has discrete nature, what demands of adequate coding schemes and does not always guarantee good improvement from points close to the minimum in the continuum space. In this sense PSO seems to be more ade- 
quate. A good comparison between both methods can be found in [954]. From these techniques it is highlighted that convergence velocity can be extremely slow and poor solutions are obtained unless the algorithm is well fitted to the problem. This implies that the user must know quite well the mechanisms underlying the algorithm to properly program it.

Finally, hybrid techniques using surrogate models such as the well-known Space Mapping (SM) methods [850], [955]-[956], or the use rational models [852], must be mentioned apart. More in general, Neural Networks (NN) [957]-[959] belong to this category of strategies. These techniques exploit a priori knowledge of the problem and take advantage of simplified models (analytical in simple problems or empirical in more complex ones) and how they relate to precise full-wave simulation (space mapping). As a result, accurate optimization can be carried out faster by using any of the previously mentioned optimization techniques in the optimization space of the simplified model, where the evaluation of the error function is quick.

From the experience in this part of the thesis, the Nelder-Mead simplex method has been found very useful in practice, providing robustness, ease of use and good precision. In addition, when encountering convergence problems, hybridization with simple PSO provides good improvement. It is found as well that using surrogate models is a powerful technique that gives good control of the optimization besides physical insight. In general, it can be said that success is guaranteed by the knowledge of the problem and the capability of tailoring the optimization techniques for it. The use of surrogate models naturally follows this premise, whereas for the underlying optimization algorithm (which must be properly chosen), the engineer is quite responsible of the algorithm performance. Finally it is recalled that good starting point(s) facilitates noticeably the convergence and may even become indispensable to achieve it in some cases.

\subsubsection{GGW all-pole filter topology}

In the previous section, the fundamental variables of a bandpass filter (synchronous tuning frequency of each resonator $f_{\text {res }}$, coupling between adjacent resonators $k_{i}$, and external quality factor of the first and last resonators $Q_{e x}$ ) have been introduced and related with the Chebyshev filter specifications. In addition, a procedure to relate these variables with the actual filter geometry has been detailed. In this section, the real GGW topologies implementing the functionalities described by such variables are presented. These topologies conform the later presented GGW filters.

\subsubsection{Resonating element: GGW cavities}

The resonators are implemented by GGW cavities, Fig. 10.18. These cavities are created in a similar way than in the rectangular waveguide case, however, the resonator boundaries are delimited by pins instead of metallic walls. The EBG effect provided by the periodic structure avoids the waves to escape so that resonances are created inside the structure. As it has been explained in section 10.1, three row of pins are generally enough (see Fig. 10.18). However, since the number of rows increases the total device size, sometimes only two rows may be considered as long as the leakage does not affect the response in a notably way. The two geometrical 
parameters $C$ and $L$ defining the cavity are adjusted by moving the corresponding blocks of pins at the sides. Beyond the differences between GGW and rectangular waveguide, the properties and the way to proceed in the design this kind of cavities is the same for both waveguides.

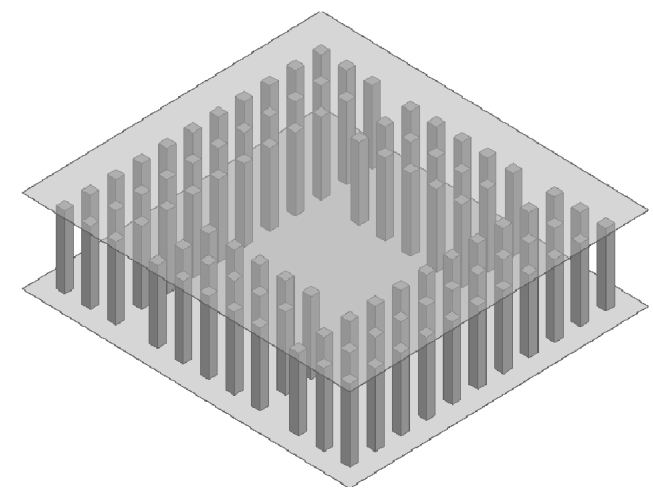

(a)

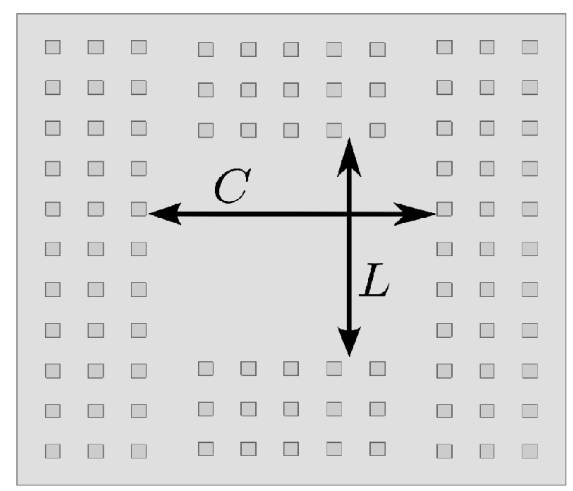

(b)

Figure 10.18: Resonating element: GGW cavities. a) $3 D$ view; b) $2 D$ view.

\subsubsection{Coupling element: GGW inductive windows}

The chosen coupling element is the GGW inductive window of Fig. 10.19. As commented in the GW technology review, there are several alternative topologies [835], [836]. However, that shown in Fig. 10.19 has been preferred to keep similarity with rectangular waveguide designs. As in the case of the resonators, in the GGW, the inductive window is implemented with pins instead of metallic walls, but the geometrical shape is very similar. The parameter to adjust the coupling is the window width $w$, that can be varied by moving the pin blocks defining it.

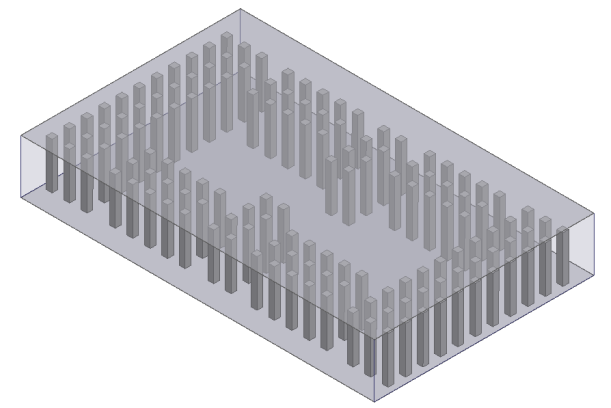

(a)

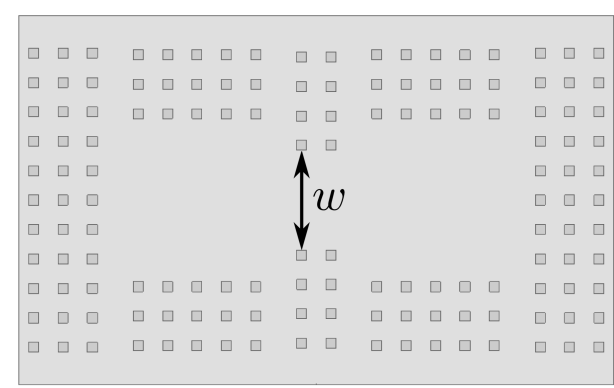

(b)

Figure 10.19: Coupling element: GGW inductive windows. a) $3 D$ view; b) $2 D$ view. 


\subsubsection{Feeding element: Cavity inserted coaxial cable}

The feeding to the structure is provided by means of a coaxial cable inserted in the cavity, either by the top or by the bottom, however, the posterior sections show that this last option is more suitable for practical implementations. In Figs. 10.20 (a) and 10.20 (b) it is shown a GGW cavity fed by a coaxial cable from the bottom plate. To adjust the grade of perturbation the location of the feeding can be adjusted by choosing its position $\left(x_{0}, y_{0}\right)$ relative to the cavity center, $C_{C_{1}}$ in the schematic of Fig. 10.20(b). Although this adjustment can be done with only one parameter, to consider the second one is a suitable option to control the grade of variation in the tuning process. Fig. 10.20(c) shows a detail of the coaxial connector. The employed connector consist on a coaxial cable with internal diameter $d_{c o n}$ and external diameter $d_{\text {teflon }}$. The inner conductor of the coaxial cable is terminated with a protrusive metallic pin of diameter $d_{p i n}$. The length of this pin is $T$. As an additional parameter, it is possible to consider the extra height of the coaxial insertion $z_{0}$, see Fig. 10.20 (c), so that the perturbation can be increased if that produced by the protrusive metallic pin is not enough.

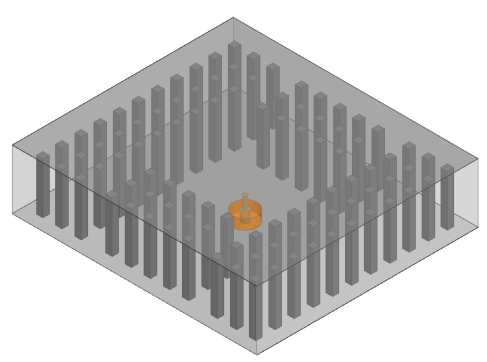

(a)

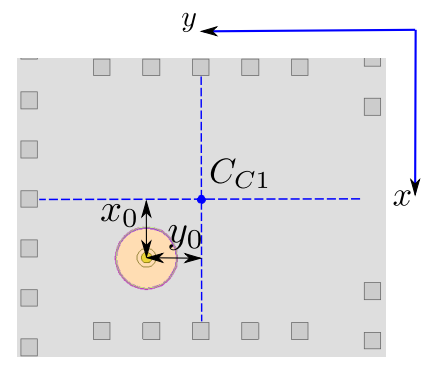

(b)

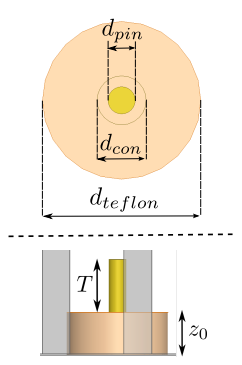

(c)

Figure 10.20: Feeding element: Cavity inserted coaxial cable. a) $3 D$ view; b) $2 D$ top view; c) Coaxial cable detail. 


\subsubsection{Design 1: $4^{\text {th }}$-order Ka-band filter at $f=40 \mathrm{GHz}$}

The first design consists in a $4^{\text {th }}$-order Chebyshev filter operating in the Ka-band, specifically, at $f=40 \mathrm{GHz}$. In this way, accordingly to the review of mm-wave applications, the proposal is in the context of some future mobile applications and, predominantly, of new-generation satellite communications. The work described in this section is mainly contained in [840]. At the time of that publication, design of GGW components in the Ka-band was pioneer and it could be said that this design was a first contact with a work line that continues with the designs 2 and 3 in this chapter, in which further advances, consequence of the conclusions in this first design, are applied.

First, the GGW to be the topological basis of the filter is designed. By applying the geometrical parameters listed in Table 10.4, the dispersion diagram of Fig. 10.21 is obtained. In such plot, it can be appreciated a stopband between $26.4 \mathrm{GHz}$ and $53 \mathrm{GHz}$, delimited by the unwanted modes of the parallel-plate waveguide formed by the upper and bottom plates. The propagation constant of the GGW fundamental mode is plotted in solid dark yellow, exhibiting the usual $\mathrm{TE}_{10}$ mode behavior in rectangular waveguides. The propagation constant of a plane wave is plotted in blue for reference purposes.

\begin{tabular}{cccccc} 
Parameter & $p$ & $w_{p}$ & $h_{p}$ & $h_{a}$ & $a$ \\
\hline Value $(\mathrm{mm})$ & 0.9 & 0.3 & 2.4 & 0.375 & 4.7 \\
\hline Table 10.4: & $G G W$ & geometrical parameters.
\end{tabular}

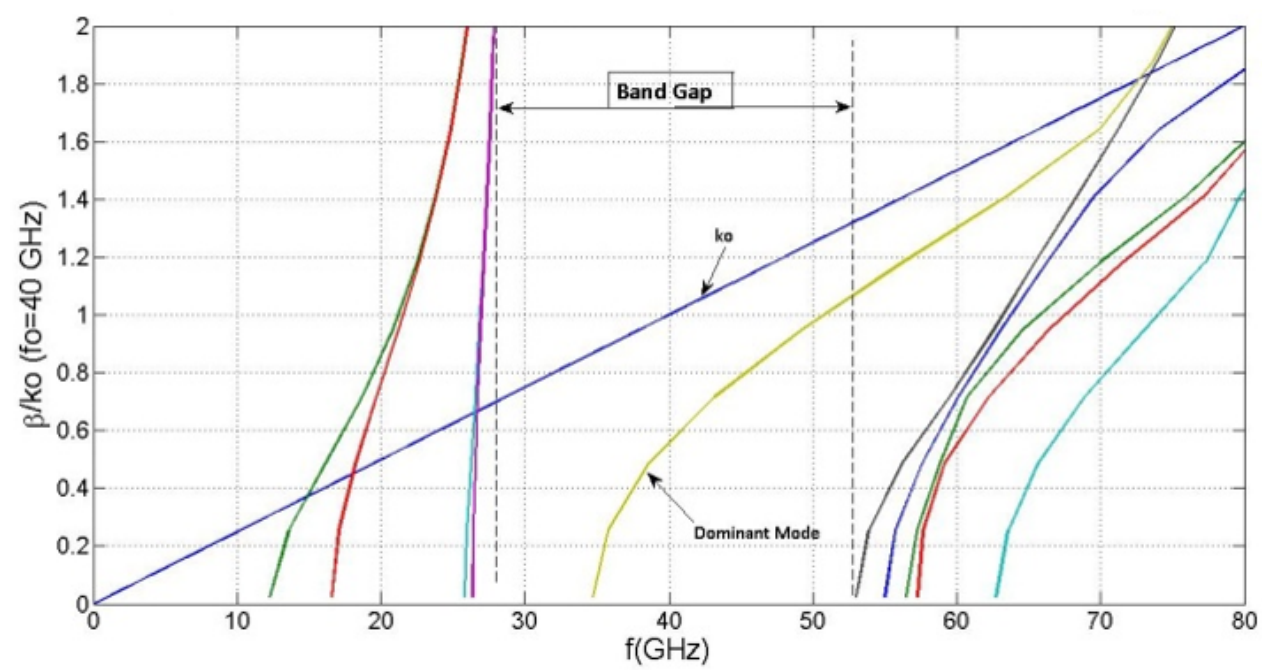

Figure 10.21: Dispersion diagram of the designed GGW. The stopband is delimited by the unwanted parallel-plate cavity modes of the structure. The propagation constant of the dominant mode is shown in dark yellow and the propagation constant of a plane wave, $k_{0}$, is shown in blue as reference.

Fig. 10.22(a) shows the E-field lines (orientation and magnitude) on a a plane transversal to the propagation. The usual $\mathrm{TE}_{10}$ sinusoidal form of a rectangular 
waveguide is obtained between the pins delimiting the propagation channel, with a slight presence of local effects over the first row of pins, which are not influencing the GGW behavior for the filter design purposes. Similarly, a plot of the electric current on the top plate is displayed in Fig. 10.22(b). Note the particular feature of the current, which, inherently to the design, does not exhibit any flow between top and bottom pieces. Analysis of the fields inside the structure reveals that the field is transversely attenuated $28 \mathrm{~dB}$ after the second row of pins and $52 \mathrm{~dB}$ after the third row of pins. These values, together with previous considerations in GGW filter design (the work [835] in $\mathrm{Ku}$-band) lead to a first approach with a periodic structure truncated after the second row of pins.

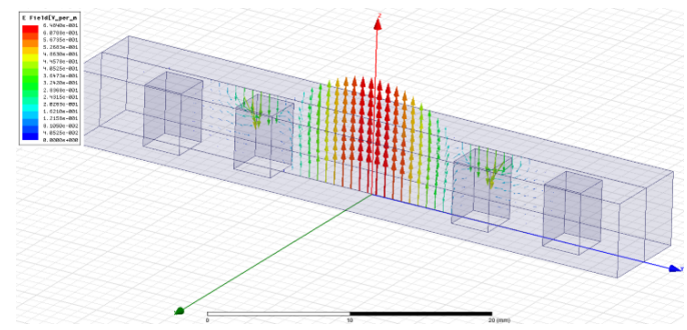

(a)

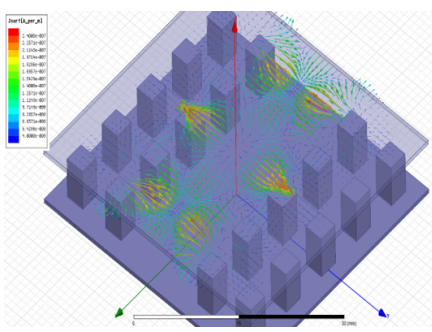

(b)

Figure 10.22: E-field and electric currents in the designed GGW. a) E-field on the plane transversal to propagation; b) Electric currents on the top metal plate.

\subsubsection{Filter specifications}

The filter specifications on the response are shown in Table 10.5. As it can be observed, since the response is narrow-band $(R B W=2.5 \%)$ the bandwidth extending around the central frequency does not represent any challenge regarding the stopband coverage, usually presenting a much wider $R B W$. A ripple of $R=0.1 \mathrm{~dB}$ has been chosen, which translates in return losses of $R L>16.42 \mathrm{~dB}$. A moderate good return loss figure for lower frequencies that may result more challenging to achieve in the Ka-band. The ideal response with such specifications is shown in Fig. 10.23. By following the procedure described in section 10.3.1 the normalized elements of the low pass prototype are obtained:

\begin{tabular}{cccc} 
Parameter & Frequency $f_{0}$ & Relative Bandwidth $(\mathrm{RBW})$ & BP Ripple $R(R L)$ \\
\hline Value & $40 \mathrm{GHz}$ & {$[39.5 \mathrm{GHz}-40.5 \mathrm{GHz}](2.5 \%)$} & $0.1 \mathrm{~dB}(16.42 \mathrm{~dB})$ \\
\hline
\end{tabular}

Table 10.5: Filter response specifications. 


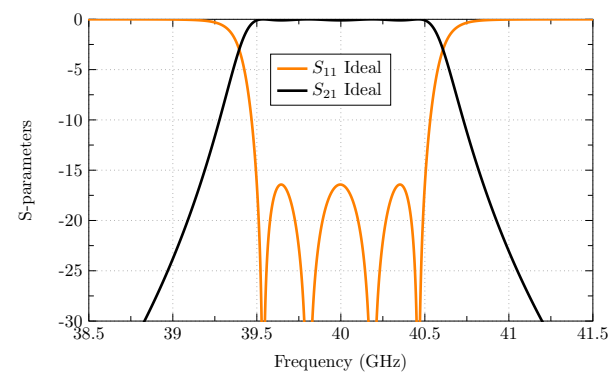

(a)

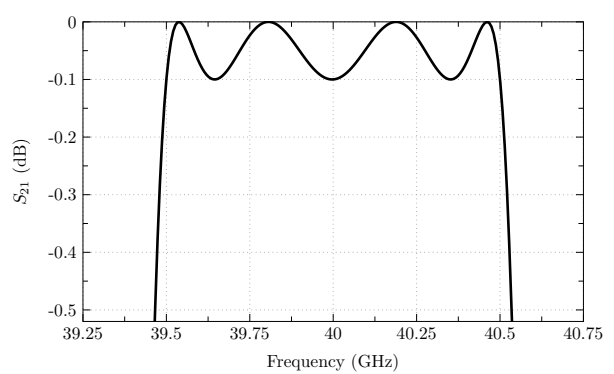

(b)

Figure 10.23: Ideal response. a) S-parameters; b) $S_{21}$ (dB) detail.

$$
\begin{aligned}
& g_{0}=1 \\
& g_{1}=1.109 \\
& g_{2}=1.306 \\
& g_{3}=1.770 \\
& g_{4}=0.818 \\
& g_{5}=1.355
\end{aligned}
$$

These values imply through (10.21)-(10.23) that the required coupling coefficients and external quality factors are:

$$
\begin{aligned}
Q_{e x_{1}} & =44.35 \\
k_{12} & =0.021 \\
k_{23} & =0.016 \\
k_{34} & =0.021 \\
Q_{e x_{N}} & =44.35
\end{aligned}
$$

Note that $Q_{e x_{1}}=Q_{e x_{N}}$ and $k_{12}=k_{34}$, i. e., the filter is symmetric, due to the imposed filter characteristics and specifications.

\subsubsection{Filter design}

\section{Resonant cavity}

To implement the resonators, the GGW cavity described in Fig. 10.18 is considered. In order to synthesize the cavity, the dimension in $\hat{x}$ is firstly fixed to $L=4.7 \mathrm{~mm}$, which is the width assigned to the GGW analyzed in Figs. 10.21 and 10.22. Then, the cavity length is tuned until the resonance frequency is $f=40 \mathrm{GHz}$. This is provided by $C=6.1 \mathrm{~mm}$.

\section{Coupling elements}

The coupling elements are the inductive windows described in Fig. 10.19, but implemented with only two rows, see Fig. 10.24(a). The procedure of section 10.3.1.3 
is used to obtain the coupling factor as a function of the window size $w$. Results are shown in Fig. 10.24(b). As usual, wider windows allow for larger coupling factors. The required coupling factors are easily obtained inside the considered range of widths. Specifically, the required windows are $w_{12}=w_{34}=3.034 \mathrm{~mm}$ and $w_{23}=2.919 \mathrm{~mm}$.

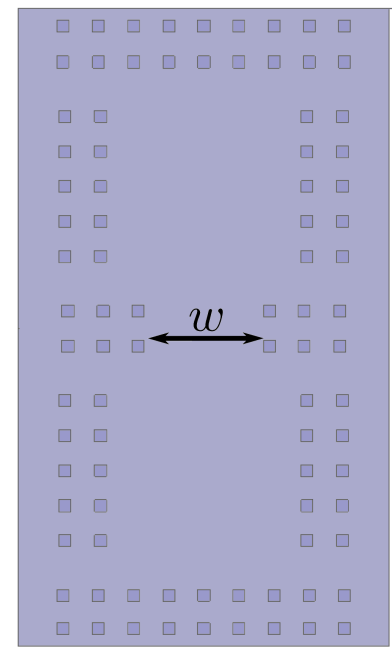

(a)

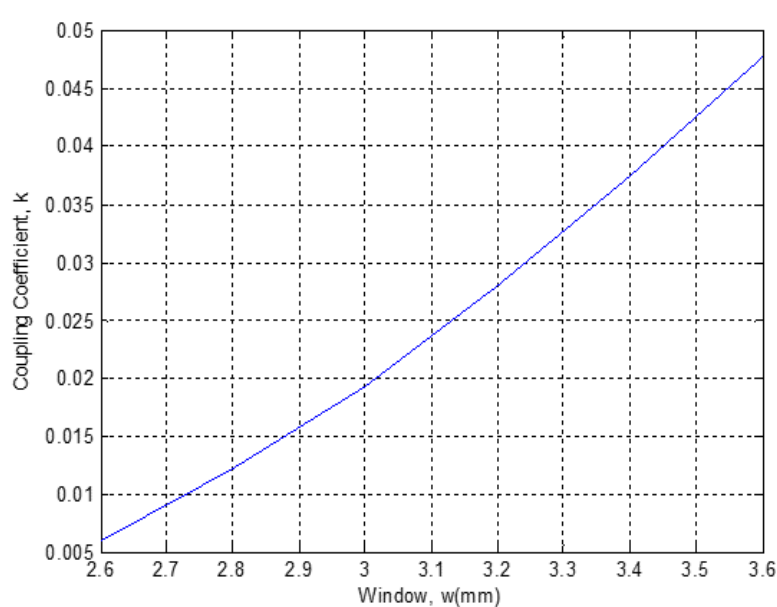

(b)

Figure 10.24: Coupling factor $k$ synthesis. a) Coupling structure; b) Coupling factor $k$ vs. window size $w$.

\section{Feeding elements}

A $2.4 \mathrm{~mm}$ coaxial connector (SouthWest Microwave1412-02SF) is selected as input/output filter excitation. The inserted pin of this connector presents a diameter $d_{\text {pin }}=0.254 \mathrm{~mm}$ and has a penetrating height of $T=1.27 \mathrm{~mm}$ (see Fig. 10.20(c)). In this design, the filter is fed from the the top plate. The location in the $\hat{y}$ direction is firstly fixed between the $2^{\text {nd }}$ and $3^{\text {rd }}$ (starting from the left) pin columns of the upper and bottom blocks of the cavity, see Fig. 10.25(a). Then, the offset $x_{0}$ from the central position in the $\hat{x}$ direction is adjusted to vary $Q_{e x}$, see Fig. 10.25(a). Results of this study are plotted in Fig. 10.25(b). The required $Q_{e x}$ is achieved with $x_{0}=1.53 \mathrm{~mm}$. As an additional comment, note that $\partial Q_{e x} / \partial x_{0}$ is low on $x_{0}$, otherwise $Q_{e x}$ would be excessively sensitive to $x_{0}$ what could difficult the response synthesis and increase the manufacturing errors effects. If that was the case, other position in the $\hat{y}$, displacing the connector to the cavity center (where the fields are maximum) would be required so that lower $Q_{e x}$ values were obtained for the same range of $x_{0}$.

\section{Complete filter}

The complete filter structure is shown in Fig. 10.26. Since several parameters have been fixed parameters and the filter presents symmetry, the only parameters 


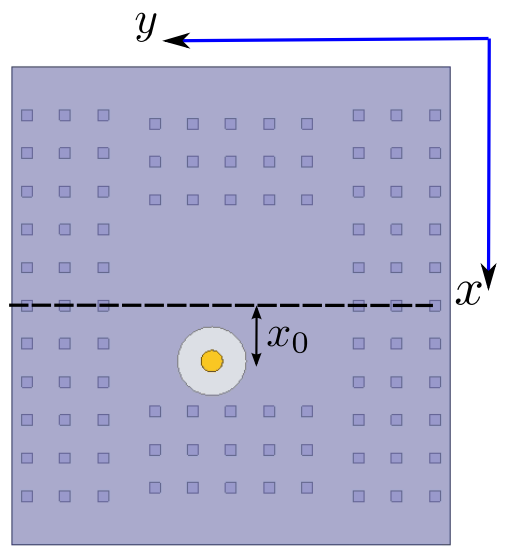

(a)

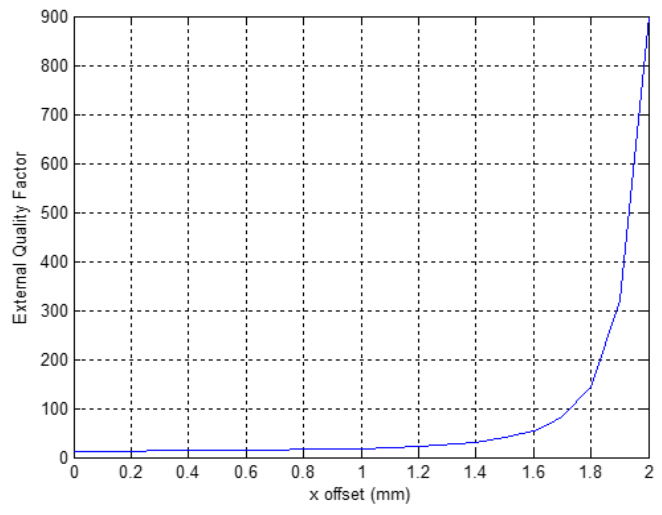

(b)

Figure 10.25: External quality factor $Q_{e x}$ synthesis. a) Feeding structure (top view); b) External quality factor $Q_{e x}$ vs. connector position $x_{0}$.

\begin{tabular}{lccccc} 
Parameter & $C_{1}$ & $C_{2}$ & $w_{12}$ & $w_{23}$ & $x_{0}$ \\
\hline Initial value $(\mathrm{mm})$ & 6.1 & 6.1 & 3.034 & 2.919 & 1.53 \\
\hline Optimum value $(\mathrm{mm})$ & 5.73 & 5.36 & 3.08 & 2.97 & 1.31 \\
\hline
\end{tabular}

Table 10.6: Filter optimization parameters.

included in the optimization process are those shown in Fig. 10.26(b). As it can be appreciated, the feeding locations present a double symmetry one from the other, i. e., the input is closer to one of the sides and the output is closer to the other. This has not influence on the filter performance (single symmetry could have been used), however, this allows from a slight larger separation between the connectors, what facilitates the measurement process. The previous procedure has provided an initial design with the parameter values listed in the first row of Table 10.6. Since the procedure presents some implicit approximations, simulation of the filter with the initial geometric values provides a response that does not satisfy the proposed specifications. Nevertheless, the optimization process results simple and fast being that such initial design is close enough to the optimum one. In a first approach ${ }^{1}$ it is used FEST3D ${ }^{\circledR}$ [960], which implements the BI-RME method [544], [575], hence results very efficient. With a small CPU effort, a filter design with a suitable response is obtained. The optimum values for the optimization variables are listed in the second row of Table 10.6. The optimum simulated response, which satisfy the specifications, is represented in Fig. 10.27.

\subsubsection{Manufactured prototype and measurements}

A prototype has been manufactured in aluminum by means of an in-house process using a Datron ${ }^{\circledR}$ M25 milling machine system. Figs. 10.28(a) and 10.28(b) show

\footnotetext{
${ }^{1}$ Note that the presented design is previous to the analysis method exposed in section 10.2 so that method is not used here.
} 


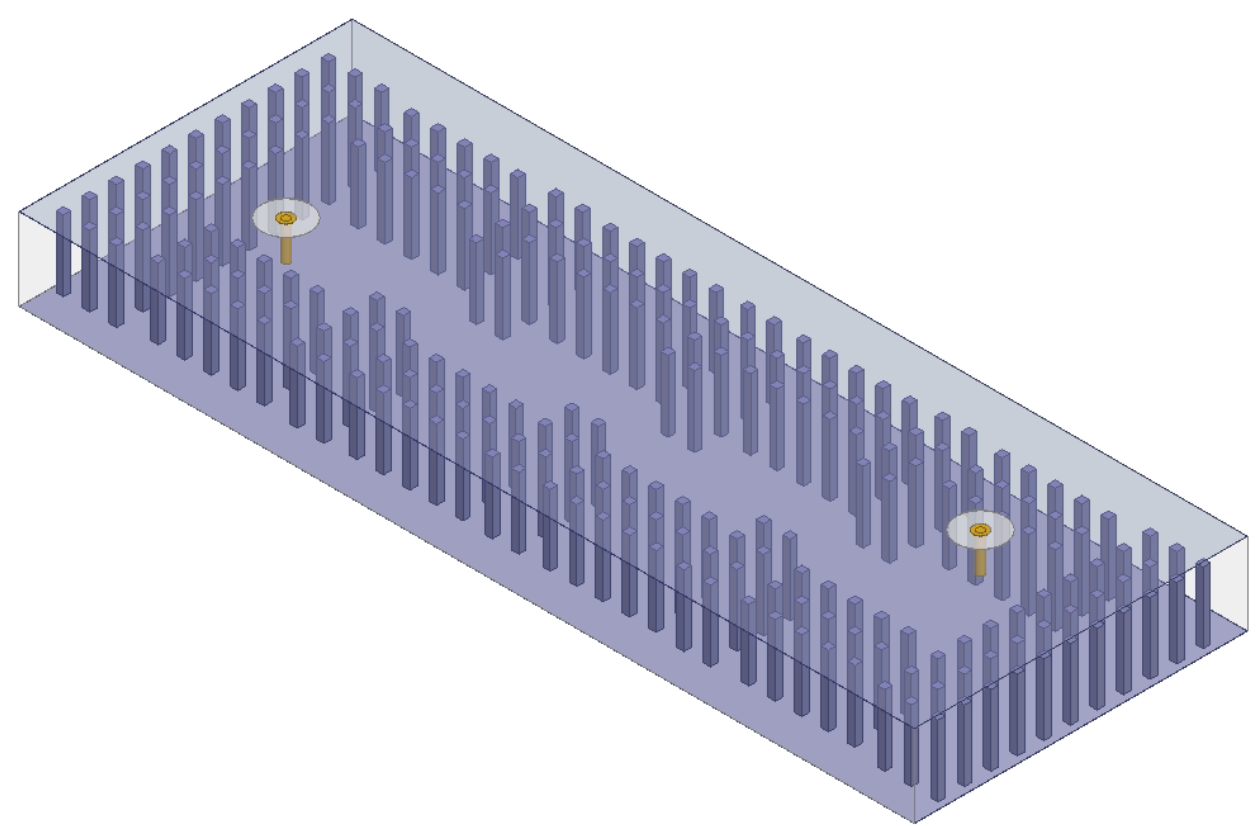

(a)

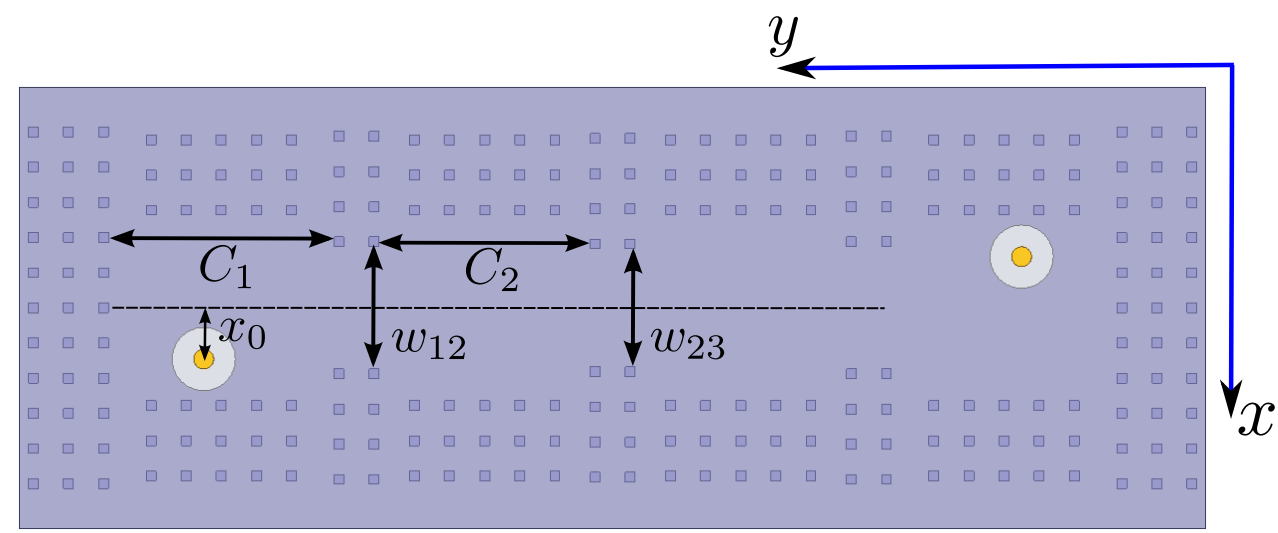

(b)

Figure 10.26: Final structure: four cavity $G G W$ filter. a) $3 D$ view; b) Top view.

pictures of this prototype. As it can be appreciated in Fig. 10.28(a), the filter is made of two parts: a lower metal plate that contains the pins surface and an upper metal flat plate with two holes for the input and ouput connectors. These two parts are distant by four supports located at the filter corners, which means that the end walls are kept open, see Fig. 10.28(b). Although this is not necessary, i. e., the filter could be totally packaged without any negative effect (note that pins prevent from field leakage), this design has been preferred to explicitly show that the fields are confined inside the filter layout defined by the pins without significant 


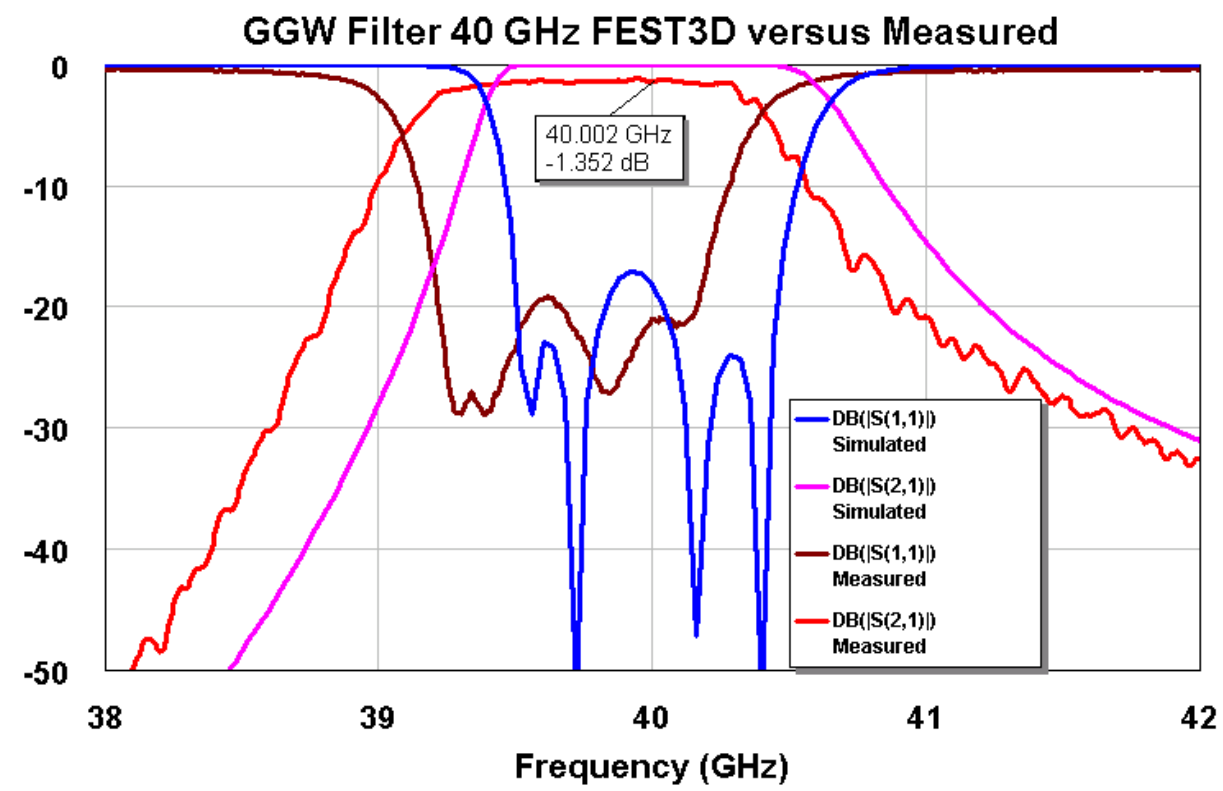

Figure 10.27: Comparison between the optimum simulated response and measured results.

leakage. In addition, the system of screws is a simple one, implemented for fixing and alignement purposes, contrary to those employed for rectangular waveguide filters, which require more screws and careful pressure to ensure contact between pieces along all the metallic surface joint between the pieces.

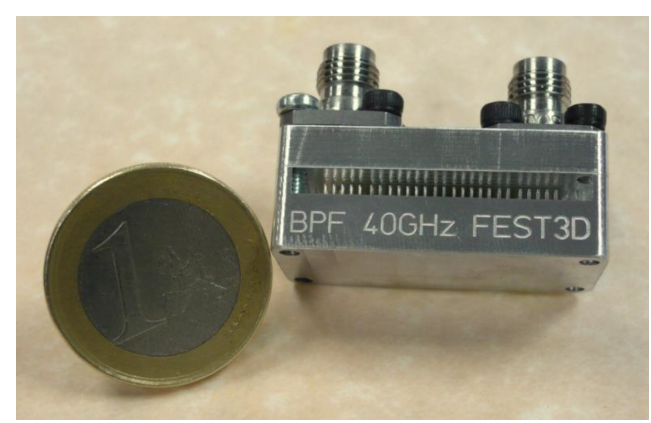

(a)

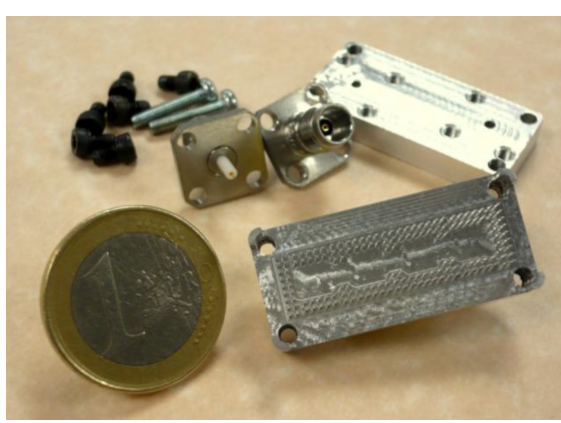

(b)

Figure 10.28: Manufactured prototype. a) Assembled filter; b) Detail of the different pieces that compose the structure.

The measured response is as well plotted in Fig. 10.27 to be compared with the previous simulations. Although the S-parameters aspect is close to what is expected, including good return losses, it is found a frequency displacement of about $\Delta f=0.4$ $\mathrm{GHz}$ towards lower frequencies. In addition, measurement reveals insertion losses of $I L=1.352 \mathrm{~dB}(I L=1.091 \mathrm{~dB}$ subtracting the losses introduced by the coaxial 
connectors) which are not present in the FEST3D ${ }^{\circledR}$ simulation, being that ohmic losses and potential residual leakage of the structure are not taken into account by the BI-RME method. The measured value of insertion losses implies through (10.25) an equivalent quality factor of $Q=960$, which, although moderate, is far from the expectations since a rectangular waveguide could reach (in theory) $Q=3928$ at the considered central frequency $(f=40 \mathrm{GHz})$.

In order to identify the source of the frequency displacement issue, a dimensional analysis has been performed with the manufactured pieces by means of a SEM microscope. Fig. 10.29(a) shows the microscope top view image of the bottom piece with the pins, where the roughness caused by the milling machine can be clearly appreciated. In Fig. 10.29(b), a detail of one of the manufactured pins is shown. As it can be read in the indicators of the left corner of the image, the pin width is approximately $w_{p}=0.25 \mathrm{~mm}$ instead of the supposed $w_{p}=0.3 \mathrm{~mm}$. Observation of several randomly chosen pins confirms this systematic deviation, which seems to be caused by vibration movements of the milling cutter that lead to excessive cutting of the pins. This explains the obtained response: since the pins are cut in excess and made narrower, the obtained GGW cavities are larger than the simulated ones, what implies a frequency displacement of the response towards lower frequencies.

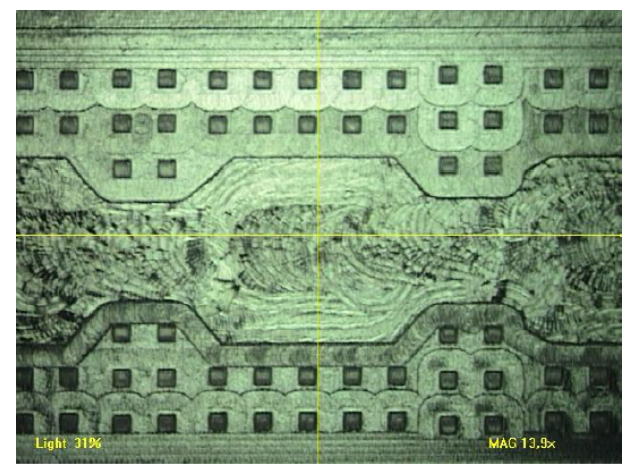

(a)

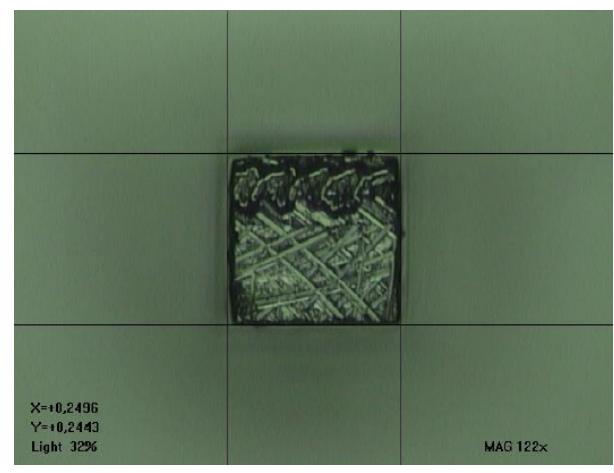

(b)

Figure 10.29: Manufacturing errors analysis. a) Top view image of the manufactured bottom piece; b) Detail of a manufactured pin.

With the aim of taking into account this effect, together with ohmic and leakage losses, a simulation using the commercial solver HFSS ${ }^{\circledR}$ is carried out. Results of this new simulation are shown in Fig. 10.30 together with the measurements. With this simulation, more precise, good agreement with the measured response is observed. This agreement suggests that, since the identified manufacturing errors have an important systematic component, taking control over them may lead to experimental results satisfying the specifications. For instance, inasmuch as it has been observed that the milling cutter is overcutting the pins, a larger $w_{p}$ can be sent to the machine to compensate this effect.

Regarding to the insertion losses, on the one hand, the obtained value of approximately $I L=1.1 \mathrm{~dB}$ results competitive with the state of the art in the Ka-band given the filter order and $R B W$ (see for instance current results with microstrip technol- 


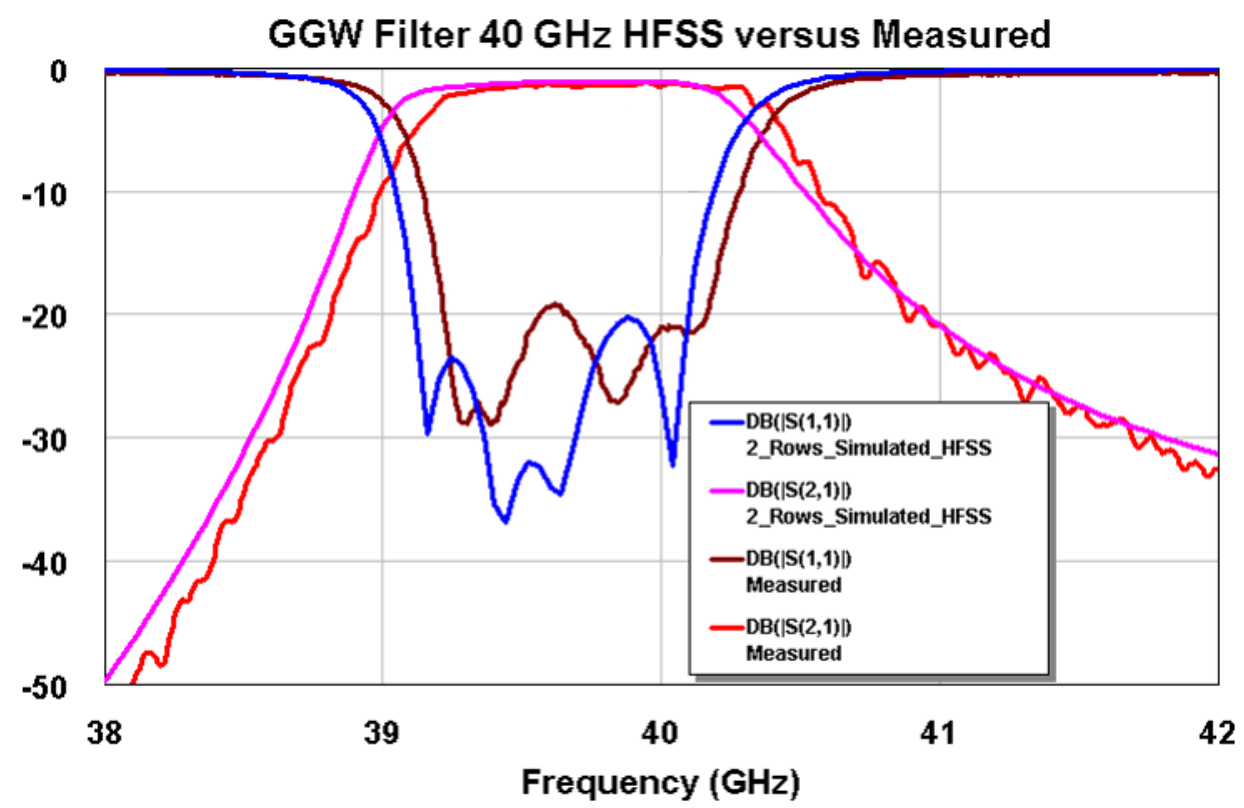

Figure 10.30: Comparison between the simulated response taking into account the manufacturing errors and the measured results.

ogy [961]-[962] or SIW technology [919]-[920], [963]-[964]). Obviously, the non-planar feature of GGW structure and the absence of dielectrics in them provides inherently a better performance in terms of losses compared to planar topologies. On the other hand, the obtained equivalent quality factor for the GGW resonators is still far from the quality factor for rectangular waveguide cavities (even taken into account the manufactured rectangular waveguide cavities reach only $65 \%-80 \%$ of their theoretical value [704]). This suggests a further study to determine the source of this large difference between GGW and rectangular waveguide in terms of insertion losses.

Inspection of the possible leakage due to the truncation of the periodic structure reveals that this is, indeed, the cause of the obtained insertion losses, higher than expected for a GGW design. To reveal the effect of this leakage, full-wave simulations considering three and four rows of pins have been done and a prototype incorporating three rows has been manufactured. These new results are plotted in Fig. 10.31 together with the initial simulation with two pin rows and the measured results. The simulated results indicate that values of only $I L=0.42 \mathrm{~dB}$ and $I L=0.39$ $\mathrm{dB}$ are obtained with three and four pin rows, respectively. This is a considerable improvement from the simulated $I L=1.1 \mathrm{~dB}$ of the original filter with a periodic structure truncated after two pin rows. In addition, note that the agreement between simulation and measurements is very good regarding the insertion loss level. This suggests that avoiding possible leakage by proper choosing an enough number of pin rows may lead to very competitive filters. Note that $I L=0.42$ would imply an equivalent quality factor of $Q=2630$.

The previous results indicate, on the one hand, that the manufacturing process must be supervised to eliminate, at least, systematic errors. This occurs due to 


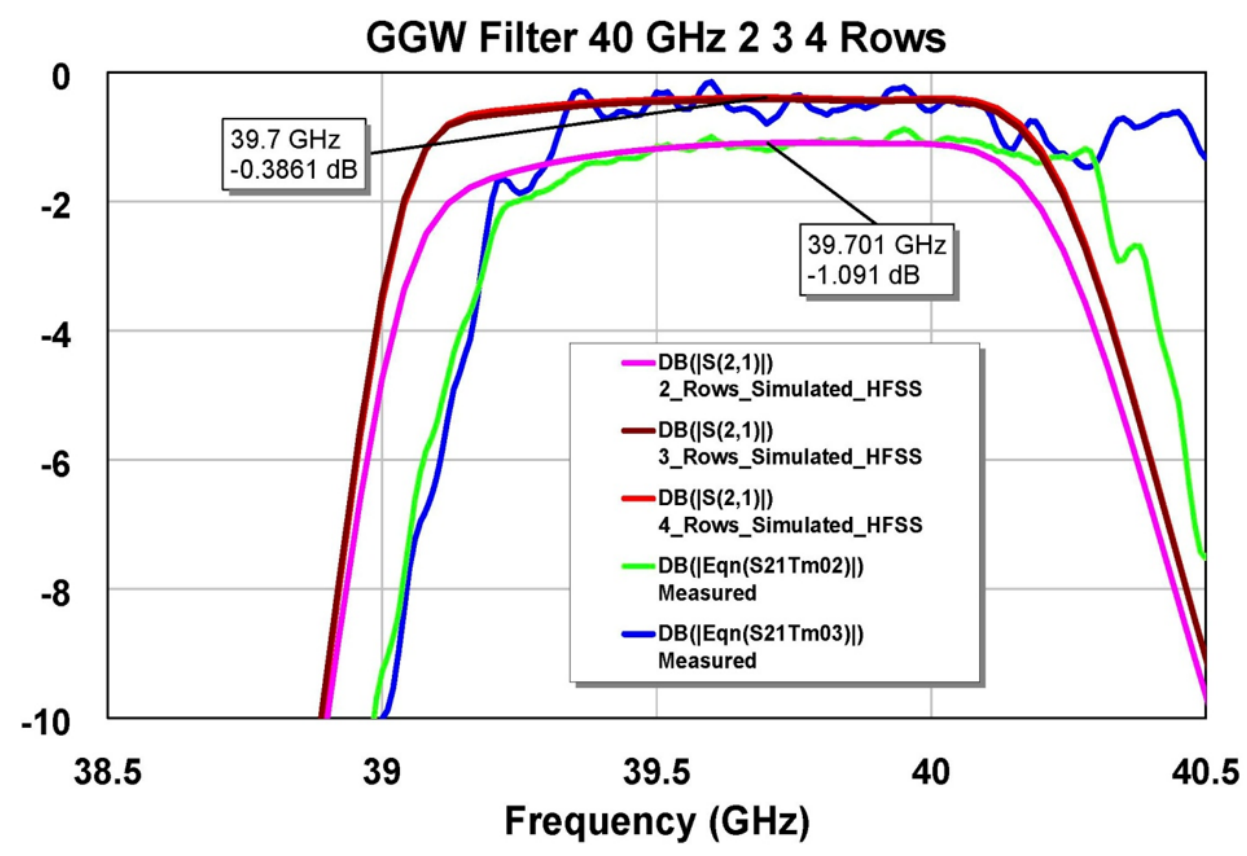

Figure 10.31: Analysis of the effect of the number of rows on the insertion loss of the filter.

the high sensibility of high-frequency devices to manufacturing errors. On the other hand, three or more rows seem necessary for high-frequency (Ka-band and beyond) GGW devices. If these premises are followed and the observed issues are avoided, GGW narrow-band filters could reach a very competitive performance. In order to evaluate the suitability of GGW topology front other options such classical technologies and alternative GWs, a study of losses has been performed at $f=40 \mathrm{GHz}$. Several cavities of length $L=3 / 2 \lambda_{g}$ have been considered. From the obtained quality factor, the propagation losses has been calculated by the quality factor method (see [796], [800] for details). Results of this study are shown in table 10.7. As it can be observed, in the Ka-band, the simulated GGW cavities quality factor reach almost $75 \%$ of their rectangular waveguide counterparts, a percentage that may considerably be increased (even to the point of having $Q_{G G W}>Q_{R W G}$ ) in manufactured prototypes according to the conclusions in [800], and previous observed degradation of manufactured rectangular waveguides [704]. However, a reliable proof of this predicted fact is necessary in order to rightfully confirm GGW as a suitable alternative to the rectangular waveguide for implementing mm-wave narrow-band filters. In addition, results in Table 10.7 also indicate that GGW is clearly the most suitable structure among GWs in terms of losses. Nevertheless, RGW and SSGW show competitive values given the operation frequency, with a performance much higher than their reference classical counterpart structures such as the microstrip line.

Finally, in order to remark the competitiveness of the present design regarding the current state of the art, Table 10.8 shows a comparison between several recent 


\begin{tabular}{cccc} 
Structure & & Quality Factor $(Q)$ & Losses $(\mathrm{dB} / \mathrm{cm})$ \\
\cline { 1 - 1 } Rectangular Waveguide & & 3928 & 1.4 \\
GGW (four rows) & & 2893 & 2.1 \\
Microstrip Line & & 243 & 29.2 \\
RGW & & 976 & 3.9 \\
SSGW & 686 & 5.7 \\
\hline
\end{tabular}

Table 10.7: Comparison of losses for several waveguides (simulated).

narrow-band filter proposals in the Ka-band, including different technologies: microstrip line, SIW, rectangular waveguide (WR-28), and the proposed Design 1 using GGW. The main indicator of performance is the equivalent quality factor $Q_{e q}$ which is obtained from the order of the filter $N$, the insertion losses $I L$ and the relative bandwidth $R B W$ and allows to realize a proper comparison between the different designs $^{2}$. Return losses and the evaluation of a possible frequency shifts in the response complete the features to observe when comparing the studied designs. As it can be observed, microstrip designs (see first two rows in Table 10.8) present a low $Q$ besides slightly poor $R L$ and an appreciable frequency shift. Therefore, it can be stated that NBF designs with this technology may result quite challenging at Ka-band frequencies even for permissive specifications cases. Properly manufactured, SIW technology allows for a moderate $Q$ that can be close to 500. The return losses are good as well and small frequency shifts are appreciated on the measured responses. Therefore, SIW technology results to be a reasonable option for NBF implementation in the Ka-band, providing, moreover a planar profile. However, SIW technology is inherently limited due to the use of dielectric and its moderate ohmic losses caused by its planar profile. Thus, is quite improbable that SIW technology reach the high performance levels that low-loss NBFs implemented with hollow metallic waveguides reach at lower frequencies.

Regarding the rectangular waveguide topology, it is hard to find recent scientific works dealing with Ka-band NBF designs, but the technology is enough mature to be accepted by the industry. Therefore, the designs offered in the catalogs of three companies, Pasternack ${ }^{\circledR}$ [965], A-INFO [966] and RTx Technology Co. [967], are evaluated. According to the catalog specifications, a remarkable good feature of these designs is that, in principle, any frequency shift is present in the response. In the case of the Pasternack ${ }^{\circledR}$, the equivalent quality factor is, surprisingly, of only $Q=400$, a quite low value for a rectangular waveguide design. In the case of AINFO, the filter is manufactured in H-plane, what allows the placement of tuning screws. Such filter reaches a quite high quality factor: $Q_{e q}=1400$ which results competitive for the Ka-band. The return loss figure is slightly poor, however. The best design is that offered by RTx Technology Co. which obtains a quality factor of $Q=1900$, very competitive, besides a good return losses figure. These results show that, actually, rectangular waveguide technology still represent the highest

\footnotetext{
${ }^{2}$ Note that insertion losses $I L$ are directly proportional to the filter order $N$ and inversely proportional to the relative bandwidth $R B W$ by means of (10.25). Therefore, a direct comparison regarding only $I L$ results inappropriate and the equivalent quality factor of each resonator is used instead.
} 


\begin{tabular}{ccccccccc} 
Filter & & Technology & $N$ & $I L$ & $R B W$ & $Q_{e q}$ & $R L$ & $\Delta f$ \\
\cline { 1 - 5 }$[961]$ & & Microstrip & 2 & $2.68 \mathrm{~dB}$ & $3.22 \%$ & 74 & $10 \mathrm{~dB}$ & Medium \\
{$[962]$} & & Microstrip & 5 & $3.5 \mathrm{~dB}$ & $4.9 \%$ & 178 & $10 \mathrm{~dB}$ & Medium \\
{$[963]$} & & SIW & 4 & $2.95 \mathrm{~dB}$ & $4.66 \%$ & 158 & $13.5 \mathrm{~dB}$ & Small \\
{$[964]$} & & LTCC SIW & 4 & $2.95 \mathrm{~dB}$ & $4.66 \%$ & 158 & $13.5 \mathrm{~dB}$ & Small \\
{$[919]$} & & SIW & 6 & $2 \mathrm{~dB}$ & $4 \%$ & 468 & $14 \mathrm{~dB}$ & Small \\
{$[920]$} & & SIW & 4 & $1.25 \mathrm{~dB}$ & $3.71 \%$ & 464 & $14 \mathrm{~dB}$ & Small \\
{$[965]$} & & WR-28 & 5 & $1.5 \mathrm{~dB}$ & $5.12 \%$ & 400 & $14 \mathrm{~dB}$ & No \\
{$[966]$} & & WR-28 & 6 & $0.5 \mathrm{~dB}$ & $5.7 \%$ & 1400 & $10 \mathrm{~dB}$ & No \\
{$[967]$} & & WR-28 & 5 & $1.5 \mathrm{~dB}$ & $1 \%$ & 1900 & $17 \mathrm{~dB}$ & No \\
Design 1 & & GGW & $\mathbf{4}$ & $\mathbf{1 . 1} \mathbf{d B}$ & $\mathbf{2 . 5 \%}$ & $\mathbf{9 6 0}$ & $\mathbf{2 0 ~ d B}$ & Medium \\
\hline
\end{tabular}

Table 10.8: Comparison between several recently proposed Ka-band narrow-band filters. $N:=$ Filter order; $I L:=$ Insertion losses; $R B W:=$ Relative bandwidth; $Q:=$ Equivalent quality factor; $R L:=$ Return Losses; $\Delta f:=$ Frequency shift;

performance option. However, note that the obtained figures are poorer than for lower bands. In fact, for most of vendors, Ka-band filters are the highest frequency offered ones, which is an indicator of the difficulty involved in the design of highperformance NBF at mm-wave frequencies. The presented filter (see last row of Table 10.8 ) reaches a $Q$ as high as 960 , which is a moderate-high value for the Ka-band. In addition, it has been shown that this value may be increased (by a factor 2.6) if a larger number of pin rows is used in the filter layout. Regarding the loss performance of the rectangular filters offered by the industry, it seems evident that an eye must be put in the presented GGW topology. However, the frequency shift issue is an important one to be solved. Probably, if a high-performance manufacturing process was used instead of the employed in-house one, the problem could be alleviated, being that this issue mainly depends on the manufacturing tolerances.

In this first design, several issues have been observed so that several premises are established. In the posterior designs the number of pin rows will be set to three since the field leakage with two pin rows produce a noticeable degradation of the insertion losses and to use more rows does not produces appreciable improvement but increases the total size of the structure. In addition, an observed practical issue is commented now. In the presented design, the coaxial connectors are placed in the top metal plate. This implies that, to place them in their precise locations, a good alignment between top and bottom pieces must be ensured. High sensitive has been appreciated in the manufactured prototype. Note that, if the coaxial connectors were placed in the bottom piece, the whole filter layout would be contained in this piece. Since the only functionality of the top plate is to be located at a proper distance from the pins to ensure the stopband, the alignment of both pieces is not critical any more ${ }^{3}$. This modification alleviates the manufacturing requirements and leads to a structure inherently more resistant to vibrations, which is a suitable feature

\footnotetext{
${ }^{3}$ Horizontal displacements of the top plate does not affect the structure since the GGW filter layout is not modified and vertical displacements will have only slight influence on the response due to the vertical invariance of the quasi- $\mathrm{TE}_{10}$ mode of the GGW.
} 
given the possibles context of application of GGW NBFs, e. g., automotive radars, satellite front-end, portable imaging cameras, etc. In this way, much more profit can be taken from the GGW feature of being formed by two independent pieces.

\subsubsection{Design 2: $5^{\text {th }}$-order V-band filter at $f=61 \mathbf{G H z}$}

The second design consists in a $5^{\text {th }}$-order Chebyshev filter operating in the V-band, specifically at $f=61 \mathrm{GHz}$. There are three main reasons to move up to the $\mathrm{V}$ band for the second design and apply the improvements derived from the Design 1 conclusions. First, as the literature indicates, V-band is currently more under the focus than the Ka-band for the incoming next generation applications. In this way, the presented design could find a good niche of application in several mmwave communication systems. Second, the state of the art in the topic of V-band narrow-band filters clearly reveals that, at V-band, filter design becomes quite more challenging that at Ka-band, being that, in several novel proposed topologies for the V-band, experimental results rarely meet the specifications [365], [853], [968][970]. In addition, rectangular waveguide NBFs at V-band are not offered by most of the commercial houses (see for instance catalogs of Pasternack ${ }^{\circledR}$ [965] or A-INFO [966]). Third, the higher frequencies of the V-band are more suitable to prove the potentials of the GGW as an alternative to the rectangular waveguide. Note that, by topological reasons, if the same manufacturing process is used, rectangular waveguide designs will, in principle, experience a higher response degradation. Most of the work described next can be found summarized in [841], publication that received the Young Engineer Prize at the $44^{\text {th }}$ Microwave European Conference. This was, actually, the first work in which a V-band GGW component manufactured with conventional techniques was presented, encouraging the use of higher frequencies in GGW technology. Several posterior V-band proposals came later, mainly in 2015 and 2016 [822], [838]-[839], [843].

As before, the first step in the design is to establish the geometry of the GGW to the topological basis of the filter. The geometrical parameters listed in Table 10.9 provide the required stopband. Fig. 10.32(a) shows the dispersion diagram of the designed GGW. The undesired modes supported by the parallel-plate formed by upper and bottom metal plates are depicted in red. It is clearly observed a stopband in the range $[43 \mathrm{GHz}-75 \mathrm{GHz}]$. As it can be appreciated, the $\mathrm{GGW}$ is designed in such way that the the stopband covers the propagating region of the fundamental mode. Under this conditions, the operating band of the filter is located far from cutoff, in a less dispersive zone, what provides a better group delay response. The propagation constant of a rectangular waveguide with same propagation channel dimensions and the propagation constant of a plane wave $\left(k_{0}\right)$ are as well plotted as a reference.

\begin{tabular}{cccccc} 
Parameter & $p$ & $w_{p}$ & $h_{p}$ & $h_{a}$ & $a$ \\
\hline Value $(\mathrm{mm})$ & 0.59 & 0.2 & 1.59 & 0.25 & 4 \\
\hline Table 10.9: & GGW geometrical parameters.
\end{tabular}

In this design at $f=61 \mathrm{GHz}$, following the conclusions of Design 1, three rows have been used to provide the desired confinement without requiring a large periodic 


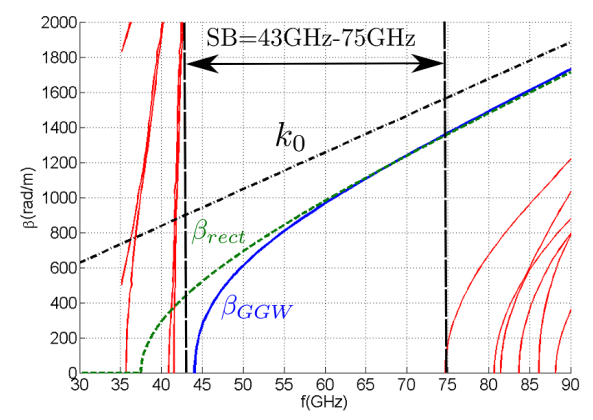

(a)
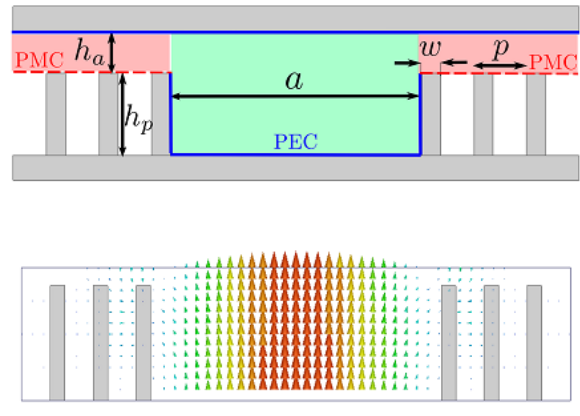

(b)

Figure 10.32: Basis GGW. a) Dispersion diagram of the designed GGW. The stopband is delimited by the unwanted parallel-plate cavity modes of the structure, in red. As a reference, they are depicted the propagation constant of a rectangular waveguide with same channel dimensions (green dashed line) and the propagation constant of a plane wave $k_{0}$ (black point-dashed line); b) GGW geometrical parameters and E-field (orientation and magnitude) at $f=f_{0}=61 \mathrm{GHz}$ on a plane transversal to the propagation.

structure. Fig. 10.32(b) shows the transversal cut of the GGW structure and its main parameters (top image), and the E-field (orientation and magnitude) at the central frequency $f=f_{0}=61 \mathrm{GHz}$ on a plane transversal to propagation. The expected field pattern, exhibiting confinement in the propagation channel and typical sinusoidal distribution plus some negligible local effects in the neighborhood of the first row of pins, is obtained. This confirms the suitability of the designed GGW to be the base waveguiding structure of the filter to be design.

\subsubsection{Filter specifications}

The filter specifications on the response are shown in Table 10.10. Regarding to the bandwidth, $R B W=2.5 \%$ is again considered, therefore, in this aspect, the design 2 is a scaled version at $f_{0}=61 \mathrm{GHz}$ of the Design 1 . The bandpass ripple $R$ (hence return losses $R L$ ) are as well identical to the Design 1. However, the order is increased to $N=5$ with the aim of providing a step further selectivity than in the previous design. Being that the operation takes places in the $\mathrm{V}$-band, the proposed specifications imply a design with a rather high difficulty to be successfully realized. The ideal response with the proposed specifications is shown in Fig. 10.33. Again, the procedure treated in section 10.3.1, gives the required normalized elements of the low pass prototype:

\begin{tabular}{cccc} 
Parameter & Frequency $f_{0}$ & Relative Bandwidth $(\mathrm{RBW})$ & BP Ripple $R(R L)$ \\
\hline Value & $61 \mathrm{GHz}$ & {$[60.25 \mathrm{GHz}-61.75 \mathrm{GHz}](\approx 2.5 \%)$} & $0.1 \mathrm{~dB}(16.42 \mathrm{~dB})$ \\
\hline
\end{tabular}

Table 10.10: Filter response specifications. 


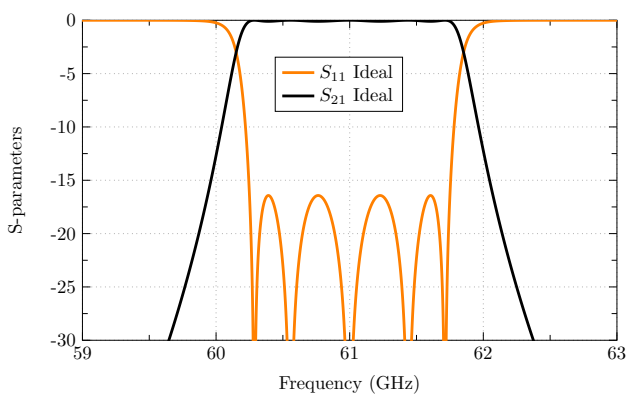

(a)

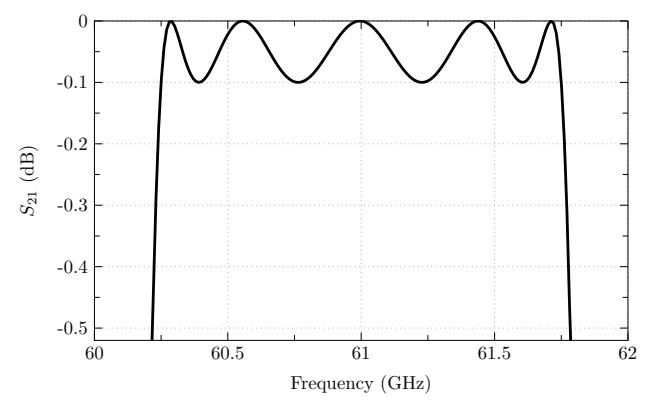

(b)

Figure 10.33: Ideal response. a) S-parameters; b) $S_{21}$ (dB) detail.

$$
\begin{aligned}
& g_{0}=1 \\
& g_{1}=1.147 \\
& g_{2}=1.371 \\
& g_{3}=1.975 \\
& g_{4}=1.371 \\
& g_{5}=1.147 \\
& g_{6}=1
\end{aligned}
$$

These values imply through (10.21)-(10.23) that the required coupling coefficients and external quality factors are:

$$
\begin{aligned}
Q_{e x_{1}} & =45.87 \\
k_{12} & =0.02 \\
k_{23} & =0.015 \\
k_{34} & =0.015 \\
k_{45} & =0.02 \\
Q_{e x_{N}} & =45.87
\end{aligned}
$$

Again, due to the filter characteristics and specifications, symmetry is present in the design (note that $Q_{e x_{1}}=Q_{e x_{N}}, k_{12}=k_{45}$ and $k_{23}=k_{34}$ ). Since the order is odd, symmetry can be appreciated even in the $g_{i}$ elements.

\subsubsection{Filter design}

\section{Resonant cavity:}

The topology of employed resonant cavities is that described in Fig. 10.18, with periodic structure truncated after the third row of pins. The pair $(C=4 \mathrm{~mm}, L=$ $3.27 \mathrm{~mm}$ ) provides exactly a resonant frequency of $f_{0}=61 \mathrm{GHz}$. The commercial 
solver HFSS ${ }^{\circledR}[411]$ has been used to perform the synthesis. In this way, ohmic losses has been considered, hence the unloaded quality factor has been taken into account. It has been obtained a value of $Q_{u}=1450$, which, considering (10.25), in turn implies a minimum theoretical insertion loss of:

$$
I_{L}=\frac{4.343 f_{0}}{\Delta f Q_{u}} \sum_{i=1}^{N} g_{i}=0.84 \mathrm{~dB}
$$

\section{Coupling elements:}

The coupling elements are the same as Design 1, but with one extra pin row to prevent residual leakage, see Fig. 10.34(a). Again, the procedure of section 10.3.1.3 leads to the relationship between the physical window width $w$ and the coupling factor, see Fig. 10.34(b). In this plot, red circles mark the required values for the design: $w_{12}=w_{34}=2 \mathrm{~mm}$ and $w_{23}=w_{32}=1.93 \mathrm{~mm}$.

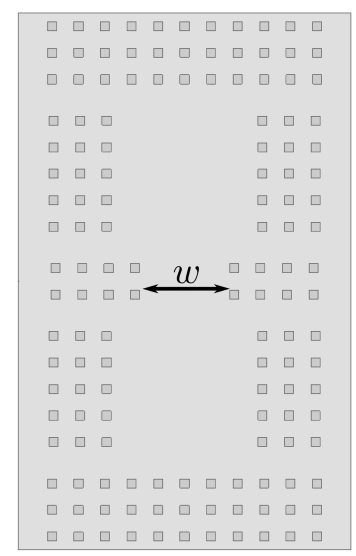

(a)

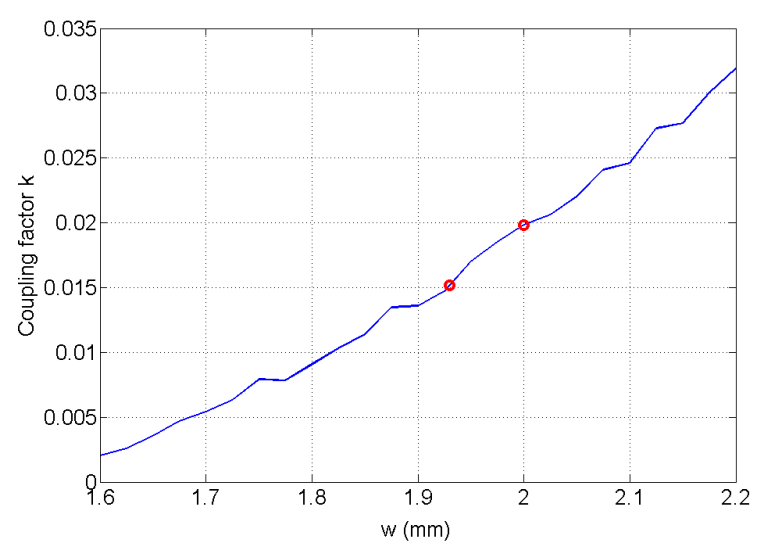

(b)

Figure 10.34: Coupling factor $k$ synthesis. a) Coupling structure; b) Coupling factor $k$ vs. window size $w$. Red circles indicate the required widths to meet the coupling requirements of the design.

\section{Feeding elements:}

As commented before, taking advantage of the experience with the first design, in the present filter, coaxial feeding probes are integrated in the bottom piece instead of the top one. Unnecessary positioning requirements are, therefore, avoided, and the top plate becomes a rather dummy piece which deformation results much less critical. In this way, the structure is better prepared for vibrations, an extra advantage to highlight front rectangular waveguide structures.

In addition, since the frequency is higher in this second design, other connectors, suitable for the operation frequency range, are required. Two $1.85 \mathrm{~mm}$ coaxial connectors (SouthWest Microwave1490-12G) have been used for the filter feeding. These connectors have a launch pin of diameter $d_{p i n}=0.127 \mathrm{~mm}$ and a penetration 
depth of $T=0.381 \mathrm{~mm}$. Note that in the previous design the connectors were $\left(d_{c}=\right.$ $0.254 \mathrm{~mm}, T=1.27 \mathrm{~mm}$ ). Therefore, not only in absolute terms, but in relative terms, the connectors in the present design are smaller. This has an important implication. Whereas in the Design 1 the required perturbation to achieve the desired $Q_{e x}$ value was easily obtained, in this case, the perturbation caused by the connector results to be insufficient in such way that $Q_{e x}$ remains higher than the requested value even when the feeding is placed in the cavity center $\left(C_{C_{1}}\right.$ in the bottom image of Fig. 10.35(a)), the location of maximum perturbation.
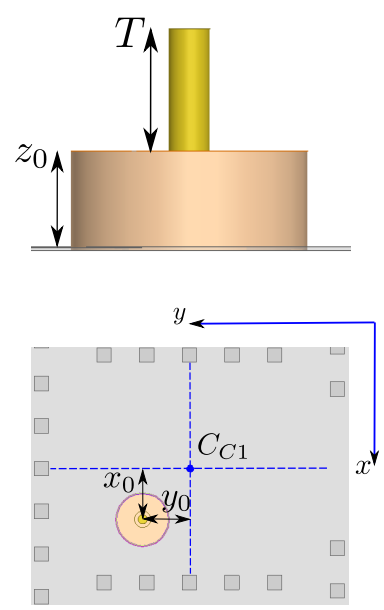

(a)

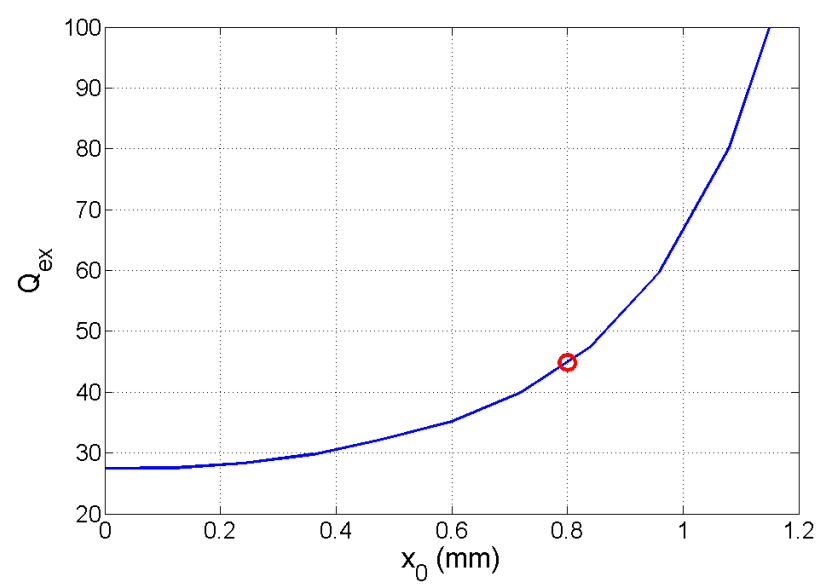

(b)

Figure 10.35: External quality factor $Q_{e x}$ synthesis. a) Feeding structure: connector detail (top image) and top view of the feeding area in the first cavity (bottom image); b) External quality factor $Q_{e x}$ vs. connector position $x_{0}$. The other two position parameters are fixed to $y_{0}=0.5 \mathrm{~mm}$ and $z_{0}=0.3 \mathrm{~mm}$. Red circles indicate the required $x_{0}$ position to meet the coupling requirements of the design.

To solve the previous problem, a larger insertion of the connector is proposed. Attached to the launch pin, the next part of the connector consists on a conductor of diameter $d_{\text {con }}=0.228 \mathrm{~mm}$, surrounded by a Teflon coating, with total external diameter of $d_{\text {teflon }}=0.737 \mathrm{~mm}$, see Fig. 10.20 (c) for details. This part can be introduced a distance $z_{0}$ as the top image of Fig. 10.35(a) shows. It has been checked that a value of $z_{0}=0.3 \mathrm{~mm}$ results suitable to provide reasonable values of $Q_{e x}$ and stable enough variation of this parameter in the offset $\left(x_{0}, y_{0}\right)$ domain. Once fixed the value of $z_{0}$, it has been fixed $y_{0}=0.5 \mathrm{~mm}$, and, finally, $x_{0}$ has been parametrized in order to obtain the plot of Fig. 10.35(b). The red circle in the plot marks the value $x_{0}=0.8 \mathrm{~mm}$ that provides the desired $Q_{e x} \approx 45.87$.

\section{Complete filter:}

A 3D view schematic of the complete filter structure is shown in Fig. 10.36(a). Fig. 10.36(b) shows a top view of part of the filter with the main geometrical parameters. Note that, due to the filter symmetry (from the center of the cavity 3 ), the complete structure can be totally described with the geometrical parameters shown in Fig. 


\begin{tabular}{lccccc} 
Parameter & $C_{1}$ & $C_{2}$ & $C_{3}$ & $w_{12}$ & $w_{23}$ \\
\hline Initial value $(\mathrm{mm})$ & 4 & 4 & 4 & 2 & 1.93 \\
\hline Optimum value $(\mathrm{mm})$ & 3.926 & 3.875 & 3.926 & 2.01 & 1.94 \\
\hline
\end{tabular}

\begin{tabular}{lccccc} 
Parameter & $L_{1}$ & $L_{2}$ & $L_{3}$ & $x_{0}$ & $y_{0}$ \\
\hline Initial value $(\mathrm{mm})$ & 3.27 & 3.27 & 3.27 & 0.8 & 0.5 \\
\hline Optimum value $(\mathrm{mm})$ & 2.97 & 2.97 & 2.97 & 0.705 & 0.151 \\
\hline
\end{tabular}

Table 10.11: Filter optimization parameters.

10.36(b), together with the connector position $\left(x_{0}, y_{0}\right)$. Table 10.11 lists all this parameters, which are the degrees of freedom in the optimization process. The previous procedure has given the initial set of values in the table, which provide a good initial point that has, nevertheless, to be optimized. Since the filter order is larger, the optimization process becomes more complicated. It is well-known that the larger number of optimization variables, the larger the harder to find the location of a solution satisfying the specifications since the domain becomes exponentially larger with the increment of dimensions and the error function of the optimization problem becomes more ill-posed [528], [971], [972]. Therefore, the followed strategy has been to restrict the number of variables to the minimum as possible and progressively increase it. For instance, in the first steps of the optimization, it has been fixed $C_{1}=C_{2}=C_{3}$ and $L_{1}=L_{2}=L_{3}$, and $y_{0}$ is not used. The found optimum values are as well listed in Table 10.11. As it can be appreciated, $C_{2}$ finally differs from $C_{1}$ and $C_{3}$, and a considerable modification in $y_{0}$ has been required to reach a satisfactory response, probably induced by the modification of the first cavity dimensions. It is highlighted that the optimization process of this design has involved non-negligible difficulties that might be alleviated with the analysis method presented in section 10.2 and some procedures described in the design 3. Comments will be given then.

Fig. 10.37(a) shows the simulated response with the optimum parameters of Table 10.11. S-parameters of the ideal response are added for comparison. As it can be appreciated, the simulated response is slightly narrower than the goal $(1.40 \mathrm{GHz}$ instead of $1.52 \mathrm{GHz}$ ), but has better return loss figure (19.5 dB instead of $16.42 \mathrm{~dB}$ ). The optimization process has been stopped in this response since along this process it has been observed that the return losses are very sensitive to the geometrical parameters variation. Generally, small geometrical variations around the optimal values cause an increase of return losses and a widening of the response. Thus, the obtained response has been considered a candidate with certain robustness against the manufacturing process. In addition, the obtained insertion losses are quite flat on the passband. Fig. 10.37(b) shows a detail of the $S_{21}$ in the passband. As it can be appreciated, the obtained response is on the expected level $(I L=0.84 \mathrm{~dB})$ according to (10.35).

\subsubsection{Manufacturing errors sensitivity analysis}

Since already in the Ka-band design, high sensitivity to the manufacturing process was observed, for the present design in the V-band, it has been considered opportune 


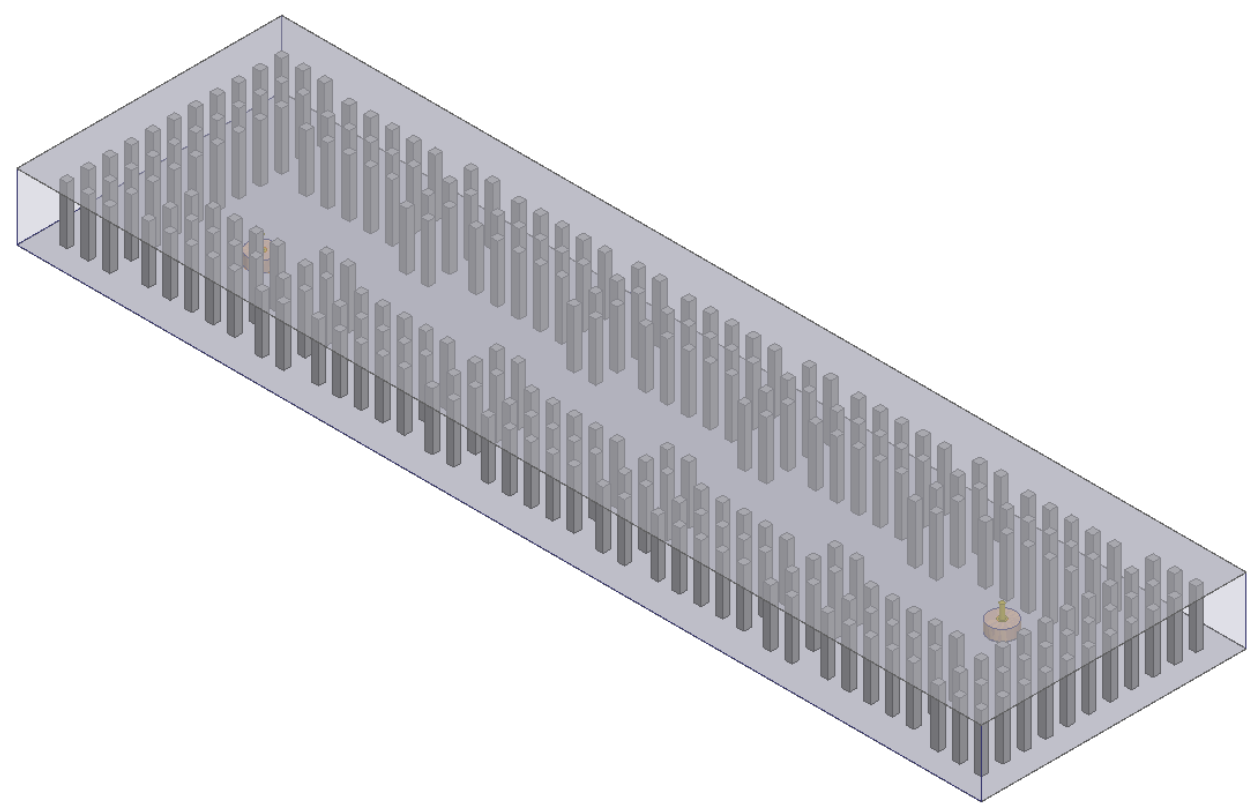

(a)

\section{$y$}

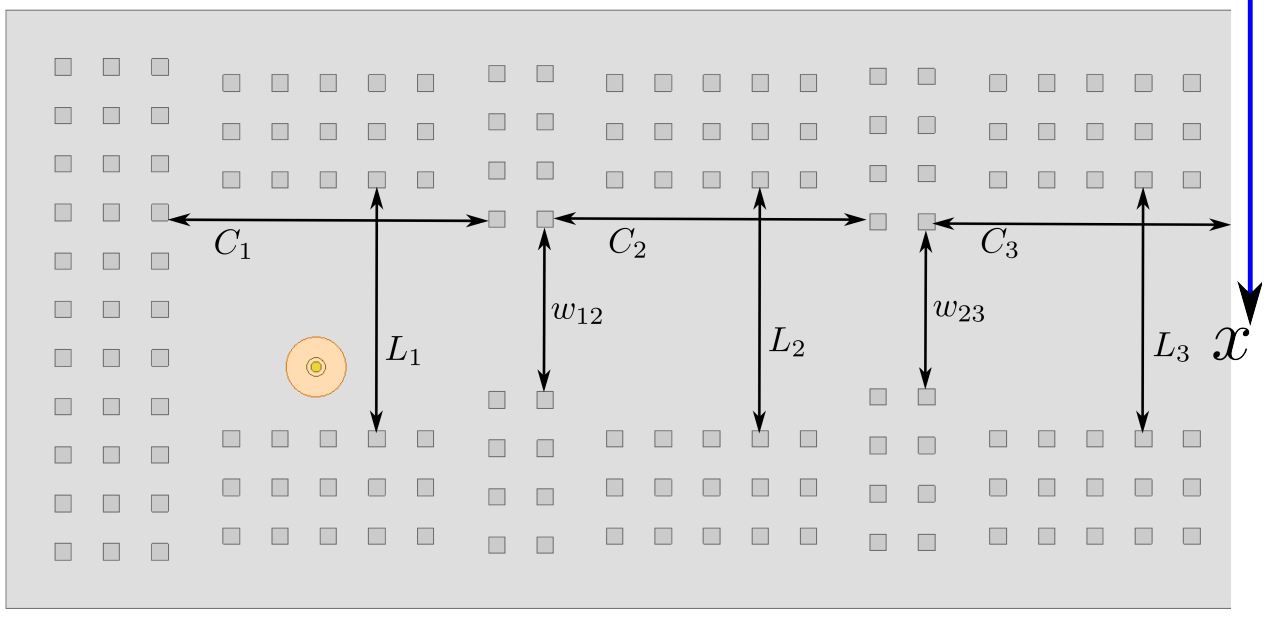

(b)

Figure 10.36: Final structure: five cavity GGW filter with bottom feeding. a) $3 D$ view; b) Top view.

to carry out a manufacturing errors sensitivity analysis. In this way, weak points of the structure regarding the errors in the manufacturing process can be determined so that some preventive actions can be taken. For this study, three possible sources of error that cover those scenarios most suitable to occur have been considered. The 


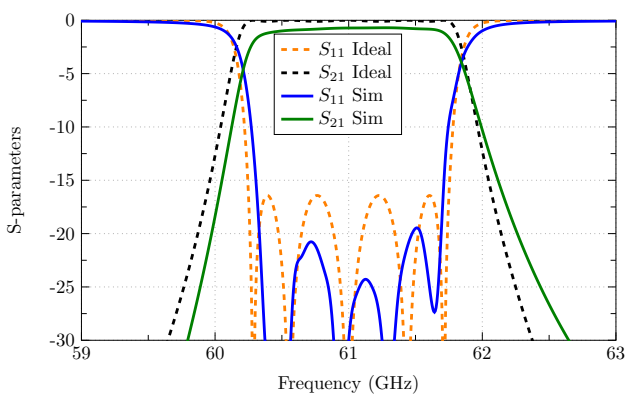

(a)

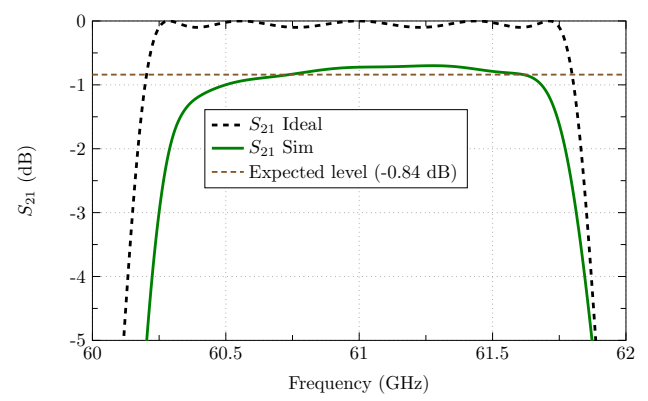

(b)

Figure 10.37: Comparison between the optimum simulated response and the ideal (lossless) response. a) $S$-parameters; b) $S_{21}(d B)$ detail.

different considered errors are schematized in Figs. 10.38(a)-10.38(c). Fig. 10.38(a) illustrates the error in the pin width $\Delta w$, Fig. 10.38(b) schematizes the error in the horizontal positioning of the feeding connector $\left(\Delta x_{0}, \Delta y_{0}\right)$, and Fig. 10.38(c) describes the error in the vertical positioning of the feeding connector, i. e., in the penetration of the connector $\Delta z_{0}$. Particular details about how these errors are modeled and how they may appear, as well as the analysis results are described next for each source of error. In all cases, the analysis carried out through the $S_{11}$ parameter. Notice that, since the considered sources of error have negligible influence in the ohmic losses, hence in the insertion losses, all the necessary information is contained in the $S_{11}$ parameter.

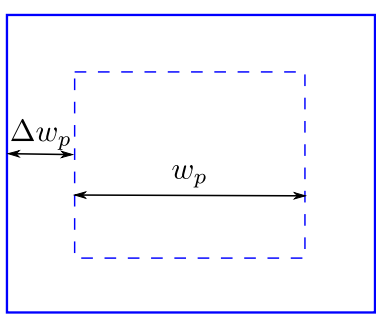

(a)

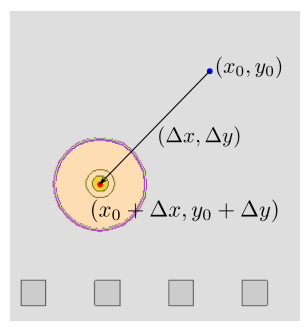

(b)

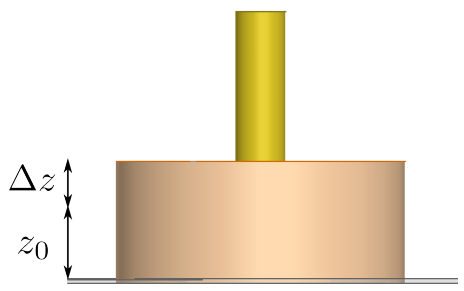

(c)

Figure 10.38: Several possible errors caused by the manufacturing process of the filter; a) Error in the pin width $\Delta w_{p}$. Values $\Delta w_{p}>0$ indicate that manufactured pins are greater than expected whereas cases $\Delta w_{p}<0$ indicate that manufactured pins are smaller than expected; b) Error in the horizontal positioning $\left(\Delta x_{0}, \Delta y_{0}\right)$ of the feeding. The vector in the schematic correspond to $\left(\Delta x_{0}>0, \Delta y_{0}>0\right)$. Other signs follow the usual Cartesian geometry orientation; c) Error in the vertical positioning of the feeding (penetration) $\Delta z_{0} . \Delta z_{0}>0$ indicates that the manufactured prototype presents larger penetration than expected whereas $\Delta z_{0}<0$ indicates that the manufactured prototype presents smaller penetration than expected.

\section{Pin width error $\Delta w_{p}$}

The error in the the pin width $\Delta w_{p}$ models an average systematic deviation that is considered to be present in every pin. Of course, random individual deviations will 
occur, however, they are assumed as a second order, negligible when compared with the average deviation. In this way, the term $\Delta w_{p}$ refers to all pins of the structure. This error may be easily caused by the mechanical vibrations of the milling cutter during the milling process. In the Design $1, \Delta w_{p}=-50 \mu \mathrm{m}$ was observed, an error that, although small, caused an appreciable deviation. By studying the influence of this error, the expected response given the precision of the milling machine can be estimated. Note that, although systematic errors such as those observed in the Design 1 may be mostly avoided by passing compensated dimensions to the milling machine, however, some residual tolerance error will be always present. In the case of the in-house process with the Datron ${ }^{\circledR}$ M25 milling machine system, a minimum tolerance error of $\pm 10 \mu \mathrm{m}$ will be easily found.

Results of this study are shown in Fig. 10.39. Several cases between $\Delta w_{p}=$ $\pm 5 \mu \mathrm{m}$ and $\Delta w_{p}= \pm 20 \mu \mathrm{m}$ have been analyzed. This source of error concentrates its influence on the frequency shift of the response, return losses and passband bandwidth are practically unaffected. Note that, changing the pin width automatically changes the cavity dimensions. Since the response is narrow-band and the operation frequency is considerably high, a large sensitivity is observed. Even small values of $\Delta w_{p}$ shift the resonance frequency of the cavities. Observe that with only $\Delta w_{p}= \pm 5 \mu \mathrm{m}$, Fig. 10.39 (a), a frequency shift is already observable, and with $\Delta w_{p}= \pm 10 \mu \mathrm{m}$ (estimated lowest achievable precision with the Datron ${ }^{\circledR}$ M25 milling machine system), Fig. 10.39(b), the frequency shift is not longer a minor problem. With $\Delta w_{p}= \pm 20 \mu \mathrm{m}$, which is a reasonable tolerance, the response is shifted practically half of the passband bandwidth, Fig. 10.39(d). Actually, the observed frequency shift follows a $35 \mathrm{MHz} / \mu \mathrm{m}$ lineal law. These results indicate that the scenario is quite challenging and that efforts must be put in minimizing as maximum this error. Unfortunately, as commented before, several microns of error will be always present, so that post-processing techniques may result very welcome. This aspect is treated in Design 3. Finally, it is worth to mention that the observed high sensitivity is not a particular feature arising from the small geometrical dimension of the pins or similar reasons of the particular GGW characteristics. In fact, this is caused by the elevated operation frequency so that any kind of structure is suitable to experience similar results in an error sensitivity analysis. Note that, compared to the these days widely used $L, S$ or $C$ microwave bands, the precision required for the $V$ band is 10 to 30 times higher.

\section{Feeding position errors $\Delta x_{0}, \Delta y_{0}$ and $\Delta z_{0}$}

The error in the feeding position models the possible errors of positioning of the milling cutter during the manufacturing process and affects exclusively to two elements: input and output connectors. The feeding position error is split in its horizontal component $\left(\Delta x_{0}, \Delta y_{0}\right)$ and its vertical component. In addition, regarding the horizontal component error, influence of $\Delta x_{0}$ and $\Delta y_{0}$ is studied separately.

First, the error $\Delta x_{0}$ is evaluated through Figs. 10.40(a)-10.40(f). As it can be appreciated in the plots, for this source of error, contrarily to the previous case, any frequency shift is observed. However, it affects the return losses. Nevertheless, the response sensitivity is low to this error. For instance, with an error of $\Delta x_{0}=$ $\pm 20 \mu \mathrm{m}$, Fig. 10.40(b), the filter response specifications are still meet. Even more, the response with $\Delta x_{0}= \pm 40 \mu \mathrm{m}$, Fig. $10.40(\mathrm{c})$, is still acceptable $(R L<15$ 


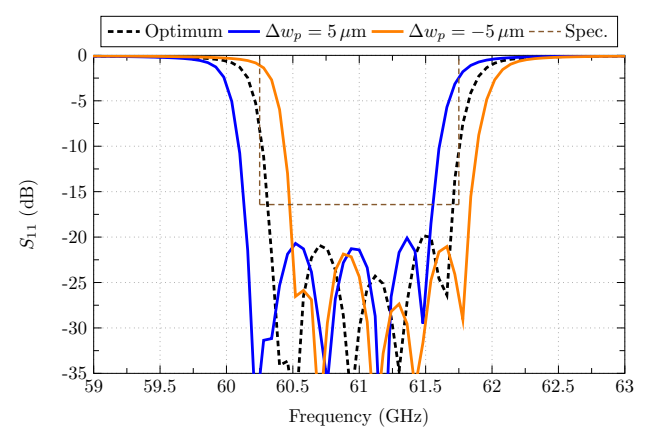

(a)

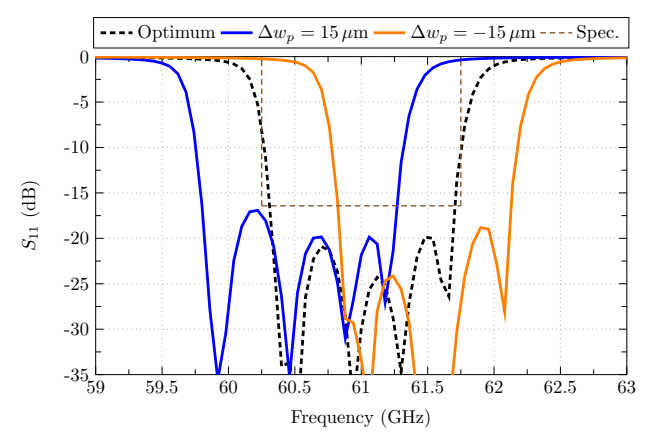

(c)

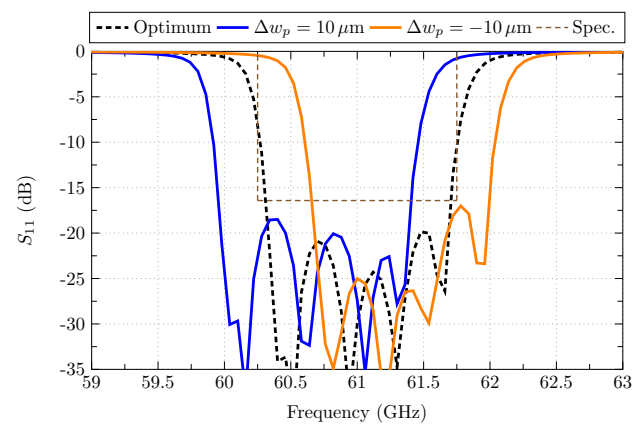

(b)

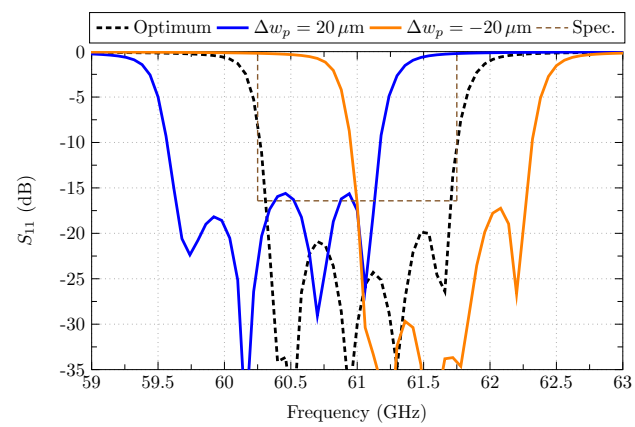

(d)

Figure 10.39: Filter response sensitivity to pin width errors $\Delta w_{p}$. a) $\left.\Delta w_{p}= \pm 5 \mu m ; b\right) \Delta w_{p}=$ $\pm 10 \mu \mathrm{m}$; c) $\Delta w_{p}= \pm 15 \mu \mathrm{m}$; d) $\Delta w_{p}= \pm 20 \mu \mathrm{m}$;

dB). Furthermore, note that when dealing with this error, since only return losses are affected (the passband remains satisfying the specifications), only the bandpass ripple is affected. In the worst considered case, $\Delta x_{0}= \pm 100 \mu \mathrm{m}$ (rarely this poor tolerance will be obtained in practice), $R L>10 \mathrm{~dB}$, which, although far from the specifications, is still a commonly found value in the literature for filter prototypes, all the more, if it refers to a V-band narrow-band filter. Therefore, it can be stated that the influence of this error will generally remain as a second order effect.

Now, the influence of the error $\Delta y_{0}$ is studied in Figs. 10.41(a)-10.41(f). As the plots reflect, the influence of this source of error is even weaker than that of $\Delta x_{0}$. In fact, even for the worst considered case, $\Delta y_{0}= \pm 100 \mu \mathrm{m}$, the response is practically not affected and still meets the specifications, see Fig. 10.41(f). The reason for this is easy to understand. The horizontal positioning error affects the return losses through its influence in the external cavity factor $Q_{e x}$ (bandwidth modification and frequency shift do not appear since these features depend on the filter layout geometrical dimensions). When moving in the $\hat{x}$ direction, the variation in $Q_{e x}$ is larger since the field intensity at the position of the probe has larger variation. Actually, note $x_{0}$ and $y_{0}$ are the distances in each direction $(\hat{x}$ and $\hat{y}$ ) to the cavity center (maximum field) and $x_{0}=0.8 \mathrm{~mm}>0.5 \mathrm{~mm}=y_{0}$. Therefore, without entering in deeper mathematical formalisms, it is clear that, according to the bi-dimensional sinusoidal 


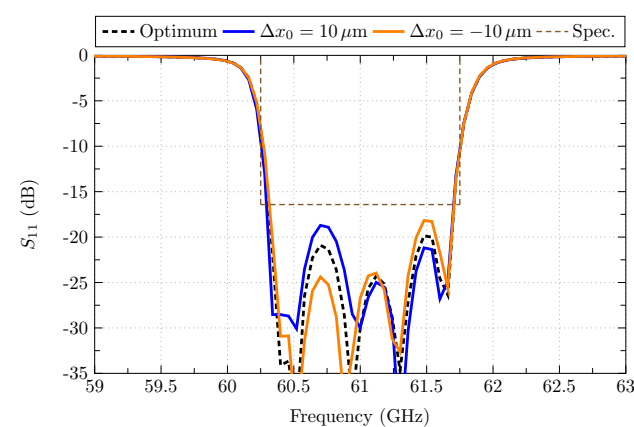

(a)

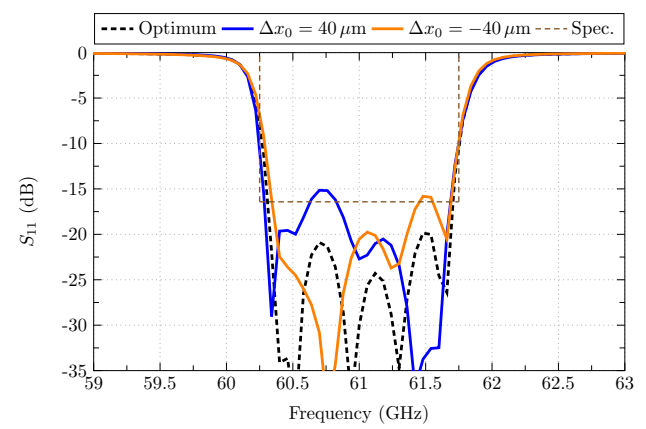

(c)

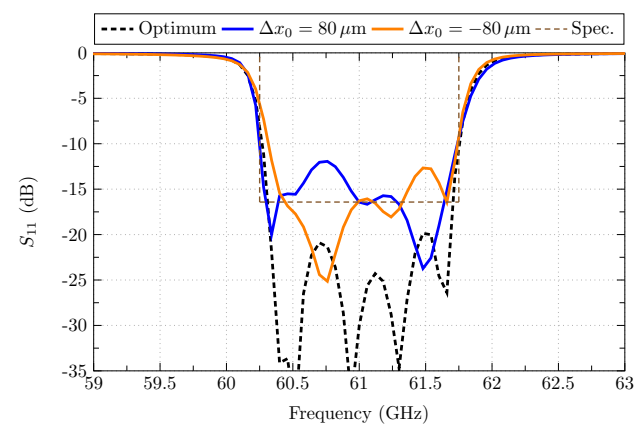

(e)

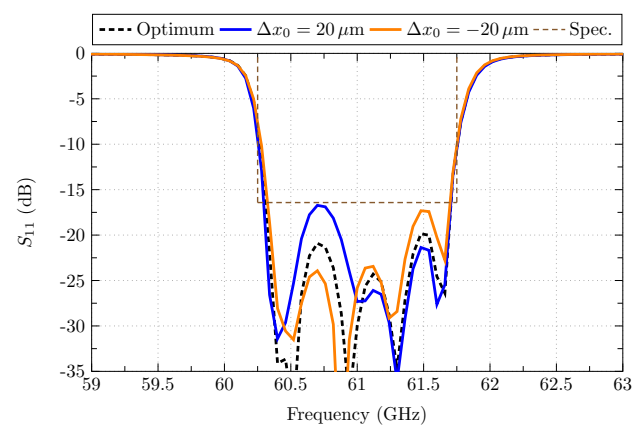

(b)

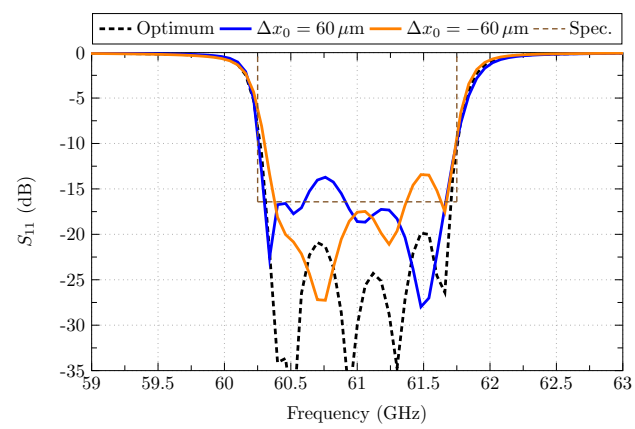

(d)

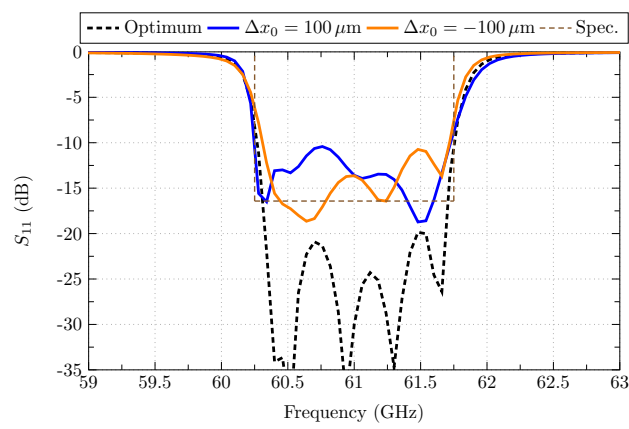

(f)

Figure 10.40: Filter response sensitivity to pin width errors $\Delta x_{0}$. a) $\left.\Delta x_{0}=10 \mu m ; b\right) \Delta x_{0}=$ $20 \mu \mathrm{m}$; c) $\Delta x_{0}=40 \mu \mathrm{m}$; d) $\Delta x_{0}=60 \mu \mathrm{m}$; e) $\Delta x_{0}=80 \mu \mathrm{m}$; f) $\Delta x_{0}=100 \mu \mathrm{m}$;

distribution of the E-field magnitude in the cavity, $\partial Q_{e x} / \partial x_{0}>\partial Q_{e x} / \partial x_{0}$. Consequently, these results alleviate even more the required precision in the horizontal positioning of the connector. In addition, through this analysis, an interesting conclusion is extracted regarding the employed feeding system: the larger the original $x_{0}$ and $y_{0}$ (the further the connector from the center, i. e., located near the cavity corners), the weaker the influence of the horizontal positioning error. Therefore, whenever possible it is convenient to preferably choose large $x_{0}$ and $y_{0}$. 


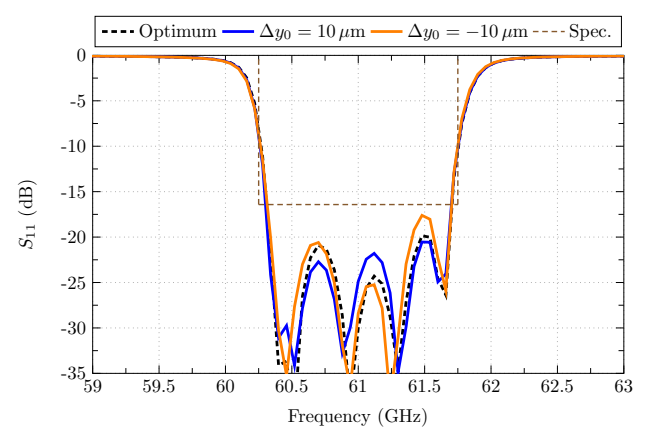

(a)

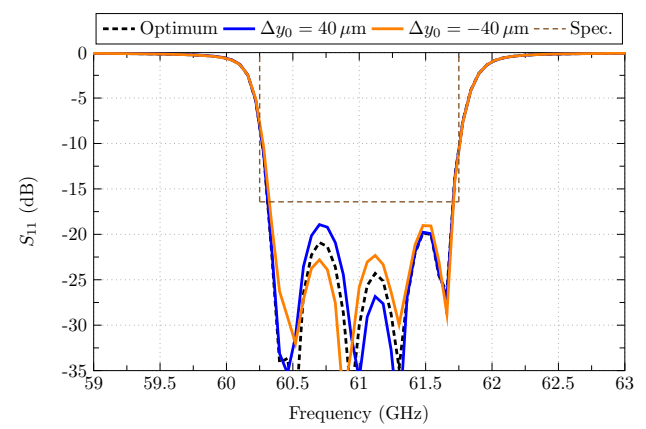

(c)

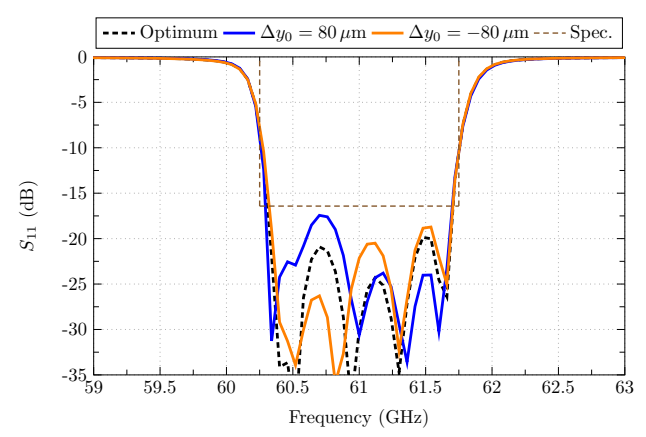

(e)

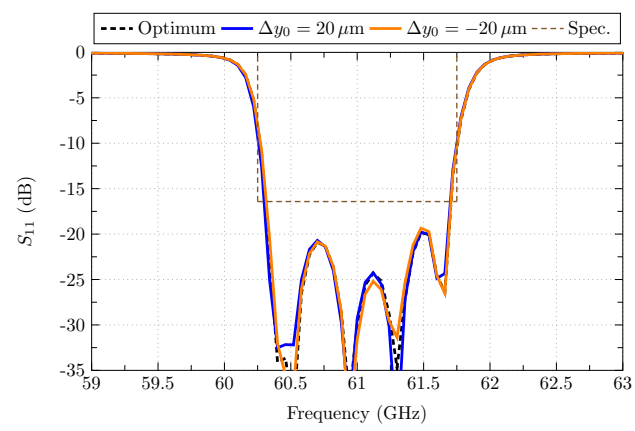

(b)

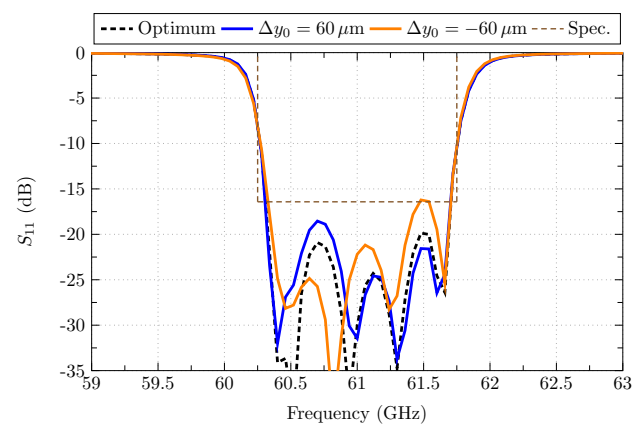

(d)

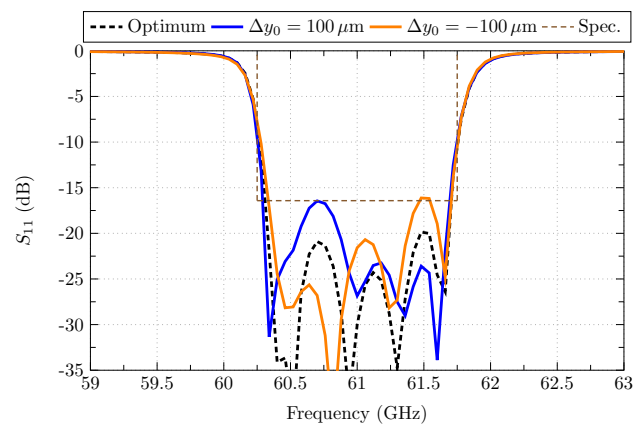

(f)

Figure 10.41: Filter response sensitivity to pin width errors $\Delta y_{0}$. a) $\Delta y_{0}=10 \mu \mathrm{m}$; b) $\Delta y_{0}=$ $\pm 20 \mu \mathrm{m}$; c) $\Delta y_{0}= \pm 40 \mu \mathrm{m}$; d) $\Delta y_{0}= \pm 60 \mu \mathrm{m}$; e) $\Delta y_{0}= \pm 80 \mu \mathrm{m}$; f) $\Delta y_{0}= \pm 100 \mu \mathrm{m}$;

Finally, the influence of $\Delta z_{0}$ is analyzed. Figs. 10.42(a)-10.42(d), where errors from $\Delta z_{0}= \pm 10 \mu \mathrm{m}$ to $\Delta z_{0}= \pm 40 \mu \mathrm{m}$ are studied, show the effect of this source of error on the filter response. As in the horizontal case, only return losses are affected for this source of error. However, the vertical positioning error (or penetration) clearly has a more critical influence than the horizontal positioning. Specifications are lost if $\Delta z_{0}= \pm 20 \mu \mathrm{m}$, see Fig. $10.42(\mathrm{~b})$, and return losses rapidly degrade as $\Delta z_{0}$ increases. With $\Delta z_{0}= \pm 40 \mu \mathrm{m}, R L>12 \mathrm{~dB}$ and, with $\Delta z_{0}= \pm 50 \mu \mathrm{m}$ (not shown), 
$R L>10 \mathrm{~dB}$. As in the previous case, these results can be easily understood if one regards the influence of $z_{0}$ on the external quality factor. Note first that the original value $z_{0}=300 \mu \mathrm{m}$ is larger than the considered displacements $\Delta z_{0}$. This meas that $\Delta z_{0}$ indicates that more (less) section of the bulkier part of the connector enters, $i$. e., the section with a dielectric surrounding $\left(d_{\text {con }}=0.228 \mathrm{~mm}, d_{\text {teflon }}=0.737 \mathrm{~mm}\right)$. It can be qualitatively intuited that perturbation variation is quite higher with this movements, that noticeably modify the total metallic volume inside the cavity, than with horizontal movements.

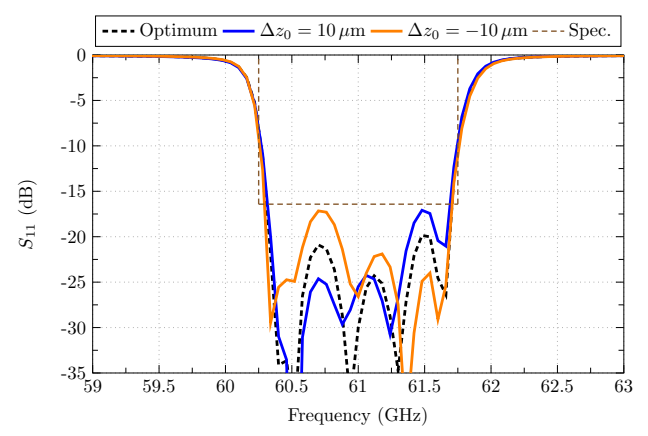

(a)

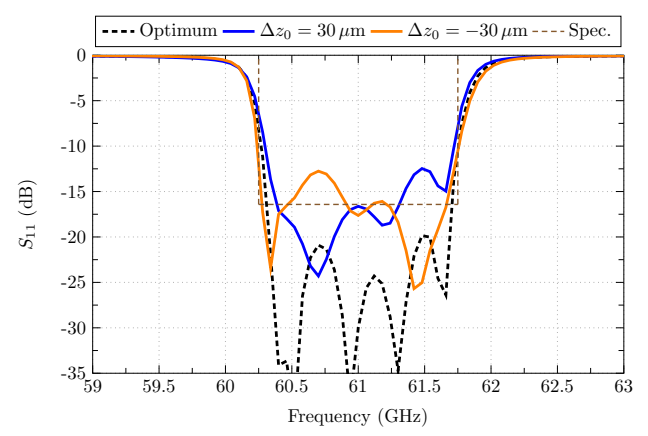

(c)

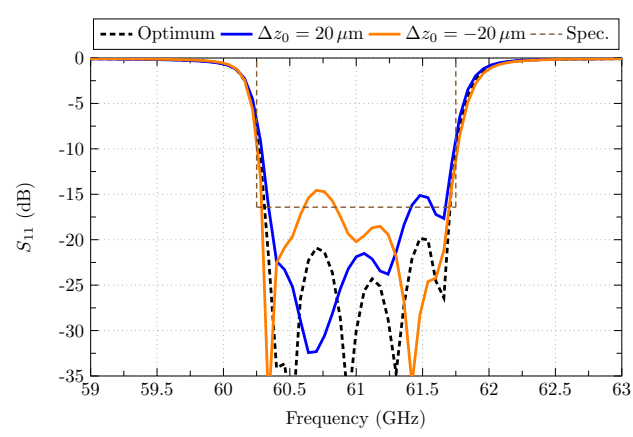

(b)

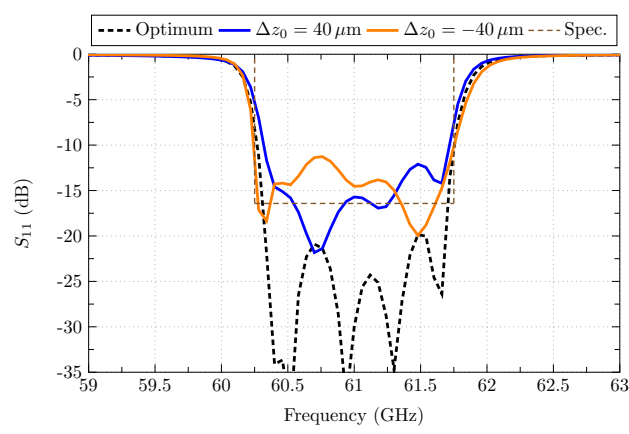

(d)

Figure 10.42: Filter response sensitivity to pin width errors $\Delta z_{0}$. a) $\Delta z_{0}= \pm 10 \mu \mathrm{m}$; b) $\Delta z_{0}=$ $\pm 20 \mu \mathrm{m}$; c) $\Delta z_{0}= \pm 30 \mu \mathrm{m}$; d) $\Delta z_{0}= \pm 40 \mu \mathrm{m}$.

Nevertheless, contrarily that what can be though at first view, the problem of this source of error does not reside in the absolute values of the maximum admitted tolerance to keep the response close to the specifications. Actually, it is the difficulty in controlling the connector penetration what makes this source of error a critical one to give treatment. Note that, the horizontal positioning is an automatized task that depends on the milling machine whereas the penetration is rather manually adjusted during the filter assembling. Since the critical distances are of few tens of microns some strategy seems necessary to prevent an important degradation depending on the technician abilities. The adopted solution will be exposed later in the section 10.3.7.4, dedicated to the filter manufacturing process and experimental results. 
In summary, the carried out sensitivity analysis regarding manufacturing errors concludes in that the most critical errors regard to the pin width $\Delta w_{p}$ and the connector penetration $\Delta z_{0}$. The case of $\Delta w_{p}$ results specially delicate inasmuch as a frequency shift in narrow-band filters is always more critical than a slight worsening of return losses. In addition, it seems not possible to reduce $\Delta w_{p}$ till the required levels due to the inherent limitations of the in-house fabrication process. In the case of $\Delta z_{0}$ the challenge comes from the required small tolerances the an adjustment process that is rather manual. However, some techniques and elements will be introduced to have a more precise control on the precision related with this source of error.

\subsubsection{Manufactured prototype and measurements}

With the optimum dimensions indicated in Table 10.11 and taking into account the conclusions obtained through the previous error sensitivity analysis, the manufacturing of the filter is finally tackled. Fig. 10.43(a) shows a photography taken during the milling process carried out with the Datron ${ }^{\circledR}$ M25 milling machine system. The final assembled filter, including the two $1.85 \mathrm{~mm}$ coaxial connectors, is shown in Fig. 10.43(b). The two independent parts composing the filter are shown in Fig. $10.43(\mathrm{c})$. In this photography it can be appreciated how all the elements and design complexity resides in the bottom piece. The four supporting blocks at the corners of this piece guarantee the required distance $h_{a}$ between pins and top plate. Only four screws are employed to assemble the piece and guarantee proper alignment. Note that the practical implementation of this second design improves that of the first design (see Fig. 10.28) in terms of compactness and weight inasmuch as the bottom piece width has been restricted to the filter layout and the supports, adopting a bone shape. By construction, the filter has not metallic lateral walls and the sides are exposed to the outside, which results convenient for temperature dissipation either passive of driven by a ventilation system. Finally, a detail of the bottom piece is shown in Fig. 10.43(d) in order to evince the filter elements size.

After the manufacturing process, a dimensional study has been carried out by means of a SEM system. A detail of a pin can be appreciated in Fig. 10.44(a), whereas a detail of the feeding hole for the coaxial connector is shown in Fig. 10.44(b). Certain imperfections are easily appreciated in this last image. Fig. 10.44(c) shows the detail of an auxiliary piece manufactured ad hoc to ensure the proper penetration $z_{0}$ of the feeding connector. Attending to the conclusions of the manufacturing error sensitivity analysis of section 10.3.7.3, the use of this piece seems very recommendable. Note that, in this way, the tolerance error is not longer produced by a human but by the precision of the milling machine in the manufacturing of this control piece. Finally, Fig. 10.44(d) show the post-processing procedure of the images, which gives the final dimensional measurements. This analysis indicates that, approximately, $\Delta w_{p}=-10 \mu \mathrm{m}, \Delta x_{0}=20 \mu \mathrm{m}, \Delta y_{0}=40 \mu \mathrm{m}$, and $\Delta z_{0}=10 \mu \mathrm{m}$. Therefore, recovering the results of the manufacturing error sensitivity analysis, the main expected drawback is certain displacement of the response towards lower frequencies. In addition, the accumulated contribution of the different sources of error is expected to show some small influence in the return losses. 


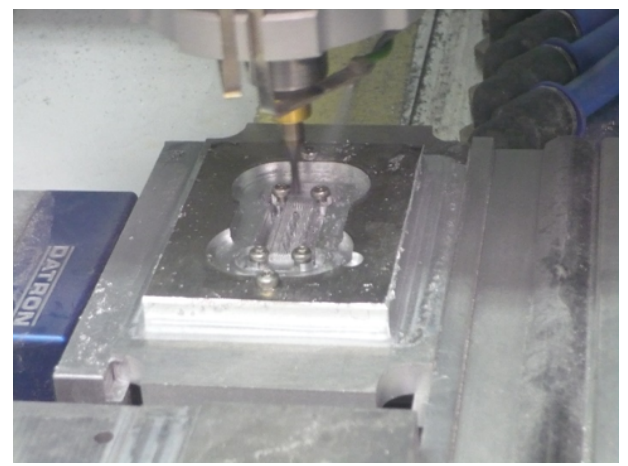

(a)

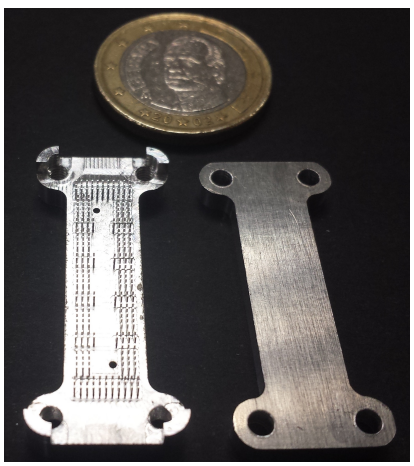

(c)

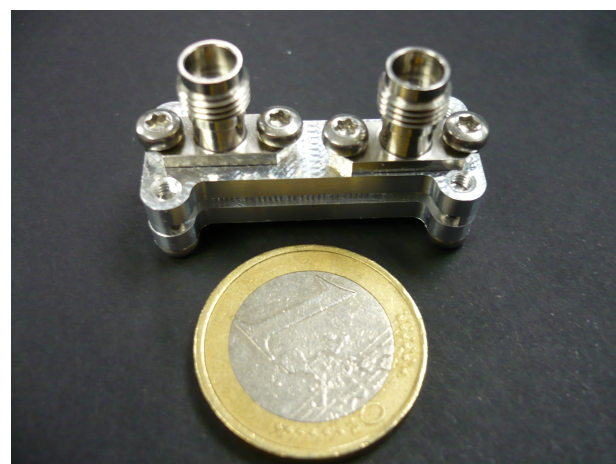

(b)

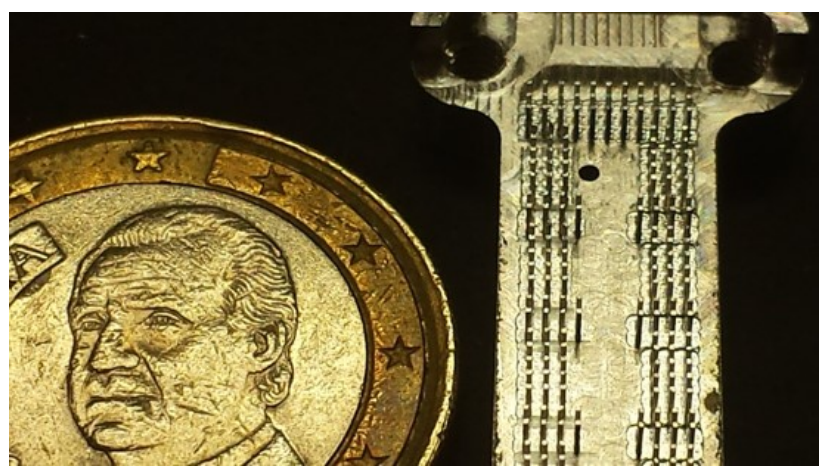

(d)

Figure 10.43: Manufactured prototype. a) Milling process with the Datron ${ }^{\circledR}$ M25 milling machine; b) Assembled filter; c) Bottom (left) and top (right) pieces of the filter; d) Detail of the filter elements.

The experimental results are finally treated now. Fig. 10.45(a) shows the measured response (curves with marks) and compares it with the simulated one (solid curves). As expected by the previous analysis, a displacement towards lower frequencies is appreciated. The curves are moved $\Delta f=-0.79 \mathrm{GHz}$, which represents only $1.3 \%$ respect to the central frequency, however, the narrow-band nature of the filter makes this displacement to be relevant. Return losses show good aspect. The only remarkable degradation, of minor importance, is a peak with level $-13 \mathrm{~dB}$ at the left side of the passband, that originates certain chamfering of the transmission response. This effect can be better appreciated in Fig. 10.45(b), which shows a detail of the $S_{21}$ parameter. It can be said that the obtained response shows a quite flat aspect in the passband despite of the commented slight chamfering. They must be highlighted the achieved low insertion losses $(I L=1.5 \mathrm{~dB})$ and the sharp cutoffs at the sides, with same sharpness as in the simulated response. Differences between simulated and measured results may be arising from the accumulated effect of several sources of error such as residual leakage or lower conductivity due to metal roughness, an effect to take into account at high frequencies. Finally, Fig. 10.45(c) shows the experimental response on a frequency range spanned to $[40 \mathrm{GHz}-70 \mathrm{GHz}]$. 


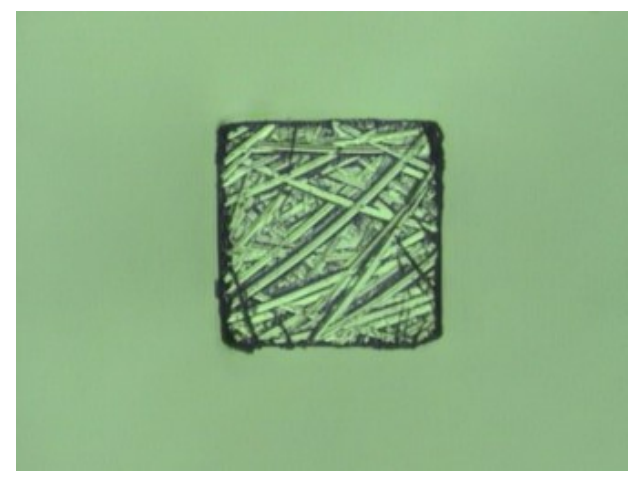

(a)

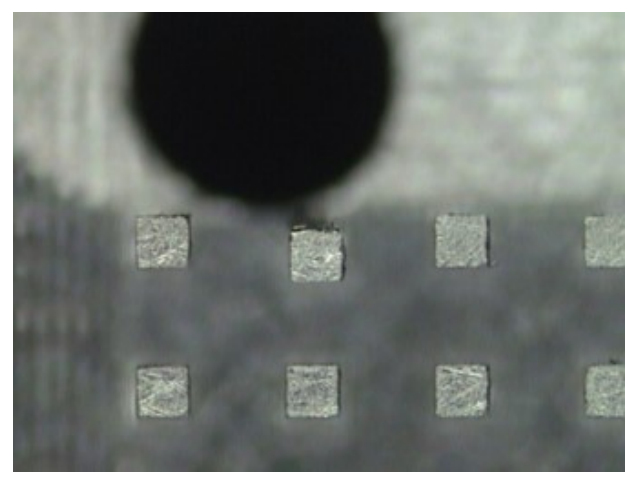

(b)

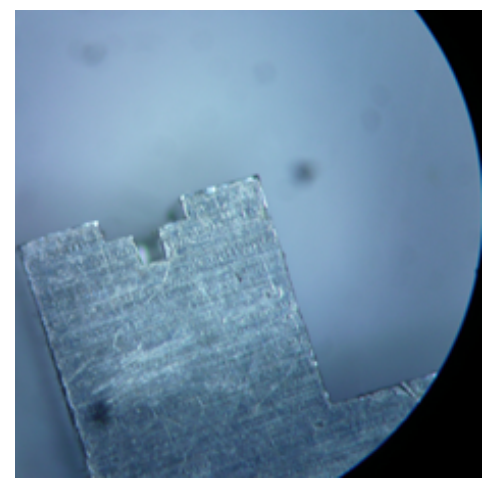

(c)

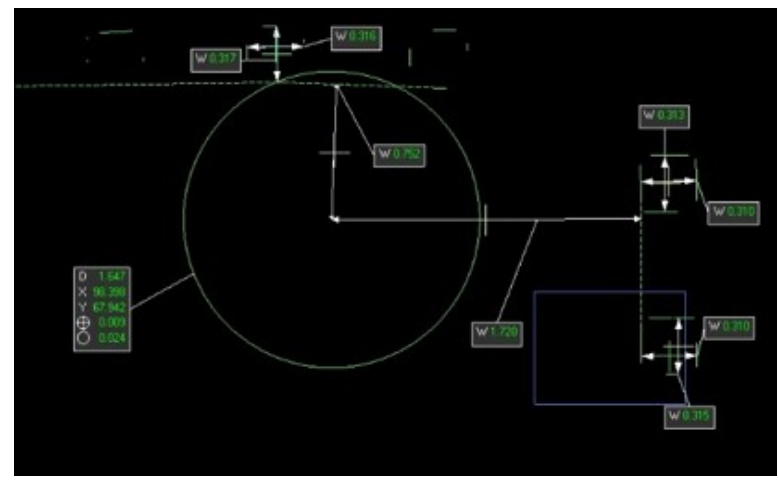

(d)

Figure 10.44: Dimensional analysis. a) Detail of a pin; b) Detail of the feeding hole for the coaxial connector; c) Detail of the piece manufactured to ensure the proper penetration of the connector, $z_{0}$; d) Post-processing of the images to determine the elements size.

As it can be appreciated, no spurious is present and the rejection level is kept below $50 \mathrm{~dB}$ at the right side of the response and below $60 \mathrm{~dB}$ at the left side. This is an experimental evidence of the stopband ([43 GHz-75 GHz] in this design) effect of the GGW structure.

Despite of the observed frequency shift, a common degradation in V-band prototypes [973]-[975], the presented prototype results competitive for the V-band due to its outstanding low insertion losses. In addition, good selectivity and a flat response are obtained. GGW proves by means of Design 2, a high potential to implement V-band NBFs. The conclusions of Design 1 have lead to satisfactory improvements in this second design. However, the main problem, which is the frequency shift, still remains. Overcoming it would lead the presented topology to achieve a very competitive performance. Of course, better manufacturing tolerances are a way to solve it. Nevertheless, solutions to this problem that do not rely on the manufacturing precision are welcome inasmuch as the fabrication cost is reduced. The design 3, presented next, faces the frequency shift issue in a thoughtful way, exploiting the 


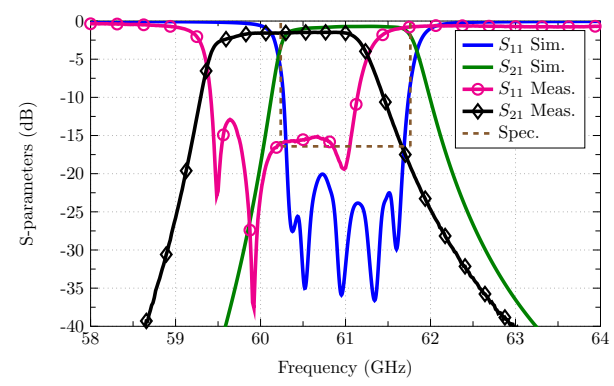

(a)

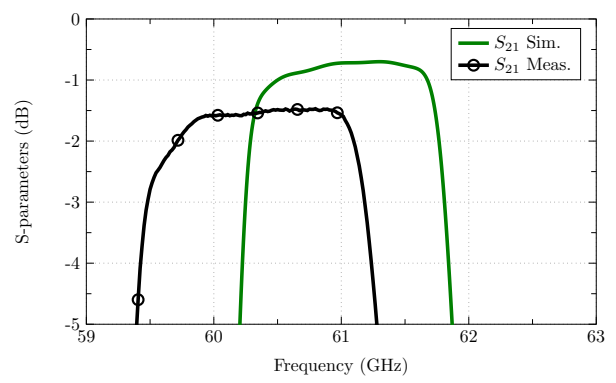

(b)

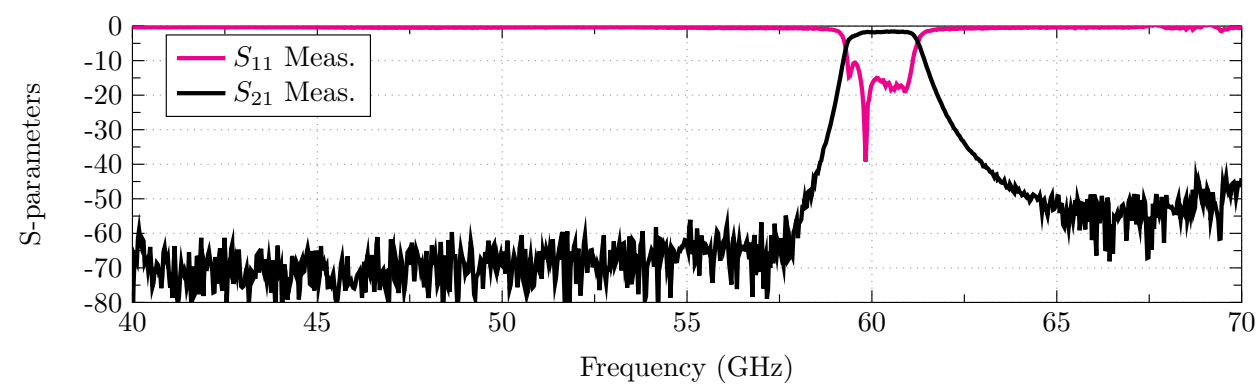

(c)

Figure 10.45: Experimental results. Comparison between the measured response a the optimum simulated response. a) $S$-parameters; b) $S_{21}(\mathrm{~dB})$ detail. c) Frequency span in the range [40 $\mathrm{GHz}-70 \mathrm{GHz}]$.

unique GGW topological characteristics to provide an improved response with the same manufacturing system.

\subsubsection{Design 3: $5^{\text {th }}$-order V-band filter at $f=60 \mathrm{GHz}$, in- cluding post-manufacture tuning metal insertions}

The third design consists in a $5^{\text {th }}$-order Chebyshev filter operating at $f=60 \mathrm{GHz}$, in the V-band, with post-manufacturing tuning possibilities. Therefore, the design is pretty similar to the previous one, however, certain elements are included in the topology in order to correct the response if required. In this way, Design 3 faces mainly the remaining problem of the response frequency shift due to manufacturing tolerances. In addition, the possibility of correcting the response allows as well to wide or to narrow the response, or to improve return losses (which are rather secondary issues regarding the previous two designs), if this is desired.

The tuning functionality is done by placing some metallic insertions on the top plate piece, which act over the filter elements, performing adjustment of the basic parameters of the filter (resonance frequency, coupling and external quality factor of the feeding elements). This solution is more appropriate than using tuning screws, whose size would hardly fit the design requirements, and might cause PIM or multipaction effects [976]-[977]. Of course, this choice implies that any change of the 
tuning element dimensions require a new top plate with fixed insertions. Nevertheless, this piece is a simple and cheap one compared to the bottom piece with the filter layout. In addition, in order to minimize the number of required top plates with insertions, an efficient algorithm is applied: the Aggressive Space Mapping (ASM) [851], [955]. Following, [729], [978], ASM is applied assuming full-wave simulation with HFSS ${ }^{\circledR}$ as coarse model and manufacturing process as fine model. Since ASM is designed to minimize the number of evaluations of the fine model to converge, a reduced number of manufactured top plate with insertions will be required.

\subsubsection{Filter specifications}

The filter specifications are listed in Table 10.12 and the ideal response is shown in Fig. 10.46. The only difference regarding the previous case is the central frequency which now is $f_{0}=60 \mathrm{GHz}$ instead of $f_{0}=61 \mathrm{GHz}$. Therefore, the normalized elements of the low pass prototype remain exactly the same. The synthesis procedure is omitted being that is practically identical to the previous design with the only exception of taking into account the presence of the insertions in every step, i. e., the insertions are already present when synthesizing the cavities, the coupling and the feeding. The reason for this is given next.

\begin{tabular}{cccc} 
Parameter & Frequency $f_{0}$ & Relative Bandwidth $(\mathrm{RBW})$ & BP Ripple $R(R L)$ \\
\hline Value & $60 \mathrm{GHz}$ & {$[59.25 \mathrm{GHz}-60.75 \mathrm{GHz}](2.5 \%)$} & $0.1 \mathrm{~dB}(16.42 \mathrm{~dB})$ \\
\hline
\end{tabular}

Table 10.12: Filter response specifications.

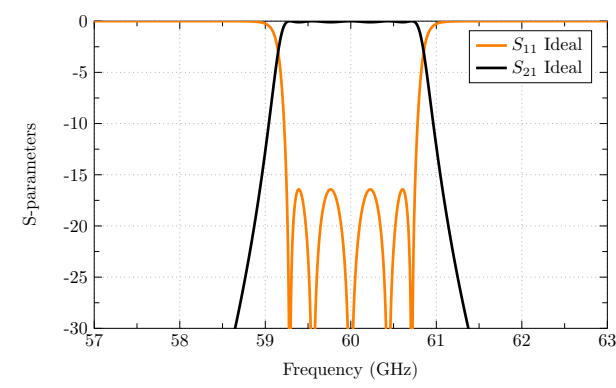

(a)

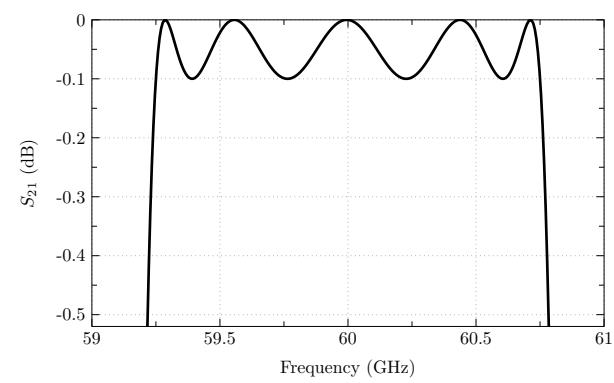

(b)

Figure 10.46: Ideal response. a) S-parameters; b) $S_{21}$ (dB) detail.

\subsubsection{Tuning insertions topology}

The physical magnitudes that control the filter response are the cavities resonance frequency, the coupling between cavities and the external quality factor of the feeding. Consequently, it is appropriate to dedicate one insertion for every cavity, every coupling window and every feeding point. For the employed filter topology this makes a total of 11 insertions: 5 cavity insertions, 4 coupling window insertions and 2 feeding insertions, see Fig. 10.47(a). However, thanks to the filter symmetry, in 
principle, it is only necessary to adjust 6 of them, those for the first 3 cavities, the first 2 windows, and the input feeding element, see Fig. 10.47(b).

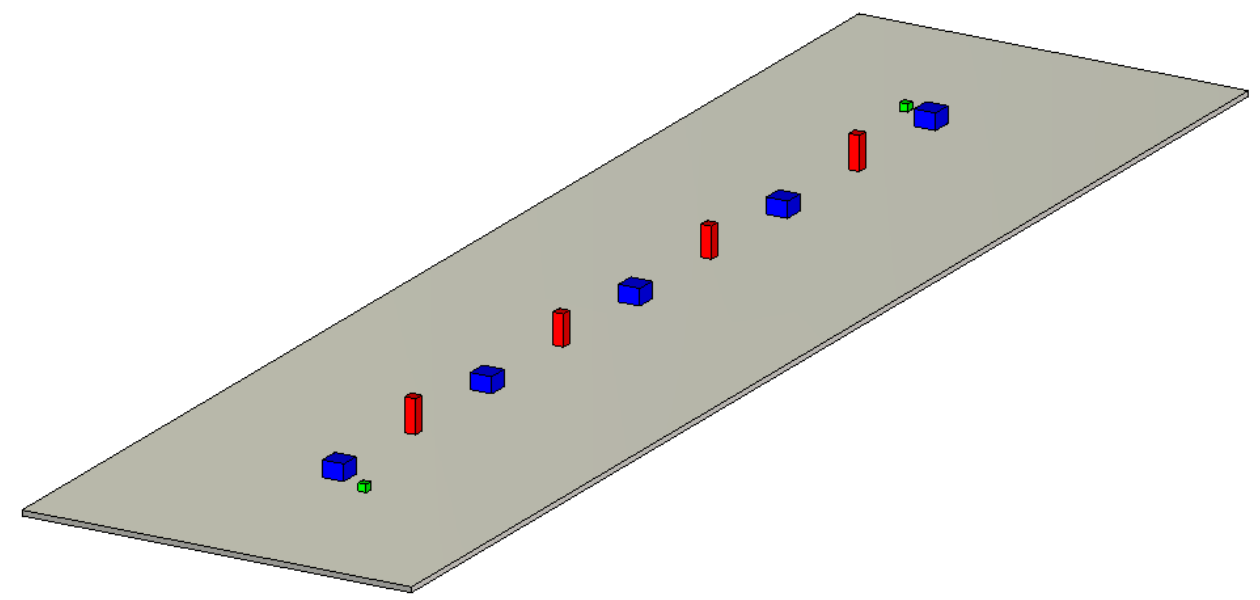

(a)

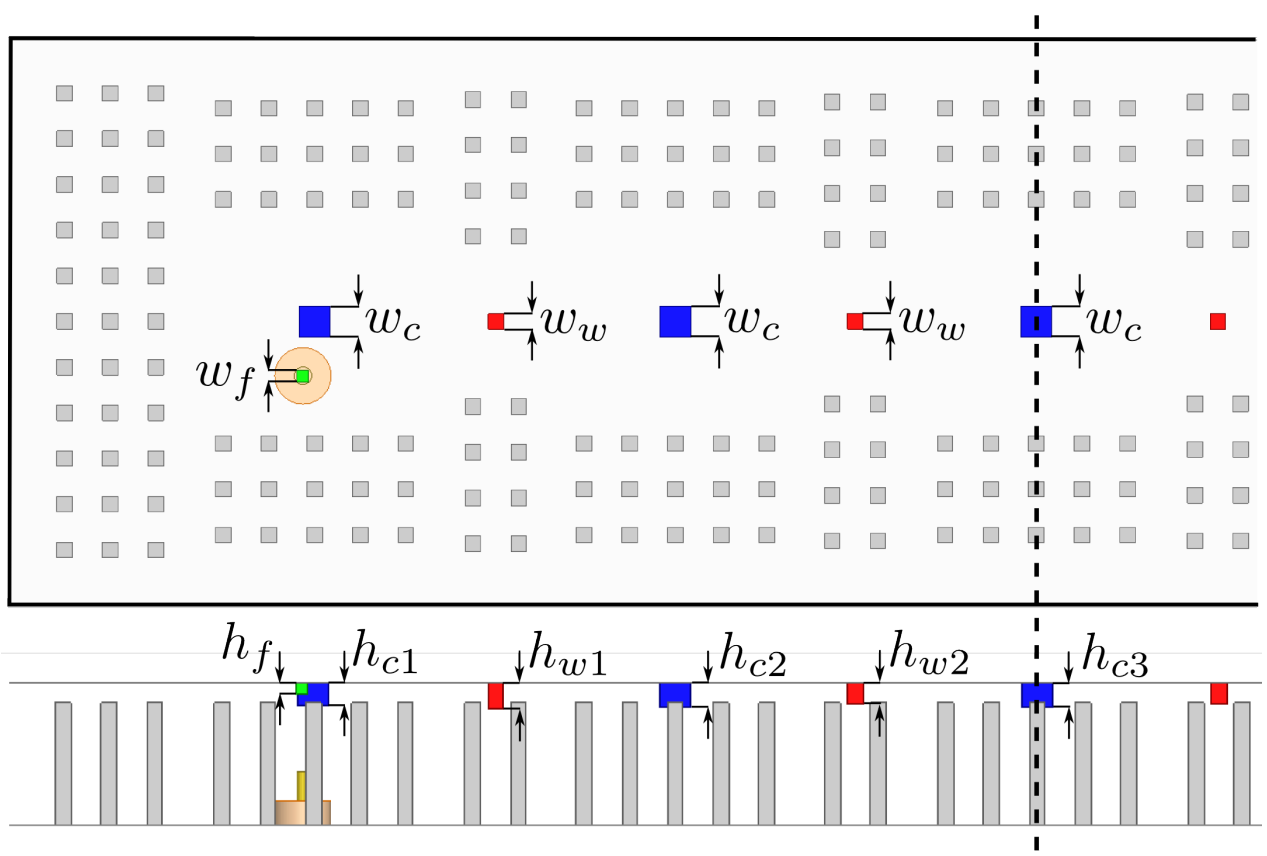

(b)

Figure 10.47: Tuning insertions topology. a) $3 D$ view schematic of the milled top plate with the metallic insertions; b) $S_{21}(\mathrm{~dB})$ detail. b) $2 D$ top and lateral views of the insertions in the filter layout. The shape of all insertions is square.

Regarding to the synthesis process, note the following important detail. At the first manufactured prototype, insertions of certain size must be present since every 
of the tunable physical parameters may require to be increased or decreased. For instance, note that if the obtained response required to be shift up in frequency, the next top plate with the insertions would require smaller insertions than the current top plate. If any insertion is present or if they are not large enough, any correction could be applied and the process would fail. This implies that insertions of certain size must be present during the synthesis procedure.

The width of the insertions has been fixed to $w_{c}=0.4 \mathrm{~mm}, w_{w}=0.2 \mathrm{~mm}$ and $w_{f}=0.15 \mathrm{~mm}$, so only 6 variables (the height of the insertions) are considered in the ASM process: $h_{c 1}, h_{c 2}, h_{c 3}, h_{w 1}, h_{w 2}$ and $h_{f}$. This leads to an optimization with a good trade-off between degrees of freedom (number of optimization variables) and complexity. Note that ASM is going to be applied using a full-wave simulation of a complex structure as a coarse model, which is evaluated a considerable number of times. Actually, if the number of iterations to converge is $N$, the computational cost will be $N$ times of that to design a single filter. Therefore, to choose a reduced number of optimization variables becomes critical in the process.

\subsubsection{Aggressive Space Mapping (ASM)}

The main concepts and procedure of the Aggressive Space Mapping (ASM) are explained now. ASM was firstly proposed in [955], as a particular version of the general case classic Space Mapping (SM) [850] very suitable for those case in which fine model evaluations are noticeably costly. Leaving apart for the moment, mathematical formalities, as its name indicates, a SM is a mapping that give correspondence between points of two spaces. In the context treated here, two points, one from the coarse domain space and other from the fine domain space, have correspondence through the mapping if they provide the same image. This will be better understood next by particularizing the case to the design of interest.

Consider Fig. 10.48. The domain of both spaces, coarse and fine, is defined by the design parameters as variables, in this case, the heights of the insertions $\left(h_{c 1}, h_{c 2}, h_{c 3}, h_{w 1}, h_{w 2}, h_{f}\right)$. In the same way, image of both spaces is the filter response. The difference between both spaces is the function (mathematical model) that maps a point from the domain to the image, i. e., how the filter response is obtained from the design parameters. In the case of the coarse space, this is done by full-wave simulations, whereas in the case of the fine space it is done by manufacturing a prototype and measuring its response. Since the measured response is, indeed, the real one given certain dimensions, and the simulated one is only one approximation, it is clear why the terms coarse and fine are employed. Note now that, as the function (model) employed in each of the spaces is different, the same domain point (same filter parameter values) does not produce the same response in each domain. What the SM provides is a correspondence that links the point of the coarse space with the point of the fine space that provides exactly the same response. A complete SM gives this correspondence for every point in the domain.

By the previous argumentation, it is clear that the coarse model is quite faster to evaluate than the fine model, but gives inexact results. It can be also foreseen that if the SM was known, the following strategy would result noticeably efficient: to optimize in the coarse domain until the optimal design parameters are found and then, through the SM, to obtain the actual design parameters that will ensure 


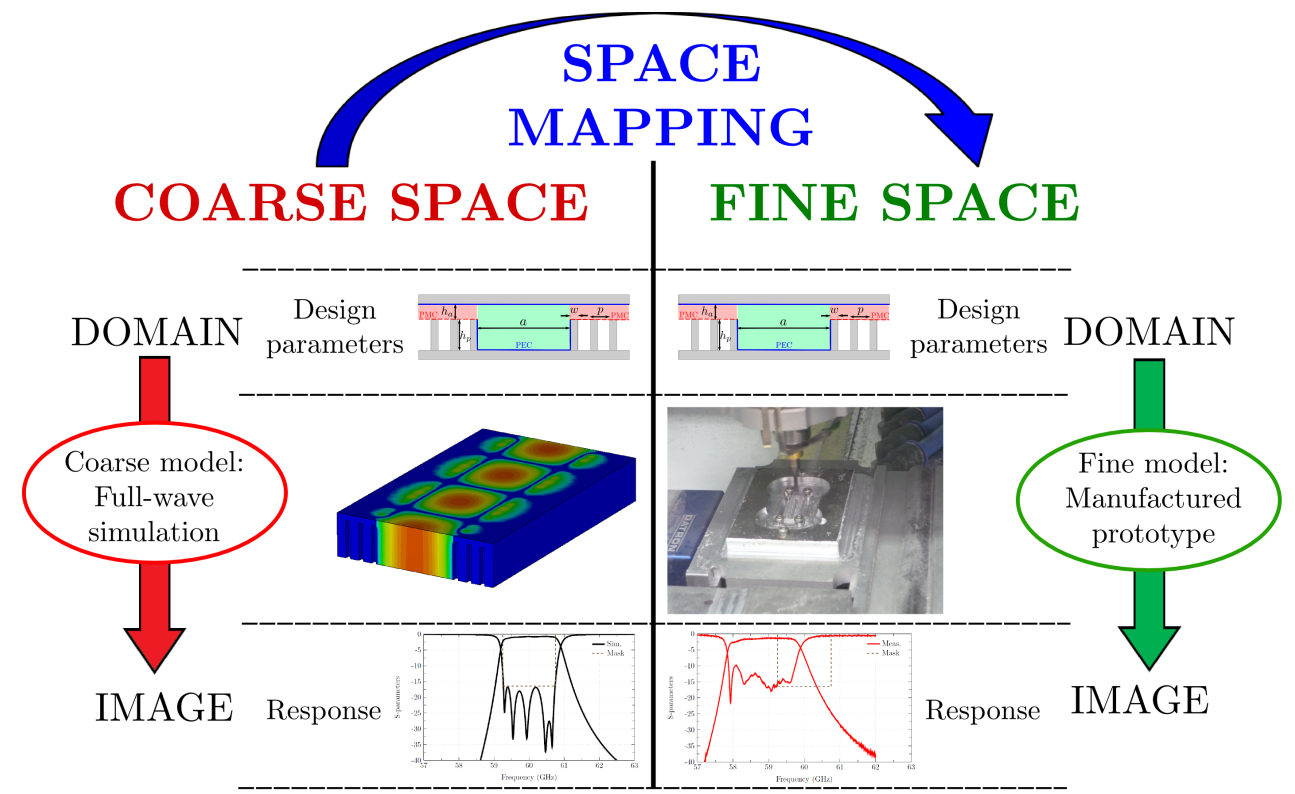

Figure 10.48: Conceptual schematic of the Space Mapping used for the Design 3.

that the response meet the specifications in the real world. In the context of the present design this means that optimization is done by simulation (as usual), but the parameters passed to the manufacturing process are corrected by the SM. For instance, a trivial case could be that the manufacturing process makes every element dimension larger by a factor 1.25. Then the SM would be known, and it would apply a correction factor of 0.8 to every dimension.

The reality is, generally, more ill-posed so that the SM between spaces is not evident but noticeably complex. In fact, to know the SM at every point of the domain is a much more complex problem than that of finding the optimum in the fine space. Therefore, what is done is to estimate the SM to know it at several points and their neighborhood through the Jacobian of the SM, this last also estimated. This process can be done in several ways, each one defining a concrete SM strategy. In the original SM [850], it is found first the SM for an initial set of $N_{0}$ points. Then, the Jacobian of the SM is estimated by the Broyden method [979]. Later, optimization is done in the coarse space and mappings to the fine model are done until the optimum is found in the fine space. In every failed attempt, as one expensive fine model response has been evaluated, the corresponding coarse point is obtained so a new point in the SM is known, and a better estimation of the Jacobian is obtained. The ASM [955] works identically with the exception of the initial SM estimation through $N_{0}$ points. In this way, the algorithms prevents a overevaluation of the fine model. The ASM is recommended for those cases in which cost difference between coarse and fine model is very accused, with a very expensive evaluation of the fine model, as it is the case under study.

The ASM algorithm works as follows. In every iteration $j$, the parameters of the coarse model $L_{c}^{(j)}$ are found so that the coarse model response matches the 
fine model response of the iteration $j-1$. Then, the new parameters of the fine model $L_{f}^{(j)}$, are obtained by the following procedure. First, the incremental $f^{(j)}$ is calculated as

$$
f^{(j)}=L_{c}^{(j)}-L_{c}^{(0)}
$$

with $L_{c}^{(0)}$ the initial parameters of the coarse model. The new $f^{(j)}$ leads to the update of the Broyden matrix

$$
B^{(j)}=B^{(j-1)}+\frac{f^{(j)} h^{(j-1) T}}{h^{(j-1) T} h^{(j-1)}}
$$

where $T$ denotes transpose, i. e., a row vector, and the incremental $h^{(j-1)}$ of the fine model is

$$
h^{(j)}=-\left(B^{(j)}\right)^{-1} f^{(j)}
$$

At the first iteration $j=1$, since there is not information available it is assumed $B^{(0)}=I$, with $I$ the identity matrix. Then, the new incremental $h^{(j)}$ is calculated from (10.38) and, finally, the new fine parameters are obtained:

$$
L_{f}^{(j)}=L_{f}^{(j-1)}+h^{(j)}
$$

The ASM method is a rather simple algorithm that may be obscured at first view by the involved mathematics. Understanding the roots of the method may require careful study, however, to apply it requires much less effort. In order to give a practical view of the method, Fig. 10.49 schematizes conceptually the case of a ASM process that finish after two iterations:

1. The goal response, marked with a blue star is pursued. Optimization is carried out on the coarse domain (the coarse model is evaluated several times until an optimum is found). This gives $L_{c}^{(0)}$, named as point 1 .

2. Since it is the initial iteration, $f^{(0)}=0$ by $(10.36)$, what implies $L_{f}^{(0)}=L_{c}^{(0)}$, which is marked as point 2 . This pass says no more that, at the initial iteration (iteration 0 ), the optimum values found in the coarse domain space are directly used in the fine space.

3. Evaluation of this last point in the fine space gives $F_{f}\left(L_{f}^{(0)}\right)$, named as point 3. Since the obtained response is not the goal, the process continues.

4. The previous failed response is considered in the coarse image response: $F_{c}\left(L_{c}^{(1)}\right)$, named as point 4 .

5. Now, a new search is carried out in the coarse domain to find $L_{c}^{(1)}$, the antiimage of $F_{c}\left(L_{c}^{(1)}\right)$. It is named as point 5 . Note now, that $F_{c}\left(L_{c}^{(1)}\right)=$ $F_{f}\left(L_{f}^{(0)}\right)$, hence $L_{c}^{(1)}$ and $L_{f}^{(0)}$ provide the same response. Therefore, the SM is known at one point. 


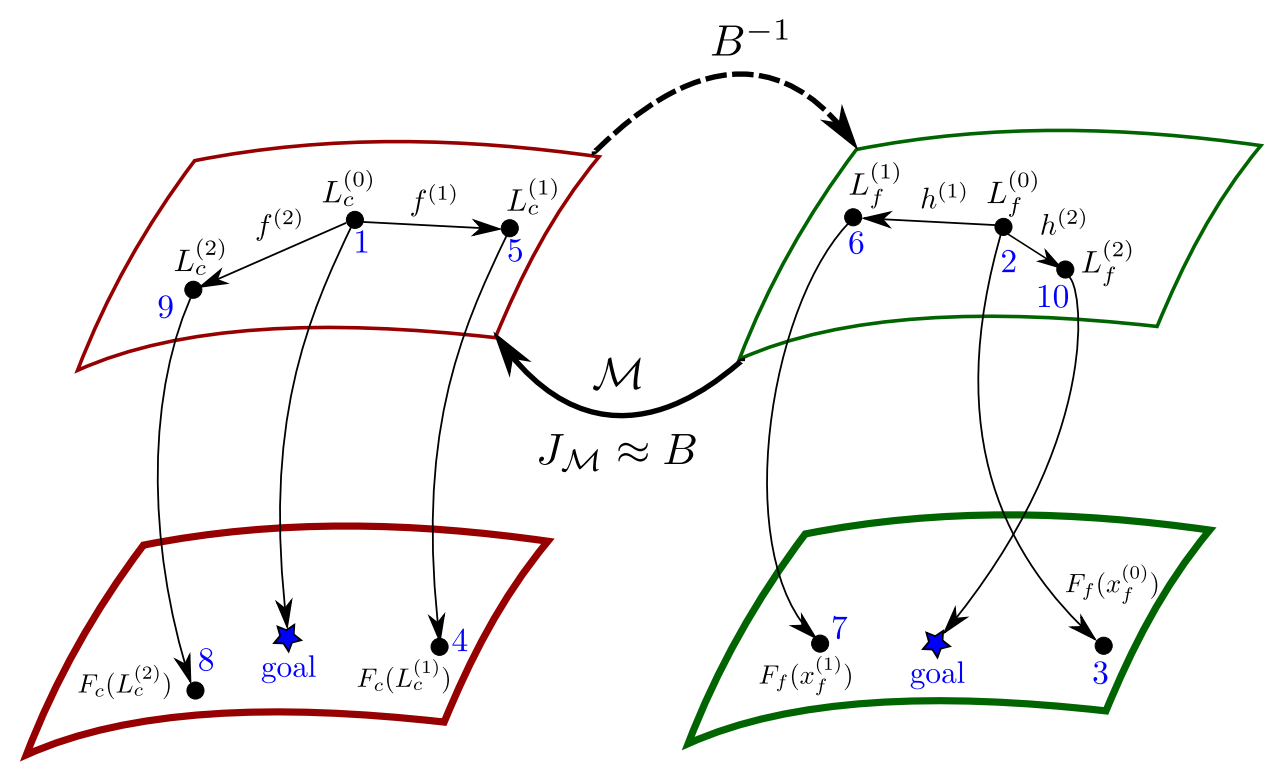

Figure 10.49: Conceptual explanation of ASM process.

6. It is possible to calculate now $f^{(1)}=L_{c}^{(1)}-L_{c}^{(0)}$. Since only one point is known for the SM, the Jacobian cannot be estimated yet (at least two points are necessary). Therefore, it is assumed that $B=I$, the identity matrix. By (10.38) and then $(10.39), L_{f}^{(1)}$ is obtained, see point 6 . Note here that, in this first correction, since $B=I, h^{(1)}=-f^{(1)}$, i. e., the algorithm treats to directly compensate the estimated deviation in a linear way. This means that if the SM is actually linear (e. g., al dimensions larger by a factor $k$ ), the obtained $L_{f}^{(1)}$ will be the optimum.

7. A second evaluation in the fine space is carried out to give $F_{f}\left(L_{f}^{(1)}\right)$, point 7 . Since the obtained response is not the goal, the process continues.

8. The previous failed response is considered in the coarse image response: $F_{c}\left(L_{c}^{(1)}\right)$, named as point 8 .

9. Now, a new search is carried out in the coarse domain to find $L_{c}^{(2)}$, the antiimage of $F_{c}\left(L_{c}^{(2)}\right)$. It is named as point $9 . \quad F_{c}\left(L_{c}^{(2)}\right)=F_{f}\left(L_{f}^{(1)}\right)$, hence $L_{c}^{(2)}$ and $L_{f}^{(1)}$ provide the same response. Therefore, the SM is known at one additional point, and it is known in a total of two points.

10. It is possible to calculate now $f^{(2)}=L_{c}^{(2)}-L_{c}^{(0)}$. Two points are known for the SM so that the Jacobian $B$ is estimated by (10.37). Using (10.38) and then (10.39), $L_{f}^{(2)}$ is obtained, see point 10. In this second correction, the Jacobian applies over the estimated deviation taking into account the information of the 


\begin{tabular}{clllll} 
& \multicolumn{2}{c}{ Iteration 0 } & & \multicolumn{2}{c}{ Iteration 1 } \\
\cline { 2 - 3 } \cline { 6 - 7 } & $L_{c}^{(0)}$ & $L_{f}^{(0)}$ & & $L_{c}^{(1)}$ & $L_{f}^{(1)}$ \\
\hline$h_{c 1}(\mathrm{~mm})$ & 0.288 & 0.288 & & 0.421 & 0.155 \\
$h_{c 2}(\mathrm{~mm})$ & 0.295 & 0.295 & & 0.434 & 0.156 \\
$h_{c 3}(\mathrm{~mm})$ & 0.303 & 0.303 & & 0.419 & 0.187 \\
$h_{v 1}(\mathrm{~mm})$ & 0.577 & 0.577 & & 0.740 & 0.414 \\
$h_{v 2}(\mathrm{~mm})$ & 0.620 & 0.620 & & 0.825 & 0.415 \\
$h_{f}(\mathrm{~mm})$ & 0.144 & 0.144 & & 0.148 & 0.140 \\
\hline
\end{tabular}

Table 10.13: Insertion heights obtained during the ASM process.

SM functional. In the considered example, $F_{f}\left(L_{f}^{(2)}\right)$ provides the goal so that the ASM algorithm finish after the second iteration.

In the previous procedure the steps 1-4 represent the loop of the iteration 0 , the steps 5-8 represent the loop of iteration 1 . After these two special iterations, general iterations follow equations (10.36)-(10.39) in their more general form, but following an identical algorithmic loop. Next, the synthesis of Design 3 is presented, starting with the usual steps of simulation and direct manufacturing (iteration 0). After them, the response is corrected through the previously described ASM algorithm.

\subsubsection{Simulated response}

The optimization process of the complete structure with the insertions is carried out in the same way as in the previous filter designs. The set $L_{c}^{(0)}$ of insertions heights of this initial (iteration 0) design are displayed in Table 10.13. With these values, the response of Fig. 10.50(a) has been obtained. In this case, taking advantage of the acquired knowledge in the optimization of the GGW filter topology, an optimum with very good agreement respect to the ideal response has been met (nevertheless, measurements will show later that stop on a narrower response as it was done in the Design 2 paradoxically lead to better experimental results). Fig. 10.50(b) shows a detail of the $S_{21}$ in the passband. The response is flat with the expected ripple, an it is in agreement with the expected level. Therefore, the complete filter is sent to manufacture. Following ASM, as the iteration 0 indicates, $L_{f}^{(0)}=L_{c}^{(0)}$, so that the set of optimum dimensions obtained in the full-wave simulation optimization process are directly passed to the manufacturing process, see the second column of Table 10.13 .

\subsubsection{Manufacturing and correction process results}

\section{Initial prototype:}

In the initial prototype, both, the bottom piece with the filter layout and the top piece with the metal insertions are manufactured. Fig. 10.51(a) shows the milling process with the Datron ${ }^{\circledR}$ M25 milling machine system. A detail of the first top plate with insertions is shown in Fig. 10.51(b). In the image it can be appreciated the small size of the insertions, and it can be understood that the use of tuning 


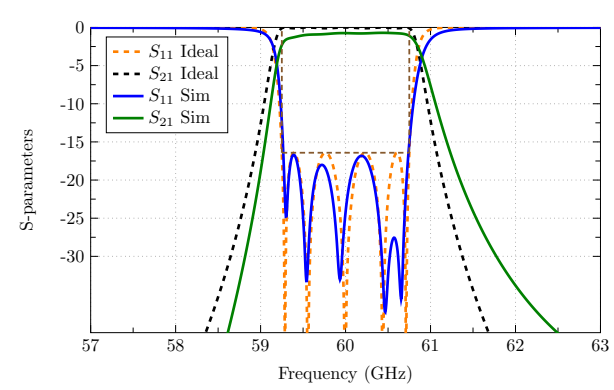

(a)

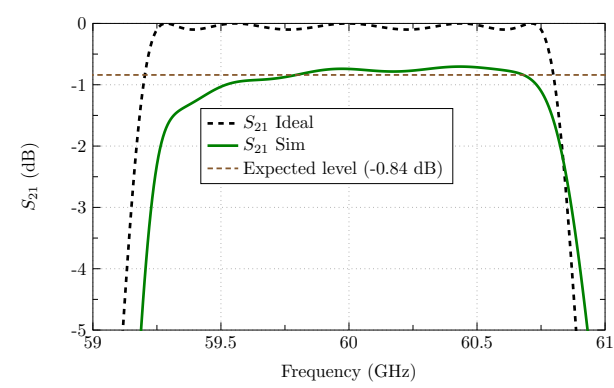

(b)

Figure 10.50: Comparison between the optimum simulated response and the ideal (lossless) response. a) S-parameters; b) $S_{21}(d B)$ detail.

screws makes no sense at this high frequencies. The manufactured top plate with the insertions is compared with the conventional smooth plate in Fig. 10.51(c). Comparing both pieces it is reasonable to argue that the additional cost of including 11 insertions is minimum, and both pieces are cheap and simple to manufacture. More specifically, the cost of manufacturing the top plate with the insertions is 50 times less than the cost of manufacturing the filter body, shown in Fig. 10.51(d), i. e., the top plate with the insertions represents only $2 \%$ of the total process. Note that this feature makes the employed strategy to be very efficient: even if several top plates are required by the ASM process, the cost and the result will clearly overcome that of manufacturing a complete new filter. Amazingly enough, ASM usually converge with very few iterations [729], [978].

The first measured response, $L_{f}^{(1)}$ in the ASM nomenclature, is that of Fig. 10.52(a). A detail of the insertion losses can be found in Fig. 10.52(b). Again, the problem of frequency shift ruins a response that could be quite good otherwise. A shift of $1.2 \mathrm{GHz}$ ( $2 \%$ respect $f_{0}$ and $80 \%$ of the passband bandwidth) towards lower frequencies is observed. Additionally, certain widening of the passband is obtained. On the other hand, the insertion loss is less than $1.7 \mathrm{~dB}$ in the passband, with a best value of $1.25 \mathrm{~dB}$, which is a remarkable good value (note that the widening of the response allows for better $I L$ through equation (10.25)). Return loss is quite good except for a peak of $-10 \mathrm{~dB}$, that degrades the left side of the passband. Therefore, results are coherent with the experience with Design 2: good $I L$ and $R L$, frequency displacement to lower frequencies and widening of the response.

By the above some systematization is observed in the process so that success with ASM is foreseen as very probable. Note in addition that, in the following, the only manufactured piece will be the top plate with the insertions. This implies that the random component of the manufacturing error has even lower influence, since a smaller number of elements (the insertions) are manufactured. It is worth to mention that if the source of error was totally random, ASM would loss all is sense, being that in every iteration the fine space model would change randomly.

\section{Iterative procedure:}

The iterative procedure of the ASM starts now to give correction to the response. In 


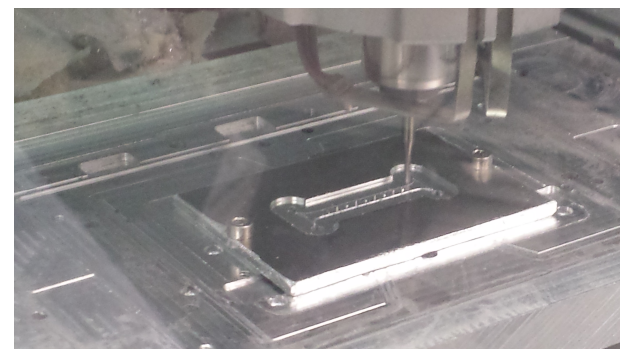

(a)

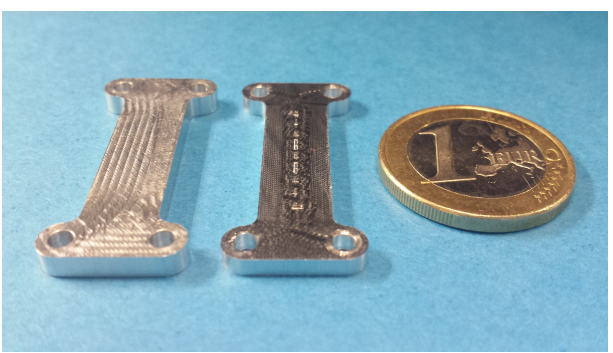

(c)

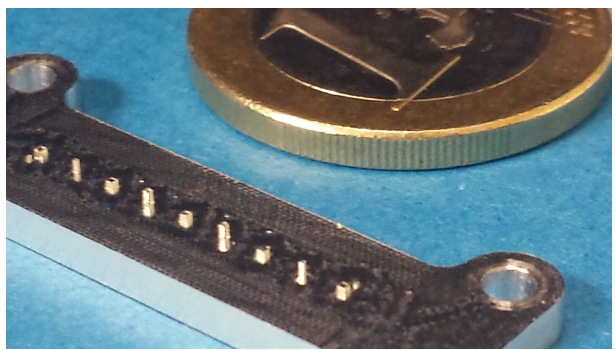

(b)

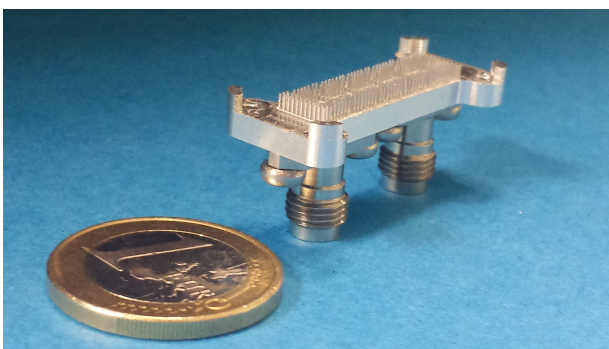

(d)

Figure 10.51: Manufactured prototype. a) Milling process with the Datron ${ }^{\circledR}$ M25 milling machine; b) Detail of the manufactured top plate with the insertions c) Comparison between the conventional smooth top plate and the top plate with the insertions; d) Filter base with the connectors. This piece is manufactured only one time.

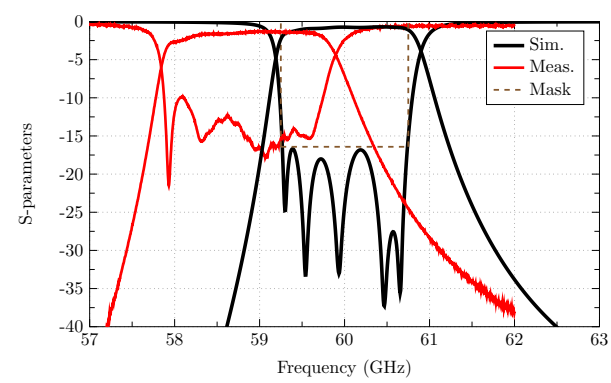

(a)

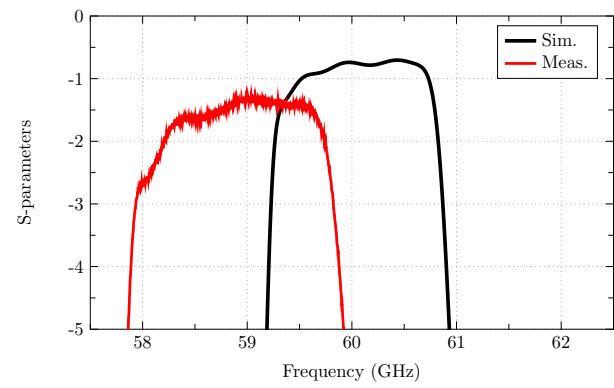

(b)

Figure 10.52: Comparison between simulated and the initial (iteration 0) measured results. a) S-parameters; b) $S_{21}(d B)$ detail.

the iteration 1 , the first goal is to find $L_{c}^{(1)}$, i. e., the parameters that, according to full-wave simulation, give the measured response, red curve of Fig. 10.52(a). This process is not more than an optimization process where the goal response is that with a particular shape instead of the classical ideal one. However, it is true that, since some effects are not captured by the full-wave simulation, a response that perfectly match the measured one its not possible to be obtained. Taking into account this 
fact, the optimization procedure must be carefully guided by the designer, i. e., use adequate error functions, in order to find suitable optimums.

The previous optimization process gives the values of the third column in Table 10.13 for the domain point $L_{c}^{(1)}$. Now, the values that must be sent to the manufacturing process $L_{f}^{(1)}$, are calculated by the ASM algorithm at iteration 1 . Note that the difference of $L_{f}^{(1)}$ respect to $L_{f}^{(0)}$ is exactly ${ }^{4}$ the negative of the difference between $L_{c}^{(1)}$ and $L_{c}^{(0)}$ as previously detailed in the ASM algorithm explanation. The result makes sense: the algorithm is asking for larger insertions in the cavities (a shift towards higher frequencies) and larger insertions in the windows (a decrement of the coupling, thus a narrowing of the response).

The measured response with the same body filter with the new top plate with the insertions is shown in Fig. 10.53(a). It can be observed that the frequency shift problem disappears and the response fits now the bandpass bandwidth much better than the previous one. Furthermore, return losses are improved, practically meeting the specifications. In this case, insertion losses are of $I L=1.5 \mathrm{~dB}$, see Fig. 10.53(b), quite flat on the passband. This implies and outstanding quality factor for the cavities of $Q_{u} \approx 930$. Advert that, the larger insertion losses of this second response respect to the previous one is totally caused by the narrowing of the response, following the law described by (10.25), so any worsening is introduced by the method in this aspect.

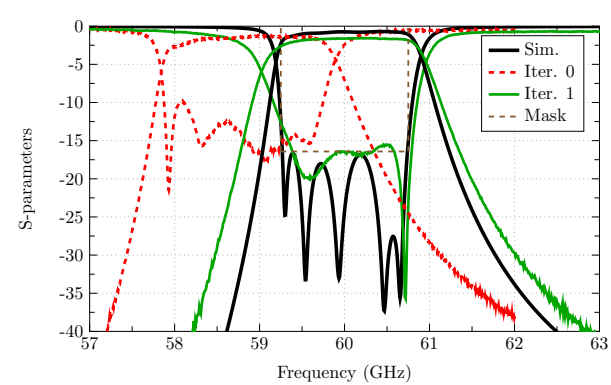

(a)

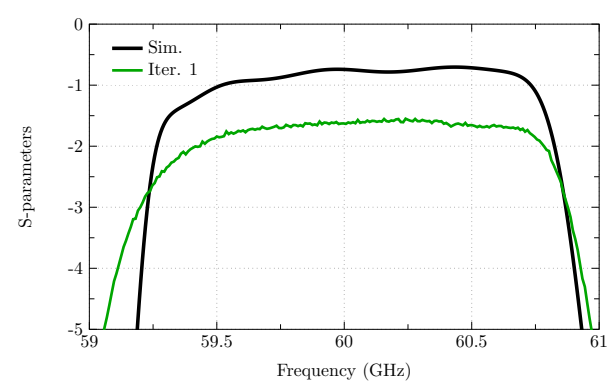

(b)

Figure 10.53: Comparison between simulated and the initial (iteration 1) measured results. a) S-parameters; b) $S_{21}(d B)$ detail.

Although some improvement may be achieved by further iterations, the response may be very reasonably considered as a proper one meeting the specs. Most severe errors are corrected at this point and the residual disagreement between simulated and measured results seems to be rather caused mainly by unavoidable mismatch between the simulation model and the reality, which, conversely to the case at lower frequencies, seems to be relevant at the mm-wave band. Additionally, randomness of the manufacturing process will always prevent of a perfect match. Nevertheless, to the authors knowledge, a filter with similar comparable performance is not found for the $\mathrm{V}$-band in the literature. An extensive review of the state of the art in V-band

\footnotetext{
${ }^{4}$ Note that values are rounded to the 3 decimal digit.
} 
NBFs will be carried out by means of a table, being this filter the most outstanding one among considered.

In this third design, the ASM algorithm has resulted very efficient to solve the problem of frequency shift, very prone in NBFs at the V-band. Only 1 iteration, i. e., a total of two manufactured top plates with insertions has been necessary. This implies an incremental cost of only $2 \%$ respect to a conventional design. Therefore, it can be affirmed that the performance of the employed strategy has been very satisfactory. Nevertheless, some issues regarding to the employed process could be improved for posterior designs. The main bottleneck in the process is found in the coarse domain search. Note that the coarse model is a full-wave simulation of a complex structure, hence time-consuming. In this way, thoughtful procedure could be to use a multi-level ASM strategy. This is, in the top level, the ASM would be the same as described here: full-wave simulations as coarse model and measurements as fine model. However, in the bottom level, the fine model would be the coarse model of the top level, i. e., full-wave simulations of the actual GGW filter. The coarse model of the bottom level would be, therefore, a simpler model. For instance, the a rectangular waveguide filter could be used as coarse model. Or even better, a rectangular waveguide filter incorporating the information of the GGW analysis method of section 10.1. In this aspect, note that, the better the coarse model, the faster the convergence. In the described multi-level ASM strategy, three different models are employed. Notice that the inclusion of a lower level consisting in a very fast model drastically reduces the number of evaluations of the former coarse model, now in a intermedium position. According to the obtained results, very few evaluations would be necessary. In addition, a SM between simulation models is expected to be less ill-posed than that between simulation and measurements, thus being able to find better matching between responses.

Regarding to this last aspect, it arises another possible issue to work with in order to improve the presented strategy. There are some aspects that the full-wave simulation does not capture. For instance, insertion losses are always larger in the experiment than those predicted by the full-wave simulation. High frequency (mm-wave and $\mathrm{THz}$ ) literature indicates that reconsidering the conductivity may be necessary quite often. A deep study on the simulation model of the actual GGW filter could give answer to this question, providing a better coarse model, hence a faster process. Note that matching coarse and fine space images during the process, is an essential step to discover the SM between both spaces.

Finally, to conclude this section, dedicated to Design 3, it seems appropriate to highlight that the obtained results here point ASM as a very suitable algorithm in the design of not only GGW NBFs but, more generally, any GW component. Note that ASM faces directly the main problem of GW component design, which is the time-consuming design process due to complex full-wave simulations (consider them as fine model). Since some simplified analysis model start to appear, and better ones are expected in the next years, great benefit may be obtained of using ASM with them as coarse model. Proper strategies in this sense may revolution GW component design scenario in a near future. 


\subsubsection{Comparative with rectangular waveguide technology at V-band}

Until now, suitability of GGW topology for the implementation of NBFs at mmwave frequencies has been proven. Design 1 exhibits good performance at Ka-band except for the frequency shift of the response. Design 2 improves some features of Design 1, providing a response that is even more competitive taking into account the design frequency, in the V-band. Finally, with Design 3 it has been demonstrated that GGW topology allows for response correction thanks due to its topological properties. This means that, with GGW topology, demanding specifications can be afforded. However, despite of the endorsement given by the previous three designs, the study carried out in this chapter would not be totally complete without a direct and fair comparison with the rectangular waveguide. Advert that the only reason to consider GGW instead of rectangular waveguide as the basis structure of mmwave NBFs is the possibility of that GGW perform better in real implementations, either regarding insertion losses, response correction possibilities, prototype weight, robustness front vibrations, temperature stability, etc. The following work is mainly contained in [842].

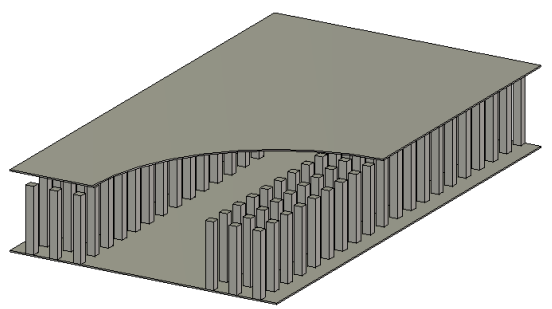

(a)

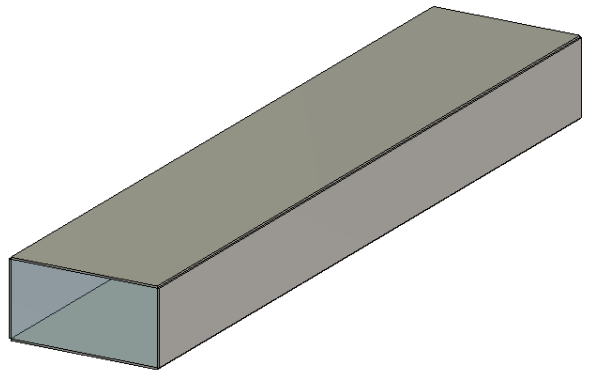

(b)

Figure 10.54: $3 D$ view of the topologies under study and comparison: a) Groove Gap Waveguide (GGW); b) Rectangular waveguide (RWG).

By the previous reason, this section tackles such demanded comparison between GGW and rectangular waveguide. In order to obtain reliable conclusions, both topologies are challenged with the same design and treated with the same manufacturing conditions. Specifically, the Design 2 will be implemented in rectangular waveguide topology. The choice is firstly justified by the operation frequency: Vband involve frequencies high enough to make arise rectangular waveguide technological problems (remember that at Ka-band rectangular waveguide still gives quite good performance, see again Table 10.8). Second, Design 2 is preferred to Design 3 for simplicity and because, as it will be shown later, rectangular waveguide topology is prone to fail with Design 3 procedure. Specifications and ideal response plots of Design 2 are included in Table 10.14 and Fig. 10.55, respectively, as the reference goal to be pursued in the rectangular waveguide filter design carried out next in this section. 


\begin{tabular}{cccc} 
Parameter & Frequency $f_{0}$ & Relative Bandwidth $(\mathrm{RBW})$ & BP Ripple $R(R L)$ \\
\hline Value & $61 \mathrm{GHz}$ & {$[60.25 \mathrm{GHz}-61.75 \mathrm{GHz}](\approx 2.5 \%)$} & $0.1 \mathrm{~dB}(16.42 \mathrm{~dB})$ \\
\hline
\end{tabular}

Table 10.14: Filter response specifications.

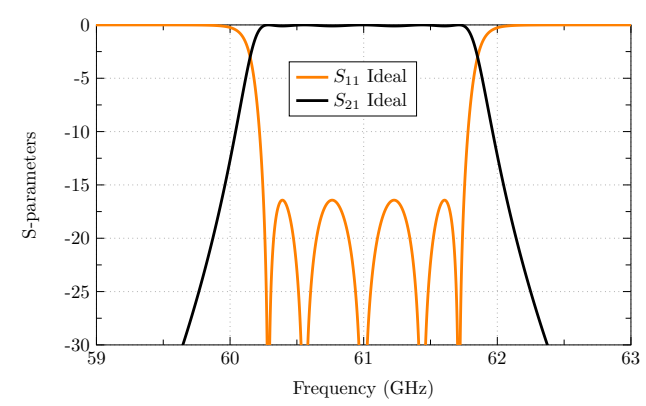

(a)

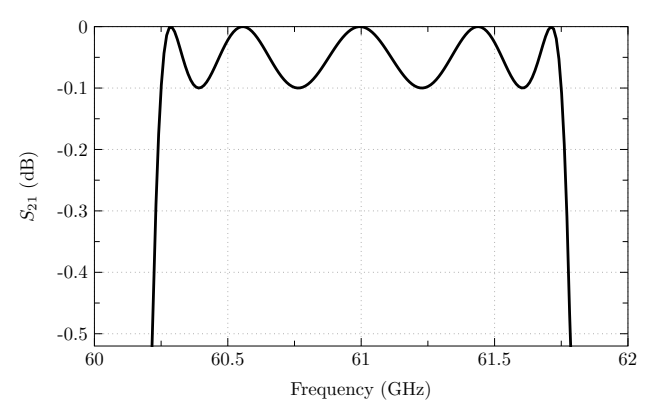

(b)

Figure 10.55: Ideal response. a) S-parameters; b) $S_{21}$ (dB) detail.

For the presented comparison, H-plane manufacturing has been chosen to implement the rectangular waveguide filter. In this way, both topologies are implemented with the same assembling procedure so that equity of conditions is kept in the comparison, hence is more fair. Of course, E-plane rectangular waveguide filters present lower insertion losses than their H-plane counterparts [344], [714]-[715]. However, E-plane manufacturing makes very challenging the use of any tuning element and it is not surprising that $\mathrm{H}$-plane option is preferred for practical high-frequency designs offered by hardware companies [966], [967]. In addition, note that, one of the basic objectives in this part of the thesis is to prove the suitability of GGW front rectangular waveguide for $\mathrm{H}$-plane structures (it is recovered that several components can only be implemented with this assembling option, e. g., Slot Array Antennas). Therefore, the essential information is recovered in the present study. Further comparisons, including E-plane rectangular waveguide filters are left as a possible future work line that may provide interesting, but not imperative information. The section concludes with a review of V-band NBFs state of the art, giving comparison with all the presented designs along this chapter, and extracting final conclusions in the scope of this part of the thesis.

\subsubsection{Rectangular waveguide filter topology}

The rectangular waveguide filter topology that has been considered in the study is the classical one for all-pole filters. The topology keeps parallelism with the GGW filter topology (actually, GGW topology is derived from classical rectangular waveguide all-pole filter implementations) in order to keep equity in the comparative. The only particularity that must be mentioned is that, in the rectangular waveguide, cavity corners are considered rounded with curvature radius $r_{c}=0.4 \mathrm{~mm}$ in order to take into account the finite radius of the milling machine cutter. This strategy is quite usual in order to avoid disagreement between simulation and measurements in 
such way that even several efficient strategies to analyze the topology has been not so far considered [980], [981].

The different elements of the rectangular waveguide filter topology are shown in Fig. 10.56. Filter cavities follow the shape shown in Fig. 10.56(a). Coupling is done by inductive windows such as that shown in Fig. 10.56(b). Finally, feeding is done by a coaxial inserted from the bottom wall of the filter, exactly in the same way as in Design 2. The final filter has the aspect of Fig. 10.57 (top plate not shown in order to appreciate the filter layout). In all the previous schematics, the aforementioned rounded corners can be appreciated.

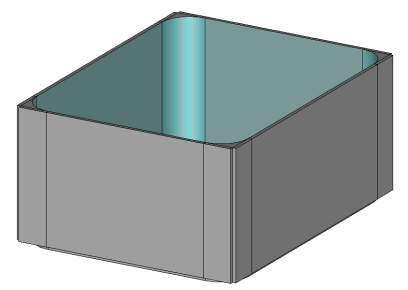

(a)

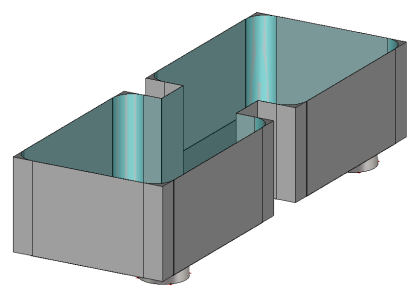

(b)

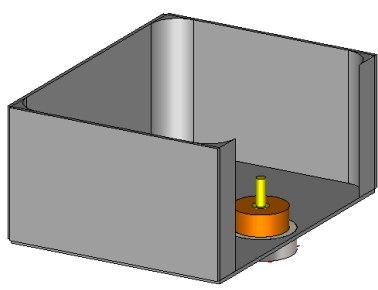

(c)

Figure 10.56: Rectangular waveguide filter topology. Rounded corners with curvature radius of $r_{c}=0.4 \mathrm{~mm}$ are considered in the model taking into account the milling cutter radius. a) Cavity $(G G W)$; b) Coupling windows; c) Coaxial connector feeding.

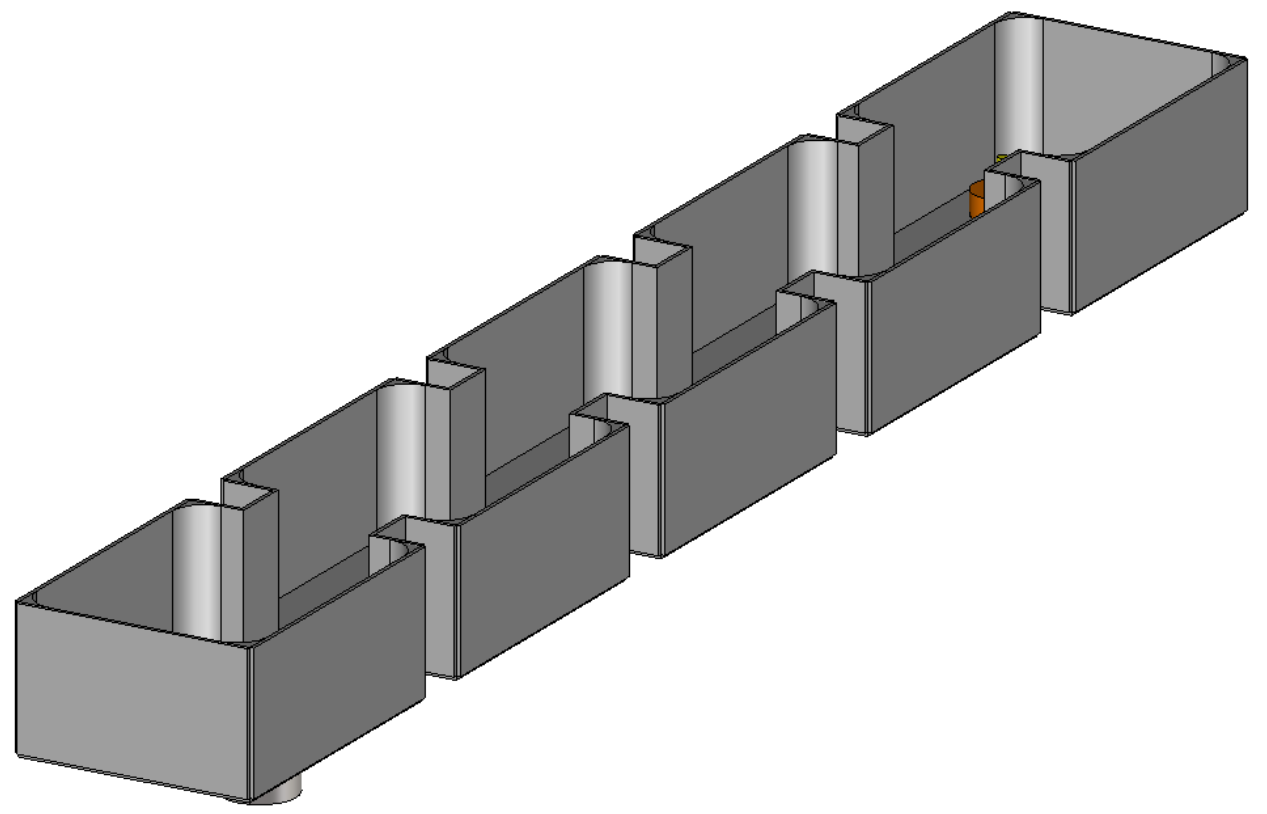

Figure 10.57: Complete rectangular waveguide filter topology. 


\subsubsection{Filter design}

Since filter specifications are those of Design 2 (Table 10.10), ideal response and low pass prototype normalized elements are exactly the same. Therefore, filter design procedure of section 10.3.1 is directly applied next.

\section{Resonant cavity}

As usual, to determine the cavity dimensions in order to synthesize the desired frequency $\left(f_{\text {res }}=61 \mathrm{GHz}\right.$ for this design) one dimension is fixed and the other is tuned. In this case, $C=4 \mathrm{~mm}$ is fixed and $L$ has been tuned, see Fig. 10.58(a). Fig. $10.58(\mathrm{~b})$ shows $f_{\text {res }}$ vs. $L$, with a red circle marker at $L=3.115 \mathrm{~mm}$, value which gives the desired resonance frequency. With the same $C$, the GGW cavity required $L=3.27 \mathrm{~mm}$ to provide the same $f_{r e s}$, i .e., GGW is equivalently smaller in aperture than the rectangular waveguide, what is in concordance with results of section 10.1 .

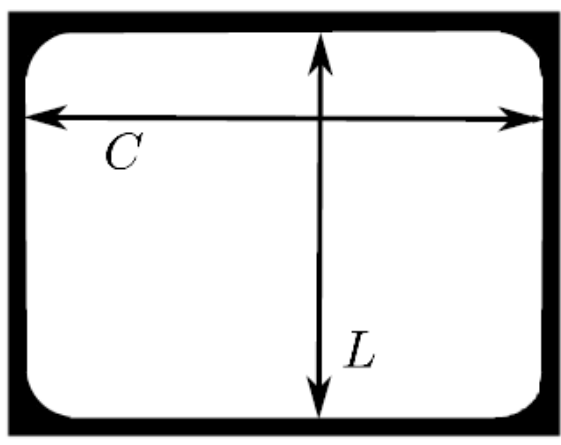

(a)

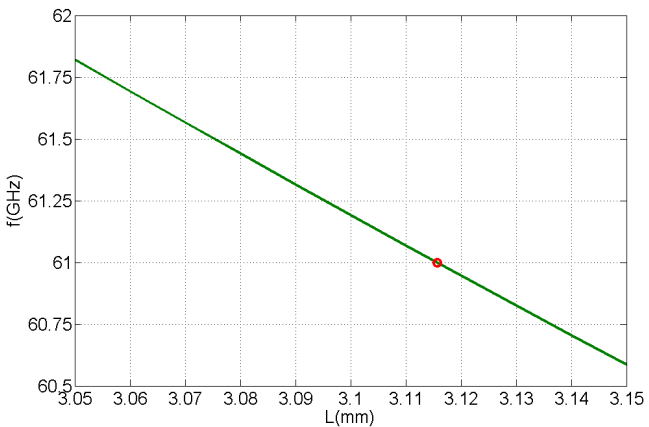

(b)

Figure 10.58: Resonance frequency synthesis. a) Cavity dimensions. $C=4 \mathrm{~mm}$ is fixed; b) $f_{\text {res }}$ vs. $C$.

\section{Coupling elements}

Coupling elements are conventional inductive coupling windows in which coupling is controlled by adjusting the window width $w$, see Fig. 10.59(a). The design plot of coupling factor $k$ vs. window size $w$ is shown in Fig, 10.59(b). The two required widths are marked with red circles in the plot: $w_{12}=w_{45}=1.59 \mathrm{~mm}$ to provide $k_{12}=k_{45}=0.02$, and $w_{23}=w_{32}=1.48 \mathrm{~mm}$ to provide $k_{23}=k_{32}=0.015$. Note how the required window widths are smaller than those required for the GGW design $\left(w_{12}=w_{45}=2 \mathrm{~mm}\right.$ and $\left.w_{23}=w_{32}=1.93 \mathrm{~mm}\right)$, a result that can surprise at first sight since rectangular waveguide windows are topologically more opaque than GGW ones. The size increment is about $25 \%$, which is quite significant. Again, differences between both waveguides endorse the argumentation given in this chapter regarding the possible errors of assuming both waveguides to be directly equivalent.

\section{Feeding elements}

Finally, the external quality factor design plot is derived. The feeding is done in 


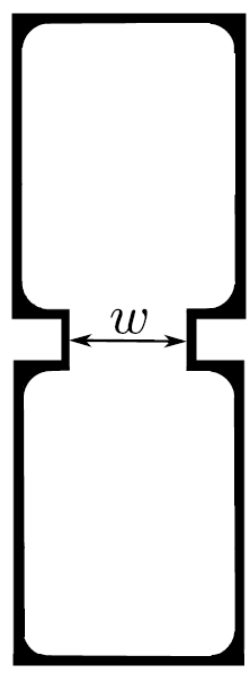

(a)

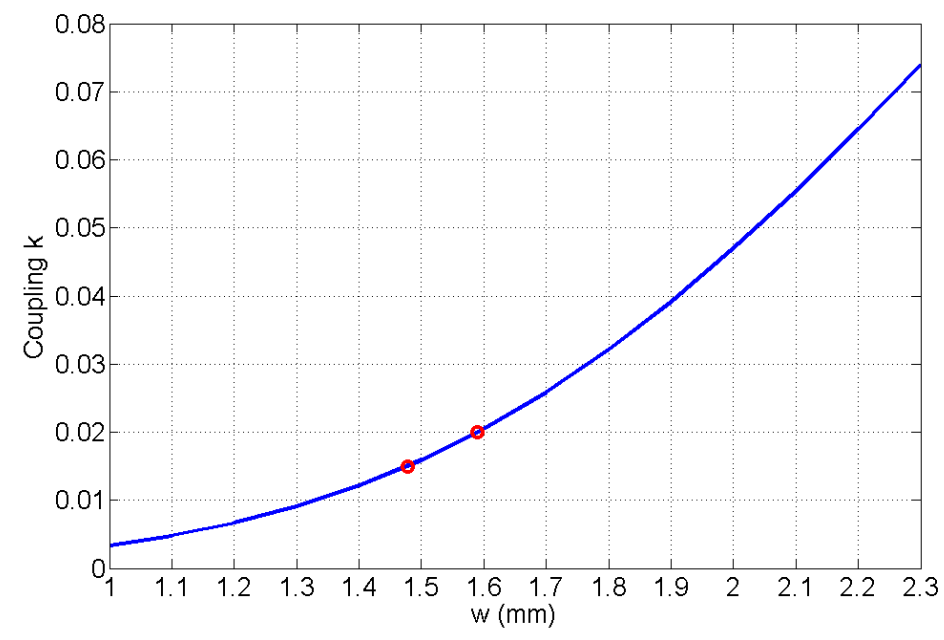

(b)

Figure 10.59: Coupling factor $k$ synthesis. a) Coupling structure; b) Coupling factor $k$ vs. window size $w$.

the same way as it was presented in Design 2 with GGW topology, i. e., two 1.85 mm coaxial connectors (SouthWest Microwave1490-12 G) have been inserted at the bottom wall. Taking advantage of the conclusions of Design 2 regarding the necessity of larger perturbation of the external cavities, a vertical insertion of $z_{0}=0.3 \mathrm{~mm}$ has been considered, see top image of Fig. 10.60(a). Then, it has been fixed $y=0.5$ $\mathrm{mm}$ and $x_{0}$ has been tuned (see bottom image of Fig. 10.60(a)) to provide the plot of Fig. 10.60(b). As it can be appreciated, the choice of $y_{0}$ and $z_{0}$ provides a very stable external quality factor around the desired value of $Q_{e x}=45.87$, obtained with $x_{0}=0.825 \mathrm{~mm}$, marked with a red circle in the plot. In this case, the similitude with the rectangular waveguide is larger, what indicates that in terms of E-field distribution both waveguides are quite similar.

\section{Complete filter}

The complete rectangular waveguide filter layout with its main geometrical parameters is shown in Fig. 10.61(a). For comparison purposes, the GGW filter layout with its main geometrical parameters is shown in Fig. 10.61(b). As usual, the filter symmetry, marked with a vertical blue dashed line, allows to consider only part of the geometrical variables in the optimization process. The obtained optimal values for the rectangular waveguide filter are shown in the first row of each of the two parts of Table 10.15. The optimum values of Design 2 with GGW has been added as well in the second row of each of the two parts of Table 10.15 for comparison purposes. If the two sets are compared, it is observed that the GGW filter is slightly larger in dimensions. This fact together with the presence of pin blocks cause the total GGW filter size to be significantly larger than that of the rectangular waveguide. Nevertheless, the size feature will be shown to revert in the practical implementa- 


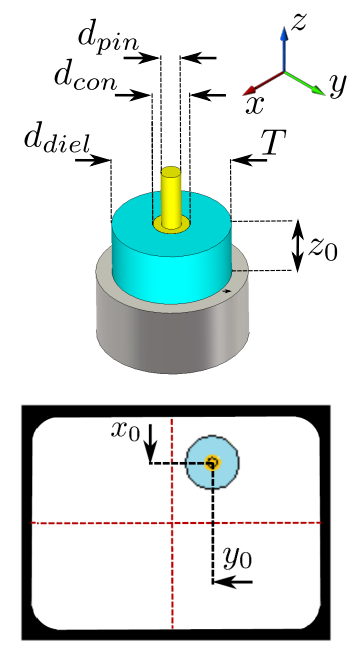

(a)

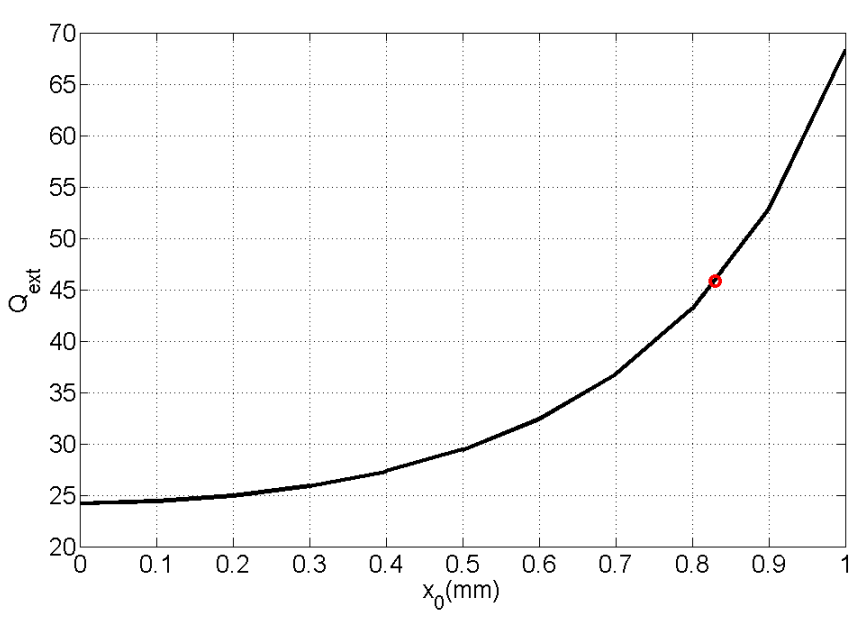

(b)

Figure 10.60: External quality factor $Q_{e x}$ synthesis. a) Feeding structure: $2 D$ view (bottom image) and $3 D$ view (top image); b) External quality factor $Q_{e x}$ vs. connector position $x_{0}$ when $y_{0}=0.5 \mathrm{~mm}$ is fixed.

\begin{tabular}{lccccc} 
Parameter & $C_{1}$ & $C_{2}$ & $C_{3}$ & $w_{12}$ & $w_{23}$ \\
\hline Rectangular waveguide $(\mathrm{mm})$ & 3.84 & 3.74 & 3.79 & 1.60 & 1.51 \\
\hline GGW $(\mathrm{mm})$ & 3.92 & 3.88 & 3.92 & 2.01 & 1.94 \\
\hline
\end{tabular}

\begin{tabular}{lccccc} 
Parameter & $L_{1}$ & $L_{2}$ & $L_{3}$ & $x_{0}$ & $y_{0}$ \\
\hline Rectangular waveguide $(\mathrm{mm})$ & 2.95 & 2.95 & 2.95 & 0.78 & 0.45 \\
\hline GGW (mm) & 2.97 & 2.97 & 2.97 & 0.71 & 0.15 \\
\hline
\end{tabular}

Table 10.15: Filter optimization parameters.

tion. The main remarkable difference between both layouts is found in that, in order to achieve the response specifications, the rectangular waveguide topology demand from narrower coupling windows, an already appreciated effect during the coupling analysis. Deeper study of this fact may reveal interesting local effects that may found their application in future innovative designs.

With the values of Table 10.15, the simulated response of Fig. 10.62(a) has been obtained for the designed filter. In the plot, the ideal response according to the specifications is shown for comparison. As it can be appreciated, good agreement is found. It must be noted that, compared with previous designs using GGW structures, the optimization with the rectangular waveguide topology is considerable faster. This causes the optimization process to be more straightforward and increases the possibility of success with a very good response, as it is the case. This is a good proof of the importance of developing good analysis methods and optimization strategies for GGW structures. The use of the analysis method of section 10.1 combined with efficient optimization strategies such as ASM, applied in Design 


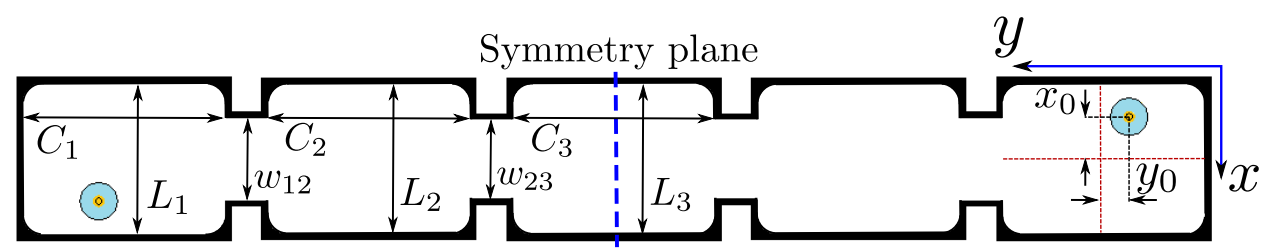

(a)

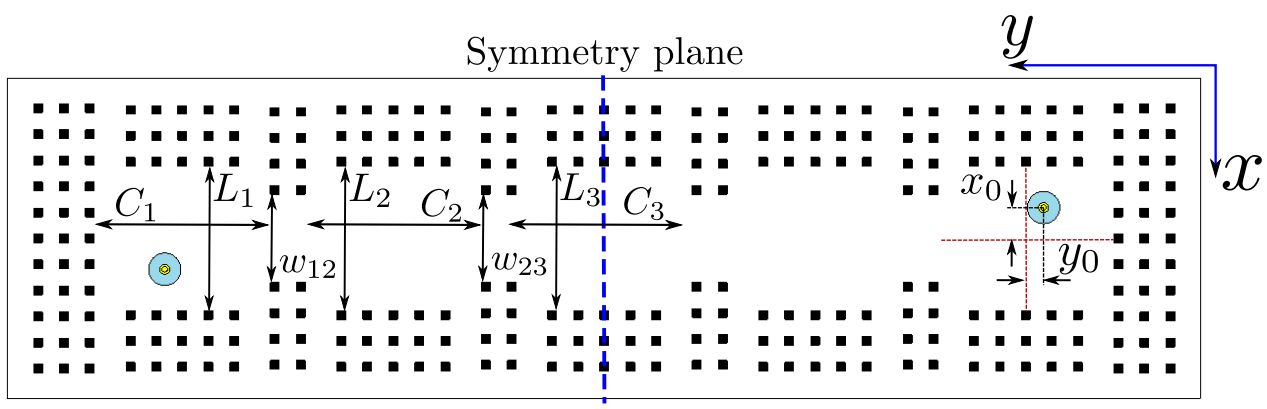

(b)

Figure 10.61: Complete filter schematics and geometrical parameters. a) Rectangular waveguide filter; b) $G G W$ filter.

3, may dispose an scenario with complexity of similar level to that observed here for the rectangular waveguide in future designs.

Regarding insertion losses, it must be noted that, in the case of the rectangular waveguide filter, the expected level according to simulations is $I L=0.6 \mathrm{~dB}$, which is lower than that of the GGW filter $(I L=0.8 \mathrm{~dB})$. The equivalent quality factor of rectangular waveguide cavities is, in simulation, $25 \%$ higher than that of GGW cavities.

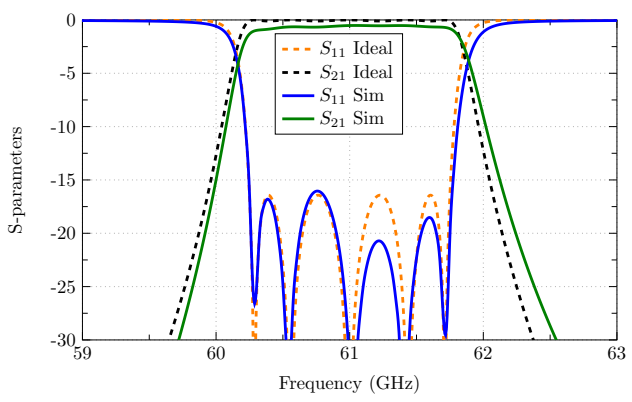

(a)

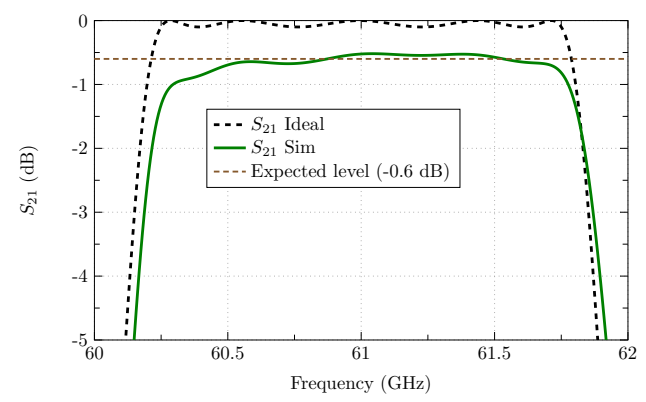

(b)

Figure 10.62: Comparison between the optimum simulated response and the ideal (lossless) response. a) $S$-parameters; b) $S_{21}(d B)$ detail. 


\subsubsection{Manufactured prototype, measurements and comparative}

The rectangular waveguide filter prototype has been manufactured in the same conditions as its GGW equivalent: an in-house process with the milling driven Datron ${ }^{\circledR}$ M25. Photographies of the prototype can be found in Fig. 10.63. More specifically, Fig. 10.63(a) shows the two separately manufactured pieces of the H-plane implementation of the filter. The bottom piece contains the filter layout and a surrounding metallic block to give allocation to consistent screw holes to ensure proper pressure besides alignment, essential in this filter due to current flow requirements. The top piece consist in a simple metal block with the screws. The aspect of the assembled filter joining these two pieces can be observed in Fig. 10.63(b).

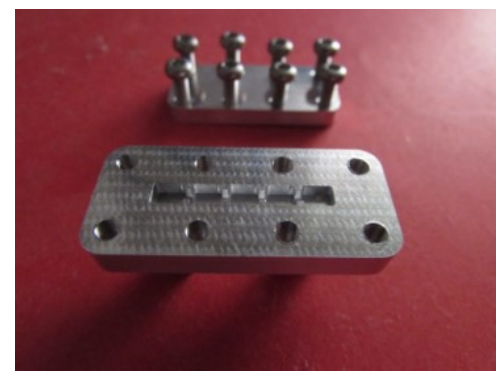

(a)

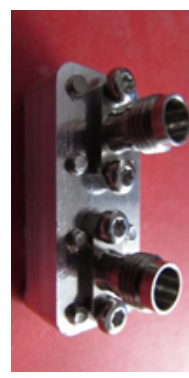

(b)

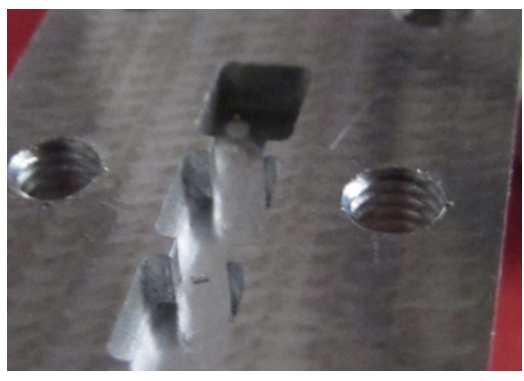

(c)

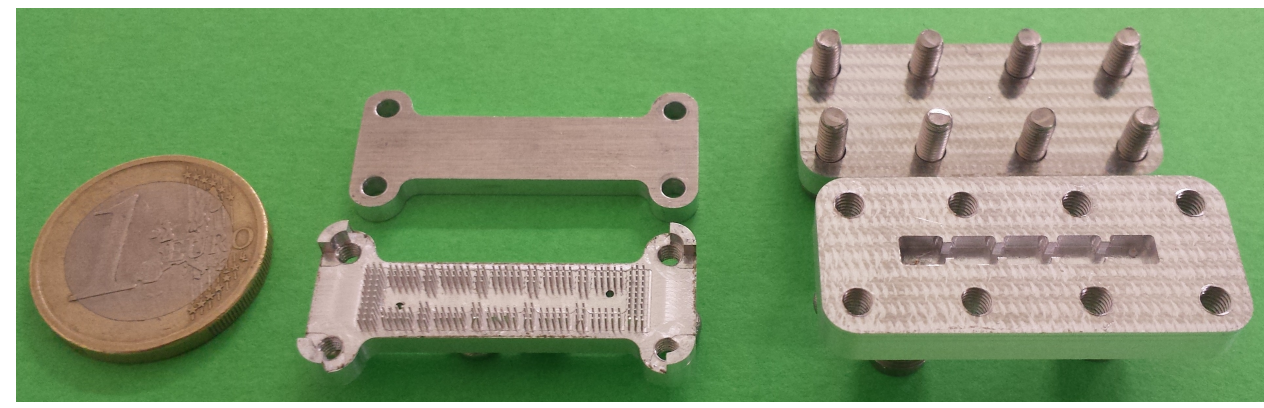

(d)

Figure 10.63: Manufactured rectangular waveguide filter. a) Manufactured pieces: top piece (metal plate with pressure screws) and bottom piece (rectangular waveguide filter layout and support for the pressure screws); b) Assembled filter; c) Detail of the filter layout; d) Comparison between the two $61 \mathrm{GHz} 5^{\text {th }}$-order filter prototypes, GGW (left) and rectangular waveguide (right).

A detail of the milled filter layout can be appreciated in Fig. 10.63(c). Round corners of the cavity can be well identified in this image. At first sight, the milling appears quite fine. Note also the detail of the large screw hole size. These holes have a size comparable to the designed cavities. This fact, together with mechanical stability requirements cause the rectangular waveguide filter to result bulkier than the GGW filter despite of the fact that the actual filter design is more compact for the former topology. This can be well observed in Fig. 10.63(d) where both filters (unmounted) are shown. The larger size, hence weight of the rectangular waveguide filter, as well as its more complex screws system are evident in the photography. 
The two manufactured filters have been measured next with the Keysight PNA N5227A Network Analyzer of Fig. 10.64(a). Experimental results are shown in Fig. 10.64(b), where responses of both filters are compared in the same graph. For completeness, Figs. 10.65(a) and 10.65(b) compare simulated and measured response for each one of the filters. The response of the GGW filter was already analyzed in the section dedicated to Design 2. Main aspects to highlight are insertions losses $(I L=1.5 \mathrm{~dB})$, return losses (satisfying the specification except for a $-13 \mathrm{~dB}$ peak at the left side of the passband), passband width $(B W=1.5 \mathrm{GHz}$, satisfying the specifications) and frequency shift $(\Delta f=-0.79 \mathrm{GHz}$, what implies $1.3 \%$ respect $f_{0}=61 \mathrm{GHz}$ ).

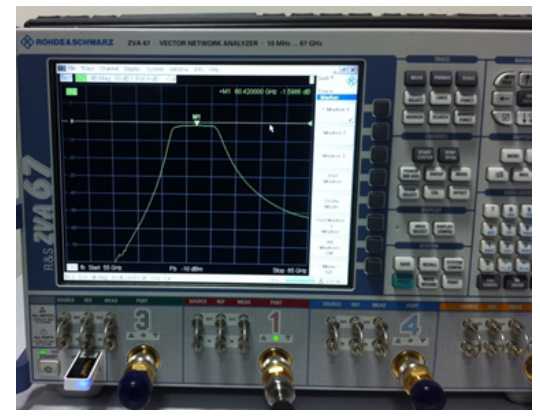

(a)

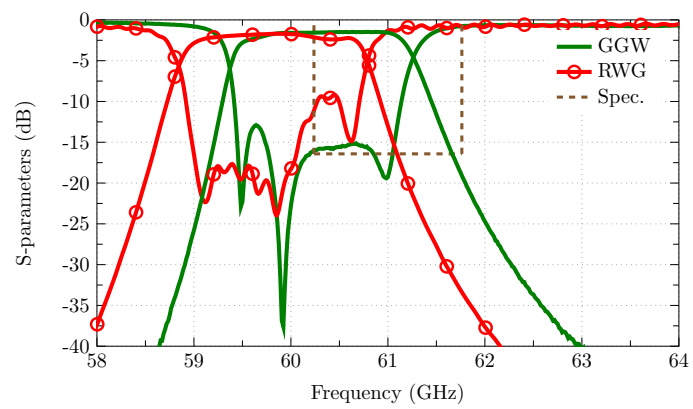

(b)

Figure 10.64: Experimental results. a) Measurement equipment: Keysight PNA N5227A Network Analyzer; b) Comparison between the measured response of the rectangular waveguide filter and the $G G W$ filter.

The response of the rectangular waveguide filter is analyzed now. The best value of insertion losses is quite good: $I L=1.6 \mathrm{~dB}$, only $0.1 \mathrm{~dB}$ higher than the obtained with the GGW filter. However, some observations must be highlighted. First, note that the simulated value was $I L=0.6 \mathrm{~dB}$ (front $I L=0.8 \mathrm{~dB}$ of GGW topology). This confirms that the GGW structure is more robust front the manufacturing process (0.7 dB degradation front $1 \mathrm{~dB}$ degradation). In addition, insertion losses of the rectangular waveguide filter present an appreciable ripple in the passband, lacking of the desired flatness. This is well appreciated through the $S_{11}$ peak of $-9 \mathrm{~dB}$ at the right side of the bandpass. Therefore, in terms insertion and return losses, GGW filter gives better performance.

The rectangular waveguide filter also presents certain widening of the response and and appreciable frequency shift. The measured bandwidth is about $B W=1.6$ $\mathrm{GHz}, 6.67 \%$ larger than the expected. Regarding the frequency shift, a movement of $1.15 \mathrm{GHz}$ ( $1.9 \%$ respect to the central frequency) towards lower frequencies is observed. Also in the rectangular waveguide filter, the frequency shift remains as the main issue, being even more accentuated than in the GGW case. This fact indicates that the observed frequency displacement of the previously designed GGW filters is not originated by the fact of that GGW topologies have small elements to be milled (what may be understood as barrier at first sight). Results are a clear proof that the high frequency of design together with the demanded narrow-band response, make 


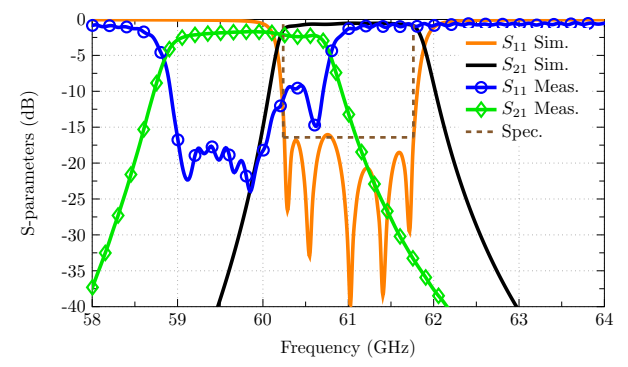

(a)

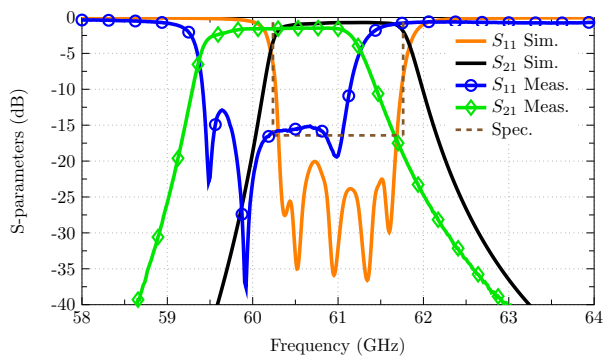

(b)

Figure 10.65: Comparison between the measured response and the optimum simulated response. a) Rectangular waveguide filter; b) GGW filter.

the manufacturing tolerances become appreciable in the response. It is evident that cavities are manufactured larger than desired.

On the other hand, the obtained result does not mean that GGW is less affected by the manufacturing process than the rectangular waveguide in terms of frequency shift. In fact, the first manufactured GGW prototype in Design 3, presents a response with similar passband widening and frequency displacement than those observed here for the rectangular waveguide. Therefore, it can be concluded that any structure implementing a filter of similar characteristics will suffer from the same problem, which is directly related to the physical dimensions of the filter. Consequently, it can affirmed that tuning elements become essential at these frequencies. In this sense, procedures such as that implemented for the Design 3 are very suitable. Such post-processing tuning has been applied successfully to GGW topology. In the case of the rectangular waveguide, although similar insertions may be displayed in the top plate, it must be first checked that the topology accepts well the required re-assembling process. This is studied in the next section.

\subsubsection{Re-assembling robustness test:}

An interesting test to carry out with the manufactured filters is presented next. It consist in a re-assembling test in which each one of the filters is measured several times. Between measurements, each one of the filters is disassembled (top plate is removed) and assembled again (top plate is placed and screws are pressed) in the way that Fig. 10.66 describes. This simple test provides first stage reliable information about the robustness of the filter front vibrations, its suitability to carry out a post-processing correction procedure based on different top plates with insertions (Design 3), or the robustness of the design from randomness of mass production chains, among others. These features are very relevant to be characterized in the context of new generation applications. Think for instance in automotive radars. Robustness front vibrations, simple design processes and reliable standard mass production process are clearly demanded in such scenario.

Results of this study are shown in Fig. 10.67. First, $S_{11}$ is analyzed in Fig. 10.67(a). In the plot it can be observed how, whereas curves of $1^{\text {st }}$ and $2^{\text {nd }}$ measurements totally overlap in the case of GGW, for the rectangular waveguide filter a 


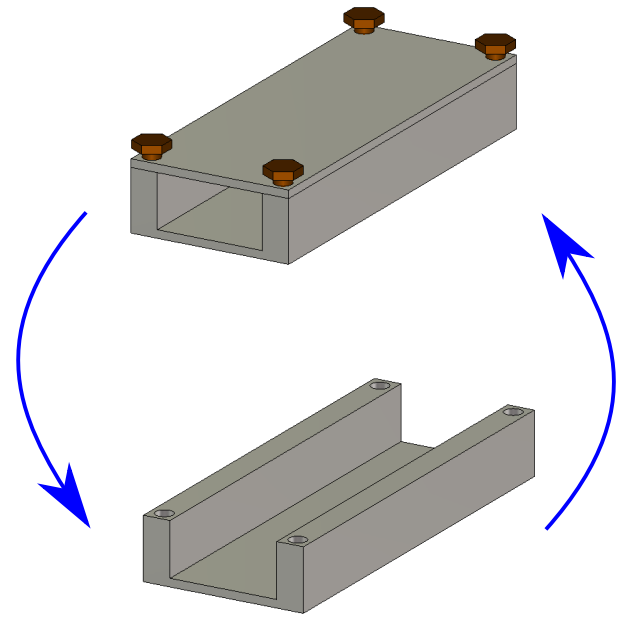

(a)

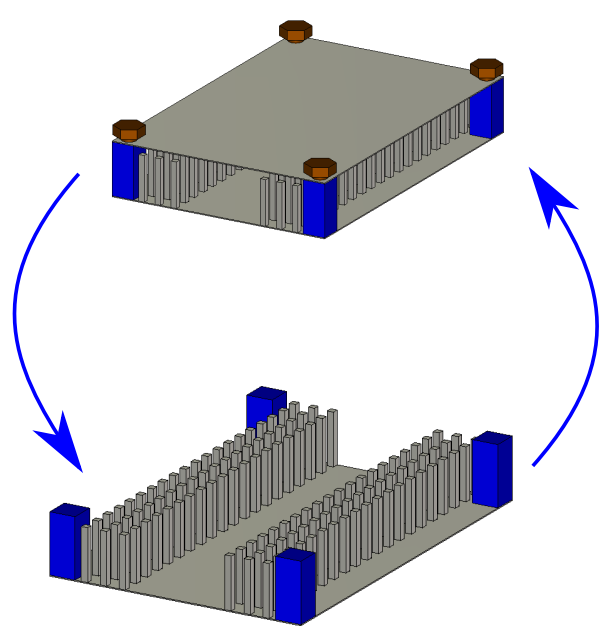

(b)

Figure 10.66: Re-assembling robustness test: several measurements are performed; in between of them the two H-plane manufactured parts of the filter are disassembled and assembled again. a) Rectangular wavaguide; b) GGW.

degradation is observed between both measurements. Return losses increase in the left side of the passband. However, the most remarkable effect is observed in the $S_{21}$, Fig. 10.67(b). Observe first that the initial response of the rectangular waveguide filter presents worse insertion losses in the passband than the GGW filter, mainly due to the lack of flatness. Now, after the re-assembling insertion losses of the rectangular increase $1 \mathrm{~dB}$, which is an important degradation. The response is as well less flat due to the worsening of return losses. Note that the increment of insertion losses is not produced by an important degradation of return losses but because certain leakage originated by perturbation of the current flow across the metallic joints is causing the loss. Contrarily, GGW response remains totally invariant, with any appreciable difference between curves corresponding to the different measurements.

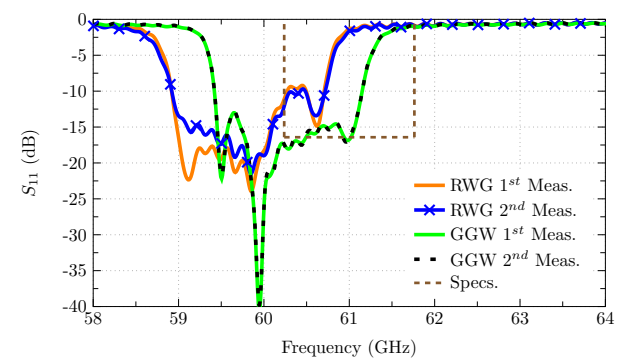

(a)

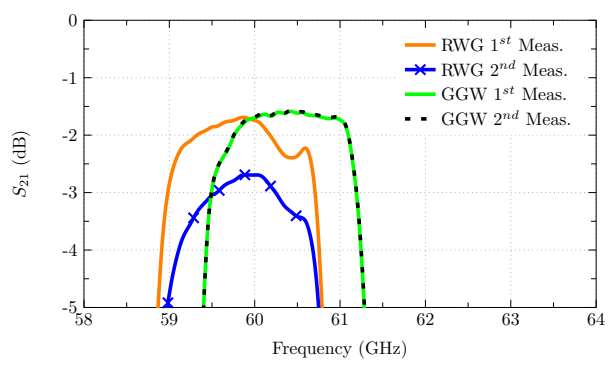

(b)

Figure 10.67: Re-assembling robustness test results. Comparison of the response of both filters. $2^{\text {nd }}$ measurement correspond to a second re-assembling of the filters. a) $S_{11}$; b) $S_{21}$. 
The same re-assembling process has been done several times obtaining the same results: the GGW filter response remains invariant whereas the RGW filter response becomes degraded. It is noted that in the case of the GGW filter, without taking much care about the assembling process works well (screws are just making alignment functions) whereas in the case of the rectangular waveguide, even though the best result has been pursued, strong limitations have been irreparably found. The observed changes may be originated by the different pressure levels of the screws that are used to joint the two pieces. Small changes in the force exercised by these screws may cause tiny shape deformations that may result important at these very small, millimetric, wavelengths. It is important to highlight this observed effect since it indicates that the pressure and mechanical forces distribution is critical in the rectangular waveguide at the mm-wave band. The GGW seems to be insensitive to this random deviation of the structure screws pressure, what makes the GGW and ideal technology for mm-wave applications.

The carried out comparative has provided valuable information and reliable conclusions concerning the choice of GGW front rectangular waveguide in certain design scenarios. First, the comparative of the S-parameter response of both filter concludes in that, in general terms, GGW filter overcome the rectangular waveguide filter. Therefore, experimental results confirm the suitability of GGW topology for V-band filter design as an alternative to rectangular waveguide, being that, despite of the fact that the later presents better simulated response (lower insertion losses), it is also more affected by the manufacturing process, presenting a quite worsened measured response respect to the simulated one. It is pointed out that both topologies experience similarly manufacturing tolerances. The observed similar frequency shift is a good proof of this.

Second, and even more important, GGW has been shown to allow a postprocessing procedure based on the use of iteratively designed top plates with insertions. A high grade of success has been obtained with this technique (results of Design 3). The re-assembling test has shown that the rectangular waveguide filter, unlike the GGW design, presents important changes in its response after being re-assembled. This makes really challenging to apply such correction procedure to rectangular waveguide topologies, being that the response is very unstable front top plate replacements. Therefore, whereas with GGW topology a NBF response satisfying frequency specifications is affordable at $\mathrm{V}$-band, with the rectangular waveguide seems quite difficult, at least, with current manufacturing tolerances. Under the same conditions, GGW has shown to be more suitable in a significant way.

\subsubsection{Results in the context of V-band NBFs state of the art}

By means of the involved synthesis procedures and experimental results of Design 2, Design 3 and, finally, of the comparative with rectangular waveguide (Design 2 implemented in this topology), several conclusions have been obtained. This information is now contrasted with external works in the literature in order to contextualize it and give further credibility to the results. In this way, the contribution of the work in this thesis will be easier to be evaluated. The next study is similar to that carried 
out in section 10.3.6 for Ka-band filters, however, it is deeper, with a larger number of reviewed works.

Table 10.16 shows a summary of the most relevant works regarding V-band NBFs. Several technologies are included. Obviously, integrated technologies such as CMOS cannot be fairly compared in terms of performances with SIW filters, or even more, with GGW or rectangular waveguide cavity filters. However, to give vision of the complete scenario results quite helpful to understand the development state of the technology so that results in each technology can be better evaluated (for instance, comparing the complete scenario with its counterpart at lower frequencies it is possible to have an estimation of the expected grade of worsening, so that a reference is kept in mind). In general terms, frequency shift and response widening is a recurrent problem no matter the technology. The narrower the bandpass of the filter the more enhanced results this effect in relative terms, becoming the main design problem. The column $\Delta f$ gives a qualitative estimation of the frequency shift of the response. Widening of the response, which is slightly less common than frequency shift has been also considered to contribute to the qualitative value of $\Delta f$, e. g., if the frequency deviation is medium but an important widening is observed, the $\Delta f$ assessment is set as high. Experimental responses of some of the filters of Table 10.16 are shown in Fig. 10.68 in order to give a general idea of the main issues and challenges encountered in the performance of practical implementations of V-band filters. An image of the prototypes is included for each plot inside a the blue-marked insets.

Integrated technologies suffer from very high insertion losses induced by the low quality factor of the involved resonators, which experience both, high ohmic and dielectric losses. The response of such filters is still far from real environment specifications except for very simple, low-cost applications. Integrated solutions of SSGW following [871]-[873] could be useful to improve results in this area. SIW or pseudo-SIW (similar concept but with differences, e. g., via holes more separated than usual, and authors avoid to typecast them as SIW), and advanced designs with classical transmission lines, give an intermedium performance. However, in order to achieve certain performance, close to $Q=500$, cavity filters are required. The employed structures use via-holes in a similar way than SIW concept, however, planar profiles are avoided in order to reduce losses (cavities are coupled vertically). Thanks to the employed low-loss dielectrics losses are kept under acceptable levels which leads to a reasonable good performance.

However, in order to achieve high performance with a $Q$ beyond 700, the structure must be irremediably based in rectangular waveguide type topologies (either classical rectangular waveguide or GGW). As it has been commented in the previous section, GGW gives better performance, on the one hand regarding losses (equivalent $Q$ is $11.5 \%$ larger) and return loss figure, and on the other hand due to the possibility of totally avoid frequency shift with the procedure implemented in Design 3. In order to contrast this information, a commercial V-band $\left(f_{0}=60.6\right.$ $\mathrm{GHz}$ ) rectangular waveguide NBF offered by RTx Technology Co. [967] has been analyzed. This product includes tuning screws incorporated with advanced manufacturing technology (non-affordable with the employed in-house process for the experimental prototypes of this thesis) that allow to obtain a response meeting the specifications. It is fair then, to compare this filter with Design 3. With both filters 


\begin{tabular}{|c|c|c|c|c|c|c|c|}
\hline Filter & Technology & $N$ & $I L$ & $R B W$ & $Q_{e q}$ & $R L$ & $\Delta f$ \\
\hline [968] & $\mu$ strip BiCMOS & 2 & $1.5 \mathrm{~dB}$ & $14.1 \%$ & 33 & $8.5 \mathrm{~dB}$ & Medium \\
\hline$[969]$ & $\mu$ strip CMOS & 3 & $5 \mathrm{~dB}$ & $4.9 \%$ & 33 & $10 \mathrm{~dB}$ & Medium \\
\hline$[970]$ & LTCC Stripline & 3 & $5 \mathrm{~dB}$ & $8.3 \%$ & 40 & $10 \mathrm{~dB}$ & Small \\
\hline$[982]$ & LTCC SIW & 4 & $7 \mathrm{~dB}$ & $3.4 \%$ & 94 & $20 \mathrm{~dB}$ & Small \\
\hline$[983]$ & LTCC SIW & 4 & $1.8 \mathrm{~dB}$ & $11 \%$ & 112 & $10.2 \mathrm{~dB}$ & Medium \\
\hline$[984]$ & Pseudo-SIW & 4 & $2.8 \mathrm{~dB}$ & $5.1 \%$ & 151 & $11 \mathrm{~dB}$ & High \\
\hline$[853]$ & Pseudo-SIW & 3 & $3 \mathrm{~dB}$ & $3 \%$ & 155 & $15 \mathrm{~dB}$ & Medium \\
\hline$[365] \mathrm{A}$ & Microstrip & 4 & $2.8 \mathrm{~dB}$ & $2.7 \%$ & 266 & $27 \mathrm{~dB}$ & Small \\
\hline [985] & Air-Stripline & 4 & $1.1 \mathrm{~dB}$ & $10 \%$ & 198 & $9 \mathrm{~dB}$ & Medium \\
\hline$[986]$ & Microstrip & 3 & $3.1 \mathrm{~dB}$ & $2 \%$ & 210 & $23 \mathrm{~dB}$ & No \\
\hline$[973]$ & LTCC Cavity & 4 & $2.8 \mathrm{~dB}$ & $3.4 \%$ & 225 & $13 \mathrm{~dB}$ & High \\
\hline$[365] \mathrm{B}$ & Microstrip & 5 & $3.4 \mathrm{~dB}$ & $4.3 \%$ & 195 & $23 \mathrm{~dB}$ & Small \\
\hline$[974]$ & LTCC Cavity & 4 & $3 \mathrm{~dB}$ & $2 \%$ & 362 & $15 \mathrm{~dB}$ & Medium \\
\hline [975] & LTCC Cavity & 3 & $2.2 \mathrm{~dB}$ & $1.5 \%$ & 432 & $16.3 \mathrm{~dB}$ & Medium \\
\hline D2' & WR-15 & 5 & $1.6 \mathrm{~dB}$ & $2.6 \%$ & 726 & $9 \mathrm{~dB}$ & Medium \\
\hline$[967]$ & WR-15 & 5 & $2 \mathrm{~dB}$ & $2 \%$ & 760 & $17 \mathrm{~dB}$ & No \\
\hline D2 & GGW & 5 & $1.5 \mathrm{~dB}$ & $2.5 \%$ & 810 & $13 \mathrm{~dB}$ & Medium \\
\hline D3 & GGW & 5 & $1.5 \mathrm{~dB}$ & $2.5 \%$ & 810 & $16.4 \mathrm{~dB}$ & No \\
\hline
\end{tabular}

Table 10.16: Comparison between several recently purposed $V$-band narrow-band filters. $N:=$ Filter order; $I L:=$ Insertion losses; $R B W:=$ Relative bandwidth; $Q:=$ Equivalent quality factor; $R L:=$ Return Losses; $\Delta f:=$ Frequency shift. D2' refers to Design 2 with rectangular waveguide, D2 refers to Design 2, and D3 refers to Design 3. 


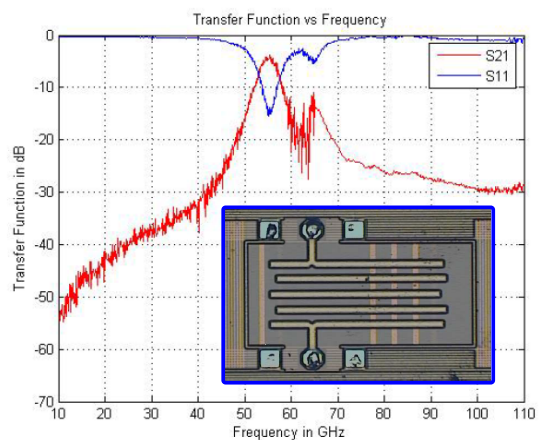

(a)

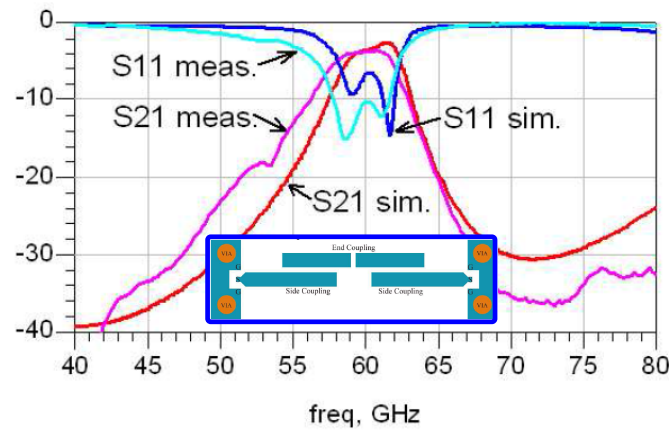

(b)

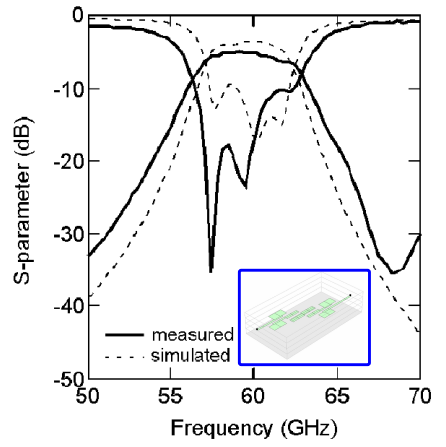

(c)

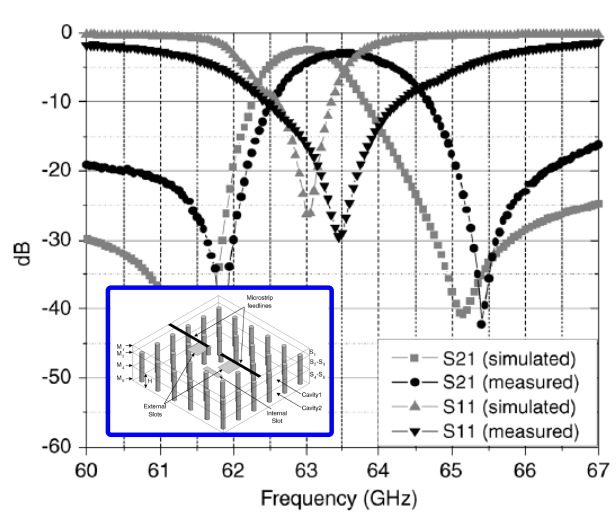

(e)

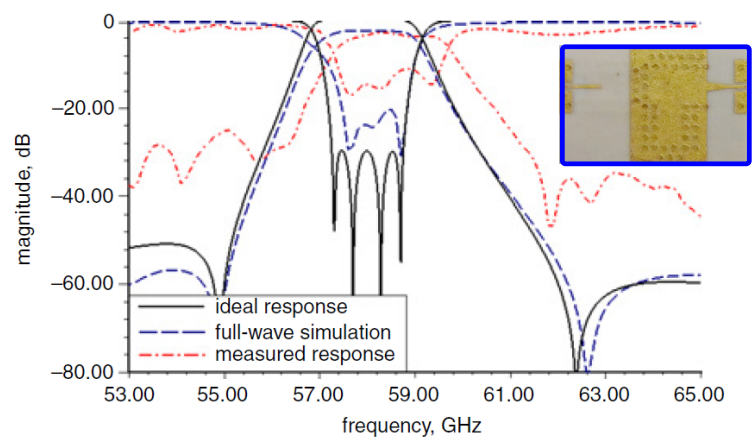

(d)

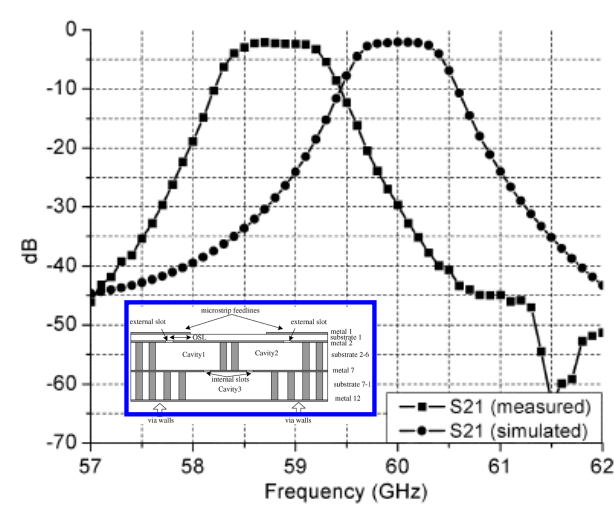

(f)

Figure 10.68: Examples of experimental response of some of the filters of Table 10.16. A photograph of the manufactured prototype is shown in each graph inside the blue-marked inset. a) Microstrip CMOS filter of [968]; b) Microstrip BiCMOS filter of [969]; c) LTCC Stripline filter of [970]; d) LTCC cavity filter of [973]; e) LTCC cavity filter of [974]; f) LTCC cavity filter of [975].

satisfying the specifications without any appreciable frequency shift, Design 3 with GGW presents an equivalent $Q 6.5 \%$ higher than the obtained one with the rectan- 
gular waveguide filter of RTx Technology Co. Taking into account the limitations of an in-house process front the advanced manufactured facilities of a commercial vendor, makes evident the advantages of GGW and its suitability to be considered instead of rectangular waveguide topology to implement V-band NBFs. It is noted also that the analysis of the rectangular waveguide prototype offered by RTx Technology Co. gives credibility to the obtained results with the rectangular waveguide prototype presented in this thesis. Notice that, leaving appart return losses and frequency shift issues, both rectangular waveguide filters present a similar maximum equivalent $Q$, obtained with the lowest insertion loss value in the passband. This indicates that, indeed, rectangular waveguide topologies are limited so that overcome the obtained $Q$ values is rather challenging.

The previous results suggest that advanced manufacturing industrial techniques applied to GGW topology may open the door to a jump in performance reaching values close to $Q=1000$, a level which is simple to reach in frequencies as close to those of Ka-band, but that seems not affordable at V-band. Thoughtful disposition of the coupled cavities, including innovative designs such as that presented [845], where GGW cavities are vertically coupled (a distribution rather complex and prone to leakage in $\mathrm{H}$-plane rectangular waveguide manufacturing) may facilitate reaching such high performance. Therefore, it seems clear that to explore the use of advanced high-performance manufacturing techniques currently available in industrial houses to GGW filters, to carry out vibration and temperature tests, and to explore advanced filter topologies with GGW (response with transmission zeros by means of cross-coupled resonators, hybrid horizontal-vertical coupling structures, etc.) are three mandatory future work lines to undertake soon. The high performance of GGW structures should not be neglected by RF houses in the next years, being that most of them still do not incorporate $\mathrm{V}$-band filters in the offer (see for instance catalogs of Pasternack ${ }^{\circledR}$ [965]a nd AINFO. Inc [966]), and a huge demand is to come proximately, driven by new generation $\mathrm{V}$-band applications. An eye must be kept on new proposals using GGW, as they may determine massive industrial adoption of this technology which up to now, has been show to be very promising. The work in this part of the thesis is a small contribution in this sense, therefore, satisfying one of the most important objectives of this part of the thesis. 
Chapter 10. Groove Gap Waveguide (GGW) components 


\section{Chapter 11}

\section{Conclusions and future Work}

The conclusions derived along this part of the thesis are collected in this chapter in order to transmit them in a clear and ordered way. In addition, the work procedure as well as the conclusions have pointed towards several possible future work lines that are also described in this chapter.

\subsection{Conclusions}

All the imposed objectives in the scope of this part of the thesis have been tackled and worked deeply until they have been accomplished with the expected grade of success. During the followed procedure, several concepts and remarks with special importance have appeared. From the perspective obtained at this point of the thesis, these elements are able to generate well-founded conclusions that are given next, classified by chapters.

In Chapter 8, current mm-wave applications and technological problems have been studied and a deep review regarding Gap Waveguide technology has been carried out. From this broad research work it is concluded that:

- After two failed attempts, mm-wave technology is receiving these days its definitive progress to maturity. Everything in the scenario indicates an imminent consolidation and universal application of mm-waves. Although automotive radar applications are developing with intensity, they are not longer the battleship of mm-wave applications, but mobile communications lead the progress these days. Synergy between both areas is also arising with new generation vehicular communications. General users will experience and increase of security and comfort in the next years. Imaging applications are also under the focus these days. Regarding imaging properties, the mm-wave band has not the same potential than the $\mathrm{THz}$ band, however, in the very near future, mm-wave will impose in commercial scenarios due technological reasons, mainly justified by MMIC performance.

- A careful and deep review of the literature involving conventional waveguides and transmission lines operating at high microwave frequencies and mm-wave 
band confirms that appreciable degradation takes place for such structures. $\mathrm{H}$-plane rectangular waveguides suffer from leakage due to the cut of currents between the manufactured pieces which do not experience the necessary good metallic contact by cause of manufacturing roughness. E-plane rectangular waveguide as well as circular waveguide experience alignment problems. Similarly, common transmission lines experience a large number of negative effects as the frequency increase including ohmic, dielectric and radiation losses, as well as parasitic modes. Several modifications have been proposed, but they have complex implementation and low-performance. Revolutionary advances in materials are hardly expected and technological advances in the manufacturing process are quite conditioned: photoresist techniques are better suited for higher frequencies and milling techniques have little empty room to be improved. Therefore, to explore new topologies seems to be the best solution. Such new topologies must be not affected by poor metallic contact or dielectric losses, and they must facilitate packaging and integration.

- SIW structures have shown interesting characteristics for the lowest frequencies of the mm-wave band. They have a planar profile, exhibiting features between conventional planar and hollow waveguides, and a simple manufacturing process. However, for higher frequencies (V-band and beyond), this topology is noticeably challenged by performance degradation, mainly caused by dielectric losses and, to a lesser degree, by dispersion. Alternative topologies must be considered. Regarding to the objective of GW technology adoption, it must be highlighted that it has been observed that the evolution of SIW technology in the research community from novelty to adoption shows that consolidation of a new technology demands from: 1) efficient analysis methods, easy to use, conceptually clear and that establish equivalence or relationship with well-known structures and techniques ; 2) technological consistence, achieved through the proposal of a large variety of devices, this including experimental validation in order to give evidence of the introduced advantages.

- The review on Gap Waveguide technology literature proves that the different proposed topologies provide similar functionality than conventional waveguides and transmission lines at the same time that they avoid the main technological problems encountered at mm-wave frequencies. As product of the review, the following conclusions can be affirmed:

> The number of papers in Gap Waveguide technology topic have increased exponentially in the past decade. GW Researchers tend to face more elaborated devices and GGW has gained lot of terrain to RGW as the structure with more projected potential. Current workforce is concentrated these days on V-band.

> Antennas has been the most prolific element, headed by Slot Array Antennas (SAA) proposals. Specially in 2D arrays, disadvantages of rectangular waveguide topologies (leakage due to bad metal contact) and microstrip (high losses as well as packaging and substrate resonances) are avoided by using GW technology. 
- Filters and diplexers have also received good attention. To date mainly basic configurations have been explored with some recent research in novel filter topologies. Nevertheless, the obtained results point towards a promising future in which advanced responses will be synthesized by means more complex GW filter topologies.

- The work in transitions and flanges has been intense and necessary being that these elements are indispensable to: 1) provide interconnection with conventional technology in order to validate the prototypes; 2) allow interconnection between different GW devices, what is mandatory to reach a scenario of GW technology consolidation, in which advanced mm-wave systems can be totally or almost totally implemented on GW topologies.

> Consistent work at higher frequencies in the mm-wave band starts to appear. Although this area is still not mature, the near future will probably come with highly competitive devices being that technological problems of conventional structures are enhanced as the frequency increases.

> Modeling of GWs has been a great and pursued challenge since the first GW proposals. During a first stage, approximate methods based on homogenization of the pin structure were common. Regarding to the impedance, IP formulation was preferred. More recent work points to a future scenario where methods will fully consider the pin geometry, and ease of practical application will be disposed as a premise. Transmission line techniques will gain terrain and modal methods may appear soon, thus the scenario becoming quite similar to that encountered for conventional waveguiding structures.

- GWs not only show extraordinary self-packaging properties, but this feature has been progressively applied to other structures. GW based packaging structures have improved performance of microstrip, CPW and even SIW circuits, and they also show good properties to manage isolation in power amplifier chains. In fact, the packaging topic has experience a great increase of attention these last years.

In Chapter 9 a method to characterize single and coupled SSGW lines in terms of their impedance and propagation constant has been proposed and validated. Moreover, procedures to design SSGW with stable impedances have been established. Then, a design chart for SSGW coupled lines in the Ka-band has been generated, which has facilitate the posterior design of a coupled-line filter. Main conclusions extracted along the involved processes and by analyzing the obtained results are:

- Calculation of GW transmission lines (RGW, MRGW, SSGW) characteristic impedance results ambiguous due to the periodic nature of the waveguide and the lack of uniqueness in the impedance definition. From a practical design point of view it seems more adequate to treat the transmission line as a component, in terms of its scattering parameters regarding a reference structure. 
- Although it is not the first time that a transmission line approach is employed to characterize SSGW impedance, several errors found in previous works have been identified and corrected. Furthermore, the analysis method has been rigorously presented, introducing a formal mathematical treatment of the error that allows to quantify the introduced output error in terms input error. It has been found that the error in the calculated impedance is of the same order of that present in the S-parameter calculated by full-wave simulation whenever reference port and waveguide are similar enough, what can be quite easily guaranteed.

- To date, it has been a common procedure to prioritize the maximization of the stopband of the GW component under design. In the case of SSGWs, this leads to periodic structures of dimensions such that, for common impedances, the relationship between strip width and period satisfies $w<p$. This cause two effects that noticeably increase the encountered difficulties during the design process:

- It is found a strong dependence of the impedance value on the relative position of the strip respect to the pin rows.

- There is a minimum achievable impedance since, when the strip is made narrower than the underlying pins, electromagnetic fields spread, leading the fundamental mode to experience a wider effective width.

- The solution of rotating the strip $45^{\circ}$ reduces the effective period experienced by the strip by a factor $\sqrt{2}$. However, although this solution may introduce certain advantage in simple circuits, with the involved strips predominantly oriented in certain direction, it results ineffective for more complex circuits. In addition, even in the best case, a factor $\sqrt{2}$ seems to be insufficient to achieve a suitable stability.

- SSGWs with stable impedance are achievable by using dense pin lattices at the cost of a slight reduction of the stopband bandwidth. Nevertheless, it is found that, since the device bandwidth is generally limited by other elements than the periodic structure (e. g. transitions, radiating slots, filter passband), a slight reduction of it has not any implication on the device performance. Increments of density by a factor 5- 6 while keeping $80 \%$ of the stopband bandwidth have been reported in this thesis, still achieving manufacturable pins according to previous experience with the in-house milling machine. In addition, it is found that, with the same argumentation, it could be suitable to allow a further reduction of the stopband in order to have less restrictions on the periodic structure parameters, what could alleviate the manufacturing process complexity. In this sense, future designs may consider a stopband strictly adjusted for the proposed specifications either to cover a complete band or designed ad hoc for certain component.

- The use of dense periodic structures with low period implies:

> Larger number of pins to manufacture. However, it is observed that, unlike RGW or GGW structures, the function of pins in SSGW structures 
is merely to provide a PMC condition underlying the substrate with the strip. In this sense they may be roughly manufactured without excessive implications. Actually, it is the manufacturing precision of the circuit layout on the substrate what mainly determines the circuit performance.

- More complex full-wave analysis. However, for such periodic structures, homogenization of the pin structure may be applicable. This would imply an even faster analysis than that required for conventional SSGWs. In such case, full-wave simulations incorporating an anisotropic substrate instead of the pins structure could be applied, with the corresponding advantages that this implies.

- Assuming that the bandwidth of a GW transmission line (RGW, MRGW, SSGW) coincides with the stopband has been a common practice in the literature. However, if low impedances values are required in the circuit, wide ridges/strips must be used, what may enable the propagation of the second ridge/strip mode inside the stopband, hence reducing the effective bandwidth to that described by the monomode operation.

- The characteristic impedance of a SSGW experiences certain dispersion, larger than that of conventional quasi-TEM transmission lines (microstrip, CPW, etc.), inherent to the use of a periodic structure. However, the obtained results indicate that this dispersion is low enough to carry out designs of moderate bandwidth. Contrarily to conventional transmission lines, higher frequencies experiences lower impedances. This phenomena is caused by the fact that, for higher frequencies, the PMC condition is created at a lower height. Therefore, PEC and PMC planes are more separated and fields spreads more laterally, hence the fundamental mode experiences a wider effective width.

- For a given value of impedance, the SSGW requires wider strips than conventional transmission lines. This implies an additional advantage in terms of ohmic losses and facilitates the circuit layout manufacturing, generally limited by the narrowest strip or strip separation width.

- SSGW coupled lines exhibit similar behavior than conventional coupled transmission lines. Therefore, the analysis method presented in this part of the thesis to calculate SSGWs impedance has been directly applicable to model SSGW coupled lines by means of the odd/even mode impedance analysis, and design charts have been shown to be straightforwardly achievable. Dispersion of SSGW coupled lines is very similar to that of isolated lines, thus allowing for moderate bandwidth designs.

- A simple SSGW coupled-line filter has been designed by using coupled-line filter theory together with the design chart developed. Simulation results predict outstanding low insertion losses, 5-6 lower than those predicted for microstrip designs.

- The filter response is practically insensitive to the circuit layout position over the pin structure thanks to the density of this last. The small variation of the response suggest a large deterioration if a non-dense pin structure was used. 
- Study of RGW/SSGW/SIW resonators in the NBF context reveals that SSGW performance clearly overcomes SIW performance since dielectric losses are noticeably alleviated with the former topology. On the other hand, SSGW performance is even more clearly overcome by RGW performance, which totally lacks of dielectric elements.

In Chapter 10, propagation characteristics of GGW below and above cutoff have been studied, and an analysis method providing an accurate equivalent between GGW and rectangular waveguide has been presented. Then, several filters have been designed and experimentally validated with increasing grade of performance. Finally, an experimental comparative between GGW and rectangular waveguide has been carried out by means of filter prototypes. From the involved work and results it can be affirmed that:

- From the propagation characteristics analysis of the GGW several properties of the waveguide have been clearly realized and described for the first time:

- Above cutoff. The GGW is more dispersive than the rectangular waveguide. Depending on the propagation channel width $a$, the relationship between the cutoff frequencies of the fundamental modes of both waveguides is different: for smaller $a, f_{c_{G G W}}>f_{c_{\text {rect }}}$ whereas for larger $a$, $f_{c_{G G W}}>f_{c_{\text {rect }}}$. The analysis of the dispersion diagram of both waveguides suggests a frequency-dependent relationship between GGW and rectangular waveguide. Therefore, a direct, pure geometric equivalent between both waveguides cannot be found. The assumption of that propagation characteristics of both waveguides are equivalent only holds for certain range of $a$ and for certain bandwidth.

> Below cutoff. The GGW is able to operate below cutoff in a similar manner than the rectangular waveguide. Below cutoff differences between both waveguides are much more evident so that assuming equivalence between them is totally wrong and will not contribute with any information to the design process of an evanescent-mode device.

- EM fields in the structure. In a GGW, EM fields exhibit a very similar pattern to that of a rectangular waveguide of same propagation channel width. However, a strong concentration of fields occur over the pins, mainly for those delimiting the propagation channel. As the frequency increases, the field pattern of both waveguides becomes more similar, with EM fields concentrated in the propagation channel. As the frequency decreases, fields spread and local effects are enhanced. This behaviour is originated by the larger interaction of propagating waves in the structure with the lateral pin walls:

* Above cutoff. Fundamental mode decomposing plane waves reach lateral pin walls with larger angle of incidence for lower frequencies.

* Below cutoff. Both, the lateral condition imposed by the periodic structure and the axial propagation experienced by the fundamental mode are of evanescent nature. At lower frequencies, propagation 
becomes more evanescent in the axial direction so that the mode is forced towards the waveguiding channel laterals.

- A method based on transmission line theory has been successfully applied to obtain the impedance observed towards the lateral pin walls of the propagation channel. The analysis (lossless) reveals that this impedance is a pure reactance that grows with the frequency (Foster's Theorem). By applying transmission line theory, it has been determined that the same impedance condition would be observed is PEC walls were placed at a certain distance from the actual pin walls. This leads to an equivalent between GGW and rectangular waveguide that depends on the frequency. Since the reactance grows with the frequency, the virtual width of GGW does, which implies that a GGW is, in terms of the propagation constant, equivalent to a rectangular waveguide which width increases with the frequency.

- During the robustness test of the method two extreme pin structure configurations were studied, discovering that GGW has enormous potential for dispersion engineering tasks. In particular, it has been found that:

> Low density lattices of thin pins and large separation between pins and top plate lead to a very dispersive GGW

- Lattices with thick pins and small separation between pins and top plate lead to GGW with practically the same dispersion than a rectangular waveguide.

- The proposed analysis method has been shown to be:

- Efficient: Given a GGW, full-wave simulation is only required for a small structure containing three pins, with the rest of calculations done analytical. It is possible to vary $a$ without any additional full-wave simulation. For a given $a$ the presented method is two orders of magnitude faster than full-wave simulations.

- Accurate: Results provided by the proposed analysis method have provided excellent coincidence with both, full-wave simulations and measurements carried out with two manufactured TRL calibration kits.

> Conceptually perceptive: An equivalence with a well-known structure, such as it is the rectangular waveguide, is provided.

- Robust: Propagation characteristics of the GGW have been recovered with accuracy for a variety of propagation channel widths and periodic structure dimensions.

- Narrow-band filters state of the art in Ka-band and V-band reveals that frequency shift of the response is a common encountered problem caused by the high sensitivity to dimensional errors at such small wavelengths. In addition, insertion losses are sensitively larger than those encountered in usual microwave filters. These negative effects are specially evident in V-band designs. With increasing order of performance (and decreasing order of compactness) 
one founds: conventional transmission line filters, SIW filters, LTCC cavity filters and rectangular waveguide filters.

- All-pole cavity filters with low insertion loss are straightforwardly implementable with GGW structures using similar topologies to those employed in rectangular waveguide filters but using pin walls instead of metallic walls for the filter layout.

- Three GGW filters have been designed, manufactured and measured. The following conclusions are extracted from the process and results:

> Design $1,4^{\text {th }}$-order Ka-band filter at $f=40 \mathrm{GHz}$ :

* Good insertion and return losses are achieved, however certain frequency shift, caused by manufactured errors, is observed in the response.

* The design obtain good results with the periodic structure truncated after the second row of pins. However, simulations predict better performance it truncation is done after the third row of pins. Including more pin rows do not introduce any improvement.

* The filter response results competitive in the Ka-band, however, commercial rectangular waveguide filters perform better.

> Design 2, $5^{\text {th }}$-order V-band filter at $f=61 \mathrm{GHz}$ :

* Due to frequency requirements, smaller coaxial cables are required for the feeding. Because of the small coaxial cable dimensions, it is necessary to insert them beyond their first section (metallic pin) in order to perturb enough the external cavities.

* A manufacturing sensitivity error analysis has been carried out concluding that:

- Errors in the pin width, $\Delta w$, affects almost exclusively to the frequency shift in the response. There is large sensitivity to this error that easily causes the response move far away laterally from the specifications. This error is hardly avoidable given current manufacturing tolerances.

- Errors in the horizontal position of the connector, $\Delta\left(x_{0}, y_{0}\right)$, influences exclusively return losses with a secondary order effect. Influence of each coordinate depends on the grade of variation of the external quality factor. Cases with $x_{0}>y_{0}$ will exhibit larger sensitivity to $\Delta x_{0}$ since the external quality factor experiences greater variations in this coordinate. This error is of minor importance given current manufacturing tolerances.

- Errors in the vertical position of the connector, $\Delta z_{0}$, influences exclusively return losses with a primary order effect. This error is important but it can be well controlled with the aid of an auxiliary piece to regulate the connector penetration. 
* A good return losses figure with competitive, low insertion losses is found in the response. However, frequency shift remains as an important problem, even enhanced respect from the Ka-band response: sensitivity increase whereas manufacturing precision is the same.

* The filter response is free of spurious and presents a high rejection level in a large bandwidth thanks to the stopband effect. This suggests the engineering of the stopband to cover the passband surrounding spectrum accordingly to possible spurious locations in those designs threatened by this problem.

> Design $3,5^{\text {th }}$-order $\mathrm{V}$-band filter at $f=60 \mathrm{GHz}$, including post-manufacture tuning metal insertions:

* It has been shown that GGW topology allows post-manufacturing correction of a filter response by a simple procedure which consist of exclusively replacing the top plate of the filter, where some tuning metallic insertions are displayed strategically.

* The initial design must contain a top plate with insertions of certain dimensions in order to be able to reduce them in the next iteration top plate if necessary.

* Aggressive Space Mapping algorithm using full-wave simulations as coarse model and manufacturing process as fine model has been found extremely efficient for the iterative correction of the response. By applying this algorithm, the number of required manufactured top plates with insertions is very reduced.

* The presented procedure is very efficient insomuch as, in the presented filter, the top plate with insertions represents only $2 \%$ of the total manufacturing cost.

* The filter response satisfies the imposed specifications. Frequency shift and widening of the response are corrected and return losses are improved to required level while insertion losses are kept as low as in Design 2.

* The presented filter is highly competitive with a performance overcoming even rectangular waveguide commercial designs.

- A comparative between GGW and rectangular has been carried out by comparing the performance of both topologies when implementing a $\mathrm{H}$-plane $5^{\text {th }}$-order V-band filter at $f=61 \mathrm{GHz}$. From this study it can be affirmed that:

- Frequency shifts affect in a similar way both topologies.

> Return losses of the GGW filter are better, however, previous experience with GGW filters suggests that, in general, similar figures will be obtained for both topologies.

> Insertion losses of the GGW filter are slightly lower than those of the rectangular waveguide filter even though the response of this last presents a slight passband widening. In terms of equivalent quality factor, the better performance of the GGW filter front the rectangular waveguide filter become certainly evident. 
- The GGW filter exhibits noticeably larger robustness than the rectangular waveguide filter being that the response of the former remains practically invariant front a re-assembling test whereas the response of the last exhibits an important degradation in terms of insertion losses and return losses. This has the following implications:

* GGW topology is more robust front mechanical vibrations.

* GGW topology will be less affected by random errors during the assembling process.

* Rectangular waveguide filters cannot be corrected through the presented post-manufacturing correction procedure since the response is very unstable front the re-assembling process.

> In general, it is expected better performance using GGW topology than using rectangular waveguide topology when implementing H-plane devices.

\subsection{Future Lines}

The work in this part of the thesis has reached significant entity to be finished here, and pretends to put itself a bit on the progress of mm-wave technology. However, as any research work, it has been stopped at some point, and several issues remain for future research. Some future work lines have arisen during the research process as issues to solve or to improve, whereas others are the natural continuation of the research flow. All of them are compiled in the following list:

- As a general scope line, research in the characterization of the mm-wave propagation channel would be very suitable. Modeling of new and more complex scenarios together with improved simulation techniques would provide more quantity and quality data for the radiofrequency engineer, hence leading to high-level device optimization. In addition, research in order to minimize the technological gap between microwave and mm-wave, pursuing homogenization and integration, is a valuable path to follow.

- Regarding to the technological problems affecting passive components, a valuable work line would be to continue exploring new topologies that alleviate the natural drawbacks appearing at mm-wave frequencies. To improve manufacturing techniques is obviously necessary as well. Nevertheless, synergy between both research lines, i. e., not only adapt the topologies to the manufacturing method but the manufacturing method to the topologies, is the key point to success.

- A natural continuation with the modeling of SSGWs is to derive closed-form expressions that totally characterize the waveguide in terms of propagation constant and impedance. Two ways to reach this goal are foreseen:

- To apply, either a similar analysis method to that applied for the GGW in order to remove the pin structure in turn of an impedance condition, 
or homogenization given that the pin structure will be dense. Then, from that point, to find a microstrip equivalent given the topological similitude.

- To use the presented analysis method to extensively parametrize the SSGW structure. Then, by data fitting, empirical expressions could be derived.

Of course, this can be directly extended to SSGW coupled lines.

- In the line of some techniques derived in this part of the thesis it would be interesting to find an equivalent between SSGW and RGW in such way that designs from SSGW could be directly mapped on RGW. In this way, complex designs could be prototyped in SSGW during the design stage and finally implemented in RGW taking advantage of its higher performance.

- A pretty direct work line is to face the manufacturing of the designed SSGW coupled line filter. Attending to the possible encountered issues, the design of a higher order filter with a more complex response could be well afforded. The final goal would be to develop advanced planar filters.

- As a general expansion of the work with the SSGW, analysis, design and manufacturing of more advanced SSGW components taking advantage of the analysis and design techniques developed in this part of the thesis is, clearly, a future work line to address. In this way SSGW technology could reach the necessary technological consistence to be adopted by the industry.

- An interesting work line dealing with the practical implementation of SSGWs would be to explore both, modifications on the pin structure and manufacturing techniques, that alleviate the manufacturing cost and increase the SSGW mechanical robustness.

- As it has been done between GGW and rectangular waveguide, an interesting comparative would be to challenge microstrip, SIW, SSGW and RGW to experimentally implement the same design. Such a work line would provide very useful quantitative information for the comparison between those waveguides, nowadays somehow diffuse.

- The equivalent between GGW and rectangular waveguide could be upgraded if information about the electromagnetic field pattern of the fundamental mode is included. This work line would reach is very final goal if rectangular waveguide designs could be almost directly mapped to GGW, what could be revolutionary.

- A good work line would be the research on the dispersion engineering capabilities of the Groove Gap Waveguide. The influence of each periodic structure parameter on the dispersion characteristics of the GGW should be determined. The very final goal in this line would be to empirically model with closed form expressions such influences, i. e., to provide a pure analytical model for the propagation characteristics of the GGW. 
- An almost mandatory future work line is to face complex designs with the GGW topology by using the analysis method proposed in this part of the thesis. In this way, all the potential of the method in the optimization stage would be realized and, additionally, the method could be improved from possible encountered issues.

- A quite necessary future work line regarding the state of the art is to carry out a deep analysis of propagation characteristics of GGW-HP, less known than those for GGW-VP, and to search for a proper equivalent with the rectangular waveguide. In the same way, it would be desirable to find an equivalent between RGW and the classical ridge waveguide.

- Regarding to the GGW filters topic, it seems obvious that new filter topologies and more advanced response must be pursued. This includes to explore new ways to excite and couple the cavities, incorporate zeros in the response or consider dual mode cavities, for instance.

- It would be quite interesting to extend the post-manufacturing correction procedure to other GGW components such as antennas, dividers, diplexers, etc., and to other GWs such RGW or GGW-HP. High performance designs could be reached according to what has been observed in this part of the thesis.

- A direct future work line is to carry out design procedures in which a multilevel ASM is used. A three-level ASM is conceived if the current coarse model becomes fine model in a surrogate ASM routine which would use the GGW equivalent with the rectangular waveguide as coarse model. In this way, the main optimization task would be done over a rectangular waveguide structure, very fast to simulate and taking advantage of a vast quantity of available methods. This multi-level ASM procedure may be used even without requiring the equivalent between GGW and rectangular waveguide although, in such case, the converge velocity would be affected.

- It seems quite desirable as well to carry out a further comparison between GGW and rectangular waveguide in which vibrational and temperature test are carried out specifically, with proper equipment. In the same way, it would be interesting to consider PIM and multipactor in tests. In addition, it would be desirable to realize the prototypes with last-generation industrial manufacturing processes in order to provide a more reliable comparison from the commercial manufacturer point of view. Good results could lead to a rapid adoption of GGW technology by the industry. It is as well possible to include in this line a comparative with E-plane rectangular waveguide devices.

- With the research inertia of these days, it seems that an obvious future step is to face designs at higher frequencies, focused in W-band at a first stage. Such high-frequency scenario could be the definitive proof of the potential of GW front conventional alternatives. 


\section{General Conclusions}

A good number of objectives were proposed before undertaking the research involved in this thesis, some of them really challenging. From this point and looking back the work, results and conclusions previous to these closing general conclusions, it can be certainly affirmed that they have been satisfied with the expected grade of success. With no little effort a wide scope thesis, facing state-of-the-art issues from $\mathrm{mm}$-wave and $\mathrm{THz}$ bands, approaching both analysis and design techniques, and realizing comprehensive classification of a large quantity and variety of information, has been completed. Passion for electromagnetics and underlying mathematics has been a motivation of great importance in this sense. From the sidelines, the fruit of this effort are the good results and conclusions obtained this thesis as well as its objective utility for the future researcher dealing with the pioneer topics treated therein, which expect intense evolution in the next years. Towards inside, as the author, gained knowledge, expedience and skills are the big reward.

Regarding to the research related with $\mathrm{THz}$ components, it has been found a quite arduous labour to assimilate such a large quantity of information regarding collateral topics such applications, sources and sensors. In all them, concepts generally outside of conventional radiofrequency engineering are found, being sources and sensors specially demanding in terms of technical knowledge. Furthermore, at $\mathrm{THz}$ frequencies, information is specially disperse in a large variety or sources. It has been, nevertheless, an interesting apprenticeship. On the other hand, the development of analytical tools for wire-type waveguides has been considerably challenging not only in terms of the mathematical and numerical complexity, the most relevant in this thesis, but also in conceptual terms. Design procedures have had its own complexity as well, specially when it has been required the use of general-purpose software, non well-prepared for wire-type structures analysis. Finally, experimental test with wire-type waveguides have been not straightforward given the exotic nature of these waveguides compared with the standard facilities. Specially at $\mathrm{THz}$ frequencies, a special care has been required to implement the experimental setups and recover the measurements.

Regarding to the research related with mm-wave components, it can be said that the research scope results inherently narrowed to more common radiofrequency concepts. In addition, as a posterior part, good advantage has been taken from the gained knowledge with the first part of the thesis. The documentation research task has been relatively straightforward but requiring important effort, mainly to the quantity of managed information and numerical data. It has been specially nice, notwithstanding, to see all-together the GW literature, endorsing well the consol- 
idation of GW technology. Concerning the technical work, from the analysis side, the development of the GGW analysis method, providing equivalence with rectangular waveguide, has required an appreciable analysis task and creativity. On the other hand, synthesis tasks have led to experience in first person the arduousness of a standard design procedure using general purpose software and how the contribution of this thesis in both analysis techniques and synthesis procedures is of real significance. For its part, those task requiring from advanced optimization has been challenging given the complexity of some of the designed filters and the fact that non-linear optimization is a complex topic per se. Valuable knowledge has been gained thanks to that, nevertheless. Finally, it can be affirmed that manufacturing and measurement process have been more beholden and straightforward to be carried out than at $\mathrm{THz}$ frequencies, even though mm-wave band is challenging in this aspect. Besides the inherent lower complexity of mm-wave prototypes, more adequate facilities and the know-how have played certain role in this aspect.

With high probability, results and conclusions of this thesis will play certain role in the future of GW technology. A good few future work lines have been proposed, may be some of them being satisfied soon by someone using part of the information delivered in this thesis. From this last paragraph, I would like to desire all the best luck and encouragement from the incoming researcher, with the hope that all the effort put in this thesis can help in the best way to progress in the treated areas. 


\section{Appendix A}

\section{Publications}

\section{A.1 Indexed JCR journals}

- A. Berenguer, D. Sanchez-Escuderos, B. Bernardo-Bernardo, M. BaqueroEscudero, V. E. Boria, "Groove Gap Waveguide as an alternative to rectangular waveguide for H-plane components", IEEE Microwave and Wireless Components Letters, 2016, pp. 1-2.

doi:10.1049/el.2016.0093.

- A. Berenguer, V. Fusco, D. E. Zelenchuk, D. Sanchez-Escuderos, M. Baquero-Escudero, V. E. Boria-Esbert, "Propagation Characteristics of Groove Gap Waveguide Below and Above Cutoff", IEEE Transactions on Microwave Theory and Techniques, vol. 64, no. 1, pp. 27-36.

doi:10.1109/TMTT.2015.2504501.

- D. Sanchez-Escuderos, M. Ferrand-Bataller, M. Baquero-Escudero, A. ValeroNogueira, A. Berenguer, "Dielectric Bed of Nails in Gap-Waveguide Technology at Millimeter-Wave Frequencies", IEEE Microwave and Wireless Components Letters, vol. 24, no. 8, pp. 515-517.

doi:10.1109/LMWC.2014.2321496.

\section{A.2 International Conferences}

- A. Berenguer, V. Fusco, M. Baquero-Escudero, V. E. Boria-Esbert, "A Frequency-Dependent Equivalence Between Groove Gap Waveguide and Rectangular Waveguide", IEEE Antennas and Propagation Society Symposium APS-URSI 2016, Fajardo, Puerto Rico, 26 June-1 July 2016. doi:10.1109/APS.2016.7696255.

- A. Berenguer, M. Baquero-Escudero, M. Ferrando-Rocher, B. BernardoBernardo, V. E. Boria-Esbert, "An Effective Post-Manufactured Tuning Method for Gap Waveguide Components", IEEE Antennas and Propagation Society Symposium APS-URSI 2016, Fajardo, Puerto Rico, 26 June-1 July 
2016 .

doi:10.1109/APS.2016.7695955.

- M. Ferrando-Rocher, A. Valero-Nogueira, J. I. Herranz-Herruzo, A. Berenguer, "V-band single-layer slot array fed by Ridge Gap Waveguide", IEEE Antennas and Propagation Society Symposium APS-URSI 2016, Fajardo, Puerto Rico, 26 June-1 July 2016.

doi:10.1109/APS.2016.7695903

- A. Berenguer, V. Fusco, M. Ferrando-Rocher, V. E. Boria, "A Fast Analysis Method for the Groove Gap Waveguide Using Transmission Line Theory", IEEE European Conference on Antennas and Propagation (EuCAP 2016), Davos, Switzerland, 10-15 April 2016. doi:10.1109/EuCAP.2016.7481433.

- A. Berenguer, M. Baquero-Escudero, D. Sanchez-Escuderos, V. E. Boria, Felipe Vico, "A Procedure to Correct the Response of Manufactured Groove Gap Waveguide Components", IEEE European Conference on Antennas and Propagation (EuCAP 2016), Davos, Switzerland, 10-15 April 2016. doi:10.1109/EuCAP.2016.7481138.

- M. Ferrando-Rocher, A. Valero-Nogueira, J. I. Herranz-Herruzo, A. Berenguer, "Groove Gap Waveguides: A Contactless Solution for Multilayer Slotted-Waveguide Array Antenna Assembly", IEEE European Conference on Antennas and Propagation (EuCAP 2016), Davos, Switzerland, 10-15 April 2016.

doi:10.1109/EuCAP.2016.7481568.

- A. Berenguer, M. Baquero-Escudero, D. Sánchez-Escuderos, B. BernardoClemente, V. E. Boria-Esbert, "Response Correction of a V-band Narrow-Band Filter Using Tuning Metal Insertions and Aggressive Space Mapping" IEEE 45th European Microwave Week, Paris, France, 6-11 November 2015. doi:10.1109/EuMC.2015.7345818.

- A. Berenguer, M. Baquero-Escudero, D. Sánchez-Escuderos, F. Vico, T. Bernabeu-Jimenez, "Design of coupled-line components with the SuspendedStrip Gap Waveguide at mm-wave frequencies", IEEE Antennas and Propagation Society Symposium APS-URSI 2015, Vancouver, Canada, 19-25 July 2015 .

doi:10.1109/USNC-URSI.2015.7303319.

- A. Berenguer, M. Baquero-Escudero, D. Sánchez-Escuderos, F. Vico, "Suspended-Strip Gap Waveguide Coupled-line Properties for Ka-band component design", IEEE European Conference on Antennas and Propagation (EuCAP 2015), Lisbon, Portugal, 12-17 April 2015.

- A. Berenguer, M. Baquero-Escudero, D. Sánchez-Escuderos, B. BernardoClemente, V. E. Boria-Esbert, "Low insertion loss $61 \mathrm{GHz}$ narrow-band filter implemented with Groove Gap Waveguides" IEEE 44th European Microwave 
Week, Rome, Italy, 5-10 October 2014.

doi:10.1109/EuMC.2014.6986402.

- A. Berenguer, M. Baquero-Escudero, D. Sánchez-Escuderos, F. Vico "Reduction of the impedance dependence on the Suspended-Strip Gap Waveguide", IEEE Antennas and Propagation Society Symposium APS-URSI 2014, Memphis, USA, 6-12 July 2014.

doi:0.1109/USNC-URSI.2014.6955533.

- A. Berenguer, M. Baquero-Escudero, D. Sánchez-Escuderos, F. Vico, "Rigorous Method for Calculating Gap Waveguides Impedance Using Transmission Line Theory", IEEE European Conference on Antennas and Propagation (EuCAP 2014), The Hague, The Netherlands, 7-11 April 2014. doi:10.1109/EuCAP.2014.6902328.

- A. Berenguer, M. Baquero-Escudero, D. Sánchez-Escuderos, M. Ferrando "Reduction of radiation losses of the single-wire waveguide at $\mathrm{THz}$ frequencies", IEEE Antennas and Propagation Society Symposium APS-URSI 2013, Orlando, USA, 7-13 July 2013. doi:10.1109/USNC-URSI.2013.6715476.

- A. del Olmo-Olmeda, M. Baquero-Escudero, V. E. Boria-Esbert, A. ValeroNogueira, A. Berenguer, "A novel Band-Pass Filter Topology for MillimeterWave Applications based on the Groove Gap Waveguide", IEEE International Microwave Symposium (IMS 2013), Seatle, USA, 2-7 June 2013. doi:10.1109/MWSYM.2013.6697780.

- A. Berenguer, M. Baquero-Escudero, D. Sánchez-Escuderos, M. FerrandoBataller, "Study of Transitions for the Single-Wire Waveguide at $\mathrm{THz}$ Frequencies", IEEE European Conference on Antennas and Propagation (EuCAP 2013), Göteborg, Sweden, 8-12 April 2013.

- D. Sanchez-Escuderos, M. Ferrando-Bataller, M. Baquero-Escudero, A. Berenguer, "Leaky-Wave Antenna On Planar Goubau Line at $\mathrm{THz}$ Frequencies", IEEE European Conference on Antennas and Propagation (EuCAP 2013), Göteborg, Sweden, 8-12 April 2013.

- A. Berenguer, M. Baquero-Escudero, D. Sanchez-Escuderos, M. FerrandoBataller, "Study of losses and high-order modes coupling on bends for wiretype waveguides at $\mathrm{THz}$ frequencies", IEEE Antennas and Propagation Society Symposium and USNC-URSI National Radio Science Meeting APS/URSI 2012, Chicago, USA, 8-14 July 2012.

- A. Berenguer, M. Baquero-Escudero, D. Sánchez-Escuderos, M. FerrandoBataller, "Reduction of High-Order Modes Coupling on Bends in the Dielectric-Coated Single Wire Waveguide", IEEE European Conference on Antennas and Propagation (EuCAP 2012), Prague, Czech Republic, 26-30 March 2012 .

doi:10.1109/EuCAP.2012.6206155. 
- D. Sanchez-Escuderos, M. Ferrando-Bataller, A. Berenguer, M. BaqueroEscudero, "Design of an H-Plane Horn Array Antenna Using the Complete 1D/3D-EBG Waveguide in the THz Band", IEEE European Conference on Antennas and Propagation (EuCAP 2012), Prague, Czech Republic, 26-30 March 2012.

doi:10.1109/EuCAP.2012.6206078.

- A. Berenguer, M. Baquero-Escudero, D. Sánchez-Escuderos, M. FerrandoBataller, "Study of the effect of coating the single wire waveguide with a dielectric", 36th International Conference on Infrared, Millimeter-wave and THz waves (IRMMW-THz 2011), Houston, USA, 2-7 October 2012. doi:10.1109/irmmw-THz.2011.6105124.

- D. Sanchez, M. Ferrando-Bataller, A. Berenguer, M. Baquero-Escudero, "Design of low-loss waveguides and devices at $\mathrm{THz}$ frequencies using EBG structures", 36th International Conference on Infrared, Millimeter-wave and THz waves (IRMMW-THz 2011), Houston, USA, 2-7 October 2012. doi:10.1109/irmmw-THz.2011.6105126.

- D. Sanchez-Escuderos, M. Ferrando-Bataller, M. Baquero-Escudero, J.I. Herranz, A. Berenguer, "H-plane horn array using low-loss rectangular waveguide at THz frequencies", IEEE European Conference on Antennas and Propagation (EuCAP 2011), Rome, Italy, 11-15 April 2011.

\section{A.3 National Conferences}

- A. Berenguer, V. Fusco, M. Baquero-Escudero, V. E. Boria-Esbert, "An accurate, efficient simplified model for the Groove Gap Waveguide", XXXI Simposium Nacional de la Unión Científica Internacional de Radio (URSI 2015), Madrid, Spain, 5-7 September 2016.

- A. Berenguer, M. Baquero-Escudero, D. Sanchez-Escuderos, B. BernardoClemente, V. E. Boria-Esbert, "Using Aggressive Space Mapping to correct the response of a narrow-band V-band filter through metal insertions", $X X X$ Simposium Nacional de la Unión Científica Internacional de Radio (URSI 2015), Pamplona, Spain, 2-4 September 2015.

- A. Berenguer, M. Baquero-Escudero, D. Sanchez-Escuderos, F. Vico, "Diseño de componentes en la banda Ka mediante líneas Suspended-strip gap waveguide acopladas", XXX Simposium Nacional de la Unión Científica Internacional de Radio (URSI 2015), Pamplona, Spain, 2-4 September 2015.

- A. Berenguer, M. Baquero-Escudero, D. Sanchez-Escuderos, B. BernardoClemente, V. E. Boria-Esbert, "V-band low insertion loss narrow-band filter using Groove Gap Waveguide Technology", XXIX Simposium Nacional de la Unión Científica Internacional de Radio (URSI 2014), Valencia, Spain, 3-5 September 2014. 
- A. Berenguer, M. Baquero-Escudero, D. Sanchez-Escuderos, F. Vico, "Análisis de impedancias basado en teoría de líneas de transmisión aplicado a las Gap waveguides", XXIX Simposium Nacional de la Unión Científica Internacional de Radio (URSI 2014), Valencia, Spain, 3-5 September 2014.

- J. Romeu, A. Broquetas, L. Jofre, M. Alonso, J. Abril, E. Nova, D. SanchezEscuderos, M. Ferrando-Bataller, A. Berenguer, M. Baquero-Escudero, F. Las-Heras, J. Laviada, Y. Alvarez-Lopez, C. Garcia-Gonzalez, A. Arboleya, L. E. Garcia, D. Segovia "TeraSense: THz Tomographic Biospectrometer", XXIX Simposium Nacional de la Unión Científica Internacional de Radio (URSI 2014), Convened contribution, Valencia, Spain, 3-5 September 2014.

- A. Berenguer, M. Baquero-Escudero, D. Sanchez-Escuderos, M. FerrandoBataller, "Transiciones en guía de hilo metálico para la reducción de radiación en curvas a THz", XXVIII Simposium Nacional de la Unión Científica Internacional de Radio (URSI 2013),, Santiago de Compostela, Spain, 11-13 September 2013.

- J. Romeu, A. Broquetas, L. Jofre, M. Alonso, J. Abril, E. Nova, D. SanchezEscuderos, M. Ferrando-Bataller, A. Berenguer, F. Las-Heras, J. Laviada, Y. Alvarez-Lopez, C. Garcia-Gonzalez, A. Arboleya, L. E. Garcia, D. Segovia, "TeraSense: THz Tomographic Biospectrometer", XXVIII Simposium Nacional de la Unión Científica Internacional de Radio (URSI 2013), Santiago de Compostela, Spain, 11-13 September 2013.

- A. Berenguer, M. Baquero-Escudero, D. Sanchez-Escuderos, M. FerrandoBataller, "Modal analysis of cylindrical surface waveguides for $\mathrm{THz}$ applications", IX Iberian Meeting on Computational Electromagnetics (EIEC 2013), Denia, Spain, 14-17 May 2013.

- A. Berenguer, M. Baquero-Escudero, D. Sanchez-Escuderos, M. FerrandoBataller, "Optimización de curvas en la guía de hilo metálico a frecuencias de THz", XXVII Simposium Nacional de la Unión Científica Internacional de Radio (URSI 2012), Elche, Spain, 12-14 September 2012.

- D. Sanchez-Escuderos, M. Ferrando-Bataller, A. Berenguer, M. BaqueroEscudero, "Antena Leakyk-Wave a Frecuencias de THz", XXVII Simposium Nacional de la Unión Científica Internacional de Radio (URSI 2012),, Elche, Spain, 12-14 September 2012.

- A. Berenguer, M. Baquero-Escudero, D. Sanchez-Escuderos, M. FerrandoBataller, "Study of surface waveguides at THz frequencies", XXVII Simposium Nacional de la Unión Científica Internacional de Radio (URSI 2011), Leganés, Spain, 13-15 September 2011.

- D. Sanchez-Escuderos, M. Ferrando-Bataller, A. Berenguer, M. BaqueroEscudero, "Application of EBG structures for the design of low-loss rectangular waveguides in the $\mathrm{THz}$ band", XXVI Simposium Nacional de la Unión Científica Internacional de Radio (URSI 2011),, Leganés, Spain, 1315 September 2011. 


\section{A.4 Other Publications}

\section{A.4.1 Indexed JCR journals}

- F. Vico, M. Ferrando-Bataller, A. Valero-Nogueira, A. Berenguer, "A High Order Local Corrected Nystrom Scheme For Charge-Current Integral Equations", IEEE Transactions on Antennas and Propagation, vol. 63, no. 4, pp. 1678-1685.

doi:10.1109/TAP.2015.2392127

- J. Izquierdo, J. Benitez, A. Berenguer, Concepcion Lago-Alonso, "I Decide, Therefore I Am (Relevant!): A Project-Based Learning Experience in Linear Algebra", Computer Applications in Engineering Eduction, vol. 24, no. 1, pp. 481-492.

doi:10.1002/cae. 21725

\section{A.4.2 International Conferences}

- F. Vico, M. Ferrando-Bataller, T. Bernabeu-Jimenez, A. Berenguer, "Decoupled Potential Integral Equation Applied to Complex Geometries" IEEE Antennas and Propagation Society Symposium APS-URSI 2015, Vancouver, Canada, 19-25 July 2015.

doi:10.1109/APS.2015.7304760.

- F. Vico, M. Ferrando-Bataller, T. Bernabeu-Jimenez, A. Berenguer, "A High Order Locally Corrected Nyström Implementation of the Decoupled Potential Integral Equation" IEEE European Conference on Antennas and Propagation (EuCAP 2015), Lisbon, Portugal, 12-17 April 2015.

- F. Vico, M. Ferrando-Bataller, A. Berenguer, D. Sánchez-Escuderos, "Integral Equation for Low Frequency Scattering Problem of Perfect Electric Conductors in Quasi-static Regime", IEEE Antennas and Propagation Society Symposium APS-URSI 2014, Memphis, USA, 6-12 July 2014.

doi:10.1109/APS.2014.6905420.

- F. Vico, M. Ferrando-Bataller, A. Valero-Nogueria, A. Berenguer, "High Order Integral Equation Methods for the Scattering of a Superellipsoid", IEEE European Conference on Antennas and Propagation (EuCAP 2014), The Hague, The Netherlands, 7-11 April 2014.

doi:10.1109/EuCAP.2014.6901870.

- D. Sanchez-Escuderos, M. Ferrando-Bataller, A. Berenguer, J. I. Herranz "Circularly-Polarized Periodic Leaky-wave Antenna at Millimeter-Wave Frequencies", textitIEEE Antennas and Propagation Society Symposium APSURSI 2013, Orlando, USA, 7-13 July 2013.

doi:10.1109/APS.2013.6710740.

- D. Sanchez-Escuderos, M. Ferrando-Bataller, J. I. Herranz, A. Berenguer, "Planar single-wire antennas at millimeter-wave frequencies", IEEE Antennas 
and Propagation Society Symposium and USNC-URSI National Radio Science Meeting APS/URSI 2012, Chicago, USA, 8-14 July 2012.

doi:10.1109/APS.2012.6348477.

\section{A.4.3 National Conferences}

- J. Izquierdo, J. Benitez, A. Berenguer, "Decido, luego existo", Congreso de Innovación Educativa y Docencia en Red (INRED 2015), Valencia, Spain, 1-2 July 2015.

- F. Vico, M. Ferrando-Bataller, A. Valero-Nogueira, A. Berenguer, "Efficient Integral Equation Methods in the low frequency regime", IX Iberian Meeting on Computational Electromagnetics (EIEC 2013), Denia, Spain, 14-17 May 2013. 


\section{References}

[1] P. Siegel, "Terahertz technology," IEEE Transactions on Microwave Theory and Techniques, vol. 50, no. 3, pp. 910-928, 2002.

[2] I. Hosako, N. Sekine, M. Patrashin, S. Saito, K. Fukunaga, Y. Kasai, P. Baron, T. Seta, J. Mendrok, S. Ochiai et al., "At the dawn of a new era in Terahertz technology," Proceedings of the IEEE, vol. 95, no. 8, pp. 1611-1623, 2007.

[3] B. Ferguson and X.-C. Zhang, "Materials for Terahertz science and technology," Nature materials, vol. 1, no. 1, pp. 26-33, 2002.

[4] J. M. Chamberlain and R. E. Miles, New directions in Terahertz technology. Kluwer Academic Publishers, 1997.

[5] D. van der Weide, "Applications and outlook for electronic terahertz technology," Optics and photonics news, vol. 14, no. 4, pp. 48-53, 2003.

[6] E. J. Nichols and J. D. Tear, "Joining the infrared and electric wave spectra," Astrophysics Journal, vol. 61, no. 4, pp. 17-37, 1925.

[7] I. Kaufman, "The Band between Microwave and Infrared Regions," Proceedings of the IRE, vol. 47, no. 3, pp. 381-396, March 1959.

[8] P. Coleman and R. Becker, "Present State of the Millimeter Wave Generation and Technique Art-1958," IRE Transactions on Microwave Theory and Techniques, vol. 7, no. 1, pp. 42-61, 1959.

[9] P. D. Coleman, "State of the art: background and recent developmentsmillimeter and submillimeter waves," IEEE Transactions on Microwave Theory and Techniques, vol. 11, no. 5, pp. 271-288, 1963.

[10] A. J. Kerecman, "The tungsten-P type silicon point contact diode," in 1973 IEEE G-MTT International Microwave Symposium, 1973, pp. 30-34.

[11] J. R. Ashley and F. Palka, "Transmission cavity and injection stabilization of an X-band transferred electron oscillator," in IEEE G-MTT International Microwave Symposium. IEEE, 1973, pp. 181-182.

[12] J. Fleming, "High-resolution submillimeter-wave Fourier-transform spectrometry of gases," IEEE Transactions on Microwave Theory Techniques, vol. 22, pp. 1023-1025, 1974. 
[13] F. De Lucia, "Science and technology in the submillimeter region," Optics and photonics news, vol. 14, no. 8, pp. 44-50, 2003.

[14] V. Podobedov, D. Plusquellic, and G. Fraser, "Investigation of the water-vapor continuum in the $\mathrm{THz}$ region using a multipass cell," Journal of Quantitative Spectroscopy and Radiative Transfer, vol. 91, no. 3, pp. 287-295, 2005.

[15] A. Danylov, "THz laboratory measurements of atmospheric absorption between $6 \%$ and $52 \%$ relative humidity," Submillimeter-Wave Technology Laboratory University of Massachusetts Lowell, vol. 175, 2006.

[16] V. Podobedov, D. Plusquellic, K. Siegrist, G. Fraser, Q. Ma, and R. Tipping, "New measurements of the water vapor continuum in the region from 0.3 to $2.7 \mathrm{THz}, "$ Journal of Quantitative Spectroscopy and Radiative Transfer, vol. 109, no. 3, pp. 458-467, 2008.

[17] D. M. Slocum, E. J. Slingerland, R. H. Giles, and T. M. Goyette, "Atmospheric absorption of terahertz radiation and water vapor continuum effects," Journal of Quantitative Spectroscopy and Radiative Transfer, vol. 127, pp. 49-63, 2013.

[18] Y.-S. Lee, Principles of terahertz science and technology. Springer Science \& Business Media, 2009, vol. 170.

[19] J.-H. Son, "Terahertz electromagnetic interactions with biological matter and their applications," Journal of Applied Physics, vol. 105, no. 10, p. 102033, 2009.

[20] T. Kampfrath, K. Tanaka, and K. A. Nelson, "Resonant and nonresonant control over matter and light by intense terahertz transients," Nature Photonics, vol. 7, no. 9, pp. 680-690, 2013.

[21] C. Yeh and F. Shimabukuro, The essence of dielectric waveguides. Springer Verlag, 2008.

[22] D. Pozar, "Microwave Engineering, 3rd," 2005.

[23] G. Keiser, Optical fiber communications. Wiley Online Library, 2003.

[24] K. Kawase, "Terahertz imaging for drug detection and large-scale integrated circuit inspection," Optics and photonics news, vol. 15, no. 10, pp. 34-39, 2004.

[25] T. G. Phillips and J. Keene, "Submillimeter astronomy [heterodyne spectroscopy]," Proceedings of the IEEE, vol. 80, no. 11, pp. 1662-1678, 1992.

[26] T. Greve, P. Papadopoulos, Y. Gao, and S. Radford, "Molecular gas in extreme star-forming environments: the starbursts Arp 220 and NGC 6240 as case studies," The Astrophysical Journal, vol. 692, no. 2, p. 1432, 2009.

[27] F. Walter, R. Decarli, C. Carilli, D. Riechers, F. Bertoldi, A. Weiß, P. Cox, R. Neri, R. Maiolino, M. Ouchi et al., "Evidence for low extinction in actively star-forming galaxies at $z>6.5$," The Astrophysical Journal, vol. 752, no. 2, p. $93,2012$. 
[28] S. Matsushita, K. Asada, R. Blundell, C.-C. Chang, M.-T. Chen, P. Grimes, J. Han, H. Hirashita, P. T. Ho, T. Huang et al., "Greenland Telescope: Imaging Black Hole Shadow and THz Astronomy," IAU General Assembly, vol. 22, p. $51138,2015$.

[29] D. T. Leisawitz, W. C. Danchi, M. J. DiPirro, L. D. Feinberg, D. Y. Gezari, M. Hagopian, W. D. Langer, J. C. Mather, S. H. Moseley Jr, M. Shao et al., "Scientific motivation and technology requirements for the SPIRIT and SPECS far-infrared/submillimeter space interferometers," in Astronomical Telescopes and Instrumentation. International Society for Optics and Photonics, 2000, pp. 36-46.

[30] T. Nakagawa, "Observations of the Universe in the Terahertz Range: From AKARI to SPICA," IEEE Transactions on Terahertz Science and Technology, vol. 5, no. 6, pp. 1133-1139, 2015.

[31] C. Risacher, R. Guesten, J. Stutzki, H.-W. Huebers, D. Buechel, U. U. Graf, S. Heyminck, C. E. Honingh, K. Jacobs, B. Klein et al., "First supra-THz Heterodyne Array Receivers for Astronomy with the SOFIA Observatory," arXiv preprint arXiv:1512.02908, 2015.

[32] M. Verdant and G. Schwehm, "The international Rosetta mission," ESA bulletin, vol. 93, pp. 39-50, 1998.

[33] K.-H. Glassmeier, H. Boehnhardt, D. Koschny, E. Kührt, and I. Richter, "The Rosetta mission: flying towards the origin of the solar system," Space Science Reviews, vol. 128, no. 1-4, pp. 1-21, 2007.

[34] P. H. Siegel, "THz for space: The golden age," in Microwave Symposium Digest (MTT), 2010 IEEE MTT-S International. IEEE, 2010, pp. 816-819.

[35] P. Hartogh, E. Lellouch, R. Moreno, D. Bockelée-Morvan, N. Biver, T. Cassidy, M. Rengel, C. Jarchow, T. Cavalié, J. Crovisier et al., "Direct detection of the Enceladus water torus with Herschel," Astronomy \& Astrophysics, vol. 532, p. L2, 2011.

[36] P. Roelfsema, F. Helmich, D. Teyssier, V. Ossenkopf, P. Morris, M. Olberg, R. Shipman, C. Risacher, M. Akyilmaz, R. Assendorp et al., "In-orbit performance of Herschel-HIFI," Astronomy \& Astrophysics, vol. 537, p. A17, 2012.

[37] P. Kaufmann, J.-P. Raulin, C. G. De Castro, H. Levato, D. E. Gary, J. E. Costa, A. Marun, P. Pereyra, A. V. Silva, and E. Correia, "A new solar burst spectral component emitting only in the terahertz range," The Astrophysical Journal Letters, vol. 603, no. 2, p. L121, 2004.

[38] J. Waters, "Submillimeter-wavelength heterodyne spectroscopy and remote sensing of the upper atmosphere," Proceedings of the IEEE, vol. 80, no. 11, pp. 1679-1701, 2002.

[39] J. S. Melinger, Y. Yang, M. Mandehgar, and D. Grischkowsky, "THz detection of small molecule vapors in the atmospheric transmission windows," Optics express, vol. 20, no. 6, pp. 6788-6807, 2012. 
[40] Y. Yang, M. Mandehgar, and D. R. Grischkowsky, "Understanding THz pulse propagation in the atmosphere," IEEE Transactions on Terahertz Science and Technology, vol. 2, no. 4, pp. 406-415, 2012.

[41] Y. Yang, M. Mandehgar, and D. Grischkowsky, "Determination of water vapor continuum absorption, using long-path THz-TDS without the cross-term," in Infrared, Millimeter, and Terahertz waves (IRMMW-THz), 2014 39th International Conference on. IEEE, 2014, pp. 1-2.

[42] D. M. Slocum, T. M. Goyette, R. H. Giles, and W. E. Nixon, "Experimental determination of terahertz atmospheric absorption parameters," in SPIE Sensing Technology + Applications. International Society for Optics and Photonics, 2015, pp. 94830O-94830O.

[43] H. Pickett, R. Poynter, E. Cohen, M. Delitsky, J. Pearson, and H. Müller, "Submillimeter, millimeter, and microwave spectral line catalog," Journal of Quantitative Spectroscopy and Radiative Transfer, vol. 60, no. 5, pp. 883-890, 1998.

[44] H. J. Liebe, "An atmospheric millimeter wave propagation model," DTIC Document, Tech. Rep., 1983.

[45] L. S. Rothman, D. Jacquemart, A. Barbe, D. C. Benner, M. Birk, L. Brown, M. Carleer, C. Chackerian, K. Chance, L. e. a. Coudert et al., "The HITRAN 2004 molecular spectroscopic database," Journal of Quantitative Spectroscopy and Radiative Transfer, vol. 96, no. 2, pp. 139-204, 2005.

[46] S. Clough, F. Kneizys, and R. Davies, "Line shape and the water vapor continuum," Atmospheric Research, vol. 23, no. 3, pp. 229-241, 1989.

[47] M. J. Rosker and H. B. Wallace, "Imaging through the atmosphere at terahertz frequencies," in Microwave Symposium, 200\%. IEEE/MTT-S International. IEEE, 2007, pp. 773-776.

[48] Y. Yang, M. Mandehgar, and D. Grischkowsky, "THz-TDS characterization of the digital communication channels of the atmosphere and the enabled applications," Journal of Infrared, Millimeter, and Terahertz Waves, vol. 36, no. 2, pp. 97-129, 2015.

[49] J. C. Wiltse, "History of millimeter and submillimeter waves," IEEE Transactions on Microwave Theory and Techniques, vol. 32, no. 9, pp. 1118-1127, 1984.

[50] P. Helminger, J. K. Messer, and F. C. De Lucia, "Continuously tunable coherent spectroscopy for the 0.1-1.0-THz region," Applied Physics Letters, vol. 42, no. 4, pp. 309-310, 1983.

[51] P. R. Griffiths and J. A. De Haseth, Fourier transform infrared spectrometry. John Wiley \& Sons, 2007, vol. 171. 
[52] M. Van Exter, C. Fattinger, and D. Grischkowsky, "Terahertz time-domain spectroscopy of water vapor," Optics letters, vol. 14, no. 20, pp. 1128-1130, 1989.

[53] L. Duvillaret, F. Garet, and J.-L. Coutaz, "A reliable method for extraction of material parameters in terahertz time-domain spectroscopy," IEEE Journal of Selected Topics in Quantum Electronics, vol. 2, no. 3, pp. 739-746, 1996.

[54] F. D'Angelo, Z. Mics, M. Bonn, and D. Turchinovich, "Ultra-broadband THz time-domain spectroscopy of common polymers using THz air photonics," $O p$ tics express, vol. 22, no. 10, pp. 12 475-12 485, 2014.

[55] M. C. Smith, Principles of pharmaceutical marketing. Routledge, 2014.

[56] J. Rogers, L. Kirby, S. Hempelman, D. Berry, P. McGeer, A. Kaszniak, J. Zalinski, M. Cofield, L. Mansukhani, P. Willson et al., "Clinical trial of indomethacin in Alzheimer's disease," Neurology, vol. 43, no. 8, pp. 1609-1609, 1993.

[57] C. J. Strachan, P. F. Taday, D. A. Newnham, K. C. Gordon, J. A. Zeitler, M. Pepper, and T. Rades, "Using terahertz pulsed spectroscopy to quantify pharmaceutical polymorphism and crystallinity," Journal of Pharmaceutical Sciences, vol. 94, no. 4, pp. 837-846, 2005.

[58] J. A. Zeitler, P. F. Taday, D. A. Newnham, M. Pepper, K. C. Gordon, and T. Rades, "Terahertz pulsed spectroscopy and imaging in the pharmaceutical setting-a review," Journal of Pharmacy and Pharmacology, vol. 59, no. 2, pp. 209-223, 2007.

[59] H.-B. Liu and X.-C. Zhang, "Dehydration kinetics of D-glucose monohydrate studied using THz time-domain spectroscopy," Chemical physics letters, vol. 429, no. 1, pp. 229-233, 2006.

[60] K. I. Zaytsev, K. G. Kudrin, S. A. Koroleva, I. N. Fokina, S. I. Volodarskaya, E. V. Novitskaya, A. N. Perov, V. E. Karasik, and S. O. Yurchenko, "Medical diagnostics using terahertz pulsed spectroscopy," in Journal of Physics: Conference Series, vol. 486, no. 1. IOP Publishing, 2014, p. 012014.

[61] M. Hirmer, S. N. Danilov, S. Giglberger, J. Putzger, A. Niklas, A. Jäger, K.-A. Hiller, S. Löffler, G. Schmalz, B. Redlich et al., "Spectroscopic study of human teeth and blood from visible to terahertz frequencies for clinical diagnosis of dental pulp vitality," Journal of Infrared, Millimeter, and Terahertz Waves, vol. 33, no. 3, pp. 366-375, 2012.

[62] K. Kamburoğlu, N. Ö. Yetimoĝlu, and H. Altan, "Characterization of primary and permanent teeth using terahertz spectroscopy," Dentomaxillofacial Radiology, vol. 43, no. 6, p. 20130404, 2014.

[63] E. Pickwell, B. Cole, A. Fitzgerald, M. Pepper, and V. Wallace, "In vivo study of human skin using pulsed terahertz radiation," Physics in Medicine and Biology, vol. 49, no. 9, p. 1595, 2004. 
[64] M. H. Arbab, S. C. Henry, A. Warsen, N. Farr, D. P. Winebrenner, A. M. McClintic, N. Shubin, A. M. Hocking, and S. Arbabi, "Diagnosis of burn wounds using terahertz time-domain spectroscopy," in 39th International Conference on Infrared, Millimeter, and Terahertz waves (IRMMW-THz). IEEE, 2014, pp. 1-1.

[65] A. J. Fitzgerald, V. P. Wallace, M. Jimenez-Linan, L. Bobrow, R. J. Pye, A. D. Purushotham, and D. D. Arnone, "Terahertz pulsed imaging of human breast tumors," Radiology, vol. 239, no. 2, pp. 533-540, 2006.

[66] P. C. Ashworth, E. Pickwell-MacPherson, E. Provenzano, S. E. Pinder, A. D. Purushotham, M. Pepper, V. P. Wallace et al., "Terahertz pulsed spectroscopy of freshly excised human breast cancer," Optics express, vol. 17, no. 15, pp. $12444-12454,2009$.

[67] F. Wahaia, G. Valusis, L. M. Bernardo, A. Almeida, J. A. Moreira, P. C. Lopes, J. Macutkevic, I. Kasalynas, D. Seliuta, R. Adomavicius et al., "Detection of colon cancer by terahertz techniques," Journal of Molecular Structure, vol. 1006, no. 1, pp. 77-82, 2011.

[68] D. B. Bennett, Z. D. Taylor, P. Tewari, R. S. Singh, M. O. Culjat, W. S. Grundfest, D. J. Sassoon, R. D. Johnson, J.-P. Hubschman, and E. R. Brown, "Terahertz sensing in corneal tissues," Journal of biomedical optics, vol. 16, no. 5, pp. $057003-057003,2011$.

[69] B. M. Fischer, H. Helm, and P. U. Jepsen, "Chemical recognition with broadband THz spectroscopy," Proceedings of the IEEE, vol. 95, no. 8, pp. 15921604, 2007.

[70] M. Leahy-Hoppa, M. Fitch, X. Zheng, L. Hayden, and R. Osiander, "Wideband terahertz spectroscopy of explosives," Chemical Physics Letters, vol. 434, no. 4, pp. 227-230, 2007.

[71] H.-B. Liu, Y. Chen, G. J. Bastiaans, and X.-C. Zhang, "Detection and identification of explosive RDX by $\mathrm{THz}$ diffuse reflection spectroscopy," Optics Express, vol. 14, no. 1, pp. 415-423, 2006.

[72] K. Kawase, Y. Ogawa, Y. Watanabe, and H. Inoue, "Non-destructive terahertz imaging of illicit drugs using spectral fingerprints," Optics express, vol. 11, no. 20, pp. 2549-2554, 2003.

[73] S. Jin-Hai, S. Jing-Ling, L. Lai-Shun, X. Xiao-Yu, L. Hai-Bo, and Z. CunLin, "Experimental investigation on terahertz spectra of amphetamine type stimulants," Chinese Physics Letters, vol. 22, no. 12, p. 3176, 2005.

[74] H. Hoshina, Y. Sasaki, A. Hayashi, C. Otani, and K. Kawase, "Non-invasive mail inspection system with terahertz radiation," in SPIE Defense, Security, and Sensing. International Society for Optics and Photonics, 2009, pp. $731103-731103$. 
[75] M. W. Burke, "Terahertz Spectroscopic Imaging for Standoff Detection of High Explosives," Engineering Research and Technology Report, pp. 48-49, 2008.

[76] F. Platte and H. M. Heise, "Substance identification based on transmission THz spectra using library search," Journal of Molecular Structure, vol. 1073, pp. 3-9, 2014.

[77] M. Brucherseifer, M. Nagel, P. H. Bolivar, H. Kurz, A. Bosserhoff, and R. Büttner, "Label-free probing of the binding state of DNA by time-domain terahertz sensing," Applied Physics Letters, vol. 77, no. 24, pp. 4049-4051, 2000 .

[78] A. Markelz, A. Roitberg, and E. Heilweil, "Pulsed terahertz spectroscopy of DNA, bovine serum albumin and collagen between 0.1 and $2.0 \mathrm{THz}$," Chemical Physics Letters, vol. 320, no. 1, pp. 42-48, 2000.

[79] A. Menikh, R. MacColl, C. A. Mannella, and X.-C. Zhang, "Terahertz biosensing technology: Frontiers and progress," ChemPhysChem, vol. 3, no. 8, pp. $655-658,2002$.

[80] M. Nagel, P. H. Bolivar, M. Brucherseifer, H. Kurz, A. Bosserhoff, and R. Büttner, "Integrated THz technology for label-free genetic diagnostics," Applied Physics Letters, vol. 80, no. 1, pp. 154-156, 2002.

[81] B. Born, S. J. Kim, S. Ebbinghaus, M. Gruebele, and M. Havenith, "The terahertz dance of water with the proteins: the effect of protein flexibility on the dynamical hydration shell of ubiquitin," Faraday discussions, vol. 141, pp. 161-173, 2009.

[82] B. Breitenstein, M. Scheller, M. K. Shakfa, T. Kinder, T. Müller-Wirts, M. Koch, and D. Selmar, "Introducing terahertz technology into plant biology: A novel method to monitor changes in leaf water status," Journal of Applied Botany and Food Quality, vol. 84, no. 2, p. 158, 2012.

[83] N. Born, D. Behringer, S. Liepelt, S. Beyer, M. Schwerdtfeger, B. Ziegenhagen, and M. Koch, "Monitoring plant drought stress response using terahertz timedomain spectroscopy," Plant physiology, vol. 164, no. 4, pp. 1571-1577, 2014.

[84] Z. Yan, Y. Ying, H. Zhang, and H. Yu, "Research progress of terahertz wave technology in food inspection," in Optics East 2006. International Society for Optics and Photonics, 2006, pp. 63730R-63730R.

[85] Y. Hu, L. Guo, X. Wang, and X. C. Zhang, "THz time-domain spectroscopy on plant oils and animal fats," in Photonics Asia 2004. International Society for Optics and Photonics, 2005, pp. 334-340.

[86] J. B. Jackson, J. Bowen, G. Walker, J. Labaune, G. Mourou, M. Menu, K. Fukunaga et al., "A survey of terahertz applications in cultural heritage conservation science," IEEE Transactions on Terahertz Science and Technology, vol. 1, no. 1, pp. 220-231, 2011. 
[87] M. Mizuno, K. Fukunaga, S. Saito, and I. Hosako, "Analysis of calcium carbonate for differentiating between pigments using terahertz spectroscopy," Journal of the European Optical Society-Rapid publications, vol. 4, 2009.

[88] K. Fukunaga and M. Picollo, "Terahertz spectroscopy applied to the analysis of artists' materials," Applied Physics A, vol. 100, no. 3, pp. 591-597, 2010.

[89] K. Fukunaga, Y. Ogawa, S. Hayashi, and I. Hosako, "Application of terahertz spectroscopy for character recognition in a medieval manuscript," IEICE Electronics Express, vol. 5, no. 7, pp. 223-228, 2008.

[90] J. A. Zeitler, Y. Shen, C. Baker, P. F. Taday, M. Pepper, and T. Rades, "Analysis of coating structures and interfaces in solid oral dosage forms by three dimensional terahertz pulsed imaging," Journal of pharmaceutical sciences, vol. 96, no. 2, pp. 330-340, 2007.

[91] A. J. Fitzgerald, B. E. Cole, and P. F. Taday, "Nondestructive analysis of tablet coating thicknesses using terahertz pulsed imaging," Journal of pharmaceutical sciences, vol. 94, no. 1, pp. 177-183, 2005.

[92] L. Maurer and H. Leuenberger, "Terahertz pulsed imaging and near infrared imaging to monitor the coating process of pharmaceutical tablets," International journal of pharmaceutics, vol. 370, no. 1, pp. 8-16, 2009.

[93] K. Su, R. K. May, I. S. Gregory, P. F. Taday, Y.-C. Shen, and J. A. Zeitler, "Terahertz sensor for non-contact thickness measurement of car paints," in 38th International Conference on Infrared, Millimeter, and Terahertz Waves (IRMMW-THz). IEEE, 2013, pp. 1-2.

[94] T. Yasui, T. Yasuda, K.-i. Sawanaka, and T. Araki, "Terahertz paintmeter for noncontact monitoring of thickness and drying progress in paint film," Applied Optics, vol. 44, no. 32, pp. 6849-6856, 2005.

[95] C. Jördens and M. Koch, "Detection of foreign bodies in chocolate with pulsed terahertz spectroscopy," Optical Engineering, vol. 47, no. 3, pp. $037003-$ $037003,2008$.

[96] Y. DEMARTY, B. M. FISCHER, P. ESPEAU, Y. CORVIS, S. MICHEL, H. DUFAT, L. LEFORT, and P.-H. PIOGER, "Alypotec: Analysis of pharmaceutical counterfeits using terahertz technology," 2013.

[97] N. Luhmann Jr and W. Peebles, "Instrumentation for magnetically confined fusion plasma diagnostics," Review of scientific instruments, vol. 55, no. 3, pp. 279-331, 1984.

[98] N. Luhmann Jr, "Instrumentation and techniques for plasma diagnostics: An overview," Infrared and Millimeter Waves, vol. 2, 2014.

[99] M. Bornatici, R. Cano, O. De Barbieri, and F. Engelmann, "Electron cyclotron emission and absorption in fusion plasmas," Nuclear Fusion, vol. 23, no. 9, p. 1153, 1983. 
[100] W. Knap, J. Lusakowski, T. Parenty, S. Bollaert, A. Cappy, V. Popov, and M. Shur, "Terahertz emission by plasma waves in $60 \mathrm{~nm}$ gate high electron mobility transistors," Applied Physics Letters, vol. 84, no. 13, pp. 2331-2333, 2004.

[101] C. W. Domier, N. Luhmann Jr, H. K. Park, Z. Xia, and P. Zhang, "Advances in millimeter wave/THz plasma diagnostics instrumentation," in Joint 32nd International Conference on Infrared and Millimeter Waves, 2007 and the 2007 15th International Conference on Terahertz Electronics. IRMMW-THz. IEEE, 2007, pp. 8-11.

[102] M. Zerbini, F. Causa, A. Doria, G. Gallerano, E. Giovenale, A. Tuccillo, G. Galatola-Teka, F. Cuttaia, and M. Johnston, "Progress on THz applications for Plasma Diagnostics," in Conference on Infrared, Millimeter, and Terahertz waves (IRMMW-THz), 2015 40th International. IEEE, 2015, pp. 1-2.

[103] C. Paoloni, R. Letizia, M. Mineo, A. Malek Abadi, N. C. Luhmann Jr, B. Popovic, L. Himes, R. Barchfeld, D. Gamzina, L. Yue et al., "Magnetic fusion energy plasma diagnostic needs novel THz BWOs," IVEC 2015, 2015.

[104] Teraview ${ }^{\circledR}$. (2016). [Online]. Available: http://www.teraview.com

[105] TeraSense ${ }^{\circledR}$. (2016). [Online]. Available: http://terasense.com/company

[106] X. Zhang, "Terahertz wave imaging: horizons and hurdles," Physics in Medicine and Biology, vol. 47, no. 21, p. 3667, 2002.

[107] D. Arnone, C. Ciesla, M. Pepper et al., "Terahertz imaging comes into view," Physics World, vol. 4, pp. 35-40, 2000.

[108] R. Woodward, V. Wallace, D. Arnone, E. Linfield, and M. Pepper, "Terahertz pulsed imaging of skin cancer in the time and frequency domain," Journal of Biological Physics, vol. 29, no. 2-3, pp. 257-259, 2003.

[109] J. Labaune, J. Jackson, S. Pages-Camagna, I. Duling, M. Menu, and G. Mourou, "Papyrus imaging with terahertz time domain spectroscopy," $A p$ plied Physics A, vol. 100, no. 3, pp. 607-612, 2010.

[110] N. Karpowicz, H. Zhong, J. Xu, K.-I. Lin, J.-S. Hwang, and X. Zhang, "Comparison between pulsed terahertz time-domain imaging and continuous wave terahertz imaging," Semiconductor Science and Technology, vol. 20, no. 7, p. S293, 2005.

[111] M. C. Kemp, P. Taday, B. E. Cole, J. Cluff, A. J. Fitzgerald, and W. R. Tribe, "Security applications of terahertz technology," in AeroSense 2003. International Society for Optics and Photonics, 2003, pp. 44-52.

[112] W. R. Tribe, D. A. Newnham, P. F. Taday, and M. C. Kemp, "Hidden object detection: security applications of terahertz technology," in Integrated Optoelectronic Devices 2004. International Society for Optics and Photonics, 2004, pp. $168-176$. 
[113] E. L. Jacobs, S. Moyer, C. C. Franck, F. C. DeLucia, C. Casto, D. T. Petkie, S. R. Murrill, and C. E. Halford, "Concealed weapon identification using terahertz imaging sensors," in Defense and Security Symposium. International Society for Optics and Photonics, 2006, pp. 62 120J-62 120J.

[114] D. M. Sheen, T. E. Hall, R. H. Severtsen, D. L. McMakin, B. K. Hatchell, and P. L. Valdez, "Active wideband $350 \mathrm{GHz}$ imaging system for concealed-weapon detection," in SPIE Defense, Security, and Sensing. International Society for Optics and Photonics, 2009, pp. 73 090I-73 090I.

[115] E. Grossman, C. Dietlein, J. Ala-Laurinaho, M. Leivo, L. Gronberg, M. Gronholm, P. Lappalainen, A. Rautiainen, A. Tamminen, and A. Luukanen, "Passive terahertz camera for standoff security screening," Applied optics, vol. 49, no. 19, pp. E106-E120, 2010.

[116] K. B. Cooper, R. J. Dengler, N. Llombart, B. Thomas, G. Chattopadhyay, and P. H. Siegel, "THz imaging radar for standoff personnel screening," IEEE Transactions on Terahertz Science and Technology, vol. 1, no. 1, pp. 169-182, 2011.

[117] A. Luukanen, R. Appleby, M. Kemp, and N. Salmon, "Millimeter-wave and terahertz imaging in security applications," in Terahertz Spectroscopy and Imaging. Springer, 2013, pp. 491-520.

[118] R. Knipper, A. Brahm, E. Heinz, T. May, G. Notni, H.-G. Meyer, A. Tunnermann, and J. Popp, "THz Absorption in Fabric and Its Impact on Body Scanning for Security Application," IEEE Transactions on Terahertz Science and Technology, vol. 5, no. 6, pp. 999-1004, 2015.

[119] G. P. Williams and G. R. Neil, "Method for large and rapid terahertz imaging," Jan. 29 2013, uS Patent 8,362,430.

[120] F. Mohamadi, "TERAHERTZ SCREENING APPARATUS FOR DETECTION OF CONCEALED WEAPONS," Oct. 8 2015, uS Patent 20,150,285,907.

[121] S. D. Caruthers, S. A. Wickline, and G. M. Lanza, "Nanotechnological applications in medicine," Current opinion in Biotechnology, vol. 18, no. 1, pp. 26-30, 2007.

[122] J. K. Han, "Terahertz medical imaging," in Convergence of Terahertz Sciences in Biomedical Systems. Springer, 2012, pp. 351-371.

[123] E. Pickwell and V. Wallace, "Biomedical applications of terahertz technology," Journal of Physics D: Applied Physics, vol. 39, p. R301, 2006.

[124] W. L. Chan, J. Deibel, and D. M. Mittleman, "Imaging with terahertz radiation," Reports on progress in physics, vol. 70, no. 8, p. 1325, 2007.

[125] Y.-C. Shen, "Terahertz pulsed spectroscopy and imaging for pharmaceutical applications: A review," International Journal of Pharmaceutics, vol. 417, no. 1, pp. 48-60, 2011. 
[126] Y. Sun, M. Y. Sy, Y.-X. J. Wang, A. T. Ahuja, Y.-T. Zhang, and E. PickwellMacPherson, "A promising diagnostic method: Terahertz pulsed imaging and spectroscopy," World journal of radiology, vol. 3, no. 3, p. 55, 2011.

[127] A. W. Lee, Q. Qin, S. Kumar, B. S. Williams, Q. Hu, and J. L. Reno, "Real-time terahertz imaging over a standoff distance (> 25 meters)," $A p$ plied Physics Letters, vol. 89, no. 14, pp. 141 125-141 125, 2006.

[128] S. Caorsi, A. Massa, M. Pastorino, and M. Donelli, "Improved microwave imaging procedure for nondestructive evaluations of two-dimensional structures," IEEE Transactions on Antennas and Propagation, vol. 52, no. 6, pp. 1386-1397, 2004.

[129] N. Smith, L. Rivera, N. Burford, T. Bowman, M. El-Shenawee, and G. DeSouza, "Towards root phenotyping in situ using $\mathrm{THz}$ imaging," in 40th International Conference on Infrared, Millimeter, and Terahertz waves (IRMMWTHz). IEEE, 2015, pp. 1-2.

[130] M. Herrmann, M. Tani, K. Sakai, and R. Fukasawa, "Terahertz imaging of silicon wafers," Journal of applied physics, vol. 91, no. 3, pp. 1247-1250, 2002.

[131] F. Rutz, M. Koch, S. Khare, M. Moneke, H. Richter, and U. Ewert, "Terahertz quality control of polymeric products," International Journal of Infrared and Millimeter Waves, vol. 27, no. 4, pp. 547-556, 2006.

[132] S. Wietzke, C. Jördens, N. Krumbholz, B. Baudrit, M. Bastian, and M. Koch, "Terahertz imaging: a new non-destructive technique for the quality control of plastic weld joints," Journal of the European Optical Society-Rapid publications, vol. 2, 2007.

[133] M. Koch, "Terahertz technology: a land to be discovered," Optics and Photonics News, vol. 18, no. 3, pp. 20-25, 2007.

[134] I. Duling and D. Zimdars, "Terahertz imaging: Revealing hidden defects," Nature Photonics, vol. 3, no. 11, pp. 630-632, 2009.

[135] B. Pradarutti, S. Riehemann, G. Notni, and A. Tünnermann, "Terahertz imaging for styrofoam inspection," in Optics East 200\%. International Society for Optics and Photonics, 2007, pp. 67 720P-67 720P.

[136] J. A. Zeitler and Y.-C. Shen, "Industrial applications of terahertz imaging," in Terahertz Spectroscopy and Imaging. Springer, 2012, pp. 451-489.

[137] S. Wietzke, C. Jansen, C. Jördens, N. Krumbholz, N. Vieweg, M. Scheller, M. Shakfa, D. Romeike, T. Hochrein, M. Mikulics et al., "Industrial applications of THz systems," in International Symposium on Photoelectronic Detection and Imaging 2009. International Society for Optics and Photonics, 2009, pp. $738506-738506$.

[138] T. Kiwa, M. Tonouchi, M. Yamashita, and K. Kawase, "Laser terahertzemission microscope for inspecting electrical faults in integrated circuits," $O p$ tics Letters, vol. 28, no. 21, pp. 2058-2060, 2003. 
[139] E. Cristofani, F. Friederich, S. Wohnsiedler, C. Matheis, J. Jonuscheit, M. Vandewal, and R. Beigang, "Nondestructive testing potential evaluation of a terahertz frequency-modulated continuous-wave imager for composite materials inspection," Optical Engineering, vol. 53, no. 3, pp. 031 211-031 211, 2014.

[140] C. Ferraris and R. Carveth, "NASA and the Columbia disaster: decisionmaking by groupthink?" in Proceedings of the 2003 Association for Business Communication Annual Convention, 2003, p. 12.

[141] J. Kauffman, "Lost in space: A critique of NASA's crisis communications in the Columbia disaster," Public Relations Review, vol. 31, no. 2, pp. 263-275, 2005.

[142] E. Matias and B. Rao, "3D printing: On its historical evolution and the implications for business," in International Conference on Management of Engineering and Technology (PICMET), Portland. IEEE, 2015, pp. 551-558.

[143] I. H. Lee and Y. G. Kim, "The recent patent analysis and industrial trend of 3D printing," Indian Journal of Science and Technology, vol. 8, no. S8, pp. 70-73, 2015.

[144] C. Jansen, S. Wietzke, O. Peters, M. Scheller, N. Vieweg, M. Salhi, N. Krumbholz, C. Jördens, T. Hochrein, and M. Koch, "Terahertz imaging: applications and perspectives," Applied optics, vol. 49, no. 19, pp. E48-E57, 2010.

[145] Y. Oyama, L. Zhen, T. Tanabe, and M. Kagaya, "Sub-terahertz imaging of defects in building blocks," NDT $\&$ E International, vol. 42, no. 1, pp. 28-33, 2009.

[146] M. G. Baillie, A slice through time: dendrochronology and precision dating. Routledge, 2012.

[147] N. Oda, A. W. Lee, T. Ishi, I. Hosako, and Q. Hu, "Proposal for real-time terahertz imaging system with palm-size terahertz camera and compact quantum cascade laser," in SPIE Defense, Security, and Sensing. International Society for Optics and Photonics, 2012, pp. 83630A-83630A.

[148] L. Öhrström, A. Bitzer, M. Walther, and F. J. Rühli, "Technical note: terahertz imaging of ancient mummies and bone," American journal of physical anthropology, vol. 142, no. 3, pp. 497-500, 2010.

[149] M. Bessou, H. Duday, J.-P. Caumes, S. Salort, B. Chassagne, A. Dautant, A. Ziéglé, and E. Abraham, "Advantage of terahertz radiation versus X-ray to detect hidden organic materials in sealed vessels," Optics Communications, vol. 285, no. 21, pp. 4175-4179, 2012.

[150] E. Abraham, A. Younus, J.-C. Delagnes, and P. Mounaix, "Non-invasive investigation of art paintings by terahertz imaging," Applied Physics A, vol. 100, no. 3, pp. 585-590, 2010. 
[151] C. Seco-Martorell, V. López-Domínguez, G. Arauz-Garofalo, A. Redo-Sanchez, J. Palacios, and J. Tejada, "Goya's artwork imaging with Terahertz waves," Optics express, vol. 21, no. 15, pp. 17800-17805, 2013.

[152] R. J. Gettens and G. L. Stout, Painting materials: a short encyclopaedia. Courier Corporation, 1966.

[153] G. P. Gallerano, A. Doria, M. Germini, E. Giovenale, G. Messina, and I. P. Spassovsky, "Phase-Sensitive reflective imaging device in the mm-wave and Terahertz regions," Journal of Infrared, Millimeter, and Terahertz Waves, vol. 30, no. 12, pp. 1351-1361, 2009.

[154] A. García Pino, J. Gutiérrez Asueta, J. Montero de Paz, J. Parrón Granados, J. Romeu Robert, R. Vera Rodriguez, P. d. Paco Sánchez, L. E. García Muñoz, B. González Valdés, M. Moreno Moreno et al., "TERASENSE: THz device technology laboratory," 2013.

[155] R. J., L. Jofre, M. Alonso, J. Abril, E. Nova, D. Sánchez-Escuderos, A. Berenguer, F. Las-Heras, J. Laviada, Y. Álvarez López, C. García-González, A. Arboleya, L. García, and D. Segovia, "TeraSense: THz Tomographic Biospectrometer," in URSI, 2013, pp. 1-4.

[156] R. J., A. Broquetas, L. Jofre, D. Sánchez-Escuderos, M. Ferrando-Bataller, A. Berenguer, M. A. Baquero-Escudero, J. Laviada, Y. álvarez López, R. Camblor, S. Ver-Hoeye, F. Las-Heras, C. García-González, C. Vázquez-Antuña, A. Arboleya, M. Fernández, G. Hotopan, L. García, D. Segovia, and J. Montero de Paz, "TeraSense: THz Tomographic Biospectrometer," in URSI, 2013, 2014, pp. 1-4.

[157] M. Vickers, "Dextro-chlorpheniramine (polaramine) in allergy: preliminary report of 75 patients and comparison with racemic chlorpheniramine (chlortrimeton) in 39 patients." The Journal of the Maine Medical Association, vol. 50, no. 1, pp. 16-20, 1959.

[158] A. Danylov, T. Goyette, J. Waldman, M. Coulombe, A. Gatesman, R. Giles, X. Qian, N. Chandrayan, S. Vangala, K. Termkoa et al., "Terahertz inverse synthetic aperture radar (ISAR) imaging with a quantum cascade laser transmitter," Optics Express, vol. 18, no. 15, pp. 16 264-16 272, 2010.

[159] J. Byrnes and G. Ostheimer, Advances in Sensing with Security Applications. Springer, 2006.

[160] K. Iwaszczuk, H. Heiselberg, and P. U. Jepsen, "Terahertz radar cross section measurements," Optics express, vol. 18, no. 25, pp. 26 399-26 408, 2010.

[161] R. W. McGowan, R. A. Cheville, and D. R. Grischkowsky, "Experimental study of the surface waves on a dielectric cylinder via terahertz impulse radar ranging," IEEE Transactions on Microwave Theory and Techniques, vol. 48, no. 3 , pp. 417-422, 2000. 
[162] H.-Y. Li, Q. Li, Z.-W. Xia, Y.-P. Zhao, D.-Y. Chen, and Q. Wang, "Influence of gaussian beam on terahertz radar cross section of a conducting sphere," Journal of Infrared, Millimeter, and Terahertz Waves, vol. 34, no. 1, pp. 8896, 2013.

[163] J. Li, Y. Pi, and X. Yang, "Micro-Doppler signature feature analysis in terahertz band," Journal of Infrared, Millimeter, and Terahertz Waves, vol. 31, no. 3, pp. 319-328, 2010.

[164] Y.-W. Huang, T.-F. Tseng, C.-C. Kuo, Y.-J. Hwang, and C.-K. Sun, "Fiberbased swept-source terahertz radar," Optics letters, vol. 35, no. 9, pp. 1344$1346,2010$.

[165] N. Llombart, K. B. Cooper, R. J. Dengler, T. Bryllert, G. Chattopadhyay, and P. H. Siegel, "Time-delay multiplexing of two beams in a terahertz imaging radar," IEEE Transactions on Microwave Theory and Techniques, vol. 58, no. 7, pp. 1999-2007, 2010.

[166] M. J. Fitch and R. Osiander, "Terahertz waves for communications and sensing," Johns Hopkins APL technical digest, vol. 25, no. 4, pp. 348-355, 2004.

[167] K. R. Jha and G. Singh, "Terahertz planar antennas for future wireless communication: A technical review," infrared physics \& Technology, vol. 60, pp. 71-80, 2013.

[168] H.-J. Song and T. Nagatsuma, "Present and future of terahertz communications," IEEE Transactions on Terahertz Science and Technology, vol. 1, no. 1, pp. 256-263, 2011.

[169] M. Toyoshima, "Trends in satellite communications and the role of optical free-space communications [invited]," Journal of Optical Networking, vol. 4, no. 6, pp. 300-311, 2005.

[170] H. Han, J. Yuan, and J. Tong, "Design of THz space application system," Journal of Computer and Communications, vol. 3, no. 03, p. 61, 2015.

[171] W. Dou, L. Zhang, H. Meng, and Z. Wang, "Tracking antennas for intersatellite communications at sub-millimeter wavelengths," in 3rd Asia-Pacific Conference on Antennas and Propagation (APCAP). IEEE, 2014, pp. 11491152.

[172] J. Federici and L. Moeller, "Review of terahertz and subterahertz wireless communications," Journal of Applied Physics, vol. 107, no. 11, p. 111101, 2010 .

[173] T. Kleine-Ostmann and T. Nagatsuma, "A review on terahertz communications research," Journal of Infrared, Millimeter, and Terahertz Waves, vol. 32, no. 2, pp. 143-171, 2011.

[174] S. Ergün and S. Sönmez, "Terahertz technology for military applications," Journal of Military and Information Science, vol. 3, no. 1, pp. 13-16, 2015. 
[175] N. Breuil, "Les applications civiles et militaires des ondes THz," REE. Revue de l'électricité et de l'électronique, no. 1, 2008.

[176] M. Koch, "Terahertz communications: A 2020 vision," in Terahertz Frequency Detection and Identification of Materials and Objects. Springer, 2007, pp. $325-338$.

[177] J. Y. Suen, M. T. Fang, S. P. Denny, and P. M. Lubin, "Modeling of Terabit Geostationary Terahertz Satellite Links From Globally Dry Locations," IEEE Transactions on Terahertz Science and Technology, vol. 5, no. 2, pp. 299-313, 2015 .

[178] F. A. Fernández, "Inter-satellite ranging and inter-satellite communication links for enhancing GNSS satellite broadcast navigation data," Advances in Space Research, vol. 47, no. 5, pp. 786-801, 2011.

[179] R. E. Everly, D. C. Limmer, and C. A. MacKenzie, "16 cost-effectiveness analysis of autonomous aerial platforms and communication payloads," Military Cost-Benefit Analysis: Theory and practice, 2015.

[180] S. Cherry, "RESOURCES-Telecom-Do telecom data rates mimic Moore's Law?" IEEE Spectrum, vol. 41, no. 7, pp. 50-51, 2004.

[181] M. Jacob, S. Priebe, T. Kurner, C. Jastrow, T. Kleine-Ostmann, and T. Schrader, "An Overview of ongoing activities in the field of channel modeling, spectrum allocation and standardization for mm-wave and $\mathrm{THz}$ indoor communications," in 2009 IEEE Globecom Workshops, 2009.

[182] R. Piesiewicz, T. Kleine-Ostmann, N. Krumbholz, D. Mittleman, M. Koch, and T. Kürner, "Terahertz characterisation of building materials," Electronics Letters, vol. 41, no. 18, pp. 1002-1004, 2005.

[183] S. Priebe, M. Jacob, C. Jastrow, T. Kleine-Ostmann, T. Schrader, and T. Kurner, "A comparison of indoor channel measurements and ray tracing simulations at $300 \mathrm{ghz}$, , in 35th International Conference on Infrared, Millimeter, and Terahertz Waves, 2010.

[184] C. Roberts, "Bicmos process and process for forming bipolar transistors on wafers also containing fets," Jan. 22 1991, uS Patent 4,987,089.

[185] I. F. Akyildiz, J. M. Jornet, and C. Han, "Terahertz band: Next frontier for wireless communications," Physical Communication, vol. 12, pp. 16-32, 2014.

[186] I. Akyildiz, J. M. Jornet, and C.-C. Han, "TeraNets: ultra-broadband communication networks in the terahertz band," IEEE Wireless Communications, vol. 21, no. 4, pp. 130-135, 2014.

[187] M. Strasser, C. Pöpper, and S. Čapkun, "Efficient uncoordinated FHSS antijamming communication," in Proceedings of the tenth ACM international symposium on Mobile ad hoc networking and computing. ACM, 2009, pp. 207-218. 
[188] F. Sizov, "Thz radiation sensors," Opto-electronics review, vol. 18, no. 1, pp. 10-36, 2010.

[189] A. Rogalski and F. Sizov, "Terahertz detectors and focal plane arrays," Optoelectronics review, vol. 19, no. 3, pp. 346-404, 2011.

[190] M. Tonouchi, "Cutting-edge terahertz technology," Nature photonics, vol. 1, no. 2, pp. 97-105, 2007.

[191] N. Llombart, A. Mazzinghi, P. Siegel, and A. Freni, "Design of a Low Loss Metallo-Dielectric EBG Waveguide at Submillimeter Wavelengths," IEEE Microwave and Wireless Components Letters, vol. 19, no. 7, pp. 437-439, 2009.

[192] G. Gallot, S. Jamison, R. McGowan, and D. Grischkowsky, "Terahertz waveguides," JOSA B, vol. 17, no. 5, pp. 851-863, 2000.

[193] R. C. Jones, "The general theory of bolometer performance," JOSA, vol. 43, no. 1, pp. 1-14, 1953.

[194] D. Grbovic and G. Karunasiri, "Fabrication of Bi-material MEMS detector arrays for THz imaging," in SPIE Defense, Security, and Sensing. International Society for Optics and Photonics, 2009, pp. 731 108-731 108.

[195] P. Jayaweera, S. Matsik, A. Perera, Y. Paltiel, A. Sher, A. Raizman, H. Luo, and H. Liu, "GaSb homojunctions for far-infrared (terahertz) detection," Applied physics letters, vol. 90, no. 11, p. 111109, 2007.

[196] S. Komiyama, O. Astafiev, V. Antonov, and T. Kutsuwa, "Single-photon detection of THz-waves using quantum dots," Microelectronic engineering, vol. 63, no. 1, pp. 173-178, 2002.

[197] Y. Kawano, T. Fuse, S. Toyokawa, T. Uchida, and K. Ishibashi, "Terahertz photon-assisted tunneling in carbon nanotube quantum dots," Journal of Applied Physics, vol. 103, no. 3, p. 034307, 2008.

[198] J. Wei, D. Olaya, B. S. Karasik, S. V. Pereverzev, A. V. Sergeev, and M. E. Gershenson, "Ultrasensitive hot-electron nanobolometers for terahertz astrophysics," Nature nanotechnology, vol. 3, no. 8, pp. 496-500, 2008.

[199] M. Tarasov and L. Kuzmin, "Concept of a mixer based on a cold-electron bolometer," Journal of Experimental and Theoretical Physics Letters, vol. 81, no. 10 , pp. $538-541,2005$.

[200] M. Shur, "Terahertz technology: devices and applications," in Proceedings of the 31st European Solid-State Circuits Conference (ESSCIRC). IEEE, 2005, pp. 13-21.

[201] A. Lisauskas, D. Glaab, H. Roskos, E. Oejefors, and U. Pfeiffer, "Terahertz imaging with Si MOSFET focal-plane arrays," in SPIE OPTO: Integrated Optoelectronic Devices. International Society for Optics and Photonics, 2009, pp. $72150 \mathrm{~J}-72150 \mathrm{~J}$. 
[202] J. Grade, P. Haydon, and D. Van der Weide, "Electronic terahertz antennas and probes for spectroscopic detection and diagnostics," Proceedings of the IEEE, vol. 95, no. 8, pp. 1583-1591, 2007.

[203] H. M. Manohara, E. W. Wong, E. Schlecht, B. D. Hunt, and P. H. Siegel, "Carbon nanotube Schottky diodes using Ti-Schottky and Pt-Ohmic contacts for high frequency applications," Nano letters, vol. 5, no. 7, pp. 1469-1474, 2005 .

[204] M. Tarasov, J. Svensson, J. Weis, L. Kuzmin, and E. Campbell, "Carbon nanotube based bolometer," JETP letters, vol. 84, no. 5, pp. 267-270, 2006.

[205] V. Dobrovolsky, F. Sizov, Y. Kamenev, and A. Smirnov, "Ambient temperature or moderately cooled semiconductor hot electron bolometer for $\mathrm{mm}$ and sub-mm regions," Opto-Electronics Review, vol. 16, no. 2, pp. 172-178, 2008.

[206] S. Matsuura, M. Tani, and K. Sakai, "Generation of coherent terahertz radiation by photomixing in dipole photoconductive antennas," Applied Physics Letters, vol. 70, no. 5, pp. 559-561, 1997.

[207] M. Tani, Y. Hirota, C. T. Que, S. Tanaka, R. Hattori, M. Yamaguchi, S. Nishizawa, and M. Hangyo, "Novel terahertz photoconductive antennas," International journal of infrared and millimeter waves, vol. 27, no. 4, pp. 531546, 2006.

[208] M. Tani, M. Herrmann, and K. Sakai, "Generation and detection of terahertz pulsed radiation with photoconductive antennas and its application to imaging," Measurement science and technology, vol. 13, no. 11, p. 1739, 2002.

[209] A. Jelenski, A. Grüb, V. Krozer, and H. L. Hartnagel, "New approach to the design and the fabrication of THz Schottky barrier diodes," IEEE Transactions on Microwave Theory and Techniques, vol. 41, no. 4, pp. 549-557, 1993.

[210] R. Han, Y. Zhang, Y. Kim, D. Y. Kim, H. Shichijo, E. Afshari, and O. Kenneth, "280GHz and $860 \mathrm{GHz}$ image sensors using Schottky-barrier diodes in $0.13 \mu \mathrm{m}$ digital CMOS," in Solid-State Circuits Conference Digest of Technical Papers (ISSCC), 2012 IEEE International. IEEE, 2012, pp. 254-256.

[211] U. R. Pfeiffer, C. Mishra, R. M. Rassel, S. Pinkett, and S. K. Reynolds, "Schottky barrier diode circuits in silicon for future millimeter-wave and terahertz applications," IEEE Transactions on Microwave Theory and Techniques, vol. 56, no. 2, pp. 364-371, 2008.

[212] J. Kooi, J. Stern, G. Chattopadhyay, H. LeDuc, B. Bumble, and J. Zmuidzinas, "Low-loss NbTiN films for THz SIS mixer tuning circuits," International journal of infrared and millimeter waves, vol. 19, no. 3, pp. 373-383, 1998.

[213] A. Karpov, D. Miller, F. Rice, J. Stern, B. Bumble, H. LeDuc, and J. Zmuidzinas, "Low Noise $1 \mathrm{THz}-1.4 \mathrm{THz}$ Mixers Using Nb/Al-AlN/NbTiN SIS Junctions," IEEE Transactions on Applied Superconductivity, vol. 17, no. 2, pp. 343-346, 2007. 
[214] P. K. Day, H. G. LeDuc, B. A. Mazin, A. Vayonakis, and J. Zmuidzinas, "A broadband superconducting detector suitable for use in large arrays," Nature, vol. 425 , no. 6960 , pp. 817-821, 2003.

[215] A. Sergeev, V. Mitin, and B. Karasik, "Ultrasensitive hot-electron kineticinductance detectors operating well below the superconducting transition," Applied physics letters, vol. 80, no. 5, pp. 817-819, 2002.

[216] A. Baryshev, J. J. Baselmans, A. Freni, G. Gerini, H. Hoevers, A. Iacono, and A. Neto, "Progress in antenna coupled kinetic inductance detectors," IEEE Transactions on Terahertz Science and Technology, vol. 1, no. 1, pp. 112-123, 2011.

[217] S. Komiyama, "Single-photon detectors in the terahertz range," IEEE Journal of Selected Topics in Quantum Electronics, vol. 17, no. 1, pp. 54-66, 2011.

[218] Y. Kajihara, T. Nakajima, Z. Wang, and S. Komiyama, "Terahertz singlephoton detectors based on quantum wells," Journal of Applied Physics, vol. 113, no. 13, p. 136506, 2013.

[219] K. Ikushima, A. Ito, and S. Okano, "Generation, transmission, and detection of terahertz photons on an electrically driven single chip," Applied Physics Letters, vol. 104, no. 5, p. 052112, 2014.

[220] T. W. Crowe, D. W. Porterfield, J. L. Hesler, W. L. Bishop, D. S. Kurtz, and K. Hui, "Terahertz sources and detectors," in Defense and Security. International Society for Optics and Photonics, 2005, pp. 271-280.

[221] N. R. A. Observatory. (2016). [Online]. Available: https://science.nrao.edu/ facilities $/ \mathrm{cdl} / \mathrm{mm}$-submm-devices

[222] Astrobites/Microwave Kinetic Inductance Detectors. (2016). [Online]. Available: http://astrobites.org/2011/12/06/ microwave-kinetic-inductance-detectors/

[223] Q. Wu and X.-C. Zhang, "Design and characterization of traveling-wave electrooptic terahertz sensors," IEEE Journal of Selected Topics in Quantum Electronics, vol. 2, no. 3, pp. 693-700, 1996.

[224] C. W. Berry and M. Jarrahi, "Principles of impedance matching in photoconductive antennas," Journal of Infrared, Millimeter, and Terahertz Waves, vol. 33, no. 12, pp. 1182-1189, 2012.

[225] M. Tani, P. Gu, M. Hyodo, K. Sakai, and T. Hidaka, "Generation of coherent terahertz radiation by photomixing of dual-mode lasers," Optical and Quantum Electronics, vol. 32, no. 4-5, pp. 503-520, 2000.

[226] F. Miyamaru, Y. Saito, M. Takeda, L. Liu, B. Hou, W. Wen, and P. Sheng, "Emission of terahertz radiations from fractal antennas," Applied physics letters, vol. 95, no. 22, p. 221111, 2009. 
[227] T. W. Crowe, W. L. Bishop, D. W. Porterfield, J. L. Hesler, and R. M. Weikle, "Opening the terahertz window with integrated diode circuits," IEEE Journal of Solid-State Circuits, vol. 40, no. 10, pp. 2104-2110, 2005.

[228] H.-W. Hubers, "Terahertz heterodyne receivers," IEEE journal of selected topics in quantum electronics, vol. 2, no. 14, pp. 378-391, 2008.

[229] N. E. Booth and D. J. Goldie, "Superconducting particle detectors," Superconductor Science and Technology, vol. 9, no. 7, p. 493, 1996.

[230] M. Griffin, J. Baselmans, A. Baryshev, S. Doyle, M. Grim, P. Hargrave, T. Klapwijk, J. Martin-Pintado, A. Monfardini, A. Neto et al., "SPACEKIDS: Kinetic inductance detector arrays for space applications," in 40th International Conference on Infrared, Millimeter, and Terahertz waves (IRMMWTHz). IEEE, 2015, pp. 1-2.

[231] A. Denisov, J. H. Qiu, S. Lan, and A. Gudkov, "Some opinion about matrix terahertz imaging system based on Josephson junctions," in SPIE Defense+ Security. International Society for Optics and Photonics, 2015, pp. 94620B$94620 \mathrm{~B}$.

[232] N. Nishioka, P. Richards, and D. Woody, "Composite bolometers for submillimeter wavelengths," Applied optics, vol. 17, no. 10, pp. 1562-1567, 1978.

[233] S. M. Wentworth and D. P. Neikirk, "Composite microbolometers with tellurium detector elements," IEEE Transactions on Microwave Theory and Techniques, vol. 40, no. 2, pp. 196-201, 1992.

[234] T. Phillips and D. Woody, "Millimeter-and submillimeter-wave receivers," Annual Review of Astronomy and Astrophysics, vol. 20, pp. 285-321, 1982.

[235] S. Cherednichenko, P. Khosropanah, E. Kollberg, M. Kroug, and H. Merkel, "Terahertz superconducting hot-electron bolometer mixers," Physica $\mathrm{C}: \mathrm{Su}$ perconductivity, vol. 372, pp. 407-415, 2002.

[236] M. S. Vitiello, L. Viti, L. Romeo, D. Ercolani, G. Scalari, J. Faist, F. Beltram, L. Sorba, and A. Tredicucci, "Semiconductor nanowires for highly sensitive, room-temperature detection of terahertz quantum cascade laser emission," $A p$ plied physics letters, vol. 100, no. 24, p. 241101, 2012.

[237] R. Müller, B. Gutschwager, J. Hollandt, M. Kehrt, C. Monte, R. Müller, and A. Steiger, "Characterization of a Large-Area Pyroelectric Detector from $300 \mathrm{GHz}$ to $30 \mathrm{THz}$," Journal of Infrared, Millimeter, and Terahertz Waves, vol. 36, no. 7, pp. 654-661, 2015.

[238] E. Hack, L. Valzania, G. Gäumann, M. Shalaby, C. P. Hauri, and P. Zolliker, "Comparison of Thermal Detector Arrays for Off-Axis THz Holography and Real-Time THz Imaging," Sensors, vol. 16, no. 2, p. 221, 2016. 
[239] H. Roehle, R. Dietz, H. Hensel, J. Böttcher, H. Künzel, D. Stanze, M. Schell, and B. Sartorius, "Next generation $1.5 \mu \mathrm{m}$ terahertz antennas: mesa-structuring of InGaAs/InAlAs photoconductive layers," Optics express, vol. 18, no. 3, pp. 2296-2301, 2010.

[240] J. Bjarnason, T. Chan, A. Lee, M. Celis, and E. Brown, "Millimeter-wave, terahertz, and mid-infrared transmissionthrough common clothing," Applied Physics Letters, vol. 85, no. 4, pp. 519-521, 2004.

[241] W. Stillman and M. Shur, "Closing the gap: plasma wave electronic terahertz detectors," Journal of Nanoelectronics and Optoelectronics, vol. 2, no. 3, pp. 209-221, 2007.

[242] G. B. Airy, "On the diffraction of an object-glass with circular aperture," Transactions of the Cambridge Philosophical Society, vol. 5, p. 283, 1835.

[243] R. Ozhegov, K. Gorshkov, Y. B. Vachtomin, K. Smirnov, M. Finkel, G. Goltsman, O. Kiselev, N. Kinev, L. Filippenko, and V. Koshelets, "Terahertz Imaging System Based on Superconducting Heterodyne Integrated Receiver," in THz and Security Applications. Springer, 2014, pp. 113-125.

[244] T. W. Crowe, R. J. Mattauch, H. P. Röser, W. L. Bishop, W. C. Peatman, and X. Liu, "GaAs Schottky diodes for THz mixing applications," Proceedings of the IEEE, vol. 80, no. 11, pp. 1827-1841, 1992.

[245] K. D. Irwin and G. C. Hilton, "Transition-edge sensors," in Cryogenic Particle Detection. Springer, 2005, pp. 63-150.

[246] F. Sizov, V. Reva, O. Golenkov, V. Petriakov, A. Shevchik-Shekera, S. Korinets, M. Sakhno, I. Lysiuk, V. Zabudsky, S. Bunchuk et al., "THz/sub-THz direct detector challenges: Rectification and thermal detectors for active imaging," in 20th International Conference on Microwaves, Radar, and Wireless Communication (MIKON). IEEE, 2014, pp. 1-4.

[247] G. Gallerano, S. Biedron et al., "Overview of terahertz radiation sources," in Proceedings of the 2004 FEL Conference, 2004, pp. 216-221.

[248] A. V. Räisänen, "Frequency multipliers for millimeter and submillimeter wavelengths," Proceedings of the IEEE, vol. 80, no. 11, pp. 1842-1852, 1992.

[249] A. Maestrini, B. Thomas, H. Wang, C. Jung, J. Treuttel, Y. Jin, G. Chattopadhyay, I. Mehdi, and G. Beaudin, "Schottky diode-based terahertz frequency multipliers and mixers," Comptes Rendus Physique, vol. 11, no. 7, pp. 480-495, 2010.

[250] J. Faist, F. Capasso, D. L. Sivco, C. Sirtori, A. L. Hutchinson, and A. Y. Cho, "Quantum cascade laser," Science, vol. 264, no. 5158, pp. 553-556, 1994.

[251] B. S. Williams, "Terahertz quantum-cascade lasers," Nature photonics, vol. 1, no. 9, pp. 517-525, 2007. 
[252] J. H. Booske, R. J. Dobbs, C. D. Joye, C. L. Kory, G. R. Neil, G.-S. Park, J. Park, and R. J. Temkin, "Vacuum electronic high power terahertz sources," IEEE Transactions on Terahertz Science and Technology, vol. 1, no. 1, pp. $54-75,2011$.

[253] H. R. Johnson, "Backward-wave oscillators," Proceedings of the IRE, vol. 43, no. 6 , pp. 684-697, 1955.

[254] E. Borie, Review of gyrotron theory. Kernforschungszentrum, 1991.

[255] E. Rouvalis, C. C. Renaud, D. G. Moodie, M. J. Robertson, and A. J. Seeds, "Continuous wave terahertz generation from ultra-fast InP-based photodiodes," IEEE Transactions on Microwave Theory and Techniques, vol. 60, no. 3, pp. 509-517, 2012.

[256] K. Reimann, "Table-top sources of ultrashort THz pulses," Reports on Progress in Physics, vol. 70, no. 10, p. 1597, 2007.

[257] J. Danielson, A. Jameson, J. Tomaino, H. Hui, J. Wetzel, Y.-S. Lee, and K. Vodopyanov, "Intense narrow band terahertz generation via type-II difference-frequency generation in ZnTe using chirped optical pulses," Journal of Applied Physics, vol. 104, no. 3, p. 033111, 2008.

[258] P. Kirawanich, S. J. Yakura, and N. E. Islam, "Study of high-power wideband terahertz-pulse generation using integrated high-speed photoconductive semiconductor switches," IEEE Transactions on Plasma Science, vol. 37, no. 1, pp. 219-228, 2009.

[259] A. J. Seeds, M. J. Fice, K. Balakier, M. Natrella, O. Mitrofanov, M. Lamponi, M. Chtioui, F. van Dijk, M. Pepper, G. Aeppli et al., "Coherent terahertz photonics," Optics express, vol. 21, no. 19, pp. 22 988-23 000, 2013.

[260] N. Shimizu, K. Matsuyama, and H. Uchida, "Incoherent sub-terahertz radiation source with a photomixer array for active imaging in smoky environments," in SPIE OPTO. International Society for Optics and Photonics, 2015, pp. $936204-936204$.

[261] A. J. Deninger, T. Göbel, D. Schönherr, T. Kinder, A. Roggenbuck, M. Köberle, F. Lison, T. Müller-Wirts, and P. Meissner, "Precisely tunable continuous-wave terahertz source with interferometric frequency control," Review of Scientific Instruments, vol. 79, no. 4, p. 044702, 2008.

[262] A. Moretti, G. Moruzzi, F. Strumia, J. C. S. Moraes, and G. Carelli, "New Terahertz Laser Lines From Pumped by Regular and Hot Bands Laser," IEEE Journal of Quantum Electronics, vol. 44, no. 11, pp. 1104-1106, 2008.

[263] L. Li, L. Chen, J. Zhu, J. Freeman, P. Dean, A. Valavanis, A. G. Davies, and E. H. Linfield, "Terahertz quantum cascade lasers with $>1$ W output powers," Electronics Letters, vol. 50, no. 4, pp. 309-311, 2014.

[264] H. Eisele, "State of the art and future of electronic sources at terahertz frequencies," Electronics Letters, vol. 46, no. 26, pp. s8-s11, 2010. 
[265] D. W. Porterfield, "High-efficiency terahertz frequency triplers," in Microwave Symposium, 2007. IEEE/MTT-S International. IEEE, 2007, pp. 337-340.

[266] I. Mehdi, G. Chattopadhyay, E. Schlecht, J. Ward, J. Gill, F. Maiwald, and A. Maestrini, THz multiplier circuits. Citeseer, 2006.

[267] H. Wang, L. Samoska, T. Gaier, A. Peralta, H.-H. Liao, Y. Leong, S. Weinreb, Y. C. Chen, M. Nishimoto, and R. Lai, "Power-amplifier modules covering 70-113 GHz using MMICs," IEEE Transactions on Microwave Theory and Techniques, vol. 49, no. 1, pp. 9-16, 2001.

[268] L. A. Samoska, T. C. Gaier, A. Peralta, S. Weinreb, J. Bruston, I. Mehdi, Y. Chen, H. H. Liao, M. Nishimoto, R. Lai et al., "MMIC power amplifiers as local oscillator drivers for FIRST," in Astronomical Telescopes and Instrumentation. International Society for Optics and Photonics, 2000, pp. 275-284.

[269] L. Samoska, V. Radisic, M. Micovic, M. Hu, P. Janke, and C. Ngo, InP MMIC chip set for power sources covering 86-170 GHz. Pasadena, CA: Jet Propulsion Laboratory, National Aeronautics and Space Administration, 2001.

[270] H. Eisele and G. I. Haddad, "Active microwave diodes," Modern semiconductor devices, vol. 6, pp. 343-407, 1998.

[271] A. Acharyya and J. Banerjee, "Potentiality of IMPATT devices as terahertz source: an Avalanche response time-based approach to determine the upper cut-off frequency limits," IETE Journal of Research, vol. 59, no. 2, pp. 118127, 2013.

[272] J. Nishizawa, P. Płotka, T. Kurabayashi, and H. Makabe, "706-GHz GaAs CW fundamental-mode TUNNETT diodes fabricated with molecular layer epitaxy," physica status solidi (c), vol. 5, no. 9, pp. 2802-2804, 2008.

[273] H. Eisele, "InP gunn devices for 400-425 GHz," Electronics Letters, vol. 42, no. 6, pp. 358-359, 2006.

[274] J.-i. Nishizawa, K. Motoya, and Y. Okuno, "GaAs TUNNETT diodes," IEEE Transactions on Microwave Theory and Techniques, vol. 26, no. 12, pp. 10291035, 1978.

[275] J.-S. Rieh, D. Yoon, and J. Yun, "An overview of solid-state electronic sources and detectors for Terahertz imaging," in 12th IEEE International Conference on Solid-State and Integrated Circuit Technology (ICSICT). IEEE, 2014, pp. 1-4.

[276] L. A. Samoska, "An overview of solid-state integrated circuit amplifiers in the submillimeter-wave and THz regime," IEEE Transactions on Terahertz Science and Technology, vol. 1, no. 1, pp. 9-24, 2011.

[277] V. Radisic, K. M. Leong, X. Mei, S. Sarkozy, W. Yoshida, and W. R. Deal, "Power amplification at $0.65 \mathrm{THz}$ using INP HEMTs," IEEE Transactions on Microwave Theory and Techniques, vol. 60, no. 3, pp. 724-729, 2012. 
[278] V. Radisic, K. M. Leong, X. Mei, S. Sarkozy, W. Yoshida, P.-H. Liu, J. Uyeda, R. Lai, and W. R. Deal, "A $50 \mathrm{~mW} 220 \mathrm{GHz}$ power amplifier module," in IEEE MTT-S International Microwave Symposium Digest (MTT). IEEE, 2010, pp. 45-48.

[279] V. Radisic, W. R. Deal, K. M. Leong, X. Mei, W. Yoshida, P.-H. Liu, J. Uyeda, A. Fung, L. Samoska, T. Gaier et al., "A 10-mW submillimeter-wave solidstate power-amplifier module," IEEE Transactions on Microwave Theory and Techniques, vol. 58, no. 7, pp. 1903-1909, 2010.

[280] J. Schellenberg, "The evolution of power combining techniques: From the 60s to today," in IEEE MTT-S International Microwave Symposium (IMS). IEEE, 2015, pp. 1-4.

[281] J. Wang, A. Ofiare, K. Alharbi, R. Brown, A. Khalid, D. Cumming, and E. Wasige, "MMIC resonant tunneling diode oscillators for THz applications," in Ph. D. Research in Microelectronics and Electronics (PRIME), 2015 11th Conference on. IEEE, 2015, pp. 262-265.

[282] J. V. Siles, E. Schlecht, R. Lin, C. Lee, and I. Mehdi, "High-efficiency planar Schottky diode based submillimeter-wave frequency multipliers optimized for high-power operation," in 40th International Conference on Infrared, Millimeter, and Terahertz waves (IRMMW-THz). IEEE, 2015, pp. 1-1.

[283] G. Kantorowicz and P. Palluel, "Backward wave oscillators," Infrared and millimeter waves, vol. 1, pp. 185-212, 1979.

[284] K. Sakamoto, A. Kasugai, K. Takahashi, R. Minami, N. Kobayashi, and K. Kajiwara, "Achievement of robust high-efficiency $1 \mathrm{MW}$ oscillation in the hardself-excitation region by a $170 \mathrm{GHz}$ continuous-wave gyrotron," Nature physics, vol. 3, no. 6, pp. 411-414, 2007.

[285] M. Y. Glyavin and A. G. Luchinin, "Powerful terahertz gyrotrons based on pulsed magnets," Terahertz Science Technology, vol. 2, pp. 150-155, 2009.

[286] P.-K. Liu, L. Luo, and C.-H. Du, "Broadband THz gyrotron based on a pulse magnet," in 40th International Conference on Infrared, Millimeter, and Terahertz waves (IRMMW-THz). IEEE, 2015, pp. 1-2.

[287] M. Thumm, A. Arnold, E. Borie, O. Braz, G. Dammertz, O. Dumbrajs, K. Koppenburg, M. Kuntze, G. Michel, and B. Piosczyk, "Frequency steptunable (114-170 GHz) megawatt gyrotrons for plasma physics applications," Fusion Engineering and Design, vol. 53, no. 1, pp. 407-421, 2001.

[288] T. Idehara, T. Saito, I. Ogawa, S. Mitsudo, Y. Tatematsu, L. Agusu, H. Mori, and S. Kobayashi, "Development of terahertz FU CW gyrotron series for DNP," Applied Magnetic Resonance, vol. 34, no. 3-4, pp. 265-275, 2008.

[289] V. Bajaj, C. Farrar, M. Hornstein, I. Mastovsky, J. Vieregg, J. Bryant, B. Elena, K. Kreischer, R. Temkin, and R. Griffin, "Dynamic nuclear polarization at 9T using a novel $250 \mathrm{GHz}$ gyrotron microwave source," Journal of Magnetic Resonance, vol. 160, no. 2, pp. 85-90, 2003. 
[290] W. He, L. Zhang, D. Bowes, H. Yin, K. Ronald, A. Phelps, and A. Cross, "Generation of broadband terahertz radiation using a backward wave oscillator and pseudospark-sourced electron beam," Applied Physics Letters, vol. 107, no. 13, p. 133501, 2015.

[291] L. Zhang, Y. Wei, B. Wang, W. Shen, J. Xu, Y. Gong, and G.-S. Park, "Investigation of $0.38 \mathrm{THz}$ backward-wave oscillator based on slotted sine waveguide and pencil electron beam," Physics of Plasmas (1994-present), vol. 23, no. 3, p. $033111,2016$.

[292] G. Liu, J. Wang, G. Shu, L. Yang, and Y. Luo, "1.2 THz backward wave radiation generated by double bunched sheet beams," in IEEE International Vacuum Electronics Conference (IVEC). IEEE, 2015, pp. 1-2.

[293] C. Paoloni, L. Yue, X. Tang, F. Zhang, B. Popovic, L. Himes, R. Barchfeld, D. Gamzina, R. Letizia, M. Mineo et al., "Thz backward-wave oscillators for plasma diagnostic in nuclear fusion," in IEEE International Conference on Plasma Sciences (ICOPS). IEEE, 2015, pp. 1-1.

[294] A. Dobroiu, M. Yamashita, Y. N. Ohshima, Y. Morita, C. Otani, and K. Kawase, "Terahertz imaging system based on a backward-wave oscillator," Applied optics, vol. 43, no. 30, pp. 5637-5646, 2004.

[295] A. Y. Pawar, D. D. Sonawane, K. B. Erande, and D. V. Derle, "Terahertz technology and its applications," Drug Invention Today, vol. 5, no. 2, pp. 157-163, 2013.

[296] M. Van Exter and D. R. Grischkowsky, "Characterization of an optoelectronic terahertz beam system," IEEE Transactions on Microwave Theory and Techniques, vol. 38, no. 11, pp. 1684-1691, 1990.

[297] C. Fattinger and D. Grischkowsky, "Terahertz beams," Applied Physics Letters, vol. 54, no. 6, pp. 490-492, 1989.

[298] M. Tani, O. Morikawa, S. Matsuura, and M. Hangyo, "Generation of terahertz radiation by photomixing with dual-and multiple-mode lasers," Semiconductor science and technology, vol. 20, no. 7, p. S151, 2005.

[299] E. Peytavit, S. Lepilliet, F. Hindle, C. Coinon, T. Akalin, G. Ducournau, G. Mouret, and J.-F. Lampin, "Milliwatt-level output power in the subterahertz range generated by photomixing in a GaAs photoconductor," Applied Physics Letters, vol. 99, no. 22, p. 223508, 2011.

[300] F. Hindle, A. Cuisset, R. Bocquet, and G. Mouret, "Continuous-wave terahertz by photomixing: applications to gas phase pollutant detection and quantification," Comptes Rendus Physique, vol. 9, no. 2, pp. 262-275, 2008.

[301] V. Podobedov, D. Plusquellic, and G. Fraser, "THz laser study of self-pressure and temperature broadening and shifts of water vapor lines for pressures up to $1.4 \mathrm{kPa}$, " Journal of Quantitative Spectroscopy and Radiative Transfer, vol. 87, no. 3, pp. 377-385, 2004. 
[302] S. Preu, G. Döhler, S. Malzer, L. Wang, and A. Gossard, "Tunable, continuouswave terahertz photomixer sources and applications," Journal of Applied Physics, vol. 109, no. 6, p. 061301, 2011.

[303] C. W. Berry, M. R. Hashemi, S. Preu, H. Lu, A. C. Gossard, and M. Jarrahi, "High power terahertz generation using $1550 \mathrm{~nm}$ plasmonic photomixers," $A p$ plied Physics Letters, vol. 105, no. 1, p. 011121, 2014.

[304] S. Verghese, K. McIntosh, and E. Brown, "Highly tunable fiber-coupled photomixers with coherent terahertz output power," IEEE Transactions on Microwave Theory and Techniques, vol. 45, no. 8, pp. 1301-1309, 1997.

[305] H. Tanoto, J. Teng, Q. Wu, M. Sun, Z. Chen, S. Maier, B. Wang, C. Chum, G. Si, A. Danner et al., "Greatly enhanced continuous-wave terahertz emission by nano-electrodes in a photoconductive photomixer," Nature photonics, vol. 6 , no. 2, pp. 121-126, 2012.

[306] A. G. Stepanov, A. A. Melnikov, V. O. Kompanets, and S. V. Chekalin, "Spectral modification of femtosecond laser pulses in the process of highly efficient generation of terahertz radiation via optical rectification," JETP Letters, vol. 85, no. 5, pp. 227-230, 2007.

[307] K. Yeh, M. Hoffmann, J. Hebling, and K. A. Nelson, "Generation of $10 \mathrm{~J}$ ultrashort terahertz pulses by optical rectification," Applied Physics Letters, vol. 90, no. 17, p. 171121, 2007.

[308] H. Hirori, A. Doi, F. Blanchard, and K. Tanaka, "Single-cycle terahertz pulses with amplitudes exceeding $1 \mathrm{MV} / \mathrm{cm}$ generated by optical rectification in LiNbO3," Applied Physics Letters, vol. 98, no. 9, p. 091106, 2011.

[309] S. B. Bodrov, I. E. Ilyakov, B. V. Shishkin, and A. N. Stepanov, "Efficient terahertz generation by optical rectification in Si-LiNbO3-air-metal sandwich structure with variable air gap," Applied Physics Letters, vol. 100, no. 20, p. 201114, 2012.

[310] M. Tsarev, D. Ehberger, and P. Baum, "High-average-power, intense THz pulses from a LiNbO3 slab with silicon output coupler," Applied Physics B, vol. 122, no. 2, pp. 1-5, 2016.

[311] K. L. Vodopyanov, "Optical THz-wave generation with periodically-inverted GaAs," Laser \&3 Photonics Reviews, vol. 2, no. 1-2, pp. 11-25, 2008.

[312] C. D Amico, A. Houard, M. Franco, B. Prade, A. Mysyrowicz, A. Couairon, and V. Tikhonchuk, "Conical forward thz emission from femtosecond-laserbeam filamentation in air," Physical review letters, vol. 98, no. 23, p. 235002, 2007.

[313] C. Zhang, Y. Avetisyan, A. Glosser, I. Kawayama, H. Murakami, and M. Tonouchi, "Bandwidth tunable $\mathrm{THz}$ wave generation in large-area periodically poled lithium niobate," Optics express, vol. 20, no. 8, pp. 8784-8790, 2012. 
[314] E. R. Mueller, "Submillimeter wave lasers," Wiley Encyclopedia of Electrical and Electronics Engineering, 1999.

[315] G. Chin, "Optically pumped submillimeter laser heterodyne receivers: Astrophysical observations and recent technical developments," Proceedings of the IEEE, vol. 80, no. 11, pp. 1788-1799, 1992.

[316] S. D. Ganichev and W. Prettl, Intense terahertz excitation of semiconductors. Oxford University Press Oxford, 2006, vol. 14.

[317] E. Gornik and A. Andronov, "Far-infrared semiconductor lasers," Optical and Quantum Electronics, vol. 23, no. 2, pp. v-v, 1991.

[318] E. Bründermann, A. Linhart, L. Reichertz, H. Röser, O. Dubon, W. Hansen, G. Sirmain, and E. Haller, "Double acceptor doped ge: A new medium for inter-valence-band lasers," Applied physics letters, vol. 68, no. 22, pp. 30753077, 1996.

[319] E. Bründermann, D. Chamberlin, and E. Haller, "Novel design concepts of widely tunable germanium terahertz lasers," Infrared physics \& technology, vol. 40, no. 3, pp. 141-151, 1999.

[320] A. Liu and C.-Z. Ning, "Terahertz optical gain based on intersubband transitions in optically pumped semiconductor quantum wells: Coherent pumpprobe interactions," Applied physics letters, vol. 75, no. 9, pp. 1207-1209, 1999.

[321] Y. Chassagneux, R. Colombelli, W. Maineult, S. Barbieri, H. Beere, D. Ritchie, S. Khanna, E. Linfield, and A. Davies, "Electrically pumped photonic-crystal terahertz lasers controlled by boundary conditions," Nature, vol. 457, no. 7226, pp. 174-178, 2009.

[322] S. Pavlov, V. Shastin et al., "Terahertz lasers based on germanium and silicon," Semiconductor science and technology, vol. 20, no. 7, p. S211, 2005.

[323] A. Bergner, U. Heugen, E. Bründermann, G. Schwaab, M. Havenith, D. R. Chamberlin, and E. E. Haller, "New p-Ge THz laser spectrometer for the study of solutions: $\mathrm{THz}$ absorption spectroscopy of water," Review of scientific instruments, vol. 76, no. 6, p. 063110, 2005.

[324] D. J. Paul, "The progress towards terahertz quantum cascade lasers on silicon substrates," Laser \& Photonics Reviews, vol. 4, no. 5, pp. 610-632, 2010.

[325] A. Kavokin, I. Shelykh, T. Taylor, and M. Glazov, "Vertical cavity surface emitting terahertz laser," Physical review letters, vol. 108, no. 19, p. 197401, 2012.

[326] R. Köhler, A. Tredicucci, F. Beltram, H. E. Beere, E. H. Linfield, A. G. Davies, D. A. Ritchie, R. C. Iotti, and F. Rossi, "Terahertz semiconductorheterostructure laser," Nature, vol. 417, no. 6885, pp. 156-159, 2002.

[327] C. Sirtori, S. Barbieri, and R. Colombelli, "Wave engineering with THz quantum cascade lasers," Nature Photonics, vol. 7, no. 9, pp. 691-701, 2013. 
[328] J. Ulrich, R. Zobl, W. Schrenk, G. Strasser, K. Unterrainer, and E. Gornik, "Terahertz quantum cascade structures: Intra-versus interwell transition," $A p$ plied Physics Letters, vol. 77, no. 13, pp. 1928-1930, 2000.

[329] P. Shumyatsky and R. R. Alfano, "Terahertz sources," Journal of biomedical optics, vol. 16, no. 3, pp. $033001-033$ 001, 2011.

[330] M. Razeghi, Q. Lu, N. Bandyopadhyay, S. Slivken, and Y. Bai, "Room temperature continuous wave $\mathrm{THz}$ quantum cascade laser source with high power operation," in SPIE Optical Engineering + Applications. International Society for Optics and Photonics, 2014, pp. 919 902-919 902.

[331] D. Turčinková, M. I. Amanti, G. Scalari, M. Beck, and J. Faist, "Electrically tunable terahertz quantum cascade lasers based on a two-sections interdigitated distributed feedback cavity," Applied Physics Letters, vol. 106, no. 13, p. $131107,2015$.

[332] B. S. Williams, S. Kumar, Q. Hu, and J. L. Reno, "Operation of terahertz quantum-cascade lasers at $164 \mathrm{~K}$ in pulsed mode and at $117 \mathrm{~K}$ in continuouswave mode," Optics Express, vol. 13, no. 9, pp. 3331-3339, 2005.

[333] Q. Lu, N. Bandyopadhyay, S. Slivken, Y. Bai, and M. Razeghi, "Continuous operation of a monolithic semiconductor terahertz source at room temperature," Applied Physics Letters, vol. 104, no. 22, p. 221105, 2014.

[334] S. Suphakul, K. Damminsak, H. Zen, T. Kii, and H. Ohgaki, "Development of compact seeded terahertz free-electron laser amplifier system at Kyoto University," in Infrared, Millimeter, and Terahertz waves (IRMMW-THz), 2015 40th International Conference on. IEEE, 2015, pp. 1-1.

[335] D. Oepts, A. Van der Meer, and P. Van Amersfoort, "The free-electron-laser user facility FELIX," Infrared Physics \& Technology, vol. 36, no. 1, pp. 297308, 1995.

[336] C. A. Brau, "Free-electron lasers," 1990.

[337] C. Collins, R. Miles, J. Digby, G. Parkhurst, R. Pollard, J. Chamberlain, D. Steenson, N. Cronin, S. Davies, and J. W. Bowen, "A new micro-machined millimeter-wave and terahertz snap-together rectangular waveguide technology," IEEE microwave and guided wave letters, vol. 9, no. 2, pp. 63-65, 1999.

[338] J. W. Digby, C. E. McIntosh, G. M. Parkhurst, B. M. Towlson, S. Hadjiloucas, J. W. Bowen, J. M. Chamberlain, R. D. Pollard, R. E. Miles, D. P. Steenson et al., "Fabrication and characterization of micromachined rectangular waveguide components for use at millimeter-wave and terahertz frequencies," IEEE Transactions on Microwave Theory and Techniques, vol. 48, no. 8, pp. 1293$1302,2000$.

[339] R. E. Collin, Field Theory of Guided Waves. John Wiley \& Sons, 1991. 
[340] L. Chen, A. Arsenovic, J. R. Stanec, T. J. Reck, A. W. Lichtenberger, R. M. Weikle, N. S. Barker et al., "A micromachined terahertz waveguide 90 twist," IEEE Microwave and Wireless Components Letters, vol. 21, no. 5, pp. 234-236, 2011.

[341] X. Shang, M. Ke, Y. Wang, and M. J. Lancaster, "Micromachined W-band waveguide and filter with two embedded H-plane bends," IET Microwaves, Antennas 85 Propagation, vol. 5, no. 3, pp. 334-339, 2011.

[342] — , "Micromachined WR-3 waveguide filter with embedded bends," Electronics Letters, vol. 47, no. 9, pp. 545-547, 2011.

[343] —, "WR-3 band waveguides and filters fabricated using SU8 photoresist micromachining technology," IEEE Transactions on Terahertz Science and Technology, vol. 2, no. 6, pp. 629-637, 2012.

[344] C. A. Leal-Sevillano, T. J. Reck, C. Jung-Kubiak, G. Chattopadhyay, J. A. Ruiz-Cruz, J. R. Montejo-Garai, and J. M. Rebollar, "Silicon Micromachined Canonical-Plane and-Plane Bandpass Filters at the Terahertz Band," IEEE Microwave and Wireless Components Letters, vol. 23, no. 6, pp. 288-290, 2013.

[345] X. Shang, Y. Tian, M. J. Lancaster, and S. Singh, " SU8 micromachined WR-1.5 band waveguide filter," IEEE Microwave and Wireless Components Letters, vol. 23, no. 6, pp. 300-302, 2013.

[346] H. Lorenz, M. Despont, N. Fahrni, N. LaBianca, P. Renaud, and P. Vettiger, "SU-8: a low-cost negative resist for MEMS," Journal of Micromechanics and Microengineering, vol. 7, no. 3, p. 121, 1997.

[347] M. Ordal, R. Bell, R. Alexander Jr, L. Long, and M. Querry, "Optical properties of fourteen metals in the infrared and far infrared: $\mathrm{Al}, \mathrm{Co}, \mathrm{Cu}, \mathrm{Au}, \mathrm{Fe}$, Pb, Mo, Ni, Pd, Pt, Ag, Ti, V, and W." Applied optics, vol. 24, no. 24, pp. 4493-4499, 1985.

[348] C. A. Leal-Sevillano, J. A. Ruiz-Cruz, J. R. Montejo-Garai, and J. M. Rebollar, "Field propagation in circular hollow waveguides with non-ideal metallic conductors from microwaves to terahertz frequencies," IEEE Transactions on Microwave Theory and Techniques, vol. 59, no. 12, pp. 3013-3022, 2011.

[349] W. Chow, A. Champion, and D. Steenson, "Measurements to $320 \mathrm{GHz}$ of millimetre-wave waveguide components made by high precision and economic micro-machining techniques," in High Frequency Postgraduate Student Colloquium. IEEE, 2003, pp. 90-93.

[350] Y.-S. Liao and Y.-T. Chen, "Precision fabrication of an arrayed micro metal probe by the laser-LIGA process," Journal of Micromechanics and Microengineering, vol. 15, no. 12, p. 2433, 2005.

[351] C. H. Smith, X. HAIYONG, and N. S. Barker, "Development of a multilayer SU-8 process for terahertz frequency waveguide blocks," in IEEE MTT-S International Microwave Symposium Digest, vol. 1. Institute of Electrical and Electronics Engineers, 2005, pp. 439-442. 
[352] M. Mineo and C. Paoloni, "Corrugated rectangular waveguide tunable backward wave oscillator for terahertz applications," IEEE Transactions on Electron Devices, vol. 57, no. 6, pp. 1481-1484, 2010.

[353] X. Shang, M. J. Lancaster, M. Ke, and Y. Wang, "Measurements of micromachined submillimeter waveguide circuits," in 76th Microwave Measurement Symposium (ARFTG). IEEE, 2010, pp. 1-4.

[354] Z. Wang, "The influence of surface roughness on conductor at terahertz frequencies," Optik-International Journal for Light and Electron Optics, vol. 125, no. 13, pp. 3237-3240, 2014.

[355] Y. Zhou and S. Lucyszyn, "HFSS® modelling anomalies with THz metal-pipe rectangular waveguide structures at room temperature," Piers Online, vol. 5, no. 3, pp. 201-211, 2009.

[356] C. Leal-Sevillano, J. Ruiz-Cruz, J. Montejo-Garai, and J. Rebollar, "Simple models for the analysis of waveguiding systems at the terahertz band using classical microwave approaches," in 4th European Conference on Antennas and Propagation (EuCAP). IEEE, 2010, pp. 1-5.

[357] D. Sanchez-Escuderos, M. Ferrando-Bataller, J. I. Herranz, and M. BaqueroEscudero, "Optimization of the e-plane loaded rectangular waveguide for lowloss propagation," Progress In Electromagnetics Research, vol. 135, pp. 411433, 2013.

[358] Standard Rectangular Waveguide Dimensions. Microwaves101.com. (2016). [Online]. Available: http://www.microwaves101.com/encyclopedias/ rectangular-waveguide-dimensions

[359] Z. Wang, Y. Zhang, R. Xu, and W. Lin, "Investigation of terahertz wave propagation along shielded dielectric multiple-slot waveguide," Journal of Infrared, Millimeter, and Terahertz Waves, vol. 32, no. 2, pp. 204-215, 2011.

[360] P.-S. Kildal, "Three metamaterial-based gap waveguides between parallel metal plates for mm/submm waves," in 3rd European Conference on Antennas and Propagation (EuCAP), 2009, pp. 28-32.

[361] A. Berenguer, V. Fusco, D. E. Zelenchuk, D. Sánchez-Escuderos, M. BaqueroEscudero, and V. E. Boria-Esbert, "Propagation characteristics of groove gap waveguide below and above cutoff," IEEE Transactions on Microwave Theory and Techniques, vol. 64, no. 1, pp. 27-36, 2016.

[362] A. Wakatsuki, T. Furuta, Y. Muramoto, T. Yoshimatsu, and H. Ito, "Highpower and broadband sub-terahertz wave generation using a J-band photomixer module with rectangular-waveguide output port," in 33rd International Conference on Infrared, Millimeter and Terahertz Waves, IRMMWTHz. IEEE, 2008, pp. 1-2.

[363] Y. Koren, Computer control of manufacturing systems. McGraw-Hill New York et al., 1983. 
[364] X. Shang, M. Ke, Y. Wang, and M. Lancaster, "Micromachined W-band waveguide and filter with two embedded H-plane bends," IET Microwaves, Antennas \& Propagation, vol. 5, no. 3, pp. 334-339, 2011.

[365] P. Blondy, A. R. Brown, D. Cros, and G. M. Rebeiz, "Low-loss micromachined filters for millimeter-wave communication systems," IEEE Transactions on Microwave Theory and Techniques, vol. 46, no. 12, pp. 2283-2288, 1998.

[366] R. Arnaudov, B. Avdjiiski, A. Kostov, V. Videkov, S. Andreev, and N. Yordanov, "Novel microcontacts in microwave chip carriers developed by UVLIGA process," IEEE Transactions on Advanced Packaging, vol. 29, no. 1, pp. 122-130, 2006.

[367] R. Kishikawa, M. Horibe, and M. Shida, "Evaluation of complex residual error in vector network analyzer measurement system in the range of millimeterwave and submillimeter-wave frequencies," in 77th Microwave Measurement Conference (ARFTG). IEEE, 2011, pp. 1-7.

[368] D. F. Williams, "500 GHz-750 GHz Rectangular-Waveguide Vector-NetworkAnalyzer Calibrations," IEEE Transactions on Terahertz Science and Technology, vol. 1, no. 2, pp. 364-377, 2011.

[369] V. Desmaris, D. Dochev, D. Meledin, A. Pavolotsky, and V. Belitsky, "Waveguide packaging technology for thz components and systems," in Proceedings of 6th ESA Workshop on Millimetre-Wave Technology and Applications. IEEE, 2011.

[370] S. Liu, J. Hu, Y. Zhang, Z. Zheng, Y. Liu, R. Xu, and Q. Xue, "1 THz Micromachined Waveguide Band-Pass Filter," Journal of Infrared, Millimeter, and Terahertz Waves, pp. 1-13, 2015.

[371] C. D. Nordquist, M. C. Wanke, A. Rowen, C. L. Arrington, A. D. Grine, and C. T. Fuller, "Properties of surface metal micromachined rectangular waveguide operating near $3 \mathrm{THz}$," IEEE Journal of Selected Topics in Quantum Electronics, vol. 17, no. 1, pp. 130-137, 2011.

[372] D. Sanchez-Escuderos, M. Ferrando-Bataller, J. Herranz-Herruzo, and M. Baquero-Escudero, "Rectangular waveguide with low metallic losses on side walls at THz," in 35th International Conference on Infrared Millimeter and Terahertz Waves (IRMMW-THz). IEEE, 2010, pp. 1-2.

[373] D. Sanchez-Escuderos, M. Ferrando-Bataller, M. Baquero-Escudero, J. Herranz-Herruzo, and A. Berenguer, "H-plane horn array using lowloss rectangular waveguide at THz frequencies," in 4th European Conference on Antennas and Propagation (EuCAP). IEEE, 2010, pp. 452-456.

[374] Q. Tang, L. Zhou, Y.-P. Zhang, and J.-F. Mao, "Propagation characteristics of graphene-based rectangular waveguides in terahertz band," in 3rd AsiaPacific Conference on Antennas and Propagation (APCAP). IEEE, 2014, pp. $745-748$. 
[375] G. Shkerdin, H. Alkorre, H. Guoqiang, and J. Stiens, "Modified TE modes of metal waveguide with integrated graphene structure in the sub-terahertz frequency range," IET Microwaves, Antennas $\&$ Propagation, vol. 10, no. 6, pp. 692-699, 2016.

[376] C. Balanis, Advanced engineering electromagnetics. Wiley New York, 1989.

[377] M. Navarro-Cia, C. M. Bledt, M. S. Vitiello, H. E. Beere, D. A. Ritchie, J. A. Harrington, and O. Mitrofanov, "Modes in silver-iodide-lined hollow metallic waveguides mapped by terahertz near-field time-domain microscopy," JOSA $B$, vol. 30, no. 1, pp. 127-135, 2013.

[378] H.-S. Yang, J. Ma, and Z.-Z. Lu, "Circular groove guide for short millimeter and submillimeter waves," IEEE Transactions on Microwave Theory and Techniques, vol. 43, no. 2, pp. 324-330, 1995.

[379] F. Tischer, "The groove guide, a low-loss waveguide for millimeter waves," IEEE Transactions on Microwave Theory and Techniques, vol. 11, no. 5, pp. 291-296, 1963.

[380] R. McGowan, G. Gallot, and D. Grischkowsky, "Propagation of ultrawideband short pulses of terahertz radiation through submillimeter-diameter circular waveguides," Optics letters, vol. 24, no. 20, pp. 1431-1433, 1999.

[381] J. Harrington, R. George, P. Pedersen, and E. Mueller, "Hollow polycarbonate waveguides with inner $\mathrm{Cu}$ coatings for delivery of terahertz radiation," Opt. Express, vol. 12, no. 21, pp. 5263-5268, 2004.

[382] B. Bowden, J. Harrington, and O. Mitrofanov, "Silver/polystyrene-coated hollow glass waveguides for the transmission of terahertz radiation," Optics letters, vol. 32, no. 20, pp. 2945-2947, 2007.

[383] — - "Low-loss modes in hollow metallic terahertz waveguides with dielectric coatings," Applied physics letters, vol. 93, p. 181104, 2008.

[384] L. Hao, M. Ji, J. Yuan, D. An, M. Li, X. Zhou, Y. Huang, H. Sun, Q. Zhu, F. Rudau et al., "Compact Superconducting Terahertz Source Operating in Liquid Nitrogen," Physical Review Applied, vol. 3, no. 2, p. 024006, 2015.

[385] T. Ito, Y. Matsuura, M. Miyagi, H. Minamide, and H. Ito, "Flexible terahertz fiber optics with low bend-induced losses," JOSA B, vol. 24, no. 5, pp. 1230$1235,2007$.

[386] Y. Matsuura and E. Takeda, "Hollow optical fibers loaded with an inner dielectric film for terahertz broadband spectroscopy," JOSA B, vol. 25, no. 12, pp. 1949-1954, 2008.

[387] J. Tucek, D. Gallagher, K. Kreischer, and R. Mihailovich, "A compact, high power, $0.65 \mathrm{THz}$ source," in IEEE International Vacuum Electronics Conference, IVEC 2008. IEEE, 2008, pp. 16-17. 
[388] H. Wang, C. He, and W. Liu, "Corrugated cylindrical waveguide with an axial magnetized degenerate plasma rod for terahertz vacuum devices," in IEEE International Vacuum Electronics Conference (IVEC). IEEE, 2015, pp. 1-2.

[389] W. Zhang, E. Brown, M. Rahman, and M. Norton, "Observation of terahertz absorption signatures in microliter DNA solutions," Applied Physics Letters, vol. 102, no. 2, p. 023701, 2013.

[390] E. C. Jameson, Electrical discharge machining. Society of Manufacturing Engineers, 2001.

[391] Y. Huang, K. Chu, and M. Thumm, "Self-consistent modeling of terahertz waveguide and cavity with frequency-dependent conductivity," Physics of Plasmas (1994-present), vol. 22, no. 1, p. 013108, 2015.

[392] I. Llamas-Garro, M. Lancaster, and P. Hall, "Air-filled square coaxial transmission line and its use in microwave filters," in IEE Proceedings Microwaves, Antennas and Propagation, vol. 152, no. 3. IET, 2005, pp. 155-159.

[393] X. Lu, J. Han, and W. Zhang, "Transmission field enhancement of terahertz pulses in plasmonic, rectangular coaxial geometries," Optics letters, vol. 35, no. 7 , pp. 904-906, 2010.

[394] N. Talebi and M. Shahabadi, "Spoof surface plasmons propagating along a periodically corrugated coaxial waveguide," Journal of Physics D: Applied Physics, vol. 43, no. 13, p. 135302, 2010.

[395] M. Bieler, M. Spitzer, H. Lecher, G. Hein, and U. Siegner, "Transfer of sub-5 ps electrical test pulses to coplanar and coaxial electronic devices," Electronics Letters, vol. 38, no. 3, p. 1, 2002.

[396] T. Jeon and D. Grischkowsky, "Direct optoelectronic generation and detection of sub-ps-electrical pulses on sub-mm-coaxial transmission lines," Applied physics letters, vol. 85, p. 6092, 2004.

[397] D. M. Mittleman and K. Wang, "Method for coupling terahertz pulses into a coaxial waveguide," Nov. 3 2015, uS Patent 9,178,282.

[398] X. Wang, W. Xiong, W. Sun, and Y. Zhang, "Coaxial waveguide mode reconstruction and analysis with THz digital holography," Optics express, vol. 20, no. 7, pp. 7706-7715, 2012.

[399] X.-s. YUAN, Y. YAN, and S.-g. LIU, "Theoretical Study on a Large Radius Coaxial Cavity Terahertz Gyrotron [J]," Acta Electronica Sinica, vol. 2, p. 016, 2009 .

[400] K. Gupta, R. Garg, I. Bahl, and P. Bhartia, Microstrip lines and slotlines. Artech House Norwood, MA, 1996.

[401] L. Cao, A.-S. Grimault-Jacquin, and F. Aniel, "Comparison and Optimization of Dispersion, and Losses of Planar Waveguides on Benzocyclobutene (BCB) at $\mathrm{THz}$ Frequencies: Coplanar Waveguide (CPW), Microstrip, Stripline and Slotline," Progress In Electromagnetics Research B, vol. 56, pp. 161-183, 2013. 
[402] E. Perret, N. Zerounian, S. David, and F. Aniel, "Complex permittivity characterization of benzocyclobutene for terahertz applications," Microelectronic engineering, vol. 85, no. 11, pp. 2276-2281, 2008.

[403] A. Kumar and M. V. Kartikeyan, "A Design of a Terahertz Microstrip Bandstop Filter with Defected Ground Structure," Active and Passive Electronic Components, vol. 2013, 2013.

[404] A. G. Engel Jr and L. P. Katehi, "Low-loss monolithic transmission lines for submillimeter and terahertz frequency applications," IEEE Transactions on Microwave Theory and Techniques, vol. 39, no. 11, pp. 1847-1854, 1991.

[405] H. Roskos, M. C. Nuss, K. W. Goossen, D. W. Kisker, A. E. White, K. T. Short, D. C. Jacobson, and J. M. Poate, "Propagation of picosecond electrical pulses on a silicon-based microstrip line with buried cobalt silicide ground plane," Applied physics letters, vol. 58, no. 23, pp. 2604-2606, 1991.

[406] H.-M. Heiliger, M. Nagel, H. Roskos, H. Kurz, F. Schnieder, W. Heinrich, R. Hey, and K. Ploog, "Low-dispersion thin-film microstrip lines with cyclotene (benzocyclobutene) as dielectric medium," Applied physics letters, vol. 70, no. 17, pp. 2233-2235, 1997.

[407] W. Ganzevles, J. Gao, P. de Korte, and T. Klapwijk, "Direct response of microstrip line coupled Nb THz hot-electron bolometer mixers," Applied Physics Letters, vol. 79, no. 15, pp. 2483-2485, 2001.

[408] M. Byrne, J. Cunningham, K. Tych, A. Burnett, M. Stringer, C. Wood, L. Dazhang, M. Lachab, E. Linfield, and A. Davies, "Terahertz vibrational absorption spectroscopy using microstrip-line waveguides," Applied Physics Letters, vol. 93, no. 18, p. 182904, 2008.

[409] L. Cao, A.-S. Grimault-Jacquin, N. Zerounian, and F. Aniel, "Design and VNA-measurement of coplanar waveguide (CPW) on benzocyclobutene (BCB) at THz frequencies," Infrared Physics \& Technology, vol. 63, pp. 157-164, 2014.

[410] D. Ahn, J.-S. Park, C.-S. Kim, J. Kim, Y. Qian, and T. Itoh, "A design of the low-pass filter using the novel microstrip defected ground structure," IEEE Transactions on Microwave Theory and Techniques, vol. 49, no. 1, pp. 86-93, 2001.

[411] U. Ansys Corporation Suite, v13 Pittsburg (PA), "HFSS (high frequency structural simulator)," 2017, http://www.ansys.com/products/electronics/ansyshfss.

[412] D. Systemes, "CST (computer simulation technology)," 2017, https://www.cst.com/.

[413] K. Technologies, "ADS (advanced design simulator)," 2017, http://www.keysight.com. 
[414] X. Shen, T. Jiang, Y. Liu, X. Tang, and G. Chen, "Suspended microstrip line covered with dielectric in the ground plate for THz transmission," in 3rd AsiaPacific Conference on Antennas and Propagation (APCAP). IEEE, 2014, pp. 201-202.

[415] D. R. Grischkowsky, "Optoelectronic characterization of transmission lines and waveguides by terahertz time-domain spectroscopy," IEEE Journal of Selected Topics in Quantum Electronics, vol. 6, no. 6, pp. 1122-1135, 2000.

[416] M. Y. Frankel, S. Gupta, J. A. Valdmanis, and G. A. Mourou, "Terahertz attenuation and dispersion characteristics of coplanar transmission lines," IEEE Transactions on Microwave Theory and Techniques, vol. 39, no. 6, pp. 910-916, 1991.

[417] H.-J. Cheng, J. F. Whitaker, T. M. Weller, and L. P. Katehi, "Terahertzbandwidth characteristics of coplanar transmission lines on low permittivity substrates," IEEE Transactions on Microwave Theory and Techniques, vol. 42, no. 12, pp. 2399-2406, 1994.

[418] A.-S. Grimault-Jacquin, B. Tissafi, E. Perret, and F. Aniel, "Consideration to minimize losses in terahertz coplanar waveguide on indium phosphide," Microwave and Optical Technology Letters, vol. 54, no. 1, pp. 213-219, 2012.

[419] A. Endo, S. Yates, J. Bueno, D. Thoen, V. Murugesan, A. Baryshev, T. Klapwijk, P. Werf, and J. Baselmans, "Superconducting Coplanar Waveguide Filters for Submillimeter Wave On-Chip Filterbank Spectrometers," Journal of Low Temperature Physics, vol. 184, no. 1-2, pp. 412-417, 2016.

[420] R. Mendis and D. Grischkowsky, "Thz interconnect with low-loss and lowgroup velocity dispersion," IEEE Microwave and Wireless Components Letters, vol. 11, no. 11, pp. 444-446, 2001.

[421] —, "Undistorted guided-wave propagation of subpicosecond terahertz pulses," Optics letters, vol. 26, no. 11, pp. 846-848, 2001.

[422] S. Coleman and D. Grischkowsky, "Parallel plate THz transmitter," Applied physics letters, vol. 84, p. 654, 2004.

[423] M. Wächter, M. Nagel, and H. Kurz, "Metallic slit waveguide for dispersionfree low-loss terahertz signal transmission," Applied physics letters, vol. 90, p. 061111, 2007.

[424] R. Mendis and D. M. Mittleman, "An investigation of the lowest-order transverse-electric (TE 1) mode of the parallel-plate waveguide for $\mathrm{THz}$ pulse propagation," JOSA B, vol. 26, no. 9, pp. A6-A13, 2009.

[425] A. Bingham, Y. Zhao, and D. Grischkowsky, "Thz parallel plate photonic waveguides," Applied Physics Letters, vol. 87, p. 051101, 2005.

[426] E. S. Lee, J.-K. So, G.-S. Park, D. Kim, C.-S. Kee, and T.-I. Jeon, "Terahertz band gaps induced by metal grooves inside parallel-plate waveguides," Optics express, vol. 20, no. 6, pp. 6116-6123, 2012. 
[427] A. Malekabadi, S. A. Charlebois, and D. Deslandes, "Parallel plate waveguide with anisotropic graphene plates: Effect of electric and magnetic biases," Journal of Applied Physics, vol. 113, no. 11, p. 113708, 2013.

[428] M. Razanoelina, I. Kawayama, X. Zhang, R. Vajtai, J. Kono, D. Mittleman, M. Tonouchi et al., "Terahertz Parallel Plate Waveguide to Evaluate Electrical Transport Properties of 2D Materials," in CLEO: Science and Innovations. Optical Society of America, 2016, pp. STh4I-6.

[429] M. Razanoelina, F. R. Bagsican, I. Kawayama, X. Zhang, L. Ma, H. Murakami, R. Vajtai, P. M. Ajayan, J. Kono, D. M. Mittleman et al., "Parallel plate waveguide time domain spectroscopy to study terahertz conductivity of utltrathin materials," in SPIE Commercial+ Scientific Sensing and Imaging. International Society for Optics and Photonics, 2016, pp. 985 604-985604.

[430] L. Novotny and C. Hafner, "Light propagation in a cylindrical waveguide with a complex, metallic, dielectric function," Physical review E, vol. 50, no. 5, p. 4094, 1994.

[431] E. Snitzer, "Cylindrical dielectric waveguide modes," JOSA, vol. 51, no. 5, pp. 491-498, 1961.

[432] K. Kao and G. Hockham, "Dielectric-fiber Surface Waveguides for Optical Fiber," Proc. IEE, vol. 133, p. 1151, 1966.

[433] S. Atakaramians, S. Afshar, T. M. Monro, and D. Abbott, "Terahertz dielectric waveguides," Advances in Optics and Photonics, vol. 5, no. 2, pp. 169-215, 2013.

[434] S. R. Andrews, "Microstructured terahertz waveguides," Journal of Physics D: Applied Physics, vol. 47, no. 37, p. 374004, 2014.

[435] K. Benaissa and A. Nathan, "Silicon anti-resonant reflecting optical waveguides for sensor applications," Sensors and Actuators A: Physical, vol. 65, no. 1, pp. 33-44, 1998.

[436] S. Jamison, R. McGowan, and D. Grischkowsky, "Single-mode waveguide propagation and reshaping of sub-ps terahertz pulses in sapphire fibers," Applied Physics Letters, vol. 76, no. 15, pp. 1987-1989, 2000.

[437] P. Bolivar, M. Brucherseifer, J. Rivas, R. Gonzalo, I. Ederra, A. Reynolds, M. Holker, and P. de Maagt, "Measurement of the dielectric constant and loss tangent of high dielectric-constant materials at terahertz frequencies," IEEE Transactions on Microwave Theory and Techniques, vol. 51, no. 4, pp. 10621066, 2003.

[438] M. Nagel, A. Marchewka, and H. Kurz, "Low-index discontinuity terahertz waveguides," Optics express, vol. 14, no. 21, pp. 9944-9954, 2006. 
[439] A. Berenguer, M. Baquero-Escudero, D. Sánchez-Escuderos, and M. FerrandoBataller, "Reduction of High-Order Modes Coupling on Bends in the Dielectric-Coated Single Wire Waveguide," in 6th European Conference on Antennas and Propagation (EuCAP), 2012.

[440] A. Berenguer, M. Baquero-Escudero, D. Sanchez Escuderos, and M. FerrandoBataller, "Study of surface waveguides at THz frequencies," in XXVI Simposium Nacional de la Unión Científica Internacional de Radio (URSI), 2011. IEEE, 2011.

[441] A. Berenguer, M. Baquero-Escudero, D. Sánchez-Escuderos, and M. FerrandoBataller, "Study of the effect of coating the single wire waveguide with a dielectric," in 36th International Conference on Infrared Millimeter and Terahertz Waves (IRMMW-THz), 2011, pp. 1-2.

[442] L.-J. Chen, H.-W. Chen, T.-F. Kao, J.-Y. Lu, and C.-K. Sun, "Low-loss subwavelength plastic fiber for terahertz waveguiding," Optics Letters, vol. 31, no. 3, pp. 308-310, 2006.

[443] V. R. Almeida, Q. Xu, C. A. Barrios, and M. Lipson, "Guiding and confining light in void nanostructure," Optics letters, vol. 29, no. 11, pp. 1209-1211, 2004.

[444] Q. Xu, V. R. Almeida, R. R. Panepucci, and M. Lipson, "Experimental demonstration of guiding and confining light in nanometer-size low-refractive-index material," Optics letters, vol. 29, no. 14, pp. 1626-1628, 2004.

[445] D. Grischkowsky, S. Keiding, M. Van Exter, and C. Fattinger, "Far-infrared time-domain spectroscopy with terahertz beams of dielectrics and semiconductors," JOSA B, vol. 7, no. 10, pp. 2006-2015, 1990.

[446] C.-H. Lai, Y.-C. Hsueh, H.-W. Chen, Y.-j. Huang, H.-c. Chang, and C.-K. Sun, "Low-index terahertz pipe waveguides," Optics letters, vol. 34, no. 21, pp. 3457-3459, 2009.

[447] H. Schmidt, D. Yin, J. P. Barber, and A. R. Hawkins, "Hollow-core waveguides and 2-D waveguide arrays for integrated optics of gases and liquids," Selected Topics in Quantum Electronics, IEEE Journal of, vol. 11, no. 2, pp. 519-527, 2005.

[448] S. Atakaramians, S. A. Vahid, B. M. Fischer, D. Abbott, and T. M. Monro, "Loss mechanisms for T-ray microwires," in Joint 32st International IEEE Conference on Infrared and Millimeter Waves and 15th International Conference on Terahertz Electronics, 2007, pp. 811-812.

[449] J.-T. Lu, Y.-C. Hsueh, Y.-R. Huang, Y.-J. Hwang, and C.-K. Sun, "Bending loss of terahertz pipe waveguides," Optics express, vol. 18, no. 25, pp. $26332-$ $26338,2010$.

[450] H. Bao, K. Nielsen, O. Bang, and P. U. Jepsen, "Dielectric tube waveguides with absorptive cladding for broadband, low-dispersion and low loss THz guiding," Scientific reports, vol. 5, 2015. 
[451] S. Gross, D. Camozzo, V. Di Noto, L. Armelao, and E. Tondello, "PMMA: A key macromolecular component for dielectric low- $\kappa$ hybrid inorganic-organic polymer films," European polymer journal, vol. 43, no. 3, pp. 673-696, 2007.

[452] A. A. Danylov, J. Waldman, T. M. Goyette, A. J. Gatesman, R. H. Giles, K. J. Linden, W. R. Neal, W. E. Nixon, M. C. Wanke, and J. L. Reno, "Transformation of the multimode terahertz quantum cascade laser beam into a gaussian, using a hollow dielectric waveguide," Applied Optics, vol. 46, no. 22, pp. 5051$5055,2007$.

[453] J. P. Pousi, D. V. Lioubtchenko, S. N. Dudorov, and A. V. Räisänen, "High permittivity dielectric rod waveguide as an antenna array element for millimeter waves," IEEE Transactions on Antennas and Propagation, vol. 58, no. 3, pp. 714-719, 2010.

[454] D. W. Vogt, J. Anthony, and R. Leonhardt, "Metallic and 3D-printed dielectric helical terahertz waveguides," Optics Express, vol. 23, no. 26, pp. 33359 $33369,2015$.

[455] S. Atakaramians, S. Afshar, B. M. Fischer, D. Abbott, and T. M. Monro, "Porous fibers: a novel approach to low loss THz waveguides," Optics Express, vol. 16, no. 12, pp. 8845-8854, 2008.

[456] J.-Y. Lu, C.-P. Yu, H.-C. Chang, H.-W. Chen, Y.-T. Li, C.-L. Pan, and C.K. Sun, "Terahertz air-core microstructure fiber," Applied Physics Letters, vol. 92 , no. 6, p. 064105, 2008.

[457] A. Hassani, A. Dupuis, and M. Skorobogatiy, "Porous polymer fibers for lowloss Terahertz guiding," Optics express, vol. 16, no. 9, pp. 6340-6351, 2008.

[458] A. Dupuis, J.-F. Allard, D. Morris, K. Stoeffler, C. Dubois, and M. Skorobogatiy, "Fabrication and $\mathrm{THz}$ loss measurements of porous subwavelength fibers using a directional coupler method," Optics express, vol. 17, no. 10, pp. 8012$8028,2009$.

[459] S. Atakaramians, S. Afshar, H. Ebendorff-Heidepriem, M. Nagel, B. M. Fischer, D. Abbott, and T. M. Monro, "THz porous fibers: design, fabrication and experimental characterization," Optics express, vol. 17, no. 16, pp. 14053 $14062,2009$.

[460] C. A. Volkert and A. M. Minor, "Focused ion beam microscopy and micromachining," MRS bulletin, vol. 32, no. 05, pp. 389-399, 2007.

[461] S. Ali, N. Ahmed, S. Aljunid, and B. Ahmad, "Ultra-flat low material loss porous core THz waveguide with near zero flat dispersion," Electronics Letters, vol. 52, no. 10, pp. 863-865, 2016.

[462] A. Sengupta, A. Bandyopadhyay, B. Bowden, J. Harrington, and J. Federici, "Characterisation of olefin copolymers using terahertz spectroscopy," Electron. Lett, vol. 42, no. 25, pp. 1477-1479, 2006. 
[463] R. Mendis and D. Grischkowsky, "Plastic ribbon THz waveguides," Journal of Applied Physics, vol. 88, no. 7, pp. 4449-4451, 2000.

[464] C. G. Poulton, C. Koos, M. Fujii, A. Pfrang, T. Schimmel, J. Leuthold, and W. Freude, "Radiation modes and roughness loss in high index-contrast waveguides," IEEE Journal of selected topics in quantum electronics, vol. 12, no. 6 , pp. 1306-1321, 2006.

[465] R. A. Soref, S. J. Emelett, and W. R. Buchwald, "Silicon waveguided components for the long-wave infrared region," Journal of Optics A: Pure and Applied Optics, vol. 8, no. 10, p. 840, 2006.

[466] N. Ranjkesh, M. Basha, A. Taeb, and S. Safavi-Naeini, "Silicon-on-glass dielectric waveguide, Part II: For THz applications," IEEE Transactions on Terahertz Science and Technology, vol. 5, no. 2, pp. 280-287, 2015.

[467] L. Brillouin, Wave propagation in periodic structures: electric filters and crystal lattices. Courier Corporation, 2003.

[468] E. Yablonovitch, "Inhibited spontaneous emission in solid-state physics and electronics," Physical review letters, vol. 58, no. 20, p. 2059, 1987.

[469] —- "Photonic band-gap structures," JOSA B, vol. 10, no. 2, pp. 283-295, 1993.

[470] R.-J. Yu, B. Zhang, Y.-Q. Zhang, C.-Q. Wu, Z.-G. Tian, and X.-Z. Bai, "Proposal for ultralow loss hollow-core plastic Bragg fiber with cobweb-structured cladding for terahertz waveguiding," IEEE Photonics Technology Letters, vol. 19, no. 12, pp. 910-912, 2007.

[471] P. Yeh, A. Yariv, and E. Marom, "Theory of Bragg fiber," JOSA, vol. 68, no. 9, pp. 1196-1201, 1978.

[472] K. Nielsen, H. K. Rasmussen, A. J. Adam, P. C. Planken, O. Bang, and P. U. Jepsen, "Bendable, low-loss topas fibers for the terahertz frequency range," Optics Express, vol. 17, no. 10, pp. 8592-8601, 2009.

[473] A. Dupuis, K. Stoeffler, B. Ung, C. Dubois, and M. Skorobogatiy, "Transmission measurements of hollow-core THz Bragg fibers," JOSA B, vol. 28, no. 4, pp. 896-907, 2011.

[474] M. I. Hasan, S. A. Razzak, G. Hasanuzzaman, and M. S. Habib, "Ultra-low material loss and dispersion flattened fiber for THz transmission," IEEE Photonics Technology Letters, vol. 26, no. 23, pp. 2372-2375, 2014.

[475] R. Islam, G. Hasanuzzaman, M. S. Habib, S. Rana, and M. Khan, "Low-loss rotated porous core hexagonal single-mode fiber in THz regime," Optical Fiber Technology, vol. 24, pp. 38-43, 2015.

[476] W. Xing, J. Bai, and Y. Li, "Mode classification and calculation in all-solid photonic bandgap fibers," Journal of Lightwave Technology, vol. 30, no. 6, pp. 821-828, 2012. 
[477] D. Sánchez-Escuderos, M. Ferrando-Bataller, J. Herranz-Herruzo, M. BaqueroEscudero, and A. Berenguer Verdu, "H-plane horn array antenna using lowloss rectangular waveguide at THz frequencies," in 5th European Conference on Antennas and Propagation (EuCAP), 2011.

[478] D. Sánchez-Escuderos, M. Ferrando-Bataller, M. Baquero-Escudero, and J. Herranz, "EBG structures for antenna design at thz frequencies," in IEEE Antennas and Propagation Society International Symposium, July 2011.

[479] D. Sánchez-Escuderos, M. Ferrando-Bataller, A. Berenguer, and M. BaqueroEscudero, "Application of EBG structures for the design of low-loss rectangular waveguides in the THz band," in Simposium Nacional de la URSI 2011, Leganés (Madrid), 2011.

[480] — - "Design of low-loss waveguides and devices at $\mathrm{THz}$ frequencies using EBG structures," in 36th International Conference on Infrared, Millimeter, and Terahertz Waves (IRMMW-THz), 2011.

[481] D. Sanchez-Escuderos, M. Ferrando-Bataller, A. Berenguer, and M. BaqueroEscudero, "Design of an H-plane horn array antenna using the complete 1D/3D-EBG waveguide in the THz band," in 6th European Conference on Antennas and Propagation (EUCAP). IEEE, 2012, pp. 2343-2347.

[482] H. Sözüer and J. P. Dowling, "Photonic band calculations for woodpile structures," Journal of Modern Optics, vol. 41, no. 2, pp. 231-239, 1994.

[483] I. Ederra, I. Khromova, R. Gonzalo, N. Delhote, D. Baillargeat, A. Murk, B. E. Alderman, and P. De Maagt, "Electromagnetic-bandgap waveguide for the millimeter range," IEEE Transactions on Microwave Theory and Techniques, vol. 58, no. 7, pp. 1734-1741, 2010.

[484] E. Degirmenci, F. Surre, S. Philippe, R. Maldonado-Basilio, and P. Landais, "Improved bend waveguide design for terahertz transmission," IEEE Transactions on Terahertz Science and Technology, vol. 2, no. 1, pp. 137-143, 2012.

[485] K. Tsuruda, M. Fujita, and T. Nagatsuma, "Extremely low-loss terahertz waveguide based on silicon photonic-crystal slab," Optics express, vol. 23, no. 25 , pp. 31977-31990, 2015.

[486] E. Ozbay, "Plasmonics: merging photonics and electronics at nanoscale dimensions," science, vol. 311, no. 5758, pp. 189-193, 2006.

[487] A. Sommerfeld, "Über die fortpflanzung elektrodynamischer wellen längs eines drahtes," Annalen der Physik, vol. 303, no. 2, pp. 233-290, 1899.

[488] — , "An oscillating dipole above a finitely conducting plane," Ann. der Physik, vol. 28, p. 665, 1909.

[489] J. Stratton, Electromagnetic theory. McGraw-Hill, New York, 1941.

[490] G. Goubau, "Surface waves and their application to transmission lines," Journal of Applied Physics, vol. 21, p. 1119, 1950. 
[491] T. Rao and M. Hamid, "Propagation characteristics of a dielectric-coated conducting surface-wave transmission line with an intervening airgap," Electrical Engineers, Proceedings of the Institution of, vol. 123, no. 10, pp. 973-980, 1976.

[492] K. Wang and D. Mittleman, "Metal wires for terahertz wave guiding," Nature, vol. 432, no. 7015, pp. 376-379, 2004.

[493] H. Cao and A. Nahata, "Coupling of terahertz pulses onto a single metal wire waveguide using milled grooves," Optics Express, vol. 13, no. 18, pp. 7028$7034,2005$.

[494] J. C. Wiltse, "Low-loss surface-wave propagation on coated or uncoated cylindrical conductor from 0.1 to $1 \mathrm{THz}$," in Antennas and Propagation Society International Symposium. IEEE, 2007, pp. 4657-4660.

[495] J. Deibel, K. Wang, M. Escarra, and D. Mittleman, "Enhanced coupling of terahertz radiation to cylindrical wire waveguides," Optics express, vol. 14, no. 1, pp. 279-290, 2006.

[496] J. Zenneck, "Über die Fortpflanzung ebener elektromagnetischer Wellen längs einer ebenen Leiterfläche und ihre Beziehung zur drahtlosen Telegraphie," Annalen der Physik, vol. 328, no. 10, pp. 846-866, 1907.

[497] G. Goubau, "Single-conductor surface-wave transmission lines," Proceedings of the IRE, vol. 39, no. 6, pp. 619-624, 1951.

[498] — "On the excitation of surface waves," Proceedings of the IRE, vol. 7, no. 40, pp. 865-868, 1952.

[499] —-, "Open wire lines," IRE Transactions on Microwave Theory and Techniques, vol. 4, no. 4, pp. 197-200, 1956.

[500] M. King and J. Wiltse, "Surface-wave propagation on coated or uncoated metal wires at millimeter wavelengths," IEEE Transactions on Antennas and Propagation, vol. 10, pp. 246-254, 1962.

[501] J. Chiba, "Experimental Studies of the Losses and Radiations Due to Bends in the Goubau Line," IEEE Transactions on Microwave Theory and Techniques, vol. 25, no. 2, pp. 94-100, 1977.

[502] H. Rao, "Mode spectrum of the Modified Goubau Line," Proc. IEE, pp. 122732, 1979 .

[503] J. Fikioris and J. Roumeliotis, "Cutoff wavenumbers of Goubau lines," IEEE Transactions on Microwave Theory and Techniques, vol. 27, no. 6, pp. 570-573, 1979.

[504] R. Harman and A. Gagnon, "Cable for use as a distributed antenna," Dec. 5 1995, US Patent 5,473,336. 
[505] K. Alonso and M. J. Hagmann, "Simulations of tapered Goubau line for coupling microwave signals generated by resonant laser-assisted field emission," Journal of Vacuum Science \& Technology B, vol. 18, no. 2, pp. 1009-1013, 2000 .

[506] T. Jeon, J. Zhang, and D. Grischkowsky, "THz Sommerfeld wave propagation on a single metal wire," Applied Physics Letters, vol. 86, p. 161904, 2005.

[507] M. Wätchter, M. Nagel, and H. Kurz, "Frequency-dependent characterization of $\mathrm{THz}$ Sommerfeld wave propagation on single-wires," Optics Express, vol. 13, no. 26, pp. $10815-10822,2005$.

[508] M. Walther, M. Freeman, and F. Hegmann, "Metal-wire terahertz time-domain spectroscopy," Applied Physics Letters, vol. 87, p. 261107, 2005.

[509] K. Wang and D. Mittleman, "Guided propagation of terahertz pulses on metal wires," Journal of the Optical Society of America B, vol. 22, no. 9, pp. 20012008, 2005.

[510] S. Maier, S. Andrews, L. Martin-Moreno, and F. Garcia-Vidal, "Terahertz surface plasmon-polariton propagation and focusing on periodically corrugated metal wires," Physical review letters, vol. 97, no. 17, p. 176805, 2006.

[511] Z. Wang, Y. Zhang, R.-M. Xu, and W. Lin, "A novel broadband coaxial probe to conical wire transition at $\mathrm{THz}$ frequency," Progress In Electromagnetics Research, vol. 16, pp. 31-45, 2011.

[512] K. Wang and D. Mittleman, "Dispersion of surface plasmon polaritons on metal wires in the terahertz frequency range," Physical review letters, vol. 96, no. 15, p. 157401, 2006.

[513] Y. B. Ji, E. S. Lee, J. S. Jang, and T.-I. Jeon, "Enhancement of the detection of THz Sommerfeld wave using a conical wire waveguide," Optics express, vol. 16, no. 1, pp. 271-278, 2008.

[514] V. Astley, R. Mendis, and D. Mittleman, "Characterization of terahertz field confinement at the end of a tapered metal wire waveguide," Applied Physics Letters, vol. 95, p. 031104, 2009.

[515] X.-Y. He, "Investigation of terahertz sommerfeld wave propagation along conical metal wire," JOSA B, vol. 26, no. 9, pp. A23-A28, 2009.

[516] X. He, "Investigation of terahertz surface waves of a metallic nanowire," JOSA $B$, vol. 27, no. 11, pp. 2298-2303, 2010.

[517] V. Astley, J. Scheiman, R. Mendis, and D. M. Mittleman, "Bending and coupling losses in terahertz wire waveguides," Optics letters, vol. 35, no. 4, pp. 553-555, 2010.

[518] J.-M. Liu, H.-W. Liang, M. Zhang, and H. Su, "Coupling of Sommerfeld waves using odd TM mode of double-dielectric-slab waveguide," Journal of Optics, vol. 44, no. 1, pp. 53-58, 2015. 
[519] Y. Xu and R. Bosisio, "Study of Goubau lines for submillimetre wave and terahertz frequency applications," in Microwaves, Antennas and Propagation, IEE Proceedings-, vol. 151, no. 5. IET, 2004, pp. 460-464.

[520] Y. Chen, Z. Song, Y. Li, M. Hu, Q. Xing, Z. Zhang, L. Chai, and C. Wang, "Effective surface plasmon polaritons on the metal wire with arrays of subwavelength grooves," Optics Express, vol. 14, no. 26, pp. 13 021-13 029, 2006.

[521] P. Overfelt, K. Halterman, S. Feng, and D. Bowling, "Mode bifurcation and fold points of complex dispersion curves for the metamaterial goubau line," arXiv preprint arXiv:0909.0535, 2009.

[522] Z. Wang, Y. Zhang, R. Xu, and W. Lin, "Accurate calculation of terahertz attenuation of a dielectric-coated metal wire with an intervening air gap using the engineering approach," Optics Communications, vol. 284, no. 16, pp. 39903995, 2011.

[523] P. Padhy, P. K. Sahu, and R. Jha, "Metal wire waveguide based all plasmonic refractive index sensor for terahertz frequencies," Sensors and Actuators B: Chemical, vol. 225, pp. 115-120, 2016.

[524] J. Yang, Q. Cao, and C. Zhou, "An explicit formula for metal wire plasmon of terahertz wave," Optics Express, vol. 17, no. 23, pp. 20 806-20 815, 2009.

[525] M. Mbonye, R. Mendis, and D. Mittleman, "A terahertz two-wire waveguide with low bending loss," Applied Physics Letters, vol. 95, p. 233506, 2009.

[526] H. Pahlevaninezhad, T. Darcie, and B. Heshmat, "Two-wire waveguide for terahertz," Optics Express, vol. 18, no. 7, pp. 7415-7420, 2010.

[527] H. Pahlevaninezhad and T. Darcie, "Coupling of terahertz waves to a two-wire waveguide," Optics Express, vol. 18, no. 22, pp. 22 614-22 624, 2010.

[528] W. H. Press, B. P. Flannery, W. T. Teukolsky, and W. T. Vetterling, Numerical recipes in $C$, the art of scientific computing. Cambridge University Press, New York, 1988.

[529] A. Markov and M. Skorobogatiy, "Two-wire terahertz fibers with porous dielectric support," Optics express, vol. 21, no. 10, pp. 12 728-12 743, 2013.

[530] A. Mazhorova, M. Mridha, M. Daneau, M. Clerici, M. Peccianti, P.-L. Lavertu, X. Ropagnol, F. Vidal, and R. Morandotti, "Low Dispersion Propagation of Broad-Band THz Pulses in a Two-Wire Waveguide," in Nano-Structures for Optics and Photonics. Springer, 2015, pp. 533-535.

[531] C. Yeh, F. Shimabukuro, and P. Siegel, "Low-loss terahertz ribbon waveguides," Applied optics, vol. 44, no. 28, p. 5937, 2005.

[532] T. Akalin, A. Treizebré, and B. Bocquet, "Single-wire transmission lines at terahertz frequencies," IEEE Transactions on Microwave Theory and Techniques, vol. 54, no. 6, pp. 2762-2767, 2006. 
[533] A. Treizebré, B. Bocquet, Y. Xu, and R. Bosisio, "New THz excitation of planar Goubau line," Microwave and Optical Technology Letters, vol. 50, no. 11, pp. 2998-3001, 2008.

[534] T.-I. Jeon and D. Grischkowsky, "THz Zenneck surface wave ( $\mathrm{THz}$ surface plasmon) propagation on a metal sheet," Applied physics letters, vol. 88, no. 6, p. 061113, 2006.

[535] M. Gong, T. Jeon, and D. Grischkowsky, "THz surface wave collapse on coated metal surfaces," Opt. Express, vol. 17, no. 19, pp. 17 088-17 101, 2009.

[536] X. Li, J. Song, and J. X. Zhang, "Design of terahertz metal-dielectric-metal waveguide with microfluidic sensing stub," Optics Communications, vol. 361, pp. 130-137, 2016.

[537] R. DeglInnocenti, Y. D. Shah, R. Wallis, A. Klimont, Y. Ren, D. S. Jessop, H. E. Beere, and D. A. Ritchie, "A hybrid plasmonic waveguide terahertz quantum cascade laser," Applied Physics Letters, vol. 106, no. 8, p. 082101, 2015 .

[538] C. Yeh, "Boundary conditions in electromagnetics," Physical Review E, vol. 48, no. 2, p. 1426, 1993.

[539] C. Gray, "Multipole expansions of electromagnetic fields using Debye potentials," American Journal of Physics, vol. 46, no. 2, pp. 169-179, 1978.

[540] D. Kajfez and P. Guillon, Dielectric resonators. MA, Artech House, Inc, 1986.

[541] A. Kapoor and G. Singh, "Mode classification in cylindrical dielectric waveguides," Journal of lightwave technology, vol. 18, no. 6, pp. 849-852, 2000.

[542] M. Abramowitz and I. A. Stegun, Handbook of mathematical functions. Dover publications Inc., New Ýork, 1965.

[543] T. Jablonski, "Complex modes in open lossless dielectric waveguides," JOSA $A$, vol. 11, no. 4, pp. 1272-1282, 1994 .

[544] G. Conciauro, M. Bressan, and C. Zuffada, "Waveguide modes via an integral equation leading to a linear matrix eigenvalue problem," IEEE Transactions on Microwave Theory and Techniques, vol. 32, no. 11, pp. 1495-1504, 1984.

[545] M. Mongiardo, P. Russer, R. Sorrentino, and C. Tomassoni, "Spherical modal expansion for arrays of flange-mounted rectangular waveguides," in European Microwave Conference. IEEE, 2007, pp. 32-35.

[546] —_ "Spherical mode expansions for flange-mounted waveguide apertures," in International Conference on Electromagnetics in Advanced Applications (ICEAA). IEEE, 2007, pp. 41-44.

[547] C. Tomassoni, M. Mongiardo, P. Russer, and R. Sorrentino, "Rigorous modal analysis of radiation from coaxial waveguide mounted on finite circular flange," in 38th European Microwave Conference (EuMC). IEEE, 2008, pp. 654-657. 
[548] S. Lucyszyn, "Investigation of anomalous room temperature conduction losses in normal metals at terahertz frequencies," IEE Proceedings-Microwaves, Antennas and Propagation, vol. 151, no. 4, pp. 321-329, 2004.

[549] E. Episkopou, S. Papantonis, W. J. Otter, and S. Lucyszyn, "Defining material parameters in commercial EM solvers for arbitrary metal-based $\mathrm{THz}$ structures," IEEE Transactions on Terahertz Science and Technology, vol. 2, no. 5, pp. 513-524, 2012.

[550] M. Silveirinha and N. Engheta, "Tunneling of electromagnetic energy through subwavelength channels and bends using $\varepsilon$-near-zero materials," Physical review letters, vol. 97, no. 15, p. 157403, 2006.

[551] A. Alù, M. G. Silveirinha, A. Salandrino, and N. Engheta, "Epsilon-nearzero metamaterials and electromagnetic sources: Tailoring the radiation phase pattern," Physical Review B, vol. 75, no. 15, p. 155410, 2007.

[552] B. Edwards, A. Alù, M. E. Young, M. Silveirinha, and N. Engheta, "Experimental verification of epsilon-near-zero metamaterial coupling and energy squeezing using a microwave waveguide," Physical review letters, vol. 100, no. 3, p. $033903,2008$.

[553] A. Snyder and J. Love, Optical waveguide theory. Springer, 1983, vol. 190.

[554] B. Prade and J. Vinet, "Guided optical waves in fibers with negative dielectric constant," Journal of Lightwave Technology, vol. 12, no. 1, pp. 6-18, 1994.

[555] J. Strube and F. Arndt, "Rigorous hybrid-mode analysis of the transition from rectangular waveguide to shielded dielectric image guide," IEEE Transactions on Microwave Theory and Techniques, vol. 33, no. 5, pp. 391-401, 1985.

[556] J. Rhodes, "General constraints on propagation characteristics of electromagnetic waves in uniform inhomogeneous waveguides," Proceedings of the Institution of Electrical Engineers, vol. 118, no. 7, pp. 849-856, 1971.

[557] K. Ogusu, "Numerical analysis of the rectangular dielectric waveguide and its modifications," IEEE Transactions on Microwave Theory and Techniques, vol. 25, no. 11, pp. 874-885, 1977.

[558] K. Bierwirth, N. Schulz, and F. Arndt, "Finite-difference analysis of rectangular dielectric waveguide structures," IEEE Transactions on Microwave Theory and Techniques, vol. 34, no. 11, pp. 1104-1114, 1986.

[559] E. Hansen and M. Patrick, "A family of root finding methods," Numerische Mathematik, vol. 27, no. 3, pp. 257-269, 1976.

[560] M. Lang and B.-C. Frenzel, "Polynomial root finding," IEEE Signal Processing Letters, vol. 1, no. 10, pp. 141-143, 1994.

[561] P. Kravanja and M. Van Barel, "A derivative-free algorithm for computing zeros of analytic functions," Computing, vol. 63, no. 1, pp. 69-91, 1999. 
[562] S. Amat, S. Busquier, and S. Plaza, "Review of some iterative root-finding methods from a dynamical point of view," Scientia, vol. 10, no. 3, p. 35, 2004.

[563] V. Y. Pan and A.-L. Zheng, "New progress in real and complex polynomial root-finding," Computers \& Mathematics with Applications, vol. 61, no. 5, pp. 1305-1334, 2011.

[564] E. Anemogiannis and E. N. Glytsis, "Multilayer waveguides: efficient numerical analysis of general structures," Journal of Lightwave Technology, vol. 10, no. 10, pp. 1344-1351, 1992.

[565] R. Rodríguez-Berral, F. Mesa, and F. Medina, "Systematic and efficient root finder for computing the modal spectrum of planar layered waveguides," International Journal of RF and Microwave Computer-Aided Engineering, vol. 14, no. 1, pp. 73-83, 2004.

[566] _ _ "Appropriate formulation of the characteristic equation for open nonreciprocal layered waveguides with different upper and lower half-spaces," IEEE Transactions on Microwave Theory and Techniques, vol. 53, no. 5, pp. 1613$1623,2005$.

[567] C. Pfeiffer, E. Economou, and K. Ngai, "Surface polaritons in a circularly cylindrical interface: surface plasmons," Physical review $B$, vol. 10, no. 8, p. 3038, 1974 .

[568] G. Aers, B. Paranjape, and A. Boardman, "Non-radiative surface plasmapolariton modes of inhomogeneous metal circular cylinders," Journal of Physics F: Metal Physics, vol. 10, no. 1, p. 53, 1980.

[569] H. Khosravi, D. Tilley, and R. Loudon, "Surface polaritons in cylindrical optical fibers," JOSA A, vol. 8, no. 1, pp. 112-122, 1991.

[570] A. R. Zakharian, J. V. Moloney, and M. Mansuripur, "Surface plasmon polaritons on metallic surfaces," Optics express, vol. 15, no. 1, pp. 183-197, 2007.

[571] M. Mansuripur, A. R. Zakharian, and J. V. Moloney, "Surface plasmon polaritons on metallic surfaces," Optics and Photonics News, vol. 18, no. 4, pp. 44-49, 2007.

[572] J. Catalá-Civera, A. J. Canós, F. Peñaranda-Foix, and E. de los Reyes, "Microwave Non-Destructive Evaluation of Moisture Content in Liquid Composites in a Cylindrical Cavity at a Single Frequency," in Advances in Microwave and Radio Frequency Processing. Springer, 2006, pp. 138-148.

[573] A. Barannik, N. Cherpak, Y. V. Prokopenko, Y. F. Filipov, E. Shaforost, and I. Shipilova, "Two-layered disc quasi-optical dielectric resonators: electrodynamics and application perspectives for complex permittivity measurements of lossy liquids," Measurement science and technology, vol. 18, no. 7, p. 2231, 2007. 
[574] F. L. Penaranda-Foix, M. D. Janezic, J. M. Catala-Civera, and A. J. Canos, "Full-wave analysis of dielectric-loaded cylindrical waveguides and cavities using a new four-port ring network," IEEE Transactions on Microwave Theory and Techniques, vol. 60, no. 9, pp. 2730-2740, 2012.

[575] G. Conciauro, P. Arcioni, M. Bressan, and L. Perregrini, "Wideband modeling of arbitrarily shaped h-plane waveguide components by the "boundary integral-resonant mode expansion method"," IEEE Transactions on Microwave Theory and Techniques, vol. 44, no. 7, pp. 1057-1066, 1996.

[576] S. Cogollos, S. Marini, V. E. Boria, P. Soto, A. Vidal, H. Esteban, J. V. Morro, and B. Gimeno, "Efficient modal analysis of arbitrarily shaped waveguides composed of linear, circular, and elliptical arcs using the BI-RME method," IEEE Transactions on Microwave Theory and Techniques, vol. 51, no. 12, pp. 2378-2390, 2003.

[577] R. Scarmozzino, A. Gopinath, R. Pregla, and S. Helfert, "Numerical techniques for modeling guided-wave photonic devices," IEEE Journal of Selected Topics in Quantum Electronics, vol. 6, no. 1, pp. 150-162, 2000.

[578] S. Selleri, L. Vincetti, A. Cucinotta, and M. Zoboli, "Complex fem modal solver of optical waveguides with pml boundary conditions," Optical and Quantum Electronics, vol. 33, no. 4-5, pp. 359-371, 2001.

[579] A. Sihvola, "Metamaterials in electromagnetics," Metamaterials, vol. 1, no. 1, pp. 2-11, 2007.

[580] J. Pendry, A. Holden, W. Stewart, and I. Youngs, "Extremely low frequency plasmons in metallic mesostructures," Physical review letters, vol. 76, no. 25, p. $4773,1996$.

[581] D. R. Smith, J. B. Pendry, and M. C. Wiltshire, "Metamaterials and negative refractive index," Science, vol. 305, no. 5685, pp. 788-792, 2004.

[582] M. N. Afsar and K. J. Button, "Millimeter-wave dielectric measurement of materials," Proceedings of the IEEE, vol. 73, no. 1, pp. 131-153, 1985.

[583] J. Birch, J. Dromey, and J. Lesurf, "The optical constants of some common low-loss polymers between 4 and $40 \mathrm{~cm}$ - 1," Infrared Physics, vol. 21, no. 4, pp. 225-228, 1981.

[584] A. Berenguer, M. Baquero-Escudero, D. Sanchez-Escuderos, and M. FerrandoBataller, "Optimización de curvas en la guía de hilo metálico a frecuencias de thz," in Simposium Nacional de la URSI, 2012.

[585] —, "Study of losses and high-order modes coupling on bends for wire-type waveguides at thz frequencies," in USNC/URSI National Science Meeting. IEEE, 2012, p. 1.

[586] — "Study of transitions for the single-wire waveguide at THz frequencies," in 7th European Conference on Antennas and Propagation (EUCAP), 2013, pp. 3426-3430. 
[587] — - "Reduction of radiation losses of the single-wire waveguide at $\mathrm{THz}$ frequencies," in USNC/URSI National Science Meeting. IEEE, 2013, p. 1.

[588] — - "Transiciones en gu 'ia de hilo metálico para la reducción de radiación en curvas a THz," in URSI, 2013, pp. 1-4.

[589] V. Larin, A. Torcunov, A. Zhukov, J. Gonzalez, M. Vazquez, and L. Panina, "Preparation and properties of glass-coated microwires," Journal of Magnetism and Magnetic Materials, vol. 249, no. 1, pp. 39-45, 2002.

[590] T. Itoh, Numerical techniques for microwave and millimeter-wave passive structures. Wiley-Interscience, 1989.

[591] K. Yee, "Protective coatings for metals by chemical vapour deposition," International Metals Reviews, 2013.

[592] M. O. Aziz-Zanjani and A. Mehdinia, "A review on procedures for the preparation of coatings for solid phase microextraction," Microchimica Acta, vol. 181, no. 11-12, pp. 1169-1190, 2014.

[593] J. Capmany and D. Novak, "Microwave photonics combines two worlds," $\mathrm{Na}$ ture photonics, vol. 1, no. 6, pp. 319-330, 2007.

[594] J. E. Roman and K. A. Winick, "Waveguide grating filters for dispersion compensation and pulse compression," IEEE journal of quantum electronics, vol. 29, no. 3, pp. 975-982, 1993.

[595] Micro-coax ${ }^{\circledR}$. (2016). [Online]. Available: http://www.micro-coax.com

[596] Toptica Photonics AG. (2016). [Online]. Available: http://www.toptica.com/

[597] C. Roman, O. Ichim, L. Sarger, V. Vigneras, and P. Mounaix, "Terahertz dielectric characterisation of polymethacrylimide rigid foam:: The perfect sheer plate?" Electronics Letters, vol. 40, no. 19, p. 1, 2004.

[598] A. Olver, "Millimetrewave systems-past, present and future," in IEE Proceedings F-Radar and Signal Processing, vol. 136, no. 1. IET, 1989, pp. 35-52.

[599] International Telecommunication Union (ITU). (2016). [Online]. Available: http://www.itu.int

[600] Z. Pi and F. Khan, "An introduction to millimeter-wave mobile broadband systems," IEEE Communications Magazine, vol. 49, no. 6, pp. 101-107, 2011.

[601] T. S. Rappaport, S. Sun, R. Mayzus, H. Zhao, Y. Azar, K. Wang, G. N. Wong, J. K. Schulz, M. Samimi, and F. Gutierrez, "Millimeter wave mobile communications for $5 \mathrm{G}$ cellular: It will work!" Access, IEEE, vol. 1, pp. 335$349,2013$.

[602] A. I. Sulyman, A. T. Nassar, M. K. Samimi, G. R. Maccartney, T. S. Rappaport, and A. Alsanie, "Radio propagation path loss models for $5 \mathrm{G}$ cellular networks in the $28 \mathrm{GHz}$ and $38 \mathrm{GHz}$ millimeter-wave bands," IEEE Communications Magazine, vol. 52, no. 9, pp. 78-86, 2014. 
[603] Y. Niu, Y. Li, D. Jin, L. Su, and A. V. Vasilakos, "A survey of millimeter wave communications (mmWave) for 5G: opportunities and challenges," Wireless Networks, vol. 21, no. 8, pp. 2657-2676, 2015.

[604] M. Schneider, "Automotive radar-status and trends," in German microwave conference, 2005, pp. 144-147.

[605] J. Hasch, E. Topak, R. Schnabel, T. Zwick, R. Weigel, and C. Waldschmidt, "Millimeter-wave technology for automotive radar sensors in the $77 \mathrm{GHz}$ frequency band," IEEE Transactions on Microwave Theory and Techniques, vol. 60, no. 3, pp. 845-860, 2012.

[606] W. Menzel and A. Moebius, "Antenna concepts for millimeter-wave automotive radar sensors," Proceedings of the IEEE, vol. 100, no. 7, pp. 2372-2379, 2012.

[607] R. Rouveure, P. Faure, and M.-O. Monod, "PELICAN: Panoramic millimeterwave radar for perception in mobile robotics applications, Part 1: Principles of FMCW radar and of 2D image construction," Robotics and Autonomous Systems, vol. 81, pp. 1-16, 2016.

[608] J. Wells, "Faster than fiber: The future of multi-G/s wireless," IEEE Microwave Magazine, vol. 10, no. 3, pp. 104-112, 2009.

[609] A. M. Niknejad, "Siliconization of $60 \mathrm{GHz}$," IEEE MICROWAVE MAGAZINE, vol. 11, no. 2, pp. 93-93, 2010.

[610] J. Curry, P. Hobbs, M. King, D. Randall et al., "Fire artic clouds experiment," Bulletin of the American Meteorological Society, vol. 81, no. 1, p. 5, 2000.

[611] H. Hertz, "Memoirs. Letters. Diaries. Hertz J, Hertz M, Süsskind C eds. San Francisco," San Francisco Press, Inc, vol. 165, p. 315, 1977.

[612] A. Righi, "L'ottica delle oscillazioni elettriche," Bologna, Italy: N. Zanichelli, 1897.

[613] O. Lodge, Signalling Through Space Without Wires: Being a Description of the Work of Hertz $\&$ His Successors. The Electrician, 1903.

[614] J. A. Fleming, The principles of electric wave telegraphy. Longmans, Green, and Company, 1906.

[615] J. Bose, W. Bragg, and G. Porter, "Electromagnetic radiation and the polarisation of the electric ray," Friday Evening Discourse, The Royal Institution, vol. $29,1897$.

[616] G. Marconi, “Apparatus for wireless telegraphy." Jun. 11 1901, uS Patent 676,332 .

[617] S. S. Swords, Technical history of the beginnings of radar. IET, 1985.

[618] W. Webb, "Sources of Tracking Error in Millimeter Wavelength Missile Guidance Systems," DTIC Document, Tech. Rep., 1979. 
[619] J. J. Renier, A. B. Bishop III, J. M. Gavin, D. R. Heebner, and R. L. Hess, "Military Operations in Built-Up Areas (MOBA)," DTIC Document, Tech. Rep., 1979.

[620] P. W. Kruse, "Why the military interest in near-millimeter wave imaging?" in Proc. SPIE, vol. 259, 1980, pp. 94-97.

[621] H. H. Meinel, "Millimeter wave applications and technology trends," in Annales des télécommunications, vol. 47, no. 11-12. Springer, 1992, pp. 456-468.

[622] — - "Commercial applications of millimeterwaves: history, present status, and future trends," IEEE Transactions on Microwave Theory and Techniques, vol. 43, no. 7, pp. 1639-1653, 1995.

[623] — - "Applications of microwaves and millimeterwaves for vehicle communications and control in europe," in Microwave Symposium Digest, 1992., IEEE MTT-S International. IEEE, 1992, pp. 609-612.

[624] S. Tokoro, "Automotive application systems of a millimeter-wave radar," in Intelligent Vehicles Symposium, 1996., Proceedings of the 1996 IEEE. IEEE, 1996, pp. 260-265.

[625] S. Tokoro, K. Kuroda, A. Kawakubo, K. Fujita, and H. Fujinami, "Electronically scanned millimeter-wave radar for pre-crash safety and adaptive cruise control system," in Intelligent Vehicles Symposium, 2003. Proceedings. IEEE. IEEE, 2003, pp. 304-309.

[626] Y. Takimoto and T. Ihara, "Research activities on millimeter wave indoor communication systems in japan," in Microwave Symposium Digest, 1993., IEEE MTT-S International. IEEE, 1993, pp. 673-676.

[627] Y. Takimoto, "Recent activities on millimeter wave indoor LAN system development in Japan," in Microwave Symposium Digest, 1995., IEEE MTT-S International. IEEE, 1995, pp. 405-408.

[628] T. Manabe, K. Sato, H. Masuzawa, K. Taira, T. Ihara, Y. Kasashima, and K. Yamaki, "Polarization dependence of multipath propagation and high-speed transmission characteristics of indoor millimeter-wave channel at $60 \mathrm{GHz}$," IEEE Transactions on Vehicular Technology, vol. 44, no. 2, pp. 268-274, 1995.

[629] I. Gresham, N. Jain, T. Budka, A. Alexanian, N. Kinayman, B. Ziegner, S. Brown, and P. Staecker, "A compact manufacturable 76-77-GHz radar module for commercial ACC applications," IEEE Transactions on Microwave Theory and Techniques, vol. 49, no. 1, pp. 44-58, 2001.

[630] G. K. Felic, R. Evans, H. T. Duong, H. V. Le, J. Li, and E. Skafidas, "Singlechip Millimeter Wave Radar," Microwave Journal, pp. 108-116, 2015.

[631] J. Choi, N. Gonzalez-Prelcic, R. Daniels, C. R. Bhat, and R. W. Heath Jr, "Millimeter wave vehicular communication to support massive automotive sensing," arXiv preprint arXiv:1602.06456, 2016. 
[632] N. Guo, R. C. Qiu, S. S. Mo, and K. Takahashi, "60-GHz millimeter-wave radio: Principle, technology, and new results," EURASIP Journal on Wireless Communications and Networking, vol. 2007, no. 1, pp. 48-48, 2007.

[633] Y. J. Guo, D. Liu, and N. C. Bird, "Guest Editorial for the Special Issue on Antennas and Propagation Aspects of 60-90 GHz Wireless Communications," IEEE Transactions on Antennas and Propagation, vol. 10, no. 57, pp. 28172819, 2009.

[634] C. Dehos, J. L. González, A. De Domenico, D. Kténas, and L. Dussopt, "Millimeter-wave access and backhauling: the solution to the exponential data traffic increase in $5 \mathrm{G}$ mobile communications systems?" IEEE Communications Magazine, vol. 52, no. 9, pp. 88-95, 2014.

[635] C.-X. Wang, F. Haider, X. Gao, X.-H. You, Y. Yang, D. Yuan, H. M. Aggoune, H. Haas, S. Fletcher, and E. Hepsaydir, "Cellular architecture and key technologies for 5G wireless communication networks," IEEE Communications Magazine, vol. 52, no. 2, pp. 122-130, 2014.

[636] F. Aryanfar, J. Pi, H. Zhou, T. Henige, G. Xu, S. Abu-Surra, D. Psychoudakis, and F. Khan, "Millimeter-wave base station for mobile broadband communication," in IEEE MTT-S International Microwave Symposium. IEEE, 2015, pp. $1-3$.

[637] S. E. Clark, J. A. Lovberg, C. A. Martin, and V. G. Kolinko, "Passive millimeter-wave imaging for airborne and security applications," in AeroSense 2003. International Society for Optics and Photonics, 2003, pp. 16-21.

[638] R. Doyle, B. Lyons, A. Lettington, T. McEnroe, J. Walshe, J. McNaboe, and P. Curtin, "Illumination strategies to achieve effective indoor millimeter wave imaging for personnel screening applications," in Defense and Security. International Society for Optics and Photonics, 2005, pp. 101-108.

[639] V. G. Kolinko, S.-H. Lin, A. Shek, W. Manning, C. Martin, M. Hall, O. Kirsten, J. Moore, and D. A. Wikner, "A passive millimeter-wave imaging system for concealed weapons and explosives detection," in Defense and Security. International Society for Optics and Photonics, 2005, pp. 85-92.

[640] L. Yujiri, "Passive millimeter wave imaging," in 2006 IEEE MTT-S International Microwave Symposium Digest. IEEE, 2006, pp. 98-101.

[641] J. A. Lovberg, C. Martin, and V. Kolinko, "Video-rate passive millimeterwave imaging using phased arrays," in IEEE MTT-S International Microwave Symposium. IEEE, 2007, pp. 1689-1692.

[642] H. Sato, K. Sawaya, K. Mizuno, J. Uemura, M. Takeda, J. Takahashi, K. Yamada, K. Morichika, T. Hasegawa, H. Hirai et al., "Development of $77 \mathrm{GHz}$ millimeter wave passive imaging camera," in Sensors, 2009 IEEE. IEEE, 2009, pp. 1632-1635. 
[643] C. Adams, D. Holbrook, and R. Sengsten, "A handheld active millimeter wave camera," in IEEE International Conference on Technologies for Homeland Security (HST). IEEE, 2010, pp. 283-286.

[644] G. Brooker and D. G. Johnson, "Low-Cost Millimeter Wave Imaging Using a Commercial Plasma Display," IEEE Sensors Journal, vol. 15, no. 6, pp. 3557-3564, 2015.

[645] C. A. Balanis, Antenna Theory: Analysis and desing. John Wiley \& Sons, 1997.

[646] D. Lockie and D. Peck, "High-data-rate millimeter-wave radios," IEEE Microwave Magazine, vol. 10, no. 5, pp. 75-83, 2009.

[647] IBM $^{\circledR}$. (2016). [Online]. Available: https://www.ibm.com

[648] F. DOMENE OLTRA, "Evaluation of precoding and feedback quantization schemes for multiuser communications systems," Ph.D. dissertation, Editorial Universitat Politècnica de València, 2015.

[649] M. E. Russell, A. Crain, A. Curran, R. A. Campbell, C. A. Drubin, and W. F. Miccioli, "Millimeter-wave radar sensor for automotive intelligent cruise control (ICC)," IEEE Transactions on microwave theory and techniques, vol. 45, no. 12 , pp. 2444-2453, 1997.

[650] E. Clothiaux, M. Miller, B. Albrecht, T. Ackerman, J. Verlinde, D. Babb, R. Peters, and W. Syrett, "An evaluation of a 94-GHz radar for remote sensing of cloud properties," Journal of Atmospheric and Oceanic Technology, vol. 12, no. 2, pp. 201-229, 1995.

[651] W. Szyrmer, A. Tatarevic, and P. Kollias, "Ice clouds microphysical retrieval using 94-GHz Doppler radar observations: Basic relations within the retrieval framework," Journal of Geophysical Research: Atmospheres, vol. 117, no. D14, 2012 .

[652] ITU, "Specific Attenuation Model for Rain for Use in Prediction Methods," ITR-R Standard P.838-3, 2005.

[653] J. V. Evans and A. Dissanayake, "The prospects for commercial satellite services at Q-and V-band," Space communications, vol. 15, no. 1, pp. 1-19, 1998.

[654] E. Suematsu, Y. Amano, A. Yamada, Y. Zhu, H. Sato, N. Hashizume, F. Kuroki, and T. Yoneyama, "Digital and analog satellite/millimeter-wave transmission link," in IEEE MTT-S International Microwave Symposium Digest, vol. 3. IEEE, 1999, pp. 1047-1050.

[655] H. Ohyama, T. Nagahama, A. Mizuno, H. Nakane, and H. Ogawa, "Observations of stratospheric and mesospheric O3 with a millimeter-wave radiometer at Rikubetsu, Japan," Earth, Planets and Space, vol. 68, no. 1, pp. 1-14, 2016. 
[656] M. Agiwal, F. Jabeen, and K. Ahmed, "Feasibility Study of Millimeter Wave Transmission," International Journal of Computer Science and Information Security, vol. 11, no. 3, p. 45, 2013.

[657] G.-M. Muntean, Wireless Multi-Access Environments and Quality of Service Provisioning: Solutions and Application: Solutions and Application. IGI Global, 2012.

[658] North Atlantic Treaty Organization (NATO). (2016). [Online]. Available: http://www.nato.int

[659] H. H. Meinel, "Evolving automotive radar. from the very beginnings into the future," in The 8th European Conference on Antennas and Propagation (EuCAP 2014). IEEE, 2014, pp. 3107-3114.

[660] D. M. Sheen, J. L. Fernandes, J. R. Tedeschi, D. L. McMakin, A. M. Jones, W. M. Lechelt, and R. H. Severtsen, "Wide-bandwidth, wide-beamwidth, highresolution, millimeter-wave imaging for concealed weapon detection," in SPIE Defense, Security, and Sensing. International Society for Optics and Photonics, 2013, pp. 871 509-871509.

[661] W. Johannes, H. Essen, S. Stanko, R. Sommer, A. Wahlen, J. Wilcke, C. Wagner, M. Schlechtweg, and A. Tessmann, "Miniaturized high resolution Synthetic Aperture Radar at $94 \mathrm{GHz}$ for microlite aircraft or UAV," in IEEE Sensors. IEEE, 2011, pp. 2022-2025.

[662] M. Caris, S. Stanko, R. Sommer, A. Wahlen, A. Leuther, A. Tessmann, M. Malanowski, P. Samczynski, K. Kulpa, M. Cohen et al., "SARape-synthetic aperture radar for all weather penetrating UAV application," in 14th International Radar Symposium (IRS), vol. 1. IEEE, 2013, pp. 41-46.

[663] D. A. Robertson, D. G. Macfarlane, and T. Bryllert, "220GHz wideband 3D imaging radar for concealed object detection technology development and phenomenology studies," in SPIE Defense+ Security. International Society for Optics and Photonics, 2016, pp. 983 009-983009.

[664] G. Prigent, E. Rius, R. Plana, and T. M. Vu, Trend on Silicon Technologies for Millimetre-Wave Applications up to $220 \mathrm{GHz}$. INTECH Open Access Publisher, 2010.

[665] H. Essen, S. Stanko, R. Sommer, A. Wahlen, R. Brauns, J. Wilcke, W. Johannes, A. Tessmann, and M. Schlechtweg, "A high performance 220-GHz broadband experimental radar," in 33rd International Conference on Infrared, Millimeter and Terahertz Waves (IRMWW-THz). IEEE, 2008, pp. 1-1.

[666] H. H. Meinel, "Millimeter-wave technology advances since 1985 and future trends," IEEE transactions on microwave theory and techniques, vol. 39, no. 5, pp. 759-767, 1991.

[667] R. Alemany, M. Ayuso, and M. Guillén, "Impact of road traffic injuries on disability rates and long-term care costs in Spain," Accident Analysis 86 Prevention, vol. 60, pp. 95-102, 2013. 
[668] Q. Luo, S. Gao, and L. Zhang, "Millimeter-wave smart antennas for advanced satellite communications," in IEEE MTT-S International Microwave Symposium. IEEE, 2015, pp. 1-4.

[669] R. N. Simons and E. G. Wintucky, "Multi-Band Multi-Tone Tunable Millimeter-Wave Frequency Synthesizer For Satellite Beacon Transmitter," in IEEE MTT-S International Microwave Symposium, 2016.

[670] K. Tsukamoto, A. Hashimoto, Y. Aburakawa, and M. Matsumoto, "The case for free space," IEEE Microwave Magazine, vol. 10, no. 5, pp. 84-92, 2009.

[671] H. Ahmad, M. Soltanian, I. Amiri, S. Alavi, A. Othman, and A. Supa'at, "Carriers generated by mode-locked laser to increase serviceable channels in radio over free space optical systems," IEEE Photonics Journal, vol. 7, no. 5, pp. 1-12, 2015.

[672] H. Al-Raweshidy and S. Komaki, Radio over fiber technologies for mobile communications networks. Artech House, 2002.

[673] V. A. Thomas, M. El-Hajjar, and L. Hanzo, "Performance improvement and cost reduction techniques for radio over fiber communications," IEEE Communications Surveys \& Tutorials, vol. 17, no. 2, pp. 627-670, 2015.

[674] 1 Velocity. (2016). [Online]. Available: http://www.velocity1.com

[675] A. Sadri, "802.15. 3c Usage Model Document (UMD)," IEEE Draft, vol. 802, 2006.

[676] K. Ohata, K. Maruhashi, M. Ito, S. Kishimoto, K. Ikuina, T. Hashiguchi, K. Ikeda, and N. Takahashi, "1.25 Gbps wireless Gigabit Ethernet link at 60 GHz-band," in IEEE MTT-S International Microwave Symposium Digest, vol. 1. IEEE, 2003, pp. 373-376.

[677] A. Aijaz, H. Aghvami, and M. Amani, "A survey on mobile data offloading: technical and business perspectives," IEEE Wireless Communications, vol. 20, no. 2, pp. 104-112, 2013.

[678] R. Rom and M. Sidi, Multiple access protocols: performance and analysis. Springer Science \& Business Media, 2012.

[679] E. Amyotte and L. Martins Camelo, "Antennas for satellite communications," Space Antenna Handbook, pp. 466-510, 2012.

[680] Q. Luo, S. Gao, C. Zhang, D. Zhou, T. Chaloun, W. Menzel, V. Ziegler, and M. Sobhy, "Design and analysis of a reflectarray using slot antenna elements for Ka-band SatCom," IEEE Transactions on Antennas and Propagation, vol. 63, no. 4, pp. 1365-1374, 2015.

[681] K.-J. Koh, J. W. May, and G. M. Rebeiz, "A millimeter-wave (40-45 GHz) 16-element phased-array transmitter in $0.18 \mu \mathrm{m}$ SiGe BiCMOS technology," IEEE Journal of Solid-State Circuits, vol. 44, no. 5, pp. 1498-1509, 2009. 
[682] H.-T. Dabag, B. Hanafi, F. Golcuk, A. Agah, J. F. Buckwalter, and P. M. Asbeck, "Analysis and design of stacked-FET millimeter-wave power amplifiers," IEEE Transactions on Microwave Theory and Techniques, vol. 61, no. 4, pp. 1543-1556, 2013.

[683] J. Blumenstein, T. Mikulasek, T. Zemen, C. Mecklenbrauker, R. Marsalek, and A. Prokes, "In-vehicle mm-wave channel model and measurement," in 2014 IEEE 80th Vehicular Technology Conference (VTC2014-Fall). IEEE, 2014, pp. 1-5.

[684] V. Va, T. Shimizu, G. Bansal, R. W. Heath Jr et al., "Millimeter Wave Vehicular Communications: A Survey," Foundations and Trends® in Networking, vol. 10, no. 1, 2016.

[685] T. Nitsche, C. Cordeiro, A. B. Flores, E. W. Knightly, E. Perahia, and J. C. Widmer, "IEEE 802.11 ad: directional $60 \mathrm{GHz}$ communication for multiGigabit-per-second Wi-Fi [Invited Paper]," IEEE Communications Magazine, vol. 52, no. 12, pp. 132-141, 2014.

[686] J. Louberg, V. Kolinko, E. Woodbridge, and R. C. Saunders, "Imaging millimeter wave radar system," Mar. 28 2006, uS Patent 7,019,682.

[687] J. A. Lovberg and V. Kolinko, "Security system with metal detection and mm-wave imaging," Jul. 24 2007, uS Patent 7,248,204.

[688] A. Babakhani, D. Liu, S. K. Reynolds, and M. A. Sanduleanu, "Near-field millimeter wave imaging," Feb. 23 2016, uS Patent 9,268,017.

[689] S. Oka, H. Togo, N. Kukutsu, and T. Nagatsuma, "Latest trends in millimeterwave imaging technology," Progress In Electromagnetics Research Letters, vol. 1, pp. 197-204, 2008.

[690] — - "Latest trends in millimeter-wave imaging technology," Progress In Electromagnetics Research Letters, vol. 1, pp. 197-204, 2008.

[691] S. Shylo and S. Harmer, "Millimeter-Wave Imaging for Recycled Paper Classification," IEEE Sensors Journal, vol. 16, no. 8, pp. 2361-2366, 2016.

[692] Transportation Security Administration (TSA). (2016). [Online]. Available: https://www.tsa.gov

[693] D. R. Vizard and R. Doyle, "Invited paper: Advances in millimeter wave imaging and radar systems for civil applications," in IEEE MTT-S International Microwave Symposium Digest. IEEE, 2006, pp. 94-97.

[694] J. Paul, F. Fortuin, and H. Kelder, "An ozone climatology based on ozonesonde and satellite measurements," Journal of Geophysical Research: Atmospheres, vol. 103, no. D24, pp. 31 709-31 734, 1998.

[695] M. N. Deeter and K. F. Evans, "A novel ice-cloud retrieval algorithm based on the Millimeter-Wave Imaging Radiometer (MIR) 150-and 220-GHz channels," Journal of Applied Meteorology, vol. 39, no. 5, pp. 623-633, 2000. 
[696] T. J. Hewison and S. J. English, "Airborne retrievals of snow and ice surface emissivity at millimeter wavelengths," IEEE Transactions on Geoscience and Remote Sensing, vol. 37, no. 4, pp. 1871-1879, 1999.

[697] S. Reising, P. Kangaslahti, A. Tanner, S. Padmanabhan, O. Montes, C. Parashare, X. Bosch-Lluis, V. Hadel, T. Johnson, S. Brown et al., "Airborne Demonstration of Microwave and Wide-Band Millimeter-Wave Radiometers to Provide High-Resolution Wet-Tropospheric Path Delay Corrections for Coastal and Inland Water Altimetry," in EGU General Assembly Conference Abstracts, vol. 16, 2014, p. 9200.

[698] G. Valladeau, P. Thibaut, B. Picard, J. Poisson, N. Tran, N. Picot, and A. Guillot, "Using SARAL/AltiKa to improve Ka-band altimeter measurements for coastal zones, hydrology and ice: The PEACHI prototype," Marine Geodesy, vol. 38, no. sup1, pp. 124-142, 2015.

[699] J. Vieira, T. Crawford, E. Switzer, P. A. Ade, K. Aird, M. Ashby, B. Benson, L. Bleem, M. Brodwin, J. Carlstrom et al., "Extragalactic millimeterwave sources in South Pole Telescope survey data: source counts, catalog, and statistics for an 87 square-degree field," The Astrophysical Journal, vol. 719, no. 1, p. $763,2010$.

[700] M. Hoefle, A. Penirschke, O. Cojocari, T. Decoopman, M. Trier, P. Piironen, M. Périchaud, and R. Jakoby, "89 GHz zero-bias Schottky detector for direct detection radiometry in European satellite programme MetOp-SG," Electronics Letters, vol. 50, no. 8, pp. 606-608, 2014.

[701] E. Zabolotskikh and B. Chapron, "Neural network-based method for the estimation of the rain rate over oceans by measurements of the satellite radiometer AMSR2," Izvestiya, Atmospheric and Oceanic Physics, vol. 52, no. 1, pp. 8288, 2016.

[702] J. Sayers, S. Golwala, P. A. Ade, J. Aguirre, J. Bock, S. Edgington, J. Glenn, A. Goldin, D. Haig, A. Lange et al., "Studies of millimeter-wave atmospheric noise above mauna kea," The Astrophysical Journal, vol. 708, no. 2, p. 1674, 2009.

[703] A. Deepak, T. D. Wilkerson, and L. H. Ruhnke, Atmospheric water vapor. Elsevier, 2013.

[704] C. Kudsia, R. Cameron, and T. WAI-CHEUNG, "Innovations in microwave filters and multiplexing networks for communications satellite systems," IEEE Transactions on Microwave Theory and Techniques, vol. 40, no. 6, pp. 11331149, 1992.

[705] Thales Alenia Space Spain. (2016). [Online]. Available: https://www. thalesgroup.com/es/homepage/espana

[706] V. E. Boria, P. Soto, and S. Cogollos, "Distributed models for filter synthesis," IEEE Microwave Magazine, vol. 12, no. 6, pp. 87-100, 2011. 
[707] M. D’Auria, W. J. Otter, J. Hazell, B. T. Gillatt, C. Long-Collins, N. M. Ridler, and S. Lucyszyn, "3-D printed metal-pipe rectangular waveguides," IEEE Transactions on Components, Packaging and Manufacturing Technology, vol. 5, no. 9, pp. 1339-1349, 2015.

[708] G. Bertin, B. Piovano, L. Accatino, and M. Mongiardo, "Analysis and Design of Circular Waveguide Polarizers with Elliptical Irises," in 30th European Microwave Conference (EuMC). IEEE, 2000, pp. 1-4.

[709] J. Hesler, "A photonic crystal joint (PCJ) for metal waveguides," in IEEE MTT-S International Microwave Symposium Digest, vol. 2. IEEE, 2001, pp. $783-786$.

[710] C. Vicente and H. L. Hartnagel, "Passive-intermodulation analysis between rough rectangular waveguide flanges," IEEE Transactions on Microwave Theory and Techniques, vol. 53, no. 8, pp. 2515-2525, 2005.

[711] E. Bayar and A. J. Booth, "Waveguide and method of manufacture," Oct. 9 2007, uS Patent 7,280,011.

[712] P.-S. Kildal, E. Alfonso, A. Valero-Nogueira, and E. Rajo-Iglesias, "Local Metamaterial-Based Waveguides in Gaps Between Parallel Metal Plates," Antennas and Wireless Propagation Letters, IEEE, vol. 8, pp. 84-87, 2009.

[713] J. Becker, J. East, and L. Katehi, "Performance of silicon micromachined waveguide at W-band," Electronics Letters, vol. 38, no. 13, pp. 638-639, 2002.

[714] E. Ofli, R. Vahldieck, and S. Amari, "Novel E-plane filters and diplexers with elliptic response for millimeter-wave applications," IEEE Transactions on $\mathrm{Mi}$ crowave Theory and Techniques, vol. 53, no. 3, pp. 843-851, 2005.

[715] J. A. Ruiz-Cruz, J. R. Montejo-Garai, J. M. Rebollar Machain, and S. Sobrino, "Compact full Ku-band triplexer with improved E-plane power divider," Progress In Electromagnetics Research, vol. 86, pp. 39-51, 2008.

[716] Y. Miura, J. Hirokawa, M. Ando, Y. Shibuya, and G. Yoshida, "Double-layer full-corporate-feed hollow-waveguide slot array antenna in the 60-GHz band," IEEE Transactions on Antennas and Propagation, vol. 59, no. 8, pp. 28442851, 2011.

[717] T. Tomura, Y. Miura, M. Zhang, J. Hirokawa, and M. Ando, "A 45 linearly polarized hollow-waveguide corporate-feed slot array antenna in the $60-\mathrm{GHz}$ band," IEEE Transactions on Antennas and Propagation, vol. 60, no. 8, pp. 3640-3646, 2012.

[718] D. Kim, J. Hirokawa, M. Ando, J. Takeuchi, and A. Hirata, "64x64Element and 32x32-Element Slot Array Antennas Using Double-Layer HollowWaveguide Corporate-Feed in the $120 \mathrm{GHz}$ Band," IEEE Transactions on Antennas and Propagation, vol. 62, no. 3, pp. 1507-1512, 2014. 
[719] D. Busuioc, A. Borji, M. Shahabadi, and S. Safavi-Naeini, "Low Loss Integrated Waveguide Feed Network for Planar Antenna AiTays," in IEEE International Symposium on Antennas and Propagation \& USNC/URSI National Radio Science Meeting, 2005.

[720] A. Valero-Nogueira, E. Alfonso, J. Herranz, and M. Baquero, "Planar slotarray antenna fed by an oversized quasi-TEM waveguide," Microwave and Optical Technology Letters, vol. 49, no. 8, pp. 1875-1877, 2007.

[721] X.-P. Chen, K. Wu, L. Han, and F. He, "Low-cost high gain planar antenna array for $60-\mathrm{GHz}$ band applications," IEEE transactions on Antennas and Propagation, vol. 58, no. 6, pp. 2126-2129, 2010.

[722] A. Navarrini and R. L. Plambeck, "A turnstile junction waveguide orthomode transducer," IEEE Transactions on Microwave Theory and Techniques, vol. 54, no. 1, pp. 272-277, 2006.

[723] V. Furtula and M. Salewski, "W-band waveguide bandpass filter with E-plane cut," Review of Scientific Instruments, vol. 85, no. 7, p. 074703, 2014.

[724] Y. Rong, H.-W. Yao, K. A. Zaki, and T. G. Dolan, "Millimeter-wave Ka-band H-plane diplexers and multiplexers," IEEE Transactions on Microwave Theory and Techniques, vol. 47, no. 12, pp. 2325-2330, 1999.

[725] L. Accatino, G. Bertin, and M. Mongiardo, "A four-pole dual mode elliptic filter realized in circular cavity without screws," IEEE Transactions on Microwave Theory and Techniques, vol. 44, no. 12, pp. 2680-2687, 1996.

[726] J. Pawlan, "Compact and easy to manufacture dual mode feed horn with ultra-low backlobes," in German Microwave Conference. IEEE, 2015, pp. $355-358$.

[727] S. Cogollos, M. Brumos, V. Boria, C. Vicente, B. Gimeno, and M. Guglielmi, "New distributed model for synthesis of classical dual mode filters," in IEEE MTT-S International Microwave Symposium Digest (MTT). IEEE, 2010, pp. $437-440$.

[728] S. Cogollos, M. Brumos, V. E. Boria, C. Vicente, J. Gil, B. Gimeno, and M. Guglielmi, "A systematic design procedure of classical dual-mode circular waveguide filters using an equivalent distributed model," IEEE Transactions on Microwave Theory and Techniques, vol. 60, no. 4, pp. 1006-1017, 2012.

[729] M. Brumos, V. E. Boria, M. Guglielmi, and S. Cogollos, "Correction of manufacturing deviations in circular-waveguide dual-mode filters using aggressive space mapping," in Microwave Conference (EuMC), 2014 44th European. IEEE, 2014, pp. 624-627.

[730] J. R. Garner, L. Zhang, C. R. Donaldson, A. W. Cross, and W. He, "Design Study of a 372-GHz Higher Order Mode Input Coupler," IEEE Transactions on Electron Devices, vol. 63, no. 8, pp. 3284-3290, 2016. 
[731] C. Oleson and A. Denning, "Millimeter wave vector analysis calibration and measurement problems caused by common waveguide irregularities," in ARFTG Conference Digest-Fall, 56th, vol. 38. IEEE, 2000, pp. 1-9.

[732] E. L. Holzman, "A simple circular-to-rectangular waveguide transition," IEEE Microwave and Wireless Components Letters, vol. 15, no. 1, pp. 25-26, 2005.

[733] H. Esteban, J. Morro, V. Boria, C. Bachiller, B. Gimeno, and L. Conde, "Hybrid full-wave simulator for the multipaction modelling of low-cost H-plane filters 4th International Workshop on Multipactor, Corona and Passive Intermodulation in Space RF Hardware," in 4th International Workshop on Multipactor, Corona and Passive Intermodulation in Space RF Hardware, 2003.

[734] I. C. Hunter, L. Billonet, B. Jarry, and P. Guillon, "Microwave filtersapplications and technology," IEEE Transactions on Microwave Theory and Techniques, vol. 50, no. 3, pp. 794-805, 2002.

[735] V. Semenov, E. Rakova, D. Anderson, M. Lisak, and J. Puech, "Multipactor in rectangular waveguides," Physics of Plasmas (1994-present), vol. 14, no. 3, p. $033501,2007$.

[736] M. Yüceer, "Emerging technologies for communication satellite payloads," Progress in Aerospace Sciences, vol. 50, pp. 27-34, 2012.

[737] F. Piro and Y. Brand, "PIM and multipactor considerations for future highRF power space missions," in The 8th European Conference on Antennas and Propagation (EuCAP 2014). IEEE, 2014, pp. 1643-1646.

[738] Y.-C. Shih, T.-N. Ton, and L. Q. Bui, "Waveguide-to-microstrip transitions for millimeter-wave applications," in IEEE MTT-S International Microwave Symposium Digest. IEEE, 1988, pp. 473-475.

[739] D. Deslandes and $\mathrm{K}$. Wu, "Integrated microstrip and rectangular waveguide in planar form," IEEE Microwave and Wireless Components Letters, vol. 11, no. 2, pp. 68-70, 2001.

[740] T. Itoh and J. Rivera, "A Comparative Study of Millimeter-Wave Transmission Lines," Infrared and Millimeter Waves V9: Millimeter Components and Techniques, p. 95, 1983.

[741] F. Benson and F. Tischer, "Some guiding structures for millimetre waves," IEE Proceedings A-Physical Science, Measurement and Instrumentation, Management and Education-Reviews, vol. 131, no. 7, pp. 429-449, 1984.

[742] D. Lioubtchenko, S. Tretyakov, and S. Dudorov, Millimeter-wave waveguides. Springer Science \& Business Media, 2003, vol. 114.

[743] R. Garg, I. Bahl, and M. Bozzi, Microstrip lines and slotlines. Artech house, 2013.

[744] T. C. Edwards, T. Edwards, and M. Steer, Foundations for microstrip circuit design. John Wiley \& Sons, 2016. 
[745] D. Liu, U. Pfeiffer, J. Grzyb, and B. Gaucher, Advanced millimeter-wave technologies: antennas, packaging and circuits. John Wiley \& Sons, 2009.

[746] M. K. Chirala and C. Nguyen, "Multilayer design techniques for extremely miniaturized cmos microwave and millimeter-wave distributed passive circuits," IEEE transactions on microwave theory and techniques, vol. 54, no. 12, pp. 4218-4224, 2006.

[747] R. W. Jackson, "Considerations in the use of coplanar waveguide for millimeter-wave integrated circuits," IEEE Transactions on microwave theory and techniques, vol. 34, no. 12, pp. 1450-1456, 1986.

[748] C. Schollhorn, W. Zhao, M. Morschbach, and E. Kasper, "Attenuation mechanisms of aluminum millimeter-wave coplanar waveguides on silicon," IEEE Transactions on Electron Devices, vol. 50, no. 3, pp. 740-746, 2003.

[749] D. Li, F. Xu, and J. Xu, "An improved coplanar waveguide to slotline transition for multi-layer circuit structures," in 2015 Asia-Pacific Microwave Conference $(A P M C)$, vol. 3. IEEE, 2015, pp. 1-3.

[750] A. U. Zaman, "Gap Waveguide for High Frequency Microwave ApplicationValidation of Concept, Filter Design \& Packaging Aspects," Ph.D. dissertation, Chalmers University of Technology, 2011.

[751] T. M. Weller, R. M. Henderson, K. J. Herrick, S. Robertson, R. Kihm, and L. P. Katehi, "Three-dimensional high-frequency distribution networks I: Optimization of CPW discontinuities," IEEE Transactions on Microwave Theory and Techniques, vol. 48, no. 10, pp. 1635-1642, 2000.

[752] E. Rius, G. Prigent, H. Happy, G. Dambrine, S. Boret, and A. Cappy, "Wideand narrow-band bandpass coplanar filters in the w-frequency band," IEEE Transactions on microwave theory and techniques, vol. 51, no. 3, pp. 784-791, 2003.

[753] D. Deslandes, M. Bozzi, P. Arcioni, and K. Wu, "Substrate integrated slab waveguide (SISW) for wideband microwave applications," in IEEE MTT-S International Microwave Symposium Digest, vol. 2. IEEE, 2003, pp. 11031106.

[754] Y. Cassivi and K. Wu, "Substrate integrated nonradiative dielectric waveguide," IEEE Microwave and Wireless Components Letters, vol. 14, no. 3, pp. 89-91, 2004.

[755] W. Hong, B. Liu, Y. Wang, Q. Lai, H. Tang, X. X. Yin, Y. D. Dong, Y. Zhang, and $\mathrm{K} . \mathrm{Wu}$, "Half mode substrate integrated waveguide: A new guided wave structure for microwave and millimeter wave application," in Joint 31st International Conference on Infrared Millimeter Waves and 14th International Conference on Teraherz Electronics. IEEE, 2006, pp. 219-219. 
[756] A. Belenguer, H. Esteban, and V. E. Boria, "Novel empty substrate integrated waveguide for high-performance microwave integrated circuits," IEEE Transactions on Microwave Theory and Techniques, vol. 62, no. 4, pp. 832-839, 2014 .

[757] Y. Cassivi, L. Perregrini, P. Arcioni, M. Bressan, K. Wu, and G. Conciauro, "Dispersion characteristics of substrate integrated rectangular waveguide," IEEE Microwave and Wireless Components Letters, vol. 12, no. 9, pp. 333-335, 2002.

[758] F. Xu and K. Wu, "Guided-wave and leakage characteristics of substrate integrated waveguide," IEEE Transactions on microwave theory and techniques, vol. 53, no. 1, pp. 66-73, 2005.

[759] D. Deslandes and K. Wu, "Accurate modeling, wave mechanisms, and design considerations of a substrate integrated waveguide," IEEE Transactions on microwave theory and techniques, vol. 54, no. 6, pp. 2516-2526, 2006.

[760] X. H. Wu and A. A. Kishk, "Hybrid of method of moments and cylindrical eigenfunction expansion to study substrate integrated waveguide circuits," IEEE Transactions on Microwave Theory and Techniques, vol. 56, no. 10, pp. 2270-2276, 2008.

[761] A. Belenguer, H. Esteban, E. Diaz, C. Bachiller, J. Cascon, and V. E. Boria, "Hybrid technique plus fast frequency sweep for the efficient and accurate analysis of substrate integrated waveguide devices," IEEE Transactions on Microwave Theory and Techniques, vol. 59, no. 3, pp. 552-560, 2011.

[762] E. D. Caballero, H. Esteban, Á. Belenguer, and V. Boria, "Efficient analysis of substrate integrated waveguide devices using hybrid mode matching between cylindrical and guided modes," IEEE Transactions on Microwave Theory and Techniques, vol. 60, no. 2, pp. 232-243, 2012.

[763] Z. Kordiboroujeni, J. Bornemann, and T. Sieverding, "Mode-matching design of substrate-integrated waveguide couplers," in 2012 Asia-Pacific Symposium on Electromagnetic Compatibility. IEEE, 2012, pp. 701-704.

[764] L. Yan, W. Hong, G. Hua, J. Chen, K. Wu, and T. J. Cui, "Simulation and experiment on SIW slot array antennas," IEEE Microwave and Wireless Components Letters, vol. 14, no. 9, pp. 446-448, 2004.

[765] B. S. Izquierdo, P. Young, N. Grigoropoulos, J. Batchelor, and R. Langley, "Substrate-integrated folded waveguide slot antenna," Proc. IEEE Small Antennas Novel Meta Mater, no. 9, pp. 307-309, 2005.

[766] M. Henry, C. Free, B. S. Izqueirdo, J. Batchelor, and P. Young, "Millimeter wave substrate integrated waveguide antennas: Design and fabrication analysis," IEEE Transactions on Advanced Packaging, vol. 32, no. 1, pp. 93-100, 2009 . 
[767] Z.-C. Hao, W. Hong, J.-X. Chen, X.-P. Chen, and K. Wu, "Compact superwide bandpass substrate integrated waveguide (siw) filters," IEEE Transactions on Microwave Theory and Techniques, vol. 53, no. 9, pp. 2968-2977, 2005 .

[768] Y. L. Zhang, W. Hong, K. Wu, J. X. Chen, and H. J. Tang, "Novel substrate integrated waveguide cavity filter with defected ground structure," IEEE Transactions on Microwave Theory and Techniques, vol. 53, no. 4, pp. 1280-1287, 2005 .

[769] W. Che, K. Deng, D. Wang, and Y. Chow, "Analytical equivalence between substrate-integrated waveguide and rectangular waveguide," IET microwaves, antennas $\&$ propagation, vol. 2, no. 1, pp. 35-41, 2008.

[770] L.-S. Wu, X.-L. Zhou, and W.-Y. Yin, "Evanescent-mode bandpass filters using folded and ridge substrate integrated waveguides (SIWs)," IEEE Microwave and Wireless Components Letters, vol. 19, no. 3, pp. 161-163, 2009.

[771] Y. D. Dong, T. Yang, and T. Itoh, "Substrate integrated waveguide loaded by complementary split-ring resonators and its applications to miniaturized waveguide filters," IEEE Transactions on Microwave Theory and Techniques, vol. 57, no. 9, pp. 2211-2223, 2009.

[772] X.-P. Chen and K. Wu, "Substrate integrated waveguide filters: Design techniques and structure innovations," IEEE Microwave Magazine, vol. 15, no. 6, pp. 121-133, 2014.

[773] J.-X. Chen, W. Hong, Z.-C. Hao, H. Li, and K. Wu, "Development of a low cost microwave mixer using a broad-band substrate integrated waveguide (SIW) coupler," IEEE microwave and wireless components letters, vol. 16, no. 2, pp. 84-86, 2006.

[774] Y. Cassivi and K. Wu, "Low cost microwave oscillator using substrate integrated waveguide cavity," IEEE Microwave and Wireless Components Letters, vol. 13 , no. 2 , pp. 48-50, 2003.

[775] D. Deslandes and K. Wu, "Analysis and design of current probe transition from grounded coplanar to substrate integrated rectangular waveguides," IEEE Transactions on Microwave Theory and Techniques, vol. 53, no. 8, pp. 24872494, 2005.

[776] D. Deslandes, "Design equations for tapered microstrip-to-substrate integrated waveguide transitions," in IEEE MTT-S International Microwave Symposium Digest. IEEE, 2010, pp. 704-707.

[777] E. D. Caballero, A. B. Martinez, H. E. Gonzalez, O. M. Belda, and V. B. Esbert, "A novel transition from microstrip to a substrate integrated waveguide with higher characteristic impedance," in IEEE MTT-S International Microwave Symposium Digest (IMS). IEEE, 2013, pp. 1-4. 
[778] D. F. Williams, "Damping of the resonant modes of a rectangular metal package [MMICs]," IEEE Transactions on Microwave Theory and Techniques, vol. 37 , no. 1, pp. 253-256, 1989.

[779] J. T. Gear, "Microwave absorbers manage military electronics RF interference," RF DESIGN, vol. 27, no. 8; SUPP, pp. 6-9, 2004.

[780] P. Dixon, "Cavity-resonance dampening," IEEE Microwave magazine, vol. 6, no. 2, p. 74, 2005.

[781] A. Algaba Brazález, Gap Waveguide Technology-Electromagnetic Packaging and Transitions. Chalmers University of Technology, 2015.

[782] D. G. Swanson, "Narrow-band microwave filter design," IEEE Microwave Magazine, vol. 8, no. 5, pp. 105-114, 2007.

[783] P.-S. Kildal, "Definition of artificially soft and hard surfaces for electromagnetic waves," Electronics Letters, vol. 24, pp. 168-170, 1988.

[784] —_ "Artificially soft and hard surfaces in electromagnetics," IEEE Transactions on Antennas and Propagation, vol. 38, no. 10, pp. 1537-1544, 1990.

[785] E. Alfonso, P.-S. Kildal, A. Valero, and J. I. Herranz, "Study of local quasiTEM waves in oversized waveguides with one hard wall for killing higher order global modes," in IEEE Antennas and Propagation Society International Symposium. IEEE, 2008, pp. 1-4.

[786] D. Sievenpiper, L. Zhang, R. F. Broas, N. G. Alexopolous, and E. Yablonovitch, "High-impedance electromagnetic surfaces with a forbidden frequency band," IEEE Transactions on Microwave Theory and Techniques, vol. 47, no. 11, pp. 2059-2074, 1999.

[787] H. Raza, J. Yang, P.-S. Kildal, and E. A. Alós, "Microstrip-Ridge Gap Waveguide-Study of Losses, Bends, and Transition to WR-15," IEEE Transactions on Microwave Theory and Techniques, vol. 62, no. 9, pp. 1943-1952, 2014.

[788] E. Pucci, E. Rajo-Iglesias, and P.-S. Kildal, "New microstrip gap waveguide on mushroom-type EBG for packaging of microwave components," IEEE Microwave and Wireless Components Letters, vol. 22, no. 3, pp. 129-131, 2012.

[789] E. Alfonso, P.-S. Kildal, A. Valero-Nogueira, and J. Herranz, "Numerical analysis of a metamaterial-based ridge gap waveguide with a bed of nails as parallelplate mode killer," in 3rd European Conference on Antennas and Propagation (EuCAP), 2009, pp. 23-27.

[790] P.-S. Kildal, A. U. Zaman, E. Rajo-Iglesias, E. Alfonso, and A. ValeroNogueira, "Design and experimental verification of ridge gap waveguide in bed of nails for parallel-plate mode suppression," IET Microwaves, Antennas \& Propagation, vol. 5, no. 3, pp. 262-270, 2011. 
[791] G. F. Engen and C. A. Hoer, "Thru-reflect-line: An improved technique for calibrating the dual six-port automatic network analyzer," IEEE Transactions on Microwave Theory and Techniques, vol. 27, no. 12, pp. 987-993, 1979.

[792] E. Rajo-Iglesias and P.-S. Kildal, "Numerical studies of bandwidth of parallelplate cut-off realised by a bed of nails, corrugations and mushroom-type electromagnetic bandgap for use in gap waveguides," IET Microwaves, Antennas 6 Propagation, vol. 5, no. 3, pp. 282-289, 2011.

[793] E. Rajo-Iglesias, M. Caiazzo, L. Inclan-Sanchez, and P.-S. Kildal, "Comparison of bandgaps of mushroom-type EBG surface and corrugated and strip-type soft surfaces," IET microwaves, antennas $\&$ propagation, vol. 1, no. 1, pp. 184-189, 2007.

[794] E. Rajo-Iglesias and P.-S. Kildal, "Groove gap waveguide: A rectangular waveguide between contactless metal plates enabled by parallel-plate cut-off," in 4 th European Conference on Antennas and Propagation (EuCAP). IEEE, 2010, pp. 1-4.

[795] A. Valero-Nogueira, M. Baquero, J. I. Herranz, J. Domenech, E. Alfonso, and A. Vila, "Gap Waveguides Using a Suspended Strip on a Bed of Nails," IEEE Antennas and Wireless Propagation Letters, vol. 10, pp. 1006-1009, 2011.

[796] E. Pucci, A. U. Zaman, E. Rajo-Iglesias, and P.-S. Kildal, "New low loss inverted microstrip line using gap waveguide technology for slot antenna applications," in 5th European Conference on Antennas and Propagation (EUCAP). IEEE, 2011, pp. 979-982.

[797] D. Sánchez-Escuderos, M. Ferrando-Bataller, A. Berenguer, M. BaqueroEscudero, and A. Valero-Nogueira, "Dielectric Bed of Nails in Gap-Waveguide Technology at Millimeter-Wave Frequencies," IEEE Microwave and Wireless Components Letters, vol. 24, no. 8, pp. 515-517, 2014.

[798] E. Pucci, A. Zaman, E. Rajo-Iglesias, P.-S. Kildal, and A. Kishk, "Losses in ridge gap waveguide compared with rectangular waveguides and microstrip transmission lines," in 4th European Conference on Antennas and Propagation (EuCAP), 2010, pp. 1-4.

[799] E. Pucci, P.-S. Kildal, and E. Rajo-Iglesias, "Evaluation of losses in microstrip gap waveguide for slot antennas applications," in Antennas and Propagation Society International Symposium (APSURSI), 2012 IEEE, 2012, pp. 1-2.

[800] E. Pucci, A. Zaman, E. Rajo-Iglesias, P.-S. Kildal, and A. Kishk, "Study of Q-factors of ridge and groove gap waveguide resonators," IET Microwaves, Antennas Propagation, vol. 7, no. 11, pp. 900-908, 2013.

[801] A. U. Zaman, P.-S. Kildal, M. Ferndahl, and A. Kishk, "Validation of ridge gap waveguide performance using in-house TRL calibration kit," in 4th European Conference on Antennas and Propagation (EuCAP). IEEE, 2010, pp. 1-4. 
[802] H. Kirino and K. Ogawa, "A 76 GHz Multi-Layered Phased Array Antenna Using a Non-Metal Contact Metamaterial Waveguide," IEEE Transactions on Antennas and Propagation, vol. 60, no. 2, pp. 840-853, 2012.

[803] — - "A fast and slow wave combined-mode metamaterial ridged waveguide for array antenna applications," in 7th European Conference on Antennas and Propagation (EuCAP). IEEE, 2013, pp. 2878-2882.

[804] S. M. Giner, A. Valero-Nogueira, J. I. H. Herruzo, and M. B. Escudero, "Excitation of untilted narrow-wall slot in groove gap waveguide by using a parasitic dipole," in 7th European Conference on Antennas and Propagation (EuCAP). IEEE, 2013, pp. 3082-3085.

[805] J. I. H. Herruzo, A. Valero-Nogueira, S. M. Giner, and A. V. Jiménez, "Untilted Narrow-Wall Slots Excited by Parasitic Dipoles in Groove Gap Waveguide Technology," IEEE Transactions on Antennas and Propagation, vol. 63, no. 11, pp. 4759-4765, 2015.

[806] A. U. Zaman and P.-S. Kildal, "Slot antenna in ridge gap waveguide technology," in 6th European Conference on Antennas and Propagation (EUCAP). IEEE, 2012, pp. 3243-3244.

[807] — - "Ku band linear slot-array in ridge gapwaveguide technology," in 7th European Conference on Antennas and Propagation (EuCAP). IEEE, 2013, pp. 3078-3081.

[808] — , "Wide-band slot antenna arrays with single-layer corporate-feed network in ridge gap waveguide technology," IEEE Transactions on Antennas and Propagation, vol. 62, no. 6, pp. 2992-3001, 2014.

[809] Z. Talepour, S. Esmaili, and A. Khaleghi, "Ridge gap waveguide antenna array using integrated coaxial power divider," in Antennas \& Propagation Conference (LAPC), 2015 Loughborough. IEEE, 2015, pp. 1-5.

[810] A. Algaba-Brazalez and E. Rajo-Iglesias, "Design of a Butler matrix at $60 \mathrm{GHz}$ in inverted microstrip gap waveguide technology," in IEEE International Symposium on Antennas and Propagation \& USNC/URSI National Radio Science Meeting. IEEE, 2015, pp. 2125-2126.

[811] A. Sahu, V. Devabhaktuni, and P. H. Aaen, "A slot anntenna designed in ridge gap waveguide technology for V-band applications," in 2015 IEEE MTT$S$ International Microwave and RF Conference (IMaRC). IEEE, 2015, pp. 385-387.

[812] A. Vosoogh and P.-S. Kildal, "Corporate-fed planar $60 \mathrm{GHz}$ slot array made of three unconnected metal layers using AMC pin surface for the gap waveguide," IEEE Antennas and Wireless Propagation Letters, 2015.

[813] D. Zarifi, A. Farahbakhsh, A. Zaman, and P.-S. Kildal, "A high gain ridge gap waveguide fed slot antenna array for $60 \mathrm{GHz}$ applications," in 10th European Conference on Antennas and Propagation (EuCAP). IEEE, 2016, pp. 1-3. 
[814] A. U. Zaman and P.-S. Kildal, "Different gap waveguide slot array configurations for mmwave fixed beam antenna application," in 2016 10th European Conference on Antennas and Propagation (EuCAP). IEEE, 2016, pp. 1-4.

[815] S. A. Razavi, P.-S. Kildal, L. Xiang, E. A. Alós, and H. Chen, "2x2-Slot Element for $60 \mathrm{GHz}$ Planar Array Antenna Realized on Two Doubled-Sided PCBs Using SIW Cavity and EBG-Type Soft Surface fed by Microstrip-Ridge Gap Waveguide," IEEE Transactions on Antennas and Propagation, vol. 62, no. 9, pp. 4564-4573, 2014.

[816] B. Cao, H. Wang, Y. Huang, and J. Zheng, "High-gain L-probe excited substrate integrated cavity antenna array with LTCC-based gap waveguide feeding network for W-band application," IEEE Transactions on Antennas and Propagation, vol. 63, no. 12, pp. 5465-5474, 2015.

[817] B. Cao, H. Wang, and Y. Huang, "W-Band High-Gain TE-Mode Slot Antenna Array With Gap Waveguide Feeding Network," IEEE Antennas and Wireless Propagation Letters, vol. 15, pp. 988-991, 2016.

[818] M. Ferrando-Rocher, A. Valero-Nogueira, J. I. Herranz-Herruzo, A. Berenguer, and B. Bernardo-Clemente, "Groove Gap Waveguides: A contactless solution for multilayer slotted-waveguide array antenna assembly," in 10th European Conference on Antennas and Propagation (EuCAP). IEEE, 2016, pp. 1-4.

[819] A. J. Sáez, A. Valero-Nogueira, J. I. Herranz, and V. M. Rodrigo, "Ring resonances in groove gap waveguides with application to slot array antennas," in IEEE International Symposium on Antennas and Propagation \& USNC/URSI National Radio Science Meeting. IEEE, 2015, pp. 260-261.

[820] A. J. Sáez, A. Valero-Nogueira, J. I. Herranz, and B. Bernardo, "Single-Layer Cavity-Backed Slot Array Fed by Groove Gap Waveguide," IEEE Antennas and Wireless Propagation Letters, vol. 15, pp. 1402-1405, 2016.

[821] E. Pucci, E. Rajo-Iglesias, J.-L. Vazquez-Roy, and P.-S. Kildal, "Planar dualmode horn array with corporate-feed network in inverted microstrip gap waveguide," IEEE Transactions on Antennas and Propagation, vol. 62, no. 7, pp. 3534-3542, 2014.

[822] A. U. Zaman and P.-S. Kildal, "A new $2 \times 2$ microstrip patch sub-array for 60 $\mathrm{GHz}$ wideband planar antenna with ridge gap waveguide distribution layer," in 9th European Conference on Antennas and Propagation (EuCAP). IEEE, 2015, pp. 1-4.

[823] M. Vukomanovic, J.-L. Vazquez-Roy, O. Quevedo-Teruel, E. Rajo-Iglesias, and Z. Sipus, "Gap Waveguide Leaky-Wave Antenna," IEEE Transactions on Antennas and Propagation, vol. 64, no. 5, pp. 2055-2060, 2016.

[824] M. Al Sharkawy, A. Foroozesh, A. A. Kishk, and R. Paknys, "A robust horn ridge gap waveguide launcher for metal strip grating leaky wave antenna," IEEE Transactions on Antennas and Propagation, vol. 62, no. 12, pp. 60196026, 2014. 
[825] F. C. Suarez, D. N. Mendez, and M. Baquero-Escudero, "Rotman lens with Ridge Gap Waveguide technology for millimeter wave applications," in 7th European Conference on Antennas and Propagation (EuCAP), 2013.

[826] L. F. C. Suárez, D. V. N. Méndez, M. Baquero-Escudero, B. BernardoClemente, and S. M. Giner, "Transitions between Gap Waveguides for use in a phased array antenna fed by a Rotman lens," in 8th European Conference on Antennas and Propagation (EuCAP 2014). IEEE, 2014, pp. 774-777.

[827] S. Park, Y. Tsunemitsu, J. Hirokawa, and M. Ando, "Center feed single layer slotted waveguide array," IEEE Transactions on Antennas and Propagation, vol. 54, no. 5, pp. 1474-1480, 2006.

[828] M. Ando, Y. Tsunemitsu, M. Zhang, J. Hirokawa, and S. Fujii, "Reduction of long line effects in single-layer slotted waveguide arrays with an embedded partially corporate feed," IEEE Transactions on Antennas and Propagation, vol. 58, no. 7, pp. 2275-2280, 2010.

[829] H. Kirino and K. Ogawa, "A 76GHz Dielectric Loaded Slot Array Antenna fed by a Ridge Waveguide using Waffle-iron Structure," in Proceedings of the IEICE General Conference, 2009.

[830] —, "A Ridge Waveguide Phase Shifter using Waffle-iron Structure for a 76GHz Slot Array," in Proceedings of the IEICE General Conference, 2009.

[831] A. Valero-Nogueira, J. I. Herranz-Herruzo, M. Baquero, R. Hernández-Murcia, and V. Rodrigo, "Practical derivation of slot equivalent admittance in periodic waveguides," IEEE Transactions on Antennas and Propagation, vol. 61, no. 4, pp. 2321-2324, 2013.

[832] X.-J. Tang, S.-q. Xiao, B.-Z. Wang, and J. Wang, "A 60-GHz wideband slot antenna based on substrate integrated waveguide cavity," International Journal of Infrared and Millimeter Waves, vol. 28, no. 4, pp. 275-281, 2007.

[833] Y. Imanaka, Multilayered low temperature cofired ceramics (LTCC) technology. Springer Science \& Business Media, 2005.

[834] A. E. Lamminen, J. Saily, and A. R. Vimpari, "60-GHz patch antennas and arrays on LTCC with embedded-cavity substrates," IEEE Transactions on Antennas and Propagation, vol. 56, no. 9, pp. 2865-2874, 2008.

[835] A. U. Zaman, P.-S. Kildal, and A. A. Kishk, "Narrow-band microwave filter using high-Q groove gap waveguide resonators with manufacturing flexibility and no sidewalls," IEEE Transactions on Components, Packaging and Manufacturing Technology, vol. 2, no. 11, pp. 1882-1889, 2012.

[836] E. Alfonso, A. Zaman, and P. Kildal, "Ka-Band Gap Waveguide CoupledResonator Filter for Radio Link Diplexer Application," IEEE Transactions on Components, Packaging and Manufacturing Technology, vol. 3, no. 5, pp. 870-879, 2013. 
[837] F. Fan, Z. Yan, J. Wang, and X. Song, "Ka band-pass filter based on the microstrip-groove gap waveguide technology," in IET International Radar Conference. IET, 2015, pp. 1-4.

[838] M. Rezaee, A. U. Zaman, and P.-S. Kildal, "A groove gap waveguide iris filter for V-band application," in 23rd Iranian Conference on Electrical Engineering. IEEE, 2015, pp. 462-465.

[839] — - "V-band groove gap waveguide diplexer," in 9th European Conference on Antennas and Propagation (EuCAP). IEEE, 2015, pp. 1-4.

[840] A. del Olmo-Olmeda, M. Baquero-Escudero, V. E. Boria-Esbert, A. ValeroNogueira, and A. Berenguer, "A Novel Band-Pass Filter Topology For Millimeter-Wave Applications Based On The Groove Gap Waveguide," in International Microwave Symposium. IEEE, 2013, pp. 1-4.

[841] A. Berenguer, M. Baquero-Escudero, D. Sanchez-Escuderos, B. BernardoClemente, and V. E. Boria-Esbert, "Low insertion loss $61 \mathrm{GHz}$ narrow-band filter implemented with Groove Gap Waveguides," in IEEE 45th European Microwave Conference (EuMC), 2014, pp. 1-4.

[842] A. Berenguer, D. Sánchez-Escuderos, B. Bernardo-Clemente, M. BaqueroEscudero, and V. Boria, "Groove gap waveguide as an alternative to rectangular waveguide for H-plane components," Electronics Letters, vol. 52, no. 11, pp. 939-941, 2016.

[843] A. Berenguer, M. Baquero-Escudero, D. Sanchez-Escuderos, B. BernardoClemente, and V. E. Boria-Esbert, "Response Correction of a V-band Narrowband Filter Using Tuning Metal Insertions and Aggressive Space Mapping," in IEEE 46th European Microwave Conference (EuMC), 2015, pp. 1-4.

[844] A. Berenguer, M. Baquero-Escudero, D. Sanchez-Escuderos, V. E. Boria, and F. Vico, "A procedure to correct the response of manufactured Groove Gap Waveguide components," in 2016 10th European Conference on Antennas and Propagation (EuCAP). IEEE, 2016, pp. 1-5.

[845] B. Ahmadi and A. Banai, "Direct Coupled Resonator Filters Realized by Gap Waveguide Technology," IEEE Transactions on Microwave Theory and Techniques, vol. 63, no. 10, pp. 3445-3452, 2015.

[846] M. S. Sorkherizi, A. Khaleghi, and P.-S. Kildal, "Direct-coupled cavity filter in ridge gap waveguide," IEEE Transactions on Components, Packaging and Manufacturing Technology, vol. 4, no. 3, pp. 490-495, 2014.

[847] C. S. Cabello and E. Rajo-Iglesias, "Optimized self-diplexed antenna in gap waveguide technology," in IEEE International Symposium on Antennas and Propagation \& USNC/URSI National Radio Science Meeting. IEEE, 2015, pp. $460-461$. 
[848] A. Berenguer, M. Baquero-Escudero, D. Sanchez-Escuderos, and F. Vico, "Suspended-strip gap waveguide coupled-line properties for Ka-band component design," in 9th European Conference on Antennas and Propagation (EUCAP), 2015, pp. 1-5.

[849] A. Vosoogh, A. A. Brazález, and P.-S. Kildal, "A V-Band Inverted Microstrip Gap Waveguide End-Coupled Bandpass Filter," IEEE Microwave and Wireless Components Letters, vol. 26, no. 4, pp. 261-263, 2016.

[850] J. W. Bandler, R. M. Biernacki, S. H. Chen, P. A. Grobelny, and R. H. Hemmers, "Space mapping technique for electromagnetic optimization," IEEE Transactions on Microwave Theory and Techniques, vol. 42, no. 12, pp. 25362544, 1994.

[851] J. W. Bandler, Q. S. Cheng, S. A. Dakroury, A. S. Mohamed, M. H. Bakr, K. Madsen, and J. Sondergaard, "Space mapping: the state of the art," IEEE Transactions on Microwave theory and techniques, vol. 52, no. 1, pp. 337-361, 2004.

[852] A. García-Lampérez, S. Llorente-Romano, M. Salazar-Palma, and T. K. Sarkar, "Efficient electromagnetic optimization of microwave filters and multiplexers using rational models," IEEE transactions on microwave theory and techniques, vol. 52, no. 2, pp. 508-521, 2004.

[853] S. T. Choi, K. S. Yang, K. Tokuda, and Y. H. Kim, "A V-band planar narrow bandpass filter using a new type integrated waveguide transition," IEEE Microwave and Wireless Components Letters, vol. 14, no. 12, pp. 545-547, 2004.

[854] E. Pucci and P.-S. Kildal, "Contactless non-leaking waveguide flange realized by bed of nails for millimeter wave applications," in 6th European Conference on Antennas and Propagation (EUCAP). IEEE, 2012, pp. 3533-3536.

[855] A. U. Zaman, E. Rajo-Iglesias, E. Alfonso, and P.-S. Kildal, "Design of transition from coaxial line to ridge gap waveguide," in 2009 IEEE Antennas and Propagation Society International Symposium. IEEE, 2009, pp. 1-4.

[856] A. A. Brazález, A. U. Zaman, and P.-S. Kildal, "Design of a coplanar waveguide-to-ridge gap waveguide transition via capacitive coupling," in 6th European Conference on Antennas and Propagation (EUCAP). IEEE, 2012, pp. 3524-3528.

[857] A. Algaba Brazález, A. U. Zaman, and P.-S. Kildal, "Investigation of a Microstrip-to-Ridge Gap Waveguide transition by electromagnetic coupling," in IEEE Antennas and Propagation Society, AP-S International Symposium (Digest), 2012, pp. 1-2.

[858] A. U. Zaman, T. Vukusic, M. Alexanderson, and P.-S. Kildal, "Design of a simple transition from microstrip to ridge gap waveguide suited for MMIC and antenna integration," IEEE Antennas and Wireless Propagation Letters, vol. 12, pp. 1558-1561, 2013. 
[859] C. Gahete Arias, M. Baquero Escudero, A. Valero Nogueira, and A. Vila Jimenez, "Test-Fixture for Suspended-Strip Gap-Waveguide Technology on Ka-Band," IEEE Microwave and Wireless Components Letters, vol. 23, no. 6, pp. 321-323, 2013.

[860] A. A. Brazález, E. Rajo-Iglesias, J. L. Vázquez-Roy, A. Vosoogh, and P.-S. Kildal, "Design and validation of microstrip gap waveguides and their transitions to rectangular waveguide, for millimeter-wave applications," IEEE Transactions on Microwave Theory and Techniques, vol. 63, no. 12, pp. 4035-4050, 2015 .

[861] A. A. Brazález, E. Rajo-Iglesias, and P.-S. Kildal, "Design of millimeter-wave wideband gap waveguide transitions considering integration into the antenna system," in 9th European Conference on Antennas and Propagation (EuCAP). IEEE, 2015, pp. 1-5.

[862] A. A. Brazález, J. Flygare, J. Yang, V. Vassilev, M. Baquero-Escudero, and P.S. Kildal, "Design of-Band Transition From Microstrip to Ridge Gap Waveguide Including Monte Carlo Assembly Tolerance Analysis," IEEE Transactions on Microwave Theory and Techniques, vol. 64, no. 4, pp. 1245-1254, 2016.

[863] L. F. Carrera-Suárez, D. V. Navarro-Méndez, M. Baquero-Escudero, S. Martinez, and A. Valero-Nogueira, "A novel twist between Gap Waveguides for compact slot-array antennas," in IEEE Antennas and Propagation Society International Symposium (APSURSI), 2014.

[864] B. Molaei and A. Khaleghi, "A novel wideband microstrip line to ridge gap waveguide transition using defected ground slot," IEEE Microwave and Wireless Components Letters, vol. 25, no. 2, pp. 91-93, 2015.

[865] W. Rotman and R. Turner, "Wide-angle microwave lens for line source applications," IEEE Transactions on Antennas and Propagation, vol. 11, no. 6, pp. 623-632, 1963.

[866] T. Katagi, S. Mano, and S.-I. Sato, "An improved design method of rotman lens antennas," IEEE Transactions on Antennas and Propagation, vol. 32, no. 5 , pp. 524-527, 1984.

[867] A. A. Brazález, E. R. Iglesias, and P.-S. Kildal, "Investigation of transitions for use in inverted microstrip gap waveguide antenna arrays," in 8th European Conference on Antennas and Propagation (EuCAP 2014). IEEE, 2014, pp. 995-999.

[868] N. Metropolis and S. Ulam, "The monte carlo method," Journal of the American statistical association, vol. 44, no. 247, pp. 335-341, 1949.

[869] S. Rahiminejad, A. U. Zaman, E. Pucci, H. Raza, V. Vassilev, S. Haasl, P. Lundgren, P.-S. Kildal, and P. Enoksson, "Micromachined ridge gap waveguide and resonator for millimeter-wave applications," Sensors and Actuators A: Physical, vol. 186, pp. 264-269, 2012. 
[870] S. Rahiminejad, H. Raza, A. Zaman, S. Haasl, P. Enoksson, and P.-S. Kildal, "Micromachined gap waveguides for $100 \mathrm{GHz}$ applications," in 7th European Conference on Antennas and Propagation (EuCAP), 2013, pp. 1935-1938.

[871] C. A. Buendia, F. Gallée, A. Valero-Nogueira, and C. Person, "Gap waveguide structure in LTCC for millimeter-wave applications," in 8th European Conference on Antennas and Propagation (EuCAP 2014). IEEE, 2014, pp. 982-986.

[872] C. Arenas-Buendia, F. Gall, A. Valero-Nogueira, C. Person et al., "LTCC technology for microfluidic applications based on the gap waveguide technology," in European Microelectronics Packaging Conference (EMPC). IEEE, 2015, pp. $1-5$.

[873] C. Arenas-Buendia, F. Gallée, A. Valero-Nogueira, and C. Person, "RF sensor based on gap waveguide technology in LTCC for liquid sensing," in 9th European Conference on Antennas and Propagation (EuCAP). IEEE, 2015, pp. 1-4.

[874] A. Saleem, S. Rahiminejad, V. Desmaris, and P. Enoksson, "Carbon nanotubes as base material for fabrication of gap waveguide components," Sensors and Actuators A: Physical, vol. 224, pp. 163-168, 2015.

[875] S. Rahiminejad, A. Zaman, S. Haasl, P. Kildal, and P. Enoksson, "Demonstration of a micromachined planar distribution network in gap waveguide technology for a linear slot array antenna at $100 \mathrm{GHz}$," Journal of Micromechanics and Microengineering, vol. 26, no. 7, p. 074001, 2016.

[876] G. M. Rebeiz, RF MEMS: theory, design, and technology. John Wiley \& Sons, 2004.

[877] S. Iijima et al., "Helical microtubules of graphitic carbon," nature, vol. 354, no. 6348 , pp. 56-58, 1991.

[878] A. D. McNaught, A. Wilkinson et al., Compendium of chemical terminology. IUPAC recommendations. Oxford (United Kingdom) Blackwell, 1997.

[879] S. Rahiminejad, E. Pucci, S. Haasl, and P. Enoksson, "SU8 ridge-gap waveguide resonator," International Journal of Microwave and Wireless Technologies, vol. 6, no. 05, pp. 459-465, 2014.

[880] M. G. Silveirinha, C. A. Fernandes, and J. R. Costa, "Electromagnetic characterization of textured surfaces formed by metallic pins," IEEE Transactions on Antennas and Propagation, vol. 56, no. 2, pp. 405-415, 2008.

[881] M. Bosiljevac, Z. Sipus, and P.-S. Kildal, "Efficient spectral domain green's function analysis of novel metamaterial bandgap guiding structures," in 4th European Conference on Antennas and Propagation. IEEE, 2010, pp. 1-4. 
[882] A. Polemi and S. Maci, "Closed form expressions for the modal dispersion equations and for the characteristic impedance of a metamaterial-based gap waveguide," IET Microwaves, Antennas 6 Propagation, vol. 4, no. 8, pp. 1073$1080,2010$.

[883] M. Bosiljevac, Z. Sipus, and P.-S. Kildal, "Construction of green's functions of parallel plates with periodic texture with application to gap waveguides - a plane-wave spectral-domain approach," IET Microwaves, Antennas Propagation, vol. 4, no. 11, pp. 1799-1810, 2010.

[884] M. Bosiljevac and Z. Sipus, "Controlling the path of electromagnetic waves inside parallel plate waveguides using periodic surfaces," in ELMAR, 2011 Proceedings. IEEE, 2011, pp. 337-340.

[885] M. Bosiljevac, Z. Sipus, and P.-S. Kildal, "Analytical modeling and experimental verification of coupling between transmission lines in gap-waveguides," in Proceedings of 6th European Conference on Antennas and Propagation, EuCAP 2012. Prague, 26-30 March 2012, 2012, pp. 1402-1406.

[886] Z. Sipus, M. Bosiljevac, and P.-S. Kildal, "Evaluation of cross-coupling inside gap-waveguides," in 7th European Conference on Antennas and Propagation (EuCAP), 2013, pp. 3090-3091.

[887] Z. Sipus and M. Bosiljevac, "Simplifying the design of complex electromagnetic structures using green's functions based on asymptotic boundary conditions," in The 8th European Conference on Antennas and Propagation (EuCAP 2014). IEEE, 2014, pp. 750-753.

[888] M. Vukomanović, M. Bosiljevac, and Z. Šipuš, "Efficient analysis of gapwaveguide structures through a rigorous mode-matching approach," in Proceedings ELMAR-2014. IEEE, 2014, pp. 1-4.

[889] E. Alfonso, P.-S. Kildal, A. Valero-Nogueira, and M. Baquero, "Study of the characteristic impedance of a ridge gap waveguide," in IEEE Antennas and Propagation Society International Symposium, 2009. APSURSI '09., 2009, pp. $1-4$.

[890] H. Raza, J. Yang, P.-S. Kildal, and E. Alfonso, "Resemblance between gap waveguides and hollow waveguides," IET Microwaves, Antennas Propagation, pp. 1-7, 2013.

[891] H. Raza, J. Yang, and P. Kildal, "Study of the characteristic impedance of gap waveguide microstrip line realized with square metal pins," in 7th European Conference on Antennas and Propagation (EuCAP), 2013, pp. 3001-3005.

[892] A. Berenguer, M. Baquero-Escudero, D. Sanchez-Escuderos, and F. Vico, "Rigorous Method for Calculating Gap Waveguides Impedance Using Transmission Line Theory," in 8th European Conference on Antennas and Propagation (EUCAP), 2014, pp. 1-5. 
[893] — - "Reduction of the impedance dependence on the suspended-strip gap waveguide," in USNC/URSI National Science Meeting. IEEE, 2014, p. 1.

[894] A. Berenguer, M. Baquero-Escudero, D. Sánchez-Escuderos, T. BernabeuJiménez, and F. Vico, "Design of coupled-line components with the SuspendedStrip Gap Waveguide at mm-wave frequencies," in USNC/URSI National Science Meeting. IEEE, 2015, p. 1.

[895] R. E. Collin, Foundations for microwave engineering. John Wiley \& Sons, 2007.

[896] O. Luukkonen, C. Simovski, G. Granet, G. Goussetis, D. Lioubtchenko, A. V. Raisanen, and S. A. Tretyakov, "Simple and accurate analytical model of planar grids and high-impedance surfaces comprising metal strips or patches," IEEE Transactions on Antennas and Propagation, vol. 56, no. 6, pp. 1624$1632,2008$.

[897] A. Polemi, S. Maci, and P.-S. Kildal, "Dispersion characteristics of metamaterial-based parallel plate ridge waveguides," 3rd European Conference on Antennas and Propagation (EuCAP), 2009.

[898] — - "Dispersion characteristics of a metamaterial-based parallel-plate ridge gap waveguide realized by bed of nails," IEEE Transactions on Antennas and Propagation, vol. 59, no. 3, pp. 904-913, 2011.

[899] M. Bosiljevac, A. Polemi, S. Maci, and Z. Sipus, "Analytic approach to the analysis of ridge and groove gap waveguides-comparison of two methods," in 5th European Conference on Antennas and Propagation (EUCAP). IEEE, 2011, pp. 1886-1889.

[900] K. Gupta, R. Garg, I. Bahl, and P. Bhartia, "Microstrip Lines and Slotlines," Arthech House, 1996.

[901] T.-S. Chen, "Calculation of the parameters of ridge waveguides," IRE Transactions on Microwave Theory and Techniques, vol. 5, no. 1, pp. 12-17, 1957.

[902] J. Pyle, "The cutoff wavelength of the TE 10 mode in ridged rectangular waveguide of any aspect ratio," IEEE Transactions on Microwave Theory and Techniques, vol. 14, no. 4, pp. 175-183, 1966.

[903] M. McKay and J. Helszajn, "Voltage-current definition of impedance of singleridge waveguide," IEEE Microwave and Guided Wave Letters, vol. 9, no. 2, pp. 66-68, 1999.

[904] J. Liu, A. U. Zaman, and P.-S. Kildal, "Optimizing the numerical port for inverted microstrip gap waveguide in full-wave simulators," in 10th European Conference on Antennas and Propagation (EuCAP). IEEE, 2016, pp. 1-5.

[905] R. Tomar and P. Bhartia, "New quasi-static models for the computer-aided design of suspended and inverted microstrip lines (short paper)," IEEE Transactions on Microwave Theory and Techniques, vol. 35, no. 4, pp. 453-457, 1987. 
[906] E. Rajo-Iglesias, A. U. Zaman, and P.-S. Kildal, "Parallel plate cavity mode suppression in microstrip circuit packages using a lid of nails," IEEE Microwave and Wireless Components Letters, vol. 20, no. 1, pp. 31-33, 2010.

[907] A. A. Brazález, A. U. Zaman, E. Pucci, E. Rajo-Iglesias, P.-S. Kildal, and A. Kishk, "Improving microstrip filters with gap waveguide packaging," in 5th European Conference on Antennas and Propagation (EUCAP). IEEE, 2011, pp. 1080-1084.

[908] H. Raza and J. Yang, "Compact UWB power divider packaged by using gapwaveguide technology," in 6th European Conference on Antennas and Propagation (EUCAP). IEEE, 2012, pp. 2938-2942.

[909] A. Kishk, A. U. Zaman, and P.-S. Kildal, "Numerical prepackaging with PMC lid-efficient and simple design procedure for microstrip circuits including the packaging," Applied Computational Electromagnetics Society Journal, vol. 27, no. 5, pp. 389-398, 2012.

[910] A. A. Brazalez, A. U. Zaman, and P.-S. Kildal, "Improved microstrip filters using PMC packaging by lid of nails," IEEE Transactions on Components, Packaging and Manufacturing Technology, vol. 2, no. 7, pp. 1075-1084, 2012.

[911] A. U. Zaman, M. Alexanderson, T. Vukusic, and P.-S. Kildal, "Gap waveguide PMC packaging for improved isolation of circuit components in highfrequency microwave modules," IEEE Transactions on Components, Packaging and Manufacturing Technology, vol. 4, no. 1, pp. 16-25, 2014.

[912] J. Gao and G. Lu, "Improved gap waveguide PMC packaging for zeroth-order resonator," in 2015 IEEE 6th International Symposium on Microwave, Antenna, Propagation, and EMC Technologies (MAPE). IEEE, 2015, pp. 429431.

[913] J. Zhang, X. Zhang, D. Shen, and K. Wu, "Gap Waveguide PMC Packaging for a SIW-GCPW-Based Filter," IEEE Microwave and Wireless Components Letters, vol. 26, no. 3, pp. 159-161, 2016.

[914] J. Zhang, X. Zhang, D. Shen, T. Liu, and K. Wu, "Gap Waveguide-Based PMC Packaging for via Holes-Caused Nonsmooth PEC Surface," IEEE Transactions on Components, Packaging and Manufacturing Technology, vol. 5, no. 12, pp. $1828-1838,2015$.

[915] A. Sanada, M. Kimura, I. Awai, C. Caloz, and T. Itoh, "A planar zeroth-order resonator antenna using a left-handed transmission line," in 34th European Microwave Conference, vol. 3. IEEE, 2004, pp. 1341-1344.

[916] L. N. Trefethen and D. Bau III, Numerical linear algebra. Siam, 1997, vol. 50.

[917] M. Kirschning and R. H. Jansen, "Accurate wide-range design equations for the frequency-dependent characteristic of parallel coupled microstrip lines," IEEE Transactions on Microwave Theory and Techniques, vol. 32, no. 1, pp. 83-90, 1984. 
[918] X.-P. Chen, K. Wu, and D. Drolet, "Substrate integrated waveguide filter with improved stopband performance for satellite ground terminal," IEEE Transactions on Microwave Theory and Techniques, vol. 57, no. 3, pp. 674$683,2009$.

[919] X. Chen and K. Wu, "Self-equalised pseudo-elliptical filter made of substrate integrated waveguide," Electronics Letters, vol. 45, no. 2, p. 112, 2009.

[920] X.-P. Chen and K. Wu, "Self-packaged millimeter-wave substrate integrated waveguide filter with asymmetric frequency response," IEEE Transactions on Components, Packaging and Manufacturing Technology, vol. 2, no. 5, pp. 775$782,2012$.

[921] V. Egorov, V. Masalov, Y. A. Nefyodov, A. Shevchun, and M. Trunin, "Measuring microwave properties of laminated dielectric substrates," Review of scientific instruments, vol. 75, no. 11, pp. 4423-4433, 2004.

[922] G. F. Craven and C. Mok, "The design of evanescent mode waveguide bandpass filters for a prescribed insertion loss characteristic," IEEE Transactions on Microwave Theory and Techniques, vol. 19, no. 3, pp. 295-308, 1971.

[923] P. Ludlow and V. Fusco, "Reconfigurable small-aperture evanescent waveguide antenna," IEEE Transactions on Antennas and Propagation, vol. 59, no. 12, pp. 4815-4819, 2011.

[924] P. Ludlow, V. Fusco, G. Goussetis, and D. E. Zelenchuk, “Applying band-pass filter techniques to the design of small-aperture evanescent-mode waveguide antennas," IEEE Transactions on Antennas and Propagation, vol. 61, no. 1, pp. 134-142, 2013.

[925] H. Auda and R. F. Harrington, "Inductive posts and diaphragms of arbitrary shape and number in a rectangular waveguide," IEEE Transactions on Microwave Theory and Techniques, vol. 32, no. 6, pp. 606-613, 1984.

[926] R. M. Foster, "A reactance theorem," Bell System Technical Journal, vol. 3, no. 2, pp. 259-267, 1924.

[927] S. Hrabar, I. Krois, I. Bonic, and A. Kiricenko, "Basic concepts of active dispersionless metamaterial based on non-foster elements," in ICECom Conference Proceedings. IEEE, 2010, pp. 1-4.

[928] E. Ugarte-Munoz, S. Hrabar, D. Segovia-Vargas, and A. Kiricenko, "Stability of non-foster reactive elements for use in active metamaterials and antennas," IEEE Transactions on Antennas and Propagation, vol. 60, no. 7, pp. 34903494, 2012.

[929] S. Saadat, M. Adnan, H. Mosallaei, and E. Afshari, "Composite metamaterial and metasurface integrated with non-foster active circuit elements: A bandwidth-enhancement investigation," IEEE Transactions on Antennas and Propagation, vol. 61, no. 3, pp. 1210-1218, 2013. 
[930] M. Dishal, "Alignment and adjustment of synchronously tuned multipleresonant-circuit filters," Proceedings of the IRE, vol. 39, no. 11, pp. 1448-1455, 1951.

[931] K. V. Puglia, "A General Design Procedure for Bandpass Filters Derived from Lowpass Prototype Elements: Part I," Microwave Journal -Euroglobal Edition, vol. 43, no. 12 , pp. 22-38, 2000.

[932] K. Puglia, "A General Design Procedure for Bandpass Filters Derived from Lowpass Prototype Elements: Part II," Microwave Journal -Euroglobal Edition-, vol. 44, no. 1, pp. 114-137, 2001.

[933] J.-S. G. Hong and M. J. Lancaster, Microstrip filters for RF/microwave applications. John Wiley \& Sons, 2004, vol. 167.

[934] Y. Matthaei, "Jones, Microwave Filters, Impedance-Matching Networks and Coupling Structures, Artech House," ISBN 0-89006-099-1, Tech. Rep., 1980.

[935] D. H. Wolpert and W. G. Macready, "No free lunch theorems for optimization," IEEE Transactions on Evolutionary Computation, vol. 1, no. 1, pp. 67-82, 1997.

[936] R. Fletcher, Practical methods of optimization. John Wiley \& Sons, 2013.

[937] D. P. Bertsekas, Nonlinear programming. Athena scientific Belmont, 1999.

[938] A. Gottvald, K. Preis, C. Magele, O. Biro, and A. Savini, "Global optimization methods for computational electromagnetics," IEEE Transactions on Magnetics, vol. 28, no. 2, pp. 1537-1540, 1992.

[939] D. Budimir, Generalized filter design by computer optimization. Artech House, Inc., 1998.

[940] D. Swanson and G. Macchiarella, "Microwave filter design by synthesis and optimization," IEEE Microwave Magazine, vol. 8, no. 2, pp. 55-69, 2007.

[941] R. Fletcher and C. M. Reeves, "Function minimization by conjugate gradients," The computer journal, vol. 7, no. 2, pp. 149-154, 1964.

[942] E. Polak and G. Ribiere, "Note sur la convergence de méthodes de directions conjuguées," Revue française d'informatique et de recherche opérationnelle, série rouge, vol. 3, no. 1, pp. 35-43, 1969.

[943] C. G. Broyden, "Quasi-Newton methods and their application to function minimization," Mathematics of Computation, vol. 21, no. 99, pp. 368-381, 1967.

[944] D. F. Shanno, "Conditioning of quasi-newton methods for function minimization," Mathematics of Computation, vol. 24, no. 111, pp. 647-656, 1970.

[945] J. A. Nelder and R. Mead, "A simplex method for function minimization," The Computer Journal, vol. 7, no. 4, pp. 308-313, 1965. 
[946] J. Dréo, A. Pétrowski, P. Siarry, and E. Taillard, Metaheuristics for hard optimization: methods and case studies. Springer Science \& Business Media, 2006.

[947] T. Weise, "Global optimization algorithms-theory and application," Selfpublished, vol. 2, 2009.

[948] T. A. Cusick, S. Iezekiel, and R. E. Miles, "All-optical microwave filter design employing a genetic algorithm," IEEE Photonics Technology Letters, vol. 10, no. 8, pp. 1156-1158, 1998.

[949] Y. Rahmat-Samii and E. Michielssen, "Electromagnetic optimization by genetic algorithms," Microwave Journal, vol. 42, no. 11, pp. 232-232, 1999.

[950] S. Chahravarty and R. Mittra, "Design of microwave filters using a binary coded genetic algorithm," in Antennas and Propagation Society International Symposium, vol. 1. IEEE, 2000, pp. 144-147.

[951] L. Matekovits, M. Mussetta, P. Pirinoli, S. Selleri, and R. Zich, "Particle swarm optimization of microwave microstrip filters," in Antennas and Propagation Society International Symposium, vol. 3. IEEE, 2004, pp. 2731-2734.

[952] W. Wang, Y. Lu, J. S. Fu, and Y. Z. Xiong, "Particle swarm optimization and finite-element based approach for microwave filter design," IEEE Transactions on Magnetics, vol. 41, no. 5, pp. 1800-1803, 2005.

[953] F. Viani, F. Robol, M. Salucci, and R. Azaro, "Automatic EMI Filter Design Through Particle Swarm Optimization," IEEE Transactions on Electromagnetic Compatibility, 2017.

[954] R. A. Vural, T. Yildirim, T. Kadioglu, and A. Basargan, "Performance evaluation of evolutionary algorithms for optimal filter design," IEEE Transactions on Evolutionary Computation, vol. 16, no. 1, pp. 135-147, 2012.

[955] J. W. Bandler, R. M. Biernacki, S. H. Chen, R. H. Hemmers, and K. Madsen, "Electromagnetic optimization exploiting aggressive space mapping," IEEE Transactions on Microwave Theory and Techniques, vol. 43, no. 12, pp. 28742882, 1995.

[956] M. H. Bakr, J. W. Bandler, K. Madsen, J. E. Rayas-Sanchez, and J. Sondergaard, "Space-mapping optimization of microwave circuits exploiting surrogate models," IEEE Transactions on Microwave Theory and Techniques, vol. 48, no. 12, pp. 2297-2306, 2000.

[957] P. Burrascano, M. Dionigi, C. Fancelli, and M. Mongiardo, "A neural network model for CAD and optimization of microwave filters," in IEEE MTT-S International Microwave Symposium Digest, vol. 1. IEEE, 1998, pp. 13-16.

[958] H. Kabir, Y. Wang, M. Yu, and Q.-J. Zhang, "Neural network inverse modeling and applications to microwave filter design," IEEE Transactions on Microwave Theory and Techniques, vol. 56, no. 4, pp. 867-879, 2008. 
[959] — - "High-dimensional neural-network technique and applications to microwave filter modeling," IEEE Transactions on Microwave Theory and Techniques, vol. 58, no. 1, pp. 145-156, 2010.

[960] A. Software and Testing, "FEST3D," 2016, www.fest3d.com.

[961] H.-C. Chen and C.-K. Tzuang, "All-planar dual-mode asymmetric filters at ka-band," IEEE microwave and wireless components letters, vol. 13, no. 3, pp. 111-113, 2003.

[962] A. S. M. Marzuki, A. Naemat, S. Selamat, A. T. M. A. Tee, K. Khalil, and Y. C. Lee, "Design and fabrication of $40 \mathrm{GHz}$ hairpin filter using Printed Circuit Board," in RF and Microwave Conference (RFM), 2013 IEEE International. IEEE, 2013, pp. 156-160.

[963] H. J. Tang, W. Hong, J.-X. Chen, G. Q. Luo, and K. Wu, "Development of millimeter-wave planar diplexers based on complementary characters of dualmode substrate integrated waveguide filters with circular and elliptic cavities," IEEE Transactions on Microwave Theory and Techniques, vol. 55, no. 4, pp. 776-782, 2007.

[964] K. Ahn and I. Yom, "A Ka-band multilayer LTCC 4-pole bandpass filter using dual-mode cavity resonators," in Microwave Symposium Digest, 2008 IEEE MTT-S International. IEEE, 2008, pp. 1235-1238.

[965] PASTERNACK ${ }^{\circledR}$. (2016). [Online]. Available: http://www.pasternack.com

[966] AINFO Inc. (2016). [Online]. Available: http://www.ainfoinc.com

[967] RTx Technology Co., Ltd. (2016). [Online]. Available: http://www.rtxtech. com

[968] B. Yang, E. Skafidas, and R. Evans, "60 GHz compact integrated cross-coupled SIR-MH bandpass filter on bulk CMOS," Electronics Letters, vol. 44, no. 12, pp. 738-740, 2008.

[969] S. Glisic and C. Scheytt, "Integrated compact microstrip filters for $60 \mathrm{ghz}$ applications," in 38th European Microwave Conference (EuMC). IEEE, 2008, pp. $1386-1389$.

[970] K. Nishikawa, T. Seki, I. Toyoda, and S. Kubota, "Compact 60-GHz LTCC stripline parallel-coupled bandpass filter with parasitic elements for millimeterwave system-on-package," in IEEE/MTT-S International Microwave Symposium. IEEE, 2007, pp. 1649-1652.

[971] J. Nocedal and S. Wright, Numerical optimization. Springer Science \& Business Media, 2006.

[972] S. Amari, "Synthesis of cross-coupled resonator filters using an analytical gradient-based optimization technique," IEEE Transactions on Microwave Theory and Techniques, vol. 48, no. 9, pp. 1559-1564, 2000. 
[973] H. Chu, Y. Guo, and X. Shi, "60 GHz LTCC 3D cavity bandpass filter with two finite transmission zeros," Electronics Letters, vol. 47, no. 5, pp. 324-326, 2011.

[974] K. Ha, C. Lee, J. Kim, R. Krishnan, and S.-G. Oh, "Design and development of low-cost and high-efficiency variable-speed drive system with switched reluctance motor," IEEE Transactions on Industry Applications, vol. 43, no. 3, pp. 703-713, 2007.

[975] J.-H. Lee, S. Pinel, J. Papapolymerou, J. Laskar, and M. M. Tentzeris, "Lowloss LTCC cavity filters using system-on-package technology at $60 \mathrm{GHz}$," IEEE Transactions on Microwave Theory and Techniques, vol. 53, no. 12, pp. 38173824, 2005.

[976] S. H. Yun, M. S. Uhm, and I. B. Yom, "Design of the multipaction free high power Ka-band diplexer with an E-plane T-junction," in 2005 Asia-Pacific Conference on Communications. IEEE, 2005, pp. 582-585.

[977] X. Yu, X. Tang, J. Wang, D. Tang, and X. He, "A kind of coaxial resonator structure with low multipactor risk," Progress In Electromagnetics Research Letters, vol. 39, pp. 127-132, 2013.

[978] M. Brumos Vicente, "Design of microwave filters and multiplexers in waveguide technology using distributed models," Ph.D. dissertation, Universidad Politécnica de Valencia, 2015.

[979] C. G. Broyden, "A class of methods for solving nonlinear simultaneous equations," Mathematics of computation, vol. 19, no. 92, pp. 577-593, 1965.

[980] J. Morro, H. Esteban, V. Boria, C. Bachiller, S. Cogollos, and A. Coves, "Efficient automated design of $\mathrm{H}$ plane filters with rounded corners using ASM with a segmentation strategy and hybrid optimization techniques," in Antennas and Propagation Society International Symposium, vol. 3. IEEE, 2004, pp. 2735-2738.

[981] S. Cogollos, V. Boria, P. Soto, B. Gimeno, and M. Guglielmi, "Efficient cad tool for inductively coupled rectangular waveguide filters with rounded corners," in 31st European Microwave Conference (EuMC). IEEE, 2001, pp. 1-4.

[982] G. Zhang, J. Wang, J. Pan, and H. Gu, "Compact 60 GHz LTCC balun bandpass filter with two transmission zeroes," Electronics Letters, vol. 51, no. 8, pp. 637-638, 2015.

[983] G.-H. Lee, C.-S. Yoo, J.-G. Yook, and J.-C. Kim, "SIW (substrate integrated waveguide) quasi-elliptic filter based on LTCC for $60-\mathrm{GHz}$ application," in European Microwave Integrated Circuits Conference (EuMIC). IEEE, 2009, pp. 204-207.

[984] M. Ito, K. Maruhashi, K. Ikuina, T. Hashiguchi, S. Iwanaga, and K. Ohata, "A 60-GHz-band planar dielectric waveguide filter for flip-chip modules," IEEE Transactions on Microwave Theory and Techniques, vol. 49, no. 12, pp. 24312436, 2001. 
[985] Y. Wang, M. Ke, M. J. Lancaster et al., "Micromachined $60 \mathrm{GHz}$ air-filled interdigital bandpass filter," ESA. University of Greenwich, 2009.

[986] D. S. Jun, H. Y. Lee, D.-Y. Kim, S. S. Lee, and E. S. Nam, "A narrow bandwidth microstrip band-pass filter with symmetrical frequency characteristics," ETRI Journal, vol. 27, no. 5, pp. 643-646, 2005. 